Cochrane Database of Systematic Reviews

\title{
Exercise for preventing falls in older people living in the community
} (Review)

Sherrington C, Fairhall NJ, Wallbank GK, Tiedemann A, Michaleff ZA, Howard K, Clemson L, Hopewell S, Lamb SE

Sherrington C, Fairhall NJ, Wallbank GK, Tiedemann A, Michaleff ZA, Howard K, Clemson L, Hopewell S, Lamb SE. Exercise for preventing falls in older people living in the community.

Cochrane Database of Systematic Reviews 2019, Issue 1. Art. No.: CD012424.

DOI: 10.1002/14651858.CD012424.pub2.

www.cochranelibrary.com 
TABLE OF CONTENTS

ABSTRACT

PLAIN LANGUAGE SUMMARY

SUMMARY OF FINDINGS

BACKGROUND

OBJECTIVES

METHODS

RESULTS

Figure 1.

Figure 2.

Figure 3.

Figure 4.

Figure 5.

Figure 6.

Figure 7.

Figure 8.

Figure 9.

DISCUSSION

AUTHORS' CONCLUSIONS

ACKNOWLEDGEMENTS

REFERENCES

CHARACTERISTICS OF STUDIES

DATA AND ANALYSES

Analysis 1.1. Comparison 1 Exercise versus control (rate of falls), Outcome 1 Rate of falls - overall analysis.

Analysis 1.2. Comparison 1 Exercise versus control (rate of falls), Outcome 2 Rate of falls - subgrouped by baseline falls risk. ...

Analysis 1.3. Comparison 1 Exercise versus control (rate of falls), Outcome 3 Rate of falls - subgrouped by age (threshold 75 years).

Analysis 1.4. Comparison 1 Exercise versus control (rate of falls), Outcome 4 Rate of falls - subgrouped by personnel. .............

Analysis 1.5. Comparison 1 Exercise versus control (rate of falls), Outcome 5 Rate of falls - subgrouped by group or individual exercise.

Analysis 1.6. Comparison 1 Exercise versus control (rate of falls), Outcome 6 Rate of falls - subgrouped by exercise type. ........ Analysis 1.7. Comparison 1 Exercise versus control (rate of falls), Outcome 7 Rate of falls - long-term follow-up by exercise type. Analysis 2.1. Comparison 2 Exercise versus control (number of fallers), Outcome 1 Number of fallers - overall analysis. ........... Analysis 2.2. Comparison 2 Exercise versus control (number of fallers), Outcome 2 Number of fallers - subgrouped by baseline fall risk.

Analysis 2.3. Comparison 2 Exercise versus control (number of fallers), Outcome 3 Number of fallers - subgrouped by age (threshold 75 years).

Analysis 2.4. Comparison 2 Exercise versus control (number of fallers), Outcome 4 Number of fallers - subgrouped by personnel.

Analysis 2.5. Comparison 2 Exercise versus control (number of fallers), Outcome 5 Number of fallers - subgrouped by group or individual exercise.

Analysis 2.6. Comparison 2 Exercise versus control (number of fallers), Outcome 6 Number of fallers - subgrouped by exercise type.

Analysis 2.7. Comparison 2 Exercise versus control (number of fallers), Outcome 7 Number of fallers - long-term follow-up by exercise type.

Analysis 3.1. Comparison 3 Exercise versus control (number of people with fractures), Outcome 1 Number of people who experienced one or more fall-related fractures- overall analysis.

Analysis 3.2. Comparison 3 Exercise versus control (number of people with fractures), Outcome 2 Number of people who experienced one or more fall-related fractures - subgrouped by baseline falls risk.

Analysis 3.3. Comparison 3 Exercise versus control (number of people with fractures), Outcome 3 Number of people who experienced one or more fall-related fractures - subgrouped by age (threshold 75 years).

Analysis 3.4. Comparison 3 Exercise versus control (number of people with fractures), Outcome 4 Number of people who experienced one or more fall-related fractures - subgrouped by exercise type. 
Analysis 3.5. Comparison 3 Exercise versus control (number of people with fractures), Outcome 5 Number of people who experienced one or more fall-related fractures - long-term follow-up by exercise type.

Analysis 4.1. Comparison 4 Exercise versus control (number of people with falls that resulted in hospital admission), Outcome 1 Number of people who experienced one or more falls that resulted in hospital admission - overall analysis.

Analysis 5.1. Comparison 5 Exercise versus control (number of people with falls that required medical attention), Outcome 1 Number of people who experienced one or more falls that required medical attention- overall analysis.

Analysis 5.2. Comparison 5 Exercise versus control (number of people with falls that required medical attention), Outcome 2 Number of people who experienced one or more falls that required medical attention - subgrouped by exercise type.

Analysis 5.3. Comparison 5 Exercise versus control (number of people with falls that required medical attention), Outcome 3 Number of people who experienced one or more falls that required medical attention - long-term follow-up pooled.

Analysis 6.1. Comparison 6 Exercise versus control (health-related quality of life), Outcome 1 Health-related quality of lifeoverall analysis.

Analysis 6.2. Comparison 6 Exercise versus control (health-related quality of life), Outcome 2 Health-related quality of life subgrouped by baseline fall risk.

Analysis 7.1. Comparison 7 Exercise versus control (number of people who died), Outcome 1 Number of people who diedoverall analysis.

Analysis 7.2. Comparison 7 Exercise versus control (number of people who died), Outcome 2 Number of people who died subgrouped by baseline fall risk.

Analysis 8.1. Comparison 8 Balance and functional exercises versus control: subgroup analyses, Outcome 1 Rate of falls, subgrouped by baseline fall risk.

Analysis 8.2. Comparison 8 Balance and functional exercises versus control: subgroup analyses, Outcome 2 Number of fallers, subgrouped by baseline fall risk.

Analysis 8.3. Comparison 8 Balance and functional exercises versus control: subgroup analyses, Outcome 3 Rate of falls, subgrouped by personnel.

Analysis 8.4. Comparison 8 Balance and functional exercises versus control: subgroup analyses, Outcome 4 Number of fallers, subgrouped by personnel.

Analysis 8.5. Comparison 8 Balance and functional exercises versus control: subgroup analyses, Outcome 5 Rate of falls, subgrouped by group or individual exercise.

Analysis 8.6. Comparison 8 Balance and functional exercises versus control: subgroup analyses, Outcome 6 Number of fallers, subgrouped by group or individual exercise.

Analysis 9.1. Comparison 9 Multiple categories of exercise versus control: subgroup analyses, Outcome 1 Rate of falls, subgrouped by baseline fall risk.

Analysis 9.2. Comparison 9 Multiple categories of exercise versus control: subgroup analyses, Outcome 2 Number of fallers, subgrouped by baseline fall risk.

Analysis 9.3. Comparison 9 Multiple categories of exercise versus control: subgroup analyses, Outcome 3 Rate of falls, subgrouped by personnel.

Analysis 9.4. Comparison 9 Multiple categories of exercise versus control: subgroup analyses, Outcome 4 Number of fallers, subgrouped by personnel.

Analysis 9.5. Comparison 9 Multiple categories of exercise versus control: subgroup analyses, Outcome 5 Rate of falls, subgrouped by group or individual exercise.

Analysis 9.6. Comparison 9 Multiple categories of exercise versus control: subgroup analyses, Outcome 6 Number of fallers, subgrouped by group or individual exercise.

Analysis 10.1. Comparison 10 Exercise versus control (by exercise type, in people after hospital stays), Outcome 1 Rate of falls. .

Analysis 10.2. Comparison 10 Exercise versus control (by exercise type, in people after hospital stays), Outcome 2 Number of fallers.

Analysis 10.3. Comparison 10 Exercise versus control (by exercise type, in people after hospital stays), Outcome 3 Health-related quality of life.

Analysis 10.4. Comparison 10 Exercise versus control (by exercise type, in people after hospital stays), Outcome 4 Number of people who died.

Analysis 11.1. Comparison 11 Exercise versus exercise, Outcome 1 Rate of falls, different types of exercise compared.

Analysis 11.2. Comparison 11 Exercise versus exercise, Outcome 2 Rate of falls $>18$ months, different types of exercise compared.

Analysis 11.3. Comparison 11 Exercise versus exercise, Outcome 3 Number of fallers, different types of exercise compared. ..... Analysis 11.4. Comparison 11 Exercise versus exercise, Outcome 4 Number of people who experienced one or more fall-related fractures, different types of exercise compared. 
Analysis 11.5. Comparison 11 Exercise versus exercise, Outcome 5 Number of people who experienced one or more falls that required medical attention, different types of exercise compared.

Analysis 11.6. Comparison 11 Exercise versus exercise, Outcome 6 Quality of life, different types of exercise compared. ......... Analysis 11.7. Comparison 11 Exercise versus exercise, Outcome 7 Number of people who died, different types of exercise compared.

Analysis 11.8. Comparison 11 Exercise versus exercise, Outcome 8 Rate of falls, group vs individual exercise delivery within the same type of exercise.

Analysis 11.9. Comparison 11 Exercise versus exercise, Outcome 9 Number of fallers, group vs individual exercise delivery within the same type of exercise.

Analysis 11.10. Comparison 11 Exercise versus exercise, Outcome 10 Number of people who experienced one or more falls requiring hospital admission, group vs individual exercise delivery within the same type of exercise.

Analysis 11.11. Comparison 11 Exercise versus exercise, Outcome 11 Health-related quality of life, group vs individual exercise delivery within the same type of exercise.

Analysis 11.12. Comparison 11 Exercise versus exercise, Outcome 12 Number of people who died, group vs individual exercise delivery within the same type of exercise.

Analysis 11.13. Comparison 11 Exercise versus exercise, Outcome 13 Rate of falls, higher vs lower dose within the same type of exercise.

Analysis 11.14. Comparison 11 Exercise versus exercise, Outcome 14 Number of fallers, higher vs lower dose within the same type of exercise.

Analysis 11.15. Comparison 11 Exercise versus exercise, Outcome 15 Number of people who died, higher vs lower dose within the same type of exercise.

Analysis 12.1. Comparison 12 Sensitivity analysis 1: exercise versus control excluding studies that included people $<65$ years, Outcome 1 Rate of falls: pooled data.

Analysis 12.2. Comparison 12 Sensitivity analysis 1: exercise versus control excluding studies that included people $<65$ years, Outcome 2 Rate of falls: grouped by exercise type.

Analysis 12.3. Comparison 12 Sensitivity analysis 1: exercise versus control excluding studies that included people $<65$ years, Outcome 3 Number of fallers: pooled data.

Analysis 12.4. Comparison 12 Sensitivity analysis 1 : exercise versus control excluding studies that included people $<65$ years, Outcome 4 Number of fallers: grouped by exercise type.

Analysis 12.5. Comparison 12 Sensitivity analysis 1: exercise versus control excluding studies that included people $<65$ years, Outcome 5 Number of people who experienced one or more fall-related fractures: pooled data.

Analysis 12.6. Comparison 12 Sensitivity analysis 1: exercise versus control excluding studies that included people $<65$ years, Outcome 6 Number of people who experienced one or more fall-related fractures: by exercise type.

Analysis 12.7. Comparison 12 Sensitivity analysis 1 : exercise versus control excluding studies that included people $<65$ years, Outcome 7 Number of people who experienced one or more falls requiring medical attention: pooled data. ........................... Analysis 12.8. Comparison 12 Sensitivity analysis 1: exercise versus control excluding studies that included people $<65$ years, Outcome 8 Number of people who experienced one or more falls requiring medical attention - subgrouped by exercise type. .. Analysis 13.1. Comparison 13 Sensitivity analysis 2: exercise versus control excluding studies at a high risk of bias, Outcome 1 Rate of falls - overall analysis.

Analysis 13.2. Comparison 13 Sensitivity analysis 2: exercise versus control excluding studies at a high risk of bias, Outcome 2 Rate of falls - subgrouped by exercise type.

Analysis 13.3. Comparison 13 Sensitivity analysis 2: exercise versus control excluding studies at a high risk of bias, Outcome 3 Number of fallers - overall analysis.

Analysis 13.4. Comparison 13 Sensitivity analysis 2: exercise versus control excluding studies at a high risk of bias, Outcome 4 Number of fallers - subgrouped by exercise type.

Analysis 13.5. Comparison 13 Sensitivity analysis 2: exercise versus control excluding studies at a high risk of bias, Outcome 5 Number of people who experienced one or more fall-related fractures - overall analysis.

Analysis 14.1. Comparison 14 Sensitivity analysis 3: exercise versus control excluding studies with unclear or high risk of bias due to allocation concealment (rate of falls), Outcome 1 Rate of falls - overall analysis.

Analysis 15.1. Comparison 15 Sensitivity analysis 4: exercise versus control excluding studies with unclear or high risk of bias due to assessor blinding (rate of falls), Outcome 1 Rate of falls - overall analysis.

Analysis 16.1. Comparison 16 Sensitivity analysis 5: exercise versus control excluding studies with unclear or high risk of bias due to incomplete outcome data (rate of falls), Outcome 1 Rate of falls - overall analysis.

Analysis 17.1. Comparison 17 Sensitivity analysis 6: exercise versus control excluding cluster trials (rate of falls), Outcome 1 Rate of falls - overall analysis. 
Analysis 18.1. Comparison 18 Sensitivity analysis 7: exercise versus control with fixed-effect meta-analysis (rate of falls), Outcome 1 Rate of falls - overall analysis.

Analysis 19.1. Comparison 19 Sensitivity analysis 8: multiple categories of exercise versus control excluding trials that do not include balance and strength training, Outcome 1 Rate of falls.

Analysis 19.2. Comparison 19 Sensitivity analysis 8: multiple categories of exercise versus control excluding trials that do not include balance and strength training, Outcome 2 Number of fallers.

Analysis 20.1. Comparison 20 Sensitivity analysis 9: different exercise type coding, Outcome 1 Rate of falls - subgrouped by exercise type (OEP as multiple intervention).

Analysis 20.2. Comparison 20 Sensitivity analysis 9: different exercise type coding, Outcome 2 Number of fallers - subgrouped by exercise type (OEP as multiple intervention).

Analysis 20.3. Comparison 20 Sensitivity analysis 9: different exercise type coding, Outcome 3 Rate of falls - subgrouped by exercise type (any balance+strength as multiple intervention).

Analysis 20.4. Comparison 20 Sensitivity analysis 9: different exercise type coding, Outcome 4 Number of fallers - subgrouped by exercise type (any balance+strength as multiple intervention).

ADDITIONAL TABLES

APPENDICES

CONTRIBUTIONS OF AUTHORS 
[Intervention Review]

\section{Exercise for preventing falls in older people living in the community}

Catherine Sherrington ${ }^{1}$, Nicola J Fairhall ${ }^{1}$, Geraldine K Wallbank ${ }^{1}$, Anne Tiedemann ${ }^{1}$, Zoe A Michaleff ${ }^{1}$, Kirsten Howard², Lindy Clemson 3 , Sally Hopewell4, Sarah E Lamb4

1Institute for Musculoskeletal Health, School of Public Health, Faculty of Medicine and Health, The University of Sydney, Sydney, Australia. ${ }^{2}$ School of Public Health, The University of Sydney, Sydney, Australia. ${ }^{3}$ Faculty of Health Sciences, The University of Sydney, Lidcombe, Australia. ${ }^{4}$ Nuffield Department of Orthopaedics, Rheumatology and Musculoskeletal Sciences (NDORMS), University of Oxford, Oxford, UK

Contact: Catherine Sherrington, Institute for Musculoskeletal Health, School of Public Health, Faculty of Medicine and Health, The University of Sydney, PO Box 179, Missenden Road, Sydney, NSW, 2050, Australia. cathie.sherrington@sydney.edu.au.

Editorial group: Cochrane Bone, Joint and Muscle Trauma Group.

Publication status and date: New, published in Issue 1, 2019.

Citation: Sherrington C, Fairhall NJ, Wallbank GK, Tiedemann A, Michaleff ZA, Howard K, Clemson L, Hopewell S, Lamb SE. Exercise for preventing falls in older people living in the community. Cochrane Database of Systematic Reviews 2019, Issue 1. Art. No.: CD012424. DOI: 10.1002/14651858.CD012424.pub2.

Copyright @ 2019 The Cochrane Collaboration. Published by John Wiley \& Sons, Ltd.

\section{A B S T R A C T}

\section{Background}

At least one-third of community-dwelling people over 65 years of age fall each year. Exercises that target balance, gait and muscle strength have been found to prevent falls in these people. An up-to-date synthesis of the evidence is important given the major long-term consequences associated with falls and fall-related injuries

\section{Objectives}

To assess the effects (benefits and harms) of exercise interventions for preventing falls in older people living in the community.

\section{Search methods}

We searched CENTRAL, MEDLINE, Embase, three other databases and two trial registers up to 2 May 2018, together with reference checking and contact with study authors to identify additional studies.

\section{Selection criteria}

We included randomised controlled trials (RCTs) evaluating the effects of any form of exercise as a single intervention on falls in people aged $60+$ years living in the community. We excluded trials focused on particular conditions, such as stroke.

\section{Data collection and analysis}

We used standard methodological procedures expected by Cochrane. Our primary outcome was rate of falls.

\section{Main results}

We included 108 RCTs with 23,407 participants living in the community in 25 countries. There were nine cluster-RCTs. On average, participants were 76 years old and $77 \%$ were women. Most trials had unclear or high risk of bias for one or more items. Results from four trials focusing on people who had been recently discharged from hospital and from comparisons of different exercises are not described here.

\section{Exercise (all types) versus control}


Eighty-one trials (19,684 participants) compared exercise (all types) with control intervention (one not thought to reduce falls). Exercise reduces the rate of falls by $23 \%$ (rate ratio $(\mathrm{RaR}) 0.77,95 \%$ confidence interval $(\mathrm{Cl}) 0.71$ to $0.83 ; 12,981$ participants, 59 studies; high-certainty evidence). Based on an illustrative risk of 850 falls in 1000 people followed over one year (data based on control group risk data from the 59 studies), this equates to 195 ( $95 \% \mathrm{Cl} 144$ to 246) fewer falls in the exercise group. Exercise also reduces the number of people experiencing one or more falls by $15 \%$ (risk ratio (RR) $0.85,95 \% \mathrm{Cl} 0.81$ to $0.89 ; 13,518$ participants, 63 studies; high-certainty evidence). Based on an illustrative risk of 480 fallers in 1000 people followed over one year (data based on control group risk data from the 63 studies), this equates to 72 (95\% Cl 52 to 91 ) fewer fallers in the exercise group. Subgroup analyses showed no evidence of a difference in effect on both falls outcomes according to whether trials selected participants at increased risk of falling or not.

The findings for other outcomes are less certain, reflecting in part the relatively low number of studies and participants. Exercise may reduce the number of people experiencing one or more fall-related fractures (RR 0.73, $95 \% \mathrm{Cl} 0.56$ to $0.95 ; 4047$ participants, 10 studies; low-certainty evidence) and the number of people experiencing one or more falls requiring medical attention (RR $0.61,95 \% \mathrm{Cl} 0.47$ to 0.79 ; 1019 participants, 5 studies; low-certainty evidence). The effect of exercise on the number of people who experience one or more falls requiring hospital admission is unclear (RR $0.78,95 \% \mathrm{Cl} 0.51$ to $1.18 ; 1705$ participants, 2 studies, very low-certainty evidence). Exercise may make little important difference to health-related quality of life: conversion of the pooled result (standardised mean difference (SMD) $-0.03,95 \% \mathrm{Cl}-0.10$ to $0.04 ; 3172$ participants, 15 studies; low-certainty evidence) to the EQ-5D and SF-36 scores showed the respective $95 \%$ Cls were much smaller than minimally important differences for both scales.

Adverse events were reported to some degree in 27 trials (6019 participants) but were monitored closely in both exercise and control groups in only one trial. Fourteen trials reported no adverse events. Aside from two serious adverse events (one pelvic stress fracture and one inguinal hernia surgery) reported in one trial, the remainder were non-serious adverse events, primarily of a musculoskeletal nature. There was a median of three events (range 1 to 26 ) in the exercise groups.

\section{Different exercise types versus control}

Different forms of exercise had different impacts on falls (test for subgroup differences, rate of falls: $P=0.004, I^{2}=71 \%$ ). Compared with control, balance and functional exercises reduce the rate of falls by $24 \%$ (RaR $0.76,95 \% \mathrm{Cl} 0.70$ to $0.81 ; 7920$ participants, 39 studies; highcertainty evidence) and the number of people experiencing one or more falls by $13 \%$ (RR $0.87,95 \% \mathrm{Cl} 0.82$ to $0.91 ; 8288$ participants, 37 studies; high-certainty evidence). Multiple types of exercise (most commonly balance and functional exercises plus resistance exercises) probably reduce the rate of falls by $34 \%$ (RaR $0.66,95 \% \mathrm{Cl} 0.50$ to $0.88 ; 1374$ participants, 11 studies; moderate-certainty evidence) and the number of people experiencing one or more falls by $22 \%$ (RR $0.78,95 \% \mathrm{Cl} 0.64$ to $0.96 ; 1623$ participants, 17 studies; moderate-certainty evidence). Tai Chi may reduce the rate of falls by $19 \%$ (RaR $0.81,95 \% \mathrm{Cl} 0.67$ to $0.99 ; 2655$ participants, 7 studies; low-certainty evidence) as well as reducing the number of people who experience falls by $20 \%$ (RR $0.80,95 \% \mathrm{Cl} 0.70$ to $0.91 ; 2677$ participants, 8 studies; highcertainty evidence). We are uncertain of the effects of programmes that are primarily resistance training, or dance or walking programmes on the rate of falls and the number of people who experience falls. No trials compared flexibility or endurance exercise versus control.

\section{Authors' conclusions}

Exercise programmes reduce the rate of falls and the number of people experiencing falls in older people living in the community (highcertainty evidence). The effects of such exercise programmes are uncertain for other non-falls outcomes. Where reported, adverse events were predominantly non-serious.

Exercise programmes that reduce falls primarily involve balance and functional exercises, while programmes that probably reduce falls include multiple exercise categories (typically balance and functional exercises plus resistance exercises). Tai Chi may also prevent falls but we are uncertain of the effect of resistance exercise (without balance and functional exercises), dance, or walking on the rate of falls.

\section{PLAIN LANGUAGE SUMMARY}

\section{Exercise for preventing falls in older people living in the community}

\section{Background}

At least one-third of community-dwelling people over 65 years of age fall each year. Exercises that target balance, gait and muscle strength have previously been found to prevent falls in these people.

\section{Review aim}

To assess the effects (benefits and harms) of exercise interventions for preventing falls in older people living in the community.

\section{Search date}

We searched the healthcare literature for reports of randomised controlled trials relevant to this review up to 2 May 2018. In such studies, people are allocated at random to receive one of two or more interventions being compared in the study. Leaving group allocation to chance helps ensure the participant populations are similar in the intervention groups. 


\section{Study characteristics}

This review includes 108 randomised controlled trials with 23,407 participants. These were carried out in 25 countries. On average, participants were 76 years old and $77 \%$ were women.

\section{Certainty of the evidence}

The majority of trials had unclear or high risk of bias, mainly reflecting lack of blinding of trial participants and personnel to the interventions. This could have influenced how the trial was conducted and outcome assessment. The certainty of the evidence for the overall effect of exercise on falls was high. Risk of fracture, hospitalisation, medical attention and adverse events were not well reported and, where reported, the evidence was low- to very low-certainty. This leads to uncertainty regarding drawing conclusions from the evidence for these outcomes.

\section{Key results}

Eighty-one trials compared exercise (all types) versus a control intervention that is not thought to reduce falls in people living in the community (who also had not recently been discharged from hospital). Exercise reduces the number of falls over time by around onequarter (23\% reduction). By way of an example, these data indicate that if there were 850 falls in 1000 people followed over one year, exercise would result in 195 fewer falls. Exercise also reduces the number of people experiencing one or more falls (number of fallers) by around one-sixth (15\%) compared with control. For example, if there were 480 fallers who fell in 1000 people followed over one year, exercise would result in 72 fewer fallers. The effects on falls were similar whether the trials selected people who were at an increased risk of falling or not.

We found exercise that mainly involved balance and functional training reduced falls compared with an inactive control group. Programmes involving multiple types of exercise (most commonly balance and functional exercises plus resistance exercises) probably reduced falls, and Tai Chi may also reduce falls. We did not find enough evidence to determine the effects of exercise programmes classified as being mainly resistance exercises, dance, or walking programmes. We found no evidence to determine the effects of programmes that were mainly flexibility or endurance exercise.

There was considerably less evidence for non-fall outcomes. Exercise may reduce the number of people experiencing fractures by over one-quarter $(27 \%)$ compared with control. However, more studies are needed to confirm this. Exercise may also reduce the risk of a fall requiring medical attention. We did not find enough evidence to determine the effects of exercise on the risk of a fall requiring hospital admission. Exercise may make very little difference to health-related quality of life. The evidence for adverse events related to exercise was also limited. Where reported, adverse events were usually non-serious events of a musculoskeletal nature; exceptionally one trial reported a pelvic stress fracture and a hernia. 
SUMMARY OF FINDINGS

Summary of findings for the main comparison. Summary of findings: exercise (all types) versus control (e.g. usual activities)

Exercise (all types) versus control (e.g. usual activities) for preventing falls in older people living in the community

Patient or population: Older people living in the community (trials focusing on people recently discharged from hospital were not included)

Settings: Community, either at home or in places of residence that, on the whole, do not provide residential health-related care

Intervention: Exercise of all types ${ }^{a}$

Comparison: Usual care (no change in usual activities) or a control (non-active) interventionb

\begin{tabular}{|c|c|c|c|c|c|c|}
\hline \multirow[t]{3}{*}{ Outcomes } & \multicolumn{2}{|c|}{$\begin{array}{l}\text { Illustrative comparative risks* } \\
(95 \% \mathrm{CI})\end{array}$} & \multirow[t]{3}{*}{$\begin{array}{l}\text { Relative effect } \\
(95 \% \mathrm{CI})\end{array}$} & \multirow{3}{*}{$\begin{array}{l}\text { No of partici- } \\
\text { pants } \\
\text { (studies) }\end{array}$} & \multirow{3}{*}{$\begin{array}{l}\text { Certainty of } \\
\text { the evidence } \\
\text { (GRADE) }\end{array}$} & \multirow[t]{3}{*}{ Comments } \\
\hline & Assumed risk & $\begin{array}{l}\text { Corresponding } \\
\text { risk }\end{array}$ & & & & \\
\hline & Control & $\begin{array}{l}\text { Exercise (all } \\
\text { types) }\end{array}$ & & & & \\
\hline \multirow{6}{*}{$\begin{array}{l}\text { Rate of falls } \\
\text { (falls per per- } \\
\text { son-years) } \\
\text { Follow-up: } \\
\text { range } 3 \text { to } 30 \\
\text { months }\end{array}$} & \multicolumn{2}{|c|}{ All studies population } & \multirow{6}{*}{$\begin{array}{l}\text { Rate ratio } 0.77 \\
(0.71 \text { to } 0.83)^{d}\end{array}$} & \multirow{6}{*}{$\begin{array}{l}12,981 \\
\text { (59 RCTs) }\end{array}$} & \multirow{6}{*}{$\begin{array}{l}\oplus \oplus \oplus \oplus \\
\text { highe }\end{array}$} & \multirow{6}{*}{$\begin{array}{l}\text { Overall, there is a reduction of } 23 \% \text { ( } 95 \% \mathrm{Cl} 17 \% \text { to } 29 \%) \\
\text { in the number of falls } \\
\text { Guide to the data: } \\
\text { If } 1000 \text { people were followed over } 1 \text { year, the number of } \\
\text { falls in the overall population would be } 655 \text { ( } 95 \% \mathrm{Cl} 604 \\
\text { to } 706 \text { ) compared with } 850 \text { in the group receiving usual } \\
\text { care or attention control. } \\
\text { In the unselected population, the corresponding data } \\
\text { are } 466 \text { ( } 95 \% \mathrm{Cl} 430 \text { to } 503 \text { ) compared with } 605 \text { in the } \\
\text { group receiving usual care or attention control. } \\
\text { In the selected higher-risk population, the correspond- } \\
\text { ing data are } 924 \text { ( } 95 \% \mathrm{Cl} 852 \text { to } 996) \text { compared with } 1200 \\
\text { in the control group }\end{array}$} \\
\hline & 850 per $1000 C$ & $\begin{array}{l}\mathbf{6 5 5} \text { per } \mathbf{1 0 0 0} \\
\text { (604 to } 706)\end{array}$ & & & & \\
\hline & \multicolumn{2}{|c|}{$\begin{array}{l}\text { Not selected for high risk popula- } \\
\text { tion }\end{array}$} & & & & \\
\hline & 605 per $1000^{C}$ & $\begin{array}{l}466 \text { per } 1000 \\
(430 \text { to } 503)\end{array}$ & & & & \\
\hline & \multicolumn{2}{|c|}{ Selected for high risk population } & & & & \\
\hline & 1200 per $1000 c$ & $\begin{array}{l}\mathbf{9 2 4} \text { per } \mathbf{1 0 0 0} \\
\text { (852 to } 996)\end{array}$ & & & & \\
\hline \multirow{2}{*}{$\begin{array}{l}\text { Number of peo- } \\
\text { ple who expe- } \\
\text { rienced one or } \\
\text { more falls }\end{array}$} & \multicolumn{2}{|c|}{ All studies population } & \multirow{2}{*}{$\begin{array}{l}\text { RR } 0.85 \\
(0.81 \text { to } 0.89) g\end{array}$} & \multirow{2}{*}{$\begin{array}{l}13,518 \\
\text { (63 RCTs) }\end{array}$} & \multirow{2}{*}{$\begin{array}{l}\oplus \oplus \oplus \oplus \\
\text { highe }\end{array}$} & \multirow{2}{*}{$\begin{array}{l}\text { Overall, there is a reduction of } 15 \%(95 \% \mathrm{Cl} 11 \% \text { to } 19 \%) \\
\text { in the number of people who experienced one or more } \\
\text { falls }\end{array}$} \\
\hline & 480 per $1000^{f}$ & $\begin{array}{l}\mathbf{4 0 8} \text { per } 1000 \\
(389 \text { to } 428)\end{array}$ & & & & \\
\hline
\end{tabular}


Follow-up: range 3 to 25

months

Not selected for high risk popula-

tion

380 per $1000^{f} \quad \begin{aligned} & 323 \text { per } 1000 \\ & \text { (308 to 339) }\end{aligned}$

Selected for high risk population

500 per $1000^{f} \quad 425$ per 1000

(405 to 445)

Number of peo-

ple who expe-

rienced one or

more fall-relat-

ed fractures

Follow-up:

range 4 to 42

months

\section{All studies population $h$}

64 per 1000

47 per

47 per 1000

(36 to 61 )
RR $0.73(0.56 \quad 4047$

to 0.95 )

(10 RCTs)

If 1000 people were followed over 1 year, the number of people who experienced one or more falls in the unselected population would be 408 ( $95 \% \mathrm{Cl} 389$ to 428 ) compared with 480 in the group receiving usual care or attention control.

In the unselected population, the corresponding data are $323(95 \% \mathrm{Cl} 308$ to 339$)$ compared with 380 in the group receiving usual care or attention control.

In the selected higher-risk population, the corresponding data are 425 (95\% Cl 405 to 445) compared with 500 in the control group.

Overall, there may be a reduction of $27 \%(95 \% \mathrm{Cl} 5 \%$ to $44 \%$ ) in the number of people who experienced one or more fall-related fractures

Guide to the data:

If 1000 people were followed over 1 year, the number of people who experienced one or more fall-related fractures may be 47 ( $95 \% \mathrm{Cl} 36$ to 61$)$ compared with 64 in the control group

Number of people who experienced one of more falls that resulted in hospital admission

All studies population ${ }^{h}$

57 per 1000

45 per 1000

(29 to 68)

Follow-up:

range 3 to 42

months

\section{RR $0.78(0.51 \quad 1705$}

to 1.18 )

(2 RCTs) $\oplus \ominus \ominus \ominus$

very lowj

The evidence is very low certainty, hence we are uncertain of the findings of a reduction of $22 \%(95 \% \mathrm{Cl} 49 \%$

reduction to $18 \%$ increase) in the number of people who experienced one or more falls that required hospital admission. Of note is that the $95 \% \mathrm{Cl}$ includes the possibility of both reduced and increased hospitalisation.

Guide to the data:

If 1000 people were followed over 1 year, the number of people who experience one or more falls that required hospital admission in the general risk population may be 45 ( $95 \% \mathrm{Cl} 30$ to 68 ) compared with 57 in the group receiving usual care or attention control

\begin{tabular}{|c|c|c|}
\hline \multirow{2}{*}{$\begin{array}{l}\text { Number of peo- } \\
\text { ple who expe- } \\
\text { rienced one or } \\
\text { more falls that } \\
\text { required med- } \\
\text { ical attention. }\end{array}$} & \multicolumn{2}{|c|}{ All studies populationh } \\
\hline & 211 per 1000 & $\begin{array}{l}\mathbf{1 2 9} \text { per } \mathbf{1 0 0 0} \\
(100 \text { to } 167)\end{array}$ \\
\hline
\end{tabular}

$\begin{array}{llll}\begin{array}{l}\text { RR 0.61 (0.47 } \\ \text { to 0.79) }\end{array} & 1019 & \begin{array}{l}\oplus \oplus \ominus \ominus \\ \text { lowk }\end{array} & \begin{array}{l}\text { Overall, there may be a reduction of } 39 \%(95 \% \mathrm{Cl} 21 \% \text { to } \\ 53 \%) \text { in the number of people who experienced one or } \\ \text { more falls that required medical attention }\end{array}\end{array}$

Guide to the data:

If 1000 people were followed over 1 year, the number of people who experienced one or more falls that required medical attention may be $129(95 \% \mathrm{Cl} 100$ to 167$)$ com- 


\begin{tabular}{|c|c|c|c|c|c|c|}
\hline $\begin{array}{l}\text { Health-related } \\
\text { quality of life } \\
\text { Follow-up: } \\
\text { range } 3 \text { to } 24 \\
\text { months } \\
\text { (A higher score } \\
\text { indicates better } \\
\text { quality of life) }\end{array}$ & - & $\begin{array}{l}\text { The mean } \\
\text { health-related } \\
\text { quality of life } \\
\text { score in the in- } \\
\text { tervention } \\
\text { groups was } \\
0.03 \text { standard } \\
\text { deviations low- } \\
\text { er } \\
\text { (0.10 lower to } \\
0.04 \text { higher) }\end{array}$ & - & $\begin{array}{l}3172 \\
\text { (15 RCTs) }\end{array}$ & $\begin{array}{l}\oplus \oplus \odot \odot \\
\text { lowl }\end{array}$ & $\begin{array}{l}\text { SMD was calculated from } 4 \text { trials with EQ-5D, } 5 \text { trials } \\
\text { with SF-36, } 3 \text { trials with SF } 12,1 \text { trial with QUALEFFO- } 41 \text {, } \\
1 \text { trial with WHOQOL-BREF, and } 1 \text { with Assessment of } \\
\text { QOL } \\
\text { EQ-5D: Mean difference }=-0.0026 \text { ( } 95 \% \mathrm{Cl}-0.0086 \text { to } \\
\text { 0.0034). SMD was converted back to MD using EQ-5D } \\
\text { scale ( } 0 \text { to } 1 \text { ), based on data for } 4 \text { trials ( } 6 \text { comparisons) } \\
\text { reporting endpoint scores. } \mathrm{m} \text { MID for the EQ-5D is typi- } \\
\text { cally } 0.074 \text { (Walters 2005) } \\
\text { SF36: Mean difference }=-0.36 \text { ( } 95 \% \mathrm{Cl}-1.20 \text { to } 0.48 \text { ). } \\
\text { SMD was converted back to MD using SF-36 scale, based } \\
\text { on data for } 5 \text { trials. } \mathrm{m} \text { MID for the SF-36 is typically } 3 \text { to } 5 \text { ( } \\
\text { Walters 2003) }\end{array}$ \\
\hline Adverse events & See comment & & Not estimable & $\begin{array}{l}6019 \\
\text { (27 RCTs) }\end{array}$ & $\begin{array}{l}\oplus \odot \odot \ominus^{n} \\
\text { very low }\end{array}$ & $\begin{array}{l}\text { Adverse events were reported to various degrees, but } \\
\text { predominantly in the intervention groups, in the } 27 \\
\text { RCTs, } 14 \text { of which reported no adverse events. Aside } \\
\text { from } 2 \text { serious adverse events ( } 1 \text { pelvic stress fracture } \\
\text { and } 1 \text { inguinal hernia surgery) reported in } 1 \text { trial, the rest } \\
\text { were non-serious adverse events, primarily of a muscu- } \\
\text { loskeletal nature. There was a median of } 3 \text { events (range } \\
1 \text { to } 26 \text { ) in the exercise groups }\end{array}$ \\
\hline
\end{tabular}

*The basis for the assumed risk (e.g. the median control group risk across studies) is provided in footnotes. The corresponding risk (and its $95 \%$ confidence interval) is based on the assumed risk in the comparison group and the relative effect of the intervention (and its $95 \% \mathrm{Cl}$ ).

CI: confidence interval; MID: minimally important difference; RR: risk ratio; SMD: standardised mean difference

\section{GRADE Working Group grades of evidence}

High certainty: We are very confident that the true effect lies close to that of the estimate of the effect

Moderate certainty: We are moderately confident in the effect estimate: The true effect is likely to be close to the estimate of the effect, but there is a possibility that it is substantially different

Low certainty: Our confidence in the effect estimate is limited: The true effect may be substantially different from the estimate of the effect

Very low certainty: We have very little confidence in the effect estimate: The true effect is likely to be substantially different from the estimate of effect

${ }^{a}$ Exercise is a physical activity that is planned, structured and repetitive and aims to improve or maintain physical fitness. There is a wide range of possible types of exercise, and exercise programmes often include one or more types of exercise. We categorised exercise based on the Prevention of Falls Network Europe (ProFaNE) taxonomy that classifies exercise type as: i) gait, balance, and functional [task] training; ii) strength/resistance (including power); iii) flexibility; iv) three-dimensional (3D) exercise (e.g. Tai Chi, Qigong, dance); v) general physical activity; vi) endurance; and vii) other kind of exercises. The taxonomy allows for more than one type of exercise to be delivered within a programme. 
bA control intervention is one that is not thought to reduce falls, such as general health education, social visits, very gentle exercise, or 'sham' exercise not expected to impact on falls.

CThe all-studies population risk was based on the number of events and the number of participants in the control group for this outcome over the 59 RCTs. We calculated the risk in the control group using the median falls per person-year for the subgroups of trials for which a) an increased risk of falls was not an inclusion criterion (29 RCTs, 6123 participants), or b) increased risk of falls was an inclusion criterion (30 RCTs, 6858 participants).

dSubgroup analysis found no difference based on whether risk of falls was an inclusion criterion or not (test for subgroup differences: $\mathrm{Chi} 2=0.90, \mathrm{df}=1, \mathrm{P}=0.34, \mathrm{I}=0 \%$ ).

eThere was no downgrading, including for risk of bias, as results were essentially unchanged with removal of the trials with a high risk of bias on one or more items.

fThe all-studies population risk was based on the number of events and the number of participants in the control group for this outcome over the 63 RCTs. We calculated the risk in the control group using the median proportion of fallers for the subgroups of trials for which a) an increased risk of falls was not an inclusion criterion ( 28 RCTs, 6347 participants), or b) increased risk of falls was an inclusion criterion (35 RCTs, 7171 participants).

gSubgroup analysis found no difference based on whether risk of falls was an inclusion criterion or not (test for subgroup differences: $\mathrm{Chi} 2=0.94, \mathrm{df}=1, \mathrm{P}=0.33, \mathrm{I}=0 \%$ ).

hWe calculated the risk in the control group based on the number of events and the number of participants in the control group for this outcome.

i Downgraded by two levels due to imprecision (few events and wide $\mathrm{Cl}$ due to small sample size), and risk of publication bias (likelihood of reporting fractures only if there was a treatment effect; with some indication on viewing the funnel plot).

jDowngraded by two levels due to imprecision (low event rate and wide confidence intervals) and because most of the 81 studies included in the review for this comparison do not contribute to the outcome. We further downgraded the evidence by one level for risk of bias because the evidence was dominated by one trial that was at high risk of bias in one or more items.

kDowngraded by two levels due to imprecision and the high probability of publication bias (only 5 of 89 RCTs included in the review reported the outcome). We did not downgrade for risk of bias as results were essentially unchanged with removal of the trials at a high risk of bias in one or more items.

IDowngraded by two levels due to inconsistency (there was considerable heterogeneity $\left(1^{2}=76 \%\right)$ ) and risk of bias (removing studies with high risk of bias in one or more items had a marked impact on results).

$\mathrm{m} / \mathrm{n}$ order to express the MD in the unit-specific measurement instruments (ED-5D and SF-36), we multiplied the SMD by a typical among-person standard deviation for that scale, using the pooled standard deviation of baseline scores in the largest study in the analysis. For EQ-5D, Iliffe 2015 has a combined SD of 0.086; for SF36, Dangour 2011 has combined SD of 12.04 .

nDowngraded by three levels due to limitations in design of studies, suggesting a very serious risk of bias and incomplete data. Only one trial measured the number of people experiencing adverse events in both groups throughout the trial period (Iliffe 2015).

\section{Summary of findings 2. Summary of findings: balance and functional exercises versus control (e.g. usual activities)}

Balance, and functional exercises versus control (e.g. usual activities) for preventing falls in older people in the community

Patient or population: Older people living in the community (trials focusing on people recently discharged from hospital were not included)

Settings: Community, either at home or in places of residence that, on the whole, do not provide residential health-related care

Intervention: Exercise, type = gait, balance, and functional (task) training $a$

Comparison: Usual care (no change in usual activities) or a control (non-active) interventionb

\begin{tabular}{|c|c|c|c|c|c|c|}
\hline \multirow[t]{2}{*}{ Outcomes } & \multicolumn{2}{|c|}{$\begin{array}{l}\text { Illustrative comparative risks* } \\
(95 \% \mathrm{Cl})\end{array}$} & \multirow[t]{2}{*}{$\begin{array}{l}\text { Relative effect } \\
(95 \% \mathrm{Cl})\end{array}$} & \multirow{2}{*}{$\begin{array}{l}\text { No of partici- } \\
\text { pants } \\
\text { (studies) }\end{array}$} & \multirow{2}{*}{$\begin{array}{l}\text { Certainty of } \\
\text { the evidence } \\
\text { (GRADE) }\end{array}$} & \multirow[t]{2}{*}{ Comments } \\
\hline & Assumed risk & $\begin{array}{l}\text { Corresponding } \\
\text { risk }\end{array}$ & & & & \\
\hline
\end{tabular}




\begin{tabular}{ll} 
Control & $\begin{array}{l}\text { Exercise (gait, } \\
\text { balance, and } \\
\text { functional } \\
\text { [task] training) }\end{array}$ \\
\hline
\end{tabular}

\section{Rate of falls}

(falls per per-

son-years) Fol-

low-up: range 3

to 30 months

\begin{tabular}{|c|c|}
\hline All studies pop & tion \\
\hline 850 per $1000^{C}$ & $\begin{array}{l}\mathbf{6 4 6} \text { per } \mathbf{1 0 0 0} \\
\text { (595 to } 689)\end{array}$ \\
\hline
\end{tabular}

\section{Rate ratio $0.76 \quad 7920$}

$(0.70$ to 0.81$)$

\section{0}

(39 RCTs)

Specific exercise population

930 per $1000 \mathrm{C} \quad 707$ per 1000 (651 to 754 )

Number of people who experienced one of more falls

Follow-up: range 3 to 24

months

\begin{tabular}{ll}
\multicolumn{2}{l|}{ All studies population } \\
\hline $\mathbf{4 8 0}$ per 1000e & $\begin{array}{l}\mathbf{4 1 8} \text { per } 1000 \\
\text { (394 to } 437)\end{array}$ \\
\hline Specific exercise population \\
\hline $\mathbf{5 4 9}$ per 1000 & $\begin{array}{l}\mathbf{4 7 8} \text { per } 1000 \\
\text { (451 to } 500)\end{array}$ \\
\hline
\end{tabular}

\section{RR 0.87 (0.82}

to 0.91 )

\section{(37 RCTs)}

$\oplus \oplus \oplus \oplus^{\mathrm{d}}$

high

$\oplus \oplus \oplus \oplus^{\mathrm{d}}$

high

Overall, there is a reduction of $24 \%(95 \% \mathrm{Cl} 19 \%$ to $30 \%)$ in the number of falls

Guide to the data based on the all-studies estimate.

If 1000 people were followed over 1 year, the number of falls would be 646 ( $95 \% \mathrm{Cl} 595$ to 689) compared with 850 in the group receiving usual care or attention control

verall, there is a reduction of $13 \%(95 \% \mathrm{Cl} 9 \%$ to $18 \%)$ in the number of people who experienced one or more falls.

Guide to the data based on the all-studies estimate.

If 1000 people were followed over 1 year, the number of people who experienced one or more falls would be 418 $(95 \% \mathrm{Cl} 394$ to 437$)$ compared with 480 in the group receiving usual care or attention control

\section{Number of peo-} ple who experienced one or more fall-related fractures.

All studies population

64 per $1000^{f}$

29 per 1000

Follow-up:

range 6 to 30 months

Adverse events

See comment

(16 to 49)

\section{RR 0.44 (0.25}

to 0.76 )

2139

(7 RCTs)

$\oplus \oplus \ominus \ominus \mathrm{g}$

low

Overall, there may be a reduction of $56 \%$ (95\% Cl 24\% to $75 \%$ ) in the number of people who experienced one or more fall-related fractures

Guide to the data.

If 1000 people were followed over 1 year, the number of people who experienced one or more fall-related fractures may be 29 ( $95 \% \mathrm{Cl} 16$ to 49) compared with 64 in the group receiving usual care or attention control

Adverse events were reported on in 15 of the 48 trials with gait, balance, and functional (task) training as the primary intervention in exercise versus control analyses in trials. Adverse events were reported for both intervention and control groups (11 trials) or just the intervention group (4 trials). 200 adverse events were reported; most were non-serious adverse events of a musculoskeletal nature; 173 were in a single study including 2 intervention groups. Other adverse events included 
*The basis for the assumed risk (e.g. the median control group risk across studies) is provided in footnotes. The corresponding risk (and its $95 \%$ confidence interval) is based on the assumed risk in the comparison group and the relative effect of the intervention (and its $95 \% \mathrm{Cl}$ ).

Cl: confidence interval; RR: risk ratio

\section{GRADE Working Group grades of evidence}

High certainty: We are very confident that the true effect lies close to that of the estimate of the effect

Moderate certainty: We are moderately confident in the effect estimate: The true effect is likely to be close to the estimate of the effect, but there is a possibility that it is substantially different

Low certainty: Our confidence in the effect estimate is limited: The true effect may be substantially different from the estimate of the effect

Very low certainty: We have very little confidence in the effect estimate: The true effect is likely to be substantially different from the estimate of effect

a Using Prevention of Falls Network Europe (ProFaNE) taxonomy, gait, balance, and functional [task] training is: gait training = specific correction of walking technique, and changes of pace, level and direction; balance training = transferring bodyweight from one part of the body to another or challenging specific aspects of the balance systems; functional training = functional activities, based on the concept of task specificity. Training is assessment-based, tailored and progressed. Exercise programs included in this analysis contained a single primary exercise category (gait, balance, and functional [task] training); these exercise programs may also include secondary categories of exercise. bA control intervention is one that is not thought to reduce falls, such as general health education, social visits, very gentle exercise, or 'sham' exercise not expected to impact on falls.

c The all-studies population risk was based on the number of events and the number of participants in the control group for this outcome over the 59 all-exercise types RCTs. The specific exercise population risk was based on the number of events and the number of participants in the control group for this outcome over the 39 RCTs.

dWe did not downgrade for risk of bias, as results were essentially unchanged with the removal of the trials with a high risk of bias in one or more items.

eThe all-studies population risk was based on the number of events and the number of participants in the control group for this outcome over the 63 all-exercise types RCTs. The specific exercise population risk was based on the number of events and the number of participants in the control group for this outcome over the 37 RCTs.

fThe all-studies population risk was based on the number of events and the number of participants in the control group for this outcome over the 10 all-exercise types RCTs. Based on the number of events and the number of participants in the control group for this outcome over the seven RCTs, the assumed risk in the control group was 43 per 1000.

gDowngraded by two levels due to risk of bias (removing studies with high risk of bias on one or more items had a marked impact on results), and imprecision (few events and wide Cl due to small sample size).

hDowngraded by three levels due to limitations in design of studies, suggesting a high likelihood of bias (no trials in this analysis measured the number of participants experiencing adverse events in both groups throughout the trial period).

\section{Summary of findings 3. Summary of findings: resistance exercises versus control (e.g. usual activities)}

\section{Resistance exercises versus control (e.g. usual activities) for preventing falls in older people in the community}

Patient or population: Older people living in the community (trials focusing on people recently discharged from hospital were not included)

Settings: Community, either at home or in places of residence that, on the whole, do not provide residential health-related care

Intervention: Exercise, type $=$ resistance training $a$ 
Comparison: Usual care (no change in usual activities) or a control (non-active) interventionb

\begin{tabular}{|c|c|c|c|c|c|c|}
\hline \multirow[t]{3}{*}{ Outcomes } & \multicolumn{2}{|c|}{$\begin{array}{l}\text { Illustrative comparative risks* } \\
(95 \% \mathrm{CI})\end{array}$} & \multirow[t]{3}{*}{$\begin{array}{l}\text { Relative effect } \\
(95 \% \mathrm{CI})\end{array}$} & \multirow[t]{3}{*}{$\begin{array}{l}\text { No of partici- } \\
\text { pants } \\
\text { (studies) }\end{array}$} & \multirow[t]{3}{*}{$\begin{array}{l}\text { Certainty of } \\
\text { the evidence } \\
\text { (GRADE) }\end{array}$} & \multirow[t]{3}{*}{ Comments } \\
\hline & Assumed risk & $\begin{array}{l}\text { Corresponding } \\
\text { risk }\end{array}$ & & & & \\
\hline & Control & $\begin{array}{l}\text { Exercise (resis- } \\
\text { tance training) }\end{array}$ & & & & \\
\hline \multirow{4}{*}{$\begin{array}{l}\text { Rate of falls } \\
\text { (falls per per- } \\
\text { son-years) } \\
\text { Follow-up: } \\
\text { range } 4 \text { to } 12 \\
\text { months }\end{array}$} & \multicolumn{2}{|c|}{ All studies population } & \multirow{4}{*}{$\begin{array}{l}\text { Rate ratio } 1.14 \\
(0.67 \text { to } 1.97)\end{array}$} & \multirow{4}{*}{$\begin{array}{l}327 \\
\text { (5 RCTs) }\end{array}$} & \multirow{4}{*}{$\begin{array}{l}\oplus \ominus \Theta^{d} \\
\text { very low }\end{array}$} & \multirow{4}{*}{$\begin{array}{l}\text { The evidence is of very low certainty, hence we are un- } \\
\text { certain of the findings of an increase of } 14 \%(95 \% \mathrm{Cl} \\
33 \% \text { reduction to } 97 \% \text { increase) in the number of falls. } \\
\text { Guide to the data based on the all-studies estimate. } \\
\text { If } 1000 \text { people were followed over } 1 \text { year, the number of } \\
\text { falls would be } 969 \text { ( } 95 \% \mathrm{Cl} 570 \text { to } 1675) \text { compared with } \\
850 \text { in the group receiving usual care or attention con- } \\
\text { trol }\end{array}$} \\
\hline & 850 per $1000 c$ & $\begin{array}{l}\mathbf{9 6 9} \text { per } \mathbf{1 0 0 0} \\
(570 \text { to } 1675)\end{array}$ & & & & \\
\hline & \multicolumn{2}{|c|}{ Specific exercise population } & & & & \\
\hline & 630 per $1000 c$ & $\begin{array}{l}719 \text { per } 1000 \\
\text { (423 to } 1242)\end{array}$ & & & & \\
\hline \multirow{2}{*}{$\begin{array}{l}\text { Number of peo- } \\
\text { ple who experi- } \\
\text { enced } 1 \text { or more } \\
\text { falls }\end{array}$} & \multicolumn{2}{|c|}{ All studies population } & \multirow{4}{*}{$\begin{array}{l}\text { RR } 0.81(0.57 \\
\text { to } 1.15)\end{array}$} & \multirow{4}{*}{$\begin{array}{l}163 \\
(2 \text { RCTs })\end{array}$} & \multirow{4}{*}{$\begin{array}{l}\oplus \ominus \Theta \ominus^{f} \\
\text { very low }\end{array}$} & \multirow{4}{*}{$\begin{array}{l}\text { The evidence is of very low certainty, hence we are un- } \\
\text { certain of the findings of a decrease of } 19 \% \text { ( } 95 \% \mathrm{Cl} 43 \% \\
\text { reduction to } 15 \% \text { increase) in the number of people who } \\
\text { experienced one or more falls } \\
\text { Guide to the data based on the all-studies estimate. } \\
\text { If } 1000 \text { people were followed over } 1 \text { year, the number of } \\
\text { people who experienced one or more falls would be } 389 \\
\text { ( } 95 \% \mathrm{Cl} 274 \text { to } 552 \text { ) compared with } 480 \text { in the group re- } \\
\text { ceiving usual care or attention control }\end{array}$} \\
\hline & 480 per 1000 & $\begin{array}{l}389 \text { per } 1000 \\
\text { (274 to } 552)\end{array}$ & & & & \\
\hline \multirow{2}{*}{$\begin{array}{l}\text { Follow-up: } \\
\text { range } 4 \text { to } 12 \\
\text { months }\end{array}$} & \multicolumn{2}{|c|}{ Specific exercise population } & & & & \\
\hline & $\begin{array}{l}864 \\
\text { per } 1000^{\circ}\end{array}$ & $\begin{array}{l}700 \text { per } 1000 \\
\text { (493 to } 994)\end{array}$ & & & & \\
\hline \multirow{2}{*}{$\begin{array}{l}\text { Number of peo- } \\
\text { ple who experi- } \\
\text { enced } 1 \text { or more } \\
\text { fall-related frac- } \\
\text { tures }\end{array}$} & \multicolumn{2}{|c|}{ All studies population } & \multirow{2}{*}{$\begin{array}{l}\text { RR } 0.97 \\
(0.14 \text { to } 6.49)\end{array}$} & \multirow{2}{*}{$\begin{array}{l}73 \\
(1 \mathrm{RCT})\end{array}$} & \multirow{2}{*}{$\begin{array}{l}\oplus \ominus \Theta \Theta^{h} \\
\text { very low }\end{array}$} & \multirow{2}{*}{$\begin{array}{l}\text { The evidence is of very low certainty, hence we are un- } \\
\text { certain of the findings of a decrease of } 3 \% \text { ( } 95 \% \mathrm{Cl} 86 \% \\
\text { reduction to } 549 \% \text { increase) } \\
\text { The very small number of events ( } 3 \text { fractures in all) } \\
\text { means that these data are not informative }\end{array}$} \\
\hline & 64 per $1000 \mathrm{~g}$ & $\begin{array}{l}63 \text { per } 1000 \\
(9 \text { to } 416)\end{array}$ & & & & \\
\hline Adverse events & See comment & & Not estimable & $\begin{array}{l}64 \\
(1 \mathrm{RCT})\end{array}$ & $\begin{array}{l}\oplus \Theta \Theta \Theta^{i} \\
\text { very low }\end{array}$ & $\begin{array}{l}\text { Adverse events were reported on in one of the five tri- } \\
\text { als with resistance training as the primary intervention } \\
\text { in exercise versus control analyses. The study report- } \\
\text { ed } 10 \text { musculoskeletal complaints in the intervention }\end{array}$ \\
\hline
\end{tabular}


${ }^{*}$ The basis for the assumed risk (e.g. the median control group risk across studies) is provided in footnotes. The corresponding risk (and its $95 \%$ confidence interval) is based on the assumed risk in the comparison group and the relative effect of the intervention (and its 95\% Cl).

Cl: confidence interval; RR: risk ratio

\section{GRADE Working Group grades of evidence}

High certainty: We are very confident that the true effect lies close to that of the estimate of the effect

Moderate certainty: We are moderately confident in the effect estimate: The true effect is likely to be close to the estimate of the effect, but there is a possibility that it is substantially different

Low certainty: Our confidence in the effect estimate is limited: The true effect may be substantially different from the estimate of the effect

Very low certainty: We have very little confidence in the effect estimate: The true effect is likely to be substantially different from the estimate of effect

a Using Prevention of Falls Network Europe (ProFaNE) taxonomy, resistance training is any type of weight training (contraction of muscles against resistance to induce a training effect in the muscular system). Resistance is applied by body weight or external resistance. Training is assessment-based, tailored and progressed. Exercise programmes included in this analysis had resistance training as the single primary exercise category; these exercise programmes may also include secondary categories of exercise.

bA control intervention is one that is not thought to reduce falls, such as general health education, social visits, very gentle exercise, or 'sham' exercise not expected to impact on falls.

CThe all-studies population risk was based on the number of events and the number of participants in the control group for this outcome over the 59 all-exercise types RCTs. The specific exercise population risk was based on the number of events and the number of participants in the control group for this outcome over the 5 RCTs.

dDowngraded by three levels due to risk of inconsistency (there was substantial heterogeneity $\left(I^{2}=67 \%\right)$ ), imprecision (wide $\mathrm{Cl}$ due to small sample size), and risk of bias (removing studies with high risk of bias in one or more items had a marked impact on results).

eThe all-studies population risk was based on the number of events and the number of participants in the control group for this outcome over the 63 all-exercise types RCTs. The specific exercise population risk was based on the number of events and the number of participants in the control group for this outcome over the 2 RCTs.

fDowngraded by one level due to risk of bias (removing studies with high risk of bias on one or more items had a marked impact on results), and downgraded by two levels due to imprecision (small number of trials and participants, wide $\mathrm{Cl}$ ).

gThe all-studies population risk was based on the number of events and the number of participants in the control group for this outcome over the 10 all-exercise types RCTs. Based on the number of events and the number of participants in the control group for this outcome in the sole RCT, the assumed risk in the control group was 28 per 1000 howngraded by three levels for imprecision (wide $\mathrm{Cl}$, single study, very few events).

iDowngraded by three levels due to only one study reporting adverse events and limitations in design of studies, suggesting a high likelihood of bias (number of participants experiencing adverse events was not reported in the same manner in both groups throughout the trial period)

\section{Summary of findings 4. Summary of findings: 3D (Tai Chi) exercise versus control (e.g. usual activities)}

\section{D (Tai Chi) exercise versus control (e.g. usual activities) for preventing falls in older people in the community}

Patient or population: Older people living in the community (trials focusing on people recently discharged from hospital were not included)

Settings: Community, either at home or in places of residence that, on the whole, do not provide residential health-related care

Intervention: Exercise, type $=3 \mathrm{D}($ Tai Chi) training $a$ 
Comparison: Usual care (no change in usual activities) or a control (non-active) interventionb

\begin{tabular}{|c|c|c|c|c|c|c|}
\hline \multirow[t]{3}{*}{ Outcomes } & \multicolumn{2}{|c|}{$\begin{array}{l}\text { Illustrative comparative risks* } \\
(95 \% \mathrm{Cl})\end{array}$} & \multirow[t]{3}{*}{$\begin{array}{l}\text { Relative effect } \\
(95 \% \mathrm{CI})\end{array}$} & \multirow{3}{*}{$\begin{array}{l}\text { No of partici- } \\
\text { pants } \\
\text { (studies) }\end{array}$} & \multirow{3}{*}{$\begin{array}{l}\text { Certainty of } \\
\text { the evidence } \\
\text { (GRADE) }\end{array}$} & \multirow[t]{3}{*}{ Comments } \\
\hline & Assumed risk & $\begin{array}{l}\text { Corresponding } \\
\text { risk }\end{array}$ & & & & \\
\hline & Control & $\begin{array}{l}\text { Exercise (3D (Tai } \\
\text { Chi)) }\end{array}$ & & & & \\
\hline \multirow{4}{*}{$\begin{array}{l}\text { Rate of falls (falls } \\
\text { per person-year) } \\
\text { Follow-up: range } \\
6 \text { to } 17 \text { months }\end{array}$} & \multicolumn{2}{|c|}{ All studies population } & \multirow{4}{*}{$\begin{array}{l}\text { Rate ratio } 0.81 \\
(0.67 \text { to } 0.99)\end{array}$} & \multirow{4}{*}{$\begin{array}{l}2655 \\
\text { (7 RCTs) }\end{array}$} & \multirow{4}{*}{$\begin{array}{l}\oplus \oplus \ominus{ }^{d} \\
\text { low }\end{array}$} & \multirow{4}{*}{$\begin{array}{l}\text { Overall, there may be a reduction of } 19 \% \text { ( } 95 \% \mathrm{Cl} 1 \% \\
\text { to } 33 \%) \text { in the number of falls. } \\
\text { Guide to the data based on the all-studies estimate. } \\
\text { If } 1000 \text { people were followed over } 1 \text { year, the num- } \\
\text { ber of falls may be } 689 \text { ( } 95 \% \mathrm{Cl} 570 \text { to } 842) \text { compared } \\
\text { with } 850 \text { in the group receiving usual care or atten- } \\
\text { tion control }\end{array}$} \\
\hline & 850 per $1000 c$ & $\begin{array}{l}\mathbf{6 8 9} \text { per } 1000 \\
(570 \text { to } 842)\end{array}$ & & & & \\
\hline & \multicolumn{2}{|c|}{ Specific exercise population } & & & & \\
\hline & 1020 per $1000 c$ & $\begin{array}{l}827 \text { per } 1000 \\
(684 \text { to } 1010)\end{array}$ & & & & \\
\hline \multirow{2}{*}{$\begin{array}{l}\text { Number of peo- } \\
\text { ple who expe- } \\
\text { rienced one or } \\
\text { more falls }\end{array}$} & \multicolumn{2}{|c|}{ All studies population } & \multirow{4}{*}{$\begin{array}{l}\mathrm{RR} 0.80(0.70 \\
\text { to } 0.91)\end{array}$} & \multirow{4}{*}{$\begin{array}{l}2677 \\
\text { (8 RCTs) }\end{array}$} & \multirow{4}{*}{$\begin{array}{l}\oplus \oplus \oplus \oplus^{f} \\
\text { high }\end{array}$} & \multirow{4}{*}{$\begin{array}{l}\text { Overall, there is a reduction of } 20 \% \text { ( } 95 \% \mathrm{Cl} 9 \% \text { to } \\
30 \%) \text { in the number of people who experienced one } \\
\text { or more falls } \\
\text { Guide to the data based on the all-studies estimate. } \\
\text { If } 1000 \text { people were followed over } 1 \text { year, the number } \\
\text { of people who experienced one or more falls would } \\
\text { be } 384 \text { ( } 95 \% \mathrm{Cl} 336 \text { to } 437 \text { ) compared with } 480 \text { in the } \\
\text { group receiving usual care or attention control }\end{array}$} \\
\hline & 480 per $1000^{e}$ & $\begin{array}{l}\mathbf{3 8 4} \text { per } \mathbf{1 0 0 0} \\
\text { (336 to } 437)\end{array}$ & & & & \\
\hline \multirow[t]{2}{*}{$\begin{array}{l}\text { Follow-up: range } \\
5 \text { to } 17 \text { months }\end{array}$} & \multicolumn{2}{|c|}{ Specific exercise population } & & & & \\
\hline & 437 per $1000^{e}$ & $\begin{array}{l}\mathbf{3 5 0} \text { per } \mathbf{1 0 0 0} \\
\text { (306 to } 398)\end{array}$ & & & & \\
\hline $\begin{array}{l}\text { Number of peo- } \\
\text { ple who expe- } \\
\text { rienced one or } \\
\text { more fall-related } \\
\text { fractures }\end{array}$ & See comment & & Not estimable & See comment & - & This outcomes was not reported \\
\hline Adverse events & See comment & & Not estimable & $\begin{array}{l}474 \\
(2 \mathrm{RCTs})\end{array}$ & $\begin{array}{l}\oplus \ominus \ominus \ominus \mathrm{g} \\
\text { very low }\end{array}$ & $\begin{array}{l}\text { Adverse events were reported in two of } 10 \text { trials ( } 474 \\
\text { participants) with 3D (Tai Chi) as the primary in- } \\
\text { tervention. There were no occurrences of adverse } \\
\text { events }\end{array}$ \\
\hline
\end{tabular}

*The basis for the assumed risk (e.g. the median control group risk across studies) is provided in footnotes. The corresponding risk (and its $95 \%$ confidence interval) is based on the assumed risk in the comparison group and the relative effect of the intervention (and its $95 \% \mathrm{Cl}$ ). 
GRADE Working Group grades of evidence

High certainty: We are very confident that the true effect lies close to that of the estimate of the effect

Moderate certainty: We are moderately confident in the effect estimate: The true effect is likely to be close to the estimate of the effect, but there is a possibility that it is substantially different

Low certainty: Our confidence in the effect estimate is limited: The true effect may be substantially different from the estimate of the effect

Very low certainty: We have very little confidence in the effect estimate: The true effect is likely to be substantially different from the estimate of effect

aUsing Prevention of Falls Network Europe (ProFaNE) taxonomy, 3D (Tai Chi) training uses upright posture, specific weight transferences and movements of the head and gaze, during constant movement in a fluid, repetitive, controlled manner through three spatial planes. Exercise programmes included in this analysis had 3D (Tai Chi) training as the single primary exercise category; these exercise programmes may also include secondary categories of exercise.

bA control intervention is one that is not thought to reduce falls, such as general health education, social visits, very gentle exercise, or 'sham' exercise not expected to impact on falls.

CThe all-studies population risk was based on the number of events and the number of participants in the control group for this outcome over the 59 all-exercise types RCTs. The specific exercise population risk was based on the number of events and the number of participants in the control group for this outcome over the seven RCTs.

dDowngraded by two levels due to inconsistency (there was substantial heterogeneity $\left(I^{2}=74 \%\right)$ ), and risk of bias (removing studies with high risk of bias in one or more items had a marked impact on results).

eThe all-studies population risk was based on the number of events and the number of participants in the control group for this outcome over the 63 all-exercise types RCTs. The specific exercise population risk was based on the number of events and the number of participants in the control group for this outcome over the eight RCTs.

fWe did not downgrade for risk of bias, as results were essentially unchanged with removal of the trials with a high risk of bias in one or more items.

gDowngraded by three levels due to only $30 \%$ of trials reporting adverse events to any degree, and limitations in the design of studies suggesting a high likelihood of bias (no trials in this analysis measured the number of participants experiencing adverse events in both groups throughout the trial period).

Summary of findings 5. Summary of findings: 3D (dance) exercise versus control (e.g. usual activities)

\section{D (dance) exercise versus control (e.g. usual activities) for preventing falls in older people in the community}

Patient or population: Older people living in the community (trials focusing on people recently discharged from hospital were not included)

Settings: Community, either at home or in places of residence that, on the whole, do not provide residential health-related care

Intervention: Exercise, type $=3 \mathrm{D}$ (dance) training ${ }^{a}$

Comparison: Usual care (no change in usual activities) or a control (non-active) interventionb

\begin{tabular}{|c|c|c|c|c|c|c|}
\hline \multirow[t]{2}{*}{ Outcomes } & \multicolumn{2}{|c|}{$\begin{array}{l}\text { Illustrative comparative risks* } \\
(95 \% \mathrm{Cl})\end{array}$} & \multirow[t]{2}{*}{$\begin{array}{l}\text { Relative effect } \\
(95 \% \mathrm{CI})\end{array}$} & \multirow[t]{2}{*}{$\begin{array}{l}\text { No of partici- } \\
\text { pants } \\
\text { (studies) }\end{array}$} & \multirow[t]{2}{*}{$\begin{array}{l}\text { Certainty of } \\
\text { the evidence } \\
\text { (GRADE) }\end{array}$} & \multirow[t]{2}{*}{ Comments } \\
\hline & Assumed risk & $\begin{array}{l}\text { Corresponding } \\
\text { risk }\end{array}$ & & & & \\
\hline
\end{tabular}




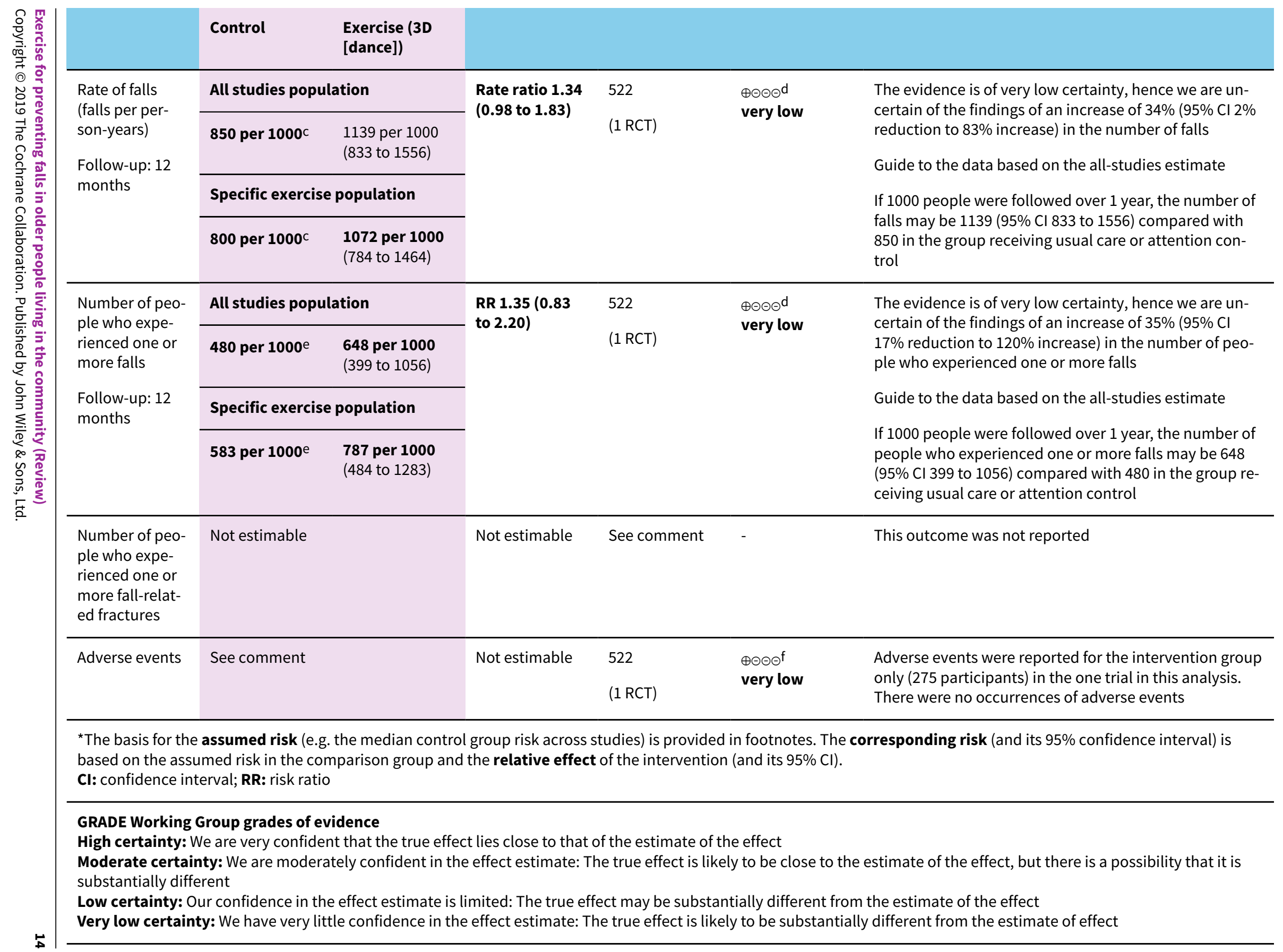


aUsing Prevention of Falls Network Europe (ProFaNE) taxonomy, 3D (dance) training uses dynamic movement qualities, patterns and speeds whilst engaged in constant movement in a fluid, repetitive, controlled manner through three spatial planes. Exercise programmes included in this analysis had 3D (dance) training as the single primary exercise category; these exercise programmes may also include secondary categories of exercise.

bA control intervention is one that is not thought to reduce falls, such as general health education, social visits, very gentle exercise, or 'sham' exercise not expected to impact on falls.

CThe all-studies population risk was based on the number of events and the number of participants in the control group for this outcome over the 59 all-exercise types RCTs. The specific exercise population risk was based on the number of events and the number of participants in the control group for this outcome in the sole RCT.

dGraded very low due to serious imprecision (only one cluster-RCT, with a wide $\mathrm{Cl}$ due to small sample size).

eThe all-studies population risk was based on the number of events and the number of participants in the control group for this outcome over the 63 all-exercise types RCTs. The specific exercise population risk was based on the number of events and the number of participants in the control group for this outcome in the sole RCT.

fDowngraded by three levels due to limitations in the design of studies, suggesting a high likelihood of bias (the trial measured the number of participants experiencing adverse events in the exercise group)

\section{Summary of findings 6. Summary of findings: walking programme (general physical activity) versus control (e.g. usual activities)}

\section{General physical activity (including walking) training versus control (e.g. usual activities) for preventing falls in older people in the community}

Patient or population: Older people living in the community (trials focusing on people recently discharged from hospital were not included)

Settings: Community, either at home or in places of residence that, on the whole, do not provide residential health-related care

Intervention: Exercise, type = general physical activity (including walking) training $a$

Comparison: Usual care (no change in usual activities) or a control (non-active) intervention ${ }^{b}$

\begin{tabular}{|c|c|c|c|c|c|c|}
\hline \multirow[t]{3}{*}{ Outcomes } & \multicolumn{2}{|c|}{$\begin{array}{l}\text { Illustrative comparative risks } \\
(95 \% \mathrm{CI})\end{array}$} & \multirow[t]{3}{*}{$\begin{array}{l}\text { Relative effect } \\
(95 \% \mathrm{Cl})\end{array}$} & \multirow{3}{*}{$\begin{array}{l}\text { No of partici- } \\
\text { pants } \\
\text { (studies) }\end{array}$} & \multirow{3}{*}{$\begin{array}{l}\text { Certainty of } \\
\text { the evidence } \\
\text { (GRADE) }\end{array}$} & \multirow[t]{3}{*}{ Comments } \\
\hline & Assumed risk & $\begin{array}{l}\text { Corresponding } \\
\text { risk }\end{array}$ & & & & \\
\hline & Control & $\begin{array}{l}\text { Exercise (gen- } \\
\text { eral physical } \\
\text { activity [in- } \\
\text { cluding walk- } \\
\text { ing]) }\end{array}$ & & & & \\
\hline \multirow{2}{*}{$\begin{array}{l}\text { Rate of falls } \\
\text { (falls per per- } \\
\text { son-years) }\end{array}$} & \multicolumn{2}{|c|}{ All studies population } & \multirow{4}{*}{$\begin{array}{l}\text { Rate ratio } 1.14 \\
\text { (0.66 to } 1.97 \text { ) }\end{array}$} & \multirow{4}{*}{$\begin{array}{l}441 \\
\text { (2 RCTs) }\end{array}$} & \multirow{4}{*}{$\begin{array}{l}\oplus \ominus \Theta{ }^{d} \\
\text { very low }\end{array}$} & \multirow{4}{*}{$\begin{array}{l}\text { The evidence is of very low certainty, hence we are un- } \\
\text { certain of the findings of an increase of } 14 \%(95 \% \mathrm{Cl} \\
34 \% \text { reduction to } 97 \% \text { increase) in the number of falls } \\
\text { Guide to the data based on the all-studies estimate }\end{array}$} \\
\hline & 850 per $1000 c$ & $\begin{array}{l}969 \text { per } 1000 \\
\text { (561 to } 1675 \text { ) }\end{array}$ & & & & \\
\hline \multirow{2}{*}{$\begin{array}{l}\text { Follow-up: } \\
\text { range } 12 \text { to } 24 \\
\text { months }\end{array}$} & & & & & & \\
\hline & \multicolumn{2}{|c|}{ Specific exercise population } & & & & \\
\hline
\end{tabular}




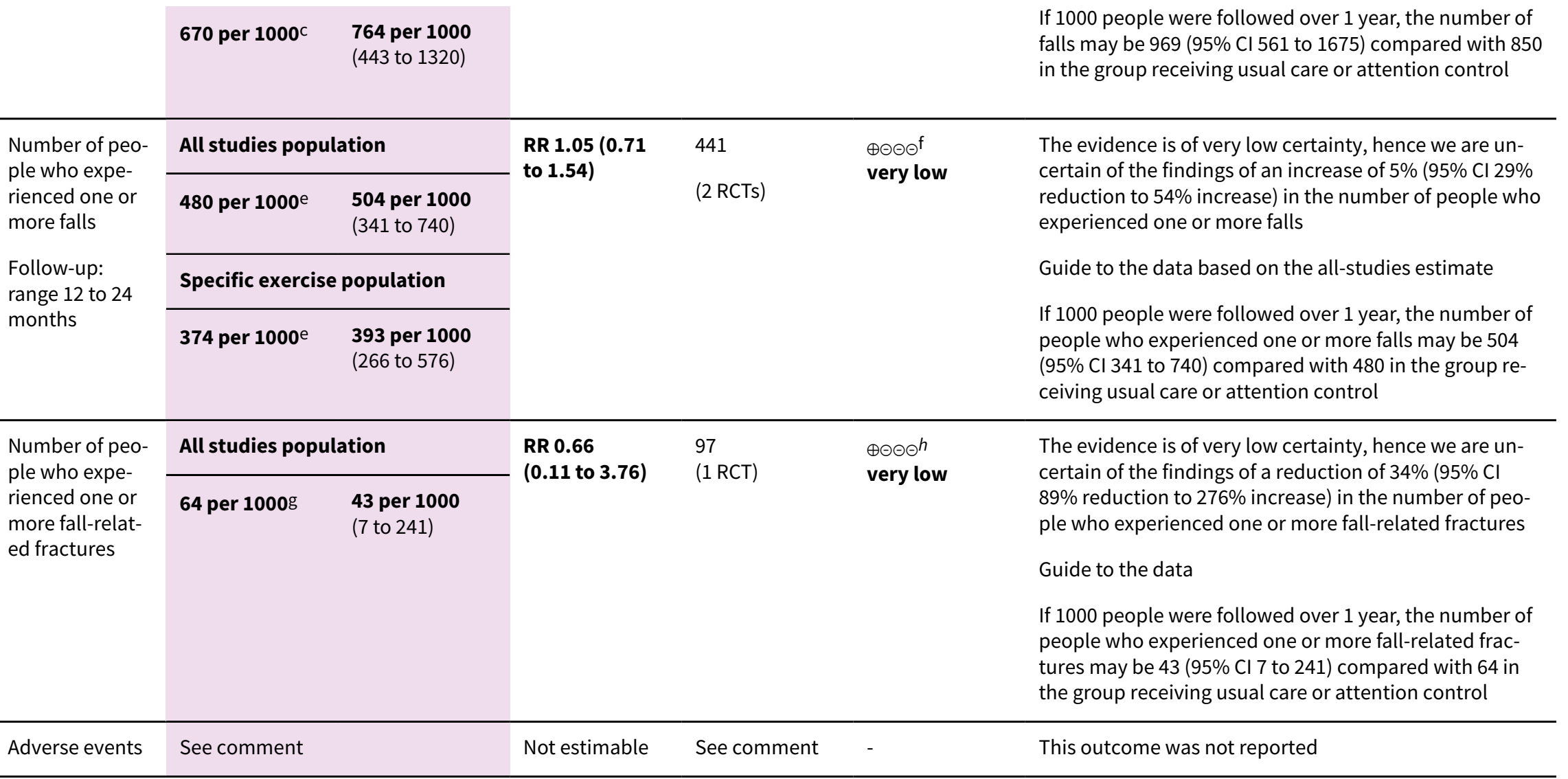

*The basis for the assumed risk (e.g. the median control group risk across studies) is provided in footnotes. The corresponding risk (and its $95 \%$ confidence interval) is based on the assumed risk in the comparison group and the relative effect of the intervention (and its $95 \% \mathrm{Cl}$ ).

Cl: confidence interval; RR: risk ratio

\section{GRADE Working Group grades of evidence}

High certainty: We are very confident that the true effect lies close to that of the estimate of the effect

Moderate certainty: We are moderately confident in the effect estimate: The true effect is likely to be close to the estimate of the effect, but there is a possibility that it is substantially different

Low certainty: Our confidence in the effect estimate is limited: The true effect may be substantially different from the estimate of the effect

Very low certainty: We have very little confidence in the effect estimate: The true effect is likely to be substantially different from the estimate of effect

a Using Prevention of Falls Network Europe (ProFaNE) taxonomy, physical activity is any movement of the body, produced by skeletal muscle, that causes energy expenditure to be substantially increased. Recommendations regarding intensity, frequency and duration are required in order to increase performance. Exercise programmes included in this analysis had general physical activity (including walking) training as the single primary exercise category; these exercise programmes may also include secondary categories 
bA control intervention is one that is not thought to reduce falls, such as general health education, social visits, very gentle exercise, or 'sham' exercise not expected to impact on falls.

CThe all-studies population risk was based on the number of events and the number of participants in the control group for this outcome over the 59 all-exercise types RCTs. The specific exercise population risk was based on the number of events and the number of participants in the control group for this outcome in the two RCTs.

dDowngraded by three levels due to inconsistency (there was substantial heterogeneity $\left(I^{2}=67 \%\right)$ ), imprecision (wide $\mathrm{Cl}$ ), and risk of bias (removing studies with high risk of bias on one or more items had a marked impact on results).

eThe all-studies population risk was based on the number of events and the number of participants in the control group for this outcome over the 63 all-exercise types RCTs. The specific exercise population risk was based on the number of events and the number of participants in the control group for this outcome in the two RCTs.

fDowngraded by three levels due to inconsistency (there was moderate heterogeneity $\left(I^{2}=50 \%\right)$, imprecision (wide Cl), and risk of bias (removing studies with high risk of bias on one or more items had a marked impact on results).

gThe all-studies population risk was based on the number of events and the number of participants in the control group for this outcome over the 10 all-exercise types RCTs. Based on the number of events and the number of participants in the control group for this outcome in the only RCT, the assumed risk in the control group was 84 per 1000.

hDowngraded three levels due to risk of bias and imprecision (single study, wide $\mathrm{Cl}$ ).

\section{Summary of findings 7. Summary of findings: multiple categories of exercise versus control (e.g. usual activities)}

Multiple categories of exercise (often including, as primary interventions: gait, balance, and functional (task) training plus resistance training) versus control (e.g. usual activities) for preventing falls in older people in the community

Patient or population: Older people living in the community (trials focusing on people recently discharged from hospital were not included)

Settings: Community, either at home or in places of residence that, on the whole, do not provide residential health-related care

Intervention: Exercise, type $=$ Multiple types of exercise ${ }^{a}$

Comparison: Usual care (no change in usual activities) or a control (non-active) interventionb

\begin{tabular}{|c|c|c|c|c|c|c|}
\hline \multirow[t]{3}{*}{ Outcomes } & \multicolumn{2}{|c|}{$\begin{array}{l}\text { Illustrative comparative risks }{ }^{\star} \\
(95 \% \mathrm{Cl})\end{array}$} & \multirow[t]{3}{*}{$\begin{array}{l}\text { Relative effect } \\
(95 \% \mathrm{CI})\end{array}$} & \multirow{3}{*}{$\begin{array}{l}\text { No of partici- } \\
\text { pants } \\
\text { (studies) }\end{array}$} & \multirow{3}{*}{$\begin{array}{l}\text { Certainty of } \\
\text { the evidence } \\
\text { (GRADE) }\end{array}$} & \multirow[t]{3}{*}{ Comments } \\
\hline & Assumed risk & $\begin{array}{l}\text { Corresponding } \\
\text { risk }\end{array}$ & & & & \\
\hline & Control & $\begin{array}{l}\text { Exercise (mul- } \\
\text { tiple types (in- } \\
\text { cluding, as } \\
\text { primary in- } \\
\text { terventions: } \\
\text { gait, balance, } \\
\text { and functional } \\
\text { (task) training, } \\
\text { plus resistance } \\
\text { training)) }\end{array}$ & & & & \\
\hline
\end{tabular}




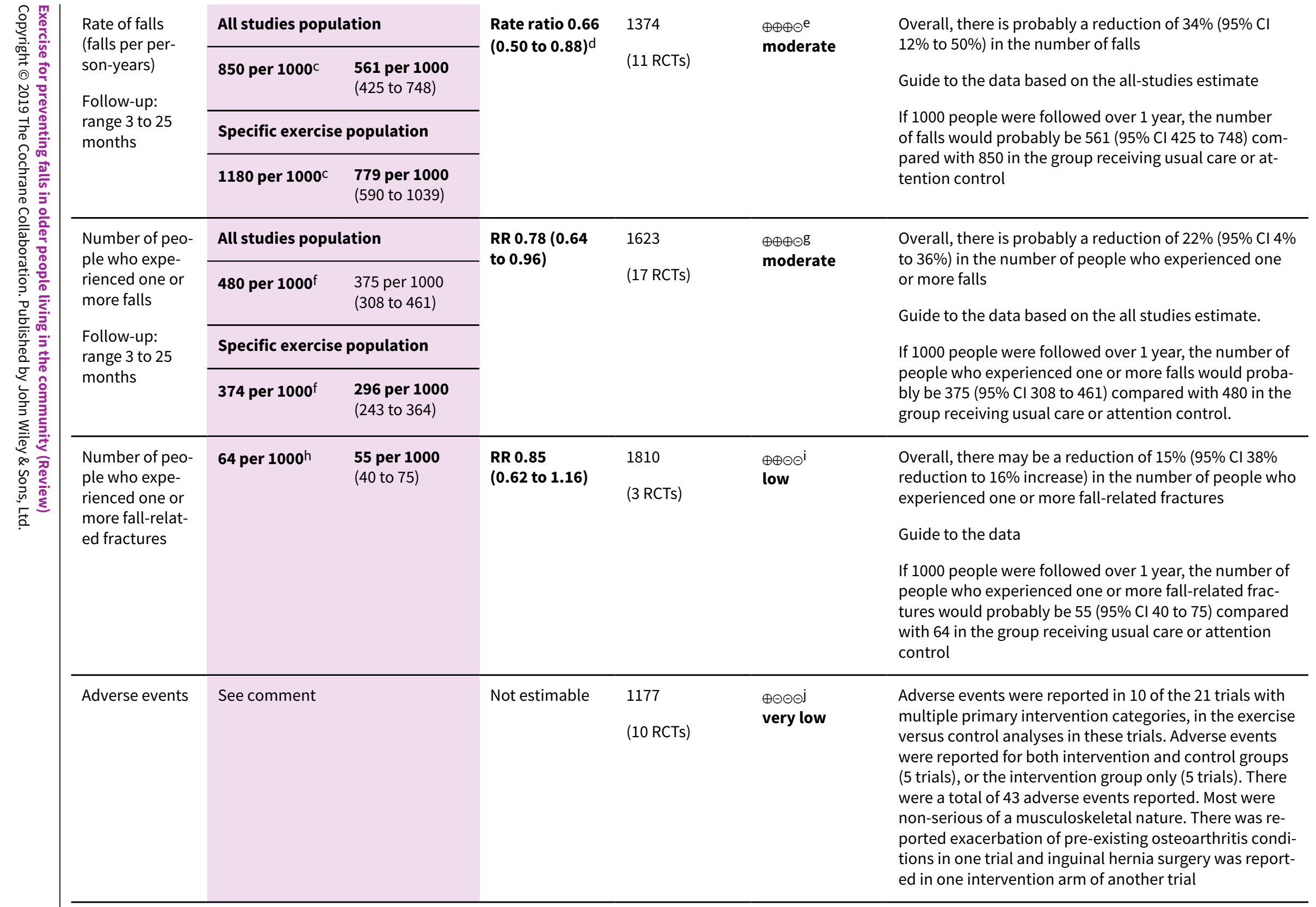

${ }^{*}$ The basis for the assumed risk (e.g. the median control group risk across studies) is provided in footnotes. The corresponding risk (and its $95 \%$ confidence interval) is $\stackrel{\leftrightarrow}{\infty}$ 


\section{GRADE Working Group grades of evidence}

High certainty: We are very confident that the true effect lies close to that of the estimate of the effect

Moderate certainty: We are moderately confident in the effect estimate: The true effect is likely to be close to the estimate of the effect, but there is a possibility that it is substantially different

Low certainty: Our confidence in the effect estimate is limited: The true effect may be substantially different from the estimate of the effect

Very low certainty: We have very little confidence in the effect estimate: The true effect is likely to be substantially different from the estimate of effect

${ }^{a}$ Exercise programmes included in this analysis had more than one primary exercise category. We categorised exercise based on the Prevention of Falls Network Europe (ProFaNE) taxonomy that classifies exercise type as: i) gait, balance, and functional (task) training; ii) strength/resistance (including power); iii) flexibility; iv) three-dimensional (3D) exercise (e.g. Tai Chi, Qigong, dance); v) general physical activity; vi) endurance; and vii) other kind of exercises. The programmes often included, as the primary intervention, gait, balance, and functional (task) training plus resistance training. The exercise programmes may also include secondary categories of exercise.

bA control intervention is one that is not thought to reduce falls, such as general health education, social visits, very gentle exercise, or 'sham' exercise not expected to impact on falls.

CThe all-studies population risk was based on the number of events and the number of participants in the control group for this outcome over the 59 all-exercise types RCTs. The specific exercise population risk was based on the number of events and the number of participants in the control group for this outcome over the 11 RCTs.

dSensitivity analyses revealed little difference in the results when only trials that include the most common two components (balance and functional exercises plus resistance exercises) were pooled ( $R a R 0.69,95 \% \mathrm{Cl} 0.48$ to $0.97 ; 1084$ participants; 8 studies; $I^{2}=72 \%$ ).

eDowngraded by one level due to inconsistency (there was substantial heterogeneity $\left(I^{2}=65 \%\right)$ ). We did not downgrade for risk of bias, as results were essentially unchanged with removal of the trials at a high risk of bias in one or more items.

fThe all-studies population risk was based on the number of events and the number of participants in the control group for this outcome over the 63 all-exercise types RCTs. The specific exercise population risk was based on the number of events and the number of participants in the control group for this outcome over the 17 RCTs.

gDowngraded by one level due to risk of bias (removing studies with high risk of bias in one or more items had a marked impact on results).

hThe all-studies population risk was based on the number of events and the number of participants in the control group for this outcome over the 10 all-exercise types RCTs. Based on the number of events and the number of participants in the control group for this outcome over three RCTs, the assumed risk in the control group was 87 per 1000. iDowngraded by one level due to risk of bias and by one level due to imprecision.

jDowngraded by three levels for limitations in the design of studies, suggesting a high likelihood of bias (no trials in this analysis measured the number of participants experiencing adverse events in both groups throughout the trial period). 


\section{B A C K G R O U N D}

\section{Description of the condition}

At least one-third of community-dwelling people over 65 years of age fall each year (Campbell 1990; Tinetti 1988), and the rate of fall-related injuries increases with age (Peel 2002). Falls can have serious consequences, such as fractures and head injuries (Peel 2002). Around $10 \%$ of falls result in a fracture (Campbell 1990; Tinetti 1988); fall-associated fractures in older people are a significant source of morbidity and mortality (Burns 2016). Although most fall-related injuries, such as bruising, lacerations and sprains, are less serious, they can still lead to pain, reduced function and substantial healthcare costs (Burns 2016).

Falls are associated with reduced quality of life (Stenhagen 2014), and can have psychological consequences: fear of falling and loss of confidence that can result in self-restricted activity levels leading to a reduction in physical function and social interactions (Yardley 2002). Paradoxically, this restriction of activities may increase the risk of further falls by contributing to deterioration in physical abilities. Both injurious and non-injurious falls can have these psychological and subsequent physical impacts.

Despite early attempts to achieve a consensus definition of a 'fall' (Anonymous 1987), many definitions still exist in the literature. It is particularly important for studies to use a clear, simple definition of a fall. An international researchers' consensus statement defines a fall as "an unexpected event in which the participant comes to rest on the ground, floor, or lower level" (Lamb 2005). The wording recommended when asking study participants is: "In the past month, have you had any fall including a slip or trip in which you lost your balance and landed on the floor or ground or lower level?" (Lamb 2005). 'Lower level' refers to a surface lower than the person's starting position so, for example, falling from a standing position to unintentionally sitting on a bed would be considered a fall.

In addition to the physical and psychological consequences for individuals and their families, falls can have important financial impacts on individuals, families and health and community care systems (Burns 2016). For example, falling is an independent predictor of admission to residential aged care facilities (Tinetti 1997).

\section{Description of the intervention}

Exercise is a physical activity that is planned, structured and repetitive and aims to improve or maintain physical fitness (Caspersen 1985). There is a wide range of possible types of exercise, such as strengthening exercise, balance and co-ordination exercise and aerobic exercise. Exercise programmes often include one or more types of exercise. The Prevention of Falls Network Europe (ProFaNE) developed a taxonomy that classifies exercise type as: i) gait, balance, and functional (task) training; ii) strength/ resistance (including power); iii) flexibility; iv) three-dimensional (3D) exercise (e.g. Tai Chi, Qigong, dance); v) general physical activity; vi) endurance; and vii) other kinds of exercises (Lamb 2011). The taxonomy allows for more than one type of exercise to be delivered within a programme.

Formal exercise programmes are delivered by a wide range of individuals ranging from health professionals (such as physiotherapists, also known as physical therapists) and exercise professionals (such as trained fitness leaders) to trained volunteers. Exercise programmes may be supervised, unsupervised or involve a mixture of both.

This review considers all types of exercise and all delivery methods.

Exercise can also be delivered as part of a multiple component intervention, where people also receive one or more other fall or fracture prevention interventions, such as home-hazard modification and vitamin D supplementation. The effects of multiple component interventions that include exercise are assessed in Hopewell 2018.

\section{How the intervention might work}

Many aspects of physical functioning deteriorate with increased age and inactivity. Impairments in muscle strength, balance control and gait are particularly strong risk factors for falls (Tinetti 1988). For example, those with poor leg extensor strength were found to be $43 \%$ more likely to fall at home than their stronger counterparts (Menant 2017). Systematic reviews have found that those with gait problems have twice the odds of falling than those without (Deandrea 2010), and that measures of balance and mobility such as the Berg Balance Scale, Timed Up and Go Test, and Five Times Sit-to-Stand Test can identify individuals at greater risk of future falls (Lusardi 2017).

Exercises that address these impairments are therefore likely to reduce the risk of falling. As Cochrane Reviews have now found that exercise improves both strength (Liu 2009), and balance (Howe 2011) in older people, exercise is likely to have a fall prevention effect through its impact on these key fall risk factors. A Cochrane Review found that exercise reduces the fear of falling (Kendrick 2014), which is also a strong predictor of falls.

A previous Cochrane Review found exercise as a single intervention, prevents falls (Gillespie 2012), and to be the most commonly tested single fall prevention intervention. Economic evaluations accompanying randomised trials have found exercise to be a costeffective fall-prevention strategy (Davis 2010).

Exercise interventions have been found to be effective when delivered in a group-based setting or on an individual basis. The optimal features of successful fall prevention exercise programmes are not yet clear, but programmes that are multicomponent (e.g. target both strength and balance; Gillespie 2012), and programmes that include balance training, appear to be particularly effective (Sherrington 2017).

Different approaches to exercise will have advantages and disadvantages in terms of cost, 'enjoyability', accessibility and impacts on various body systems and outcomes. These advantages and disadvantages are likely to vary between individuals and in different settings.

Exercise has the potential to lead to adverse events such as cardiovascular episodes and musculoskeletal injuries if not carefully prescribed and undertaken (Thompson 2013). Exercise may also increase the risk of falls, particularly in higher risk individuals. For example, exercise interventions aiming to improve balance and ultimately lessen the risk of falling, often involve a 'challenge' to balance that simultaneously puts the person at greater risk of falling (Sherrington 2017). The risk may be increased if an exercise participant becomes fatigued (due to 
deconditioning or as a result of comorbidities or medications) or are not encouraged to use support when needed (Skelton 2001). Trials and reviews should therefore record and report adverse events.

As the majority of fractures in older people involve falls, exercise has the potential to prevent fractures. Systematic reviews have suggested that exercise may prevent fractures (Gillespie 2012), and fall-related injuries (Robertson 2002).

\section{Why it is important to do this review}

An update of the effects of exercise interventions on falls is warranted given the number of new trials published, the increasing number of older people living in the community and the major longterm consequences associated with falls and fall-related injuries to both the individual and to society.

It is also important to understand to what extent interventions designed to prevent falls will also prevent fall-associated fractures, the need for medical attention and improve quality of life. Different exercise programmes may have different effects on falls and so careful analysis of the impact of different programmes is crucial to optimise the prescription of exercise interventions and inform public health promotion initiatives for healthy ageing. Additionally, looking for adverse events associated with the different exercise programmes, such as exercise-related falls and muscle strains, is also important.

\section{OB JECTIVES}

To assess the effects (benefits and harms) of exercise interventions for preventing falls in older people living in the community.

\section{METHODS}

\section{Criteria for considering studies for this review}

\section{Types of studies}

We included randomised controlled trials (RCTs), either individual or cluster randomised, evaluating the effects of exercise interventions on falls or fall-related fractures in older people living in the community. We excluded trials that explicitly used methods of quasi-randomisation (e.g. allocation to groups by alternation or date of birth).

\section{Types of participants}

We included trials if they specified an inclusion criterion of 60 years of age or over. Trials that included younger participants were included if the mean age minus one standard deviation was more than 60 years. We included trials where the majority of participants were living in the community, either at home or in places of residence that, on the whole, do not provide residential healthrelated care or rehabilitative services; for example, retirement villages, or sheltered housing. Trials with mixed populations (community and higher dependency places of residence) were eligible for inclusion if data were provided for subgroups based on setting or the numbers in higher dependency residences were very few and balanced in the comparison groups.

We excluded studies that only included participants affected by particular clinical conditions that increase the risk of falls, such as stroke, Parkinson's disease, multiple sclerosis, dementia, hip fracture and severe visual impairment. Several of these topic areas are covered by other Cochrane Reviews (Canning 2015; Verheyden 2013). We acknowledge that some individuals with these (and other) health conditions may be included in studies of the general community; these we included.

As in our protocol, we also included trials recruiting participants in hospital if the majority were discharged to the community, where the majority of the intervention was delivered and falls recorded. As we considered such trials, whose participants were recently discharged from hospital, to be a distinct category we reported them separately.

\section{Types of interventions}

This review included all exercise interventions tested in trials that measured falls in older people. The intention was to include trials where exercise was a single intervention as opposed to a component of a broader intervention. We included trials where an additional low-contact intervention (e.g. information on fall prevention) was given to one or both groups if we judged that the main purpose of the study was to investigate the role of exercise.

We classified exercise programmes on the basis of the primary exercise category and noted the presence of additional, secondary, exercise categories. Based on the Prevention of Falls Network Europe (ProFaNE) taxonomy (Lamb 2011), as shown in Appendix 1 , we classified exercise programmes in the included trials as primarily involving the following exercise categories: i) gait, balance, co-ordination and functional task training (referred to as 'balance and functional exercises' for simplicity); ii) strength/ resistance training (including power training, using resistance so referred to as 'resistance exercises'); iii) flexibility; iv) threedimensional (3D) exercise (with separate Tai Chi and dance subcategories); iv) general physical activity (walking programmes); v) endurance; and vi) other kinds of exercises. We also formed another category for exercise programmes that included more than one of the above categories as the primary exercise category, e.g. a programme with 15 minutes of gait, balance, coordination and functional task training followed by 15 minutes of strength/resistance training. We examined the descriptions of interventions used in individual trials and categorised the intervention accordingly. For example, some forms of yoga may have been categorised as flexibility exercise and others as 3D exercise.

We compared each of these types of exercise with control, comprising either 'usual care' (i.e. no change in usual activities) or a control intervention (i.e. an intervention that is not thought to reduce falls, such as general health education, social visits, very gentle exercise, or 'sham' exercise not expected to impact on falls).

We first undertook an 'umbrella' comparison of 'exercise (all types) versus control', explored the impact of the use of an increased risk of falls as a trial inclusion criterion and the impact of participant age on the overall impact of exercise on falls, then set out the following comparisons.
1. Balance and functional exercises versus control.
2. Resistance exercises versus control.
3. Flexibility training versus control.
4. 3D (including Tai Chi, Qigong) exercise versus control.
5. 3D (dance) exercise versus control. 
6. Walking programme versus control.

7. Endurance training versus control.

8. Other kinds of exercise versus control.

9. Multiple categories of exercise versus control (i.e. exercise programmes including more than one of the above categories versus control).

We also planned to undertake the following secondary comparisons of different exercise programmes.

1. Different types of exercise, based on the above categories.

2. Different modes of delivery (e.g. group versus individual) of the same type of exercise.

3. Different doses (e.g. higher intensity versus lower intensity) of the same type of exercise.

\section{Types of outcome measures}

\section{Primary outcomes}

1. Rate of falls (falls per person-year)

\section{Secondary outcomes}

1. Number of people who experienced one or more falls (risk of falling)

2. Number of people who experienced one or more fall-related fractures

3. Number of people who experienced one or more falls that resulted in hospital admission (newly listed outcome April 2018)

4. Number of people who experienced one or more falls that required medical attention

5. Health-related quality of life, measured using validated scale, e.g. EQ-5D or similar (newly listed outcome April 2018)

6. Number of people who experienced one or more adverse events (see below)

We chose 'rate of falls' as the single primary outcome for ease of interpretation of the results of the review. Furthermore, the rate of falls is likely to be more sensitive to change than the proportion of fallers, especially in samples with high fall rates. As falls are count data, dichotomisation to falling versus not falling represents a loss of information. Therefore, many trials use the rate of falls as their primary outcome and use negative binomial regression to compare the rates between intervention and control groups, as recommended in Robertson 2005.

Adverse events needed to be monitored closely in all groups using the same methods over the entire study period to be included in the analysis.

\section{Other outcomes}

We recorded and reported mortality data, distinguishing where possible, between those who were lost to the trials because they had died and those whose death was explicitly linked to trial participation.

We recorded and reported data regarding intervention adherence, cost and cost-effectiveness, where available.

\section{Timing of outcome measurement}

The primary outcome included one time point from each study. For studies with outcomes measured at multiple time points, we used the closest to 18 months in the primary analysis. We included a separate longer-term outcome for studies with follow-up at more than 18 months after randomisation. To maximise the use of available information, we also included studies with just one time point that was longer than 18 months in the primary analysis.

\section{Search methods for identification of studies}

\section{Electronic searches}

Our search extended the searches performed up to February 2012 in Gillespie 2012. We searched: the Cochrane Bone, Joint and Muscle Trauma Group Specialised Register (February 2012 to 2 May 2018); the Cochrane Central Register of Controlled Trials (CENTRAL) (Cochrane Register of Studies Online) (2012 Issue 2 to 2018 Issue 5); MEDLINE (including Epub Ahead of Print, In-Process \& Other NonIndexed Citations and MEDLINE Daily) (January 2012 to 30 April 2018); Embase (March 2012 to 2018 Week 18); the Cumulative Index to Nursing and Allied Health Literature (CINAHL) (February 2012 to 2 May 2018); and the Physiotherapy Evidence Database (PEDro) (2012 to 2 May 2018), using tailored search strategies. We did not apply any language restrictions.

In MEDLINE, we combined subject-specific search terms with the sensitivity- and precision-maximising version of the Cochrane Highly Sensitive Search Strategy for identifying randomised trials (Lefebvre 2011). The search strategies for CENTRAL, MEDLINE, Embase, CINAHL and PEDro are shown in Appendix 2.

We also searched the World Health Organisation International Clinical Trials Registry Platform (WHO ICTRP) and ClinicalTrials.gov for ongoing and recently completed trials (May 2018) (see Appendix 2).

\section{Searching other resources}

We checked reference lists of other systematic reviews as well as contacting researchers in the field to assist in the identification of ongoing and recently completed trials.

\section{Data collection and analysis}

The intended methodology for data collection and analysis was described in our published protocol (Sherrington 2016), which was based on the Cochrane Handbook for Systematic Reviews of Interventions (Higgins 2011).

\section{Selection of studies}

Pairs of review authors (CS, AT, NJF, ZAM) screened the title, abstract and descriptors of identified studies for possible inclusion. From the full text, two review authors (CS, AT, NJF, ZAM) independently assessed potentially eligible trials for inclusion and resolved any disagreement through discussion. We contacted authors for additional information as necessary.

\section{Data extraction and management}

Pairs of review authors (CS, AT, NJF, ZAM, GW) independently extracted data using a pretested data extraction form (based on the one used in Gillespie 2012). We extracted data from both newly included trials and those included in Gillespie 2012. For 
the latter trials, however, we primarily extracted information and data for additional outcomes that were not collected previously for Gillespie 2012. Disagreement was resolved by consensus or third party adjudication. Review authors were not blinded to authors and sources. Review authors did not assess their own trials.

We used the standardised data extraction form to record the following items.

1. General information: review author's name; date of data extraction; study ID; first author of study; author's contact address (if available); citation of paper; and trial objectives.

2. Trial details: trial design; location; setting; sample size; inclusion and exclusion criteria (with particular note of whether there was exclusion for cognitive impairment); comparability of groups; length of follow-up; stratification; stopping rules; and funding source.

3. 'Risk of bias' assessment and justification for this judgement: sequence generation; allocation concealment; blinding (participants, personnel, outcome assessors); incomplete outcome data; selective outcome reporting; and other bias (recall bias).

4. Characteristics of participants: age; gender; ethnicity; the number randomised, analysed and lost to follow-up; and dropouts in each arm (with reasons).

5. Interventions: experimental and control interventions; details of exercise programme (duration, frequency, intensity and individual- or group-based delivery, level of supervision); timing of intervention; uptake of intervention (acceptance of exercise intervention), whether studies assessed adherence (compliance) with interventions and associated data (e.g. number of sessions attended); and additional co-interventions (such as motivational strategies, additional information or support given to participants).

6. Outcomes measured: rate of falls; number of people experiencing one or more falls; number of people who experienced one or more fall-related fractures; number of people who experienced one or more falls requiring medical attention; and number of people who experienced adverse events.

7. Other details: cost and cost-effectiveness information related to fall outcomes.

We retrieved data from both full-text and abstract reports of studies. Where these sources did not provide sufficient information, we contacted study authors for additional details. We also used data sourced from personal communication reported by Gillespie 2012.

In response to feedback on an earlier draft of this review we extended our data extraction to extract data on the number of people who experienced one or more falls resulting in hospital admission, mortality and health-related quality of life (Differences between protocol and review).

We recorded and reported data on fracture, hospitalisation, medical attention, and health-related quality of life only where separate data were available by intervention group.

\section{Assessment of risk of bias in included studies}

Pairs of two review authors (CS, AT, NJF, ZAM, GW) independently assessed risk of bias using Cochrane's 'Risk of bias' tool as described in the Cochrane Handbook for Systematic Reviews of Interventions (Higgins 2011). Review authors were not blinded to authors and sources. Review authors did not assess their own trials. Disagreement was resolved by consensus or third party adjudication (CS)

As outlined in Appendix 3 we assessed the following domains: random sequence generation (selection bias); allocation concealment (selection bias); blinding of participants and personnel (performance bias); blinding of outcome assessment (detection bias); incomplete outcome data (attrition bias); and selective outcome reporting bias. We also assessed bias in the recall of falls due to less reliable methods of ascertainment (Hannan 2010). We rated risk of bias as either low, high or unclear for each domain.

Specifically for trials using cluster-randomisation, we considered the risk of additional bias relating to recruitment, baseline imbalance, loss of clusters, incorrect analysis and comparability with individually-randomised trials, as described in Chapter 16 of the Cochrane Handbook for Systematic Reviews of Interventions (Higgins 2011).

\section{Measures of treatment effect}

We reported the treatment effects for rate of falls as rate ratios (RaRs) with 95\% confidence intervals (Cls). For the number of fallers, number of participants experiencing fallrelated fractures, fall-related hospital admission, falls that required medical attention and adverse events, we reported risk ratios (RRs) and $95 \% \mathrm{Cls}$.

The rate of falls is the total number of falls per unit of persontime that falls were monitored (e.g. falls per person-year). The RaR compares the rate of falls in any two groups during each trial. We used a RaR (for example, incidence RaR or hazard ratio (HR) for all falls) with $95 \% \mathrm{Cl}$ if these were reported in the paper. If both adjusted and unadjusted RaRs were reported, we used the unadjusted estimate unless the adjustment was for clustering. If a RaR was not reported, but appropriate raw data were available, we used Excel to calculate a RaR and $95 \% \mathrm{Cl}$. We used the reported rate of falls (falls per person-year) in each group and the total number of falls for participants contributing data, or we calculated the rate of falls in each group from the total number of falls and the actual total length of time falls were monitored (person-years) for participants contributing data. In cases where data were only available for people who had completed the study, or where the trial authors had stated there were no losses to follow-up, we assumed that these participants had been followed up for the maximum possible period.

The risk ratio (RR) compares the number of people who fell once or more (fallers) between groups. We used a reported estimate of the RR, HR for first fall, or odds ratio (OR)) and $95 \% \mathrm{Cl}$ if available. If both adjusted and unadjusted estimates were reported we used the unadjusted estimate, unless the adjustment was for clustering. If an OR was reported, or an effect estimate and $95 \% \mathrm{Cl}$ was not, and appropriate data were available, we calculated a RR and 95\% $\mathrm{Cl}$ using the 'csi' command in Stata. For the calculations, we used the number of participants contributing data in each group, if 
this was known; if not reported, we used the number randomised to each group. The same approach was used for the number of people experiencing fractures, falls requiring medical attention and adverse events. Data regarding the number of people in each group experiencing the additional variables of falls resulting in hospitalisation and death were entered into Review Manager 5 directly (Review Manager 2014).

For continuous outcomes (health-related quality of life), we presented the mean difference (MD) with $95 \% \mathrm{Cls}$ where the same outcome measure was used, or standardised mean difference (SMD) with $95 \% \mathrm{Cls}$ for outcomes measured using different scales. Final values, which were used in preference to change scores, were always available where these outcomes were reported.

\section{Unit of analysis issues}

For trials which were cluster randomised, for example by medical practice, we performed adjustments for clustering, as described in Higgins 2011, if this was not done in the published report. We used an intraclass correlation coefficient (ICC) of 0.01 as reported in Smeeth 2002. We ignored the possibility of a clustering effect in trials that randomised by household. We anticipated that trials would be unlikely to report details of clustering by household and that the clustering effect by household would be very small (if any).

The pooled exercise versus control comparisons necessitated the inclusion of more than one pair-wise comparison (intervention versus control) from the same trial in the same meta-analysis. Where multiple comparisons from the same trial were included in the same meta-analysis the standard errors were inflated by $25 \%$ and the number of control participants shown in the analyses was 'shared' between different comparisons by dividing by the number of intervention groups in the same analysis. For example, if a trial had 100 participants in a control group, 100 participants in a resistance training group, and 100 participants in a balance training group, the standard errors in the resistance versus control and balance versus control comparisons would be inflated by $25 \%$ and the number of control participants would be shown as 50 in both the resistance versus control and balance versus control comparisons.

We did not include outcomes collected at different time points in the same trial in the same analysis.

\section{Dealing with missing data}

Some missing data are inevitable in studies of older people, given the increased risk of ill health and death, and the length of delivery of the intervention in fall prevention trials. We attempted to contact study investigators for any key missing or unclear data or information in their trial; clarification on outcome data was only sought for number of falls and number of people who experienced falls. We undertook sensitivity analyses excluding trials with more than $20 \%$ loss to follow-up or where the loss to follow-up was unclear.

\section{Assessment of heterogeneity}

Where we considered study interventions to be sufficiently similar to be combined in meta-analyses, we assessed heterogeneity of treatment effects by visual inspection of forest plots and by using the $\mathrm{Chi}^{2}$ test (with a significance level at $P<0.10$ ) and the $\mathrm{I}^{2}$ statistic. We based our interpretation of the $I^{2}$ results on that suggested by Higgins 2011: $0 \%$ to $40 \%$ might not be important; $30 \%$ to $60 \%$ may represent moderate heterogeneity; $50 \%$ to $90 \%$ may represent substantial heterogeneity; and $75 \%$ to $100 \%$ may represent very substantial ('considerable') heterogeneity.

\section{Assessment of reporting biases}

We constructed and visually inspected funnel plots for outcomes that included more than 10 data points.

\section{Data synthesis}

For our primary comparison, we pooled data from all relevant trials without stratification. We originally planned to present the umbrella comparison of exercise versus control subgrouped by the main exercise categories (Sherrington 2016). This change was made in response to editorial input and the request for additional subgroup and sensitivity analyses in a commissioning brief relating to the National Institute for Health and Care Excellence (NICE) guideline CG161 (NICE 2013).

We presented separate analyses for studies that recruited people in hospitals and delivered interventions after discharge as we considered these were a distinct population compared with general community-dwelling older adults.

We grouped similar exercise interventions using the fall prevention classification system (taxonomy) developed by the Prevention of Falls Network Europe (ProFaNE) (Lamb 2011). Full details are available in Appendix 1 and the ProFaNE Taxonomy Manual.

When considered appropriate, we pooled results of comparable studies using random-effects models. We used $95 \% \mathrm{Cls}$ throughout. We planned not to pool data where there was considerable heterogeneity $\left(I^{2} \geq 75 \%\right)$ that could not be explained by the diversity of methodological or clinical features among trials.

When considered appropriate, we pooled data using the generic inverse variance method in Review Manager 5 (Review Manager 2014). This method enables pooling of the adjusted and unadjusted treatment effect estimates (rate ratios or risk ratios) reported in the individual studies or which can be calculated from data presented in the published article (see Measures of treatment effect). The generic inverse variance option in Review Manager 5 requires entering the natural logarithm of the rate ratio or risk ratio and its standard error for each trial; we calculated these in Excel. For continuous outcomes (health-related quality of life), we presented MDs with $95 \% \mathrm{Cls}$ where the same outcome measure was used, or SMDs with $95 \% \mathrm{Cls}$ for outcomes measured using different scales.

Where it was inappropriate to pool data, we present trial-level data in the analyses and tables for illustrative purposes.

The statistician was not blind to study or group.

\section{Subgroup analysis and investigation of heterogeneity}

We undertook subgroup analyses for the fall and fracture outcomes for the pooled (all-exercise types) versus control analyses to compare the effect of exercise on falls and fractures in trials that did and did not use an increased risk of falls as an inclusion criterion. In response to a request (Differences between protocol and review) to explore the potential effects of stratification by age (based on a threshold of 75 years), we undertook subgroup analyses for the falls and fracture outcomes for the pooled (all-exercise types) versus 
control analyses. We compared the effects on falls outcomes in trials with predominantly older populations (defined by inclusion criteria 75 years or above, lower range limit more than 75 years, or mean age minus one standard deviation more than 75 years) and those with predominantly younger populations.

Prompted by feedback at editorial review, we extended the following subgroup analyses (originally established for different exercise categories) to the all-exercise types versus control for fall outcomes: a) individual versus group-based exercise; and b) exercise delivered by people with different qualifications (e.g. health professionals versus trained fitness leaders).

We presented separate analyses stratified by the different ProFaNE exercise intervention categories outlined above, and performed subgroup analyses for the fall and fracture outcomes. We then used subgroup analyses to explore effects within the different ProFaNE exercise intervention categories. When there were at least 10 trials in a comparison, we carried out subgroup analyses to compare effects in trials of: a) higher versus lower falls risk at enrolment (i.e. trials with participants selected for inclusion based on history of falling or other specific risk factors for falling versus trials with unselected participants); b) individual versus groupbased exercise; and c) exercise delivered by people with different qualifications.

We used the test for subgroup differences available in Review Manager 2014 to determine whether there was evidence for a difference in treatment effect between subgroups.

\section{Sensitivity analysis}

We carried out 10 sensitivity analyses to explore the stability of the results.

\section{Sensitivity analysis 1 (participant age)}

In response to a specific request (Differences between protocol and review) to explore the potential effects of changing the age threshold from 60 to 65 years for inclusion into the review, we set out a series of sensitivity analyses to explore the effects of removing trials that would have been excluded from the review if a 65 year or older inclusion threshold had been applied.

\section{Sensitivity analyses 2-5 (risk of bias in included trials)}

To assist with the GRADE rating we undertook sensitivity analyses for all outcomes in the 'Summary of findings' table by removing trials with a high risk of bias in any item.

To explore the possible impact of risk of bias on the primary pooled estimates of treatment effect, we examined the effects of the following.

1. Inclusion of trials at high or unclear risk of selection bias from inadequate concealment of allocation.

2. Inclusion of trials at high or unclear risk of detection bias from inadequate blinding of outcome assessors.

3. Inclusion of trials at high or unclear risk of attrition bias from incomplete outcome data.

\section{Sensitivity analyses 6-7 (meta-analysis decisions)}

We also examined the impact on the results of the removal of the cluster-randomised trials and the use of fixed-effect rather than random-effects models for data pooling.

\section{Sensitivity analysis 8 (multiple exercise category components)}

In order to assist in the interpretation of the results of the type of exercise subgroup 'multiple categories of exercise' comparisons, we undertook a sensitivity analyses for both falls outcomes which only included trials that were coded as having the two primary components balance/functional exercises and resistance exercises.

\section{Sensitivity analyses $9 a$ and $9 b$ (different exercise type coding)}

To explore the possible impact of how we classified exercise interventions, we examined the effects of the following for both falls outcomes.

1. Classification of interventions based on the Otago Exercise Program as multiple categories of exercise.

2. Classification of any intervention that included balance and functional exercises plus strength exercises as multiple categories of exercise.

\section{Assessing the certainty of evidence and 'Summary of findings' tables}

We used the GRADE approach to assess the quality of evidence related to all outcomes listed in the Types of outcome measures (Schünemann 2017). Using GRADEpro GDT (GRADEPro GDT 2015), we assessed the certainty of the evidence as 'high', 'moderate', 'low' or 'very low' depending on the presence and extent of five factors: risk of bias; inconsistency of effect; indirectness; imprecision; and publication bias. We prepared 'Summary of finding' tables featuring the seven listed outcomes for the umbrella comparison (exercise (all types) versus control) and for the rate of falls, risk of falling, fall-related fractures and adverse events for the primary exercise categories versus control comparisons, where data were available (Types of interventions). We used standardised qualitative statements to describe the different combinations of effect size and the certainty of evidence (Cochrane Norway 2017).

\section{RES U L T S}

\section{Description of studies}

\section{Results of the search}

A total of 8007 records were downloaded from the following databases: Cochrane Bone, Joint and Muscle Trauma Group Specialised Register (7), CENTRAL (1650), MEDLINE (1601), Embase (2998), CINAHL (1104), PEDro (139), the WHO ICTRP (317), and ClinicalTrials.gov (191). We identified 359 studies from a prior Cochrane Review (Gillespie 2012), and other systematic reviews. We also found one study after the search process in September 2018 ( $\mathrm{Li}$ 2018b)

Removal of duplicates and spurious records resulted in 4006 references. Upon screening of these, we excluded 3541 records and we obtained copies of 465 papers for consideration. A screening of these led to the removal of a further 230 records. The final round of study selection based on 235 reports resulted in the inclusion of 108 studies (194 reports), the exclusion of 21 studies (23 reports) (see Characteristics of excluded studies) and identification 
of 16 ongoing studies (Ongoing studies). Two further studies await classification (Jagdhane 2016; Li 2018b).

We contacted authors of two studies to request additional details to assess eligibility, and received responses from both studies; we included Hamrick 2017 and excluded Hinrichs 2016.
A flow diagram summarising the study selection process is shown in Figure 1. 
Figure 1. Study flow diagram.

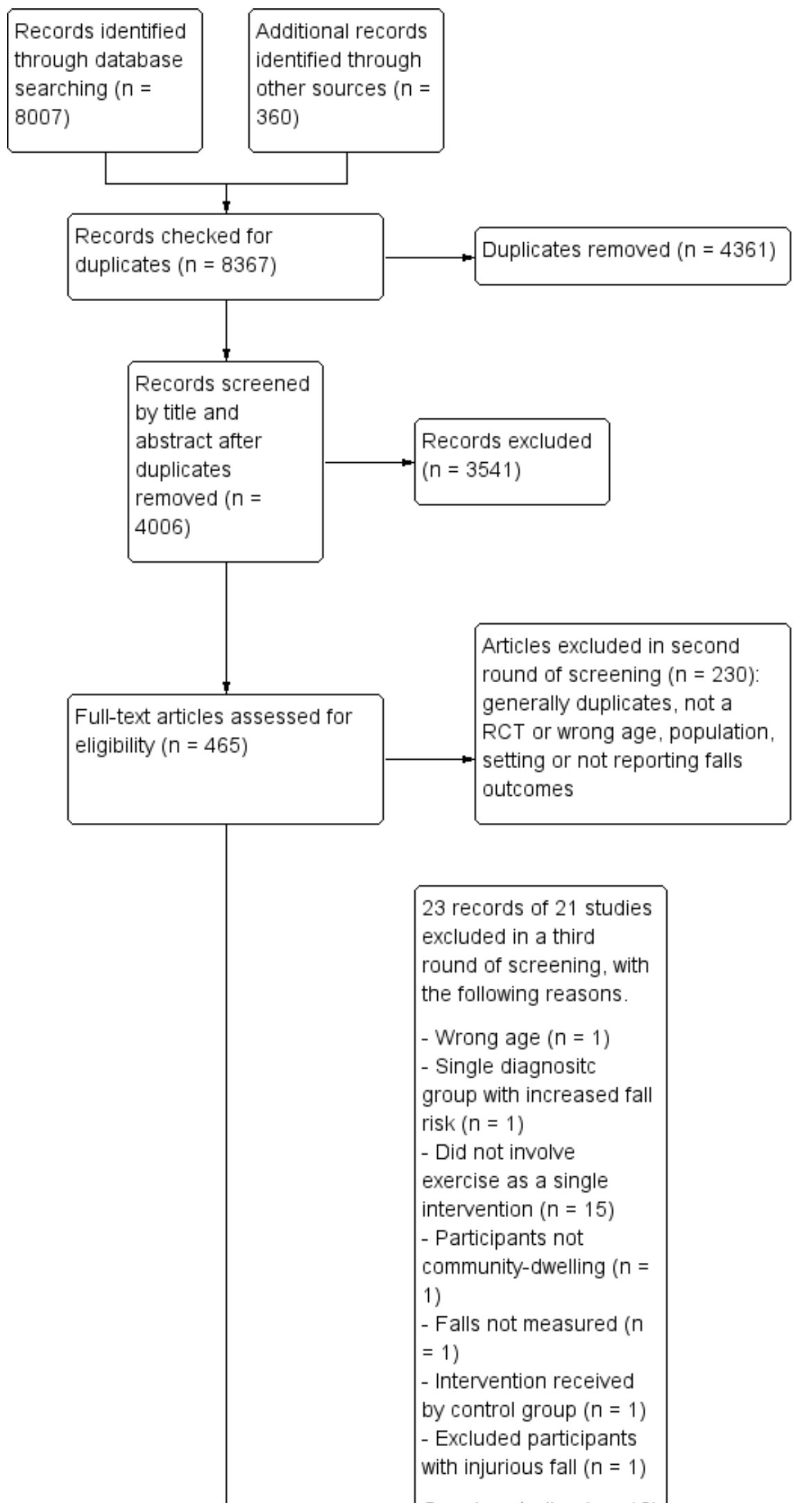


Figure 1. (Continued)

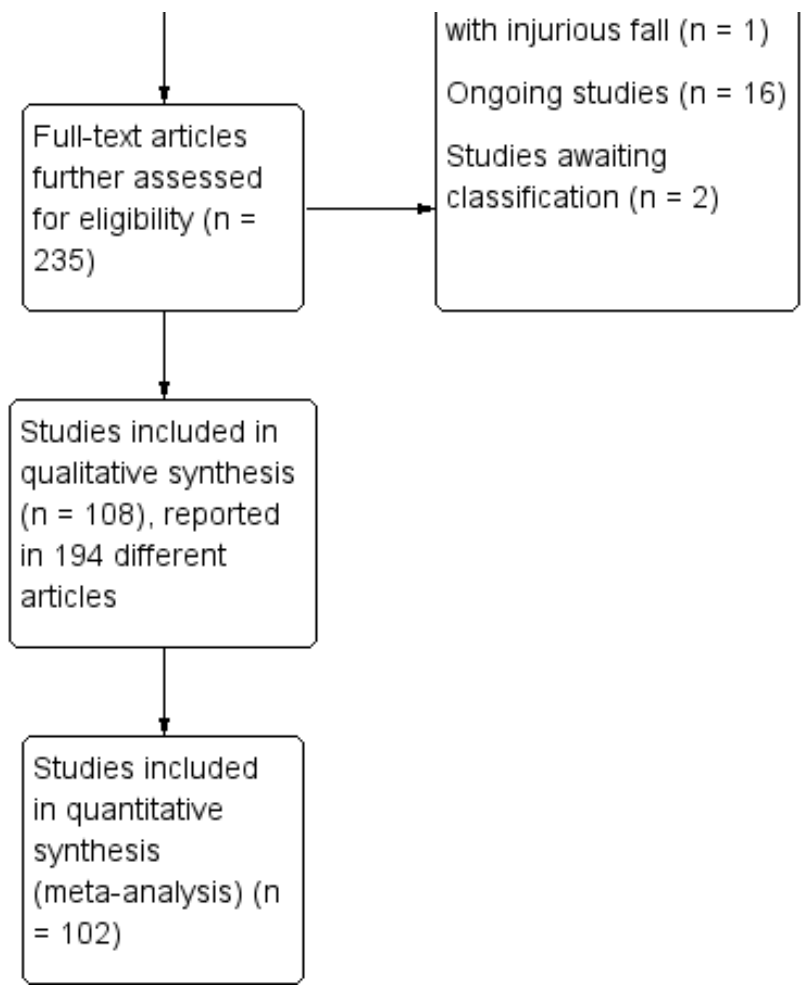

\section{Included studies}

This review includes 108 trials with 23,407 participants. Details are provided in the Characteristics of included studies and are briefly summarised below. Due to the size of the review, not all links to references have been inserted in the following text but can be viewed in Appendix 4. Characteristics of the included studies are summarised in Table 1 and Table 2.

We contacted authors of 49 included studies to request additional details regarding study design and outcome data and received responses for 26 trials; this resulted in additional information that is used in the review for 10 studies (Arkkukangas 2015; Clegg 2014; Dadgari 2016; Hamrick 2017; Kerse 2010; Kovacs 2013; Lord 2003; Morrison 2018; Sales 2017; Siegrist 2016). Trialists of the other 16 studies either reported they had no data to supply or they supplied data that could not be used in the review (Ansai 2015; Beyer 2007; Cerny 1998; Dangour 2011; Davis 2011; Duque 2013; Gschwind 2015; Huang 2010; Kyrdalen 2014; LaStayo 2017; Lurie 2013; Morgan 2004; Morone 2016; Okubo 2016; Park 2008; Resnick 2002). This account does not include the studies for which further information or data were sought or supplied regarding trials included in Gillespie 2012.

\section{Trial design}

All included studies were randomised controlled trials (RCTs). The majority of trials were individually randomised and nine were cluster randomised; either by unit of residence (Huang 2010; Lord 2003; Merom 2016; Wolf 2003), health centre (Dadgari 2016; Dangour 2011; Iliffe 2015; Siegrist 2016), or senior centre (Reinsch 1992). The included trials had 230 groups. Most trials $(n=95)$ had two groups included in this review (usually intervention and control), 10 studies had three groups (two intervention and one control: Almeida 2013; Ansai 2015; Clemson 2012; Halvarsson 2016;
Hirase 2015; Iliffe 2015; Liu-Ambrose 2004; Vogler 2009; Wolf 1996; Woo 2007; all intervention: Davis 2011; Wu 2010), and one study had four groups (3 intervention, 1 control) (Karinkanta 2007).

\section{Trial size}

The median number of participants randomised per trial was 134 (interquartile range (IQR) 65 to 262). The trials ranged in sample size from 20 participants in Resnick 2002 to 1635 participants in Gill 2016.

\section{Trial setting}

The included trials were carried out in 25 countries, the most common being Australia (19 trials), USA (18 trials), Japan (11 trials), the UK ( 7 trials), Finland (5 trials), Brazil (4 trials), Canada (4 trials), Germany (4 trials), New Zealand (4 trials), Sweden (4 trials), the Netherlands ( 3 trials), and Taiwan ( 3 trials). The remaining trials were conducted in Chile ( 2 trials), France ( 2 trials), Hungary ( 2 trials), Italy (2 trials), Norway (2 trials), Singapore (2 trials), China (1 trial), Denmark (1 trial), Iran (1 trial), Korea (1 trial), Switzerland (1 trial), Thailand ( 1 trial) and Turkey ( 1 trial). Of the three multinational trials, Gschwind 2015 included participants in Germany, Spain and Australia; Mirelman 2016 recruited from Belgium, Israel, Italy, Netherlands and the UK and Latham 2003 from Australia and New Zealand. See Appendix 4.

\section{Participants}

There were 23,407 participants randomised and 20,007 with fall data at follow-up. Overall, $77 \%$ of included participants were women. All participants were women in 28 trials (see Appendix 4), and men in one trial (Rubenstein 2000). The average participant age in the included trials was 76 years. 
The inclusion/exclusion criteria and other participant details are listed for each study in the Characteristics of included studies.

Sixteen trials (15\%) would have been excluded if the review inclusion criteria had been set at $65+$ years of age (see Appendix 4).

Sixty included studies (56\%) specified a history of falling or evidence of one or more risk factors for falling in their inclusion criteria (see Appendix 4).

Seventy-two trials (67\%) excluded participants with cognitive impairment, either defined as an exclusion criterion or implied by the stated requirement to be able to give informed consent and/or to follow instructions (see Appendix 4).

Four trials (4\%) only included people who had recently been discharged from hospital (Haines 2009; Latham 2003; Sherrington 2014; Vogler 2009). It is possible other trials also included some participants who had been recently discharged from hospital or the emergency department, however this was not quantified.

\section{Interventions}

Exercise was compared with a control intervention (one that is not thought to reduce falls, such as general health education, social visits, very gentle exercise, or 'sham' exercise) in 81 trials $(19,684$ participants) in people not recently discharged from hospital, and four trials (816 participants) in people who were recently discharged from hospital (Haines 2009; Latham 2003; Sherrington 2014; Vogler 2009). Twenty-three trials, with 3527 participants, compared the effect of different types of exercise in people not recently discharged from hospital, and one trial (180 participants) compared the effect of different types of exercise in the posthospital population (Vogler 2009). Four trials (1021 participants) compared group versus individual exercise (Barker 2016; Helbostad 2004; Iliffe 2015; Kyrdalen 2014), and three trials (879 participants) compared high-versus low-dose exercise (Ballard 2004; Davis 2011; Taylor 2012); see Appendix 4).

When interventions are grouped by the type of intervention (descriptors), as described in Data synthesis, there were 230 groups; 146 intervention arms and 84 control arms. There were 13 multiarm studies included in the review; 12 trials had three arms (Almeida 2013; Ansai 2015; Clemson 2012; Davis 2011; Halvarsson 2016; Hirase 2015; Iliffe 2015; Liu-Ambrose 2004; Vogler 2009; Wolf 1996; Woo 2007; Wu 2010), and one trial had four arms (Karinkanta 2007). Buchner 1997 had four arms; however, because fall data were not available for individual intervention groups we made an a priori decision to report fall outcomes for all three exercise groups combined compared with control group. In 76 (52\%) intervention arms, the exercise intervention was delivered in a group setting; in $43(29 \%)$ intervention arms, it was delivered individually; and 27 (18\%) intervention arms involved a combination of group-based and individual exercise (see Appendix 4). In 67 (46\%) intervention arms, the intervention was delivered by a health professional; in the $77(53 \%)$ intervention arms where the intervention was not delivered by a trained health professional, personnel included trained physical educators, trained exercise leaders and Tai Chi instructors; in one intervention arm, the intervention was delivered by both types of personnel (Sales 2017); and in one trial the personnel were not specified (Park 2008).
The intervention arms were grouped by their primary exercise modality into six categories (Appendix 5) using the ProFaNE taxonomy (Appendix 1).

1. Most intervention arms ( $n=78 ; 53 \%$ ) included balance and functional exercises as the primary intervention (ProFaNE taxonomy code gait/balance/co-ordination/functional task training).

2. Strength/resistance training was the primary component of 9 (6\%) intervention arms.

3. Flexibility training was the primary component of one $(1 \%)$ intervention arms.

4. $3 \mathrm{D}$ training (constant repetitive movement through all three spatial planes) was the primary component of $15(10 \%)$ intervention arms.

5. General physical activity (walking groups) was the primary component of $6(4 \%)$ intervention arms.

6. Endurance training alone was the primary component of one $(1 \%)$ intervention arm.

7. Multiple categories of ProFaNE taxonomy were the primary intervention in $37(25 \%)$ intervention arms. The majority (n $=19,51 \%$ ) of these intervention arms included balance and functional exercise as well as resistance training.

The number of studies, and how many of these are clusterRCTs, for the main exercise versus control comparison for each primary exercise category is summarised below, with further details including numbers of participants presented in Table 3 , and associated study IDs in Appendix 6 (all trials) and Appendix 7 (trials contributing data to the rate of falls analysis). Note that these do not include the four post-hospital discharge RCTs.

1. Exercise (all types) versus control: 81 RCTs (9 cluster-RCTs).

2. Balance and functional exercises versus control: 48 RCTs (6 cluster-RCTs).

3. Resistance exercises versus control: 7 RCTs.

4. Flexibility versus control: 0 RCTs.

5. 3D exercise (Tai Chi) versus control: $10 \mathrm{RCTs}$ (2 cluster-RCTs).

6. 3D exercise (dance) versus control: 1 RCTs (1 cluster-RCT).

7. General physical activity (walking programme) versus control: 3 RCTs.

8. Endurance training versus control: 0 RCTs.

9. Other kinds of exercise versus control: 0 RCTs.

10. Multiple categories of exercise versus control: 21 RCTs.

The duration of the exercise intervention in these 81 trials ranged from 5 to 130 weeks; it was one year or more in 24 trials (30\%) and two years or more in five trials (6\%) (Table 2).

Additional details of the number of studies and number of participants included in the primary analysis (exercise versus control on rate of falls) for each primary category of exercise are shown in Appendix 8.

\section{Outcomes}

The source of data used for calculating outcomes for each trial for generic inverse variance analysis is shown in Appendix 9. Rate of falls was reported in 34 trials, and could be calculated from a further 43 trials. Data on risk of falling (number of fallers) were available in 17 trials and could be calculated for a further 61 . Raw 
data for rate of falls and number of fallers, when available, are shown in Appendix 10. Six trials met our inclusion criteria but did not include data that could be included in these analyses (Almeida 2013; Fiatarone 1997; Mirelman 2016; Morone 2016; Morrison 2018; Resnick 2002). Two of these trials contained inadequate data to include in an analysis (Fiatarone 1997; Resnick 2002), but reported no significant between-group difference in number of falls, and two trials reported zero falls in each group (Almeida 2013; Morrison 2018). Morone 2016 did not present fall data, but found balance training using Wii-fit may have a greater effect on balance outcomes compared with conventional balance training. Mirelman 2016 found treadmill plus virtual reality training may be more effective in preventing falls than treadmill alone, six months after the end of a six-week training period. The raw data for non-fall outcomes for these studies are shown in Appendix 11.

Eleven trials reported a fracture outcome, two trials reported number of falls requiring hospitalisation, and five trials reported the number of people experiencing a fall requiring medical attention. Death was recorded in 40 trials and was listed as a reason for loss to follow-up in all of these trials except Wolf 2003, which also assessed death as an adverse event. Deaths were not reported by group in two trials (Day 2002; Lord 1995; Appendix 12). None of the deaths were explicitly linked to the trial participation.

\section{Adverse events}

Two trials, including one in the post-hospital population, measured the number of people experiencing adverse events in both groups throughout the trial period (Iliffe 2015; Latham 2003). No other studies reported adverse events that were monitored closely in all groups over the entire study period. Adverse events reported to any degree are described in Appendix 13. Adverse events were reported to a degree in the intervention and control groups in 16 trials, in the intervention group only in 13 trials, in two intervention groups in seven trials, and in two intervention plus control group in five trials.

\section{Adherence}

Adherence was measured in 78 studies and adherence data were reported in 77 studies (Appendix 14). The measures used to quantify adherence varied: the majority of studies summarised proportion of classes attended $(n=53)$ or proportion of scheduled sessions completed $(n=20)$, three studies quantified the amount of exercise performed (Boongrid 2017; Okubo 2016; Sherrington 2014), and two studies described the proportion of participants who started the programme (El-Khoury 2015; Skelton 2005).

\section{Excluded studies}

We eliminated 253 reports on full-text review. We retained 21 studies (23 reports) as excluded studies as they initially appeared to meet the inclusion criteria but were subsequently excluded (see Excluded studies for links to references, and the Characteristics of excluded studies and Appendix 15 for details). Of the identified trials:
1. one trial did not meet the review's inclusion criterion for age (Pereira 1998);

2. one trial included participants with a particular clinical condition that increases the risk of falls (Hsu 2017);

3. one trial included participants who were not communitydwelling (DeSure 2013);

4. 15 trials did not involve exercise as a single intervention;

5. one trial included an ineligible comparator (Ohtake 2013);

6. one trial did not measure falls (Hinrichs 2016);

7. one trial withdrew three of the six fallers from the study because the falls resulted in injuries (Morris 2008).

\section{Studies awaiting classification}

Two studies are awaiting classification. Li 2018b is a large study (n =670) comparing the effect of Tai Ji Quan, multimodal exercise and stretching in older people at high risk of falls. The other is a small $(n=6)$ study (Jagdhane 2016).

\section{Ongoing studies}

We identified 16 ongoing trials (see the Characteristics of ongoing studies). Seven trials are currently open to recruitment CTRI/2018/01/011214; NCT02617303; NCT02926105; NCT03211429; NCT03320668; NCT03417531; NCT03462654), and nine are ongoing but no longer recruiting (ACTRN 12613001161718; ACTRN 12615000138583; ACTRN 12615000865516; ISRCTN71002650; NCT01029171; NCT02126488; NCT02287740; NCT03404830; NCT03455179).

The median target sample size is 402 (IQR 280-670) and two of the ongoing trials are cluster randomised (ACTRN 12613001161718; ISRCTN71002650). Half of the trials (8/16, 50\%) specify increased fall-risk as an inclusion criterion. Eight studies are investigating the effect of a programme of multiple categories of exercise (ACTRN 12615000865516; CTRI/2018/01/011214; ISRCTN71002650; NCT01029171; NCT02287740; NCT02617303; NCT02926105; NCT03455179), including four using the Otago Exercise Program (ACTRN 12615000865516; NCT01029171; NCT02617303; NCT02926105). There are three trials on resistance training (ACTRN 12613001161718; NCT03404830; NCT03455179), one on Tai Chi (NCT03211429), one on balance training (ACTRN 12615000138583), and a study evaluating slip training on the treadmill (NCT02126488). Two studies compare group versus individual delivery, using the LiFE Program (NCT03462654) and Otago Exercise Program (NCT03320668). There are no studies investigating the effect of flexibility training, general physical activity or endurance training alone.

\section{Risk of bias in included studies}

Details of the 'Risk of bias' assessment across all included trials and for each individual item in the included trials are shown in Characteristics of included studies, Figure 2 and Figure 3. 
Figure 2. Risk of bias graph: review authors' judgements about each risk of bias item presented as percentages across all included studies.

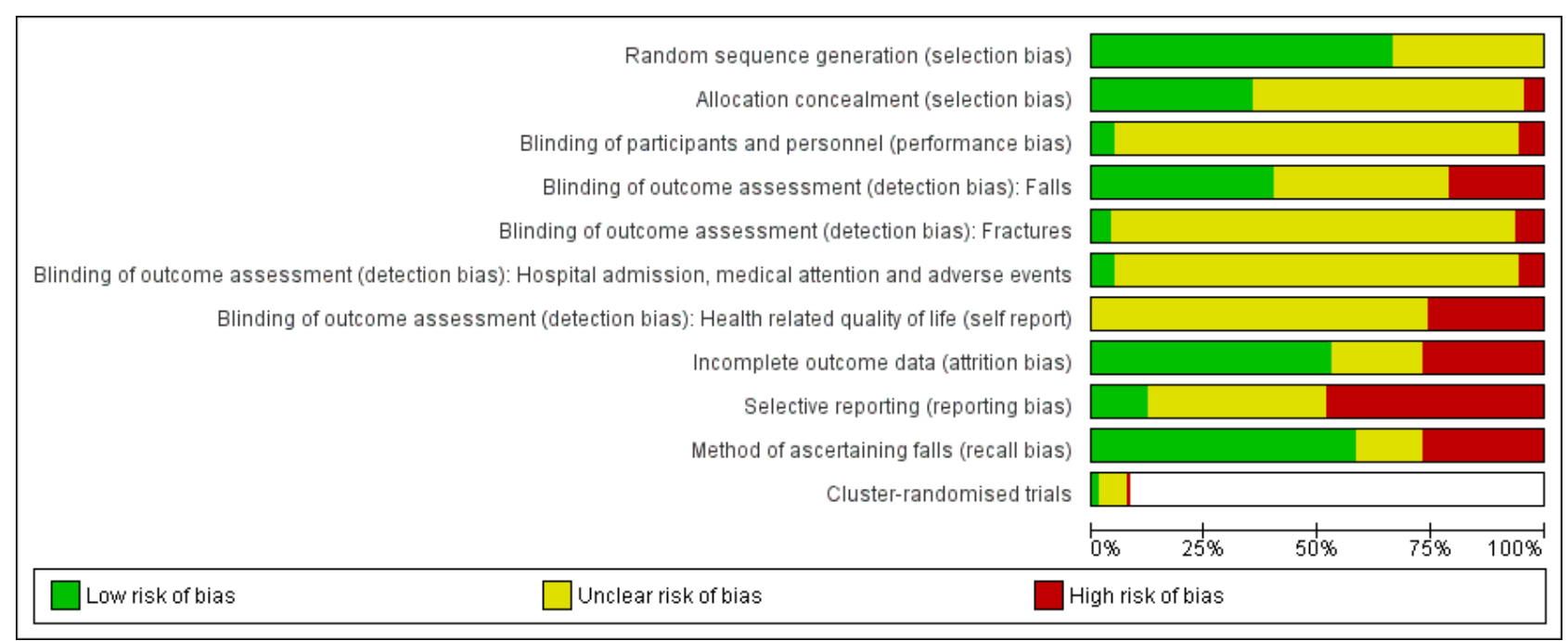


Figure 3. Risk of bias summary: review authors' judgements about each risk of bias item for each included study.

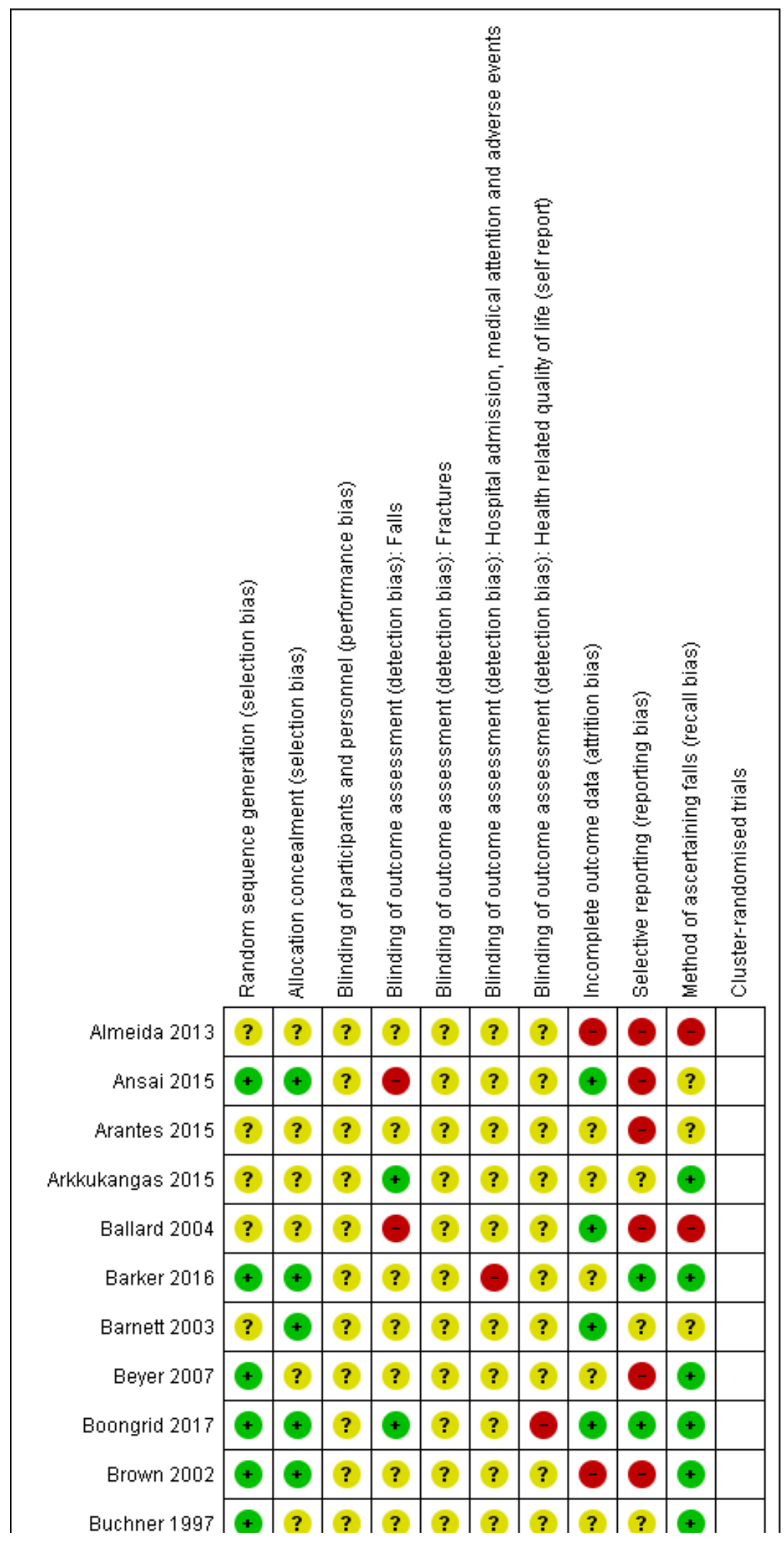


Figure 3. (Continued)

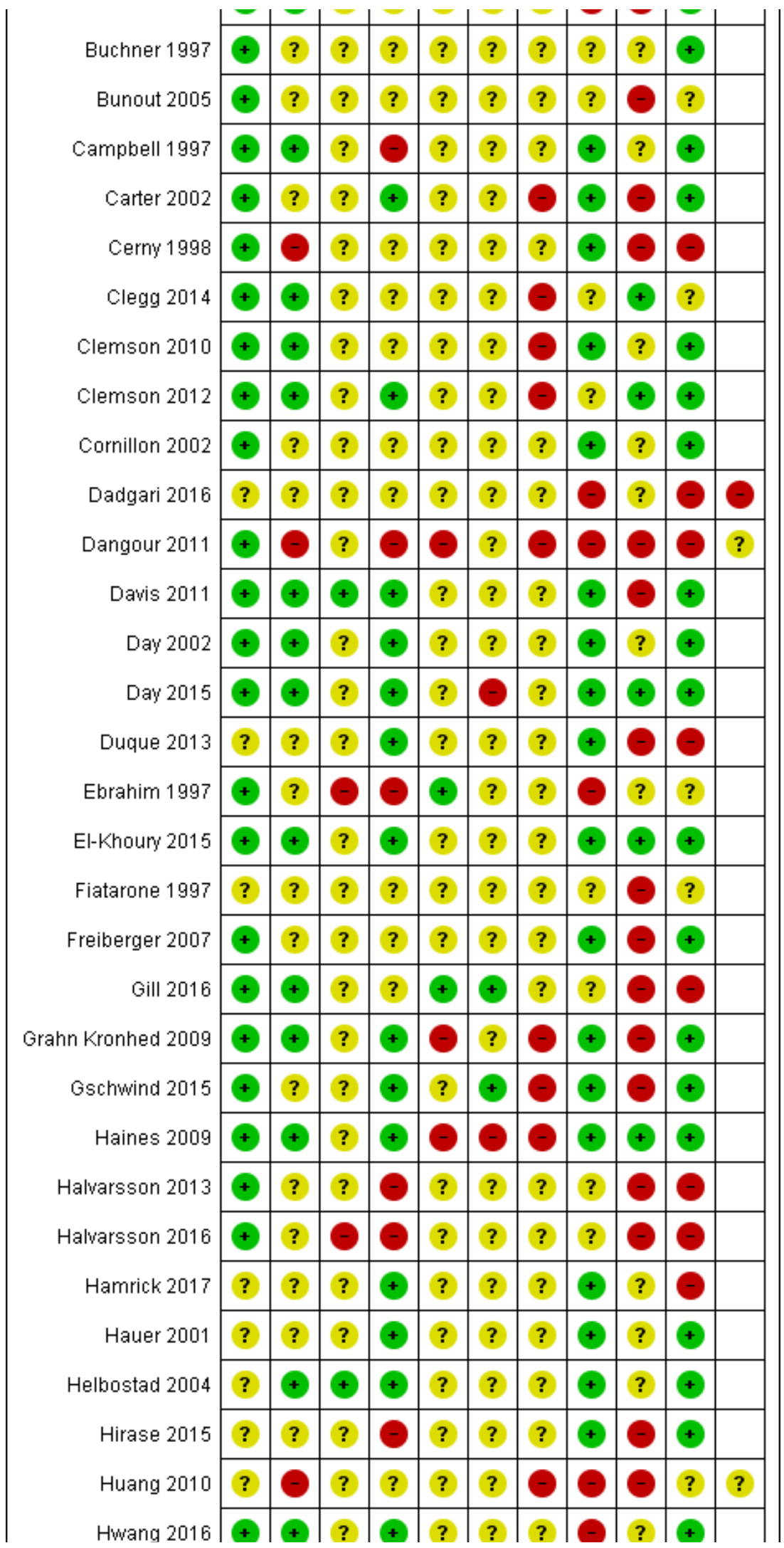


Figure 3. (Continued)

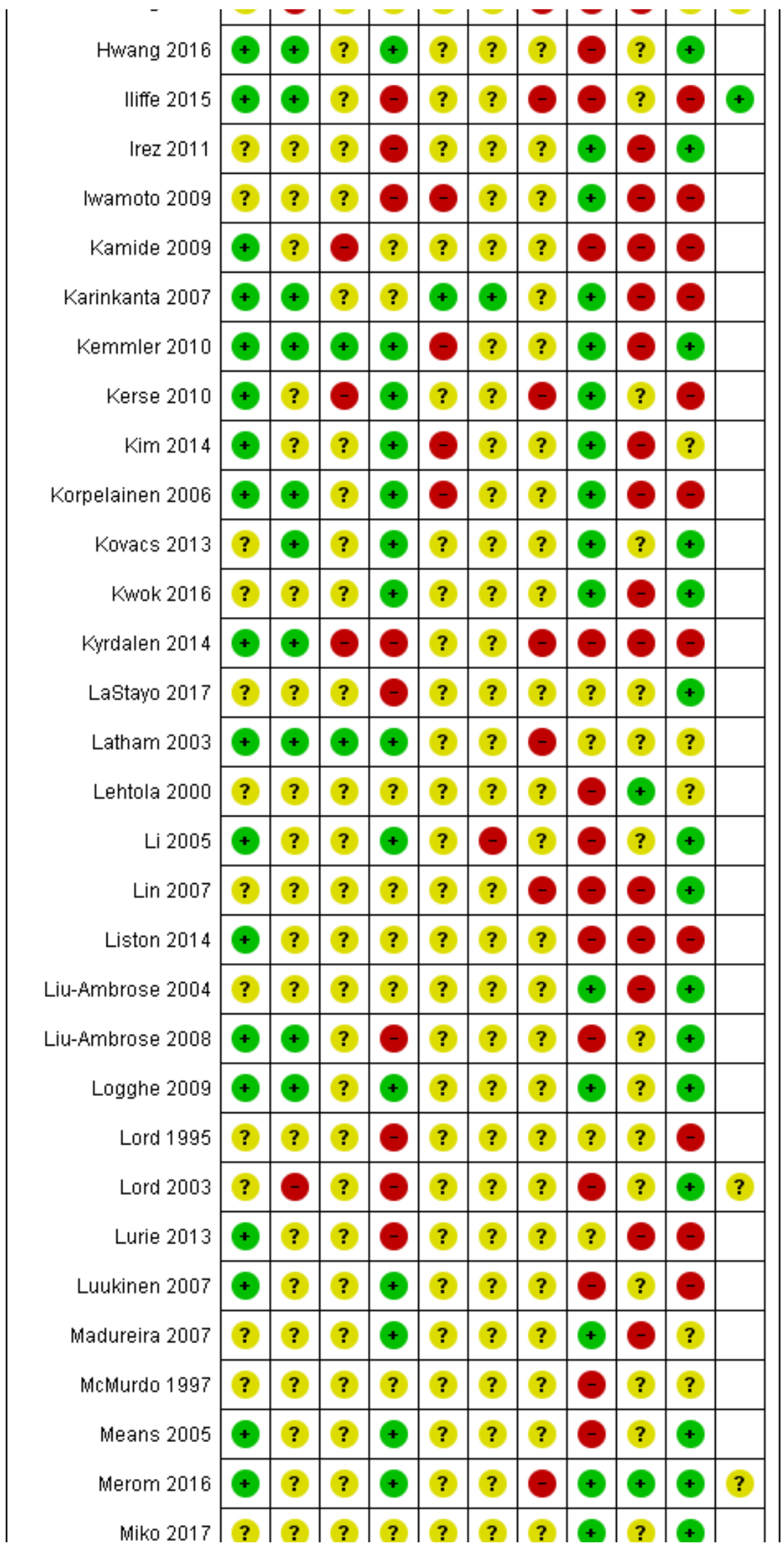


Figure 3. (Continued)

\begin{tabular}{|c|c|c|c|c|c|c|c|c|c|c|c|}
\hline Miko 2017 & $?$ & $?$ & $?$ & $?$ & $?$ & $?$ & $?$ & $\odot$ & $?$ & + & \\
\hline Mirelman 2016 & $\oplus$ & + & $?$ & + & $?$ & $?$ & $\odot$ & + & O & $\oplus$ & \\
\hline Morgan 2004 & $?$ & $?$ & $?$ & $?$ & $?$ & $?$ & $?$ & O & - & + & \\
\hline Morone 2016 & 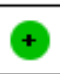 & + & $?$ & $?$ & $?$ & $?$ & $\odot$ & $\odot$ & 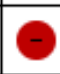 & $?$ & \\
\hline Morrison 2018 & + & $?$ & $?$ & $?$ & $?$ & $?$ & $?$ & - & - & $\odot$ & \\
\hline Ng 2015 & + & + & $?$ & + & $?$ & - & $?$ & + & - & $\odot$ & \\
\hline Nitz 2004 & $\odot$ & $?$ & $?$ & $\odot$ & $?$ & $?$ & $?$ & $\odot$ & O & + & \\
\hline Okubo 2016 & $\odot$ & $?$ & $?$ & $\odot$ & $?$ & $?$ & $?$ & $?$ & 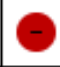 & + & \\
\hline Park 2008 & + & $?$ & $?$ & $?$ & $?$ & $?$ & $?$ & + & - & $\odot$ & \\
\hline Reinsch 1992 & $?$ & $\odot$ & $?$ & $?$ & $?$ & $?$ & $?$ & $\odot$ & - & $\odot$ & $?$ \\
\hline Resnick 2002 & + & $?$ & $?$ & $?$ & $?$ & $?$ & $\odot$ & $\odot$ & - & $?$ & \\
\hline Robertson 2001a & + & + & $?$ & $\odot$ & + & + & $?$ & + & $?$ & + & \\
\hline Rubenstein 2000 & + & $?$ & $?$ & - & $?$ & $?$ & $?$ & + & $?$ & $?$ & \\
\hline Sakamoto 2013 & $\odot$ & $?$ & $?$ & $?$ & $?$ & $?$ & $?$ & $\odot$ & $?$ & $\oplus$ & \\
\hline Sales 2017 & + & $?$ & $?$ & - & $?$ & $\odot$ & $\odot$ & $\odot$ & + & + & \\
\hline Sherrington 2014 & $\odot$ & + & $?$ & + & $?$ & + & $\odot$ & + & + & + & \\
\hline Shigematsu 2008 & $\oplus$ & $?$ & + & $\odot$ & $?$ & $?$ & $?$ & + & $?$ & + & \\
\hline Siegrist 2016 & $\oplus$ & $?$ & $?$ & + & $?$ & $?$ & $?$ & + & + & + & $?$ \\
\hline Skelton 2005 & $\odot$ & $?$ & $?$ & $\odot$ & $?$ & $?$ & $?$ & $\odot$ & $?$ & $\odot$ & \\
\hline Smulders 2010 & $?$ & $?$ & $?$ & + & $?$ & $?$ & $\odot$ & + & $?$ & + & \\
\hline Steadman 2003 & $\oplus$ & $?$ & $?$ & + & $?$ & $?$ & $\odot$ & $\odot$ & - & $\odot$ & \\
\hline Suzuki 2004 & $?$ & $?$ & $?$ & $?$ & $?$ & $?$ & $?$ & $?$ & $?$ & $\odot$ & \\
\hline Taylor 2012 & $\odot$ & + & $?$ & + & $?$ & $?$ & $?$ & + & $?$ & + & \\
\hline Trombetti 2011 & + & + & $?$ & $?$ & $?$ & $?$ & $?$ & + & $?$ & + & \\
\hline Uusi-Rasi 2015 & $\odot$ & $?$ & $?$ & $?$ & $?$ & $?$ & $?$ & $?$ & $?$ & $\odot$ & \\
\hline Verrusio 2017 & 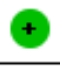 & $?$ & $?$ & $?$ & $?$ & $?$ & $\odot$ & + & - & + & \\
\hline Vogler 2009 & $\odot$ & $\odot$ & $?$ & $\odot$ & $?$ & $?$ & $\odot$ & + & - & + & \\
\hline Voukelatos 2007 & $\odot$ & $?$ & $?$ & + & $?$ & $?$ & $?$ & $\oplus$ & $?$ & + & \\
\hline Voukelatos 2015 & $\odot$ & + & $?$ & $?$ & $?$ & $?$ & $\odot$ & $?$ & $?$ & $\odot$ & \\
\hline Weerdesteyn 2006 & $?$ & $?$ & $?$ & $\odot$ & $?$ & $?$ & $?$ & + & $?$ & 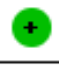 & \\
\hline Wolf 1996 & $\oplus$ & $?$ & $?$ & $?$ & $?$ & $?$ & $?$ & + & O & + & \\
\hline
\end{tabular}


Figure 3. (Continued)

\begin{tabular}{|c|c|c|c|c|c|c|c|c|c|c|c|}
\hline Wolf 1996 & + & $?$ & $?$ & $?$ & $?$ & $?$ & $?$ & + & $O$ & + & \\
\hline Wolf 2003 & $?$ & $?$ & $?$ & $\oplus$ & $?$ & $?$ & $?$ & $\odot$ & $?$ & 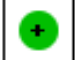 & $?$ \\
\hline Woo 2007 & + & $?$ & $?$ & - & $?$ & $?$ & $?$ & - & - & - & \\
\hline Wu 2010 & $?$ & $?$ & $?$ & $?$ & $?$ & $?$ & - & + & - & - & \\
\hline Yamada 2010 & $?$ & $\odot$ & $?$ & $?$ & $?$ & $?$ & $?$ & $?$ & $?$ & + & \\
\hline Yamada 2012 & $?$ & $\odot$ & $\ominus$ & $?$ & $?$ & $?$ & $?$ & + & $?$ & + & \\
\hline Yamada 2013 & $?$ & $?$ & $?$ & $?$ & $?$ & $?$ & $?$ & $?$ & + & + & \\
\hline Yang 2012 & + & $?$ & $?$ & + & $?$ & $?$ & $\theta$ & $\theta$ & $\theta$ & $\theta$ & \\
\hline
\end{tabular}

\section{Allocation}

We judged the risk of bias in generation of the allocation sequence as low in $67 \%(n=72 / 108)$ of trials, unclear in 33\% $(n=36 / 108)$ and high in zero trials. We assessed the methods of concealment of the allocation prior to group assignment as low risk of bias in $35 \%$ ( $n=$ $38 / 108)$, unclear in $60 \%(n=65 / 108)$ and high in the remaining $5 \%$ (5/108) of trials (Cerny 1998; Dangour 2011; Huang 2010; Lord 2003; Reinsch 1992).

\section{Blinding}

\section{Blinding of participants and personnel}

In the majority of studies $(90 \%, n=97 / 108)$ it was not possible to blind the personnel and participants to group allocation. As the likelihood of awareness of group allocation introducing performance bias was not clear, we assessed the risk of bias for non-blinding as unclear for these trials. We judged the impact of performance bias as low in $5 \%(n=5 / 108)$ of trials, unclear in $89 \%$ $(97 / 108)$ of trials and high in $6 \%(6 / 108)$ of trials.

\section{Blinding of outcome assessment}

We assessed the risk of bias for blinding of outcome assessment separately for the following outcomes.

\section{Rate of falls and risk of falling}

a. We judged the risk of detection bias in relation to the methods of ascertainment of the rate and/or risk of falls to be low in $40 \%(n=43 / 108)$, high in $21 \%(n=23 / 108)$ and unclear in $39 \%(n=42 / 108)$ of the included trials.

2. Risk of fractures

a. In trials reporting on the risk of fracture, we assessed the risk of bias for blinding of outcome assessment for the rate of fractures. We judged the risk of detection bias in relation to the methods of ascertainment of fractures to be low in $20 \%$ $(n=4 / 20)$, high in $35 \%(n=7 / 20)$ and unclear in $45 \%(n=9 / 20)$ of the included trials that measured fractures.

3. Requiring hospital admission/medical attention, adverse events a. In trials reporting on the risk of hospital admission and/ or requiring medical attention and/or adverse events, we judged the risk of detection bias in relation to the method of ascertainment of these outcomes to be low in 15\% (5/33) of trials, unclear in $67 \%(22 / 33)$ and high in $18 \%(6 / 33)$ of trials.
4. Health-related quality of life

a. In trials reporting on health-related quality of life we judged the risk of detection bias in relation to the method of ascertainment of health-related quality of life to be high in all studies (23/23), due to participants in these studies being unblinded to their allocated group and health-related quality of life being a self-reported outcome.

\section{Incomplete outcome data}

We judged the risk of bias due to incomplete outcome data to be low in $53 \%(n=57 / 108)$, unclear in $20 \%(n=22 / 108)$ and high in the remaining $27 \%$ of trials $(n=29 / 108)$.

\section{Selective reporting}

We assessed the risk of bias due to selective reporting of falls outcomes as low in $12 \%(n=13 / 108)$ of studies, unclear in $40 \%(n=$ $43 / 108)$ and high in 48\% (52/108).

\section{Other potential sources of bias}

\section{Bias in the recall of falls due to less reliable methods of ascertainment}

We assessed $58 \%$ of included studies $(n=63 / 108)$ as being at low risk of bias in the recall of falls (i.e. falls were recorded concurrently using recommended methods of monthly diaries or postcards). We judged the risk of bias to be high in $27 \%$ of trials $(n=29 / 108)$, in that ascertainment of falling episodes was by participant recall, at intervals during the study or at its conclusion. In $15 \%$ of trials ( $n=$ $16 / 108$ ) the risk of bias was unclear, as retrospective recall was for a short period only, or details of ascertainment were not described.

\section{Bias due to cluster-randomisation}

We assessed the nine cluster-randomised trials for risk of bias associated with recruitment methods, baseline imbalance, loss of clusters, incorrect analysis and comparability with individuallyrandomised trials. We judged the risk of bias due to factors associated with cluster-randomised trials to be low in one $(11 \%)$ trial, unclear in seven trials (78\%) and high in the remaining trial (11\%, Dadgari 2016). 


\section{Effects of interventions}

See: Summary of findings for the main comparison Summary of findings: exercise (all types) versus control (e.g. usual activities); Summary of findings 2 Summary of findings: balance and functional exercises versus control (e.g. usual activities); Summary of findings 3 Summary of findings: resistance exercises versus control (e.g. usual activities); Summary of findings 4 Summary of findings: 3D (Tai Chi) exercise versus control (e.g. usual activities); Summary of findings 5 Summary of findings: 3D (dance) exercise versus control (e.g. usual activities); Summary of findings 6 Summary of findings: walking programme (general physical activity) versus control (e.g. usual activities); Summary of findings 7 Summary of findings: multiple categories of exercise versus control (e.g. usual activities)

\section{Exercise (all types) versus control}

\section{Overview of results reporting format}

For each outcome described below we report the overall pooled effects of all exercise interventions (including the subgroup analyses for age, baseline risk of falling, personnel, and group delivery, for the falls outcomes) then the effects in studies testing interventions within each exercise category of the ProFaNE taxonomy (Appendix 1; Appendix 5), as well as the results of studies of exercise interventions that included multiple categories. For analyses with more than 10 included comparisons (both rate of falls and number of people experiencing one or more falls comparisons for balance and functional exercises, and multiple categories of exercise) we also report the results of the three prespecified subgroup analyses (increased fall risk as a study entry criterion, exercise delivery by a health professional, group versus individual delivery).

The findings are summarised and the absolute impact of interventions illustrated in 'Summary of findings' tables for the overall 'exercise versus control' comparison and for separate primary exercise categories for which there are data. No trials compared primarily flexibility exercise, endurance exercise or other exercise type versus control.

The results for the four trials comparing exercise (all types) versus control in people who had been recently discharged from hospital are presented separately, after this main comparison.

\section{Rate of falls (falls per person-year)}

Exercise (all types) reduces the rate of falls by $23 \%$ compared with control (rate ratio (RaR) 0.77 , 95\% confidence interval $(\mathrm{Cl}) 0.71$ to $0.83 ; 12,981$ participants, 59 studies, $I^{2}=55 \%$; high-certainty evidence; Analysis 1.1).

Subgroup analysis by falls risk at baseline, found there was probably little or no difference in the effect of exercise (all types) on the rate of falls in trials where all participants were at an increased risk of falling (RaR $0.80,95 \% \mathrm{Cl} 0.72$ to 0.88 ; 6858 participants, 30 studies, $I^{2}=56 \%$ ) compared with trials that did not use increased risk of falling as an entry criterion ( $\mathrm{RaR} 0.74,95 \% \mathrm{Cl} 0.65$ to 0.84; 6123 participants, 29 studies, $I^{2}=53 \%$ ); test for subgroup differences: $\mathrm{Chi}^{2}=0.90, \mathrm{df}=1, \mathrm{P}=0.34, \mathrm{I}^{2}=0 \%$ (Analysis 1.2).

Subgroup analysis by participant age found there was probably little or no difference in the effect of exercise (all types) on the rate of falls in trials where participants were aged 75 years or older ( $R a R$
$0.83,95 \% \mathrm{Cl} 0.72$ to $0.97 ; 3376$ participants, 13 studies, $\mathrm{I}^{2}=54 \%$ ) compared with trials where participants were aged less than 75 years (RaR $0.75,95 \% \mathrm{Cl} 0.69$ to $0.82 ; 9605$ participants, 46 studies, $\left.\mathrm{I}^{2}=55 \%\right)$; test for subgroup differences: $\mathrm{Chi}^{2}=1.36, \mathrm{df}=1, \mathrm{P}=0.24$, $\mathrm{I}^{2}=27 \%$ (Analysis 1.3).

Subgroup analyses found a larger effect of exercise (all types) in trials where interventions were delivered by a health professional (RaR $0.69,95 \% \mathrm{Cl} 0.61$ to $0.79 ; 4511$ participants, 25 studies, $\mathrm{I}^{2}$ $=47 \%$ ) than in trials where the interventions were delivered by trained instructors who were not health professionals (RaR 0.82, $95 \% \mathrm{Cl} 0.75$ to $0.90 ; 8470$ participants, 34 studies, $I^{2}=57 \%$ ); test for subgroup differences: $\mathrm{Chi}^{2}=4.44, \mathrm{df}=1, \mathrm{P}=0.04, \mathrm{I}^{2}=78 \%$ (Analysis 1.4). Notably, both approaches resulted in reductions in the rate of falls.

Subgroup analyses found there may be no difference in the effect of exercise (all types) on the rate of falls where interventions were delivered in a group setting ( $\mathrm{RaR} 0.76,95 \% \mathrm{Cl} 0.69$ to $0.85 ; 8163$ participants, 40 studies, $\mathrm{I}^{2}=62 \%$ ) compared with trials where interventions were delivered individually $(\mathrm{RaR} 0.79,95 \% \mathrm{Cl} 0.71$ to 0.88 ; 4818 participants, 21 studies, $I^{2}=35 \%$ ); test for subgroup differences: Chi $^{2}=0.21, d f=1, P=0.65, I^{2}=0 \%$ (Analysis 1.5). Two three-group studies, appear in both subgroups (Iliffe 2015; Wolf 1996).

Subgroup analysis by exercise type showed a variation in the effects of the different types of exercise on rate of falls, the visual impression being confirmed by the statistically significant test for subgroup differences: $\mathrm{Chi}^{2}=17.18, \mathrm{df}=5, \mathrm{P}=0.004, \mathrm{I}^{2}=70.9 \%$ (Analysis 1.6).

\section{Different categories of primary exercise versus control}

\section{Balance and functional exercises versus control}

Exercise interventions that were classified as being primarily gait, balance, co-ordination or functional task training using the ProFaNE taxonomy, reduce the rate of falls by $24 \%$ compared with control (RaR $0.76,95 \% \mathrm{Cl} 0.70$ to $0.81 ; 7920$ participants, 39 studies, $\mathrm{I}^{2}=29 \%$, high-certainty evidence; Analysis 1.6).

Subgroup analyses found little or no difference in the effect of balance and functional exercises on the rate of falls in trials where all participants were at an increased risk of falling (RaR $0.72,95 \% \mathrm{Cl}$ 0.65 to $0.80 ; 4602$ participants, 21 studies, $\mathrm{I}^{2}=38 \%$ ) compared with trials that did not use increased risk of falling as an entry criterion (RaR $0.80,95 \% \mathrm{Cl} 0.72$ to $0.90 ; 3355$ participants, 18 studies, $\mathrm{I}^{2}=$ $17 \%)$; test for subgroup differences: $\mathrm{Chi}^{2}=1.99, \mathrm{df}=1, \mathrm{P}=0.16, \mathrm{I}^{2}=$ 50\% (Analysis 8.1).

Subgroup analyses found a larger effect of balance and functional exercises in trials where interventions were delivered by a health professional ( $\mathrm{RaR} 0.67,95 \% \mathrm{Cl} 0.58$ to $0.65 ; 2960$ participants, 20 studies, $I^{2}=37 \%$ ) than in trials where the interventions were delivered by trained instructors who were not health professionals (RaR 0.82, 95\% $\mathrm{Cl} 0.76$ to $0.88 ; 4997$ participants, 19 studies, $\mathrm{I}^{2}=9 \%$ ); test for subgroup differences: $\mathrm{Chi}^{2}=6.72, \mathrm{df}=1, \mathrm{P}=0.01, \mathrm{I}^{2}=85 \%$ (Analysis 8.3). Notably, both approaches resulted in statistically significant reductions in the rate of falls.

Subgroup analyses found little or no difference in the effect of balance and functional exercises on the rate of falls in trials where interventions were delivered in a group setting ( $\mathrm{RaR} 0.73,95 \% \mathrm{Cl}$ 
0.65 to $0.82 ; 3620$ participants, 20 studies, $I^{2}=34 \%$ ) compared with trials where interventions were delivered individually (RaR 0.77, $95 \% \mathrm{Cl} 0.70$ to $0.85 ; 4589$ participants, 20 studies, $\mathrm{I}^{2}=28 \%$ ); test for subgroup differences: $\mathrm{Chi}^{2}=0.47, \mathrm{df}=1, \mathrm{P}=0.50, \mathrm{I}^{2}=0 \%$ (Analysis 8.5).

\section{Resistance exercises versus control}

We are uncertain whether exercises, classified as being primarily resistance or strength exercises using the ProFaNE taxonomy, reduce the rate of falls compared with control $(\mathrm{RaR} 1.14,95 \% \mathrm{Cl}$ 0.67 to $1.97 ; 327$ participants, 5 studies, $I^{2}=67 \%$; very low-certainty evidence; Analysis 1.6).

\section{D (Tai Chi) exercise versus control}

Exercise interventions that were classified as 3D (Tai Chi or similar) may reduce the rate of falls by $19 \%$ compared with control (RaR $0.81,95 \% \mathrm{Cl} 0.67$ to $0.99 ; 2655$ participants, 7 studies, $\mathrm{I}^{2}=74 \%$; lowcertainty evidence; Analysis 1.6).

\section{D (dance) exercise versus control}

We are uncertain whether exercises, classified as being primarily 3D (dance) using the ProFaNE taxonomy, reduce the rate of falls compared with control (RaR 1.34, $95 \% \mathrm{Cl} 0.98$ to $1.83 ; 522$ participants, 1 study; very low-certainty evidence; Analysis 1.6).

\section{Walking programme versus control}

We are uncertain whether exercises, classified as being primarily walking programmes using the ProFaNE taxonomy, reduce the rate of falls compared with control (RaR $1.14,95 \% \mathrm{Cl} 0.66$ to 1.97 ; 441 participants, 2 studies; $I^{2}=67 \%$; very low-certainty evidence; Analysis 1.6).

\section{Multiple categories of exercise versus control}

Exercise interventions that include multiple categories of the ProFaNE taxonomy (most commonly balance and functional exercises plus resistance exercises) probably reduce the rate of falls by $34 \%$ compared with controls ( $\mathrm{RaR} 0.66,95 \% \mathrm{Cl} 0.50$ to $0.88 ; 1374$ participants, 11 studies; $I^{2}=65 \%$; moderate-certainty evidence; Analysis 1.6).

Sensitivity analyses revealed little difference in the results when we pooled only trials that include the most common two components (balance and functional exercises plus resistance exercises) (RaR $0.69,95 \% \mathrm{Cl} 0.48$ to $0.97 ; 1084$ participants, 8 studies; $I^{2}=72 \%$; Analysis 19.1).

Subgroup analyses found there is probably little or no difference in the effect of exercise interventions that included multiple categories on the rate of falls in trials where all participants were at an increased risk of falling ( $\mathrm{RaR} 0.77,95 \% \mathrm{Cl} 0.63$ to $0.94 ; 618$ participants, 5 studies, $\mathrm{I}^{2}=0 \%$ ) compared with trials that did not use increased risk of falling as an entry criterion ( $\mathrm{RaR} 0.54,95 \% \mathrm{Cl}$ 0.29 to $0.99 ; 763$ participants, 6 studies, $I^{2}=79 \%$ ); test for subgroup differences: $\mathrm{Chi}^{2}=1.19, \mathrm{df}=1, \mathrm{P}=0.27, \mathrm{I}^{2}=16.2 \%$ (Analysis 9.1).

Subgroup analyses found there is probably little or no difference in the effect of exercise interventions that included multiple categories on rate of falls in trials where interventions were delivered by health professionals $(\mathrm{RaR} 0.65,95 \% \mathrm{Cl} 0.43$ to 0.99 ; 653 participants, 3 studies, $I^{2}=72 \%$ ) compared with trials where interventions were delivered by trained instructors who were not health professionals ( $\mathrm{RaR} 0.66,95 \% \mathrm{Cl} 0.44$ to $0.99 ; 751$ participants; 8 studies, $I^{2}=67 \%$ ); test for subgroup differences: $\mathrm{Chi}^{2}=0, \mathrm{df}=1, \mathrm{P}$ $=0.96, \mathrm{I}^{2}=0 \%$ (Analysis 9.3).

Subgroup analyses found there is probably little or no difference in the effect of exercise interventions that included multiple categories on the rate of falls in trials where interventions were delivered in a group setting ( $\mathrm{RaR} 0.64,95 \% \mathrm{Cl} 0.46$ to $0.89 ; 1194$ participants, 10 studies, $I^{2}=67 \%$ ) compared with trials where interventions were delivered individually (RaR $0.81,95 \% \mathrm{Cl} 0.56$ to 1.18; 210 participants, 1 study); test for subgroup differences: $\mathrm{Chi}^{2}$ $=0.86, \mathrm{df}=1, \mathrm{P}=0.35, \mathrm{I}^{2}=0 \%$ (Analysis 9.5).

\section{Long-term follow-up rate of falls (secondary outcome)}

Five studies reported the rate of falls at more than 18 months after randomisation. Data from four studies, pooled by exercise category, are presented in Analysis 1.7. Balance and functional exercises may reduce the rate of falls in the long term (RaR 0.82 , $95 \% \mathrm{Cl} 0.66$ to $1.01 ; 858$ participants, 2 studies; $\left.\right|^{2}=41 \%$; lowcertainty evidence). The long-term effects of a walking programme tested in Ebrahim 1997 (97 participants) and a multiple exercise programme, including balance and strength training tested in UusiRasi 2015 (175 participants) are unclear (Analysis 1.7). Data from Iliffe 2015 were not included in Analysis 1.7 because the followup period, which differed from the other four studies, was a oneyear period started six months after programme completion. There was no evidence of a difference in rate of falls for either exercise programme (FaME programme: RaR $0.94,95 \% \mathrm{Cl} 0.62$ to $1.41 ; 202$ participants; Otago Exercise Program: RaR 1.04, 95\% Cl 0.69 to 1.55; 201 participants).

\section{Number of people who experienced one or more falls (risk of falling)}

Exercise (all types) reduces the number of people experiencing one or more falls by $15 \%$ compared with control (risk ratio (RR) 0.85 , $95 \% \mathrm{Cl} 0.81$ to 0.89 ; 13,518 participants, 63 studies, $\mathrm{I}^{2}=26 \%$; highcertainty evidence; Analysis 2.1).

Subgroup analysis by falls risk at baseline found there was little or no difference in the effect of exercise (all types) on the number of people experiencing one or more falls in trials where all participants were at an increased risk of falling (RR $0.87,95 \% \mathrm{Cl} 0.83$ to 0.91 ; 7171 participants, 35 studies, $\mathrm{I}^{2}=1 \%$ ) compared with trials that did not use increased risk of falling as an entry criterion (RR 0.82 , $95 \% \mathrm{Cl} 0.73$ to $0.92 ; 6347$ participants, 28 studies, $I^{2}=45 \%$ ); test for subgroup differences: $\mathrm{Chi}^{2}=0.94, \mathrm{df}=1, \mathrm{P}=0.33, \mathrm{I}^{2}=0 \%$ (Analysis 2.2).

Subgroup analysis by participant age found there was little or no difference in the effect of exercise (all types) on the number of people experiencing one or more falls in trials where participants were aged 75 years or older (RR $0.86,95 \% \mathrm{Cl} 0.80$ to $0.92 ; 3172$ participants, 13 studies, $\mathrm{I}^{2}=0 \%$ ) compared with trials where participants were aged less than 75 years (RR $0.85,95 \% \mathrm{Cl} 0.79$ to 0.91; 10,346 participants, 50 studies, $\mathrm{I}^{2}=33 \%$ ); test for subgroup differences: $\mathrm{Chi}^{2}=0.07, \mathrm{df}=1, \mathrm{P}=0.79, \mathrm{I}^{2}=0 \%$ (Analysis 2.3 ).

Subgroup analyses by personnel delivering exercise found there was little or no difference in the effect of exercise (all types) on the number of people experiencing one or more falls in trials where interventions were delivered by a health professional (RR 0.82, 95\% $\mathrm{Cl} 0.74$ to $0.91 ; 3747$ participants, 26 studies, $\mathrm{I}^{2}=25 \%$ ) than in trials 
where the interventions were delivered by trained instructors who were not health professionals (RR $0.86,95 \% \mathrm{Cl} 0.81$ to $0.92 ; 9726$ participants, 36 studies, $\left.I^{2}=29 \%\right)$; test for subgroup differences: $\mathrm{Chi}^{2}=0.63, \mathrm{df}=1(\mathrm{P}=0.43), \mathrm{I}^{2}=0 \%$ (Analysis 2.4). The personnel providing the exercise programme was not identified in Park 2008.

Subgroup analyses found there may be no difference in the effect of exercise (all types) on the number of people experiencing one or more falls in trials where interventions were delivered in a group setting (RR $0.83,95 \% \mathrm{Cl} 0.78$ to 0.90 ; 9219 participants, 48 studies, $\left.1^{2}=33 \%\right)$ compared with trials where interventions were delivered individually (RR $0.88,95 \% \mathrm{Cl} 0.83$ to $0.93 ; 4299$ participants, 16 studies; $I^{2}=0 \%$ ); test for subgroup differences: Chi $^{2}=1.14, \mathrm{df}=1$, $\mathrm{P}=0.29, \mathrm{I}^{2}=12 \%$ (Analysis 2.5 ). One three-group study appears in both subgroups (Iliffe 2015).

The subgroup analysis by exercise type provided a visual impression of potential subgroup differences of effect of different exercises on the numbers of fallers, but the test for subgroup differences did not show a statistically significant result: test for subgroup differences: $\mathrm{Chi}^{2}=6.45, \mathrm{df}=5, \mathrm{P}=0.26, \mathrm{I}^{2}=22.5 \%$ (Analysis 2.6).

\section{Different categories of primary exercise versus control}

\section{Balance and functional exercises versus control}

Exercise interventions that were classified as being primarily gait, balance, co-ordination or functional task training using the ProFaNE taxonomy, reduce the number of people experiencing one or more falls by $13 \%$ compared with control (RR $0.87,95 \% \mathrm{Cl} 0.82$ to $0.91 ; 8288$ participants, 37 studies, $I^{2}=9 \%$; high-certainty evidence; Analysis 2.6).

Subgroup analyses found little or no difference in the effect of balance and functional exercises on the number of people experiencing one or more falls in trials where all participants were at an increased risk of falling (RR $0.86,95 \% \mathrm{Cl} 0.81$ to $0.91 ; 4639$ participants, 22 studies, $\mathrm{I}^{2}=6 \%$ ) compared with trials that did not use increased risk of falling as an entry criterion (RR $0.88,95 \% \mathrm{Cl}$ 0.80 to $97 ; 3649$ participants, 15 studies, $I^{2}=18 \%$ ); test for subgroup differences: $\mathrm{Chi}^{2}=0.21, \mathrm{df}=1, \mathrm{P}=0.65, \mathrm{I}^{2}=0 \%$ (Analysis 8.2).

Subgroup analyses found little or no difference in the effect of balance and functional exercises on the number of people experiencing one or more falls in trials where interventions were delivered by health professionals (RR $0.82,95 \% \mathrm{Cl} 0.75$ to 0.90 ; 2894 participants, 19 studies, $\mathrm{I}^{2}=5 \%$ ) compared with trials where interventions were delivered by trained instructors who were not health professionals (RR $0.89,95 \% \mathrm{Cl} 0.84$ to $0.94 ; 5394$ participants, 18 studies, $\left.\mathrm{I}^{2}=11 \%\right)$; test for subgroup differences: $\mathrm{Chi}^{2}=1.71, \mathrm{df}=$ $1, \mathrm{P}=0.19, \mathrm{I}^{2}=41 \%$ (Analysis 8.4 ).

Subgroup analyses also found little or no difference in the effect of balance and functional exercises on the number of people experiencing one or more falls in trials where interventions were delivered in a group setting (RR $0.87,95 \% \mathrm{Cl} 0.80$ to $0.95 ; 4465$ participants, 22 studies, $\left.\mathrm{I}^{2}=19 \%\right)$ compared with trials where interventions were delivered individually (RR $0.87,95 \% \mathrm{Cl} 0.82$ to 0.92 ; 4075 participants, 16 studies, $\mathrm{I}^{2}=0 \%$ ); test for subgroup differences: $\mathrm{Chi}^{2}=0.01, \mathrm{df}=1(\mathrm{P}=0.92), \mathrm{I}^{2}=0 \%$ (Analysis 8.6).

\section{Resistance exercises versus control}

We are uncertain whether exercise, classified as being primarily resistance or strength exercises, reduces the number of people experiencing one or more falls compared with control (RR 0.81, 95\% $\mathrm{Cl} 0.57$ to 1.15 ; 163 participants, 2 studies, $\mathrm{I}^{2}=0 \%$; very low-certainty evidence; Analysis 2.6).

\section{D (Tai Chi) exercise versus control}

Exercise interventions that were classified as 3D (Tai Chi or similar) reduce the number of people experiencing one or more falls by $20 \%$ compared with control (RR $0.80,95 \% \mathrm{Cl} 0.70$ to $0.91 ; 2677$ participants, 8 studies, $\mathrm{I}^{2}=42 \%$; high-certainty evidence; Analysis 2.6).

\section{D (dance) exercise versus control}

We are uncertain whether exercise, classified as being primarily $3 \mathrm{D}$ (dance), reduces the number of people experiencing one or more falls compared with control (RR $1.35,95 \% \mathrm{Cl} 0.83$ to 2.20; 522 participants, 1 study; very low-certainty evidence; Analysis 2.6). We assessed the certainty of the evidence as very low due to there being wide $\mathrm{Cl}$ in the single trial.

\section{Walking programme versus control}

We are uncertain whether exercise, classified as being primarily walking programmes, reduces the number of people experiencing one or more falls compared with control (RR 1.05, 95\% Cl 0.71 to 1.54 ; 441 participants, 2 studies, $\mathrm{I}^{2}=50 \%$; Analysis 2.6 ), as we assessed the certainty of the evidence as very low.

\section{Multiple categories of exercise versus control}

Exercise interventions that included multiple categories of the ProFaNE taxonomy probably reduce the number of people experiencing one or more falls by $22 \%$ compared with control (RR $0.78,95 \% \mathrm{Cl} 0.64$ to $0.96 ; 1623$ participants, 17 studies, $\mathrm{I}^{2}=48 \%$; moderate-certainty evidence; Analysis 2.6).

Sensitivity analyses revealed little difference in the results when we pooled only trials that included the two most common components (balance and functional exercises plus resistance exercises) (RR $0.76,95 \% \mathrm{Cl} 0.61$ to $0.95 ; 1375$ participants, 13 studies; $I^{2}=53 \%$; Analysis 19.2).

Subgroup analyses found there may be little or no difference in the effect of exercise interventions that included multiple categories on the number of people experiencing one or more falls in trials where all participants were at an increased risk of falling (RR $0.84,95 \% \mathrm{Cl}$ 0.71 to 1.00 ; 913 participants, 10 studies, $I^{2}=19 \%$ ) compared with trials that did not use increased risk of falling as an entry criterion (RR $0.70,95 \% \mathrm{Cl} 0.41$ to $1.19 ; 710$ participants, 7 studies, $\mathrm{I}^{2}=67 \%$ ); test for subgroup differences: $\mathrm{Chi}^{2}=0.42, \mathrm{df}=1, \mathrm{P}=0.52, \mathrm{I}^{2}=0 \%$ (Analysis 9.2).

Subgroup analyses found there may be little or no difference in the effect of exercise interventions that included multiple categories on the number of people experiencing one or more falls in trials where interventions were delivered by health professionals (RR 0.81, 95\% $\mathrm{Cl} 0.65$ to $1.02 ; 867$ participants, 8 studies, $\mathrm{I}^{2}=50 \%$ ) compared with trials where interventions were delivered by trained instructors who were not health professionals (RR $0.70,95 \% \mathrm{Cl} 0.45$ to $1.10 ; 711$ participants, 8 studies, $I^{2}=57 \%$ ); test for subgroup differences: $\mathrm{Chi}^{2}$ $=0.34, \mathrm{df}=1, \mathrm{P}=0.56, \mathrm{I}^{2}=0 \%$ (Analysis 9.4). 
Subgroup analyses found there may be little or no difference in the effect of exercise interventions that included multiple categories on the number of people experiencing one or more falls in trials where interventions were delivered in a group setting (RR $0.77,95 \% \mathrm{Cl} 0.60$ to $1.00 ; 1301$ participants, 14 studies, $I^{2}=57 \%$ ) compared with trials where interventions were delivered individually (RR $0.86,95 \% \mathrm{Cl}$ 0.72 to 1.03 ; 322 participants, 3 studies, $I^{2}=0 \%$ ); test for subgroup differences: $\mathrm{Chi}^{2}=0.45, \mathrm{df}=1(\mathrm{P}=0.50), \mathrm{I}^{2}=0 \%$ (Analysis 9.6).

\section{Long-term follow-up}

Data from the three studies reporting on the number of people experiencing one or more falls at more than 18 months after randomisation are shown in Analysis 2.7. Balance and functional exercises may reduce the number of fallers in the long term (RR $0.86,95 \% \mathrm{Cl} 0.78$ to $0.94 ; 1325$ participants, 2 studies; $I^{2}=0 \%$; low-certainty evidence) but there is no evidence of difference for a multiple exercise programme (including balance and strength training) tested in Uusi-Rasi 2015 (RR 1.01, 95\% Cl 0.74 to 1.38; 175 participants; low-certainty evidence).

\section{Number of people who experienced one or more fall-related fractures}

Exercise (all types) may reduce the number of people experiencing one or more fall-related fractures by $27 \%$ compared with control (RR $0.73,95 \% \mathrm{Cl} 0.56$ to $0.95 ; 4047$ participants, 10 studies, $\mathrm{I}^{2}=0 \%$; low-certainty evidence; Analysis 3.1).

Subgroup analysis by falls risk at baseline found there may be little or no difference in the effect of exercise (all types) on the number of people experiencing one or more fall-related fractures in trials where all participants were at an increased risk of falling (RR 0.80 , $95 \% \mathrm{Cl} 0.60$ to $1.07 ; 2792$ participants, 5 studies, $\mathrm{I}^{2}=0$ ) compared with trials that did not use increased risk of falling as an entry criterion (RR $0.48,95 \% \mathrm{Cl} 0.26$ to $0.91 ; 1255$ participants, 5 studies, $\mathrm{I}^{2}=0 \%$ ); test for subgroup differences: $\mathrm{Chi}^{2}=2.05, \mathrm{df}=1, \mathrm{P}=0.15$, $\mathrm{I}^{2}=50.6 \%$ (Analysis 3.2 ).

Subgroup analyses found there may be little or no difference in the effect of exercise (all types) on the number of people experiencing one or more fall-related fractures in trials where participants were aged 75 years or older (RR $0.61,95 \% \mathrm{Cl} 0.31$ to 1.20; 2740 participants, 3 studies, $I^{2}=42 \%$ ) compared with trials where participants were aged less than 75 years (RR $0.53,95 \% \mathrm{Cl}$ 0.29 to $0.96 ; 1308$ participants, 7 studies, $I^{2}=0 \%$ ); test for subgroup differences: $\mathrm{Chi}^{2}=0.1, \mathrm{df}=1, \mathrm{P}=0.75, \mathrm{I}^{2}=0 \%$ (Analysis 3.3).

The subgroup analysis by exercise type did not show subgroup differences on the effects on fall-related fractures: test for subgroup differences: $\mathrm{Chi}^{2}=4.22, \mathrm{df}=3, \mathrm{P}=0.24, \mathrm{I}^{2}=28.9 \%$ (Analysis 3.4).

\section{Different categories of primary exercise versus control}

\section{Balance and functional exercises versus control}

Exercise interventions that were classified as being primarily gait, balance, co-ordination or functional task training using the ProFaNE taxonomy, may reduce the number of people experiencing one or more fall-related fractures by $56 \%$ compared with control (RR $0.44,95 \% \mathrm{Cl} 0.25$ to $0.76 ; 2139$ participants, 7 studies, $\mathrm{I}^{2}=0 \%$; low-certainty evidence; Analysis 3.4).

\section{Resistance exercises versus control}

We are uncertain whether exercises, classified as being primarily resistance or strength exercises using the ProFaNE taxonomy, reduce the number of people experiencing one or more fall-related fractures compared with control (RR $0.97,95 \% \mathrm{Cl} 0.14$ to $6.49 ; 73$ participants; 1 study; very low-certainty of evidence due to single study with very wide $\mathrm{Cl}$; Analysis 3.4).

\section{D exercise versus control}

We did not find any studies that looked at the impact of 3D exercises (Tai Chi or dance) on the number of people experiencing one or more fall-related fractures compared with control.

\section{Walking programme versus control}

We are uncertain whether exercises, classified as being primarily walking programmes using the ProFaNE taxonomy, reduce the number of people experiencing one or more fall-related fractures compared with control (RR 0.66, $95 \% \mathrm{Cl} 0.11$ to 3.76 ; 97 participants, 1 study; very low-certainty evidence due to a single study with very wide $\mathrm{Cl}$; Analysis 3.4).

\section{Multiple categories of exercise versus control}

Exercise interventions that include multiple categories of the ProFaNE taxonomy, may slightly reduce the number of people experiencing one or more fall-related fractures compared with control; however, the $95 \% \mathrm{Cl}$ includes the possibility of both reduced and increased numbers of people experiencing fall-related fractures (RR $0.85,95 \% \mathrm{Cl} 0.62$ to $1.16 ; 1810$ participants, 3 studies, $\mathrm{I}^{2}=0 \%$; low-certainty evidence; Analysis 3.4).

\section{Long-term follow-up}

Three studies, each testing a different exercise category, reported the number of people who experienced fractures more than 18 months after randomisation (Dangour 2011; Ebrahim 1997; Gill 2016). The effect of exercise on fractures at long-term follow-up is unclear (RR 0.93, 95\% Cl 0.69 to 1.25; 2351 participants, 3 studies; very low-certainty; Analysis 3.5). Only the data ( 6 versus 4 fractures at 24 months compared with 2 versus 3 at 12 months) for Ebrahim 1997 differed from that presented in the main analysis (Analysis 3.1).

\section{Number of people who experienced one or more falls that resulted in hospital admission}

Only two studies reported this outcome (Clegg 2014; Gill 2016). We are uncertain of the finding that exercise (all types) makes little or no difference to the number of people who experience one or more falls requiring hospital admission compared with control (RR $0.78,95 \% \mathrm{Cl} 0.51$ to $1.18 ; 1705$ participants, 2 studies, $\mathrm{I}^{2}=0 \%$; very low-certainty evidence, downgraded three levels due to high risk of bias, imprecision (wide $\mathrm{Cl}$ ) and because a large number of studies included in the review do not contribute data to the outcome; Analysis 4.1).

\section{Number of people who experienced one or more falls that required medical attention}

Exercise (all types) may reduce the number of people who experience one or more falls requiring medical attention by $39 \%$ compared with control (RR $0.61,95 \% \mathrm{Cl} 0.47$ to $0.79 ; 1019$ participants, 5 studies ( 7 comparisons), $\mathrm{I}^{2}=3 \%$; low-certainty 
evidence downgraded due to imprecision and risk of publication bias; Analysis 5.1).

\section{Different categories of primary exercise versus control}

\section{Balance and functional exercises versus control}

Exercise interventions that were classified as being primarily gait, balance, co-ordination or functional task training using the ProFaNE taxonomy, may make little or no difference to the number of people who experienced one or more falls requiring medical attention compared with control (RR $0.76,95 \% \mathrm{Cl} 0.54$ to $1.09 ; 583$ participants, 3 studies, $\mathrm{I}^{2}=0 \%$; low-certainty evidence; Analysis 5.2).

\section{Resistance exercises versus control}

Exercises classified as being primarily resistance or strength exercises using the ProFaNE taxonomy, may make little or no difference to the number of falls requiring medical attention compared with control (RR 0.92, 95\% $\mathrm{Cl} 0.47$ to $1.80 ; 73$ participants, 1 study; very low-certainty evidence; Analysis 5.2).

\section{D (Tai Chi) exercise versus control}

Exercise interventions that were classified as 3D (Tai Chi or similar) may reduce the number of falls requiring medical attention by $65 \%$ compared with control (RR $0.35,95 \% \mathrm{Cl} 0.13$ to $0.93 ; 188$ participants, 1 study; low-certainty evidence; Analysis 5.2).

\section{Walking programme versus control}

This outcome was not reported.

\section{Multiple categories of exercise versus control}

Exercise interventions that include multiple categories of the ProFaNE taxonomy, may reduce the rate of falls requiring medical attention (RR $0.44,95 \% \mathrm{Cl} 0.29$ to $0.66 ; 247$ participants, 2 studies, $\mathrm{I}^{2}=0 \%$; low-certainty evidence; Analysis 5.2).

\section{Long-term follow-up}

Two studies reported on this outcome at more than 18 months after randomisation (Karinkanta 2007; Uusi-Rasi 2015). Pooled data from these two studies showed exercise (all types) may reduce the number of people who experience one or more falls requiring medical attention in the long term ( $\mathrm{RR} 0.54,95 \% \mathrm{Cl} 0.37$ to $0.78 ; 319$ participants, 2 studies; low-certainty evidence; Analysis 5.3). The same data from both studies were used in Analysis 5.1 and Analysis 5.3.

\section{Health-related quality of life}

We were able to pool data from 15 of the 23 trials that assessed health-related quality of life in people not recently discharged from hospital. Based on pooled standardised mean difference (SMD) results from the 15 trials (17 comparisons) that reported final scores, exercise interventions may make little or no difference to people's reported health-related quality of life compared with those who received usual care or an attention control; however, the $95 \% \mathrm{Cl}$ includes the possibility of both increased and reduced quality of life (SMD $-0.03,95 \% \mathrm{Cl}-0.10$ to $0.04 ; 3172$ participants, 15 studies; $\left.\right|^{2}=76 \%$; low-quality evidence downgraded two levels due to inconsistency (there was considerable heterogeneity, $76 \%$ ), and risk of bias (removing studies with high risk of bias on two or more items had a marked impact on results; Analysis 6.1).
Four trials (6 comparisons) reported end point scores using the EQ-5D; the SMD converted back to mean difference (MD) -0.0026 points $(95 \% \mathrm{Cl}-0.0086$ to 0.0034$)$ on the 0 to $1 \mathrm{EQ}-5 \mathrm{D}$ scale, which is less than the minimally important difference of 0.074 (Walters 2005). For the five trials that measured health-related quality of life using SF-36, converting these data to the SF-36 scale (0 worst to 100 best) indicates that the estimated MD of $0.36(95 \% \mathrm{Cl}-1.20$ to 0.47$)$ is not clinically important, as the minimally important difference is usually 3 to 5 (Walters 2003).

Appendix 16 provides summary information for all 23 trials including three post-hospital studies and those which we could not include in the meta-analysis (e.g. because they used unique outcome measures or reported median, IQR or P value), the results of which are similar to the above.

\section{Number of people who experienced one or more adverse events}

Twenty-seven trials reported on adverse event to some degree (Appendix 13). Fourteen of the trials reporting on adverse events stated there were no adverse events.

Iliffe 2015 measured the number of people experiencing adverse events in both groups throughout the trial period and reported 59 events classified as 'adverse reactions' or 'possible adverse reactions' in the group receiving FaME intervention, 60 in the OEP group and 45 in the control group; the majority were reports of musculoskeletal pain and none were serious. No other studies reported adverse events that were monitored closely in all groups over the entire study period. A serious adverse effect was a pelvic stress fracture reported in Clemson 2012. The remaining trials reported non-serious adverse events of a musculoskeletal nature, with a median of three events (range 1 to 26) in the intervention group. The majority of reported adverse events were of a musculoskeletal nature and not serious. Of the studies that reported adverse events, a greater proportion of the strength-only exercises were associated with adverse events than in the gait, balance and functional training or multiple exercise categories.

\section{Different categories of primary exercise}

\section{Balance and functional exercises versus control}

Adverse events were reported in 15 of the 48 trials, including exercise interventions that were classified as being primarily gait, balance, co-ordination or functional task training using the ProFaNE taxonomy. Two hundred adverse events were reported; most were non-serious adverse events of a musculoskeletal nature, one trial (two intervention arms) reported 128 of these adverse events (Iliffe 2015), one intervention arm reported shortness of breath in four participants (Liu-Ambrose 2004), another trial reported palpitations in a participant (Sakamoto 2013), and one trial reported a pelvic stress fracture (Clemson 2012). See Appendix 13.

\section{Resistance exercises versus control}

Adverse events were reported in one trial, including exercises classified as being primarily resistance or strength exercises using the ProFaNE taxonomy (Liu-Ambrose 2004). The study reported 10 musculoskeletal complaints in the intervention group and one musculoskeletal complaint in the control group. 


\section{D (Tai Chi) exercise versus control}

Adverse events were reported in two of 10 trials with 3D (Tai Chi) as the primary intervention. There were zero occurrences of adverse events.

\section{D (dance) exercise versus control}

Adverse events were reported in the one trial in this analysis, in the intervention group only. There were zero occurrences of adverse events.

\section{Walking programme versus control}

This outcome was not reported.

\section{Multiple categories of exercise versus control}

Adverse events were reported in 10 of the 21 trials of exercise interventions that include multiple categories of the ProFaNE taxonomy. Adverse events were reported for both intervention and control groups ( 5 trials), or the intervention group only ( 5 trials). There was a total of 43 adverse events reported. The majority were non-serious and of a musculoskeletal nature. There was reported exacerbation of pre-existing osteoarthritis conditions (Uusi-Rasi 2015), and inguinal hernia surgery was reported in one intervention arm (Clemson 2012).

\section{Number of people who died}

Death was primarily reported as a reason for loss to follow-up in all 30 trials with separate group data. Exercise (all types) may reduce the number of people who died compared with control; however, the $95 \% \mathrm{Cl}$ includes the possibility of both reduced death and increased death with exercise (RR $0.86,95 \% \mathrm{Cl} 0.66$ to 1.12 10,037 participants, 30 studies, $I^{2}=0 \%$; low-certainty evidence (downgraded one level due to risk of bias, as results changed, becoming statistically significant, with removal of the 14 trials with a high risk of bias on one or more items; and one level for indirectness, as the outcome was assessed indirectly as a reason for loss to follow-up; Analysis 7.1). The risk of death did not differ between the trials including people selected or not-selected for risk of falling: test for subgroup differences: $\mathrm{Chi}^{2}=0.19, \mathrm{df}=1, \mathrm{P}=0.67$, $1^{2}=0 \%$ (Analysis 7.2). None of the deaths were explicitly linked to trial participation.

\section{Exercise (all types) versus control tested in people who had recently been discharged from hospital}

Four studies investigated outcomes in people who had recently been discharged from hospital (Haines 2009; Latham 2003; Sherrington 2014; Vogler 2009). Results of individual studies for rate of falls (3 trials) are shown in Analysis 10.1; number of falls (4 trials) in Analysis 10.2; health-related quality of life (3 trials) in Analysis 10.3; and mortality (4 trials) in Analysis 10.4. Given the diversity of interventions, we did not pool data. It is noted that overall, the effects of exercise on falls appear smaller (or in the opposite direction in the case of Sherrington 2014) in these studies compared with studies in the general older population (very lowcertainty evidence).

All four studies reported on adverse events to some degree (Appendix 13). Latham 2003 measured the number of people experiencing adverse events in both groups throughout the trial period and reported that 18 participants had back and knee pain directly attributable to the exercise programme; there were no details of the five participants with adverse events in the control group. The remaining trials reported non-serious adverse events of a musculoskeletal nature.

\section{Exercise versus exercise}

\section{Comparisons of different types of exercise}

The results of individual trials directly comparing different types of exercise are shown for rate of falls in Analysis 11.1, with longterm rate of falls data in Analysis 11.2; number of fallers in Analysis 11.3; number with fall-related fractures in Analysis 11.4; number requiring medical attention in Analysis 11.5; quality of life in Analysis 11.6; and mortality in Analysis 11.7. Given the variability between programmes, we did not undertake any meta-analyses for these comparisons for any of the outcomes. Overall there is very low-certainty evidence for each comparison.

Most of the trials in these analyses did not find significant differences in the fall prevention effects of different programmes, but most were not likely to be adequately powered to detect differences between different exercise programmes.

A few studies did find greater effects of particular programmes. For example, Kemmler 2010 found greater effects on the rate of falls of a more intensive programme delivered twice a week compared with a low intensity programme delivered once a week. Studies by Yamada et al found greater fall prevention effects of complex obstacle negotiation training compared with simple training (Yamada 2012), and greater effects of multidimensional stepping compared with walking (Yamada 2013). Both these interventions were delivered in addition to group exercise primarily targeting balance. Hwang 2016 found greater effects of Tai Chi than supervised balance and strength training on the rate of falls and the number of people falling. All these findings require confirmation in different and larger studies.

\section{Different modes of delivery (e.g. group versus individual) of the same type of exercise}

The results of individual trials that provided direct comparisons between the same programmes being delivered in group-based settings and individually are shown for rate of falls in Analysis 11.8; number of fallers in Analysis 11.9; number requiring hospital admission in Analysis 11.10; quality of life in Analysis 11.11; and mortality in Analysis 11.12. All results were inconclusive; the five trials were too small to draw conclusions (Barker 2016; Helbostad 2004; Iliffe 2015; Kyrdalen 2014; Wu 2010).

\section{Different doses (e.g. higher intensity versus lower intensity) of the same type of exercise}

The results of the individual trials that directly compared higher with lower doses of the same type of exercise are shown for rate of falls in Analysis 11.13, number of fallers in Analysis 11.14, and mortality in Analysis 11.15. Taylor 2012 found a greater impact on the rate of falls when Tai Chi classes were delivered twice rather than once per week. The other two trials were too small to draw conclusions (Ballard 2004; Davis 2011).

\section{Number of people who experienced one or more adverse events}

No studies reported adverse events that were monitored closely in all groups over the entire study period. Adverse events reported to any degree are described in Appendix 13. Three of the 10 trials 
reporting on adverse events stated there were no adverse events. The remaining trials reported non-serious adverse events of a musculoskeletal nature.

\section{Economic data}

We identified 12 out of the 108 studies that reported economic data. These included reports of costs of intervention or health service use and/or the results of trial-based cost-effectiveness or cost-utility analyses (Appendix 17).

As in Gillespie 2012, the perspectives taken, the cost items measured and valued, and the type of healthcare resources included in the calculation of incremental cost-effectiveness ratios (ICERs) all varied, so that comparison of ICERs for the interventions remains difficult even for evaluations carried out within similar health systems.

Nonetheless, the results from several studies demonstrate the potential cost-effectiveness of fall prevention interventions. One trial of the Otago Exercise Program showed cost savings in those aged 80 years and over resulting from fewer hospital admissions (Robertson 2001a). Davis 2011 reported that both once and twice weekly resistance training dominated control (balance and tone) classes in terms of both falls and quality-adjusted life years (i.e. were less costly and more effective).

Other studies provide information on the cost per fall prevented from the delivery of exercise interventions. For example, Voukelatos 2007 reported AUD 1683 per fall prevented from groupbased Tai Chi and Davis 2009 reports a cost of CAD 247 per fall prevented from a group-based exercise programme compared with guideline-based care.

\section{Sensitivity analyses}

For each of these, the impact on the pooled exercise versus control fall rate outcome is summarised in Appendix 18. The results of the sensitivity analyses can be seen in Analyses 12 to 20 .

1. Sensitivity analysis 1 , removing trials that included participants aged < 65 years: Analysis 12.1 (rate of falls: pooled data); Analysis 12.2 (rate of falls: grouped by exercise); Analysis 12.3 (number of fallers: pooled data); Analysis 12.4 (number of fallers: grouped by exercise); Analysis 12.5 (fracture: pooled data); Analysis 12.6 (fracture: grouped by exercise type); Analysis 12.7 (medical attention: pooled data); Analysis 12.8 (medical attention: subgrouped by exercise).

2. Sensitivity analysis 2 , removing trials with high risk of bias on any item: Analysis 13.1 (rate of falls: pooled data); Analysis 13.2 (rate of falls: subgrouped by exercise); Analysis 13.3 (number of fallers: pooled data); Analysis 13.4 (number of fallers: subgrouped by exercise type); Analysis 13.5 (fracture: pooled data).

3. Sensitivity analysis 3, removing trials with unclear or high risk of bias on allocation concealment: Analysis 14.1 (rate of falls: pooled data).

4. Sensitivity analysis 4, removing trials with unclear or high risk of bias on assessor blinding: Analysis 15.1 (rate of falls: pooled data).

5. Sensitivity analysis 5, removing trials with unclear or high risk of bias on incomplete outcome data: Analysis 16.1 (rate of falls: pooled data).
6. Sensitivity analysis 6 , removing cluster-randomised trials: Analysis 17.1 (rate of falls: pooled data).

7. Sensitivity analysis 7, all trials, fixed-effect meta-analysis: Analysis 18.1 (rate of falls: pooled data).

8. Sensitivity analysis 8 , multiple categories of exercise versus control, removing trials that do not include balance and strength training: Analysis 19.1 (rate of falls: pooled data); Analysis 19.2 (number of fallers: pooled data).

9. Sensitivity analysis $9 a$, classification of interventions based on the Otago Exercise Program as multiple categories of exercise: Analysis 20.1 (rate of falls: pooled data); Analysis 20.2 (number of fallers: pooled data).

10.Sensitivity analysis 9b, classification of interventions that included balance and functional exercises plus strength exercises as multiple categories of exercise: Analysis 20.3 (rate of falls: pooled data); Analysis 20.4 (number of fallers: pooled data).

As shown in Appendix 18; the nine sensitivity analyses (based on age of included participants, risk of bias, cluster trials, fixedeffect analyses, and categorisation of interventions) made little difference to the results of the primary pooled analysis. This indicates the robustness of the review's primary findings and methods.

In undertaking the GRADE assessment we downgraded the certainty of evidence based on sensitivity analysis (removal of trials with one or more items at high risk of bias) for the following comparisons.

1. Fall outcome: resistance exercises versus control, Tai Chi versus control, walking programme versus control.

2. Faller outcome: resistance exercises versus control, walking programme versus control, multiple categories of exercise versus control.

3. Fracture outcome: exercise (all types) versus control, balance and functional exercises versus control, multiple versus control.

4. Health-related quality of life outcome: exercise (all types) versus control.

\section{Heterogeneity}

This review's primary analyses display minimal to substantial heterogeneity with $\mathrm{P}<0.05$ for the $\mathrm{Chi}^{2}$ test and $\mathrm{I}^{2}$ values up to $74 \%$. This variability was not explained by our subgroup analyses. We consider this likely to represent between-study differences in the exact nature of programmes (e.g. dose, intensity, adherence) and target populations, which requires ongoing investigation. Given the overall positive impact of the programmes and the stability of results, we do not consider this to preclude the meta-analyses we have undertaken.

\section{Funnel plots}

The funnel plots in Figure 4; Figure 5; Figure 6; Figure 7; Figure 8 and Figure 9 do show some asymmetry, particularly for the fracture outcomes. We used this information in the GRADE assessment to downgrade the strength of the evidence for the fracture outcomes but did not consider the asymmetry sufficient to downgrade the level of evidence for the other outcomes. 
Figure 4. Funnel plot of comparison: 1 Exercise versus control (rate of falls), outcome: 1.1 Rate of falls - overall analysis.

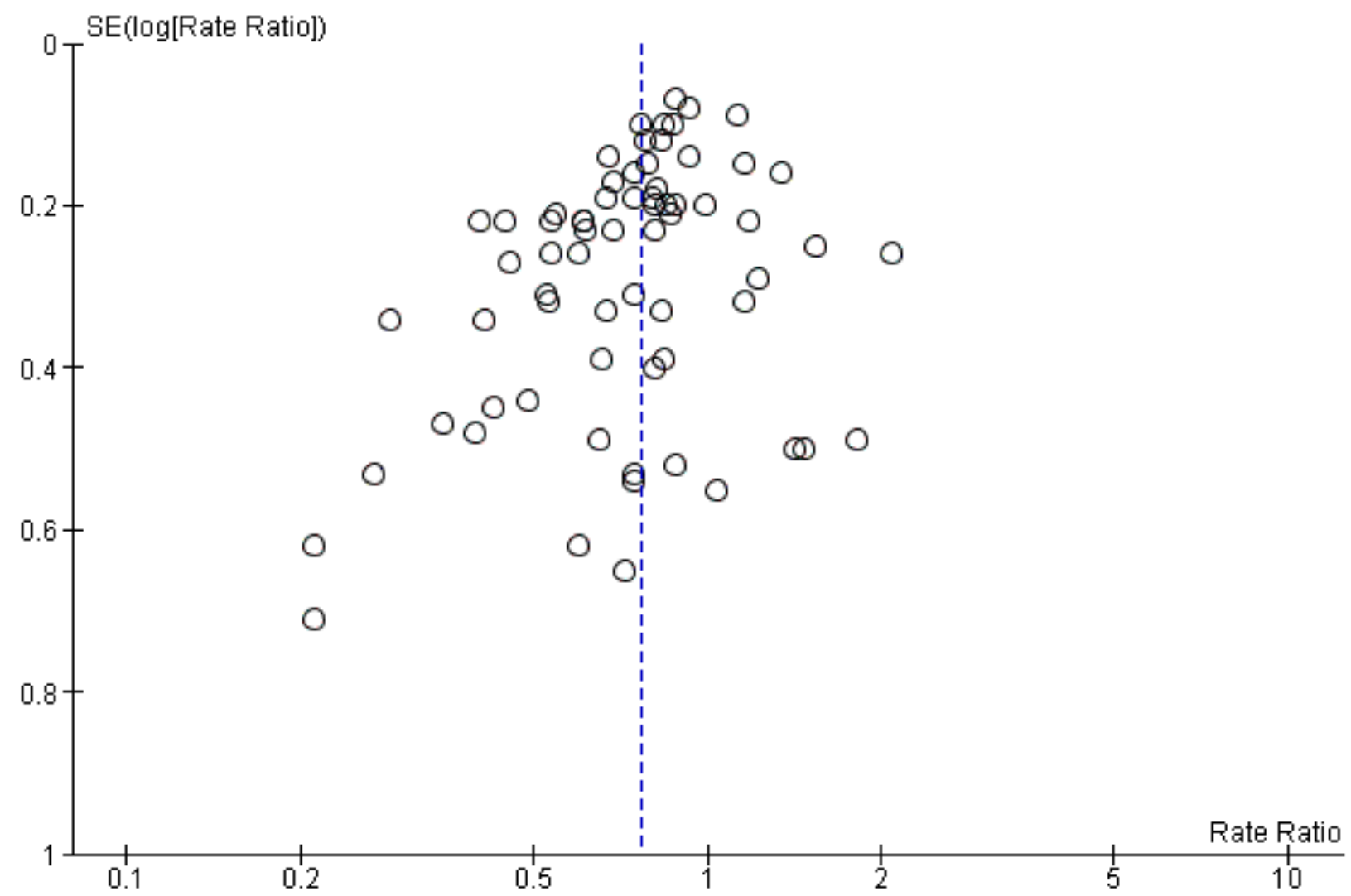


Figure 5. Funnel plot of comparison: 2 Exercise versus control (number of fallers), outcome: 2.1 Number of fallers overall analysis.

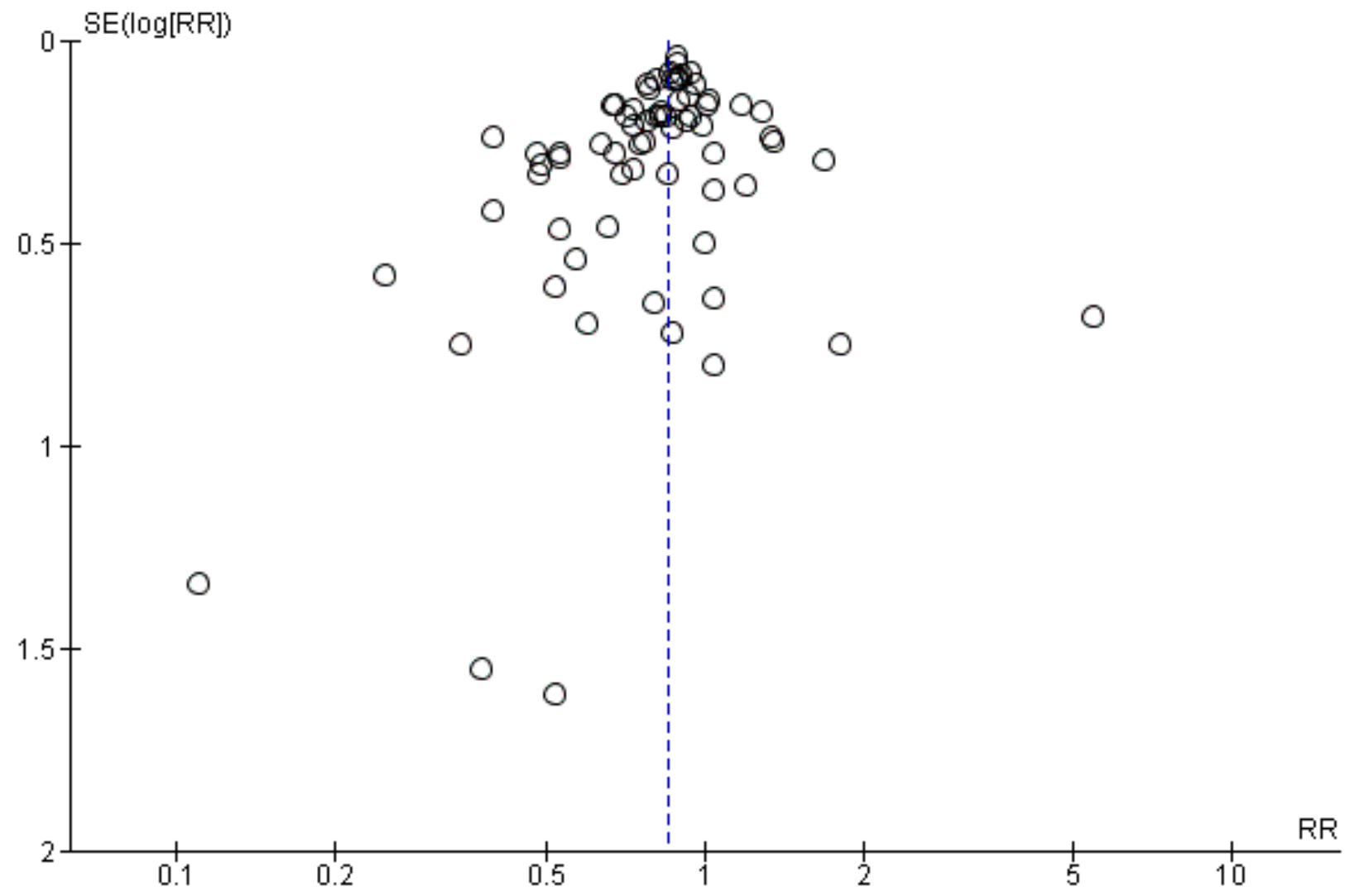


Figure 6. Funnel plot of comparison: 3 Exercise versus control (number of people with fractures), outcome: 3.1 Number of people who experienced one or more fall-related fractures- overall analysis.

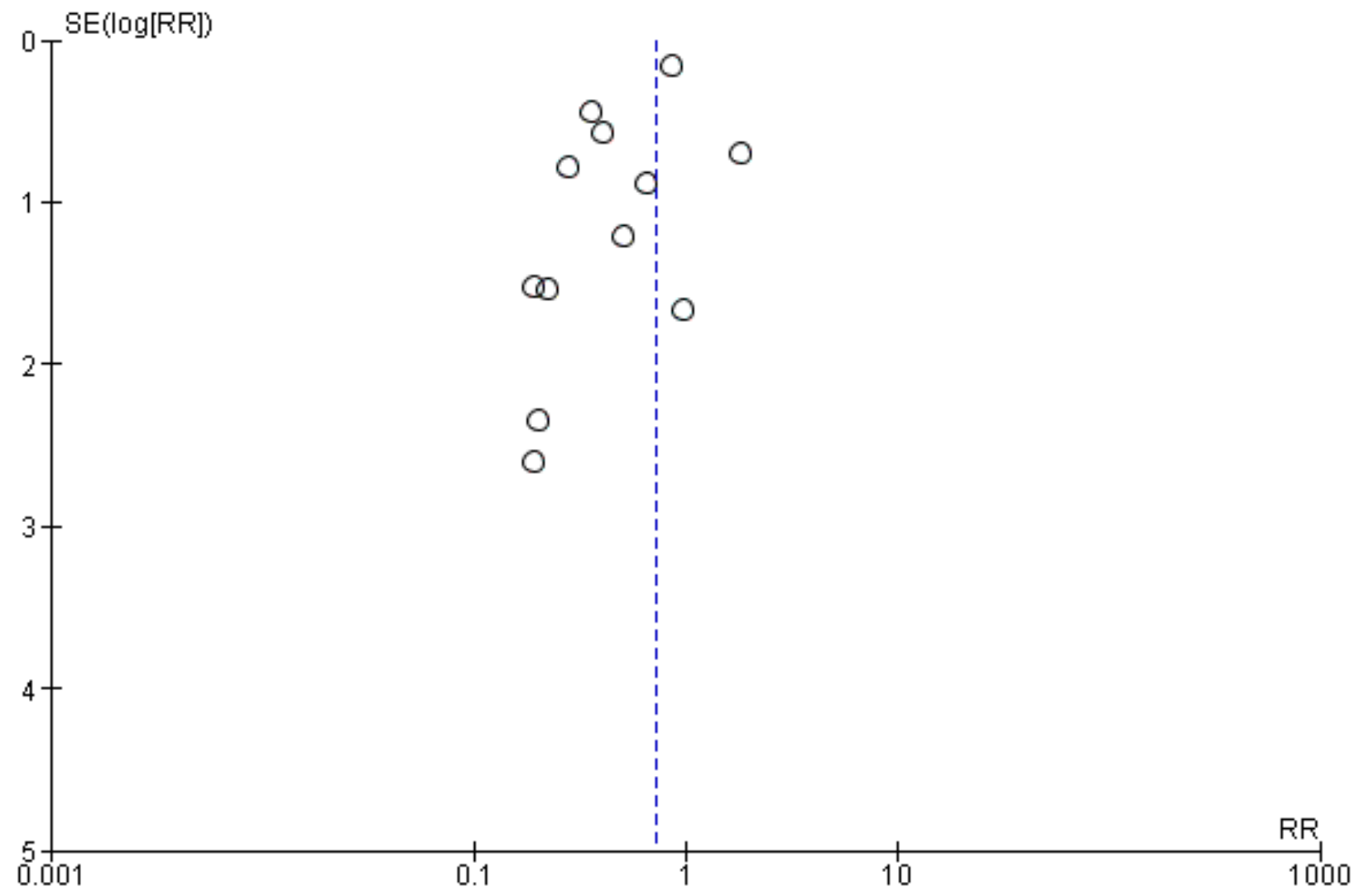


Figure 7. Funnel plot of comparison: 6 Exercise versus control (health-related quality of life), outcome: 6.1 Healthrelated quality of life- overall analysis.

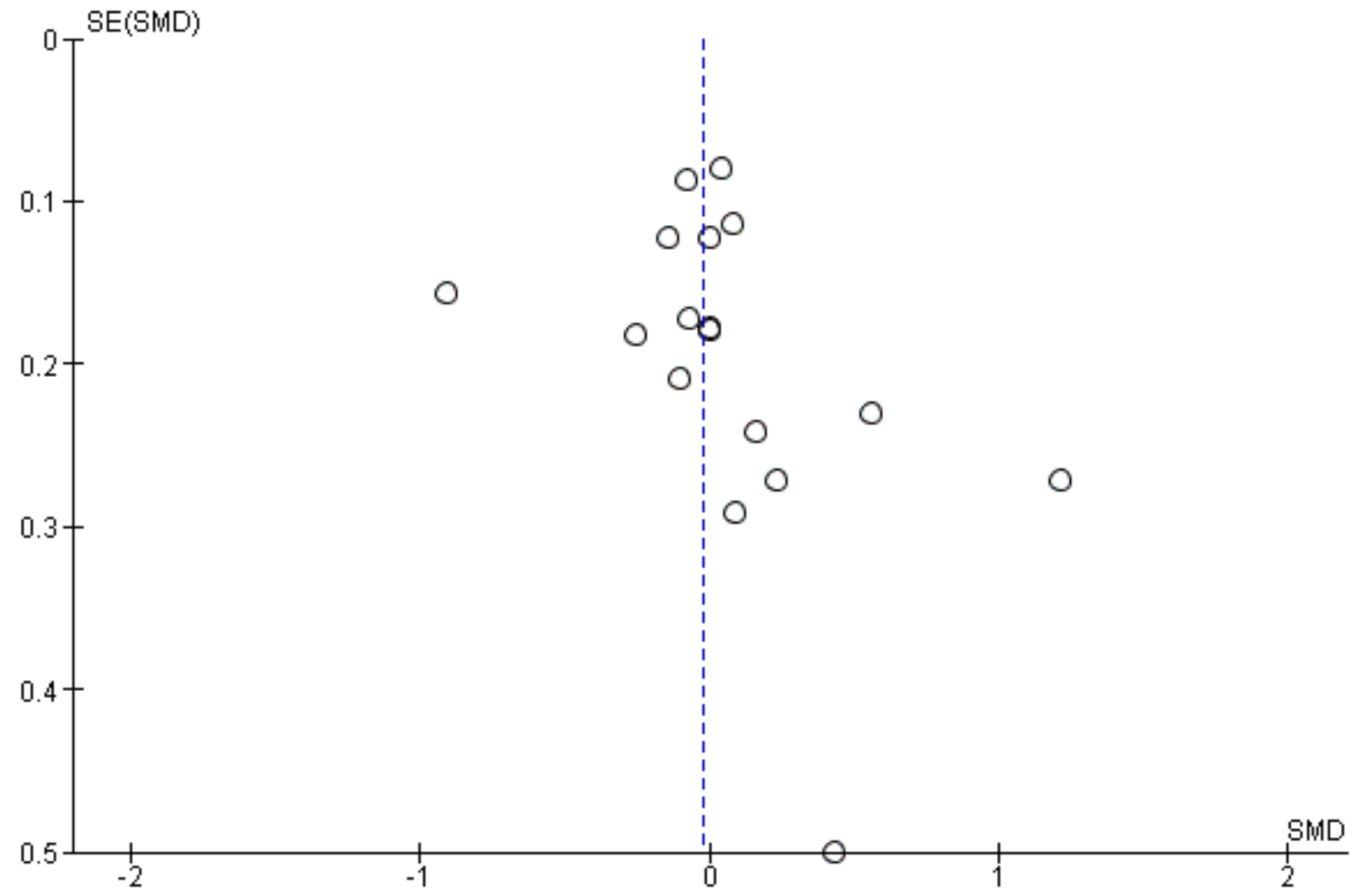


Figure 8. Funnel plot of comparison: 1 Exercise versus control (rate of falls), outcome: 1.6 Rate of falls - subgrouped by exercise type.

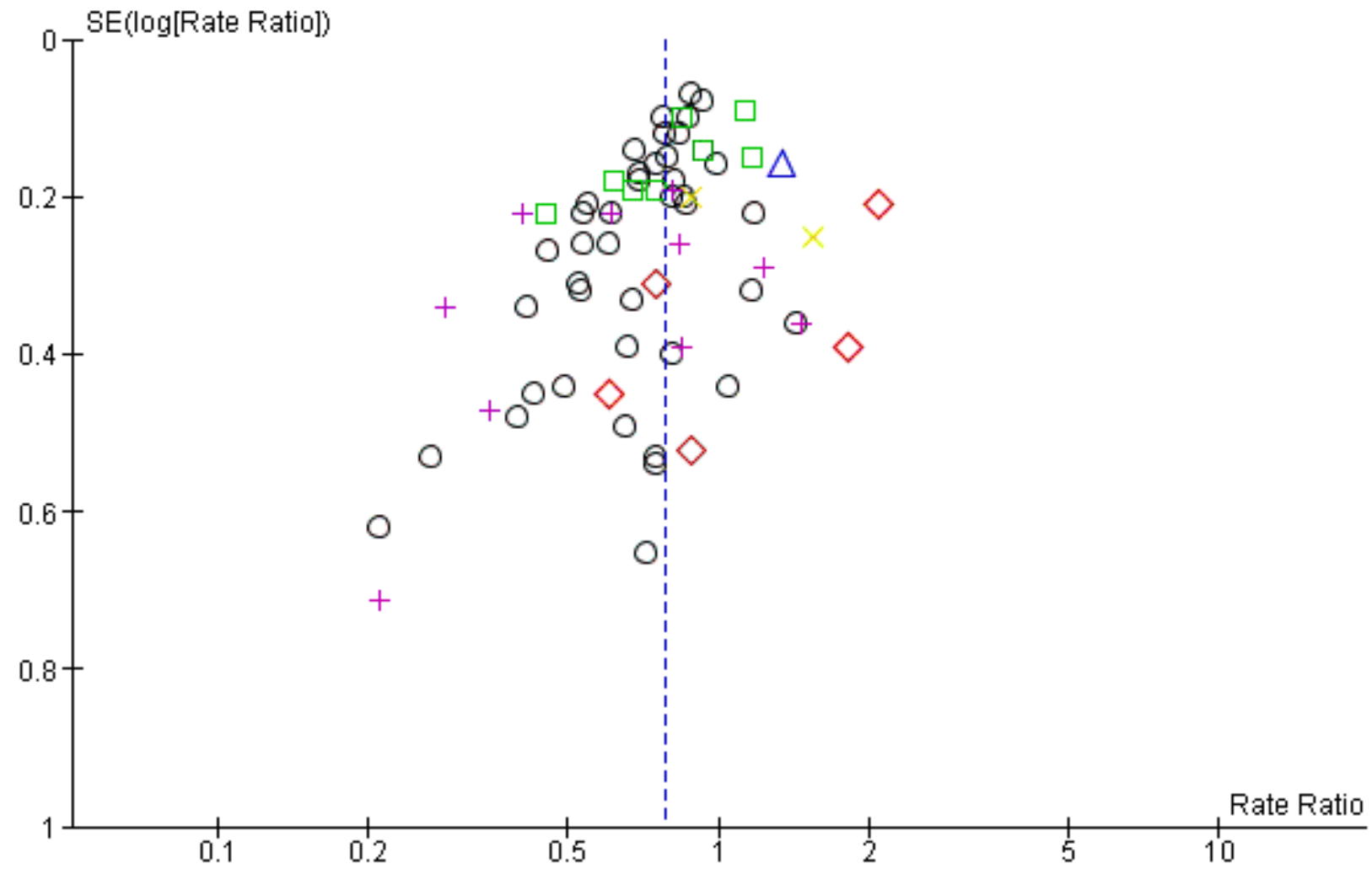

Subgroups

$\begin{array}{ll}\bigcirc \text { Balance and functional exercises vs control } & \triangle \text { 3D exercise (dance) vs control } \\ \diamond \text { Resistance exercise vs control } & \text { Walking programme vs control } \\ \square \text { 3D exercise (Tai Chi) vs control } & + \text { Multiple categories of exercise vs control }\end{array}$


Figure 9. Funnel plot of comparison: 2 Exercise versus control (number of fallers), outcome: 2.6 Number of fallers subgrouped by exercise type.

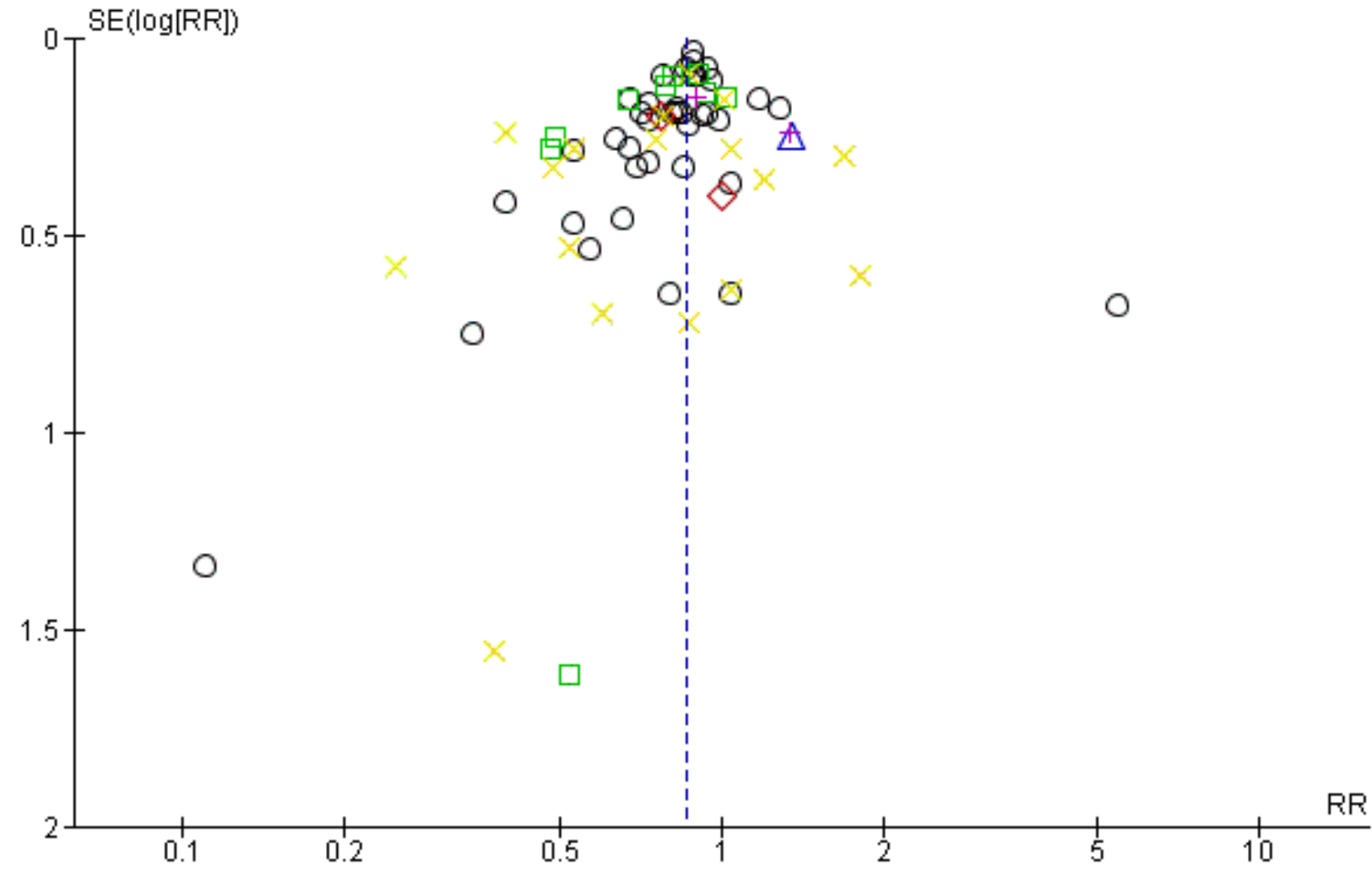

Subgroups

Balance and functional exercises vs control

\section{$\triangle 3 \mathrm{D}$ exercise (dance) vs control Multiple categories of exercise vs control + Walking programme vs control}

\section{DISCUSSION}

\section{Summary of main results}

This review includes 108 trials with 23,407 participants, who were older people living in the community. Of these, 81 trials $(19,684$ participants) contributed the evidence for the main 'exercise versus control' intervention (one that is not thought to reduce falls) comparison; these did not include the four trials that included only people who had been recently discharged from hospital. After summarising the results for this comparison, we summarised the evidence for the primary exercise categories versus control comparisons, where data were available. Our illustrative risks for dichotomous outcomes presented in Summary of findings for the main comparison, are based on counts (number of events divided by the number of participants) for those trials included in the analysis for that outcome. In Summary of findings for the main comparison, we also based our illustrative risks for falls outcomes on the median values obtained from the subgroups of trials for which: a) an increased risk of falls was not an inclusion criterion (not selected population); or b) increased risk of falls was an inclusion criterion. In the other 'Summary of findings' tables, we used the 'all-exercise versus control' studies risks to illustrate the absolute risks for falls and fracture outcomes; we supplemented the falls outcomes by illustrative risks based on count data for the specific exercise category summarised.

\section{Exercise (all types) versus control}

There is high-certainty evidence that falls can be prevented by exercise programmes, as summarised in Summary of findings for the main comparison. Exercise reduces both the rate of falls (reported in 59 randomised controlled trials (RCTs)) and the number of people experiencing falls (reported in 63 RCTs). Subgroup analyses did not reveal differences in effect on both falls outcomes according to whether trials were selected for high risk of falling or not. Hence, the overall rate of falls and number of fallers results were applied when estimating absolute risks in the following lower and higher risk of falls categories. As shown below, the absolute numbers of falls or numbers of fallers prevented are greater in the higher risk populations.

1. For the overall risk category, based on an illustrative risk of 850 falls per 1000 person-years in the control group, there were 195 $(23 \%)$ fewer falls per 1000 person-years in the exercise group (95\% confidence interval (CI) 144 (17\%) to 246 (29\%) fewer). Based on an illustrative risk of 480 fallers per 1000 older people in the control group, there were 72 (15\%) fewer fallers per 1000 
older people in the exercise group (95\% Cl 52 (11\%) to 91 (19\%) fewer).

2. For the non-selected lower risk category, based on an illustrative risk of 605 falls per 1000 person-years in the control group, there were 139 (23\%) fewer falls per 1000 person-years in the exercise group (95\% Cl $102(17 \%)$ to 175 (29\%) fewer). Based on an illustrative risk of 380 fallers per 1000 older people in the control group, there were 57 (15\%) fewer fallers per 1000 older people in the exercise group $(95 \% \mathrm{Cl} 41$ (11\%) to $72(19 \%)$ fewer).

3. For the selected higher risk category, based on an illustrative risk of 1200 falls per 1000 person-years in the control group, there were $276(23 \%)$ fewer falls per 1000 person-years in the exercise group (95\% Cl 204 (17\%) to 348 (29\%) fewer). Based on an illustrative risk of 500 fallers per 1000 older people in the control group, there were $75(15 \%)$ fewer fallers per 1000 older people in the exercise group $(95 \% \mathrm{Cl} 55(11 \%)$ to $95(19 \%)$ fewer).

Subgroup analyses did not reveal differences in effect on both falls outcomes according to whether trials included younger and older populations based on a 75 year cut-off. There was, however, a greater reduction on the rate of falls from exercises (all types) in trials where interventions were delivered by a health professional than in trials where trained instructors who were not health professionals delivered the interventions; however, both approaches reduced the rate of falls. This finding did not apply to the subgroup analysis for number of fallers. Subgroup analyses did not reveal differences in effect on both falls outcomes according to whether interventions were delivered in a group setting or delivered individually.

The test for subgroup differences for when subgrouped by exercise type revealed significant subgroup differences for rate of falls, a finding that endorsed our prespecified intention to report separate analyses by primary exercise type (see below).

Far fewer studies reported on number of people who experienced fall-related fractures (10 RCTs), fall-related hospital admission (2 RCTs) and medical attention (5 RCTs). Exercise may reduce the number of people with fall-related fractures: $27 \%$ reduction, $95 \%$ $\mathrm{Cl} 5 \%$ to $44 \%$ reduction. Based on an illustrative risk, derived from the study data, of 64 people with fall-related fractures per 1000 older people in the control group, there were 17 fewer people with fall-related fractures per 1000 older people in the exercise group (95\% $\mathrm{Cl} 3$ to 28 fewer). Exercise may make little or no difference to the number of people who experience one or more falls requiring hospital admission; reduction $22 \%, 95 \% \mathrm{Cl} 49 \%$ reduction to $18 \%$ increase. Based on an illustrative risk of 57 people with fall-related hospital admission per 1000 older people in the control group, there were 12 fewer people with fall-related hospital admissions per 1000 older people in the exercise group $(95 \% \mathrm{Cl} 28$ fewer to 11 more). Exercise may reduce the number of people who experience one or more falls requiring medical attention: $39 \%$ reduction, $95 \%$ $\mathrm{Cl} 21 \%$ to $53 \%$ reduction. Based on an illustrative risk of 211 people with falls that required medical attention per 1000 older people in the control group, there were 82 fewer people with fall-related medical attention per 1000 older people in the exercise group (95\% $\mathrm{Cl} 44$ to 111 fewer).

Exercise may make little important difference to people-reported health-related quality of life compared with control: conversion of the pooled result (standardised mean difference (SMD) $-0.03,95 \%$ $\mathrm{Cl}-0.10$ to $0.04 ; 15 \mathrm{RCTs}$ ) to the EQ-5D and SF-36 scores showed the respective $95 \% \mathrm{Cls}$ were much smaller than minimally important differences for both scales.

We are uncertain of the evidence for adverse events, which were incompletely reported and mainly for the exercise groups only in 27 RCTs (6019 participants). Fourteen trials reported no adverse events. Aside from two serious adverse events ( 1 pelvic stress fracture and 1 inguinal hernia surgery) reported in one trial, the remainder were non-serious adverse events, primarily of a musculoskeletal nature.

\section{Different exercise types versus control}

'Summary of findings' tables, summarising the evidence for the rate of falls, risk of falling, fall-related fractures and adverse events, are presented for the primary exercise categories for which data are available. There are no data available for flexibility exercise or endurance exercise versus control. The following should be viewed in terms of the data available for each exercise type. The few direct comparisons of different exercise types were clinically heterogeneous and we did not undertake any meta-analyses for these comparisons for any of the outcomes.

\section{Balance and functional exercises}

This was compared with control in 48 trials. As summarised in Summary of findings 2, there is high-certainty evidence that balance and functional exercises reduce the rate of falls and the number of people who experience falls. There is lowcertainty evidence that this type of exercise programme may help prevent fall-related fractures. Adverse events, which were incompletely reported, were mainly non-serious adverse events of a musculoskeletal nature.

\section{Resistance (strength) exercises}

This was compared with control in seven trials. As summarised in Summary of findings 3, we are uncertain of the effects of resistance training on the rate of falls and number of fallers. We are uncertain of the effects on fall-related fractures; only three participants had fractures in the single trial reporting this outcome. Adverse events, which were incompletely reported, were nonserious adverse events of a musculoskeletal nature.

\section{D exercise: Tai Chi}

This was compared with control in 10 trials. As summarised in Summary of findings 4, there is low-certainty evidence that Tai Chi may reduce the rate of falls and high-certainty evidence that Tai $C$ hi reduces the number of people who experience falls. Fall-related fractures were not reported. The two trials reporting on adverse events, reported none.

\section{D exercise: dance}

This was compared with control in one trial. As summarised in Summary of findings 5, we uncertain of findings of little effect of dance training on rate of falls or numbers of fallers. Fall-related fractures were not reported. The trials reported there had been no adverse events in the dance group.

\section{General physical activity: walking programme}

This was compared with control in three trials. As summarised in Summary of findings 6 , we are uncertain of the effects of walking programmes on rate of falls and number of people who experience 
falls. We are uncertain of the effects on fall-related fractures; only 10 participants had fractures in the single trial reporting this outcome. All three trials reported there had been no adverse events.

\section{Multiple categories of exercise}

Multiple categories of exercise (most commonly balance and functional exercises plus resistance exercises) were compared with control in 21 trials. As summarised in Summary of findings 7 , there is moderate-certainty evidence that these interventions probably reduce rate of falls and number of fallers. Sensitivity analyses revealed little difference in the results when only the trials that included the most commonly two components (balance and functional exercises plus resistance exercises) as primary outcomes were pooled. Sensitivity analyses also revealed little difference in the results when any intervention that included balance and functional exercises plus strength exercises, as primary or secondary interventions, was classified as multiple types of exercise (Appendix 18). There is low-certainty evidence that these interventions may have little effect on fall-related fractures. Adverse events, which were incompletely reported, were mainly non-serious adverse events of a musculoskeletal nature.

\section{Subgroup analyses}

Our prespecified subgroup analyses were performed on falls outcomes for balance and functional exercises and multiple categories of exercise. As for the overall exercise versus control comparison, subgroup analysis did not suggest a difference in effects on falls outcomes between trials that used increased risk of falls as an inclusion criterion to those in trials that did not. Also consistent with the overall exercise versus control comparison, there was greater reduction on the rate of falls from balance and functional exercises in trials where interventions were delivered by a health professional than in trials where the interventions were delivered by trained instructors who were not health professionals; although both approaches resulted in reductions in the rate of falls. There was no difference in the reduction on rate of falls from multiple primary types of exercise in trials where interventions were delivered by a health professional than in trials where the intervention was not delivered by a health professional. Other subgroup analyses did not detect differences in effects of exercises in trials where interventions were delivered in a group setting compared with trials where interventions were delivered individually. We did not explore the interaction between subgroups. For example, higher risk people are likely to require health professional input for safe exercise prescription.

\section{Adverse events}

Forty-one of the 108 included trials reported on adverse events to some degree (31 exercise versus control trials, of which four trials included people recently discharged from hospital, and 10 exercise versus exercise trials). Seventeen trials reported an absence of adverse events, one trial reported a pelvic fracture and an inguinal hernia surgery (Clemson 2012), and the remaining trials primarily reported non-serious musculoskeletal events. Only two trials, one of which included post-discharge from hospital participants, reported adverse events in both exercise and control groups over the whole trial period, perhaps reflecting the cost and complexity of such monitoring.

\section{Exercise (all types) versus control in people who had recently been discharged from hospital}

Four heterogeneous studies investigated outcomes in people who had recently been discharged from hospital. We did not pool the data available for rate of falls, number of fallers and health-related quality of life given the small numbers of trials and diversity of the interventions. Overall, the very low-certainty evidence, downgraded for risk of bias, inconsistency and imprecision evidence is insufficient to draw any conclusions.

\section{Comparisons of different types, modes of delivery and doses of exercise}

Given the variability between programmes, we did not undertake any meta-analyses of comparisons between different types of exercise. Most of the trials in these analyses did not find significant differences in the fall prevention effects of different programmes, but most were not likely to be adequately powered to detect differences between different exercise programmes. When comparing different exercise types delivered within the same studies we found some indication that higher doses of exercise were associated with a greater impact on the rate of falls and the number of people falling.

\section{Economic data}

Of the 12 studies included in this review that reported economic evaluation, some give an indication of value for money for the interventions tested. Variations in the methods used, however, made comparisons across studies difficult. There was some, although limited, evidence that fall prevention strategies can be cost-saving during the trial period, and may also be costeffective over the participants' remaining lifetime; however, it should be noted that these analyses usually fail to include the cost of identifying the target population, which can be substantial and can impact on cost-effectiveness measures (Eldridge 2005). Additional studies have modelled the impact and cost-effectiveness of a public health falls prevention programme in Australia (Farag 2015), undertaken secondary analyses to estimate cost-effectiveness of implementing the Otago Exercise Program in Norway (Hektoen 2009), performed cost-benefit analysis of fall prevention interventions (Campbell 1999; Carande-Kulis 2015; Clemson 2004a; Li 2005), and undertaken a literature review and developed a tool to estimate the cost-effectiveness of fall prevention interventions in the community (Public Health England 2018).

\section{Overall completeness and applicability of evidence}

\section{Trial design and participants}

The 108 trials included in this review included 23,407 communitydwelling older people, who were predominantly women (77\%). A wide range of ages were included as few trials set upper age limits. Participant characteristics varied greatly due to the recruitment methods used, and the inclusion and exclusion criteria applied. Participants in most trials were healthy volunteers; however, some trials recruited people who were attending outpatient clinics. Sixty trials $(56 \%)$ recruited participants with a history of falls or one or more risk factors for falling.

We excluded trials that tested exercise interventions for preventing falls in people affected by particular conditions, such as stroke, Parkinson's disease, multiple sclerosis, hip fracture and dementia 
from this review as we considered that the results of interventions for these conditions were not necessarily applicable to older people as a whole. Fall prevention trials in these populations also often include a wider age range which would result in some being excluded from this review; Cochrane Reviews for each of these specific groups (including all age groups) would be preferable for summarising the evidence. The majority of trials $(67 \%)$ excluded older people who were cognitively impaired, therefore the results of this review may not be applicable to this high risk group.

Most trials were relatively small (median $=134$ participants), with a mean age of 76 (ranging from a mean age of 65 to a maximum mean age of 88 years). Thirty-seven trials reported 12 -month follow-up, with 49 reporting less than 12 months and 22 reporting more than 12 months follow-up. Trials were undertaken over 25 years from 1992 to 2017.

\section{Setting}

Exercise-based fall prevention interventions tested in a further 58 RCTs were included in this review compared with Gillespie 2012. The included trials were conducted in 25 countries using a variety of healthcare models. These different healthcare systems and structures may have impacted upon the effectiveness of some interventions. There remains a paucity of studies undertaken in low-income economies.

\section{Interventions}

We classified the exercise interventions using the ProFanE guidelines. This classification system is clearly described(Lamb 2011; Appendix 1); however, we acknowledge there is a degree of subjectivity in the classification of exercise interventions based on brief descriptions in trial reports. We conducted post-hoc sensitivity analyses to explore the effects of recategorising trials with a secondary component of strength training as having multiple primarily exercise categories and found this made little difference to the results (Appendix 18). The duration of exercise intervention in the 81 exercise versus control trials ranged from 5 to 130 weeks; it being one year or more in $30 \%$ of these.

\section{Outcomes}

We sought data for rate of falls, number of people falling, number of people sustaining a fall-related fracture, number of people who experienced falls leading to medical attention, number of people who had a fall-related hospital admission, health-related quality of life and number of people who experienced adverse events. However, few studies provided fracture, medical attention, hospital admission, health-related quality of life and full adverse events data. As the analyses and Appendix 10 demonstrate, some studies provided data for both falls and fallers, as recommended in Lamb 2005, and others provided data for one or other falls outcomes.

The outcome of interest, falling, was not always clearly defined, which is a source of concern. Comparability of future research findings would be enhanced by the adoption of the consensus definition of a fall developed for trials in community-dwelling populations by the Prevention of Falls Network Europe (ProFaNE) (Lamb 2005). The included studies also varied in the methods used for falls ascertainment, recording, analysing and reporting. Studies should use accepted protocols for recording of falls data, including daily recording of falls with monthly or more frequent follow-up by the researchers who are blind to group allocation (Lamb 2005). At least $26 \%$ of included trials did not do this despite evidence of a $25 \%$ underreporting of falls when data were collected retrospectively by telephone at the end of a three-month period, compared with data collected daily and returned monthly over the same period (Hannan 2010). There are difficulties in using fall diaries over long time periods however, with trial dropouts due to over-burden of paperwork reported by lliffe 2015.

The lack of consistent measurement of adverse events in trials requires attention by trialists. We found just two studies that measured adverse events in both groups throughout the trial period. Although it is worth noting that the burden on trial resources and participants of full documentation of adverse events is probably a key reason this has not been done to date. Trials of exercise interventions do not tend to be as well-resourced as trials of pharmacological interventions in which adverse event monitoring is routine.

This review only included data for the risk of fractures and injurious falls, rather than for the rates of fractures and injurious falls; however, it is important to note that several trials have identified an impact of exercise on rates of fall-related fracture (Karinkanta 2007; Korpelainen 2006; Kemmler 2010), as well as rates of injurious falls (Uusi-Rasi 2015). There is also evidence of an impact of exercise on the rate of falls requiring medical care, over and above the impact from other types of interventions (Fitzharris 2010).

\section{Other considerations relating to applicability}

We decided not to pool studies undertaken in people who had recently been discharged from hospital with studies undertaken among general older populations. It is well documented that people who have recently been discharged form hospital are at a particularly high risk of falls (Mahoney 1994), and as such may require different intervention approaches. There is increasing awareness that many older people deteriorate physically during a hospital admission (Oliver 2017). We note that a number of recent studies of interventions have been undertaken in this population and among emergency department attendees (Harper 2017; Matchar 2017; Oliver 2017); however, there is still uncertainly of the best treatment for this population and a separate review may be needed in future.

For the control groups of the trials that did not have increased risk of falls as an inclusion criterion, the median rate of falls (if 1000 people were followed over 1 year, there would be 605 falls) and the median proportion of fallers (if 1000 people were followed over 1 year, 380 would experience one or more falls) are similar to estimates of fall risk and rate in the general community derived from large population studies (AIHW 2018; Lord 2011; NICE 2018). This indicates that participants in trials that do not recruit based on fall risk, are representative of the general community, rather than being at low risk of falls.

Subgroup analyses comparing the effects on falls outcomes in trials with predominantly older populations and those with predominantly younger populations should be interpreted with some caution, as implementation of one of the categorisation criteria (mean age minus 1 SD $>75$ ) may result in some younger people in the older group and vice versa. 


\section{Ongoing studies}

The 16 identified ongoing studies may contribute to research priorities. Six ongoing studies, two of which have a larger sample size (exceeding 400 participants), will evaluate the relative impact of different exercise programmes (NCT02126488; NCT03211429; NCT03404830; NCT03455179; $\mathrm{n}>400$ (NCT02287740; NCT02926105). Two studies will investigate individual versus group delivery of the LiFE programme (NCT03462654), and Otago Exercise Program (NCT03320668). Also, one large trial awaiting classification studied the difference between three types of exercise, including flexibility exercise (Li 2018b). Fall-related fractures are listed as outcomes in only two trials (ISRCTN71002650; NCT02617303). Two trials, in India (CTRI/2018/01/011214), and Columbia (NCT03211429), will contribute to the understanding of the effect of exercise on falls in emerging economies. In addition, research is underway to investigate strategies for optimal translation of effective exercise programmes from the research setting to clinical and community settings (Carpenter 2018; Hawley-Hague 2017).

\section{Certainty of the evidence}

This review, containing 108 trials (23,407 participants) provides moderate- to high-certainty evidence of the effectiveness of exercise-based interventions for preventing falls among community-dwelling people aged 60 years and over.

We have summarised the GRADE certainty of evidence in seven 'Summary of findings' tables: Summary of findings for the main comparison (Exercise (all types) versus control); Summary of findings 2 (Balance and functional exercises versus control); Summary of findings 3 (Resistance exercises versus control); Summary of findings 4 (3D (Tai Chi) exercise versus control)); Summary of findings 5 (3D (dance) exercise versus control)); Summary of findings 6 (Walking programme versus control); Summary of findings 7 (Multiple categories of exercise versus control).

The certainty of the evidence ranged from high to very low. We downgraded outcomes by one level for risk of bias if the results changed with removal of the trials with a high risk of bias on one or more items. We downgraded one level for inconsistency where heterogeneity was greater than $60 \%$. In addition, we downgraded the level of evidence for imprecision by one or two levels due to the wide confidence intervals, often reflecting the small number of participants and trials. We downgraded where the risk of small sample bias was evident on funnel plot and downgraded one level for fall-related hospital admission and fall-related medical attention because a large number of studies included in the review do not contribute to the outcome.

Sensitivity analyses revealed the results for the falls outcomes to be stable (see Appendix 18) suggesting that the results are robust to key risks of bias and essentially unchanged by methodological choices in the conduct of the review. In undertaking the GRADE assessment we downgraded the certainty of evidence based on sensitivity analysis (removal of trials with one or more items at high risk of bias) for one or both falls outcomes for several types of exercise (resistance, Tai Chi, walking, multiple) and for the overall fracture and quality of life outcomes. It is noteworthy that many of the sensitivity analyses undertaken regarding risk of bias revealed a stability of the results of this review.
Rates of fractures and injurious falls were not prespecified outcomes in this review. More trials reported the outcome in this way than anticipated. We would be in favour of reporting these outcomes in future versions of this review.

\section{Potential biases in the review process}

We conducted a comprehensive search of the published literature using multiple databases and also searched clinical trial registries for completed trials for which full reports had not been identified. Two review authors who were blinded to each other's results performed screening and data extraction in duplicate to minimise bias. Despite this thorough search strategy, we acknowledge the possibility that some relevant trials may have been missed, especially if they were published in languages other than English.

Two review authors independently classified the exercise interventions using the ProFaNE guidelines (Lamb 2011), including assigning intervention categories to primary or secondary status. We recognise there is some subjectivity in this classification system, particularly for those interventions containing more than one category of exercise. Sensitivity analyses that tested the effects of recategorising primary balance and functional exercise trials with a secondary component of strength training indicated that this did not importantly affect the results.

We recorded and reported data on fracture, hospitalisation, medical attention and health-related quality of life only where it was reported by intervention group. To check whether this could be a source of potential bias, we conducted an audit of fracture reporting in the 48 trials with balance, function and gait exercise interventions. Of the 10 trials reporting fracture outcomes, we included seven reporting fracture outcomes by intervention group in the analysis. We did not include the three other studies in the analysis because they either did not report fractures by group (Skelton 2005), they reported fractures during the intervention period but not during follow-up (Iliffe 2014), or they just reported a fracture (1 pelvic stress fracture) as an adverse event (Clemson 2012). This provided some reassurance that our approach for these secondary and generally under-reported outcomes did not have an important impact on the results.

\section{Agreements and disagreements with other studies or reviews}

Our review adds to the existing body of evidence and supports the findings of Gillespie 2012, whereby multiple component groupbased exercise was found to reduce the rate of falls (rate ratio (RaR) $0.71,95 \%$ confidence interval $(\mathrm{Cl}) 0.63$ to $0.82 ; 16$ trials, 3622 participants) and the risk of falling (risk ratio (RR) $0.85,95 \% \mathrm{Cl} 0.76$ to $0.96 ; 22$ trials, 5333 participants). Similar results were found for individually-delivered multiple component exercise that reduced the rate of falls (RaR $0.68,95 \% \mathrm{Cl} 0.58$ to $0.80 ; 951$ participants, 7 trials) and the number of people falling (RR $0.78,95 \% \mathrm{Cl} 0.64$ to $0.94 ; 714$ participants, 6 trials). The review by Gillespie 2012, also found that Tai Chi reduced the rate of falls ( $\mathrm{RaR} 0.72,95 \% \mathrm{Cl}$ 0.52 to $1.00 ; 1563$ participants, 5 trials) and the number of people falling (RR $0.71,95 \% \mathrm{Cl} 0.57$ to $0.87 ; 1625$ participants, 6 trials). Group-based balance or functional exercises also demonstrated a statistically significant reduction in the rate of falls (RaR $0.72,95 \%$ $\mathrm{Cl} 0.55$ to $0.94 ; 519$ participants, 4 trials) but not in the number of people falling (RR $0.81,0.62$ to 1.07; 453 participants, 3 trials). 
This influential review has informed, and been the basis of, many guidelines and policy documents internationally.

We extended the findings of Gillespie 2012 by recoding intervention programmes (Appendix 1), in an attempt to identify a primary exercise component for each included study and reserving the 'multiple component' category for trials in which the intervention programme had an equal focus on each of the multiple components. As a result, more studies in our review are classified as balance and functional exercises and fewer as multiple component programmes. We hope that this change will be of assistance to those seeking to design exercise intervention programmes.

The present review also adds to our previous non-Cochrane review (Sherrington 2017), that used different methodology (multivariable metaregression) yet reached similar conclusions about the importance of the inclusion of exercises that safely challenge balance in fall prevention exercise programmes. Other recent analyses have reached similar findings, including a large network meta-analysis (Tricco 2017).

The importance of exercise in fall prevention suggests that greater attention be given to the widespread implementation of a life course approach to healthy ageing, i.e. lifelong exercise to maximise physical functioning in older age, as suggested by the World Health Organization (WHO 2015).

\section{AUTHORS' CONCLUSIONS}

\section{Implications for practice}

Well-designed exercise programmes reduce the rate of falls and the number of people experiencing falls amongst older people living in the community (high-certainty evidence).

The effects of exercise programmes are uncertain for other nonfalls outcomes, mainly reflecting the considerable under-reporting of these outcomes in the included trials. Exercise may reduce the number of people experiencing one or more fall-related fractures and the number of people experiencing one or more falls requiring medical attention (low-certainty evidence). We are uncertain about the effect of exercise programmes on the number of people who experience one or more falls requiring hospital admission. Exercise may make little important difference to health-related quality of life (low-certainty evidence). The reporting of adverse events was poor; where reported these were usually non-serious and predominantly musculoskeletal.

Effective exercise programmes that reduce both falls outcomes primarily involve balance and functional exercises (high-certainty evidence) or include multiple exercise categories, most commonly balance and functional exercises plus resistance exercises (moderate-certainty evidence). Tai Chi reduces the number of people experiencing falls (high-certainty evidence) and may reduce the rate of falls (low-certainty evidence). We are uncertain about the effect of programmes involving primarily resistance exercises, dance or walking, as there is insufficient evidence on these. There are no data available for flexibility exercise or endurance exercise versus control.

Exercise programmes were effective regardless of whether they were delivered individually or in groups, by health professionals or trained non-health professionals, to younger or older populations (based on a 75 year age threshold) or to those identified at a higher risk of falls or not selected for risk of falls. There is likely to be a greater absolute impact in people identified at increased risk of falling, but there is benefit also for those who are at more general risk in the community. Although trial follow-up ranged from 3 to 18 months in the main comparison, there may also be longer-term benefits of introducing fall prevention exercise habits in people in the general community. Notably too, the duration of most of the exercise programmes was 12 weeks or over and nearly one-third lasted a year or more. These findings highlight the importance of primary prevention.

There is currently insufficient evidence to determine the effects of exercise programmes for people recently discharged from hospital. There is also insufficient information from direct comparisons to determine whether there are differences in the effectiveness of different types, modes of delivery and doses of exercise.

\section{Implications for research}

Further work is needed to understand the relative impact of different exercise programmes. Such studies will need to be very large to be adequately powered to detect effects between interventions.

Large studies are also needed to establish the impact of fall prevention interventions on fall-related fractures and falls requiring medical attention, as such falls are particularly costly to health systems and impactful for individuals.

During the development of priority topics for future research, the current evidence base should be considered in conjunction with the areas studied in the ongoing trials.

Individual participant data meta-analysis could contribute further to the investigation of differential effects of exercise in people of different ages and baseline fall risks, as these are individual-level rather than trial-level characteristics. We recommend researchers follow the Prevention of Falls Network Europe (ProFaNE) guidelines for the conduct of falls trials (Lamb 2005).

Further research is required to establish the effectiveness of fall prevention programmes in emerging economies, where the burden of falls is increasing more rapidly than in high-income countries due to rapidly ageing populations (WHO 2015).

There is an urgent need to investigate strategies to enhance implementation of effective exercise-based fall prevention interventions into routine care of older people by healthcare professionals and community organisations.

As it is possible that interventions designed to increase physical activity could increase falls due to increased exposure to risk, we suggest that those undertaking trials of physical activity interventions in older people consider monitoring falls.

Future studies should use the consensus definition of a fall developed for trials in community-dwelling populations by ProFaNE (Lamb 2005), consistent methods of falls ascertainment, and consistent measurement of adverse events in both groups throughout the trial period. Future research should use the ProFaNE descriptors to categorise interventions (Lamb 2011), but should be clear how this was operationalised. Appendix 1 outlines how this guide was operationalised in the present review and may provide a useful resource. 


\section{ACKN OWLEDGEMENTS}

We are very grateful for helpful feedback from editors Liz Bickerdike, Helen Handoll and Helen Wakeford; and external referees Elizabeth Burns, Helen Hawley, Dawn Skelton and Edgar Ramos Vieir on drafts of the review. We also thank Joanne Elliott and Helen Wakeford for editorial support on the review and Kate Cahill and Clare Dooley for copy-editing. We thank Joanne Elliott for assistance with developing the search strategy. We would like to acknowledge the helpful feedback on the review from consumer peer reviewers: Federica Davolio, Auxiliadora Fraiz and Marina Sartini.
We are also grateful to the authors of Gillespie 2012, particularly Lesley Gillespie and Clare Robertson, for the development of methods and procedures and assistance with this review.

We are grateful to Courtney West, Connie Jensen and Christoper Ng for assistance with searching and data extraction.

This project was partly funded by the National Institute for Health Research (NIHR) via Cochrane Infrastructure funding to the Cochrane Bone, Joint and Muscle Trauma Group. Additional funding for the review was via the NIHR (UK): NIHR Cochrane Reviews of National Institute for Care and Excellence (NICE) Priority scheme, project reference: NIHR127512. The views and opinions expressed therein are those of the authors and do not necessarily reflect those of the Systematic Reviews Programme, NIHR, National Health Service (NHS) or the Department of Health. 


\section{REFERE N CE S}

\section{References to studies included in this review}

Almeida 2013 \{published data only\}

Almeida TL, Alexander NB, Nyquist LV, Montagnini ML, Santos AC, Rodrigues GH, et al. Minimally supervised multimodal exercise to reduce falls risk in economically and educationally disadvantaged older adults. Journal of Aging and Physical Activity 2013;21(3):241-59.

Ansai 2015 \{published data only (unpublished sought but not used)\}

Ansai JH, Rebelatto JR. Effect of two physical exercise protocols on cognition and depressive symptoms in oldest-old people: A randomized controlled trial. Geriatrics and Gerontology International 2015;15(9):1127-34.

Arantes 2015 \{published data only (unpublished sought but not used)\}

Arantes PM, Dias JM, Fonseca FF, Oliveira AM, Oliveira MC, Pereira LS, et al. Effect of a program based on balance exercises on gait, functional mobility, fear of falling, and falls in prefrail older women: a randomized clinical trial. Topics in Geriatric Rehabilitation 2015;31(2):113-20. [DOI: 10.1097/ TGR.0000000000000056]

\section{Arkkukangas 2015 \{published and unpublished data\}}

Arkkukangas M. Fall data [personal communication]. Email to: Z. Michaleff. 18 August 2015.

* Arkkukangas M, Johnson ST, Hellström K, Söderlund A, Eriksson S, Johansson AC. A feasibility study of a randomised controlled trial comparing fall prevention using exercise with or without the support of motivational interviewing. Preventive Medicine Reports 2015;2:134-40.

Ballard 2004 \{published data only (unpublished sought but not used)\}

Ballard JE, McFarland C, Wallace LS, Holiday DB, Roberson G. The effect of 15 weeks of exercise on balance, leg strength, and reduction in falls in 40 women aged 65 to 89 years. Journal of the American Medical Women's Association 2004;59(4):255-61. [MEDLINE: 16845754]

\section{Barker 2016 \{published data only\}}

Barker AL, Talevski J, Bohensky MA, Brand CA, Cameron PA, Morello RT. Feasibility of Pilates exercise to decrease falls risk: a pilot randomized controlled trial in community-dwelling older people. Clinical Rehabilitation 2016;30(10):984-96.

\section{Barnett 2003 \{published data only\}}

Barnett A, Smith B, Lord SR, Williams M, Baumand A. Community-based group exercise improves balance and reduces falls in at-risk older people: a randomised controlled trial. Age and Ageing 2003;32(4):407-14.

\section{Beyer 2007 \{published data only (unpublished sought but not used)\}}

Beyer N, Simonsen L, Bulow J, Lorenzen T, Jensen D V, Larsen L, et al. Old women with a recent fall history show improved muscle strength and function sustained for six months after finishing training. Aging Clinical and Experimental Research 2007;19(4):300-9.

\section{Boongrid 2017 \{published data only\}}

Boongrid C, Keesukphan P, Phiphadthakusolkul S, Rattanasiri S, Thakkinstian A. Effects of a simple home-based exercise program on fall prevention in older adults: A 12-month primary care setting, randomized controlled trial. Geriatrics and Gerontology International 2017;17(11):2157-63. [DOI: 10.1111/ ggi.13052]

\section{Brown 2002 \{published data only\}}

Brown A P. Reducing falls in elderly people: A review of exercise interventions. Physiotherapy Theory and Practice 1999;15(2):59-68

* Brown Al. Functional Adaptation to Exercise in Elderly Subjects [thesis]. Perth (Australia): Curtin University of Technology, 2002 (espace.curtin.edu.au/ handle/20.500.11937/401).

Piotrowski A, Cole J, Allison G. The influence of functional ability and physical and social intervention on falls in elderly subjects. XVIth Congress of the International Association of Gerontology; 1997 August 19-23; Adelaide (Australia). 2002.

Buchner 1997 \{published data only\}

* Buchner DM, Cress ME, De Lateur BJ, Esselman PC, Margherita AJ, Price R, et al. The effect of strength and endurance training on gait, balance, fall risk, and health services use in community-living older adults. Journals of Gerontology. Series A, Biological Sciences and Medical Sciences 1997;52(4):M218-24

Buchner DM, Cress ME, Wagner EH, De Lateur BJ. The role of exercise in fall prevention: Developing targeting criteria for exercise programs. In: Vellas B, Toupet M, Rubenstein L, Albarede JL, Christen Y editor(s). Falls, Balance and Gait Disorders in the Elderly. Amsterdam: Elsevier, 1992:55-68.

Buchner DM, Cress ME, Wagner EH, De Lateur BJ, Price R, Abrass IB. The Seattle FICSIT/Movelt study: the effect of exercise on gait and balance in older adults. Journal of the American Geriatrics Society 1993;41(3):321-5.

Bunout 2005 \{published and unpublished data\}

Bunout D. [Personal communication]. Personal communication reported by Gillespie 2012.

* Bunout D, Barrera G, Avendano M, De la Maza P, Gattas V, Leiva L, et al. Results of a community-based weight-bearing resistance training programme for healthy Chilean elderly subjects. Age and Ageing 2005;34(1):80-3.

\section{Campbell 1997 \{published data only\}}

Campbell AJ, Robertson MC, Gardner MM, Norton RN, Buchner DM. Falls prevention over 2 years: a randomized controlled trial in women 80 years and older. Age and Ageing 1999;28(6):513-8. 
Campbell AJ, Robertson MC, Gardner MM, Norton RN, Buchner DM. Psychotropic medication withdrawal and a home-based exercise program to prevent falls: a randomized, controlled trial. Journal of the American Geriatrics Society 1999;47(7):850-3.

* Campbell AJ, Robertson MC, Gardner MM, Norton RN, Tilyard MW, Buchner DM. Randomised controlled trial of a general practice programme of home based exercise to prevent falls in elderly women. BMJ 1997;315(7115):1065-9.

Gardner M. Home-based exercises to prevent falls in elderly women. New Zealand Journal of Physiotherapy 1998;26(3):6.

Gardner MM, Buchner DM, Robertson MC, Campbel AJ. Practical implementation of an exercise-based falls prevention programme. Age and Ageing 2001;30(1):77-83.

Robertson MC. Development of a falls prevention programme for elderly people: evaluation of efficacy, effectiveness, and efficiency [thesis]. Dunedin (NZ): University of Otago, 2001.

Robertson MC, Campbell AJ, Gardner MM, Devlin N. Preventing injuries in older people by preventing falls: a meta-analysis of individual-level data. Journal of the American Geriatrics Society 2002;50(5):905-11.

Robertson MC, Devlin N, Scuffham P, Gardner MM, Buchner DM, Campbell AJ. Economic evaluation of a community based exercise programme to prevent falls. Journal of Epidemiology and Community Health 2001;55(8):600-6.

Carter 2002 \{published data only (unpublished sought but not used)\}

* Carter ND, Khan KM, McKay HA, Petit MA, Waterman C, Heinonen $A$, et al. Community-based exercise program reduces risk factors for falls in 65- to 75-year-old women with osteoporosis: randomized controlled trial. Canadian Medical Association Journal 2002;167(9):997-1004.

Carter ND, Khan KM, Petit MA, Heinonen A, Waterman C, Donaldson MG, et al. Results of a 10 week community based strength and balance training programme to reduce fall risk factors: a randomised controlled trial in 65-75 year old women with osteoporosis. British Journal of Sports Medicine 2001;35(5):348-51.

\section{Cerny 1998 \{published and unpublished data\}}

Cerny K. [Personal communication]. Personal communication reported by Gillespie 2012.

* Cerny K, Blanks R, Mohamed O, Schwab D, Robinson B, Russo $A$, et al. The effect of a multidimensional exercise program on strength, range of motion, balance and gait in the well elderly. Gait and Posture 1998;7(2):185-6.

Clegg 2014 \{published and unpublished data\}

Clegg A. Faller outcome data [personal communication]. Email to: C. West. 4 June 2018.

* Clegg A, Barber S, Young J, Iliffe S, Forster A. The Homebased Older People's Exercise (HOPE) trial: a pilot randomised controlled trial of a home-based exercise intervention for older people with frailty. Age and Ageing 2014;43(5):687-95.

Clemson 2010 \{published data only\}

Clemson L, Singh MF, Bundy A, Cumming RG, Weissel E, Munro J, et al. LiFE Pilot Study: a randomised trial of balance and strength training embedded in daily life activity to reduce falls in older adults. Australian Occupational Therapy Journal 2010;57(1):42-50.

\section{Clemson 2012 \{published data only\}}

Clemson L, Fiatarone Singh MA, Bundy A, Cumming RG, Manollaras K, O'Loughlin P, et al. Integration of balance and strength training into daily life activity to reduce rate of falls in older people (the LiFE study): randomised parallel trial. BMJ 2012;345:e4547.

\section{Cornillon 2002 \{published data only\}}

Cornillon E, Blanchon MA, Ramboatsisetraina P, Braize C, Beauchet $O$, Dubost $V$. Effectiveness of falls prevention strategies for elderly subjects who live in the community with performance assessment of physical activities (before-after) [Impact d'un programme de prevention multidisciplinaire de la chute chez le sujet age autonome vivant a domicile, avec analyse avant-apres des performances physiques]. Annales de Readaptation et de Medecine Physique 2002;45(9):493-504.

\section{Dadgari 2016 \{published and unpublished data\}}

Dadgari A. Fall outcomes [personal communication]. Email to: $\mathrm{N}$ Fairhall. 29 August 2017.

Dadgari A. Number of males / females [personal communication]. Email to: C Ng. 4 September 2017.

Dadgari A. Number of study participants per health centre [personal communication]. Email to: C Sherrington. 1 January 2018.

* Dadgari A, Aizan Hamid T, Hakim MN, Chaman R, Mousavi SA, Poh Hin L, et al. Randomized control trials on Otago Exercise Program (OEP) to reduce falls among elderly community dwellers in Shahroud, Iran. Iran Red Crescent Medical Journal 2016;18(5):e26340.

\section{Dangour 2011 \{published data only (unpublished sought but not used)\}}

Dangour AD, Albala C, Aedo C, Elbourne D, Grundy E, Walker D, et al. A factorial-design cluster randomised controlled trial investigating the cost-effectiveness of a nutrition supplement and an exercise programme on pneumonia incidence, walking capacity and body mass index in older people living in Santiago, Chile: the CENEX study protocol. Nutrition Journal 2007;6:14.

* Dangour AD, Albala C, Allen E, Grundy E, Walker DG, Aedo C, et al. Effect of a nutrition supplement and physical activity program on pneumonia and walking capacity in Chilean older people: a factorial cluster randomized trial. PLoS Medicine 2011;8(4):e1001023.

ISRCTN48153354. Cost-effectiveness of nutritional supplementation and exercise programme among older people 
in Santiago, Chile. controlled-trials.com/ISRCTN48153354 (first received 22 July 2005).

Walker DG, Aedo C, Albala C, Allen E, Dangour AD, Elbourne D, et al. Methods for economic evaluation of a factorial-design cluster randomised controlled trial of a nutrition supplement and an exercise programme among healthy older people living in Santiago, Chile: the CENEX study. BMC Health Services Research 2009;9:85.

Davis 2011 \{published data only (unpublished sought but not used)\}

Davis JC, Marra CA, Beattie BL, Robertson MC, Najafzadeh M, Graf $P$, et al. Sustained cognitive and economic benefits of resistance training among community-dwelling senior women: a 1-year follow-up study of the Brain Power study. Archives of Internal Medicine 2010;170(22):2036-8.

Davis JC, Marra CA, Liu-Ambrose TY. Falls-related self-efficacy is independently associated with quality-adjusted life years in older women. Age and Ageing 2011;40(3):340-6.

* Davis JC, Marra CA, Robertson MC, Khan KM, Najafzadeh M, Ashe MC, et al. Economic evaluation of dose-response resistance training in older women: a cost-effectiveness and cost-utility analysis. Osteoporosis International 2011;22(5):1355-66.

Davis JC, Marra CA, Robertson MC, Najafzadeh M, LiuAmbrose T. Sustained economic benefits of resistance training in community-dwelling senior women. Journal of American Geriatrics Society 2011;59(7):1232-7.

Liu-Ambrose T, Davis JC, Nagamatsu LS, Hsu CL, Katarynych LA, Khan KM. Changes in executive functions and self-efficacy are independently associated with improved usual gait speed in older women. BMC Geriatrics 2010;10:25.

Liu-Ambrose T, Nagamatsu LS, Graf P, Beattie BL, Ashe MC, Handy TC. Resistance training and executive functions: a 12month randomized controlled trial. Archives of Internal Medicine 2010;170(2):170-8.

\section{Day 2002 \{published data only\}}

* Day L, Fildes B, Gordon I, Fitzharris M, Flamer H, Lord S. Randomised factorial trial of falls prevention among older people living in their own homes. BMJ 2002;325(7356):128.

Fitzharris MP, Day L, Lord SR, Gordon I, Fildes B. The Whitehorse NoFalls trial: effects on fall rates and injurious fall rates. Age and Ageing 2010;39(6):728-33.

McLean K, Day L, Dalton A. Economic evaluation of a groupbased exercise program for falls prevention among the older community-dwelling population. BMC Geriatrics 2015;15(1):33.

\section{Day 2015 \{published data only\}}

Day L, Hill KD, Stathakis VZ, Flicker L, Segal L, Cicuttini F, et al. Impact of Tai-Chi on falls among preclinically disabled older people: a randomized controlled trial. Journal of the American Medical Directors Association 2015;16(5):420-6.
Duque 2013 \{published data only (unpublished sought but not used)\}

Duque G, Boersma D, Loza-Diaz G, Hassan S, Suarez H, Geisinger D, et al. Effects of balance training using a virtualreality system in older fallers. Clinical Interventions in Aging 2013;8:257-63.

\section{Ebrahim 1997 \{published data only\}}

Ebrahim S, Thompson PW, Baskaran V, Evans K. Randomized placebo-controlled trial of brisk walking in the prevention of postmenopausal osteoporosis. Age and Ageing 1997;26(4):253-60.

\section{El-Khoury 2015 \{published data only\}}

El-Khoury F, Cassou B, Latouche A, Aegerter P, Charles MA, Dargent-Molina P. Effectiveness of two year balance training programme on prevention of fall induced injuries in at risk women aged 75-85 living in community: Ossebo randomised controlled trial. BMJ 2015;351:h3830.

\section{Fiatarone 1997 \{published data only\}}

Fiatarone MA, O'Neill EF, Doyle Ryan N, Clements K. Efficacy of home-based resistance training in frail elders. World Congress of Gerontology, 16th Congress of the International Association of Gerontology; 1997 August 19-23; Adelaide, South Australia. 1997:323 (abstract no. 985). [CN-00847936]

\section{Freiberger 2007 \{published data only\}}

* Freiberger E, Haberle L, Spirduso WW, Zijlstra GA. Longterm effects of three multicomponent exercise interventions on physical performance and fall-related psychological outcomes in community-dwelling older adults: a randomized controlled trial. Journal of the American Geriatrics Society 2012;60(3):437-46.

Freiberger E, Menz H B, Abu-Omar K, Rutten A. Preventing falls in physically active community-dwelling older people: a comparison of two intervention techniques. Gerontology 2007;53(5):298-305.

\section{Gill 2016 \{published data only\}}

Gill TM, Pahor M, Guralnik JM, McDermott MM, King AC, Buford TW, et al. Effect of structured physical activity on prevention of serious fall injuries in adults aged 70-89: randomized clinical trial (LIFE Study). BMJ 2016;352:i245.

Grahn Kronhed 2009 \{published data only (unpublished sought but not used)\}

Grahn Kronhed AC, Hallberg I, Odkvist L, Moller M. Effect of training on health-related quality of life, pain and falls in osteoporotic women. Advances in Physiotherapy 2009;11(3):154-65.

Gschwind 2015 \{published data only (unpublished sought but not used)\}

Gschwind YJ, Eichberg S, Ejupi A, De Rosario H, Kroll M, Marston HR, et al. ICT-based system to predict and prevent falls (iStoppFalls): results from an international multicenter randomized controlled trial. European Review of Aging and Physical Activity 2015;12:10. 
Haines 2009 \{published data only\}

Haines TP, Russell T, Brauer SG, Erwin S, Lane P, Urry S, et al. Effectiveness of a video-based exercise programme to reduce falls and improve health-related quality of life among older adults discharged from hospital: a pilot randomized controlled trial. Clinical Rehabilitation 2009;23(11):973-85.

Halvarsson 2013 \{published data only (unpublished sought but not used)\}

* Halvarsson A, Franzén E, Farén E, Olsson E, Oddsson L, Ståhle A. Long-term effects of new progressive group balance training for elderly people with increased risk of falling - a randomized controlled trial. Clinical Rehabilitation 2013;27(5):450-8.

Halvarsson A, Oddsson L, Olsson E, Farén E, Pettersson A, Ståhle A. Effects of new, individually adjusted, progressive balance group training for elderly people with fear of falling and tend to fall: a randomized controlled trial.[Erratum in Clinical Rehabilitation 2012 Nov;26(11):1055]. Clinical Rehabilitation 2011;25(11):1021-31.

Halvarsson 2016 \{published data only (unpublished sought but not used)\}

Halvarsson A, Franzén E, Ståhle A. Balance training with multi-task exercises improves fall-related self-efficacy, gait, balance performance and physical function in older adults with osteoporosis: a randomized controlled trial. Clinical Rehabilitation 2015;29(4):365-75.

* Halvarsson A, Oddsson L, Franzén E, Ståhle A. Longterm effects of a progressive and specific balance-training programme with multi-task exercises for older adults with osteoporosis: a randomized controlled study. Clinical Rehabilitation 2016;30(11):1049-59.

\section{Hamrick 2017 \{published and unpublished data\}}

Hamrick I. Sample size, instructor:participant ratio and faller outcome data [personal communication]. Email to: N Fairhall. 14 December 2017, 19 May 2018.

* Hamrick I, Mross P, Christopher N, Smith PD. Yoga's effect on falls in rural, older adults. Complementary Therapies in Medicine 2017;35:57-63.

\section{Hauer 2001 \{published and unpublished data\}}

Hauer K. [Personal communication]. Personal communication reported by Gillespie 2012.

Hauer K, Pfisterer M, Schuler M, Bartsch P, Oster P. Two years later: a prospective long-term follow-up of a training intervention in geriatric patients with a history of severe falls. Archives of Physical Medicine and Rehabilitation 2003;84(10):1426-32.

* Hauer K, Rost B, Rutschle K, Opitz H, Specht N, Bartsch P, et al. Exercise training for rehabilitation and secondary prevention of falls in geriatric patients with a history of injurious falls. Journal of the American Geriatrics Society 2001;49(1):10-20.
Hauer K, Specht N, Schuler M, Bartsch P, Oster P. Intensive physical training in geriatric patients after severe falls and hip surgery. Age and Ageing 2002;31(1):49-57.

Oster P, Hauer K, Specht N, Rost B, Baertsch P, Schlierf G. Strength and coordination training for prevention of falls in the elderly [Kraft- und koordinationstraining zur sturzpravention im alter]. Zeitschrift fur Gerontologie und Geriatrie 1997;30(4):289-92.

\section{Helbostad 2004 \{published data only\}}

Helbostad JL, Moe-Nilssen R, Sletvold O. Comparison of two types of exercise regimes on selected functional abilities for community-dwelling elderly at risk of falling. XVI Conference of the International Society for Postural Gait Research; 2003 March 23-27; Sydney (Australia). 2004.

* Helbostad JL, Sletvold O, Moe-Nilssen R. Effects of home exercises and group training on functional abilities in homedwelling older persons with mobility and balance problems. A randomized study. Aging Clinical and Experimental Research 2004;16(2):113-21.

Helbostad JL, Sletvold O, Moe-Nilssen R. Home training with and without additional group training in physically frail old people living at home: effect on health-related quality of life and ambulation. Clinical Rehabilitation 2004;18(5):498-508.

Hirase 2015 \{published data only (unpublished sought but not used)\}

Hirase T, Inokuchi S, Matsusaka N, Okita M. Effects of a balance training program using a foam rubber pad in community-based older adults: a randomized controlled trial. Journal of Geriatric Physical Therapy 2015;38(2):62-70.

Huang 2010 \{published data only (unpublished sought but not used)\}

Huang HC, Liu CY, Huang YT, Kernohan WG. Community-based interventions to reduce falls among older adults in Taiwan long time follow-up randomised controlled study. Journal of Clinical Nursing 2010;19(7-8):959-68.

\section{Hwang 2016 \{published data only\}}

Hwang HF, Chen SJ, Lee-Hsieh J, Chien DK, Chen CY, Lin MR. Effects of home-based tai chi and lower extremity training and self-practice on falls and functional outcomes in older fallers from the emergency department - a randomized controlled trial. Journal of the American Geriatrics Society 2016;64(3):518-25.

\section{Iliffe 2015 \{published data only\}}

Gawler S, Skelton DA, Dinan-Young S, Masud T, Morris RW, Griffin M, et al. Reducing falls among older people in general practice: The ProAct65+ exercise intervention trial. Archives of Gerontology and Geriatrics 2016;67:46-54.

* Iliffe S, Kendrick D, Morris R, Griffin M, Haworth D, Carpenter $\mathrm{H}$, et al. Promoting physical activity in older people in general practice: ProAct65+ cluster randomised controlled trial. British Journal of General Practice 2015;65(640):e731-8. 
Iliffe S, Kendrick D, Morris R, Masud T, Gage H, Skelton D, et al. Multicentre cluster randomised trial comparing a community group exercise programme and home-based exercise with usual care for people aged 65 years and over in primary care. Health Technology Assessment 2014;18(49):vii-xxvii, 1-105.

Irez 2011 \{published data only\} Irez GB, Ozdemir RA, Evin R, Irez SG, Korkusuz F. Integrating pilates exercise into an exercise program for $65+$ year-old women to reduce falls. Journal of Sports Science and Medicine 2011;10(1):105-11.

\section{Iwamoto 2009 \{published data only\}}

Iwamoto J, Suzuki H, Tanaka K, Kumakubo T, Hirabayashi H, Miyazaki Y, et al. Preventative effect of exercise against falls in the elderly: A randomized controlled trial. Osteoporosis International 2009;20(7):1233-40.

\section{Kamide 2009 \{published data only\}}

Kamide N, Shiba Y, Shibata H. Effects on balance, falls, and bone mineral density of a home-based exercise program without home visits in community-dwelling elderly women: a randomized controlled trial. Journal of Physiological Anthropology 2009;28(3):115-22.

Karinkanta 2007 \{published data only (unpublished sought but not used)\}

* Karinkanta S, Heinonen A, Sievanen H, Uusi-Rasi K, Pasanen M, Ojala K, et al. A multi-component exercise regimen to prevent functional decline and bone fragility in homedwelling elderly women: randomized, controlled trial. Osteoporosis International 2007;18(4):453-62.

Karinkanta S, Kannus P, Uusi-Rasi K, Heinonen A, Sievanen H. Combined resistance and balance-jumping exercise reduces older women's injurious falls and fractures: 5-year follow-up study. Age and Ageing 2015;44(5):784-9.

Karinkanta S, Nupponen R, Heinonen A, Pasanen M, Sievanen $H$, Uusi-Rasi K, et al. Effects of exercise on health-related quality of life and fear of falling in home-dwelling older women. Journal of Aging and Physical Activity 2012;20(2):198-214.

\section{Kemmler 2010 \{published data only\}}

* Kemmler W, Von Stengel S, Engelke K, Haberle L, Kalender WA. Exercise effects on bone mineral density, falls, coronary risk factors, and health care costs in older women: the randomized controlled senior fitness and prevention (SEFIP) study. Archives of Internal Medicine 2010;170(2):179-85.

Kemmler W, Von Stengel S, Engelke K, Haberle L, Mayhew JL, Kalender WA. Exercise, body composition, and functional ability: a randomized controlled trial. American Journal of Preventive Medicine 2010;38(3):279-87.

Kemmler W, Von Stengel S, Engelke K, Kalender WA. Exercise decreases the risk of metabolic syndrome in elderly females. Medicine and Science in Sports and Exercise 2009;41(2):297-305.

NCT00267839. Effect of exercise on risk-factors of elderly women. clinicaltrials.gov/show/NCT00267839 (first received 21 December 2005).
Kerse 2010 \{published and unpublished data\}

ACTRN12605000475640. Late life intervention to improve function in elderly patients with depression. www.anzctr.org.au/trial_view.aspx?ID=736 (first received 23 September 2005).

Kerse N. Fall outcome data [personal communication]. Email to: C West Vol. 18 June 2018.

Kerse N, Falloon K, Moyes SA, Hayman KJ, Dowell T, Kolt GS, et al. DeLLITE depression in late life: an intervention trial of exercise. Design and recruitment of a randomised controlled trial. BMC Geriatrics 2008;8:12.

* Kerse N, Hayman KJ, Moyes SA, Peri K, Robinson E, Dowell A, et al. Home-based activity program for older people with depressive symptoms: DeLLITE--a randomized controlled trial. Annals of Family Medicine 2010;8(3):214-23.

Kim 2014 \{published data only (unpublished sought but not used)\} Kim H, Yoshida H, Suzuki T. Falls and fractures in participants and excluded non-participants of a fall prevention exercise program for elderly women with a history of falls: 1-year follow-up study. Geriatrics and Gerontology International 2014;14(2):285-92.

Korpelainen 2006 \{published data only (unpublished sought but not used)\}

Korpelainen R, Keinanen-Kiukaanniemi S, Heikkinen J, Vaananen K, Korpelainen J. Effect of exercise on extraskeletal risk factors for hip fractures in elderly women with low BMD: a population-based randomized controlled trial. Journal of Bone and Mineral Research 2006;21(5):772-9.

* Korpelainen R, Keinanen-Kiukaanniemi S, Heikkinen J, Vaananen K, Korpelainen J. Effect of impact exercise on bone mineral density in elderly women with low BMD: a populationbased randomized controlled 30-month intervention. Osteoporosis International 2006;17(1):109-18.

Korpelainen R, Keinanen-Kiukaanniemi S, Nieminen P, Heikkinen J, Vaananen K, Korpelainen J. Long-term outcomes of exercise: follow-up of a randomized trial in older women with osteopenia. Archives of Internal Medicine 2010;170(17):1548-56.

NCT00655577. Exercise and prevention of hip fractures. clinicaltrials.gov/show/NCT00655577 (first received 10 April 2008).

\section{Kovacs 2013 \{published and unpublished data\}}

Kovacs E. Fall outcome data [personal communication]. Email to: C West 18 June 2018.

${ }^{*}$ Kovacs E, Prokai L, Meszaros L, Gondos T. Adapted physical activity is beneficial on balance, functional mobility, quality of life and fall risk in community-dwelling older women: a randomized single-blinded controlled trial. European Journal of Physical and Rehabilitation Medicine 2013;49(3):301-10.

\section{Kwok 2016 \{published data only\}}

Kwok BC, Pua YH. Effects of WiiActive exercises on fear of falling and functional outcomes in community-dwelling older adults: a randomised control trial. Age and Ageing 2016;45(5):621-7. 
Kyrdalen 2014 \{published data only (unpublished sought but not used)\}

Kyrdalen IL, Moen K, Rõysland AS, Helbostad JL. The Otago Exercise Program performed as group training versus home training in fall-prone older people: a randomized controlled trial. Physiotherapy Research International 2014;19(2):108-16.

LaStayo 2017 \{published data only (unpublished sought but not used)\}

LaStayo P, Marcus R, Dibble L, Wong B, Pepper G. Eccentric versus traditional resistance exercise for older adult fallers in the community: a randomized trial within a multi-component fall reduction program. BMC Geriatrics 2017;17:149. [DOI: 10.1186/s12877-017-0539-8]

\section{Latham 2003 \{published data only\}}

Latham NK, Anderson CS, Lee A, Bennett DA, Moseley A, Cameron ID. A randomized, controlled trial of quadriceps resistance exercise and vitamin $D$ in frail older people: The Frailty Interventions Trial in Elderly Subjects (FITNESS). Journal of the American Geriatrics Society 2003;51(3):291-9.

\section{Lehtola 2000 \{published data only (unpublished sought but not used)\}}

Lehtola S, Hanninen L, Paatalo M. The incidence of falls during a six-month exercise trial and four-month followup among home dwelling persons aged 70-75 years [Kaatumistapaturmien ilmaantuvuus 70-75-vuotiailla oululaisilla liikuntaintervention ja sen jälkeisen seurannan aikana]. Liikuntatiede 2000;6:41-6.

\section{Li 2005 \{published data only\}}

* Li F, Harmer P, Fisher K J, McAuley E, Chaumeton N, Eckstrom E, et al. Tai Chi and fall reductions in older adults: a randomized controlled trial. Journals of Gerontology, Series A: Biological Sciences and Medical Sciences 2005;60(2):187-94.

Li F, Harmer P, Fisher KJ, McAuley E. Tai Chi: improving functional balance and predicting subsequent falls in older persons. Medicine and Science in Sports and Exercise 2004;36(12):2046-52.

Lin 2007 \{published data only (unpublished sought but not used)\} Lin MR, Wolf SL, Hwang HF, Gong SY, Chen CY. A randomized, controlled trial of fall prevention programs and quality of life in older fallers. Journal of the American Geriatrics Society 2007;55(4):499-506.

\section{Liston 2014 \{published data only\}}

Liston MB, Alushi L, Bamiou DE, Martin FC, Hopper A, Pavlou M. Feasibility and effect of supplementing a modified OTAGO intervention with multisensory balance exercises in older people who fall: a pilot randomized controlled trial. Clinical Rehabilitation 2014;28(8):784-93.

\section{Liu-Ambrose 2004 \{published data only\}}

* Liu-Ambrose T, Khan KM, Eng JJ, Janssen PA, Lord SR, McKay HA. Resistance and agility training reduce fall risk in women aged 75 to 85 with low bone mass: a 6-month randomized, controlled trial. Journal of the American Geriatrics Society 2004;52(5):657-65.
Liu-Ambrose T, Khan KM, Eng JJ, Lord SR, McKay HA. Balance confidence improves with resistance or agility training. Increase is not correlated with objective changes in fall risk and physical abilities. Gerontology 2004;50(6):373-82.

Liu-Ambrose T, Khan KM, Eng JJ, Lord SR, McKay HA. Strength or agility training significantly reduces fall risk compared to posture training in 75 to 85 year old women with low bone density: a six month RCT. XVIth conference of the International Society for Postural and Gait Research; 2003 March 23-27;Sydney (Australia). 2004.

Liu-Ambrose TY, Khan KM, Eng JJ, Gillies GL, Lord SR, McKay HA. The beneficial effects of group-based exercises on fall risk profile and physical activity persist 1 year postintervention in older women with low bone mass: follow-up after withdrawal of exercise. Journal of the American Geriatrics Society 2005;53(10):1767-73.

\section{Liu-Ambrose 2008 \{published data only\}}

Davis J, Guy P, Liu-Ambrose T, Donaldson M, Robertson M, Khan K, et al. Cost-effectiveness analysis of the Otago homebased strength and balance retraining in senior fallers. Journal of Nutrition, Health and Aging 2009;13(Suppl 1):S436. [DOI: 10.1007/s12603-009-0095-9]

Donaldson MG. Falls risk in frail seniors: clinical and methodological studies [thesis]. Vancouver (CA): University of British Columbia, 2007.

* Liu-Ambrose T, Donaldson MG, Ahamed Y, Graf P, Cook WL, Close J, et al. Otago home-based strength and balance retraining improves executive functioning in older fallers: a randomized controlled trial. Journal of the American Geriatrics Society 2008;56(10):1821-30.

NCT00323596. Trial of a home based strength and balance retraining program in reducing falls risk factors. clinicaltrials.gov/show/NCT00323596 (first received 9 May 2006).

\section{Logghe 2009 \{published data only\}}

Logghe IH, Verhagen AP, Rademaker AC, Zeeuwe PE, BiermaZeinstra SM, van Rossum E, et al. Explaining the ineffectiveness of a Tai Chi fall prevention training for community-living older people: A process evaluation alongside a randomized clinical trial (RCT). Archives of Gerontology and Geriatrics 2011;52(3):357-62.

* Logghe IH, Zeeuwe PE, Verhagen AP, Wijnen-Sponselee RM, Willemsen SP, Bierma-Zeinstra SM, et al. Lack of effect of Tai Chi Chuan in preventing falls in elderly people living at home: a randomized clinical trial. Journal of the American Geriatrics Society 2009;57(1):70-5.

Zeeuwe PE, Verhagen AP, Bierma-Zeinstra SM, Van Rossum E, Faber MJ, Koes BW. The effect of Tai Chi Chuan in reducing falls among elderly people: design of a randomized clinical trial in the Netherlands. BMC Geriatrics 2006;6:6.

\section{Lord 1995 \{published data only\}}

* Lord SR, Ward JA, Williams P, Strudwick M. The effect of a 12-month exercise trial on balance, strength, and falls in older 
women: a randomized controlled trial. Journal of the American Geriatrics Society 1995;43(11):1198-206.

Lord SR, Ward JA, Williams P, Zivanovic E. The effects of a community exercise program on fracture risk factors in older women. Osteoporosis International 1996;6(5):361-7.

Lord 2003 \{published and unpublished data\}

Lord S. Number of fallers outcome data [personal

communication]. Email to: C West Vol. 18 June 2018.

* Lord SR, Castell S, Corcoran J, Dayhew J, Matters B, Shan A, et al. The effect of group exercise on physical functioning and falls in frail older people living in retirement villages: a randomized, controlled trial. Journal of the American Geriatrics Society 2003;51(12):1685-92.

Lurie 2013 \{published data only (unpublished sought but not used)\}

Lurie JD, Zagaria AB, Pidgeon DM, Forman JL, Spratt KF. Pilot comparative effectiveness study of surface perturbation treadmill training to prevent falls in older adults. BMC Geriatrics 2013;13:49.

\section{Luukinen 2007 \{published data only\}}

* Luukinen H, Lehtola S, Jokelainen J, Vaananen-Sainio R, Lotvonen S, Koistinen P. Pragmatic exercise-oriented prevention of falls among the elderly: a populationbased, randomized, controlled trial. Preventive Medicine 2007;44(3):265-71.

Luukinen H, Lehtola S, Jokelainen J, Vaananen-Sainio R, Lotvonen S, Koistinen P. Prevention of disability by exercise among the elderly: a population-based, randomized, controlled trial. Scandanavian Journal of Primary Health Care 2006;24(4):199-205.

Madureira 2007 \{published data only (unpublished sought but not used)\}

Madureira MM, Bonfá E, Takayama L, Pereira RM. A 12-month randomized controlled trial of balance training in elderly women with osteoporosis: improvement of quality of life. Maturitas 2010;66(2):206-11.

Madureira MM, Pereira RM. A 12-month randomized controlled trial of balance training in elderly women with osteoporosis: Improvement of quality of life and reduction of falls. Arthritis and Rheumatism 2009;10:1496.

* Madureira MM, Takayama L, Gallinaro AL, Caparbo VF, Costa RA, Pereira RM. Balance training program is highly effective in improving functional status and reducing the risk of falls in elderly women with osteoporosis: a randomized controlled trial. Osteoporosis International 2007;18(4):419-25.

\section{McMurdo 1997 \{published data only\}}

McMurdo ME, Mole PA, Paterson CR. Controlled trial of weight bearing exercise in older women in relation to bone density and falls. BMJ 1997;314(7080):569.
Means 2005 \{published data only\}

Means KM, Rodell DE, O'Sullivan PS. Balance, mobility, and falls among community-dwelling elderly persons: effects of a rehabilitation exercise program. American Journal of Physical Medicine and Rehabilitation 2005;84(4):238-50.

\section{Merom 2016 \{published data only\}}

Merom D, Mathieu E, Cerin E, Morton RL, Simpson JM, Rissel C, et al. Social dancing and incidence of falls in older adults: a cluster randomised controlled trial. PLoS Medicine 2016;13(8):e1002112.

\section{Miko 2017 \{published data only\}}

Miko I, Szerb I, Szerb A, Poor G. Effectiveness of balance training programme in reducing the frequency of falling in established osteoporotic women: a randomized controlled trial. Clinical Rehabilitation 2017;31(2):217-24.

Mirelman 2016 \{published data only (unpublished sought but not used)\}

Mirelman A, Rochester L, Maidan I, Del D, Alcock L, Nieuwhof F, et al. Addition of a non-immersive virtual reality component to treadmill training to reduce fall risk in older adults (V-TIME): a randomised controlled trial. Lancet 2016;388(10050):1170-82.

Morgan 2004 \{published data only (unpublished sought but not used)\}

DeVito CA, Morgan RO, Duque M, Abdel-Moty E, Virnig BA. Physical performance effects of low-intensity exercise among clinically defined high-risk elders. Gerontology 2003;49(3):146-54

* Morgan RO, Virnig BA, Duque M, Abdel-Moty E, Devito CA. Lowintensity exercise and reduction of the risk for falls among atrisk elders. Journals of Gerontology. Series A, Biological Sciences and Medical Sciences 2004;59(10):1062-7.

NCT00013078. Safe-Grip fall/injuries intervention: a randomized controlled trial. clinicaltrials.gov/show/NCT00013078 (first received 16 March 2001).

Morone 2016 \{published data only (unpublished sought but not used)\}

Morone G, Paolucci T, Luziatelli S, losa M, Piermattei C, Zangrando $\mathrm{F}$, et al. Wii Fit is effective in women with bone loss condition associated with balance disorders: a randomized controlled trial. Aging Clinical and Experimental Research 2016;28(6):1187-93.

\section{Morrison 2018 \{published and unpublished data\}}

Morrison S. Fall outcome data, randomisation and intervention details [personal communication]. Email to: N Fairhall. 15 June 2018.

* Morrison S, Simmons R, Colberg SR, Parson HK, Vinik I. Supervised balance training and Wii Fit-based exercises lower falls risk in older adults with type 2 diabetes. Journal of the American Medical Directors Association 2018;19(2):185.e7-185.e13. 
Ng 2015 \{published data only (unpublished sought but not used)\} Ng TP, Feng L, Nyunt MS, Feng L, Niti M, Tan BY, et al. Nutritional, physical, cognitive, and combination interventions and frailty reversal among older adults: a randomized controlled trial. American Journal of Medicine 2015;128(11):1225-36 e1.

Nitz 2004 \{published and unpublished data\}

Nitz JC. [Personal communication]. Personal communication reported by Gillespie 2012 .

* Nitz JC, Choy NL. The efficacy of a specific balancestrategy training programme for preventing falls among older people: A pilot randomised controlled trial. Age and Aging 2004;33(1):52-8

Okubo 2016 \{published data only (unpublished sought but not used)\}

Okubo Y, Osuka Y, Jung S, Rafael F, Tsujimoto T, Aiba T, et al. Walking can be more effective than balance training in fall prevention among community-dwelling older adults. Geriatrics and Gerontology International 2016;16(1):118-25.

Park 2008 \{published data only (unpublished sought but not used)\} Park H, Kim KJ, Komatsu T, Park SK, Mutoh Y. Effect of combined exercise training on bone, body balance, and gait ability: a randomized controlled study in community-dwelling elderly women. Journal of Bone and Mineral Metabolism 2008; Vol. 26, issue $3: 254-9$.

Reinsch 1992 \{published data only (unpublished sought but not used)\}

El-Faizy M, Reinsch S. Home safety intervention for the prevention of falls. Physical and Occupational Therapy in Geriatrics 1994;12(3):33-49.

MacRae PG, Feltner ME, Reinsch S. A 1-year exercise program for older women: effects on falls, injuries, and physical performance. Journal of Aging and Physical Activity 1994;2:127-42.

* Reinsch S, MacRae P, Lachenbruch PA, Tobis JS. Attempts to prevent falls and injury: a prospective community study. Gerontologist 1992;32(4):450-6.

Tobis J, Reinsch S, McRae P, Lachenbruch T. Experimental intervention at senior centres for the prevention of falls. Journal of the American Geriatrics Society 1990;38(8):A28.

Resnick 2002 \{published data only (unpublished sought but not used)\}

Resnick B. Testing the effect of the WALC intervention on exercise adherence in older adults. Journal of Gerontological Nursing 2002;28(6):40-9.

\section{Robertson 2001a \{published data only\}}

Gardner MM, Buchner DM, Robertson MC, Campbell AJ. Practical implementation of an exercise-based falls prevention programme. Age and Ageing 2001;30(1):77-83.

Robertson MC. Development of a falls prevention programme for elderly people: evaluation of efficacy, effectiveness, and efficiency [thesis]. Dunedin (NZ): University of Otago, 2001.
Robertson MC, Campbell AJ, Gardner MM, Devlin N. Preventing injuries in older people by preventing falls: a meta-analysis of individual-level data. Journal of the American Geriatrics Society 2002;50(5):905-11.

* Robertson MC, Devlin N, Gardner MM, Campbell AJ.

Effectiveness and economic evaluation of a nurse delivered home exercise programme to prevent falls. 1: Randomised controlled trial. BMJ 2001;322(7288):697-701.

\section{Rubenstein 2000 \{published data only\}}

Rubenstein LZ, Josephson KR, Trueblood PR, Loy S, Harker JO, Pietruszka FM, et al. Effects of a group exercise program on strength, mobility, and falls among fall-prone elderly men. Journals of Gerontology. Series A, Biological Sciences and Medical Sciences 2000;55(6):M317-21.

\section{Sakamoto 2013 \{published data only\}}

Sakamoto K, Endo N, Harada A, Sakada T, Tsushita K, Kita K, et al. A randomized, controlled trial of balance therapy to prevent falls and fractures for elderly people who can stand on one leg for $\leq 15$ s. Journal of Orthopaedic Science 2013;18(1):110-20.

\section{Sales 2017 \{published and unpublished data\}}

Sales M. Fall outcome data [personal communication]. Email to: N Fairhall. 23 May 2018.

* Sales M, Polman R, Hill KD, Levinger P. A novel exercise initiative for seniors to improve balance and physical function. Journal of Aging and Health 2017;29(8):1424-43.

Sherrington 2014 \{published data only\}

* Sherrington C, Lord SR, Vogler CM, Close JC, Howard K, Dean CM, et al. A post-hospital home exercise program improved mobility but increased falls in older people: a randomised controlled trial. PLoS One 2014;9(9):e104412.

Sherrington C, Lord SR, Vogler CM, Close JC, Howard K, Dean CM, et al. Minimising disability and falls in older people through a post-hospital exercise program: a protocol for a randomised controlled trial and economic evaluation. BMC Geriatrics 2009;26:8. [DOI: 10.1186/1471-2318-9-8]

Shigematsu 2008 \{published data only\}

* Shigematsu R, Okura T, Nakagaichi M, Tanaka K, Sakai T, Kitazumi S, et al. Square-stepping exercise and fall risk factors in older adults: a single-blind, randomized controlled trial. Journals of Gerontology. Series A, Biological Sciences and Medical Sciences 2008;63(1):76-82.

Shigematsu R, Okura T, Sakai T, Rantanen T. Square-stepping exercise versus strength and balance training for fall risk factors. Aging Clinical and Experimental Research 2008;20(1):19-24.

\section{Siegrist 2016 \{published and unpublished data\}}

Siegrist M. Fall and injury data [personal communication]. Email to: N Fairhall. 2 February 2018.

* Siegrist M, Freiberger E, Geilhof B, Salb J, Hentschke C, Landendoerfer $\mathrm{P}$, et al. Fall prevention in a primary care setting. Deutsches Ärzteblatt International 2016;113(21):365-72. 
Skelton 2005 \{published and unpublished data\}

Skelton D. [Personal communication]. Personal communication reported by Gillespie 2012 February 12005.

* Skelton D, Dinan S, Campbell M, Rutherford O. Tailored group exercise (Falls Management Exercise -- FaME) reduces falls in community-dwelling older frequent fallers (an RCT). Age and Ageing 2005;34(6):636-9.

Skelton DA, Dinan SM. Exercise for falls management: Rationale for an exercise programme aimed at reducing postural instability. Physiotherapy Theory and Practice 1999;15(2):105-20.

Skelton DA, Dinan SM, Campbell M, Rutherford OM. FaME (Falls Management Exercise): An RCT on the effects of a 9-month group exercise programme in frequently falling community dwelling women aged 65 and over. Journal of Aging and Physical Activity 2004;12(3):457-8.

Skelton DA, Stranzinger K, Dinan S, Rutherford OM. BMD improvements following FaME (Falls Management Exercise) in frequently falling women age 65 and over: an RCT. Journal of Aging and Physical Activity 2008;16 Suppl:S89-90.

\section{Smulders 2010 \{published data only\}}

NCT00432692. Falls prevention in osteoporosis. clinicaltrials.gov/show/NCT00432692 (first received 8 February 2007).

Smulders E, Weerdesteyn V, Duysens J, Laan R, Lankveld W. Falls prevention in persons with osteoporosis: A randomized clinical trial. Arthritis and Rheumatism 2009;10:1883.

* Smulders E, Weerdesteyn V, Groen BE, Duysens J, Eijsbouts A, Laan R, et al. Efficacy of a short multidisciplinary falls prevention program for elderly persons with osteoporosis and a fall history: a randomized controlled trial. Archives of Physical Medicine and Rehabilitation 2010;91(11):1705-11.

\section{Steadman 2003 \{published and unpublished data\}}

Kalra L. [Personal communication]. Personal communication reported by Gillespie 201227 March 2006.

* Steadman J, Donaldson N, Kalra L. A randomized controlled trial of an enhanced balance training program to improve mobility and reduce falls in elderly patients. Journal of the American Geriatrics Society 2003;51(6):847-52.

\section{Suzuki 2004 \{published data only\}}

Suzuki T, Kim H, Yoshida H, Ishizaki T. Randomized controlled trial of exercise intervention for the prevention of falls in community-dwelling elderly Japanese women. Journal of Bone and Mineral Metabolism 2004;22(6):602-11.

\section{Taylor 2012 \{published data only\}}

Taylor D, Hale L, Schluter P, Waters DL, Binns EE, McCracken H, et al. Effectiveness of tai chi as a community-based falls prevention intervention: a randomized controlled trial. Journal of the American Geriatrics Society 2012;60(5):841-8.

\section{Trombetti 2011 \{published data only\}}

NCT01107288. Effects of a music-based multitask exercises program on gait, balance and fall risk in the elderly.
clinicaltrials.gov/show/NCT01107288 (first received 20 April 2010).

Trombetti A, Hars M, Herrmann F, Kressig R, Ferrari S, Rizzoli R. "Jaques-Dalcroze eurhythmics" improves gait and prevents falls in the elderly [Prevention des chutes par une methode d'exercice en musique (rythmique Jaques-Dalcroze)]. Revue Medicale Suisse 2011;7(299):1305-8.

Trombetti A, Hars M, Herrmann F, Kressig R, Ferrari S, Rizzoli R. A randomized controlled trial of music-based multitask training on gait, balance and fall risk. Osteoporosis International 2011;22(Suppl 1):S119.

* Trombetti A, Hars M, Herrmann FR, Kressig RW, Ferrari S, Rizzoli R. Effect of music-based multitask training on gait, balance, and fall risk in elderly people: a randomized controlled trial. Archives of Internal Medicine 2011;171(6):525-33.

\section{Uusi-Rasi 2015 \{published data only\}}

Patil R, Kolu P, Raitanen J, Valvanne J, Kannus P, Karinkanta S, et al. Cost-effectiveness of vitamin D supplementation and exercise in preventing injurious falls among older home-dwelling women: findings from an RCT. Osteoporosis International 2016;27(1):193-201.

Uusi-Rasi K, Patil R, Karinkanta S, Kannus P, Tokola K, LambergAllardt C, et al. A 2-year follow-up after a 2-year RCT with vitamin $D$ and exercise: effects on falls, injurious falls and physical functioning among older women. Journals of Gerontology. Series A, Biological Sciences and Medical Sciences 2017;72(9):1239-45.

* Uusi-Rasi K, Patil R, Karinkanta S, Kannus P, Tokola K, Lamberg-Allardt $C$, et al. Exercise and vitamin $D$ in fall prevention among older women: a randomized clinical trial. JAMA Internal Medicine 2015;175(5):703-11.

\section{Verrusio 2017 \{published data only\}}

Verrusio W, Gianturco V, Cacciafesta M, Marigliano V, Troisi G, Ripani M. Fall prevention in the young old using an exoskeleton human body posturizer: a randomized controlled trial. Aging Clinical and Experimental Research 2017;29(2):207-14.

\section{Vogler 2009 \{published data only\}}

Vogler CM, Sherrington C, Ogle SJ, Lord SR. Reducing risk of falling in older people discharged from hospital: a randomized controlled trial comparing seated exercises, weight-bearing exercises, and social visits. Archives of Physical Medicine and Rehabilitation 2009;90(8):1317-24.

\section{Voukelatos 2007 \{published and unpublished data\}}

Haas M. Economic analysis of tai chi as a means of preventing falls and related injuries among older adults. CHERE working paper 2006/4. Sydney, Australia: Centre for Health Economics Research and Evaluation, University of Technology. https:// www.uts.edu.au/sites/default/files/wp2006_3.pdf (accessed 24 January 2019).

Rissel C, Voukelatos A, Cumming B, Lord S. Central Sydney Tai Chi Trial. Canberra: Australian Resource Centre for Health Care Innovations, 2005. 
Voukelatos A. [Personal communication]. Personal communication reported by Gillespie 201225 July 2003.

* Voukelatos A, Cumming RG, Lord SR, Rissel C. A randomized, controlled trial of tai chi for the prevention of falls: the Central Sydney tai chi trial. Journal of the American Geriatrics Society 2007;55(8):1185-91.

Voukelatos A, Metcalfe A. Central Sydney Tai Chi Trial: methodology. New South Wales Public Health Bulletin 2002;13(1-2):19.

Voukelatos A, Rissel C, Cumming R, Lord S. The Central Sydney Tai Chi Trial: a randomised controlled trial of the effectiveness of tai chi in reducing risk of falls in older people. Sydney: NSW Department of Health, 2006 https://www.health.nsw.gov.au/ research/Publications/2000-tai-chi.pdf (accessed 24 January 2019).

\section{Voukelatos 2015 \{published data only\}}

Voukelatos A, Merom D, Sherrington C, Rissel C, Cumming RG, Lord SR. The impact of a home-based walking programme on falls in older people: the Easy Steps randomised controlled trial. Age and Ageing 2015;44(3):377-83.

\section{Weerdesteyn 2006 \{published and unpublished data\}}

Weerdesteyn V. [Personal communication]. Personal communication reported by Gillespie 201206 September 2006.

* Weerdesteyn V, Rijken H, Geurts AC, Smits-Engelsman BC, Mulder T, Duysens J. A five-week exercise program can reduce falls and improve obstacle avoidance in the elderly. Gerontology 2006;52(3):131-41.

Wolf 1996 \{published data only (unpublished sought but not used)\} Kutner NG, Barnhart H, Wolf SL, McNeely E, Xu T. Self-report benefits of Tai Chi practice by older adults. Journals of Gerontology. Series B, Psychological Sciences and Social Sciences 1997;52(5):P242-6.

McNeely E, Clements SD, Wolf SL. A program to reduce frailty in the elderly. In: Funk SG, Tornquist EM, Champagne MT, Weise RA editor(s). Key Aspects of Elder Care: Managing Falls, Incontinence, and Cognitive Impairment. New York: Springer, 1992:89-96

O'Grady M, Wolf SL, Barnhart HX, Kutner N, McNeely E. Tai Chi effect on falls in frail older adults. Archives of Physical Medicine and Rehabilitation 1997; Vol. 78:1028. [CN-00716674]

Wolf SL, Barnhart HX, Ellison GL, Coogler CE. The effect of Tai Chi Quan and computerized balance training on postural stability in older subjects. Atlanta FICSIT Group. Frailty and Injuries: Cooperative Studies on Intervention Techniques. Physical Therapy 1997;77(4):371-81; discussion 382-4.

* Wolf SL, Barnhart HX, Kutner NG, McNeely E, Coogler C, Xu T. Reducing frailty and falls in older persons: an investigation of Tai Chi and computerized balance training. Atlanta FICSIT Group. Frailty and Injuries: Cooperative Studies of Intervention Techniques. Journal of the American Geriatrics Society 1996;44(5):489-97.
Wolf SL, Kutner NG, Green RC, McNeely E. The Atlanta FICSIT study: two exercise interventions to reduce frailty in elders. Journal of the American Geriatrics Society 1993;41(3):329-32.

\section{Wolf 2003 \{published data only\}}

Greenspan AI, Wolf SL, Kelley ME, O'Grady M. Tai chi and perceived health status in older adults who are transitionally frail: a randomized controlled trial. Physical Therapy 2007;87(5):525-35.

Sattin RW, Easley KA, Wolf SL, Chen Y, Kutner MH. Reduction in fear of falling through intense tai chi exercise training in older, transitionally frail adults. Journal of the American Geriatrics Society 2005;53(7):1168-78.

Wolf SL, O'Grady M, Easley KA, Guo Y, Kressig RW, Kutner M. The influence of intense Tai Chi training on physical performance and hemodynamic outcomes in transitionally frail, older adults. Journals of Gerontology. Series A, Biological Sciences and Medical Sciences 2006;61(2):184-9.

* Wolf SL, Sattin RW, Kutner M, O'Grady M, Greenspan AI, Gregor RJ. Intense tai chi exercise training and fall occurrences in older, transitionally frail adults: a randomized, controlled trial. Journal of the American Geriatrics Society 2003;51(12):1693-701.

Wolf SL, Sattin RW, O'Grady M, Freret N, Ricci L, Greenspan A I, et al. A study design to investigate the effect of intense Tai Chi in reducing falls among older adults transitioning to frailty. Controlled Clinical Trials 2001;22(6):689-704.

\section{Woo 2007 \{published and unpublished data\}}

Woo J. [Personal communication]. Personal communication reported by Gillespie 2012 May 62008.

* Woo J, Hong A, Lau E, Lynn H. A randomised controlled trial of Tai Chi and resistance exercise on bone health, muscle strength and balance in community-living elderly people. Age and Ageing 2007;36(3):262-8

\section{Wu 2010 \{published data only\}}

Wu G, Keyes L, Callas P, Ren X, Bookchin B. Comparison of telecommunication, community, and home-based Tai Chi exercise programs on compliance and effectiveness in elders at risk for falls. Archives of Physical Medicine and Rehabilitation 2010;91(6):849-56.

\section{Yamada 2010 \{published data only\}}

Yamada M, Tanaka B, Nagai K, Aoyama T, Ichihashi N. Trail-walking exercise and fall risk factors in communitydwelling older adults: preliminary results of a randomized controlled trial. Journal of the American Geriatrics Society 2010;58(10):1946-51.

\section{Yamada 2012 \{published data only\}}

Yamada M, Aoyama T, Arai H, Nagai K, Tanaka B, Uemura K, et al. Complex obstacle negotiation exercise can prevent falls in community-dwelling elderly Japanese aged 75 years and older. Geriatrics and Gerontology International 2012;12(3):461-7. 
Yamada 2013 \{published data only\}

Yamada M, Higuchi T, Nishiguchi S, Yoshimura K, Kajiwara Y, Aoyama T. Multitarget stepping program in combination with a standardized multicomponent exercise program can prevent falls in community-dwelling older adults: a randomized, controlled trial. Journal of the American Geriatrics Society 2013;61(10):1669-75.

Yang 2012 \{published data only (unpublished sought but not used)\} Yang XJ, Hill K, Moore K, Williams S, Dowson L, Borschmann K, et al. Effectiveness of a targeted exercise intervention in reversing older people's mild balance dysfunction: a randomized controlled trial. Physical Therapy 2012;92(1):24-37.

\section{References to studies excluded from this review}

Alkan 2011 \{published data only\}

Alkan H, Topuz O, Yildiz, Alkan S, Sarsan A, Ardıç F. Efficacy of home based exercise program and postural biofeedback therapy in reducing risk of falling among osteoporotic women over 65 years of age. Turkish Journal of Geriatrics 2011;14(1):26-34.

\section{Beling 2009 \{published data only\}}

Beling J, Roller M. Multifactorial intervention with balance training as a core component among fall-prone older adults. Journal of Geriatric Physical Therapy 2009;32(3):125-33.

\section{Clemson 2004b \{published data only\}}

Clemson L, Cumming RG, Kendig H, Swann M, Heard R, Taylor K. The effectiveness of a community-based program for reducing the incidence of falls in the elderly: a randomized trial. Journal of the American Geriatrics Society 2004;52(9):1487-94.

\section{DeSure 2013 \{published data only\}}

DeSure AR, Peterson K, Gianan FV, Pang L. An exercise program to prevent falls in institutionalized elderly with cognitive deficits: a crossover pilot study. Hawaii Journal of Medicine and Public Health 2013;72(11):391-5.

\section{Fahlström 2017 \{published data only\}}

Fahlström G, Kamwendo K, Forsberg J, Bodin L. Fall prevention by nursing assistants among community-living elderly people. A randomised controlled trial. Scandinavian Journal of Caring Sciences 2017 Aug 29 [Epub ahead of print]. [DOI: 10.1111/ scs.12481]

\section{Gianoudis 2014 \{published data only\}}

Gianoudis J, Bailey CA, Ebeling PR, Nowson CA, Sanders KM, Hill K, et al. Effects of a targeted multimodal exercise program incorporating high-speed power training on falls and fracture risk factors in older adults: a community-based randomized controlled trial. Journal of Bone and Mineral Research 2014;29(1):182-91.

\section{Hinrichs 2016 \{published and unpublished data\}}

Hinrichs T. Outcome data [personal communication]. Email to: N Fairhall. 29 August 2017.

* Hinrichs T, Bücker B, Klaaßen-Mielke R, Brach M, Wilm S, Platen $\mathrm{P}$, et al. Home-based exercise supported by general practitioner practices: Ineffective in a sample of chronically IIl, mobility-limited older adults (the HOMEfit randomized controlled trial). Journal of the American Geriatrics Society 2016;64(11):2270-9.

\section{Hsu 2017 \{published data only\}}

Hsu CL, Best JR, Wang S, Voss MW, Hsiung RG, Munkacsy M, et al. The impact of aerobic exercise on fronto-parietal network connectivity and its relation to mobility: An exploratory analysis of a 6-month randomized controlled trial. Frontiers in Human Neuroscience 2017;11:344. [DOI: 10.3389/fnhum.2017.00344]

Iwamoto 2012 \{published data only\}

Iwamoto J, Sato Y, Takeda T, Matsumoto H. Whole body vibration exercise improves body balance and walking velocity in postmenopausal osteoporotic women treated with alendronate: Galileo and Alendronate Intervention Trail (GAIT). Journal of Musculoskeletal \& Neuronal Interactions 2012;12(3):136-43.

Lee 2013 \{published data only\}

Lee HC, Chang KC, Tsauo JY, Hung JW, Huang YC, Lin SI. Effects of a multifactorial fall prevention program on fall incidence and physical function in community-dwelling older adults with risk of falls. Archives of Physical Medicine and Rehabilitation 2013;94(4):606-15.

\section{Leung 2014 \{published data only\}}

Leung KS, Li CY, Tse YK, Choy TK, Leung PC, Hung VW, et al. Effects of 18-month low-magnitude high-frequency vibration on fall rate and fracture risks in 710 community elderly - A cluster-randomized controlled trial. Osteoporosis International 2014;25(6):1785-95.

\section{Li 2018a \{published data only\}}

Li Z, Wang XX, Liang YY, Chen SY, Sheng J, Ma SJ. Effects of the visual-feedback-based force platform training with functional electric stimulation on the balance and prevention of falls in older adults: A randomized controlled trial. PeerJ 2018;6:e4244. [DOI: $10.7717 /$ peerj.4244]

Morris 2008 \{published data only (unpublished sought but not used)\}

Morris D. An evaluation of yoga for the reduction of fall risk factors in older adults. purl.flvc.org/fsu/fd/FSU_migr_etd-2257 (accessed 7 June 2018).

Ohtake 2013 \{published data only\}

Ohtake M, Morkiagi Y, Suzuki I, Kanoya Y, Sato C. Effects of exercise on the prevention of conditions leading to the need for long-term care. Aging Clinical and Experimental Research 2013;25(1):49-57.

\section{Olsen 2014 \{published data only\}}

Olsen CF, Bergland A. The effect of exercise and education on fear of falling in elderly women with osteoporosis and a history of vertebral fracture: results of a randomized controlled trial. Osteoporosis International 2014;25(8):2017-25. 
Pai 2014 \{published data only\}

Pai YC, Bhatt T, Yang F, Wang E. Perturbation training can reduce community-dwelling older adults' annual fall risk: a randomized controlled trial. Journals of Gerontology Series A, Biological Sciences and Medical Sciences 2014;69(12):1586-94.

Pereira 1998 \{published data only\}

Kriska AM, Bayles C, Cauley JA, LaPorte RE, Sandler RB, Pambianco G. A randomized exercise trial in older women: increased activity over two years and the factors associated with compliance. Medicine and Science in Sports and Exercise 1986;18(5):557-62.

Pereira MA. Ten year follow-up of a randomized exercise trial in post-menopausal women [thesis]. Pittsburgh (USA): Univ. of Pittsburgh, 1996. [Proquest Digital Dissertations Publication Number AAT 97 16627]

* Pereira MA, Kriska AM, Day RD, Cauley JA, LaPorte RE, Kuller $\mathrm{LH}$. A randomized walking trial in postmenopausal women: effects on physical activity and health 10 years later. Archives of Internal Medicine 1998;158(15):1695-701.

\section{Rossi-Izquierdo 2017 \{published data only\}}

Rossi-Izquierdo M, Gayoso-Diz P, Santos-Pérez S, Del-RíoValeiras M, Faraldo-García A, Vaamonde-Sanchez-Andrade I, et al. Short-term effectiveness of vestibular rehabilitation in elderly patients with postural instability: a randomized clinical trial. European Archives of Oto-Rhino-Laryngology 2017;274(6):2395-403.

\section{Steinberg 2000 \{published data only\}}

Steinberg M, Cartwright C, Peel N, Williams G. A sustainable programme to prevent falls and near falls in community dwelling older people: results of a randomised trial. Journal of Epidemiology and Community Health 2000;54(3):227-32.

\section{Swanenburg 2007 \{published data only\}}

Swanenburg J, De Bruin ED, Stauffacher M, Mulder T, Uebelhart D. Effects of exercise and nutrition on postural balance and risk of falling in elderly people with decreased bone mineral density: randomized controlled trial pilot study. Clinical Rehabilitation 2007;21(6):523-34.

\section{Ueda 2017 \{published data only\}}

Ueda T, Higuchi Y, Imaoka M, Todo E, Kitagawa T, Ando S. Tailored education program using home floor plans for falls prevention in discharged older patients: A pilot randomized controlled trial. Archives of Gerontology and Geriatrics 2017;71:9-13.

\section{References to studies awaiting assessment}

Jagdhane 2016 \{published data only\}

Jagdhane S, Kanekar N, Aruin AS. The effect of a four-week balance training program on anticipatory postural adjustments in older adults: a pilot feasibility study. Current Aging Science 2016;9(4):295-300

\section{Li 2018b \{published data only\}}

Li F, Harmer P, Fitzgerald K, Eckstrom E, Akers L, Chu L, et al. Effectiveness of a therapeutic Tai Ji Quan intervention vs a multimodal exercise intervention to prevent falls among older adults at high risk of falling. JAMA Internal Medicine 2018; Vol. 178, issue 10:1301-10. [DOI: 10.1001/jamaintemmed.2018.3915]

\section{References to ongoing studies}

\section{ACTRN 12613001161718 \{published data only\}}

ACTRN12613001161718. Effects of dual-task functional power training on falls in the elderly? An 18-month communitybased randomised controlled trial. www.anzctr.org.au/Trial/ Registration/TrialReview.aspx?id=364361 (first received 21 October 2013).

\section{ACTRN 12615000138583 \{published data only\}}

ACTRN12615000138583. Standing Tall - a home-based exercise program using mobile technology for preventing falls in older people [Evaluating the effect of a home-based exercise program delivered through mobile technology for preventing falls in older community-dwelling people over 2 years, compared to a health promotion education 'control' program. The 'Standing Tall' randomized control trial]. www.anzctr.org.au/Trial/ Registration/TrialReview.aspx?id=367746 (first received 13 February 2015).

\section{ACTRN 12615000865516 \{published data only\}}

ACTRN12615000865516. Balance Exercise and Strength Training (BEST) program for older people living at home [Lower limb home-based exercise program compared with upper limb home-based exercise program to prevent falls and upper limb dysfunction in older community-dwelling people: a randomised controlled trial]. www.anzctr.org.au/Trial/Registration/ TrialReview.aspx?id=367886 (first received 19 August 2015).

\section{CTRI/2018/01/011214 \{published data only\}}

CTRI/2018/01/011214. Role of physiotherapy in prevention of falls and fractures in elder population [Falls \& fractures a physiotherapy approach to prediction \& prevention in Healthcare]. www.ctri.nic.in/Clinicaltrials/pmaindet2.php? trialid=22175 (first received 9 January 2018).

\section{ISRCTN71002650 \{published data only\}}

ISRCTN71002650. Prevention of fall injury trial [Prevention of fall injury trial: a parallel group cluster randomised controlled trial and economic evaluation]. www.isrctn.com/ ISRCTN71002650 (first received 13 April 2010).

\section{NCT01029171 \{published data only\}}

NCT01029171. Action Seniors! Exercise to prevent falls [Action Seniors!: A 12 month randomized controlled trial of a home based strength and balance retraining program in reducing falls]. clinicaltrials.gov/ct2/show/NCT01029171 (first received 9 December 2009)

NCT02126488 \{published data only\}

NCT02126488. Effect of adaptive training for balance recovery [Perturbation training for fall-risk reduction among older 
adults]. clinicaltrials.gov/ct2/show/NCT02126488 (first received 30 April 2014).

\section{NCT02287740 \{published data only\}}

NCT02287740. Prevention of falls among older adults in community settings [Comparative effectiveness and costeffectiveness of a fall prevention intervention: Tai Ji Quan: moving for better balance]. clinicaltrials.gov/ct2/show/ NCT02287740 (first received 11 November 2014).

\section{NCT02617303 \{published data only\}}

NCT02617303. Prevention of falls and its consequences in elderly people [Effectiveness of an intervention through physical exercise for the prevention of falls and its consequences in elderly people (75-89 years) performed in primary care: study protocol for a randomized controlled trial]. clinicaltrials.gov/ct2/show/NCT02617303 (first received 30 November 2015).

\section{NCT02926105 \{published data only\}}

NCT02926105. Comparison of home-based exercise programmes for falls prevention and quality of life in older adults [Comparison of the effects of three home-based exercise programmes regarding falls, quality of life and exerciseadherence in older adults at risk of falling: protocol for a randomised controlled trial]. clinicaltrials.gov/ct2/show/ NCT02926105 (first received 6 October 2016).

\section{NCT03211429 \{published data only\}}

NCT03211429. Effectiveness of three interventions to reduce fear of falling and improve functionality in the elderly [Randomized clinical trial evaluating the effectiveness of three interventions programs to improve functionality and decrease fear of falling]. clinicaltrials.gov/ct2/show/NCT03211429 (first received 7 July 2017).

\section{NCT03320668 \{published data only\}}

NCT03320668. Efficacy of the Otago Exercise Program delivered as group training versus individually tailored training [Efficacy of the Otago Exercise Program (OEP) delivered as group training versus individually tailored training in community-dwelling adults between 65 and 80 years old]. clinicaltrials.gov/ct2/ show/NCT03320668 (first received 25 October 2017).

\section{NCT03404830 \{published data only\}}

NCT03404830. High intensity training to reduce the risk of falls in older people [Effects of a program of high intensity exercise by intervals on the risk of falls the physical condition and the state of health in people over 60 years]. clinicaltrials.gov/ct2/ show/NCT03404830 (first received 19 January 2018).

\section{NCT03417531 \{published data only\}}

NCT03417531. Sarcopenia prevention with a targeted exercise and protein supplementation program. clinicaltrials.gov/ct2/ show/NCT03417531 (first received 31 January 2018).

\section{NCT03455179 \{published data only\}}

NCT03455179. Effects of slow-speed traditional resistance training, high-speed resistance training and multicomponent training with variable resistances on molecular, body composition, neuromuscular, physicalfFunction and quality of life variables in older adults. clinicaltrials.gov/ct2/show/ NCT03455179 (first received 6 March 2018).

NCT03462654 \{published data only\}

NCT03462654. Comparison of a group-delivered vs. individually delivered 'LiFE' Program LiFE-is-LiFE [Comparison of a groupdelivered and individually delivered lifestyle-integrated functional exercise (LiFE) program in older persons]. clinicaltrials.gov/ct2/show/NCT03462654 (first received 12 March 2018).

\section{Additional references}

\section{AlHW 2018}

Australian Institute of Health and Welfare: Pointer S. Trends in Hospitalised Injury due to Falls in Older People, 2002-03 to 2014-15. Injury and Statistics Series, no 111.. Canberra: AIWH, 2018.

\section{Anonymous 1987}

Anonymous. The prevention of falls in later life. A report of the Kellogg International Work Group on the Prevention of Falls by the Elderly. Danish Medical Bulletin 1987;34 Suppl 4:1-24.

\section{Burns 2016}

Burns ER, Stevens JA, Lee R. The direct costs of fatal and nonfatal falls among older adults - United States. Journal of Safety Research 2016;58:99-103.

\section{Campbell 1990}

Campbell AJ, Borrie MJ, Spears GF, Jackson SL, Brown JS, Fitzgerald JL. Circumstances and consequences of falls experienced by a community population 70 years and over during a prospective study. Age and Ageing 1990;19(2):136-41.

\section{Campbell 1999}

Campbell AJ, Robertson MC, Gardner MM, Norton RN, Buchner DM. Falls prevention over 2 years: a randomized controlled trial in women 80 years and older. Age and Ageing 1999;28(6):513-8.

\section{Canning 2015}

Canning CG, Allen NE, Bloem BR, Keus SH, Munneke M, Nieuwboer $A$, et al. Interventions for preventing falls in Parkinson's disease. Cochrane Database of Systematic Reviews 2015, Issue 3. [DOI: 10.1002/14651858.CD011574]

\section{Carande-Kulis 2015}

Carande-Kulis V, Stevens JA, Florence CS, Beattie BL, Arias I. A cost-benefit analysis of three older adult fall prevention interventions. Journal of Safety Research 2015;52:65-70.

\section{Carpenter 2018}

Carpenter H, Audsley S, Coupland C, Gladman J, Kendrick D, Lafond N, et al. PHysical activity Implementation Study In Community-dwelling AduLts (PHISICAL): study protocol. Injury Prevention 2018 [Epub ahead of print]:pii: injuryprev-2017-042627. [DOI: 0.1136/injuryprev-2017-042627] 


\section{Caspersen 1985}

Caspersen CJ, Powell KE, Christenson GM. Physical activity, exercise, and physical fitness: definitions and distinctions for health-related research. Public Health Reports 1985;100(2):126-31.

\section{Clemson 2004a}

Clemson L, Cumming RG, Kendig H, Swann M, Heard R, Taylor K. The effectiveness of a community-based program for reducing the incidence of falls in the elderly: a randomized trial. Journal of the American Geriatrics Society 2004;52(9):1487-94.

\section{Cochrane Norway 2017}

Cochrane Norway. How to write a plain language summary of a Cochrane intervention review. www.cochrane.no/sites/ cochrane.no/files/public/uploads/checklist_for_cochrane_pls_ 28th_feb_2017_0.pdf (accessed 15 November 2018).

\section{Davis 2009}

Davis J, Guy P, Liu-Ambrose T, Donaldson M, Robertson M, Khan K. Cost-effectiveness analysis of the Otago home-based strength and balance retraining in senior fallers. Journal of Nutrition, Health \& Aging 2009;13(1):S436.

\section{Davis 2010}

Davis JC, Robertson MC, Ashe MC, Liu-Ambrose T, Khan KM, Marra CA. Does a home-based strength and balance programme in people aged $>$ or $=80$ years provide the best value for money to prevent falls? A systematic review of economic evaluations of falls prevention interventions. British Journal of Sports Medicine 2010;44(2):80-9.

\section{Deandrea 2010}

Deandrea S, Lucenteforte E, Bravi F, Foschi R, La VC, Negri E. Risk factors for falls in community-dwelling older people: a systematic review and meta-analysis. Epidemiology 2010;21(5):658-68.

\section{Farag 2015}

Farag I, Howard K, Ferreira ML, Sherrington C. Economic modelling of a public health programme for fall prevention. Age and Ageing 2015;44(3):409-14.

\section{Farag 2015a}

Farag I, Howard K, Hayes A J, Ferreira M L, Lord S R, Close J T, et al. Cost-effectiveness of a home-exercise program among older people after hospitalization. Journal of the American Medical Directors Association 2015;16(6):490-6.

\section{Fitzharris 2010}

Fitzharris MP, Day L, Lord SR, Gordon I, Fildes B. The Whitehorse NoFalls trial: effects on fall rates and injurious fall rates. Age and Ageing 2010;39(6):728-33.

\section{Fried 2001}

Fried LP, Tangen CM, Walston J, Newman AB, Hirsch C, Gottdiener J, et al. Frailty in older adults: evidence for a phenotype. Journals of Gerontology. Series A, Biological Sciences and Medical Sciences 2001;56(3):M146-56.

\section{Gillespie 2012}

Gillespie LD, Robertson MC, Gillespie WJ, Sherrington C, Gates S, Clemson LM, et al. Interventions for preventing falls in older people living in the community. Cochrane Database of Systematic Reviews 2012, issue 9. [DOI: 10.1002/14651858.CD007146.pub3]

\section{GRADEPro GDT 2015 [Computer program]}

McMaster University (developed by Evidence Prime). GRADEpro GDT. Hamilton (ON): McMaster University (developed by Evidence Prime), 2015.

\section{Haas 2006}

Haas M. Economic analysis of tai chi as a means of preventing falls and falls related injuries among older adults. CHERE working paper 2006/4. Sydney, Australia: Centre for Health Economics Research and Evaluation, University of Technology. https://www.uts.edu.au/sites/default/files/wp2006_3.pdf. ., (accessed 24 January 2019).

\section{Hannan 2010}

Hannan MT, Gagnon MM, Aneja J, Jones RN, Cupples LA, Lipsitz LA, et al. Optimizing the tracking of falls in studies of older participants: comparison of quarterly telephone recall with monthly falls calendars in the MOBILIZE Boston Study. American Journal of Epidemiology 2010;171(9):1031-6.

\section{Harper 2017}

Harper KJ, Barton AD, Arendts G, Edwards DG, Petta AC, Celenza A. Controlled clinical trial exploring the impact of a brief intervention for prevention of falls in an emergency department. Emergency Medicine Australasia 2017;29:524-30.

\section{Hawley-Hague 2017}

Hawley-Hague H, Roden A, Abbott J. The evaluation of a strength and balance exercise program for falls prevention in community primary care. Physiotherapy Theory and Practice 2017;33(8):611-621.

\section{Hektoen 2009}

Hektoen LF, Aas E, Luras H. Cost-effectiveness in fall prevention for older women. Scandanavian Journal of Public Health 2009;37(6):584-9.

\section{Higgins 2011}

Higgins JP, Green S, editor(s). Cochrane Handbook for Systematic Reviews of Interventions. Version 5.1.0 (updated March 2011). The Cochrane Collaboration, 2011. Available from www.handbook.cochrane.org.

\section{Hopewell 2018}

Hopewell S, Adedire O, Copsey BJ, Sherrington C, Clemson LM, Close JCT, et al. Multifactorial and multiple component interventions for preventing falls in older people living in the community. Cochrane Database of Systematic Reviews 2018, Issue 7. [DOI: 10.1002/14651858.CD012221.pub2]

\section{Howe 2011}

Howe TE, Rochester L, Neil F, Skelton DA, Ballinger C. Exercise for improving balance in older people. Cochrane 
Database of Systematic Reviews 2011, Issue 11. [DOI: 10.1002/14651858.CD004963]

\section{Iliffe 2014}

Iliffe S, Kendrick D, Morris R, Masud T, Gage H, Skelton D, et al. Multicentre cluster randomised trial comparing a community group exercise programme and home-based exercise with usual care for people aged 65 years and over in primary care. Health Technology Assessment 2014;18(49):1-105.

\section{Kendrick 2014}

Kendrick D, Kumar A, Carpenter H, Zijlstra GA, Skelton DA, Cook JR, et al. Exercise for reducing fear of falling in older people living in the community. Cochrane Database of Systematic Reviews 2014, Issue 11. [DOI: 10.1002/14651858.CD009848.pub2]

\section{Lamb 2005}

Lamb SE, Jorstad-Stein EC, Hauer K, Becker C, Prevention of Falls Network Europe and Outcomes Consensus Group. Development of a common outcome data set for fall injury prevention trials: the Prevention of Falls Network Europe consensus. Journal of the American Geriatrics Society 2005;53(9):1618-22.

\section{Lamb 2011}

Lamb SE, Becker C, Gillespie LD, Smith JL, Finnegan S, Potter R, et al. Reporting of complex interventions in clinical trials: development of a taxonomy to classify and describe fallprevention interventions. Trials 2011;12:125.

\section{Lefebvre 2011}

Lefebvre C, Manheimer E, Glanville J. Chapter 6: Searching for studies. In: Higgins JP, Green S, editor(s). Cochrane Handbook for Systematic Reviews of Interventions Version 5.1.0 (updated March 2011). The Cochrane Collaboration, 2011. Available from www.handbook.cochrane.org.

\section{Liu 2009}

Liu CJ, Latham N. Progressive resistance strength training for improving physical function in older adults. Cochrane Database of Systematic Reviews 2009, Issue 3. [DOI: 10.1002/14651858.CD002759.pub2]

\section{Liu-Ambrose 2010}

Liu-Ambrose T, Nagamatsu LS, Graf P, Beattie BL, Ashe MC, Handy TC. Resistance training and executive functions: a 12month randomized controlled trial. Archives of Internal Medicine 2010;170(2):170-8

\section{Lord 2011}

Lord SR, Sherrington C, Menz HB, Close J. Falls in Older People: Risk Factors and Strategies for Prevention. 2nd Edition. Cambridge University Press, 2011.

\section{Lusardi 2017}

Lusardi MM, Fritz S, Middleton A, Allison L, Wingood M, Phillips $\mathrm{E}$, et al. Determining risk of future falls in communitydwelling older adults: a systematic review and meta-analysis using posttest probability. Journal of Geriatric Physical Therapy 2017; Vol. 40, issue 1:1-36.

\section{Mahoney 1994}

Mahoney J, Sager M, Dunham NC, Johnson J. Risk of falls after hospital discharge. Journal of American Geriatrics Society 1994; Vol. 42, issue 3:269-74.

\section{Matchar 2017}

Matchar DB, Duncan PW, Lien CT, Ong MEH, Lee M, Gao F, et al. Randomized controlled trial of screening, risk modification, and physical Tterapy to prevent falls among the elderly recently discharged from the emergency department to the community: The Steps to Avoid Falls in the Elderly Study. Archives of Physical Medicine and Rehabilitation 2017;98(6):1086-96.

\section{McLean 2015}

McLean K, Day L, Dalton A. Economic evaluation of a groupbased exercise program for falls prevention among the older community-dwelling population. BMC Geriatrics 2015;15(1):33.

\section{Menant 2017}

Menant JC, Weber F, Lo J, Sturnieks DL, Close JC, Sachdev PS, et al. Strength measures are better than muscle mass measures in predicting health-related outcomes in older people: time to abandon the term sarcopenia?. Osteoporosis International 2017; Vol. 28, issue 1:59-70.

\section{NICE 2013}

National Institute for Health and Care Excellence. Falls in older people: assessing risk and prevention CG161. www.nice.org.uk/ guidance/cg161/resources/falls-in-older-people-assessingrisk-and-prevention-pdf-35109686728645 (accessed 10 August 2018).

\section{NICE 2018}

National Institute for Health and Care Excellence. NICEimpact falls and fragility fractures. www.nice.org.uk/media/default/ about/what-we-do/into practice/measuring-uptake/niceimpact-falls-and-fragility-fractures.pdf (accessed 5 August 2018).

Oliver 2017

Oliver D. Fighting pyjama paralysis in hospital wards. BMJ 2017;357:j2096.

\section{Patil 2016}

Patil R, Karinkanta S, Tokola K, Kannus P, Sievanen H, UusiRasi K. Effects of vitamin $D$ and exercise on the wellbeing of older community-dwelling women: a randomized controlled trial. Gerontology. 2016;62(4):401-8.

\section{Peel 2002}

Peel NM, Kassulke DJ, McClure RJ. Population based study of hospitalised fall related injuries in older people. Injury Prevention 2002;8(4):280-3.

\section{Public Health England 2018}

Public Health England. Guidance Falls prevention: costeffective commissioning. A resource to help commissioners and communities provide cost-effective falls prevention activities. www.gov.uk/government/publications/falls-prevention-costeffective-commissioning (accessed 15 November 2018). 


\section{Review Manager 2014 [Computer program]}

Nordic Cochrane Centre, The Cochrane Collaboration. Review Manager 5 (RevMan 5). Version 5.3. Copenhagen: Nordic Cochrane Centre, The Cochrane Collaboration, 2014.

\section{Robertson 2001b}

Robertson MC, Gardner MM, Devlin N, McGee R, Campbell AJ. Effectiveness and economic evaluation of a nurse delivered home exercise programme to prevent falls. 2: Controlled trial in multiple centres. BMJ 2001;322(7288):701-4.

\section{Robertson 2002}

Robertson MC, Campbell AJ, Gardner MM, Devlin N. Preventing injuries in older people by preventing falls: a meta-analysis of individual-level data. Journal of the American Geriatrics Society 2002;50(5):905-11.

\section{Robertson 2005}

Robertson MC, Campbell AJ, Herbison P. Statistical analysis of efficacy in falls prevention trials. Journals of Gerontology. Series A, Biological Sciences and Medical Sciences 2005;60(4):530-4.

\section{Schünemann 2017}

Schünemann HJ, Oxman AD, Higgins JP, Vist GE, Glasziou P, Akl E, et al. on behalf of the Cochrane GRADEing Methods Group (formerly Applicability and Recommendations Methods Group) and the Cochrane Statistical Methods Group. Chapter 11: Completing 'Summary of findings' tables and grading the confidence in or quality of the evidence. In: Higgins JPT, Churchill R, Chandler J, Cumpston MS (editors), Cochrane Handbook for Systematic Reviews of Interventions version 5.2.0 (updated June 2017). Cochrane, 2017. Available from www.training.cochrane.org/handbook.

\section{Sherrington 2017}

Sherrington C, Michaleff ZA, Fairhall N, Paul SS, Tiedemann A, Whitney J, et al. Exercise to prevent falls in older adults: an updated systematic review and meta-analysis. British Journal of Sports Medicine 2017;51(24):1750-8.

\section{Skelton 2001}

Skelton DA. Effects of physical activity on postural stability. Age and Ageing 2001;30 Suppl 4:33-9. [PUBMED: 11769787]

\section{Smeeth 2002}

Smeeth L, Ng ES. Intraclass correlation coefficients for cluster randomized trials in primary care: data from the MRC Trial of the Assessment and Management of Older People in the Community. Controlled Clinical Trials 2002;23(4):409-21.

\section{Speechley 1991}

Speechley M, Tinetti M. Falls and injuries in frail and vigorous community elderly persons. Journal of the American Geriatrics Society 1991;39(1):46-52.

\section{Stenhagen 2014}

Stenhagen M, Ekström H, Nordell E, Elmståhl S. Accidental falls, health-related quality of life and life satisfaction: A prospective study of the general elderly population. Archives of Gerontology and Geriatrics 2014;58(1):95-100.

\section{Sterne 2011}

Sterne JA, Sutton AJ, Ioannidis JP, Terrin N, Jones DR, Lau J, et al. Recommendations for examining and interpreting funnel plot asymmetry in meta-analyses of randomised controlled trials. BMJ 2011; Vol. 343:d4002.

\section{Thompson 2013}

Thompson PD, Arena R, Riebe D, Pescatello LS. ACSM's new preparticipation health screening recommendations from ACSM's guidelines for exercise testing and prescription, 9th edition. Current Sports Medicine Reports 2013;12(4):215-7.

\section{Tinetti 1988}

Tinetti ME, Speechley M, Ginter SF. Risk factors for falls among elderly persons living in the community. New England Journal of Medicine 1988;319(26):1701-7.

\section{Tinetti 1997}

Tinetti ME, Williams CS. Falls, injuries due to falls, and the risk of admission to a nursing home. New England Journal of Medicine 1997;337(18):1279-84

\section{Tricco 2017}

Tricco AC, Thomas SM, Veroniki AA, Hamid JS, Cogo E, Strifler L, et al. Comparisons of interventions for preventing falls in older adults: a systematic review and meta-analysis. Journal of the American Medical Association 2017; Vol. 318, issue 7:1687-99.

\section{Verheyden 2013}

Verheyden GS, Weerdesteyn V, Pickering RM, Kunkel D, Lennon S, Geurts ACH, et al. Interventions for preventing falls in people after stroke. Cochrane Database of Systematic Reviews 2013, Issue 5. [DOI: 10.1002/14651858.CD008728.pub2]

\section{Walker 2009}

Walker DG, Aedo C, Albala C, Allen E, Dangour AD, Elbourne D, et al. Methods for economic evaluation of a factorial-design cluster randomised controlled trial of a nutrition supplement and an exercise programme among healthy older people living in Santiago, Chile: the CENEX study. BMC Health Service Research 2009;9:85.

\section{Walters 2003}

Walters SJ, Brazier JE. What is the relationship between the minimally important difference and health state utility values? The case of the SF-6D. Health and Quality of Life Outcomes 2003;1(4):10.1186/1477-7525-1-4.

\section{Walters 2005}

Walters SJ, Brazier JE. Comparison of the minimally important difference for two health state utility measures: EQ-5D and SF-6D. Quality of Life Research 2005;14(6):1523-32.

\section{WHO 2015}

World Health Organization. World Report on Ageing and Health. www.who.int/ageing/events/world-report-2015-launch/en/. World Health Organization, 2015 (accessed Jaunuary 25 2018).

\section{Yardley 2002}

Yardley L, Smith H. A prospective study of the relationship between feared consequences of falling and avoidance of 
activity in community-living older people. Gerontologist 2002;42(1):17-23.

\section{References to other published versions of this review}

\section{Sherrington 2016}

Sherrington C, Tiedemann A, Fairhall NJ, Hopewell S, Michaleff ZA, Howard K, Clemson L, Lamb SE. Exercise for

\section{CHARACTERISTICS OF STUDIES}

Characteristics of included studies [ordered by study ID]

Almeida 2013

\begin{tabular}{ll}
\hline Methods & Study design: RCT \\
Number of study arms: 3 \\
Length of follow-up: 4 months \\
Setting: Sao Paulo, Brazil \\
Number of participants: 119 \\
Number analysed: 76 \\
Number lost to follow-up: 43 \\
Sample: community-dwelling \\
Age (years): mean 79.1 (SD 4.6) \\
Sex: $83 \%$ female \\
Inclusion criteria: non-institutionalised, able to walk independently, had at least 1 fall in the previous \\
year, not enrolled in a regular exercise programme \\
Exclusion criteria: any self-reported conditions that would preclude exercise prescription and physical \\
activity for older people, systolic or diastolic BP > 170 and 130 mm Hg, respectively, inability to follow \\
written instructions and unable to obtain constant support for that task
\end{tabular}

Interventions preventing falls in older people living in the community. Cochrane Database of Systematic Reviews 2016, Issue 11. [DOI: 10.1002/14651858.CD012424]

* Indicates the major publication for the study
1. Fully-supervised group-based balance and strength training: own body weight used for strength

\section{training, received home hazard reduction information and monthly phone calls; 50-minute sessions, 3 a week for 4 months \\ 2. Minimally-supervised group-based balance and strength training: own body weight used for strength training, received home hazard reduction information and monthly phone calls; 1 ×50-minute session, alternate weeks for 4 months. Brochure provided with same exercises to be performed at home $3 \times a$ week for 4 months \\ 3. Control: no exercise intervention, participants asked not to engage in any other exercise programme during the study}
Outcomes
1. Rate of falls
2. Number of people who experienced 1 or more falls (risk of falling)

\begin{tabular}{ll}
\hline Duration of the study & 16 weeks \\
\hline Adherence & None reported \\
\hline Notes & Source of funding: São Paulo State Funding Agency
\end{tabular}


Almeida 2013 (Continued)

Economic information: not reported

Data could not be analysed due to zero events for falls (and thus fallers)

\section{Risk of bias}

\begin{tabular}{lll}
\hline Bias & Authors' judgement & Support for judgement \\
\hline $\begin{array}{ll}\text { Random sequence genera- } \\
\text { tion (selection bias) }\end{array}$ & Unclear risk & $\begin{array}{l}\text { Quote: "After baseline assessments, participants were randomly assigned } \\
\text { to one of the 3 groups". Insufficient information about sequence generation } \\
\text { process }\end{array}$
\end{tabular}

\begin{tabular}{lll}
\hline $\begin{array}{l}\text { Allocation concealment } \\
\text { (selection bias) }\end{array}$ & Unclear risk & Concealment not described \\
\hline $\begin{array}{l}\text { Blinding of participants } \\
\text { and personnel (perfor- }\end{array}$ & Unclear risk & Participants and personnel unblinded but impact of unblinding unknown \\
mance bias) & & \\
All outcomes &
\end{tabular}

\begin{tabular}{lll}
\hline $\begin{array}{l}\text { Blinding of outcome as- } \\
\text { sessment (detection bias) } \\
\text { Falls }\end{array}$ & Unclear risk & $\begin{array}{l}\text { Information about falls collected at 4-month assessment in both groups. } \\
\text { Blinding of assessors was not stated. Insufficient information to permit judge- } \\
\text { ment }\end{array}$ \\
\hline $\begin{array}{l}\text { Blinding of outcome as- } \\
\text { sessment (detection bias) } \\
\text { Fractures }\end{array}$ & Unclear risk & Not applicable \\
\hline
\end{tabular}

\begin{tabular}{ll}
\hline Blinding of outcome as- & Unclear risk \\
sessment (detection bias) & \\
Hospital admission, med- & \\
ical attention and adverse & \\
events & \\
\hline
\end{tabular}

\begin{tabular}{ll}
\hline Blinding of outcome as- & Unclear risk \\
sessment (detection bias) & \\
Health related quality of & \\
life (self report) & \\
\hline
\end{tabular}

\begin{tabular}{ll}
\hline $\begin{array}{l}\text { Incomplete outcome data } \\
\text { (attrition bias) }\end{array}$ & High risk \\
Falls and fallers & $\begin{array}{l}\text { More than } 20 \% \text { missing outcome data, unbalanced losses across groups and } \\
\text { reasons for missing data across groups not specified }\end{array}$ \\
\hline
\end{tabular}

\begin{tabular}{|c|c|c|}
\hline $\begin{array}{l}\text { Selective reporting (re- } \\
\text { porting bias) }\end{array}$ & High risk & $\begin{array}{l}\text { Falls were measured but number of falls not reported. Fall outcomes and ad- } \\
\text { verse events were not prespecified in the Methods section. There was no pro- } \\
\text { tocol or trial registration }\end{array}$ \\
\hline
\end{tabular}

Method of ascertaining High risk Information about falls collected at 4-month assessment
falls (recall bias)

falls (recall bias)

Ansai 2015

\begin{tabular}{ll}
\hline Methods & $\begin{array}{l}\text { Study design: RCT } \\
\text { Number of study arms: } 3 \\
\text { Length of follow-up: } 4 \text { months }\end{array}$ \\
\hline Participants & Setting: São Paulo, Brazil
\end{tabular}

Exercise for preventing falls in older people living in the community (Review) 
Ansai 2015 (Continued)

Number of participants: 69

Number analysed: 68

Number lost to follow-up: 1

Sample: community-dwelling

Age (years): mean 82.4 (SD 2.4)

Sex: $68 \%$ female

Inclusion criteria: aged $>80$, community-dwelling, sedentary, able to walk alone, available to attend training site 3 a week

Exclusion criteria: presence of any injury listed in the absolute contraindications of the Physical Activity Readiness Medical Examination, relative cognition, neurological or musculoskeletal contraindications making participation in protocols impossible, MMSE score below the cut-off designated by educational level minus 1 SD

Interventions

1. Group-based balance, strength and aerobic training: cycle ergometer used for aerobic training, strength exercises (upper limbs, abdominals, squats, ankles) progressed using Borg scale and increments of $1 \mathrm{~kg}$, balance activities with increasing difficulty; 1 hour, 3 a week for 16 weeks

2. Group-based progressive strength training: leg press, chest press, calf raise, back extension, abdominal and rowing, 3 sets of 10 - 12 RM using gym equipment; 1 hour, 3 a week, 16 weeks

3. Control: no intervention

Outcomes $\quad \begin{aligned} & \text { 1. Rate of falls } \\ & \text { 2. Number of people who experienced } 1 \text { or more falls (risk of falling) }\end{aligned}$

\begin{tabular}{ll}
\hline Duration of the study & 23 weeks \\
\hline Adherence & $\begin{array}{l}\text { 1. Group-based balance, strength and aerobic training group: } 35 \% \text { performed } \geq 24 \text { sessions for } 16 \\
\text { weeks (50\% intervention) }\end{array}$ \\
& $\begin{array}{l}\text { 2. Group-based progressive strength training group: } 56 \% \text { performed } \geq 24 \text { sessions for } 16 \text { weeks (50\% in- } \\
\text { tervention) }\end{array}$
\end{tabular}

Notes Source of funding: Federal University of São Carlos

Economic information: not reported

16-week data used due to proportion of fallers not being clear for longer follow-up periods

Email communication regarding fall data, response received, data not included in review

\section{Risk of bias}

\begin{tabular}{lll}
\hline Bias & Authors' judgement & Support for judgement \\
\hline $\begin{array}{l}\text { Random sequence genera- } \\
\text { tion (selection bias) }\end{array}$ & Low risk & Computerised random-number generator \\
\hline $\begin{array}{l}\text { Allocation concealment } \\
\text { (selection bias) }\end{array}$ & Low risk & Opaque, sealed envelope \\
\hline $\begin{array}{l}\text { Blinding of participants } \\
\text { and personnel (perfor- } \\
\text { mance bias) } \\
\begin{array}{l}\text { All outcomes } \\
\hline\end{array}\end{array}$ & Unclear risk & Participants and personnel unblinded but impact of unblinding unknown \\
\hline
\end{tabular}


Ansai 2015 (Continued)

Blinding of outcome as- High risk Blinding of assessor not specified; as falls were reported by telephone or dursessment (detection bias)

Falls

Blinding of outcome as-
sessment (detection bias) Unclear risk Not applicable

Blinding of outcome as-
sessment (detection bias)
Hospital admission, med-
ical attention and adverse
events

\begin{tabular}{|c|c|c|}
\hline $\begin{array}{l}\text { Blinding of outcome as- } \\
\text { sessment (detection bias) } \\
\text { Health related quality of } \\
\text { life (self report) }\end{array}$ & Unclear risk & Not applicable \\
\hline
\end{tabular}

\begin{tabular}{lll}
\hline $\begin{array}{l}\text { Incomplete outcome data } \\
\text { (attrition bias) } \\
\text { Falls and fallers }\end{array}$ & Low risk & $\begin{array}{l}\text { Less than 20\% of outcome data are missing (6\%) and losses are balanced } \\
\text { across groups }\end{array}$ \\
\hline $\begin{array}{l}\text { Selective reporting (re- } \\
\text { porting bias) }\end{array}$ & High risk & $\begin{array}{l}\text { Falls were measured, but number of falls and adverse events were not report- } \\
\text { ed }\end{array}$ \\
\hline $\begin{array}{l}\text { Method of ascertaining } \\
\text { falls (recall bias) }\end{array}$ & Unclear risk & $\begin{array}{l}\text { Provided with fall calendar, falls reported by retrospective recall once a } \\
\text { month, by telephone or during training }\end{array}$ \\
\hline
\end{tabular}

\section{Arantes 2015}

\begin{tabular}{ll}
\hline Methods & Study design: RCT \\
& Number of study arms: 2 \\
& Length of follow-up: 3 months
\end{tabular}

Participants

Setting: Belo Horizonte, Brazil

Number of participants: 30

Number analysed: 28

Number lost to follow-up: 2

Sample: community-dwelling

Age (years): Intervention mean = 73.9 (SD 7.7); Control mean = $72.2($ SD 5.7)

Sex: $100 \%$ female

Inclusion criteria: age 65 years + , history of 1 or more falls in the previous year, at risk for falling (at least 2 risk factors assessed by the QuickScreen Falls Risk Assessment), classified as prefrail (phenotype proposed by Fried 2001), able to walk $6 \mathrm{~m}$ independently

Exclusion criteria: cognitive impairment (evaluated by MMSE), presence of neurological disease, acute vestibular dysfunction in past month, initiation of any other intervention during study period
Interventions
1. Group-based balance training: exercises increased in difficulty; 1 hour, 2 a week, 12 weeks
2. Control group: neck and upper limb stretches and movements; 1 hour, 1 a week, 12 weeks 
Arantes 2015 (Continued)

Outcomes 1. Number of people who experienced 1 or more falls (risk of falling)

\begin{tabular}{ll}
\hline Duration of the study & 12 weeks \\
\hline Adherence & Adherence measured by session attendance \\
& 1. Group-based balance training group: average number of sessions attended: 22.1 (range; 20 - 24) \\
2. Control group: average number of sessions attended: 10.8 (range $10-12)$
\end{tabular}

$\begin{array}{ll}\text { Notes } & \text { Source of funding: CNPq and FAPEMIG } \\ \text { Economic information: not reported }\end{array}$

Paper states "falls were registered for 1 year after randomisation" but these results not reported

\section{Risk of bias}

\begin{tabular}{|c|c|c|}
\hline Bias & Authors' judgement & Support for judgement \\
\hline $\begin{array}{l}\text { Random sequence genera- } \\
\text { tion (selection bias) }\end{array}$ & Unclear risk & Quote: "The allocation was made through a computer program" \\
\hline $\begin{array}{l}\text { Allocation concealment } \\
\text { (selection bias) }\end{array}$ & Unclear risk & Concealment not described \\
\hline $\begin{array}{l}\text { Blinding of participants } \\
\text { and personnel (perfor- } \\
\text { mance bias) } \\
\text { All outcomes }\end{array}$ & Unclear risk & $\begin{array}{l}\text { Participants and personnel implementing the intervention not blind to allocat- } \\
\text { ed group, but impact of non-blinding unclear }\end{array}$ \\
\hline $\begin{array}{l}\text { Blinding of outcome as- } \\
\text { sessment (detection bias) } \\
\text { Falls }\end{array}$ & Unclear risk & $\begin{array}{l}\text { Quote: "The assessments were performed before and immediately after the } \\
\text { end of intervention, always by the same evaluators, and they were blinded in } \\
\text { all the moments of the study". } \\
\text { Unclear whether these same assessors made monthly telephone calls to col- } \\
\text { lect fall data. }\end{array}$ \\
\hline $\begin{array}{l}\text { Blinding of outcome as- } \\
\text { sessment (detection bias) } \\
\text { Fractures }\end{array}$ & Unclear risk & Not applicable \\
\hline $\begin{array}{l}\text { Blinding of outcome as- } \\
\text { sessment (detection bias) } \\
\text { Hospital admission, med- } \\
\text { ical attention and adverse } \\
\text { events }\end{array}$ & Unclear risk & Not applicable \\
\hline $\begin{array}{l}\text { Blinding of outcome as- } \\
\text { sessment (detection bias) } \\
\text { Health related quality of } \\
\text { life (self report) }\end{array}$ & Unclear risk & Not applicable \\
\hline $\begin{array}{l}\text { Incomplete outcome data } \\
\text { (attrition bias) } \\
\text { Falls and fallers }\end{array}$ & Unclear risk & $\begin{array}{l}\text { Less than } 20 \% \text { of outcome data are missing }(7 \%) \text {, with losses only from control } \\
\text { group, due to starting another intervention }(n=1) \text { and family problems }(n=1)\end{array}$ \\
\hline $\begin{array}{l}\text { Selective reporting (re- } \\
\text { porting bias) }\end{array}$ & High risk & $\begin{array}{l}\text { Falls were measured, but number of falls and adverse events were not report- } \\
\text { ed }\end{array}$ \\
\hline
\end{tabular}


Arantes 2015 (Continued)

Method of ascertaining Unclear risk Quote: "The subjects were contacted monthly by telephone and asked about falls (recall bias)

$\begin{array}{ll}\text { Methods } & \text { Study design: RCT } \\ & \text { Number of study arms: } 2 \\ & \text { Length of follow-up: } 3 \text { months }\end{array}$

Participants

Setting: 3 different municipalities, Sweden

Number of participants: 45

Number analysed: 40

Number lost to follow-up: 5

Sample: community-dwelling

Age (years): mean 83 (range 75 - 103)

Sex: $71 \%$ female

Inclusion criteria: $\geq 75 \mathrm{yrs}$, walk independently in home, understand written and oral information in Swedish language

Exclusion criteria: < 25 MMSE, ongoing regular physical therapy due to injury \pm illness, terminal care

Interventions

Randomised into 3 groups: 2 intervention groups (1 Individual Otago Exercise Programme, 1 Otago Exercise Programme + Motivational Interview group) and 1 control group. The Individual Otago Exercise Programme and Otago Exercise Programme + Motivational Interviewing groups were combined in this review

1. Individual Otago Exercise Programme: home-based programme 3 a week, walking programme 4 a week, for 12 weeks, received written recommendations for falls prevention

2. Control group: no intervention, received written recommendations for falls prevention

\begin{tabular}{ll}
\hline Outcomes & Rate of falls \\
2. Number of people who experienced 1 or more falls (risk of falling)
\end{tabular}

Duration of the study 12 weeks

Adherence Not reported

Notes Source of funding: Mälardalen University

Economic information: not reported

Email communication to obtain fall data, response received, data included in review

\section{Risk of bias}

\begin{tabular}{lll}
\hline Bias & Authors' judgement & Support for judgement \\
\hline $\begin{array}{l}\text { Random sequence genera- } \\
\text { tion (selection bias) }\end{array}$ & Unclear risk & $\begin{array}{l}\text { Quote: "Predetermined randomisation list made by an independent statisti- } \\
\text { cian". Blocks of 3,6, 9, or 12 participants. Method of generating the randomi- } \\
\text { sation list not described }\end{array}$ \\
\hline
\end{tabular}


Arkkukangas 2015 (Continued)

Allocation concealment Unclear risk Concealment of predetermined list not described
(selection bias)

\begin{tabular}{|c|c|c|}
\hline $\begin{array}{l}\text { Blinding of participants } \\
\text { and personnel (perfor- }\end{array}$ & Unclear risk & $\begin{array}{l}\text { Participants and personnel not blind to allocated group but impact of non- } \\
\text { blinding unclear }\end{array}$ \\
\hline
\end{tabular}

mance bias)

All outcomes

\begin{tabular}{|c|c|c|}
\hline Blinding of outcome as- & Low risk & Falls collected by fall calendar in both groups \\
\hline Falls & & $\begin{array}{l}\text { Quote: "fall calendar, which was followed up by the physiotherapist every } \\
\text { month". "Four physiotherapists performed the measurements single blindly." } \\
\text { Assume fall calendar was followed up by } 1 \text { of the blinded physiotherapists }\end{array}$ \\
\hline
\end{tabular}

\begin{tabular}{|c|c|c|}
\hline $\begin{array}{l}\text { Blinding of outcome as- } \\
\text { sessment (detection bias) } \\
\text { Fractures }\end{array}$ & Unclear risk & Not applicable \\
\hline
\end{tabular}

Blinding of outcome as- $\quad$ Unclear risk $\quad$ Not applicable
sessment (detection bias)
Hospital admission, med-
ical attention and adverse
events

\begin{tabular}{|c|c|c|}
\hline $\begin{array}{l}\text { Blinding of outcome as- } \\
\text { sessment (detection bias) } \\
\text { Health related quality of } \\
\text { life (self report) }\end{array}$ & Unclear risk & Not applicable \\
\hline $\begin{array}{l}\text { Incomplete outcome data } \\
\text { (attrition bias) } \\
\text { Falls and fallers }\end{array}$ & Unclear risk & $\begin{array}{l}\text { Less than } 20 \% \text { of outcome data are missing }(11 \%) \text {. Unbalanced losses in inter- } \\
\text { vention }(n=4) \text { and control }(n=0) \text { groups, but reason for missing data not spec- } \\
\text { ified }\end{array}$ \\
\hline $\begin{array}{l}\text { Selective reporting (re- } \\
\text { porting bias) }\end{array}$ & Unclear risk & $\begin{array}{l}\text { Adverse events were not a prespecified outcome and were not reported for all } \\
\text { groups. No trial protocol or prospective trial registration }\end{array}$ \\
\hline $\begin{array}{l}\text { Method of ascertaining } \\
\text { falls (recall bias) }\end{array}$ & Low risk & $\begin{array}{l}\text { Fall calendar, followed up monthly by physiotherapist. } \\
\text { Quote: "Four PTs performed the measurements single blindly" }\end{array}$ \\
\hline
\end{tabular}

\section{Ballard 2004}

\begin{tabular}{ll}
\hline Methods & $\begin{array}{l}\text { Study design: RCT } \\
\text { Number of study arms: } 2 \\
\text { Length of follow-up: } 16 \text { months }\end{array}$ \\
\hline Participants & Setting: USA \\
& Number of participants: 40 \\
& Number analysed: 39 \\
& Number lost to follow-up: 1 \\
& Sample: community-dwelling \\
& Age (years): mean 72.9 (SD 6) \\
& Sex: $100 \%$ female
\end{tabular}


Ballard 2004 (Continued)

Inclusion criteria: aged $\geq 65$; ambulatory; community-dwelling; history of falling in previous year or fear of future fall; able to undertake moderate exercise

Exclusion criteria: cardiovascular disease or extreme vertigo that might prohibit moderate exercise; requiring walker for support

\begin{tabular}{|c|c|}
\hline Interventions & $\begin{array}{l}\text { 1. Group-based balance, strength and aerobic training for } 15 \text { weeks: elastic bands used for strength } \\
\text { training, } 6 \text { home-safety education classes; } 1 \text { hour, } 3 \text { a week, for } 15 \text { weeks }\end{array}$ \\
\hline & $\begin{array}{l}\text { 2. Group-based balance, strength and aerobic training for } 2 \text { weeks: elastic bands used for strength } \\
\text { training, } 6 \text { home-safety education classes; } 1 \text { hour, } 3 \text { a week, for } 2 \text { weeks }\end{array}$ \\
\hline
\end{tabular}

\begin{tabular}{|c|c|}
\hline Outcomes & 1. Rate of falls \\
\hline Duration of the study & 64 weeks \\
\hline \multirow[t]{7}{*}{ Adherence } & Adherence measured by session attendance, exercising at 1 year, frequency of exercise at 1 year \\
\hline & Participants attended $83 \%$ ( $\pm 9 \%$ ) of the exercise sessions \\
\hline & At 1-year follow-up: \\
\hline & $\begin{array}{l}\text { 1. Group-based balance, strength and aerobic training for } 15 \text { weeks plus home practice group: Contin- } \\
\text { ued exercise format as in intervention group: } \mathrm{No}=7, \text { Yes }=13\end{array}$ \\
\hline & Exercise format performed 2 a week $=5$; performed $\geq 3$ a week $=8$. \\
\hline & $\begin{array}{l}\text { 2. Group-based balance, strength and aerobic training for } 2 \text { weeks plus home practice with videotape } \\
\text { group: Started exercise format as in intervention group: No }=17, \text { Yes }=2\end{array}$ \\
\hline & Exercise format performed 2 a week $=1$; performed $3 \times$ ar week $=1$ \\
\hline
\end{tabular}

$\begin{array}{ll}\text { Notes } & \text { Source of funding: not reported } \\ & \text { Economic information: not reported } \\ & \text { Data not used for number of people falling as not clear on total proportion of fallers }\end{array}$

\section{Risk of bias}

\begin{tabular}{|c|c|c|}
\hline Bias & Authors' judgement & Support for judgement \\
\hline $\begin{array}{l}\text { Random sequence genera- } \\
\text { tion (selection bias) }\end{array}$ & Unclear risk & $\begin{array}{l}\text { Quote: "assigned to exercise and control groups using stratified randomisa- } \\
\text { tion" }\end{array}$ \\
\hline $\begin{array}{l}\text { Allocation concealment } \\
\text { (selection bias) }\end{array}$ & Unclear risk & Insufficient information to permit judgement \\
\hline $\begin{array}{l}\text { Blinding of participants } \\
\text { and personnel (perfor- } \\
\text { mance bias) } \\
\text { All outcomes }\end{array}$ & Unclear risk & $\begin{array}{l}\text { Participants and personnel not blind to allocated group but impact of non- } \\
\text { blinding unclear }\end{array}$ \\
\hline $\begin{array}{l}\text { Blinding of outcome as- } \\
\text { sessment (detection bias) } \\
\text { Falls }\end{array}$ & High risk & $\begin{array}{l}\text { Falls data were collected in both groups at the } 6 \text { home-safety education ses- } \\
\text { sions, assume assessors not blinded. Fall data also collected by telephone at } 1 \\
\text { year; blinding of telephone assessors not reported }\end{array}$ \\
\hline $\begin{array}{l}\text { Blinding of outcome as- } \\
\text { sessment (detection bias) } \\
\text { Fractures }\end{array}$ & Unclear risk & Not applicable \\
\hline
\end{tabular}


Ballard 2004 (Continued)

\author{
Blinding of outcome as- \\ Unclear risk Not applicable \\ sessment (detection bias) \\ Hospital admission, med- \\ ical attention and adverse \\ events

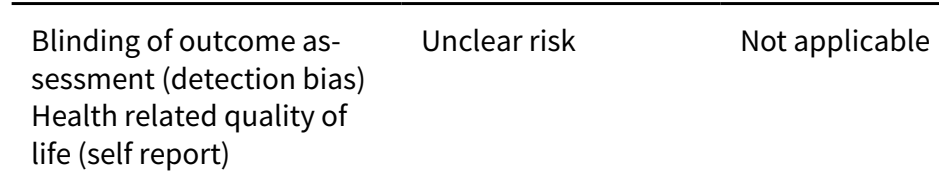

\begin{tabular}{lll}
\hline $\begin{array}{l}\text { Incomplete outcome data } \\
\text { (attrition bias) } \\
\text { Falls and fallers }\end{array}$ & Low risk & $\begin{array}{l}\text { Less than 20\% of outcome data are missing (3\%). Missing data are from 1 exer- } \\
\text { cise group participant and unlikely to be related to outcome }\end{array}$ \\
\hline $\begin{array}{l}\text { Selective reporting (re- } \\
\text { porting bias) }\end{array}$ & High risk & $\begin{array}{l}\text { Number of fallers was reported in only 1 group. Adverse events were not pre- } \\
\text { specified or reported }\end{array}$ \\
\hline $\begin{array}{l}\text { Method of ascertaining } \\
\text { falls (recall bias) }\end{array}$ & High risk & $\begin{array}{l}\text { Falls identified retrospectively during intervention at each home-safety class } \\
\text { (every 2 months), and by telephone follow-up 1 year after end of intervention }\end{array}$ \\
\hline
\end{tabular}

Barker 2016

\begin{tabular}{ll}
\hline Methods & $\begin{array}{l}\text { Study design: RCT } \\
\text { Number of study arms: } 2 \\
\text { Length of follow-up: } 6 \text { months }\end{array}$ \\
\hline Participants & Setting: Melbourne, Australia \\
Number of participants: 53 \\
Number analysed: 44 \\
Number lost to follow-up: 9 \\
Sample: community-dwelling \\
Age (years): mean 69 \\
Sex: $88 \%$ female \\
Inclusion criteria: $\geq 60$ years, at risk of sustaining a fall injury based on a telephone screen developed by \\
the research team, able to negotiate a set of 10 stairs independently without a gait aid \\
Exclusion criteria: cognitive impairment (telephone MMSE $<17$ ), acute medical condition that impaired \\
safe performance of exercise (e.g. unstable BP, chronic back pain, acute MI), cancer diagnosis with- \\
in the past 5 years or receiving active treatment for cancer, uncontrolled chronic conditions (e.g. dia- \\
betes, hypertension), already participating in Pilates or other formal exercise ( $\geq 60$ minutes a week for $\geq$ \\
4 weeks during the 12 weeks prior to screening for eligibility)
\end{tabular}

Interventions

1. Group-based Pilates focused on balance and strength plus home practice: group performed predominantly in standing with minimal-to-no upper limb support, used Pilates equipment; 1 hour, 2 a week, 12 weeks, and tailored home exercises performed 20 minutes daily; participants paid AUD 36.50 per class

2. Individual strength and balance: tailored home exercise performed 20 minutes daily for 12 weeks

Both groups received a fall and fracture prevention information and exercise brochure

Outcomes 1 Rate of falls


Barker 2016 (Continued)

2. Number of people who experienced 1 or more falls (risk of falling)

\begin{tabular}{ll}
\hline Duration of the study & 24 weeks \\
\hline Adherence & Adherence measured by class attendance, time recorded exercising \\
& $\begin{array}{l}\text { 1. Group-based Pilates focused on balance and strength plus home practice group: } 95 \% \text { attended over } \\
\text { 2. Individual strength and balance group: mean hours of exercise recorded at } 24 \text { weeks }=40.8\end{array}$ \\
\hline Notes & $\begin{array}{l}\text { Source of funding: Monash University Faculty of Medicine, Nursing and Health Sciences Strategic Grant } \\
\text { Scheme } \\
\text { Economic information: not reported }\end{array}$ \\
\hline
\end{tabular}

\section{Risk of bias}

\begin{tabular}{lll}
\hline Bias & Authors' judgement & Support for judgement \\
\hline $\begin{array}{l}\text { Random sequence genera- } \\
\text { tion (selection bias) }\end{array}$ & Low risk & Computer-generated, permuted, block randomisation schedule \\
\hline $\begin{array}{l}\text { Allocation concealment } \\
\text { (selection bias) }\end{array}$ & Low risk & Sealed opaque envelopes \\
\hline $\begin{array}{l}\text { Blinding of participants } \\
\text { and personnel (perfor- } \\
\text { mance bias) }\end{array}$ & Unclear risk & $\begin{array}{l}\text { Participants and personnel not blind to allocated group but impact of non- } \\
\text { All outcomes }\end{array}$ \\
\end{tabular}

\begin{tabular}{lll}
\hline $\begin{array}{l}\text { Blinding of outcome as- } \\
\text { sessment (detection bias) }\end{array}$ & Unclear risk & $\begin{array}{l}\text { Falls assessed by monthly calendar and telephone calls in all groups. Blinding } \\
\text { of assessors of fall calendars / phone calls was not stated. Insufficient informa- } \\
\text { Falls }\end{array}$
\end{tabular}

\begin{tabular}{ll}
\hline Blinding of outcome as- & Unclear risk \\
sessment (detection bias) & \\
Fractures & \\
\hline
\end{tabular}

\section{Blinding of outcome as- High risk} sessment (detection bias) Hospital admission, medical attention and adverse events

\begin{abstract}
Adverse events were "monitored by therapists delivering pilates classes or spontaneously reported by participants to the research staff", therefore assessors not blinded
\end{abstract}

Blinding of outcome as- $\quad$ Unclear risk Not applicable
sessment (detection bias)
Health related quality of
life (self report)

\begin{tabular}{|c|c|c|}
\hline $\begin{array}{l}\text { Incomplete outcome data } \\
\text { (attrition bias) } \\
\text { Falls and fallers }\end{array}$ & Unclear risk & $\begin{array}{l}\text { Less than } 20 \% \text { of outcome data are missing }(17 \%) \text {. Unbalanced losses in in- } \\
\text { tervention }(n=4) \text { and control }(n=9) \text { groups, with reasons for missing data in- } \\
\text { consistent across groups. Missing data have been imputed using appropriate } \\
\text { methods (last observation carried forward) }\end{array}$ \\
\hline
\end{tabular}

\begin{tabular}{lll}
\hline $\begin{array}{l}\text { Selective reporting (re- } \\
\text { porting bias) }\end{array}$ & Low risk & Prespecified falls outcomes reported in prospective trial protocol \\
\hline $\begin{array}{l}\text { Method of ascertaining } \\
\text { falls (recall bias) }\end{array}$ & Low risk & Monthly calendar and telephone calls \\
\hline \hline
\end{tabular}


Barnett 2003

\begin{tabular}{ll}
\hline Methods & $\begin{array}{l}\text { Study design: RCT } \\
\text { Number of study arms: } 2 \\
\text { Length of follow-up: } 12 \text { months }\end{array}$ \\
\hline Participants & Setting: Sydney, Australia \\
& Number of participants: 163 \\
& Number analysed: 150 \\
& Number lost to follow-up: 13 \\
& Sample: older people identified as at risk of falling by general practitioner or hospital physiotherapist \\
& using assessment tool \\
& Age (years): mean 74.9 (SD 10.9) \\
& Sex: $67 \%$ female \\
Inclusion criteria: age > 65 years; identified as 'at risk' of falling (1 or more of the following risk factors: \\
lower limb weakness, poor balance, slow reaction time) \\
Exclusion criteria: cognitive impairment; degenerative conditions, e.g. Parkinson's disease or medical \\
condition involving neuromuscular, skeletal, or cardiovascular system that precluded taking part in ex- \\
ercise programme
\end{tabular}

$\begin{array}{ll}\text { Interventions } & \text { Group-based balance, strength and aerobic training: exercises increased in difficulty, strength train- } \\ \text { ing using own body weight; } 1 \text { hour a week for } 4 \text { terms for } 1 \text { year ( } 37 \text { classes) plus home exercise based } \\ \text { on class content + diaries to record participation }\end{array}$

2. Control: no exercise intervention

Both groups received information on strategies for avoiding falls, e.g. hand and foot placement if loss of balance occurred

Outcomes
$\begin{aligned} & \text { 2. Rate of falls } \\ & \text { 3. Number of people who experienced } 1 \text { or more falls (risk of falling) }\end{aligned}$

\begin{tabular}{ll}
\hline Duration of the study & 52 weeks \\
\hline Adherence & Adherence measured by class attendance, frequency of home programme \\
& 1. Group-based balance, strength and aerobic training group: \\
& Median number of classes attended: 23 (range $0-36)$ \\
& Number attended 30 or more classes: $28(34 \%)$ \\
& $\begin{array}{l}\text { Attending exercise classes at end of trial and performing home programme } \geq 1 \text { a week: } 91 \%, \text { with } 13 \% \\
\text { performing exercises daily }\end{array}$
\end{tabular}

Notes Source of funding: Bankstown-Lidcombe hospital

Economic information: not reported

\section{Risk of bias}

\begin{tabular}{lll}
\hline Bias & Authors' judgement & Support for judgement \\
\hline $\begin{array}{l}\text { Random sequence genera- } \\
\text { tion (selection bias) }\end{array}$ & Unclear risk & Quote: "randomised in matched blocks" (N =6) \\
\hline $\begin{array}{l}\text { Allocation concealment } \\
\text { (selection bias) }\end{array}$ & Low risk & Consecutively-numbered, opaque envelopes \\
\hline
\end{tabular}




\section{Barnett 2003 (Continued)}

Blinding of participants and personnel (perfor-

Unclear risk mance bias)

All outcomes
Both groups received information on strategies for avoiding falls and intervention group also received structured weekly exercise sessions. Blinding not reported, but impact of non-blinding unclear

\begin{tabular}{|c|c|c|}
\hline $\begin{array}{l}\text { Blinding of outcome as- } \\
\text { sessment (detection bias) } \\
\text { Falls }\end{array}$ & Unclear risk & $\begin{array}{l}\text { Falls reported by participants who were aware of their group allocation, by } \\
\text { postal surveys monthly in both groups. Telephone interview if not returned by } \\
2 \text { weeks. Unclear whether those conducting telephone check were unblinded }\end{array}$ \\
\hline
\end{tabular}

\begin{tabular}{lll}
\hline Blinding of outcome as- & Unclear risk $\quad$ Not applicable
\end{tabular}

sessment (detection bias)

Fractures

Blinding of outcome as-
sessment (detection bias)
Hospital admission, med-
ical attention and adverse
events

\begin{tabular}{|c|c|c|}
\hline $\begin{array}{l}\text { Blinding of outcome as- } \\
\text { sessment (detection bias) } \\
\text { Health related quality of } \\
\text { life (self report) }\end{array}$ & Unclear risk & Not applicable \\
\hline $\begin{array}{l}\text { Incomplete outcome data } \\
\text { (attrition bias) } \\
\text { Falls and fallers }\end{array}$ & Low risk & $\begin{array}{l}\text { Less than } 20 \% \text { of outcome data are missing }(8 \%) \text {. Balanced losses in interven- } \\
\text { tion }(n=7) \text { and control }(n=6) \text { groups, with reasons for missing fall data un- } \\
\text { clear }\end{array}$ \\
\hline $\begin{array}{l}\text { Selective reporting (re- } \\
\text { porting bias) }\end{array}$ & Unclear risk & $\begin{array}{l}\text { Minimum set of expected outcomes not reported (adverse events not report- } \\
\text { ed) }\end{array}$ \\
\hline $\begin{array}{l}\text { Method of ascertaining } \\
\text { falls (recall bias) }\end{array}$ & Unclear risk & $\begin{array}{l}\text { Interval recall. Falls identified by postal survey at the end of each calendar } \\
\text { month. Phoned if not returned within } 2 \text { weeks }\end{array}$ \\
\hline
\end{tabular}

Beyer 2007

\begin{tabular}{ll} 
Methods & Study design: RCT \\
& Number of study arms: 2 \\
& Length of follow-up: 12 months \\
\hline Participants & Setting: Copenhagen, Denmark \\
& Number of participants: 65 \\
& Number analysed: 53 \\
& Number lost to follow-up: 12 \\
& Sample: women with a history of a fall identified from hospital records \\
& Age (years): range 70 - 90 \\
& Sex: $100 \%$ female \\
& Inclusion criteria: community-dwelling; at a relatively high risk of falls, defined as either $\geq 80$ years old \\
& or $\geq 65$ years with history of a fall in the previous 12 months or a timed 'up and go' test score of at least \\
& 15 seconds; home-dwelling; aged 70 - 90 years; history of a fall requiring treatment in ED but not hospi- \\
talisation; able to come to training facility \\
Exclusion criteria: lower limb fracture in last 6 months; neurological diseases, unable to understand \\
Danish; cognitively impaired (MMSE < 24)
\end{tabular}


Beyer 2007 (Continued)

Interventions $\quad$ 1. Group-based balance, strength and flexibility training: gym equipment used for strengthening, 1

hour, 2 a week, for 6 months

2. Control: no intervention; offered intervention after 1 year

\begin{tabular}{ll}
\hline Outcomes & 1. Number of people who experienced 1 or more falls (risk of falling) \\
\hline Duration of the study & 52 weeks
\end{tabular}

\begin{tabular}{ll}
\hline Adherence & Adherence measured by training compliance \\
1. Group-based balance, strength and flexibility training group: mean training compliance $79 \%$ (42 - \\
$100 \%)$
\end{tabular}

\section{Risk of bias}

\begin{tabular}{|c|c|c|}
\hline Bias & Authors' judgement & Support for judgement \\
\hline $\begin{array}{l}\text { Random sequence genera- } \\
\text { tion (selection bias) }\end{array}$ & Low risk & $\begin{array}{l}\text { Quote: "...using the minimization method with the aid of a computer program } \\
\text { for randomization" }\end{array}$ \\
\hline $\begin{array}{l}\text { Allocation concealment } \\
\text { (selection bias) }\end{array}$ & Unclear risk & Insufficient information to permit judgement \\
\hline $\begin{array}{l}\text { Blinding of participants } \\
\text { and personnel (perfor- } \\
\text { mance bias) } \\
\text { All outcomes }\end{array}$ & Unclear risk & $\begin{array}{l}\text { Participants and personnel not blinded to allocated group but impact of non- } \\
\text { blinding unclear }\end{array}$ \\
\hline $\begin{array}{l}\text { Blinding of outcome as- } \\
\text { sessment (detection bias) } \\
\text { Falls }\end{array}$ & Unclear risk & $\begin{array}{l}\text { Falls were recorded in both allocated groups using the same method (a } \\
\text { monthly falls calendar), but no mention of blinding of personnel confirming } \\
\text { falls or carrying out data entry. Insufficient information to make a judgement }\end{array}$ \\
\hline $\begin{array}{l}\text { Blinding of outcome as- } \\
\text { sessment (detection bias) } \\
\text { Fractures }\end{array}$ & Unclear risk & Not applicable \\
\hline $\begin{array}{l}\text { Blinding of outcome as- } \\
\text { sessment (detection bias) } \\
\text { Hospital admission, med- } \\
\text { ical attention and adverse } \\
\text { events }\end{array}$ & Unclear risk & Not applicable \\
\hline $\begin{array}{l}\text { Blinding of outcome as- } \\
\text { sessment (detection bias) } \\
\text { Health related quality of } \\
\text { life (self report) }\end{array}$ & Unclear risk & Not applicable \\
\hline $\begin{array}{l}\text { Incomplete outcome data } \\
\text { (attrition bias) } \\
\text { Falls and fallers }\end{array}$ & Unclear risk & $\begin{array}{l}\text { Less than } 20 \% \text { of outcome data are missing }(18 \%) \text {. Unbalanced losses in inter- } \\
\text { vention }(n=10) \text { and control }(n=4) \text { groups, with reasons for missing fall data } \\
\text { differing between the } 2 \text { groups (intervention group: } n=3 \text { did not start training, } \\
4=\text { ill, } 1=\text { fracture, } 2=\text { lost to follow-up; control group: } n=1 \text { dropped out as un- } \\
\text { happy with group allocation, } 1=i l l, 1=\text { fracture, } 1=\text { spouse ill) }\end{array}$ \\
\hline
\end{tabular}


Beyer 2007 (Continued)

Selective reporting (re- High risk $\quad$ The study prespecified falls "were monitored in all participants during the porting bias)

Method of ascertaining Low risk

falls (recall bias) study period", but number of falls was not reported

Quote: "A falls calendar was sent to every participant on the first day of each month" for 1 year

Boongrid 2017

$\begin{array}{ll}\text { Methods } & \text { Study design: RCT } \\ & \text { Number of study arms: } 2 \\ & \text { Length of follow-up: } 12 \text { months }\end{array}$

\section{Participants}

\section{Setting: Bangkok, Thailand}

Number of participants: 439

Number analysed: 437

Number lost to follow-up: 2

Sample: community-dwelling

Age (years): mean 73.8 (SD 6.7)

Sex: $83 \%$ female

Inclusion criteria: $\geq 65$ years, mild-to-moderate balance dysfunction, able to provide written informed consent.

Exclusion criteria: moderate-to severe cognitive problems, a neurological condition that severely influenced their gait and mobility (e.g. Parkinson's disease, stroke with hemiparesis), acute arthritis, any unstable or terminal illnesses that would preclude the planned exercises and were unlikely to resolve, unable to communicate well in Thai, already participating in regular strengthening exercise (e.g. yoga, Tai Chi)

Interventions 1. Individual Otago Exercise Programme and walking plan; video disk, manuals and weekly calendars provided, telephone calls every 2 weeks, and home visit in 3, 6, 9, 12 months

2. Control group: no intervention

Both groups received fall prevention education and home safety information through video disk recorder media and books

1. Rate of falls
2. Number of people who experienced 1 or more falls (risk of falling)
3. Number of people who died

\begin{tabular}{ll}
\hline Duration of the study & 52 weeks \\
\hline Adherence & Adherence measured by proportion exercising $\geq 120$ minutes a week at 3 months \\
& $\begin{array}{l}\text { 1. Individual Otago Exercise Programme and walking plan group: } 30 \% \text { exercised } \geq 120 \text { minutes a week } \\
\text { at } 3 \text { months; } 32 \% \text { exercised } \geq 120 \text { minutes a week at } 6 \text { month; } 57 \% \text { exercised } \geq 120 \text { minutes a week at } 3 \\
\text { months }\end{array}$
\end{tabular}

Notes

Source of funding: Development potentials of Thai People Project, Mahidol University Economic information: not reported 
Boongrid 2017 (Continued)

Risk of bias

\begin{tabular}{|c|c|c|}
\hline Bias & Authors' judgement & Support for judgement \\
\hline $\begin{array}{l}\text { Random sequence genera- } \\
\text { tion (selection bias) }\end{array}$ & Low risk & $\begin{array}{l}\text { Quote: "A block randomization was applied to generate random sequence lists } \\
\text { by an investigator who was not involved in data collection or administering in- } \\
\text { terventions" }\end{array}$ \\
\hline $\begin{array}{l}\text { Allocation concealment } \\
\text { (selection bias) }\end{array}$ & Low risk & Opaque sealed envelopes and sequence kept confidential \\
\hline $\begin{array}{l}\text { Blinding of participants } \\
\text { and personnel (perfor- } \\
\text { mance bias) } \\
\text { All outcomes }\end{array}$ & Unclear risk & $\begin{array}{l}\text { Participants and personnel implementing the intervention not blinded to allo- } \\
\text { cated group, but impact of non-blinding unclear }\end{array}$ \\
\hline
\end{tabular}

\begin{tabular}{lll}
\hline $\begin{array}{l}\text { Blinding of outcome as- } \\
\text { sessment (detection bias) } \\
\text { Falls }\end{array}$ & Low risk & $\begin{array}{l}\text { Falls were recorded on daily calendar in all groups. Research assistants who } \\
\text { conducted interviews were blinded to group allocation }\end{array}$ \\
\hline $\begin{array}{l}\text { Blinding of outcome as- } \\
\text { sessment (detection bias) } \\
\text { Fractures }\end{array}$ & Unclear risk & Not applicable \\
\hline
\end{tabular}

\begin{tabular}{|c|c|c|}
\hline $\begin{array}{l}\text { Blinding of outcome as- } \\
\text { sessment (detection bias) } \\
\text { Hospital admission, med- } \\
\text { ical attention and adverse } \\
\text { events }\end{array}$ & Unclear risk & Method of ascertaining adverse events is unclear \\
\hline
\end{tabular}

\begin{tabular}{|c|c|c|}
\hline $\begin{array}{l}\text { Blinding of outcome as- } \\
\text { sessment (detection bias) } \\
\text { Health related quality of } \\
\text { life (self report) }\end{array}$ & High risk & Participants were not blind to allocated group \\
\hline
\end{tabular}

\begin{tabular}{ll}
\hline $\begin{array}{l}\text { Incomplete outcome data } \\
\text { (attrition bias) }\end{array}$ & Low risk \\
Falls and fallers & $\begin{array}{l}\text { Less than } 20 \% \text { of outcome data are missing }(<1 \%) . \text { Balanced losses in inter- } \\
\text { vention and control groups }\end{array}$
\end{tabular}

\begin{tabular}{ll}
\hline $\begin{array}{l}\text { Selective reporting (re- } \\
\text { porting bias) }\end{array}$ & Low risk
\end{tabular}

\begin{tabular}{|c|c|c|}
\hline $\begin{array}{l}\text { Method of ascertaining } \\
\text { falls (recall bias) }\end{array}$ & Low risk & $\begin{array}{l}\text { Falls were self-recorded on a daily calendar, plus interviews by blinded re- } \\
\text { search assistants at } 3,6,9 \text { and } 12 \text { months }\end{array}$ \\
\hline
\end{tabular}

\section{Brown 2002}

\begin{tabular}{ll}
\hline Methods & $\begin{array}{l}\text { Study design: RCT } \\
\text { Number of study arms: } 2 \\
\text { Length of follow-up: } 14 \text { months }\end{array}$ \\
\hline Participants & Setting: Perth, Western Australia \\
& Number of participants: 99 \\
& Number analysed: 71 \\
& Number lost to follow-up: 28
\end{tabular}


Brown 2002 (Continued)

Sample: men and women recruited by press releases in 11 newspapers and information brochures distributed to organisations, GPs, etc; 6 pairs of people with the same residential address randomised to the same group

Age (years): $N=101$ aged 75 to $84, N=48$ aged 85 to 94

Sex: $79 \%$ female

Inclusion criteria: age $\geq 75$; community-living; independent in basic ADL; able to walk $20 \mathrm{~m}$ without personal assistance

Exclusion criteria: cognitive impairment (MMSE $\leq 24$ ); various conditions, e.g. angina, claudication, cerebrovascular disease, low or high blood pressure, major systemic disease, mental illness

Interventions

Randomised into 3 groups: 2 intervention groups (1 group-based balance, strength and aerobic training, and 1 social intervention group) and 1 control group. Only group-based balance, strength and aerobic training and control group included in this review

1. Group-based balance, strength and aerobic training: individualised and progressed, elastic tubing and free weights used for strength training, home practice of a functional task; 1 hour, 2 a week, 16 weeks

2. Control group: no intervention

\begin{tabular}{ll} 
Outcomes & 1. Number of people who experienced 1 or more falls (risk of falling) \\
& 2. Number of people who died \\
\hline Duration of the study & 56 weeks \\
\hline Adherence & $\begin{array}{l}\text { Adherence measured by session attendance } \\
\text { 1. Group-based balance, strength and aerobic training group: mean attendance; } 85 \% \text { (22-26 sessions), } \\
\text { range of } 62-100 \% \text { (16 sessions) }\end{array}$
\end{tabular}

Notes Source of funding: not reported

Economic information: not reported

Only group-based balance, strength and aerobic training and control group included in this review

\section{Risk of bias}

\begin{tabular}{lll}
\hline Bias & Authors' judgement & Support for judgement \\
\hline $\begin{array}{l}\text { Random sequence genera- } \\
\text { tion (selection bias) }\end{array}$ & Low risk & $\begin{array}{l}\text { Quote: "randomised into one of three groups using a table of random num- } \\
\text { bers" }\end{array}$ \\
\hline $\begin{array}{l}\text { Allocation concealment } \\
\text { (selection bias) }\end{array}$ & Low risk & $\begin{array}{l}\text { Randomised into one of } 3 \text { groups "by a physiotherapist uninvolved in the } \\
\text { study." }\end{array}$ \\
\hline $\begin{array}{l}\text { Blinding of participants } \\
\text { and personnel (perfor- } \\
\text { mance bias) }\end{array}$ & Unclear risk & $\begin{array}{l}\text { Participants and personnel not blind to allocated group but impact of non- } \\
\text { blinding unclear }\end{array}$ \\
\hline $\begin{array}{l}\text { Blinding of outcome as- } \\
\text { sessment (detection bias) } \\
\text { Falls }\end{array}$ & Unclear risk & $\begin{array}{l}\text { Fall data collected in same manner in each group. Study reports outcome as- } \\
\text { sessors were blinded, but it is unclear whether blinded assessors conducted } \\
\text { the telephone follow-ups for falls }\end{array}$ \\
\hline $\begin{array}{l}\text { Blinding of outcome as- } \\
\text { sessment (detection bias) } \\
\begin{array}{l}\text { Fractures } \\
\hline\end{array}\end{array}$ & Unclear risk & Not applicable \\
\hline
\end{tabular}


Brown 2002 (Continued)
Blinding of outcome as-
Unclear risk
Not applicable sessment (detection bias) Hospital admission, medical attention and adverse events

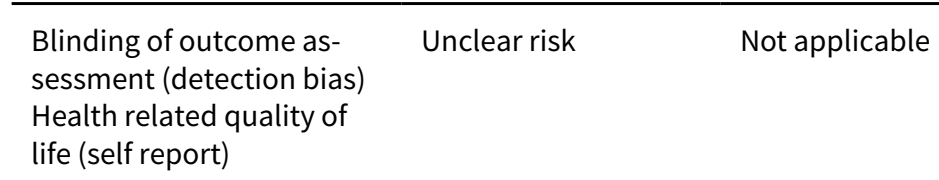

\begin{tabular}{lll}
\hline $\begin{array}{l}\text { Incomplete outcome data } \\
\text { (attrition bias) } \\
\text { Falls and fallers }\end{array}$ & High risk & $\begin{array}{l}\text { More than 20\% of outcome data are missing (28\%). Unbalanced losses in inter- } \\
\text { vention and control groups }\end{array}$ \\
\hline $\begin{array}{l}\text { Selective reporting (re- } \\
\text { porting bias) }\end{array}$ & High risk & Fall data were collected but number of falls not reported \\
\hline $\begin{array}{l}\text { Method of ascertaining } \\
\text { falls (recall bias) }\end{array}$ & Low risk & $\begin{array}{l}\text { Participants provided details of falls in monthly report sheet returned in re- } \\
\text { ply-paid addressed envelopes. No mention of telephone calls }\end{array}$ \\
\hline
\end{tabular}

Buchner 1997

\begin{tabular}{ll} 
Methods & $\begin{array}{l}\text { Study design: RCT } \\
\text { Number of study arms: } 2 \text { for analysis } \\
\text { Length of follow-up: } 25 \text { months }\end{array}$ \\
\hline Participants & Setting: Seattle, USA \\
& Number of participants: 105 \\
& Number analysed: 100 \\
& Number lost to follow-up: 5 \\
& Sample: random sample of HMO members (FICSIT intervention groups only) \\
& Age (years): mean 75 \\
& Sex: $51 \%$ female \\
Inclusion criteria: aged 68 - $85 ;$ unable to do 8-step tandem gait test without errors; below 50th per- \\
centile in knee extensor strength for height and weight \\
Exclusion criteria: active cardiovascular, pulmonary, vestibular, and bone disease; positive cardiac \\
stress test; body weight > 180\% ideal; major psychiatric illness; active metabolic disease; chronic \\
anaemia; amputation; chronic neurological or muscle disease; inability to walk; dependency in eating, \\
dressing, transfer or bathing; terminal illness; inability to speak English or complete written forms
\end{tabular}

Interventions

Randomised into 7 groups: 6 intervention groups (3 FICSIT trial - group-based stationary cycling, groupbased strength training, group-based combined endurance and strength training; and 3 MovelT trial), and 1 control group. This paper reports on the 3 FICSIT groups and the control group 1. Group-based stationary cycling: stationary cycles used for arms and legs, supervised classes; 1 hour (30 - 35 minutes endurance exercise), 3 a week for 6 months followed by unsupervised exercise 2. Group-based strength training: weight machines used for upper and lower body ( 2 sets of 10 reps per set, $50-60 \% 1$ RM for set 1 and $75 \%$ of 1 RM for set 2), supervised classes; 1 hour, 3 a week for 6 months followed by unsupervised exercise

3. Group-based combined endurance and strength training: 20 minutes of endurance training and 1 set of strength training exercises ( $75 \% 1 \mathrm{RM}$ )

4. Control: usual activity levels but "allowed to exercise after 6 months" 
Buchner 1997 (Continued)

2. Number of people who experienced 1 or more falls (risk of falling)

"A priori decision" to report fall outcomes for "any exercise" (all 3 exercise groups combined) compared with control group

\begin{tabular}{|c|c|}
\hline Duration of the study & Up to 100 weeks, median 72 weeks \\
\hline Adherence & $\begin{array}{l}\text { Exercise groups: } 14 \text { dropouts (19\%), participants who remained in the study attended } 95 \% \text { sessions } \\
\text { Control group; } 1 \text { dropout (3\%) }\end{array}$ \\
\hline Notes & $\begin{array}{l}\text { Source of funding: National Institute on Aging, Centers for Disease Control and Prevention, Department } \\
\text { of Veterans Affairs } \\
\text { Economic information: Healthcare service costs: hospitalised control participants more likely to have } \\
\text { hospital costs > USD } 5000(P<0.05) \text {; no significant difference in ancillary outpatient costs between } \\
\text { groups at } 7-18 \text { months } \\
\text { Seattle FICSIT trial. Only } 1.3 \% \text { of original sample randomised. Falls not primary outcome. Other out- } \\
\text { comes assessed at end of intervention ( } 6 \text { months) then "control group allowed to exercise after } 6 \\
\text { months" ( } 7 / 30 \text { participants did). Cost analysis reported in primary reference }\end{array}$ \\
\hline
\end{tabular}

\section{Risk of bias}

\begin{tabular}{|c|c|c|}
\hline Bias & Authors' judgement & Support for judgement \\
\hline $\begin{array}{l}\text { Random sequence genera- } \\
\text { tion (selection bias) }\end{array}$ & Low risk & Randomised, quote: "using a variation of randomly permuted blocks" \\
\hline $\begin{array}{l}\text { Allocation concealment } \\
\text { (selection bias) }\end{array}$ & Unclear risk & Insufficient information to permit judgement \\
\hline $\begin{array}{l}\text { Blinding of participants } \\
\text { and personnel (perfor- } \\
\text { mance bias) } \\
\text { All outcomes }\end{array}$ & Unclear risk & $\begin{array}{l}\text { Participants and personnel not blinded to allocated group but impact of non- } \\
\text { blinding unclear }\end{array}$ \\
\hline $\begin{array}{l}\text { Blinding of outcome as- } \\
\text { sessment (detection bias) } \\
\text { Falls }\end{array}$ & Unclear risk & $\begin{array}{l}\text { Falls reported by participants who were aware of their group allocation. } \\
\text { Quote: "Most study outcomes were measured by blinded examiners..." but un- } \\
\text { clear whether this applies to personnel carrying out telephone follow-up of } \\
\text { falls }\end{array}$ \\
\hline $\begin{array}{l}\text { Blinding of outcome as- } \\
\text { sessment (detection bias) } \\
\text { Fractures }\end{array}$ & Unclear risk & Not applicable \\
\hline $\begin{array}{l}\text { Blinding of outcome as- } \\
\text { sessment (detection bias) } \\
\text { Hospital admission, med- } \\
\text { ical attention and adverse } \\
\text { events }\end{array}$ & Unclear risk & Not applicable \\
\hline $\begin{array}{l}\text { Blinding of outcome as- } \\
\text { sessment (detection bias) } \\
\text { Health related quality of } \\
\text { life (self report) }\end{array}$ & Unclear risk & Not applicable \\
\hline $\begin{array}{l}\text { Incomplete outcome data } \\
\text { (attrition bias) } \\
\text { Falls and fallers }\end{array}$ & Unclear risk & $\begin{array}{l}\text { Less than } 20 \% \text { of outcome data are missing }(5 \%) \text {. Unbalanced losses between } \\
\text { intervention groups ( } n=2 \text { in each of the } 3 \text { groups) and control }(n=0) \text { group. } \\
\text { Reason for missing data unclear }\end{array}$ \\
\hline
\end{tabular}


Buchner 1997 (Continued)

Selective reporting (re- Unclear risk Minimum set of expected outcomes not reported (adverse events not reportporting bias)

ed)

Method of ascertaining Low risk

Falls reported immediately by mail, also monthly postcard return; telephone

falls (recall bias)

follow-up if no postcard received

Bunout 2005

$\begin{array}{ll}\text { Methods } & \text { Study design: RCT } \\ & \text { Number of study arms: } 2 \\ & \text { Length of follow-up: } 12 \text { months }\end{array}$

\begin{tabular}{ll}
\hline Participants & Setting: Santiago, Chile \\
& Number of participants: 298 \\
& Number analysed: 241 \\
& Number lost to follow-up: 57 \\
Sample: men and women & Age (years): mean 75 (SD 5) \\
Sex: $70 \%$ female \\
Inclusion criteria: "elderly subjects" consenting to participate; able to reach community centre \\
Exclusion criteria: severe disabling condition; cognitive impairment (MMSE $<20$ )
\end{tabular}

Interventions $\begin{aligned} & \text { Group-based balance, strength and walking: moderate intensity strength training using functional } \\ & \text { weight-bearing exercises, progressive resistance TheraBands; } 1 \text { hour, } 2 \text { a week, } 1 \text { year } \\ & \text { 2. Control: no intervention }\end{aligned}$

1. Rate of falls
2. Number of people who experienced 1 or more falls (risk of falling)
3. Number of people who died

Duration of the study 52 weeks

Adherence Adherence measured by attendance at $>50 \%$ sessions

1. Group-based balance, strength and walking group: $42 \%$ non-compliant (attended $<50 \%$ sessions)

\begin{tabular}{ll}
\hline Notes & Source of funding: University of Chile \\
& Economic information: not reported \\
& Journal website for supplementary data www.ageing.oupjournals.org. Additional data obtained from \\
& author
\end{tabular}

\section{Risk of bias}

\begin{tabular}{lll}
\hline Bias & Authors' judgement & Support for judgement \\
\hline $\begin{array}{l}\text { Random sequence genera- } \\
\text { tion (selection bias) }\end{array}$ & Low risk & Randomised using computer-generated random-number table \\
\hline $\begin{array}{l}\text { Allocation concealment } \\
\text { (selection bias) }\end{array}$ & Unclear risk & Insufficient information to permit judgement \\
\hline
\end{tabular}


Bunout 2005 (Continued)

Blinding of participants Unclear risk Participants and personnel not blinded to allocated group but impact of nonand personnel (perforblinding unclear

All outcomes

\begin{tabular}{|c|c|c|}
\hline $\begin{array}{l}\text { Blinding of outcome as- } \\
\text { sessment (detection bias) } \\
\text { Falls }\end{array}$ & Unclear risk & $\begin{array}{l}\text { Falls reported at follow-up clinics by participants who were aware of their } \\
\text { group allocation. Blinding of researchers at follow-up not reported }\end{array}$ \\
\hline
\end{tabular}

Plindin

\begin{tabular}{ll}
\hline Blinding of outcome as- & Unclear risk
\end{tabular}

\section{Fractures}

\begin{tabular}{|c|c|c|}
\hline $\begin{array}{l}\text { Blinding of outcome as- } \\
\text { sessment (detection bias) } \\
\text { Hospital admission, med- } \\
\text { ical attention and adverse } \\
\text { events }\end{array}$ & Unclear risk & Not applicable \\
\hline
\end{tabular}

\begin{tabular}{lll}
\hline $\begin{array}{l}\text { Blinding of outcome as- } \\
\text { sessment (detection bias) } \\
\text { Health related quality of } \\
\text { life (self report) }\end{array}$ & Unclear risk & Not applicable \\
\hline $\begin{array}{l}\text { Incomplete outcome data } \\
\text { (attrition bias) }\end{array}$ & Unclear risk & $\begin{array}{l}\text { Less than 20\% of outcome data are missing (19\%). Number lost from each } \\
\text { group is unclear }\end{array}$ \\
\hline $\begin{array}{l}\text { Selective reporting (re- } \\
\text { porting bias) }\end{array}$ & High risk & $\begin{array}{l}\text { Falls data were collected but number of fallers was not reported; adverse } \\
\text { events were not reported }\end{array}$ \\
\hline $\begin{array}{l}\text { Method of ascertaining } \\
\text { falls (recall bias) }\end{array}$ & Unclear risk & Interval recall. Falls ascertained at monthly outpatient clinic or by telephone \\
\hline
\end{tabular}

\section{Campbell 1997}

\begin{tabular}{ll} 
Methods & $\begin{array}{l}\text { Study design: RCT } \\
\text { Number of study arms: } 2 \\
\text { Length of follow-up: } 24 \text { months }\end{array}$ \\
\hline Participants & Setting: Dunedin, New Zealand \\
& Number of participants: 233 \\
& Number analysed: 233 \\
& Number lost to follow-up: 0 \\
& Sample: women identified from general practice registers \\
& Age (years): mean 84.1 (SD 3.1) \\
& Sex: $100 \%$ female \\
& Inclusion criteria: at least 80 years old; community-living \\
& Exclusion criteria: cognitive impairment; not ambulatory in own residence; already receiving physio- \\
therapy
\end{tabular}

Interventions

1. Individual Otago Exercise Programme: home-based programme prescribed in $4 \times 1$-hour visits in first 2 months, 30-minute exercise, 3 a week plus walk outside home 3 a week. Regular phone contact after first 2 months 
Campbell 1997 (Continued)

2. Control: social visit by research nurse $x 4$ in first 2 months. Regular phone contact

$\begin{array}{ll}\text { Outcomes } & \text { 1. Rate of falls } \\ \text { 2. Number of people who experienced } 1 \text { or more falls (risk of falling) }\end{array}$

\begin{tabular}{|c|c|}
\hline Duration of the study & 52 weeks. 2-year data reported in Campbell 1999 \\
\hline Adherence & Not reported \\
\hline Notes & $\begin{array}{l}\text { Source of funding: Accident Rehabilitation and Compensation Insurance Corporation of New Zealand, } \\
\text { Department of Veterns Affairs, USA } \\
\text { Economic information: Mean cost per person (intervention): NZD } 173 \text { in year 1, NZD } 22 \text { in year } 2 \text {. } \\
\text { Healthcare service costs: no difference between the } 2 \text { groups resulting from falls or for total healthcare } \\
\text { costs, } 27 \% \text { hospital admission costs resulted from fall. Incremental cost per fall prevented/per QALY } \\
\text { gained: at } 1 \text { year = NZD } 314 \text { (programme implementation costs only); at } 2 \text { years = NZD } 265 \text { (programme } \\
\text { implementation costs only) }\end{array}$ \\
\hline & $\begin{array}{l}\text { Otago Exercise Programme manual can be obtained from www.cdc.gov/HomeandRecreationalSafe- } \\
\text { ty/Falls/compendium/1.2_otago.html. Cost-effectiveness analysis reported (Robertson 2001ac). }\end{array}$ \\
\hline
\end{tabular}

\section{Risk of bias}

\begin{tabular}{|c|c|c|}
\hline Bias & Authors' judgement & Support for judgement \\
\hline $\begin{array}{l}\text { Random sequence genera- } \\
\text { tion (selection bias) }\end{array}$ & Low risk & Allocation schedule developed using computer-generated numbers \\
\hline $\begin{array}{l}\text { Allocation concealment } \\
\text { (selection bias) }\end{array}$ & Low risk & Assignment by independent person off-site \\
\hline $\begin{array}{l}\text { Blinding of participants } \\
\text { and personnel (perfor- } \\
\text { mance bias) } \\
\text { All outcomes }\end{array}$ & Unclear risk & $\begin{array}{l}\text { Participants and personnel not blinded to allocated group but impact of non- } \\
\text { blinding unclear }\end{array}$ \\
\hline $\begin{array}{l}\text { Blinding of outcome as- } \\
\text { sessment (detection bias) } \\
\text { Falls }\end{array}$ & High risk & $\begin{array}{l}\text { Falls reported by participants who were aware of group allocation. Blinding of } \\
\text { adjudicator reported, but researcher making telephone contact was aware of } \\
\text { group allocation as she also did social visits (personal communication report- } \\
\text { ed by Gillespie 2012) }\end{array}$ \\
\hline $\begin{array}{l}\text { Blinding of outcome as- } \\
\text { sessment (detection bias) } \\
\text { Fractures }\end{array}$ & Unclear risk & Not applicable \\
\hline $\begin{array}{l}\text { Blinding of outcome as- } \\
\text { sessment (detection bias) } \\
\text { Hospital admission, med- } \\
\text { ical attention and adverse } \\
\text { events }\end{array}$ & Unclear risk & Not applicable \\
\hline
\end{tabular}

\begin{tabular}{|c|c|c|}
\hline $\begin{array}{l}\text { Blinding of outcome as- } \\
\text { sessment (detection bias) } \\
\text { Health related quality of } \\
\text { life (self report) }\end{array}$ & Unclear risk & Not applicable \\
\hline
\end{tabular}

Incomplete outcome data Low risk No missing outcome data for falls
(attrition bias)

Falls and fallers 
Campbell 1997 (Continued)

Selective reporting (re- Unclear risk Minimum set of expected outcomes not reported (adverse events not reportporting bias)

ed)

Method of ascertaining Low risk

falls (recall bias)

Falls recorded daily on postcard calendars, mail registration monthly by postcard, telephone follow-up

Carter 2002

\begin{tabular}{ll}
\hline Methods & Study design: RCT \\
& Number of study arms: 2 \\
& Length of follow-up: 5 months
\end{tabular}

Setting: Vancouver, Canada
Pumber of participants: 93
Number analysed: 80
Number lost to follow-up: 13
Sample: community-dwelling osteoporotic women
Age (years): mean 69 (SD 3)
Sex: $100 \%$ female
Inclusion criteria: aged 65 - 75 years; residents of greater Vancouver; osteoporotic (based on BMD)
Exclusion criteria: < 5 years post-menopause; weighed > 130\% ideal body weight; other contraindica-
tions to exercising; already doing > 8 hours/week moderate-to-hard exercise; planning to be out of city
>4 week during 20 -week programme

Interventions 1. Group-based Osteofit strength and gait training: strengthening and stretching exercises using progressive resistance Theraband elastic bands and small free weights, 40 minutes, 2 a week, for 20 weeks, bimonthly social seminar

2. Control: usual activities, bimonthly social seminar separate from intervention group

\begin{tabular}{ll}
\hline Outcomes & 1. Rate of falls \\
\hline Duration of the study & 20 weeks \\
\hline
\end{tabular}

\begin{tabular}{ll}
\hline Adherence & Adherence measured by class attendance \\
& 1. Group-based Osteofit strength and gait training class: $89 \%$ \\
\hline Notes & $\begin{array}{l}\text { Source of funding: BC Medical Services Foundation of the Vancouver Foundation, British Columbia } \\
\text { Sports Medicine Foundation, RBC Foundation } \\
\text { Economic information: not reported }\end{array}$ \\
\hline
\end{tabular}

\section{Risk of bias}

\begin{tabular}{lll}
\hline Bias & Authors' judgement & Support for judgement \\
\hline $\begin{array}{l}\text { Random sequence genera- } \\
\text { tion (selection bias) }\end{array}$ & Low risk & Randomised by computer-generated programme \\
\hline $\begin{array}{l}\text { Allocation concealment } \\
\text { (selection bias) }\end{array}$ & Unclear risk & Insufficient information to permit judgement \\
\hline $\begin{array}{l}\text { Blinding of participants } \\
\text { and personnel (perfor- } \\
\text { mance bias) }\end{array}$ & Unclear risk & $\begin{array}{l}\text { Participants and personnel not blinded to allocated group but impact of non- } \\
\text { blinding unclear }\end{array}$ \\
\end{tabular}


Carter 2002 (Continued)

All outcomes

\begin{tabular}{|c|c|c|}
\hline Blinding of outcome as- & Low risk & Falls recorded in falls calendars in both groups. \\
\hline Falls & & $\begin{array}{l}\text { Quote: "All data were collected by trained researchers blinded to group assign- } \\
\text { ment" }\end{array}$ \\
\hline
\end{tabular}

\begin{tabular}{l} 
Blinding of outcome as- \\
sessment (detection bias) \\
Fractures \\
\hline Blinding of outcome as- \\
sessment (detection bias) \\
Hospital admission, med- \\
ical attention and adverse \\
events
\end{tabular}
Unclear risk
Not applicable

sessment (detection bias)

\begin{tabular}{lll}
\hline $\begin{array}{l}\text { Blinding of outcome as- } \\
\text { sessment (detection bias) } \\
\text { Health related quality of } \\
\text { life (self report) }\end{array}$ & High risk & Participants not blind to allocated group \\
\hline $\begin{array}{l}\text { Incomplete outcome data } \\
\text { (attrition bias) }\end{array}$ & Low risk & $\begin{array}{l}\text { Less than } 20 \% \text { of outcome data are missing }(5 \%) \text {. Minor imbalance in with- } \\
\text { drawals in intervention }(n=5) \text { and control }(n=8) \text { groups, with balanced rea- } \\
\text { salls and fallers }\end{array}$ \\
\hline
\end{tabular}

\begin{tabular}{lll}
\hline $\begin{array}{l}\text { Selective reporting (re- } \\
\text { porting bias) }\end{array}$ & High risk & $\begin{array}{l}\text { Fall data were collected but number of fallers was not reported; adverse } \\
\text { events were not reported }\end{array}$ \\
\hline $\begin{array}{l}\text { Method of ascertaining } \\
\text { falls (recall bias) }\end{array}$ & Low risk & Falls recorded in falls calendars returned monthly \\
\hline
\end{tabular}

\section{Cerny 1998}

\begin{tabular}{|c|c|}
\hline Methods & $\begin{array}{l}\text { Study design: RCT } \\
\text { Number of study arms: } 2 \\
\text { Length of follow-up: } 6 \text { months }\end{array}$ \\
\hline \multirow[t]{8}{*}{ Participants } & Setting: California, USA \\
\hline & Number of participants: 28 \\
\hline & Number analysed: 28 \\
\hline & Number lost to follow-up: 0 \\
\hline & $\begin{array}{l}\text { Sample: community-dwelling "well elderly" (proportion of women not stated); some pairs of people } \\
\text { randomised to the same group where they were (e.g. dependent on the other for transport) }\end{array}$ \\
\hline & Age (years): mean 71 (SD 4) \\
\hline & Inclusion criteria: none described \\
\hline & Exclusion criteria: none described \\
\hline \multirow[t]{2}{*}{ Interventions } & $\begin{array}{l}\text { 1. Group-based balance, strength, flexibility, aerobic training and brisk walking: } 1 \frac{1}{2} \text { hours, } 3 \text { a week, } 6 \\
\text { months }\end{array}$ \\
\hline & 2. Control: no intervention \\
\hline
\end{tabular}

Outcomes 1. Number of people who experienced 1 or more falls (risk of falling)

Duration of the study 24 weeks


Cerny 1998 (Continued)

\begin{tabular}{ll} 
Adherence & Not reported \\
\hline Notes & Source of funding: not reported \\
Economic information: not reported \\
Contact with lead author but no full paper or report prepared \\
Email communication about fall data, response received, data not included in review
\end{tabular}

\section{Risk of bias}

\begin{tabular}{|c|c|c|}
\hline Bias & Authors' judgement & Support for judgement \\
\hline $\begin{array}{l}\text { Random sequence genera- } \\
\text { tion (selection bias) }\end{array}$ & Low risk & $\begin{array}{l}\text { Randomised by coin toss. Individually randomised but some clusters, e.g. cou- } \\
\text { ples or } 2 \text { women where } 1 \text { was dependent on the other for transport (personal } \\
\text { communication reported in Gillespie 2012) }\end{array}$ \\
\hline $\begin{array}{l}\text { Allocation concealment } \\
\text { (selection bias) }\end{array}$ & High risk & Coin toss on site \\
\hline $\begin{array}{l}\text { Blinding of participants } \\
\text { and personnel (perfor- } \\
\text { mance bias) } \\
\text { All outcomes }\end{array}$ & Unclear risk & $\begin{array}{l}\text { Participants and personnel not blinded to allocated group but impact of non- } \\
\text { blinding unclear }\end{array}$ \\
\hline
\end{tabular}

\begin{tabular}{|c|c|c|}
\hline $\begin{array}{l}\text { Blinding of outcome as- } \\
\text { sessment (detection bias) } \\
\text { Falls }\end{array}$ & Unclear risk & $\begin{array}{l}\text { Unclear if assessors were blinded, insufficient information to permit judge- } \\
\text { ment }\end{array}$ \\
\hline
\end{tabular}

\begin{tabular}{|c|c|c|}
\hline $\begin{array}{l}\text { Blinding of outcome as- } \\
\text { sessment (detection bias) } \\
\text { Fractures }\end{array}$ & Unclear risk & Not applicable \\
\hline
\end{tabular}

\begin{tabular}{|c|c|c|}
\hline $\begin{array}{l}\text { Blinding of outcome as- } \\
\text { sessment (detection bias) } \\
\text { Hospital admission, med- } \\
\text { ical attention and adverse } \\
\text { events }\end{array}$ & Unclear risk & Not applicable \\
\hline $\begin{array}{l}\text { Blinding of outcome as- } \\
\text { sessment (detection bias) } \\
\text { Health related quality of } \\
\text { life (self report) }\end{array}$ & Unclear risk & Not applicable \\
\hline $\begin{array}{l}\text { Incomplete outcome data } \\
\text { (attrition bias) } \\
\text { Falls and fallers }\end{array}$ & Low risk & No missing outcome data for falls \\
\hline $\begin{array}{l}\text { Selective reporting (re- } \\
\text { porting bias) }\end{array}$ & High risk & $\begin{array}{l}\text { Fall data were collected but number of falls was not reported; adverse events } \\
\text { were not reported }\end{array}$ \\
\hline $\begin{array}{l}\text { Method of ascertaining } \\
\text { falls (recall bias) }\end{array}$ & High risk & Assume retrospective recall and 3- and 6-month assessment \\
\hline
\end{tabular}


Clegg 2014

\begin{tabular}{ll}
\hline Methods & $\begin{array}{l}\text { Study design: RCT } \\
\text { Number of study arms: } 2 \\
\text { Length of follow-up: } 3 \text { months }\end{array}$ \\
\hline Participants & Setting: Bradford, United Kingdom \\
& Number of participants: 84 \\
& Number analysed: 70 \\
Number lost to follow-up: 14 & Sample: community-dwelling \\
& Age (years): mean 79 (SD 9.2) \\
& Sex: $71 \%$ female \\
Inclusion criteria: living at home in assisted-living sites, housebound, recently discharged from elderly \\
medicine outpatient clinic, had a case manager, attending a day centre or respite care \\
Exclusion criteria: unable to stand and walk independently, currently participating in exercise pro- \\
gramme, registered blind, poorly-controlled angina, another household member in the trial, severe de- \\
mentia, palliative care
\end{tabular}

Interventions

1. Individual balance and strength training: no special equipment required and manual provided, leg strengthening for basic mobility tasks, 5 face-to-face home visits, 7 telephone calls, $<15$ minutes exercise sessions, 3 a day, 5 a week, 12 weeks

2. Control group: usual care

Outcomes
$\begin{aligned} & \text { 2. Number of people who experienced } 1 \text { or more falls (risk of falling) } \\ & \text { 3. Number of people who experienced } 1 \text { or more falls requiring hospital admission } \\ & \text { 4. Health-related quality of life } \\ & \text { 5. Number of people who died }\end{aligned}$

Duration of the study Adherence

\section{2 weeks}

Adherence measured by completion of programme, diary completion

1. Individual balance and strength training group: $70 \%$ completed the 12 -week programme $(n=28)$;

$27 / 28(96 \%)$ diaries returned

mean diary completion $=64 \%$

mean recorded total adherence $=46 \%$

mean recorded partial or total intervention adherence $=67 \%$

\begin{tabular}{ll}
\hline Notes & Source of funding: Dunhill Medical Trust, Royal College of Physicians Joint Research Fellowship \\
Economic information: not reported
\end{tabular}

Email communication to obtain fall data, response received, data included in review

\section{Risk of bias}

\section{Bias}

Authors' judgement Support for judgement

Random sequence genera- Low risk tion (selection bias) 
Clegg 2014 (Continued)

\begin{tabular}{|c|c|c|}
\hline $\begin{array}{l}\text { Allocation concealment } \\
\text { (selection bias) }\end{array}$ & Low risk & Storage of randomsation sequence by independent research unit \\
\hline $\begin{array}{l}\text { Blinding of participants } \\
\text { and personnel (perfor- } \\
\text { mance bias) } \\
\text { All outcomes }\end{array}$ & Unclear risk & $\begin{array}{l}\text { Participants and personnel not blinded to allocated group, but impact of non- } \\
\text { blinding unclear }\end{array}$ \\
\hline $\begin{array}{l}\text { Blinding of outcome as- } \\
\text { sessment (detection bias) } \\
\text { Falls }\end{array}$ & Unclear risk & $\begin{array}{l}\text { Unclear whether falls were confirmed using the same method in both groups } \\
\text { and unclear who assessed falls. Assessors of performance/questionnaire out- } \\
\text { comes intended to be blinded but Quote: "were frequently unblinded". Impact } \\
\text { of unblinding unknown }\end{array}$ \\
\hline
\end{tabular}

\begin{tabular}{|c|c|c|}
\hline $\begin{array}{l}\text { Blinding of outcome as- } \\
\text { sessment (detection bias) } \\
\text { Fractures }\end{array}$ & Unclear risk & Not applicable \\
\hline
\end{tabular}

\begin{tabular}{|c|c|c|}
\hline $\begin{array}{l}\text { Blinding of outcome as- } \\
\text { sessment (detection bias) }\end{array}$ & Unclear risk & $\begin{array}{l}\text { Assessors of performance/questionnaire outcomes intended to be blinded, } \\
\text { but }\end{array}$ \\
\hline $\begin{array}{l}\text { Hospital admission, med- } \\
\text { ical attention and adverse } \\
\text { events }\end{array}$ & & Quote: "were frequently unblinded". Impact of unblinding unknown \\
\hline
\end{tabular}

\begin{tabular}{|c|c|c|}
\hline $\begin{array}{l}\text { Blinding of outcome as- } \\
\text { sessment (detection bias) } \\
\text { Health related quality of } \\
\text { life (self report) }\end{array}$ & High risk & Participants not blind to group allocation \\
\hline $\begin{array}{l}\text { Incomplete outcome data } \\
\text { (attrition bias) } \\
\text { Falls and fallers }\end{array}$ & Unclear risk & $\begin{array}{l}\text { Less than } 20 \% \text { of outcome data are missing }(17 \%) \text {. Minor unbalance in with- } \\
\text { drawals in intervention }(n=5) \text { and control }(n=9) \text { groups, with some unbalance } \\
\text { in reasons for withdrawal between the groups (intervention: } 3=\text { withdrew, } 1= \\
\text { lost to follow-up, } 1=\text { died; control: } 4=\text { withdrew, } 2=\text { lost to follow-up, } 3=\text { died) }\end{array}$ \\
\hline $\begin{array}{l}\text { Selective reporting (re- } \\
\text { porting bias) }\end{array}$ & Low risk & $\begin{array}{l}\text { Falls outcomes were prospectively specified in trial registery. Adverse events } \\
\text { reported }\end{array}$ \\
\hline $\begin{array}{l}\text { Method of ascertaining } \\
\text { falls (recall bias) }\end{array}$ & Unclear risk & Method of fall recording not stated \\
\hline
\end{tabular}

\section{Clemson 2010}

\begin{tabular}{ll}
\hline Methods & $\begin{array}{l}\text { Study design: RCT } \\
\text { Number of study arms: } 2 \\
\text { Length of follow-up: } 6 \text { months }\end{array}$ \\
\hline Participants & Setting: Sydney, Australia \\
& Number of participants: 34 \\
& Number analysed: 34 \\
& Number lost to follow-up: 0 \\
& Sample: volunteer community-dwelling men and women recruited by various strategies \\
& Age (years): mean 82 (SD 5.9 ) \\
& Sex: $47 \%$ female \\
Inclusion criteria: aged $>70$ years; $\geq 2$ falls or an injurious fall in previous year
\end{tabular}


Exclusion criteria: cognitive impairment; no conversational English; unable to walk independently; resident in nursing home or hostel; unstable or terminal illness that would preclude planned exercises; neurological conditions, e.g. Parkinson's disease

\begin{tabular}{ll}
\hline Interventions & $\begin{array}{l}\text { 1. LiFE (Lifestyle approach to reducing Falls through Exercise) programme - progressive balance and } \\
\text { strength training embedded in daily life activities: taught in } 5 \text { home visits }+2 \text { booster visits over } 3 \\
\text { months + } 2 \text { phone calls; } 6 \text {-month programme } \\
\text { 2. Control group: no intervention }\end{array}$ \\
\hline Outcomes & $\begin{array}{l}\text { 1. Rate of falls } \\
\text { 2. Number of people who experienced } 1 \text { or more falls (risk of falling) }\end{array}$ \\
\hline Duration of the study & 24 weeks \\
\hline Adherence & Not reported \\
\hline Notes & $\begin{array}{l}\text { Source of funding: University of Sydney Bridging Grant } \\
\text { Economic information: not reported }\end{array}$ \\
\hline
\end{tabular}

\section{Risk of bias}

\begin{tabular}{lll}
\hline Bias & Authors' judgement & Support for judgement \\
\hline $\begin{array}{l}\text { Random sequence genera- } \\
\text { tion (selection bias) }\end{array}$ & Low risk & Quote: "Randomisation was conducted ... using a random numbers table" \\
\hline $\begin{array}{l}\text { Allocation concealment } \\
\text { (selection bias) }\end{array}$ & Low risk & $\begin{array}{l}\text { Quote: "Randomisation was conducted by an investigator not involved in as- } \\
\text { sessment or intervention ..." "Once baseline assessments were completed by } \\
\text { the research assistant (RA), participants were then allocated in order of com- } \\
\text { pletion from the generated lists by the blinded investigator" }\end{array}$
\end{tabular}

Blinding of participants Unclear risk Participants and personnel unblinded but impact of unblinding unknown and personnel (performance bias)

All outcomes

\begin{tabular}{|c|c|c|}
\hline $\begin{array}{l}\text { Blinding of outcome as- } \\
\text { sessment (detection bias) } \\
\text { Falls }\end{array}$ & Unclear risk & $\begin{array}{l}\text { Quote: "An RA who was not involved in the intervention and masked to the } \\
\text { group allocation conducted all assessments. Falls surveillance was by daily } \\
\text { calendar, which participants mailed monthly, using pre-addressed envelopes } \\
\text { to the RA. An investigator telephoned any participant who failed to return the } \\
\text { calendar or who reported a fall." } \\
\text { Unclear whether the investigator carrying out the telephone calls was blind to } \\
\text { group allocation }\end{array}$ \\
\hline
\end{tabular}

\begin{tabular}{ll}
\hline Blinding of outcome as- & Unclear risk \\
sessment (detection bias) & \\
Fractures & \\
\hline
\end{tabular}

\begin{tabular}{lll}
\hline $\begin{array}{l}\text { Blinding of outcome as- } \\
\text { sessment (detection bias) } \\
\text { Hospital admission, med- }\end{array}$ & Unclear risk & Not applicable \\
$\begin{array}{l}\text { ical attention and adverse } \\
\text { events }\end{array}$ & \\
\hline $\begin{array}{l}\text { Blinding of outcome as- } \\
\text { sessment (detection bias) } \\
\begin{array}{l}\text { Health related quality of } \\
\text { life (self report) }\end{array}\end{array}$ & High risk & \\
\hline
\end{tabular}




\section{Clemson 2010 (Continued)}

Incomplete outcome data Low risk (attrition bias)

Falls and fallers

Selective reporting (reporting bias)

Unclear risk

Method of ascertaining Low risk

falls (recall bias)
Less than $20 \%$ of fall outcome data are missing $(9 \%)$. Balance in withdrawals in intervention $(n=1)$ and control $(n=2)$ groups, with balanced reasons for withdrawal between the groups

\section{Clemson 2012}

\begin{tabular}{ll}
\hline Methods & $\begin{array}{l}\text { Study design: RCT } \\
\text { Number of study arms: } 3 \\
\text { Length of follow-up: } 12 \text { months }\end{array}$ \\
\hline Participants & Setting: Sydney, Australia \\
& Number of participants: 317 \\
& Number analysed: 317 \\
& Number lost to follow-up: 0 \\
& Sample: community-dwelling \\
& Age (years): mean 83.4 \\
& Sex: $55 \%$ female \\
& Inclusion criteria: men and women $\geq 70$ yrs, $\geq 2$ falls or 1 injurious fall in past 12 months determined by \\
& self-report \\
& Exclusion criteria: moderate to severe cognitive problems, no conversational English, inability to walk \\
independently, neurological condition severely influencing gait and mobility, resident in a nursing \\
home or hostel, unstable or terminal medical illness precluding the planned exercises and unlikely to \\
resolve
\end{tabular}

Interventions 1. LiFE (Lifestyle approach to reducing Falls through Exercise) programme - progressive balance and strength training embedded in daily life activities: performed throughout the day, taught in 5 home visits +2 booster visits over 3 months +2 phone calls. Manual provided for increasing intensity and challenge. 6-month programme.

2. Individual balance and strength training: progressive exercises performed 3 a week, taught in 5 home visits +2 booster visits over 3 months +2 phone calls. 6 -month programme.

3. Control: Low-intensity flexibility and balance training; gentle and flexibility exercises in sitting, lying down, or standing while holding on, not progressed, 2 sessions +1 booster session +6 follow-up phone calls. 6 months

1. Rate of falls
2. Number of people who experienced 1 or more falls (risk of falling)
3. Health-related quality of life
4. Number of people who died


Clemson 2012 (Continued)

Adherence

Adherence measured by sessions performed. Mean adherence to programme over first 6 months for each group/still exercising at 6 months reported:

1. LiFE (Lifestyle approach to reducing Falls through Exercise) programme group: 47\% (SD 33)/81 (76\%)

2. Individual balance and strength training group: 35\% (SD 29)/63 (60\%)

3. Control group: $47 \%$ (SD 34$) / 74(71 \%)$

Notes

Source of funding: Australian National Health and Medical Research Council

Economic information: not reported

\section{Risk of bias}

\begin{tabular}{|c|c|c|}
\hline Bias & Authors' judgement & Support for judgement \\
\hline $\begin{array}{l}\text { Random sequence genera- } \\
\text { tion (selection bias) }\end{array}$ & Low risk & Computer-generated randomisation \\
\hline $\begin{array}{l}\text { Allocation concealment } \\
\text { (selection bias) }\end{array}$ & Low risk & $\begin{array}{l}\text { Quote: "Randomisation was ... concealed by using an automated secure web- } \\
\text { site that was operated by an off-site independent service" }\end{array}$ \\
\hline $\begin{array}{l}\text { Blinding of participants } \\
\text { and personnel (perfor- } \\
\text { mance bias) } \\
\text { All outcomes }\end{array}$ & Unclear risk & Participants and personnel unblinded but impact of unblinding unknown \\
\hline $\begin{array}{l}\text { Blinding of outcome as- } \\
\text { sessment (detection bias) } \\
\text { Falls }\end{array}$ & Low risk & $\begin{array}{l}\text { Fall data collected using same method in each group. Fall event surveillance } \\
\text { was conducted by a research assistant blinded to group allocation }\end{array}$ \\
\hline $\begin{array}{l}\text { Blinding of outcome as- } \\
\text { sessment (detection bias) } \\
\text { Fractures }\end{array}$ & Unclear risk & Not applicable \\
\hline $\begin{array}{l}\text { Blinding of outcome as- } \\
\text { sessment (detection bias) } \\
\text { Hospital admission, med- } \\
\text { ical attention and adverse } \\
\text { events }\end{array}$ & Unclear risk & Not applicable \\
\hline $\begin{array}{l}\text { Blinding of outcome as- } \\
\text { sessment (detection bias) } \\
\text { Health related quality of } \\
\text { life (self report) }\end{array}$ & High risk & Participants unblinded to group allocation \\
\hline $\begin{array}{l}\text { Incomplete outcome data } \\
\text { (attrition bias) } \\
\text { Falls and fallers }\end{array}$ & Unclear risk & $\begin{array}{l}\text { Less than } 20 \% \text { of fall outcome data are missing }(10 \%) \text {. Minor imbalance in } \\
\text { withdrawals in LiFE }(n=8) \text {, structured programme }(n=9) \text { and control }(n=14) \\
\text { groups, with reasons for loss of fall data unclear }\end{array}$ \\
\hline $\begin{array}{l}\text { Selective reporting (re- } \\
\text { porting bias) }\end{array}$ & Low risk & $\begin{array}{l}\text { Falls outcomes were prospectively specified in trial registry. Adverse events re- } \\
\text { ported }\end{array}$ \\
\hline $\begin{array}{l}\text { Method of ascertaining } \\
\text { falls (recall bias) }\end{array}$ & Low risk & $\begin{array}{l}\text { Daily calendar mailed monthly, follow-up phone call for missing calendars or } \\
\text { fall reported by blinded researcher }\end{array}$ \\
\hline
\end{tabular}


Cornillon 2002

\begin{tabular}{|c|c|}
\hline Methods & $\begin{array}{l}\text { Study design: RCT } \\
\text { Number of study arms: } 2 \\
\text { Length of follow-up: } 12 \text { months }\end{array}$ \\
\hline \multirow[t]{9}{*}{ Participants } & Setting: St Étienne, France \\
\hline & Number of participants: 303 \\
\hline & Number analysed: 303 \\
\hline & Number lost to follow-up: 0 \\
\hline & Sample: community-dwelling and independent in ADL \\
\hline & Age (years): mean 71 \\
\hline & Sex: $83 \%$ female \\
\hline & Inclusion criteria: aged > 65; living at home; ADL-independent; consented \\
\hline & Exclusion criteria: cognitively impaired (MMSE $<20$ ); obvious disorder of walking or balance \\
\hline Interventions & $\begin{array}{l}\text { 1. Group-based balance and gait training, information on fall risk, and balance and sensory training, } 1 \text { a } \\
\text { week, } 8 \text { weeks }\end{array}$ \\
\hline \multirow[t]{3}{*}{ Outcomes } & 1. Rate of falls \\
\hline & 2. Number of people who experienced 1 or more falls (risk of falling) \\
\hline & 3. Number of people who died \\
\hline Duration of the study & 52 weeks \\
\hline Adherence & $\begin{array}{l}102 \text { people (68\%) participated in at least } 6 \text { sessions, } 14(9 \%) \text { participated in } 1-5 \text { sessions and } 34(23 \%) \\
\text { did not participate in any sessions (due to refusal, health, or dissatisfaction with the proposed pro- } \\
\text { gramme) }\end{array}$ \\
\hline Notes & $\begin{array}{l}\text { Source of funding: not reported } \\
\text { Economic information: not reported }\end{array}$ \\
\hline
\end{tabular}

\section{Risk of bias}

\begin{tabular}{|c|c|c|}
\hline Bias & Authors' judgement & Support for judgement \\
\hline $\begin{array}{l}\text { Random sequence genera- } \\
\text { tion (selection bias) }\end{array}$ & Low risk & Randomised by random-number tables \\
\hline $\begin{array}{l}\text { Allocation concealment } \\
\text { (selection bias) }\end{array}$ & Unclear risk & Insufficient information to permit judgement \\
\hline $\begin{array}{l}\text { Blinding of participants } \\
\text { and personnel (perfor- } \\
\text { mance bias) } \\
\text { All outcomes }\end{array}$ & Unclear risk & $\begin{array}{l}\text { Participants and personnel not blind to allocated group but impact of non- } \\
\text { blinding unclear }\end{array}$ \\
\hline $\begin{array}{l}\text { Blinding of outcome as- } \\
\text { sessment (detection bias) } \\
\text { Falls }\end{array}$ & Unclear risk & $\begin{array}{l}\text { Falls recorded on } 6 \text {-monthly falls calendars in both groups. No telephone con- } \\
\text { tact described. Blinding of study personnel recording data from the calendars } \\
\text { not described }\end{array}$ \\
\hline $\begin{array}{l}\text { Blinding of outcome as- } \\
\text { sessment (detection bias) } \\
\text { Fractures }\end{array}$ & Unclear risk & Not applicable \\
\hline $\begin{array}{l}\text { Blinding of outcome as- } \\
\text { sessment (detection bias) }\end{array}$ & Unclear risk & $\begin{array}{l}\text { Method of ascertaining adverse events unclear. Blinding of study personnel } \\
\text { not described }\end{array}$ \\
\hline
\end{tabular}


Cornillon 2002 (Continued) Hospital admission, medical attention and adverse events

\begin{tabular}{|c|c|c|}
\hline $\begin{array}{l}\text { Blinding of outcome as- } \\
\text { sessment (detection bias) } \\
\text { Health related quality of } \\
\text { life (self report) }\end{array}$ & Unclear risk & Not applicable \\
\hline $\begin{array}{l}\text { Incomplete outcome data } \\
\text { (attrition bias) } \\
\text { Falls and fallers }\end{array}$ & Low risk & No missing fall data \\
\hline $\begin{array}{l}\text { Selective reporting (re- } \\
\text { porting bias) }\end{array}$ & Unclear risk & $\begin{array}{l}\text { Prespecified falls outcomes reported, adverse events reported. No trial proto- } \\
\text { col or prospective trial registration }\end{array}$ \\
\hline $\begin{array}{l}\text { Method of ascertaining } \\
\text { falls (recall bias) }\end{array}$ & Low risk & Prospective. Falls recorded on monthly falls calendars \\
\hline
\end{tabular}

Methods Study design: Cluster-RCT

Number of study arms: 2

Number of clusters: 25

Length of follow-up: 6 months

\section{Participants}

Setting: Shahroud, Iran

Number of participants: 551

Number analysed: 317

Number lost to follow-up: 234

Sample: community-dwelling

Age (years): mean 70.6 (SD 5.1)

Sex: $49 \%$ female

Inclusion criteria: $\geq 60$ years, able to walk $\geq 10 \mathrm{~m}$, permanent residency in an urban area in past 12 months, previous falls, had a female family member (to maintain homogeneity) as a caregiver (aged 18 - 50) with health literacy (able to read instructional booklet and explaining the content to the researchers)

Exclusion criteria: acute or chronic disease restricting exercise, unable to walk independently for $10 \mathrm{~m}$, hip replacement surgery or lower extremity fracture/s in past 12 months, orthopaedic surgeon recommending not to participate due to severe articular involvement limiting physical activity or any other reason, elderly people with high level of activity in past 12 months

Interventions

1. Individual Otago Exercise Programme: home programme with monthly visits in the presence of family caregiver/s, 45-minute sessions, $3 \mathrm{x}$ ar week, 6 months

2. Control group: given a booklet on general health for elderly people published by the 'Iranian Ministry of Health, Treatment and Medical Education'
Outcomes
1. Rate of falls
2. Number of people who experienced 1 or more falls (risk of falling) 
Dadgari 2016 (Continued)

\begin{tabular}{ll} 
Duration of the study & 24 weeks \\
\hline Adherence & Not reported \\
\hline Notes & $\begin{array}{l}\text { Source of funding: Shahroud University of Medical Sciences } \\
\text { Economic information: not reported } \\
\text { Email communication to obtain fall data, response received, data included in review }\end{array}$ \\
\hline
\end{tabular}

\section{Risk of bias}

\begin{tabular}{lll}
\hline Bias & Authors' judgement & Support for judgement \\
\hline $\begin{array}{l}\text { Random sequence genera- } \\
\text { tion (selection bias) }\end{array}$ & Unclear risk & Only mentions block randomisation \\
\hline $\begin{array}{l}\text { Allocation concealment } \\
\text { (selection bias) }\end{array}$ & Unclear risk & $\begin{array}{l}\text { Cluster-RCT. Individual participant recruitment was undertaken after group } \\
\text { allocation. The method of concealment is not described and it is unclear } \\
\text { whether recruitment was undertaken by a person who was unblinded and may } \\
\text { have had knowledge of participant characteristics }\end{array}$ \\
\hline
\end{tabular}

\begin{tabular}{|c|c|c|}
\hline $\begin{array}{l}\text { Blinding of participants } \\
\text { and personnel (perfor- }\end{array}$ & Unclear risk & $\begin{array}{l}\text { Participants and personnel not blinded to allocated group, but impact of non- } \\
\text { blinding unclear }\end{array}$ \\
\hline
\end{tabular}

mance bias)

All outcomes

\begin{tabular}{|c|c|c|}
\hline $\begin{array}{l}\text { Blinding of outcome as- } \\
\text { sessment (detection bias) } \\
\text { Falls }\end{array}$ & Unclear risk & $\begin{array}{l}\text { Method of ascertaining falls was not clear in either group. Blinding of assessors } \\
\text { not described }\end{array}$ \\
\hline $\begin{array}{l}\text { Blinding of outcome as- } \\
\text { sessment (detection bias) } \\
\text { Fractures }\end{array}$ & Unclear risk & Not applicable \\
\hline $\begin{array}{l}\text { Blinding of outcome as- } \\
\text { sessment (detection bias) } \\
\text { Hospital admission, med- } \\
\text { ical attention and adverse } \\
\text { events }\end{array}$ & Unclear risk & Not applicable \\
\hline
\end{tabular}

\begin{tabular}{|c|c|c|}
\hline $\begin{array}{l}\text { Blinding of outcome as- } \\
\text { sessment (detection bias) } \\
\text { Health related quality of } \\
\text { life (self report) }\end{array}$ & Unclear risk & Not applicable \\
\hline $\begin{array}{l}\text { Incomplete outcome data } \\
\text { (attrition bias) } \\
\text { Falls and fallers }\end{array}$ & High risk & $\begin{array}{l}\text { More than } 20 \% \text { of fall outcome data are missing }(42 \%) \text {. Balanced withdrawals } \\
\text { in intervention }(n=119) \text { and control }(n=115) \text { groups; reasons for loss of fall } \\
\text { data unclear }\end{array}$ \\
\hline $\begin{array}{l}\text { Selective reporting (re- } \\
\text { porting bias) }\end{array}$ & Unclear risk & $\begin{array}{l}\text { Minimum set of expected outcomes not reported (adverse events not report- } \\
\text { ed) }\end{array}$ \\
\hline $\begin{array}{l}\text { Method of ascertaining } \\
\text { falls (recall bias) }\end{array}$ & High risk & $\begin{array}{l}\text { Falls outcome: Quote: "was examined before and after the exercise training } \\
\text { program" ( } 6 \text { months). Method of ascertaining falls at } 6 \text { months was not clear }\end{array}$ \\
\hline Cluster-randomised trials & High risk & $\begin{array}{l}\text { Individuals were recruited to the trial after the clusters were randomised and } \\
\text { personnel recruiting participants were not blinded to cluster; baseline com- } \\
\text { parability of clusters was not reported; missing outcomes for clusters or with- }\end{array}$ \\
\hline
\end{tabular}




$\begin{array}{ll}\text { Methods } & \text { RCT (cluster-randomised by health centre, } 2 \text { × } 2 \text { factorial design) } \\ \text { Study design: Cluster-RCT } \\ \text { Number of study arms: } 2 \\ \text { Number of clusters: } 28 \text { (20 clusters only for fallers and fractures) } \\ \text { Length of follow-up: } 24 \text { months } \\ \text { Setting: Santiago, Chile } \\ \text { Number of participants: } 984 \\ \text { Number analysed: } 619 \\ \text { Number lost to follow-up: } 365 \\ \text { Sample: randomly sampled households in health centre catchment areas and health centre registries } \\ \text { Age (years): range } 65 \text { - } 68 \\ \text { Sex: } 68 \% \text { female } \\ \text { Inclusion criteria (clusters): health centres with > } 400 \text { residents aged } 65 \text { - } 67.9 \text { years in low-middle eco- } \\ \text { nomic status municipalities } \\ \text { Exclusion criteria (individuals): unable to walk unaided; seeking medical advice for unplanned } 3 \mathrm{~kg} \\ \text { weight loss over } 3 \text { months; planning to move house within } 3 \text { month; already enrolled in national } \\ \text { Programme of Complementary Feeding for the Older Population (PACAM) or consuming PACAM pro- } \\ \text { gramme supplements; scoring } \geq 6 \text { on Pfeffer screen (poor cognitive function) }\end{array}$

Interventions

Randomised into 3 groups: 2 intervention groups (1 group-based balance and strength, and 1 nutritional supplements group) and 1 control group. Only group-based balance and strength and control group included in this review

1. Group-based balance and strength: supervised sessions for functional weight-bearing exercises; 1 hour, 2 a week, 24 months

2. Control group: no intervention

Outcomes Number of people who experienced 1 or more falls (risk of falling)
$\begin{aligned} & \text { 2. Number of people who experienced } 1 \text { or more fall-related fractures } \\ & \text { 3. Health-related quality of life } \\ & \text { 4. Number of people who died }\end{aligned}$

Duration of the study 108 weeks

Adherence Adherence measured as attendance at sessions offered

1. Group-based balance and strength group: $38 \%$

Notes

Source of funding: London School of Hygiene and Tropical Medicine, London, UK

Economic information: Mean cost per person (intervention) USD 164 for physical activity intervention. Incremental cost per fall prevented/per QALY gained: cost effectiveness of physical activity intervention reported as USD 4.84 per extra metre walked

Cost analysis reported in primary reference

Number of clusters allocated to intervention: 5; number of clusters allocated to control: 5; number of clusters analysed (intervention): 5 ; number of clusters analysed (control): 5 


\section{Risk of bias}

\begin{tabular}{lll}
\hline Bias & Authors' judgement & Support for judgement \\
\hline $\begin{array}{ll}\text { Random sequence genera- } \\
\text { tion (selection bias) }\end{array}$ & Low risk & $\begin{array}{l}\text { Drawing of lots } \\
\text { Quote: "The center names (clusters) were put into a hat. The four treatment } \\
\text { arms (nutritional supplementation, nutritional supplementation+physical ac- } \\
\text { tivity, physical activity, control) were randomly numbered 1-4. As each name } \\
\text { was drawn out of the hat by a member of the study team, it was assigned to } \\
\text { the next treatment number until each arm contained five clusters" }\end{array}$ \\
\end{tabular}

\begin{tabular}{ll}
\hline $\begin{array}{l}\text { Allocation concealment } \\
\text { (selection bias) }\end{array}$ & High risk \\
& $\begin{array}{l}\text { Cluster RCT. Individual participant recruitment was undertaken after group } \\
\text { allocation. The method of concealment is not described and it is unclear } \\
\text { whether recruitment was undertaken by a person who was unblinded and may } \\
\text { have had knowledge of participant characteristics }\end{array}$
\end{tabular}

\begin{tabular}{|c|c|c|}
\hline $\begin{array}{l}\text { Blinding of participants } \\
\text { and personnel (perfor- }\end{array}$ & Unclear risk & $\begin{array}{l}\text { Participants and personnel not blinded to allocated group but impact of non- } \\
\text { blinding unclear }\end{array}$ \\
\hline
\end{tabular}

mance bias)

All outcomes

\begin{tabular}{|c|c|c|}
\hline $\begin{array}{l}\text { Blinding of outcome as- } \\
\text { sessment (detection bias) } \\
\text { Falls }\end{array}$ & High risk & $\begin{array}{l}\text { Falls assessed via participant recall in both groups. Although assessors of the } \\
\text { primary outcomes (pneumonia, physical function) were blind to group alloca- } \\
\text { tion, this was not mentioned, therefore assumed not to apply, for secondary } \\
\text { outcomes (included fallers) }\end{array}$ \\
\hline
\end{tabular}

\begin{tabular}{lll}
\hline $\begin{array}{l}\text { Blinding of outcome as- } \\
\text { sessment (detection bias) }\end{array}$ & High risk & $\begin{array}{l}\text { Fractures were self-reported, not confirmed by the results of radiological ex- } \\
\text { amination or from primary care case record }\end{array}$ \\
\hline $\begin{array}{l}\text { Fractures }\end{array}$ &
\end{tabular}

\begin{tabular}{l} 
Fractures \\
\hline Blinding of outcome as- \\
sessment (detection bias) \\
Hospital admission, med- \\
ical attention and adverse \\
events
\end{tabular}

\begin{tabular}{|c|c|c|}
\hline $\begin{array}{l}\text { Blinding of outcome as- } \\
\text { sessment (detection bias) } \\
\text { Health related quality of } \\
\text { life (self report) }\end{array}$ & High risk & Participants were not blinded to allocated group \\
\hline
\end{tabular}

\begin{tabular}{lll}
\hline $\begin{array}{l}\text { Incomplete outcome data } \\
\text { (attrition bias) } \\
\text { Falls and fallers }\end{array}$ & High risk & $\begin{array}{l}\text { More than 20\% of fall outcome data are missing (37\%). Unbalanced with- } \\
\text { drawals in intervention }(\mathrm{n}=155) \text { and control ( } \mathrm{n}=209) \text { groups; reasons for loss } \\
\text { of fall data unclear }\end{array}$ \\
\hline $\begin{array}{l}\text { Selective reporting (re- } \\
\text { porting bias) }\end{array}$ & High risk & $\begin{array}{l}\text { Fall data were collected but number of falls was not reported; adverse events } \\
\text { were not reported }\end{array}$ \\
\hline $\begin{array}{l}\text { Method of ascertaining } \\
\text { falls (recall bias) }\end{array}$ & High risk & $\begin{array}{l}\text { Participant recall for falls was at } 12 \text { and } 24 \text { months. For secondary outcomes } \\
\text { including } \\
\text { Quote: "self-reported incidence of falls" ... "Participants in the original 20 clus- } \\
\text { ters were re-interviewed after } 12 \text { and 24 mo for outcome data" }\end{array}$ \\
\hline Cluster-randomised trials & Unclear risk & $\begin{array}{l}\text { Individuals were recruited to the trial after the clusters were randomised and } \\
\text { personnel recruiting participants were not blind to cluster; baseline character- } \\
\text { istics of clusters and participants were similar between trial arms; missing out- } \\
\text { comes for clusters or within clusters were not reported; accounted for the clus- }\end{array}$ \\
\hline
\end{tabular}




\begin{tabular}{ll}
\hline Methods & $\begin{array}{l}\text { Study design: RCT } \\
\text { Number of study arms: } 3 \\
\text { Length of follow-up: } 9 \text { months }\end{array}$ \\
\hline Participants & Setting: Vancouver, Canada \\
& Number of participants: 155 \\
& Number analysed: 155 \\
& Number lost to follow-up: 0 \\
& Sample: community-dwelling women \\
& Age (years): mean 70 (range 65 - 75) \\
& Sex: $100 \%$ female \\
& Inclusion criteria: aged 65 - 75; cognitively intact; visual acuity 20/40 or better \\
& Exclusion criteria: resistance training in the last 6 months; medical condition for which exercise is con- \\
traindicated; neurogenerative disease; taking cholinesterase inhibitors; depression; on hormone re- \\
placement therapy during previous 12 months
\end{tabular}

I. Group-based progressive high-intensity resistance training classes: gym equipment and free weights
used with a "progressive, high intensity protocol", 1 a week, 1 year
2. Group-based progressive high-intensity resistance training classes: gym equipment and free weights
used with a "progressive, high intensity protocol", 2 a week, 1 year
3. Group-based balance and tone: stretching, range of motion, pelvic floor, balance, relaxation exercis-
es using body weight alone, 2 a week, 1 year

\begin{tabular}{ll}
\hline Outcomes & 1. Rate of falls \\
\hline Duration of the study & 52 weeks \\
\hline Adherence & Not reported \\
\hline Notes & $\begin{array}{l}\text { Source of funding: The Vancouver Foundation, Natural Sciences and Engineering Research Council of } \\
\text { Canada, Michael Smith Foundation for Health Research, the Canada Foundation for Innovation }\end{array}$ \\
& $\begin{array}{l}\text { Economic information: Mean cost per person (intervention): CAD 353 once-weekly resistance train- } \\
\text { ing, CAD 706 twice-weekly resistance training, CAD 706 twice-weekly balance and tone classes. Mean } \\
\text { healthcare costs resulting from falls, mean total healthcare costs respectively: CAD 547, CAD 1379 once- } \\
\text { weekly resistance training; CAD 184, CAD 1684 twice-weekly resistance training; CAD 162, CAD 1772 } \\
\text { twice-weekly balance and tone classes. Incremental cost per fall prevented/per QALY gained: both } \\
\text { once- and twice-weekly resistance training less costly and more effective than balance and tone class- } \\
\text { es } \\
\text { Cost-effectiveness analysis and cost utility analysis reported in primary reference } \\
\text { Email communication about fall data, response received, data not included in review }\end{array}$ \\
\hline
\end{tabular}

\section{Risk of bias}

\begin{tabular}{lll} 
Bias & Authors' judgement & Support for judgement \\
\hline $\begin{array}{l}\text { Random sequence genera- } \\
\text { tion (selection bias) }\end{array}$ & Low risk & $\begin{array}{l}\text { Quote: "The randomization sequence was generated by www.randomiza- } \\
\text { tion.com." }\end{array}$ \\
\hline
\end{tabular}


Davis 2011 (Continued)

$\begin{array}{ll}\begin{array}{l}\text { Allocation concealment } \quad \text { Low risk } \\ \text { (selection bias) }\end{array} & \begin{array}{l}\text { Quote: "The randomization sequence ... was concealed until interventions } \\ \text { were assigned. This sequence was held independently and remotely by the re- } \\ \text { search coordinator" }\end{array}\end{array}$

\begin{tabular}{|c|c|c|}
\hline $\begin{array}{l}\text { Blinding of participants } \\
\text { and personnel (perfor- }\end{array}$ & Low risk & $\begin{array}{l}\text { Not possible to blind participants or personnel but both groups received an ex- } \\
\text { ercise intervention so unlikely to introduce bias }\end{array}$ \\
\hline
\end{tabular}
mance bias)

All outcomes

\begin{tabular}{|c|c|c|}
\hline $\begin{array}{l}\text { Blinding of outcome as- } \\
\text { sessment (detection bias) } \\
\text { Falls }\end{array}$ & Low risk & $\begin{array}{l}\text { Fall calendars used to assess falls in all groups. } \\
\text { Quote: "The assessors were blinded to the participants' assignments" }\end{array}$ \\
\hline $\begin{array}{l}\text { Blinding of outcome as- } \\
\text { sessment (detection bias) } \\
\text { Fractures }\end{array}$ & Unclear risk & Not applicable \\
\hline $\begin{array}{l}\text { Blinding of outcome as- } \\
\text { sessment (detection bias) } \\
\text { Hospital admission, med- } \\
\text { ical attention and adverse } \\
\text { events }\end{array}$ & Unclear risk & Method of ascertaining adverse events unclear \\
\hline $\begin{array}{l}\text { Blinding of outcome as- } \\
\text { sessment (detection bias) } \\
\text { Health related quality of } \\
\text { life (self report) }\end{array}$ & Unclear risk & Not applicable \\
\hline $\begin{array}{l}\text { Incomplete outcome data } \\
\text { (attrition bias) } \\
\text { Falls and fallers }\end{array}$ & Low risk & No missing fall data \\
\hline $\begin{array}{l}\text { Selective reporting (re- } \\
\text { porting bias) }\end{array}$ & High risk & Fall data were collected but number of fallers was not reported \\
\hline $\begin{array}{l}\text { Method of ascertaining } \\
\text { falls (recall bias) }\end{array}$ & Low risk & $\begin{array}{l}\text { Quote: "We used monthly fall diary calendars to track all falls for each partici- } \\
\text { pant during the } 12 \text {-month study period." }\end{array}$ \\
\hline
\end{tabular}

Day 2002

\begin{tabular}{ll}
\hline Methods & $\begin{array}{l}\text { Study design: RCT } \\
\text { Number of study arms: } 2 \\
\text { Length of follow-up: } 18 \text { months }\end{array}$ \\
\hline Participants & Setting: Melbourne, Australia \\
& Number of participants: 272 \\
& Number analysed: 272 \\
& Number lost to follow-up: 0 \\
& Sample: community-dwelling men and women identified from electoral roll \\
& Age (years): mean 76.1 (SD 5.0) \\
& Sex: $60 \%$ female \\
Inclusion criteria: aged $\geq 70 ;$ community-dwelling and able to make modifications; expected to remain \\
in area for 2 years (except for short absences); have approval of family physician \\
Exclusion criteria: undertaken regular to moderate exercise with a balance component in previous 2 \\
months; unable to walk 10 to 20 m without rest or help or having angina; severe respiratory or cardiac
\end{tabular}


Day 2002 (Continued)

disease; psychiatric illness prohibiting participation; dysphasia; recent major home modifications; education and language adjusted score $>4$ on the short portable mental status questionnaire

Interventions

Randomised into 8 groups: only 1 intervention group (group-based balance and strength) and 1 control group included in this review

1. Group-based balance and strength, plus daily home exercises tailored by physiotherapist: 1-hour

class a week, 15 weeks

2. Control group: no intervention. Received brochure on eye care for over-40-year olds

1. Rate of falls
2. Number of people who experienced 1 or more falls (risk of falling)
3. Number of people who experienced 1 or more falls requiring medical attention

\begin{tabular}{ll}
\hline Duration of the study & 18 months \\
\hline Adherence & Adherence measured by class attendance, frequency of home programme \\
& $\begin{array}{l}\text { 1. Group-based balance and strength group: } 401 / 541 \text { participants started a class; mean number of ses- } \\
\text { sions attended, } 10 \text { (SD 3.8); } 328 / 401 \text { attended }>50 \% \text { of their sessions; mean number of additional home } \\
\text { exercise sessions, } 9 \text { a month }\end{array}$
\end{tabular}

Notes

Source of funding: Australian National Health and Medical Research Council, Victorian Department of Human Services (Aged Care), City of Whitehorse, Victorian Health Promotioin Foundation, Rotary, National Safety Council

Economic information: Mean cost per person (intervention) AUD 52, AUD 33 for exercise group, AUD 39 for control group. Incremental cost per fall prevented/per QALY gained: ICER per fall prevented AUD 652, injurious fall prevented AUD 1176, fracture prevented AUD 26,236, QALY AUD 51,483

\section{Risk of bias}

\begin{tabular}{|c|c|c|}
\hline Bias & Authors' judgement & Support for judgement \\
\hline $\begin{array}{l}\text { Random sequence genera- } \\
\text { tion (selection bias) }\end{array}$ & Low risk & $\begin{array}{l}\text { Randomised by "adaptive biased coin" technique, to ensure balanced group } \\
\text { numbers }\end{array}$ \\
\hline $\begin{array}{l}\text { Allocation concealment } \\
\text { (selection bias) }\end{array}$ & Low risk & Computer-generated by an independent third party contacted by telephone \\
\hline $\begin{array}{l}\text { Blinding of participants } \\
\text { and personnel (perfor- } \\
\text { mance bias) } \\
\text { All outcomes }\end{array}$ & Unclear risk & $\begin{array}{l}\text { Participants and personnel not blinded to allocated group but impact of non- } \\
\text { blinding unclear }\end{array}$ \\
\hline $\begin{array}{l}\text { Blinding of outcome as- } \\
\text { sessment (detection bias) } \\
\text { Falls }\end{array}$ & Low risk & $\begin{array}{l}\text { All participants used monthly falls diary, with telephone contact from a re- } \\
\text { searcher blinded to group allocation if not returned in } 5 \text { days }\end{array}$ \\
\hline $\begin{array}{l}\text { Blinding of outcome as- } \\
\text { sessment (detection bias) } \\
\text { Fractures }\end{array}$ & Unclear risk & Not applicable \\
\hline $\begin{array}{l}\text { Blinding of outcome as- } \\
\text { sessment (detection bias) } \\
\text { Hospital admission, med- } \\
\text { ical attention and adverse } \\
\text { events }\end{array}$ & Unclear risk & Not applicable \\
\hline
\end{tabular}


Day 2002 (Continued)
Blinding of outcome as-
Unclear risk
Not applicable sessment (detection bias)
Health related quality of
life (self report)

\begin{tabular}{lll}
\hline $\begin{array}{l}\text { Incomplete outcome data } \\
\text { (attrition bias) } \\
\text { Falls and fallers }\end{array}$ & Low risk & No missing fall data \\
\hline $\begin{array}{l}\text { Selective reporting (re- } \\
\text { porting bias) }\end{array}$ & Unclear risk & $\begin{array}{l}\text { Minimum set of expected outcomes not reported (adverse events not report- } \\
\text { ed) }\end{array}$ \\
\hline $\begin{array}{l}\text { Method of ascertaining } \\
\text { falls (recall bias) }\end{array}$ & Low risk & $\begin{array}{l}\text { Falls reported using monthly postcard to record daily falls. Telephone fol- } \\
\text { low-up if calendar not returned within 5 working days of the end of each } \\
\text { month, or reporting a fall }\end{array}$ \\
\hline
\end{tabular}

\section{Day 2015}

\begin{tabular}{ll}
\hline Methods & Study design: RCT \\
& Number of study arms: 2 \\
& Length of follow-up: 12 months
\end{tabular}

Setting: Melbourne, Australia
Number of participants: 503
Number analysed: 409
Number lost to follow-up: 94
Sample: community-dwelling men and women
Age (years): mean 70
Sex: $70 \%$ female
Inclusion criteria: $\geq 70$ years and older, community residents, and preclinically disabled as defined by
Fried 2001.
Exclusion criteria: already participating in Tai Chi or a vigorous exercise programme (other physical ac-
tivity was allowed), adjusted score $>4$ on the Short Portable Mental Status Questionnaire, major unsta-
ble cardiopulmonary disease, life-threatening illness, major psychiatric illness unless stable on treat-
ment, or did not have approval to participate from their local doctor

Interventions

1. Group based Tai Chi (Modified Sun style Tai-Chi): 1-hour session, 2 a week, up to 48 weeks. Participants paid AUD 3 a class

2. Control: Group-based flexibility training conducted primarily in the seated position with some leg exercises performed in standing, holding on to the back of a chair, 1-hour session, 2 a week, up to 48 weeks. Participants paid AUD 3 a class
Outcomes
1. Rate of falls
2. Number of people who experienced 1e or more falls (risk of falling)
3. Number of people who experienced 1 or more falls requiring hospital admission
4. Number of people who died

\begin{tabular}{ll}
\hline Duration of the study & 48 weeks \\
\hline Adherence & Adherence measured by class attendance
\end{tabular}


1. Group-based Tai Chi group: mean number of classes attended during the first 24-week period, 25.8 (SD 15.9), median 30; mean number of classes attended during the full 48 weeks, 34.4 (SD 26.9), median 33.5

2. Group-based flexibility training group: mean number of classes attended during the first 24-week period, 27.4 (SD 13.4), median 30; mean number of classes attended during the full 48 weeks, 41.3 (SD 26.1), median 39.0

Notes

Source of funding: Australian National Health and Medical Research Council

Economic information: not reported

\section{Risk of bias}

\begin{tabular}{lll}
\hline Bias & Authors' judgement & Support for judgement \\
\hline $\begin{array}{l}\text { Random sequence genera- } \\
\text { tion (selection bias) }\end{array}$ & Low risk & $\begin{array}{l}\text { Quote: "participants were randomized by the study statistician (D.J.) by using } \\
\text { a computerized random number generator and a minimization algorithm" }\end{array}$ \\
\hline $\begin{array}{l}\text { Allocation concealment } \\
\text { (selection bias) }\end{array}$ & Low risk & $\begin{array}{l}\text { Quote: "The allocation list was e-mailed directly to the exercise program ad- } \\
\text { ministrator who managed exercise class delivery, independent of the research } \\
\text { staff involved in the data collection" }\end{array}$ \\
\hline
\end{tabular}

\begin{tabular}{|c|c|c|}
\hline $\begin{array}{l}\text { Blinding of participants } \\
\text { and personnel (perfor- } \\
\text { mance bias) } \\
\text { All outcomes }\end{array}$ & Unclear risk & $\begin{array}{l}\text { Participants and personnel not blinded to group allocation } \\
\text { Quote: "Although class leaders and participants were not blinded to group as- } \\
\text { signment, they were told that we were comparing the } 2 \text { exercise programs" }\end{array}$ \\
\hline
\end{tabular}

\begin{tabular}{|c|c|c|}
\hline $\begin{array}{l}\text { Blinding of outcome as- } \\
\text { sessment (detection bias) } \\
\text { Falls }\end{array}$ & Low risk & $\begin{array}{l}\text { Participants reported falls for up to } 48 \text { weeks using a monthly post-card calen- } \\
\text { dar system, supplemented with telephone follow up for missing calendars } \\
\text { Quote: "The interviewer was blind to group assignment" }\end{array}$ \\
\hline
\end{tabular}

\begin{tabular}{ll}
\hline Blinding of outcome as- & Unclear risk $\quad$ Not applicable \\
sessment (detection bias) & \\
Fractures &
\end{tabular}

\begin{tabular}{|c|c|c|}
\hline $\begin{array}{l}\text { Blinding of outcome as- } \\
\text { sessment (detection bias) } \\
\text { Hospital admission, med- } \\
\text { ical attention and adverse } \\
\text { events }\end{array}$ & High risk & A blinded interviewer ascertained injury from participant self-report \\
\hline $\begin{array}{l}\text { Blinding of outcome as- } \\
\text { sessment (detection bias) } \\
\text { Health related quality of } \\
\text { life (self report) }\end{array}$ & Unclear risk & Not applicable \\
\hline $\begin{array}{l}\text { Incomplete outcome data } \\
\text { (attrition bias) } \\
\text { Falls and fallers }\end{array}$ & Low risk & $\begin{array}{l}\text { Less than } 20 \% \text { of fall outcome data are missing }(18 \%) \text {. Loss of fall data was bal- } \\
\text { anced in intervention }(n=46) \text { and control }(n=48) \text { groups; reason for loss of fall } \\
\text { data was 'refused calendars' in all in both groups }\end{array}$ \\
\hline $\begin{array}{l}\text { Selective reporting (re- } \\
\text { porting bias) }\end{array}$ & Low risk & Prespecified falls outcomes reported. Prospective trial registration \\
\hline $\begin{array}{l}\text { Method of ascertaining } \\
\text { falls (recall bias) }\end{array}$ & Low risk & $\begin{array}{l}\text { Quote: "Participants reported falls for up to } 48 \text { weeks using a monthly post- } \\
\text { card calendar system, supplemented with telephone follow up for missing cal- } \\
\text { endars. Reported falls were followed up with a telephone interview to record } \\
\text { the circumstances of the fall and any resulting injuries and subsequent treat- } \\
\text { ment. Interviews were completed for } 96.3 \% \text { of reported falls." }\end{array}$ \\
\hline
\end{tabular}


Duque 2013

$\begin{array}{ll}\text { Methods } & \text { Study design: RCT } \\ & \text { Number of study arms: } 2 \\ & \text { Length of follow-up: } 9 \text { months }\end{array}$

\section{Participants}

Setting: Penrith, Australia

Number of participants: 60

Number analysed: 60

Number lost to follow-up: 0

Sample: community-dwelling people attending the Falls and Fractures Clinic, Nepean Hospital

Age (years): Intervention mean $=79.33($ SD 10), control mean $=75($ SD 8)

Sex: $62 \%$ female

Inclusion criteria: fallen within 6 months of assessment, poor performance in balance assessed using posturography component of the Balance Rehabilitaion Unit (BRU) virtual reality system

Exclusion criteria: severe visual impairment, inability to walk independently with a cane or walker, inability to stand unaided for 60 secs, score of $<22 / 30$ in MMSE, PD or any neuromuscular conditions, Geriatric Depression Scale (GDS) $>8 / 15$, inability to understand or answer the study questionnaires

\begin{tabular}{ll}
\hline Interventions & $\begin{array}{l}\text { 1. Virtual reality balance training: performed in standing, } 30 \text {-minute session, } 2 \text { a week, } 6 \text { weeks } \\
\text { 2. Control group: usual care, general recommendations and care plan on falls prevention }\end{array}$ \\
\hline Outcomes & 1. Rate of falls \\
\hline Duration of the study & 36 weeks \\
\hline Adherence & $\begin{array}{l}\text { Adherence not defined. Proportion that progressed through levels reported: } \\
\text { 1. Virtual reality balance training group: } 97 \% \text {; most of the participants (91\%) reached } \geq 10 / 15 \text { possible } \\
\text { levels in every group of virtual exercises }\end{array}$ \\
\hline Notes & $\begin{array}{l}\text { Source of funding: Nepean Medical Research Foundation, Department of Geriatric Medicine at Nepean } \\
\text { Hospital } \\
\text { Economic information: not reported } \\
\text { Email communication regarding fall data, response received, data not included in review }\end{array}$
\end{tabular}

\section{Risk of bias}

\section{Bias}

Random sequence genera- Unclear risk tion (selection bias)

\begin{tabular}{lll}
\hline $\begin{array}{l}\text { Allocation concealment } \\
\text { (selection bias) }\end{array}$ & Unclear risk & Insufficient information to permit judgement \\
\hline $\begin{array}{l}\text { Blinding of participants } \\
\begin{array}{l}\text { and personnel (perfor- } \\
\text { mance bias) }\end{array}\end{array}$ & Unclear risk & $\begin{array}{l}\text { Participants and personnel not blinded to allocated group, but impact of non- } \\
\text { blinding unclear }\end{array}$ \\
All outcomes & \\
\hline
\end{tabular}


Duque 2013 (Continued)

$\begin{array}{ll}\begin{array}{l}\text { Blinding of outcome as- } \\ \text { sessment (detection bias) }\end{array} & \text { Low risk }\end{array} \quad \begin{aligned} & \text { Fall outcomes were recorded using the same method in both groups } \\ & \text { Falls }\end{aligned} \quad \begin{aligned} & \text { Quote: "to prevent any assessment bias, different physiotherapists with no ac- } \\ & \text { cess to the subjects' data were specifically assigned to perform either assess- } \\ & \text { ment or training" }\end{aligned}$

\begin{tabular}{ll}
\hline Blinding of outcome as- & Unclear risk
\end{tabular}

\begin{tabular}{|c|c|c|}
\hline $\begin{array}{l}\text { Blinding of outcome as- } \\
\text { sessment (detection bias) } \\
\text { Hospital admission, med- } \\
\text { ical attention and adverse } \\
\text { events }\end{array}$ & Unclear risk & Not applicable \\
\hline $\begin{array}{l}\text { Blinding of outcome as- } \\
\text { sessment (detection bias) } \\
\text { Health related quality of } \\
\text { life (self report) }\end{array}$ & Unclear risk & Not applicable \\
\hline $\begin{array}{l}\text { Incomplete outcome data } \\
\text { (attrition bias) } \\
\text { Falls and fallers }\end{array}$ & Low risk & No missing fall data \\
\hline $\begin{array}{l}\text { Selective reporting (re- } \\
\text { porting bias) }\end{array}$ & High risk & $\begin{array}{l}\text { Fall data were collected but number of fallers was not reported. Adverse } \\
\text { events not reported }\end{array}$ \\
\hline $\begin{array}{l}\text { Method of ascertaining } \\
\text { falls (recall bias) }\end{array}$ & High risk & $\begin{array}{l}\text { The occurrence of falls was retrospectively assessed by asking the participant } \\
\text { (1) whether they have suffered a fall, and (2) the number of falls during the } 6 \\
\text { months prior to the assessment }\end{array}$ \\
\hline
\end{tabular}

Ebrahim 1997

\begin{tabular}{ll}
\hline Methods & Study design: RCT \\
& Number of study arms: 2 \\
& Length of follow-up: 24 months (also 12 months)
\end{tabular}

Setting: London, UK
Pumber of participants: 165
Number analysed: 102
Number lost to follow-up: 63
Sample: community-dwelling women
Age (years): Intervention mean $=66.4$ (SD 7.8), Control mean $=68.1$ (SD 7.8)
Sex: $100 \%$ female
Inclusion criteria: postmenopausal women who had sustained a fracture in the upper arm in the past 2
years recruited from 2 East London Hospitals
Exclusion criteria: women being treated with bisphosphonates, if expected survival was $<1$ year, cogni-
tive impairment, too frail to withstand brisk walking or travelling for measurements

Interventions

1. Individual Brisk Walking: intensity progressed, monthly telephone contact, advice from nurse about general health and balanced diet, walked 40 minutes, 3 a week, 2 years 
Ebrahim 1997 (Continued)

2. Control group: simple upper limb exercises, monthly telephone contact, advice from nurse about general health and balanced diet

1. Rate of falls
2. Number of people who experienced 1 or more falls (risk of falling)
3. Number of people who experienced 1 or more fall-related fractures

\begin{tabular}{ll}
\hline Duration of the study & 2 years \\
\hline Adherence & Adherence not defined. Participation in programme reported: \\
& $\begin{array}{l}\text { 1. Individual Brisk Walking group: adherence not defined, } 49 / 81(60.5 \%) \text { continued programme, with all } \\
\text { remaining participants exercising } \geq 40 \text { min, } 3 \text { a week } \\
\text { 2. Control group: adherence not defined, } 48 / 84(57.14 \%) \text { continued programme }\end{array}$
\end{tabular}

\begin{tabular}{ll}
\hline Notes & Source of funding: The Wolfson Family Trust \\
& Economic information: not reported
\end{tabular}

\begin{tabular}{|c|c|c|}
\hline \multicolumn{3}{|l|}{ Risk of bias } \\
\hline Bias & Authors' judgement & Support for judgement \\
\hline $\begin{array}{l}\text { Random sequence genera- } \\
\text { tion (selection bias) }\end{array}$ & Low risk & "Randomly assigned" using "computer generated" allocation \\
\hline $\begin{array}{l}\text { Allocation concealment } \\
\text { (selection bias) }\end{array}$ & Unclear risk & Series of prepared envelopes but did not mention "opaque" or "sealed" \\
\hline $\begin{array}{l}\text { Blinding of participants } \\
\text { and personnel (perfor- } \\
\text { mance bias) } \\
\text { All outcomes }\end{array}$ & High risk & $\begin{array}{l}\text { Participants not blind to allocated group. Research personnel were not blind } \\
\text { to group, yet delivered the intervention to both groups and assessed fall out- } \\
\text { come, which increases the risk of bias }\end{array}$ \\
\hline $\begin{array}{l}\text { Blinding of outcome as- } \\
\text { sessment (detection bias) } \\
\text { Falls }\end{array}$ & High risk & $\begin{array}{l}\text { Falls ascertained by the same method in both groups. The research nurse de- } \\
\text { livering intervention to groups also conducted the monthly telephone calls to } \\
\text { monitor the occurrence of falls, therefore was not blinded }\end{array}$ \\
\hline $\begin{array}{l}\text { Blinding of outcome as- } \\
\text { sessment (detection bias) } \\
\text { Fractures }\end{array}$ & Low risk & $\begin{array}{l}\text { Fracutres were assessed in all groups using radiological examination, by per- } \\
\text { sonnel blinded to group allocation }\end{array}$ \\
\hline $\begin{array}{l}\text { Blinding of outcome as- } \\
\text { sessment (detection bias) } \\
\text { Hospital admission, med- } \\
\text { ical attention and adverse } \\
\text { events }\end{array}$ & Unclear risk & Not applicable \\
\hline $\begin{array}{l}\text { Blinding of outcome as- } \\
\text { sessment (detection bias) } \\
\text { Health related quality of } \\
\text { life (self report) }\end{array}$ & Unclear risk & Not applicable \\
\hline $\begin{array}{l}\text { Incomplete outcome data } \\
\text { (attrition bias) } \\
\text { Falls and fallers }\end{array}$ & High risk & $\begin{array}{l}\text { More than } 20 \% \text { of fall outcome data are missing }(38 \%) \text {. Loss of fall data was un- } \\
\text { balanced in intervention }(n=17) \text { and control }(n=12) \text { groups; reason for loss of } \\
\text { fall data was unclear }\end{array}$ \\
\hline
\end{tabular}


Ebrahim 1997 (Continued)

\begin{tabular}{|c|c|c|}
\hline $\begin{array}{l}\text { Selective reporting (re- } \\
\text { porting bias) }\end{array}$ & Uncl & $\begin{array}{l}\text { Minimum set of expected outcomes not reported (adverse events not report- } \\
\text { ed) }\end{array}$ \\
\hline
\end{tabular}

Method of ascertaining Unclear risk Monthly telephone calls

falls (recall bias)

\section{El-Khoury 2015}

$\begin{array}{ll}\text { Methods } & \text { Study design: RCT } \\ & \text { Number of study arms: } 2 \\ & \text { Length of follow-up: } 24 \text { months }\end{array}$

\begin{tabular}{|c|c|}
\hline \multirow[t]{9}{*}{ Participants } & Setting: France \\
\hline & Number of participants: 706 \\
\hline & Number analysed: 706 \\
\hline & Number lost to follow-up: 0 \\
\hline & Sample: community-dwelling women \\
\hline & Age (years): Intervention mean $=79.8(S D 2.8)$, Control mean $=79.6(S D 2.8)$ \\
\hline & Sex: $100 \%$ female \\
\hline & $\begin{array}{l}\text { Inclusion criteria: Women aged } 75 \text { - } 85 \text { living in the community, diminished balance or gait capacities } \\
\text { (assessed by } 6 \mathrm{~m} \text { walking time and tandem walk test) }\end{array}$ \\
\hline & $\begin{array}{l}\text { Exclusion criteria: > } 12.5 \text { seconds to walk } 6 \mathrm{~m} \text {, unable to stand for } 10 \mathrm{sec} \text { with feet together, medical } \\
\text { conditions precluding exercise, expected to move away in next } 6 \text { months, difficulty attending exercise } \\
\text { classes regularly, already attending exercise classes }\end{array}$ \\
\hline \multirow[t]{3}{*}{ Interventions } & $\begin{array}{l}\text { 1. Group-based balance and strength, } 1 \text { hour a week for } 2 \text { years, plus tailored home practice performed } \\
\text { weekly }\end{array}$ \\
\hline & 2. Control group: no intervention, offered 4 exercise sessions at end of trial \\
\hline & Both groups offered fall prevention brochures and newsletters \\
\hline \multirow[t]{3}{*}{ Outcomes } & 1. Rate of falls \\
\hline & 2. Number of people who experienced 1 or more falls (risk of falling) \\
\hline & 3. Number of people who died \\
\hline Duration of the study & 104 weeks \\
\hline \multirow[t]{2}{*}{ Adherence } & Adherence measured by programme attendance \\
\hline & $\begin{array}{l}\text { 1. Group-based balance and strength group: } 58 / 352(16 \%) \text { never started the programme; 38/352 (11\%) } \\
\text { attended a few classes in the first month only }\end{array}$ \\
\hline Notes & $\begin{array}{l}\text { Source of funding: "Assistance Publique-Hôpitaux de Paris" (AP-HP), French Ministry of Health, French } \\
\text { National Research Agency, National Institute of Health Prevention and Education, Council of the Ile-de- } \\
\text { France region } \\
\text { Economic information: not reported }\end{array}$ \\
\hline
\end{tabular}

\section{Risk of bias}


El-Khoury 2015 (Continued)

\section{Bias Authors' judgement Support for judgement}

Random sequence genera- Low risk tion (selection bias)

Allocation concealment Low risk
(selection bias)

Quote: "randomisation lists were computer generated, based on randomly permuted blocks of varying size $(2,4$ or 6 , randomly sampled with equal probability)...stratified for study centre and body weight"
Baseline assessment and randomisation lists installed on assessors laptop, where

Quote: "at the end of the baseline examination, the programme automatically determined the eligibility of each woman, based on her examination results; if she was eligible and agreed to participate, it randomly assigned her into the experimental intervention or the control group"

\begin{tabular}{|c|c|c|}
\hline $\begin{array}{l}\text { Blinding of participants } \\
\text { and personnel (perfor- } \\
\text { mance bias) } \\
\text { All outcomes }\end{array}$ & Unclear risk & Participants and personnel unblinded but impact of unblinding unknown \\
\hline $\begin{array}{l}\text { Blinding of outcome as- } \\
\text { sessment (detection bias) } \\
\text { Falls }\end{array}$ & Low risk & $\begin{array}{l}\text { Falls ascertained by the same method in both groups } \\
\text { Quote: "Investigator blinded to group assignment" phoned those who report- } \\
\text { ed falls }\end{array}$ \\
\hline $\begin{array}{l}\text { Blinding of outcome as- } \\
\text { sessment (detection bias) } \\
\text { Fractures }\end{array}$ & Unclear risk & Not applicable \\
\hline $\begin{array}{l}\text { Blinding of outcome as- } \\
\text { sessment (detection bias) } \\
\text { Hospital admission, med- } \\
\text { ical attention and adverse } \\
\text { events }\end{array}$ & Unclear risk & $\begin{array}{l}\text { In both groups } \\
\text { Quote: "if a fracture of admission to hospital was reported, a copy of the radi- } \\
\text { ologist's report or medical record was requested to confirm the severity of the } \\
\text { injuries". Blinding of assessor unclear }\end{array}$ \\
\hline
\end{tabular}

\begin{tabular}{|c|c|c|}
\hline $\begin{array}{l}\text { Blinding of outcome as- } \\
\text { sessment (detection bias) } \\
\text { Health related quality of } \\
\text { life (self report) }\end{array}$ & Unclear risk & Not applicable \\
\hline $\begin{array}{l}\text { Incomplete outcome data } \\
\text { (attrition bias) } \\
\text { Falls and fallers }\end{array}$ & Low risk & No missing fall data \\
\hline $\begin{array}{l}\text { Selective reporting (re- } \\
\text { porting bias) }\end{array}$ & Low risk & $\begin{array}{l}\text { Fall outcomes prespecified in prospective trial registratio were reported, ad- } \\
\text { verse events reported }\end{array}$ \\
\hline $\begin{array}{l}\text { Method of ascertaining } \\
\text { falls (recall bias) }\end{array}$ & Low risk & $\begin{array}{l}\text { Quote: "Participants were asked to mark the exact date of any fall on pre-ad- } \\
\text { dressed, prepaid monthly calendar postcards, and to return the cards at the } \\
\text { end of the corresponding month". A blinded assessor telephoned those who } \\
\text { reported falls. }\end{array}$ \\
\hline
\end{tabular}

Fiatarone 1997

$\begin{array}{ll}\text { Methods } & \text { Study design: RCT } \\ & \text { Number of study arms: } 2 \\ & \text { Length of follow-up: } 4 \text { months }\end{array}$


Fiatarone 1997 (Continued)

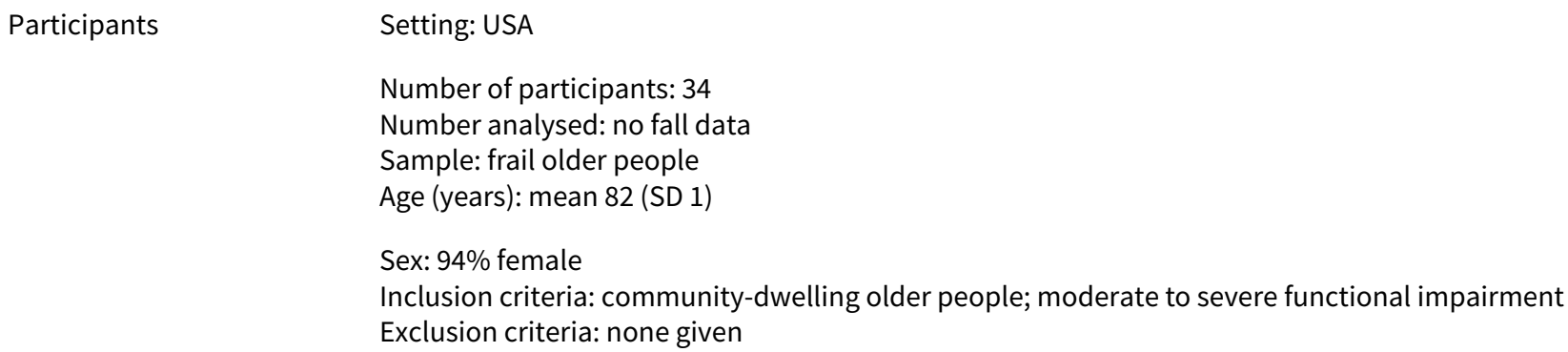

1. Individual high-intensity progressive resistance training, 11 different upper and lower limb exercises with arm and leg weights, 2 weeks instruction and then weekly phone calls, performed 3 a week, 16 weeks

2. Control: wait-list control. Weekly phone calls

\begin{tabular}{ll}
\hline Outcomes & Reported number of people sustaining 1 or more adverse effects of intervention \\
\hline Duration of the study & 16 weeks \\
\hline Adherence & Not reported \\
\hline Notes & $\begin{array}{l}\text { Source of funding: not reported } \\
\text { Economic information: not reported } \\
\end{array}$ \\
& Abstract only \\
\hline
\end{tabular}

\section{Risk of bias}

\section{Bias}

Authors' judgement Support for judgement

Random sequence genera- Unclear risk Method of randomisation not described
tion (selection bias)

Allocation concealment Unclear risk Insufficient information to permit judgement
(selection bias)

Blinding of participants Unclear risk Insufficient information to permit judgement
and personnel (perfor-
mance bias)
All outcomes

\begin{tabular}{lll}
\hline $\begin{array}{l}\text { Blinding of outcome as- } \\
\text { sessment (detection bias) } \\
\text { Falls }\end{array}$ & Unclear risk & Insufficient information to permit judgement \\
\hline $\begin{array}{l}\text { Blinding of outcome as- } \\
\text { sessment (detection bias) }\end{array}$ & Unclear risk & Not applicable \\
Fractures & \\
\hline
\end{tabular}

Blinding of outcome as- $\quad$ Unclear risk
sessment (detection bias)
Hospital admission, med-
ical attention and adverse
events

\begin{tabular}{lll}
\hline Blinding of outcome as- & Unclear risk & Not applicable \\
sessment (detection bias) &
\end{tabular}


Fiatarone 1997 (Continued) Health related quality of life (self report)

\begin{tabular}{|c|c|c|}
\hline $\begin{array}{l}\text { Incomplete outcome data } \\
\text { (attrition bias) } \\
\text { Falls and fallers }\end{array}$ & Unclear risk & Insufficient information to permit judgement. \\
\hline $\begin{array}{l}\text { Selective reporting (re- } \\
\text { porting bias) }\end{array}$ & High risk & $\begin{array}{l}\text { Falls not mentioned in Methods, fall outcome mentioned in results, adverse } \\
\text { events not reported }\end{array}$ \\
\hline $\begin{array}{l}\text { Method of ascertaining } \\
\text { falls (recall bias) }\end{array}$ & Unclear risk & Interval recall. Falls identified weekly by phone call \\
\hline
\end{tabular}

Freiberger 2007

\begin{tabular}{ll}
\hline Methods & Study design: RCT \\
& Number of study arms: 2 \\
& Length of follow-up: 24 months
\end{tabular}

\begin{tabular}{|c|c|}
\hline \multirow[t]{9}{*}{ Participants } & Setting: Erlangen, Germany \\
\hline & Number of participants: 134 \\
\hline & Number analysed: 127 \\
\hline & Number lost to follow-up: 7 \\
\hline & Sample: community-dwelling \\
\hline & Age (years): mean 76.1 (SD 4.1) \\
\hline & Sex: $44 \%$ female \\
\hline & $\begin{array}{l}\text { Inclusion criteria: } \geq 70 \text { years, fallen in past } 6 \text { months, fear of falling, signed informed consent, complet- } \\
\text { ing baseline assessment }\end{array}$ \\
\hline & $\begin{array}{l}\text { Exclusion criteria: unable to walk independently, cognitive impairment }(<25 \text { on the Digit Symbol Sub- } \\
\text { stitution Test) }\end{array}$ \\
\hline \multirow[t]{3}{*}{ Interventions } & $\begin{array}{l}\text { Randomised into } 3 \text { groups: } 2 \text { intervention groups (group-based psychomotor programme and group- } \\
\text { based balance, strength, flexibility, endurance) and } 1 \text { control group. Only the } 2 \text { intervention groups } \\
\text { were included in this review }\end{array}$ \\
\hline & $\begin{array}{l}\text { 1. Group-based psychomotor programme: strength training using dumbbells, free weights and body } \\
\text { weight, increasing difficulty of balance exercises, motor co-ordination, competence training, perceptu- } \\
\text { al training, and home exercises; sessions } 1 \text { hour, } 2 \text { a week for } 16 \text { weeks }\end{array}$ \\
\hline & $\begin{array}{l}\text { 2. Group-based balance, strength, flexibility, endurance: strength training using dumbbells, free } \\
\text { weights and body weight, plus home exercises; sessions } 1 \text { hour, } 2 \text { a week for } 16 \text { weeks }\end{array}$ \\
\hline \multirow[t]{2}{*}{ Outcomes } & 1. Rate of falls \\
\hline & 2. Number of people who experienced 1 or more falls (risk of falling) \\
\hline Duration of the study & 52 weeks \\
\hline \multirow[t]{3}{*}{ Adherence } & Adherence measured by session attendance \\
\hline & 1. Group-based psychomotor programme: $82 \%$ attended at least $24 / 32$ sessions \\
\hline & 2. Group-based balance, strength, flexibility, endurance group: $84 \%$ attended at least $24 / 32$ sessions \\
\hline
\end{tabular}


Freiberger 2007 (Continued)

Notes
Source of funding: The Robert Bosch Foundation, Siemens Health Insurance

Economic information: not reported

\section{Risk of bias}

\begin{tabular}{lll}
\hline Bias & Authors' judgement & Support for judgement \\
\hline $\begin{array}{l}\text { Random sequence genera- } \\
\text { tion (selection bias) }\end{array}$ & Low risk & Computerised random-number generator \\
\hline $\begin{array}{l}\text { Allocation concealment } \\
\text { (selection bias) }\end{array}$ & Unclear risk & Quote: "All randomizations were concealed". No other information given \\
\hline $\begin{array}{l}\text { Blinding of participants } \\
\text { and personnel (perfor- } \\
\text { mance bias) }\end{array}$ & Unclear risk & Participants and personnel unblinded but impact of unblinding unknown \\
All outcomes & & \\
\hline
\end{tabular}

\begin{tabular}{lll}
\hline Blinding of outcome as- & Unclear risk & Falls ascertained by the same method in both groups. Blinding of assessors \\
sessment (detection bias) & performing the telephone interview was not specified \\
Falls &
\end{tabular}

Falls performing the telephone interview was not specified

\begin{tabular}{|c|c|}
\hline $\begin{array}{l}\text { Blinding of outcome as- } \\
\text { sessment (detection bias) } \\
\text { Fractures }\end{array}$ & Unclear risk \\
\hline
\end{tabular}

\begin{tabular}{|c|c|c|}
\hline $\begin{array}{l}\text { Blinding of outcome as- } \\
\text { sessment (detection bias) } \\
\text { Hospital admission, med- } \\
\text { ical attention and adverse } \\
\text { events }\end{array}$ & Unclear risk & Not applicable \\
\hline $\begin{array}{l}\text { Blinding of outcome as- } \\
\text { sessment (detection bias) } \\
\text { Health related quality of } \\
\text { life (self report) }\end{array}$ & Unclear risk & Not applicable \\
\hline $\begin{array}{l}\text { Incomplete outcome data } \\
\text { (attrition bias) } \\
\text { Falls and fallers }\end{array}$ & Low risk & $\begin{array}{l}\text { Less than } 20 \% \text { of fall outcome data are missing }(5 \%) \text {. Loss of fall data was bal- } \\
\text { anced in the balance programme }(n=4) \text { and psychomotor programme }(n=3) \\
\text { groups; reason for loss of fall data was unclear }\end{array}$ \\
\hline $\begin{array}{l}\text { Selective reporting (re- } \\
\text { porting bias) }\end{array}$ & High risk & $\begin{array}{l}\text { Fall data were collected but number of falls was not reported. Adverse events } \\
\text { not reported }\end{array}$ \\
\hline $\begin{array}{l}\text { Method of ascertaining } \\
\text { falls (recall bias) }\end{array}$ & Low risk & $\begin{array}{l}\text { Quote: "falls were collected prospectively using a monthly fall calendar be- } \\
\text { tween months } 12 \text { and } 24 \text {; fall sheets were mailed in at the end of the month. } \\
\text { Up to five follow-up telephone calls were made in the event of no response af- } \\
\text { ter each month. If falls were reported, details were collected during a struc- } \\
\text { tured telephone interview" }\end{array}$ \\
\hline
\end{tabular}

$\begin{array}{ll}\text { Methods } & \text { Study design: RCT } \\ & \text { Number of study arms: } 2 \\ & \text { Length of follow-up: } 42 \text { months }\end{array}$


Gill 2016 (Continued)

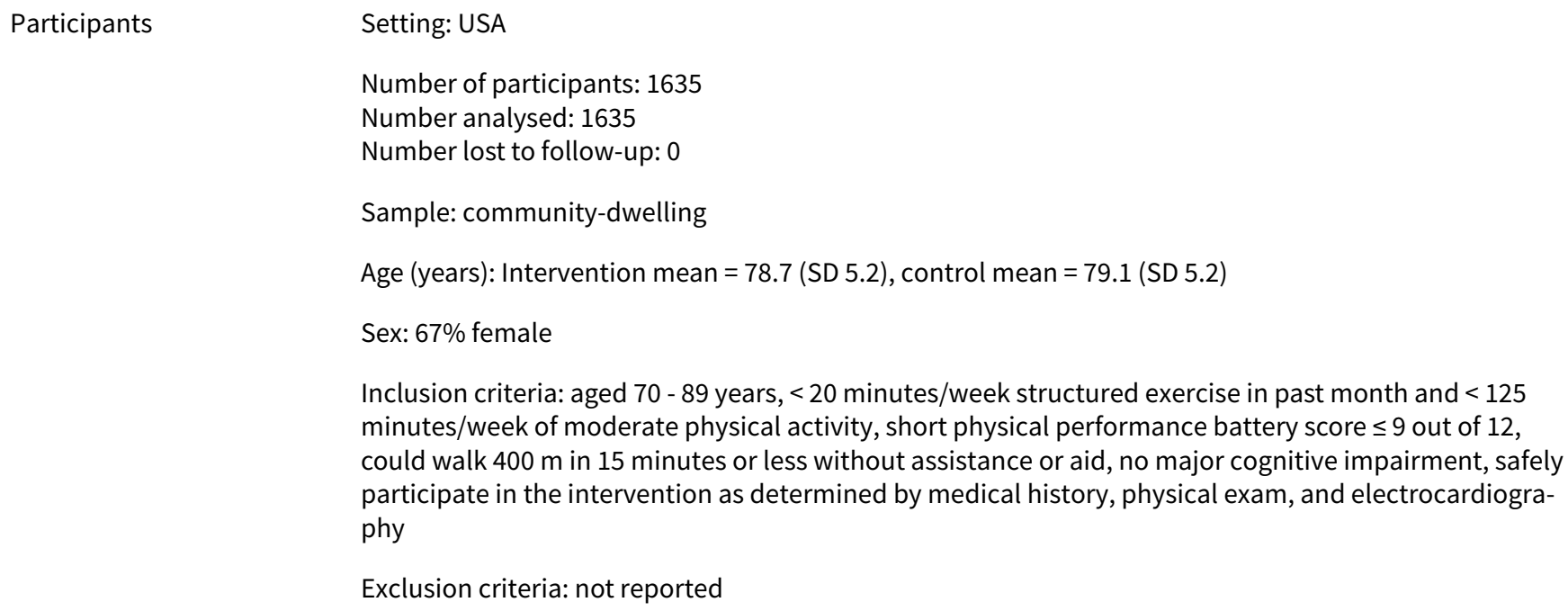

2. Control group: attended weekly health education group for 26 weeks and monthly sessions thereafter, plus 5 - 10 minutes stretching exercises

Outcomes Number of people who experienced 1 or more fall-related fractures
2. Number of people who experienced 1 of more falls requiring hospital admission
3. Number of people who died

\begin{tabular}{ll} 
Duration of the study & Up to 168 weeks \\
\hline Adherence & Adherence measured by attendance at sessions \\
& $\begin{array}{l}\text { 1. Group- and home-based balance, strength, flexibility and walking training group: attended mean of } \\
63 \% \text { of scheduled sessions, median } 71 \% \text { (interquartile range } 50-83 \%)\end{array}$ \\
& 2. Control: attended mean of $73 \%$ of the scheduled sessions, median $82 \%(63-90 \%)$ \\
\hline Notes & $\begin{array}{l}\text { Source of funding: National Institute of Health, National Institute of Aging, National Heart, Lung and } \\
\text { Blood Institute } \\
\text { Economic information: not reported }\end{array}$
\end{tabular}

Risk of bias

\begin{tabular}{lll}
\hline Bias & Authors' judgement & Support for judgement \\
\hline $\begin{array}{l}\text { Random sequence genera- } \\
\text { tion (selection bias) }\end{array}$ & Low risk & $\begin{array}{l}\text { Quote: "Randomised..through a secure web based data management system } \\
\text { using a permuted block algorithm (with random block lengths) stratified by } \\
\text { field center and sex" }\end{array}$ \\
\hline $\begin{array}{l}\text { Allocation concealment } \\
\text { (selection bias) }\end{array}$ & Low risk & Quote: "Secure web based data management system" \\
\hline $\begin{array}{l}\text { Blinding of participants } \\
\begin{array}{l}\text { and personnel (perfor- } \\
\text { mance bias) }\end{array}\end{array}$ & Unclear risk & Participants and personnel unblinded but impact of unblinding unknown \\
All outcomes & \\
\hline \hline
\end{tabular}




\section{Gill 2016 (Continued)}

Blinding of outcome assessment (detection bias)

Falls
Unclear risk Not applicable

$\begin{array}{ll}\begin{array}{l}\text { Blinding of outcome as- } \\ \text { sessment (detection bias) }\end{array} & \text { Low risk } \\ \text { Fractures } & \text { Question by blinded assessor: } \\ & \begin{array}{l}\text { Quote: "did a doctor tell you that you fractured or broke a bone?" If yes, } \\ \text { Quote: "Two experts blinded to group randomization subsequently reviewed } \\ \text { and adjudicated independently relevant medical records, including those from } \\ \text { all hospital admissions." A fall-related fracture required the fulfilment of } 4 \text { pre- } \\ \text { specified criteria }\end{array}\end{array}$

\begin{tabular}{lll}
\hline Blinding of outcome as- & Low risk & Quote: "Two experts blinded to group randomization subsequently reviewed \\
sessment (detection bias) & & $\begin{array}{l}\text { and adjudicated independently relevant medical records, including those from } \\
\text { Hospital admission, med- }\end{array}$ \\
all hospital admissions."
\end{tabular}

ical attention and adverse events

\begin{tabular}{|c|c|c|}
\hline $\begin{array}{l}\text { Blinding of outcome as- } \\
\text { sessment (detection bias) } \\
\text { Health related quality of } \\
\text { life (self report) }\end{array}$ & Unclear risk & Not applicable \\
\hline $\begin{array}{l}\text { Incomplete outcome data } \\
\text { (attrition bias) } \\
\text { Falls and fallers }\end{array}$ & Unclear risk & No fall data \\
\hline $\begin{array}{l}\text { Selective reporting (re- } \\
\text { porting bias) }\end{array}$ & High risk & The question "have you fallen?" was asked but was not prespecified \\
\hline $\begin{array}{l}\text { Method of ascertaining } \\
\text { falls (recall bias) }\end{array}$ & High risk & $\begin{array}{l}\text { Questioned by blinded assessors every } 6 \text { months: Since (last visit date), did } \\
\text { a doctor tell you that you fractured or broke a bone? (If yes) Did you break } \\
\text { a bone as a result of a fall? and Other than the conditions we just asked you } \\
\text { about, were you admitted to a hospital overnight for any other reasons since } \\
\text { (last visit date)? Since (last visit date), have you fallen? Did this fall result in an } \\
\text { inability to leave home for at least one week? }\end{array}$ \\
\hline
\end{tabular}

\section{Grahn Kronhed 2009}

\begin{tabular}{ll}
\hline Methods & Study design: RCT \\
& Number of study arms: 2 \\
& Length of follow-up: 12 months \\
\hline
\end{tabular}

Participants Setting: Linköping, Sweden

Number of participants: 65

Number analysed: 65

Number lost to follow-up: 0

Sample: women with osteoporosis identified from Linköping Hospital, Osteoporosis Unit files

Age (years): mean 71.4, range 60 to 81

Sex: $100 \%$ female

Inclusion criteria: BMD measured within previous 9 months and T-score $\leq-2.5 \mathrm{SD}$ 
Grahn Kronhed 2009 (Continued)

Exclusion criteria: enrolled in a pharmacological RCT; requiring indoor walking aids; cognitively impaired (MMSE < 20); severe heart disease, malignancy, recent arthroplasty, unhealed fractures; unable to understand Swedish

$\begin{array}{ll}\text { Interventions } & \text { 1. Group-based strength and balance training: supervised and progressed using body weight, pulleys, } \\ \text { leg press, exercises on balance boards and weight shifting on trampoline; } 1 \text { hour, } 2 \text { a week for } 4 \text { months } \\ \text { 2. Control: no intervention. Instructed not to change exercise routines for } 1 \text { year }\end{array}$

Outcomes Rate of falls
2. Health-related quality of life

\begin{tabular}{ll}
\hline Duration of the study & 52 weeks \\
\hline Adherence & Adherence measured by completion of sessions \\
& 1. Group-based strength training group: completed mean of 24/30 sessions (median = 25, range 13 - 30)
\end{tabular}

Notes

Source of funding: Ostergotland County Council and the Faculty of Health Sciences, Linköping University, Region Västra Gotaland, the Stohne's foundation, and Sanofi-AventisOstergotland County Council and the Faculty of Health Sciences, Linköping University, Region Västra Gotaland, the Stohne's foundation, and Sanofi-Aventis

Economic information: not reported

No participants sustained a fracture during follow-up

\section{Risk of bias}

\begin{tabular}{|c|c|c|}
\hline Bias & Authors' judgement & Support for judgement \\
\hline $\begin{array}{l}\text { Random sequence genera- } \\
\text { tion (selection bias) }\end{array}$ & Low risk & $\begin{array}{l}\text { Method not described but assume it was truly random, given that } \\
\text { Quote: "an independent statistical unit randomised the participants" }\end{array}$ \\
\hline $\begin{array}{l}\text { Allocation concealment } \\
\text { (selection bias) }\end{array}$ & Low risk & Quote: "An independent statistical unit randomized the participants" \\
\hline $\begin{array}{l}\text { Blinding of participants } \\
\text { and personnel (perfor- } \\
\text { mance bias) } \\
\text { All outcomes }\end{array}$ & Unclear risk & $\begin{array}{l}\text { Participants and personnel not blinded to allocated group but impact of non- } \\
\text { blinding unclear }\end{array}$ \\
\hline $\begin{array}{l}\text { Blinding of outcome as- } \\
\text { sessment (detection bias) } \\
\text { Falls }\end{array}$ & Low risk & $\begin{array}{l}\text { Falls ascertained by the same method in both groups } \\
\text { Quote: "... participants were followed-up concerning ... falls ... for } 1 \text { year by } \\
\text { the independent statistical unit." Probably blind to allocated group or at least } \\
\text { unlikely to introduce bias. }\end{array}$ \\
\hline
\end{tabular}

Blinding of outcome as-
sessment (detection bias)
Fractures

\begin{tabular}{lll}
\hline $\begin{array}{l}\text { Blinding of outcome as- } \\
\text { sessment (detection bias) }\end{array}$ & Unclear risk & Not applicable \\
$\begin{array}{l}\text { Hospital admission, med- } \\
\text { ical attention and adverse } \\
\text { events }\end{array}$ & \\
\hline $\begin{array}{l}\text { Blinding of outcome as- } \\
\text { sessment (detection bias) }\end{array}$ & High risk & Participants not blind to allocated group \\
\hline
\end{tabular}


Grahn Kronhed 2009 (Continued)

Health related quality of

life (self report)

\begin{tabular}{lll}
\hline $\begin{array}{l}\text { Incomplete outcome data } \\
\text { (attrition bias) }\end{array}$ & Low risk \\
$\begin{array}{l}\text { Falls and fallers } \\
\text { Selective reporting (re- } \\
\text { porting bias) }\end{array}$ & High risk & $\begin{array}{l}\text { Fall data obtined but number of fallers not reported. Adverse events not re- } \\
\text { ported }\end{array}$ \\
\hline $\begin{array}{l}\text { Method of ascertaining } \\
\text { falls (recall bias) }\end{array}$ & Low risk & $\begin{array}{l}\text { Quote: "... participants reported number of falls each week for the 1-year study } \\
\text { period" }\end{array}$ \\
\hline
\end{tabular}

Gschwind 2015

$\begin{array}{ll}\text { Methods } & \text { Study design: RCT } \\ & \text { Number of study arms: } 2 \\ & \text { Length of follow-up: } 6 \text { months }\end{array}$

\section{Participants}

Setting: Cologne, Germany; Valencia, Spain; Sydney, Australia

Number of participants: 153

Number analysed: 136

Number lost to follow-up: 17

Sample: community-dwelling

Age (years): mean 74.7 (SD 6.3)

Sex: $61 \%$ female

Inclusion criteria: $\geq 65$ years, living in the community, able to walk $20 \mathrm{~m}$ without a walking aid, able to watch television \pm glasses from $3 \mathrm{~m}$ distance, have enough space for system use $\left(3.5 \mathrm{~m}^{2}\right)$

Exclusion criteria: insufficient language skills to understand the study procedures, cognitive impairment, medical conditions precluding participation in a regular exercise programme (i.e. uncontrolled hypertension, severe neurological disorder, acute cancer, psychiatric disorder, acute infection)

$\begin{array}{ll}\text { Interventions } & \text { Individual balance and strength training using exergames: home programme of balance exercises } \\ \text { (Weight-bearing Exercise for Better Balance (WEBB) programme (www.webb.org.au) }+ \text { technology ex- } \\ \text { ergames and feedback, 40-minute sessions, } 3 \text { a week, and progressive strengthening exercises based }\end{array}$
on the Otago Exercise Programme, 15 - 20 minute sessions, 3 a week for 16 weeks

2. Control group: no intervention

\begin{tabular}{ll}
\hline Outcomes & 1. Rate of falls \\
2. Health-related quality of life
\end{tabular}

\begin{tabular}{ll}
\hline Duration of the study & 24 weeks \\
\hline Adherence & Adherence was monitored automatically by iStopFalls system \\
& $\begin{array}{l}\text { 1. Individual balance and strength training using exergames groups: used the iStopFalls system } 42 \\
\text { times (median, IQR }=3.9) \text { for a total duration of } 11.7 \text { hours (median, IQR }=22.0)\end{array}$
\end{tabular}

Notes

Source of funding: European Union's Seventh Framework Program, NHMRC

Economic information: not reported 


\section{Risk of bias}

\begin{tabular}{lll}
\hline Bias & Authors' judgement & Support for judgement \\
\hline $\begin{array}{ll}\text { Random sequence genera- } \\
\text { tion (selection bias) }\end{array}$ & Low risk & $\begin{array}{l}\text { Quote: "Were randomised by permuted block- randomisation (ratio1:1) using } \\
\text { a unique computer-generated random number for identification. Participants } \\
\text { who lived in the same household were treated as one unit and randomised in } \\
\text { to the same block" }\end{array}$ \\
& &
\end{tabular}

\begin{tabular}{lll}
\hline $\begin{array}{l}\text { Allocation concealment } \\
\text { (selection bias) }\end{array}$ & Unclear risk & Allocation concealment not reported \\
\hline $\begin{array}{l}\text { Blinding of participants } \\
\text { and personnel (perfor- } \\
\text { mance bias) }\end{array}$ & Unclear risk & Participants and personnel unblinded but impact of unblinding unknown \\
All outcomes & \\
\hline
\end{tabular}

$\begin{array}{ll}\begin{array}{l}\text { Blinding of outcome as- } \\ \text { sessment (detection bias) }\end{array} & \text { Low risk } \\ \text { Falls } & \text { Falls ascertained by the same method in both groups } \\ & \begin{array}{l}\text { Quote: "Falls frequency .. monitored with monthly diaries for } 6 \text { months. Partic- } \\ \text { ipants were contacted by phone when the diaries were not returned." "Staff } \\ \text { performing the assessments was.. blinded to group allocation" It is likely, al- } \\ \text { though not certain, that staff conducting follow-up calls were blinded to group }\end{array}\end{array}$

\begin{tabular}{ll}
\hline Blinding of outcome as- & Unclear risk \\
sessment (detection bias) & \\
Fractures &
\end{tabular}

\begin{tabular}{|c|c|c|}
\hline $\begin{array}{l}\text { Blinding of outcome as- } \\
\text { sessment (detection bias) }\end{array}$ & Low risk & In both groups \\
\hline $\begin{array}{l}\text { Hospital admission, med- } \\
\text { ical attention and adverse } \\
\text { events }\end{array}$ & & $\begin{array}{l}\text { Quote: "falls frequency and adverse events were monitored with monthly di- } \\
\text { aries for } 6 \text { months". "Staff performing the assessments was.. blinded to group } \\
\text { allocation" It is likely, although not certain, that staff conducting follow-up } \\
\text { calls were blinded to group }\end{array}$ \\
\hline
\end{tabular}

\begin{tabular}{lll}
\hline $\begin{array}{l}\text { Blinding of outcome as- } \\
\text { sessment (detection bias) } \\
\text { Health related quality of } \\
\text { life (self report) }\end{array}$ & High risk & Participants were unblinded to group allocation \\
\hline $\begin{array}{l}\text { Incomplete outcome data } \\
\text { (attrition bias) } \\
\text { Falls and fallers }\end{array}$ & Low risk & $\begin{array}{l}\text { Less than } 20 \% \text { of fall outcome data are missing }(11 \%) \text {. Loss of fall data was bal- } \\
\text { anced in the intervention }(n=7) \text { and control }(\mathrm{n}=10) \text { groups; reason for missing } \\
\text { data was unclear }\end{array}$ \\
\hline $\begin{array}{l}\text { Selective reporting (re- } \\
\text { porting bias) }\end{array}$ & High risk & Fall data obtined but number of fallers not reported \\
\hline $\begin{array}{l}\text { Method of ascertaining } \\
\text { falls (recall bias) }\end{array}$ & Low risk & $\begin{array}{l}\text { Falls frequency and adverse events were monitored with monthly diaries for } \\
6 \text { months Participants were contacted by phone when the diaries were not re- } \\
\text { turned }\end{array}$ \\
\hline
\end{tabular}

Haines 2009

\begin{tabular}{ll}
\hline Methods & Study design: RCT \\
& Number of study arms: 2
\end{tabular}


Haines 2009 (Continued)

Length of follow-up: 6 months

Setting: Brisbane, Australia
Number of participants: 53
Number analysed: 53
Number lost to follow-up: 0
Sample: patients in geriatric rehabilitation, medical, or surgical units in Princess Alexandra Hospital
Age (years): mean 80.7 (SD 7.7)
Sex: $60 \%$ female
Inclusion criteria: aged > 65 years; gait instability or walking with a mobility aid; discharged from hospi-
tal to community-dwelling
Exclusion criteria: unstable severe cardiac disease; cognitive impairment; aggressive behaviour; re-
stricted weight-bearing status; referred for post-discharge community rehabilitation services

Interventions $\quad$ 1. Home-based strength and balance programme with DVD/workbook: lower limb strength and balance exercises with 6 levels of difficulty, $3-7$ a week. DVD player provided if required. At least 1 home visit from project PT, then telephone contact weekly for 8 weeks, then 18 weeks without active encouragement

2. Control: did not receive programme materials, visits or telephone calls

Outcomes
$\begin{aligned} & \text { 2. Number of people who experienced } 1 \text { or more falls (risk of falling) } \\ & \text { 3. Health-related quality of life } \\ & \text { 4. Number of people who died }\end{aligned}$

\begin{tabular}{|c|c|}
\hline Duration of the study & 26 weeks \\
\hline \multirow[t]{3}{*}{ Adherence } & $\begin{array}{l}\text { Exercise group: exercise adherence monitored by weekly phone calls by the physio for } 8 \text { weeks } \\
\text { Week } 1: N=15 \text { exercised } \geq 1, N=12 \text { exercised } \geq 2 / \text { week } \\
\text { Week } 2: N=15 \text { exercised } \geq 1, N=11 \text { exercised } \geq 2 / \text { week } \\
\text { Week } 3: N=13 \text { exercised } \geq 1, N=8 \text { exercised } \geq 2 / \text { week } \\
\text { Week } 4: N=12 \text { exercised } \geq 1, N=9 \text { exercised } \geq 2 / \text { week }\end{array}$ \\
\hline & Week $5 ; N=11$ exercised $\geq 1, N=8$ exercised $\geq 2 /$ week \\
\hline & Week 6: $N=9$ exercised $\geq 1, N=4$ exercised $\geq 2 /$ week \\
\hline \multirow[t]{2}{*}{ Notes } & $\begin{array}{l}\text { Source of funding: Queensland Health, Allied Health Advisory, Community Rehabilitation Workforce } \\
\text { Project }\end{array}$ \\
\hline & Economic information: not reported \\
\hline
\end{tabular}

\section{Risk of bias}

\begin{tabular}{lll}
\hline Bias & Authors' judgement & Support for judgement \\
\hline $\begin{array}{l}\text { Random sequence genera- } \\
\text { tion (selection bias) }\end{array}$ & Low risk & $\begin{array}{l}\text { Quote: "The random allocation sequence was generated by an investigator } \\
\text { (TH) using a computerized random number generator" }\end{array}$ \\
\hline $\begin{array}{l}\text { Allocation concealment } \\
\text { (selection bias) }\end{array}$ & Low risk & $\begin{array}{l}\text { Quote: "This sequence was entered into sealed, consecutively numbered, } \\
\text { opaque envelopes. Each envelope corresponding to the participants study } \\
\text { number (allocated in the order in which participants consented to partici- } \\
\text { pate in the study) was opened following completion of the baseline assess- }\end{array}$
\end{tabular}


ment. The envelopes containing the allocation sequence were secured within a locked office."

\begin{tabular}{ll}
\hline $\begin{array}{l}\text { Blinding of participants } \\
\text { and personnel (perfor- }\end{array}$ & Unclear risk \\
mance bias) & $\begin{array}{l}\text { Participants and personnel not blinded to intervention, effect of not blinding } \\
\text { unclear }\end{array}$ \\
All outcomes &
\end{tabular}

\begin{tabular}{|c|c|c|}
\hline $\begin{array}{l}\text { Blinding of outcome as- } \\
\text { sessment (detection bias) }\end{array}$ & Low risk & $\begin{array}{l}\text { Quote: "All participants received monthly follow-up phone calls from the } \\
\text { blinded outcome assessor" }\end{array}$ \\
\hline
\end{tabular}

Falls

\begin{tabular}{|c|c|c|}
\hline $\begin{array}{l}\text { Blinding of outcome as- } \\
\text { sessment (detection bias) }\end{array}$ & High risk & The only evidence for fractures was from self-reports from participants \\
\hline
\end{tabular}

\begin{tabular}{|c|c|c|}
\hline $\begin{array}{l}\text { Blinding of outcome as- } \\
\text { sessment (detection bias) } \\
\text { Hospital admission, med- } \\
\text { ical attention and adverse } \\
\text { events }\end{array}$ & High risk & $\begin{array}{l}\text { Number of falls resulting in medical review (GP or hospital medical officer or } \\
\text { emergency department) were self-reports }\end{array}$ \\
\hline $\begin{array}{l}\text { Blinding of outcome as- } \\
\text { sessment (detection bias) } \\
\text { Health related quality of } \\
\text { life (self report) }\end{array}$ & High risk & Participants not blindde to group allocation \\
\hline $\begin{array}{l}\text { Incomplete outcome data } \\
\text { (attrition bias) } \\
\text { Falls and fallers }\end{array}$ & Low risk & $\begin{array}{l}\text { Less than } 20 \% \text { of fall outcome data are missing (6\%). Loss of data was due to } 3 \\
\text { deaths in the control group. Unlikely this was linked to outcome }\end{array}$ \\
\hline $\begin{array}{l}\text { Selective reporting (re- } \\
\text { porting bias) }\end{array}$ & Low risk & $\begin{array}{l}\text { Prespecified fall and adverse event outcomes reported. Trial prospectively } \\
\text { registered }\end{array}$ \\
\hline $\begin{array}{l}\text { Method of ascertaining } \\
\text { falls (recall bias) }\end{array}$ & Low risk & $\begin{array}{l}\text { Quote: "Participants in both groups were provided with a log for recording falls } \\
\text { and details surrounding them." "All participants received monthly follow-up } \\
\text { phone calls from the blinded outcome assessor." }\end{array}$ \\
\hline
\end{tabular}

Halvarsson 2013

\begin{tabular}{ll} 
Methods & $\begin{array}{l}\text { Study design: RCT } \\
\text { Number of study arms: } 2 \\
\text { Length of follow-up: } 15 \text { months }\end{array}$ \\
\hline Participants & Setting: Stockholm, Sweden \\
& Number of participants: 59 \\
& Number analysed: 48 \\
& Number lost to follow-up: 11 \\
& Sample: community-dwelling \\
& Age (years): mean 77 (range 67 - 93) \\
& Sex: $71 \%$ female \\
& Inclusion criteria: $\geq 65$ years, fear of falling or an experience of a fall during the previous 12 months, or \\
both, ability to walk unaided indoors and a MMSE score $\geq 24$
\end{tabular}


Halvarsson 2013 (Continued)

Exclusion criteria: severely impaired vision or hearing, severe cancer, severe pain, neurological disease or damage with symptoms, dizziness requiring medical care, or heart and respiratory problems that might affect participation
Interventions
1. Group-based progressive balance training: 45 minute sessions, 3 a week for 12 weeks
2. Control group: usual activities and offered intervention following the study period

\begin{tabular}{ll}
\hline Outcomes & 1. Number of people who experienced 1 or more falls (risk of falling) \\
\hline Duration of the study & 65 weeks \\
\hline Adherence & Adherence measured by attendance at sessions \\
& $\begin{array}{l}\text { 1. Group-based progressive balance training group: } 71-100 \%(n=24-36), \text { mean } 87 \%(n=31) \\
\text { Notes }\end{array}$ \\
$\begin{array}{l}\text { Source of funding: Stockholm County Council and Karolinska Institute, the Torsten and Ragnar Söder- } \\
\text { berg Foundation, and Johanniterorden, Sister Kenny Foundation in Minneapolis } \\
\text { Economic information: not reported }\end{array}$
\end{tabular}

\section{Risk of bias}

\begin{tabular}{lll}
\hline Bias & Authors' judgement & Support for judgement \\
\hline $\begin{array}{l}\text { Random sequence genera- } \\
\text { tion (selection bias) }\end{array}$ & Low risk & $\begin{array}{l}\text { Quote: "Randomization to group allocation was done in blocks, with a 2:1 ratio } \\
\text { in favor of the intervention group, by the subjects themselves drawing a allo- } \\
\text { cation slip" }\end{array}$ \\
\hline $\begin{array}{l}\text { Allocation concealment } \\
\text { (selection bias) }\end{array}$ & Unclear risk & Allocation concealment not reported \\
\hline $\begin{array}{l}\text { Blinding of participants } \\
\text { and personnel (perfor- } \\
\begin{array}{l}\text { mance bias) } \\
\text { All outcomes }\end{array}\end{array}$ & Unclear risk & Participants and personnel unblinded but impact of unblinding unknown \\
\hline
\end{tabular}

\begin{tabular}{lll}
\hline $\begin{array}{l}\text { Blinding of outcome as- } \\
\text { sessment (detection bias) } \\
\text { Falls }\end{array}$ & High risk & $\begin{array}{l}\text { Quote: "were told not to reveal group allocation to the assessors. However, } \\
\text { most of the participants did reveal which group they belonged to at the time of } \\
\text { the first follow-up, resulting in non-masked assessors at long-term follow-up" }\end{array}$ \\
\hline $\begin{array}{l}\text { Blinding of outcome as- } \\
\text { sessment (detection bias) } \\
\text { Fractures }\end{array}$ & Unclear risk & Not applicable \\
\hline
\end{tabular}

\begin{tabular}{|c|c|c|}
\hline $\begin{array}{l}\text { Blinding of outcome as- } \\
\text { sessment (detection bias) } \\
\text { Hospital admission, med- } \\
\text { ical attention and adverse } \\
\text { events }\end{array}$ & Unclear risk & Not applicable \\
\hline $\begin{array}{l}\text { Blinding of outcome as- } \\
\text { sessment (detection bias) } \\
\text { Health related quality of } \\
\text { life (self report) }\end{array}$ & Unclear risk & Not applicable \\
\hline $\begin{array}{l}\text { Incomplete outcome data } \\
\text { (attrition bias) } \\
\text { Falls and fallers }\end{array}$ & Unclear risk & $\begin{array}{l}\text { Less than } 20 \% \text { of fall outcome data are missing }(19 \%) \text {. Loss of fall data was un- } \\
\text { balanced in intervention }(n=8) \text { and control }(n=3) \text { groups; reason for loss of } \\
\text { fall data was unclear }\end{array}$ \\
\hline
\end{tabular}


Halvarsson 2013 (Continued)

Selective reporting (re- High risk $\quad$ Falls measured but number of falls not reported. Adverse events not reported porting bias)

Method of ascertaining High risk

falls (recall bias)
Quote: "Fall frequency was assessed at baseline and during the time between the follow-ups by asking the participants to recall if they had fallen during the last year"

\section{Halvarsson 2016}

\begin{tabular}{|c|c|}
\hline Methods & $\begin{array}{l}\text { Study design: RCT } \\
\text { Number of study arms: } 3 \\
\text { Length of follow-up: } 3 \text { months }\end{array}$ \\
\hline \multirow[t]{9}{*}{ Participants } & Setting: Stockholm, Sweden \\
\hline & Number of participants: 96 \\
\hline & Number analysed: 69 \\
\hline & Number lost to follow-up: 27 \\
\hline & Sample: community-dwelling \\
\hline & Age (years): Intervention mean 76 (range 67 - 86), Control mean 75 (range 66 - 84) \\
\hline & Sex: $98 \%$ female \\
\hline & $\begin{array}{l}\text { Inclusion criteria: age } \geq 65 \text { years afraid of falling or having experienced at least one fall in the last } 12 \\
\text { month s, or both, and independence in ambulation }\end{array}$ \\
\hline & $\begin{array}{l}\text { Exclusion criteria: fractures during the last year, MMSE score }<24 \text {, severely decreased vision, or other } \\
\text { diseases or constraints that might interfere with participation in the exercise programme }\end{array}$ \\
\hline \multirow[t]{3}{*}{ Interventions } & $\begin{array}{l}\text { 1. Group-based progressive balance training: supervised and tailored exercises, } 45 \text { minute sessions, } 3 \text { a } \\
\text { week for } 12 \text { weeks }\end{array}$ \\
\hline & $\begin{array}{l}\text { 2. Group-based progressive balance training plus walking: supervised and tailored exercises, } 45 \text {-minute } \\
\text { sessions, } 3 \text { a week for } 12 \text { weeks, plus walking (preferably with poles) for } \geq 30 \text { minutes, } 3 \text { a week for } 12 \\
\text { weeks }\end{array}$ \\
\hline & 3. Control group: no intervention, offered the same balance training at the end of the study \\
\hline Outcomes & 1. Number of people who experienced 1 or more falls (risk of falling) \\
\hline Duration of the study & 60 weeks \\
\hline \multirow[t]{3}{*}{ Adherence } & Adherence measured in sessions attended \\
\hline & $\begin{array}{l}\text { Participants attending } \geq 66 \% \text { sessions included in follow-up. Adherence rate to the training sessions } \\
\text { was } 89 \% \text { (range } 66-100 \% \text { ) }\end{array}$ \\
\hline & $\begin{array}{l}\text { 2. Group-based progressive balance training plus walking: all except } 1 \text { participant fulfilled the added } \\
\text { physical activity intervention }\end{array}$ \\
\hline \multirow[t]{2}{*}{ Notes } & $\begin{array}{l}\text { Source of funding: Stockholm County Council, Karolinska Institutet (ALF), Swedish Research Council, } \\
\text { Health Care Sciences Postgraduate School at Karolinska Institutet } \\
\text { Economic information: not reported }\end{array}$ \\
\hline & 3-month data used due to proportion of fallers not being clear for longer follow-up period \\
\hline
\end{tabular}


Halvarsson 2016 (Continued)

Risk of bias

\begin{tabular}{|c|c|c|}
\hline Bias & Authors' judgement & Support for judgement \\
\hline $\begin{array}{l}\text { Random sequence genera- } \\
\text { tion (selection bias) }\end{array}$ & Low risk & Quote: "Subjects were randomised...using web-based software" \\
\hline $\begin{array}{l}\text { Allocation concealment } \\
\text { (selection bias) }\end{array}$ & Unclear risk & Allocation concealment not reported \\
\hline $\begin{array}{l}\text { Blinding of participants } \\
\text { and personnel (perfor- } \\
\text { mance bias) } \\
\text { All outcomes }\end{array}$ & High risk & $\begin{array}{l}\text { Participants not blind to allocated group. Research personnel were not blind } \\
\text { to group, yet delivered the intervention to both groups and assessed fall out- } \\
\text { come, which increases the risk of bias }\end{array}$ \\
\hline $\begin{array}{l}\text { Blinding of outcome as- } \\
\text { sessment (detection bias) } \\
\text { Falls }\end{array}$ & High risk & $\begin{array}{l}\text { Quote: "The test leaders were blinded to group allocation at baseline; howev- } \\
\text { er, it was no longer possible after baseline testing, because some of the test } \\
\text { leaders were also involved in the balance training" }\end{array}$ \\
\hline $\begin{array}{l}\text { Blinding of outcome as- } \\
\text { sessment (detection bias) } \\
\text { Fractures }\end{array}$ & Unclear risk & Not applicable \\
\hline $\begin{array}{l}\text { Blinding of outcome as- } \\
\text { sessment (detection bias) } \\
\text { Hospital admission, med- } \\
\text { ical attention and adverse } \\
\text { events }\end{array}$ & Unclear risk & Not applicable \\
\hline $\begin{array}{l}\text { Blinding of outcome as- } \\
\text { sessment (detection bias) } \\
\text { Health related quality of } \\
\text { life (self report) }\end{array}$ & Unclear risk & Not applicable \\
\hline $\begin{array}{l}\text { Incomplete outcome data } \\
\text { (attrition bias) } \\
\text { Falls and fallers }\end{array}$ & Unclear risk & $\begin{array}{l}\text { Less than } 20 \% \text { of fall outcome data are missing }(3 \%) \text {. Loss of fall data was } \\
\text { unbalanced in balance }(n=9) \text { balance }+ \text { walking }(n=13) \text { and control }(n=5) \\
\text { groups; reason for loss of fall data was unbalanced }\end{array}$ \\
\hline $\begin{array}{l}\text { Selective reporting (re- } \\
\text { porting bias) }\end{array}$ & High risk & Falls measured but number of falls not reported. \\
\hline $\begin{array}{l}\text { Method of ascertaining } \\
\text { falls (recall bias) }\end{array}$ & High risk & $\begin{array}{l}\text { Quote: "Participants reported .. at each follow-up whether they had fallen dur- } \\
\text { ing the time since the previous follow-up session". Follow-up was at 3, } 9 \text { and } \\
15 \text { months }\end{array}$ \\
\hline Hamrick 2017 & & \\
\hline
\end{tabular}

Hamrick 2017

Methods Study design: RCT

Number of study arms: 2

Length of follow-up: 6 months

$\begin{array}{ll}\text { Participants } & \text { Setting: Wisconsin, USA } \\ & \text { Number of participants: } 43 \\ & \text { Number analysed: } 38 \\ & \text { Number lost to follow-up: } 5\end{array}$


Hamrick 2017 (Continued)

Sample: community-dwelling

Age (years): mean 69.9 (range 60 - 88)

Sex: $79 \%$ female

Inclusion: 60 years and older; able to walk 150 feet without assistive devices; cognitively intact as evidenced by correct answers to the Memory Impairment Screen; able to provide informed consent

Exclusion criteria: pelvic or lower extremity injury in the previous 6 months that required temporary use of an assistive device, including crutches, for $>7$ days; inability to provide informed consent; neurologic condition that impairs strength or balance including herniated lumbar disc with nerve root compression, previous stroke with residual lower extremity weakness, Parkinson's Disease, multiple sclerosis, muscular dystrophy and other neuromuscular diseases; cardiac or other medical condition with previous physician instructions to avoid low-intensity exercise; terminal condition with rapid progression of disease and not expected to live $>6$ months; pelvic or lower extremity orthopaedic surgery in the previous 12 months.; practised yoga at home or in a classroom setting in the past 6 months

1. Home-exercise group: instructed to practice 3 yoga home poses for 10 minutes +5 minutes of relaxation (breathing techniques) daily for 8 weeks

2. Relaxation group: instructed to practice 5 minutes of relaxation daily for 8 weeks

Both groups attended 60-minute yoga classes, 2 a week for 8 weeks

$\begin{array}{ll}\text { Outcomes } & \text { 1. Rate of falls } \\ \text { 2. Number of people who experienced } 1 \text { or more falls (risk of falling) }\end{array}$

\begin{tabular}{ll}
\hline Duration of the study & 26 weeks \\
\hline Adherence & Attendance in the 16 yoga sessions was $92 \%$ \\
\hline
\end{tabular}

Notes

Source of funding: Wisconsin Partnership Program

Economic information: not reported

Email communication to obtain fall data, response received, data included in review

\section{Risk of bias}

\begin{tabular}{|c|c|c|}
\hline Bias & Authors' judgement & Support for judgement \\
\hline $\begin{array}{l}\text { Random sequence genera- } \\
\text { tion (selection bias) }\end{array}$ & Unclear risk & $\begin{array}{l}\text { Quote: "Participants were randomly assigned } 1: 1 \text { by concealed allocation at } \\
\text { enrollment". Method of randomisation not stated }\end{array}$ \\
\hline $\begin{array}{l}\text { Allocation concealment } \\
\text { (selection bias) }\end{array}$ & Unclear risk & $\begin{array}{l}\text { Quote: "Participants were randomly assigned } 1: 1 \text { by concealed allocation at } \\
\text { enrollment". Method of concealment is not described }\end{array}$ \\
\hline $\begin{array}{l}\text { Blinding of participants } \\
\text { and personnel (perfor- } \\
\text { mance bias) } \\
\text { All outcomes }\end{array}$ & Unclear risk & $\begin{array}{l}\text { Participants and yoga instructors were not blinded to group allocation, but the } \\
\text { impact of non-blinding is unclear }\end{array}$ \\
\hline $\begin{array}{l}\text { Blinding of outcome as- } \\
\text { sessment (detection bias) } \\
\text { Falls }\end{array}$ & Low risk & $\begin{array}{l}\text { Quote: "participants underwent assessment at baseline and within } 1 \text { week of } \\
\text { completing the classes by one of the authors who was blinded to participant } \\
\text { home exercise assignment. We conducted a telephone survey about falls ... } 2 \\
\text { months and } 4 \text { months after completing the class" }\end{array}$ \\
\hline $\begin{array}{l}\text { Blinding of outcome as- } \\
\text { sessment (detection bias) }\end{array}$ & Unclear risk & Not applicable \\
\hline
\end{tabular}


Hamrick 2017 (Continued)

Fractures

\section{Blinding of outcome as- sessment (detection bias) Hospital admission, med- ical attention and adverse events}

Unclear risk Not applicable

Unclear risk Not applicable sessment (detection bias) Health related quality of life (self report)

Incomplete outcome data Low risk (attrition bias)

Falls and fallers

\section{Selective reporting (re-} porting bias)

Method of ascertaining falls (recall bias) Less than $20 \%$ of fall outcome data are missing $(11 \%)$. Loss of fall data was balanced in the treatment groups
Unclear risk

High risk
Minimum set of expected outcomes not reported (adverse events not reported)
Logs were given to inspire tracking of falls but logs were not collected. Telephone survey about falls 2 months and 4 months after completion of the intervention

\section{Hauer 2001}

\begin{tabular}{ll}
\hline Methods & Study design: RCT \\
& Number of study arms: 2 \\
& Length of follow-up: 6 months \\
\hline Participants & Setting: Germany \\
& Number of participants: 57 \\
& Number analysed: 56 \\
& Number lost to follow-up: 1 \\
& Sample: women recruited at the end of ward rehabilitation in a geriatric hospital \\
& Age (years): mean 82 (SD 4.8), range 75 - 90 \\
& Sex: $100 \%$ female \\
& Inclusion criteria: $\geq 75$ years; fall(s) as reason for admission to hospital or recent history of injurious fall \\
& leading to medical treatment; residing within study community \\
& Exclusion criteria: acute neurological impairment; severe cardiovascular disease; unstable chronic or \\
terminal illness; major depression; severe cognitive impairment; musculoskeletal impairment prevent- \\
ing participation in training regimen; falls known to be due to a single, identifiable disease, e.g. stroke \\
or hypoglycaemia
\end{tabular}

Interventions 1. Group-based progressive strength and balance training: gym equipment, pulleys and body weight used for 'high-intensity' progressive strength training; 45-minute sessions, 3 a week, for 12 weeks 2. Control group: flexibility, calisthenics, ball games, and memory tasks while seated, 60 -minute sessions, 3 a week, for 12 weeks

Both groups also received identical physiotherapy with balance and strength training components excluded (25 mins, 2 a week) 
Hauer 2001 (Continued)

\begin{tabular}{ll} 
Duration of the study & 26 weeks \\
\hline Adherence & Adherence was measured in training lists \\
& 1. Group-based strength and balance training group: $23 / 31$ completed study, $85.4 \%$ adherence \\
2. Control group: $22 / 26$ completed study, $84.2 \%$ adherence
\end{tabular}

$\begin{array}{ll}\text { Notes } & \text { Source of funding: Ministerium für Wissenschaft, Forschung und Kunst Baden-Wuerttemberg, Universi- } \\ & \text { ty of Heidelberg } \\ & \text { Economic information: not reported }\end{array}$

\section{Risk of bias}

\begin{tabular}{|c|c|c|}
\hline Bias & Authors' judgement & Support for judgement \\
\hline $\begin{array}{l}\text { Random sequence genera- } \\
\text { tion (selection bias) }\end{array}$ & Unclear risk & Stratified randomisation \\
\hline $\begin{array}{l}\text { Allocation concealment } \\
\text { (selection bias) }\end{array}$ & Unclear risk & Insufficient information to permit judgement \\
\hline $\begin{array}{l}\text { Blinding of participants } \\
\text { and personnel (perfor- } \\
\text { mance bias) } \\
\text { All outcomes }\end{array}$ & Unclear risk & $\begin{array}{l}\text { Unclear whether participants were blinded, but control group received place- } \\
\text { bo activities and both groups received identical physiotherapy sessions }\end{array}$ \\
\hline $\begin{array}{l}\text { Blinding of outcome as- } \\
\text { sessment (detection bias) } \\
\text { Falls }\end{array}$ & Low risk & $\begin{array}{l}\text { Falls ascertained by the same method in both groups. Staff documenting falls } \\
\text { were blinded to group assignment }\end{array}$ \\
\hline $\begin{array}{l}\text { Blinding of outcome as- } \\
\text { sessment (detection bias) } \\
\text { Fractures }\end{array}$ & Unclear risk & Not applicable \\
\hline
\end{tabular}

\begin{tabular}{lll}
\hline $\begin{array}{l}\text { Blinding of outcome as- } \\
\text { sessment (detection bias) } \\
\text { Hospital admission, med- } \\
\text { ical attention and adverse } \\
\text { events }\end{array}$ & Unclear risk & Method of determining adverse events was not described \\
\hline $\begin{array}{l}\text { Blinding of outcome as- } \\
\text { sessment (detection bias) } \\
\text { Health related quality of } \\
\text { life (self report) }\end{array}$ & Unclear risk & Not applicable \\
\hline $\begin{array}{l}\text { Incomplete outcome data } \\
\text { (attrition bias) }\end{array}$ & Low risk & \\
$\begin{array}{l}\text { Falls and fallers } \\
\text { Selective reporting (re- }\end{array}$ & Unclear risk & $\begin{array}{l}\text { Less than 20\% of fall outcome data are missing (2\%). 1 control participant had } \\
\text { porting bias) }\end{array}$ \\
\hline $\begin{array}{l}\text { Method of ascertaining } \\
\text { falls (recall bias) }\end{array}$ & Low risk & $\begin{array}{l}\text { Prespecified falls outcomes reported. Adverse events reported but not pre- } \\
\text { specified. No trial protocol or prospective trial registration }\end{array}$ \\
\hline
\end{tabular}


Helbostad 2004

\begin{tabular}{|c|c|}
\hline Methods & $\begin{array}{l}\text { Study design: RCT } \\
\text { Number of study arms: } 2 \\
\text { Length of follow-up: } 12 \text { months }\end{array}$ \\
\hline Participants & $\begin{array}{l}\text { Setting: } 6 \text { local districts in Trondheim, Norway } \\
\text { Number of participants: } 77 \\
\text { Number analysed: } 68 \\
\text { Number lost to follow-up: } 9 \\
\text { Sample: volunteers recruited through newspapers and invitations from health workers } \\
\text { Age (years): mean } 81 \text { (SD 4.5) } \\
\text { Sex: } 81 \% \text { female } \\
\text { Inclusion criteria: aged } \geq 75 \text {; fallen in last year and / or using walking aid indoors or outdoors } \\
\text { Exclusion criteria: exercising } 1 \text { or more times weekly; terminal illness; cognitive impairment (MMSE < } \\
\text { 22); recent stroke; unable to tolerate exercise }\end{array}$ \\
\hline Interventions & $\begin{array}{l}\text { 1. Combined group and home-based balance and strength training: individually-tailored progressive } \\
\text { resistance exercises, functional balance training, } 1 \text { hour sessions, } 2 \text { x ar week, for } 12 \text { weeks + home ex- } \\
\text { ercises as below ( } 2 \text { ) } \\
\text { 2. Individual home-balance and strength training: } 4 \text { non-progressive functional balance and strength } \\
\text { exercises using own body weight, } 2 \text { a day, for } 12 \text { weeks, plus } 3 \text { education group meetings }\end{array}$ \\
\hline Outcomes & $\begin{array}{l}\text { 1. Rate of falls } \\
\text { 2. Number of people who experienced 1or more falls (risk of falling) }\end{array}$ \\
\hline Duration of the study & 52 weeks \\
\hline Adherence & $\begin{array}{l}\text { Adherence measured as sessions participated, frequency of home sessions } \\
\text { 1. Group- and home-based balance and strength training: mean training sessions participated } 21 / 24 \\
\text { (range } 14 \text { - 24); mean home training sessions completed a day } 1.35(\mathrm{SD}=0.51) \\
\text { 2. Individual balance and strength training: mean group meetings participated } 2.5 / 3 \text { (range } 0-3) \text {; mean } \\
\text { home training sessions completed a day } 1.29(\mathrm{SD}=0.54)\end{array}$ \\
\hline Notes & $\begin{array}{l}\text { Source of funding: Norwegian Foundation for Research in Physiotherapy, Norwegian Research Council, } \\
\text { University of Bergen } \\
\text { Economic information: not reported }\end{array}$ \\
\hline
\end{tabular}

\section{Risk of bias}

\begin{tabular}{|c|c|c|}
\hline Bias & Authors' judgement & Support for judgement \\
\hline $\begin{array}{l}\text { Random sequence genera- } \\
\text { tion (selection bias) }\end{array}$ & Unclear risk & Quote: "randomised into one of two exercise programs" \\
\hline $\begin{array}{l}\text { Allocation concealment } \\
\text { (selection bias) }\end{array}$ & Low risk & Randomised by independent research office using sealed envelopes \\
\hline $\begin{array}{l}\text { Blinding of participants } \\
\text { and personnel (perfor- } \\
\text { mance bias) } \\
\text { All outcomes }\end{array}$ & Low risk & $\begin{array}{l}\text { Cluster-randomised trial comparing } 2 \text { types of exercise intervention. Low risk } \\
\text { of performance bias }\end{array}$ \\
\hline $\begin{array}{l}\text { Blinding of outcome as- } \\
\text { sessment (detection bias) } \\
\text { Falls }\end{array}$ & Low risk & $\begin{array}{l}\text { Falls ascertained by the same method in both groups. Assessors blind to par- } \\
\text { ticipants' assignment }\end{array}$ \\
\hline
\end{tabular}


Helbostad 2004 (Continued)

$\begin{array}{lll}\text { Blinding of outcome as- } & \text { Unclear risk } & \text { Not applicable } \\ \text { sessment (detection bias) } & \end{array}$

Fractures

\begin{tabular}{|c|c|c|}
\hline $\begin{array}{l}\text { Blinding of outcome as- } \\
\text { sessment (detection bias) } \\
\text { Hospital admission, med- } \\
\text { ical attention and adverse } \\
\text { events }\end{array}$ & Unclear risk & Not applicable \\
\hline
\end{tabular}

\begin{tabular}{|c|c|c|}
\hline $\begin{array}{l}\text { Blinding of outcome as- } \\
\text { sessment (detection bias) } \\
\text { Health related quality of } \\
\text { life (self report) }\end{array}$ & Unclear risk & Not applicable \\
\hline $\begin{array}{l}\text { Incomplete outcome data } \\
\text { (attrition bias) } \\
\text { Falls and fallers }\end{array}$ & Low risk & $\begin{array}{l}\text { Less than } 20 \% \text { of fall outcome data are missing }(12 \%) \text {. Loss of fall data was bal- } \\
\text { anced in the home training }(n=4) \text { and combined training }(n=5) \text { groups. Rea- } \\
\text { sons for data loss were balanced in the } 2 \text { groups }\end{array}$ \\
\hline $\begin{array}{l}\text { Selective reporting (re- } \\
\text { porting bias) }\end{array}$ & Unclear risk & $\begin{array}{l}\text { Minimum set of expected outcomes not reported (adverse events not report- } \\
\text { ed) }\end{array}$ \\
\hline $\begin{array}{l}\text { Method of ascertaining } \\
\text { falls (recall bias) }\end{array}$ & Low risk & $\begin{array}{l}\text { Monthly falls diary (prepaid postcard), telephone call if no response or fall re- } \\
\text { ported }\end{array}$ \\
\hline
\end{tabular}

Hirase 2015

$\begin{array}{ll}\text { Methods } & \text { Study design: RCT } \\ & \text { Number of study arms: } 3 \\ & \text { Length of follow-up: } 4 \text { months }\end{array}$

\title{
Participants
}

\author{
Setting: Nagasaki and Unzen, Japan \\ Number of participants: 93 \\ Number analysed: 86 \\ Number lost to follow-up: 7 \\ Sample: community-dwelling
}

Age (years): Foam rubber intervention mean $=82.1($ SD 5.5), sStable surface intervention mean $=82.0$

(SD 5.7), Control group: 82.2 (SD 6.3)

\section{Sex: $70 \%$ female}

Inclusion criteria: > 65 years, living at home, able to walk with or without a cane, assessed to be at high falls risk ( $\geq 4$ risk factors using falls assessment questionnaire)

Exclusion criteria: participated in exercise $\geq 4$ a month before the intervention, musculoskeletal, neurological, or cardiovascular disorders that may be aggravated by exercise, unable to respond to interview questions because of cognitive impairment

Interventions

1. Group-based balance training on foam rubber pad: 10 exercises performed in a standing position, 60minute sessions, weekly for 4 months; plus 3 home-based exercises performed daily

2. Group-based balance training on stable flat surface: same balance training programme as foam rubber mat group but performed on a stable flat surface; 60-minute sessions, weekly for 4 months; plus 3 home-based exercises performed daily 
Hirase 2015 (Continued)

3. Control group: weekly social programmes at a day centre for 4 months

\begin{tabular}{ll}
\hline Outcomes & 1. Rate of falls \\
\hline Duration of the study & 16 weeks \\
\hline Adherence & Adherence measured as class attendance, frequency of home programme \\
& $\begin{array}{l}\text { 1. Group-based balance training on foam rubber pad: } 96 \% \text { attendance of all possible classes. Per- } \\
\text { formed the home-based exercise programme } 3.5 \text { (SD: } 2.0) \text { days a week }\end{array}$ \\
& $\begin{array}{l}\text { 2. Group-based balance training on stable flat surface: } 93 \% \text { attendance of all possible classes. Per- } \\
\text { formed the home-based exercise programme 3.4 (SD: } 2.3) \text { days a week }\end{array}$ \\
& 3. Control group: $91 \%$ attendance of all possible programmes \\
\hline Notes & $\begin{array}{l}\text { Source of funding: NR, Department of Locomotive Rehabilitation Science, Unit of Rehabilitation } \\
\text { Sciences, Graduate School of Biomedical Sciences, Nagasaki University } \\
\text { Economic information: not reported }\end{array}$ \\
\hline
\end{tabular}

\section{Risk of bias}

\begin{tabular}{|c|c|c|}
\hline Bias & Authors' judgement & Support for judgement \\
\hline $\begin{array}{l}\text { Random sequence genera- } \\
\text { tion (selection bias) }\end{array}$ & Unclear risk & Process not reported \\
\hline $\begin{array}{l}\text { Allocation concealment } \\
\text { (selection bias) }\end{array}$ & Unclear risk & Quote: "using the sealed envelope method" \\
\hline $\begin{array}{l}\text { Blinding of participants } \\
\text { and personnel (perfor- } \\
\text { mance bias) } \\
\text { All outcomes }\end{array}$ & Unclear risk & Participants and personnel unblinded but impact of unblinding unknown \\
\hline $\begin{array}{l}\text { Blinding of outcome as- } \\
\text { sessment (detection bias) } \\
\text { Falls }\end{array}$ & High risk & $\begin{array}{l}\text { Quote: "The number of additional falls was recorded every week by a physi- } \\
\text { cal therapist working in each day center"" "Physical therapists working in the } \\
\text { day centers assessed the participants and implemented the intervention pro- } \\
\text { gram." Assume assessors not blinded }\end{array}$ \\
\hline $\begin{array}{l}\text { Blinding of outcome as- } \\
\text { sessment (detection bias) } \\
\text { Fractures }\end{array}$ & Unclear risk & Not applicable \\
\hline $\begin{array}{l}\text { Blinding of outcome as- } \\
\text { sessment (detection bias) } \\
\text { Hospital admission, med- } \\
\text { ical attention and adverse } \\
\text { events }\end{array}$ & Unclear risk & Not applicable \\
\hline $\begin{array}{l}\text { Blinding of outcome as- } \\
\text { sessment (detection bias) } \\
\text { Health related quality of } \\
\text { life (self report) }\end{array}$ & Unclear risk & Not applicable \\
\hline $\begin{array}{l}\text { Incomplete outcome data } \\
\text { (attrition bias) } \\
\text { Falls and fallers }\end{array}$ & Low risk & $\begin{array}{l}\text { Less than } 20 \% \text { of fall outcome data are missing }(7 \%) \text {. Loss of fall data was bal- } \\
\text { anced in the groups ( } n=3 \text { in foam rubber group, } n=2 \text { in stable and control } \\
\text { groups), with all withdrawals due to hospital admission }\end{array}$ \\
\hline
\end{tabular}


Hirase 2015 (Continued)

\begin{tabular}{lll}
$\begin{array}{l}\text { Selective reporting (re- } \\
\text { porting bias) }\end{array}$ & High risk & $\begin{array}{l}\text { Falls measured, but number of fallers not reported. Adverse events not report- } \\
\text { ed }\end{array}$ \\
\hline $\begin{array}{l}\text { Method of ascertaining } \\
\text { falls (recall bias) }\end{array}$ & Low risk & $\begin{array}{l}\text { Quote: "a diary with a monthly sheet to record the number of additional falls } \\
\text { during the follow-up period. The number of additional falls was recorded } \\
\text { every week by a physical therapist working in each day center" }\end{array}$ \\
\hline
\end{tabular}

Huang 2010

$\begin{array}{ll}\text { Methods } & \text { Study design: Cluster RCT } \\ & \text { Number of study arms: } 2 \\ & \text { Number of clusters: } 4 \text { (2 clusters included in this review) } \\ & \text { Length of follow-up: } 5 \text { months }\end{array}$

Setting: Taipei, Taiwan
Pumber of participants: 115
Number analysed: 78
Number lost to follow-up: 37
Sample: people registered as living in 4 randomly-selected villages
Age (years): mean 71.5 (SD 0.6) in people not lost to follow-up
Sex: $30 \%$ female
Inclusion criteria: aged > 65 years; living in a non-organised community of Taiwan
Exclusion criteria: immobile; living outside registered living area

Interventions

Randomised into 4 groups: 3 intervention groups ( 1 group-based Tai Chi, 1 education group, 1 Tai Chi plus education group) and 1 control group. Only group-based Tai Chi and control groups included in this review

1. Group-based Tai Chi: 13 simple movements, 40 -minute sessions, 3 a week for 20 weeks

2. Control group: usual care

Outcomes Number of people who experienced 1 or more falls (risk of falling)
2. Health-related quality of life

Duration of the study $\quad 20$ weeks

Adherence Not reported

Notes Source of funding: The National Science Council, Taiwan

Economic information: not reported

Reported results not adjusted for clustering. Raw data at 5 months used in the review and adjusted for clustering. No raw data for 18 months so not possible to adjust for clustering.

Number of clusters allocated to intervention: 1 ; number of clusters allocated to control: 1 ; number of clusters analysed (intervention): 1 ; number of clusters analysed (control): 1

Email communication regarding fall data, response received, data not included in review

\section{Risk of bias}

Bias Authors' judgement Support for judgement


Huang 2010 (Continued)

Random sequence genera- Unclear risk Quote: "The three intervention groups and one control group were then astion (selection bias) signed randomly to one each of the four selected villages."

Allocation concealment High risk
(selection bias)
(selection bias)

Blinding of participants and personnel (perfor-

mance bias)

All outcomes
Individual participant recruitment was undertaken after group allocation of the 4 villages. There was no mention of active blinding of research team members recruiting participants

\begin{tabular}{|c|c|c|}
\hline $\begin{array}{l}\text { Blinding of outcome as- } \\
\text { sessment (detection bias) } \\
\text { Falls }\end{array}$ & Unclear risk & $\begin{array}{l}\text { Insufficient information to determine how falls were monitored in each group } \\
\text { or whether assessors were blinded }\end{array}$ \\
\hline $\begin{array}{l}\text { Blinding of outcome as- } \\
\text { sessment (detection bias) } \\
\text { Fractures }\end{array}$ & Unclear risk & Not applicable \\
\hline $\begin{array}{l}\text { Blinding of outcome as- } \\
\text { sessment (detection bias) } \\
\text { Hospital admission, med- } \\
\text { ical attention and adverse } \\
\text { events }\end{array}$ & Unclear risk & Not applicable \\
\hline
\end{tabular}

\begin{tabular}{|c|c|c|}
\hline $\begin{array}{l}\text { Blinding of outcome as- } \\
\text { sessment (detection bias) } \\
\text { Health related quality of } \\
\text { life (self report) }\end{array}$ & High risk & Participants were not blinded to group allocation \\
\hline
\end{tabular}

\begin{tabular}{|c|c|c|}
\hline $\begin{array}{l}\text { Incomplete outcome data } \\
\text { (attrition bias) } \\
\text { Falls and fallers }\end{array}$ & High risk & $\begin{array}{l}\text { More than } 20 \% \text { of fall outcome data are missing }(32 \%) \text {. Loss of fall data was un- } \\
\text { balanced in the Tai Chi }(n=34) \text { and control }(n=3) \text { groups, with the reasons for } \\
\text { withdrawal not clear }\end{array}$ \\
\hline
\end{tabular}

\begin{tabular}{lll}
\hline $\begin{array}{l}\text { Selective reporting (re- } \\
\text { porting bias) }\end{array}$ & High risk & Falls measured, but number of falls not reported. Adverse events not reported \\
\hline $\begin{array}{l}\text { Method of ascertaining } \\
\text { falls (recall bias) }\end{array}$ & Unclear risk & No mention of how falls were monitored \\
& $\begin{array}{l}\text { Quote: "The fall or non-fall situation was checked at preintervention, postin- } \\
\text { tervention and at one and half year later with the aim of examining the effec- } \\
\text { tiveness of the interventions" }\end{array}$ \\
\hline Cluster-randomised trials & Unclear risk & $\begin{array}{l}\text { Individuals were recruited to the trial after the clusters were randomised and } \\
\text { personnel recruiting participants were not blind to cluster; clusters were not } \\
\text { comparable at baseline for gender or education level; missing outcomes for } \\
\text { clusters or within clusters were not reported; did not account for clustering in } \\
\text { analysis; results comparable with individually randomised trials }\end{array}$ \\
\hline
\end{tabular}

\section{Hwang 2016}

$\begin{array}{ll}\text { Methods } & \text { Study design: RCT } \\ & \text { Number of study arms: } 2\end{array}$


Hwang 2016 (Continued)

Length of follow-up: 18 months

Setting: Taipei, Taiwan
Number of participants: 456
Number analysed: 334
Number lost to follow-up: 122
Sample: community-dwelling
Age (years): mean 72
Sex: $67 \%$ female
Inclusion criteria: aged $\geq 60$ who received fall-related medical attention - an older person was pre-
sumed to have recovered from a fall injury within 6 months and who could walk independently were in-
vited by telephone to enrol in the study and participate in the baseline assessment
Exclusion criteria: major unstable cardiopulmonary disease (ischaemic chest pain or shortness of
breath on mild exertion), cognitive impairment (MMSE score < 24), and contraindications to physical
exercise (e.g. severe arthritis that limits exercise capability)

Interventions

1. Individually-supervised Tai Chi: taught individually each week for 24 consecutive weeks, 60-minute sessions, 1 a week for 6 months

2. Individually-supervised balance and strength training: exercises at increasing difficulty levels using own body weight; 60 -minute sessions, 1 a week for 6 months

1. Rate of falls
2. Number of people who experienced 1 or more falls (risk of falling)
3. Number of people who died

\begin{tabular}{ll}
\hline Duration of the study & 72 weeks \\
\hline Adherence & Adherence measured as participation in sessions \\
& $\begin{array}{l}\text { 1. Individually-supervised Tai Chi group: } 145(78 \%) \text { people participated in } 20 \text { or more sessions } \\
\text { 2. Supervised balance and strength training group: } 132 \text { (72\%) people participated in } 20 \text { or more ses- } \\
\text { sions }\end{array}$
\end{tabular}

Notes Source of funding: National Health Research Institute, Ministry of Science Technology

Economic information: not reported

\section{Risk of bias}

\begin{tabular}{lll}
\hline Bias & Authors' judgement & Support for judgement \\
\hline $\begin{array}{l}\text { Random sequence genera- } \\
\text { tion (selection bias) }\end{array}$ & Low risk & $\begin{array}{l}\text { Statisticans using computer-generated sequence; block-randomised in groups } \\
\text { of } 8\end{array}$ \\
\hline $\begin{array}{l}\text { Allocation concealment } \\
\text { (selection bias) }\end{array}$ & Low risk & $\begin{array}{l}\text { Using an automated secure website operated by an off-site independent ser- } \\
\text { vice }\end{array}$ \\
\hline $\begin{array}{l}\text { Blinding of participants } \\
\text { and personnel (perfor- } \\
\text { mance bias) }\end{array}$ & Unclear risk & Participants and personnel unblinded but impact of unblinding unknown \\
\hline
\end{tabular}


Hwang 2016 (Continued)

All outcomes

\begin{tabular}{|c|c|c|}
\hline $\begin{array}{l}\text { Blinding of outcome as- } \\
\text { sessment (detection bias) }\end{array}$ & Low risk & $\begin{array}{l}\text { Research assistants who conducted fall-related phone calls were blinded to al- } \\
\text { location }\end{array}$ \\
\hline
\end{tabular}

Falls

\begin{tabular}{|c|c|c|}
\hline $\begin{array}{l}\text { Blinding of outcome as- } \\
\text { sessment (detection bias) } \\
\text { Fractures }\end{array}$ & Unclear risk & Not applicable \\
\hline
\end{tabular}

\begin{tabular}{|c|c|c|}
\hline $\begin{array}{l}\text { Blinding of outcome as- } \\
\text { sessment (detection bias) } \\
\text { Hospital admission, med- } \\
\text { ical attention and adverse } \\
\text { events }\end{array}$ & Unclear risk & Not applicable \\
\hline $\begin{array}{l}\text { Blinding of outcome as- } \\
\text { sessment (detection bias) } \\
\text { Health related quality of } \\
\text { life (self report) }\end{array}$ & Unclear risk & Not applicable \\
\hline
\end{tabular}

\begin{tabular}{lll}
$\begin{array}{l}\text { Incomplete outcome data } \\
\text { (attrition bias) } \\
\text { Falls and fallers }\end{array}$ & High risk & More than 20\% of fall outcome data are missing (27\%) \\
\hline $\begin{array}{l}\text { Selective reporting (re- } \\
\text { porting bias) }\end{array}$ & Unclear risk & $\begin{array}{l}\text { Minimum set of expected outcomes not reported (adverse events not report- } \\
\text { ed) }\end{array}$ \\
\hline $\begin{array}{l}\text { Method of ascertaining } \\
\text { falls (recall bias) }\end{array}$ & Low risk & $\begin{array}{l}\text { Quote: "Falls were prospectively monitored and recorded daily using a di- } \\
\text { ary, and these records were mailed monthly to the study coordinator." "When } \\
\text { a participant failed to return the diary or provided incomplete data, two re- } \\
\text { search assistants blinded to the group assignment provided telephone re- } \\
\text { minders, making a maximum of five calls. Monthly follow-up of fall records was } \\
\text { continued in participants who were unavailable for certain periods". }\end{array}$
\end{tabular}

Iliffe 2015

\begin{tabular}{ll}
\hline Methods & Study design: Cluster-RCT \\
& Number of study arms: 3 \\
& Number of clusters: 42 \\
& Length of follow-up: 18 months \\
\hline Participants & Setting: London and Nottingham, UK \\
& Number of participants: 1254 \\
& Number analysed: 709 \\
& Number lost to follow-up: 545 \\
& Sample: community-dwelling \\
& Age (years): mean 73 (range 65 - 94) \\
& Sex: $62 \%$ female
\end{tabular}


Iliffe 2015 (Continued)

Inclusion criteria: $\geq 65$ years, registered with participating general practices, living independently (not in residential or nursing homes), physically able to attend group exercise

Exclusion criteria: $\geq 3$ falls in the past year, $\geq 150$ minutes of moderate-vigorous physical activity a week, uncontrolled medical conditions and significant cognitive impairment

Interventions 1. Individual Otago Exercise Programme: leg strengthening, balance exercises and walking plan, 30 minute, 3 a week for 24 weeks

2. Group-based FaME plus home training based on Otago Exercise Programme: leg and trunk strengthening, balance, flexibility, functional floor skills, walking plan, 1-hour group session a week for 24 weeks +30 -minute home exercises sessions, 2 a week for 24 weeks

3. Control group: no intervention

\begin{tabular}{|c|c|}
\hline \multirow[t]{4}{*}{ Outcomes } & 1. Rate of falls \\
\hline & 2. Number of people who experienced 1 or more falls (risk of falling) \\
\hline & 3. Health-related quality of life \\
\hline & 4. Number of people who died \\
\hline Duration of the study & 96 weeks \\
\hline \multirow[t]{3}{*}{ Adherence } & Adherence measured as home sessions completed, or class attendance \\
\hline & $\begin{array}{l}\text { 1. Individual Otago Exercise Programme: } 149(37 \%) \text { participants reported they achieved } \geq 75 \% \text { of the } \\
\text { home exercise prescription ( } 90 \text { minutes a week) }\end{array}$ \\
\hline & $\begin{array}{l}\text { 2. Group-based FaME plus home training based on Otago Exercise Programme: } 150 \text { participants (40\%) } \\
\text { attended } 75 \% \text { (or more) of classes }\end{array}$ \\
\hline \multirow[t]{3}{*}{ Notes } & $\begin{array}{l}\text { Source of funding: Health Technology Assessment programme of the National Institute for Health Re- } \\
\text { search }\end{array}$ \\
\hline & $\begin{array}{l}\text { Economic information: Mean cost per person (intervention) OEP London GBP } 88 \text {, Nottingham GBP 117; } \\
\text { FaME: London GBP 269, Nottingham GBP } 218 \text {. Health service cost OEP GBP 404, FaME GBP 412, usu- } \\
\text { al care GBP } 367 . \text { Incremental cost per fall prevented/per QALY gained: no between-group difference in } \\
\text { QALY. }\end{array}$ \\
\hline & $\begin{array}{l}\text { Number of clusters allocated to OEP: } 14 \text {; Number of clusters allocated to FaME: 14; number of clusters } \\
\text { allocated to control: } 14 \text {; number of clusters analysed (OEP): 14; number of clusters analysed (FaME): 14; } \\
\text { number of clusters analysed (control): } 14\end{array}$ \\
\hline
\end{tabular}

\section{Risk of bias}

\begin{tabular}{lll}
\hline Bias & Authors' judgement & Support for judgement \\
\hline $\begin{array}{l}\text { Random sequence genera- } \\
\text { tion (selection bias) }\end{array}$ & Low risk & $\begin{array}{l}\text { Quote: "Treatments will be assigned...using computer generated random } \\
\text { number tables, embedded in a computer programme for minimisation" }\end{array}$ \\
\hline $\begin{array}{l}\text { Allocation concealment } \\
\text { (selection bias) }\end{array}$ & Low risk & $\begin{array}{l}\text { Quote: "Practices were allocated to intervention or usual care, only after } \\
\text { all participants had been recruited. The practices, their patients and the re- } \\
\text { searchers undertaking baseline assessments were all blinded to allocation un- } \\
\text { til this point" }\end{array}$ \\
\hline
\end{tabular}

Blinding of participants Unclear risk Participants and personnel unblinded but impact of unblinding unknown
and personnel (perfor-
mance bias)
All outcomes


Iliffe 2015 (Continued)

Blinding of outcome as- High risk Falls were measured using the same method in all groups. The researchers assessment (detection bias) sessing outcomes were not blinded

Falls

Blinding of outcome as-
sessment (detection bias)
Fractures
Blinding of outcome as-
sessment (detection bias)
Hospital admission, med-
ical attention and adverse
events

\begin{tabular}{|c|c|c|}
\hline $\begin{array}{l}\text { Blinding of outcome as- } \\
\text { sessment (detection bias) } \\
\text { Health related quality of } \\
\text { life (self report) }\end{array}$ & High risk & Participants not blinded to group allocation \\
\hline
\end{tabular}

\begin{tabular}{lll}
\hline $\begin{array}{l}\text { Incomplete outcome data } \\
\text { (attrition bias) } \\
\text { Falls and fallers }\end{array}$ & High risk & More than 20\% of fall outcome data are missing (44\%) at 18-month follow-up \\
\hline $\begin{array}{l}\text { Selective reporting (re- } \\
\text { porting bias) }\end{array}$ & Unclear risk & $\begin{array}{l}\text { Minimum set of expected outcomes not reported (adverse events not report- } \\
\text { ed) }\end{array}$ \\
\hline $\begin{array}{l}\text { Method of ascertaining } \\
\text { falls (recall bias) }\end{array}$ & High risk & $\begin{array}{l}\text { Self-completed fall diaries (completed monthly during the 6-month interven- } \\
\text { tion period and every } 3 \text { months from } 6 \text { to } 24 \text { months follow-up). Telephone } \\
\text { contact with non-responders and fallers }\end{array}$ \\
\hline Cluster-randomised trials & Low risk & $\begin{array}{l}\text { After all participants from a practice had been recruited, the practice was in- } \\
\text { dividually allocated to a study arm by the London co-ordinating centre; base- } \\
\text { line comparability of clusters was not reported; missing outcomes for clusters } \\
\text { or within clusters were not reported; accounted for the clustered design in the } \\
\text { analysis; results comparable with individually randomised trials }\end{array}$ \\
\hline
\end{tabular}

Irez 2011

\begin{tabular}{ll}
\hline Methods & Study design: RCT \\
& Number of study arms: 2 \\
Length of follow-up: 3 months \\
\hline
\end{tabular}

\title{
Participants
}

\author{
Setting: Turkey \\ Number of participants: 60 \\ Number analysed: 60 \\ Number lost to follow-up: 0 \\ Sample: community-dwelling women \\ Age (years): Intervention mean 72.8 (SD 6.7), Control mean 78.0 (SD 5.7) \\ Sex: $100 \%$ female
}


Irez 2011 (Continued)

Inclusion criteria: Healthy, $>65$ years of age, relatively sedentary (undertaking no leisure time physical activity or $<30$ minutes of physical activity a day) for at least a year

Exclusion criteria: Any significant health problem or orthopaedic problem that would keep them from fully participating in the intervention protocol or the inability to attend at least $80 \%$ of the training sessions, or both

Interventions $\quad$ 1. Group-based Pilates: mat exercises, used TheraBand elastic resistance bands, Pilates or exercise
balls; 60 minutes, 3 a week for 12 weeks

2. Control group: usual activity

\begin{tabular}{ll}
\hline Outcomes & 1. Rate of falls \\
\hline Duration of the study & 12 weeks \\
\hline Adherence & Adherence measured as sessions completed \\
& 1. Group-based Pilates group: completed 32/36 sessions (92\% participation rate) \\
\hline Notes & Source of funding: Mugla University, School of Physical Education and Sports \\
& Economic information: not reported \\
\hline
\end{tabular}

\section{Risk of bias}

\begin{tabular}{lll}
\hline Bias & Authors' judgement & Support for judgement \\
\hline $\begin{array}{l}\text { Random sequence genera- } \\
\text { tion (selection bias) }\end{array}$ & Unclear risk & Method not reported \\
\hline $\begin{array}{l}\text { Allocation concealment } \\
\text { (selection bias) }\end{array}$ & Unclear risk & Not reported \\
\hline
\end{tabular}

Blinding of participants $\quad$ Unclear risk Participants and personnel unblinded but impact of unblinding unknown
and personnel (perfor-
mance bias)
All outcomes

\begin{tabular}{|c|c|c|}
\hline $\begin{array}{l}\text { Blinding of outcome as- } \\
\text { sessment (detection bias) }\end{array}$ & High risk & $\begin{array}{l}\text { Fall calendars were returned to the treating physiotherapist, who also con- } \\
\text { ducted follow-up phone-calls }\end{array}$ \\
\hline
\end{tabular}

Falls

\begin{tabular}{|c|c|c|}
\hline $\begin{array}{l}\text { Blinding of outcome as- } \\
\text { sessment (detection bias) } \\
\text { Fractures }\end{array}$ & Unclear risk & Not applicable \\
\hline
\end{tabular}

Blinding of outcome as-
sessment (detection bias)
Hospital admission, med-
ical attention and adverse
events

Unclear risk Not applicable

Blinding of outcome as- Unclear risk Not applicable
sessment (detection bias) Health related quality of life (self report)

Incomplete outcome data Low risk No missing fall data
(attrition bias)


Irez 2011 (Continued)

Falls and fallers

$\begin{array}{lll}\begin{array}{l}\text { Selective reporting (re- } \\ \text { porting bias) }\end{array} & \text { High risk } & \begin{array}{l}\text { Falls measured, but number of fallers not reported. Adverse events not report- } \\ \text { ed. }\end{array}\end{array}$

\begin{tabular}{ll}
\hline $\begin{array}{l}\text { Method of ascertaining } \\
\text { falls (recall bias) }\end{array}$ & Low risk \\
& $\begin{array}{l}\text { Falls calendars, completed daily. Calendars were returned to the treating } \\
\text { physiotherapist at the end of each month. Physiotherapists followed up non- } \\
\text { returns }\end{array}$ \\
\hline
\end{tabular}

Iwamoto 2009

\begin{tabular}{ll}
\hline Methods & Study design: RCT \\
Number of study arms: 2 \\
Length of follow-up: 5 months \\
\hline Setting: Tokyo, Japan \\
Number of participants: 68 \\
Number analysed: 67 \\
Number lost to follow-up: 1 Sample: volunteer patients from Department of Orthopaedic Surgery (2 hospitals) and Orthopaedic \\
Clinics (3) \\
Age (years): mean 76.4 (SD 5.6), range 66 - 88 \\
Sex: $90 \%$ female \\
Inclusion criteria: aged > 50 years; fully ambulatory; able to complete physical assessments \\
Exclusion criteria: using walking aids; severe kyphosis due to osteoporotic vertebral fractures; acute ill- \\
ness; severe cardiovascular disease
\end{tabular}

Interventions

1. Group-based balance and gait training: supervised exercise programme (calisthenics, balance, muscle power, walking ability training); 30 minutes, 3 a week for 20 weeks

2. Control group: no exercise

\begin{tabular}{ll}
\hline Outcomes & 1. Number of people who experienced 1 or more falls (risk of falling) \\
\hline Duration of the study & 20 weeks \\
\hline Adherence & Adherence not defined. Completion rate: \\
& $\begin{array}{l}\text { 1. Group-based balance and gait training group: all participants completed the 5-month trial; adher- } \\
\text { ence not defined }\end{array}$ \\
2. Control group: $33 / 34$ participants completed trial
\end{tabular}

\section{Notes}

Source of funding: Keio University School of Medicine

Economic information: not reported

Place of residence not specified, i.e. not specifically community-dwelling, but not preventing falls in hospital or specifically in an institution

\section{Risk of bias}


Iwamoto 2009 (Continued)

Random sequence genera- Unclear risk Q Quote: "The subjects were randomly divided into two groups ..." tion (selection bias)

$\begin{aligned} & \text { Allocation concealment } \\ & \text { (selection bias) }\end{aligned}$
Unclear risk Quote: "The subjects were randomly divided into two groups ..."

Blinding of participants Unclear risk Participants and personnel unblinded but impact of unblinding unknown

and personnel (perfor-

mance bias)

All outcomes

\begin{tabular}{lll}
\hline Blinding of outcome as- & High risk & Assessor blinding is unclear, but assume obtaining "information regarding falls \\
sessment (detection bias) & & and fractures .... every week by directly asking the participants" occured for \\
Falls & exercise participants during class and control participants were assessed at \\
& $21 / 2$ and 5 months
\end{tabular}

\begin{tabular}{lll}
\hline $\begin{array}{l}\text { Blinding of outcome as- } \\
\text { sessment (detection bias) }\end{array}$ & High risk & $\begin{array}{l}\text { Fractures appear to be self-reported with no confirmation from medical } \\
\text { Fractures }\end{array}$
\end{tabular}

\begin{tabular}{|c|c|c|}
\hline $\begin{array}{l}\text { Blinding of outcome as- } \\
\text { sessment (detection bias) } \\
\text { Hospital admission, med- } \\
\text { ical attention and adverse } \\
\text { events }\end{array}$ & Unclear risk & Method of ascertaining adverse events unclear \\
\hline $\begin{array}{l}\text { Blinding of outcome as- } \\
\text { sessment (detection bias) } \\
\text { Health related quality of } \\
\text { life (self report) }\end{array}$ & Unclear risk & Not applicable \\
\hline $\begin{array}{l}\text { Incomplete outcome data } \\
\text { (attrition bias) } \\
\text { Falls and fallers }\end{array}$ & Low risk & $\begin{array}{l}\text { Less than } 20 \% \text { of fall outcome data are missing }(2 \%) \text {. Only missing data are } \\
\text { from } 1 \text { control participant due to noncompliance }\end{array}$ \\
\hline $\begin{array}{l}\text { Selective reporting (re- } \\
\text { porting bias) }\end{array}$ & High risk & Falls were measured, but number of fallers not reported \\
\hline $\begin{array}{l}\text { Method of ascertaining } \\
\text { falls (recall bias) }\end{array}$ & High risk & $\begin{array}{l}\text { Quote: "The incidence of fall and fracture ... was assessed } 2.5 \text { and } 5 \text { months af- } \\
\text { ter the start of the trial. In particular, information regarding falls and fractures } \\
\text { was obtained every week by directly asking the participants." No mention of } \\
\text { diaries or calendars. Retrospective recall. Possibly only the intervention group } \\
\text { were asked every week (at class) and remainder at } 2 \frac{1}{2} \text { and } 5 \text { months. }\end{array}$ \\
\hline
\end{tabular}

Kamide 2009

\begin{tabular}{ll}
\hline Methods & Study design: RCT \\
& Number of study arms: 2 \\
& Length of follow-up: 6 months \\
\hline Participants & Setting: Kanagawa, Japan \\
& Number of participants: 57 \\
& Number analysed: 43
\end{tabular}


Kamide 2009 (Continued)

Number lost to follow-up: 14

Sample: women registered at an employment agency for older people (see Notes)

Age (years): mean 71 (SD 3.6)

Sex: $100 \%$ female

Inclusion criteria: aged $\geq 65$ years; community-dwelling; independently mobile; no restriction on physical activities

Exclusion criteria: cerebrovascular, cardiopulmonary, neuromuscular, liver, or kidney disease; hyperparathyroidism; unstable diabetes mellitus or hypertension; fracture of spine or lower limbs; taking prednisolone; exercising regularly

Interventions $\quad$ 1. Individual balance and strength training: home-based exercises, Theraband used for moderate-intensity lower-limb strength training, no home visits but monthly telephone or mail contact; performed $\geq 3$ days a week for 24 weeks

2. Control: usual activities, telephone or mail contact from PT every 3 months

\begin{tabular}{ll}
\hline Outcomes & 1. Number of people who experienced 1 or more falls (risk of falling) \\
\hline Duration of the study & 52 weeks \\
\hline Adherence & Adherence measured as frequency of sessions completed \\
& $\begin{array}{l}\text { 1. Individual balance and strength training group: } 19 \text { of } 23(83 \%) \text { intervention participants completed }> \\
3 \text { a week, } 21 \text { of } 23(91 \%) \text { intervention participants completed }>2 \text { a week }\end{array}$ \\
\hline Notes & $\begin{array}{l}\text { Source of funding: Univers Foundation, Tokyo } \\
\text { Economic information: not reported } \\
\text { Employment agency providing light work or volunteer activities for older people and encouraging so- } \\
\text { cial activities }\end{array}$
\end{tabular}

\section{Risk of bias}

\begin{tabular}{|c|c|c|}
\hline Bias & Authors' judgement & Support for judgement \\
\hline $\begin{array}{l}\text { Random sequence genera- } \\
\text { tion (selection bias) }\end{array}$ & Low risk & $\begin{array}{l}\text { Quote: "The random assignment procedure was performed using random } \\
\text { numbers generated by a computer program ..." }\end{array}$ \\
\hline $\begin{array}{l}\text { Allocation concealment } \\
\text { (selection bias) }\end{array}$ & Unclear risk & $\begin{array}{l}\text { Quote: "The subjects were randomly assigned to either the home-based exer- } \\
\text { cise group or the control group". Insufficient information to permit judgement }\end{array}$ \\
\hline \multirow[t]{2}{*}{$\begin{array}{l}\text { Blinding of participants } \\
\text { and personnel (perfor- } \\
\text { mance bias) } \\
\text { All outcomes }\end{array}$} & High risk & $\begin{array}{l}\text { Participants and therapists aware of group allocation. Intervention group: } \\
\text { Quote: "the therapist contacted each subject by telephone or mail every } \\
\text { month to maintain their motivation." Control group: }\end{array}$ \\
\hline & & $\begin{array}{l}\text { Quote: "The subjects who were assigned to the control group were instruct- } \\
\text { ed to continue with their usual daily activities, with no restrictions on their ex- } \\
\text { ercise activities. A therapist contacted them every } 3 \text { months by telephone or } \\
\text { mail." }\end{array}$ \\
\hline $\begin{array}{l}\text { Blinding of outcome as- } \\
\text { sessment (detection bias) } \\
\text { Falls }\end{array}$ & Unclear risk & $\begin{array}{l}\text { Quote: "Functional capacity, physical function, and bone mineral density were } \\
\text { assessed in all subjects in both groups before and after the } 6 \text {-month inter- } \\
\text { vention. The staff performing the assessments were blinded to each subject's } \\
\text { group assignment. Falls were also assessed before and after the } 12 \text {-month fol- } \\
\text { lowup." Unclear if assessors were blinded. Assume method of fall asessment } \\
\text { was the same in both groups }\end{array}$ \\
\hline
\end{tabular}


Kamide 2009 (Continued)

Blinding of outcome as-
sessment (detection bias) $\quad$ Unclear risk Not applicable

Fractures

\begin{tabular}{|c|c|c|}
\hline $\begin{array}{l}\text { Blinding of outcome as- } \\
\text { sessment (detection bias) } \\
\text { Hospital admission, med- } \\
\text { ical attention and adverse } \\
\text { events }\end{array}$ & Unclear risk & Not applicable \\
\hline
\end{tabular}

\begin{tabular}{|c|c|c|}
\hline $\begin{array}{l}\text { Blinding of outcome as- } \\
\text { sessment (detection bias) } \\
\text { Health related quality of } \\
\text { life (self report) }\end{array}$ & Unclear risk & Not applicable \\
\hline $\begin{array}{l}\text { Incomplete outcome data } \\
\text { (attrition bias) } \\
\text { Falls and fallers }\end{array}$ & High risk & More than $20 \%$ of fall outcome data are missing $(25 \%)$. \\
\hline $\begin{array}{l}\text { Selective reporting (re- } \\
\text { porting bias) }\end{array}$ & High risk & Falls were measured, but number of falls not reported \\
\hline $\begin{array}{l}\text { Method of ascertaining } \\
\text { falls (recall bias) }\end{array}$ & High risk & $\begin{array}{l}\text { Quote: "Falls were also assessed before and after the 12-month followup." No } \\
\text { concurrent recording described. No mention of frequent telephone monitor- } \\
\text { ing }\end{array}$ \\
\hline
\end{tabular}

\section{Karinkanta 2007}

\begin{tabular}{ll}
\hline Methods & Study design: RCT \\
Number of study arms: 4 \\
Length of follow-up: 12 months \\
\hline Setting: Tampere, Finland \\
Number of participants: 149 \\
Number analysed: 144 \\
Number lost to follow-up: 5 \\
Sample: community-dwelling women \\
Age (years): Balance group mean 72.9 (SD 2.3 ), Combined group mean 72.9 (SD 2.2 ), Resistance group \\
mean 72.7 (SD 2.5$)$, Control group mean 72.0 (SD 2.1 ) \\
Sex: $100 \%$ female \\
Inclusion criteria: Willingness to participate, aged 70 - 79 years, female, full understanding of the study \\
procedures, no history of any illness that would contraindicate exercise or limiting participation in ex- \\
ercise, no history of any illness that affects the bones or balance, No uncorrected vision problems, not \\
taking medications known to affect balance or bone metabolism (for 12 months prior to recruitment) \\
Exclusion criteria: Already involved in intense exercise > twice a week \\
BMD score T score <-2.5 in femoral neck
\end{tabular}

Interventions

1. Group-based balance and agility training: static and dynamic balance, agility training, jumps and other impacts, and changes of direction exercises, 50-minute sessions, 3 a week for 12 months 
Karinkanta 2007 (Continued)

2. Group-based balance and strength training: strength and balance training as described in (1) and (3) on alternate weeks, 50-minute sessions, 3 a week for 12 months

3. Group-based resistance training: tailored resistance exercises for large muscle groups using machines tailored up to $70-80 \%$ of 1 RM, 50 -minute sessions, 3 a week for 12 months

4. Control group: asked to maintain same level of activity

\begin{tabular}{ll}
\hline Outcomes & 1. Rate of falls \\
2. Number of people who experienced 1 or fall-related fractures \\
3. Number of people who experienced a fall requiring medical attention \\
\hline Duration of the study & 52 weeks \\
\hline Adherence & Adherence measured as attendance rate \\
1. Group-based balance and agility training: mean attendance rate $59 \%$ \\
2. Group-based balance and strength training: mean attendance rate $67 \%$ \\
3. Group-based resistance training: mean attendance rate $74 \%$
\end{tabular}

Notes Source of funding: Academy of Finland, the Finnish Ministry of Education, and the Medical Research Fund of the Tampere University Hospital

Economic information: not reported

\section{Risk of bias}

\begin{tabular}{lll}
\hline Bias & Authors' judgement & Support for judgement \\
\hline $\begin{array}{l}\text { Random sequence genera- } \\
\text { tion (selection bias) }\end{array}$ & Low risk & Quote: "Computer-generated randomization list" \\
\hline $\begin{array}{l}\text { Allocation concealment } \\
\text { (selection bias) }\end{array}$ & Low risk & Blinded statistician allocated participants \\
\hline $\begin{array}{l}\text { Blinding of participants } \\
\begin{array}{l}\text { and personnel (perfor- } \\
\text { mance bias) }\end{array}\end{array}$ & Unclear risk & Participants and personnel unblinded but impact of unblinding unknown \\
All outcomes & \\
\hline
\end{tabular}

\begin{tabular}{|c|c|c|}
\hline $\begin{array}{l}\text { Blinding of outcome as- } \\
\text { sessment (detection bias) } \\
\text { Falls }\end{array}$ & Unclear risk & $\begin{array}{l}\text { Assume falls assessed using same method for all participants. Unclear whether } \\
\text { researcher assessing files was blinded }\end{array}$ \\
\hline $\begin{array}{l}\text { Blinding of outcome as- } \\
\text { sessment (detection bias) } \\
\text { Fractures }\end{array}$ & Low risk & Medical files examined for fractures by researcher blinded to group allocation \\
\hline
\end{tabular}

Blinding of outcome as- Low risk sessment (detection bias) Hospital admission, medical attention and adverse events
Medical files examined for injurious falls by researcher blinded to group allocation

\begin{tabular}{lll}
\hline Blinding of outcome as- & Unclear risk & Not applicable \\
sessment (detection bias)
\end{tabular}


Karinkanta 2007 (Continued) Health related quality of life (self report)

\begin{tabular}{|c|c|c|}
\hline $\begin{array}{l}\text { Incomplete outcome data } \\
\text { (attrition bias) } \\
\text { Falls and fallers }\end{array}$ & Low risk & $\begin{array}{l}\text { Less than } 20 \% \text { of fall outcome data are missing }(3 \%) \text {. Missing data were bal- } \\
\text { anced between balance group }(n=2) \text {, combination group }(n=2) \text { and control ( } n \\
=1 \text { ), with } 2 \text { participants dying ( } 1 \text { balance, } 1 \text { control) and the remaining } 3 \text { losing } \\
\text { interest }\end{array}$ \\
\hline
\end{tabular}

$\begin{array}{lll}\begin{array}{l}\text { Selective reporting (re- } \\ \text { porting bias) }\end{array} & \text { High risk } & \begin{array}{l}\text { Falls were measured, but number of fallers not reported. Adverse events not } \\ \text { reported }\end{array}\end{array}$

Method of ascertaining $\quad$ High risk $\quad$ Medical files examined for injurious falls
falls (recall bias)

\section{Kemmler 2010}

\begin{tabular}{ll}
\hline Methods & Study design: RCT \\
& Number of study arms: 2 \\
& Length of follow-up: 18 months \\
\hline Participants & Setting: Erlangen-Nuremberg area, Germany \\
& Number of participants: 246 \\
& Number analysed: 227 \\
& Number lost to follow-up: 19 \\
& Sample: female members of Siemens Health Insurance living in Erlangen-Nuremberg area \\
& Age (years): mean 69 (SD 4) \\
& Sex: $100 \%$ female \\
& Inclusion criteria: aged $\geq 65 ;$ community-dwelling; consenting \\
& Exclusion criteria: diseases affecting bone metabolism or fall risk; medication affecting bone metabo- \\
lism or fall risk; history of profound coronary heart diseases (stroke, cardiac events), acute or chronic \\
inflammatory diseases, or secondary osteoporosis; participation in exercise studies during previous 2 \\
years; very low physical capacity (<50 W during ergometry)
\end{tabular}

Interventions

1. Group-based balance, gait, flexibility and strength training plus home practice: progressive highintensity exercise programme (aerobic dance, static and dynamic balance training, functional gymnastics, isometric strength training, and stretching for trunk, hip, and thigh, and upper body exercises using elastic belts), 60-minute, 2 a week; plus progressive strength and flexibility home exercises, 20minute, 2 a week for 18 months

2. Group-based low-intensity, low-frequency balance and endurance training: low- to moderate-intensity "Wellness programme" (relaxation, games/interaction, general co-ordination, endurance, balance, dances, body sensitivity, muscle strength, breathing, and flexibility); 1 hour, 1 a week for 10 weeks then 10 week rest

1. Rate of falls
2. Number of people who experienced 1 or more falls (risk of falling)
3. Number of people who died

Duration of the study

72 weeks 
Kemmler 2010 (Continued)

Adherence Adherence measured as session attendance, frequency of home training

1. Group-based balance, gait, flexibility and strength training plus home practice: mean attendance rate, $76 \%$ (SD 8\%) group training, 42\% (SD 5\%) for home training

2. Control: mean attendance rate, $72 \%$ (SD 9\%)

Notes

Source of funding: Siemens Betriebs Krankenkasse, Behinderten- und Rehabilitations- Sportverband Bayern, Netzwerk Knochengesundheit e.V., Opfermann Arzneimittel GmbH, Thera-Band, Institute of Sport Science, Institute of Medical Physics

Economic information: Mean total healthcare service costs: Exercise group EUR 2255, Control group EUR 2780

Cost analysis in primary reference

\section{Risk of bias}

\begin{tabular}{|c|c|c|}
\hline Bias & Authors' judgement & Support for judgement \\
\hline $\begin{array}{l}\text { Random sequence genera- } \\
\text { tion (selection bias) }\end{array}$ & Low risk & Quote: "Computer-generated block randomization" \\
\hline $\begin{array}{l}\text { Allocation concealment } \\
\text { (selection bias) }\end{array}$ & Low risk & $\begin{array}{l}\text { Quote: "The allocation sequence and group assignment were performed by } \\
\text { the Institute of Biometry and Epidemiology. Participants were enrolled by the } \\
\text { Institute of Medical Physics" }\end{array}$ \\
\hline $\begin{array}{l}\text { Blinding of participants } \\
\text { and personnel (perfor- } \\
\text { mance bias) } \\
\text { All outcomes }\end{array}$ & Low risk & $\begin{array}{l}\text { Quote: "The study was blinded for the outcome assessors and participants ..." } \\
\text { "To blind the participants, the control group performed a program that fo- } \\
\text { cused on well-being and was designed not to cause physical adaptations" } \\
\text { "The effectiveness of the blinding in the control group was proven in struc- } \\
\text { tured interviews conducted by the primary investigators at the end of the } 18 \\
\text { months". Assume no blinding of personnel; impact is unclear }\end{array}$ \\
\hline
\end{tabular}

\begin{tabular}{lll}
\hline $\begin{array}{l}\text { Blinding of outcome as- } \\
\text { sessment (detection bias) } \\
\text { Falls }\end{array}$ & Low risk & $\begin{array}{l}\text { Falls assessed using same method for all participants. Outcome assessors } \\
\text { were blind to allocation }\end{array}$ \\
\hline $\begin{array}{l}\text { Blinding of outcome as- } \\
\text { sessment (detection bias) }\end{array}$ & High risk & $\begin{array}{l}\text { Quote: "Injurious falls and overall fractures were monitored daily with the use } \\
\text { of fall calendars compiled by the participants. Outcome assessors contacted } \\
\text { subjects who fell and nonresponders monthly by telephone". No report of ra- } \\
\text { diological confirmation of fractures }\end{array}$ \\
\hline
\end{tabular}

Blinding of outcome as-

Unclear risk

Not applicable sessment (detection bias) Hospital admission, medical attention and adverse events

\begin{tabular}{lll}
\hline $\begin{array}{l}\text { Blinding of outcome as- } \\
\text { sessment (detection bias) } \\
\text { Health related quality of } \\
\text { life (self report) }\end{array}$ & Unclear risk & Not applicable \\
\hline $\begin{array}{l}\text { Incomplete outcome data } \\
\text { (attrition bias) }\end{array}$ & Low risk & $\begin{array}{l}\text { Less than } 20 \% \text { of fall outcome data are missing }(8 \%) \text {. Missing data were bal- } \\
\text { anced between high-intensity }(n=8) \text { and low-intensity }(n=11) \text { groups, with } \\
\text { balls and fallers }\end{array}$ \\
\hline
\end{tabular}


Kemmler 2010 (Continued)

Selective reporting (re- High risk $\quad$ Falls were measured, but number of falls was not reported. Adverse events porting bias) were not reported

Method of ascertaining Low risk

falls (recall bias)
Quote: "Injurious falls and overall fractures were monitored daily with the use of fall calendars compiled by the participants. Outcome assessors contacted subjects who fell and nonresponders monthly by telephone."

\section{Kerse 2010}

\begin{tabular}{ll}
\hline Methods & Study design: RCT \\
Number of study arms: 2 \\
Length of follow-up: 12 months \\
\hline Setting: Auckland, New Zealand \\
Number of participants: 193 \\
Number analysed: 193 \\
Number lost to follow-up: 0 \\
Sample: community-dwelling \\
Age (years): mean 81.1 (SD 4.4) \\
Sex: $58 \%$ female \\
Inclusion criteria: aged 75 years or older, were community-dwelling, were able to communicate in Eng- \\
lish to complete assessments, positive depression screen (answered yes to 2 of the 3 depression screen \\
questions) and that they had no severe dementia or unstable medical conditions precluding participa- \\
tion in a physical activity programme \\
Exclusion criteria: see inclusion criteria
\end{tabular}

Interventions

1. Individual Otago Exercise Programme: home-based programme which comprised moderate-intensity balance retraining, 'progressive resistance' lower limb-strengthening exercises, upper limb strengthening, walking, goal setting, and social enrichment; leg and arm weights used $(1,2,3 \mathrm{~kg}) ; \geq 30$ minutes, 3 a week for 6 months; total of $8 \times 1$-hour visits to discuss, adjust the programme and motivate

2. Control group: 8 social visits with standardised conversation for a similar amount of time to the intervention participants

1. Rate of falls
$\begin{aligned} & \text { 2. Number of people who experienced } 1 \text { or more falls (risk of falling) } \\ & \text { 3. Health-related quality of life } \\ & \text { 4. Number of people who died }\end{aligned}$

Duration of the study 52 weeks

Adherence
Adherence measured as number of visits received, frequency of exercises
1. Individual Otago Exercise Programme: $81 / 97$ participants (84\%) received all the intervention visits, $6 / 97$ had < 6 visits;
During the first 6 months: 
Kerse 2010 (Continued)

$29 \%$ exercised $\geq 3$ a week and $37 \%$ walked $\geq 3$ a week

$65 \%$ exercised $\geq 2$ a week and $63 \%$ walked $\geq 2$ a week

At 12 months:

$25 \%$ exercised $\geq 3$ a week and $37 \%$ walked $\geq 3$ a week

$55 \%$ exercised $\geq 2$ a week and $59 \%$ walked $\geq 2$ a week

7 participants performed the programme almost daily

2. Control group: $86 \%$ completed all visits

Notes Source of funding: New Zealand Health Research Council, University of Auckland Research Committee

Economic information: not reported

Email communication to obtain fall data, response received, data included in review

\section{Risk of bias}

\begin{tabular}{|c|c|c|}
\hline Bias & Authors' judgement & Support for judgement \\
\hline $\begin{array}{l}\text { Random sequence genera- } \\
\text { tion (selection bias) }\end{array}$ & Low risk & Computer-generated random numbers \\
\hline $\begin{array}{l}\text { Allocation concealment } \\
\text { (selection bias) }\end{array}$ & Unclear risk & Method of concealment is not described \\
\hline $\begin{array}{l}\text { Blinding of participants } \\
\text { and personnel (perfor- } \\
\text { mance bias) } \\
\text { All outcomes }\end{array}$ & High risk & Participants and personnel unblinded but impact of unblinding unknown \\
\hline $\begin{array}{l}\text { Blinding of outcome as- } \\
\text { sessment (detection bias) } \\
\text { Falls }\end{array}$ & Low risk & $\begin{array}{l}\text { Assessment of falls was the same in both groups } \\
\text { Quote: "The research nurses conducting follow-up assessments were blind- } \\
\text { ed to the participants' group allocation. To maintain this blinding, immediate- } \\
\text { ly before the follow-up visits, participants were reminded by a telephone call } \\
\text { from a researcher not to talk to the assessment nurses about the physical ac- } \\
\text { tivity program or who had been visiting them." }\end{array}$ \\
\hline $\begin{array}{l}\text { Blinding of outcome as- } \\
\text { sessment (detection bias) } \\
\text { Fractures }\end{array}$ & Unclear risk & Not applicable \\
\hline $\begin{array}{l}\text { Blinding of outcome as- } \\
\text { sessment (detection bias) } \\
\text { Hospital admission, med- } \\
\text { ical attention and adverse } \\
\text { events }\end{array}$ & Unclear risk & Not applicable \\
\hline
\end{tabular}

Blinding of outcome as- High risk Participants not blinded to group allocation

sessment (detection bias)

Health related quality of

life (self report)

Incomplete outcome data Low risk No missing falls data

(attrition bias)

Falls and fallers 
Kerse 2010 (Continued)

\begin{tabular}{|c|c|c|}
\hline $\begin{array}{l}\text { Selective reporting (re- } \\
\text { porting bias) }\end{array}$ & Uncl & $\begin{array}{l}\text { Minimum set of expected outcomes not reported (adverse events not report- } \\
\text { ed) }\end{array}$ \\
\hline
\end{tabular}

High risk

Interval recall. Falls were ascertained by self-report at 6 months and 12

Method of ascertaining

falls (recall bias) months

Kim 2014

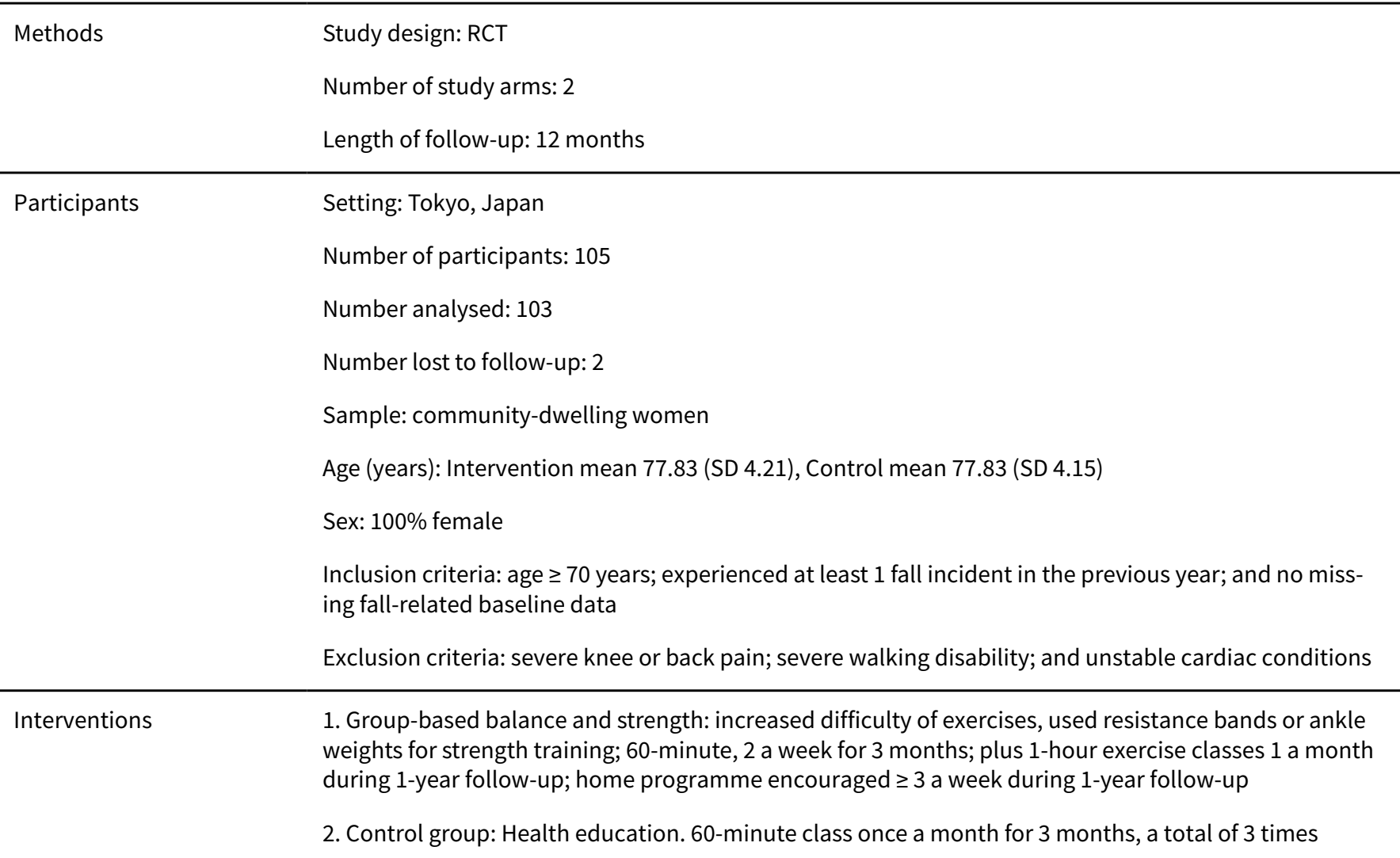

Outcomes

1. Number of people who experienced 1 or more falls (risk of falling)

2. Number of people who experienced 1 or more fall-related fractures

\begin{tabular}{ll}
\hline Duration of the study & 52 weeks \\
\hline Adherence & Adherence measured as session attendance, frequency of home exercises, mean exercise time \\
& $\begin{array}{l}\text { 1. Group-based balance and strength group: mean attendance rate during intervention, } 75 \% \text { (range } 64 \\
-86 \%) ; \text { mean frequency home exercises 3.4 a week; mean exercise time } 24.9 \text { minutes }\end{array}$
\end{tabular}

Notes

Source of funding: Ministry of Health and Welfare of Japan, Japan Society for the Promotion of Science

Economic information: not reported

\section{Risk of bias}

\section{Bias}

Authors' judgement Support for judgement 
Kim 2014 (Continued)

\begin{tabular}{|c|c|c|}
\hline $\begin{array}{l}\text { Random sequence genera- } \\
\text { tion (selection bias) }\end{array}$ & Low risk & Computer-generated random numbers \\
\hline $\begin{array}{l}\text { Allocation concealment } \\
\text { (selection bias) }\end{array}$ & Unclear risk & $\begin{array}{l}\text { Quote: "The allocation process was blinded". Insufficient information to per- } \\
\text { mit judgement. }\end{array}$ \\
\hline $\begin{array}{l}\text { Blinding of participants } \\
\text { and personnel (perfor- } \\
\text { mance bias) } \\
\text { All outcomes }\end{array}$ & Unclear risk & Participants and personnel unblinded but impact of unblinding unknown \\
\hline $\begin{array}{l}\text { Blinding of outcome as- } \\
\text { sessment (detection bias) } \\
\text { Falls }\end{array}$ & Low risk & $\begin{array}{l}\text { Fall diaries were collected at 1-year follow-up } \\
\text { Quote: "The investigators evaluating the effects of the exercise treatment were } \\
\text { blind to intervention allocations" }\end{array}$ \\
\hline $\begin{array}{l}\text { Blinding of outcome as- } \\
\text { sessment (detection bias) } \\
\text { Fractures }\end{array}$ & High risk & $\begin{array}{l}\text { Participants were asked about fractures by face-to-face interview at baseline, } 3 \\
\text { month and } 1 \text { year. No radiological confirmation }\end{array}$ \\
\hline $\begin{array}{l}\text { Blinding of outcome as- } \\
\text { sessment (detection bias) } \\
\text { Hospital admission, med- } \\
\text { ical attention and adverse } \\
\text { events }\end{array}$ & Unclear risk & Not applicable \\
\hline
\end{tabular}

\begin{tabular}{|c|c|c|}
\hline $\begin{array}{l}\text { Blinding of outcome as- } \\
\text { sessment (detection bias) } \\
\text { Health related quality of } \\
\text { life (self report) }\end{array}$ & Unclear risk & Not applicable \\
\hline $\begin{array}{l}\text { Incomplete outcome data } \\
\text { (attrition bias) } \\
\text { Falls and fallers }\end{array}$ & Low risk & $\begin{array}{l}\text { Less than } 20 \% \text { of fall outcome data are missing }(2 \%) \text {. Missing data were bal- } \\
\text { anced between the exercise }(n=2) \text { and control }(n=1) \text { groups, with reasonable } \\
\text { reasons for loss of data in the } 2 \text { groups (exercise: reduced motivation }=1 \text {, hos- } \\
\text { pitalisation }=1 \text {; control: } \text { moved house }=1 \text { ) }\end{array}$ \\
\hline
\end{tabular}

\begin{tabular}{|c|c|c|}
\hline $\begin{array}{l}\text { Selective reporting (re- } \\
\text { porting bias) }\end{array}$ & High risk & $\begin{array}{l}\text { Falls were measured, but number of falls was not reported. Adverse events } \\
\text { were not reported }\end{array}$ \\
\hline
\end{tabular}

\begin{tabular}{|c|c|c|}
\hline $\begin{array}{l}\text { Method of ascertaining } \\
\text { falls (recall bias) }\end{array}$ & Unclear risk & $\begin{array}{l}\text { Falls diary, distributed at 3-month assessment and collected at 1-year fol- } \\
\text { low-up }\end{array}$ \\
\hline
\end{tabular}

\section{Korpelainen 2006}

\begin{tabular}{ll}
\hline Methods & Study design: RCT \\
& Number of study arms: 2 \\
& Length of follow-up: 30 months \\
\hline Participants & Setting: Oulu, Finland \\
& Number of participants: 160 \\
& Number analysed: 160 \\
& Number lost to follow-up: 0 \\
Sample: birth cohort of women
\end{tabular}


Korpelainen 2006 (Continued)

Age (years): mean 73 (SD 1.2)

Sex: $100 \%$ female

Inclusion criteria: hip BMD > 2 less than the reference value

Exclusion criteria: "medical reasons"; use of a walking aid other than a stick; bilateral total hip joint re-

placement; unstable chronic illness; malignancy; medication known to affect bone density; severe cognitive impairment; involvement in other interventions

Interventions

1. Group-based balance and strength training plus home practice: exercises increased in difficulty and used no special equipment; 1-hour session, weekly, plus 20 minutes daily at home for 6 months each year; plus twice-yearly seminars on nutrition, health, medical treatment and fall prevention

2. Control: twice-yearly seminars on nutrition, health, medical treatment, and fall prevention

$\begin{array}{ll}\text { Outcomes } & \text { 2. Rate of falls } \\ \text { 2. Number of people who experienced } 1 \text { or more fall-related fractures }\end{array}$

\begin{tabular}{ll}
\hline Duration of the study & 130 weeks \\
\hline Adherence & Adherence measured as session attendance and frequency of home programme \\
& $\begin{array}{l}\text { 1. Group-based balance and strength training plus home-practice group: mean attendance at sessions; } \\
77 \% \text { during the first supervised } 6 \text {-month period, } 75 \% \text { during the second supervised period and } 74 \% \\
\text { during the last supervised } 6 \text { months; mean frequency of performing home programme was } 3 \text { a week }\end{array}$ \\
\hline Notes & $\begin{array}{l}\text { Source of funding: Finnish Ministry of Education, the Finnish Cultural Foundation, University of Oulu, } \\
\text { Deaconess Institute of Oulu, Juho Vainio Foundation, Miina Sillanpää Foundation, Research Founda- } \\
\text { tion of Orion Corporation } \\
\text { Economic information: not reported }\end{array}$
\end{tabular}

\section{Risk of bias}

\begin{tabular}{|c|c|c|}
\hline Bias & Authors' judgement & Support for judgement \\
\hline $\begin{array}{l}\text { Random sequence genera- } \\
\text { tion (selection bias) }\end{array}$ & Low risk & $\begin{array}{l}\text { Quote: "Each participant received sequentially, according to the original iden- } \\
\text { tification numbers, the next random assignment in the computer list". }\end{array}$ \\
\hline $\begin{array}{l}\text { Allocation concealment } \\
\text { (selection bias) }\end{array}$ & Low risk & $\begin{array}{l}\text { The randomisation was "provided by a technical assistant not involved in the } \\
\text { conduction of the trial" }\end{array}$ \\
\hline $\begin{array}{l}\text { Blinding of participants } \\
\text { and personnel (perfor- } \\
\text { mance bias) } \\
\text { All outcomes }\end{array}$ & Unclear risk & $\begin{array}{l}\text { Participants and personnel not blind to allocated group but impact of non- } \\
\text { blinding unclear }\end{array}$ \\
\hline $\begin{array}{l}\text { Blinding of outcome as- } \\
\text { sessment (detection bias) } \\
\text { Falls }\end{array}$ & Low risk & $\begin{array}{l}\text { Falls measured using the same method in each group } \\
\text { Quote: "The assessors in direct contact with participants during the study did } \\
\text { not know to which group they had been allocated" }\end{array}$ \\
\hline $\begin{array}{l}\text { Blinding of outcome as- } \\
\text { sessment (detection bias) } \\
\text { Fractures }\end{array}$ & High risk & No radiological evidence for fractures \\
\hline $\begin{array}{l}\text { Blinding of outcome as- } \\
\text { sessment (detection bias) } \\
\text { Hospital admission, med- } \\
\text { ical attention and adverse } \\
\text { events }\end{array}$ & Unclear risk & Not applicable \\
\hline
\end{tabular}


Korpelainen 2006 (Continued)
Blinding of outcome as-
Unclear risk
Not applicable sessment (detection bias)
Health related quality of
life (self report)

\begin{tabular}{lll}
\hline $\begin{array}{l}\text { Incomplete outcome data } \\
\text { (attrition bias) }\end{array}$ & Low risk & No missing falls data \\
Falls and fallers & \\
\hline $\begin{array}{l}\text { Selective reporting (re- } \\
\text { porting bias) }\end{array}$ & High risk & Falls were measured, but number of fallers was not reported \\
\hline $\begin{array}{l}\text { Method of ascertaining } \\
\text { falls (recall bias) }\end{array}$ & High risk & 3-monthly retrospective recall \\
\hline
\end{tabular}

Kovacs 2013

\begin{tabular}{ll} 
Methods & Study design: RCT \\
Number of study arms: 2 & Length of follow-up: 12 months \\
\hline Participants & Setting: Budapest, Hungary \\
Number of participants: 76 \\
Number analysed: 72 \\
Number lost to follow-up: 4 \\
Sample: community-dwelling women \\
Age (years): Intervention mean 68.5 (SD 5.3), Control mean 68.3 (SD 6.4) \\
Sex: $100 \%$ female \\
Inclusion criteria: Women aged 60 years of age or over, lived in community setting \\
Exclusion criteria: GP did not recommend their participation because of having progressive neurologi- \\
cal or unstable cardiovascular diseases that would limit participation in the exercise programme, hav- \\
ing severe pain in lower limb in weight-bearing positions or participation in regular physical exercise \\
programme (sport or physiotherapy) in the past 6 months
\end{tabular}

Interventions 1. Group-based balance and strength training plus home-practice: exercises and competition games with no special equipment, 60-minute sessions, 2 a week for 25 weeks

2. Control group: asked not to start any type of regular exercise programme and maintain their usual activities, offered participation in the next programme

1. Rate of falls
2. Number of people who experienced 1 or more falls (risk of falling)

\begin{tabular}{ll}
\hline Duration of the study & 52 weeks \\
\hline Adherence & $\begin{array}{l}\text { Adherence measured as the percentage of the number of sessions completed out of the total } 50 \text { ses- } \\
\text { sions }\end{array}$
\end{tabular}


Email communication to obtain fall data, response received, data included in review

\section{Risk of bias}

\begin{tabular}{|c|c|c|}
\hline Bias & Authors' judgement & Support for judgement \\
\hline $\begin{array}{l}\text { Random sequence genera- } \\
\text { tion (selection bias) }\end{array}$ & Unclear risk & $\begin{array}{l}\text { Quote: "Blocked randomisation was performed (with a block size of } 4 \text { and 6)". } \\
\text { Insufficient information about the sequence generation process to permit } \\
\text { judgement }\end{array}$ \\
\hline $\begin{array}{l}\text { Allocation concealment } \\
\text { (selection bias) }\end{array}$ & Low risk & Quote: "Consecutively numbered opaque identical sealed envelopes" \\
\hline $\begin{array}{l}\text { Blinding of participants } \\
\text { and personnel (perfor- } \\
\text { mance bias) } \\
\text { All outcomes }\end{array}$ & Unclear risk & Participants and personnel unblinded but impact of unblinding unknown \\
\hline $\begin{array}{l}\text { Blinding of outcome as- } \\
\text { sessment (detection bias) } \\
\text { Falls }\end{array}$ & Low risk & $\begin{array}{l}\text { Fall calendars were distributed and collected by a physiotherapist who was } \\
\text { not involved in the exercise programme and who was not informed about the } \\
\text { participants' group allocation. Blinding assumed }\end{array}$ \\
\hline $\begin{array}{l}\text { Blinding of outcome as- } \\
\text { sessment (detection bias) } \\
\text { Fractures }\end{array}$ & Unclear risk & Not applicable \\
\hline $\begin{array}{l}\text { Blinding of outcome as- } \\
\text { sessment (detection bias) } \\
\text { Hospital admission, med- } \\
\text { ical attention and adverse } \\
\text { events }\end{array}$ & Unclear risk & Not applicable \\
\hline $\begin{array}{l}\text { Blinding of outcome as- } \\
\text { sessment (detection bias) } \\
\text { Health related quality of } \\
\text { life (self report) }\end{array}$ & Unclear risk & Not applicable \\
\hline $\begin{array}{l}\text { Incomplete outcome data } \\
\text { (attrition bias) } \\
\text { Falls and fallers }\end{array}$ & Low risk & $\begin{array}{l}\text { Less than } 20 \% \text { of fall outcome data are missing }(5 \%) \text {. Missing data were bal- } \\
\text { anced between the exercise }(n=2) \text { and control }(n=2) \text { groups }\end{array}$ \\
\hline $\begin{array}{l}\text { Selective reporting (re- } \\
\text { porting bias) }\end{array}$ & Unclear risk & $\begin{array}{l}\text { Minimum set of expected outcomes not reported, (adverse events not report- } \\
\text { ed) }\end{array}$ \\
\hline $\begin{array}{l}\text { Method of ascertaining } \\
\text { falls (recall bias) }\end{array}$ & Low risk & Fall calendar, collected monthly \\
\hline
\end{tabular}

Kwok 2016

Methods Study design: RCT


Kwok 2016 (Continued)

Number of study arms: 2

Length of follow-up: 12 months

Setting: Singapore
Number of participants: 80
Number analysed: 80
Number lost to follow-up: 0
Sample: community-dwelling
Age (years): mean 80
Sex: $85 \%$ female
Inclusion criteria: not participating in any routine exercise programme, participants with MFES scores $\leq$
9 and could comprehend English, Mandarin or a local dialect
Exclusion criteria: people with neurological disorders

Interventions

1. Group-based balance, strength and aerobic training plus home practice: gym equipment used for cardiovascular training, strength training prescribed at 10 or 15 repetitive maximum; 1-hour sessions, weekly for 12 weeks, 20 minutes of home balance and strength exercises from week 13 on non-intervention days

2. Balance, strength and aerobic training using the Nintendo WiiActive: supervision provided for gaming exercises with the Wii balance board, calisthenics and resistance band and calisthenics used for cadiovascular training, resistance band used for strengthening; 20 minutes, weekly for 12 weeks, 20 minutes of home exercises from week 13

Outcomes
2. Rate of falls
2. Number of people who experienced 1 or more falls (risk of falling)

\begin{tabular}{ll}
\hline Duration of the study & 52 weeks \\
\hline Adherence & Adherence measured as session attendance and home exercise compliance \\
& $\begin{array}{l}\text { 1. Group-based balance, strength and aerobic training plus home-practice group: mean exercise ses- } \\
\text { sion attendance 9.4 (SD 3.2); mean home exercise compliance 2.1 days a week (SD 1.2) }\end{array}$ \\
$\begin{array}{l}\text { 2. Balance, strength and aerobic training using the Nintendo WiiActive group: mean exercise session at- } \\
\text { tendance 9.5 (SD 2.5); mean home exercise compliance 2.4 days per week (SD 1.4) }\end{array}$
\end{tabular}

Notes

Source of funding: The SingHealth Foundation, Singapore Physiotherapy Association

Economic information: not reported

\section{Risk of bias}

\begin{tabular}{lll}
\hline Bias & Authors' judgement & Support for judgement \\
\hline $\begin{array}{l}\text { Random sequence genera- } \\
\text { tion (selection bias) }\end{array}$ & Unclear risk & $\begin{array}{l}\text { Quote: "Generated the random allocation sequence". Insufficient information } \\
\text { about the sequence generation process to permit judgement }\end{array}$ \\
\hline $\begin{array}{l}\text { Allocation concealment } \\
\text { (selection bias) }\end{array}$ & Unclear risk & Consecutively-numbered, sealed envelope. Opaque not stated \\
\hline
\end{tabular}




\section{Kwok 2016 (Continued)}

Blinding of participants

Unclear risk

Participants and personnel unblinded but impact of unblinding unknown and personnel (performance bias)

All outcomes

\begin{tabular}{|c|c|c|}
\hline $\begin{array}{l}\text { Blinding of outcome as- } \\
\text { sessment (detection bias) } \\
\text { Falls }\end{array}$ & Low risk & $\begin{array}{l}\text { Quote: "Baseline and follow-up measurements were performed by trained } \\
\text { and blinded research assistants". Assume this includes monthly telephone fol- } \\
\text { low-up of fall-tracking }\end{array}$ \\
\hline $\begin{array}{l}\text { Blinding of outcome as- } \\
\text { sessment (detection bias) } \\
\text { Fractures }\end{array}$ & Unclear risk & Not applicable \\
\hline $\begin{array}{l}\text { Blinding of outcome as- } \\
\text { sessment (detection bias) } \\
\text { Hospital admission, med- } \\
\text { ical attention and adverse } \\
\text { events }\end{array}$ & Unclear risk & Not applicable \\
\hline $\begin{array}{l}\text { Blinding of outcome as- } \\
\text { sessment (detection bias) } \\
\text { Health related quality of } \\
\text { life (self report) }\end{array}$ & Unclear risk & Not applicable \\
\hline $\begin{array}{l}\text { Incomplete outcome data } \\
\text { (attrition bias) } \\
\text { Falls and fallers }\end{array}$ & Low risk & No missing fall data \\
\hline $\begin{array}{l}\text { Selective reporting (re- } \\
\text { porting bias) }\end{array}$ & High risk & Falls were measured, but number of falls was not reported \\
\hline $\begin{array}{l}\text { Method of ascertaining } \\
\text { falls (recall bias) }\end{array}$ & Low risk & $\begin{array}{l}\text { Participants tracked monthly fall incidence on a recording sheet and were con- } \\
\text { tacted monthly through telephone or mobile phone short messages to min- } \\
\text { imise recall bias }\end{array}$ \\
\hline
\end{tabular}

\section{Kyrdalen 2014}

Methods

Study design: RCT

Number of study arms: 2

Length of follow-up: 3 months

Participants

Setting: 11 communities in southeast Norway

Number of participants: 125

Number analysed: 94

Number lost to follow-up: 31

Sample: community-dwelling

Age (years): mean 82.5 (SD 5.7)

Sex: $73 \%$ female

Inclusion criteria: home-dwelling, at increased fall risk (defined as answering yes on either criterion 1 or 2 below, and in addition yes on 2 or more of criteria 3 - 9:1) had fallen at least once during the previous 
12 months; 2) had self-reported balance or gait problems; 3) had Parkinson's disease or had suffered a stroke; 4) had 4+ concomitant diseases; 5) needed a handrail or support while rising from a chair; 6 ) used 4+ prescribed medications; 7) had reduced cognitive function as assessed by a geriatrician; 8) had $\mathrm{BMI}<20$, and 9) had reduced vision for their age

Exclusion criteria: a score of $23 / 30$ or less on the MMSE or not able to walk without support from another

person

Interventions

1. Group-based Otago Exercise Programme: 45 minutes 2 a week for 12 weeks plus outdoor walking for 30 minutes, $\geq 3$ a week for 12 weeks

2. Individual Otago Exercise Programme: 30 minutes, 3 a week for 12 weeks, plus outdoor walking for 30 minutes, $\geq 3$ a week for 12 weeks

Both groups received 4 home visits to check programme plus 4 telephone calls
Outcomes
1. Number of people who experienced 1 or more falls (risk of falling)
2. Number of people who experienced 1 of more falls requiring hospital admission
3. Health-related quality of life
4. Number of people who died

\begin{tabular}{ll}
\hline Duration of the study & 12 weeks \\
\hline Adherence & Adherence measured as session attendance \\
& 1. Group-based Otago Exercise Programme: attended mean of 21.9 out of 24 sessions (SD 2.7) \\
2. Individual Otago Exercise Programme: attended mean 32.8 out of 36 recommended sessions (SD 2.8)
\end{tabular}

Source of funding: Norwegian Fund for Post-Graduate Physiotherapy Training
Economic information: not reported
Email communication regarding fall data, response received, data not included in review

\section{Risk of bias}

\begin{tabular}{lll}
\hline Bias & Authors' judgement & Support for judgement \\
\hline $\begin{array}{l}\text { Random sequence genera- } \\
\text { tion (selection bias) }\end{array}$ & Low risk & $\begin{array}{l}\text { Quote: "A Web-based block randomization procedure with varying group size, } \\
\text { developed by the Applied Clinical Research Unit at the Norwegian University of } \\
\text { Science and Technology, was used" }\end{array}$ \\
\hline $\begin{array}{l}\text { Allocation concealment } \\
\text { (selection bias) }\end{array}$ & Low risk & Centralised "web-based" randomisation procedure \\
\hline
\end{tabular}

\begin{tabular}{|c|c|c|}
\hline $\begin{array}{l}\text { Blinding of participants } \\
\text { and personnel (perfor- } \\
\text { mance bias) } \\
\text { All outcomes }\end{array}$ & High risk & Participants and personnel not blinded \\
\hline $\begin{array}{l}\text { Blinding of outcome as- } \\
\text { sessment (detection bias) } \\
\text { Falls }\end{array}$ & High risk & $\begin{array}{l}\text { Baseline to } 3 \text { months: fall calendars collected by unblinded exercise instruc- } \\
\text { tors at intervention sessions. } 3 \text { - } 6 \text { months: falls collected retrospectively at } 6 \text { - } \\
\text { month interview with blinded assessor }\end{array}$ \\
\hline $\begin{array}{l}\text { Blinding of outcome as- } \\
\text { sessment (detection bias) }\end{array}$ & Unclear risk & Not applicable \\
\hline
\end{tabular}


Kyrdalen 2014 (Continued)

Fractures

\section{Blinding of outcome as- sessment (detection bias) Hospital admission, med- ical attention and adverse events}

Unclear risk Method of ascertaining hospital admission is unclear

\begin{tabular}{lll}
\hline $\begin{array}{l}\text { Blinding of outcome as- } \\
\text { sessment (detection bias) } \\
\text { Health related quality of } \\
\text { life (self report) }\end{array}$ & High risk & Participants not blinded to group allocaiton \\
\hline $\begin{array}{l}\text { Incomplete outcome data } \\
\text { (attrition bias) } \\
\text { Falls and fallers }\end{array}$ & High risk & More than 20\% of fall outcome data are missing (25\%) \\
\hline $\begin{array}{l}\text { Selective reporting (re- } \\
\text { porting bias) }\end{array}$ & High risk & Falls were measured, but number of falls was not reported \\
\hline $\begin{array}{l}\text { Method of ascertaining } \\
\text { falls (recall bias) }\end{array}$ & High risk & $\begin{array}{l}\text { Baseline to } 3 \text { months: falls were recorded on fall calendars which were collect- } \\
\text { ed by unblinded exercise instructors during twice-weekly group sessions (in- } \\
\text { tervention group) or at home visits in weeks 1, 2, } 4 \text { and } 8 \text { (control group). Non- } \\
\text { returns or incomplete calendars were followed up with the participant or next } \\
\text { of kin; the person collecting this information unclear. } 3 \text { - } 6 \text { months: falls col- } \\
\text { lected retrospectively at 6-month interview with blinded assessor }\end{array}$ \\
\hline
\end{tabular}

\section{LaStayo 2017}

\begin{tabular}{ll}
\hline Methods & Study design: RCT \\
& Number of study arms: 2 \\
& Length of follow-up: 12 months
\end{tabular}

\section{Participants}

Setting: Utah, USA

Number of participants: 134

Number analysed: 112

Number lost to follow-up: 22

Sample: community-dwelling

Age (years): mean 76.1 (SD 7.18)

\section{Sex: $65 \%$ female}

Inclusion criteria: at least 65 years of age or older; had experienced at least 1 fall in the previous 12 months; community-dwelling; ambulatory with a gait speed ranging from of 0.42 to $1.3 \mathrm{~m} / \mathrm{s}$; able to recall all 3 items (or 1 to 2 items with a normal clock drawing test) on the Mini-CogTM instrument for dementia screening; managing 2 or more co-morbid conditions, though cleared by their physician to participate in a 60-minute (with rests) multicomponent exercise fall reduction programme (MCEFRP)

Exclusion criteria: progressive diagnosed neurologic disease (e.g. Parkinson's, multiple sclerosis, Guillain-Barre, Alzheimers); any dystrophies or rheumatologic conditions that primarily affects muscle (e.g. muscular dystrophy, polymyalgia rheumatica); already participated in a MCEFRP or if they were currently performing (or had performed) regular (3 times a week) aerobic (defined as hiking, fast-walking, jogging, running swimming or cycling) or resistance (defined as weight training with bands, cable, free- 
LaStayo 2017 (Continued)

weights or weight-machines) exercise over the past 12 months; any of the absolute contraindications for a MRI scan

Interventions

Participants trained for 60 minutes per session, 3 times a week for 3 months as part of the multicomponent exercise fall reduction program that included aerobic training (recumbent trainer, cycle erg or treadmill), flexibility exercise, 15 - 20-minute individualised balance exercises, upper-limb resistance training and lower-limb resistance training

The 2 lower-limb resistance training programmes were:

1) Traditional (TRAD) resistance exercise: 3 sets of 15 repetitions of a seated bilateral leg-press exercise at $70 \% 1 \mathrm{RM}$. Also, standing multidirectional straight-leg exercises with a weighted cuff placed just proximal to the ankle. The training loads for this exercise were increased as tolerated every 2 weeks, provided the participants could complete 3 sets of 15 repetitions with appropriate form

2) Resistance exercise by negative, eccentrically-induced, work (RENEW): progressive resistive eccentric exercise of the knee and hip extensor muscles using a recumbent stepper-ergometer. The duration of each resistance training session was progressively increased to a maximum 15-minute duration during weeks 5 - 12

Outcomes
2. Number of people who experienced 1 or more falls (risk of falling)

\begin{tabular}{ll}
\hline Duration of the study & 52 weeks \\
\hline Adherence & $\begin{array}{l}\text { All participants completed the prespecified requisite minimum } 18 \text { MCEFRP sessions and } \geq 90 \% \text { adhered } \\
\text { to at least } 29 \text { of the } 36 \text { exercise sessions }\end{array}$
\end{tabular}

Notes Source of funding: National Institute of Aging of the National Institutes of Health

Economic information: not reported

Email communication regarding fall data, response received, data not included in review

\section{Risk of bias}

\begin{tabular}{|c|c|c|}
\hline Bias & Authors' judgement & Support for judgement \\
\hline $\begin{array}{l}\text { Random sequence genera- } \\
\text { tion (selection bias) }\end{array}$ & Unclear risk & $\begin{array}{l}\text { Quote: "A randomisation process with blocks of ten insured equivalency in } \\
\text { the number of subjects adn the same proportion of men and women were as- } \\
\text { signed into each of the groups" }\end{array}$ \\
\hline $\begin{array}{l}\text { Allocation concealment } \\
\text { (selection bias) }\end{array}$ & Unclear risk & Allocation not specified \\
\hline $\begin{array}{l}\text { Blinding of participants } \\
\text { and personnel (perfor- } \\
\text { mance bias) } \\
\text { All outcomes }\end{array}$ & Unclear risk & $\begin{array}{l}\text { Blinding not specified. Assume participants and presonnel not blinded. Impact } \\
\text { of non-blinding is unknown }\end{array}$ \\
\hline $\begin{array}{l}\text { Blinding of outcome as- } \\
\text { sessment (detection bias) } \\
\text { Falls }\end{array}$ & High risk & Assessors were not blinded to group \\
\hline $\begin{array}{l}\text { Blinding of outcome as- } \\
\text { sessment (detection bias) } \\
\text { Fractures }\end{array}$ & Unclear risk & Not applicable \\
\hline
\end{tabular}


LaStayo 2017 (Continued)

Blinding of outcome as-

Unclear risk

Not applicable sessment (detection bias) Hospital admission, medical attention and adverse events

\begin{tabular}{|c|c|}
\hline $\begin{array}{l}\text { Blinding of outcome as- } \\
\text { sessment (detection bias) } \\
\text { Health related quality of } \\
\text { life (self report) }\end{array}$ & Unclear risk \\
\hline
\end{tabular}

\begin{tabular}{lll}
\hline $\begin{array}{l}\text { Incomplete outcome data } \\
\text { (attrition bias) } \\
\text { Falls and fallers }\end{array}$ & Unclear risk & $\begin{array}{l}\text { Less than 20\% of fall outcome data are missing (16\%). Missing data were not } \\
\text { balanced between the RENEW ( } \mathrm{n}=14) \text { and traditional }(\mathrm{n}=8) \text { groups, with } \\
\text { more participants dropping out in the first } 3 \text { months in the RENEW group }(9 \\
\text { dropouts compared with } 4 \text { dropouts). The reasons for the dropouts are not } \\
\text { clear }\end{array}$ \\
\hline $\begin{array}{l}\text { Selective reporting (re- } \\
\text { porting bias) }\end{array}$ & Unclear risk & $\begin{array}{l}\text { Minimum set of expected outcomes not reported (adverse events not report- } \\
\text { ed) }\end{array}$ \\
\hline $\begin{array}{l}\text { Method of ascertaining } \\
\text { falls (recall bias) }\end{array}$ & Low risk & $\begin{array}{l}\text { From } 0 \text { - 3 months intervention personnel asked about falls at weekly inter- } \\
\text { vention sessions. } 4 \text { - 12 months falls were recorded by monthly stamped post- } \\
\text { cards, with telephone contact if a fall was reported or postcards were not re- } \\
\text { turned }\end{array}$
\end{tabular}

\section{Latham 2003}

\begin{tabular}{ll} 
Methods & Study design: RCT \\
& Number of study arms: 2 \\
& Length of follow-up: 6 months \\
\hline
\end{tabular}

Setting: 5 hospitals in Auckland, New Zealand and Sydney, Australia
Number of participants: 243
Number analysed: 222
Number lost to follow-up: 21
Sample: frail older people recently discharged from hospital
Age (years): mean 79
Sex: $53 \%$ female
Inclusion criteria: aged $\geq 65$, considered frail (1 or more health problems, e.g. dependency in an ADL,
prolonged bed rest, impaired mobility, or a recent fall); no clear indication or contraindication to either
of the study treatments
Exclusion criteria: poor prognosis and unlikely to survive 6 months; severe cognitive impairment; phys-
ical limitations that would limit adherence to exercise programme; unstable cardiac status; large ulcers
around ankles that would preclude use of ankle weights; living outside hospitals' geographical zone;
not fluent in English

Interventions

1. Exercise: quadriceps exercises using adjustable ankle cuff weights 3 a week for 10 weeks. First 2 sessions in hospital, remainder at home. Monitored weekly by physiotherapist: alternating home visit with telephone calls

2. "Attention" control: frequency-matched telephone calls and home visits from research physical therapist including general enquiry about recovery, general advice on problems, support 
Latham 2003 (Continued)

3. Vitamin D: single oral dose of $6 \times 1.25 \mathrm{mg}$ calciferol $(300,000 \mathrm{IU})$

4. Vitamin D control: placebo tablets

1. Rate of falls
2. Number of people who experienced 1 or more falls (risk of falling)
3. Health-related quality of life
5. Number of people who died

\begin{tabular}{ll}
\hline Duration of the study & 26 weeks \\
\hline Adherence & Adherence was monitored through a participant diary \\
& $\begin{array}{l}\text { 1. Exercise: adhered to } 82 \% \text { of prescribed sessions (mean } 24.6 \text { of } 30 \text { sessions). Mean exercise intensity } \\
\text { at the end of training was } 51 \% \pm 13 \% \text { of } 1 \text { RM, only } 25 \% \text { of participants were able to reach the high in- } \\
\text { tensity desired by the intervention }\end{array}$
\end{tabular}

Notes

Source of funding: Health Research Council of New Zealand, Auckland University of Technology Research Fund, Lenore Wilson Estate

Economic information: not reported

Detailed description of exercise regimen given in paper

\section{Risk of bias}

\begin{tabular}{lll}
\hline Bias & Authors' judgement & Support for judgement \\
\hline $\begin{array}{l}\text { Random sequence genera- } \\
\text { tion (selection bias) }\end{array}$ & Low risk & $\begin{array}{l}\text { Study biostatistician-generated random sequence. Block randomisation tech- } \\
\text { nique }\end{array}$ \\
\hline $\begin{array}{l}\text { Allocation concealment } \\
\text { (selection bias) }\end{array}$ & Low risk & Computerised centralised randomisation scheme \\
\hline $\begin{array}{l}\text { Blinding of participants } \\
\text { and personnel (perfor- } \\
\text { mance bias) }\end{array}$ & Low risk & $\begin{array}{l}\text { Trial with 4 arms with varying risks of bias (factorial design). 2 arms dou- } \\
\text { ble-blind, placebo-controlled (low risk) and 2 arms exercise and attention con- } \\
\text { trol with matched frequency of visits where impact of non-blinding likely to be }\end{array}$ \\
\hline $\begin{array}{l}\text { Blinding of outcome as- } \\
\text { sessment (detection bias) } \\
\text { Falls }\end{array}$ & Low risk & $\begin{array}{l}\text { Placebo-controlled arms: falls reported by participants who were blinded to } \\
\text { group allocation (and assessor blinded to group allocation). Exercise and exer- } \\
\text { cise control arms: falls reported by participants who were aware of their group } \\
\text { allocation but assessor blinded to group allocation }\end{array}$ \\
\hline
\end{tabular}

\begin{tabular}{|c|c|c|}
\hline $\begin{array}{l}\text { Blinding of outcome as- } \\
\text { sessment (detection bias) } \\
\text { Fractures }\end{array}$ & Unclear risk & Not applicable \\
\hline $\begin{array}{l}\text { Blinding of outcome as- } \\
\text { sessment (detection bias) } \\
\text { Hospital admission, med- } \\
\text { ical attention and adverse } \\
\text { events }\end{array}$ & Unclear risk & $\begin{array}{l}\text { Quote: "The field research staff recorded all adverse events, and a blinded as- } \\
\text { sessor coded them". Assume field research staff were not blinded. Assume ad- } \\
\text { verse events were recorded using same methods in both groups (as visits were } \\
\text { frequency-matched) }\end{array}$ \\
\hline
\end{tabular}

\begin{tabular}{|c|c|c|}
\hline $\begin{array}{l}\text { Blinding of outcome as- } \\
\text { sessment (detection bias) }\end{array}$ & High risk & $\begin{array}{l}\text { Trial participants in exercise and placebo-controlled groups were not blinded } \\
\text { to group allocation }\end{array}$ \\
\hline
\end{tabular}


Latham 2003 (Continued) Health related quality of life (self report)

\section{Incomplete outcome data Unclear risk (attrition bias)}

Falls and fallers
Less than $20 \%$ of fall outcome data are missing $(9 \%)$. There was a minor imbalance in missing data between the resistance $(n=8)$ and control $(n=13)$ groups, with the resistance group missing data due to death $(n=6)$ and refusal $(n=2)$, and the control group missing data due to death $(n=8)$ and refusal $(n=$ 5)

\begin{tabular}{lll}
\hline $\begin{array}{l}\text { Selective reporting (re- } \\
\text { porting bias) }\end{array}$ & Unclear risk & $\begin{array}{l}\text { Minimum set of expected outcomes reported. No protocol or prospective trial } \\
\text { registration }\end{array}$ \\
\hline $\begin{array}{l}\text { Method of ascertaining } \\
\text { falls (recall bias) }\end{array}$ & Unclear risk & $\begin{array}{l}\text { Prospective. Falls recorded in fall diary with weekly reminders for first } 10 \\
\text { weeks. Nurses examined fall diaries and sought further details about each fall } \\
\text { at 3- and 6-month visits. Reminder phone call between visits }\end{array}$
\end{tabular}

Lehtola 2000

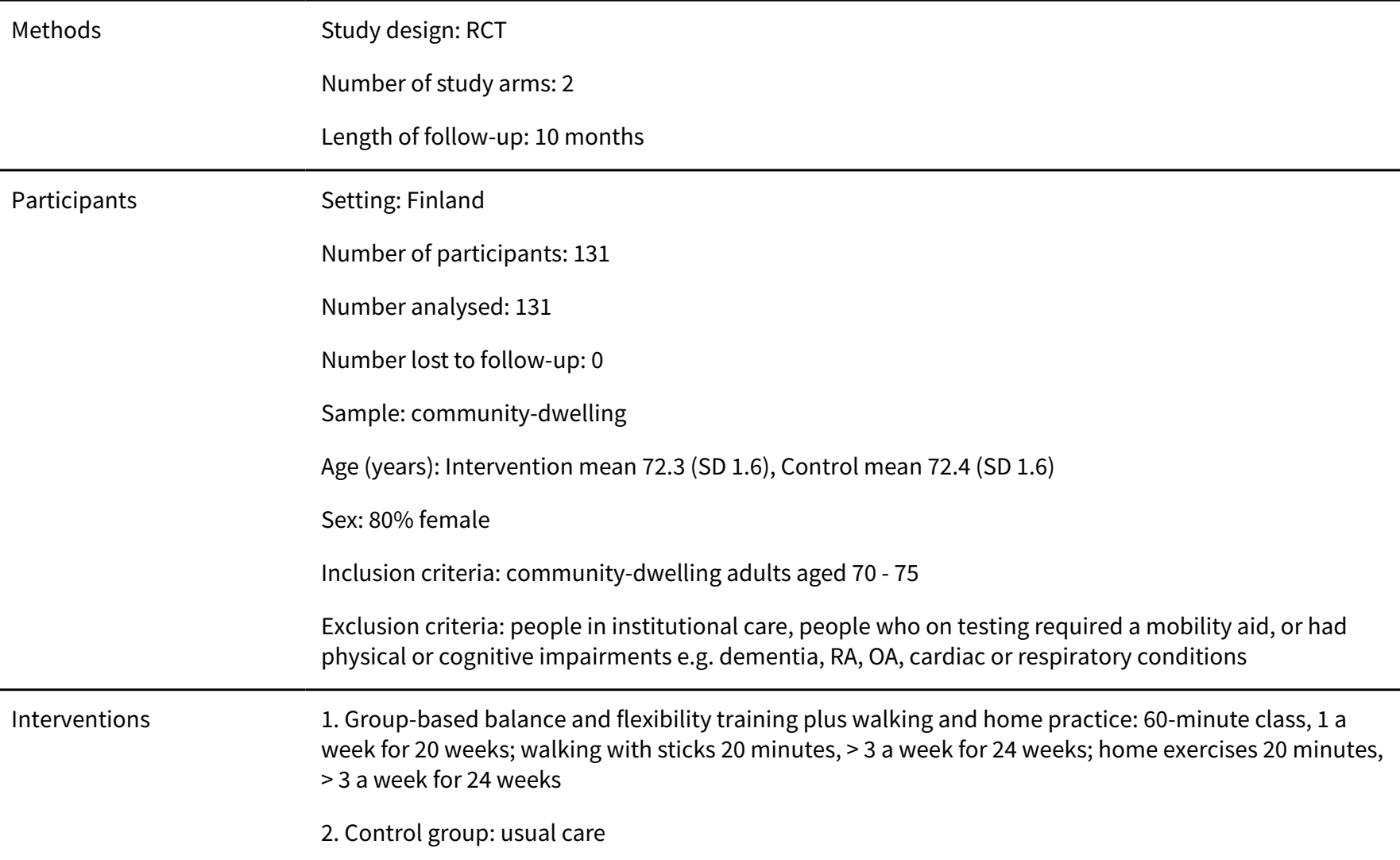

\begin{tabular}{ll}
\hline Outcomes & 1. Rate of falls \\
\hline Duration of the study & 40 weeks \\
\hline Adherence & Participants completed diary collected monthly \\
& $\begin{array}{l}\text { 1. Group-based balance and flexibility training plus walking and home practice group: 'Active' partici- } \\
\text { pants: } 52 \text { participants; 'Passive': } 20 \text { participants }\end{array}$
\end{tabular}

Notes

Source of funding: not reported 


\section{Risk of bias}

\begin{tabular}{|c|c|c|}
\hline Bias & Authors' judgement & Support for judgement \\
\hline $\begin{array}{l}\text { Random sequence genera- } \\
\text { tion (selection bias) }\end{array}$ & Unclear risk & Unable to assess due to language \\
\hline $\begin{array}{l}\text { Allocation concealment } \\
\text { (selection bias) }\end{array}$ & Unclear risk & Unable to assess due to language \\
\hline $\begin{array}{l}\text { Blinding of participants } \\
\text { and personnel (perfor- } \\
\text { mance bias) } \\
\text { All outcomes }\end{array}$ & Unclear risk & Unable to assess due to language \\
\hline $\begin{array}{l}\text { Blinding of outcome as- } \\
\text { sessment (detection bias) } \\
\text { Falls }\end{array}$ & Unclear risk & Unable to assess due to language \\
\hline $\begin{array}{l}\text { Blinding of outcome as- } \\
\text { sessment (detection bias) } \\
\text { Fractures }\end{array}$ & Unclear risk & Not applicable \\
\hline $\begin{array}{l}\text { Blinding of outcome as- } \\
\text { sessment (detection bias) } \\
\text { Hospital admission, med- } \\
\text { ical attention and adverse } \\
\text { events }\end{array}$ & Unclear risk & Not applicable \\
\hline $\begin{array}{l}\text { Blinding of outcome as- } \\
\text { sessment (detection bias) } \\
\text { Health related quality of } \\
\text { life (self report) }\end{array}$ & Unclear risk & Not applicable \\
\hline $\begin{array}{l}\text { Incomplete outcome data } \\
\text { (attrition bias) } \\
\text { Falls and fallers }\end{array}$ & High risk & Risk of falls and adverse events not reported \\
\hline $\begin{array}{l}\text { Selective reporting (re- } \\
\text { porting bias) }\end{array}$ & Low risk & No missing fall data \\
\hline $\begin{array}{l}\text { Method of ascertaining } \\
\text { falls (recall bias) }\end{array}$ & Unclear risk & Unable to assess due to language \\
\hline
\end{tabular}

\begin{tabular}{ll} 
Methods & Study design: RCT \\
& Number of study arms: 2 \\
& Length of follow-up: 6 months \\
\hline Participants & Setting: Legacy Health System, Portland, Oregon, USA \\
& Number of participants: 256
\end{tabular}


Li 2005 (Continued)

Number analysed: 188

Number lost to follow-up: 68

Sample: people enrolled in HMO

Age (years): mean 77.5 (SD 5), range 70 to 92

Sex: $70 \%$ female

Inclusion criteria: age $\geq 70$; physician clearance to participate; inactive (no moderate to strenuous activity in last 3 months); walks independently

Exclusion criteria: chronic medical problems that would limit participation; cognitive impairment

\begin{tabular}{ll}
\hline Interventions & $\begin{array}{l}\text { 1. Group-based Tai Chi: } 1 \text { hour, } 3 \text { a week for } 26 \text { weeks } \\
\text { 2. Control group: low-level stretching } 1 \text { hour, } 3 \text { a week for } 26 \text { weeks }\end{array}$ \\
\hline Outcomes & $\begin{array}{l}\text { 1. Rate of falls } \\
\text { 2. Number of people who experienced } 1 \text { or more falls (risk of falling) } \\
\text { 3. Number of people who experienced } 1 \text { or more falls requiring medical attention }\end{array}$ \\
\hline Duration of the study & $\begin{array}{l}\text { 52 weeks } \\
\text { Adherence }\end{array}$ \\
Adherence measured as class attendance \\
sessions \\
2. Control group: median compliance; 61 sessions (range 35 - 78). 87 (81\%) attended 50+ sessions \\
Source of funding: National Institutes of Health, National Institute on Aging \\
Economic information: not reported \\
6-month fall data used as total over 12 -month period not reported
\end{tabular}

\section{Risk of bias}

\begin{tabular}{|c|c|c|}
\hline Bias & Authors' judgement & Support for judgement \\
\hline $\begin{array}{l}\text { Random sequence genera- } \\
\text { tion (selection bias) }\end{array}$ & Low risk & Computer-generated random numbers \\
\hline $\begin{array}{l}\text { Allocation concealment } \\
\text { (selection bias) }\end{array}$ & Unclear risk & Insufficient information to permit judgement \\
\hline $\begin{array}{l}\text { Blinding of participants } \\
\text { and personnel (perfor- } \\
\text { mance bias) } \\
\text { All outcomes }\end{array}$ & Unclear risk & $\begin{array}{l}\text { Participants and personnel not blind to allocated group but impact of non- } \\
\text { blinding unclear }\end{array}$ \\
\hline $\begin{array}{l}\text { Blinding of outcome as- } \\
\text { sessment (detection bias) } \\
\text { Falls }\end{array}$ & Low risk & $\begin{array}{l}\text { Falls reported by participants who were aware of their group allocation, using } \\
\text { the same method on both groups. Fall diaries coded by blinded research assis- } \\
\text { tant }\end{array}$ \\
\hline $\begin{array}{l}\text { Blinding of outcome as- } \\
\text { sessment (detection bias) } \\
\text { Fractures }\end{array}$ & Unclear risk & Not applicable \\
\hline $\begin{array}{l}\text { Blinding of outcome as- } \\
\text { sessment (detection bias) }\end{array}$ & High risk & $\begin{array}{l}\text { The only evidence for requiring medical attention was from self-reports from } \\
\text { participants }\end{array}$ \\
\hline
\end{tabular}


Li 2005 (Continued) Hospital admission, medical attention and adverse events

\begin{tabular}{|c|c|c|}
\hline $\begin{array}{l}\text { Blinding of outcome as- } \\
\text { sessment (detection bias) } \\
\text { Health related quality of } \\
\text { life (self report) }\end{array}$ & Unclear risk & Not applicable \\
\hline $\begin{array}{l}\text { Incomplete outcome data } \\
\text { (attrition bias) } \\
\text { Falls and fallers }\end{array}$ & High risk & More than $20 \%$ of fall outcome data are missing $(27 \%)$ \\
\hline $\begin{array}{l}\text { Selective reporting (re- } \\
\text { porting bias) }\end{array}$ & Unclear risk & $\begin{array}{l}\text { Minimum set of expected outcomes was not reported (adverse events were } \\
\text { not reported) }\end{array}$ \\
\hline $\begin{array}{l}\text { Method of ascertaining } \\
\text { falls (recall bias) }\end{array}$ & Low risk & $\begin{array}{l}\text { Prospective. Falls recorded on daily fall calendars, collected on a monthly ba- } \\
\text { sis }\end{array}$ \\
\hline
\end{tabular}

Lin 2007

\begin{tabular}{ll} 
Methods & Study design: RCT \\
& Number of study arms: 2 \\
& Length of follow-up: 6 months \\
\hline Participants & Setting: Taiwan \\
& Number of participants: 100 \\
& Number analysed: 100 \\
& Number lost to follow-up: 0 \\
& Sample: residents of rural agricultural area \\
& Age (years): mean 76.5 \\
& Sex: $51 \%$ female \\
& Inclusion criteria: medical attention for a fall in previous 4 weeks, $\geq 65$ years \\
& Exclusion criteria: none described
\end{tabular}

Interventions

Randomised into 3 groups: 2 intervention groups ( 1 individual balance, strength and flexibility training group, 1 home safety assessment and modification group) and 1 control group. Only Individual balance, strength and flexibility training group and control group included in this review

1. Individual balance, strength and flexibility training: Home-based exercises with physiotherapist, used $1 \mathrm{~kg}$ ankle weights for strengthening if able, 40 - 60-minute sessions, $3 \mathrm{x}$ or more a week for 4 months

2. Control: 1 social visit by a public health worker 30 to 40 -minute every 2 weeks for 4 months with fall prevention pamphlets provided

1. Rate of falls
2. Health-related quality of life
3. Number of people who died

Duration of the study

16 weeks 
Liston 2014 (Continued)

Length of follow-up: 6 months

\begin{tabular}{|c|c|}
\hline \multirow[t]{9}{*}{ Participants } & Setting: London, UK \\
\hline & Number of participants: 21 \\
\hline & Number analysed: 15 \\
\hline & Number lost to follow-up: 6 \\
\hline & Sample: Secondary care-based falls clinic \\
\hline & $\begin{array}{l}\text { Age (mean): Otago Exercise Programme + multisensory mean } 77.8 \text { years; Otago Exercise Programme + } \\
\text { stretching mean } 76.7 \text { years }\end{array}$ \\
\hline & Sex: $85 \%$ female \\
\hline & $\begin{array}{l}\text { Inclusion criteria: } \geq 65 \text { years, } \geq 2 \text { non-syncopal falls during the previous } 12 \text { months, no previous diagno- } \\
\text { sis of vestibular dysfunction, referred after multifactorial assessment for the locally-provided 'routine' } \\
\text { modified Otago Exercise Programme classes }\end{array}$ \\
\hline & $\begin{array}{l}\text { Exclusion criteria: where falls were considered by the attending physician as due to acute illness with- } \\
\text { out significant underlying instability, medication side effects, or musculoskeletal or neurologic disease } \\
\text { significantly affecting postural stability }\end{array}$ \\
\hline \multirow[t]{3}{*}{ Interventions } & $\begin{array}{l}\text { Randomised into } 3 \text { groups: } 2 \text { intervention groups ( } 1 \text { group-based modified Otago Exercise Programme } \\
\text { plus individual, partiall-supervised multisensory balance training, and } 1 \text { group-based modified Otago } \\
\text { Exercise Programme plus individual, partially-supervised flexibility training) and } 1 \text { control group. Only } \\
\text { the } 2 \text { intervention groups were included in this review }\end{array}$ \\
\hline & $\begin{array}{l}\text { 1. Group-based modified Otago Exercise Programme plus individual, partially-supervised multisenso- } \\
\text { ry balance training: } 1 \text {-hour class, } 2 \text { a week, }+45 \text {-minute supervised home sessions providing additional } \\
\text { customised multisensory balance exercises for } 8 \text { weeks }\end{array}$ \\
\hline & $\begin{array}{l}\text { 2. Group-based modified Otago Exercise Programme plus individual, partially-supervised flexibility } \\
\text { training: 1-hour class, } 2 \text { a week, + 45-minute supervised home stretching programme for } 8 \text { weeks }\end{array}$ \\
\hline Outcomes & 1. Rate of falls \\
\hline Duration of the study & 24 weeks \\
\hline Adherence & Not reported \\
\hline \multirow[t]{2}{*}{ Notes } & Source of funding: King's College London PhD studentship \\
\hline & Economic information: not reported \\
\hline
\end{tabular}

\section{Risk of bias}

\begin{tabular}{lll}
\hline Bias & Authors' judgement & Support for judgement \\
\hline $\begin{array}{l}\text { Random sequence genera- } \\
\text { tion (selection bias) }\end{array}$ & Low risk & Computerised random-number generator \\
\hline $\begin{array}{l}\text { Allocation concealment } \\
\text { (selection bias) }\end{array}$ & Unclear risk & No details provided \\
\hline $\begin{array}{l}\text { Blinding of participants } \\
\text { and personnel (perfor- } \\
\text { mance bias) }\end{array}$ & Unclear risk & Participants and personnel unblinded but impact of unblinding unknown \\
All outcomes & \\
\hline
\end{tabular}


Liston 2014 (Continued)

Blinding of outcome as- Unclear risk Quote: "Outcome measures were assessed at baseline, four and eight weeks sessment (detection bias)

Falls (end of treatment), and were performed by a rater blinded to intervention group..... Six-months postintervention, a telephone follow-up recorded retrospective falls history". Unclear if falls were collected by an assessor blinded to treatment group

\begin{tabular}{lll}
\hline Blinding of outcome as- & Unclear risk & Not applicable \\
sessment (detection bias) &
\end{tabular}

Fractures

Blinding of outcome as- $\quad$ Unclear risk $\quad$ Not applicable
sessment (detection bias)
Hospital admission, med-
ical attention and adverse
events

\begin{tabular}{ll}
\hline Blinding of outcome as- & Unclear risk \\
sessment (detection bias) & \\
Health related quality of & \\
life (self report) & \\
\hline
\end{tabular}

\begin{tabular}{lll}
\hline $\begin{array}{l}\text { Incomplete outcome data } \\
\text { (attrition bias) } \\
\text { Falls and fallers }\end{array}$ & High risk & More than 20\% of fall outcome data are missing (29\%) \\
\hline $\begin{array}{l}\text { Selective reporting (re- } \\
\text { porting bias) }\end{array}$ & High risk & $\begin{array}{l}\text { Falls were measured, but number of fallers was not reported. Adverse events } \\
\text { were not reported }\end{array}$ \\
\hline
\end{tabular}

Method of ascertaining High risk

Quote: "Six-months postintervention, a telephone follow-up recorded retrofalls (recall bias) spective falls history...for the previous six-months"

Liu-Ambrose 2004

\begin{tabular}{ll} 
Methods & Study design: RCT \\
& Number of study arms: 3 \\
& Length of follow-up: 6 months \\
\hline Participants & Setting: British Colombia (BC), Canada \\
& Number of participants: 104 \\
& Number analysed: 98 \\
& Number lost to follow-up: 6 \\
& Sample: women with osteoporosis or osteopenia diagnosed at BC Women's Hospital and Health Cen- \\
tre; individuals with low BMD identified through Osteoporosis Society of Canada; advertising & Age (years): mean 79 (SD 3), range 75 - 85 \\
& Sex: $100 \%$ female \\
Inclusion criteria: women aged 75 - 85; osteoporosis or osteopenia (BMD total hip or spine T score at \\
least 1 SD below young normal sex-matched area BMD of the Lunar reference database); resident in \\
greater Vancouver \\
Exclusion criteria: living in care facility; non-white race; regularly exercising twice a week or more; his- \\
tory of illness or a condition affecting balance (stroke, Parkinson's disease); unable to safely participate \\
in exercise programme; MMSE 23 or less
\end{tabular}


Liu-Ambrose 2004 (Continued)

Interventions

1. Supervised, high-intensity resistance training: progressive strengthening using gym equipment and free weights; 50 minutes, 2 a week for 25 weeks

2. Supervised agility training: training to challenge hand-eye and foot-eye co-ordination, and dynamic, standing and leaning balance, and reaction time (ball games, relay races, dance movements, obstacle courses wearing hip protectors); 50 minutes, 2 a week for 25 weeks.

3. Control group: sham exercises (stretching, deep breathing, relaxation, posture education); 50 minutes, 2 a week for 25 weeks

\begin{tabular}{ll}
\hline Outcomes & 1. Rate of falls \\
\hline Duration of the study & 25 weeks \\
\hline Adherence & Adherence measured by class attendance. \\
& $\begin{array}{l}\text { 1. Supervised, high-intensity resistance training group: } 85 \% \text { compliance } \\
\text { 2. Supervised agility training group: } 87 \% \text { compliance } \\
\text { 3. Control group: } 79 \% \text { compliance }\end{array}$
\end{tabular}

Notes Source of funding: Vancouver Foundation (BCMSF), Canadian Institutes of Health Research, Michael Smith Foundation for Health Research, Peter Wall Institute for Advanced Studies at the University of British Columbia, Canada Foundation for Innovation

Economic information: not reported

\section{Risk of bias}

\begin{tabular}{|c|c|c|}
\hline Bias & Authors' judgement & Support for judgement \\
\hline $\begin{array}{l}\text { Random sequence genera- } \\
\text { tion (selection bias) }\end{array}$ & Unclear risk & $\begin{array}{l}\text { Method of randomisation not described but stratified by baseline performance } \\
\text { in postural sway }\end{array}$ \\
\hline $\begin{array}{l}\text { Allocation concealment } \\
\text { (selection bias) }\end{array}$ & Unclear risk & Insufficient information to permit judgement \\
\hline $\begin{array}{l}\text { Blinding of participants } \\
\text { and personnel (perfor- } \\
\text { mance bias) } \\
\text { All outcomes }\end{array}$ & Unclear risk & $\begin{array}{l}\text { Participants and personnel not blinded to allocated group but impact of non- } \\
\text { blinding unclear }\end{array}$ \\
\hline $\begin{array}{l}\text { Blinding of outcome as- } \\
\text { sessment (detection bias) } \\
\text { Falls }\end{array}$ & Unclear risk & $\begin{array}{l}\text { All participants asked to keep falls diary. Study described as "single blind" } \\
\text { which indicates that assessors were blinded, but unclear whether personnel } \\
\text { recording falls outcomes were blinded }\end{array}$ \\
\hline $\begin{array}{l}\text { Blinding of outcome as- } \\
\text { sessment (detection bias) } \\
\text { Fractures }\end{array}$ & Unclear risk & Not applicable \\
\hline $\begin{array}{l}\text { Blinding of outcome as- } \\
\text { sessment (detection bias) } \\
\text { Hospital admission, med- } \\
\text { ical attention and adverse } \\
\text { events }\end{array}$ & Unclear risk & $\begin{array}{l}\text { Assessors of adverse events were not blinded to group allocation. Participants } \\
\text { were questioned about the presence of adverse events after each exercise ses- } \\
\text { sion, therefore assume the } 3 \text { groups were assessed using the same method } \\
\text { and with the same frequency }\end{array}$ \\
\hline $\begin{array}{l}\text { Blinding of outcome as- } \\
\text { sessment (detection bias) } \\
\text { Health related quality of } \\
\text { life (self report) }\end{array}$ & Unclear risk & Not applicable \\
\hline
\end{tabular}


Liu-Ambrose 2004 (Continued)

Incomplete outcome data Low risk Less than $20 \%$ of fall outcome data are missing (6\%). The missing data were (attrition bias) balanced between groups ( 2 missing from each group at final assessment)

Falls and fallers

$\begin{array}{lll}\begin{array}{l}\text { Selective reporting (re- } \\ \text { porting bias) }\end{array} & \text { High risk } & \begin{array}{l}\text { Falls were measured, but number of fallers was not reported. Adverse events } \\ \text { were not reported }\end{array}\end{array}$

Method of ascertaining $\quad$ Low risk $\quad$ Prospective. Quote: "Falls documented using monthly falls calendars"
falls (recall bias)

\section{Liu-Ambrose 2008}

\begin{tabular}{ll}
\hline Methods & Study design: RCT \\
& Number of study arms: 2 \\
& Length of follow-up: 12 months \\
\hline
\end{tabular}

Setting: Vancouver, Canada
Number of participants: 74
Number analysed: 59
Number lost to follow-up: 15
Sample: people attending a falls clinic after presenting at ED or to GP with a fall or fall-related injury
(41/59 completing baseline assessment)
Age (years): mean 82.2 (SD 6.3) (in 59 participants completing baseline assessment)
Sex: $71 \%$ female
Inclusion criteria: aged $\geq 70 ;$ community-dwelling; attending 1 of 2 falls clinics (criteria for attending
clinic: history of a fall and considered at risk for further falls); able to walk at least 3 m; 1 additional non-
syncopal fall in previous year (if index fall was suspected to be due to carotid sinus syndrome); at risk of
further falls (TUG test $>15$ seconds or PPA z-score of $\geq 1$ )
Exclusion criteria: progressive neurological condition (e.g. Parkinson's disease); life expectancy < 12
months; cognitively impaired (MMSE score < 24)
Interventions 1. Individual Otago Exercise Programme: 30 minutes, 3 a week for 6 months plus walking for $\geq 2$ a week
2. Control: no exercise intervention; semi-structured interview about presenting fall and experience seeking care for the fall at ED
Both groups received falls risk factor assessment and comprehensive geriatric assessment followed by 'Guideline Care' through falls clinic

1. Rate of falls
2. Number of people who experienced 1 or more falls (risk of falling)
3. Number of people who died

Duration of the study 52 weeks

Adherence

Adherence measured by programme completion 
Liu-Ambrose 2008 (Continued)

1. Individual Otago Exercise Programme: $7 / 28$ (25\%) completed programme $\geq 3$ a week. 16/28 (57\%) completed programme $\geq 2$ a week. 19/28 (68\%) completed programme at $\geq 1$ a week

Source of funding: Canadian Institutes of Health Research
Economic information: Mean cost per person (intervention) CAD 14,285. Incremental cost per fall pre-
vented/per QALY gained: CAD 247 per fall prevented
Cost-effectiveness analysis reported in Davis 2009

\section{Risk of bias}

\begin{tabular}{lll}
\hline Bias & Authors' judgement & Support for judgement \\
\hline $\begin{array}{l}\text { Random sequence genera- } \\
\text { tion (selection bias) }\end{array}$ & Low risk & $\begin{array}{l}\text { Quote: "The randomization sequence was computer generated (www.random- } \\
\text { ization.com)" }\end{array}$ \\
\hline $\begin{array}{l}\text { Allocation concealment } \\
\text { (selection bias) }\end{array}$ & Low risk & $\begin{array}{l}\text { Quote: "The Family Practice Research Coordinator at the University of British } \\
\text { Columbia held this sequence independently and remotely" }\end{array}$ \\
\hline $\begin{array}{l}\text { Blinding of participants } \\
\text { and personnel (perfor- } \\
\text { mance bias) }\end{array}$ & Unclear risk & $\begin{array}{l}\text { Participants and personnel not blind to allocated group but impact of non- } \\
\text { blinding unclear }\end{array}$ \\
\hline
\end{tabular}

\begin{tabular}{|c|c|c|}
\hline Blinding of outcome as- & High risk & Falls self-reported and \\
\hline Falls & & $\begin{array}{l}\text { Quote: "A research assistant who was not blinded to treatment group" phoned } \\
\text { participants at the end of each month }\end{array}$ \\
\hline
\end{tabular}

\begin{tabular}{|c|c|c|}
\hline $\begin{array}{l}\text { Blinding of outcome as- } \\
\text { sessment (detection bias) } \\
\text { Fractures }\end{array}$ & Unclear risk & Not applicable \\
\hline
\end{tabular}

\begin{tabular}{ll}
\hline Blinding of outcome as- & Unclear risk \\
sessment (detection bias) & \\
Hospital admission, med- & \\
ical attention and adverse & \\
events & \\
\hline
\end{tabular}

\begin{tabular}{ll}
\hline Blinding of outcome as- & Unclear risk \\
sessment (detection bias) & \\
Health related quality of & \\
life (self report) & \\
\hline
\end{tabular}

\begin{tabular}{lll}
\hline $\begin{array}{l}\text { Incomplete outcome data } \\
\text { (attrition bias) } \\
\text { Falls and fallers }\end{array}$ & High risk & More than 20\% of fall outcome data are missing (30\%) \\
\hline $\begin{array}{l}\text { Selective reporting (re- } \\
\text { porting bias) }\end{array}$ & Unclear risk & $\begin{array}{l}\text { Minimum set of expected outcomes not reported (adverse events not report- } \\
\text { ed) }\end{array}$ \\
\hline $\begin{array}{l}\text { Method of ascertaining } \\
\text { falls (recall bias) }\end{array}$ & Low risk & $\begin{array}{l}\text { Quote: "Ascertainment of falls ... documented on monthly calendars that were } \\
\text { returned in prepaid preaddressed envelopes at the end of each month." "A re- } \\
\text { search assistant who was not blinded to treatment group but was unaware of } \\
\text { the study hypotheses made three attempts by telephone to contact partici- } \\
\text { pants at the end of each month. The purpose of each phone call was to inquire } \\
\text { about falls (both groups) ... for all participants regardless of whether the calen- } \\
\text { dar was returned." }\end{array}$ \\
\hline
\end{tabular}


Logghe 2009

\begin{tabular}{ll} 
Methods & Study design: RCT \\
& Number of study arms: 2 \\
& Length of follow-up: 12 months \\
\hline Participants & Setting: 2 industrial towns in the western Netherlands \\
& Number of participants: 269 \\
& Number analysed: 269 \\
& Number lost to follow-up: 0 \\
& Sample: registered with participating 23 general practices \\
Age (years): mean 77 (SD 4.6$)$ \\
Sex: $71 \%$ female \\
Inclusion criteria: aged $\geq 70 ;$ community-dwelling; high falls risk (1 or more falls in previous year or 2 or \\
more risk factors for falling (disturbed balance, mobility problems, dizziness, using benzodiazepines or \\
diuretics)) \\
Exclusion criteria: none described
\end{tabular}

\begin{tabular}{ll}
\hline Interventions & 1. Group-based Tai Chi: 1 hour, 2 a week for 13 weeks + fall-prevention brochure \\
2. Control: fall-prevention brochure
\end{tabular}

\begin{tabular}{l} 
Outcomes \\
$\begin{array}{l}\text { 2. Number of people who experienced } 1 \text { or more falls (risk of falling) } \\
\text { 3. Number of people who died }\end{array}$ \\
\hline
\end{tabular}

\begin{tabular}{ll}
\hline Duration of the study & 52 weeks \\
\hline Adherence & Adherence measured by lesson attendance \\
& 1. Group-based Tai Chi: $47 \%$ attended $80 \%$ of lessons \\
\hline Notes & Source of funding: Netherlands Organization for Health Research and Development (ZonMw) \\
& Economic information: not reported \\
\hline
\end{tabular}

\section{Risk of bias}

\begin{tabular}{lll}
\hline Bias & Authors' judgement & Support for judgement \\
\hline $\begin{array}{l}\text { Random sequence genera- } \\
\text { tion (selection bias) }\end{array}$ & Low risk & $\begin{array}{l}\text { Quote: "An independent research assistant performed a prestratified block } \\
\text { randomization using a computer-generated randomization list" }\end{array}$ \\
\hline $\begin{array}{l}\text { Allocation concealment } \\
\text { (selection bias) }\end{array}$ & Low risk & Quote: "An independent research assistant performed ... randomization" \\
\hline $\begin{array}{l}\text { Blinding of participants } \\
\text { and personnel (perfor- } \\
\text { mance bias) }\end{array}$ & Unclear risk & $\begin{array}{l}\text { Participants and personnel not blinded to allocated group but impact of non- } \\
\text { All outcomes }\end{array}$ \\
\hline $\begin{array}{l}\text { Blinding of outcome as- } \\
\text { sessment (detection bias) }\end{array}$ & Low risk & \\
\hline
\end{tabular}


Logghe 2009 (Continued)

Falls
Quote: "The blinded research assistant contacted the participant when forms were missing or incomplete, and they then completed the forms together over the telephone". Falls were recorded and confrimed using the same method in both groups

\begin{tabular}{lll}
\hline Blinding of outcome as- & Unclear risk & Not applicabl \\
sessment (detection bias) & \\
Fractures &
\end{tabular}

\begin{tabular}{|c|c|}
\hline $\begin{array}{l}\text { Blinding of outcome as- } \\
\text { sessment (detection bias) } \\
\text { Hospital admission, med- } \\
\text { ical attention and adverse } \\
\text { events }\end{array}$ & Unclear risk \\
\hline
\end{tabular}

\begin{tabular}{lll}
\hline $\begin{array}{l}\text { Blinding of outcome as- } \\
\text { sessment (detection bias) } \\
\text { Health related quality of } \\
\text { life (self report) }\end{array}$ & Unclear risk & Not applicable \\
\hline $\begin{array}{l}\text { Incomplete outcome data } \\
\text { (attrition bias) }\end{array}$ & Low risk & No missing fall data \\
$\begin{array}{l}\text { Falls and fallers } \\
\text { Selective reporting (re- }\end{array}$ & Unclear risk & $\begin{array}{l}\text { Minimum set of expected outcomes not reported (adverse events not report- } \\
\text { porting bias) }\end{array}$ \\
\hline $\begin{array}{l}\text { Method of ascertaining } \\
\text { falls (recall bias) }\end{array}$ & Low risk & $\begin{array}{l}\text { Quote: "At baseline, the participants received a falls calendar and the instruc- } \\
\text { tion to fill it out on a daily basis for 1 year ... The fall calendars were collect- } \\
\text { ed monthly by mail. The blinded research assistant contacted the participant } \\
\text { when forms were missing or incomplete, and they then completed the forms } \\
\text { together over the telephone" }\end{array}$ \\
\hline
\end{tabular}

\section{Lord 1995}

\begin{tabular}{ll}
\hline Methods & Study design: RCT \\
& Number of study arms: 2 \\
& Length of follow-up: 12 months \\
\hline Participants & Setting: Australia \\
& Number of participants: 197 \\
& Number analysed: 169 \\
& Number lost to follow-up: 28 \\
& Sample: women recruited from a schedule from a previous epidemiologic study. Fitness level not de- \\
fined & Age (years): mean 71.6 (SD 5.4), range 60 - 85 \\
& Sex: $100 \%$ female \\
& Inclusion criteria: living independently in the community \\
& Exclusion criteria: unable to speak English
\end{tabular}


Lord 1995 (Continued)

Interventions $\quad$ 1. Group-based balance, strength, gait training: exercise class not requiring any special equipment; 1 hour, 2 a week for 4 x 10 - 12-week terms, with 2-week inter-term breaks and 5-week Christmas/summer break

2. Control: no intervention

1. Rate of falls
2. Number of people who experienced 1 or more falls (risk of falling)
3. Number of people who died (not reported by group)

\begin{tabular}{ll}
\hline Duration of the study & 52 weeks \\
\hline Adherence & Adherence measured by class attendance \\
& $\begin{array}{l}\text { 1. Group-based balance, strength, gait training: } 75 / 100 \text { attended } 26+\text { classes; of those } 75, \text { mean of } 60 \\
\text { classes }(73 \%), \text { range } 26-82 \text { classes (max classes }=82)\end{array}$
\end{tabular}

Notes Source of funding: National Health and Medical Research Council of Australia

Economic information: not reported

\section{Risk of bias}

\begin{tabular}{|c|c|c|}
\hline Bias & Authors' judgement & Support for judgement \\
\hline $\begin{array}{l}\text { Random sequence genera- } \\
\text { tion (selection bias) }\end{array}$ & Unclear risk & Quote: "randomly assigned" \\
\hline $\begin{array}{l}\text { Allocation concealment } \\
\text { (selection bias) }\end{array}$ & Unclear risk & Insufficient information to permit judgement \\
\hline $\begin{array}{l}\text { Blinding of participants } \\
\text { and personnel (perfor- } \\
\text { mance bias) } \\
\text { All outcomes }\end{array}$ & Unclear risk & $\begin{array}{l}\text { Participants and personnel not blinded to allocated group but impact of non- } \\
\text { blinding unclear }\end{array}$ \\
\hline $\begin{array}{l}\text { Blinding of outcome as- } \\
\text { sessment (detection bias) } \\
\text { Falls }\end{array}$ & High risk & $\begin{array}{l}\text { Falls reported by participants who were aware of their group allocation. Asses- } \\
\text { sors not blinded to treatment status. }\end{array}$ \\
\hline $\begin{array}{l}\text { Blinding of outcome as- } \\
\text { sessment (detection bias) } \\
\text { Fractures }\end{array}$ & Unclear risk & Not applicable \\
\hline $\begin{array}{l}\text { Blinding of outcome as- } \\
\text { sessment (detection bias) } \\
\text { Hospital admission, med- } \\
\text { ical attention and adverse } \\
\text { events }\end{array}$ & Unclear risk & Not applicable \\
\hline $\begin{array}{l}\text { Blinding of outcome as- } \\
\text { sessment (detection bias) } \\
\text { Health related quality of } \\
\text { life (self report) }\end{array}$ & Unclear risk & Not applicable \\
\hline $\begin{array}{l}\text { Incomplete outcome data } \\
\text { (attrition bias) } \\
\text { Falls and fallers }\end{array}$ & Unclear risk & $\begin{array}{l}\text { Less than } 20 \% \text { of fall outcome data are missing }(14 \%) \text {. There was an imbalance } \\
\text { in missing data between the intervention }(n=25) \text { and control }(n=3) \text { groups. It } \\
\text { is unclear whether the reason for missing outcome data is related to true out- } \\
\text { come, but the missing intervention-group data included } 13 \text { dropouts, } 3 \text { deaths, }\end{array}$ \\
\hline
\end{tabular}


Lord 1995 (Continued)

1 stroke, 2 injurious falls and 4 medical conditions that precluded participation. Reason for missing control group data is unclear

\begin{tabular}{lll}
\hline $\begin{array}{l}\text { Selective reporting (re- } \\
\text { porting bias) }\end{array}$ & Unclear risk & $\begin{array}{l}\text { Minimum set of expected outcomes not reported (adverse events not report- } \\
\text { ed) }\end{array}$ \\
\hline $\begin{array}{l}\text { Method of ascertaining } \\
\text { falls (recall bias) }\end{array}$ & High risk & $\begin{array}{l}\text { Interval recall. Fall ascertainment questionnaires sent out every } 2 \text { months. } \\
\text { Telephone call if questionnaire not returned }\end{array}$ \\
\hline
\end{tabular}

Lord 2003

RCT. Cluster-randomised by village. Stratified by accommodation (self-care or intermediate care) and
by cluster size (<75 or at least 75 residents)
Study design: Cluster-RCT
Number of study arms: 2
Number of clusters: 20
Length of follow-up: 12 months

Participants Setting: retirement villages, Sydney, Australia

Number of participants: 551

Number analysed: 508

Number lost to follow-up: 43

Sample: recruited from self-care apartment villages (78\%) and intermediate-care hostels (22\%)

Age (years): mean 79.5 (SD 6.4), range 62 - 95

Sex: $86 \%$ female

Inclusion criteria: resident in one of 20 retirement villages

Exclusion criteria: MMSE < 20; already attending exercise classes of equivalent intensity; medical conditions that precluded participation as determined by nurse or physician (neuromuscular, skeletal, cardiovascular); in hospital or away at recruitment time

Interventions

Randomised into 3 groups: 1 intervention group (group-based balance, strength, gait training) and 2 control groups (1 seated flexibility and relaxation activities, 1 no group activity). Only the intervention group and control group with no activity included in this review

1. Group-based balance, strength, gait training: within village site, instructor-led class not requiring any special equipment; 1 hour, 2 a week for 52 weeks

2. Control: no group activity
Outcomes
1. Rate of falls
2. Number of people who experienced 1 or more falls (risk of falling)
3. Number of people who died

\begin{tabular}{|c|c|}
\hline Duration of the study & 52 weeks \\
\hline \multirow[t]{2}{*}{ Adherence } & Adherence measured by class attendance, range for both groups $0-100 \%$. \\
\hline & $\begin{array}{l}\text { 1. Group-based balance, strength, gait training: mean number of classes attended 42\%; IQR: } 10-62 \\
\text { classes }\end{array}$ \\
\hline
\end{tabular}


Lord 2003 (Continued)

2. Control group: mean number of classes attended 45\%; IQR: 6 - 50 classes

Notes Source of funding: National Health and Medical Research Council of Australia, New South Wales Health, MBF (Australia)

Economic information: not reported

Number of clusters allocated to intervention: 10; number of clusters allocated to control: 10; number of clusters analysed (intervention): 10; number of clusters analysed (control): 10

Email communication to obtain fall data, response received, data included in review

\section{Risk of bias}

\begin{tabular}{lll}
\hline Bias & Authors' judgement & Support for judgement \\
\hline $\begin{array}{l}\text { Random sequence genera- } \\
\text { tion (selection bias) }\end{array}$ & Unclear risk & Method of randomisation not described \\
\hline $\begin{array}{l}\text { Allocation concealment } \\
\text { (selection bias) }\end{array}$ & High risk & $\begin{array}{l}\text { Cluster-RCT. Individual participant recruitment was undertaken after group } \\
\text { allocation. The method of concealment is not described and it is likely that } \\
\text { recruitment was undertaken by a person who was unblinded and may have } \\
\text { known participant characteristics }\end{array}$ \\
\hline
\end{tabular}

\begin{tabular}{|c|c|c|}
\hline $\begin{array}{l}\text { Blinding of participants } \\
\text { and personnel (perfor- }\end{array}$ & Unclear risk & $\begin{array}{l}\text { Participants and personnel not blinded to allocated group but impact of non- } \\
\text { blinding unclear }\end{array}$ \\
\hline
\end{tabular}
mance bias)

All outcomes

\begin{tabular}{|c|c|c|}
\hline $\begin{array}{l}\text { Blinding of outcome as- } \\
\text { sessment (detection bias) } \\
\text { Falls }\end{array}$ & High risk & $\begin{array}{l}\text { Falls reported by completion of questionnaire monthly by all participants; if } \\
\text { not returned telephone calls were made. No mention of blinding of personnel } \\
\text { carrying out phone calls, but in intermediate-care sites, falls record book was } \\
\text { kept by nursing staff (unblinded) }\end{array}$ \\
\hline
\end{tabular}

\begin{tabular}{ll}
\hline Blinding of outcome as- & Unclear risk
\end{tabular}

\begin{tabular}{|c|c|c|}
\hline $\begin{array}{l}\text { Blinding of outcome as- } \\
\text { sessment (detection bias) } \\
\text { Hospital admission, med- } \\
\text { ical attention and adverse } \\
\text { events }\end{array}$ & Unclear risk & Not applicable \\
\hline $\begin{array}{l}\text { Blinding of outcome as- } \\
\text { sessment (detection bias) } \\
\text { Health related quality of } \\
\text { life (self report) }\end{array}$ & Unclear risk & Not applicable \\
\hline $\begin{array}{l}\text { Incomplete outcome data } \\
\text { (attrition bias) } \\
\text { Falls and fallers }\end{array}$ & High risk & More than $20 \%$ of fall data were missing $(43 \%)$ \\
\hline $\begin{array}{l}\text { Selective reporting (re- } \\
\text { porting bias) }\end{array}$ & Unclear risk & $\begin{array}{l}\text { Minimum set of expected outcomes not reported (adverse events not report- } \\
\text { ed) }\end{array}$ \\
\hline $\begin{array}{l}\text { Method of ascertaining } \\
\text { falls (recall bias) }\end{array}$ & Low risk & $\begin{array}{l}\text { Retrospective. Falls ascertained by questionnaires given to residents every } \\
\text { month, with follow-up phone calls or home visit for non-responders. In addi- } \\
\text { tion nurses recorded falls in falls record book in intermediate-care hostels }\end{array}$ \\
\hline
\end{tabular}


Lord 2003 (Continued)

Cluster-randomised trials Unclear risk
Individuals were recruited to the trial after the clusters were randomised. Personnel recruiting participants were not blind to cluster; baseline comparison of the intervention arms is reported, but not baseline comparability of clusters; missing outcomes for clusters or within clusters were not reported; accounted for the clustered design in the analysis; results comparable with individually-randomised trials

Lurie 2013

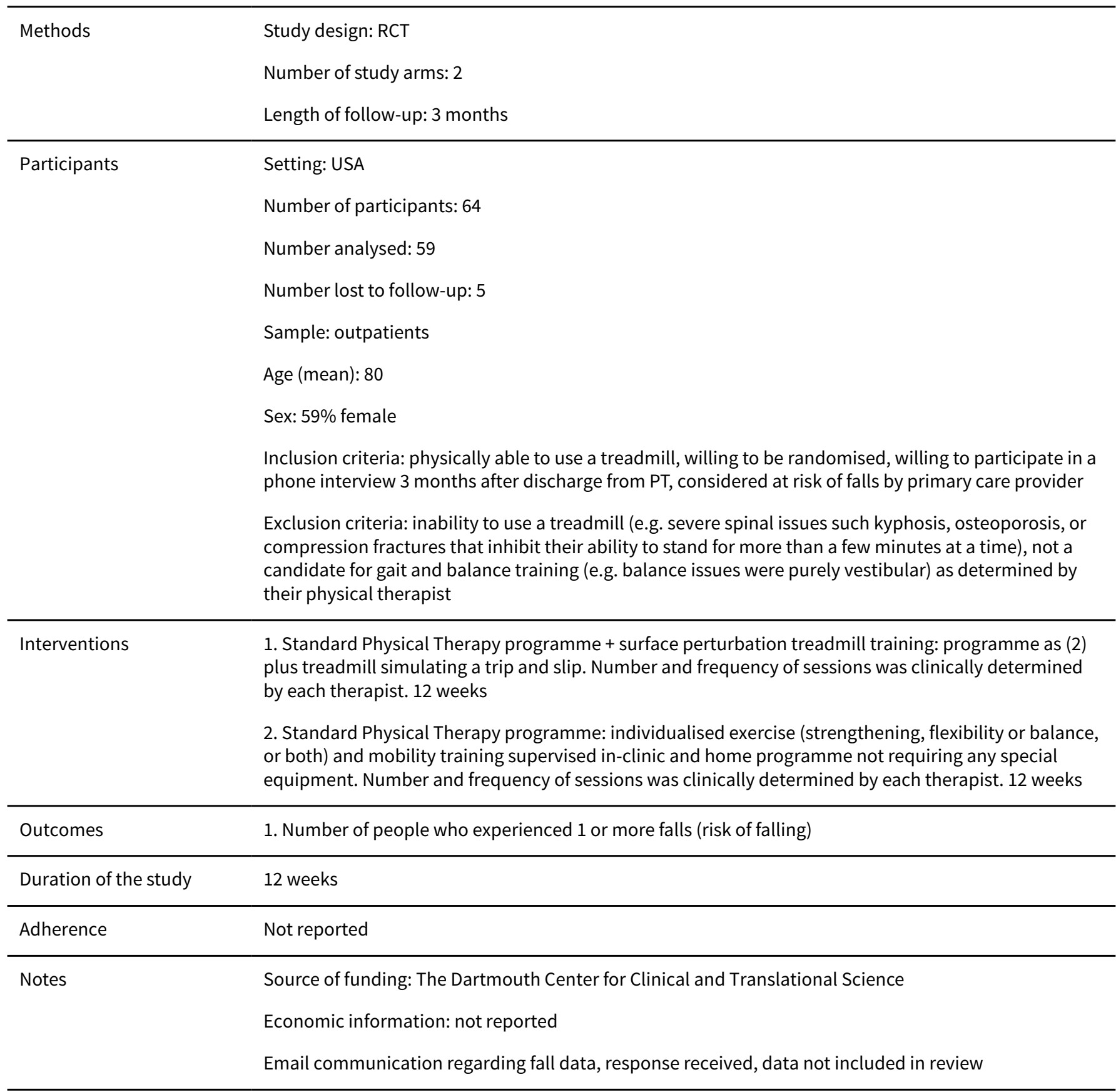

\section{Risk of bias}


Lurie 2013 (Continued)

\begin{tabular}{lll} 
Bias & Authors' judgement & Support for judgement \\
\hline $\begin{array}{l}\text { Random sequence genera- } \\
\text { tion (selection bias) }\end{array}$ & Low risk & $\begin{array}{l}\text { Quote: "Participants were assigned using permuted block randomization } \\
\text { stratified by site and gender" }\end{array}$ \\
\hline $\begin{array}{l}\text { Allocation concealment } \\
\text { (selection bias) }\end{array}$ & Unclear risk & $\begin{array}{l}\text { Quote: "Allocation concealment was ensured until after participants enrolled } \\
\text { and completed the baseline fall risk assessment". Method of allocation con- } \\
\text { cealment not specified. }\end{array}$ \\
\hline $\begin{array}{l}\text { Blinding of participants } \\
\begin{array}{l}\text { and personnel (perfor- } \\
\text { mance bias) }\end{array}\end{array}$ & Unclear risk & $\begin{array}{l}\text { Unclear risk, participants and personnel unblinded but impact of unblinding } \\
\text { unknown }\end{array}$ \\
All outcomes & &
\end{tabular}

\begin{tabular}{|c|c|c|}
\hline $\begin{array}{l}\text { Blinding of outcome as- } \\
\text { sessment (detection bias) } \\
\text { Falls }\end{array}$ & High risk & $\begin{array}{l}\text { Assessors of falls were not blinded to group allocation } \\
\text { Quote: "Another limitation of this study was the inability to blind testers to } \\
\text { treatment group allocation" }\end{array}$ \\
\hline $\begin{array}{l}\text { Blinding of outcome as- } \\
\text { sessment (detection bias) } \\
\text { Fractures }\end{array}$ & Unclear risk & Not applicable \\
\hline
\end{tabular}

\begin{tabular}{|c|c|}
\hline $\begin{array}{l}\text { Blinding of outcome as- } \\
\text { sessment (detection bias) } \\
\text { Hospital admission, med- } \\
\text { ical attention and adverse } \\
\text { events }\end{array}$ & Unclear risk \\
\hline
\end{tabular}

Blinding of outcome as- $\quad$ Unclear risk Not applicable
sessment (detection bias)
Health related quality of
life (self report)

\begin{tabular}{|c|c|c|}
\hline $\begin{array}{l}\text { Incomplete outcome data } \\
\text { (attrition bias) } \\
\text { Falls and fallers }\end{array}$ & Unclear risk & $\begin{array}{l}\text { Less than } 20 \% \text { of fall outcome data are missing }(5 \%) \text {. Missing data were not } \\
\text { balanced between groups; all missing data were from the surface perturbation } \\
\text { treadmill training programme ( } 1 \text { because they did not meet the inclusion crite- } \\
\text { ria, } 4 \text { did not return for treatment) }\end{array}$ \\
\hline
\end{tabular}

\begin{tabular}{|c|c|c|}
\hline $\begin{array}{l}\text { Selective reporting (re- } \\
\text { porting bias) }\end{array}$ & High risk & $\begin{array}{l}\text { Falls were measured, but number of falls was not reported. Adverse events } \\
\text { were not reported }\end{array}$ \\
\hline
\end{tabular}

Method of ascertaining $\quad$ High risk Asked by telephone at 3 months: " In the past 3 months have you fallen?"
falls (recall bias)

\section{Luukinen 2007}

\begin{tabular}{ll}
\hline Methods & Study design: RCT \\
& Number of study arms: 2 \\
& Length of follow-up: 16 months \\
\hline Participants & Setting: Oulu, Finland \\
Number of participants: 486
\end{tabular}


Luukinen 2007 (Continued)

Number analysed: 437

Number lost to follow-up: 49

Sample: identified from population and geriatric registers of Oulu

Age (years): mean 88 (SD 3)

Sex: $79 \%$ female

Inclusion criteria: age $\geq 85$; home-dwelling; $\geq 1$ risk factor for falling ( $\geq 2$ falls in previous year, loneliness, poor self-rated health, poor visual acuity/hearing, depression, poor cognition, impaired balance, chair rise, slow walking speed, difficulty with at least $1 \mathrm{ADL}$, able to walk outdoors, up or down stairs) Exclusion criteria: none described

\section{Interventions}

1. Individual balance and gait training: Individual plan for home exercise ( 3 a day) or group exercise, walking exercises, self-care exercises (duration and frequency not described). Interventions carried out

by OT or physiotherapist or both

2. Control: asked to visit GP without written intervention form

\begin{tabular}{ll}
\hline Outcomes & $\begin{array}{l}\text { 1. Rate of falls } \\
\text { 2. Number of people who experienced } 1 \text { or more falls (risk of falling) }\end{array}$ \\
\hline Duration of the study & 16 months median falls follow-up \\
\hline Adherence & Not reported \\
\hline Notes & Source of funding: Ministry of Health and Social Affairs of Finland \\
& Economic information: not reported \\
\hline
\end{tabular}

\section{Risk of bias}

\begin{tabular}{|c|c|c|}
\hline Bias & Authors' judgement & Support for judgement \\
\hline $\begin{array}{l}\text { Random sequence genera- } \\
\text { tion (selection bias) }\end{array}$ & Low risk & $\begin{array}{l}\text { Quote: "Randomization was done by the study statistician using a random } \\
\text { numbers table" }\end{array}$ \\
\hline $\begin{array}{l}\text { Allocation concealment } \\
\text { (selection bias) }\end{array}$ & Unclear risk & Insufficient information to permit judgement \\
\hline $\begin{array}{l}\text { Blinding of participants } \\
\text { and personnel (perfor- } \\
\text { mance bias) } \\
\text { All outcomes }\end{array}$ & Unclear risk & $\begin{array}{l}\text { Participants and personnel not blinded to allocated group but impact of non- } \\
\text { blinding unclear }\end{array}$ \\
\hline $\begin{array}{l}\text { Blinding of outcome as- } \\
\text { sessment (detection bias) } \\
\text { Falls }\end{array}$ & Low risk & $\begin{array}{l}\text { Ascertinment of falls was the same in each group and performed by blinded } \\
\text { assessor } \\
\text { Quote: "Fall recording was based on regular phone calls to all participants } \\
\text { made every second month by a research nurse ... unaware of the randomiza- } \\
\text { tion and the interventions." }\end{array}$ \\
\hline $\begin{array}{l}\text { Blinding of outcome as- } \\
\text { sessment (detection bias) } \\
\text { Fractures }\end{array}$ & Unclear risk & Not applicable \\
\hline $\begin{array}{l}\text { Blinding of outcome as- } \\
\text { sessment (detection bias) } \\
\text { Hospital admission, med- } \\
\text { ical attention and adverse } \\
\text { events }\end{array}$ & Unclear risk & Not applicable \\
\hline
\end{tabular}


Luukinen 2007 (Continued)

Blinding of outcome as- $\quad$ Unclear risk $\quad$ Not applicable
sessment (detection bias)
Health related quality of
life (self report)

\begin{tabular}{|c|c|c|}
\hline $\begin{array}{l}\text { Incomplete outcome data } \\
\text { (attrition bias) } \\
\text { Falls and fallers }\end{array}$ & High risk & More than $20 \%$ of fall data were missing $(49 \%)$ \\
\hline $\begin{array}{l}\text { Selective reporting (re- } \\
\text { porting bias) }\end{array}$ & Unclear risk & $\begin{array}{l}\text { Minimum set of expected outcomes not reported (adverse events not report- } \\
\text { ed) }\end{array}$ \\
\hline \multirow{2}{*}{$\begin{array}{l}\text { Method of ascertaining } \\
\text { falls (recall bias) }\end{array}$} & High risk & Interval recall \\
\hline & & $\begin{array}{l}\text { Quote: "Fall recording was based on regular telephone interviews once in } 2 \\
\text { months, but did not include diary reporting" }\end{array}$ \\
\hline
\end{tabular}

Madureira 2007

\begin{tabular}{ll}
\hline Methods & Study design: RCT \\
& Number of study arms: 2 \\
& Length of follow-up: 12 months \\
\hline Participants & Setting: São Paulo, Brazil \\
& Number of participants: 66 \\
& Number analysed: 60 \\
& Number lost to follow-up: 6 \\
& Sample: women attending osteometabolic disease outpatient clinic \\
& Age (years): mean 74 (SD 4.7$)$ \\
& Sex: $100 \%$ female \\
& Inclusion criteria: aged $>65$; with osteoporosis \\
& Exclusion criteria: secondary osteoporosis, visual deficiency, hearing deficiency, vestibular alteration, \\
unable to walk more than 10 m independently, contraindications for exercise training; planning to be \\
out of town for $>4$ weeks during study
\end{tabular}

Interventions
aged to continue same exercises at home, 30 minutes 3 a week
2. Control: osteoporosis treatment, "instructions to prevent falls", and 3-monthly clinic visits

\begin{tabular}{ll}
\hline Outcomes & 1. Rate of falls \\
\hline Duration of the study & 52 weeks \\
\hline Adherence & Adherence measured by class participation and frequency of home exercises \\
& $\begin{array}{l}\text { 1. Group-based balance training and walking plus home practice: } 60 \% \text { attended all exercise sessions at } \\
\text { the club; } 77 \% \text { performed home exercises } \geq 1 \text { a week, } 40 \% \text { exercised every day and } 37 \% \text { performed the } \\
\text { exercises } 1 \text { - } 4 \text { a week }\end{array}$ \\
\hline
\end{tabular}

Notes Source of funding: not reported 
Madureira 2007 (Continued)

Economic information: not reported

\section{Risk of bias}

\begin{tabular}{|c|c|c|}
\hline Bias & Authors' judgement & Support for judgement \\
\hline $\begin{array}{l}\text { Random sequence genera- } \\
\text { tion (selection bias) }\end{array}$ & Unclear risk & Quote: "The patients were randomized consecutively into two groups" \\
\hline $\begin{array}{l}\text { Allocation concealment } \\
\text { (selection bias) }\end{array}$ & Unclear risk & Insufficient information to permit judgement \\
\hline $\begin{array}{l}\text { Blinding of participants } \\
\text { and personnel (perfor- } \\
\text { mance bias) } \\
\text { All outcomes }\end{array}$ & Unclear risk & $\begin{array}{l}\text { Participants and personnel not blind to allocated group but impact of non- } \\
\text { blinding unclear }\end{array}$ \\
\hline $\begin{array}{l}\text { Blinding of outcome as- } \\
\text { sessment (detection bias) } \\
\text { Falls }\end{array}$ & Low risk & $\begin{array}{l}\text { In both groups, falls were self-reported but recorded in medical record every } \\
3 \text { months by "the Osteometabolic Outpatient Clinic physician blinded to the } \\
\text { group assignment" }\end{array}$ \\
\hline $\begin{array}{l}\text { Blinding of outcome as- } \\
\text { sessment (detection bias) } \\
\text { Fractures }\end{array}$ & Unclear risk & Not applicable \\
\hline
\end{tabular}

Blinding of outcome as- $\quad$ Unclear risk $\quad$ Not applicable
sessment (detection bias)
Hospital admission, med-
ical attention and adverse
events

\begin{tabular}{|c|c|c|}
\hline $\begin{array}{l}\text { Blinding of outcome as- } \\
\text { sessment (detection bias) } \\
\text { Health related quality of } \\
\text { life (self report) }\end{array}$ & Unclear risk & Not applicable \\
\hline $\begin{array}{l}\text { Incomplete outcome data } \\
\text { (attrition bias) } \\
\text { Falls and fallers }\end{array}$ & Low risk & $\begin{array}{l}\text { Less than } 20 \% \text { of fall data were missing }(6 \%) \text {. Missing data were balanced be- } \\
\text { tween the interention }(n=3) \text { and control }(n=3) \text { groups }\end{array}$ \\
\hline $\begin{array}{l}\text { Selective reporting (re- } \\
\text { porting bias) }\end{array}$ & High risk & $\begin{array}{l}\text { Falls were measured, but number of fallers was not reported. Adverse events } \\
\text { were not reported }\end{array}$ \\
\hline $\begin{array}{l}\text { Method of ascertaining } \\
\text { falls (recall bias) }\end{array}$ & Unclear risk & $\begin{array}{l}\text { Quote: "During the study, patients in both groups received a calendar and } \\
\text { were instructed to write down falls, which were included in the same elec- } \\
\text { tronic medical record every } 3 \text { months by the Osteometabolic Outpatient Clin- } \\
\text { ic physician blinded to the group assignment." No mention of more frequent } \\
\text { telephone follow-up }\end{array}$ \\
\hline
\end{tabular}

$\begin{array}{ll}\text { Methods } & \text { Study design: RCT } \\ & \text { Number of study arms: } 2 \\ & \text { Length of follow-up: } 24 \text { months }\end{array}$


McMurdo 1997 (Continued)

Setting: Dundee, Scotland UK
Number of participants: 118
Number analysed: 92
Number lost to follow-up: 26
Sample: women recruited by advertisement
Age (years): mean 64.5, range 60 - 73
Sex" 100\% female
Inclusion criteria: community-dwelling; post-menopausal
Exclusion criteria: conditions or drug treatment likely to affect bone

\begin{tabular}{ll}
\hline Interventions & $\begin{array}{l}\text { 1. Group-based balance training: programme of weight-bearing exercise } \\
\text { week, } 30 \text { weeks a year, over } 2 \text { years, plus } 1000 \mathrm{mg} \text { calcium carbonate } \\
\text { 2. Control: } 1000 \mathrm{mg} \text { calcium carbonate daily }\end{array}$ \\
\hline Outcomes & $\begin{array}{l}\text { 1. Rate of falls } \\
\text { 2. Number of people who experienced } 1 \text { or more falls (risk of falling) }\end{array}$ \\
3. Number of people who experienced 1 or more fall-related fractures
\end{tabular}

Duration of the study 104 weeks

Adherence $\quad$ Adherence measured by class attendance. Mean tablet complicance was $97 \%$ in both groups

1. Group-based balance training group: Mean class attendance, $76 \%$; range 46 - 100\%

Source of funding: Scottish Home and Health Department; Renacare supplied calcium carbonate
tablets
Economic information: not reported

\section{Risk of bias}

\begin{tabular}{lll}
\hline Bias & Authors' judgement & Support for judgement \\
\hline $\begin{array}{l}\text { Random sequence genera- } \\
\text { tion (selection bias) }\end{array}$ & Unclear risk & Method of randomisation not described \\
\hline $\begin{array}{l}\text { Allocation concealment } \\
\text { (selection bias) }\end{array}$ & Unclear risk & Insufficient information to permit judgement \\
\hline $\begin{array}{l}\text { Blinding of participants } \\
\text { and personnel (perfor- } \\
\text { mance bias) }\end{array}$ & Unclear risk & $\begin{array}{l}\text { Participants and personnel implementing the intervention not blind to allocat- } \\
\text { All outcomes }\end{array}$ \\
\hline $\begin{array}{l}\text { Blinding of outcome as- } \\
\text { sessment (detection bias) } \\
\text { Falls }\end{array}$ & Unclear risk & $\begin{array}{l}\text { Falls reported by participants who were aware of their group allocation. Insuf- } \\
\text { ficient information to permit judgement }\end{array}$ \\
\hline $\begin{array}{l}\text { Blinding of outcome as- } \\
\text { sessment (detection bias) }\end{array}$ & Unclear risk & Method of recording fractures is unclear \\
\begin{tabular}{l} 
Fractures \\
\hline
\end{tabular} & & \\
\hline
\end{tabular}

$\begin{array}{lll}\text { Blinding of outcome as- } & \text { Unclear risk } & \text { Not applicable } \\ \text { sessment (detection bias) }\end{array}$


McMurdo 1997 (Continued) Hospital admission, medical attention and adverse events

\begin{tabular}{|c|c|c|}
\hline $\begin{array}{l}\text { Blinding of outcome as- } \\
\text { sessment (detection bias) } \\
\text { Health related quality of } \\
\text { life (self report) }\end{array}$ & Unclear risk & Not applicable \\
\hline $\begin{array}{l}\text { Incomplete outcome data } \\
\text { (attrition bias) } \\
\text { Falls and fallers }\end{array}$ & High risk & More than $20 \%$ of fall data were missing $(26 \%)$ \\
\hline $\begin{array}{l}\text { Selective reporting (re- } \\
\text { porting bias) }\end{array}$ & Unclear risk & $\begin{array}{l}\text { Minimum set of expected outcomes not reported (adverse events not report- } \\
\text { ed) }\end{array}$ \\
\hline $\begin{array}{l}\text { Method of ascertaining } \\
\text { falls (recall bias) }\end{array}$ & Unclear risk & No description about ascertainment of falls \\
\hline
\end{tabular}

\begin{tabular}{ll}
\hline Methods & Study design: RCT \\
& Number of study arms: 2 \\
& Length of follow-up: 6 months \\
\hline Setting: Arkansas, USA \\
Number of participants: 338 \\
Number analysed: 238 \\
Number lost to follow-up: 100 \\
Sample: volunteers from 17 senior citizens' centres \\
Age (years): mean 73.5 \\
Sex: $57 \%$ female \\
Inclusion criteria: aged $\geq 65$ years; able to walk at least 30 feet without assistance from others; able to \\
follow instructions and give consent \\
Exclusion criteria: resident in a nursing home; acute medical problems; cognitive impairment
\end{tabular}

1. Group-based balance, strength, flexibility, gait training and walking: self-perceived moderate intensi-
ty, 90 -minute sessions, 3 a week for 6 weeks
2. Control: group seminars on non-health-related topics of interest to senior citizens. Same time and
frequency as intervention group

Outcomes
$\begin{aligned} & \text { 2. Rute of falls } \\ & \text { 3. Number of people who experienced } 1 \text { or more falls (risk of falling) }\end{aligned}$

\begin{tabular}{ll}
\hline Duration of the study & 26 weeks \\
\hline Adherence & Adherence measured by retention/attrition rate \\
& Attrition data:
\end{tabular}


1. Group-based balance, strength, flexibility, gait training and walking: $\mathrm{n}=12$ never attended exercise sessions after 6 weeks

2. Control: $\mathrm{n}=23$ never attended seminars after 6 weeks

Notes

Source of funding: National Institute on Aging, Rehabilitation Research and Development Service, Department of Veterans Affairs

Economic information: not reported

\section{Risk of bias}

\begin{tabular}{lll}
\hline Bias & Authors' judgement & Support for judgement \\
\hline $\begin{array}{l}\text { Random sequence genera- } \\
\text { tion (selection bias) }\end{array}$ & Low risk & Randomised by coin flip \\
\hline $\begin{array}{l}\text { Allocation concealment } \\
\text { (selection bias) }\end{array}$ & Unclear risk & Insufficient information to permit judgement \\
\hline $\begin{array}{l}\text { Blinding of participants } \\
\text { and personnel (perfor- } \\
\text { mance bias) }\end{array}$ & Unclear risk & $\begin{array}{l}\text { Blinding of participants and treatment personnel not mentioned in report, } \\
\text { All outcomes }\end{array}$ \\
\hline
\end{tabular}

\begin{tabular}{|c|c|c|}
\hline $\begin{array}{l}\text { Blinding of outcome as- } \\
\text { sessment (detection bias) } \\
\text { Falls }\end{array}$ & Low risk & $\begin{array}{l}\text { Falls reported using the same method in each group, by participants who were } \\
\text { aware of their group allocation. Assessor blinded to group allocation }\end{array}$ \\
\hline $\begin{array}{l}\text { Blinding of outcome as- } \\
\text { sessment (detection bias) } \\
\text { Fractures }\end{array}$ & Unclear risk & Not applicable \\
\hline $\begin{array}{l}\text { Blinding of outcome as- } \\
\text { sessment (detection bias) } \\
\text { Hospital admission, med- } \\
\text { ical attention and adverse } \\
\text { events }\end{array}$ & Unclear risk & $\begin{array}{l}\text { Adverse events were obtained in the same manner in each group. } \\
\text { Quote: "Research staff .. involved in collection of evaluation data did not know } \\
\text { the participants' group assignemnt at the time of their evaluation". Adverse } \\
\text { events were self-reported and were not clarified using medical records }\end{array}$ \\
\hline $\begin{array}{l}\text { Blinding of outcome as- } \\
\text { sessment (detection bias) } \\
\text { Health related quality of } \\
\text { life (self report) }\end{array}$ & Unclear risk & Not applicable \\
\hline
\end{tabular}

\begin{tabular}{lll}
\hline $\begin{array}{l}\text { Incomplete outcome data } \\
\text { (attrition bias) } \\
\text { Falls and fallers }\end{array}$ & High risk & More than 20\% of fall data were missing (30\%) \\
\hline $\begin{array}{l}\text { Selective reporting (re- } \\
\text { porting bias) }\end{array}$ & Unclear risk & $\begin{array}{l}\text { Prespecified falls outcomes reported. No prospective trial registration or pro- } \\
\text { tocol }\end{array}$ \\
\hline $\begin{array}{l}\text { Method of ascertaining } \\
\text { falls (recall bias) }\end{array}$ & Low risk & $\begin{array}{l}\text { Prospective. Recorded on pre-printed postcards weekly with telephone calls } \\
\text { to non-correspondents to optimise compliance }\end{array}$ \\
\hline
\end{tabular}

\section{Merom 2016}

Methods Study design: Cluster-RCT


Merom 2016 (Continued)

Number of study arms: 2

Number of clusters: 23

Length of follow-up: 12 months

Setting: Sydney, Australia
Number of participants: 530
Number analysed: 522
Number lost to follow-up: 8
Sample: living in retirement village
Age (years): Age > 80 years: $39 \%$
Sex: $85 \%$ female
Inclusion criteria: Eligible participants had to be a resident of the village; be able to walk at least 50 m;
agree to undergo physical and cognitive testing; plan to stay in the village for the next 12 months; and
obtain medical clearance to participate in the study
Exclusion criteria: Participants were excluded if they planned to leave the village for 3 months or more
during the trial period, or if they scored < 24 on the MMSE in the baseline assessment indicating cogni-
tive impairment

Interventions

1. Group-based social dancing: folk dancing or ballroom dancing classes with gradual increase in cognitive complexity and cardiovascular effort; 1 hour, 2 a week, for 12 months

2. Control group: usual activities, and asked not to join a dance class during the trial period, placed on a wait list for the dance classes at the end of trial.

1. Rate of falls
$\begin{aligned} & \text { 2. Number of people who experienced } 1 \text { or more falls (risk of falling) } \\ & \text { 3. Health-related quality of life } \\ & \text { 4. Number of people who died }\end{aligned}$

Duration of the study 52 weeks

Adherence
$\begin{aligned} & \text { Adherence measured by session attendance } \\ & \text { mately } 45 \text { sessions. The median attendance was lower for folk (55\%) than ballroom dancing }(60 \%)\end{aligned}$

Notes Source of funding: NHMRC

Economic information: not reported

Number of clusters allocated to intervention: 12; number of clusters allocated to control: 11; number of clusters analysed (intervention): 12; number of clusters analysed (control): 11

\section{Risk of bias}

\begin{tabular}{lll}
\hline Bias & Authors' judgement & Support for judgement \\
\hline $\begin{array}{l}\text { Random sequence genera- } \\
\text { tion (selection bias) }\end{array}$ & Low risk & Computer-generated randomisation method, constrained using minimisation \\
\hline
\end{tabular}


Merom 2016 (Continued)

Allocation concealment (selection bias)

Unclear risk

The relative timing of the randomisation of clusters and recruitment of participants is unclear. It is unclear whether personnel recruiting participants were blinded to intervention group to which the cluster was randomised

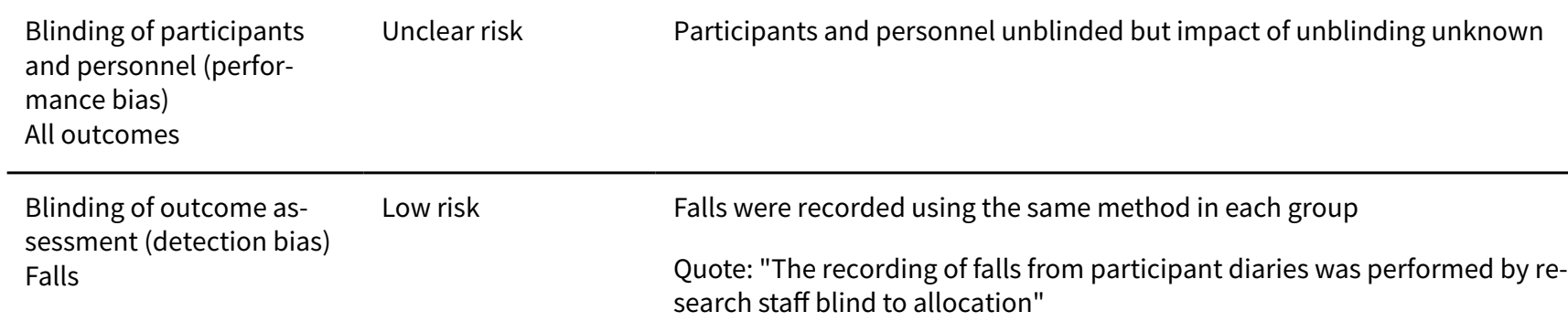

\begin{tabular}{lll}
\hline Blinding of outcome as- & Unclear risk & Not applicable \\
sessment (detection bias) & \\
Fractures &
\end{tabular}

\begin{tabular}{ll} 
Blinding of outcome as- & Unclear risk Not applicable \\
sessment (detection bias) & \\
Hospital admission, med- & \\
ical attention and adverse & \\
events & \\
\hline
\end{tabular}

Blinding of outcome as- High risk Participants were not blinded to group allocation
sessment (detection bias)

Health related quality of

life (self report)

\begin{tabular}{|c|c|c|}
\hline $\begin{array}{l}\text { Incomplete outcome data } \\
\text { (attrition bias) } \\
\text { Falls and fallers }\end{array}$ & Low risk & $\begin{array}{l}\text { Less than } 20 \% \text { of fall data were missing }(1 \%) \text {. There were missing fall data from } \\
\text { an equal number of participants in the intervention group }(n=4) \text { and the con- } \\
\text { trol group }(n=4) \text {. The reason for missing fall data was not clear }\end{array}$ \\
\hline
\end{tabular}

\begin{tabular}{lll}
\hline $\begin{array}{l}\text { Selective reporting (re- } \\
\text { porting bias) }\end{array}$ & Low risk & $\begin{array}{l}\text { Prespecified falls and adverse event outcomes reported. Prospective trial reg- } \\
\text { istration available and specifies the same fall outcomes as those in the trial re- } \\
\text { port }\end{array}$ \\
\hline $\begin{array}{l}\text { Method of ascertaining } \\
\text { falls (recall bias) }\end{array}$ & Low risk & $\begin{array}{l}\text { Participants were asked to record "F" (fall) or "N" (no fall) each day using } \\
\text { monthly calendars (diaries), which were returned by mail at the end of each } \\
\text { month. Participants who reported a fall were interviewed by telephone to ob- } \\
\text { tain details about where the fall(s) occurred; whether the fall resulted in in- } \\
\text { juries; and whether any treatment was sought. Participants who did not return } \\
\text { their calendars within } 2 \text { weeks were telephoned by study researchers and ver- } \\
\text { bal responses were recorded. At the end of the call, they were also requested } \\
\text { to return their calendar by mail to maintain completeness }\end{array}$ \\
\hline
\end{tabular}

Cluster-randomised trials Unclear risk The relative timing of the randomisation of clusters and recruitment of participants is unclear. There was attempt at concealment,

Quote: "Retirement villages were randomised by the trial statistician... The trial statistician advised the study coordinator of the village's allocation, and the study coordinator arranged the delivery of the intervention. Allocation was thus concealed from the research team that were recruiting villages and participants and performing the baseline assessments"

Baseline comparison of the intervention arms is reported, but not baseline comparability of clusters; 


\section{Miko 2017}

\begin{tabular}{ll}
\hline Methods & Study design: RCT \\
& Number of study arms: 2 \\
& Length of follow-up: 12 months \\
\hline
\end{tabular}

Setting: Budapest, Hungary
Number of participants: 100
Number analysed: 97
Number lost to follow-up: 3
Sample: community-dwelling women
Age (years): Intervention group mean 69.3 (SD 4.6), Control group mean 69.1 (SD 5.3)
Sex: $100 \%$ female
Inclusion criteria: women with osteoporosis, classified using the World Health Organization diagnostic
criteria for established osteoporosis in postmenopausal women were eligible: bone mineral density T-
score lower than -2.5 in the lumbar spine, femoral neck or total femur region, and a history of at least 1
osteoporotic fracture
Exclusion criteria: visual deficiency, severe auditiory or vestibular deficiency, advanced locomotor dis-
eases, women who used assistive walking devices or who were unable to walk independently more
than 10 metres, progressive neurological or unstable cardiovascular diseases and participation in a
regular physical exercise programme in the past 6 months

Interventions

1. Individual, partially-supervised balance training: supervised by physiotherapist in back, torso and lower-extremity muscle-strengthening exercises and balance training. Progressed through 3 levels; 30minute sessions, 2 a week, for 1 year, plus home programme 1 hour a day

2. Control group: Received osteoporosis treatment only

\begin{tabular}{ll}
\hline Outcomes & $\begin{array}{l}\text { 1. Rate of falls } \\
\text { 2. Number of people who experienced } 1 \text { or more falls (risk of falling) }\end{array}$ \\
\hline Duration of the study & 52 weeks \\
\hline Adherence & Not reported \\
\hline Notes & Source of funding: no funding received \\
& Economic information: not reported \\
\hline
\end{tabular}

\section{Risk of bias}

Bias Authors' judgement Support for judgement


Miko 2017 (Continued)

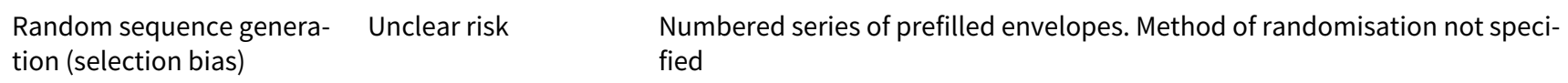

\begin{tabular}{|c|c|c|}
\hline $\begin{array}{l}\text { Allocation concealment } \\
\text { (selection bias) }\end{array}$ & Unclear risk & $\begin{array}{l}\text { Quote: "A numbered series of prefilled envelopes specifying the group". No re- } \\
\text { port of the location and whether envelopes opaque or sealed }\end{array}$ \\
\hline
\end{tabular}

(selection bias) port of the location and whether envelopes opaque or sealed

Blinding of participants Unclear risk Participants and personnel unblinded but impact of unblinding unknown
and personnel (perfor-
mance bias)
All outcomes

\begin{tabular}{|c|c|c|}
\hline $\begin{array}{l}\text { Blinding of outcome as- } \\
\text { sessment (detection bias) } \\
\text { Falls }\end{array}$ & Unclear risk & $\begin{array}{l}\text { Falls reported using same method in each group. Unclear if personnel record- } \\
\text { ing/confirming fall outcomes were blind to group allocation }\end{array}$ \\
\hline $\begin{array}{l}\text { Blinding of outcome as- } \\
\text { sessment (detection bias) } \\
\text { Fractures }\end{array}$ & Unclear risk & Not applicable \\
\hline $\begin{array}{l}\text { Blinding of outcome as- } \\
\text { sessment (detection bias) } \\
\text { Hospital admission, med- } \\
\text { ical attention and adverse } \\
\text { events }\end{array}$ & Unclear risk & Not applicable \\
\hline $\begin{array}{l}\text { Blinding of outcome as- } \\
\text { sessment (detection bias) } \\
\text { Health related quality of } \\
\text { life (self report) }\end{array}$ & Unclear risk & Not applicable \\
\hline $\begin{array}{l}\text { Incomplete outcome data } \\
\text { (attrition bias) } \\
\text { Falls and fallers }\end{array}$ & Low risk & $\begin{array}{l}\text { Less than } 20 \% \text { of fall data were missing ( } 3 \%) \text {. There were missing fall data from } \\
1 \text { intervention participant (due to loss of interest) and } 2 \text { control participants ( } 1 \\
\text { due to loss of interest, } 1 \text { without explanation). }\end{array}$ \\
\hline $\begin{array}{l}\text { Selective reporting (re- } \\
\text { porting bias) }\end{array}$ & Unclear risk & $\begin{array}{l}\text { Minimum set of expected outcomes not reported (adverse events not report- } \\
\text { ed) }\end{array}$ \\
\hline $\begin{array}{l}\text { Method of ascertaining } \\
\text { falls (recall bias) }\end{array}$ & Low risk & $\begin{array}{l}\text { Fall diary kept to record any fall and the circumstances. No follow-up phone } \\
\text { calls noted }\end{array}$ \\
\hline
\end{tabular}

\section{Mirelman 2016}

\begin{tabular}{ll}
\hline Methods & Study design: RCT \\
& Number of study arms: 2 \\
& Length of follow-up: 6 months \\
\hline
\end{tabular}

Setting: Belgium, Israel, Italy, the Netherlands, and the UK
Number of participants: 152
Number analysed: no fall data
Sample: community-dwelling
Age (years): mean 82.6




\section{Sex: $35 \%$ female}

Inclusion criteria: aged 60 - 90 years, able to walk $\geq 5$ minutes unassisted, stable medication for the past month, self-reported $\geq 2$ falls within 6 months before screening; individuals with mild cognitive impairment were included if they had a score of 0.5 on the Clinical Dementia Rating scale

Exclusion criteria: psychiatric comorbidity (e.g. major depressive disorder in accordance with DSM IV criteria); history of stroke, traumatic brain injury, or other neurological disorders (other than Parkinson's disease and mild cognitive impairment, for those groups); acute lower back or lower extremity pain; peripheral neuropathy; rheumatic and orthopaedic diseases; or a clinical diagnosis of dementia or severe cognitive impairment (MMSE score $<21$ )

Interventions Individual, supervised treadmill training: progressed with treadmill duration and speed; 45 -minute
session, 3 a week for 6 weeks
2. Individual, supervised treadmill training plus virtual reality: as (1) plus received projected images of
the virtual environment (e.g. obstacles, distractors) that necessitated continual adjustment of steps;
45-minute session, 3 a week for 6 weeks

\begin{tabular}{ll}
\hline Outcomes & 1. Health-related quality of life \\
\hline Duration of the study & 26 weeks
\end{tabular}

Adherence $\quad$ Adherence measured by number of completed sessions of the 18 sessions:

1. Individual, supervised treadmill training: $16 \cdot 82$ (SD 1·81)

2. Individual, supervised treadmill training plus virtual reality: 16.62 (SD 1.78)

Notes At baseline 130 participants had Parkinson's disease, 43 mild cognitive impairment, 109 idiopathic falls. Falls data unavailable only for non-Parkinson's disease participants

Source of funding: European Commission

Economic information: not reported

\section{Risk of bias}

\begin{tabular}{lll}
\hline Bias & Authors' judgement & Support for judgement \\
\hline $\begin{array}{l}\text { Random sequence genera- } \\
\text { tion (selection bias) }\end{array}$ & Low risk & $\begin{array}{l}\text { Quote: "By use of computer-based allocation, participants were randomly as- } \\
\text { signed" }\end{array}$ \\
\hline $\begin{array}{l}\text { Allocation concealment } \\
\text { (selection bias) }\end{array}$ & Low risk & $\begin{array}{l}\text { Group allocation performed by a third party not involved in the day-to-day } \\
\text { running of the study; treating therapist notified by e-mail to ensure concealed } \\
\text { allocation }\end{array}$ \\
\hline $\begin{array}{l}\text { Blinding of participants } \\
\text { and personnel (perfor- } \\
\text { mance bias) }\end{array}$ & Unclear risk & Participants and personnel unblinded but impact of unblinding unknown \\
$\begin{array}{l}\text { All outcomes } \\
\begin{array}{l}\text { Blinding of outcome as- } \\
\text { sessment (detection bias) }\end{array}\end{array}$ & Low risk & Falls recorded using same method in each group \\
$\begin{array}{l}\text { Falls } \\
\begin{array}{l}\text { Blinding of outcome as- } \\
\text { sessment (detection bias) }\end{array}\end{array}$ & Unclear risk & Quote: "Falls were recorded without knowledge of training group" \\
\begin{tabular}{l} 
Fractures \\
\hline
\end{tabular} & Not applicable \\
\hline
\end{tabular}


Mirelman 2016 (Continued)

Blinding of outcome assessment (detection bias) Hospital admission, medical attention and adverse events
Unclear risk The method of recording adverse events was unclear

\begin{tabular}{|c|c|c|}
\hline $\begin{array}{l}\text { Blinding of outcome as- } \\
\text { sessment (detection bias) } \\
\text { Health related quality of } \\
\text { life (self report) }\end{array}$ & High risk & Participants unblinded to intervention group \\
\hline $\begin{array}{l}\text { Incomplete outcome data } \\
\text { (attrition bias) } \\
\text { Falls and fallers }\end{array}$ & Low risk & $\begin{array}{l}\text { Less than } 20 \% \text { had missing data }(7 \%) \text { for the study. Missing data were bal- } \\
\text { anced between the treadmill training group }(n=12) \text { and treadmill plus virtual } \\
\text { reality group }(n=8) \text {, with reasons for missing data similar between groups (e.g. } \\
2 \text { adverse events in treadmill group, } 3 \text { adverse events in virtual reality group) }\end{array}$ \\
\hline $\begin{array}{l}\text { Selective reporting (re- } \\
\text { porting bias) }\end{array}$ & High risk & Falls measured, but number of fallers is not presented \\
\hline $\begin{array}{l}\text { Method of ascertaining } \\
\text { falls (recall bias) }\end{array}$ & Low risk & $\begin{array}{l}\text { Participants received a falls calendar, which they were provided as a paper } \\
\text { version, web-based calender, or a smartphone application. Research staff con- } \\
\text { tacted all participants every month to maximise compliance }\end{array}$ \\
\hline
\end{tabular}

\section{Morgan 2004}

\begin{tabular}{ll} 
Methods & Study design: RCT \\
& Number of study arms: 2 \\
& Length of follow-up: 12 months \\
\hline
\end{tabular}

Setting: community and assisted-living facilities Florida, USA
Number of participants: 294
Number analysed: 229
Number lost to follow-up: 65
Sample: recruited from Miami Department of Veterans Affairs Medical Centre, 9 assisted-living facilities,
private physical therapy clinic
Age (years): mean 80.5 (SD 7.5)
Sex: $71 \%$ female
Inclusion criteria: aged $\geq 60 ;$ hospital admission or bedrest for $\geq 2$ days in previous month
Exclusion criteria: medical conditions precluding exercise programme (angina, severe osteoporosis,
etc.); MMSE < 23 (unable to follow instructions); using oxygen therapy at home; planned inpatient treat-
ment or evaluation in 2 months following recruitment; requiring human assistance, wheelchair or arti-
ficial limbs to walk

Interventions

1. Group-based strength, balance and gait training: seated and standing exercises with no special equipment used, supervised by a physical therapist assisted by a physical therapy assistant; $45 \mathrm{~min}$ utes, 3 a week for 8 weeks

2. Control: usual activities

Outcomes 1. Number of people who experienced 1 or more falls (risk of falling)

Duration of the study

52 weeks 
Morgan 2004 (Continued)

Adherence
Adherence measured by completion of scheduled exercise sessions

1. Group-based strength, balance and gait training: completed an average of $70 \%$ of the 24 scheduled exercise sessions

Source of funding: not reported

Economic information: not reported

SAFE-GRIP (Study to Assess Falls among Elderly Geriatric Rehabilitation Intensive Program)

Email communication about fall data, response received, data not included in review

\section{Risk of bias}

\begin{tabular}{|c|c|c|}
\hline Bias & Authors' judgement & Support for judgement \\
\hline $\begin{array}{l}\text { Random sequence genera- } \\
\text { tion (selection bias) }\end{array}$ & Unclear risk & $\begin{array}{l}\text { Randomisation stratified by sex, age }(<75 \text { and } \geq 75 \text { ), falls history in previous } \\
\text { month (fall/no fall). Method of randomisation not described }\end{array}$ \\
\hline $\begin{array}{l}\text { Allocation concealment } \\
\text { (selection bias) }\end{array}$ & Unclear risk & Insufficient information to permit judgement \\
\hline $\begin{array}{l}\text { Blinding of participants } \\
\text { and personnel (perfor- } \\
\text { mance bias) } \\
\text { All outcomes }\end{array}$ & Unclear risk & $\begin{array}{l}\text { Participants and personnel implementing the intervention not blinded to allo- } \\
\text { cated group, but impact of non-blinding unclear }\end{array}$ \\
\hline $\begin{array}{l}\text { Blinding of outcome as- } \\
\text { sessment (detection bias) } \\
\text { Falls }\end{array}$ & Unclear risk & Blinding not described. Insufficient information to permit judgement \\
\hline $\begin{array}{l}\text { Blinding of outcome as- } \\
\text { sessment (detection bias) } \\
\text { Fractures }\end{array}$ & Unclear risk & Not applicable \\
\hline $\begin{array}{l}\text { Blinding of outcome as- } \\
\text { sessment (detection bias) } \\
\text { Hospital admission, med- } \\
\text { ical attention and adverse } \\
\text { events }\end{array}$ & Unclear risk & Not applicable \\
\hline $\begin{array}{l}\text { Blinding of outcome as- } \\
\text { sessment (detection bias) } \\
\text { Health related quality of } \\
\text { life (self report) }\end{array}$ & Unclear risk & Not applicable \\
\hline $\begin{array}{l}\text { Incomplete outcome data } \\
\text { (attrition bias) } \\
\text { Falls and fallers }\end{array}$ & High risk & More than $20 \%$ of fall data are missing $(22 \%)$ \\
\hline $\begin{array}{l}\text { Selective reporting (re- } \\
\text { porting bias) }\end{array}$ & High risk & $\begin{array}{l}\text { Falls were measured, but number of falls was not reported. Adverse events } \\
\text { were not reported }\end{array}$ \\
\hline $\begin{array}{l}\text { Method of ascertaining } \\
\text { falls (recall bias) }\end{array}$ & Low risk & Prospective. Pre-dated postcard diaries returned every 2 weeks \\
\hline
\end{tabular}


Morone 2016

\begin{tabular}{ll}
\hline Methods & Study design: RCT \\
Number of study arms: 2 \\
Length of follow-up: 3 months \\
\hline Setting: Italy \\
Number of participants: 38 \\
Number analysed: 38 \\
Number lost to follow-up: 0 \\
Sample: community-dwelling \\
Age (years): mean 68.93 (SD 4.18 ) \\
Sex: $100 \%$ female \\
Inclusion criteria: women; no or irregular physical or educational programmes for balance (or not per- \\
formed for the last 2 years); age $>65$ years; presence of a reduction in balance measured by the Berg \\
Balance Scale (<45); presence of bone loss (T score $>1.5$ and < 2.5) as measured by central DEXA scan \\
Exclusion criteria: presence of any orthopaedic, cardiovascular or oncologic pathology that could affect \\
the balance ability; fracture/s in past year
\end{tabular}

Interventions

1. Group-based balance training using Wii-Fit: Wii Fit programme (balance, yoga, standing leg strengthening) supervised by a physiotherapist, 1-hour session, 2 a week for 8 weeks

2. Group-based balance training: conventional balance exercises (flexibility, lying muscle strengthening, balance on unstable balance platform, postural exercises in supine) supervised by a physiotherapist, 1-hour session, 2 a week for 8 weeks

\begin{tabular}{ll}
\hline Outcomes & No outcomes included in review \\
\hline Duration of the study & 12 weeks \\
\hline Adherence & Not reported \\
\hline Notes & Source of funding: not reported \\
& $\begin{array}{l}\text { Economic information: not reported } \\
\text { No fall data in paper. Email communication about fall data, no response received. No fall data included } \\
\text { in review }\end{array}$
\end{tabular}

\section{Risk of bias}

\begin{tabular}{lll}
\hline Bias & Authors' judgement & Support for judgement \\
\hline $\begin{array}{l}\text { Random sequence genera- } \\
\text { tion (selection bias) }\end{array}$ & Low risk & Quote: "computer generated list" \\
\hline $\begin{array}{l}\text { Allocation concealment } \\
\text { (selection bias) }\end{array}$ & Low risk & $\begin{array}{l}\text { Quote: "allocation was concealed by covering each number of the list with an } \\
\text { opaque adhesive label" }\end{array}$ \\
\hline $\begin{array}{l}\text { Blinding of participants } \\
\text { and personnel (perfor- } \\
\text { mance bias) }\end{array}$ & Unclear risk & $\begin{array}{l}\text { Participants and personnel not blinded to group allocation. Effect of non- } \\
\text { blinding unclear }\end{array}$ \\
\end{tabular}


Morone 2016 (Continued)

All outcomes

\begin{tabular}{|c|c|c|}
\hline $\begin{array}{l}\text { Blinding of outcome as- } \\
\text { sessment (detection bias) }\end{array}$ & Unclear risk & $\begin{array}{l}\text { Falls were recorded using the same method in both groups. It is unclear } \\
\text { whether assessors were blinded when collecting fall data }\end{array}$ \\
\hline
\end{tabular}

Falls

\begin{tabular}{lll}
\hline $\begin{array}{l}\text { Blinding of outcome as- } \\
\text { sessment (detection bias) } \\
\text { Fractures }\end{array}$ & Unclear risk & Not applicable \\
\hline $\begin{array}{l}\text { Blinding of outcome as- } \\
\text { sessment (detection bias) }\end{array}$ & Unclear risk & Not applicable \\
$\begin{array}{l}\text { Hospital admission, med- } \\
\text { ical attention and adverse } \\
\text { events }\end{array}$ & & \\
\end{tabular}

\begin{tabular}{|c|c|c|}
\hline $\begin{array}{l}\text { Blinding of outcome as- } \\
\text { sessment (detection bias) } \\
\text { Health related quality of } \\
\text { life (self report) }\end{array}$ & High risk & Participants not blind to group allocation \\
\hline
\end{tabular}

\begin{tabular}{lll}
\hline $\begin{array}{l}\text { Incomplete outcome data } \\
\text { (attrition bias) } \\
\text { Falls and fallers }\end{array}$ & High risk & No fall data available \\
\hline $\begin{array}{l}\text { Selective reporting (re- } \\
\text { porting bias) }\end{array}$ & High risk & Fall outcome prespecified but fall data not presented \\
\hline $\begin{array}{l}\text { Method of ascertaining } \\
\text { falls (recall bias) }\end{array}$ & Unclear risk & $\begin{array}{l}\text { Quote: "participants enrolled in both groups recorded in a specific diary the } \\
\text { falls or events related to falls during the 3-month follow-up" }\end{array}$ \\
\hline
\end{tabular}

\section{Morrison 2018}

\begin{tabular}{ll}
\hline Methods & Study design: RCT \\
Number of study arms: 2 \\
Length of follow-up: 3 months \\
\hline Setting: Virginia, USA \\
Number of participants: 65 \\
Number analysed: 46 \\
Number lost to follow-up: 19 \\
Sample: community-dwelling \\
Age (years): mean 66.99 (SD 5.42) \\
Sex: $48 \%$ female \\
Inclusion criteria: Type 2 diabetes \\
Exclusion criteria: significant cardiovascular disease, unstable proliferative retinopathy, end-stage re- \\
nal disease, or uncontrolled hypertension; no balance or resistance training during the previous year
\end{tabular}


Morrison 2018 (Continued)

Interventions
1. Group-based balance training: balance and postural control exercises closely mimicking the type of training performed during unsupervised (Wii Fit) training. a) warm-up (lower-limb stretching); b) mostly balance exercises including heel-toe walking. calf raises, forward leans, single-leg balance, and basic yoga stretches (the yoga stretches selected were the same as those offered within the Wii program); 40minute sessions, 3 a week for 12 weeks

2. Home-based strength, balance and aerobic Wii Fit programme: aerobics, yoga, strength training, and balance using the Wii Fit Balance System and software programme. 1-hour interactive tutorial on using the equipment, exercised unsupervised at home, 40-minute sessions, 3 a week for 12 weeks

\begin{tabular}{ll}
\hline Outcomes & 1. Rate of falls \\
2. Number of people who experienced 1 or more falls
\end{tabular}

\begin{tabular}{ll}
\hline Duration of the study & 12 weeks \\
\hline Adherence & Not reported \\
\hline Notes & Source of funding: American Diabetes Association \\
& Economic information: not reported \\
& Email communication to obtain fall data, response received, data included in review (there were no \\
falls) & Data could not be analysed due to zero events for falls (and thus fallers)
\end{tabular}

\section{Risk of bias}

\begin{tabular}{|c|c|c|}
\hline Bias & Authors' judgement & Support for judgement \\
\hline $\begin{array}{l}\text { Random sequence genera- } \\
\text { tion (selection bias) }\end{array}$ & Low risk & Used a random-number table \\
\hline $\begin{array}{l}\text { Allocation concealment } \\
\text { (selection bias) }\end{array}$ & Unclear risk & Concealment not specified \\
\hline $\begin{array}{l}\text { Blinding of participants } \\
\text { and personnel (perfor- } \\
\text { mance bias) } \\
\text { All outcomes }\end{array}$ & Unclear risk & Blinding not specified \\
\hline $\begin{array}{l}\text { Blinding of outcome as- } \\
\text { sessment (detection bias) } \\
\text { Falls }\end{array}$ & Unclear risk & $\begin{array}{l}\text { Falls was measured using the same measures in all groups. Blinding not speci- } \\
\text { fied }\end{array}$ \\
\hline $\begin{array}{l}\text { Blinding of outcome as- } \\
\text { sessment (detection bias) } \\
\text { Fractures }\end{array}$ & Unclear risk & Not applicable \\
\hline $\begin{array}{l}\text { Blinding of outcome as- } \\
\text { sessment (detection bias) } \\
\text { Hospital admission, med- } \\
\text { ical attention and adverse } \\
\text { events }\end{array}$ & Unclear risk & Not applicable \\
\hline
\end{tabular}

\begin{tabular}{|c|c|c|}
\hline $\begin{array}{l}\text { Blinding of outcome as- } \\
\text { sessment (detection bias) } \\
\text { Health related quality of } \\
\text { life (self report) }\end{array}$ & Unclear risk & Not applicable \\
\hline
\end{tabular}


Morrison 2018 (Continued)

Incomplete outcome data High risk Large loss (>20\%) to follow-up

(attrition bias)

Falls and fallers

$\begin{aligned} & \text { Selective reporting (re- } \\ & \text { porting bias) }\end{aligned}$
High risk $\quad$ Fall outcome prespecified but fall data not presented

\begin{tabular}{ll}
\hline $\begin{array}{l}\text { Method of ascertaining } \\
\text { falls (recall bias) }\end{array}$ & High risk \\
& ing the 12-week exercise intervention"
\end{tabular}

\section{Ng 2015}

$\begin{array}{ll}\text { Methods } & \text { Study design: RCT } \\ & \text { Number of study arms: } 2 \\ & \text { Length of follow-up: } 12 \text { months }\end{array}$

Participants
$\begin{aligned} & \text { Sumber of participants: } 98 \\ & \text { Number analysed: } 92 \\ & \text { Number lost to follow-up: } 6 \\ & \text { Sample: community-dwelling } \\ & \text { Age (years): mean } 70.0 \text { (SD 4.7) } \\ & \text { Sex: } 61 \% \text { female }\end{aligned}$

Inclusion criteria: Prefrail and frail older adults were identified based on $5 \mathrm{CHS}$ criteria defining physical frailty: unintentional weight loss, slowness, weakness, exhaustion, and low activity,which were scored 1 if present and 0 if absent. The total summed scores ranging from 0 to 5 were used to classify a participant as robust (score $=0$ ), prefrail (score $=1$ to 2 ), or frail (score $=3$ to 5 ). Prefrail or frail older adults were eligible for the trial if they were aged 65 years and above, able to walk without personal assistance, and living at home

Exclusion criteria: People were excluded if they had significant cognitive impairment (MMSE score 23 or less); major depression; severe audiovisual impairment; any progressive, degenerative neurologic disease; terminal illness with life expectancy < 12 months; were participating in other interventional studies; or were unavailable to participate for the full duration of the study

Interventions

Randomised into 5 groups: 4 intervention groups (1 physical exercise group, 1 nutritional intervention group, 1 cognitive training group, 1 combination intervention group) and 1 control group. Only the physical exercise group and control group were included in this review

1. Group-based strength and balance training plus home practice: resistance and functional exercises of moderate and tailored to progress in intensity; using free weights, different floor surfaces, treadmill; 90 minutes, 2 a week for 12 weeks, and 12-week home programme

2. Control group: access to 1 standard care from health and aged care services that were normally available to older people, and given artificially sweetened liquid, 2 capsules and 1 tablet (ingredients: cornstarch, lactose, magnesium stearate) 
Ng 2015 (Continued)

\begin{tabular}{ll} 
Duration of the study & 52 weeks \\
\hline Adherence & Adherence measured by training sessions completed \\
1. Group-based strength and balance training plus home practice: $85 \%$ compliance \\
2. Control group: $94 \%$ compliance
\end{tabular}

Notes Source of funding: NHMRC

Economic information: not reported

\section{Risk of bias}

\begin{tabular}{lll}
\hline Bias & Authors' judgement & Support for judgement \\
\hline $\begin{array}{l}\text { Random sequence genera- } \\
\text { tion (selection bias) }\end{array}$ & Low risk & Quote: "Central computerized randomization procedure" \\
\hline $\begin{array}{l}\text { Allocation concealment } \\
\text { (selection bias) }\end{array}$ & Low risk & $\begin{array}{l}\text { Quote: "Treatment was allocated by a project manager not involved in the en- } \\
\text { rollment, intervention,or assessment." }\end{array}$ \\
\hline $\begin{array}{l}\text { Blinding of participants } \\
\text { and personnel (perfor- } \\
\text { mance bias) }\end{array}$ & Unclear risk & Participants and personnel unblinded but impact of unblinding unknown \\
All outcomes & \\
\hline
\end{tabular}

\begin{tabular}{|c|c|c|}
\hline $\begin{array}{l}\text { Blinding of outcome as- } \\
\text { sessment (detection bias) } \\
\text { Falls }\end{array}$ & Low risk & $\begin{array}{l}\text { Quote: "Outcome assessments were performed at baseline, } 3 \text { months, } 6 \\
\text { months, and } 12 \text { months by assessors who were blinded to the participants' } \\
\text { group allocation". Falls were self-reported at these time points. Falls were } \\
\text { measured using the same method in all groups }\end{array}$ \\
\hline
\end{tabular}

\begin{tabular}{|c|c|c|}
\hline $\begin{array}{l}\text { Blinding of outcome as- } \\
\text { sessment (detection bias) } \\
\text { Fractures }\end{array}$ & Unclear risk & Not applicable \\
\hline $\begin{array}{l}\text { Blinding of outcome as- } \\
\text { sessment (detection bias) } \\
\text { Hospital admission, med- } \\
\text { ical attention and adverse } \\
\text { events }\end{array}$ & High risk & $\begin{array}{l}\text { Adverse events were recorded by the interventional nurses who also adminis- } \\
\text { tered treatment and were therefore not blinded to group }\end{array}$ \\
\hline $\begin{array}{l}\text { Blinding of outcome as- } \\
\text { sessment (detection bias) } \\
\text { Health related quality of } \\
\text { life (self report) }\end{array}$ & Unclear risk & Not applicable \\
\hline $\begin{array}{l}\text { Incomplete outcome data } \\
\text { (attrition bias) } \\
\text { Falls and fallers }\end{array}$ & Low risk & $\begin{array}{l}\text { Less than } 20 \% \text { of fall data are missing (6\%). Missing data were blanced in the } \\
2 \text { groups (physical training: } 1 \text { withdrew, } 1 \text { unable to contact; control: } 3 \text { with- } \\
\text { drew, } 1 \text { died) }\end{array}$ \\
\hline $\begin{array}{l}\text { Selective reporting (re- } \\
\text { porting bias) }\end{array}$ & High risk & $\begin{array}{l}\text { Falls were measured, but number of fallers was not reported. Adverse events } \\
\text { were not reported }\end{array}$ \\
\hline $\begin{array}{l}\text { Method of ascertaining } \\
\text { falls (recall bias) }\end{array}$ & High risk & Falls were self-reported at 3-month, 6-month, and 12-month assessments \\
\hline
\end{tabular}




\begin{tabular}{ll}
\hline Methods & Study design: RCT \\
& Number of study arms: 2 \\
& Length of follow-up: 6 months \\
\hline Participants & Setting: Brisbane, Australia \\
& Number of participants: 73 \\
& Number analysed: 45 \\
& Number lost to follow-up: 28 \\
& Sample: volunteers recruited through advertising and fliers \\
& Age (years): mean 75.8 (SD 7.8 ) \\
& Sex: $92 \%$ female \\
& Inclusion criteria: aged $>60 ;$ living independently in the community; at least 1 fall in previous year \\
& Exclusion criteria: unstable cardiac condition, living too far from exercise class site, unable to guaran- \\
& tee regular attendance
\end{tabular}

Interventions
2. Control: Group-based gentle exercise and stretching, 1 hour a week for 10 weeks

\begin{tabular}{ll}
\hline Outcomes & 1. Rate of falls \\
\hline Duration of the study & 24 weeks \\
\hline Adherence & Adherence measured as participants who completed the study \\
& 1. Group-based balance group: 24 \\
& 2. Group-based gentle exercise and stretching group: 21 \\
\hline Notes & Source of funding: not reported \\
& Economic information: not reported
\end{tabular}

\section{Risk of bias}

\begin{tabular}{|c|c|c|}
\hline Bias & Authors' judgement & Support for judgement \\
\hline $\begin{array}{l}\text { Random sequence genera- } \\
\text { tion (selection bias) }\end{array}$ & Low risk & Quote: "Computer-generated random numbers" \\
\hline $\begin{array}{l}\text { Allocation concealment } \\
\text { (selection bias) }\end{array}$ & Unclear risk & Insufficient information to permit judgement \\
\hline $\begin{array}{l}\text { Blinding of participants } \\
\text { and personnel (perfor- } \\
\text { mance bias) } \\
\text { All outcomes }\end{array}$ & Unclear risk & $\begin{array}{l}\text { Participants and personnel implementing the intervention not blinded to allo- } \\
\text { cated group, but impact of non-blinding unclear }\end{array}$ \\
\hline $\begin{array}{l}\text { Blinding of outcome as- } \\
\text { sessment (detection bias) } \\
\text { Falls }\end{array}$ & Low risk & $\begin{array}{l}\text { Quote: "Partipants used a calendar on which each day was marked for a fall ... } \\
\text { or incident free day" } \\
\text { Quote: "The physiotherapists who undertook all assessments of the partici- } \\
\text { pants were blinded to the intervention group allocation" }\end{array}$ \\
\hline
\end{tabular}




\section{Nitz 2004 (Continued)}

$\begin{array}{lll}\text { Blinding of outcome as- } & \text { Unclear risk } & \text { Not applicable } \\ \text { sessment (detection bias) } & \end{array}$

\begin{tabular}{lll}
\hline $\begin{array}{l}\text { Blinding of outcome as- } \\
\text { sessment (detection bias) } \\
\text { Hospital admission, med- } \\
\text { ical attention and adverse } \\
\text { events }\end{array}$ & Unclear risk & Not applicable \\
\hline $\begin{array}{l}\text { Blinding of outcome as- } \\
\text { sessment (detection bias) } \\
\text { Health related quality of } \\
\text { life (self report) }\end{array}$ & Unclear risk & Not applicable \\
\hline $\begin{array}{l}\text { Incomplete outcome data } \\
\text { (attrition bias) }\end{array}$ & High risk & \\
$\begin{array}{l}\text { Falls and fallers } \\
\text { Selective reporting (re- }\end{array}$ & High risk & More than 20\% of fall data are missing (38\%) \\
\hline porting bias) & & Falls were measured, but number of fallers was not reported. Adverse events \\
\hline $\begin{array}{l}\text { Method of ascertaining } \\
\text { falls (recall bias) }\end{array}$ & Low risk & were not reported \\
\hline
\end{tabular}

Okubo 2016

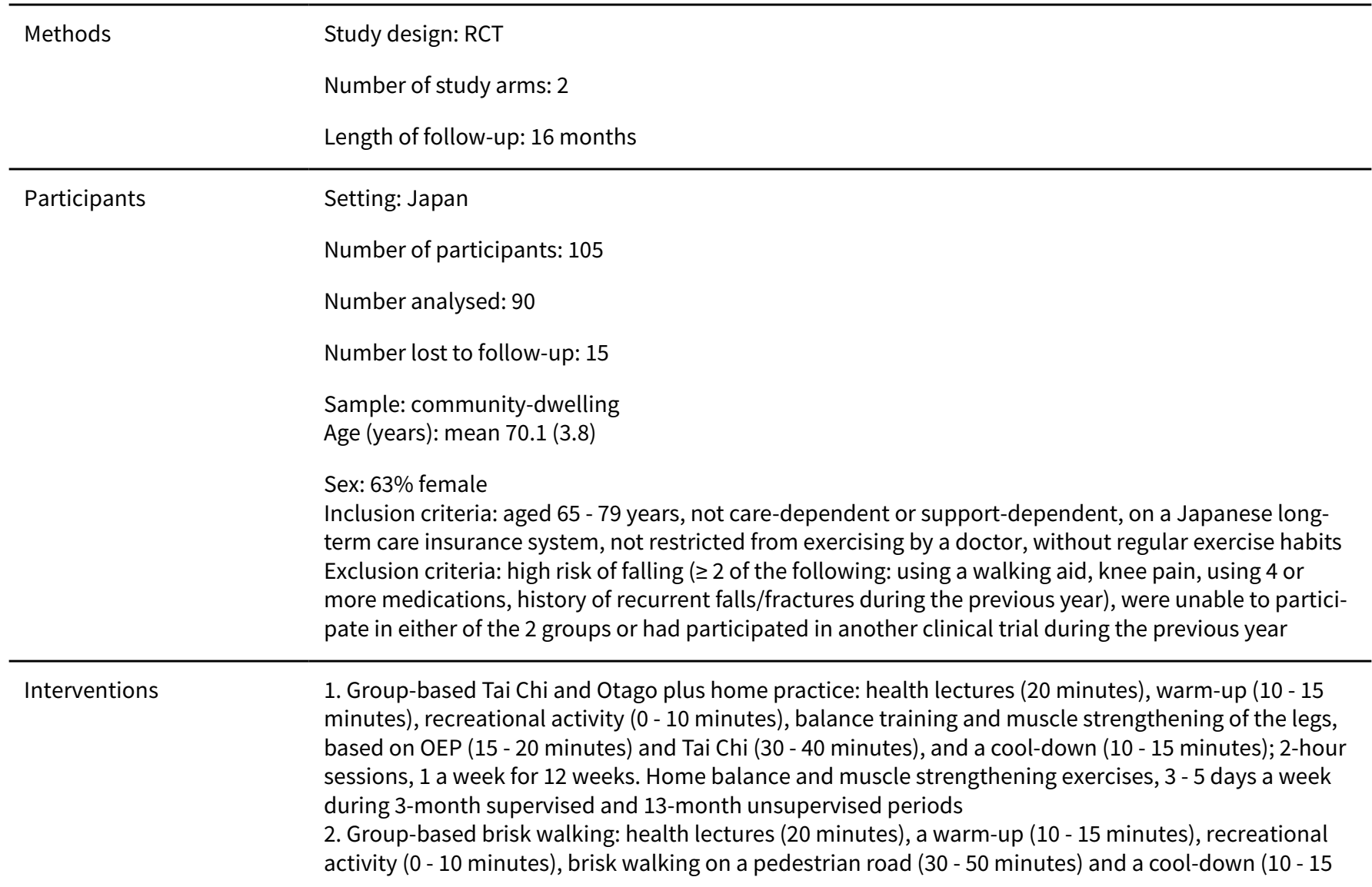


Okubo 2016 (Continued)

minutes). 2-hour sessions, 1 a week. Home exercise of walking for 30 - 50 minutes, 3 - 5 days a week was also recommended during the 3-month supervised and 13-month unsupervised follow-up periods

\begin{tabular}{ll}
\hline Outcomes & No outcomes included in the review \\
\hline Duration of the study & 56 weeks \\
\hline Adherence & $\begin{array}{l}\text { 1. Group exercise: an average of } 1.4 \pm 0.5 \text { sets/day were carried out for } 4.6 \pm 2.0 \text { days/week } \\
\text { 2. Group exercise: an average of } 45.2 \pm 24.5 \text { min/day of walking for } 4.3 \pm 1.7 \text { days/week }\end{array}$ \\
\hline Notes & Source of funding: Japan Society for the Promotion of Science \\
& $\begin{array}{l}\text { Economic information: not reported } \\
\text { Email communication about fall data, response received, data not included in review. Falls outcomes } \\
\text { reported in trial were 'falls per physically active person-day' and 'falls per person-step' }\end{array}$
\end{tabular}

\section{Risk of bias}

\begin{tabular}{|c|c|c|}
\hline Bias & Authors' judgement & Support for judgement \\
\hline $\begin{array}{l}\text { Random sequence genera- } \\
\text { tion (selection bias) }\end{array}$ & Low risk & Quote: "computer-generated random numbers" \\
\hline $\begin{array}{l}\text { Allocation concealment } \\
\text { (selection bias) }\end{array}$ & Unclear risk & Insufficient information to permit judgement \\
\hline $\begin{array}{l}\text { Blinding of participants } \\
\text { and personnel (perfor- } \\
\text { mance bias) } \\
\text { All outcomes }\end{array}$ & Unclear risk & $\begin{array}{l}\text { Participants and personnel implementing the intervention not blinded to allo- } \\
\text { cated group, but impact of non-blinding unclear }\end{array}$ \\
\hline $\begin{array}{l}\text { Blinding of outcome as- } \\
\text { sessment (detection bias) } \\
\text { Falls }\end{array}$ & High risk & No blinding was applied \\
\hline $\begin{array}{l}\text { Blinding of outcome as- } \\
\text { sessment (detection bias) } \\
\text { Fractures }\end{array}$ & Unclear risk & Not applicable \\
\hline $\begin{array}{l}\text { Blinding of outcome as- } \\
\text { sessment (detection bias) } \\
\text { Hospital admission, med- } \\
\text { ical attention and adverse } \\
\text { events }\end{array}$ & Unclear risk & Not applicable \\
\hline $\begin{array}{l}\text { Blinding of outcome as- } \\
\text { sessment (detection bias) } \\
\text { Health related quality of } \\
\text { life (self report) }\end{array}$ & Unclear risk & Not applicable \\
\hline $\begin{array}{l}\text { Incomplete outcome data } \\
\text { (attrition bias) } \\
\text { Falls and fallers }\end{array}$ & Unclear risk & $\begin{array}{l}\text { Less than } 20 \% \text { of fall data were missing }(14 \%) \text {. There were missing data from } \\
10 \text { walking-group participants (knee pain } n=3 \text {, time issue } n=6 \text {, misfortune } n= \\
1 \text { ) and } 5 \text { balance-group participants (knee pain } n=1 \text {, time issue } n=3 \text {, transfer } \\
\text { issue } n=1 \text { ) }\end{array}$ \\
\hline $\begin{array}{l}\text { Selective reporting (re- } \\
\text { porting bias) }\end{array}$ & High risk & $\begin{array}{l}\text { Falls were measured, but number of fallers was not reported. Adverse events } \\
\text { were not reported }\end{array}$ \\
\hline
\end{tabular}


Okubo 2016 (Continued)

Method of ascertaining falls (recall bias)
Low risk

Participants were asked to record the number of falls and trips daily in their fall calendars, and turn them in every month until the end of the 16th month. Falls from bicycles were excluded

\section{Park 2008}

\begin{tabular}{|c|c|}
\hline Methods & $\begin{array}{l}\text { Study design: RCT } \\
\text { Number of study arms: } 2 \\
\text { Length of follow-up: } 11 \text { months }\end{array}$ \\
\hline Participants & $\begin{array}{l}\text { Setting: Korea } \\
\text { Number of participants: } 50 \\
\text { Number analysed: } 45 \\
\text { Number lost to follow-up: } 5 \\
\text { Sample: Community-dwelling participants in a community learning centre for seniors and senior mem- } \\
\text { bers of local clubs } \\
\text { Age (years): mean } 68.35 \text { (SD 3.47) } \\
\text { Sex: } 100 \% \text { female } \\
\text { Inclusion criteria: community-dwelling (e.g. in a private dwelling, apartment, residential facility); am- } \\
\text { bulatory (with or without an aid); competent to give consent; residents of Busan, Korea; aged } 65 \text { years } \\
\text { Exclusion criteria: < } 5 \text { years after menopause; history of chronic disease that might influence BMD, } \\
\text { physical activity and balance ability; history of ovariectomy or diseases known to affect bone metabo- } \\
\text { lism (e.g. cancer, renal disease, rheumatoid arthritis); current medication with bisphosphonate, oestro- } \\
\text { gens, or other hormonal preparations; weigh > 130\% ideal body weight; other contraindications to par- } \\
\text { ticipating in a regular exercise programme; already doing moderate or hard exercise for more than } 7 \\
\text { hours a week }\end{array}$ \\
\hline
\end{tabular}

Interventions 1. Exercise group: Stretching for 9 minutes, strength training for 10 minutes followed by 23 minutes of weight-bearing exercise at an intensity above $65-75 \%$ of the maximal heart rate, and 18 minutes of balance and posture correction training. The programme was conducted 3 times a week for 48 weeks

2. Control group: retained their sedentary lifestyle participation in physical exercise

Outcomes 1. Number of people who experienced 1 or more falls (risk of falling)

\begin{tabular}{ll}
\hline Duration of the study & 48 weeks \\
\hline Adherence & Not reported \\
\hline Notes & Source of funding: Korea Science and Engineering Foundation \\
& Economic information: not reported \\
& Email communication regarding fall data, response received, data not included in review \\
\hline
\end{tabular}

\section{Risk of bias}


Park 2008 (Continued)
Random sequence genera- Low risk
Quote: "Randomly assigned (by a computer generated program)" tion (selection bias)

\begin{tabular}{lll}
\hline $\begin{array}{l}\text { Allocation concealment } \\
\text { (selection bias) }\end{array}$ & Unclear risk & Concealment not specified \\
\hline $\begin{array}{l}\text { Blinding of participants } \\
\begin{array}{l}\text { and personnel (perfor- } \\
\text { mance bias) }\end{array}\end{array}$ & Unclear risk & $\begin{array}{l}\text { Participants and personnel implementing the intervention not blinded to allo- } \\
\text { cated group, but impact of non-blinding unclear }\end{array}$ \\
All outcomes &
\end{tabular}

\begin{tabular}{|c|c|c|}
\hline $\begin{array}{l}\text { Blinding of outcome as- } \\
\text { sessment (detection bias) } \\
\text { Falls }\end{array}$ & Unclear risk & Blinding not specified \\
\hline $\begin{array}{l}\text { Blinding of outcome as- } \\
\text { sessment (detection bias) } \\
\text { Fractures }\end{array}$ & Unclear risk & Not applicable \\
\hline $\begin{array}{l}\text { Blinding of outcome as- } \\
\text { sessment (detection bias) } \\
\text { Hospital admission, med- } \\
\text { ical attention and adverse } \\
\text { events }\end{array}$ & Unclear risk & Not applicable \\
\hline $\begin{array}{l}\text { Blinding of outcome as- } \\
\text { sessment (detection bias) } \\
\text { Health related quality of } \\
\text { life (self report) }\end{array}$ & Unclear risk & Not applicable \\
\hline $\begin{array}{l}\text { Incomplete outcome data } \\
\text { (attrition bias) } \\
\text { Falls and fallers }\end{array}$ & Low risk & $\begin{array}{l}\text { Less than } 20 \% \text { of fall data were missing }(10 \%) \text {. Missing data were balanced in } \\
\text { intervention }(n=3) \text { and control }(n=2) \text { groups. The reason for missing data was } \\
\text { unclear }\end{array}$ \\
\hline $\begin{array}{l}\text { Selective reporting (re- } \\
\text { porting bias) }\end{array}$ & High risk & $\begin{array}{l}\text { Falls were measured, but number of falls was not reported. Adverse events } \\
\text { were not reported }\end{array}$ \\
\hline $\begin{array}{l}\text { Method of ascertaining } \\
\text { falls (recall bias) }\end{array}$ & High risk & $\begin{array}{l}\text { Retrospective. Participants were asked "Did you have any falls during the past } \\
\text { one year? What was the reason for the fall?" }\end{array}$ \\
\hline
\end{tabular}

\section{Reinsch 1992}

\begin{tabular}{ll}
\hline Methods & RCT (cluster-randomised by senior centre. $2 \times 2$ factorial design) \\
& Study design: Cluster-RCT \\
& Number of study arms: 2 \\
& Number of clusters: 16 \\
& Length of follow-up: 12 months
\end{tabular}

Participants

Setting: Los Angeles County and Orange County, California, USA

Number of participants: 230

Number analysed: 230 
Reinsch 1992 (Continued)

Number lost to follow-up: 0

Sample: recruited from 16 senior centres

Age (years): mean 74.2 (SD 6.0)

Sex: $80 \%$ female

Inclusion criteria: aged $>60$

Exclusion criteria: none listed

Interventions

Randomised into 4 groups: 3 intervention groups (1 group-based balance and strength training, 1 cognitive-behavioural training, 1 exercise and cognitive training) and 1 control group (discussion group). Only the group-based balance and strength training and control group were included in this review

1. Group-based balance and strength training: no special equipment used; 1 hour, 3 a week for 52 weeks

2. Control group: health and interest discussion group, 1 hour, 1 a week for 52 weeks

\begin{tabular}{ll}
\hline Outcomes & 1. Number of people who experienced 1 or more falls (risk of falling) \\
\hline Duration of the study & 52 weeks \\
\hline Adherence & Dropout/noncompliance defined as missing $1 / 3$ or more of the classes taught at their centre \\
& 1. Group-based balance and strength training: $13 / 57$ noncompliance (44/57 compliance) \\
& 2. Control group: $8 / 50$ noncompliance ( $42 / 50$ compliance) \\
\hline Notes & Source of funding: NIH, AARP Andrus Foundation, Roosevelt Warm Springs Foundation \\
& Economic information: not reported \\
& MacRae paper includes a subset of results for only 2 arms of the study, in Los Angeles county only \\
& $\begin{array}{l}\text { Number of clusters allocated to intervention: } 4 \text {; number of clusters allocated to control: } 4 ; \text { number of } \\
\text { clusters analysed (intervention): } 4 \text {; number of clusters analysed (control): } 4\end{array}$
\end{tabular}

\section{Risk of bias}

\begin{tabular}{lll}
\hline Bias & Authors' judgement & Support for judgement \\
\hline $\begin{array}{l}\text { Random sequence genera- } \\
\text { tion (selection bias) }\end{array}$ & Unclear risk & Quote: "randomly assigned to treatments" \\
\hline $\begin{array}{l}\text { Allocation concealment } \\
\text { (selection bias) }\end{array}$ & High risk & $\begin{array}{l}\text { Quote:" A biostatistician not involved in the study randomized general prac- } \\
\text { tices into the intervention or control group by using computer-generated ran- } \\
\text { dom numbers. After the randomization, the general practitioners enrolled } \\
\text { patients for the study according to the inclusion and exclusion criteria". The } \\
\text { method of concealment is not described and assume the recruiting gener- } \\
\text { al practitioners were unblinded and may have had knowledge of participant } \\
\text { characteristics }\end{array}$ \\
\hline
\end{tabular}

\begin{tabular}{|c|c|c|}
\hline $\begin{array}{l}\text { Blinding of participants } \\
\text { and personnel (perfor- } \\
\text { mance bias) } \\
\text { All outcomes }\end{array}$ & Unclear risk & $\begin{array}{l}\text { Participants and personnel implementing the intervention not blinded to allo- } \\
\text { cated group, but impact of non-blinding unclear }\end{array}$ \\
\hline $\begin{array}{l}\text { Blinding of outcome as- } \\
\text { sessment (detection bias) } \\
\text { Falls }\end{array}$ & Unclear risk & $\begin{array}{l}\text { Falls reported by participants who were aware of their group allocation. Blind- } \\
\text { ing of research assistant not described }\end{array}$ \\
\hline $\begin{array}{l}\text { Blinding of outcome as- } \\
\text { sessment (detection bias) }\end{array}$ & Unclear risk & Not applicable \\
\hline
\end{tabular}


Reinsch 1992 (Continued)

Fractures

$\begin{aligned} & \text { Blinding of outcome as- } \\ & \text { sessment (detection bias) }\end{aligned}$
$\begin{aligned} & \text { Hospital admission, med- } \\ & \text { ical attention and adverse }\end{aligned}$

\begin{tabular}{|c|c|c|}
\hline $\begin{array}{l}\text { Blinding of outcome as- } \\
\text { sessment (detection bias) } \\
\text { Health related quality of } \\
\text { life (self report) }\end{array}$ & Unclear risk & Not applicable \\
\hline $\begin{array}{l}\text { Incomplete outcome data } \\
\text { (attrition bias) } \\
\text { Falls and fallers }\end{array}$ & Low risk & No missing fall data \\
\hline $\begin{array}{l}\text { Selective reporting (re- } \\
\text { porting bias) }\end{array}$ & High risk & Falls were measured, but number of falls was not reported \\
\hline $\begin{array}{l}\text { Method of ascertaining } \\
\text { falls (recall bias) }\end{array}$ & Low risk & Prospective. Monthly diaries plus weekly phone calls or visits \\
\hline Cluster-randomised trials & Unclear risk & $\begin{array}{l}\text { Individual participant recruitment was undertaken after group allocation. The } \\
\text { method of concealment is not described and it is likely that recruitment was } \\
\text { undertaken by a person who was unblinded and may have had knowledge of } \\
\text { participant characteristics; baseline characteristics of clusters were not report- } \\
\text { ed; missing outcomes for clusters or within clusters were not reported; did not } \\
\text { account for the clustered design in the analysis; results comparable with indi- } \\
\text { vidually-randomised trials }\end{array}$ \\
\hline
\end{tabular}

\section{Resnick 2002}

\begin{tabular}{ll}
\hline Methods & Study design: RCT \\
& Number of study arms: 2 \\
& Length of follow-up: 6 months \\
\hline Participants & Setting: Baltimore, USA \\
& Number of participants: 20 \\
& Number analysed: 17 \\
& Number lost to follow-up: 3 \\
& Sample: women in a continuing-care retirement community \\
& Age (years): mean 88 (SD 3.7) \\
& Sex: $100 \%$ female \\
Inclusion criteria: able to walk 50 feet with or without assistive device; sedentary lifestyle \\
Exclusion criteria: cognitive impairment (MMSE > 20); terminal illness; medical condition precluding \\
participation in aerobic exercise
\end{tabular}


Resnick 2002 (Continued)

Outcomes 1. Health-related quality of life

\begin{tabular}{ll}
\hline Duration of the study & 26 weeks \\
\hline Adherence & Adherence measured by meeting the recommended 20 minutes, 3 a week walking programme \\
$\begin{array}{l}\text { 1. Individual or group-based walking group: } 7 \text { participants adhered to the recommended walking pro- } \\
\text { gramme. } 2 \text { engaged in a regular walking programme but did not meet the recommended } 20 \text { minutes } 3 \\
\text { a week. } 1 \text { did not engage in any exercise }\end{array}$ \\
2. Control group: 0 participants started an exercise programme during the course of the study
\end{tabular}

Source of funding: not reported
Economic information: not reported
Participants lived independently in apartments, and could walk independently. (Personal correspon-
dence). Pilot study with no usable data.
Email communication about fall data, response received, data not included in review

\section{Risk of bias}

\begin{tabular}{|c|c|c|}
\hline Bias & Authors' judgement & Support for judgement \\
\hline $\begin{array}{l}\text { Random sequence genera- } \\
\text { tion (selection bias) }\end{array}$ & Low risk & $\begin{array}{l}\text { Randomised by coin flip (personal communication as reported by Gillespie } \\
\text { 2012) }\end{array}$ \\
\hline $\begin{array}{l}\text { Allocation concealment } \\
\text { (selection bias) }\end{array}$ & Unclear risk & Insufficient information to permit judgement \\
\hline $\begin{array}{l}\text { Blinding of participants } \\
\text { and personnel (perfor- } \\
\text { mance bias) } \\
\text { All outcomes }\end{array}$ & Unclear risk & $\begin{array}{l}\text { Participants and personnel implementing the intervention not blind to allocat- } \\
\text { ed group, but impact of non-blinding unclear }\end{array}$ \\
\hline $\begin{array}{l}\text { Blinding of outcome as- } \\
\text { sessment (detection bias) } \\
\text { Falls }\end{array}$ & Unclear risk & $\begin{array}{l}\text { Falls reported by participants who were aware of their group allocation. Blind- } \\
\text { ing of research assistant not described. }\end{array}$ \\
\hline $\begin{array}{l}\text { Blinding of outcome as- } \\
\text { sessment (detection bias) } \\
\text { Fractures }\end{array}$ & Unclear risk & Not applicable \\
\hline $\begin{array}{l}\text { Blinding of outcome as- } \\
\text { sessment (detection bias) } \\
\text { Hospital admission, med- } \\
\text { ical attention and adverse } \\
\text { events }\end{array}$ & Unclear risk & Not applicable \\
\hline $\begin{array}{l}\text { Blinding of outcome as- } \\
\text { sessment (detection bias) } \\
\text { Health related quality of } \\
\text { life (self report) }\end{array}$ & High risk & Participants not blinded to group allocation \\
\hline $\begin{array}{l}\text { Incomplete outcome data } \\
\text { (attrition bias) } \\
\text { Falls and fallers }\end{array}$ & High risk & No fall data available \\
\hline
\end{tabular}


Resnick 2002 (Continued)

Selective reporting (re- High risk $\quad$ Fall outcome prespecified but fall data not presented porting bias)

Method of ascertaining Unclear risk

Quote: "based on self-report". No additional information

falls (recall bias)

\section{Robertson 2001a}

\begin{tabular}{ll} 
Methods & Study design: RCT \\
& Number of study arms: 2 \\
& Length of follow-up: 12 months \\
\hline Participants & Setting: West Auckland, New Zealand \\
& Number of participants: 240 \\
& Number analysed: 240 \\
& Number lost to follow-up: 0 \\
& Sample: identified from computerised registers at 17 general practices \\
& Age (years): mean 80.9 (SD 4.2), range 75 - 95 \\
& Sex: $68 \%$ female \\
Inclusion criteria: aged $\geq 75 ;$ living at home & Exclusion criteria: unable to walk around own residence; already receiving physiotherapy; unable to \\
understand trial requirements
\end{tabular}

Interventions

1. Individual Otago Exercise Programme: home exercises plus walking plan prescribed by nurse at 1 week ( 1 hour) and at 2, 4, 8 weeks, and 6 months (half-hour) plus monthly telephone call to maintain motivation; exercised 3 a week and walked 2 a week for 1 year

2. Control: usual care

\begin{tabular}{|c|c|}
\hline \multirow[t]{4}{*}{ Outcomes } & $\begin{array}{l}\text { 1. Rate of falls } \\
\text { 2. Number of people who experienced } 1 \text { or more falls (risk of falling) }\end{array}$ \\
\hline & 3. Number of people who experienced 1 or more fall-related fractures \\
\hline & 4. Number of people who experienced 1 or more falls requiring medical attention \\
\hline & 5. Number of people who died \\
\hline Duration of the study & 52 weeks \\
\hline \multirow[t]{3}{*}{ Adherence } & Adherence measured by completion of the trial, frequency of exercise programme \\
\hline & $\begin{array}{l}\text { 1. Individual Otago Exercise Programme: } 113 \text { participants completed the trial. } 43 \%(n=49) \text { carried out } \\
\text { their exercise programme } \geq 3 \text { times a week. } 72 \%(n=81) \text { carried out their exercise programme } \geq 2 \text { times } \\
\text { a week and } 71 \%(n=80) \text { walked at least } \geq 2 \text { times a week during the } 1 \text {-year follow-up }\end{array}$ \\
\hline & 2. Control: 98 participants completed the trial \\
\hline \multirow[t]{2}{*}{ Notes } & $\begin{array}{l}\text { Source of funding: Health Funding Authority Northern Division, Accident Rehabilitation and Compen- } \\
\text { sation Insurance Corporation of New Zealand, Trustbank Otago Community Trust medical research fel- } \\
\text { lowship }\end{array}$ \\
\hline & $\begin{array}{l}\text { Economic information: Mean cost per person (intervention) in community health service setting NZD } \\
432 \text { for } 1 \text { year. Healthcare service costs: } 5 \text { hospital admissions due to fall injuries in control group, none }\end{array}$ \\
\hline
\end{tabular}


in exercise group (cost savings of NZD 47,818). Incremental cost per fall prevented/per QALY gained: NZD 1803 per fall prevented (programme implementation costs only), NZD 7471 per injurious fall prevented (programme implementation costs only), NZD 155 per fall prevented (programme implementation costs and hospital admission cost savings), NZD 640 per injurious fall prevented (programme implementation costs and hospital admission cost savings).

District nurse had no previous experience in exercise prescription. Received 1 week's training from research group's physiotherapist, Mean who also made site visits and phone calls to monitor quality. Otago Exercise Programme manual can be obtained from www.cdc.gov/HomeandRecreationalSafety/Falls/compendium/1.2_otago.html. Cost-effectiveness analysis reported in primary reference

\section{Risk of bias}

\begin{tabular}{|c|c|c|}
\hline Bias & Authors' judgement & Support for judgement \\
\hline $\begin{array}{l}\text { Random sequence genera- } \\
\text { tion (selection bias) }\end{array}$ & Low risk & $\begin{array}{l}\text { Randomised using allocation schedule developed using computer-generated } \\
\text { numbers }\end{array}$ \\
\hline $\begin{array}{l}\text { Allocation concealment } \\
\text { (selection bias) }\end{array}$ & Low risk & Assignment by independent person off-site \\
\hline $\begin{array}{l}\text { Blinding of participants } \\
\text { and personnel (perfor- } \\
\text { mance bias) } \\
\text { All outcomes }\end{array}$ & Unclear risk & $\begin{array}{l}\text { Participants and personnel implementing the intervention not blinded to allo- } \\
\text { cated group, but impact of non-blinding unclear }\end{array}$ \\
\hline $\begin{array}{l}\text { Blinding of outcome as- } \\
\text { sessment (detection bias) } \\
\text { Falls }\end{array}$ & Low risk & $\begin{array}{l}\text { Falls reported by participants who were aware of their group allocation. } \\
\text { Phoned by independent assessor blinded to allocation. Person classifying fall } \\
\text { events also blinded to allocation }\end{array}$ \\
\hline $\begin{array}{l}\text { Blinding of outcome as- } \\
\text { sessment (detection bias) } \\
\text { Fractures }\end{array}$ & Low risk & $\begin{array}{l}\text { A blinded assessor telephoned participants who fell to record injuries as a re- } \\
\text { sult of the fall. Quote: "The circumstances of "serious" injuries were confirmed } \\
\text { from hospital and general practice records. The investigator classifying fall } \\
\text { events remained blind to group allocation" }\end{array}$ \\
\hline $\begin{array}{l}\text { Blinding of outcome as- } \\
\text { sessment (detection bias) } \\
\text { Hospital admission, med- } \\
\text { ical attention and adverse } \\
\text { events }\end{array}$ & Low risk & $\begin{array}{l}\text { A blinded assessor telephoned participants who fell to record injuries as a re- } \\
\text { sult of the fall. Quote: "The circumstances of "serious" injuries were confirmed } \\
\text { from hospital and general practice records. The investigator classifying fall } \\
\text { events remained blind to group allocation" }\end{array}$ \\
\hline $\begin{array}{l}\text { Blinding of outcome as- } \\
\text { sessment (detection bias) } \\
\text { Health related quality of } \\
\text { life (self report) }\end{array}$ & Unclear risk & Not applicable \\
\hline $\begin{array}{l}\text { Incomplete outcome data } \\
\text { (attrition bias) } \\
\text { Falls and fallers }\end{array}$ & Low risk & No missing fall data \\
\hline $\begin{array}{l}\text { Selective reporting (re- } \\
\text { porting bias) }\end{array}$ & Unclear risk & $\begin{array}{l}\text { Minimum set of expected outcomes not reported (adverse events not report- } \\
\text { ed) }\end{array}$ \\
\hline $\begin{array}{l}\text { Method of ascertaining } \\
\text { falls (recall bias) }\end{array}$ & Low risk & $\begin{array}{l}\text { Active fall registration with daily postcard calendars returned monthly, plus } \\
\text { telephone calls }\end{array}$ \\
\hline
\end{tabular}


Rubenstein 2000

\begin{tabular}{|c|c|}
\hline \multirow[t]{3}{*}{ Methods } & Study design: RCT \\
\hline & Number of study arms: 2 \\
\hline & Length of follow-up: 3 months \\
\hline \multirow[t]{9}{*}{ Participants } & Setting: California, USA \\
\hline & Number of participants: 59 \\
\hline & Number analysed: 59 \\
\hline & Number lost to follow-up: 0 \\
\hline & Sample: men recruited from Veterans Administration ambulatory care centre (volunteers) \\
\hline & Age (years): mean 74 \\
\hline & Sex: $0 \%$ female \\
\hline & $\begin{array}{l}\text { Inclusion criteria: aged } \geq 70 \text {; ambulatory; } \geq 1 \text { fall risk factor: lower limb weakness, impaired gait, im- } \\
\text { paired balance, }>1 \text { fall in previous } 6 \text { months }\end{array}$ \\
\hline & $\begin{array}{l}\text { Exclusion criteria: exercised regularly; severe cardiac or pulmonary disease; terminal illness; severe } \\
\text { joint pain; dementia; medically unresponsive depression; progressive neurological disease }\end{array}$ \\
\hline \multirow[t]{2}{*}{ Interventions } & $\begin{array}{l}\text { 1. Group-based balance, strength and endurance: using free weights, elastic bands, bicycle, treadmill; } \\
90 \text { minutes, } 3 \text { a week for } 12 \text { weeks }\end{array}$ \\
\hline & 2. Control: usual activities \\
\hline \multirow[t]{3}{*}{ Outcomes } & 1. Rate of falls \\
\hline & 2. Number of people who experienced 1 or more falls (risk of falling) \\
\hline & 3. Health-related quality of life \\
\hline Duration of the study & 12 weeks \\
\hline \multirow[t]{2}{*}{ Adherence } & Adherence measured by session attendance \\
\hline & 1. Group-based balance, strength and endurance group: attended $84 \%$ of the exercise sessions \\
\hline \multirow[t]{2}{*}{ Notes } & $\begin{array}{l}\text { Source of funding: Department of Veterans Affairs, Health Services Research and Development Service, } \\
\text { Disabled American Veterans Charities of Greater Los Angeles }\end{array}$ \\
\hline & Economic information: not reported \\
\hline
\end{tabular}

\section{Risk of bias}

\begin{tabular}{lll}
\hline Bias & Authors' judgement & Support for judgement \\
\hline $\begin{array}{l}\text { Random sequence genera- } \\
\text { tion (selection bias) }\end{array}$ & Low risk & $\begin{array}{l}\text { Randomised in blocks of } 16 \text { to } 20 \text { at 3- to 6-month intervals, using random- } \\
\text { ly-generated sequence cards in sealed envelopes }\end{array}$ \\
\hline $\begin{array}{l}\text { Allocation concealment } \\
\text { (selection bias) }\end{array}$ & Unclear risk & Cards in sealed envelopes \\
\hline $\begin{array}{l}\text { Blinding of participants } \\
\text { and personnel (perfor- } \\
\text { mance bias) }\end{array}$ & Unclear risk & $\begin{array}{l}\text { Participants and personnel implementing the intervention not blind to allocat- } \\
\text { ed group, but impact of non-blinding unclear }\end{array}$ \\
\hline $\begin{array}{l}\text { Blinding of outcome as- } \\
\text { sessment (detection bias) }\end{array}$ & High risk & $\begin{array}{l}\text { Falls reported by participants who were aware of their group allocation. Fall } \\
\text { data were gathered in different settings for the intervention and control } \\
\text { goups. The person ascertaining falls was aware of group allocation }\end{array}$ \\
\hline \hline
\end{tabular}


Rubenstein 2000 (Continued)
Blinding of outcome as-
Unclear risk
Method of recording fractures is unclear sessment (detection bias)

Fractures

\section{Blinding of outcome as- sessment (detection bias) Hospital admission, med- ical attention and adverse events}
Unclear risk
Method of recording adverse events is unclear

\begin{tabular}{|c|c|c|}
\hline $\begin{array}{l}\text { Blinding of outcome as- } \\
\text { sessment (detection bias) } \\
\text { Health related quality of } \\
\text { life (self report) }\end{array}$ & Unclear risk & Not applicable \\
\hline $\begin{array}{l}\text { Incomplete outcome data } \\
\text { (attrition bias) } \\
\text { Falls and fallers }\end{array}$ & Low risk & No missing fall data \\
\hline $\begin{array}{l}\text { Selective reporting (re- } \\
\text { porting bias) }\end{array}$ & Unclear risk & $\begin{array}{l}\text { Falls were prespecified in Methods section and reported in Results. Adverse } \\
\text { events not prespecified. No protocol paper or prospective trial registration }\end{array}$ \\
\hline $\begin{array}{l}\text { Method of ascertaining } \\
\text { falls (recall bias) }\end{array}$ & Unclear risk & $\begin{array}{l}\text { No active fall registration. Fall ascertainment for intervention group at weekly } \\
\text { classes. Controls phoned every } 2 \text { weeks }\end{array}$ \\
\hline
\end{tabular}

Sakamoto 2013

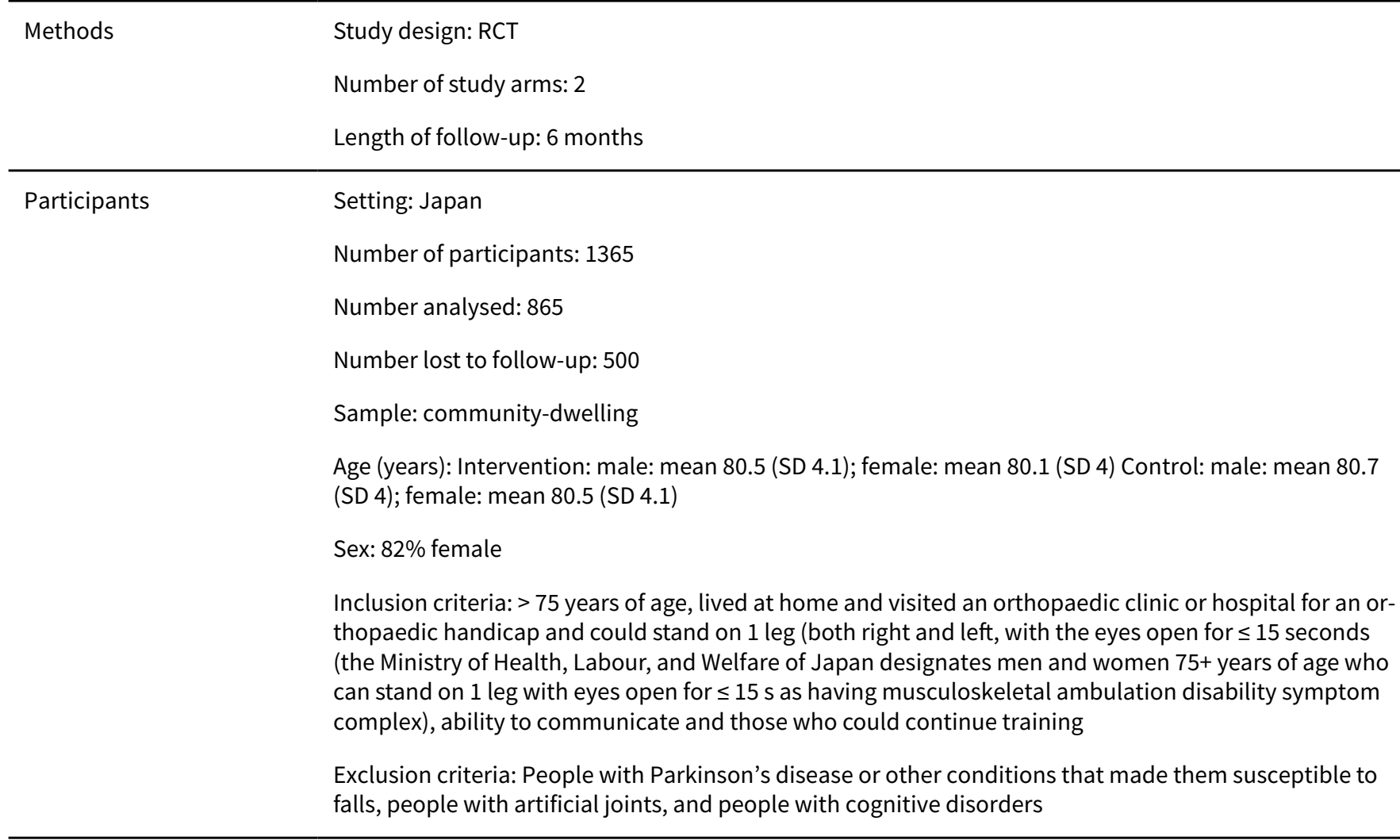

Interventions

1. 1-leg stand balance training: trained each leg with eyes open for 1 minute, 3 a day for 6 months 
Sakamoto 2013 (Continued)

2. Control group: no intervention

1. Rate of falls
2. Number of people who experienced 1 or more falls (risk of falling)
3. Number of people who experienced 1 or more fall-related fractures

\begin{tabular}{ll}
\hline Duration of the study & 26 weeks \\
\hline Adherence & Not reported \\
\hline Notes & Source of funding: Ministry of Health, Labour, and Welfare of Japan \\
& Economic information: not reported \\
\hline
\end{tabular}

\section{Risk of bias}

\begin{tabular}{lll}
\hline Bias & Authors' judgement & Support for judgement \\
\hline $\begin{array}{ll}\text { Random sequence genera- } \\
\text { tion (selection bias) }\end{array}$ & Low risk & $\begin{array}{l}\text { Quote: "The } 10 \times 5(=50) \text { random number tables with } 5 \times 5(25) \text { numbers were } \\
\text { prepared and } 2 \text { ten-faced dice (one green, one yellow) were thrown to decide } \\
\text { which table to use. Two six-faced dice were then thrown to select the num- } \\
\text { ber within the chosen random number table to decide whether the institution } \\
\text { would be designated an exercise or non-exercise institution" }\end{array}$ \\
\hline
\end{tabular}

\begin{tabular}{lll}
\hline $\begin{array}{l}\text { Allocation concealment } \\
\text { (selection bias) }\end{array}$ & Unclear risk & Insufficient information to make judgement \\
\hline $\begin{array}{l}\text { Blinding of participants } \\
\text { and personnel (perfor- }\end{array}$ & Unclear risk & $\begin{array}{l}\text { Blinding not specified but assume participants and personnel were unblinded. } \\
\text { Impact of unblinding unknown }\end{array}$ \\
$\begin{array}{l}\text { All outcomes } \\
\text { Impias) }\end{array}$ &
\end{tabular}

\begin{tabular}{|c|c|c|}
\hline $\begin{array}{l}\text { Blinding of outcome as- } \\
\text { sessment (detection bias) } \\
\text { Falls }\end{array}$ & Unclear risk & $\begin{array}{l}\text { The record of falls/exercise was checked at an outpatient orthopaedic clinic } \\
\text { monthly. Blinding not specified }\end{array}$ \\
\hline
\end{tabular}

\begin{tabular}{lll}
\hline $\begin{array}{l}\text { Blinding of outcome as- } \\
\text { sessment (detection bias) }\end{array}$ & Unclear risk & $\begin{array}{l}\text { Self-report on calendar, then fracture was confirmed and recorded by a doc- } \\
\text { tor. Unclear if doctor was blinded to group }\end{array}$ \\
\hline $\begin{array}{l}\text { Blinding of outcome as- } \\
\text { sessment (detection bias) } \\
\text { Hospital admission, med- } \\
\begin{array}{l}\text { ical attention and adverse } \\
\text { events }\end{array}\end{array}$ & Unclear risk & Participants surveyed at 6 months for adverse events. Blinding not specified \\
\hline
\end{tabular}

\begin{tabular}{|c|c|c|}
\hline $\begin{array}{l}\text { Blinding of outcome as- } \\
\text { sessment (detection bias) } \\
\text { Health related quality of } \\
\text { life (self report) }\end{array}$ & Unclear risk & Not applicable \\
\hline $\begin{array}{l}\text { Incomplete outcome data } \\
\text { (attrition bias) } \\
\text { Falls and fallers }\end{array}$ & High risk & More than $20 \%$ of fall data missing $(37 \%)$ \\
\hline $\begin{array}{l}\text { Selective reporting (re- } \\
\text { porting bias) }\end{array}$ & Unclear risk & $\begin{array}{l}\text { Falls and adverse events were prespecified in Methods section and reported in } \\
\text { Results. No protocol paper or prospective trial registration }\end{array}$ \\
\hline
\end{tabular}


Sakamoto 2013 (Continued)

Method of ascertaining Low risk_ Instructed to record exercise/falls/fracture every day. The record was checked falls (recall bias) at the time of examination at outpatient orthopaedic clinic once a month

Sales 2017

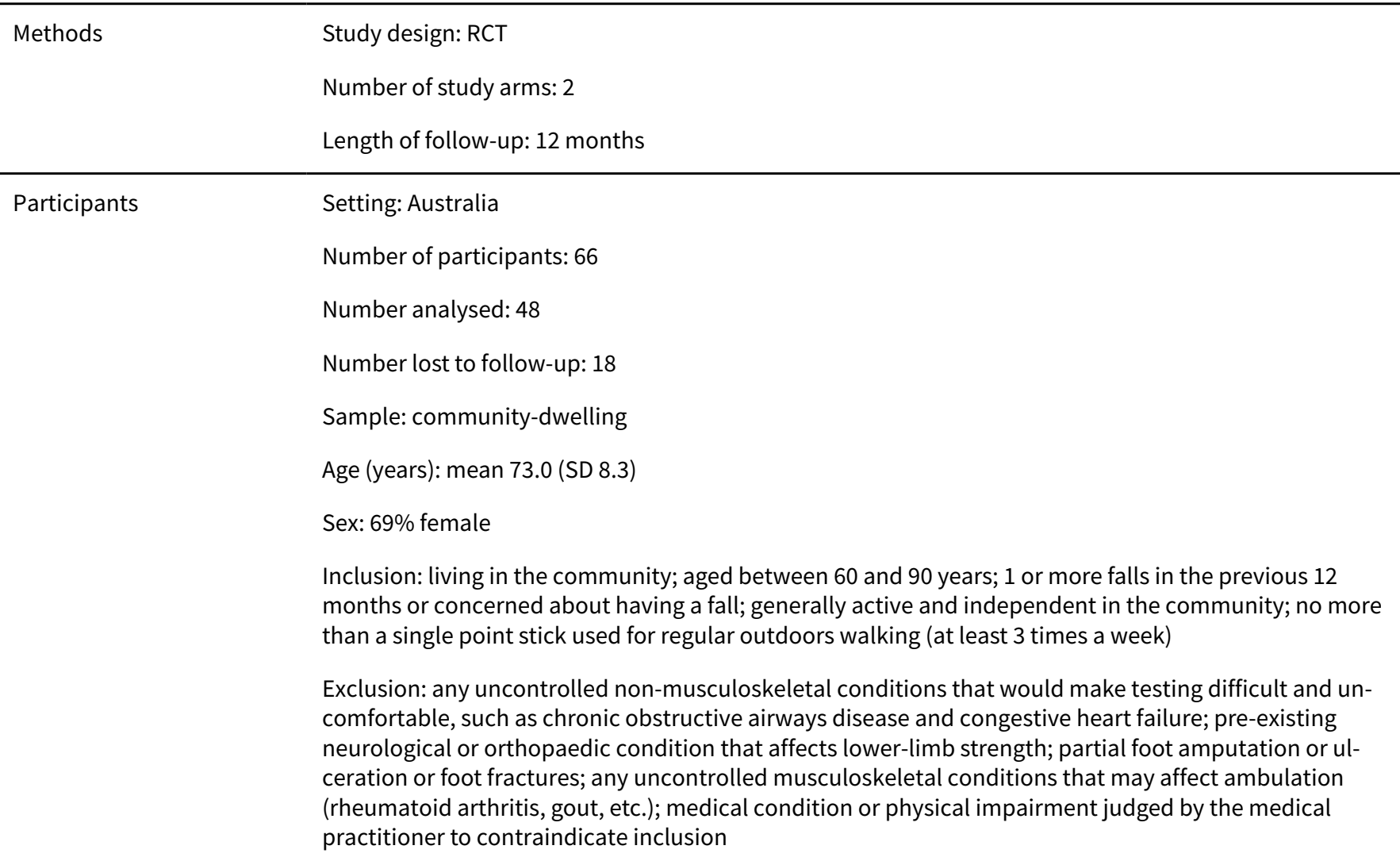

Interventions

1. Group-based strength, balance, co-ordination, mobility and flexibility: circuit-based class, 1-hour sessions, 2 a week for 18 weeks

2. Control: continue with their usual daily activities. Social activities with research team (9 meetings of 2 hours duration over 18 weeks of intervention)

Outcomes Rate of falls
$\begin{aligned} & \text { 2. Number of people who experienced } 1 \text { or more falls (risk of falling) } \\ & \text { 3. Health-related quality of life } \\ & \text { 4. Number of people who died }\end{aligned}$

Duration of the study 52 weeks

Adherence

Attendance at classes was measured. An average of 35 sessions were run for each group of participants

Notes

\author{
Source of funding: Gandel Philanthropy \\ Economic information: not reported \\ Detailed description of exercise intervention given in protocol paper
}




\section{Risk of bias}

\begin{tabular}{|c|c|c|}
\hline Bias & Authors' judgement & Support for judgement \\
\hline $\begin{array}{l}\text { Random sequence genera- } \\
\text { tion (selection bias) }\end{array}$ & Low risk & $\begin{array}{l}\text { Quote: "Block randomization stratification by gender... blocks of } 12 \text { partici- } \\
\text { pants will be recruited at a time" }\end{array}$ \\
\hline $\begin{array}{l}\text { Allocation concealment } \\
\text { (selection bias) }\end{array}$ & Unclear risk & Quote: "opaque not concealed envelopes" \\
\hline $\begin{array}{l}\text { Blinding of participants } \\
\text { and personnel (perfor- } \\
\text { mance bias) } \\
\text { All outcomes }\end{array}$ & Unclear risk & $\begin{array}{l}\text { Participants and personnel implementing the intervention not blinded to allo- } \\
\text { cated group, but impact of non-blinding unclear }\end{array}$ \\
\hline $\begin{array}{l}\text { Blinding of outcome as- } \\
\text { sessment (detection bias) } \\
\text { Falls }\end{array}$ & High risk & $\begin{array}{l}\text { Quote: "Assessors and participants will not be blinded to their respective } \\
\text { group allocation". Assume assessor collating calendars was not blinded }\end{array}$ \\
\hline $\begin{array}{l}\text { Blinding of outcome as- } \\
\text { sessment (detection bias) } \\
\text { Fractures }\end{array}$ & Unclear risk & Not applicable \\
\hline $\begin{array}{l}\text { Blinding of outcome as- } \\
\text { sessment (detection bias) } \\
\text { Hospital admission, med- } \\
\text { ical attention and adverse } \\
\text { events }\end{array}$ & High risk & $\begin{array}{l}\text { Adverse events were self-reported after undertaking exercise sessions. Asses- } \\
\text { sors not blinded }\end{array}$ \\
\hline $\begin{array}{l}\text { Blinding of outcome as- } \\
\text { sessment (detection bias) } \\
\text { Health related quality of } \\
\text { life (self report) }\end{array}$ & High risk & Participants were not blinded to group allocation \\
\hline $\begin{array}{l}\text { Incomplete outcome data } \\
\text { (attrition bias) } \\
\text { Falls and fallers }\end{array}$ & High risk & More than $20 \%$ of fall data were missing $(27 \%)$ \\
\hline $\begin{array}{l}\text { Selective reporting (re- } \\
\text { porting bias) }\end{array}$ & Low risk & $\begin{array}{l}\text { Falls, risk of falls and adverse events are reported and the prospective trial reg- } \\
\text { istration prespecifies the same fall outcomes as those in the trial report }\end{array}$ \\
\hline $\begin{array}{l}\text { Method of ascertaining } \\
\text { falls (recall bias) }\end{array}$ & Low risk & $\begin{array}{l}\text { Quote: "Participants will be requested to record any falls and physical activ- } \\
\text { ity or exercise experienced using a monthly calendar for } 12 \text { months from the } \\
\text { baseline assessment. At the end of each month the calendar will be returned } \\
\text { to the researchers in a reply paid envelope. If the calendar is not returned } \\
\text { within two weeks of the end of a month, the participant will be followed up } \\
\text { with a phone call". }\end{array}$ \\
\hline
\end{tabular}

\section{Sherrington 2014}

$\begin{array}{ll}\text { Methods } & \text { Study design: RCT } \\ & \text { Number of study arms: } 2\end{array}$


Sherrington 2014 (Continued)

Length of follow-up: 12 months

Setting: Sydney, Australia
Number of participants: 340
Number analysed: 340
Number lost to follow-up: 0
Sample: community-dwelling
Age (years): mean 81.2 (SD 8.0)
Sex: $74 \%$ female
Inclusion criteria: aged 60 years and over and had been admitted to and subsequently discharged from
9 aged care, rehabilitation and orthopaedic wards at 4 public hospitals in Sydney, Australia
Exclusion criteria: resided in a high-care residential facility (nursing home); had cognitive impairment
(a MMSE score <24); had insufficient English language to understand procedures; were unable to walk
more than 1 m even with an assistive device or the help of 1 person; or had a medical condition pre-
cluding a 12-month home exercise program (e.g. unstable cardiac disease or progressive neurological
disease)

Interventions $\quad 1$. Home-based strength and balance programme: Weight-bearing Exercise for Better Balance exercise programme + 32-page education booklet about fall prevention, home programme of lower limb balance and strengthening exercises for 20 - 30-minute sessions, up to 6 a week for 12 months; home visits: 10 over 12 months

2. Control group: Usual care from health and community services +32 -page education booklet about fall prevention

1. Rate of falls
2. Number of people who experienced 1 or more falls (risk of falling)
3. Health-related quality of life
4. Number of people who died

\begin{tabular}{ll} 
Duration of the study & 52 weeks \\
\hline Adherence & $\begin{array}{l}\text { Participants who actually exercised } \\
\text { 1. Weight-bearing Exercise group: } 1 \text { month: } 90 \%, 3 \text { months: } 81 \%, 8 \text { months: } 66 \%, 12 \text { months: } 60 \%\end{array}$ \\
\hline Notes & $\begin{array}{l}\text { Source of funding: Australian National Health and Medical Research Council, Australian National Health } \\
\text { and Medical Research Council Research Fellowships } \\
\text { Economic information: Mean cost per person (intervention): WEBB AUD 751. Healthcare service costs: } \\
\text { WEBB AUD 12,029, usual care AUD 10,327. Incremental costs per fall prevented/per QALY gained: AUD } \\
\text { 77,403 per } \\
\text { QALY gained }\end{array}$
\end{tabular}

\section{Risk of bias}

\begin{tabular}{lll}
\hline Bias & Authors' judgement & Support for judgement \\
\hline $\begin{array}{l}\text { Random sequence genera- } \\
\text { tion (selection bias) }\end{array}$ & Low risk & $\begin{array}{l}\text { Computer-generated random-number schedule with randomly-ordered } \\
\text { blocks of 2, 4, and } 6\end{array}$ \\
\hline $\begin{array}{l}\text { Allocation concealment } \\
\text { (selection bias) }\end{array}$ & Low risk & $\begin{array}{l}\text { Quote: "Ensure concealed randomisation to groups, the randomisation sched- } \\
\text { ule was generated in advance by and only accessible to the first author who } \\
\text { was not involved in participant recruitment, interviews or assessments" }\end{array}$ \\
\hline
\end{tabular}


Sherrington 2014 (Continued)

Blinding of participants Unclear risk Participants and personnel unblinded but impact of unblinding unknown and personnel (performance bias)

All outcomes

\begin{tabular}{|c|c|c|}
\hline $\begin{array}{l}\text { Blinding of outcome as- } \\
\text { sessment (detection bias) } \\
\text { Falls }\end{array}$ & Low risk & $\begin{array}{l}\text { Same method used to ascertain falls in both groups. Blinded research assis- } \\
\text { tants recorded and confirmed falls }\end{array}$ \\
\hline $\begin{array}{l}\text { Blinding of outcome as- } \\
\text { sessment (detection bias) } \\
\text { Fractures }\end{array}$ & Unclear risk & Method of ascertaining fractures not specified \\
\hline $\begin{array}{l}\text { Blinding of outcome as- } \\
\text { sessment (detection bias) } \\
\text { Hospital admission, med- } \\
\text { ical attention and adverse } \\
\text { events }\end{array}$ & Low risk & $\begin{array}{l}\text { Adverse events were monitored using the exercise diaries and recorded by } \\
\text { blinded assessors }\end{array}$ \\
\hline $\begin{array}{l}\text { Blinding of outcome as- } \\
\text { sessment (detection bias) } \\
\text { Health related quality of } \\
\text { life (self report) }\end{array}$ & High risk & Participants were not blinded to group allocation \\
\hline $\begin{array}{l}\text { Incomplete outcome data } \\
\text { (attrition bias) } \\
\text { Falls and fallers }\end{array}$ & Low risk & No missing fall data \\
\hline $\begin{array}{l}\text { Selective reporting (re- } \\
\text { porting bias) }\end{array}$ & Low risk & $\begin{array}{l}\text { Falls, risk of falls and adverse events are reported and the trial protocol paper } \\
\text { prespecifies the same fall outcomes as those in the trial report }\end{array}$ \\
\hline $\begin{array}{l}\text { Method of ascertaining } \\
\text { falls (recall bias) }\end{array}$ & Low risk & $\begin{array}{l}\text { Monthly falls calendar. Participants who did not return calendars or who re- } \\
\text { ported a fall were telphoned by blinded research assistants }\end{array}$ \\
\hline
\end{tabular}

Shigematsu 2008

\begin{tabular}{ll} 
Methods & Study design: RCT \\
& Number of study arms: 2 \\
& Length of follow-up: 8 months \\
\hline Participants & Setting: Kawage, Mie, Japan \\
& Number of participants: 68 \\
& Number analysed: 68 \\
& Number lost to follow-up: 0 \\
& Sample: randomly-selected people meeting inclusion criteria \\
& Age (years): mean 69 (SD 3) \\
& Sex: $63 \%$ female \\
Inclusion criteria: 65 - 74 years old; community-dwelling \\
Exclusion criteria: severe neurological or cardiovascular disease; mobility-limiting orthopaedic condi- \\
tions
\end{tabular}


Shigematsu 2008 (Continued)

Interventions
1. Group-based stepping training on felt mat: step direction and performance progressed on felt mat at own pace, 70-minute sessions, 2 a week for 12 weeks; group "divided" at 12 weeks and continued sessions for a further 12 weeks

2. Group-based walking: instructed to increase number of daily steps in supervised outdoor walking, 40-minute sessions, 1 a week for 12 weeks; as above, group divided and half continued walking for a further 12 weeks

$\begin{array}{ll}\text { Outcomes } & \text { 2. Rate of falls } \\ \text { 2. Number of people who experienced } 1 \text { or more falls (risk of falling) }\end{array}$

\begin{tabular}{ll}
\hline Duration of the study & 52 weeks with 32 weeks follow-up after the intervention \\
\hline Adherence & Adherence measured by session attendance \\
1. Group-based stepping training on felt mat: participants attended $21.8 \pm 2.9$ of 24 sessions ( $90.9 \% \pm$ \\
12.1\%) Dropouts: 0 . The participants conscientiously exercised for 40 minutes throughout the regimen \\
2. Group-based walking: participants attended $9.3 \pm 2.6$ of 11 sessions $(84.2 \% \pm 23.7 \%)$. Dropouts: 5
\end{tabular}

$\begin{array}{ll}\text { Notes } & \text { Source of funding: not reported } \\ \text { Economic information: not reported }\end{array}$

\section{Risk of bias}

\begin{tabular}{|c|c|c|}
\hline Bias & Authors' judgement & Support for judgement \\
\hline $\begin{array}{l}\text { Random sequence genera- } \\
\text { tion (selection bias) }\end{array}$ & Low risk & $\begin{array}{l}\text { Quote: "Randomly allocated.. by a public health nurse who used a computer- } \\
\text { ized random number generation program in which the numbers } 0 \text { and } 1 \text { corre- } \\
\text { sponded to the two groups, respectively" }\end{array}$ \\
\hline $\begin{array}{l}\text { Allocation concealment } \\
\text { (selection bias) }\end{array}$ & Unclear risk & Insufficient information to permit judgement \\
\hline $\begin{array}{l}\text { Blinding of participants } \\
\text { and personnel (perfor- } \\
\text { mance bias) } \\
\text { All outcomes }\end{array}$ & Low risk & $\begin{array}{l}\text { Study described as "single-blind", presumably meaning that participants } \\
\text { were blind to whether they were in the intervention or control groups as both } \\
\text { groups received an exercise intervention. Treatment personnel presumably } \\
\text { unblinded but judged that lack of blinding unlikely to introduce bias }\end{array}$ \\
\hline $\begin{array}{l}\text { Blinding of outcome as- } \\
\text { sessment (detection bias) } \\
\text { Falls }\end{array}$ & High risk & $\begin{array}{l}\text { Study described as "single-blind" because both groups received an exercise in- } \\
\text { tervention. Assessors presumably unblinded }\end{array}$ \\
\hline $\begin{array}{l}\text { Blinding of outcome as- } \\
\text { sessment (detection bias) } \\
\text { Fractures }\end{array}$ & Unclear risk & Not applicable \\
\hline $\begin{array}{l}\text { Blinding of outcome as- } \\
\text { sessment (detection bias) } \\
\text { Hospital admission, med- } \\
\text { ical attention and adverse } \\
\text { events }\end{array}$ & Unclear risk & Not applicable \\
\hline $\begin{array}{l}\text { Blinding of outcome as- } \\
\text { sessment (detection bias) } \\
\text { Health related quality of } \\
\text { life (self report) }\end{array}$ & Unclear risk & Not applicable \\
\hline
\end{tabular}


Shigematsu 2008 (Continued)

Incomplete outcome data Low risk No missing fall data
(attrition bias)

Falls and fallers

\begin{tabular}{lll}
\hline $\begin{array}{l}\text { Selective reporting (re- } \\
\text { porting bias) }\end{array}$ & Unclear risk & $\begin{array}{l}\text { Prespecified falls outcomes reported. Adverse events were reported but not } \\
\text { prespecified. No protocol paper or trial registration }\end{array}$ \\
\hline $\begin{array}{l}\text { Method of ascertaining } \\
\text { falls (recall bias) }\end{array}$ & Low risk & $\begin{array}{l}\text { Quote: "All the persons received a pre-paid postcard at the beginning of each } \\
\text { month, which they returned at the beginning of the next month". Instructed to } \\
\text { record falls on a daily basis. Phoned or face-to-face interview if falls reported }\end{array}$ \\
\hline
\end{tabular}

\title{
Siegrist 2016
}

\begin{tabular}{ll}
\hline Methods & Study design: Cluster-RCT \\
& Number of study arms: 2 \\
& Number of clusters: 40 \\
& Length of follow-up: 12 months
\end{tabular}

\section{Participants}

\author{
Setting: Munich, Germany \\ Number of participants: 378 \\ Number analysed: 378 \\ Number lost to follow-up: 0 \\ Sample: community-dwelling \\ Age (years): mean 78.1 (SD 5.9) \\ Sex: $75 \%$ female
}

Inclusion criteria: community-dwelling senior citizens aged $\geq 65$ years with increased physical fall risk included in the trial. Increased fall risk was defined as 1 or more falls in the past 12 months, low physical function (Timed-up-and-Go-Test or Chair-Stand-Test > 10 seconds) or subjective or objective balance deficits or fear of falling. At least 1 criterion was necessary for inclusion into the study.

Exclusion criteria: Those individuals who did not live independently or suffered from physical or mental restrictions that interfered with the assessment of physical fall risk or participation in an exercise program were excluded.

Interventions

1. Group-based balance, strength, power and gait training plus home practice: no additional equipment required, increasing levels of difficulty, behavioural aspects, a self-management programme and perceptual and functional training conducted by a fall prevention instructor (physiotherapist or sports scientist); 1 hour a week for 16 weeks

2. Control group: no guidelines for preventing falls apart from individual GP's experience

1. Rate of falls
$\begin{aligned} & \text { 2. Number of people who experienced } 1 \text { or more falls (risk of falling) } \\ & \text { 3. Number of people who died }\end{aligned}$

Duration of the study

52 weeks 
1. Group-based balance, strength, power and gait training plus home practice group: $82 \%$ participated in more than 10 training sessions. $46 \%$ of the participants performed the home-exercise programme 10 times or more (average 6.7 times)

Economic information: not reported

Number of clusters allocated to intervention: 20; number of clusters allocated to control: 20; number of clusters analysed (intervention): 17 (3 general practices dropped out after randomisation and before recruiting participants); number of clusters analysed (control): 16 (4 general practices dropped out after randomisation and before recruiting participants)

Email communication to obtain fall data, response received, data included in review

\section{Risk of bias}

\begin{tabular}{|c|c|c|}
\hline Bias & Authors' judgement & Support for judgement \\
\hline $\begin{array}{l}\text { Random sequence genera- } \\
\text { tion (selection bias) }\end{array}$ & Low risk & Quote: "...using computer-generated random numbers..". \\
\hline $\begin{array}{l}\text { Allocation concealment } \\
\text { (selection bias) }\end{array}$ & Unclear risk & $\begin{array}{l}\text { Cluster RCT. Individuals were recruited to the trial after the clusters were ran- } \\
\text { domised. It is very likely personnel recruiting participants were not blind to } \\
\text { cluster }\end{array}$ \\
\hline $\begin{array}{l}\text { Blinding of participants } \\
\text { and personnel (perfor- } \\
\text { mance bias) } \\
\text { All outcomes }\end{array}$ & Unclear risk & $\begin{array}{l}\text { Participants and personnel not blind to allocated group but impact of non- } \\
\text { blinding unclear }\end{array}$ \\
\hline $\begin{array}{l}\text { Blinding of outcome as- } \\
\text { sessment (detection bias) } \\
\text { Falls }\end{array}$ & Low risk & $\begin{array}{l}\text { Falls reported using the same method in both groups and followed-up by } \\
\text { blinded assessor }\end{array}$ \\
\hline $\begin{array}{l}\text { Blinding of outcome as- } \\
\text { sessment (detection bias) } \\
\text { Fractures }\end{array}$ & Unclear risk & Not applicable \\
\hline $\begin{array}{l}\text { Blinding of outcome as- } \\
\text { sessment (detection bias) } \\
\text { Hospital admission, med- } \\
\text { ical attention and adverse } \\
\text { events }\end{array}$ & Unclear risk & Not applicable \\
\hline
\end{tabular}

\begin{tabular}{lll}
\hline $\begin{array}{l}\text { Blinding of outcome as- } \\
\text { sessment (detection bias) } \\
\text { Health related quality of } \\
\text { life (self report) }\end{array}$ & Unclear risk & Not applicable \\
\hline $\begin{array}{l}\text { Incomplete outcome data } \\
\text { (attrition bias) } \\
\text { Falls and fallers }\end{array}$ & Low risk & No missing fall data \\
\hline $\begin{array}{l}\text { Selective reporting (re- } \\
\text { porting bias) }\end{array}$ & Low risk & Falls and adverse event outcomes were reported as prespecified in protocol \\
\hline $\begin{array}{l}\text { Method of ascertaining } \\
\text { falls (recall bias) }\end{array}$ & Low risk & Daily fall calendar, posted monthly \\
\end{tabular}


Siegrist 2016 (Continued)

Quote: "When a fall was reported, detailed information was obtained through structured telephone interviews by trained assistants"

Cluster-randomised trials Unclear risk

Individuals were recruited to the trial after the clusters were randomised. It is likely personnel recruiting participants were not blind to cluster; baseline comparability of clusters not reported; missing outcomes for clusters or within clusters were not reported (and 7 general practices dropped out after randomisation but before recruiting participants); accounted for the clustered design in the analysis; results comparable with individually-randomised trials

\section{Skelton 2005}

\begin{tabular}{ll}
\hline Methods & Study design: RCT \\
& Number of study arms: 2 \\
& Length of follow-up: 9 months \\
\hline Participants & Setting: United Kingdom \\
& Number of participants: 81 \\
& Number analysed: 81 \\
& Number lost to follow-up: 0 \\
& Sample: women recruited using posters, newspapers and radio stations \\
Age (years): mean 72.8 (SD 5.9) & Sex: $100 \%$ female \\
Inclusion criteria: aged $\geq 65 ;$ living independently in own home; $\geq 3$ falls in previous year \\
Exclusion criteria: acute rheumatoid arthritis; uncontrolled heart failure or hypertension; significant \\
cognitive impairment; significant neurological disease or impairment; previously diagnosed osteo- \\
porosis
\end{tabular}

Interventions

1. Group-based Falls Management Exercise (FaME) balance and strength training plus home practice: the exercise classes were balance-specific, individually-tailored and targeted training for dynamic balance, strength, bone, endurance, flexibility, gait and functional skills, training to improve 'righting' or 'correcting' skills to avoid a fall, backward-chaining and functional floor exercises: 1-hour sessions, 1 a week for 26 weeks; plus home exercises, 30 minutes, 2 a week for 36 weeks

2. Control: no exercise class. Home-based seated exercises 2 a week for 36 weeks
Outcomes

\section{Rate of falls}
2. Number of people who experienced 1 or more falls (risk of falling)
3. Number of people who experienced fall-related fractures (outcome not reported by group)
3. Number of people who died

\begin{tabular}{|c|c|}
\hline Duration of the study & Total of 132 weeks on average \\
\hline & 46.5 weeks (on average) of pre-intervention falls monitoring \\
\hline & 36 weeks of intervention \\
\hline & 49.7 weeks (on average) of follow-up \\
\hline
\end{tabular}

Adherence

Adherence measured by retention/attrition rate 
1. Falls Management Exercise group: $17 \%$ refused to participate, with another $10 \%$ dropping out of the exercise sessions after initial entry

Notes Source of funding: Research Into Ageing, Dunhill Medical Trust, Barnwood House Trust, Save and Prosper Educational Trust

Economic information: not reported

\section{Risk of bias}

\begin{tabular}{|c|c|c|}
\hline Bias & Authors' judgement & Support for judgement \\
\hline $\begin{array}{l}\text { Random sequence genera- } \\
\text { tion (selection bias) }\end{array}$ & Low risk & $\begin{array}{l}\text { Randomisation was performed by random-number tables by an observer un- } \\
\text { connected to the trial }\end{array}$ \\
\hline $\begin{array}{l}\text { Allocation concealment } \\
\text { (selection bias) }\end{array}$ & Unclear risk & Insufficient information to permit judgement \\
\hline $\begin{array}{l}\text { Blinding of participants } \\
\text { and personnel (perfor- } \\
\text { mance bias) } \\
\text { All outcomes }\end{array}$ & Unclear risk & $\begin{array}{l}\text { Participants and personnel implementing the intervention not blinded to allo- } \\
\text { cated group, but impact of non-blinding unclear }\end{array}$ \\
\hline $\begin{array}{l}\text { Blinding of outcome as- } \\
\text { sessment (detection bias) } \\
\text { Falls }\end{array}$ & Low risk & $\begin{array}{l}\text { Falls reported by participants who were aware of their group allocation } \\
\text { Quote: "The information from the diaries was recorded by an observer blinded } \\
\text { to the subject's group who also contacted subjects if diaries had not been re- } \\
\text { turned for two weeks or more" }\end{array}$ \\
\hline $\begin{array}{l}\text { Blinding of outcome as- } \\
\text { sessment (detection bias) } \\
\text { Fractures }\end{array}$ & Unclear risk & Not applicable \\
\hline $\begin{array}{l}\text { Blinding of outcome as- } \\
\text { sessment (detection bias) } \\
\text { Hospital admission, med- } \\
\text { ical attention and adverse } \\
\text { events }\end{array}$ & Unclear risk & Method of ascertaining adverse events is unclear \\
\hline $\begin{array}{l}\text { Blinding of outcome as- } \\
\text { sessment (detection bias) } \\
\text { Health related quality of } \\
\text { life (self report) }\end{array}$ & Unclear risk & Not applicable \\
\hline $\begin{array}{l}\text { Incomplete outcome data } \\
\text { (attrition bias) } \\
\text { Falls and fallers }\end{array}$ & Low risk & $\begin{array}{l}\text { Less than } 20 \% \text { of fall data were missing }(14 \%) \text {. Data were missing from } 7 \text { par- } \\
\text { ticipants in the intervention group (ill helath } n=4 \text {, nursing home } n=2 \text {, death } n \\
=1 \text { ), and } 4 \text { control participants (ill helath } n=2 \text {, nursing home } n=1 \text {, death } n=1 \text { ) }\end{array}$ \\
\hline $\begin{array}{l}\text { Selective reporting (re- } \\
\text { porting bias) }\end{array}$ & Unclear risk & $\begin{array}{l}\text { Fall outcomes were prespecified in protocol paper and reported. Adverse } \\
\text { events were not prespecified but were reported }\end{array}$ \\
\hline $\begin{array}{l}\text { Method of ascertaining } \\
\text { falls (recall bias) }\end{array}$ & Low risk & $\begin{array}{l}\text { Quote: "Both groups completed daily falls diaries... Diaries were returned } \\
\text { every } 2 \text { weeks by post to the investigator..." Telephone contact if dairies not } \\
\text { returned for } 2 \text { weeks or more }\end{array}$ \\
\hline
\end{tabular}


Smulders 2010

\begin{tabular}{ll}
\hline Methods & Study design: RCT \\
& Number of study arms: 2 \\
& Length of follow-up: 12 months \\
\hline Participants & Setting: Nijmegan, Netherlands \\
& Number of participants: 96 \\
& Number analysed: 92 \\
& Number lost to follow-up: 4 \\
Sample: identified from databases of DXA scans, mail out to members of Dutch Osteoporosis Patient \\
Council; advertising \\
Age (years): mean 71.0 (SD 4.7) \\
Sex: $94 \%$ female \\
Inclusion criteria: community-dwelling; aged $>65 ;$ osteoporosis (DXA; femoral neck or lower-back T \\
score $\leq-2.5) ; \geq 1$ falls in previous year; able to walk 15 minutes without walking device \\
Exclusion criteria: severe cardiac, pulmonary, or musculoskeletal disorders or disorders associated \\
with higher fall risk (e.g. neurologic disorders)
\end{tabular}

Interventions

1. Group-based balance and gait training using an obstacle avoidance course: 11 sessions between 1 -

$2 \frac{1}{2}$ hours including education, balance, gait training using obstacle course, for $51 / 2$ weeks

2. Control: usual care

Outcomes
$\begin{aligned} & \text { 2. Nute of falls } \\ & \text { 3. Number of people who experienced } 1 \text { or more falls (risk of falling) } \\ & \text { 4. Health-related quality of life }\end{aligned}$

Duration of the study 52 weeks

\begin{tabular}{ll}
\hline Adherence & Adherence measured by session attendance \\
& $\begin{array}{l}\text { 1. Group-based balance and gait training using an obstacle avoidance course group: } 93 \% \text { attendance at } \\
\text { total number of sessions. More than half }(53 \%) \text { of the participants did not miss a session }\end{array}$
\end{tabular}

Economic information: not reported

\section{Risk of bias}

\begin{tabular}{lll}
\hline Bias & Authors' judgement & Support for judgement \\
\hline $\begin{array}{l}\text { Random sequence genera- } \\
\text { tion (selection bias) }\end{array}$ & Unclear risk & $\begin{array}{l}\text { Quote: "After a baseline assessment M1, the researcher performed block ran- } \\
\text { domization using non-see-through envelopes. The probability of allocation to } \\
\text { the exercise group was independent of recruitment method" }\end{array}$ \\
\hline $\begin{array}{l}\text { Allocation concealment } \\
\text { (selection bias) }\end{array}$ & Unclear risk & Non-see-through envelopes but not sequentially numbered \\
\hline $\begin{array}{l}\text { Blinding of participants } \\
\text { and personnel (perfor- } \\
\text { mance bias) }\end{array}$ & Unclear risk & $\begin{array}{l}\text { Participants and personnel not blinded to allocated group but impact of non- } \\
\text { blinding unclear }\end{array}$
\end{tabular}


Smulders 2010 (Continued)

All outcomes

\begin{tabular}{|c|c|c|}
\hline $\begin{array}{l}\text { Blinding of outcome as- } \\
\text { sessment (detection bias) }\end{array}$ & Low risk & $\begin{array}{l}\text { Quote: "Fall calendars were scored by an independent researcher who was } \\
\text { blinded to group allocation" }\end{array}$ \\
\hline
\end{tabular}

Falls

\begin{tabular}{|c|c|c|}
\hline $\begin{array}{l}\text { Blinding of outcome as- } \\
\text { sessment (detection bias) } \\
\text { Fractures }\end{array}$ & Unclear risk & Method of reporting fractures is unclear \\
\hline $\begin{array}{l}\text { Blinding of outcome as- } \\
\text { sessment (detection bias) } \\
\text { Hospital admission, med- } \\
\text { ical attention and adverse } \\
\text { events }\end{array}$ & Unclear risk & Not applicable \\
\hline $\begin{array}{l}\text { Blinding of outcome as- } \\
\text { sessment (detection bias) } \\
\text { Health related quality of } \\
\text { life (self report) }\end{array}$ & High risk & Participants not blind to group allocation \\
\hline $\begin{array}{l}\text { Incomplete outcome data } \\
\text { (attrition bias) } \\
\text { Falls and fallers }\end{array}$ & Low risk & $\begin{array}{l}\text { Less than } 20 \% \text { of fall data were missing }(4 \%) \text { with missing data balanced be- } \\
\text { tween groups and balanced reasons for missing data }\end{array}$ \\
\hline $\begin{array}{l}\text { Selective reporting (re- } \\
\text { porting bias) }\end{array}$ & Unclear risk & $\begin{array}{l}\text { Minimum set of expected outcomes not reported (adverse events not report- } \\
\text { ed) }\end{array}$ \\
\hline $\begin{array}{l}\text { Method of ascertaining } \\
\text { falls (recall bias) }\end{array}$ & Low risk & $\begin{array}{l}\text { Quote: "After the intervention had ended, participants registered their falls for } \\
1 \text { year on fall calendars that had to be returned every month... When no fall } \\
\text { calendar was received within } 2 \text { weeks after the start of the month, the partici- } \\
\text { pant was reminded by telephone" }\end{array}$ \\
\hline
\end{tabular}

\section{Steadman 2003}

\begin{tabular}{ll}
\hline Methods & Study design: RCT \\
& Number of study arms: 2 \\
& Length of follow-up: 1 month \\
\hline Participants & Setting: London, UK \\
& Number of participants: 199 \\
& Number analysed: 133 \\
& Number lost to follow-up: 66 \\
& Sample: attendees at a hospital multidisciplinary falls clinic \\
& Age (years): mean 82.7 (SD 5.6) \\
& Sex: $82 \%$ female \\
Inclusion criteria: $\geq 60$ years; Berg Balance Scale < 45 after "adequate management of potential risk \\
factors" \\
Exclusion criteria: amputation; unable to walk 10 metres; recent stroke; progressive neurological disor- \\
der; unstable medical condition; severe cognitive impairment
\end{tabular}


Steadman 2003 (Continued)

Interventions
1. Standard, individualised physiotherapy focused on functional training plus balance training: performance of functional activities, plus repetition and progression of balance and walking exercises, 45minute sessions, 2 sessions a week for 6 weeks

2. Standard, individualised physiotherapy focused on functional training: performance of functional activities but no defined repetition or progression, 45-minute sessions, 2 sessions a week for 4 weeks plus telephone follow-up in final 2 weeks

\begin{tabular}{ll}
\hline Outcomes & 1. Rate of falls \\
\hline Duration of the study & 24 weeks \\
\hline Adherence & $\begin{array}{l}\text { Structured observation schedules were used randomly to monitor adherence to treatment protocols in } \\
\text { both groups. }\end{array}$ \\
$\begin{array}{l}\text { 1. Standard, individualised physiotherapy focused on functional training: the protocol of therapy was } \\
\text { being adhered to in all } 48 \text { participants observed receiving enhanced balance training }\end{array}$ \\
$\begin{array}{l}\text { 2. Control: the protocol of therapy was being adhered to in all } 55 \text { participants observed receiving con- } \\
\text { ventional physiotherapy alone }\end{array}$
\end{tabular}

\begin{tabular}{ll}
\hline Notes & Source of funding: not reported \\
Economic information: not reported \\
Falls reported in past month at 6 weeks used in analysis
\end{tabular}

\section{Risk of bias}

\begin{tabular}{|c|c|c|}
\hline Bias & Authors' judgement & Support for judgement \\
\hline $\begin{array}{l}\text { Random sequence genera- } \\
\text { tion (selection bias) }\end{array}$ & Low risk & Quote: "computer generated random numbers" \\
\hline $\begin{array}{l}\text { Allocation concealment } \\
\text { (selection bias) }\end{array}$ & Unclear risk & Insufficient information to permit judgement \\
\hline $\begin{array}{l}\text { Blinding of participants } \\
\text { and personnel (perfor- } \\
\text { mance bias) } \\
\text { All outcomes }\end{array}$ & Unclear risk & $\begin{array}{l}\text { Participants and personnel not blind to allocated group but impact of non- } \\
\text { blinding unclear }\end{array}$ \\
\hline $\begin{array}{l}\text { Blinding of outcome as- } \\
\text { sessment (detection bias) } \\
\text { Falls }\end{array}$ & Low risk & $\begin{array}{l}\text { Fall data collected using same method in both groups } \\
\text { Quote: "A therapist who was not involved with randomization or delivering the } \\
\text { interventions completed baseline and outcome assessments" }\end{array}$ \\
\hline $\begin{array}{l}\text { Blinding of outcome as- } \\
\text { sessment (detection bias) } \\
\text { Fractures }\end{array}$ & Unclear risk & Not applicable \\
\hline $\begin{array}{l}\text { Blinding of outcome as- } \\
\text { sessment (detection bias) } \\
\text { Hospital admission, med- } \\
\text { ical attention and adverse } \\
\text { events }\end{array}$ & Unclear risk & Not applicable \\
\hline
\end{tabular}

Blinding of outcome as- High risk Participants not blinded to intervention group

sessment (detection bias) 
Steadman 2003 (Continued) Health related quality of life (self report)

\begin{tabular}{lll}
\hline $\begin{array}{l}\text { Incomplete outcome data } \\
\text { (attrition bias) } \\
\text { Falls and fallers }\end{array}$ & High risk & More than 20\% of fall data are missing (33\%) \\
\hline $\begin{array}{l}\text { Selective reporting (re- } \\
\text { porting bias) }\end{array}$ & High risk & $\begin{array}{l}\text { Falls were measured, but number of fallers are not reported. Adverse events } \\
\text { are not reported }\end{array}$ \\
\hline $\begin{array}{l}\text { Method of ascertaining } \\
\text { falls (recall bias) }\end{array}$ & High risk & $\begin{array}{l}\text { Interval recall. Falls data collected for previous month at } 6 \text { weeks, } 12 \text { weeks } \\
\text { and } 24 \text { weeks. }\end{array}$ \\
\hline
\end{tabular}

Suzuki 2004

$\begin{array}{ll}\text { Methods } & \text { Study design: RCT } \\ \text { Number of study arms: } 2 \\ \text { Length of follow-up: } 20 \text { months } \\ \text { Setting: Tokyo, Japan } \\ \text { Number of participants: } 52 \\ \text { Number analysed: } 44 \\ \text { Number lost to follow-up: } 8 \\ \text { Age (years): mean } 78 \text { (SD 3.9), range } 73 \text { to } 90 \\ \text { Sex: } 100 \% \text { female } \\ \text { Sample and inclusion criteria: women in the Tokyo Metropolitan Institute of Gerontology Longitudi- } \\ \text { nal Interdisciplinary Study on Aging attending a comprehensive geriatric health examination; living at } \\ \text { home } \\ \text { Exclusion criteria: unable to measure muscle strength, poor mobility due to hemiplegia, poorly-con- } \\ \text { trolled blood pressure, communication difficulties due to impaired hearing }\end{array}$

Interventions
medium rubber bands used for strengthening, 1 -hour class, fortnightly for 6 months plus individual
home-based exercises 30 minutes daily, 3 a week
2. Control: pamphlet and advice on falls prevention

\begin{tabular}{ll}
\hline Outcomes & 1. Rate of falls \\
& 2. Number of people who experienced 1 or more falls (risk of falling)
\end{tabular}

\begin{tabular}{ll}
\hline Duration of the study & 87 weeks \\
\hline Adherence & Adherence measured by session attendance \\
& $\begin{array}{l}\text { 1. Group-based strength, balance and gait training plus home practice: attendance ranged from } 64 \text { - } \\
86 \% \text {, with a mean of } 75 \% .15 \text { participants (54\%) attended all } 10 \text { sessions. } 6 \text { who attended } 0-3 \text { times } \\
\text { were regarded as failing to master the exercise programme. Among the } 22 \text { participants who completed } \\
\text { the intervention, } 21(96 \%) \text { participated in }>7 \text { sessions }\end{array}$ \\
\hline
\end{tabular}

Notes

Source of funding: Tokyo Metropolitan Government

Economic information: not reported 
Suzuki 2004 (Continued)

Risk of bias

\begin{tabular}{|c|c|c|}
\hline Bias & Authors' judgement & Support for judgement \\
\hline $\begin{array}{l}\text { Random sequence genera- } \\
\text { tion (selection bias) }\end{array}$ & Unclear risk & States "randomized" but method of randomisation not described \\
\hline $\begin{array}{l}\text { Allocation concealment } \\
\text { (selection bias) }\end{array}$ & Unclear risk & Insufficient information to permit judgement \\
\hline $\begin{array}{l}\text { Blinding of participants } \\
\text { and personnel (perfor- } \\
\text { mance bias) } \\
\text { All outcomes }\end{array}$ & Unclear risk & $\begin{array}{l}\text { Participants and personnel not blinded to allocated group but impact of non- } \\
\text { blinding unclear }\end{array}$ \\
\hline $\begin{array}{l}\text { Blinding of outcome as- } \\
\text { sessment (detection bias) } \\
\text { Falls }\end{array}$ & Unclear risk & $\begin{array}{l}\text { Falls reported by participants who were aware of their group allocation, using } \\
\text { same method in each group. Does not state whether outcome assessors were } \\
\text { blind to allocation }\end{array}$ \\
\hline $\begin{array}{l}\text { Blinding of outcome as- } \\
\text { sessment (detection bias) } \\
\text { Fractures }\end{array}$ & Unclear risk & Not applicable \\
\hline $\begin{array}{l}\text { Blinding of outcome as- } \\
\text { sessment (detection bias) } \\
\text { Hospital admission, med- } \\
\text { ical attention and adverse } \\
\text { events }\end{array}$ & Unclear risk & Not applicable \\
\hline $\begin{array}{l}\text { Blinding of outcome as- } \\
\text { sessment (detection bias) } \\
\text { Health related quality of } \\
\text { life (self report) }\end{array}$ & Unclear risk & Not applicable \\
\hline $\begin{array}{l}\text { Incomplete outcome data } \\
\text { (attrition bias) } \\
\text { Falls and fallers }\end{array}$ & Unclear risk & $\begin{array}{l}<20 \% \text { of fall data are missing }(15 \%) \text {. Mild imbalance in missing data from inter- } \\
\text { vention group }(n=6) \text { and control group }(n=2) \text {. Reason for missing data in the } \\
\text { control group is unclear }\end{array}$ \\
\hline $\begin{array}{l}\text { Selective reporting (re- } \\
\text { porting bias) }\end{array}$ & Unclear risk & $\begin{array}{l}\text { Minimum set of expected outcomes not reported (adverse events not report- } \\
\text { ed) }\end{array}$ \\
\hline $\begin{array}{l}\text { Method of ascertaining } \\
\text { falls (recall bias) }\end{array}$ & High risk & $\begin{array}{l}\text { Retrospective recall. Falls and fractures recorded retrospectively at interview } \\
\text { at } 8 \text { months and } 20 \text { months after intervention }\end{array}$ \\
\hline
\end{tabular}

Taylor 2012

\begin{tabular}{ll}
\hline Methods & Study design: RCT \\
& Number of study arms: 2 \\
& Length of follow-up: 17 months \\
\hline Participants & Setting: Auckland, Christchurch and Dunedin, New Zealand \\
& Number of participants: 684 \\
& Number analysed: 684 \\
\hline
\end{tabular}


Taylor 2012 (Continued)

Number lost to follow-up: 0

Sample: community-dwelling

Age (years): mean 74.5 (SD 6.5)

Sex: $73 \%$ female

Inclusion criteria: $\geq 65$ years old ( 55 years if Ma-ori or Pacific Islander to account for ethnic disparities in health), had experienced at least 1 fall in the previous 12 months or were considered to be at risk of falling using the Falls Risk Assessment Tool (FRAT > 1).

Exclusion criteria: unable to walk independently (with or without walking aid), chronic medical condition that would limit participation in low- to moderate-intensity exercise, severe cognitive limitations (score $<23$ on the Telephone MMSE), participated in Tai Chi within the last year, or currently participating in an organized exercise programme aimed at improving strength and balance

$\begin{array}{ll}\text { Interventions } & \text { 1. Group-based Tai Chi, } 2 \text { a week: } 1 \text {-hour class, } 2 \text { a week for } 20 \text { weeks } \\ \text { 2. Group-based Tai Chi, } 1 \text { a week: } 1 \text {-hour class, } 1 \text { a week for } 20 \text { weeks } \\ \text { 3. Control: Group-based seated gentle lower-limb exercise, stretching, low-level strength, and low-level } \\ \text { cardiovascular exercise; } 1 \text {-hour class, } 1 \text { a week for } 20 \text { weeks }\end{array}$

$\begin{array}{ll}\text { Notes } & \text { Source of funding: Accident Compensation Corporation } \\ & \text { Economic information: not reported }\end{array}$

\section{Risk of bias}

\begin{tabular}{lll}
\hline Bias & Authors' judgement & Support for judgement \\
\hline $\begin{array}{l}\text { Random sequence genera- } \\
\text { tion (selection bias) }\end{array}$ & Low risk & $\begin{array}{l}\text { Quote: "Web-based, computer-generated blocked random number system } \\
\text { (generated by the study biostatistician)" }\end{array}$ \\
\hline $\begin{array}{l}\text { Allocation concealment } \\
\text { (selection bias) }\end{array}$ & Low risk & $\begin{array}{l}\text { Quote: "At the end of the baseline assessment, each participant was given a } \\
\text { sealed opaque envelope containing group allocation details and was instruct- } \\
\text { ed to open the envelope after leaving the assessment venue and not to discuss } \\
\text { the assignment with any of the assessors" }\end{array}$ \\
\hline
\end{tabular}

\begin{tabular}{|c|c|c|}
\hline $\begin{array}{l}\text { Blinding of participants } \\
\text { and personnel (perfor- }\end{array}$ & Unclear risk & $\begin{array}{l}\text { Participants and personnel implementing the intervention not blinded to allo- } \\
\text { cated group, but impact of non-blinding unclear }\end{array}$ \\
\hline
\end{tabular}
mance bias)

All outcomes 
Taylor 2012 (Continued)

\begin{tabular}{|c|c|c|}
\hline $\begin{array}{l}\text { Blinding of outcome as- } \\
\text { sessment (detection bias) } \\
\text { Falls }\end{array}$ & Low risk & $\begin{array}{l}\text { Quote: "Participants who did not return their monthly calendars had reminder } \\
\text { telephone calls within } 2 \text { weeks, and assessors blinded to group allocation col- } \\
\text { lected data related to any falls over the telephone" }\end{array}$ \\
\hline
\end{tabular}

\begin{tabular}{l} 
Blinding of outcome as- \\
sessment (detection bias) \\
Fractures \\
\hline Blinding of outcome as- \\
sessment (detection bias) \\
Hospital admission, med- \\
ical attention and adverse \\
events
\end{tabular}

\begin{tabular}{ll}
\hline Blinding of outcome as- & Unclear risk \\
sessment (detection bias) & \\
Health related quality of & \\
life (self report) & \\
\hline
\end{tabular}

\begin{tabular}{lll}
\hline $\begin{array}{l}\text { Incomplete outcome data } \\
\text { (attrition bias) } \\
\text { Falls and fallers }\end{array}$ & Low risk & No missing fall data \\
\hline $\begin{array}{l}\text { Selective reporting (re- } \\
\text { porting bias) }\end{array}$ & Unclear risk & $\begin{array}{l}\text { Prespecified falls outcomes reported. Trial registration was retrospective and } \\
\text { does not note adverse events }\end{array}$ \\
\hline $\begin{array}{l}\text { Method of ascertaining } \\
\text { falls (recall bias) }\end{array}$ & Low risk & $\begin{array}{l}\text { Quote: "Participants recorded fall incidents as they occurred on provided cal- } \\
\text { endars that they returned monthly by mail" }\end{array}$ \\
\hline
\end{tabular}

Trombetti 2011

\begin{tabular}{|c|c|}
\hline Methods & $\begin{array}{l}\text { Study design: RCT (cross-over at } 6 \text { months) } \\
\text { Number of study arms: } 2 \\
\text { Length of follow-up: } 6 \text { months }\end{array}$ \\
\hline Participants & $\begin{array}{l}\text { Setting: Geneva, Switzerland } \\
\text { Number of participants: } 134 \\
\text { Number analysed: } 134 \\
\text { Number lost to follow-up: } 0 \\
\text { Sample: volunteers recruited by advertising etc. } \\
\text { Age (years): } 75.5 \text { (SD 6.9) } \\
\text { Sex: } 96 \% \text { female } \\
\text { Inclusion criteria: aged } \geq 65 ; \text { community-dwelling; no previous experience of Jaques-Dalcroze eurhyth- } \\
\text { mics (except during childhood); high risk of falling ( } \geq 1 \text { fall after the age of } 65 \text {, impaired balance, or } \\
\text { physically frail) } \\
\text { Exclusion criteria: neurological or orthopaedic disease seriously affecting gait and balance; progres- } \\
\text { sive or unstable medical conditions limiting participation; dependent on walking aids, e.g. canes and } \\
\text { walkers }\end{array}$ \\
\hline
\end{tabular}

Interventions

1. Group-based balance and gait training: music-based multitask exercise programme gradually increasing in difficulty to challenge balance, 1 hour, 1 a week for 6 months 
Trombetti 2011 (Continued)

2. Control: received intervention after 6 months

1. Rate of falls
2. Number of people who experienced 1 or more falls (risk of falling)
3. Number of people who died

Duration of the study 26 weeks

Adherence
Adherence measured by percentage completed study, class attendance
$\begin{aligned} & \text { 1. Group-based balance and gait training: mean attendance rate; } 78 \% .83 \% \text { completed the interven- } \\ & \text { tion, of whom } 77 \% \text { attended at least } 20 \text { classes (i.e. } 80 \% \text { of the classes) }\end{aligned}$

$\begin{array}{ll}\text { Notes } & \text { Source of funding: Loterie Romande Geneva, Carigest SA, Gertrude Hirzel Foundation, Leenaards Foun- } \\ \text { dation, Oltramare Foundation, Eagle Foundation, Foundation for Geneva (Georges Junod Fund), Delta } \\ \text { réseau de soins Geneva, Helsana }\end{array}$

Economic information: not reported

Falls data from 6 months (before cross-over) used for analysis in the review

\section{Risk of bias}

\begin{tabular}{|c|c|c|}
\hline Bias & Authors' judgement & Support for judgement \\
\hline $\begin{array}{l}\text { Random sequence genera- } \\
\text { tion (selection bias) }\end{array}$ & Low risk & $\begin{array}{l}\text { Quote: "subjects were randomized ... according to a computer-generated list } \\
\ldots \text { using a permuted block randomization design" }\end{array}$ \\
\hline $\begin{array}{l}\text { Allocation concealment } \\
\text { (selection bias) }\end{array}$ & Low risk & $\begin{array}{l}\text { Quote: "subjects were randomized ... according to a computer-generated list } \\
\text { prepared by an independent statistician" }\end{array}$ \\
\hline $\begin{array}{l}\text { Blinding of participants } \\
\text { and personnel (perfor- } \\
\text { mance bias) } \\
\text { All outcomes }\end{array}$ & Unclear risk & $\begin{array}{l}\text { Participants and personnel not blinded to allocated group but impact of non- } \\
\text { blinding unclear }\end{array}$ \\
\hline $\begin{array}{l}\text { Blinding of outcome as- } \\
\text { sessment (detection bias) } \\
\text { Falls }\end{array}$ & Unclear risk & $\begin{array}{l}\text { Participants self-reported falls } \\
\text { Quote: "Participants who failed to return the diary or provided incomplete da- } \\
\text { ta were contacted by telephone." Not clear whether this assessor was blind to } \\
\text { group allocation }\end{array}$ \\
\hline $\begin{array}{l}\text { Blinding of outcome as- } \\
\text { sessment (detection bias) } \\
\text { Fractures }\end{array}$ & Unclear risk & Not applicable \\
\hline $\begin{array}{l}\text { Blinding of outcome as- } \\
\text { sessment (detection bias) } \\
\text { Hospital admission, med- } \\
\text { ical attention and adverse } \\
\text { events }\end{array}$ & Unclear risk & Method of ascertaining adverse events, and presence of blinding, unclear \\
\hline $\begin{array}{l}\text { Blinding of outcome as- } \\
\text { sessment (detection bias) } \\
\text { Health related quality of } \\
\text { life (self report) }\end{array}$ & Unclear risk & Not applicable \\
\hline
\end{tabular}


Trombetti 2011 (Continued)

Incomplete outcome data Low risk No missing fall data
(attrition bias)

Falls and fallers

\begin{tabular}{lll}
\hline $\begin{array}{l}\text { Selective reporting (re- } \\
\text { porting bias) }\end{array}$ & Unclear risk & $\begin{array}{l}\text { Fall outcomes were prespecifed in the prospective trial registration. Adverse } \\
\text { events (part of the minimum set of expected outcomes) were noted only in the } \\
\text { results }\end{array}$ \\
\hline $\begin{array}{l}\text { Method of ascertaining } \\
\text { falls (recall bias) }\end{array}$ & Low risk & $\begin{array}{l}\text { Quote: "Falls were prospectively monitored for } 12 \text { months and recorded daily } \\
\text { using a diary mailed monthly to the study coordinator. Participants who failed } \\
\text { to return the diary or provided incomplete data were contacted by telephone" }\end{array}$
\end{tabular}

Uusi-Rasi 2015

\begin{tabular}{ll} 
Methods & Study design: RCT \\
& Number of study arms: 2 \\
& Length of follow-up: 24 months \\
\hline
\end{tabular}

Setting: Tampere, Finland
Number of participants: 205
Number analysed: 186
Number lost to follow-up: 19
Sample: community-dwelling women
Age (years): mean 74 (SD 3.0)
Sex: $100 \%$ female
Inclusion criteria: 70 - 80 years, living at home independently; had fallen at least once during the previ-
ous year; no contraindication to exercise; understands the procedures of the study, voluntarily agrees
to undergo all measurements and signs informed consent
Exclusion criteria: moderate to vigorous exercise > 2 hours a week; regular use of vitamin D or calcium
+ vitamin D supplements; a recent fracture (during preceding 12 months); contraindication or inabili-
ty to participate in the exercise programme; a marked decline in the basic ADL; cognitive impairments
(MMSE, MMSE-test); primary hyperthyroidism; and degenerative conditions, such as Parkinson's dis-
ease.

Interventions

Randomised into 4 groups: 3 intervention groups ( 1 vitamin D and exercise, 1 placebo and exercise, 1 vitamin D without exercise) and 1 control group (placebo without exercise). Only the placebo and exercise and the control groups were included in this review

1. Group-based balance and strength training plus home practice: balance, weight-bearing, agility and functional exercises; weight machines, pulleys and free weights used for strength training; 2 a week for the first year, and 1 a week for the second year, plus home training 5 - 15 minutes performed on all rest days

2. Control group: usual pre-study level of physical activity

1. Rate of falls
$\begin{aligned} & \text { 2. Number of people who experienced } 1 \text { or more falls (risk of falling) } \\ & \text { 3. Number of people who experienced } 1 \text { or more falls that required medical attention }\end{aligned}$


Uusi-Rasi 2015 (Continued)

4. Number of people who died

\begin{tabular}{|c|c|}
\hline Duration of the study & 104 weeks \\
\hline \multirow[t]{2}{*}{ Adherence } & Adherence measured by session attendance, home training completion \\
\hline & $\begin{array}{l}\text { 1. Group-based balance and strength training plus home practice: attendance at all offered group train- } \\
\text { ing; } 73 \% \text { (range, } 0-97.4 \% \text { ). Attendance at all home training sessions; } 66.1 \% \text { (range } 0-100 \% \text { ) }\end{array}$ \\
\hline \multirow[t]{3}{*}{ Notes } & $\begin{array}{l}\text { Source of funding: Academy of Finland, Ministry of Education and Culture, Competitive Research Fund } \\
\text { of Pirkanmaa Hospital District, Juho Vainio Foundation }\end{array}$ \\
\hline & Hazard ratios but not numbers reported for "medically attended fallers" \\
\hline & $\begin{array}{l}\text { Economic information: Total costs (intervention and healthcare): EUR } 30.9 \text { for no exercise + placebo; } \\
\text { EUR } 206.9 \text { for no exercise + vitamin D } 800 \text { IU/day; EUR } 73.4 \text { for exercise + placebo; EUR } 188.0 \text { for exercise } \\
\text { + vitamin D } 00 \text { IU/day. Incremental costs per fall/per QALY gained: EUR } 220.7 \text { for no exercise + placebo, } \\
\text { EUR } 17,600 \text { for no exercise + vitaminD } 800 \text { IU/day, EUR } 2670 \text { for exercise + placebo, EUR } 3820 \text { for exer- } \\
\text { cise + vitamin D } 800 \text { IU/day }\end{array}$ \\
\hline
\end{tabular}

\section{Risk of bias}

\begin{tabular}{lll}
\hline Bias & Authors' judgement & Support for judgement \\
\hline $\begin{array}{l}\text { Random sequence genera- } \\
\text { tion (selection bias) }\end{array}$ & Low risk & $\begin{array}{l}\text { Quote: "The study statistician (K.T.) generated the participant list using validat- } \\
\text { ed randomization software. He was blinded to the study participants and their } \\
\text { characteristics and randomly allocated them into 4 groups (simple randomiza- } \\
\text { tion)" }\end{array}$
\end{tabular}

\begin{tabular}{lll}
\hline $\begin{array}{l}\text { Allocation concealment } \\
\text { (selection bias) }\end{array}$ & Unclear risk & Insufficient information provided \\
\hline $\begin{array}{l}\text { Blinding of participants } \\
\text { and personnel (perfor- }\end{array}$ & Unclear risk & Participants and personnel unblinded but impact of unblinding unknown \\
mance bias) & & \\
All outcomes &
\end{tabular}

\begin{tabular}{|c|c|c|}
\hline $\begin{array}{l}\text { Blinding of outcome as- } \\
\text { sessment (detection bias) }\end{array}$ & Unclear risk & $\begin{array}{l}\text { Falls ascertained by self report. Unclear whether staff conducting follow-up } \\
\text { telephone calls were blinded }\end{array}$ \\
\hline
\end{tabular}

Falls

\begin{tabular}{|c|c|c|}
\hline $\begin{array}{l}\text { Blinding of outcome as- } \\
\text { sessment (detection bias) } \\
\text { Fractures }\end{array}$ & Unclear risk & Not applicable \\
\hline
\end{tabular}

\begin{tabular}{|c|c|c|}
\hline $\begin{array}{l}\text { Blinding of outcome as- } \\
\text { sessment (detection bias) } \\
\text { Hospital admission, med- } \\
\text { ical attention and adverse } \\
\text { events }\end{array}$ & Unclear risk & Method of ascertaining adverse events and injurious falls was not clear \\
\hline $\begin{array}{l}\text { Blinding of outcome as- } \\
\text { sessment (detection bias) } \\
\text { Health related quality of } \\
\text { life (self report) }\end{array}$ & Unclear risk & Not applicable \\
\hline $\begin{array}{l}\text { Incomplete outcome data } \\
\text { (attrition bias) } \\
\text { Falls and fallers }\end{array}$ & Unclear risk & $\begin{array}{l}\text { Less than } 20 \% \text { of fall data are missing }(9 \%) \text {. Missing fall data had mild imbal- } \\
\text { ance, with intervention group }(n=12 \text {; lost interest } n=3 \text {, health reasons } n=9) \\
\text { and control group }(n=7 \text {; lost interest } n=2 \text {, health reasons } n=3 \text {, died } n=2)\end{array}$ \\
\hline
\end{tabular}


Uusi-Rasi 2015 (Continued)

Selective reporting ( $\mathrm{re}$ Unclear risk $\quad$ Fall outcomes were prespecifed in the prospective trial registration. Adverse porting bias) events (part of the minimum set of expected outcomes) were noted only in the Results

$\begin{array}{ll}\begin{array}{l}\text { Method of ascertaining } \\ \text { falls (recall bias) }\end{array} & \text { Low risk } \\ \end{array}$

\section{Verrusio 2017}

Study design: RCT
Number of study arms: 2
Length of follow-up: 12 months
Setting: Rome, Italy
Number of participants: 150
Number analysed: 147
Number lost to follow-up: 3
Sample: outpatients
Age (years): mean 64.9 (SD 4.6)
Sex: $53 \%$ female
Inclusion criteria: young old people (60 - 69 years), normal or corrected vision, Tinetti score 19 - 24
Exclusion criteria: medical condition that prevented safe participation in an exercise programme, pe-
ripheral artery occlusive disease, diabetic neuropathy, history of stroke, history of inflammatory arthri-
tis, history of vertebral fragility fractures or hip or leg fractures or both in the previous 24 months, sys-
tolic blood pressure 200 mm

Interventions

1. Individual, supervised balance and gait training using exoskeleton human body posturiser: moderate intensity, 1 hour, 3 a week for 12 months

2. Individual supervised walking, balance and posture training: moderate intensity, 1 hour, 3 a week, for 12 months

\begin{tabular}{ll}
\hline Outcomes & 1. Number of people who experienced 1 or more falls (risk of falling) \\
\hline Duration of the study & 52 weeks \\
\hline Adherence & Not reported \\
\hline Notes & Source of funding: not reported \\
& Economic information: not reported
\end{tabular}

\section{Risk of bias}

\begin{tabular}{lll}
\hline Bias & Authors' judgement & Support for judgement \\
\hline $\begin{array}{l}\text { Random sequence genera- } \\
\text { tion (selection bias) }\end{array}$ & Low risk & $\begin{array}{l}\text { Quote: "participants were randomly assigned into two groups following simple } \\
\text { randomization procedures (computerized random numbers)" }\end{array}$ \\
\hline
\end{tabular}




\section{Verrusio 2017 (Continued)}

\begin{tabular}{|c|c|c|}
\hline $\begin{array}{l}\text { Allocation concealment } \\
\text { (selection bias) }\end{array}$ & Unclear risk & No details provided \\
\hline $\begin{array}{l}\text { Blinding of participants } \\
\text { and personnel (perfor- } \\
\text { mance bias) } \\
\text { All outcomes }\end{array}$ & Unclear risk & Participants and personnel unblinded but impact of unblinding unknown \\
\hline $\begin{array}{l}\text { Blinding of outcome as- } \\
\text { sessment (detection bias) } \\
\text { Falls }\end{array}$ & Unclear risk & $\begin{array}{l}\text { It is unclear whether the assessors recording falls were blinded to group allo- } \\
\text { cation }\end{array}$ \\
\hline $\begin{array}{l}\text { Blinding of outcome as- } \\
\text { sessment (detection bias) } \\
\text { Fractures }\end{array}$ & Unclear risk & Not applicable \\
\hline $\begin{array}{l}\text { Blinding of outcome as- } \\
\text { sessment (detection bias) } \\
\text { Hospital admission, med- } \\
\text { ical attention and adverse } \\
\text { events }\end{array}$ & Unclear risk & Not applicable \\
\hline
\end{tabular}

Blinding of outcome as-

High risk

Participants were not blinded to group alloction

sessment (detection bias)

Health related quality of

life (self report)

\begin{tabular}{lll}
\hline $\begin{array}{l}\text { Incomplete outcome data } \\
\text { (attrition bias) } \\
\text { Falls and fallers }\end{array}$ & Low risk & $\begin{array}{l}\text { Less than 20\% of fall data are missing (2\%). The missing data were balanced } \\
\text { between the groups with } 2 \text { lost to follow-up in the intervention group and } 1 \text { in } \\
\text { the control group }\end{array}$ \\
\hline $\begin{array}{l}\text { Selective reporting (re- } \\
\text { porting bias) }\end{array}$ & High risk & Falls were measured, but number of falls was not reported \\
\hline $\begin{array}{l}\text { Method of ascertaining } \\
\text { falls (recall bias) }\end{array}$ & Low risk & $\begin{array}{l}\text { Quote: "The number of falls will be monitored with daily fall diaries. Diaries } \\
\text { will be collected monthly through the mail. Details of each registered fall will } \\
\text { be ascertained by the investigator" }\end{array}$
\end{tabular}

Vogler 2009

\begin{tabular}{ll}
\hline Methods & Study design: RCT \\
& Number of study arms: 3 \\
& Length of follow-up: 12 months \\
\hline Participants & Setting: Sydney, Australia \\
& Number of participants: 180 \\
& Number analysed: 171 \\
& Number lost to follow-up: 9 \\
& Sample: community-dwelling \\
& Age (years): mean 80 (SD 7) \\
& Sex: $83 \%$ female \\
& Inclusion criteria: $65+$ years hospital inpatients
\end{tabular}


Vogler 2009 (Continued)

Exclusion criteria: medical contraindications to exercise. MMSE score $<25$ out of 30 , discharge to highcare residential facility

Interventions

1. Home-based seated lower-limb strength exercises: seated exercises targeting hip flexion, extension, abduction, knee flexion and extension, and ankle plantar- and dorsiflexion; resistance via cuff weights and exercise bands with aim of 10 - 12 RM, 3 a week for 12 weeks; approximately 12 a month; checked and progressed 8 times over 12 weeks

2. Home-based strength training with weight-bearing, functional tasks: weight-bearing (WB) exercise in standing, targeting lower-limb strength, e.g. heel raises, partial squats, sit-to-stand, and stepping forward and sideways up onto blocks. Resistance by weight-loaded waist belts, with aim of $10-12 \mathrm{RM}$. Also exercise targeting WB task performance, e.g. reaching, tandem stand, 3 times a week for 12 weeks; approximately 12 times a month; checked and progressed 8 times over 12 weeks

3. Control group: social visits, frequency-matched, each 1 hour duration

Outcomes
2. Number of people who experienced 1 or more falls (risk of falling)

\begin{tabular}{ll}
\hline Duration of the study & 12 weeks \\
\hline Adherence & $\begin{array}{l}\text { 1. Seated exercise group: completed } 70 \% \text { of } 36 \text { recommended exercise sessions } \\
\text { 2. WB group: completed } 62 \% \text { of } 36 \text { recommended exercise sessions }\end{array}$ \\
\hline Notes & Source of funding: NHMRC, Good Age Trust \\
& Economic information: not reported
\end{tabular}

\section{Risk of bias}

\begin{tabular}{|c|c|c|}
\hline Bias & Authors' judgement & Support for judgement \\
\hline $\begin{array}{l}\text { Random sequence genera- } \\
\text { tion (selection bias) }\end{array}$ & Low risk & $\begin{array}{l}\text { Quote: "performed in blocks of } 15 \text { subjects by computer-generated random } \\
\text { numbers" }\end{array}$ \\
\hline $\begin{array}{l}\text { Allocation concealment } \\
\text { (selection bias) }\end{array}$ & Low risk & $\begin{array}{l}\text { Quote: "Group allocations for each subject were concealed in opaque en- } \\
\text { velopes" }\end{array}$ \\
\hline $\begin{array}{l}\text { Blinding of participants } \\
\text { and personnel (perfor- } \\
\text { mance bias) } \\
\text { All outcomes }\end{array}$ & Unclear risk & Participants and personnel unblinded but impact of unblinding unknown \\
\hline $\begin{array}{l}\text { Blinding of outcome as- } \\
\text { sessment (detection bias) } \\
\text { Falls }\end{array}$ & Low risk & $\begin{array}{l}\text { Falls were measured using the same method in each group } \\
\text { Quote: "The outcome assessor remained unaware of group allocation" }\end{array}$ \\
\hline $\begin{array}{l}\text { Blinding of outcome as- } \\
\text { sessment (detection bias) } \\
\text { Fractures }\end{array}$ & Unclear risk & Not applicable \\
\hline $\begin{array}{l}\text { Blinding of outcome as- } \\
\text { sessment (detection bias) } \\
\text { Hospital admission, med- } \\
\text { ical attention and adverse } \\
\text { events }\end{array}$ & Unclear risk & Not applicable \\
\hline
\end{tabular}

Blinding of outcome as-
sessment (detection bias) $\quad$ High risk $\quad$ Participants were not blinded to group allocation


Vogler 2009 (Continued) Health related quality of life (self report)

\begin{tabular}{lll}
\hline $\begin{array}{l}\text { Incomplete outcome data } \\
\text { (attrition bias) }\end{array}$ & Low risk & $\begin{array}{l}\text { Less than 20\% of fall data were missing (5\%). Missing data were balanced, with } \\
\text { Falls and fallers }\end{array}$ \\
\hline $\begin{array}{l}\text { Selective reporting (re- } \\
\text { porting bias) }\end{array}$ & High risk & Falls were measured but number of falls were not reported \\
\hline $\begin{array}{l}\text { Method of ascertaining } \\
\text { falls (recall bias) }\end{array}$ & Low risk & Weekly fall incidence questionnaire \\
\hline
\end{tabular}

Voukelatos 2007

\begin{tabular}{ll}
\hline Methods & Study design: RCT \\
& Number of study arms: 2 \\
& Length of follow-up: 6 months \\
\hline Participants & Setting: Sydney, Australia \\
& Number of participants: 702 \\
& Number analysed: 684 \\
& Number lost to follow-up: 18 \\
& Sample: community-dwelling \\
& Age (years): mean 69 (SD 6.5), range 69 - 70 \\
& Sex: $84 \%$ female \\
Inclusion criteria: aged $>60 ;$ community-dwelling & Exclusion criteria: degenerative neurological disease; severely debilitating stroke; metastatic cancer; \\
& severe arthritis; unable to walk across a room independently; unable to use English
\end{tabular}

\begin{tabular}{ll}
\hline Interventions & $\begin{array}{l}\text { 1. Group-based Tai Chi: style of Tai Chi differed between classes depending on Tai Chi instructor; 1-hour } \\
\text { class, } 1 \text { a week for } 16 \text { weeks. Cost AUD 44 } \\
\text { 2. Control: instructed not to take part in a Tai Chi programme and placed on 24-week waiting list, then } \\
\text { offered Tai Chi programme }\end{array}$ \\
\hline Outcomes & $\begin{array}{l}\text { 1. Rate of falls } \\
\text { 2. Number of people who experienced 1 or more falls (risk of falling) }\end{array}$ \\
\hline Duration of the study & $\begin{array}{l}\text { 24 weeks } \\
\text { Adherence }\end{array}$ \\
$\begin{array}{l}\text { 1. Group-based Tai Chi: dropout: } 6.76 \text { participants provided falls data but did not complete the 16- } \\
\text { week balance assessment }\end{array}$ \\
$\begin{array}{l}\text { 2. Control: dropout: } 12.81 \text { participants provided falls data but did not complete the 16-week balance } \\
\text { assessment }\end{array}$ \\
$\begin{array}{l}\text { Source of funding: New South Wales Health Department } \\
\text { Economic information: Mean cost per person (intervention): AUD 245 plus charged AUD 44 per partic- } \\
\text { ipant. Healthcare service costs: Tai Chi group AUD 55, control group AUD 17. Incremental cost per fall }\end{array}$
\end{tabular}


Voukelatos 2007 (Continued)

prevented/per QALY gained: AUD 1683 per fall prevented (includes cost offset by charging AUD 44 per

instruction course).Cost-effectiveness analysis reported in Haas 2006

\section{Risk of bias}

\begin{tabular}{|c|c|c|}
\hline Bias & Authors' judgement & Support for judgement \\
\hline $\begin{array}{l}\text { Random sequence genera- } \\
\text { tion (selection bias) }\end{array}$ & Low risk & $\begin{array}{l}\text { Quote: "Randomization list ... was prepared for each venue using randomly } \\
\text { permuted blocks of four or six" }\end{array}$ \\
\hline $\begin{array}{l}\text { Allocation concealment } \\
\text { (selection bias) }\end{array}$ & Unclear risk & Insufficient information to permit judgement \\
\hline $\begin{array}{l}\text { Blinding of participants } \\
\text { and personnel (perfor- } \\
\text { mance bias) } \\
\text { All outcomes }\end{array}$ & Unclear risk & $\begin{array}{l}\text { Participants and instructors conducting classes in intervention group were not } \\
\text { blinded. Control participants were asked not to take classes during the study } \\
\text { period, but may have accessed other fall-prevention interventions. Insufficient } \\
\text { evidence to make judgement on impact of lack of blinding. }\end{array}$ \\
\hline $\begin{array}{l}\text { Blinding of outcome as- } \\
\text { sessment (detection bias) } \\
\text { Falls }\end{array}$ & Low risk & $\begin{array}{l}\text { Falls were recorded using the same method in both groups. Outcome asses- } \\
\text { sors were blinded to group assignment }\end{array}$ \\
\hline $\begin{array}{l}\text { Blinding of outcome as- } \\
\text { sessment (detection bias) } \\
\text { Fractures }\end{array}$ & Unclear risk & Not applicable \\
\hline $\begin{array}{l}\text { Blinding of outcome as- } \\
\text { sessment (detection bias) } \\
\text { Hospital admission, med- } \\
\text { ical attention and adverse } \\
\text { events }\end{array}$ & Unclear risk & Not applicable \\
\hline $\begin{array}{l}\text { Blinding of outcome as- } \\
\text { sessment (detection bias) } \\
\text { Health related quality of } \\
\text { life (self report) }\end{array}$ & Unclear risk & Not applicable \\
\hline $\begin{array}{l}\text { Incomplete outcome data } \\
\text { (attrition bias) } \\
\text { Falls and fallers }\end{array}$ & Low risk & $\begin{array}{l}\text { Less than } 20 \% \text { of fall data were missing }(3 \%) \text {. Missing data were balanced } \\
\text { across groups, with } 6 / 347 \text { participants missing from the intervention group } \\
\text { and } 12 / 249 \text { missing from the control group. The reasons for missing data were } \\
\text { balanced between groups }\end{array}$ \\
\hline $\begin{array}{l}\text { Selective reporting (re- } \\
\text { porting bias) }\end{array}$ & Unclear risk & $\begin{array}{l}\text { Minimum set of expected outcomes not reported (adverse events not report- } \\
\text { ed) }\end{array}$ \\
\hline $\begin{array}{l}\text { Method of ascertaining } \\
\text { falls (recall bias) }\end{array}$ & Low risk & $\begin{array}{l}\text { Quote: "Participants were given falls calendars and were instructed to record } \\
\text { on the calendar each day for } 24 \text { weeks whether they had had a fall." Pre-paid } \\
\text { postage calendars returned at the end of each month, with telephone call if } \\
\text { not returned within } 2 \text { weeks. }\end{array}$ \\
\hline
\end{tabular}

Voukelatos 2015

\begin{tabular}{ll}
\hline Methods & Study design: RCT \\
& Number of study arms: 2
\end{tabular}


Voukelatos 2015 (Continued)

Length of follow-up: 12 months

\begin{tabular}{|c|c|}
\hline \multirow[t]{9}{*}{ Participants } & Setting: Sydney, Australia \\
\hline & Number of participants: 386 \\
\hline & Number analysed: 339 \\
\hline & Number lost to follow-up: 47 \\
\hline & Sample: community-dwelling \\
\hline & Age (years): mean 73.2 (range 65 - 90) \\
\hline & Sex: $74 \%$ female \\
\hline & $\begin{array}{l}\text { Inclusion criteria: } 65 \text { years and over community-dwelling inactive (i.e. }<120 \text { minutes of exercise a week) } \\
\text { mobile (i.e. able to walk at least } 50 \mathrm{~m} \text { with minimal aid); able to communicate in English }\end{array}$ \\
\hline & $\begin{array}{l}\text { Exclusion criteria: medical condition precluding participation in the study, participating in another } \\
\text { study }\end{array}$ \\
\hline \multirow[t]{2}{*}{ Interventions } & $\begin{array}{l}\text { 1. Individual walking programme: } 48 \text {-week self-paced walking programme by manual; focused on walk- } \\
\text { ing duration ( } 12 \text { weeks), walking intensity ( } 12 \text { weeks), maintaining the level of walking achieved in the } \\
\text { previous stages ( } 24 \text { weeks); } 6 \text { telephone calls to help modify and support adherence }\end{array}$ \\
\hline & $\begin{array}{l}\text { 2. Control group: Mailed information about health issues, } 6 \text { telephone calls to discuss health informa- } \\
\text { tion }\end{array}$ \\
\hline \multirow[t]{4}{*}{ Outcomes } & 1. Rate of falls \\
\hline & 2. Number of people who experienced 1 or more falls (risk of falling) \\
\hline & 3. Health-related quality of life \\
\hline & 4. Number of people who died \\
\hline Duration of the study & 48 weeks \\
\hline Adherence & Not reported \\
\hline \multirow[t]{2}{*}{ Notes } & Source of funding: NSW Ministry of Health \\
\hline & Economic information: not reported \\
\hline
\end{tabular}

\section{Risk of bias}

\begin{tabular}{lll}
\hline Bias & Authors' judgement & Support for judgement \\
\hline $\begin{array}{l}\text { Random sequence genera- } \\
\text { tion (selection bias) }\end{array}$ & Low risk & $\begin{array}{l}\text { Randomisation scheme used randomised permuted blocks of } 6 \text { and } 4 \text { pre- } \\
\text { pared by the chief investigator }\end{array}$ \\
\hline $\begin{array}{l}\text { Allocation concealment } \\
\text { (selection bias) }\end{array}$ & Low risk & Sequentially-numbered sealed opaque envelopes \\
\hline $\begin{array}{l}\text { Blinding of participants } \\
\text { and personnel (perfor- } \\
\text { mance bias) }\end{array}$ & Unclear risk & Participants and personnel unblinded but impact of unblinding unknown \\
All outcomes & & \\
\hline
\end{tabular}

Blinding of outcome as- Unclear risk Blinding not described. Insufficient information to permit judgement. sessment (detection bias) 
Voukelatos 2015 (Continued)

Falls

\begin{tabular}{|c|c|c|}
\hline $\begin{array}{l}\text { Blinding of outcome as- } \\
\text { sessment (detection bias) } \\
\text { Fractures }\end{array}$ & Unclear risk & Not applicable \\
\hline $\begin{array}{l}\text { Blinding of outcome as- } \\
\text { sessment (detection bias) } \\
\text { Hospital admission, med- } \\
\text { ical attention and adverse } \\
\text { events }\end{array}$ & Unclear risk & Not applicable \\
\hline $\begin{array}{l}\text { Blinding of outcome as- } \\
\text { sessment (detection bias) } \\
\text { Health related quality of } \\
\text { life (self report) }\end{array}$ & High risk & Participants not blinded to group allocation \\
\hline $\begin{array}{l}\text { Incomplete outcome data } \\
\text { (attrition bias) } \\
\text { Falls and fallers }\end{array}$ & Unclear risk & $\begin{array}{l}\text { Less than } 20 \% \text { of fall data were missing }(12 \%) \text {. Missing data were unbalanced } \\
\text { across groups, with } 33 / 192 \text { participants missing from the intervention group } \\
\text { and } 14 / 194 \text { missing from the control group. The reasons for missing fall data at } \\
24 \text { months were not clear }\end{array}$ \\
\hline $\begin{array}{l}\text { Selective reporting (re- } \\
\text { porting bias) }\end{array}$ & Unclear risk & $\begin{array}{l}\text { Minimum set of expected outcomes not reported (adverse events not report- } \\
\text { ed) }\end{array}$ \\
\hline $\begin{array}{l}\text { Method of ascertaining } \\
\text { falls (recall bias) }\end{array}$ & Low risk & $\begin{array}{l}\text { Monitored for } 48 \text { weeks through monthly calendars. When participants report- } \\
\text { ed a fall, they were contacted by telephone to confirm the fall and document } \\
\text { any fall-related injuries }\end{array}$ \\
\hline
\end{tabular}

Weerdesteyn 2006

\begin{tabular}{ll}
\hline Methods & Study design: RCT \\
& Number of study arms: 2 \\
& Length of follow-up: 7 months \\
\hline Participants & Setting: Nijmegan, The Netherlands \\
& Number of participants: 58 \\
& Number analysed: 58 \\
& Number lost to follow-up: 0 \\
& Sample: recruited using newspaper advertisements \\
& Age (years): mean 74 (SD 6) \\
& Sex: $77 \%$ female \\
Inclusion criteria: $\geq 65$ years; community-dwelling; $\geq 1$ fall in previous year; able to walk 15 minutes \\
without a walking aid \\
Exclusion criteria: severe cardiac, pulmonary, or musculoskeletal disorders; pathologies associated \\
with increased falls risk, e.g. Parkinson's disease; osteoporosis; using psychotropic drugs \\
3 arms described, but 1 not randomised. Only randomised groups were included in this review \\
1. Group-based balance and gait training using an obstacle avoidance course: daily tasks and walking \\
progressed with cognitive tasks and visual constraints, $1 \frac{1}{2}$ hours, 2 a week for 5 weeks \\
2. Control: no training
\end{tabular}


Weerdesteyn 2006 (Continued)
Outcomes
1. Rate of falls
2. Number of people who experienced 1 or more falls (risk of falling)

\begin{tabular}{ll}
\hline Duration of the study & 28 weeks \\
\hline Adherence & Adherence measured by session attendance \\
$\begin{array}{l}\text { 1. Group-based balance and gait training using an obstacle avoidance course: mean attendance rate to } \\
\text { the exercise sessions; } 87 \% \text { for both low-intensity exercise group and walking exercise group. } 51 \% \text { of ex- } \\
\text { ercise participants attended the maximum number of } 10 \text { sessions }\end{array}$
\end{tabular}

Notes $\quad \begin{aligned} & \text { Source of funding: Organization for Healthcare Research, Eurokinesis } \\ & \text { Economic information: not reported }\end{aligned}$

\section{Risk of bias}

\begin{tabular}{lll}
\hline Bias & Authors' judgement & Support for judgement \\
\hline $\begin{array}{l}\text { Random sequence genera- } \\
\text { tion (selection bias) }\end{array}$ & Unclear risk & $\begin{array}{l}\text { Quote: "Block randomization (3 blocks of 20) with gender stratification with } \\
\text { equal probability for either exercise or control group assignment" }\end{array}$ \\
\hline $\begin{array}{l}\text { Allocation concealment } \\
\text { (selection bias) }\end{array}$ & Unclear risk & $\begin{array}{l}\text { Quote: "The group allocation sequence was concealed (to both researchers } \\
\text { and participants) until assignment of interventions". "We had participants } \\
\text { draw a sealed envelope with group allocation ticket from a box containing all } \\
\text { remaining envelopes in the block" (personal communication reported in Gille- } \\
\text { spie 2012) }\end{array}$ \\
\hline
\end{tabular}

\begin{tabular}{lll}
\hline $\begin{array}{l}\text { Blinding of participants } \\
\text { and personnel (perfor- }\end{array}$ & Unclear risk & $\begin{array}{l}\text { Participants and personnel implementing the intervention not blinded to allo- } \\
\text { cated group, but impact of non-blinding unclear }\end{array}$
\end{tabular}

mance bias)

All outcomes

\begin{tabular}{lll}
\hline $\begin{array}{l}\text { Blinding of outcome as- } \\
\text { sessment (detection bias) } \\
\text { Falls }\end{array}$ & High risk & $\begin{array}{l}\text { Falls reported by participants who were aware of their group allocation. Out- } \\
\text { come assessors were not blinded to assignment (personal communication } \\
\text { from Dr Weeredesteyn, as reported in Gillespie 2012) }\end{array}$ \\
\hline $\begin{array}{l}\text { Blinding of outcome as- } \\
\begin{array}{l}\text { sessment (detection bias) } \\
\text { Fractures }\end{array}\end{array}$ & Unclear risk & Not applicable \\
\hline
\end{tabular}

\begin{tabular}{|c|c|}
\hline $\begin{array}{l}\text { Blinding of outcome as- } \\
\text { sessment (detection bias) } \\
\text { Hospital admission, med- } \\
\text { ical attention and adverse } \\
\text { events }\end{array}$ & Unclear risk \\
\hline
\end{tabular}

\begin{tabular}{lll}
\hline $\begin{array}{l}\text { Blinding of outcome as- } \\
\text { sessment (detection bias) } \\
\text { Health related quality of }\end{array}$ & Unclear risk & \\
life (self report) & \\
\hline $\begin{array}{l}\text { Incomplete outcome data } \\
\text { (attrition bias) }\end{array}$ & Low risk & No missing falls data \\
Falls and fallers & \\
\hline
\end{tabular}


Weerdesteyn 2006 (Continued)

\begin{tabular}{lll}
$\begin{array}{l}\text { Selective reporting (re- } \\
\text { porting bias) }\end{array}$ & Unclear risk & $\begin{array}{l}\text { Minimum set of expected outcomes not reported (adverse events not report- } \\
\text { ed) }\end{array}$ \\
\hline $\begin{array}{l}\text { Method of ascertaining } \\
\text { falls (recall bias) }\end{array}$ & Low risk & $\begin{array}{l}\text { Quote: "Falls were monitored monthly using pre-addressed, reply-paid fall reg- } \\
\text { istration cards." Asked whether a fall had occurred in the past month. Sent a } \\
\text { reminder if no registration card received }\end{array}$ \\
\hline
\end{tabular}

\section{Wolf 1996}

$\begin{array}{ll}\text { Methods } & \text { Study design: RCT } \\ & \text { Number of study arms: } 3 \\ & \text { Length of follow-up: } 8 \text { months }\end{array}$

Setting: Atlanta, USA
Pumber of participants: 200
Number analysed: 200
Number lost to follow-up: 0
Sample: residing in an independent living facility, recruited by advertising and direct contact
Age (years): mean 76.2 (SD 4.7)
Sex: $81 \%$ female
Inclusion criteria: aged > 70; ambulatory; living in unsupervised environment; agreeing to participate
weekly for 15 weeks with 4-month follow-up
Exclusion criteria: debilitating conditions, e.g. cognitive impairment, metastatic cancer, crippling
arthritis, Parkinson's disease, major stroke, profound visual defects

1. Group-based Tai Chi: progression to reduce base of support and towards single stance, 2 sessions a
week for 15 weeks, individual contact with instructor approximately 45 minutes a week
2. Individual, computerised balance training on force platform: increasing sway with no foot move-
ment using visual feedback from monitor with eyes open and closed, 1 a week for 15 weeks, individual
contact with instructor approximately 45 minutes a week
3. Control: group discussions of topics of interest to older people with gerontological nurse, 1 hour a
week for 15 weeks

\begin{tabular}{ll}
\hline Outcomes & 1. Rate of falls \\
\hline Duration of the study & 87 weeks \\
\hline
\end{tabular}

Adherence $\quad$ Adherence measured by attendance at sessions. Inability to make up 2 missed consecutive sessions defined as dropout

1. Group-based Tai Chi: 6/72 dropped out, $92 \%$ retention

2. Individual, computerised balance training on force platform: 4/64 dropped out, $94 \%$ retention

3. Control: $3 / 64$ dropout, $95 \%$ retention

Source of funding: NIH Cooperative Grant
Economic information: not reported
Atlanta FICSIT trial (Province 1995). 1997 paper included under this Study ID reports on a subgroup of
the trial, reporting on outcomes other than falls


Wolf 1996 (Continued)

Risk of bias

\begin{tabular}{|c|c|c|}
\hline Bias & Authors' judgement & Support for judgement \\
\hline $\begin{array}{l}\text { Random sequence genera- } \\
\text { tion (selection bias) }\end{array}$ & Low risk & Randomised using computer-generated fixed randomisation procedure \\
\hline $\begin{array}{l}\text { Allocation concealment } \\
\text { (selection bias) }\end{array}$ & Unclear risk & Insufficient information to permit judgement \\
\hline $\begin{array}{l}\text { Blinding of participants } \\
\text { and personnel (perfor- } \\
\text { mance bias) } \\
\text { All outcomes }\end{array}$ & Unclear risk & $\begin{array}{l}\text { Participants and personnel implementing the intervention not blind to allocat- } \\
\text { ed group, but impact of non-blinding unclear }\end{array}$ \\
\hline $\begin{array}{l}\text { Blinding of outcome as- } \\
\text { sessment (detection bias) } \\
\text { Falls }\end{array}$ & Unclear risk & $\begin{array}{l}\text { Falls measured using same method in each group. Does not state whether out- } \\
\text { come assessors were blind to allocation }\end{array}$ \\
\hline $\begin{array}{l}\text { Blinding of outcome as- } \\
\text { sessment (detection bias) } \\
\text { Fractures }\end{array}$ & Unclear risk & Not applicable \\
\hline $\begin{array}{l}\text { Blinding of outcome as- } \\
\text { sessment (detection bias) } \\
\text { Hospital admission, med- } \\
\text { ical attention and adverse } \\
\text { events }\end{array}$ & Unclear risk & Not applicable \\
\hline $\begin{array}{l}\text { Blinding of outcome as- } \\
\text { sessment (detection bias) } \\
\text { Health related quality of } \\
\text { life (self report) }\end{array}$ & Unclear risk & Not applicable \\
\hline $\begin{array}{l}\text { Incomplete outcome data } \\
\text { (attrition bias) } \\
\text { Falls and fallers }\end{array}$ & Low risk & No missing fall data \\
\hline $\begin{array}{l}\text { Selective reporting (re- } \\
\text { porting bias) }\end{array}$ & High risk & $\begin{array}{l}\text { Falls measured, but number of fallers not reported. Adverse events not report- } \\
\text { ed }\end{array}$ \\
\hline $\begin{array}{l}\text { Method of ascertaining } \\
\text { falls (recall bias) }\end{array}$ & Low risk & $\begin{array}{l}\text { Falls ascertained by monthly calendar, or by monthly phone call from project } \\
\text { staff }\end{array}$ \\
\hline
\end{tabular}

\section{Wolf 2003}

\begin{tabular}{ll}
\hline Methods & Study design: Cluster-RCT \\
& Number of study arms: 2 \\
& Number of clusters: 20 \\
& Length of follow-up: 11 months \\
\hline Participants & Setting: Atlanta, USA \\
Number of participants: 311 \\
\hline
\end{tabular}


Wolf 2003 (Continued)

Number analysed: 286

Number lost to follow-up: 25

Sample: congregate living facilities (independent living facilities) recruited in pairs by whether Housing and Urban Development $(\mathrm{N}=14)$ or private $(\mathrm{N}=6)$. At least 15 participants recruited per site Age (years): mean 80.9 (SD 6.2), range 70 to 97

Sex: $94 \%$ female

Inclusion criteria: aged $\geq 70 ; \geq 1$ fall in previous year; transitioning to frailty

Exclusion criteria: frail or vigorous elderly; major cardiopulmonary disease; cognitive impairment (MMSE < 24); contraindications for exercise, e.g. major orthopaedic conditions; mobility restricted to wheelchair; terminal cancer; evidence of other progressive or unstable neurological or medical conditions

1. Group-based Tai Chi: progressed from using upright support to 2 minutes of Tai Chi without support;
1-hour class progressing to 90 minutes, 2 a week for 48 weeks
2. Control group: wellness education programme (Instruction on fall prevention, exercise and bal-
ance, diet and nutrition, pharmacological management, legal issues, changes in body function, mental
health issues. Interactive material provided but no formal instruction in exercise); 1 hour a week for 48
weeks

1. Rate of falls
2. Number of people who experienced 1 or more falls (risk of falling)
3. Number of people who died

Duration of the study 48 weeks

\begin{tabular}{ll}
\hline Adherence & Adherence measured by group attendance \\
1. Group-based Tai Chi group: mean attendance rate; $76 \pm 19 \%$ (range $6-100 \%)$ \\
2. Control group: mean attendance rate; $81 \pm 17 \%($ range $10-100 \%)$ \\
\hline Sotes \\
Ecource of funding: NIH Grant \\
"Transitioning to frailty" if not vigorous or frail; based on age, gait/balance, walking activity for exer- \\
cise, other physical activity for exercise, depression, use of sedatives, vision, muscle strength, lower ex- \\
tremity disability (Speechley 1991) \\
Number of clusters allocated to intervention: $10 ;$ number of clusters allocated to control: $10 ;$ number of \\
clusters analysed (intervention): $10 ;$ number of clusters analysed (control): 10
\end{tabular}

\section{Risk of bias}

\begin{tabular}{lll}
\hline Bias & Authors' judgement & Support for judgement \\
\hline $\begin{array}{l}\text { Random sequence genera- } \\
\text { tion (selection bias) }\end{array}$ & Unclear risk & $\begin{array}{l}\text { Facilities stratified by socioeconomic status and randomised in pairs } \\
\text { Quote: "First site in the pair was randomized to an intervention. The second } \\
\text { site received the other intervention" }\end{array}$ \\
\hline $\begin{array}{l}\text { Allocation concealment } \\
\text { (selection bias) }\end{array}$ & Unclear risk & $\begin{array}{l}\text { Cluster-RCT. Insufficient information to permit judgement, although allocation } \\
\text { of second site in the pair could be predicted after the first site was randomised }\end{array}$ \\
\hline $\begin{array}{l}\text { Blinding of participants } \\
\text { and personnel (perfor- } \\
\text { mance bias) }\end{array}$ & Unclear risk & $\begin{array}{l}\text { Participants and personnel implementing the intervention not blinded to allo- } \\
\text { cated group, but impact of non-blinding unclear }\end{array}$
\end{tabular}


Wolf 2003 (Continued)

All outcomes

\begin{tabular}{|c|c|c|}
\hline $\begin{array}{l}\text { Blinding of outcome as- } \\
\text { sessment (detection bias) }\end{array}$ & Low risk & $\begin{array}{l}\text { Falls reported using the same method in each group. Outcome assessors } \\
\text { blinded to assignment }\end{array}$ \\
\hline
\end{tabular}

Falls

$\begin{array}{ll}\text { Blinding of outcome as- } & \text { Unclear risk }\end{array}$

\section{Fractures}

Blinding of outcome as-
sessment (detection bias)

Unclear risk

The method of ascertaining adverse events was unclear

Hospital admission, med-

ical attention and adverse

events

\begin{tabular}{|c|c|c|}
\hline $\begin{array}{l}\text { Blinding of outcome as- } \\
\text { sessment (detection bias) } \\
\text { Health related quality of } \\
\text { life (self report) }\end{array}$ & Unclear risk & Not applicable \\
\hline $\begin{array}{l}\text { Incomplete outcome data } \\
\text { (attrition bias) } \\
\text { Falls and fallers }\end{array}$ & Low risk & $\begin{array}{l}\text { Less than } 20 \% \text { of fall data were missing }(8 \%) \text {. Missing data were balanced } \\
\text { across groups ( } 13 / 158 \text { missing from the intervention group and } 12 / 153 \text { missing } \\
\text { from the control group) and the reasons for missing data were balanced across } \\
\text { groups }\end{array}$ \\
\hline $\begin{array}{l}\text { Selective reporting (re- } \\
\text { porting bias) }\end{array}$ & Unclear risk & $\begin{array}{l}\text { Minimum set of expected outcomes reported. No published study protocol or } \\
\text { prospective trial registration }\end{array}$ \\
\hline $\begin{array}{l}\text { Method of ascertaining } \\
\text { falls (recall bias) }\end{array}$ & Low risk & $\begin{array}{l}\text { Prospective. Falls recorded on forms and submitted to instructor weekly + } \\
\text { phone call }\end{array}$ \\
\hline Cluster-randomised trials & Unclear risk & $\begin{array}{l}\text { The relative timing of the randomisation of clusters and recruitment of partici- } \\
\text { pants is unclear; baseline characteristics of clusters not reported; missing out- } \\
\text { comes for clusters or within clusters were not reported; accounted for the clus- } \\
\text { tered design in the analysis; results comparable with individually-randomised } \\
\text { trials }\end{array}$ \\
\hline
\end{tabular}

Woo 2007

\begin{tabular}{ll}
\hline Methods & Study design: RCT \\
& Number of study arms: 3 \\
& Length of follow-up: 12 months \\
\hline Participants & Setting: Hong Kong, China \\
& Number of participants: 180 \\
& Number analysed: 176 \\
& Number lost to follow-up: 4 \\
& Sample: recruited by notices posted in 4 community centres in Shatin township \\
& Age (years): mean 69 (SD 2.6), range 65 - 74 \\
& Sex: $50 \%$ female \\
Inclusion criteria: able to walk $>8$ m without assistance
\end{tabular}


Woo 2007 (Continued)

Exclusion criteria: neurological disease which impaired mobility; shortness of breath or angina on walking up 1 flight of stairs; dementia; already performing Tai Chi or resistance training exercise

\begin{tabular}{ll}
\hline Interventions & 1. Group-based Tai Chi: Yang style Tai Chi, 3 a week for 52 weeks \\
2. Group-based resistance training: used a medium-strength Theraband for arm and leg exercises, 3 a \\
week for 52 weeks \\
3. Control: no exercise prescribed
\end{tabular}

\begin{tabular}{ll}
\hline Outcomes & 1. Number of people who experienced 1 or more falls (risk of falling) \\
\hline Duration of the study & 52 weeks \\
\hline Adherence & Adherence measured by attendance rate \\
& $\begin{array}{l}\text { 1. Group-based Tai Chi group: mean attendance rate } 81 \% \text { with no attrition between } 6 \text { and } 12 \text { months } \\
\text { 2. Group-based resistance group: mean attendance rate } 76 \% \text { with no attrition between } 6 \text { and } 12 \\
\text { months }\end{array}$
\end{tabular}

$\begin{array}{ll}\text { Notes } & \text { Source of funding: Council of Hong Kong } \\ \text { Economic information: not reported }\end{array}$

\section{Risk of bias}

\begin{tabular}{lll}
\hline Bias & Authors' judgement & Support for judgement \\
\hline $\begin{array}{l}\text { Random sequence genera- } \\
\text { tion (selection bias) }\end{array}$ & Low risk & Quote: "Computer generated blocked randomisation" \\
\hline $\begin{array}{l}\text { Allocation concealment } \\
\text { (selection bias) }\end{array}$ & Unclear risk & Insufficient information to permit judgement \\
\hline $\begin{array}{l}\text { Blinding of participants } \\
\text { and personnel (perfor- } \\
\text { mance bias) } \\
\text { All outcomes }\end{array}$ & Unclear risk & $\begin{array}{l}\text { Participants and personnel implementing the intervention not blind to allocat- } \\
\text { ed group, but impact of non-blinding unclear }\end{array}$ \\
\hline
\end{tabular}

\begin{tabular}{|c|c|c|}
\hline $\begin{array}{l}\text { Blinding of outcome as- } \\
\text { sessment (detection bias) } \\
\text { Falls }\end{array}$ & High risk & $\begin{array}{l}\text { Assessors not blinded to group allocation } \\
\text { Quote: "Falls were ascertained by diary and reported to the staff running the } \\
\text { interventions" (personal communication reported in Gillespie 2012). }\end{array}$ \\
\hline $\begin{array}{l}\text { Blinding of outcome as- } \\
\text { sessment (detection bias) } \\
\text { Fractures }\end{array}$ & Unclear risk & Not applicable \\
\hline
\end{tabular}

Blinding of outcome as-
sessment (detection bias)
Hospital admission, med-
ical attention and adverse
events

Unclear risk Not applicable

Blinding of outcome as- Unclear risk Not applicable
sessment (detection bias) Health related quality of life (self report) 
Woo 2007 (Continued)

Falls and fallers

$\begin{array}{lll}\begin{array}{l}\text { Selective reporting (re- } \\ \text { porting bias) }\end{array} & \text { High risk } & \begin{array}{l}\text { Falls were measured, but number of falls not reported. Adverse events not re- } \\ \text { ported }\end{array}\end{array}$

\begin{tabular}{|c|c|c|}
\hline $\begin{array}{l}\text { Method of ascertaining } \\
\text { falls (recall bias) }\end{array}$ & High risk & $\begin{array}{l}\text { Quote: "Falls were ascertained by diary and reported to the staff running the } \\
\text { interventions." (personal communication) but this could not apply to the con- } \\
\text { trol group (personal communication reported in Gillespie 2012) }\end{array}$ \\
\hline
\end{tabular}

Wu 2010

\begin{tabular}{ll}
\hline Methods & Study design: RCT \\
Number of study arms: 3 \\
Length of follow-up: 4 months \\
Setting: Burlington, Vermont, USA \\
Number of participants: 64 \\
Number analysed: 64 \\
Number lost to follow-up: 0 \\
Sample: volunteers recruited by advertising, referrals, flyers etc. \\
Age (years): mean 75.4 (SD 7) \\
Sex: $84 \%$ female \\
Inclusion criteria: age $\geq 65$; community-dwelling; at risk of falling ( $\geq 1$ fall in past year or $\leq 50 \%$ on ABC \\
Scale); able to walk and do weight-bearing exercises with or without assistive devices; no plans to be \\
away $>2$ weeks during study period; sufficient cognition and attention to follow directions; have a tele- \\
vision (TV) and Internet access; sufficient visual acuity to mimic instructor's movements on TV screen; \\
consenting; with primary care physician approval to participate \\
Exclusion criteria: unable to walk/exercise independently; unable to travel to community centre; hav- \\
ing certain exercise-limiting conditions including musculoskeletal, cardiac, neurological, pulmonary \\
etc
\end{tabular}

\section{Interventions Delivered by 3 methods with same content and same instructor:}

1. Individual, supervised Tai Chi delivered by videoconferencing: "Tel-ex" yang style Tai Chi home-based interactive by TV screen, live and supervised in real-time, 1 hour a day, 3 days a week for 15 weeks

2. Group-based Tai Chi: "Comm-ex" yang style Tai Chi class held in community facility, live and supervised in real-time, 1 hour a day, 3 days a week for 15 weeks

3. Individual Tai Chi with DVD instruction: "Home-ex" yang style Tai Chi exercise from home but not connected to instructor during the 15 weeks, received written instructions for DVD programme, DVD with $45 \times 1$-hour sessions, identical exercises to live class instruction groups; 1 hour a day, 3 days a week for 15 weeks

\begin{tabular}{ll}
\hline Outcomes & 1. Rate of falls \\
\hline Duration of the study & 15 weeks \\
\hline Adherence & Adherence measured by total exercise time \\
& $\begin{array}{l}1 . \text { Individual, supervised Tai Chi delivered by videoconferencing: total exercise time } 30 \pm 12 \text { hours (69 } \pm \\
\text { 2. Group-based Tai Chi: total exercise time } 31 \pm 12 \text { hours }(71 \pm 27 \%)\end{array}$ \\
\hline
\end{tabular}


Wu 2010 (Continued)

3. Individual Tai Chi with DVD instruction: total exercise time $17 \pm 21$ hours (38 $\pm 46 \%)$

Notes $\quad$ Source of funding: not reported
Economic information: not reported

\section{Risk of bias}

\begin{tabular}{lll}
\hline Bias & Authors' judgement & Support for judgement \\
\hline $\begin{array}{ll}\text { Random sequence genera- } \\
\text { tion (selection bias) }\end{array}$ & Unclear risk & $\begin{array}{l}\text { Quote: "Those who consented were enrolled in the study and were randomly } \\
\text { assigned into the Tele-ex, Commex, and Home-ex groups. To ensure balance } \\
\text { among the } 3 \text { groups on important potential confounders, randomization was } \\
\text { stratified by sex, age (65-74y vs 75y), and time expected to be away during the } \\
\text { study period (1 wk vs 1-2 wh). Blocked randomization was used within strata." }\end{array}$ \\
\end{tabular}

Allocation concealment Unclear risk Insufficient information to permit judgement

(selection bias)

\begin{tabular}{|c|c|c|}
\hline $\begin{array}{l}\text { Blinding of participants } \\
\text { and personnel (perfor- }\end{array}$ & Unclear risk & $\begin{array}{l}\text { All } 3 \text { groups received a fall-prevention intervention (Tai Chi). Unclear whether } \\
\text { there is potential for performance bias }\end{array}$ \\
\hline
\end{tabular}

mance bias)

All outcomes

\begin{tabular}{|c|c|c|}
\hline $\begin{array}{l}\text { Blinding of outcome as- } \\
\text { sessment (detection bias) }\end{array}$ & Unclear risk & $\begin{array}{l}\text { Falls were measured using the same method in each group. Unclear whether } \\
\text { assessor was blinded }\end{array}$ \\
\hline
\end{tabular}

Falls

\begin{tabular}{lll}
\hline $\begin{array}{l}\text { Blinding of outcome as- } \\
\text { sessment (detection bias) } \\
\text { Fractures }\end{array}$ & Unclear risk & Not applicable \\
\hline $\begin{array}{l}\text { Blinding of outcome as- } \\
\text { sessment (detection bias) }\end{array}$ & Unclear risk & Not applicable \\
$\begin{array}{l}\text { Hospital admission, med- } \\
\text { ical attention and adverse } \\
\text { events }\end{array}$ & \\
\end{tabular}

\begin{tabular}{lll}
\hline $\begin{array}{l}\text { Blinding of outcome as- } \\
\text { sessment (detection bias) } \\
\text { Health related quality of } \\
\text { life (self report) }\end{array}$ & High risk & Participants not blinded to group allocation \\
\hline $\begin{array}{l}\text { Incomplete outcome data } \\
\text { (attrition bias) }\end{array}$ & Low risk & No missing fall data \\
$\begin{array}{l}\text { Falls and fallers } \\
\begin{array}{l}\text { Selective reporting (re- } \\
\text { porting bias) }\end{array}\end{array}$ & High risk & $\begin{array}{l}\text { Falls were measured, but number of fallers not reported. Adverse events not } \\
\text { reported }\end{array}$ \\
\hline $\begin{array}{l}\text { Method of ascertaining } \\
\text { falls (recall bias) }\end{array}$ & High risk & $\begin{array}{l}\text { Quote: "Fall incidents were assessed by a Fall History Form that recorded the } \\
\text { number of falls in the ... past 15 weeks" }\end{array}$ \\
\hline
\end{tabular}

\section{Yamada 2010}

Methods Study design: RCT


Yamada 2010 (Continued)

Number of study arms: 2

Length of follow-up: 12 months

Setting: Kyoto, Japan
Number of participants: 60
Number analysed: 58
Number lost to follow-up: 2
Sample: people recruited using advertising in local press (proportion of women not stated)
Age (years): not stated
Inclusion criteria: aged $\geq 65$; community-dwelling; visited primary care physician in previous 3 years;
MMSE $\geq 24 ;$ able to walk independently (with or without a cane): willing to participate in group exercise
classes lasting $\geq 6$ months; access to transportation; minimal hearing and visual impairments; no regu-
lar exercise in previous 12 months
Exclusion criteria: severe cardiac pulmonary, or musculoskeletal disorders; neurological conditions as-
sociated with falling (stroke, Parkinson's disease); osteoporosis; use of psychotropic drugs

Interventions

1. Group-based trail walking training: 90-minute class (moderate intensity aerobic exercise, progressive strengthening with rubber band, flexibility and balance exercises) including trail walking between flags as quickly as possible, 1 a week for 16 weeks

2. Group-based indoor walking: 90-minute class (moderate-intensity aerobic exercise, progressive strengthening with rubber band, flexibility and balance exercises) including supervised indoor walking session at a comfortable pace (up to 30 minute on 300-foot loop); 1 a week for 16 weeks

\begin{tabular}{ll}
\hline Outcomes & 1. Rate of falls \\
2. Number of people who experienced 1 or more falls (risk of falling)
\end{tabular}

\begin{tabular}{ll}
\hline Duration of the study & 52 weeks \\
\hline Adherence & Adherence measured by completion of 16 scheduled sessions \\
& $\begin{array}{l}\text { 1. Group-based trail walking training: median relative adherence; } 100 \% \text { (25th - } 75 \text { th percentile, } 94- \\
\text { 2. Group-based indoor walking: median relative adherence; } 100 \% \text { (25th }-75 \text { th percentile, } 94-100 \%)\end{array}$
\end{tabular}

Notes Source of funding: not reported

Economic information: not reported

\section{Risk of bias}

\begin{tabular}{lll}
\hline Bias & Authors' judgement & Support for judgement \\
\hline $\begin{array}{l}\text { Random sequence genera- } \\
\text { tion (selection bias) }\end{array}$ & Unclear risk & Quote: "Participants were block randomized in blocks of four" \\
\hline $\begin{array}{l}\text { Allocation concealment } \\
\text { (selection bias) }\end{array}$ & Low risk & $\begin{array}{l}\text { Quote: "Using this sequence, opaque envelopes bearing group names were } \\
\text { numbered and the } 60 \text { participants were then randomly as signed to the TWE ( } \\
=30) \text { or walking }(W) \text { group }(n=30) " ~\end{array}$ \\
\hline $\begin{array}{l}\text { Blinding of participants } \\
\begin{array}{l}\text { and personnel (perfor- } \\
\text { mance bias) }\end{array}\end{array}$ & Unclear risk & $\begin{array}{l}\text { Both groups received an exercise intervention. Unclear whether there was any } \\
\text { risk of performance bias }\end{array}$ \\
\hline
\end{tabular}


Yamada 2010 (Continued)
Blinding of outcome as-
Unclear risk
Unclear whether person ascertaining falls was blinded to allocated group sessment (detection bias)

Falls

\begin{tabular}{|c|c|c|}
\hline $\begin{array}{l}\text { Blinding of outcome as- } \\
\text { sessment (detection bias) } \\
\text { Fractures }\end{array}$ & Unclear risk & Not applicable \\
\hline
\end{tabular}

\begin{tabular}{ll}
\hline Blinding of outcome as- & Unclear risk \\
sessment (detection bias) & \\
Hospital admission, med- & \\
ical attention and adverse & \\
events & \\
\hline
\end{tabular}

\begin{tabular}{|c|c|c|}
\hline $\begin{array}{l}\text { Blinding of outcome as- } \\
\text { sessment (detection bias) } \\
\text { Health related quality of } \\
\text { life (self report) }\end{array}$ & Unclear risk & Not applicable \\
\hline
\end{tabular}

\begin{tabular}{lll}
\hline $\begin{array}{l}\text { Incomplete outcome data } \\
\text { (attrition bias) }\end{array}$ & Unclear risk & $\begin{array}{l}\text { Less than 20\% of fall data were missing (3\%). The missing data were balanced } \\
\text { between groups, with } 1 \text { withdrawal from each group }\end{array}$ \\
\hline $\begin{array}{l}\text { Selective reporting (re- } \\
\text { porting bias) }\end{array}$ & Unclear risk & $\begin{array}{l}\text { Minimum set of expected outcomes reported. No published study protocol or } \\
\text { prospective trial registration }\end{array}$ \\
\hline $\begin{array}{l}\text { Method of ascertaining } \\
\text { falls (recall bias) }\end{array}$ & Low risk & $\begin{array}{l}\text { Quote: "The participants were asked to record any falls in fall diaries that were } \\
\text { mailed to the research assistants every month." }\end{array}$ \\
\hline
\end{tabular}

\section{Yamada 2012}

\begin{tabular}{ll}
\hline Methods & Study design: RCT \\
& Number of study arms: 2 \\
& Length of follow-up: 12 months \\
\hline
\end{tabular}

Participants
Number of participants: 157
Number analysed: 145
Number lost to follow-up: 12
Sample: community-dwelling
Age (years): mean 86
Sex: $81 \%$ female
Inclusion criteria: $\geq 75$ years old, community-dwelling, had visited a primary care physician within the
past 3 years, no severe cognitive impairment, walk independently (or with a cane), willingness to par-
ticipate in group exercise classes for at least 6 months, had access to transportation, no significant
hearing and vision impairments, no regular exercise in the past 12 months
Exclusion criteria: severe cardiac, pulmonary or musculoskeletal disorders, co-morbidities associated
with greater risk of falls, such as Parkinson disease and stroke, and use of psychotropic drugs


Yamada 2012 (Continued)

Interventions
1. Group-based balance, strength, flexibility and gait training involving complex obstacle course: 45 minute exercise session ('moderate-intensity' aerobic-dance exercise, progressive strength training using elastic band, progressive balance exercises); plus walking as quickly as possible in a progressively difficult field of obstacles 2 times a session. 1 session a week for 24 weeks

2. Group-based balance, strength, flexibility and gait training involving simple obstacle course: 45minute exercise session ('moderate-intensity' aerobic-dance exercise, progressive strength training using elastic band, progressive balance exercises); plus walking at a self-selected speed along a simple level walkway of $15 \mathrm{~m}$ with obstacles 6 times a session. 1 session a week for 24 weeks

1. Rate of falls
$\begin{aligned} & \text { 2. Number of people who experienced } 1 \text { or more falls (risk of falling) } \\ & \text { 3. Number of people who experienced } 1 \text { or more fall-related fractures }\end{aligned}$

\begin{tabular}{ll}
\hline Duration of the study & 52 weeks \\
\hline Adherence & Adherence measured by completion of programme \\
& $\begin{array}{l}\text { 1. Group-based balance, strength, flexibility and gait training involving complex obstacle course group: } \\
\text { median relative adherence; } 96 \% \text { (25th - 75th percentile, } 88-100 \%)\end{array}$ \\
& $\begin{array}{l}\text { 2. Group-based balance, strength, flexibility and gait training involving simple obstacle course group: } \\
\text { median relative adherence; } 96 \% \text { (25th - 75th percentile, } 88-100 \%)\end{array}$
\end{tabular}

Notes $\quad$ Source of funding: not reported
Economic information: not reported

\title{
Risk of bias
}

\begin{tabular}{lll}
\hline Bias & Authors' judgement & Support for judgement \\
\hline $\begin{array}{l}\text { Random sequence genera- } \\
\text { tion (selection bias) }\end{array}$ & Unclear risk & Methods not described \\
\hline $\begin{array}{l}\text { Allocation concealment } \\
\text { (selection bias) }\end{array}$ & Low risk & Quote: "Opaque envelopes bearing group names were numbered" \\
\hline $\begin{array}{l}\text { Blinding of participants } \\
\text { and personnel (perfor- } \\
\text { mance bias) }\end{array}$ & High risk & $\begin{array}{l}\text { Participants and personnel implementing the intervention not blinded to allo- } \\
\text { All outcomes }\end{array}$ \\
\hline
\end{tabular}

\begin{tabular}{|c|c|c|}
\hline $\begin{array}{l}\text { Blinding of outcome as- } \\
\text { sessment (detection bias) }\end{array}$ & Unclear risk & $\begin{array}{l}\text { Not specifically reported if the research assistants collecting fall outcomes } \\
\text { were blinded }\end{array}$ \\
\hline Falls & & $\begin{array}{l}\text { Quote: "research assistants collected fall outcomes... a physiotherapist blind- } \\
\text { ed to group allocation collected secondary outcome measures" }\end{array}$ \\
\hline
\end{tabular}

\begin{tabular}{|c|c|c|}
\hline $\begin{array}{l}\text { Blinding of outcome as- } \\
\text { sessment (detection bias) }\end{array}$ & Unclear risk & $\begin{array}{l}\text { Quote: "The diagnosis of fractures was based on radiological evidence of frac- } \\
\text { ture". Unclear if assessors were blinded to group allocation }\end{array}$ \\
\hline
\end{tabular}

Fractures

\author{
Blinding of outcome as- \\ sessment (detection bias) \\ Hospital admission, med- \\ ical attention and adverse \\ events
}

Unclear risk

Method of measuring adverse events was unclear 
Yamada 2012 (Continued)
Blinding of outcome as-
Unclear risk
Not applicable sessment (detection bias)
Health related quality of life (self report)

\begin{tabular}{lll}
\hline $\begin{array}{l}\text { Incomplete outcome data } \\
\text { (attrition bias) } \\
\text { Falls and fallers }\end{array}$ & Low risk & $\begin{array}{l}\text { Less than 20\% of fall data were missing (8\%). The missing data were balanced } \\
\text { between groups, with } 6 \text { withdrawals from each group. The reasons for with- } \\
\text { drawals were unclear }\end{array}$ \\
\hline $\begin{array}{l}\text { Selective reporting (re- } \\
\text { porting bias) }\end{array}$ & Unclear risk & $\begin{array}{l}\text { Minimum set of expected outcomes reported. No published study protocol or } \\
\text { prospective trial registration }\end{array}$ \\
\hline $\begin{array}{l}\text { Method of ascertaining } \\
\text { falls (recall bias) }\end{array}$ & Low risk & $\begin{array}{l}\text { Quote: "The participants were asked to record any falls in fall diaries mailed } \\
\text { every month by research assistants. If participants failed to send the fall di- } \\
\text { aries, research assistants collected data on falls over the telephone" }\end{array}$ \\
\hline
\end{tabular}

\section{Yamada 2013}

\begin{tabular}{ll}
\hline Methods & Study design: RCT \\
& Number of study arms: 2 \\
& Length of follow-up: 12 months \\
\hline
\end{tabular}

Participants
Number of participants: 264
Number analysed: 230
Number lost to follow-up: 34
Sample: community-dwelling
Age (years): Training group mean 76.2 (SD 8.5); Control group mean 77.2 (SD 7.6)
Sex: $57 \%$ female
Inclusion criteria: 65 years old, community-dwelling, frail, certified for long-term care insurance service
requirement, no severe cognitive impairment, ability to walk independently (or with cane), willing to
participate in group exercise classes for at least 6 months, access to transportation, no significant hear-
ing or vision impairment, and had not exercised regularly in the previous 12 months
Exclusion criteria: serious visual impairment (cataract, glaucoma, or colour blindness), severe cardiac,
pulmonary, or musculoskeletal disorders, comorbidities associated with greater risk of falls, such as
Parkinson's disease and stroke, and use of psychotropic drugs

Interventions

1. Group-based balance, strength, flexibility and gait training including stepping mat: 30-minute exercise sessions (moderate aerobic-dance warm-up, mild progressive resistance with elastic band, progressive balance exercises); plus walking on multitarget stepping mat test repeated 4 times, 2 times a week for 24 weeks

2. Group-based balance, strength, flexibility and gait training plus indoor walking: 30 -minute exercise sessions (moderate aerobic-dance warm-up, mild progressive resistance with elastic band, progressive balance exercises); plus indoor $50 \mathrm{~m}$ walking programme, 2 times a week for 24 weeks

1. Rate of falls
2. Number of people who experienced 1 or more falls (risk of falling)


Yamada 2013 (Continued)

3. Number of people who experienced 1 or more fall-related fractures

\begin{tabular}{ll}
\hline Duration of the study & 52 weeks \\
\hline Adherence & $\begin{array}{l}\text { 1. Group-based balance, strength, flexibility and gait training including stepping mat group: median rel- } \\
\text { ative adherence; } 93 \% \text { (IQR } 83-96 \%)\end{array}$ \\
$\begin{array}{l}\text { 2. Group-based balance, strength, flexibility and gait training plus indoor walking group: median rela- } \\
\text { tive adherence, } 92 \% \text { (IQR } 83-96 \%)\end{array}$
\end{tabular}

Notes Source of funding: Health Labor Sciences, Ministry of Health, Labor and Welfare

Economic information: not reported

\section{Risk of bias}

\begin{tabular}{|c|c|c|}
\hline Bias & Authors' judgement & Support for judgement \\
\hline $\begin{array}{l}\text { Random sequence genera- } \\
\text { tion (selection bias) }\end{array}$ & Unclear risk & Methods not described \\
\hline $\begin{array}{l}\text { Allocation concealment } \\
\text { (selection bias) }\end{array}$ & Unclear risk & Method not reported \\
\hline $\begin{array}{l}\text { Blinding of participants } \\
\text { and personnel (perfor- } \\
\text { mance bias) } \\
\text { All outcomes }\end{array}$ & Unclear risk & Participants and personnel not blinded. Effect of non-blinding unknown \\
\hline $\begin{array}{l}\text { Blinding of outcome as- } \\
\text { sessment (detection bias) } \\
\text { Falls }\end{array}$ & Unclear risk & $\begin{array}{l}\text { Not specifically reported if the research assistants collecting fall outcomes } \\
\text { were blinded } \\
\text { Quote: "research assistants collected fall outcomes... a physiotherapist blind- } \\
\text { ed to group allocation collected secondary outcome measures" }\end{array}$ \\
\hline $\begin{array}{l}\text { Blinding of outcome as- } \\
\text { sessment (detection bias) } \\
\text { Fractures }\end{array}$ & Unclear risk & $\begin{array}{l}\text { Quote: "All participants who had fallen were contacted by telephone and inter- } \\
\text { viewed using a structured questionnaire about the fall and its consequences. } \\
\text { Fractures were diagnosed based on radiological evidence of fracture". Unclear } \\
\text { if assessors were blinded to group allocation }\end{array}$ \\
\hline
\end{tabular}

\begin{tabular}{|c|c|c|}
\hline $\begin{array}{l}\text { Blinding of outcome as- } \\
\text { sessment (detection bias) } \\
\text { Hospital admission, med- } \\
\text { ical attention and adverse } \\
\text { events }\end{array}$ & Unclear risk & Method of measuring adverse events was unclear \\
\hline $\begin{array}{l}\text { Blinding of outcome as- } \\
\text { sessment (detection bias) } \\
\text { Health related quality of } \\
\text { life (self report) }\end{array}$ & Unclear risk & Not applicable \\
\hline $\begin{array}{l}\text { Incomplete outcome data } \\
\text { (attrition bias) } \\
\text { Falls and fallers }\end{array}$ & Unclear risk & $\begin{array}{l}\text { Less than } 20 \% \text { of fall data were missing }(13 \%) \text {. The missing data were unbal- } \\
\text { anced between groups, with } 20 \text { withdrawals from the intervention group and } \\
14 \text { from the control group. The reasons for withdrawals were unclear }\end{array}$ \\
\hline $\begin{array}{l}\text { Selective reporting (re- } \\
\text { porting bias) }\end{array}$ & Low risk & $\begin{array}{l}\text { Minimum set of expected outcomes reported. No published study protocol or } \\
\text { prospective trial registration }\end{array}$ \\
\hline
\end{tabular}


Yamada 2013 (Continued)

Method of ascertaining Low risk falls (recall bias)
Quote: "The participants were asked to record any falls in fall diaries mailed every month by research assistants. If participants failed to send the fall diaries, research assistants collected data on falls over the telephone"

Yang 2012

$\begin{array}{ll}\text { Methods } & \text { Study design: RCT } \\ \text { Number of study arms: } 2 & \\ \text { Length of follow-up: } 6 \text { months } & \text { Setting: Melbourne, Australia } \\ & \text { Number of participants: } 165 \\ \text { Participants } & \text { Number analysed: } 121 \\ & \text { Number lost to follow-up: } 44 \\ & \text { Sample: community-dwelling } \\ & \text { Age (years): Intervention mean } 81 \text { (5.9); Control mean } 80.1 \text { (6.4) } \\ & \text { Sex: } 44 \% \text { female } \\ & \text { Inclusion criteria: aged } 65 \text { years or over, living in the community, being community ambulant, requir- } \\ & \text { ing no walking aid or using a single-point stick only, experiencing no more than } 1 \text { fall in the previous } 12 \\ & \text { months, having concerns about balance, and had mild balance dysfunction (i.e. Functional Reach Test } \\ & \text { score }<26 \text { cm, Step Test score }<13 \text { steps/15 seconds, Five-Time Sit-to-Stand Test time }>17.9 \text { seconds, } \\ & \text { had }>3 \text { abnormal scores on the NeuroCom Balance Master) } \\ & \text { Exclusion criteria: balance performance within normal limits }\end{array}$

Interventions

1. Individual Otago Exercise Programme: Tailored home programme with no upper-limb support. Ankle weights and exercise manual provided. 20-minute sessions, 5 times a week, for 24 weeks, plus $\geq 30$ minutes daily walking

2. Control group: provided with a fall-prevention information booklet and continued with usual activities

1. Number of people who experienced 1 or more falls (risk of falling)
2. Health-related quality of life
3. Number of people who died

Duration of the study 24 weeks

\begin{tabular}{ll}
\hline Adherence & Adherence measured by sessions performed \\
& $\begin{array}{l}\text { 1. Individual Otago Exercise Programme: } 26(44 \%) \text { full adherence, } 8 \text { participants }(14 \%) \text { reported exer- } \\
\text { cising less than twice a week on average }\end{array}$
\end{tabular}

Notes $\quad$ Source of funding: Australian Government Department of Veterans' Affair
Economic information: not reported

Economic information: not reported

\section{Risk of bias}

\section{Bias}

Authors' judgement Support for judgement 
Yang 2012 (Continued)

\begin{tabular}{lll}
$\begin{array}{l}\text { Random sequence genera- } \\
\text { tion (selection bias) }\end{array}$ & Low risk & Computer-generated random numbers \\
\hline $\begin{array}{l}\text { Allocation concealment } \\
\text { (selection bias) }\end{array}$ & Unclear risk & $\begin{array}{l}\text { Method of concealment is not described in sufficient detail to allow a definite } \\
\text { judgement }\end{array}$
\end{tabular}

Blinding of participants Unclear risk Participants and personnel unblinded but impact of unblinding unknown
and personnel (perfor-
mance bias)
All outcomes

\begin{tabular}{|c|c|c|}
\hline $\begin{array}{l}\text { Blinding of outcome as- } \\
\text { sessment (detection bias) } \\
\text { Falls }\end{array}$ & Low risk & Quote: "Assessors were blinded to group assignment" \\
\hline $\begin{array}{l}\text { Blinding of outcome as- } \\
\text { sessment (detection bias) } \\
\text { Fractures }\end{array}$ & Unclear risk & Not applicable \\
\hline $\begin{array}{l}\text { Blinding of outcome as- } \\
\text { sessment (detection bias) } \\
\text { Hospital admission, med- } \\
\text { ical attention and adverse } \\
\text { events }\end{array}$ & Unclear risk & Not applicable \\
\hline
\end{tabular}

\begin{tabular}{lll}
\hline $\begin{array}{l}\text { Blinding of outcome as- } \\
\text { sessment (detection bias) } \\
\text { Health related quality of } \\
\text { life (self report) }\end{array}$ & High risk & Participants not blinded to group allocation \\
\hline $\begin{array}{l}\text { Incomplete outcome data } \\
\text { (attrition bias) } \\
\text { Falls and fallers }\end{array}$ & High risk & More than 20\% of fall data were missing (27\%) \\
\hline $\begin{array}{l}\text { Selective reporting (re- } \\
\text { porting bias) }\end{array}$ & High risk & $\begin{array}{l}\text { Falls were measured, but number of falls not reported. Adverse events not re- } \\
\text { ported }\end{array}$ \\
\hline $\begin{array}{l}\text { Method of ascertaining } \\
\text { falls (recall bias) }\end{array}$ & High risk & $\begin{array}{l}\text { Relied on recall over 1 month. Preliminary information on falls was collect- } \\
\text { ed based on participants' self-report (retrospective recall) at the 6-month re- } \\
\text { assessment }\end{array}$ \\
\hline
\end{tabular}

ABC Scale: Activities-specific Balance Confidence Scale

$A D L$ : activities of daily living

BMD: bone mineral density

DXA: dual-energy X-ray absorptiometry (a way of measuring bone density)

ED: emergency department

FaME: Falls Management Exercise

FICSIT: frailty and injuries: co-operative studies of intervention techniques

GP: general practitioner

HMO: health maintenance organisation

m: metres

MMSE: Mini Mental State Examination

OT: occupational therapist

PT: physical therapist/physiotherapist

$\mathrm{RCT}$ : randomised controlled trial

SD: standard deviation

TUG: Timed Up and Go test

wk: week 
$x$ : times

$<$ : less than

$>$ : more than

Characteristics of excluded studies [ordered by study ID]

\begin{tabular}{|c|c|}
\hline Study & Reason for exclusion \\
\hline Alkan 2011 & RCT. Community-dwelling women $>65$ years old. Excluded as intervention was not exercise \\
\hline Beling 2009 & RCT. Community-dwelling. Age mean 80 years. Excluded as intervention was not exercise \\
\hline Clemson 2004b & RCT. Community-dwelling. Age mean 78 years. Excluded as intervention was not exercise \\
\hline DeSure 2013 & RCT. Excluded as sample was from an assisted-living community unit \\
\hline Fahlström 2017 & RCT. Excluded due to multiple interventions delivered \\
\hline Gianoudis 2014 & RCT. Community-dwelling. Age mean 67 years. Excluded as intervention was not just exercise \\
\hline Hinrichs 2016 & RCT. Community-dwelling. Age mean 80 years. Excluded as falls not measured \\
\hline Hsu 2017 & $\begin{array}{l}\text { RCT. Excluded as an inclusion criterion was subcortical ischaemic vascular cognitive impairment, a } \\
\text { particular clinical condition that increases the risk of falls }\end{array}$ \\
\hline Iwamoto 2012 & $\begin{array}{l}\text { RCT. Community-dwelling. Age mean } 74 \text { years. Excluded as intervention whole-body vibration with- } \\
\text { out exercise }\end{array}$ \\
\hline Lee 2013 & RCT. Community-dwelling older adults. Excluded as intervention was multifactorial. \\
\hline Leung 2014 & $\begin{array}{l}\text { RCT. Community-dwelling. Age }>60 \text { years. Excluded as intervention was whole-body vibration with- } \\
\text { out exercise }\end{array}$ \\
\hline Li 2018a & RCT. Age $>60$ years. Excluded as intervention incorporated functional electrical stimulation \\
\hline Morris 2008 & $\begin{array}{l}\text { RCT. } 3 / 26 \text { participants were withdrawn from the study due to injuries resulting from a fall. This } \\
\text { equated to } 50 \% \text { of the participants who fell during the trial being excluded from the results }\end{array}$ \\
\hline Ohtake 2013 & RCT. Community-dwelling. Aged $>65$ years. Excluded due to the control group \\
\hline Olsen 2014 & RCT. Community-dwelling older women. Excluded due to multiple interventions, not just exercise \\
\hline Pai 2014 & RCT. Community-dwelling older adults. Excluded as intervention was not exercise \\
\hline Pereira 1998 & RCT. Community-dwelling. Excluded as mean age 57 (SD 4) \\
\hline Rossi-Izquierdo 2017 & RCT. Aged $>60$. Excluded as intervention involved vestibular rehabilitation \\
\hline Steinberg 2000 & RCT. Older community-dwellers. Excluded due to multiple interventions \\
\hline Swanenburg 2007 & RCT. Community-dwelling. Age mean 71 years. Excluded due to multiple interventions \\
\hline Ueda 2017 & $\begin{array}{l}\text { RCT. Community-dwelling. Excluded as the difference in intervention between groups was hazard } \\
\text { reduction using floor plans }\end{array}$ \\
\hline
\end{tabular}


Characteristics of studies awaiting assessment [ordered by study ID]

Jagdhane 2016

\begin{tabular}{ll}
\hline Methods & RCT \\
\hline Participants & 6 older adults, mean (SD) age 73.3 (5) years \\
\hline Interventions & Intervention group: 4 weeks of anticipatory postural adjustment training \\
\hline Outcomes & Timed-Up and Go, single-limb stance, and Activities-specific Balance Confidence scale \\
\hline Notes & Awaiting full-text paper to determine if falls were measured \\
\hline
\end{tabular}

\section{Li 2018b}

\begin{tabular}{|c|c|}
\hline Methods & $\mathrm{RCT}$ \\
\hline Participants & $\begin{array}{l}\text { Community-dwelling, } 70 \text { years or older, fell in past year or impaired mobility, mean (SD) age } 77.7 \\
\text { (5.6) years }\end{array}$ \\
\hline Interventions & $\begin{array}{l}3 \text { intervention groups, each with } 260 \text {-minute classes a week for } 24 \text { weeks: i) Tai Ji Quan; ii) multi- } \\
\text { modal exercise programme; iii) stretching }\end{array}$ \\
\hline Outcomes & Incidence of falls at 6 months \\
\hline Notes & $\begin{array}{l}\text { Published } 7 \text { days before Cochrane Review submitted. Results stated that at } 6 \text { months, the inci- } \\
\text { dence rate ratio (IRR) was significantly lower in the Tai Ji Quan group (IRR } 0.43,95 \% \mathrm{Cl} 0.31 \text { to } 0.56 \text {, } \\
\mathrm{P}=0.01 \text { ), and multimodal exercise (IRR } 0.60,95 \% \mathrm{Cl} 0.45 \text { to } 0.80, \mathrm{P}=0.001 \text { ), compared with the } \\
\text { stretching group }\end{array}$ \\
\hline
\end{tabular}

Characteristics of ongoing studies [ordered by study ID]

\section{ACTRN 12613001161718}

Trial name or title
Effectiveness of dual-task functional power training for preventing falls in older people: Study protocol for a cluster-randomised controlled trial

\begin{tabular}{ll}
\hline Methods & Cluster RCT \\
\hline Participants & Target sample size: 280 \\
& Inclusion criteria: aged 65 years and over, at an increased risk of falling, currently resident in re- \\
& tirement villages, able to speak English, walk unaided or with minimal assistance (walking stick or \\
& walker) or at least 50 metres and be cognitively intact; clearance from local doctor prior to exercis- \\
& ing if has any contraindicated medical conditions to exercise \\
& Exclusion criteria: current or prior participation in a structured progressive resistance training pro- \\
& gramme and/or organised balance training > 1 a week in the past 3 months, acute or terminal ill- \\
& ness likely to compromise exercise participation, unstable or ongoing cardiovascular/respiratory \\
& disorders, musculoskeletal or neurological diseases disrupting voluntary movement or that might \\
limit training, upper- or lower-extremity fracture in the past 3 months, visual impairment not cor- \\
rected with glasses
\end{tabular}

Interventions

1. Exercise programme involving dual-task functional power training (DT-FPT), 2 twice a week supervised for 6 months, 'step-down' maintenance for 6 months, follow-up after 6 months 
ACTRN 12613001161718 (Continued)

2. Usual care control group

Outcomes

1. Number of falls over the 6-, 12-, 18-month period; details of the fall location, cause, injury, treatment and the healthcare utilisation

2. Changes in lower-limb functional muscle strength and power, isometric knee extensor, dorsi-flexor and hand-grip strength, dynamic balance and reaction time, gait, Instrumental Activities of Daily Living (IADL), quality of life, cognitive function and fall-related self-efficacy

\begin{tabular}{ll}
\hline Starting date & 23 October 2015 \\
\hline Contact information & Centre for Physical Activity and Nutrition Research, \\
& School of Exercise and Nutrition Sciences, \\
& Deakin University, \\
& Burwood, Victoria, Australia \\
& Email: rmdaly@deakin.edu.au \\
\hline
\end{tabular}

Notes

\section{ACTRN 12615000138583}

Trial name or title Standing Tall - a home-based exercise programme using mobile technology for preventing falls in
older people

\begin{tabular}{ll}
\hline Methods & RCT \\
\hline Participants & Target sample size: 500 \\
& Inclusion criteria: $\geq 70$ years old, community-dwelling, English-speaking, independent in ADL, able \\
& to walk household distances without the use of a walking aid, willingness to give informed consent \\
& and comply with the study protocol \\
& Exclusion criteria: unstable or acute medical condition that precludes exercise participation, pro- \\
& gressive neurological condition (such as Parkinson's disease, multiple sclerosis, Meniere's disease), \\
cognitively-impaired, defined as a Pfeiffer Short Portable Mental Status Questionnaire (SPMSQ) \\
score $<8$, currently participating in a fall-prevention programme
\end{tabular}

Interventions

1. Balance training delivered through a tablet computer in people's homes, unsupervised for $>2$ hours a week for 2 years

2. Control group: usual care + health promotion education programme relevant to older adults delivered through the tablet computer with weekly fact sheets

\section{Outcomes}

1. Number of people falling over 12 months

2. Rate of falls over 12 months

3. Questionnaire measure of concern about falling using the iconographical Falls Efficacy Scale

4. Clinical measures of balance, gait, choice reaction stepping time, Timed Up and Go Test, Sit-toStand Test

5. Concern about falling, quality of life, depressive symptoms, acceptability and enjoyment of intervention, exercise self-efficacy, healthcare use, physical activity levels, adverse events 
ACTRN 12615000138583 (Continued)

Starting date

17 February 2015 - 15 December 2017

$\begin{array}{ll}\text { Contact information } & \text { Dr Kim Delbaere } \\ & \text { Address NeuRA } \\ \text { Barker St } & \text { Randwick 2031 NSW } \\ & \text { Australia } \\ & \text { Phone +61 } 293991066 \\ & \text { Email k.delbaere@neura.edu.au }\end{array}$

Notes

Trial name or title Balance Exercise and Strength Training (BEST) programme for older people living at home

\begin{tabular}{|c|c|}
\hline Methods & $\mathrm{RCT}$ \\
\hline \multirow[t]{3}{*}{ Participants } & Target sample size: 576 \\
\hline & $\begin{array}{l}\text { Inclusion criteria: aged } 65 \text { years and over, and living at home or independently in the community } \\
\text { (e.g. self-care unit in residential aged care facility) in the Illawarra Shoalhaven Local Health District }\end{array}$ \\
\hline & $\begin{array}{l}\text { Exclusion criteria: Residing in nursing home; cognitive impairment; inability to walk } 10 \text { metres de- } \\
\text { spite assistance from walking aid; insufficient English language skills; a progressive neurological } \\
\text { disease e.g. Parkinson's disease; recent fracture/joint replacement; a medical condition preclud- } \\
\text { ing exercise, e.g. unstable cardiac disease, uncontrolled hypertension, uncontrolled metabolic dis- } \\
\text { eases; unable to obtain a medical clearance; currently participating in an exercise programme sim- } \\
\text { ilar to either study programme } 2 \text { or more times a week }\end{array}$ \\
\hline \multirow[t]{2}{*}{ Interventions } & $\begin{array}{l}\text { 1. Lower-limb group will receive a home-based exercise programme for the lower limb based on } \\
\text { the Otago Exercise Program }\end{array}$ \\
\hline & 2. Upper-limb group will receive an exercise programme designed to improve upper limb function \\
\hline \multirow[t]{3}{*}{ Outcomes } & 1. Rate of falls \\
\hline & 2. Upper limb function \\
\hline & $\begin{array}{l}\text { 3. Strength and balance, physical activity, falls efficacy, quality of life, health service usage, atti- } \\
\text { tudes to exercise }\end{array}$ \\
\hline Starting date & 26 October 2015 \\
\hline \multirow[t]{7}{*}{ Contact information } & Professor Cathie Sherrington \\
\hline & The University of Sydney \\
\hline & Musculoskeletal Health Sydney, School of Public Health \\
\hline & PO Box M179 \\
\hline & Missenden Road NSW 2050 \\
\hline & Australia \\
\hline & Email cathie.sherrington@sydney.edu.au \\
\hline
\end{tabular}


ACTRN 12615000865516 (Continued)

Notes

CTRI/2018/01/011214

Trial name or title Falls and fractures: A physiotherapy approach to prediction and prevention in healthcare

\begin{tabular}{ll}
\hline Methods & RCT \\
\hline Participants & $\begin{array}{l}\text { Men and women, aged } 60 \text { - } 80 \text { years. Moderate risk in fracture risk assessment tool and Berg bal- } \\
\text { ance scale }\end{array}$ \\
\hline Interventions & $\begin{array}{l}\text { 1. Exercise protocol as in the standard guidelines. Physiotherapy interventions including flexibili- } \\
\text { ty, mobility, strengthening and balancing exercises } 4 \text { times a week for up to } 6 \text { weeks with follow-up } \\
\text { every week. }\end{array}$ \\
\hline 2. Lifestyle modifications and ergonomical advice \\
\hline Berg balance scale \\
Lower extremity functional scale \\
$\begin{array}{l}\text { 21 March 2017 } \\
\text { Drarting date }\end{array}$ \\
$\begin{array}{l}\text { Sainath Hospital, Physiotherapy department, Exercise therapy division, Room no 301, Bopal-Ghu- } \\
\text { ma Road, Ahmedabad-380058 } \\
\text { Ahmadabad } \\
\text { GUJARA, India }\end{array}$ \\
Ph. 9099015220 \\
Email: bhumika2207@gmail.com
\end{tabular}

Notes

\section{ISRCTN71002650}

Trial name or title

The design and development of a complex multifactorial falls assessment intervention for falls prevention: The Prevention of Falls Injury Trial (PreFIT)

Methods 3-arm cluster-RCT and economic evaluation

\begin{tabular}{ll} 
Participants & $\mathrm{N}=9821$ \\
& Inclusion criteria: $\geq 70$ years old, living in the community or in sheltered accommodation \\
& Exclusion criteria: terminally ill, residential and nursing homes \\
\hline Interventions & 3arms: \\
1. Written advice & 2. Written advice plus structured exercise \\
3. Written advice plus multifactorial fall prevention (MFFP) \\
The total duration of follow-up for all trial arms is 18 months (updated 13 August 2015: was previ- \\
ously 12 months) The total duration of treatment varies across trial arms as follows: \\
1. Advice: 30 minutes
\end{tabular}


ISRCTN71002650 (Continued)

2. Exercise: 12 weeks ( $2 \times 1$-hour sessions a week)

3. MFFP: 8 weeks (depending on individual risk factors, but typically $6 \times 30$-minute sessions over 8 weeks)
1. Number of people sustaining peripheral fractures

2. Time to first fracture

3. Rate of falls, quality of life, emotional and physical function, mortality

4. Resource use, out-of-pocket expenses

\begin{tabular}{ll}
\hline Starting date & September 2010 \\
\hline Contact information & Prof Sarah Lamb \\
& Warwick Clinical Trials Unit \\
& The University of Warwick \\
& Gibbet Hill Campus \\
& Coventry \\
& CV4 7AL \\
& United Kingdom \\
& +44 (0)24 7615 0404 \\
& Email: s.lamb@warwick.ac.uk \\
& \\
\hline Notes & \\
\hline
\end{tabular}

\section{NCT01029171}

$\begin{array}{ll}\text { Trial name or title } & \begin{array}{l}\text { Action Seniors!: A 12-month randomized controlled trial of a home-based strength and balance re- } \\ \text { training programme in reducing falls }\end{array}\end{array}$

\begin{tabular}{ll}
\hline Methods & RCT \\
\hline
\end{tabular}

Participants Target sample size: 344

Inclusion criteria: adults $\geq 70$ years old attending a Falls Prevention Clinic Service; understands, speaks, and reads English proficiently; MMSE 8 score > 24/30; had 1 documented non-syncopal fall in the last 12 months and 1 of the following: 1) A Physiological Profile Assessment (PPA) score of at least 1 SD above normal; OR 2) Timed Up and Go Test (TUG) performance of > 15 seconds; OR 3) 1 additional documented non-syncopal fall in the previous 12 months; expected to live > 12 months; community-dwelling (i.e. not residing in a nursing home, extended care unit, or assisted-care facility); able to walk 3 metres with or without an assistive device; and able to provide written informed consent

Exclusion criteria: diagnosed with a neurodegenerative disease (e.g. Parkinson's disease); diagnosed with dementia (of any type); stroke; clinically significant peripheral neuropathy or severe musculoskeletal or joint disease; or history indicative of carotid sinus sensitivity (i.e. syncopal falls)

Interventions Intervention: Otago Exercise Program; home-based balance and strength retraining programme

2. Control: Usual care as prescribed by geriatrician

\section{Outcomes}

1. Falls over a 12-month period

2. Physiological falls risk; mobility; cognitive function; and economic evaluation

Starting date November 2009


NCT01029171 (Continued)

Contact information
Teresa Liu-Ambrose

Aging, Mobility, and Cognitive Neuroscience Laboratory,

Vancouver Falls Prevention Clinic,

University of British Columbia

CANADA

Notes

\section{NCT02126488}

\begin{tabular}{ll}
\hline Trial name or title & Effect of adaptive training for balance recovery \\
\hline Methods & RCT \\
\hline Participants & $\mathrm{N}=308$ \\
& $\begin{array}{l}\text { Inclusion criteria: } \geq 65 \text { years old, healthy, no known history of musculoskeletal, neurological, car- } \\
\text { diovascular, or pulmonary impairment that may affect their ability to perform the testing proce- } \\
\text { dures } \\
\text { Exclusion criteria: Ultrasound calcaneus bone mineral density T score }<-2.5 \text { (osteoporotic), MMSE } \\
\text { Score }<25 \text { (cognitive impairment) }\end{array}$ \\
\hline
\end{tabular}

Interventions

1. Treadmill slip perturbation: perturbation training on a treadmill with precisely-controlled sliplike displacements and then encounter an unannounced novel slip during over-ground walking 2. Treadmill training placebo: placebo training (on the same treadmill for the same duration but without perturbation) but encounter an identical novel slip during their over-ground walking 3. Observation training: watching a training video and slides, so when exposed to an identical novel slip in over-ground walking, they will know where and how the slip is going to occur and how to resist a fall

\begin{tabular}{ll}
\hline Outcomes & 1. Fall incidence, 1 year \\
2. Dynamic stability, 6 months
\end{tabular}

\begin{tabular}{ll}
\hline Starting date & June 2014 \\
\hline Contact information & Yi-Chung (Clive) Pai, University of Illinois at Chicago \\
\hline
\end{tabular}

Notes

\section{NCT02287740}

\begin{tabular}{|c|c|}
\hline Trial name or title & Prevention of falls among older adults in community settings \\
\hline Methods & $\mathrm{RCT}$ \\
\hline \multirow[t]{2}{*}{ Participants } & Target sample size: 670 \\
\hline & $\begin{array}{l}\text { Inclusion criteria: } \geq 70 \text { years, living independently in the community; } \geq 1 \text { fall in the preceding } 12 \\
\text { months referral from a healthcare provider indicating the participant is at risk of falls; no partici- } \\
\text { pation in daily and/or structured vigorous physical activity or walking for exercise } \geq 15 \text { minutes or } \\
\text { muscle-strengthening activities on } 2 \text { or more days a week in previous } 3 \text { months; walking indepen- } \\
\text { dently, with or without the use of an assistive device; no severe cognitive impairment; able to exer- }\end{array}$ \\
\hline
\end{tabular}


NCT02287740 (Continued)

cise safely as determined by healthcare provider; willingness to be randomly assigned to an intervention condition and complete the 6-month intervention and 6-month follow-up Exclusion Criteria: any medical or physical condition deemed unacceptable by their physician or healthcare provider; planned to leave the study area within the next 12 months

\begin{tabular}{ll}
\hline Interventions & All training sessions are 2 a week, 6 months. \\
& $\begin{array}{l}\text { 1. Tai Ji Quan, moving for better balance: core 8-form routine training with built-in variations and a } \\
\text { subroutine of integrated therapeutic movements } \\
\text { 2. Multimodal exercise: aerobic, strength, balance, and flexibility exercises } \\
\text { 3. Stretching: primarily seated exercises accompanied by breathing, stretching, and relaxation }\end{array}$ \\
\hline Outcomes & $\begin{array}{l}\text { 1. Number of falls in } 6 \text { months } \\
\text { 2. Cost per fall prevented determined by calculating total intervention cost estimates divided by } \\
\text { number of falls observed during the 6-month intervention }\end{array}$ \\
\hline Starting date & Fovember 2014 \\
\hline Contact information & Oregon Research Institute
\end{tabular}

Notes

NCT02617303

\begin{tabular}{ll}
\hline Trial name or title & Prevention of falls and its consequences in elderly people \\
\hline Methods & RCT \\
\hline
\end{tabular}

Participants Target sample size: 402

Inclusion criteria: $\geq 75$ to 89 years, assigned to primary care team; living in the community; using assisted mobility devices are included; ranking the Folstein MMSE test; expectation of permanence in the area for at least 18 months; agree to participate in the study by informed consent; in the case of a caregiver of person with dementia who assumes the realisation of the exercise programme and the following of tips, may be included;

Exclusion Criteria: current participation in another trial or institutional programme of guided physical activity; hip or knee operation or major injury or both, or any other intervention in the last 6 months; unable to follow an aerobic physical activity programme; in Home Care Programmes or Nursing Homes at baseline or during the training phase; terminal or severe cancer cases; disabled prior to or during the study period; have not been visited in reference's Health Center in the last 2 years (displacement/transfer); very advanced dementia that precludes following the instructions in the exercise programme and nurse's instructions. In case of a caregiver who assumes the realisation of exercise programme and the following of tips, patients of the Health Center who will be displaced, or temporarily shifted ( $>2$ months/year) may be included

Interventions

1. Otago Exercise Program exercises, consisting of a set of aerobic exercises affecting gait, balance, stability and are adapted for older people to support them both in groups and individually; 3 months followed by a loyalty phase (1 year) to consolidate the exercise programme. Falls and fractures monitored quarterly for 15 months

2. Usual practice: normal medical treatment will be provided by family physicians and nurses

Outcomes

1. Reduction in falls measured with a questionnaire at baseline and quarterly over 15 months

2. Reduction of fracture, fear of falling, measured with questionnaire 
NCT02617303 (Continued)

3. Physical measures of strength, balance, motion, endurance

4. Number of appointments at the practice

5. Nursing Home admission measured through questionnaire at 15 months

6. Drug reduction

\begin{tabular}{ll}
\hline Starting date & September 2015 \\
\hline Contact information & Rafael Azagra, PhD \\
& Insitut Català de la Salut \\
& Universitat Autònoma de Barcelona, SPAIN \\
& Email: rafael.azagra@uab.cat \\
\hline
\end{tabular}

Notes

NCT02926105 $\begin{array}{ll}\text { Trial name or title } & \begin{array}{l}\text { Comparison of home-based exercise programmes for falls prevention and quality of life in older } \\ \text { adults }\end{array}\end{array}$

\begin{tabular}{|c|c|}
\hline Methods & $\mathrm{RCT}$ \\
\hline \multirow[t]{3}{*}{ Participants } & Target sample size: 405 \\
\hline & $\begin{array}{l}\text { Inclusion criteria: } \geq 65 \text { years old, living in their own home, having a history of falls in the previous } \\
12 \text { months or perceiving fear of falling ( } \geq 20 \text { points on FES-I: Falls Efficacy Scale - international ver- } \\
\text { sion), able to walk without auxiliary tools in their home, signed informed consent }\end{array}$ \\
\hline & $\begin{array}{l}\text { Exclusion criteria: having severe vision impairment that does not permit the reading of the exer- } \\
\text { cise-programme booklet and that does not permit the completion of the monthly diaries, receiving } \\
\text { physiotherapeutic treatment with balance learning, having cognitive impairment (<25 points on } \\
\text { the Folstein MMSE }\end{array}$ \\
\hline
\end{tabular}

Interventions

1. 'Test and Exercise home-based tailored balance and functional strength tests and exercises, 3 a week, 12 months +8 physiotherapist home visits

2. Otago home-based programme: tailored balance, strength, walking exercises, 3 a week over 12 months +8 physiotherapist home visits

3. Active-Control: receive the 'Helsana' booklet with recommendations and 10 exercises, 3 a week, 12 months

Outcomes
$\begin{aligned} & \text { 2. Fumber of falls, } 1 \text { year } \\ & \text { 3. Severity of falls } \\ & \text { 4. Risk of fall } \\ & \text { 5. Quality of life } \\ & \text { 6. Exercise adherence }\end{aligned}$


NCT02926105 (Continued)

Contact information
Anne-Gabrielle Mittaz Hager, MS

HES-SO Valais-Wallis

Leukerbad, Valais,

Switzerland, 3954

Telephone: +41796099063

Email: gaby.mittaz@hevs.ch

Notes

NCT03211429

\begin{tabular}{|c|c|}
\hline Trial name or title & Effectiveness of three interventions to reduce fear of falling and improve functionality in the elderly \\
\hline Methods & $\mathrm{RCT}$ \\
\hline Participants & $\begin{array}{l}N=110 \\
\text { Inclusion criteria: } \geq 60 \text { years old, healthy, community-dwelling, reported fear of falling, 'Leganés } \\
\text { Cognitive Test' } \geq 23 \text {, SPPB (short physical performance battery) } \leq 9 \\
\text { Exclusion criteria: some cognitive impairment or medical condition or both that may affect the in- } \\
\text { tervention, permanent use of wheelchair, people who have received prior protocolised manage- } \\
\text { ment for fear of falling }\end{array}$ \\
\hline Interventions & $\begin{array}{l}\text { 1. Cognitive behavioural therapy: teach participants how to deal with their concerns about falls and } \\
\text { related avoidance of activity } \\
\text { 2. Tai Chi: training in the Yang style of } 24 \text { movement } \\
\text { 3. Postural control exercise: individually-adjusted progressive, specific and functional postural } \\
\text { control training }\end{array}$ \\
\hline Outcomes & $\begin{array}{l}\text { 1. Fear of falling } \\
\text { 2. Functional mobility } \\
\text { 3. Falls } \\
\text { 4. Depression } \\
\text { 5. Handgrip } \\
\text { 6. Daily life activities } \\
\text { 7. Self-rated health } \\
\text { 8. Postural control }\end{array}$ \\
\hline Starting date & June 2016 \\
\hline Contact information & $\begin{array}{l}\text { Carmen L Curcio, PhD } \\
\text { Universidad de Caldas } \\
\text { Manizales, Caldas, Colombia, } 170004 \\
573184665019 \\
\text { Email: carmen.curcio@ucaldas.edu.co }\end{array}$ \\
\hline
\end{tabular}

Notes 
NCT03320668

$\begin{array}{ll}\text { Trial name or title } & \begin{array}{l}\text { Efficacy of the Otago Exercise Program delivered as group training versus individually-tailored } \\ \text { training }\end{array}\end{array}$

\begin{tabular}{ll}
\hline Methods & RCT \\
\hline Participants & Target sample size: 728 \\
& Inclusion criteria: $65-80$ years, people who belong (ascribed) to primary healthcare centres of the \\
& same health area, non-institutionalised, independence for walking, provide informed consent for \\
participation & Exclusion Criteria: residential period in the Health Basic Area of the primary health centre $<9$ \\
& months, or < 9 months life expectancy in the health area of the primary healthcare centre; mild and \\
& moderate cognitive impairment; sight impairment or hearing impairment which prevents follow- \\
& ing the intervention (according to the diagnosis from medical history); absolute contraindication to \\
perform physical exercise (according to the diagnosis from medical history)
\end{tabular}

\begin{tabular}{|c|c|}
\hline Interventions & $\begin{array}{l}\text { 1. Individual Otago Exercise Program (OEP): individual education in } 5 \text { sessions + telephone call to } \\
\text { follow-up }\end{array}$ \\
\hline & on to 10 people groups in 5 sessions + telephone calls to follow-up \\
\hline
\end{tabular}

\begin{tabular}{ll}
\hline Outcomes & 1. Percentage of falls, 12 months \\
& 2. Adverse events \\
& 3. Adherence \\
& 4. Participant satisfaction \\
\hline Starting date & 10 January 2017 \\
\hline Contact information & Laura Albornos-Muñoz \\
& Instituto de Salud Carlos III, SPAIN \\
Telephone: 34 918222517 & Email: lalbornos@isiii.es \\
\hline
\end{tabular}

Notes

\section{NCT03404830}

Trial name or title

Effects of a program of high intensity exercise by intervals on the risk of falls for the physical condition and the state of health in people over 60 years

\begin{tabular}{ll}
\hline Methods & RCT \\
\hline Participants & Target sample size: 45 \\
& Inclusion criteria: Men and women, aged 60 - 80 years \\
& $\begin{array}{l}\text { Exclusion criteria: diseases that may alter balance and functional activity (such as auditory or } \\
\text { vestibular alterations), central or peripheral neurological disorders, other rheumatological dis- } \\
\text { eases, or serious psychiatric or somatic diseases }\end{array}$ \\
\hline Interventions & 1 and 2: training twice a week for 12 weeks
\end{tabular}


1. High-intensity interval training (HIIT) group: Squat training with the Suspension Training System (TRX). The session will be divided into $4 \times 4$-minute intervals at an intensity of $90-95 \%$ of the maximum heart rate, followed by 3 -minute active rest intervals of $50-70 \%$. Followed by 10 minutes of exercises of joint range

2. Moderate-intensity continuous training (MICT) group: Squat training with the Suspension Training System (TRX) with an intensity close to $70 \%$ of their maximum heart rate maintained for 40 minutes. The session will conclude with a return to calm of 10 minutes of joint width and stretching.

3. No intervention group

1. Gait and balance parameters
2. Mobility
3. Balance
4. Strength
5. Balance confidence
6. Falls self-efficacy
7. Body composition
8. Health-related quality of life

\section{Starting date}

Contact information
September 2017

Agustín Aibar Almazán

University of Jaén, SPAIN

Notes

\section{NCT03417531}

\section{Trial name or title}

Methods

Participants
Sarcopenia prevention with a targeted exercise and protein supplementation program

RCT, $2 \times 2$ factorial design, triple-blinded

\section{Target sample size: 800 participants}

Inclusion: age 80+; at least 1 of 5 Cardiovascular Health Study frailty criteria (i) weight loss of $>4.5$ $\mathrm{kg}$ in the last 12 months; ii) reduced grip strength in Martin Vigorimeter test: men $\leq 64 \mathrm{kPa}$, women $\leq 42 \mathrm{kPa}$; iii) standardised question on exhaustion as published by Fried et al. (Fried 2001); iv) gait speed < $1 \mathrm{~m} / \mathrm{s}$; v) 6-minute walk test $<300$ metres; Injurious (any injury) low trauma fall in the last 12 months prior to enrolment; At risk of malnutrition or established malnutrition based on the Mini Nutritional Assessment (MNA) screening tool (score $\leq 11)$; Community-dwelling or assisted living

Exclusion: MMSE < 24; inability to come to the trial centres; inability to walk at least 3 meters with or without walking aid; severe kidney impairment; inability to follow exercise instruction or inability to take protein powder mixed in drink or food; severe gait impairment or diseases with a risk of recurrent falling; major visual or hearing impairment or other serious illness that would preclude participation (e.g. alcohol abuse, alcoholic disease); inability to read/speak/write in German; living in a nursing home; contraindication to treatment (e.g. allergy); contraindication to the vitamin $D$ standard of care therapy 
NCT03417531 (Continued)

Interventions
1. Protein supplement plus active exercise: Participants will ingest twice daily $23.7 \mathrm{~g}$ of L-leucineenriched whey protein isolate powder (equivalent to $20 \mathrm{~g}$ of protein) and perform a simple home exercise strength programme ( $3 \times 30$ minutes a week)

2. Active comparator: Protein-free supplement plus active exercise: Participants will ingest twice daily $23.7 \mathrm{~g}$ of a protein-free, isocaloric powder blend and perform a simple home exercise strength programme ( $3 \times 30$ minutes a week)

3. Active comparator: Protein supplement plus control exercise: Participants will ingest twice daily $23.7 \mathrm{~g}$ of L-leucine-enriched whey protein isolate powder (equivalent to $20 \mathrm{~g}$ of protein) and perform a joint flexibility home exercise programme ( $3 \times 30$ minutes a week)

4. Sham comparator: Protein-free supplement plus control exercise: Participants will ingest twice daily $23.7 \mathrm{~g}$ of a protein-free, isocaloric powder blend and perform a joint flexibility home exercise programme ( $3 \times 30$ minutes a week)

1. Rate of falling
2. Mobility
3. Fallers, number of people with injurious falls
4. Frailty
5. Sarcopenia
6. Institutionalisation
7. Health care utilisation

Starting date May 2018

Contact information

Heike A. Bischoff-Ferrari

University of Zurich, SWITZERLAND

Ph: +41 442552757

Email: heike.bischoff@usz.ch

\section{Notes}

\section{NCT03455179}

Trial name or title

Effects of slow-speed traditional resistance training, high-speed resistance training and multicomponent training with variable resistances on molecular, body composition, neuromuscular, physical function and quality of life variables in older adults

\begin{tabular}{ll}
\hline Methods & RCT \\
\hline Participants & Target sample size: 192 participants \\
& Inclusion criteria: Age > 60 years; physically independent (able to walk 100 meters without a walk- \\
& ing aid and climb 10 steps without rest); medical certificate of suitability or fitness to practice resis- \\
& tance training activities; no plans to leave the area during the intervention; cognitive ability to un- \\
& derstand, follow the instructions and sign the informed consent form; free of any antioxidant sup- \\
plements for at least 6 weeks before the start of this study. & \\
& Exclusion criteria: Presence of cardiovascular, musculoskeletal, renal, liver or neuromuscular dis- \\
& orders that would prevent the participant from performing the exercises; body weight changes > \\
& $10 \%$ in the previous year; intake of prescription medications that were expected to alter the re-
\end{tabular}


NCT03455179 (Continued)

sults of the study; history of malignant neoplasms; engagement in regular strength training during the previous 6 months; participating in another research project involving dietary, exercise and/or pharmaceutical intervention; MMSE < 24/30; Severe visual or hearing impairment

Interventions

1. Slow-speed traditional resistance training. Resistance training with variable resistances (elastic band) at high intensity and slow-speed ( 2 seconds of concentric contraction and 2 seconds of eccentric contraction) twice a week over 20 weeks

2. High-speed resistance training. Resistance training with variable resistances (elastic band) at low intensity and high-speed ('as fast as possible' for the concentric contraction, pause for 1 second and 2 - 3 seconds for the eccentric contraction) twice a week over 20 weeks

3. Multicomponent training. Training sessions with balance, resistance, aerobic, flexibility and coordination components twice a week over 20 weeks

4. Control. Maintain usual physical activity habits and diet

\begin{tabular}{ll}
\hline Outcomes & Muscle biochemistry \\
2. Muscle strength \\
3. Function \\
4. Mobility \\
5. Body composition \\
6. Falls
\end{tabular}

Starting date

Contact information
March 2018

Prof. Juan Carlos Colado Sánchez

Department of Physical Education and Sports

University of Valencia, SPAIN

Spain, 46010

\section{Notes}

\section{NCT03462654}

Trial name or title

Comparison of a group-delivered and individually-delivered lifestyle-integrated functional exercise (LiFE) programme in older persons

\begin{tabular}{ll}
\hline Methods & RCT \\
\hline Participants & Target sample size: 300 participants \\
& Inclusion criteria: Aged 70 years or older; speaks German; able to read newspaper; able to walk 200 \\
& meters with or without walking aid; home-dwelling; 2 or more falls in the past 12 months OR 1 inju- \\
& rous fall in the past 12 months OR subjective decline in balance and strength in the past 12 months \\
& together with Timed Up and Go Test time $>13.5$ seconds; available for intervention participation \\
& for 11 weeks \\
& Exclusion criteria: Cognitive impairment (MoCA $<23$ ); current participation in an organised exercise \\
class $>1$ a week in the past 3 months; moderate- to vigorous-intensity physical activity $\geq 150$ min- \\
utes a week in the past 3 months; a list of 8 medical conditions
\end{tabular}


NCT03462654 (Continued)

Interventions

1. Individual LiFE (iLiFE). In iLiFE, LiFE activities to increase strength, improve balance, and promote physical activity as well as habitualisation strategies are introduced and taught in 7 highly individualised, one-to-one home visits

2. Group LiFE (gLiFE). In gLiFE, the same LiFE activities as performed in iLiFE are introduced and taught in 7 group sessions with $8-12$ participants. Implementation and habitualisation strategies will be addressed within the group setting, making use of group dynamics and processes

\begin{tabular}{ll}
\hline Outcomes & $\begin{array}{l}\text { 1. Fall incidence expressed as number of falls per amount of physical activity } \\
\text { 2. Cost-effectiveness of iLiFE and gLiFE (incremental cost-effectiveness ratios (ICERs) of delivering } \\
\text { iLiFE and gLiFE) }\end{array}$ \\
\hline Starting date & April 2018 \\
\hline Contact information & Carl-Philipp Jansen \\
& Heidelberg University, Network Aging Research, GERMANY \\
ph. +496221548144 \\
Email: jansen@nar.uni-heidelberg.de
\end{tabular}

Notes

ADL: activities of daily living

$\mathrm{m}$ : metres

MMSE: Mini Mental State Examination

$\mathrm{RCT}$ : randomised controlled trial

SD: standard deviation

TUG: Timed Up and Go test

wk: week

$\mathrm{x}$ : times

$<$ : less than

$>$ : more than

z: greater than or equal to

\section{DATA AND ANALYSES}

\section{Comparison 1. Exercise versus control (rate of falls)}

\begin{tabular}{|c|c|c|c|c|}
\hline Outcome or subgroup title & No. of studies & $\begin{array}{l}\text { No. of partici- } \\
\text { pants }\end{array}$ & Statistical method & Effect size \\
\hline 1 Rate of falls - overall analysis & 59 & 12981 & $\begin{array}{l}\text { Rate Ratio (Random, 95\% } \\
\text { Cl) }\end{array}$ & $0.77[0.71,0.83]$ \\
\hline $\begin{array}{l}2 \text { Rate of falls - subgrouped by base- } \\
\text { line falls risk }\end{array}$ & 59 & & $\begin{array}{l}\text { Rate Ratio (Random, 95\% } \\
\text { Cl) }\end{array}$ & Subtotals only \\
\hline 2.1 Not selected for high risk of falling & 29 & 6123 & $\begin{array}{l}\text { Rate Ratio (Random, 95\% } \\
\mathrm{Cl} \text { ) }\end{array}$ & $0.74[0.65,0.84]$ \\
\hline 2.2 Selected for high risk of falling & 30 & 6858 & $\begin{array}{l}\text { Rate Ratio (Random, 95\% } \\
\mathrm{Cl} \text { ) }\end{array}$ & $0.80[0.72,0.88]$ \\
\hline
\end{tabular}




\begin{tabular}{|c|c|c|c|c|}
\hline Outcome or subgroup title & No. of studies & $\begin{array}{l}\text { No. of partici- } \\
\text { pants }\end{array}$ & Statistical method & Effect size \\
\hline $\begin{array}{l}3 \text { Rate of falls - subgrouped by age } \\
\text { (threshold } 75 \text { years) }\end{array}$ & 59 & & $\begin{array}{l}\text { Rate Ratio (Random, 95\% } \\
\mathrm{Cl} \text { ) }\end{array}$ & Subtotals only \\
\hline 3.1 Age $<75$ & 46 & 9605 & $\begin{array}{l}\text { Rate Ratio (Random, 95\% } \\
\mathrm{Cl} \text { ) }\end{array}$ & $0.75[0.69,0.82]$ \\
\hline 3.2 Age $75+$ & 13 & 3376 & $\begin{array}{l}\text { Rate Ratio (Random, 95\% } \\
\mathrm{Cl} \text { ) }\end{array}$ & $0.83[0.72,0.97]$ \\
\hline $\begin{array}{l}4 \text { Rate of falls - subgrouped by per- } \\
\text { sonnel }\end{array}$ & 59 & 12981 & $\begin{array}{l}\text { Rate Ratio (Random, 95\% } \\
\mathrm{Cl} \text { ) }\end{array}$ & $0.77[0.71,0.83]$ \\
\hline $\begin{array}{l}4.1 \text { Health professional delivering in- } \\
\text { tervention }\end{array}$ & 25 & 4511 & $\begin{array}{l}\text { Rate Ratio (Random, 95\% } \\
\mathrm{Cl} \text { ) }\end{array}$ & $0.69[0.61,0.79]$ \\
\hline $\begin{array}{l}4.2 \text { No health professional delivering } \\
\text { intervention }\end{array}$ & 34 & 8470 & $\begin{array}{l}\text { Rate Ratio (Random, 95\% } \\
\mathrm{Cl} \text { ) }\end{array}$ & $0.82[0.75,0.90]$ \\
\hline $\begin{array}{l}5 \text { Rate of falls - subgrouped by group } \\
\text { or individual exercise }\end{array}$ & 59 & 12981 & $\begin{array}{l}\text { Rate Ratio (Random, 95\% } \\
\mathrm{Cl} \text { ) }\end{array}$ & $0.77[0.71,0.83]$ \\
\hline 5.1 Group exercise & 40 & 8163 & $\begin{array}{l}\text { Rate Ratio (Random, 95\% } \\
\mathrm{Cl} \text { ) }\end{array}$ & $0.76[0.69,0.85]$ \\
\hline 5.2 Not group exercise & 21 & 4818 & $\begin{array}{l}\text { Rate Ratio (Random, 95\% } \\
\mathrm{Cl} \text { ) }\end{array}$ & $0.79[0.71,0.88]$ \\
\hline $\begin{array}{l}6 \text { Rate of falls - subgrouped by exer- } \\
\text { cise type }\end{array}$ & 59 & & $\begin{array}{l}\text { Rate Ratio (Random, 95\% } \\
\mathrm{Cl} \text { ) }\end{array}$ & Subtotals only \\
\hline $\begin{array}{l}6.1 \text { Balance and functional exercises } \\
\text { vs control }\end{array}$ & 39 & 7920 & $\begin{array}{l}\text { Rate Ratio (Random, 95\% } \\
\mathrm{Cl} \text { ) }\end{array}$ & $0.76[0.70,0.81]$ \\
\hline 6.2 Resistance exercise vs control & 5 & 327 & $\begin{array}{l}\text { Rate Ratio (Random, 95\% } \\
\mathrm{Cl} \text { ) }\end{array}$ & $1.14[0.67,1.97]$ \\
\hline 6.3 3D exercise (Tai Chi) vs control & 7 & 2655 & $\begin{array}{l}\text { Rate Ratio (Random, 95\% } \\
\text { Cl) }\end{array}$ & $0.81[0.67,0.99]$ \\
\hline 6.4 3D exercise (dance) vs control & 1 & 522 & $\begin{array}{l}\text { Rate Ratio (Random, 95\% } \\
\text { Cl) }\end{array}$ & $1.34[0.98,1.83]$ \\
\hline 6.5 Walking programme vs control & 2 & 441 & $\begin{array}{l}\text { Rate Ratio (Random, 95\% } \\
\mathrm{Cl} \text { ) }\end{array}$ & $1.14[0.66,1.97]$ \\
\hline $\begin{array}{l}6.6 \text { Multiple categories of exercise vs } \\
\text { control }\end{array}$ & 11 & 1374 & $\begin{array}{l}\text { Rate Ratio (Random, 95\% } \\
\mathrm{Cl} \text { ) }\end{array}$ & $0.66[0.50,0.88]$ \\
\hline $\begin{array}{l}7 \text { Rate of falls - long-term follow-up } \\
\text { by exercise type }\end{array}$ & 4 & & $\begin{array}{l}\text { Rate Ratio (Random, 95\% } \\
\text { Cl) }\end{array}$ & Subtotals only \\
\hline $\begin{array}{l}\text { 7.1 Balance and functional exercises } \\
\text { vs control }\end{array}$ & 2 & 858 & $\begin{array}{l}\text { Rate Ratio (Random, 95\% } \\
\mathrm{Cl} \text { ) }\end{array}$ & $0.82[0.66,1.01]$ \\
\hline
\end{tabular}




\begin{tabular}{lllll}
\hline Outcome or subgroup title & No. of studies & $\begin{array}{l}\text { No. of partici- } \\
\text { pants }\end{array}$ & Statistical method & Effect size \\
\hline 7.2 Walking programme vs control & 1 & 97 & $\begin{array}{l}\text { Rate Ratio (Random, 95\% } \\
\text { Cl) }\end{array}$ & $1.27[0.89,1.81]$ \\
\hline $\begin{array}{l}7.3 \text { Multiple categories of exercise vs } \\
\text { control }\end{array}$ & 1 & 175 & $\begin{array}{l}\text { Rate Ratio (Random, 95\% } \\
\text { Cl) }\end{array}$ & 0.80 [0.55, 1.16] \\
\hline
\end{tabular}

Analysis 1.1. Comparison 1 Exercise versus control (rate of falls), Outcome 1 Rate of falls - overall analysis.

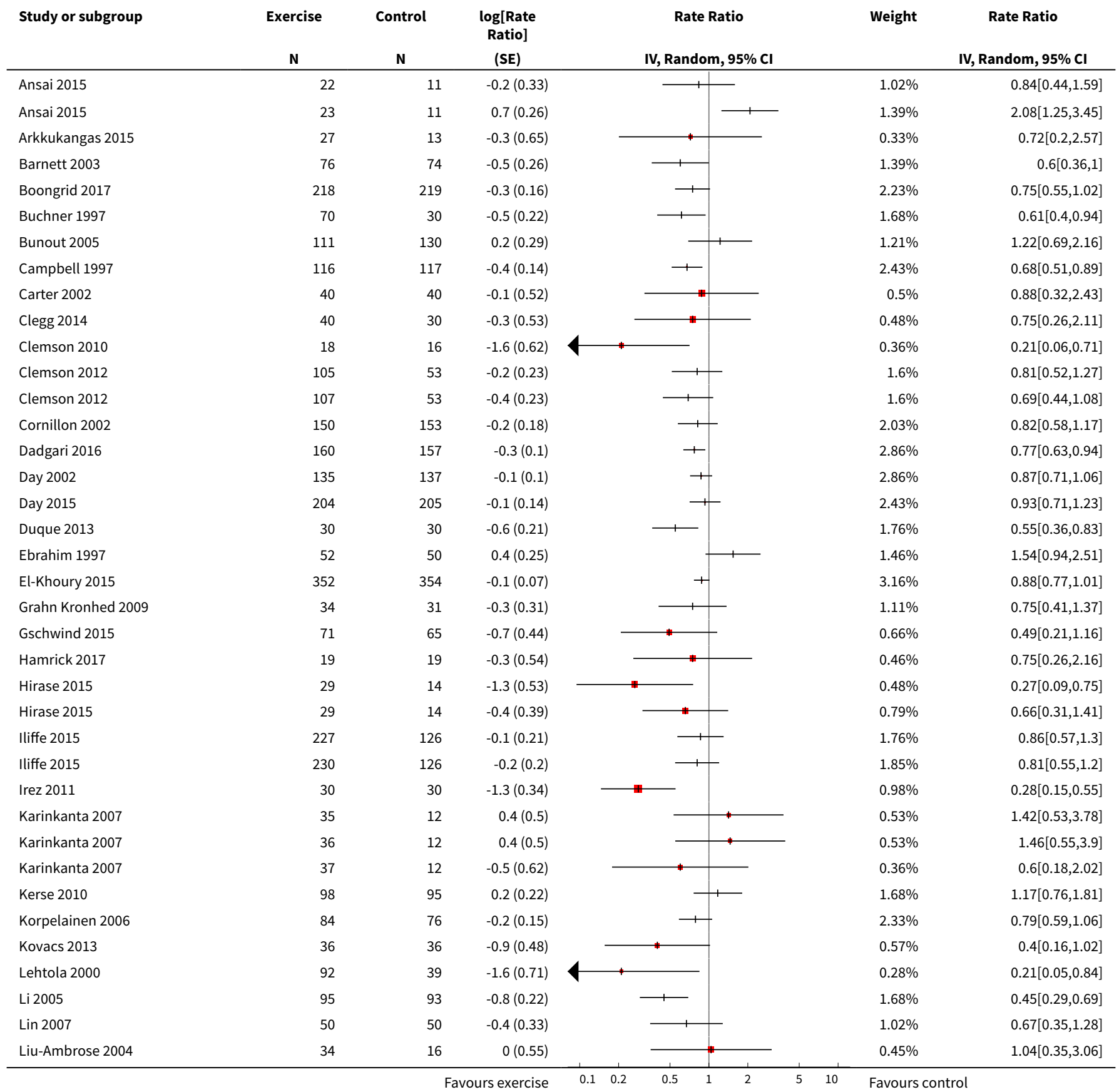




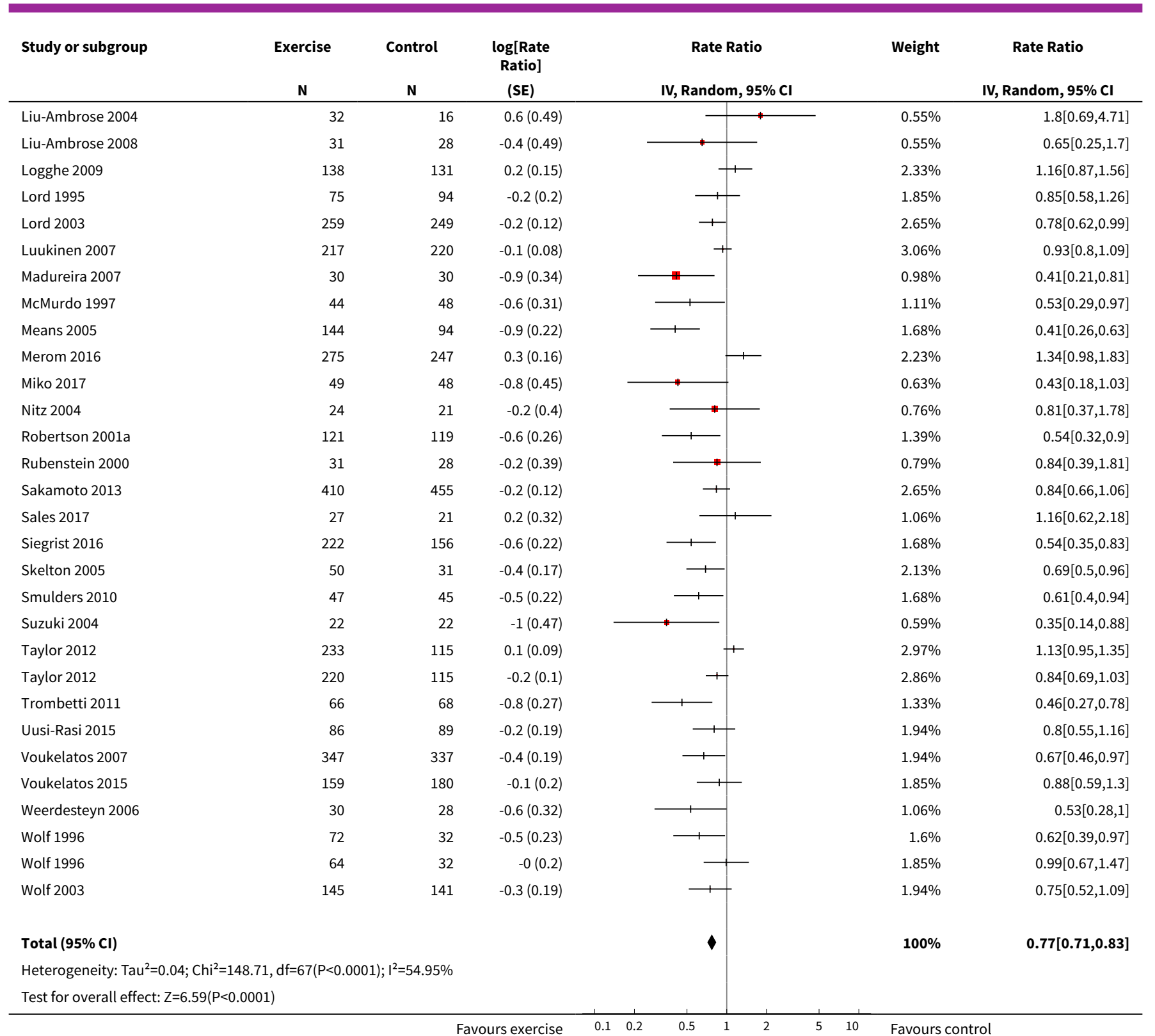

Analysis 1.2. Comparison 1 Exercise versus control (rate of falls), Outcome 2 Rate of falls - subgrouped by baseline falls risk.

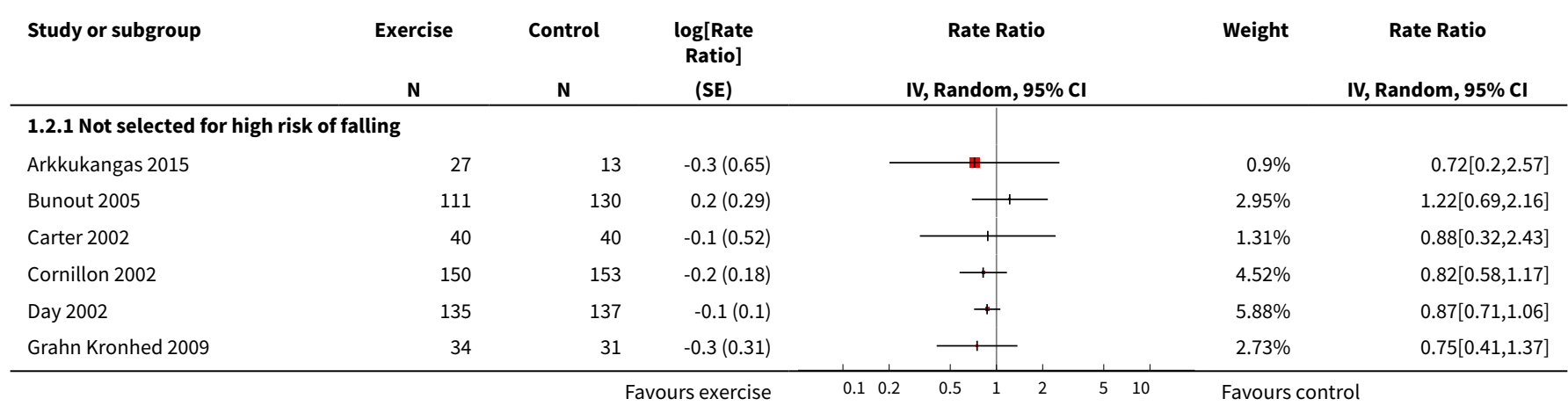




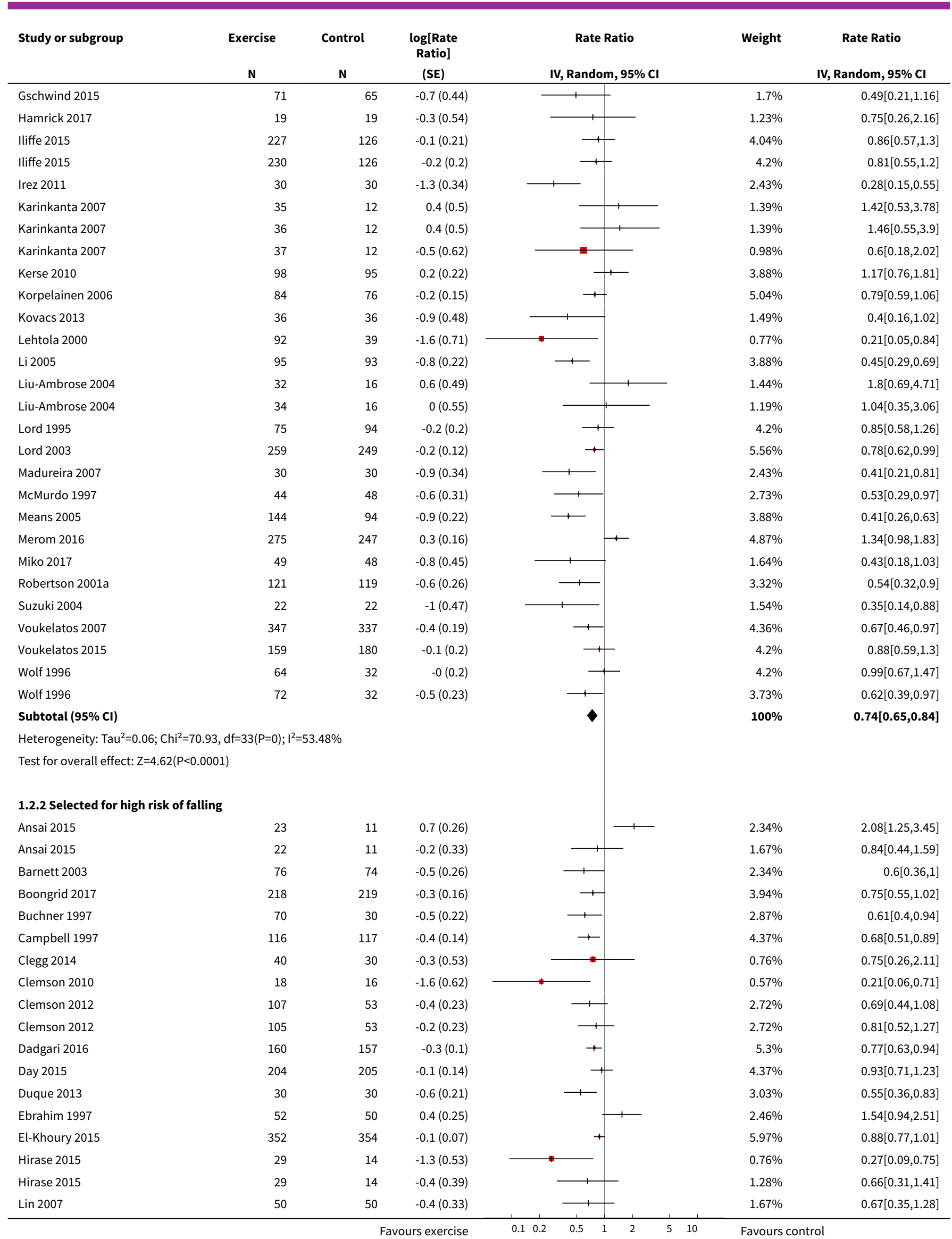




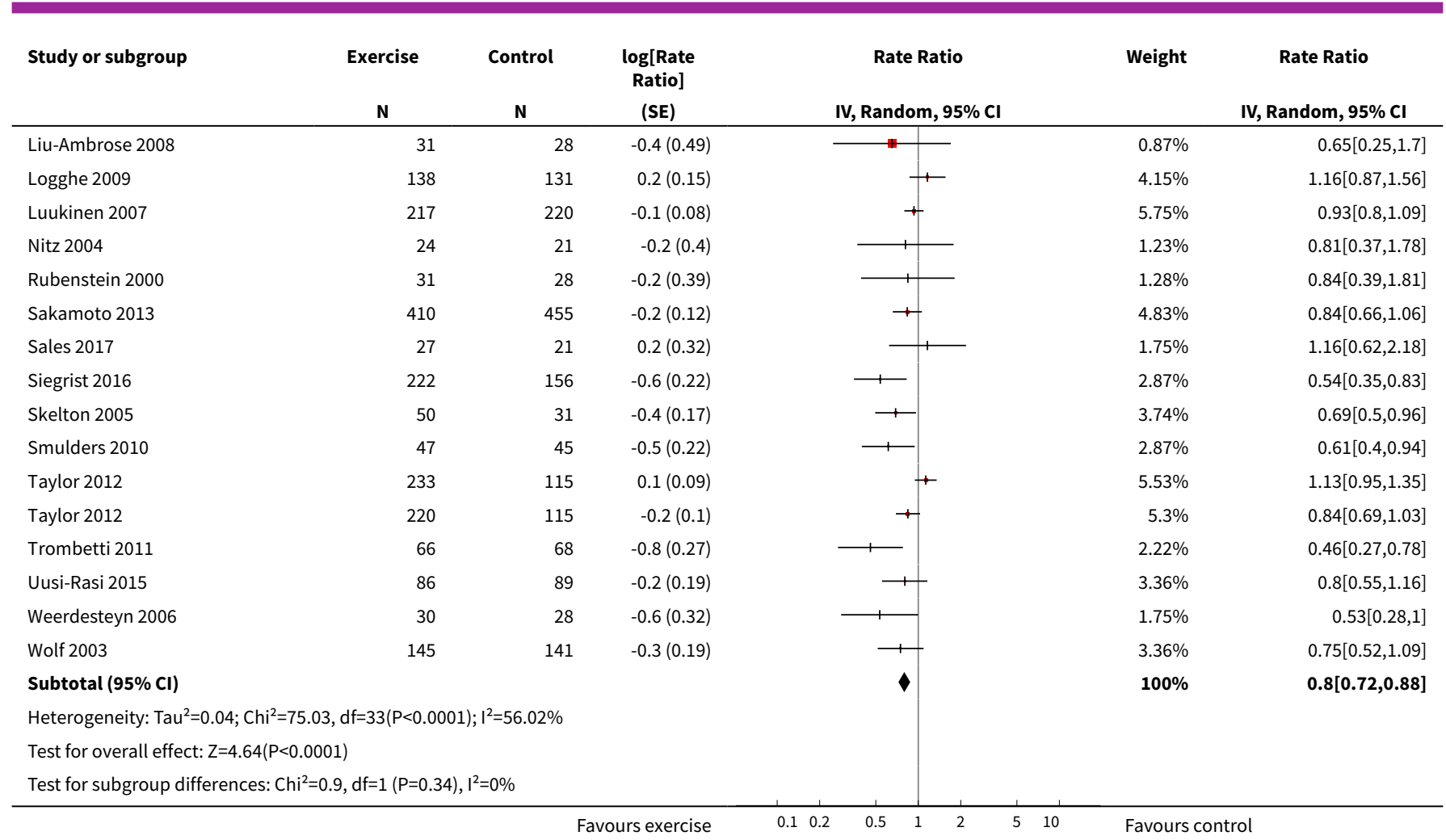

Analysis 1.3. Comparison 1 Exercise versus control (rate of falls), Outcome 3 Rate of falls - subgrouped by age (threshold 75 years).

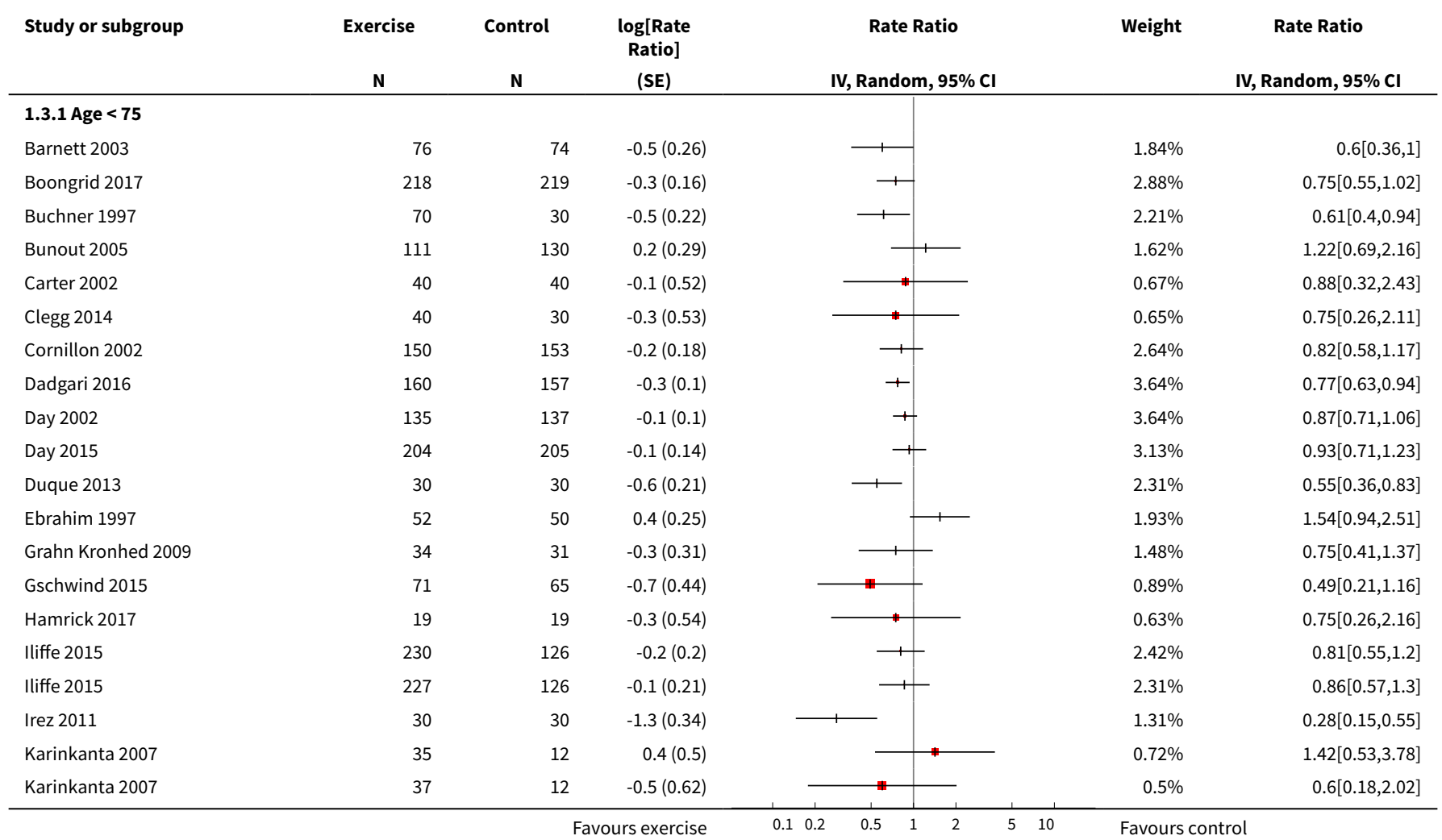




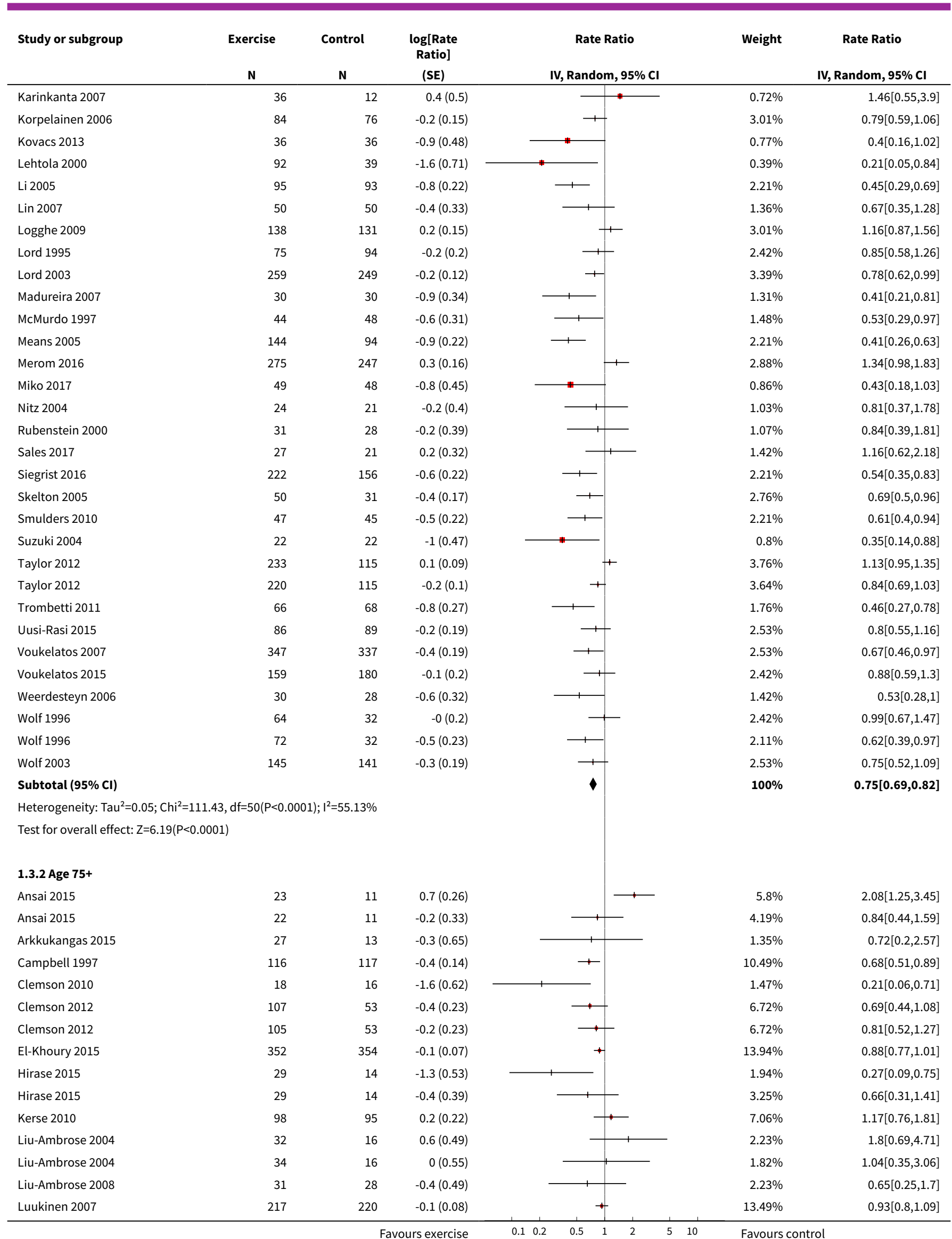




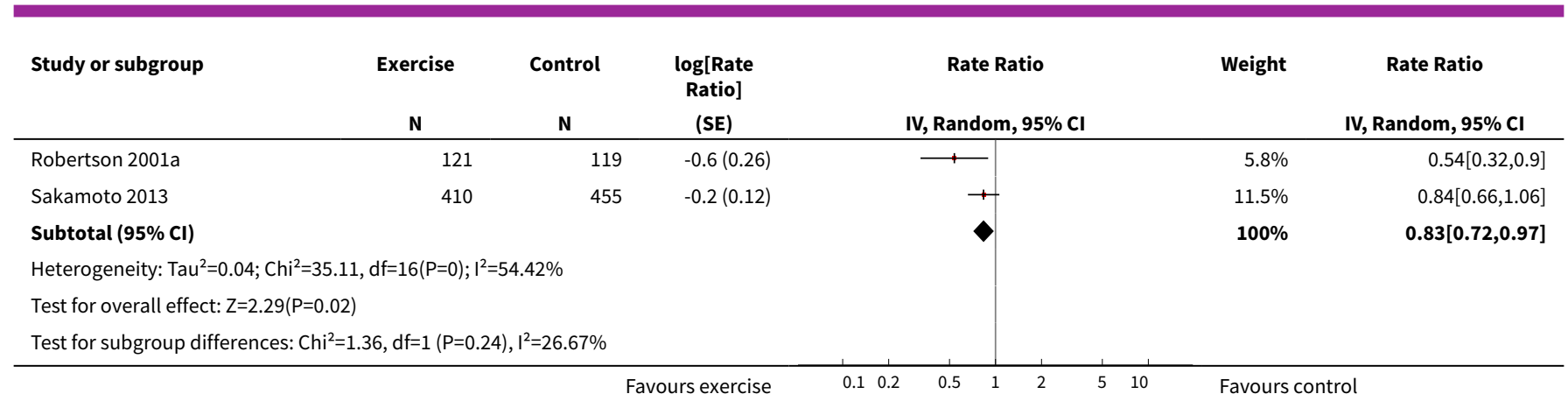

\section{Analysis 1.4. Comparison 1 Exercise versus control (rate of} falls), Outcome 4 Rate of falls - subgrouped by personnel.

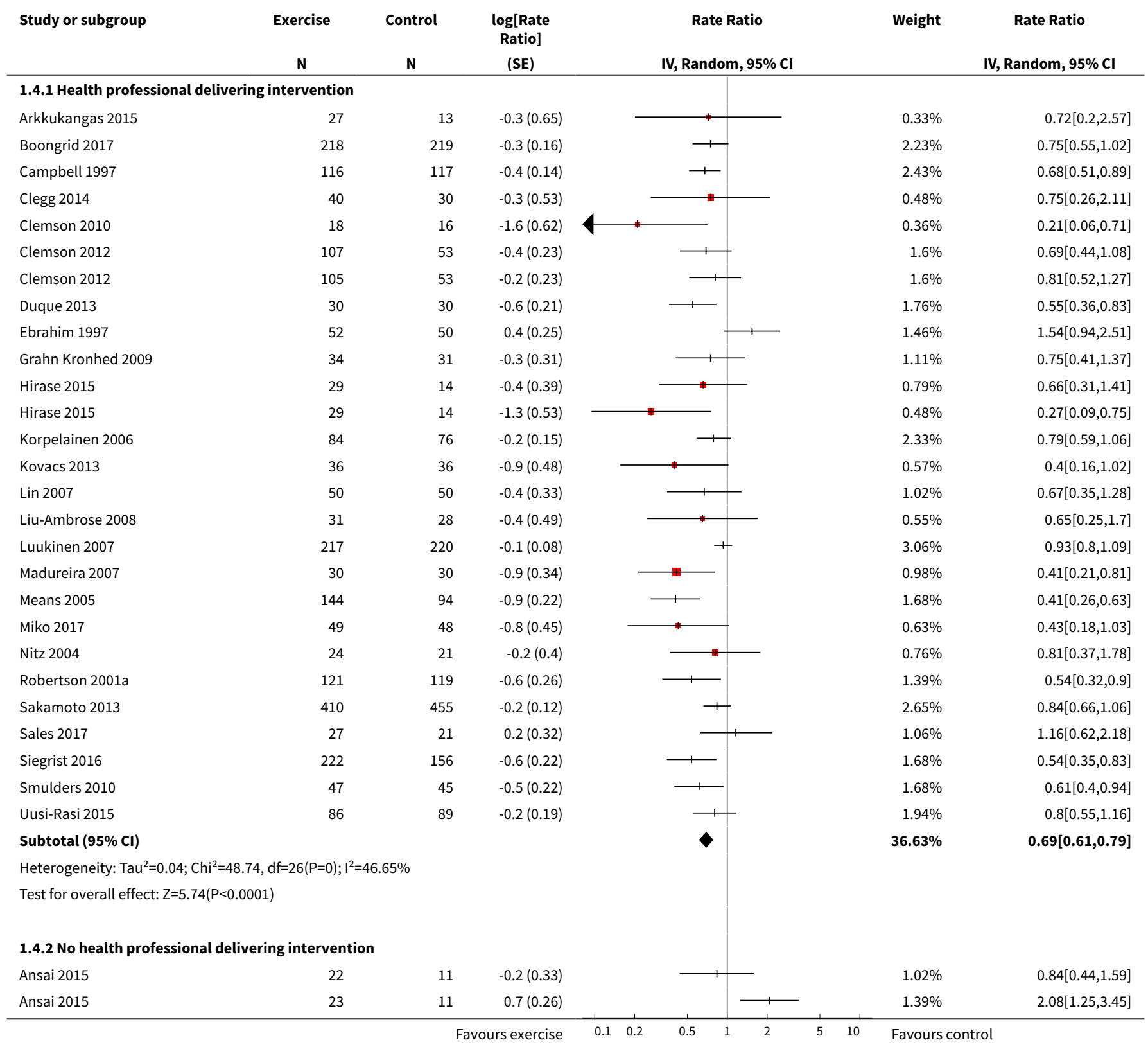




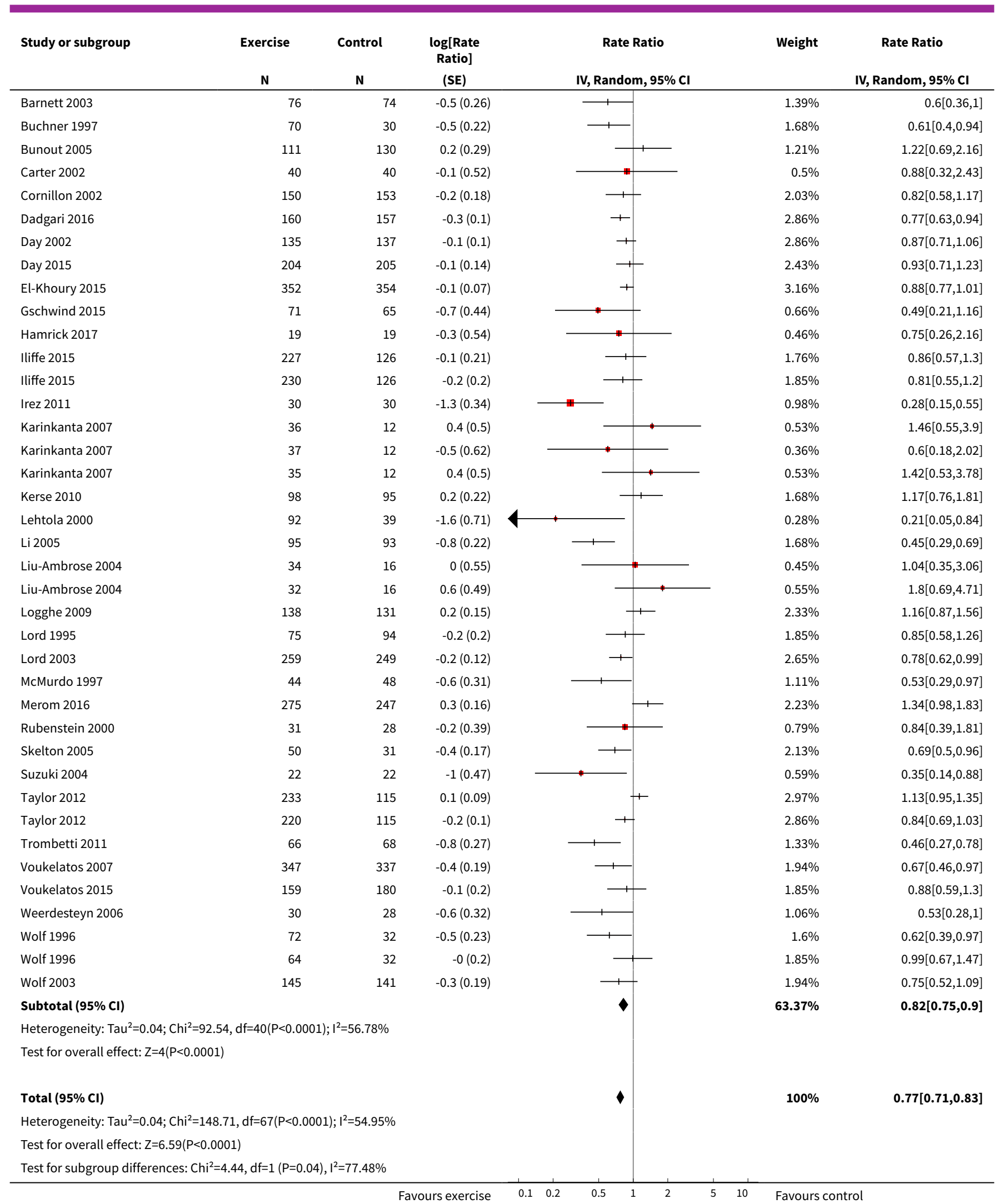


Analysis 1.5. Comparison 1 Exercise versus control (rate of falls),

Outcome 5 Rate of falls - subgrouped by group or individual exercise.

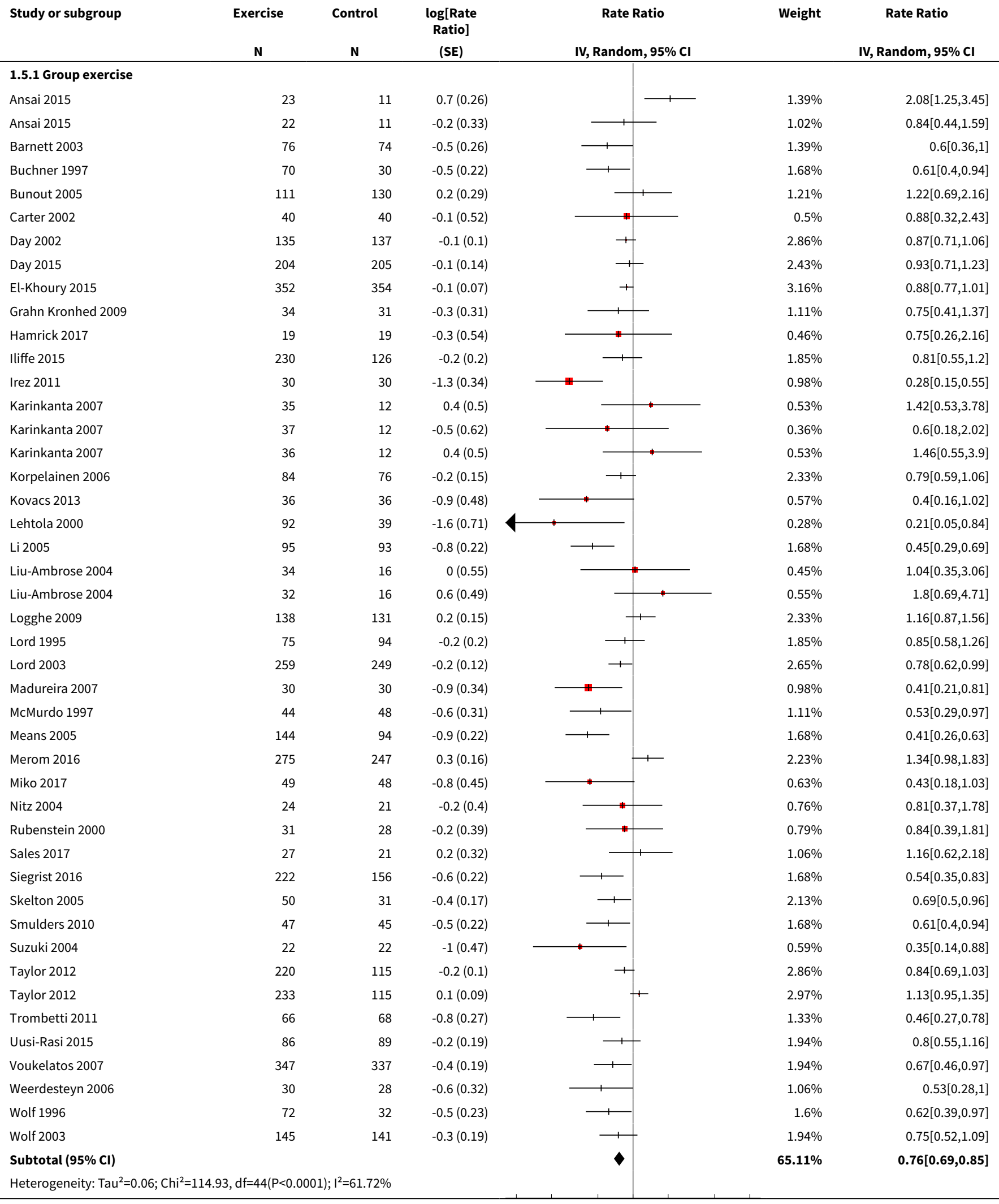




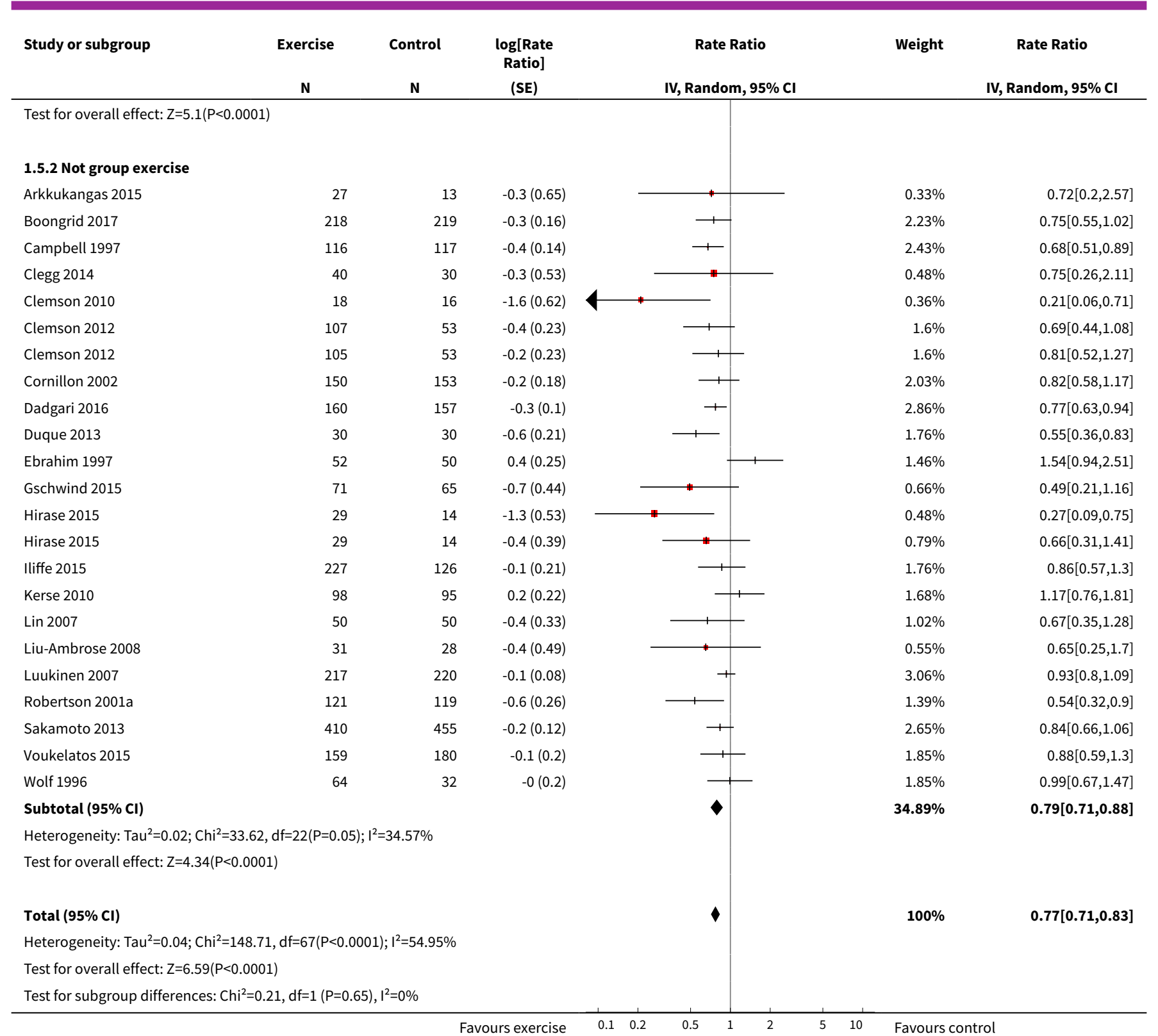

Analysis 1.6. Comparison 1 Exercise versus control (rate of falls), Outcome 6 Rate of falls - subgrouped by exercise type.

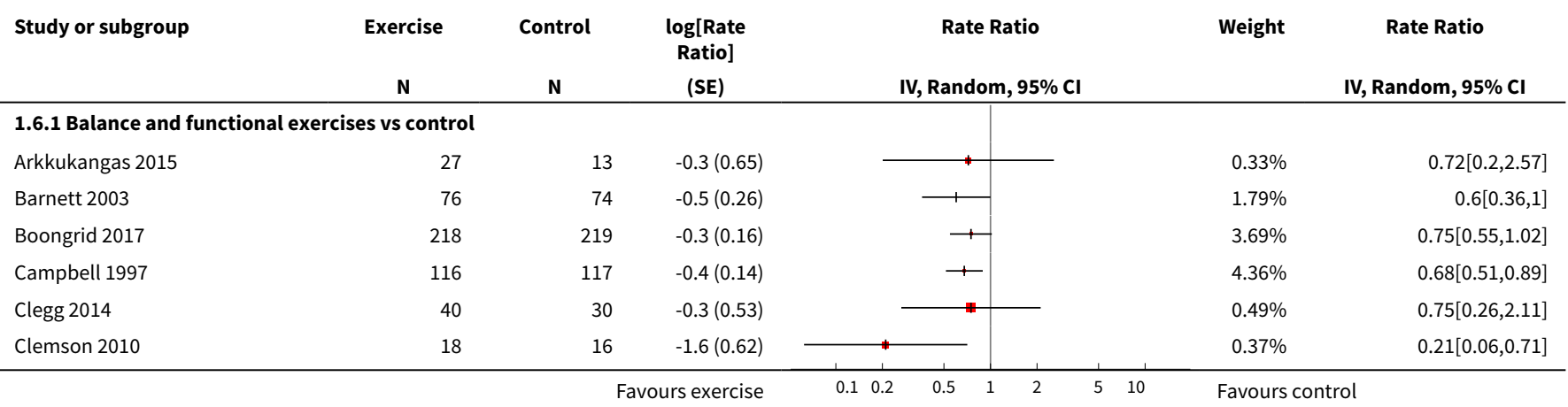




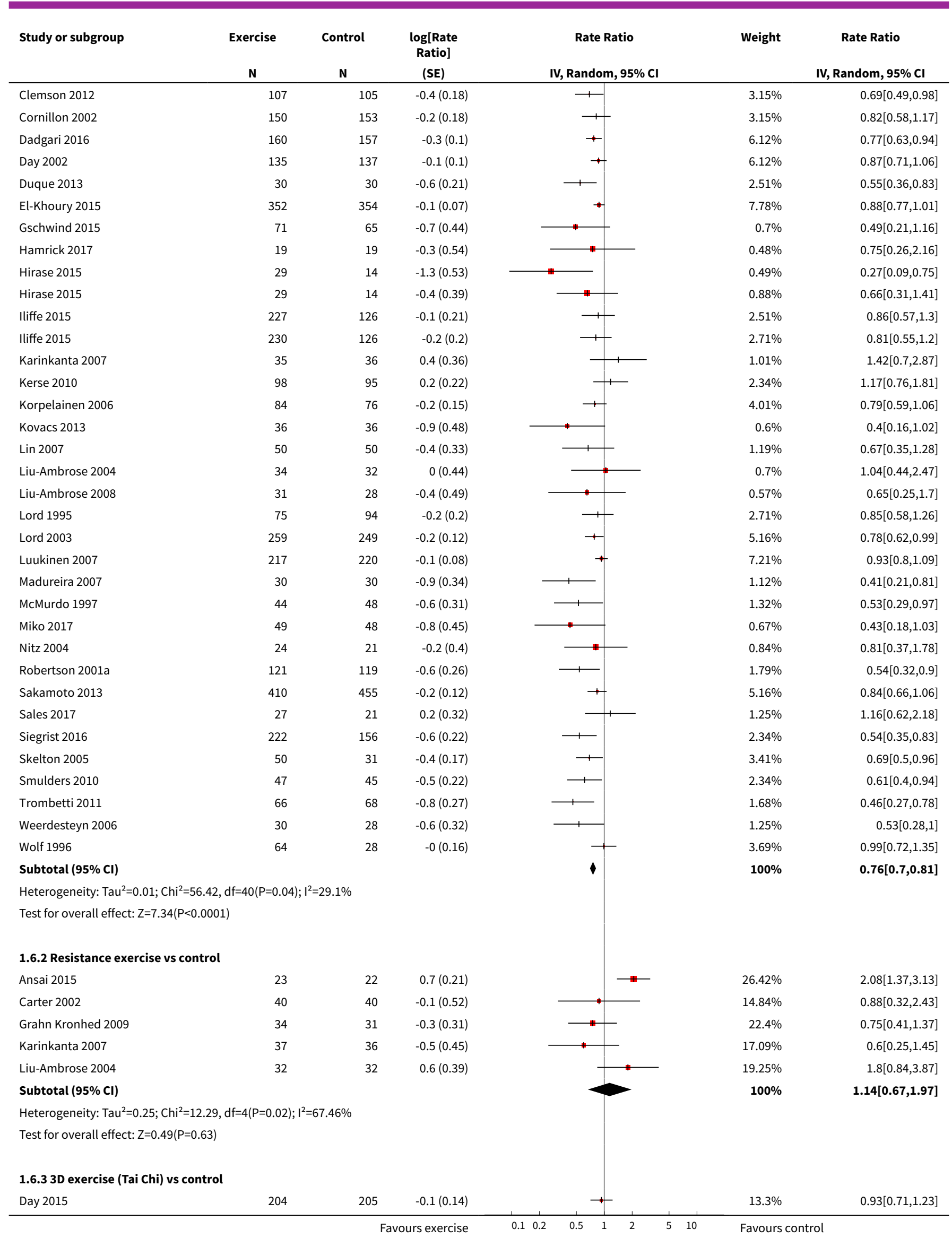




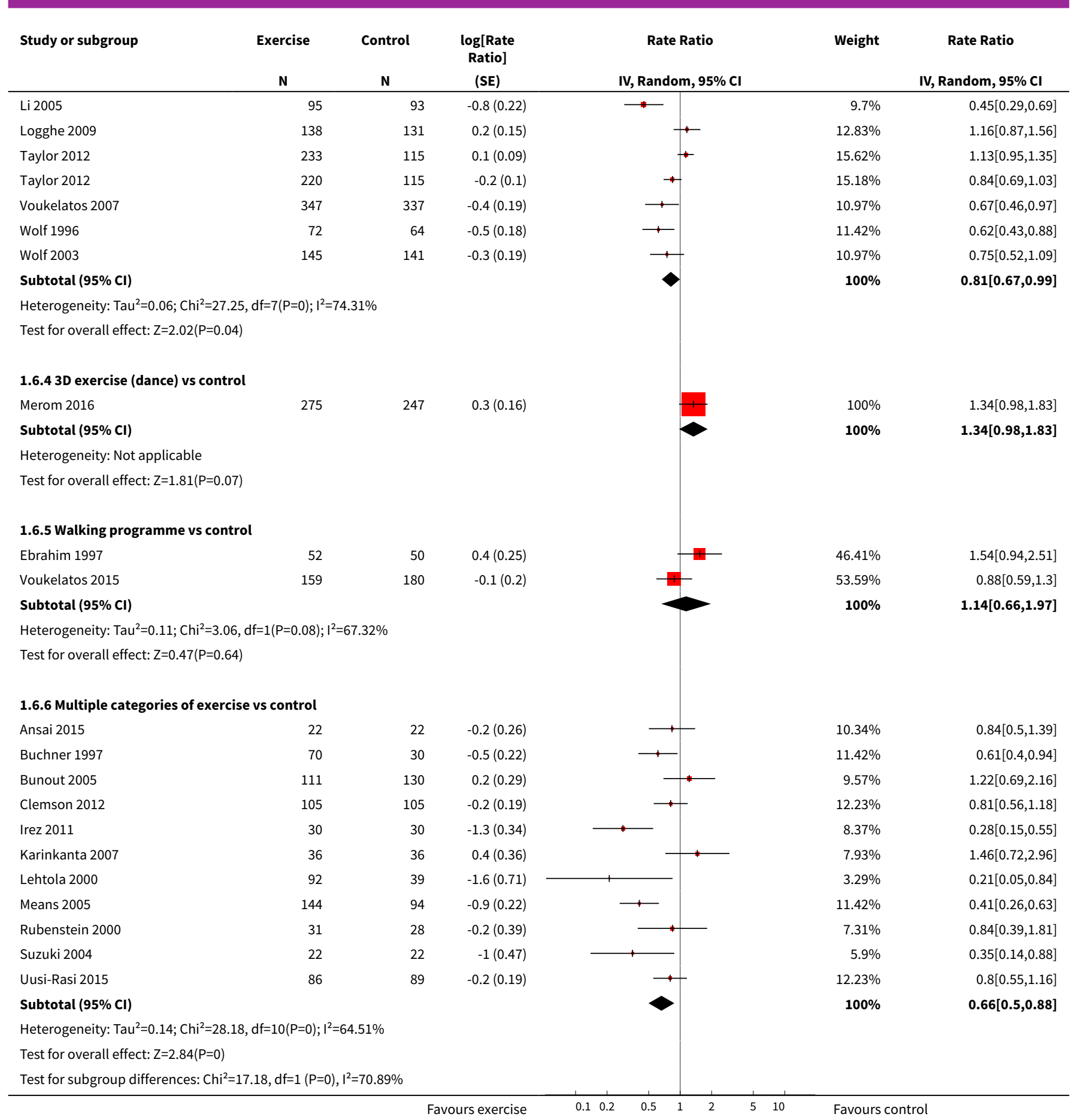

Analysis 1.7. Comparison 1 Exercise versus control (rate of falls), Outcome 7 Rate of falls - long-term follow-up by exercise type.

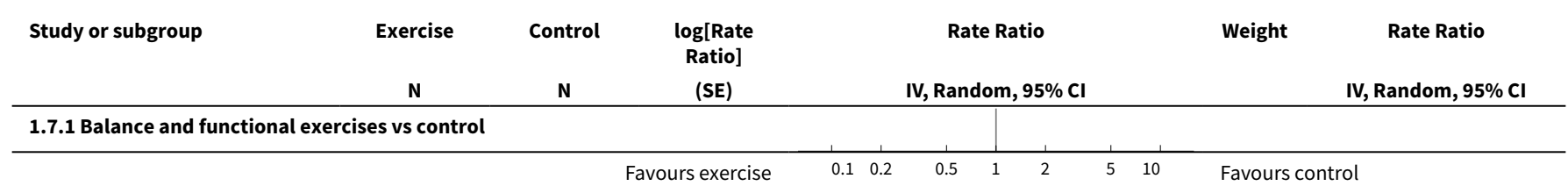




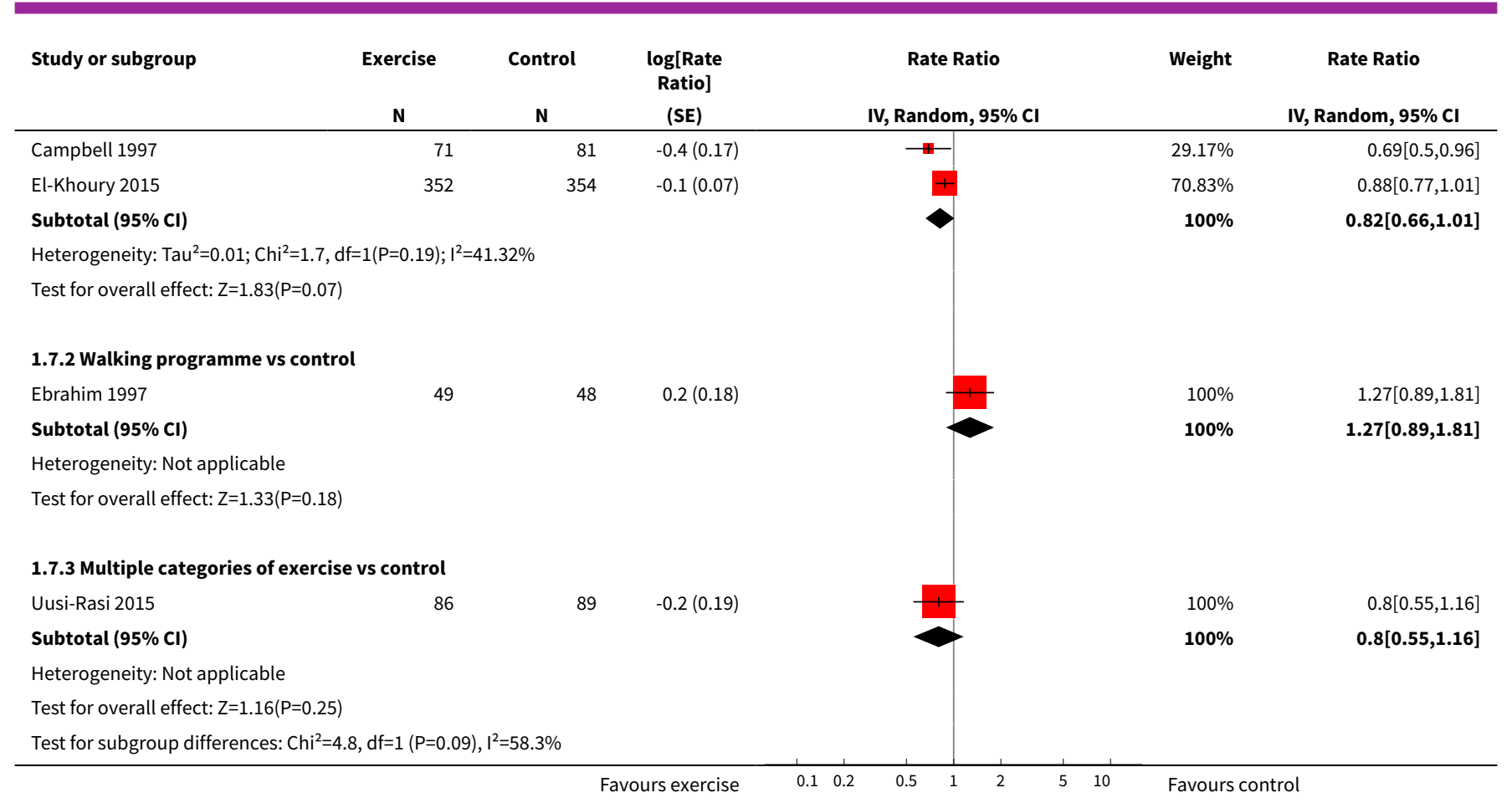

\section{Comparison 2. Exercise versus control (number of fallers)}

\begin{tabular}{|c|c|c|c|c|}
\hline Outcome or subgroup title & No. of studies & $\begin{array}{l}\text { No. of partici- } \\
\text { pants }\end{array}$ & Statistical method & Effect size \\
\hline 1 Number of fallers - overall analysis & 63 & 13518 & $\begin{array}{l}\text { Risk Ratio (Random, 95\% } \\
\mathrm{Cl} \text { ) }\end{array}$ & $0.85[0.81,0.89]$ \\
\hline $\begin{array}{l}2 \text { Number of fallers - subgrouped by } \\
\text { baseline fall risk }\end{array}$ & 63 & & $\begin{array}{l}\text { Risk Ratio (Random, 95\% } \\
\mathrm{Cl} \text { ) }\end{array}$ & Subtotals only \\
\hline 2.1 Not selected for high risk of falling & 28 & 6347 & $\begin{array}{l}\text { Risk Ratio (Random, 95\% } \\
\mathrm{Cl} \text { ) }\end{array}$ & $0.82[0.73,0.92]$ \\
\hline 2.2 Selected for high risk of falling & 35 & 7171 & $\begin{array}{l}\text { Risk Ratio (Random, 95\% } \\
\mathrm{Cl} \text { ) }\end{array}$ & $0.87[0.83,0.91]$ \\
\hline $\begin{array}{l}3 \text { Number of fallers - subgrouped by } \\
\text { age (threshold } 75 \text { years) }\end{array}$ & 63 & & $\begin{array}{l}\text { Risk Ratio (Random, 95\% } \\
\mathrm{CI} \text { ) }\end{array}$ & Subtotals only \\
\hline 3.1 Age $<75$ & 50 & 10346 & $\begin{array}{l}\text { Risk Ratio (Random, 95\% } \\
\mathrm{CI} \text { ) }\end{array}$ & $0.85[0.79,0.91]$ \\
\hline 3.2 Age $75+$ & 13 & 3172 & $\begin{array}{l}\text { Risk Ratio (Random, 95\% } \\
\mathrm{Cl} \text { ) }\end{array}$ & $0.86[0.80,0.92]$ \\
\hline $\begin{array}{l}4 \text { Number of fallers - subgrouped by } \\
\text { personnel }\end{array}$ & 62 & 13473 & $\begin{array}{l}\text { Risk Ratio (Random, 95\% } \\
\text { Cl) }\end{array}$ & $0.85[0.81,0.89]$ \\
\hline $\begin{array}{l}4.1 \text { Health professional delivering in- } \\
\text { tervention }\end{array}$ & 26 & 3747 & $\begin{array}{l}\text { Risk Ratio (Random, 95\% } \\
\text { Cl) }\end{array}$ & $0.82[0.74,0.91]$ \\
\hline
\end{tabular}




\begin{tabular}{|c|c|c|c|c|}
\hline Outcome or subgroup title & No. of studies & $\begin{array}{l}\text { No. of partici- } \\
\text { pants }\end{array}$ & Statistical method & Effect size \\
\hline $\begin{array}{l}4.2 \text { No health professional delivering } \\
\text { intervention }\end{array}$ & 36 & 9726 & $\begin{array}{l}\text { Risk Ratio (Random, 95\% } \\
\mathrm{Cl} \text { ) }\end{array}$ & $0.86[0.81,0.92]$ \\
\hline $\begin{array}{l}5 \text { Number of fallers - subgrouped by } \\
\text { group or individual exercise }\end{array}$ & 63 & 13518 & $\begin{array}{l}\text { Risk Ratio (Random, 95\% } \\
\mathrm{Cl} \text { ) }\end{array}$ & $0.85[0.81,0.89]$ \\
\hline 5.1 Group exercise & 48 & 9219 & $\begin{array}{l}\text { Risk Ratio (Random, 95\% } \\
\mathrm{Cl} \text { ) }\end{array}$ & $0.83[0.78,0.90]$ \\
\hline 5.2 Not group exercise & 16 & 4299 & $\begin{array}{l}\text { Risk Ratio (Random, 95\% } \\
\mathrm{Cl} \text { ) }\end{array}$ & $0.88[0.83,0.93]$ \\
\hline $\begin{array}{l}6 \text { Number of fallers - subgrouped by } \\
\text { exercise type }\end{array}$ & 63 & & $\begin{array}{l}\text { Risk Ratio (Random, 95\% } \\
\mathrm{Cl} \text { ) }\end{array}$ & Subtotals only \\
\hline $\begin{array}{l}\text { 6.1 Balance and functional exercises vs } \\
\text { control }\end{array}$ & 37 & 8288 & $\begin{array}{l}\text { Risk Ratio (Random, 95\% } \\
\mathrm{Cl} \text { ) }\end{array}$ & $0.87[0.82,0.91]$ \\
\hline 6.2 Resistance exercise vs control & 2 & 163 & $\begin{array}{l}\text { Risk Ratio (Random, 95\% } \\
\mathrm{Cl} \text { ) }\end{array}$ & $0.81[0.57,1.15]$ \\
\hline 6.3 3D exercise (Tai Chi) vs control & 8 & 2677 & $\begin{array}{l}\text { Risk Ratio (Random, 95\% } \\
\mathrm{Cl} \text { ) }\end{array}$ & $0.80[0.70,0.91]$ \\
\hline 6.4 3D exercise (dance) vs control & 1 & 522 & $\begin{array}{l}\text { Risk Ratio (Random, 95\% } \\
\mathrm{Cl} \text { ) }\end{array}$ & $1.35[0.83,2.20]$ \\
\hline $\begin{array}{l}6.5 \text { Multiple categories of exercise vs } \\
\text { control }\end{array}$ & 17 & 1623 & $\begin{array}{l}\text { Risk Ratio (Random, 95\% } \\
\mathrm{Cl} \text { ) }\end{array}$ & $0.78[0.64,0.96]$ \\
\hline 6.6 Walking programme vs control & 2 & 441 & $\begin{array}{l}\text { Risk Ratio (Random, 95\% } \\
\mathrm{Cl} \text { ) }\end{array}$ & $1.05[0.71,1.54]$ \\
\hline $\begin{array}{l}7 \text { Number of fallers - long-term fol- } \\
\text { low-up by exercise type }\end{array}$ & 3 & & Risk Ratio (Fixed, 95\% Cl) & Subtotals only \\
\hline $\begin{array}{l}\text { 7.1 Balance and functional exercises vs } \\
\text { control }\end{array}$ & 2 & 1325 & Risk Ratio (Fixed, 95\% Cl) & $0.86[0.78,0.94]$ \\
\hline $\begin{array}{l}7.2 \text { Multiple categories of exercise vs } \\
\text { control }\end{array}$ & 1 & 175 & Risk Ratio (Fixed, 95\% Cl) & $1.01[0.74,1.38]$ \\
\hline
\end{tabular}

\section{Analysis 2.1. Comparison 2 Exercise versus control (number of fallers), Outcome 1 Number of fallers - overall analysis.}

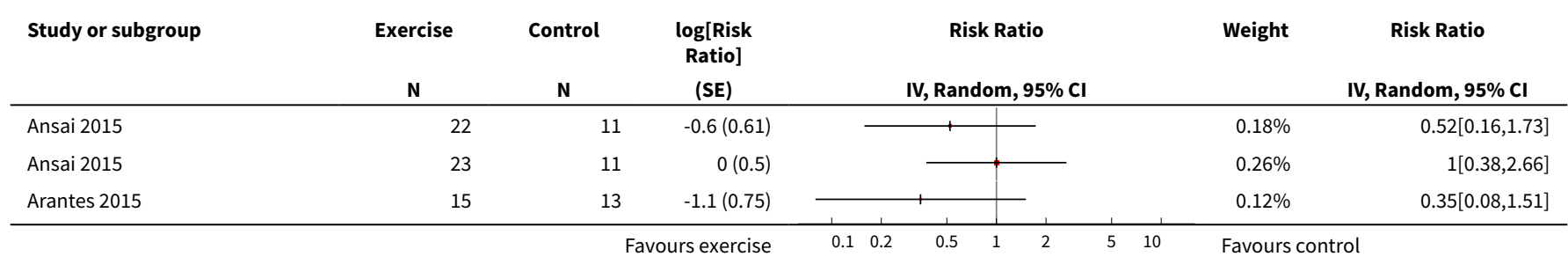




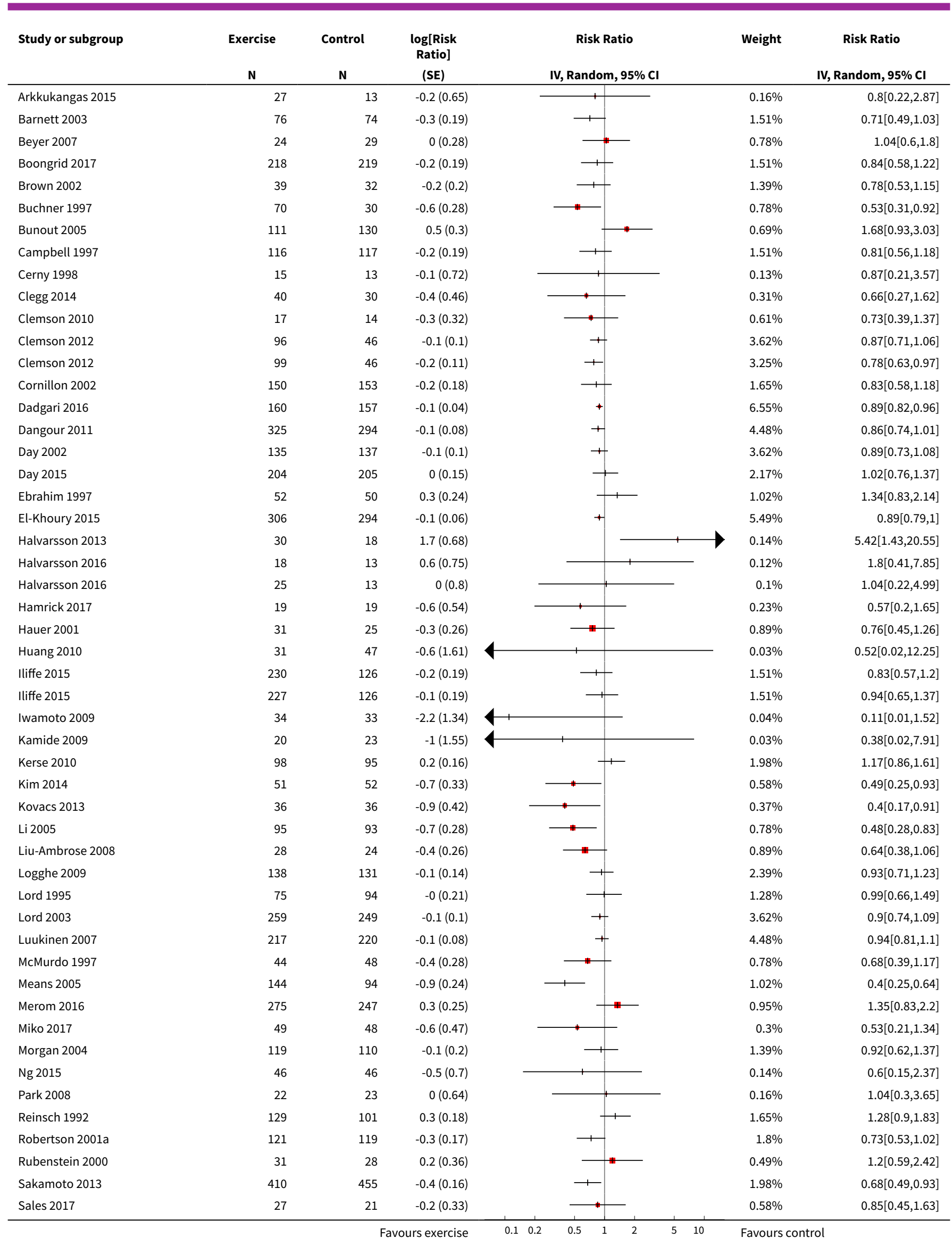




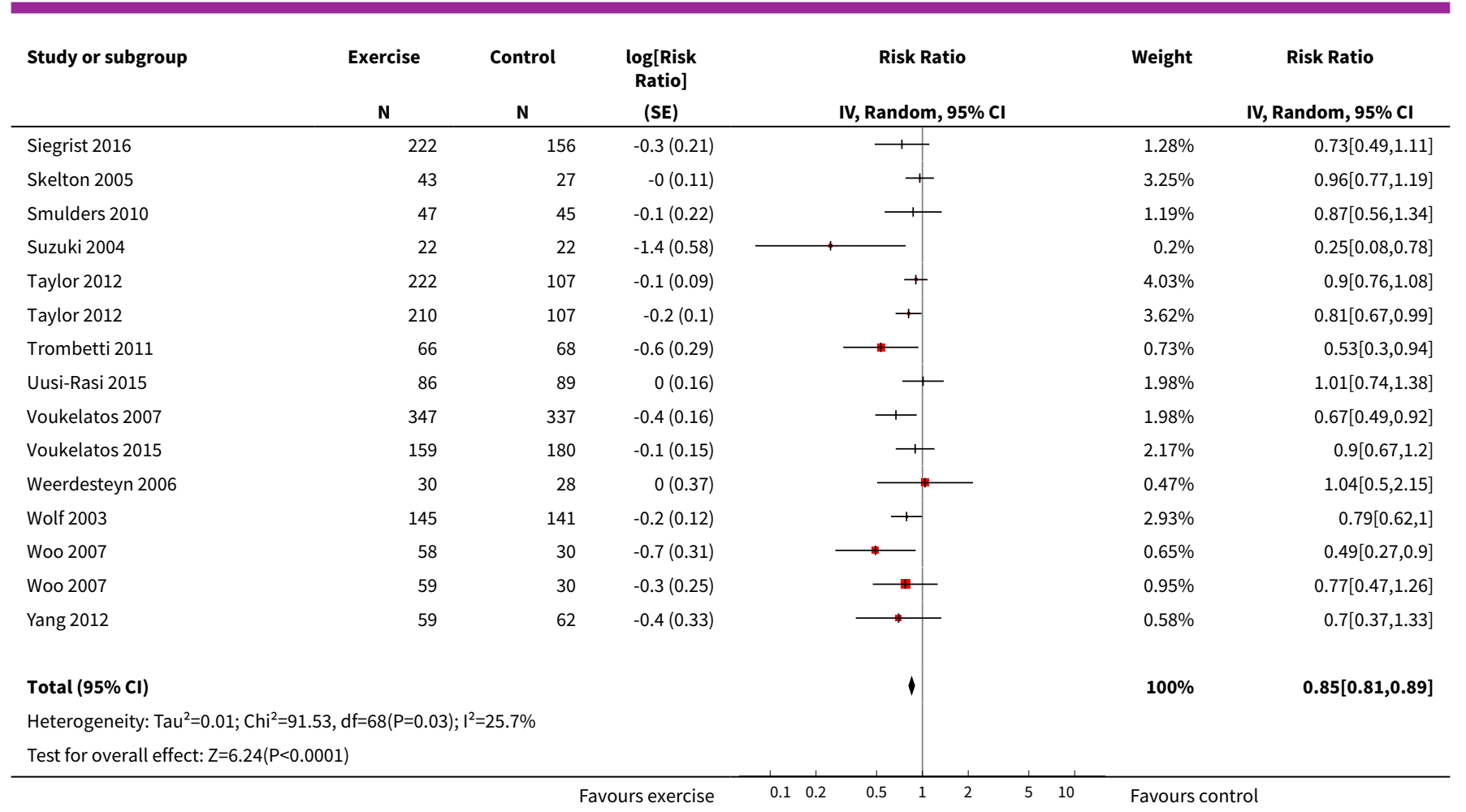

Analysis 2.2. Comparison 2 Exercise versus control (number of fallers), Outcome 2 Number of fallers - subgrouped by baseline fall risk.

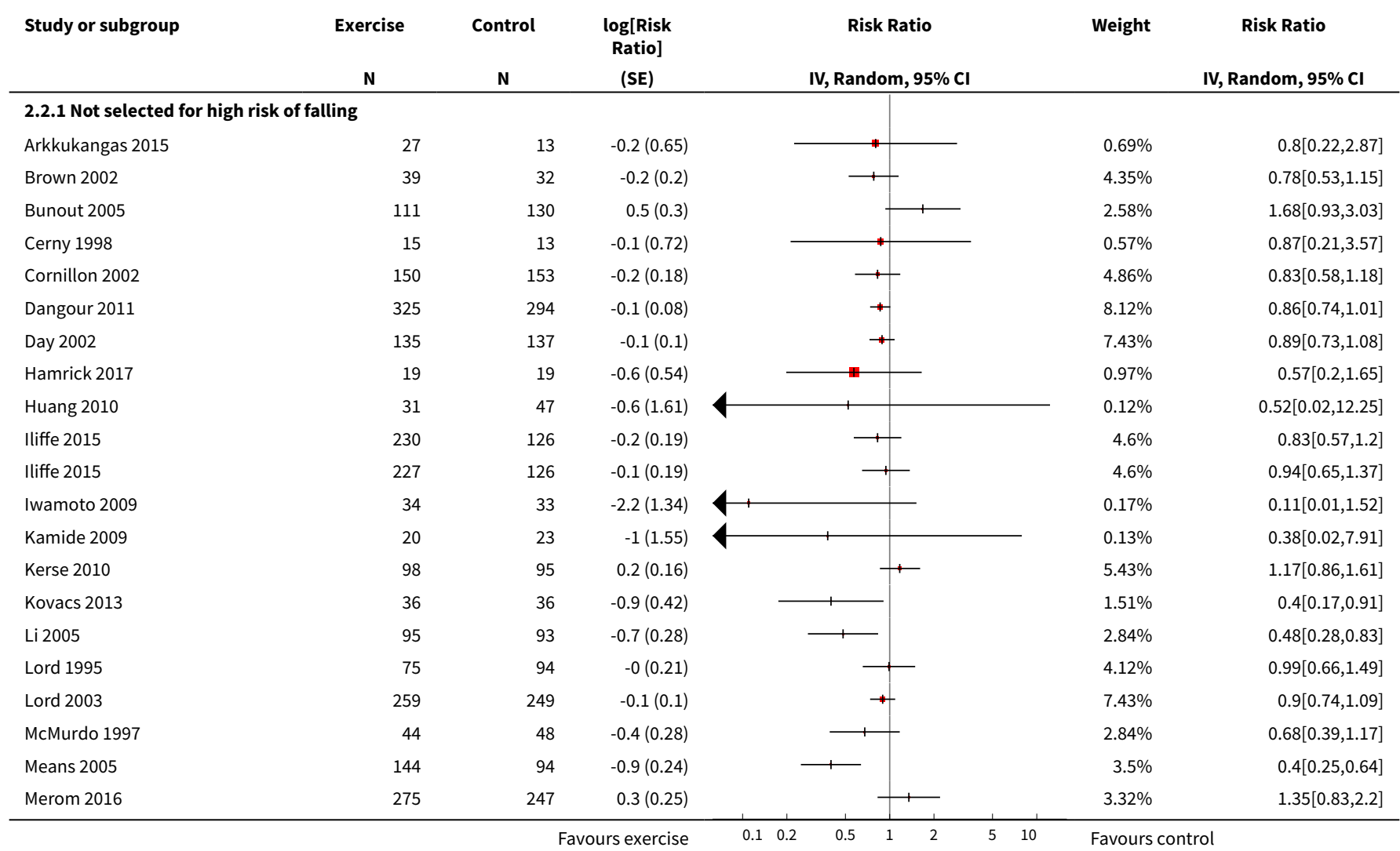




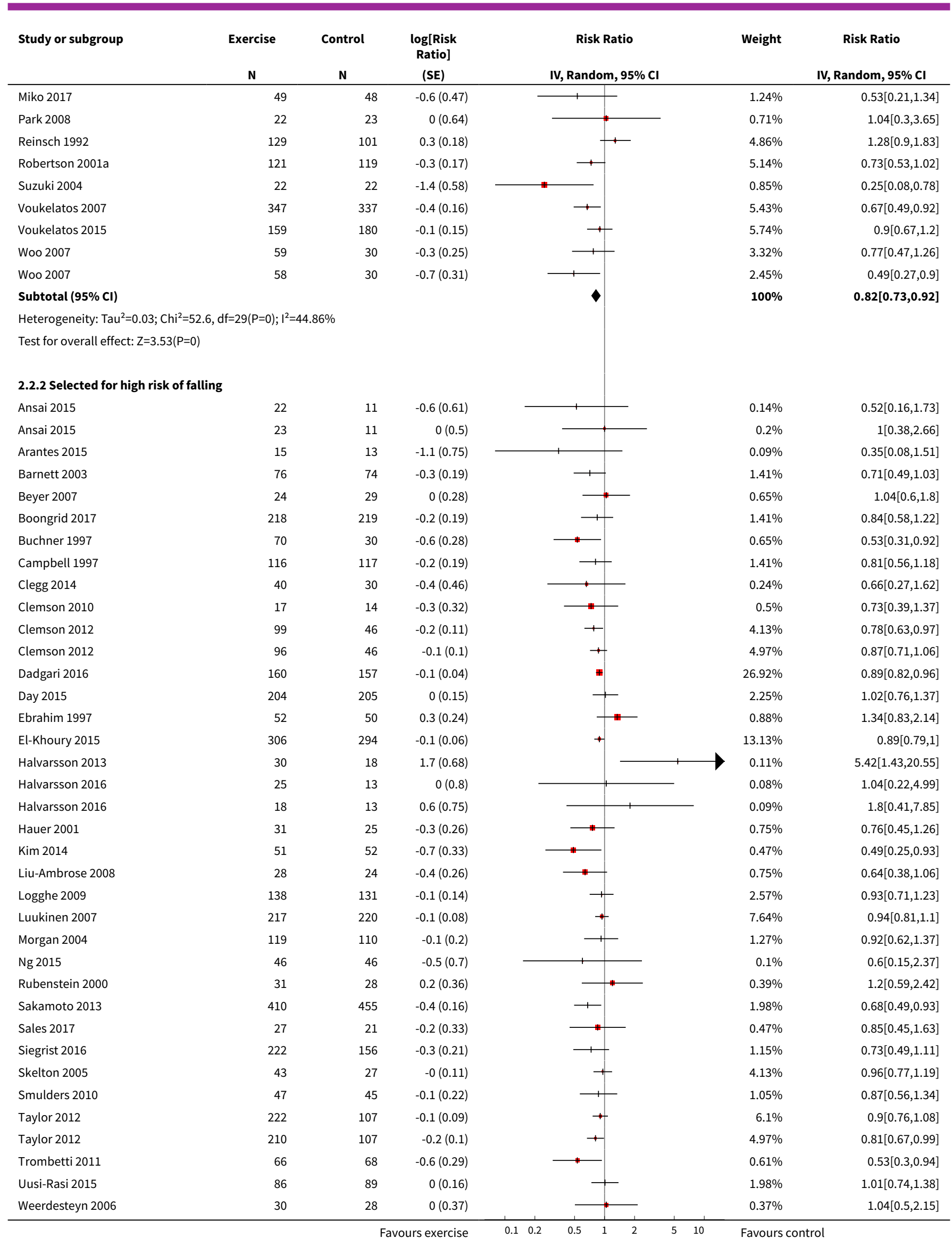




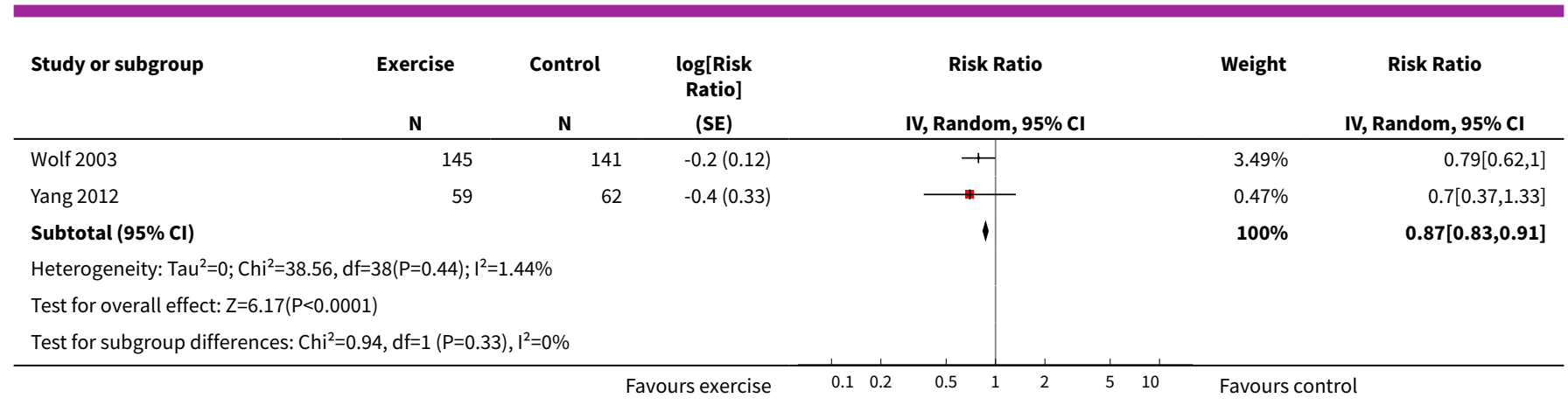

Analysis 2.3. Comparison 2 Exercise versus control (number of fallers), Outcome 3 Number of fallers - subgrouped by age (threshold 75 years).

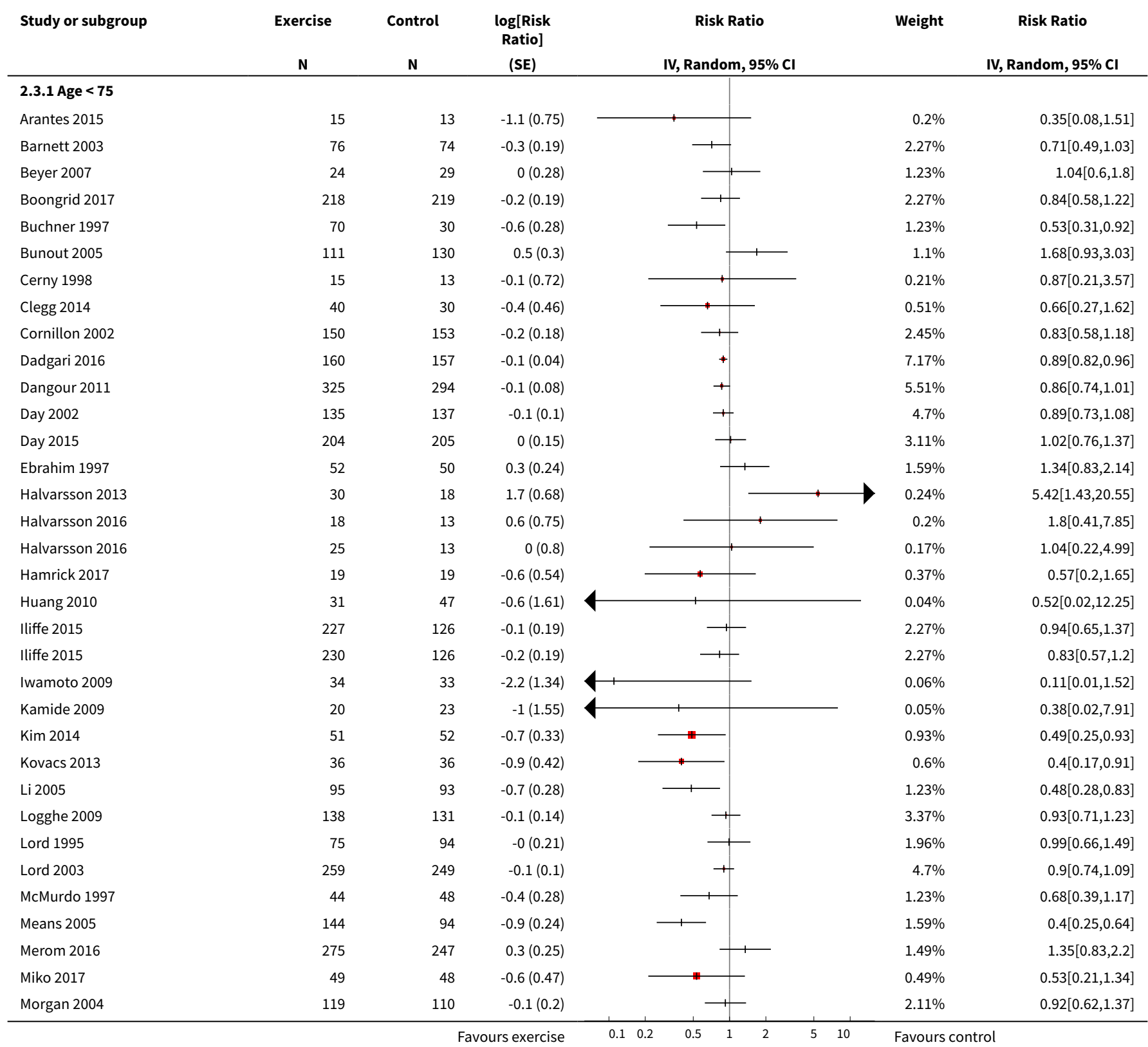




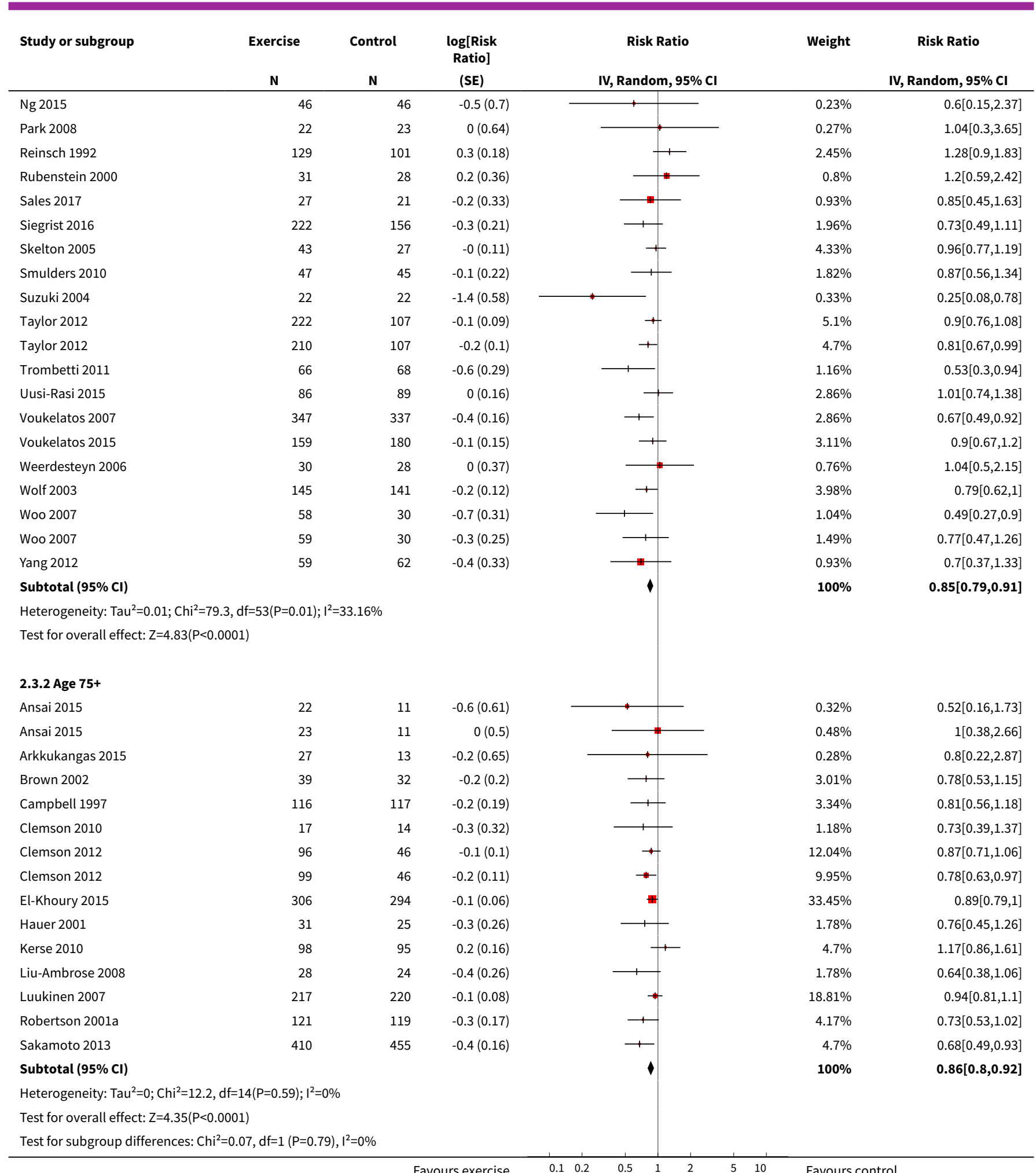

Favours exercise 
Analysis 2.4. Comparison 2 Exercise versus control (number of fallers), Outcome 4 Number of fallers - subgrouped by personnel.

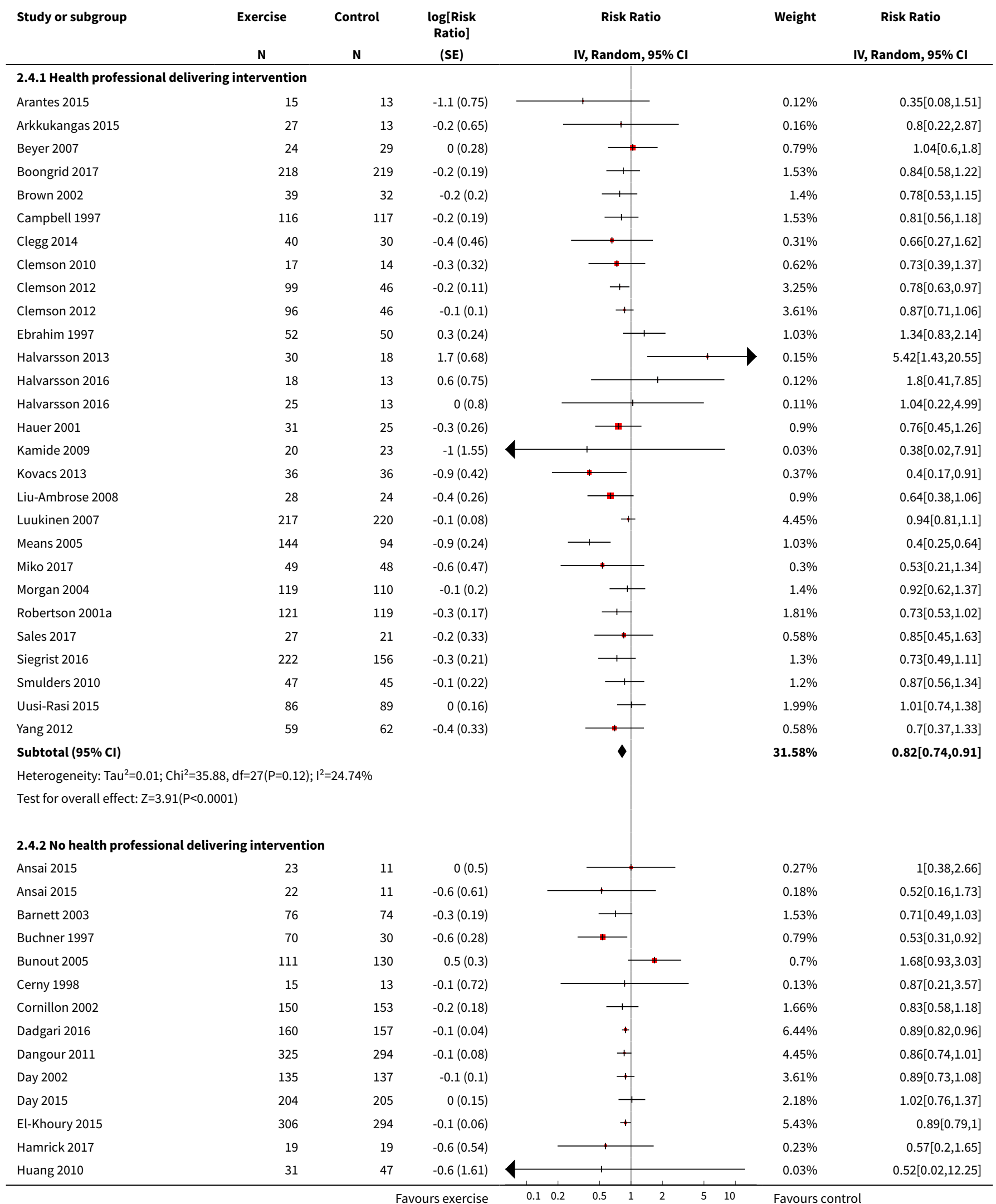




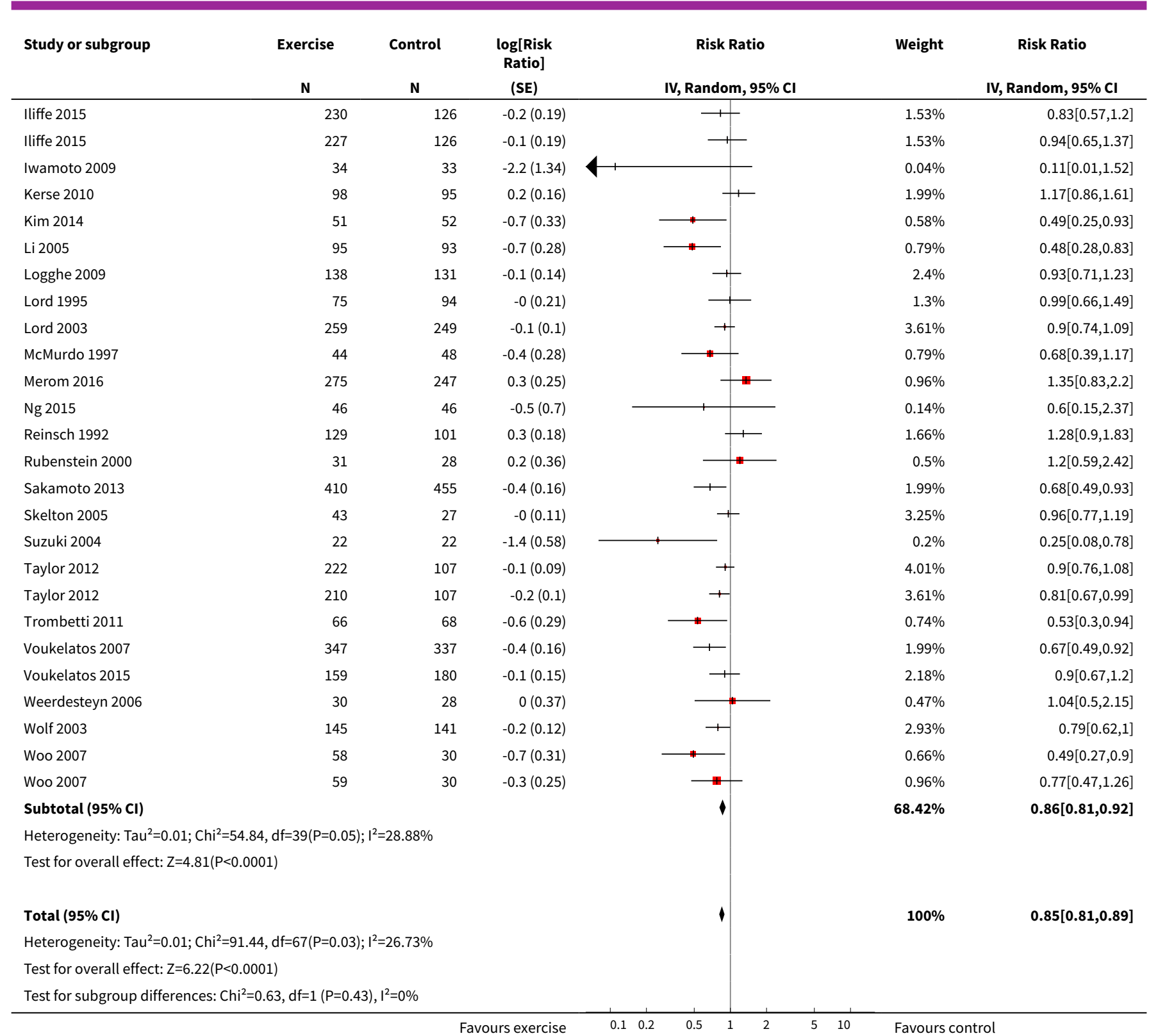

Analysis 2.5. Comparison 2 Exercise versus control (number of fallers), Outcome 5 Number of fallers - subgrouped by group or individual exercise.

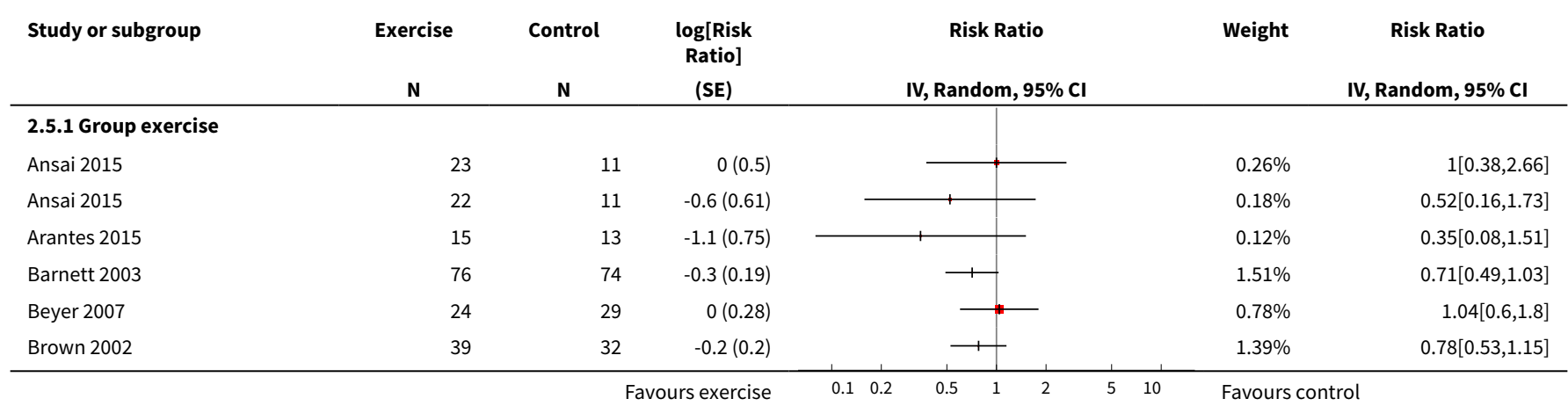




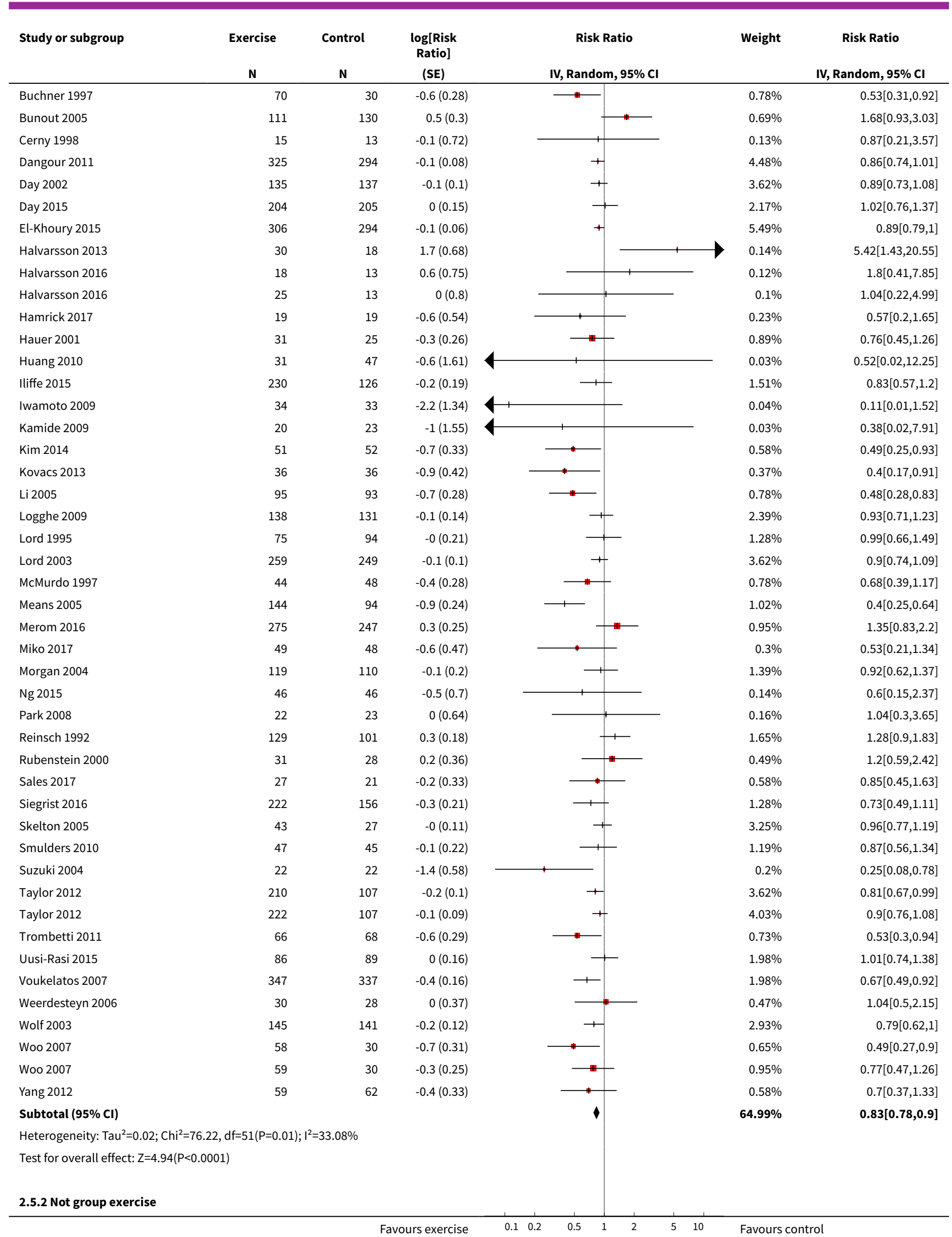




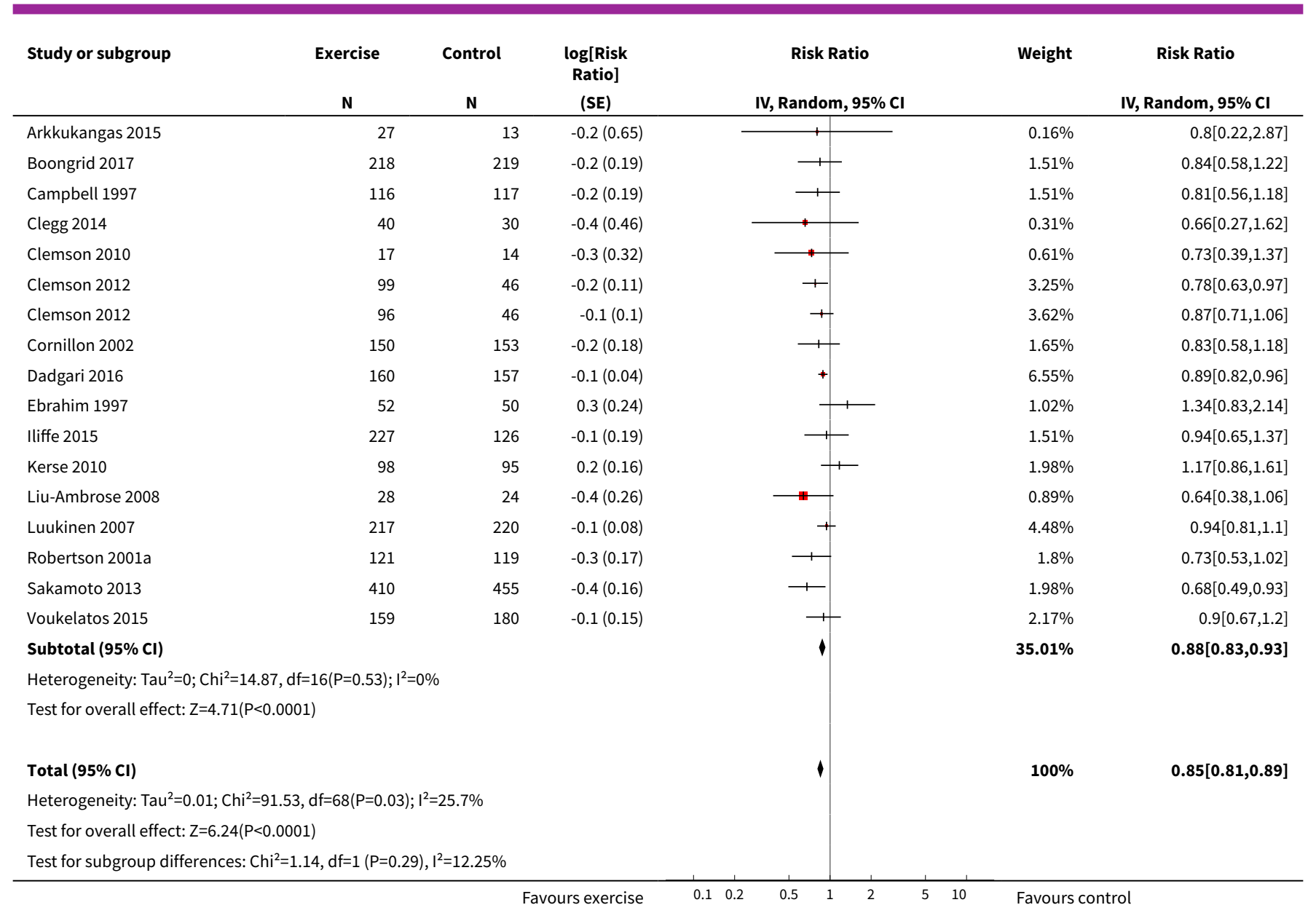

Analysis 2.6. Comparison 2 Exercise versus control (number of fallers), Outcome 6 Number of fallers - subgrouped by exercise type.

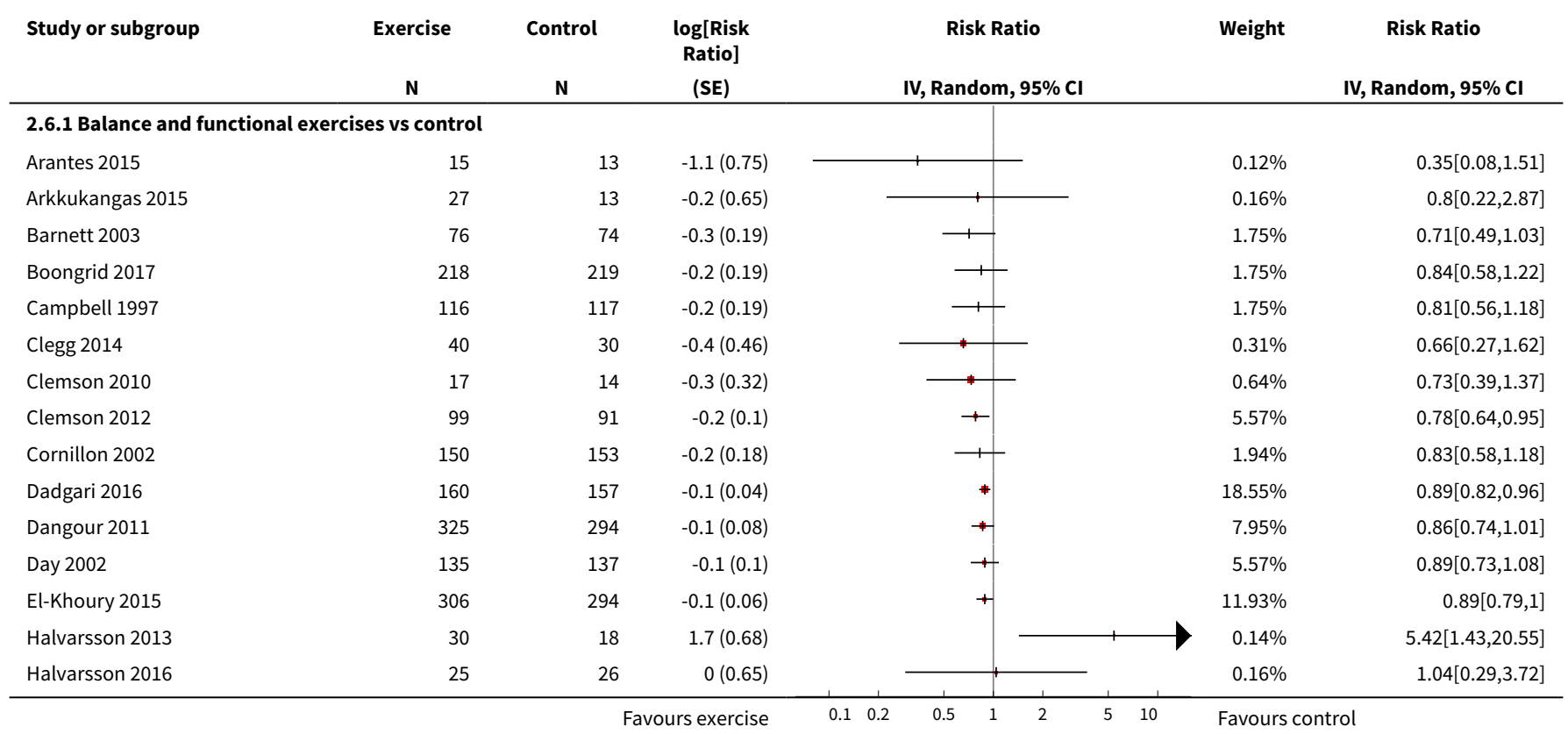




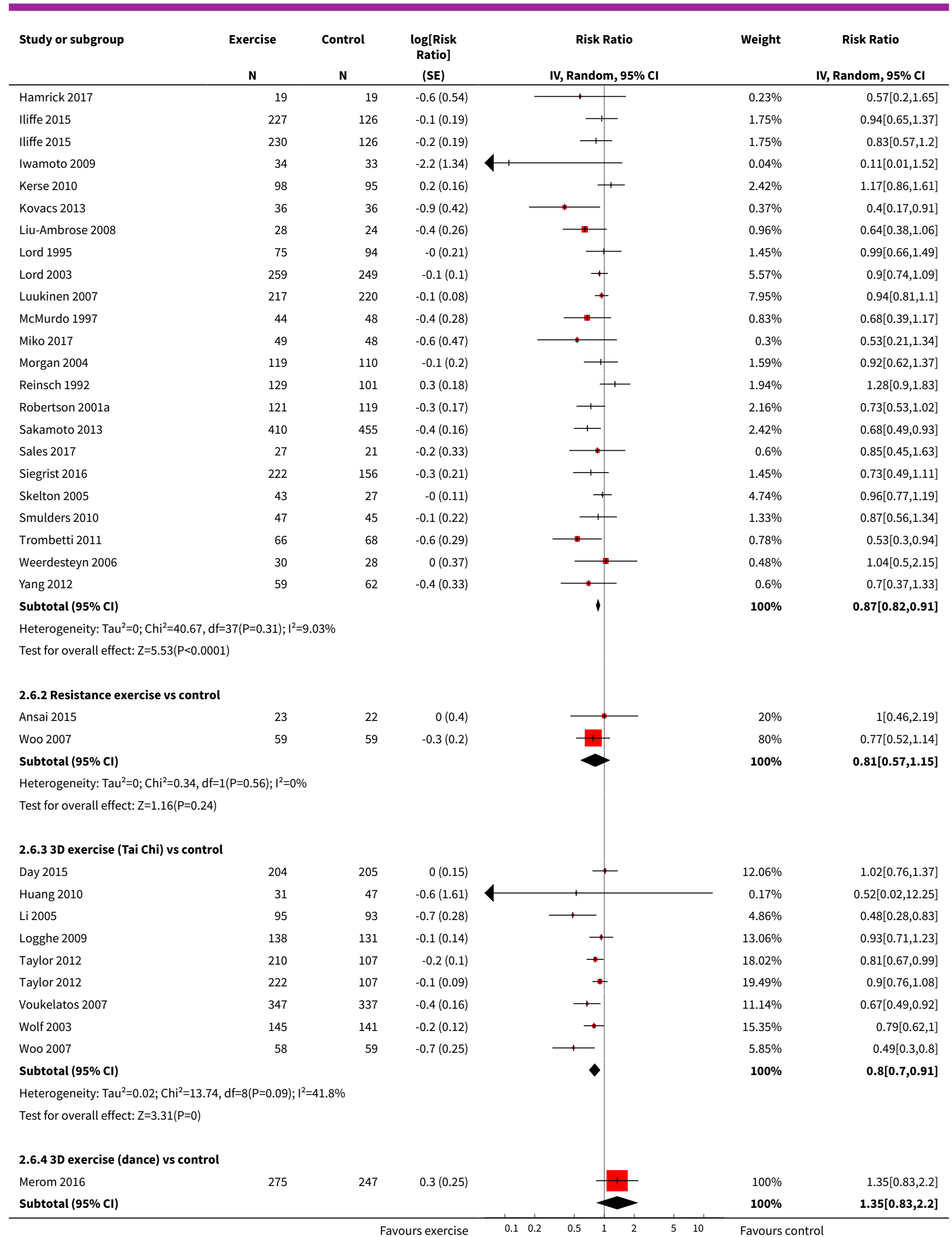




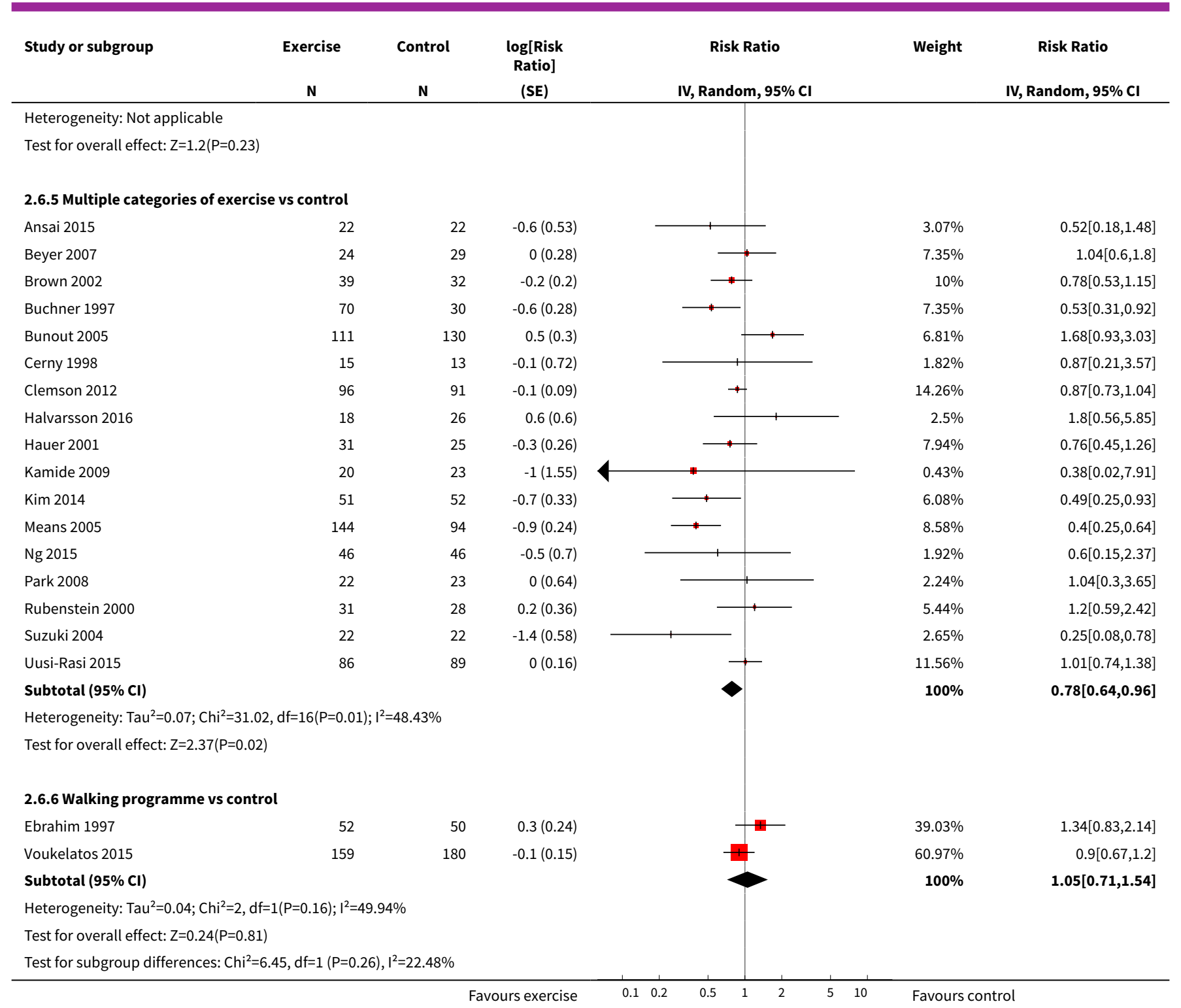

\section{Analysis 2.7. Comparison 2 Exercise versus control (number of fallers), Outcome 7 Number of fallers - long-term follow-up by exercise type.}

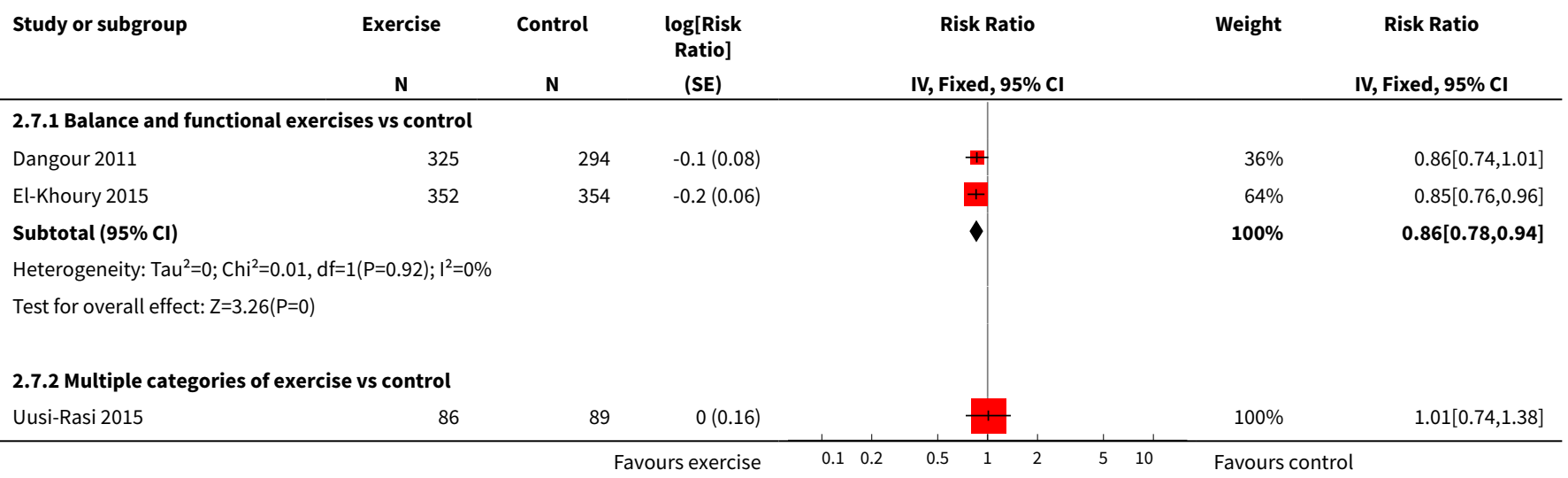




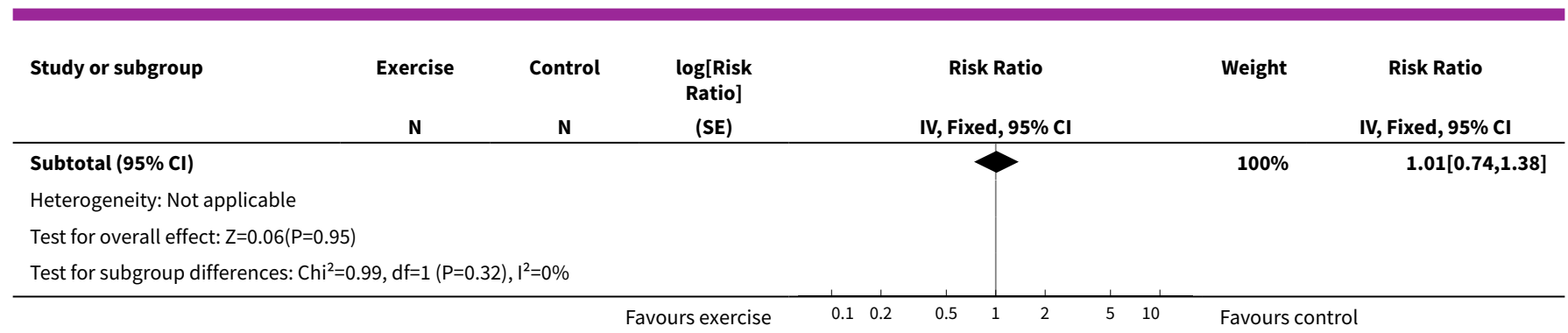

\section{Comparison 3. Exercise versus control (number of people with fractures)}

\begin{tabular}{|c|c|c|c|c|}
\hline Outcome or subgroup title & No. of studies & $\begin{array}{l}\text { No. of partici- } \\
\text { pants }\end{array}$ & Statistical method & Effect size \\
\hline $\begin{array}{l}1 \text { Number of people who experienced one or } \\
\text { more fall-related fractures- overall analysis }\end{array}$ & 10 & 4047 & $\begin{array}{l}\text { Risk Ratio (Random, } \\
95 \% \mathrm{Cl} \text { ) }\end{array}$ & $0.73[0.56,0.95]$ \\
\hline $\begin{array}{l}2 \text { Number of people who experienced one or } \\
\text { more fall-related fractures - subgrouped by } \\
\text { baseline falls risk }\end{array}$ & 10 & & $\begin{array}{l}\text { Risk Ratio (Random, } \\
95 \% \mathrm{Cl} \text { ) }\end{array}$ & Subtotals only \\
\hline 2.1 Not selected for high risk of falling & 5 & 1255 & $\begin{array}{l}\text { Risk Ratio (Random, } \\
95 \% \mathrm{Cl} \text { ) }\end{array}$ & $0.48[0.26,0.91]$ \\
\hline 2.2 Selected for high risk of falling & 5 & 2792 & $\begin{array}{l}\text { Risk Ratio (Random, } \\
95 \% \mathrm{Cl} \text { ) }\end{array}$ & $0.80[0.60,1.07]$ \\
\hline $\begin{array}{l}3 \text { Number of people who experienced one or } \\
\text { more fall-related fractures - subgrouped by } \\
\text { age (threshold } 75 \text { years) }\end{array}$ & 10 & & $\begin{array}{l}\text { Risk Ratio (Random, } \\
95 \% \mathrm{Cl} \text { ) }\end{array}$ & Subtotals only \\
\hline 3.1 Age $<75$ & 7 & 1307 & $\begin{array}{l}\text { Risk Ratio (Random, } \\
95 \% \mathrm{Cl} \text { ) }\end{array}$ & $0.53[0.29,0.96]$ \\
\hline 3.2 Age $75+$ & 3 & 2740 & $\begin{array}{l}\text { Risk Ratio (Random, } \\
95 \% \mathrm{Cl} \text { ) }\end{array}$ & $0.61[0.31,1.20]$ \\
\hline $\begin{array}{l}4 \text { Number of people who experienced one or } \\
\text { more fall-related fractures - subgrouped by } \\
\text { exercise type }\end{array}$ & 10 & & $\begin{array}{l}\text { Risk Ratio (Random, } \\
95 \% \mathrm{Cl} \text { ) }\end{array}$ & Subtotals only \\
\hline $\begin{array}{l}\text { 4.1 Balance and functional exercises vs con- } \\
\text { trol }\end{array}$ & 7 & 2139 & $\begin{array}{l}\text { Risk Ratio (Random, } \\
95 \% \mathrm{Cl} \text { ) }\end{array}$ & $0.44[0.25,0.76]$ \\
\hline 4.2 Resistance exercise vs control & 1 & 73 & $\begin{array}{l}\text { Risk Ratio (Random, } \\
95 \% \mathrm{Cl} \text { ) }\end{array}$ & $0.97[0.14,6.49]$ \\
\hline 4.3 Walking programme vs control & 1 & 97 & $\begin{array}{l}\text { Risk Ratio (Random, } \\
95 \% \mathrm{Cl} \text { ) }\end{array}$ & $0.66[0.11,3.76]$ \\
\hline 4.4 Multiple categories of exercise vs control & 3 & 1810 & $\begin{array}{l}\text { Risk Ratio (Random, } \\
95 \% \mathrm{Cl} \text { ) }\end{array}$ & $0.85[0.62,1.16]$ \\
\hline
\end{tabular}




\begin{tabular}{lllll}
\hline Outcome or subgroup title & No. of studies & $\begin{array}{l}\text { No. of partici- } \\
\text { pants }\end{array}$ & Statistical method & Effect size \\
\hline $\begin{array}{l}5 \text { Number of people who experienced one } \\
\text { or more fall-related fractures - long-term fol- } \\
\text { low-up by exercise type }\end{array}$ & 3 & 2351 & $\begin{array}{l}\text { Risk Ratio (Fixed, 95\% } \\
\text { Cl) }\end{array}$ & $0.93[0.69,1.25]$ \\
\hline $\begin{array}{l}5.1 \text { Balance and functional exercises vs con- } \\
\text { trol }\end{array}$ & 1 & 619 & Risk Ratio (Fixed, 95\% & $1.80[0.46,7.11]$ \\
\hline $\begin{array}{l}5.2 \text { Walking programme vs control } \\
\text { Cl) }\end{array}$ & Risk Ratio (Fixed, 95\% & $1.46[0.44,4.83]$ \\
\hline 5.3 Multiple categories of exercise vs control & 1 & 97 & Cl) & $\begin{array}{l}\text { Risk Ratio (Fixed, 95\% } \\
\text { Cl) }\end{array}$ \\
\hline
\end{tabular}

Analysis 3.1. Comparison 3 Exercise versus control (number of people with fractures), Outcome 1 Number of people who experienced one or more fall-related fractures- overall analysis.

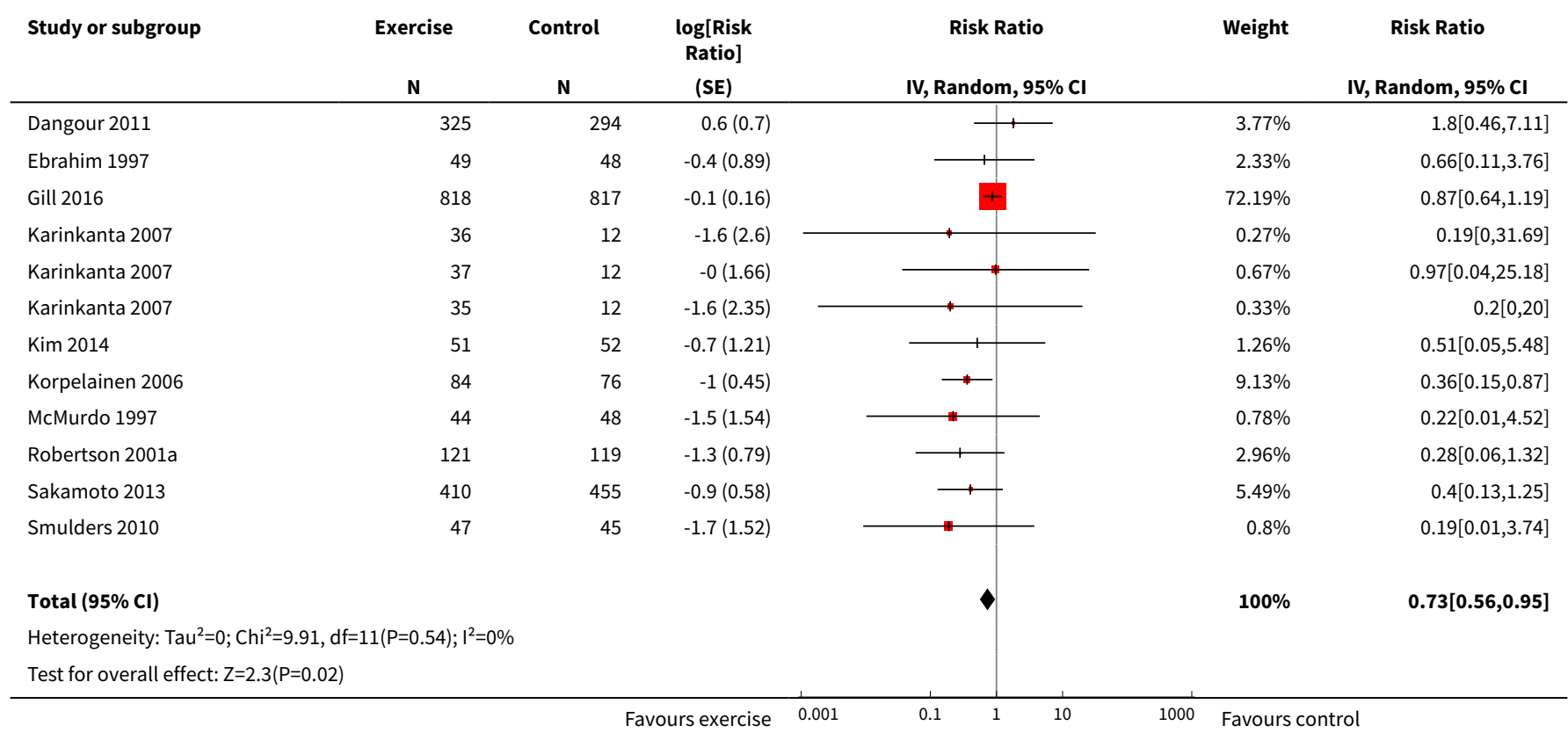

Analysis 3.2. Comparison 3 Exercise versus control (number of people with fractures), Outcome 2 Number of people who experienced one or more fall-related fractures - subgrouped by baseline falls risk.

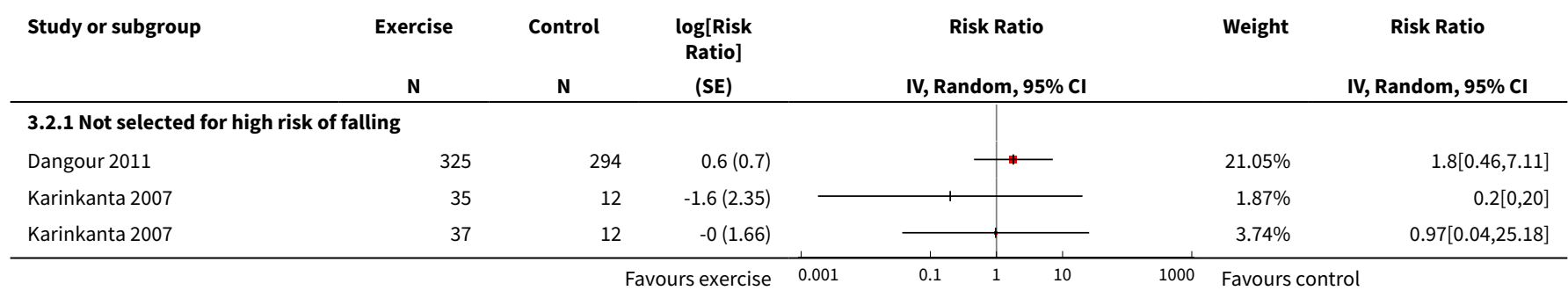




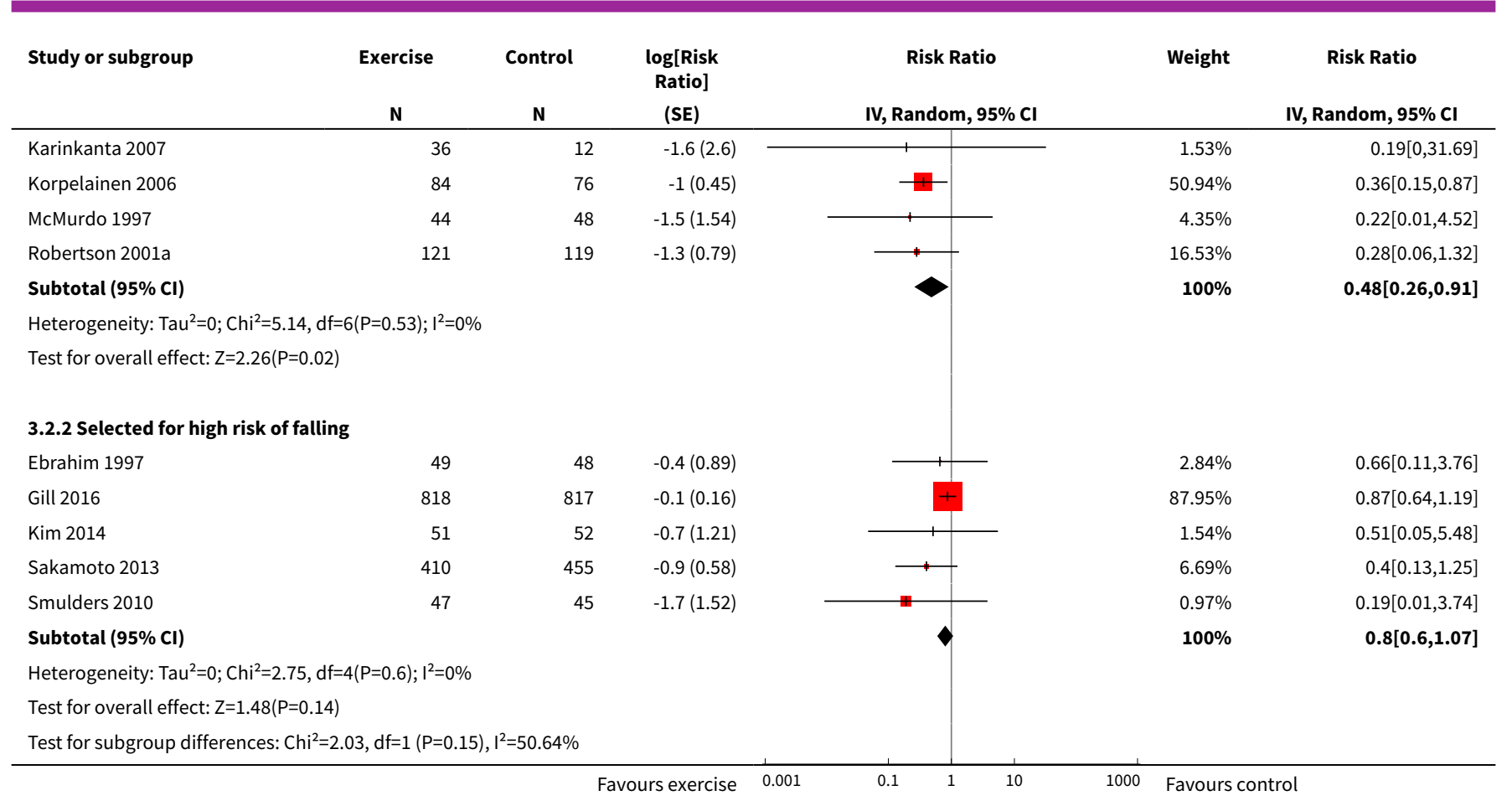

\section{Analysis 3.3. Comparison 3 Exercise versus control (number of people with fractures), Outcome 3 Number of people who experienced one or more fall-related fractures - subgrouped by age (threshold 75 years).}

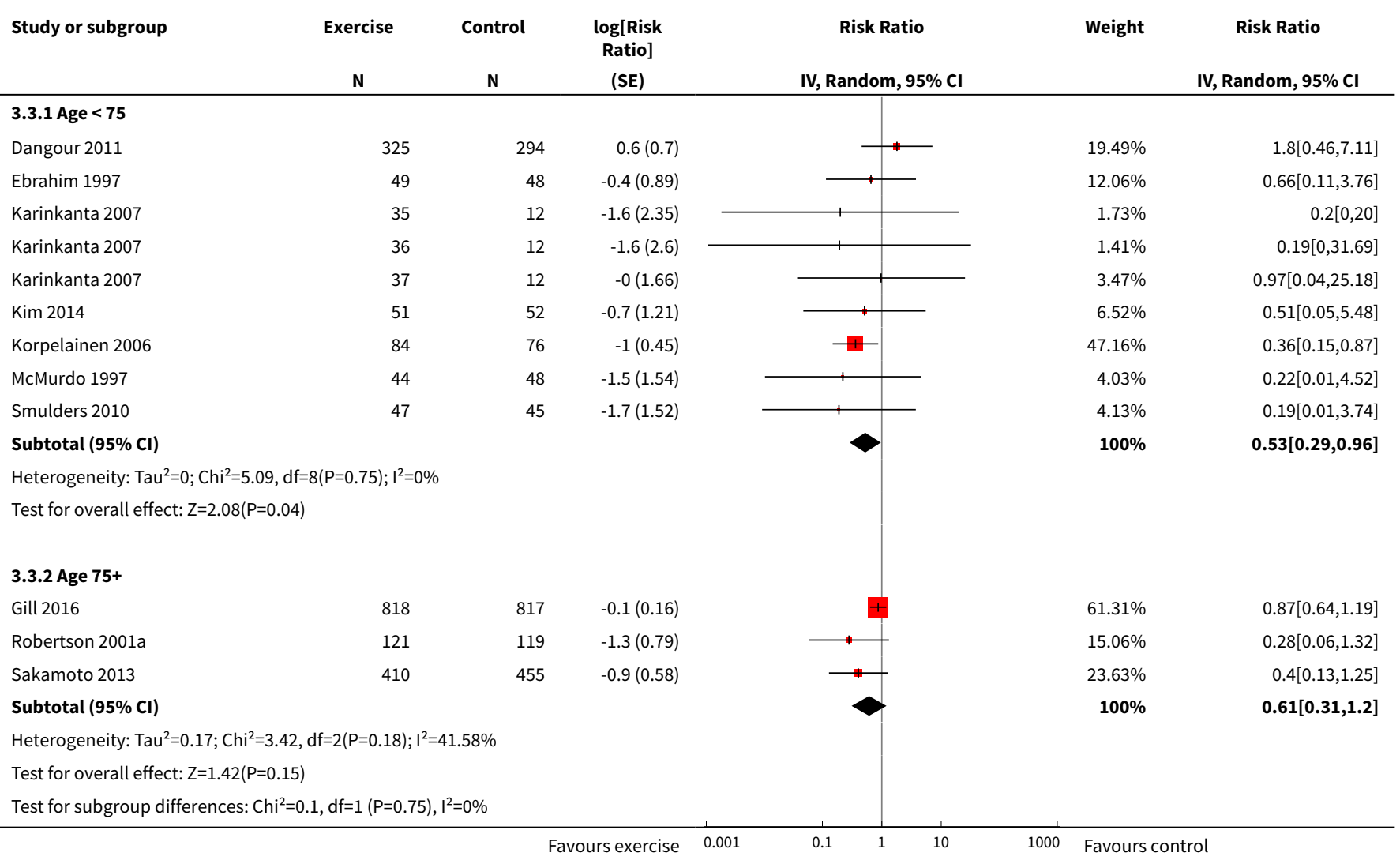


Analysis 3.4. Comparison 3 Exercise versus control (number of people with fractures), Outcome 4 Number of people who experienced one or more fall-related fractures - subgrouped by exercise type.

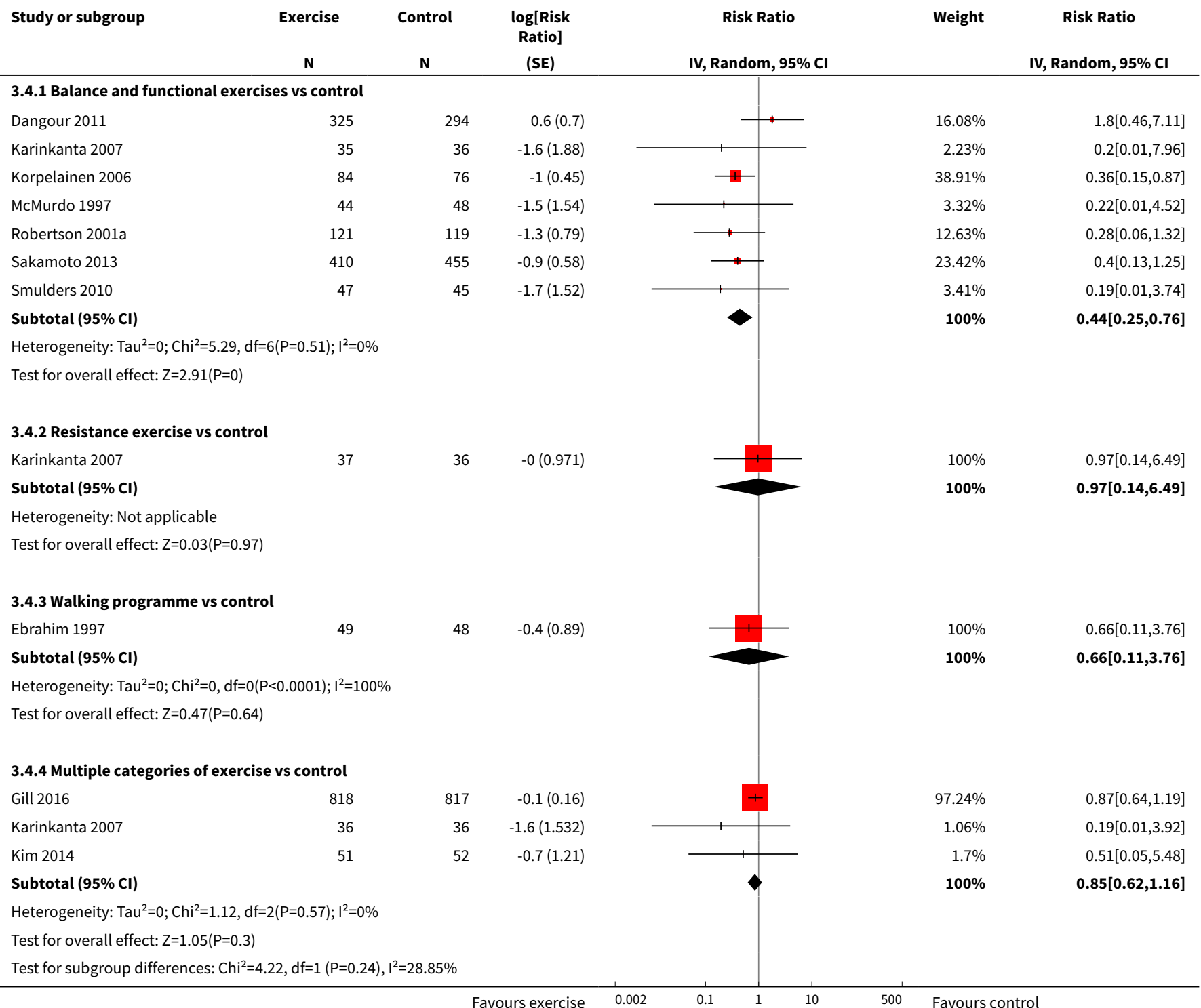

Analysis 3.5. Comparison 3 Exercise versus control (number of people with fractures), Outcome 5 Number of people who experienced one or more fall-related fractures - long-term follow-up by exercise type.

$\begin{array}{llcl}\text { Study or subgroup } & \text { Exercise } & \begin{array}{c}\log [\text { Risk } \\ \text { Ratio }\end{array} & \text { Risk Ratio }\end{array}$

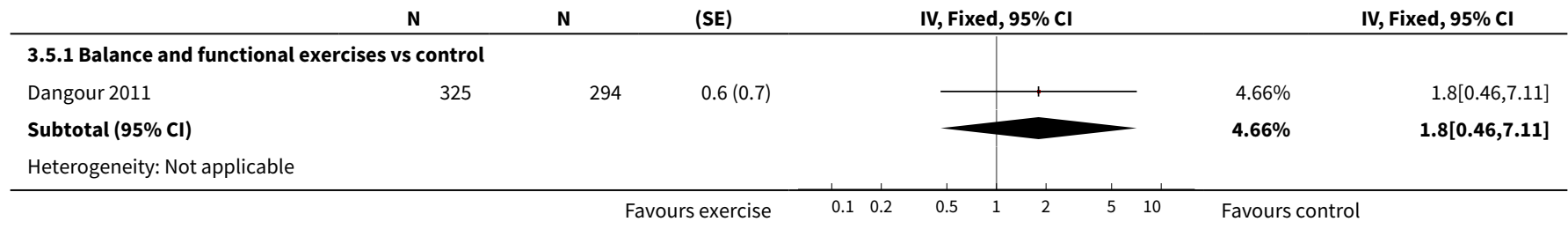




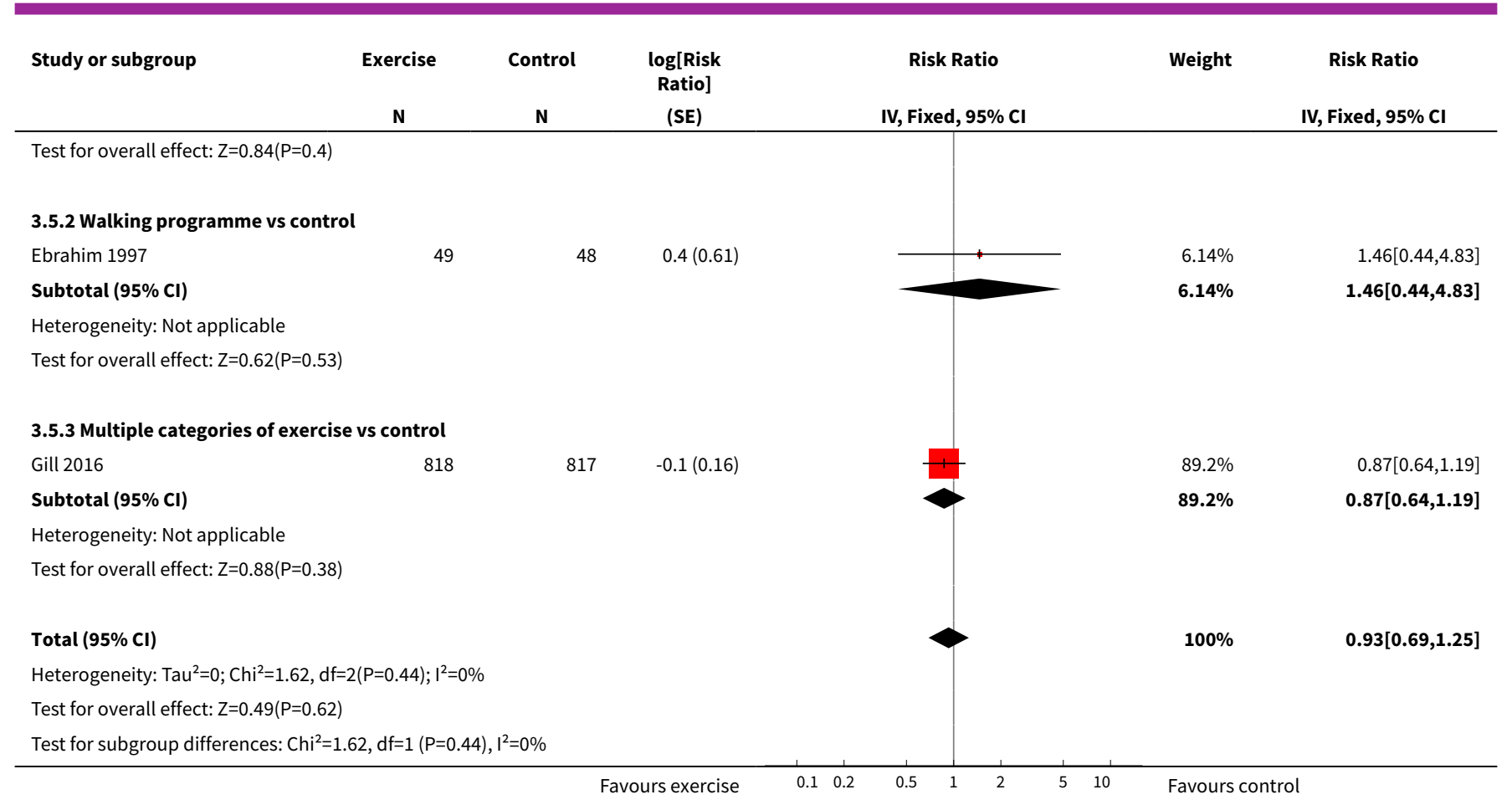

Comparison 4. Exercise versus control (number of people with falls that resulted in hospital admission)

\begin{tabular}{lllll}
\hline Outcome or subgroup title & No. of studies & $\begin{array}{l}\text { No. of partici- } \\
\text { pants }\end{array}$ & Statistical method & Effect size \\
\hline $\begin{array}{l}1 \text { Number of people who experienced one or } \\
\text { more falls that resulted in hospital admission - } \\
\text { overall analysis }\end{array}$ & 2 & 1705 & $\begin{array}{l}\text { Risk Ratio (IV, Ran- } \\
\text { dom, 95\% CI) }\end{array}$ & $0.78[0.51,1.18]$ \\
\hline
\end{tabular}

Analysis 4.1. Comparison 4 Exercise versus control (number of people with

falls that resulted in hospital admission), Outcome 1 Number of people who experienced one or more falls that resulted in hospital admission - overall analysis.

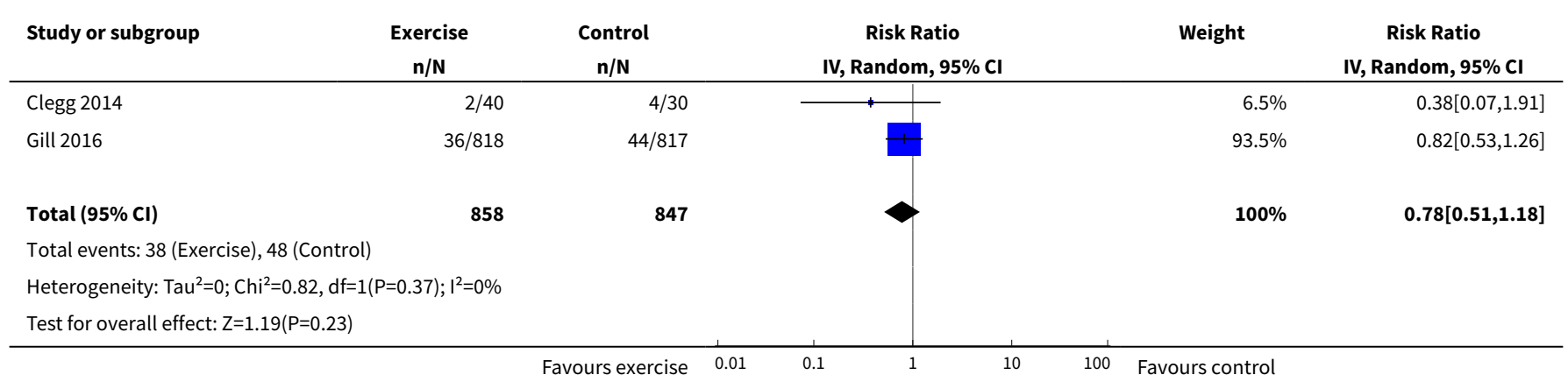


Comparison 5. Exercise versus control (number of people with falls that required medical attention)

\begin{tabular}{|c|c|c|c|c|}
\hline Outcome or subgroup title & No. of studies & $\begin{array}{l}\text { No. of partici- } \\
\text { pants }\end{array}$ & Statistical method & Effect size \\
\hline $\begin{array}{l}1 \text { Number of people who experienced one or } \\
\text { more falls that required medical attention- } \\
\text { overall analysis }\end{array}$ & 5 & 1019 & $\begin{array}{l}\text { Risk Ratio (Random, } \\
95 \% \mathrm{CI} \text { ) }\end{array}$ & $0.61[0.47,0.79]$ \\
\hline $\begin{array}{l}2 \text { Number of people who experienced one or } \\
\text { more falls that required medical attention - } \\
\text { subgrouped by exercise type }\end{array}$ & 5 & & $\begin{array}{l}\text { Risk Ratio (Random, } \\
95 \% \mathrm{Cl} \text { ) }\end{array}$ & Subtotals only \\
\hline $\begin{array}{l}2.1 \text { Balance and functional exercises vs con- } \\
\text { trol }\end{array}$ & 3 & 583 & $\begin{array}{l}\text { Risk Ratio (Random, } \\
95 \% \mathrm{Cl} \text { ) }\end{array}$ & $0.76[0.54,1.09]$ \\
\hline 2.2 Resistance exercises vs control & 1 & 73 & $\begin{array}{l}\text { Risk Ratio (Random, } \\
95 \% \mathrm{Cl} \text { ) }\end{array}$ & $0.92[0.47,1.80]$ \\
\hline 2.3 3D exercise (Tai Chi) vs control & 1 & 188 & $\begin{array}{l}\text { Risk Ratio (Random, } \\
95 \% \mathrm{Cl} \text { ) }\end{array}$ & $0.35[0.13,0.93]$ \\
\hline 2.4 Multiple categories of exercise vs control & 2 & 247 & $\begin{array}{l}\text { Risk Ratio (Random, } \\
95 \% \mathrm{Cl} \text { ) }\end{array}$ & $0.44[0.29,0.66]$ \\
\hline $\begin{array}{l}3 \text { Number of people who experienced one or } \\
\text { more falls that required medical attention - } \\
\text { long-term follow-up pooled }\end{array}$ & 2 & 319 & $\begin{array}{l}\text { Risk Ratio (Random, } \\
95 \% \mathrm{Cl} \text { ) }\end{array}$ & $0.54[0.37,0.78]$ \\
\hline
\end{tabular}

Analysis 5.1. Comparison 5 Exercise versus control (number of people with falls that required medical attention), Outcome 1 Number of people who experienced one or more falls that required medical attention- overall analysis.

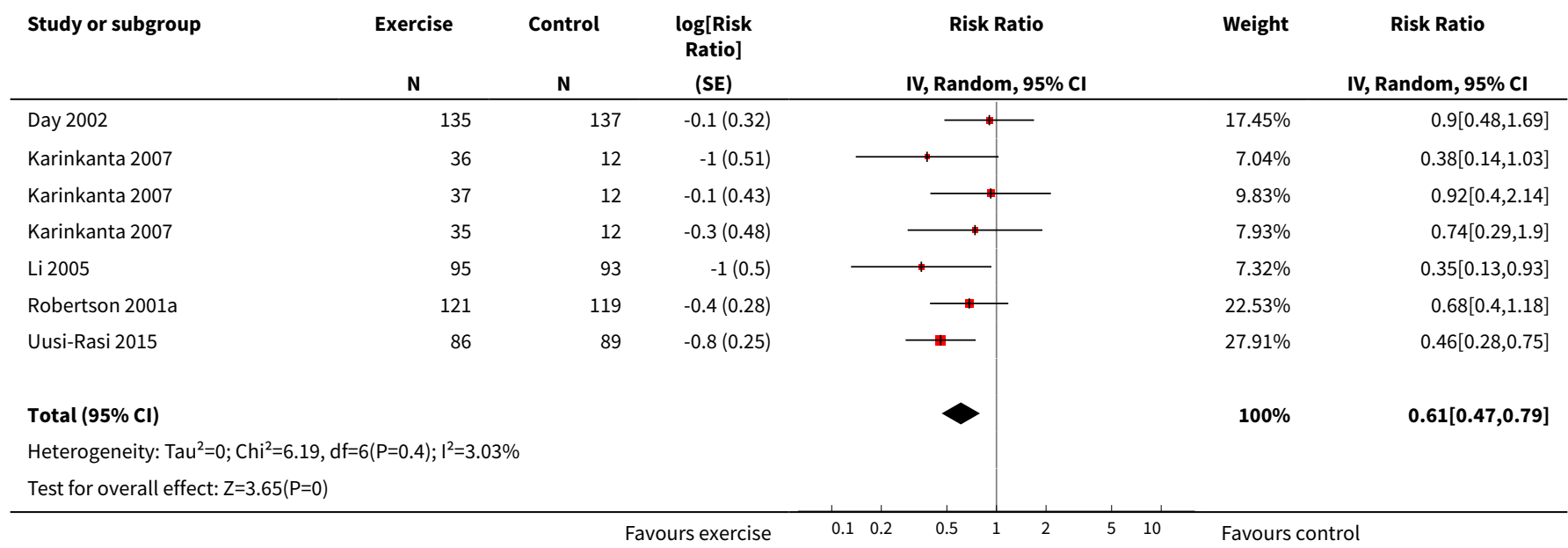


Analysis 5.2. Comparison 5 Exercise versus control (number of people with falls that required medical attention), Outcome 2 Number of people who experienced one or more falls that required medical attention - subgrouped by exercise type.

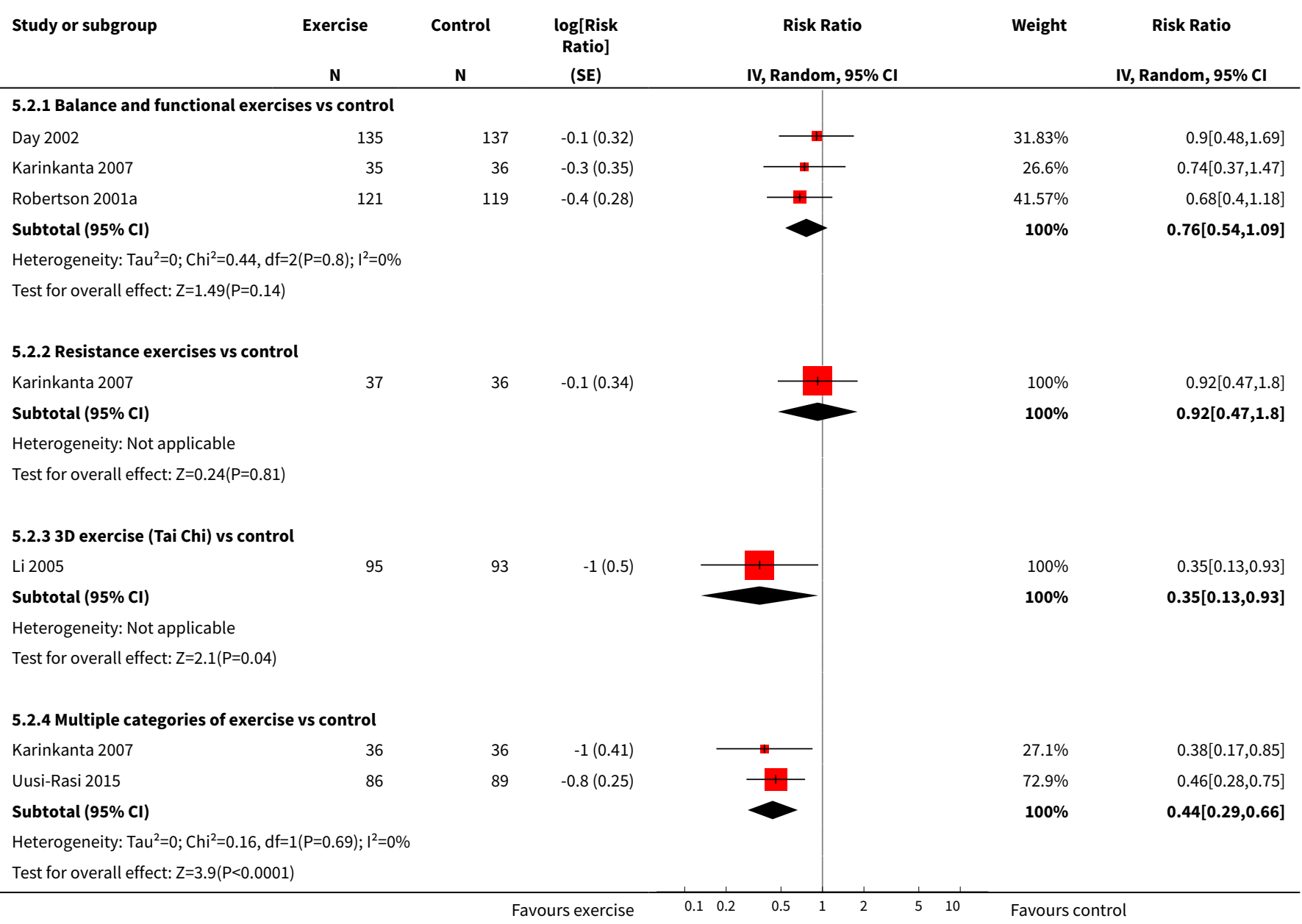

Analysis 5.3. Comparison 5 Exercise versus control (number of people with falls that required medical attention), Outcome 3 Number of people who experienced one or more falls that required medical attention - long-term follow-up pooled.

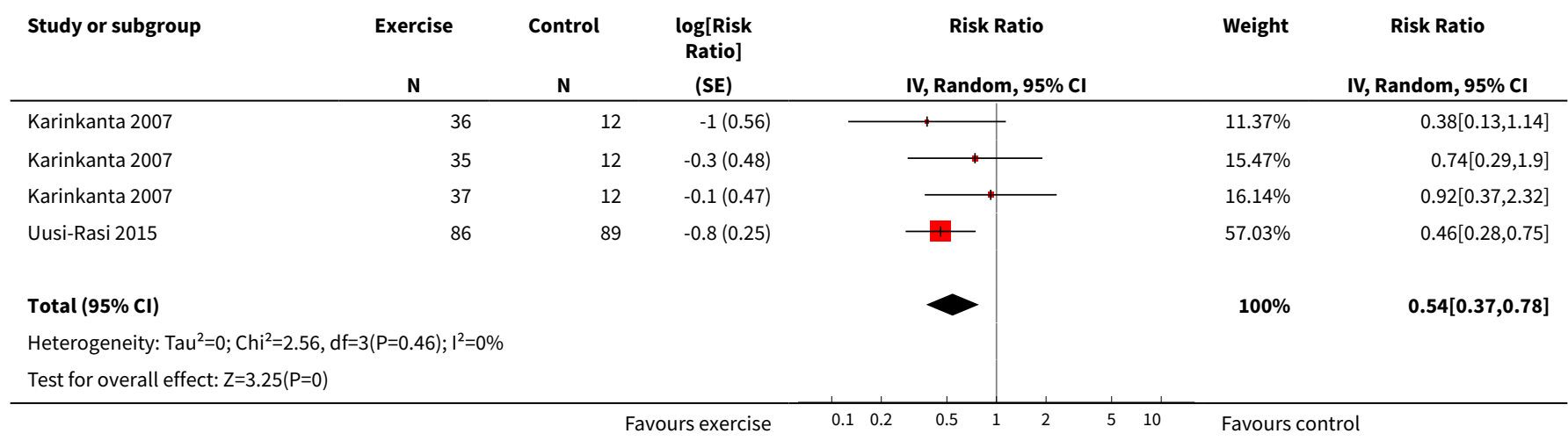


Comparison 6. Exercise versus control (health-related quality of life)

\begin{tabular}{lllll}
\hline Outcome or subgroup title & No. of studies & $\begin{array}{l}\text { No. of partici- } \\
\text { pants }\end{array}$ & Statistical method & Effect size \\
\hline $\begin{array}{l}1 \text { Health-related quality of life- over- } \\
\text { all analysis }\end{array}$ & 15 & 3172 & $\begin{array}{l}\text { Std. Mean Difference (IV, Fixed, } \\
95 \% \mathrm{CI})\end{array}$ & $-0.03[-0.10,0.04]$ \\
\hline $\begin{array}{l}2 \text { Health-related quality of life - sub- } \\
\text { grouped by baseline fall risk }\end{array}$ & 15 & & $\begin{array}{l}\text { Std. Mean Difference (IV, Ran- } \\
\text { dom, 95\% CI) }\end{array}$ & Subtotals only \\
\hline $\begin{array}{l}\text { 2.1 Not selected for high risk of falling } \\
\text { 2.2 Selected for high risk of falling }\end{array}$ & 8 & 2420 & $\begin{array}{l}\text { Std. Mean Difference (IV, Ran- } \\
\text { dom, 95\% CI) }\end{array}$ & $-0.01[-0.24,0.23]$ \\
\hline
\end{tabular}

Analysis 6.1. Comparison 6 Exercise versus control (health-related quality of life), Outcome 1 Health-related quality of life- overall analysis.

\begin{tabular}{|c|c|c|c|c|c|c|c|}
\hline \multirow[t]{2}{*}{ Study or subgroup } & \multicolumn{2}{|c|}{ Exercise } & \multicolumn{2}{|c|}{ Control } & \multirow{2}{*}{$\begin{array}{c}\text { Std. Mean Difference } \\
\text { Fixed, } 95 \% \mathrm{Cl}\end{array}$} & \multirow[t]{2}{*}{ Weight } & \multirow{2}{*}{$\begin{array}{c}\text { Std. Mean Difference } \\
\text { Fixed, } 95 \% \mathrm{Cl}\end{array}$} \\
\hline & $\mathbf{N}$ & Mean(SD) & $\mathbf{N}$ & Mean(SD) & & & \\
\hline Clegg 2014 & 40 & $0.5(0.3)$ & 30 & $0.5(0.3)$ & \begin{tabular}{l|l}
1 \\
\end{tabular} & $2.24 \%$ & $0.16[-0.31,0.63]$ \\
\hline Clemson 2012 & 99 & $6.7(1.5)$ & 46 & $6.7(1.3)$ & \begin{tabular}{l|l} 
\\
\end{tabular} & $4.11 \%$ & $0[-0.35,0.35]$ \\
\hline Dangour 2011 & 325 & $51.1(14.3)$ & 294 & $50.6(8.9)$ & - & $20.2 \%$ & $0.04[-0.12,0.2]$ \\
\hline Grahn Kronhed 2009 & 31 & $46.9(8.8)$ & 34 & $35.7(9.4)$ & & $1.78 \%$ & $1.21[0.68,1.75]$ \\
\hline Gschwind 2015 & 71 & $0.9(0.2)$ & 65 & $0.9(0.1)$ & - & $4.44 \%$ & $-0.07[-0.41,0.27]$ \\
\hline Iliffe 2015 & 179 & $0.7(0.1)$ & 106 & $0.7(0.1)$ & $\rightarrow$ & $8.69 \%$ & $-0.14[-0.38,0.1]$ \\
\hline Iliffe 2015 & 176 & $0.7(0.1)$ & 106 & $0.7(0.1)$ & $\rightarrow$ & $8.66 \%$ & $0[-0.24,0.24]$ \\
\hline Kerse 2010 & 94 & $38.3(1.2)$ & 87 & $39.4(1.2)$ & $\hookrightarrow$ & $5.35 \%$ & $-0.91[-1.22,-0.61]$ \\
\hline Lin 2007 & 39 & $62.8(9.9)$ & 40 & $55.5(15.3)$ & & $2.48 \%$ & $0.56[0.11,1.01]$ \\
\hline Merom 2016 & 275 & $41.8(10.3)$ & 247 & $42.6(9.9)$ & $\rightarrow$ & $17.02 \%$ & $-0.08[-0.25,0.09]$ \\
\hline Resnick 2002 & 10 & $33.4(4.8)$ & 7 & $31.2(4.9)$ & & $0.52 \%$ & $0.43[-0.55,1.41]$ \\
\hline Sales 2017 & 27 & $49.6(8.3)$ & 21 & $48.9(7.6)$ & & $1.54 \%$ & $0.09[-0.48,0.66]$ \\
\hline Smulders 2010 & 47 & $26.2(10.6)$ & 45 & $27.3(11)$ & - & $3.01 \%$ & $-0.1[-0.51,0.31]$ \\
\hline Voukelatos 2015 & 144 & $0.8(0.1)$ & 169 & $0.8(0.1)$ & + & $10.17 \%$ & $0.08[-0.14,0.3]$ \\
\hline Yang 2012 & 59 & $23.4(4.1)$ & 62 & $24.6(5.2)$ & $\longrightarrow$ & $3.92 \%$ & $-0.25[-0.61,0.1]$ \\
\hline 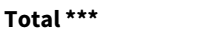 & 1740 & & 1432 & & $\diamond$ & $100 \%$ & $-0.03[-0.1,0.04]$ \\
\hline \multicolumn{8}{|c|}{ Heterogeneity: $\mathrm{Tau}^{2}=0 ; \mathrm{Chi}^{2}=66.6, \mathrm{df}=16(\mathrm{P}<0.0001) ; \mathrm{I}^{2}=75.98 \%$} \\
\hline \multicolumn{3}{|c|}{ Test for overall effect: $Z=0.76(P=0.45)$} & & & & & \\
\hline
\end{tabular}


Analysis 6.2. Comparison 6 Exercise versus control (health-related quality of life), Outcome 2 Health-related quality of life - subgrouped by baseline fall risk.

\begin{tabular}{|c|c|c|c|c|c|c|c|}
\hline \multirow[t]{2}{*}{ Study or subgroup } & \multicolumn{2}{|c|}{ Exercise } & \multicolumn{2}{|c|}{ Control } & \multirow{2}{*}{$\begin{array}{c}\text { Std. Mean Difference } \\
\text { Random, } 95 \% \mathrm{Cl}\end{array}$} & \multirow[t]{2}{*}{ Weight } & \multirow{2}{*}{$\begin{array}{c}\text { Std. Mean Difference } \\
\text { Random, } 95 \% \mathrm{Cl}\end{array}$} \\
\hline & $\mathbf{N}$ & $\operatorname{Mean}(S D)$ & $\mathbf{N}$ & $\operatorname{Mean}(S D)$ & & & \\
\hline \multicolumn{8}{|c|}{ 6.2.1 Not selected for high risk of falling } \\
\hline Dangour 2011 & 325 & $51.1(14.3)$ & 294 & $50.6(8.9)$ & + & $13.56 \%$ & $0.04[-0.12,0.2]$ \\
\hline Grahn Kronhed 2009 & 31 & $46.9(8.8)$ & 34 & $35.7(9.4)$ & \# & $8.3 \%$ & $1.21[0.68,1.75]$ \\
\hline Gschwind 2015 & 71 & $0.9(0.2)$ & 65 & $0.9(0.1)$ & & $11.14 \%$ & $-0.07[-0.41,0.27]$ \\
\hline Iliffe 2015 & 176 & $0.7(0.1)$ & 106 & $0.7(0.1)$ & - & $12.54 \%$ & $0[-0.24,0.24]$ \\
\hline Kerse 2010 & 94 & $38.3(1.2)$ & 87 & $39.4(1.2)$ & $\rightarrow$ & $11.59 \%$ & $-0.91[-1.22,-0.61]$ \\
\hline Merom 2016 & 275 & $41.8(10.3)$ & 247 & $42.6(9.9)$ & t & $13.41 \%$ & $-0.08[-0.25,0.09]$ \\
\hline Resnick 2002 & 10 & $33.4(4.8)$ & 7 & $31.2(4.9)$ & 1 & $4.11 \%$ & $0.43[-0.55,1.41]$ \\
\hline Voukelatos 2015 & 144 & $0.8(0.1)$ & 169 & $0.8(0.1)$ & + & $12.79 \%$ & $0.08[-0.14,0.3]$ \\
\hline 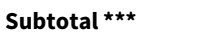 & 1305 & & 1115 & & & $100 \%$ & $-0.01[-0.24,0.23]$ \\
\hline \multicolumn{8}{|c|}{ Heterogeneity: $\operatorname{Tau}^{2}=0.1 ; \mathrm{Chi}^{2}=56.43, \mathrm{df}=8(\mathrm{P}<0.0001) ; \mathrm{I}^{2}=85.82 \%$} \\
\hline \multicolumn{8}{|c|}{ 6.2.2 Selected for high risk of falling } \\
\hline Clegg 2014 & 40 & $0.5(0.3)$ & 30 & $0.5(0.3)$ & 1 & $10.39 \%$ & $0.16[-0.31,0.63]$ \\
\hline Clemson 2012 & 96 & $6.7(1.6)$ & 46 & $6.7(1.3)$ & + & $16.42 \%$ & $0[-0.35,0.35]$ \\
\hline Clemson 2012 & 99 & $6.7(1.5)$ & 46 & $6.7(1.3)$ & $\leftarrow$ & $16.53 \%$ & $0[-0.35,0.35]$ \\
\hline Lin 2007 & 39 & $62.8(9.9)$ & 40 & $55.5(15.3)$ & + & $11.3 \%$ & $0.56[0.11,1.01]$ \\
\hline Rubenstein 2000 & 28 & $65(17.4)$ & 27 & $60.6(20.3)$ & + & $8.62 \%$ & $0.23[-0.3,0.76]$ \\
\hline Sales 2017 & 27 & $49.6(8.3)$ & 21 & $48.9(7.6)$ & $\longrightarrow$ & $7.61 \%$ & $0.09[-0.48,0.66]$ \\
\hline Smulders 2010 & 47 & $26.2(10.6)$ & 45 & $27.3(11)$ & $\rightarrow$ & $13.13 \%$ & $-0.1[-0.51,0.31]$ \\
\hline Yang 2012 & 59 & $23.4(4.1)$ & 62 & $24.6(5.2)$ & + & $16 \%$ & $-0.25[-0.61,0.1]$ \\
\hline 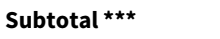 & 435 & & 317 & & $\diamond$ & $100 \%$ & $0.05[-0.12,0.22]$ \\
\hline \multicolumn{8}{|c|}{ Heterogeneity: $\mathrm{Tau}^{2}=0.01 ; \mathrm{Chi}^{2}=9.03, \mathrm{df}=7(\mathrm{P}=0.25) ; \mathrm{I}^{2}=22.46 \%$} \\
\hline \multicolumn{8}{|c|}{ Test for overall effect: $Z=0.61(P=0.54)$} \\
\hline
\end{tabular}

\section{Comparison 7. Exercise versus control (number of people who died)}

\begin{tabular}{lllll}
\hline Outcome or subgroup title & No. of studies & $\begin{array}{l}\text { No. of partici- } \\
\text { pants }\end{array}$ & Statistical method & Effect size \\
\hline $\begin{array}{l}1 \text { Number of people who died- overall } \\
\text { analysis }\end{array}$ & 30 & 10037 & $\begin{array}{l}\text { Risk Ratio (IV, Random, 95\% } \\
\text { Cl) }\end{array}$ & $0.86[0.66,1.12]$ \\
\hline $\begin{array}{l}\text { 2 Number of people who died - sub- } \\
\text { grouped by baseline fall risk }\end{array}$ & 30 & & $\begin{array}{l}\text { Risk Ratio (IV, Random, 95\% } \\
\text { Cl) }\end{array}$ & Subtotals only \\
\hline $\begin{array}{l}\text { 2.1 Not selected for high risk of falling } \\
\text { 2.2 Selected for high risk of falling }\end{array}$ & 12 & 4606 & $\begin{array}{l}\text { Risk Ratio (IV, Random, 95\% } \\
\text { Cl) }\end{array}$ & 0.94 [0.54, 1.67] \\
\hline
\end{tabular}


Analysis 7.1. Comparison 7 Exercise versus control (number of people who died), Outcome 1 Number of people who died- overall analysis.

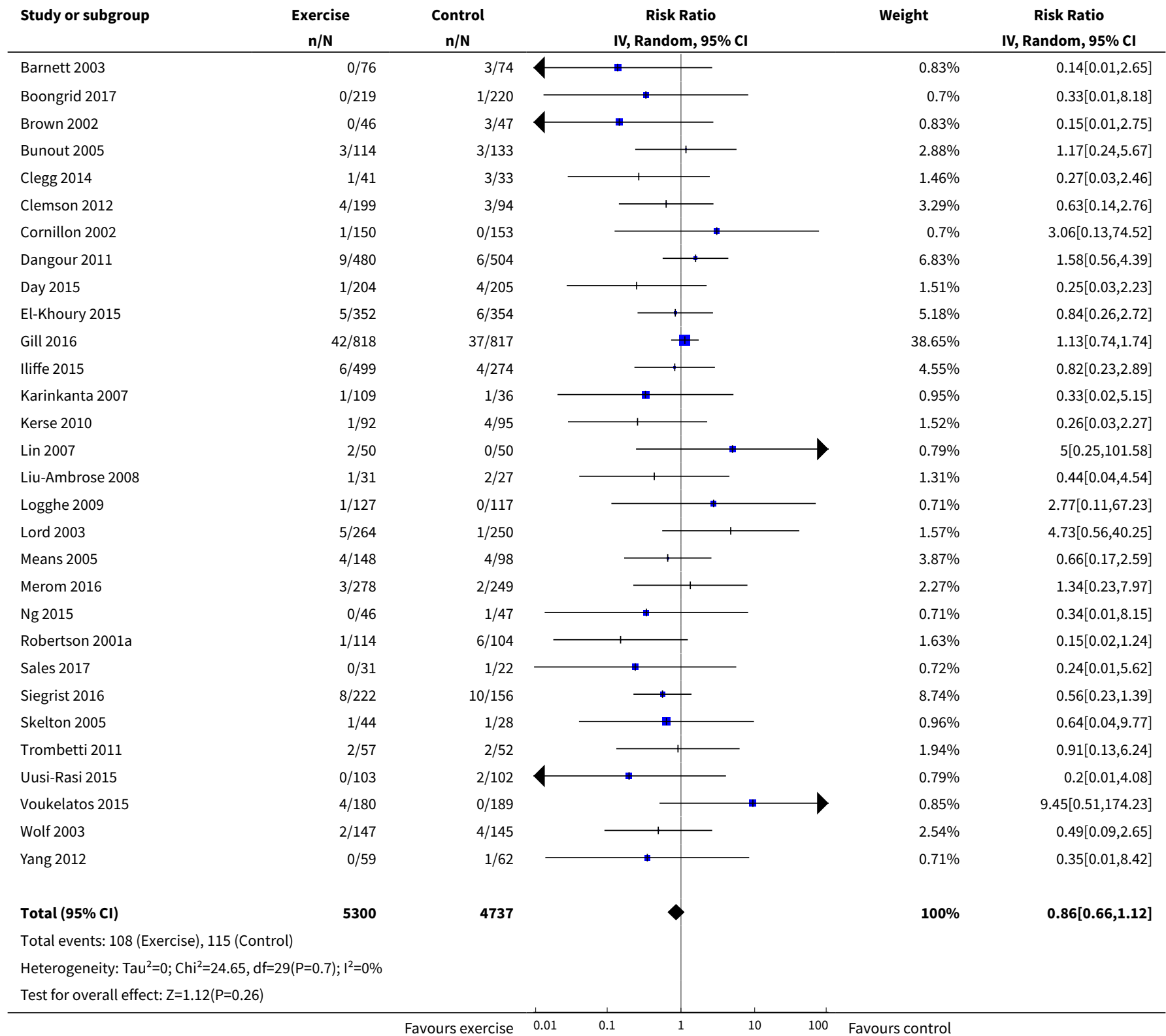

Analysis 7.2. Comparison 7 Exercise versus control (number of people who died), Outcome 2 Number of people who died - subgrouped by baseline fall risk.

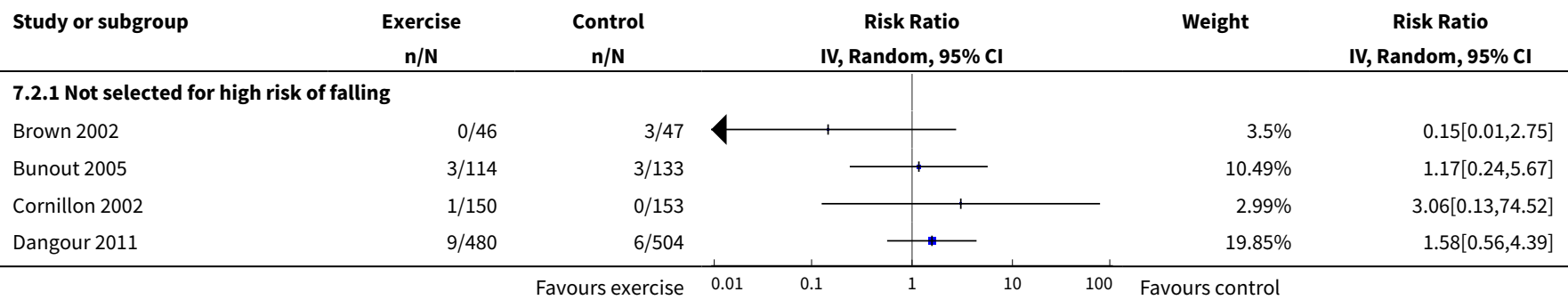




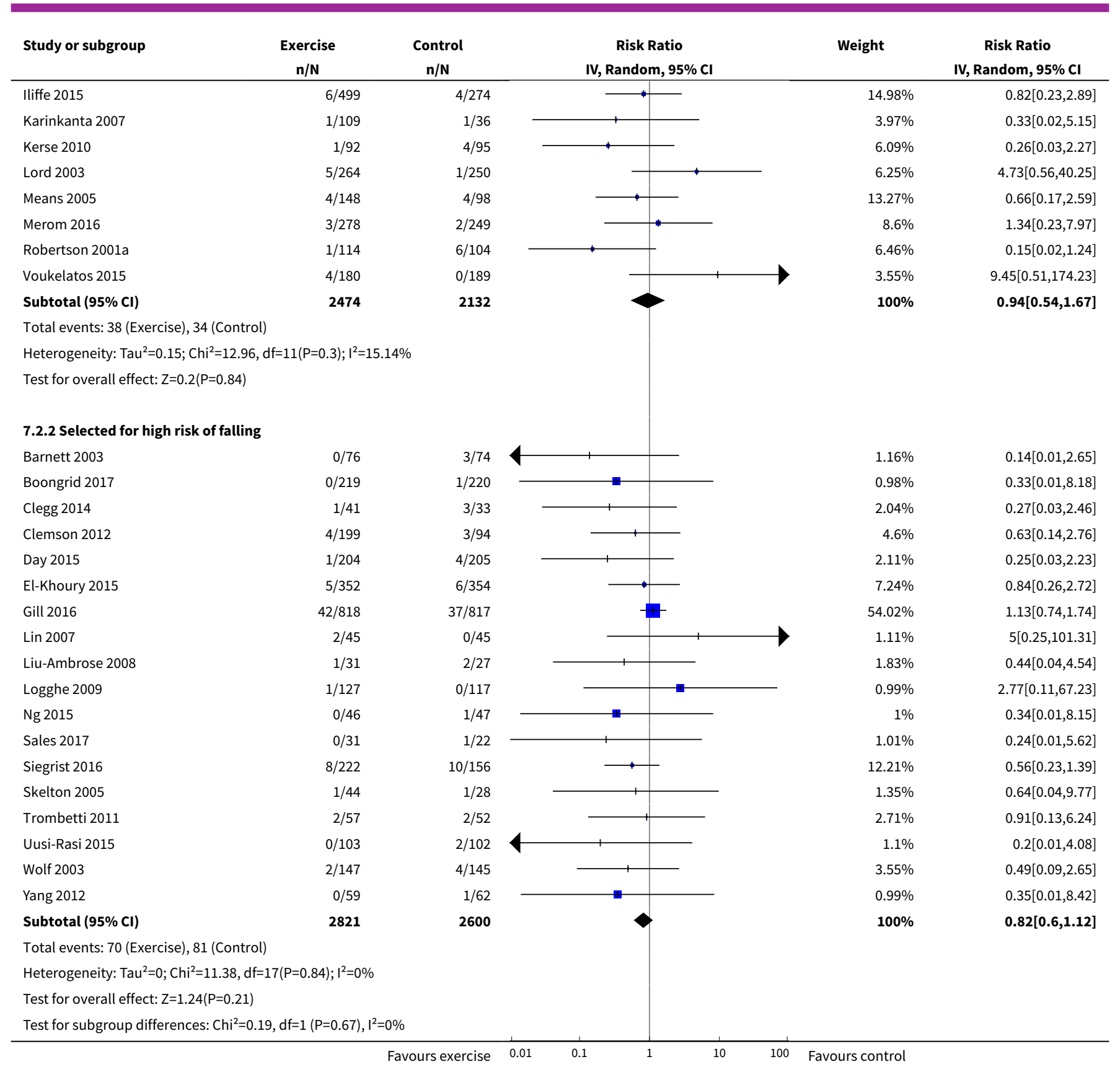

\section{Comparison 8. Balance and functional exercises versus control: subgroup analyses}

\begin{tabular}{lllll}
\hline Outcome or subgroup title & No. of studies & $\begin{array}{l}\text { No. of partici- } \\
\text { pants }\end{array}$ & Statistical method & Effect size \\
\hline $\begin{array}{l}1 \text { Rate of falls, subgrouped by baseline } \\
\text { fall risk }\end{array}$ & 39 & & $\begin{array}{l}\text { Rate Ratio (Random, 95\% } \\
\text { Cl) }\end{array}$ & Subtotals only \\
\hline 1.1 Not selected for higher risk of falling & 18 & 3355 & $\begin{array}{l}\text { Rate Ratio (Random, 95\% } \\
\text { Cl) }\end{array}$ & $0.80[0.72,0.90]$ \\
\hline
\end{tabular}




\begin{tabular}{|c|c|c|c|c|}
\hline Outcome or subgroup title & No. of studies & $\begin{array}{l}\text { No. of partici- } \\
\text { pants }\end{array}$ & Statistical method & Effect size \\
\hline 1.2 Selected for higher risk of falling & 21 & 4602 & $\begin{array}{l}\text { Rate Ratio (Random, 95\% } \\
\mathrm{Cl} \text { ) }\end{array}$ & $0.72[0.65,0.80]$ \\
\hline $\begin{array}{l}2 \text { Number of fallers, subgrouped by } \\
\text { baseline fall risk }\end{array}$ & 37 & & $\begin{array}{l}\text { Risk Ratio (Random, 95\% } \\
\text { Cl) }\end{array}$ & Subtotals only \\
\hline 2.1 Not selected for higher risk of falling & 15 & 3649 & $\begin{array}{l}\text { Risk Ratio (Random, 95\% } \\
\text { Cl) }\end{array}$ & $0.88[0.80,0.97]$ \\
\hline 2.2 Selected for higher risk of falling & 22 & 4639 & $\begin{array}{l}\text { Risk Ratio (Random, 95\% } \\
\mathrm{CI} \text { ) }\end{array}$ & $0.86[0.81,0.91]$ \\
\hline 3 Rate of falls, subgrouped by personnel & 39 & & $\begin{array}{l}\text { Rate Ratio (Random, 95\% } \\
\mathrm{Cl} \text { ) }\end{array}$ & Subtotals only \\
\hline $\begin{array}{l}3.1 \text { Health professional delivering inter- } \\
\text { vention }\end{array}$ & 20 & 2960 & $\begin{array}{l}\text { Rate Ratio (Random, 95\% } \\
\mathrm{Cl} \text { ) }\end{array}$ & $0.67[0.58,0.76]$ \\
\hline $\begin{array}{l}3.2 \text { No health professional delivering in- } \\
\text { tervention }\end{array}$ & 19 & 4997 & $\begin{array}{l}\text { Rate Ratio (Random, 95\% } \\
\mathrm{Cl} \text { ) }\end{array}$ & $0.82[0.76,0.88]$ \\
\hline $\begin{array}{l}4 \text { Number of fallers, subgrouped by per- } \\
\text { sonnel }\end{array}$ & 37 & & $\begin{array}{l}\text { Risk Ratio (Random, 95\% } \\
\mathrm{Cl} \text { ) }\end{array}$ & Subtotals only \\
\hline $\begin{array}{l}4.1 \text { Health professional delivering inter- } \\
\text { vention }\end{array}$ & 19 & 2894 & $\begin{array}{l}\text { Risk Ratio (Random, 95\% } \\
\mathrm{Cl} \text { ) }\end{array}$ & $0.82[0.75,0.90]$ \\
\hline $\begin{array}{l}\text { 4.2 No health professional delivering in- } \\
\text { tervention }\end{array}$ & 18 & 5394 & $\begin{array}{l}\text { Risk Ratio (Random, 95\% } \\
\mathrm{Cl} \text { ) }\end{array}$ & $0.89[0.84,0.94]$ \\
\hline $\begin{array}{l}5 \text { Rate of falls, subgrouped by group or } \\
\text { individual exercise }\end{array}$ & 39 & & $\begin{array}{l}\text { Rate Ratio (Random, 95\% } \\
\mathrm{Cl} \text { ) }\end{array}$ & Subtotals only \\
\hline 5.1 Group exercise & 20 & 3620 & $\begin{array}{l}\text { Rate Ratio (Random, 95\% } \\
\mathrm{Cl} \text { ) }\end{array}$ & $0.73[0.65,0.82]$ \\
\hline 5.2 Not group exercise & 20 & 4589 & $\begin{array}{l}\text { Rate Ratio (Random, 95\% } \\
\mathrm{Cl} \text { ) }\end{array}$ & $0.77[0.70,0.85]$ \\
\hline $\begin{array}{l}6 \text { Number of fallers, subgrouped by } \\
\text { group or individual exercise }\end{array}$ & 37 & & $\begin{array}{l}\text { Risk Ratio (Random, 95\% } \\
\text { Cl) }\end{array}$ & Subtotals only \\
\hline 6.1 Group exercise & 22 & 4465 & $\begin{array}{l}\text { Risk Ratio (Random, 95\% } \\
\text { Cl) }\end{array}$ & $0.87[0.80,0.95]$ \\
\hline 6.2 Not group exercise & 16 & 4075 & $\begin{array}{l}\text { Risk Ratio (Random, 95\% } \\
\text { Cl) }\end{array}$ & $0.87[0.82,0.92]$ \\
\hline
\end{tabular}


Analysis 8.1. Comparison 8 Balance and functional exercises versus control: subgroup analyses, Outcome 1 Rate of falls, subgrouped by baseline fall risk.

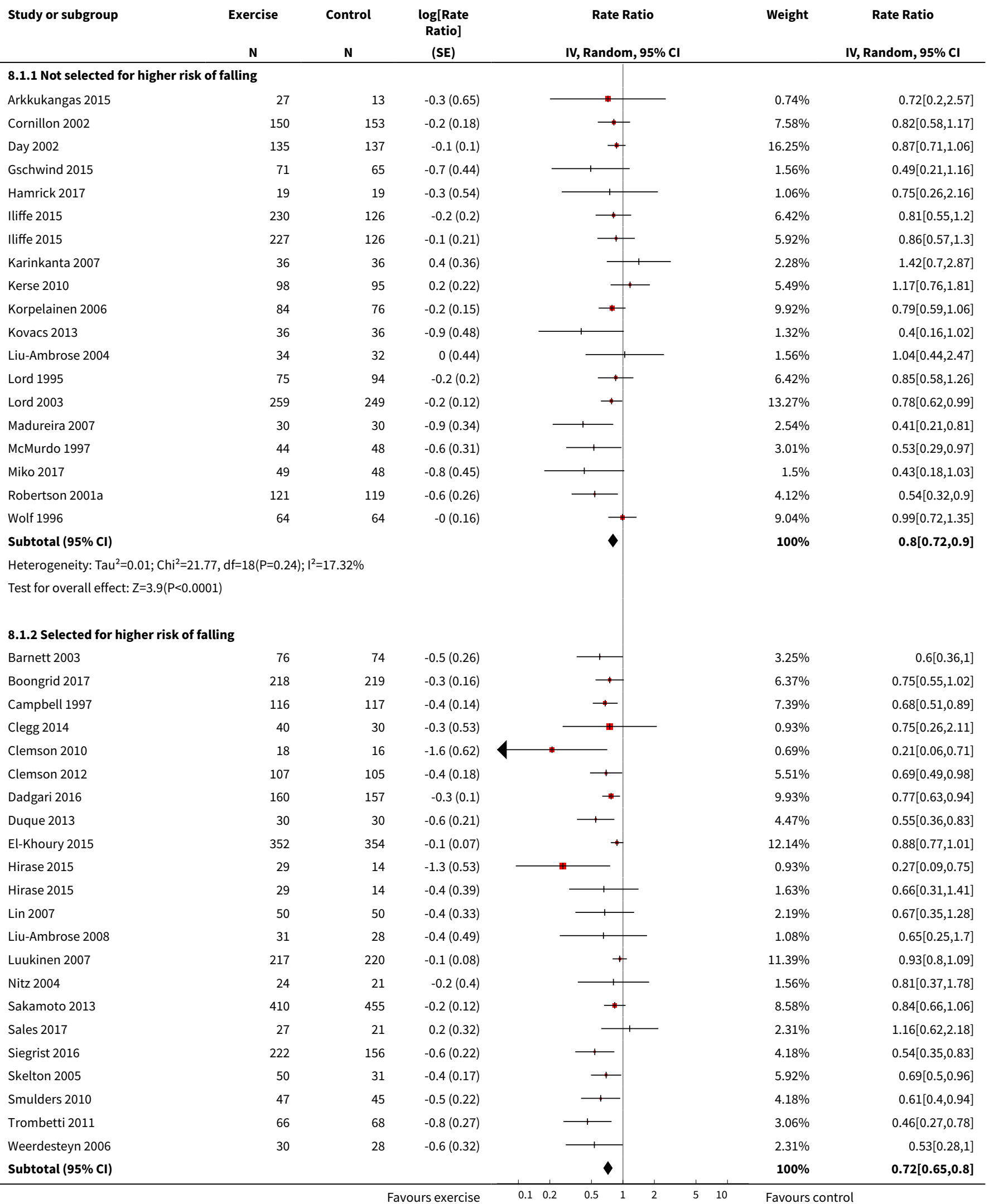




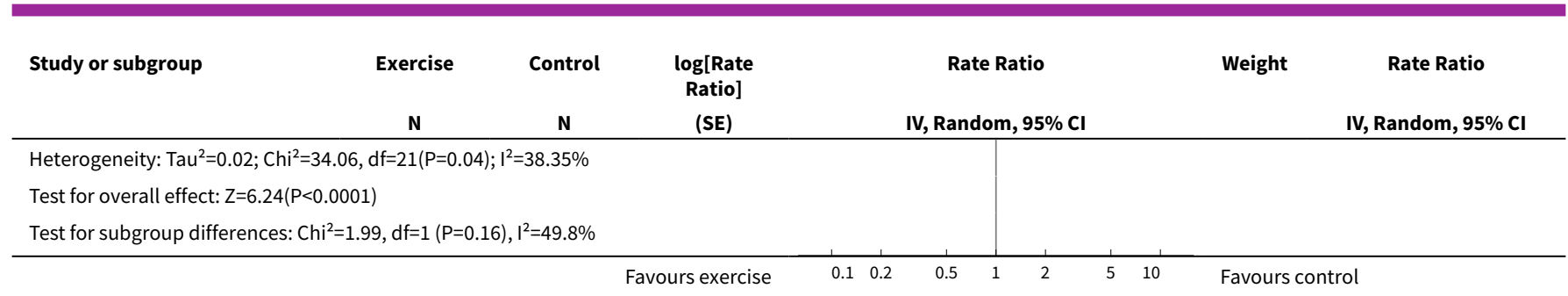

Analysis 8.2. Comparison 8 Balance and functional exercises versus control: subgroup analyses, Outcome 2 Number of fallers, subgrouped by baseline fall risk.

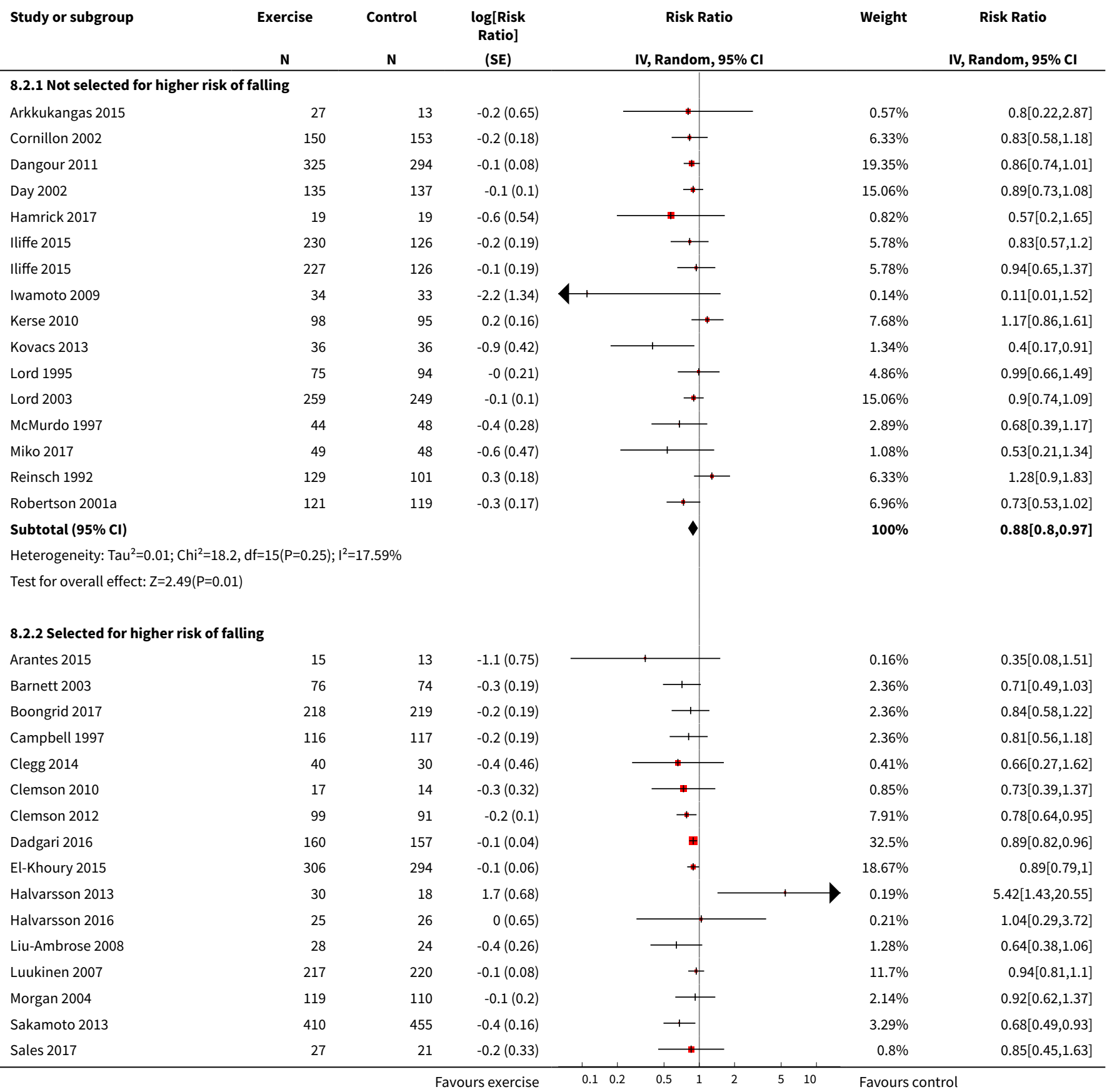




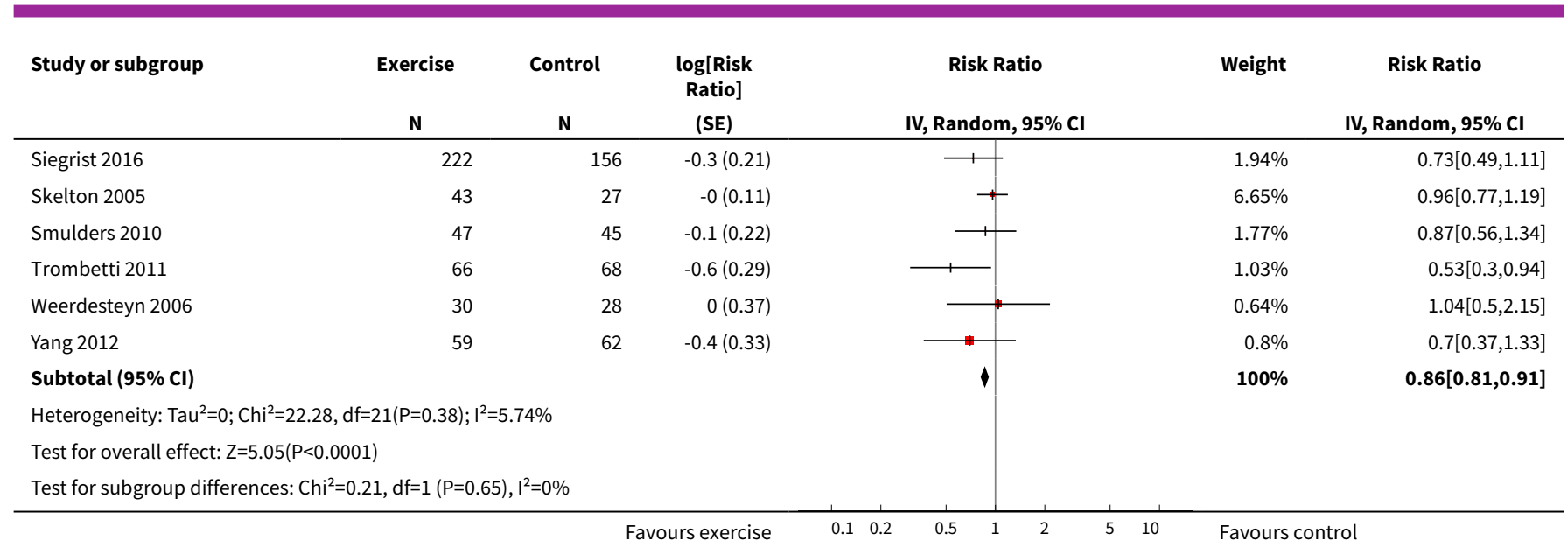

Favours exercise

$\begin{array}{llllllll}0.1 & 0.2 & 0.5 & 1 & 2 & 5 & 10 & \text { Favours control }\end{array}$

Analysis 8.3. Comparison 8 Balance and functional exercises versus control: subgroup analyses, Outcome 3 Rate of falls, subgrouped by personnel.

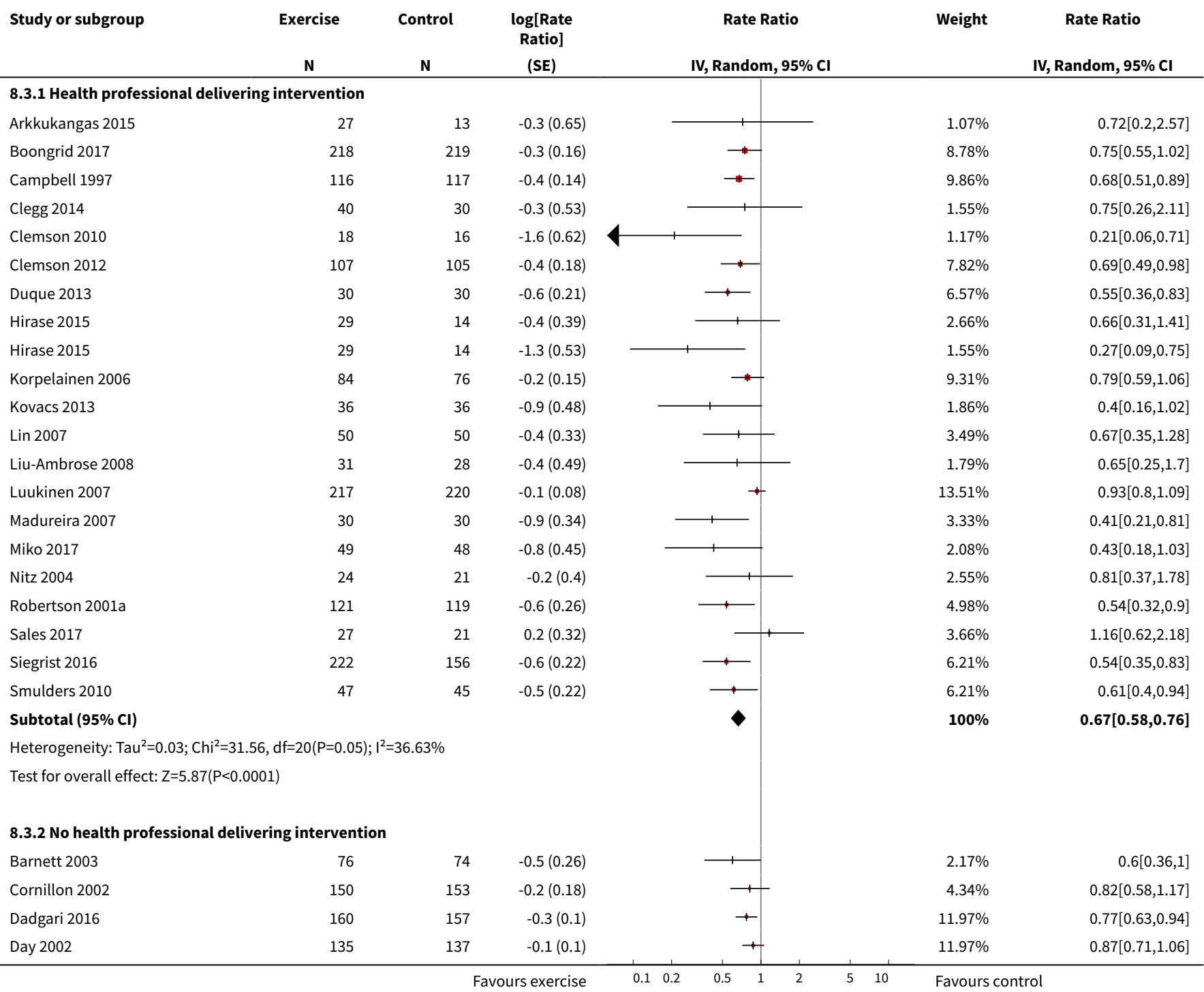




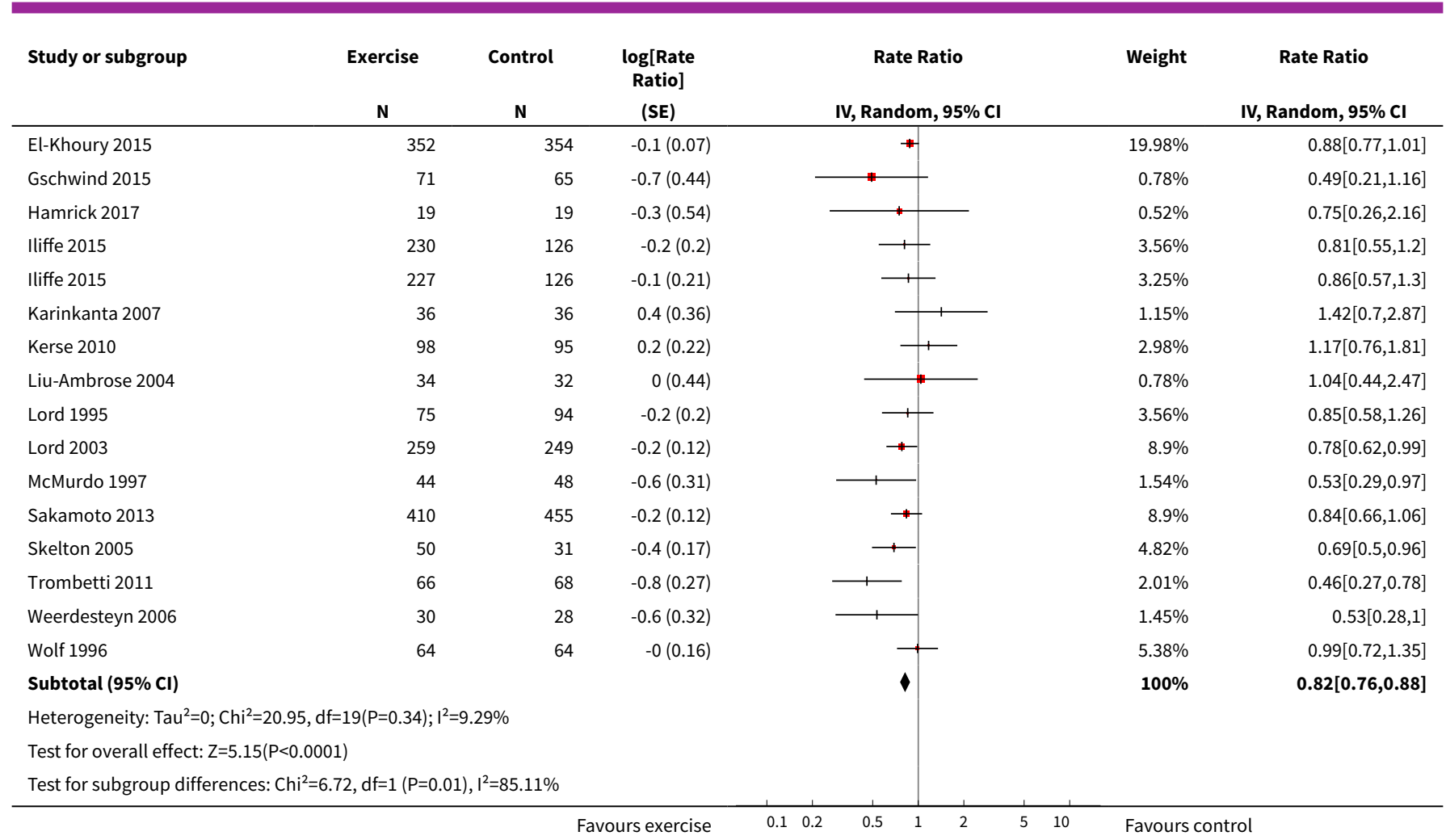

Analysis 8.4. Comparison 8 Balance and functional exercises versus control: subgroup analyses, Outcome 4 Number of fallers, subgrouped by personnel.

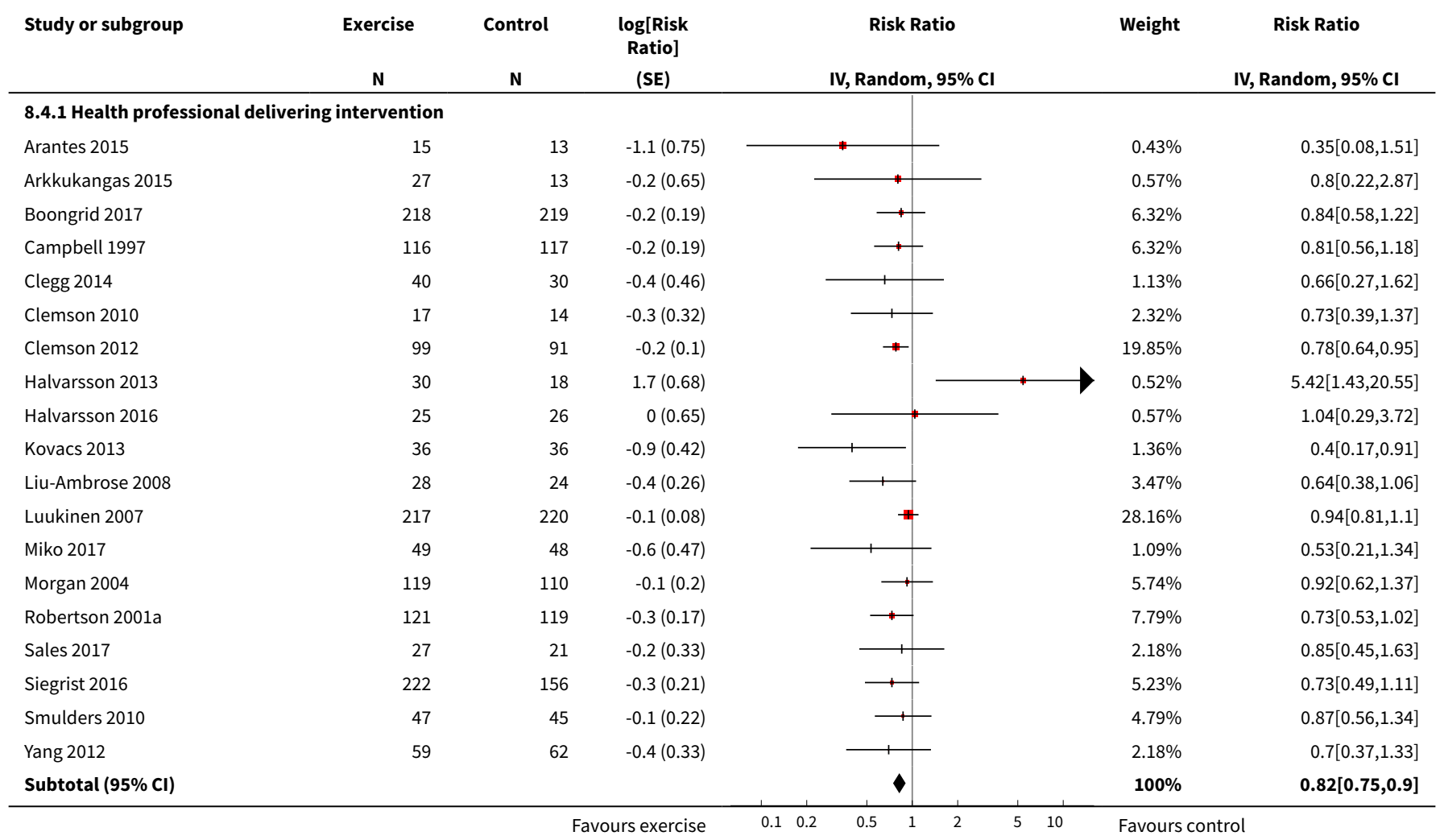




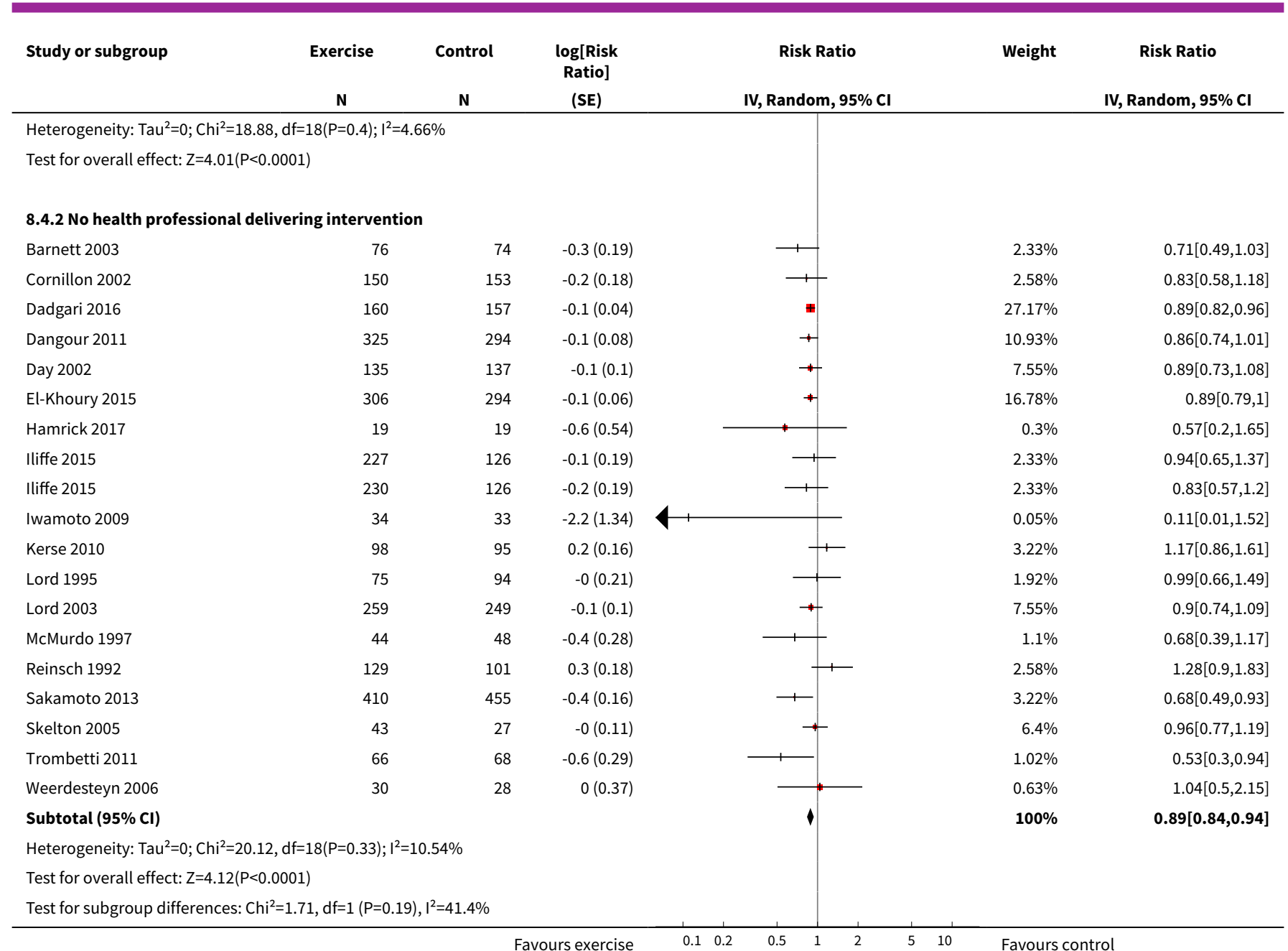

Favours exercise

$\begin{array}{lllllllll}0.1 & 0.2 & 0.5 & 1 & 2 & 5 & 10 & \text { Favours control }\end{array}$

Analysis 8.5. Comparison 8 Balance and functional exercises versus control: subgroup analyses, Outcome 5 Rate of falls, subgrouped by group or individual exercise.

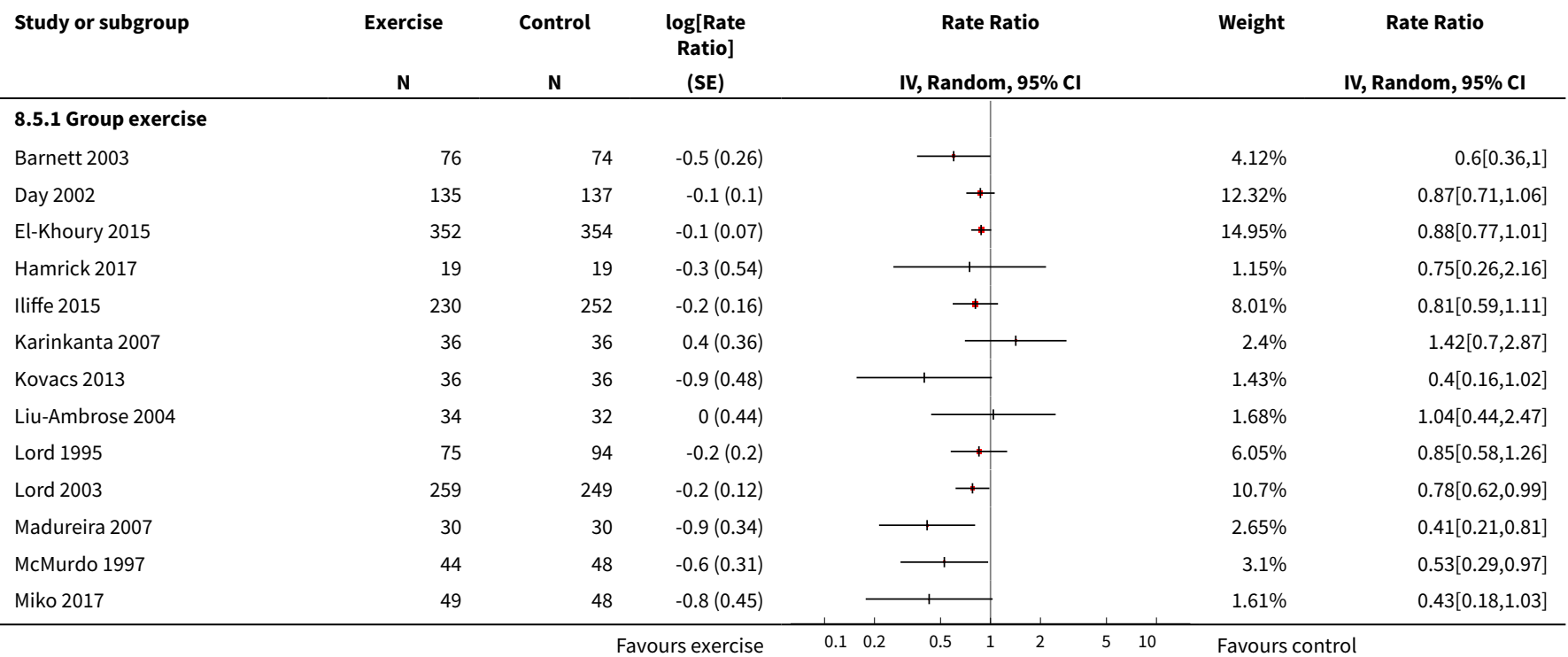




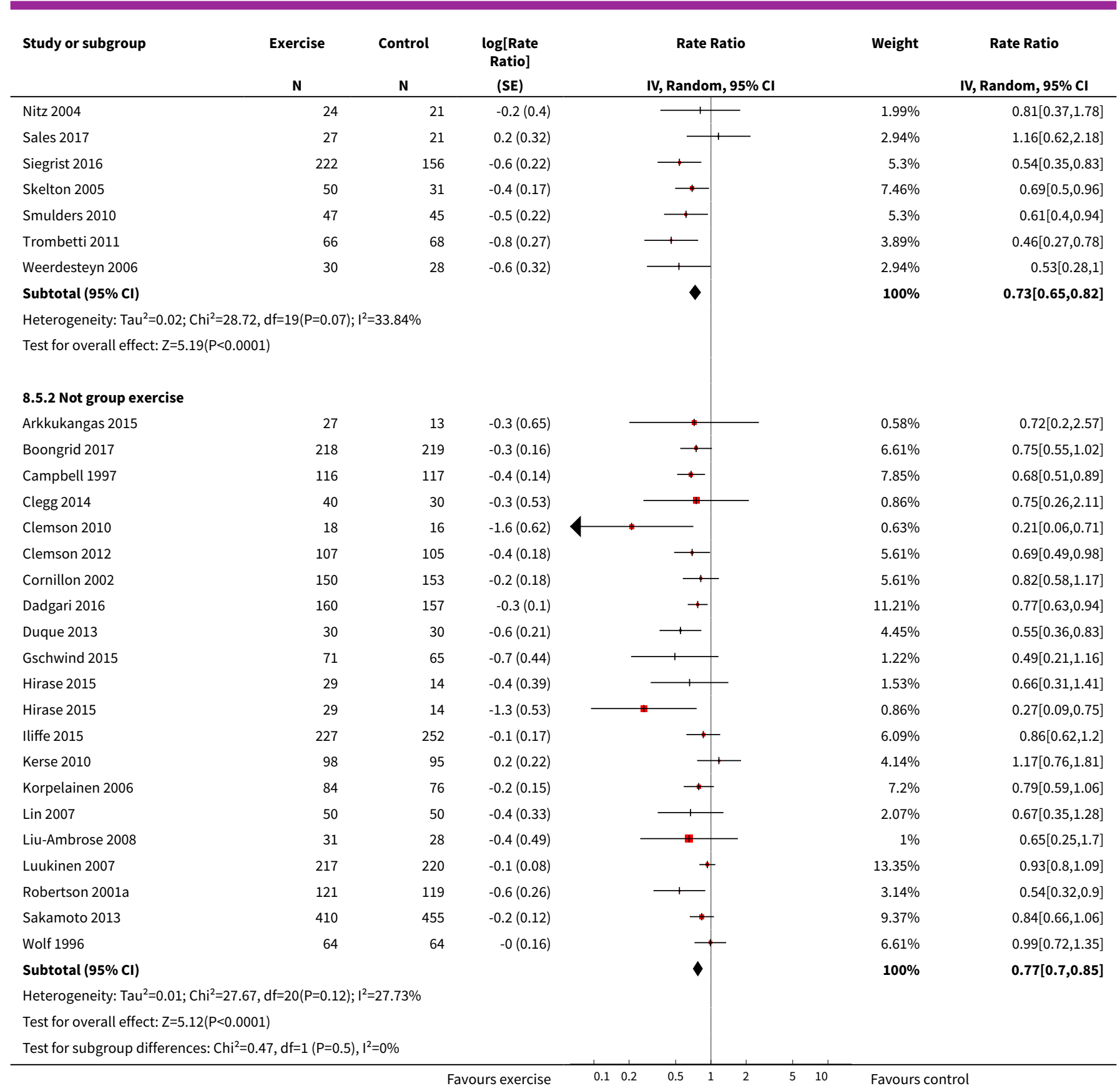

Analysis 8.6. Comparison 8 Balance and functional exercises versus control: subgroup analyses, Outcome 6 Number of fallers, subgrouped by group or individual exercise.

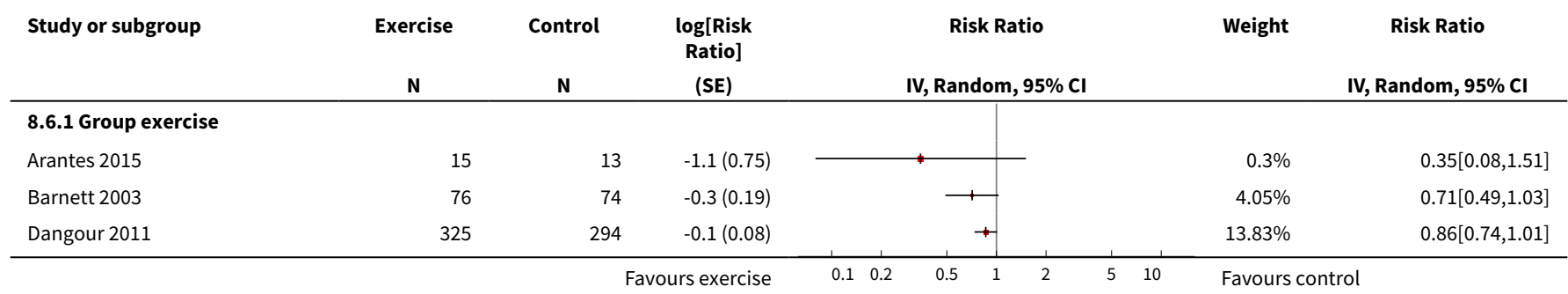




\begin{tabular}{|c|c|c|c|c|c|c|}
\hline Study or subgroup & Exercise & Control & $\begin{array}{c}\log [\text { Risk } \\
\text { Ratio] }\end{array}$ & Risk Ratio & Weight & Risk Ratio \\
\hline & $\mathbf{N}$ & $\mathbf{N}$ & (SE) & IV, Random, $95 \% \mathrm{Cl}$ & & IV, Random, 95\% CI \\
\hline Day 2002 & 135 & 137 & $-0.1(0.1)$ & + & $10.7 \%$ & $0.89[0.73,1.08]$ \\
\hline El-Khoury 2015 & 306 & 294 & $-0.1(0.06)$ & + & $17.9 \%$ & $0.89[0.79,1]$ \\
\hline Halvarsson 2013 & 30 & 18 & $1.7(0.68)$ & & $0.36 \%$ & $5.42[1.43,20.55]$ \\
\hline Halvarsson 2016 & 25 & 26 & $0(0.65)$ & 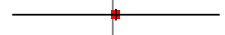 & $0.4 \%$ & $1.04[0.29,3.72]$ \\
\hline Hamrick 2017 & 19 & 19 & $-0.6(0.54)$ & \begin{tabular}{l|l} 
\\
\end{tabular} & $0.57 \%$ & $0.57[0.2,1.65]$ \\
\hline Iliffe 2015 & 230 & 252 & $-0.2(0.16)$ & $千$ & $5.4 \%$ & $0.83[0.6,1.13]$ \\
\hline Kovacs 2013 & 36 & 36 & $-0.9(0.42)$ & \pm & $0.93 \%$ & $0.4[0.17,0.91]$ \\
\hline Lord 1995 & 75 & 94 & $-0(0.21)$ & 1 & $3.4 \%$ & $0.99[0.66,1.49]$ \\
\hline Lord 2003 & 259 & 249 & $-0.1(0.1)$ & + & $10.7 \%$ & $0.9[0.74,1.09]$ \\
\hline McMurdo 1997 & 44 & 48 & $-0.4(0.28)$ & +1 & $2.02 \%$ & $0.68[0.39,1.17]$ \\
\hline Miko 2017 & 49 & 48 & $-0.6(0.47)$ & + & $0.75 \%$ & $0.53[0.21,1.34]$ \\
\hline Morgan 2004 & 119 & 110 & $-0.1(0.2)$ & -1 & $3.71 \%$ & $0.92[0.62,1.37]$ \\
\hline Reinsch 1992 & 129 & 101 & $0.3(0.18)$ & 1 & $4.44 \%$ & $1.28[0.9,1.83]$ \\
\hline Sales 2017 & 27 & 21 & $-0.2(0.33)$ & + & $1.48 \%$ & $0.85[0.45,1.63]$ \\
\hline Siegrist 2016 & 222 & 156 & $-0.3(0.21)$ & + & $3.4 \%$ & $0.73[0.49,1.11]$ \\
\hline Skelton 2005 & 43 & 27 & $-0(0.11)$ & $\rightarrow$ & $9.45 \%$ & $0.96[0.77,1.19]$ \\
\hline Smulders 2010 & 47 & 45 & $-0.1(0.22)$ & 1 & $3.13 \%$ & $0.87[0.56,1.34]$ \\
\hline Trombetti 2011 & 66 & 68 & $-0.6(0.29)$ & - & $1.89 \%$ & $0.53[0.3,0.94]$ \\
\hline Weerdesteyn 2006 & 30 & 28 & $0(0.37)$ & - & $1.19 \%$ & $1.04[0.5,2.15]$ \\
\hline Subtotal $(95 \% \mathrm{Cl})$ & & & & $\checkmark$ & $100 \%$ & $0.87[0.8,0.95]$ \\
\hline \multicolumn{7}{|c|}{ Heterogeneity: $\mathrm{Tau}^{2}=0.01 ; \mathrm{Chi}^{2}=25.88, \mathrm{df}=21(\mathrm{P}=0.21) ; \mathrm{I}^{2}=18.86 \%$} \\
\hline \multicolumn{7}{|c|}{ Test for overall effect: $Z=3.32(P=0)$} \\
\hline \multicolumn{7}{|c|}{ 8.6.2 Not group exercise } \\
\hline Arkkukangas 2015 & 27 & 13 & $-0.2(0.65)$ & 1 & $0.2 \%$ & $0.8[0.22,2.87]$ \\
\hline Boongrid 2017 & 218 & 219 & $-0.2(0.19)$ & 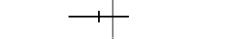 & $2.39 \%$ & $0.84[0.58,1.22]$ \\
\hline Campbell 1997 & 116 & 117 & $-0.2(0.19)$ & 1 & $2.39 \%$ & $0.81[0.56,1.18]$ \\
\hline Clegg 2014 & 40 & 30 & $-0.4(0.46)$ & 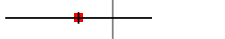 & $0.41 \%$ & $0.66[0.27,1.62]$ \\
\hline Clemson 2010 & 17 & 14 & $-0.3(0.32)$ & 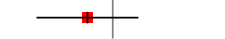 & $0.84 \%$ & $0.73[0.39,1.37]$ \\
\hline Clemson 2012 & 99 & 91 & $-0.2(0.1)$ & + & $8.62 \%$ & $0.78[0.64,0.95]$ \\
\hline Cornillon 2002 & 150 & 153 & $-0.2(0.18)$ & 1 & $2.66 \%$ & $0.83[0.58,1.18]$ \\
\hline Dadgari 2016 & 160 & 157 & $-0.1(0.04)$ & + & $53.85 \%$ & $0.89[0.82,0.96]$ \\
\hline Iliffe 2015 & 227 & 252 & $-0.1(0.16)$ & 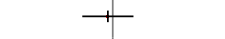 & $3.37 \%$ & $0.94[0.69,1.29]$ \\
\hline Iwamoto 2009 & 34 & 33 & $-2.2(1.34)$ & & $0.05 \%$ & $0.11[0.01,1.52]$ \\
\hline Kerse 2010 & 98 & 95 & $0.2(0.16)$ & + & $3.37 \%$ & $1.17[0.86,1.61]$ \\
\hline Liu-Ambrose 2008 & 28 & 24 & $-0.4(0.26)$ & - & $1.27 \%$ & $0.64[0.38,1.06]$ \\
\hline Luukinen 2007 & 217 & 220 & $-0.1(0.08)$ & + & $13.46 \%$ & $0.94[0.81,1.1]$ \\
\hline Robertson 2001a & 121 & 119 & $-0.3(0.17)$ & -1 & $2.98 \%$ & $0.73[0.53,1.02]$ \\
\hline Sakamoto 2013 & 410 & 455 & $-0.4(0.16)$ & $千$ & $3.37 \%$ & $0.68[0.49,0.93]$ \\
\hline Yang 2012 & 59 & 62 & $-0.4(0.33)$ & $m$ & $0.79 \%$ & $0.7[0.37,1.33]$ \\
\hline Subtotal $(95 \% \mathrm{Cl})$ & & & & 1 & $100 \%$ & $0.87[0.82,0.92]$ \\
\hline \multicolumn{7}{|c|}{ Heterogeneity: $\operatorname{Tau}^{2}=0 ; \mathrm{Chi}^{2}=14.82, \mathrm{df}=15(\mathrm{P}=0.46) ; \mathrm{I}^{2}=0 \%$} \\
\hline \multicolumn{7}{|c|}{ Test for overall effect: $Z=4.83(P<0.0001)$} \\
\hline \multicolumn{7}{|c|}{ Test for subgroup differences: $\mathrm{Chi}^{2}=0.01, \mathrm{df}=1(\mathrm{P}=0.92), \mathrm{I}^{2}=0 \%$} \\
\hline
\end{tabular}

Favours exercise 
Comparison 9. Multiple categories of exercise versus control: subgroup analyses

\begin{tabular}{|c|c|c|c|c|}
\hline Outcome or subgroup title & No. of studies & $\begin{array}{l}\text { No. of partici- } \\
\text { pants }\end{array}$ & Statistical method & Effect size \\
\hline $\begin{array}{l}1 \text { Rate of falls, subgrouped by baseline } \\
\text { fall risk }\end{array}$ & 11 & & $\begin{array}{l}\text { Rate Ratio (Random, 95\% } \\
\mathrm{Cl} \text { ) }\end{array}$ & Subtotals only \\
\hline 1.1 Not selected for higher risk of falling & 6 & 786 & $\begin{array}{l}\text { Rate Ratio (Random, 95\% } \\
\mathrm{Cl} \text { ) }\end{array}$ & $0.54[0.29,0.99]$ \\
\hline 1.2 Selected for higher risk of falling & 5 & 618 & $\begin{array}{l}\text { Rate Ratio (Random, 95\% } \\
\mathrm{Cl} \text { ) }\end{array}$ & $0.77[0.63,0.94]$ \\
\hline $\begin{array}{l}2 \text { Number of fallers, subgrouped by } \\
\text { baseline fall risk }\end{array}$ & 17 & 1623 & $\begin{array}{l}\text { Risk Ratio (Random, 95\% } \\
\mathrm{Cl} \text { ) }\end{array}$ & $0.78[0.64,0.96]$ \\
\hline 2.1 Not selected for higher risk of falling & 7 & 710 & $\begin{array}{l}\text { Risk Ratio (Random, 95\% } \\
\mathrm{Cl} \text { ) }\end{array}$ & $0.70[0.41,1.19]$ \\
\hline 2.2 Selected for higher risk of falling & 10 & 913 & $\begin{array}{l}\text { Risk Ratio (Random, 95\% } \\
\mathrm{Cl} \text { ) }\end{array}$ & $0.84[0.71,1.00]$ \\
\hline 3 Rate of falls, subgrouped by personnel & 11 & & $\begin{array}{l}\text { Rate Ratio (Random, 95\% } \\
\mathrm{Cl} \text { ) }\end{array}$ & Subtotals only \\
\hline $\begin{array}{l}\text { 3.1 Health professional delivering inter- } \\
\text { vention }\end{array}$ & 3 & 653 & $\begin{array}{l}\text { Rate Ratio (Random, 95\% } \\
\mathrm{Cl} \text { ) }\end{array}$ & $0.65[0.43,0.99]$ \\
\hline $\begin{array}{l}\text { 3.2 No health professional delivering in- } \\
\text { tervention }\end{array}$ & 8 & 751 & $\begin{array}{l}\text { Rate Ratio (Random, 95\% } \\
\mathrm{Cl} \text { ) }\end{array}$ & $0.66[0.44,0.99]$ \\
\hline $\begin{array}{l}4 \text { Number of fallers, subgrouped by per- } \\
\text { sonnel }\end{array}$ & 16 & & $\begin{array}{l}\text { Risk Ratio (Random, 95\% } \\
\text { Cl) }\end{array}$ & Subtotals only \\
\hline $\begin{array}{l}\text { 4.1 Health professional delivering inter- } \\
\text { vention }\end{array}$ & 8 & 867 & $\begin{array}{l}\text { Risk Ratio (Random, 95\% } \\
\mathrm{Cl} \text { ) }\end{array}$ & $0.81[0.65,1.02]$ \\
\hline $\begin{array}{l}4.2 \text { No health professional delivering in- } \\
\text { tervention }\end{array}$ & 8 & 711 & $\begin{array}{l}\text { Risk Ratio (Random, 95\% } \\
\mathrm{Cl} \text { ) }\end{array}$ & $0.70[0.45,1.10]$ \\
\hline $\begin{array}{l}5 \text { Rate of falls, subgrouped by group or } \\
\text { individual exercise }\end{array}$ & 11 & & $\begin{array}{l}\text { Rate Ratio (Random, 95\% } \\
\mathrm{Cl} \text { ) }\end{array}$ & Subtotals only \\
\hline 5.1 Group exercise & 10 & 1194 & $\begin{array}{l}\text { Rate Ratio (Random, 95\% } \\
\text { Cl) }\end{array}$ & $0.64[0.46,0.89]$ \\
\hline 5.2 Not group exercise & 1 & 210 & $\begin{array}{l}\text { Rate Ratio (Random, 95\% } \\
\mathrm{Cl} \text { ) }\end{array}$ & $0.81[0.56,1.18]$ \\
\hline $\begin{array}{l}6 \text { Number of fallers, subgrouped by } \\
\text { group or individual exercise }\end{array}$ & 17 & & $\begin{array}{l}\text { Risk Ratio (Random, 95\% } \\
\mathrm{Cl} \text { ) }\end{array}$ & Subtotals only \\
\hline 6.1 Group exercise & 14 & 1301 & $\begin{array}{l}\text { Risk Ratio (Random, 95\% } \\
\mathrm{Cl} \text { ) }\end{array}$ & $0.77[0.60,1.00]$ \\
\hline 6.2 Not group exercise & 3 & 322 & $\begin{array}{l}\text { Risk Ratio (Random, 95\% } \\
\text { Cl) }\end{array}$ & $0.86[0.72,1.03]$ \\
\hline
\end{tabular}


Analysis 9.1. Comparison 9 Multiple categories of exercise versus control: subgroup analyses, Outcome 1 Rate of falls, subgrouped by baseline fall risk.

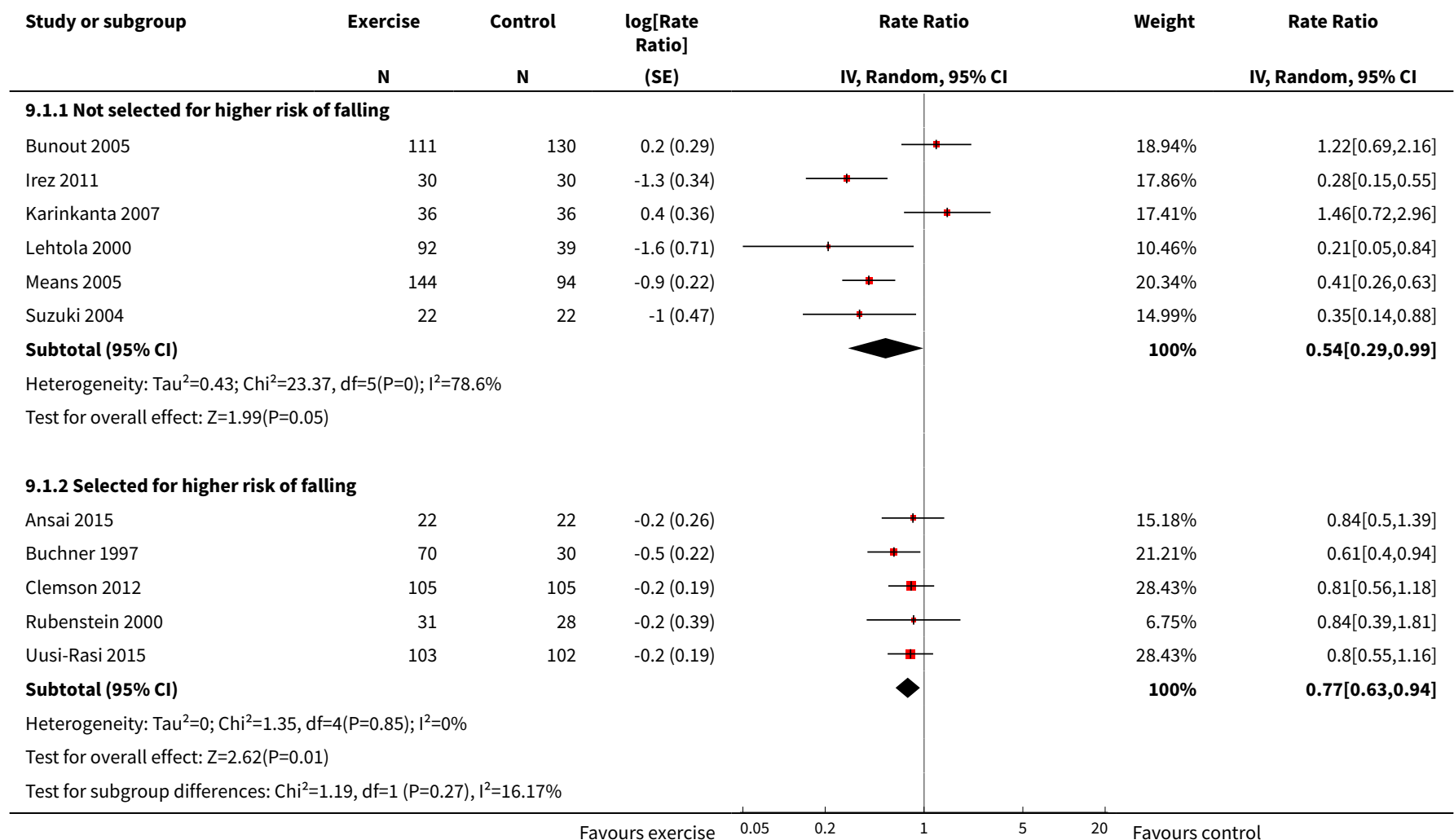

Analysis 9.2. Comparison 9 Multiple categories of exercise versus control: subgroup analyses, Outcome 2 Number of fallers, subgrouped by baseline fall risk.

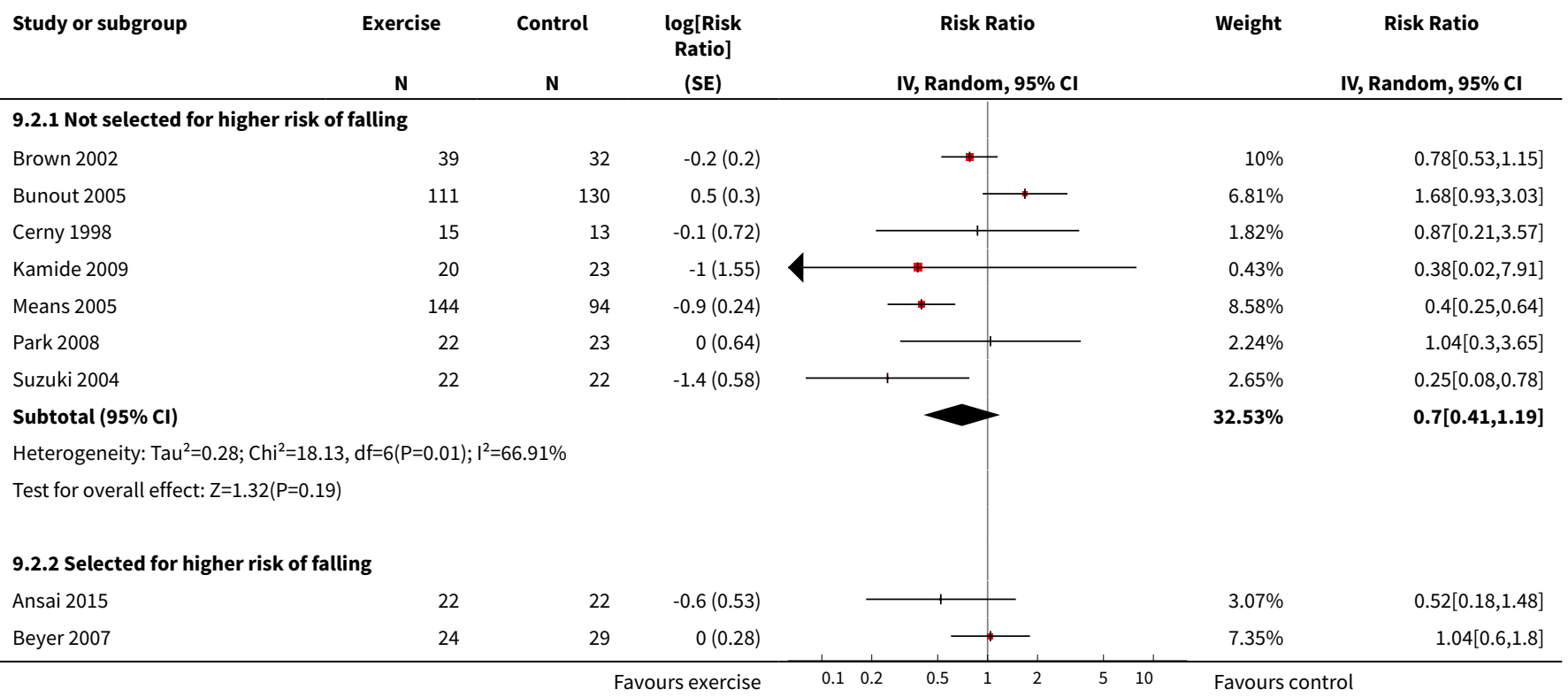




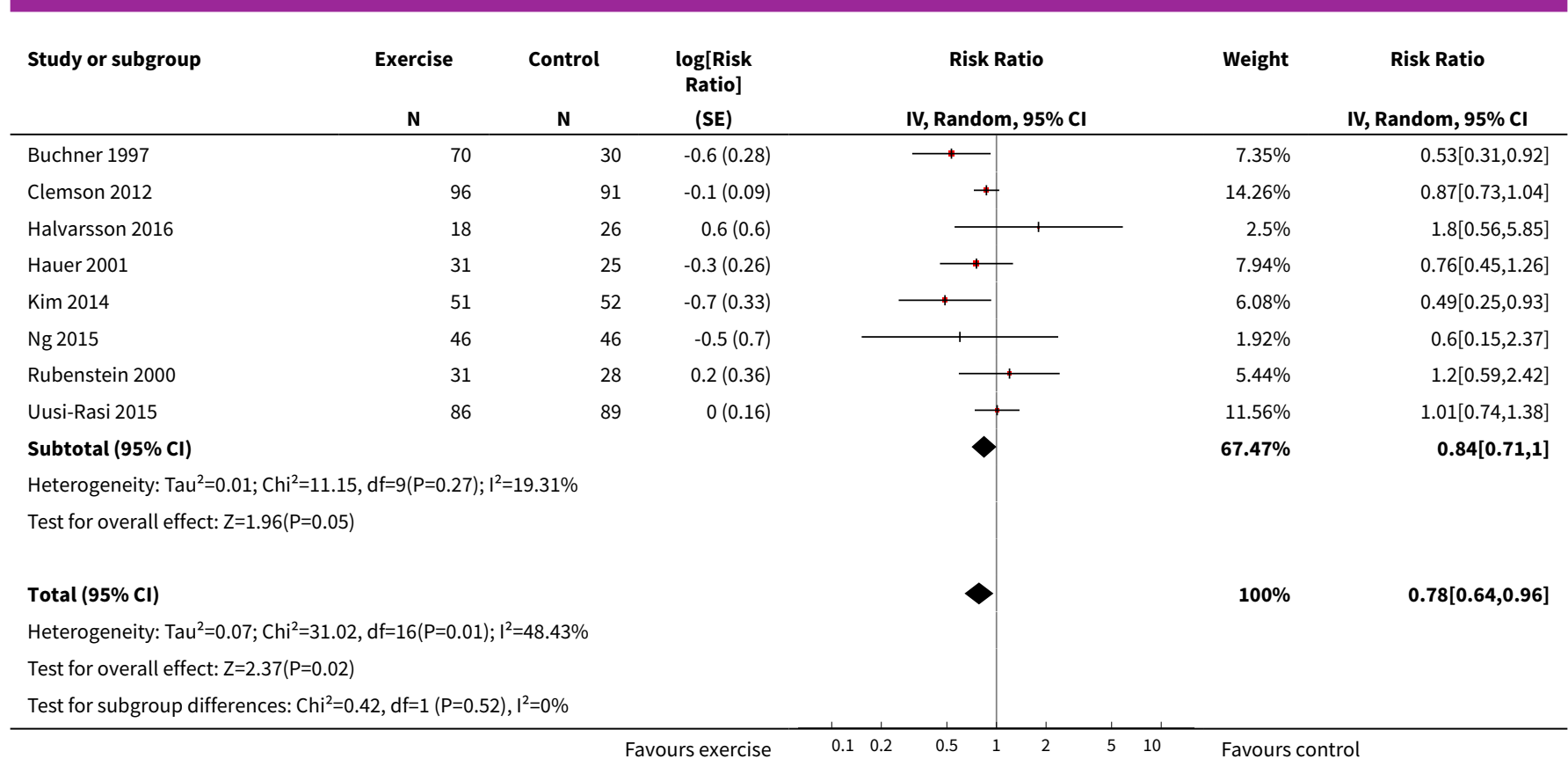

Analysis 9.3. Comparison 9 Multiple categories of exercise versus control: subgroup analyses, Outcome 3 Rate of falls, subgrouped by personnel.

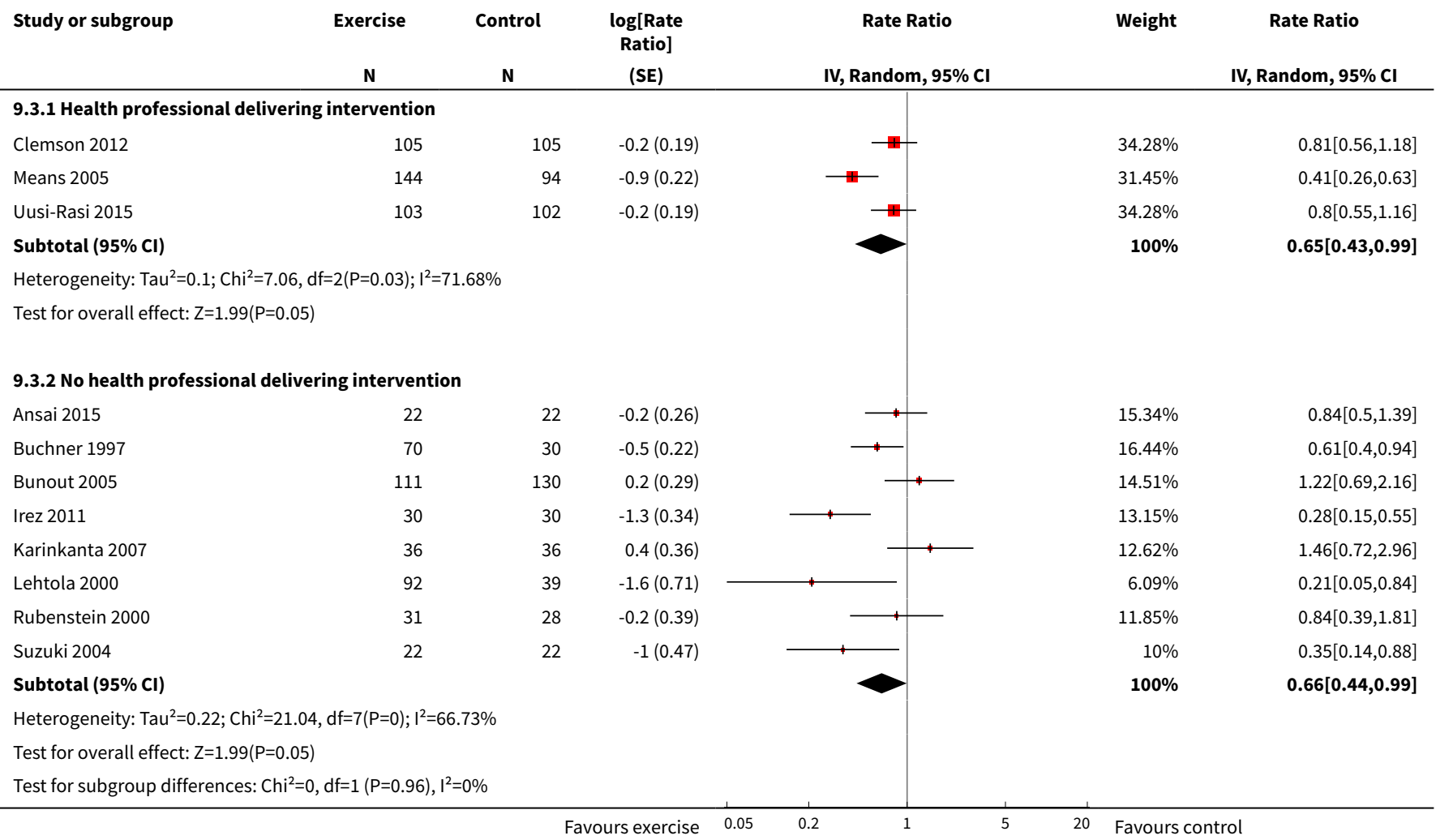


Analysis 9.4. Comparison 9 Multiple categories of exercise versus control: subgroup analyses, Outcome 4 Number of fallers, subgrouped by personnel.

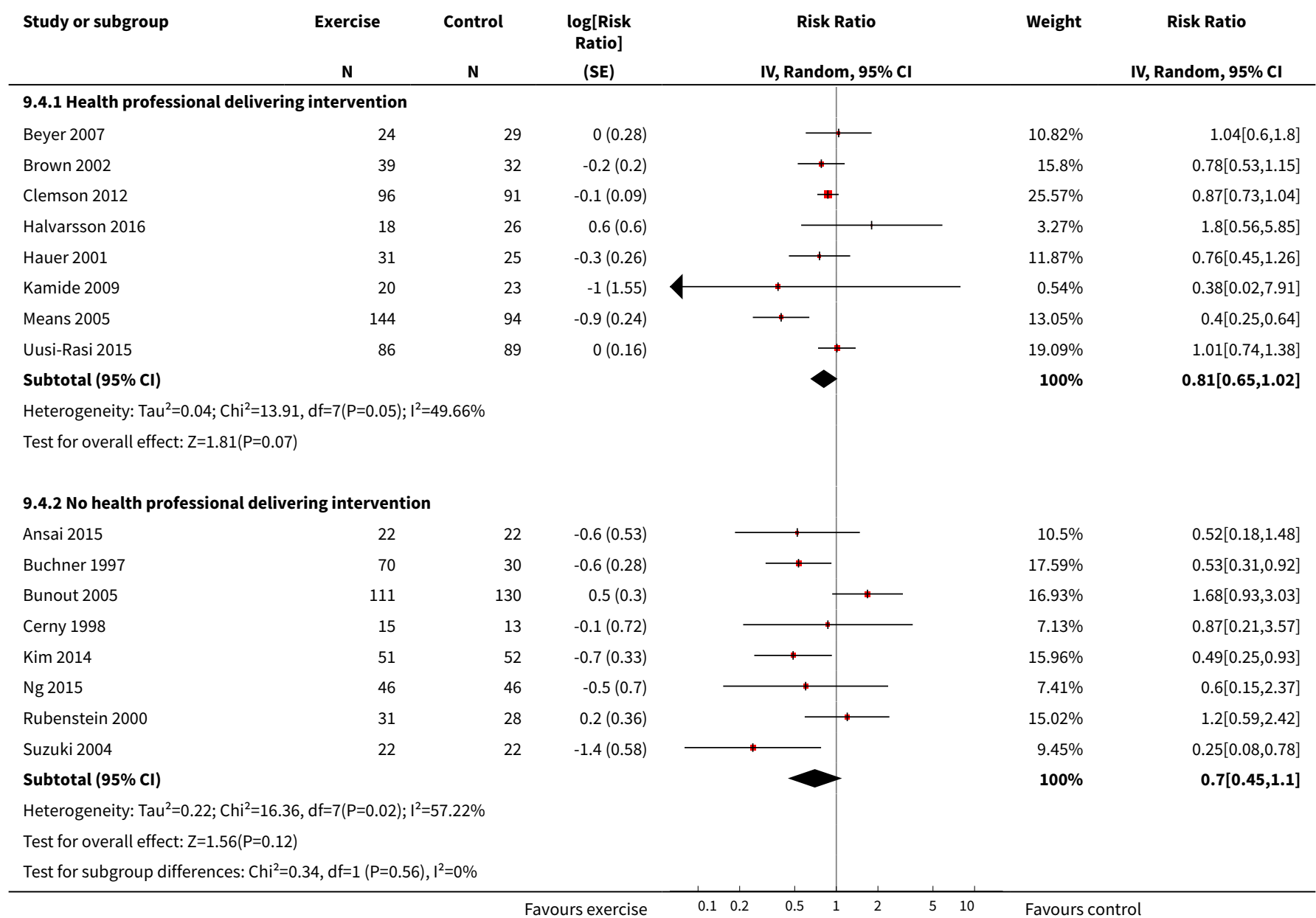

\section{Analysis 9.5. Comparison 9 Multiple categories of exercise versus control: subgroup analyses, Outcome 5 Rate of falls, subgrouped by group or individual exercise.}

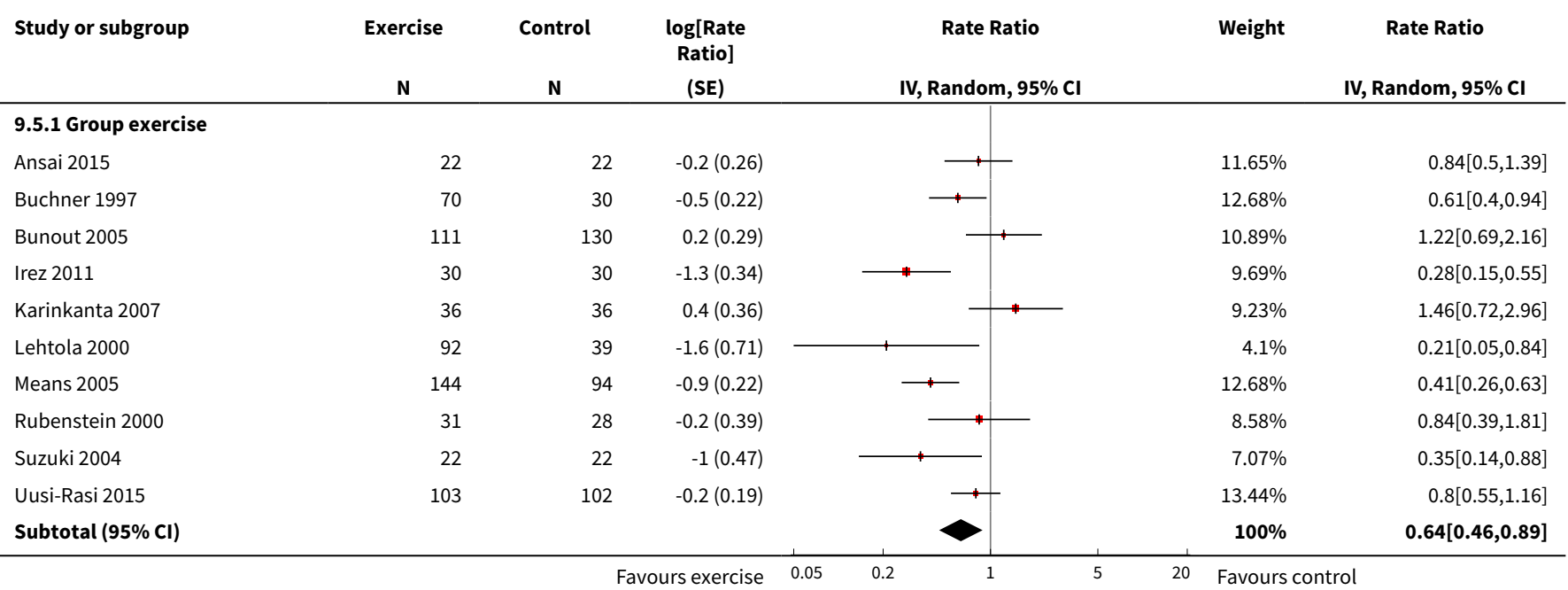




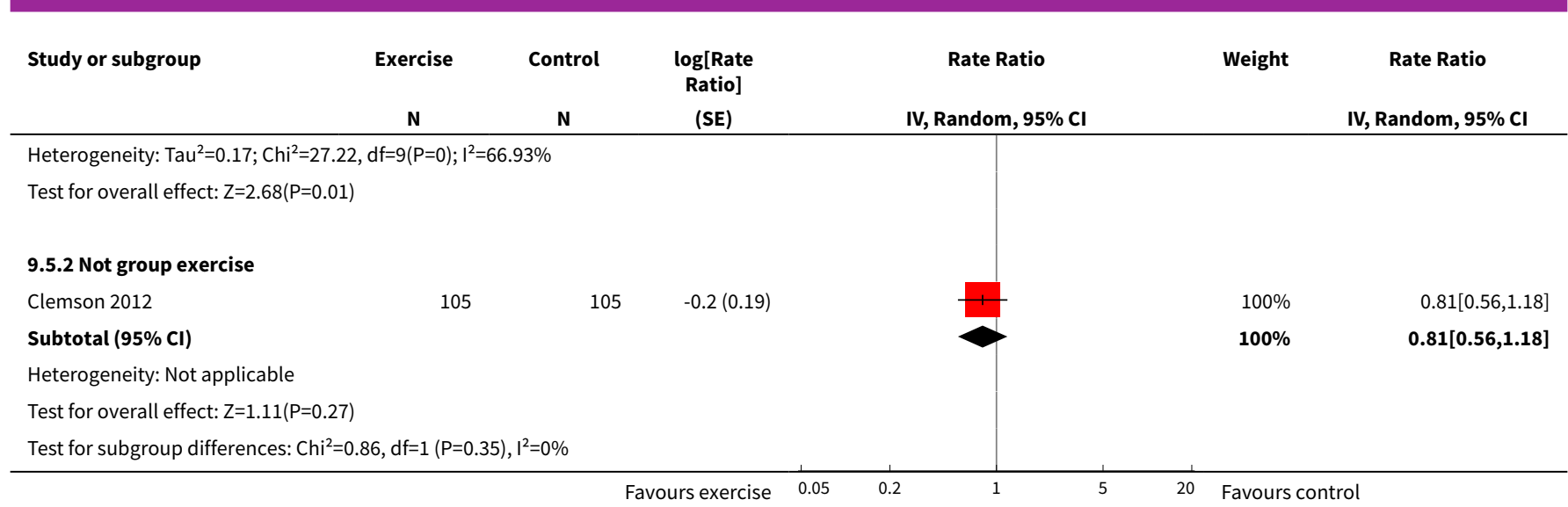

Analysis 9.6. Comparison 9 Multiple categories of exercise versus control: subgroup analyses, Outcome 6 Number of fallers, subgrouped by group or individual exercise.

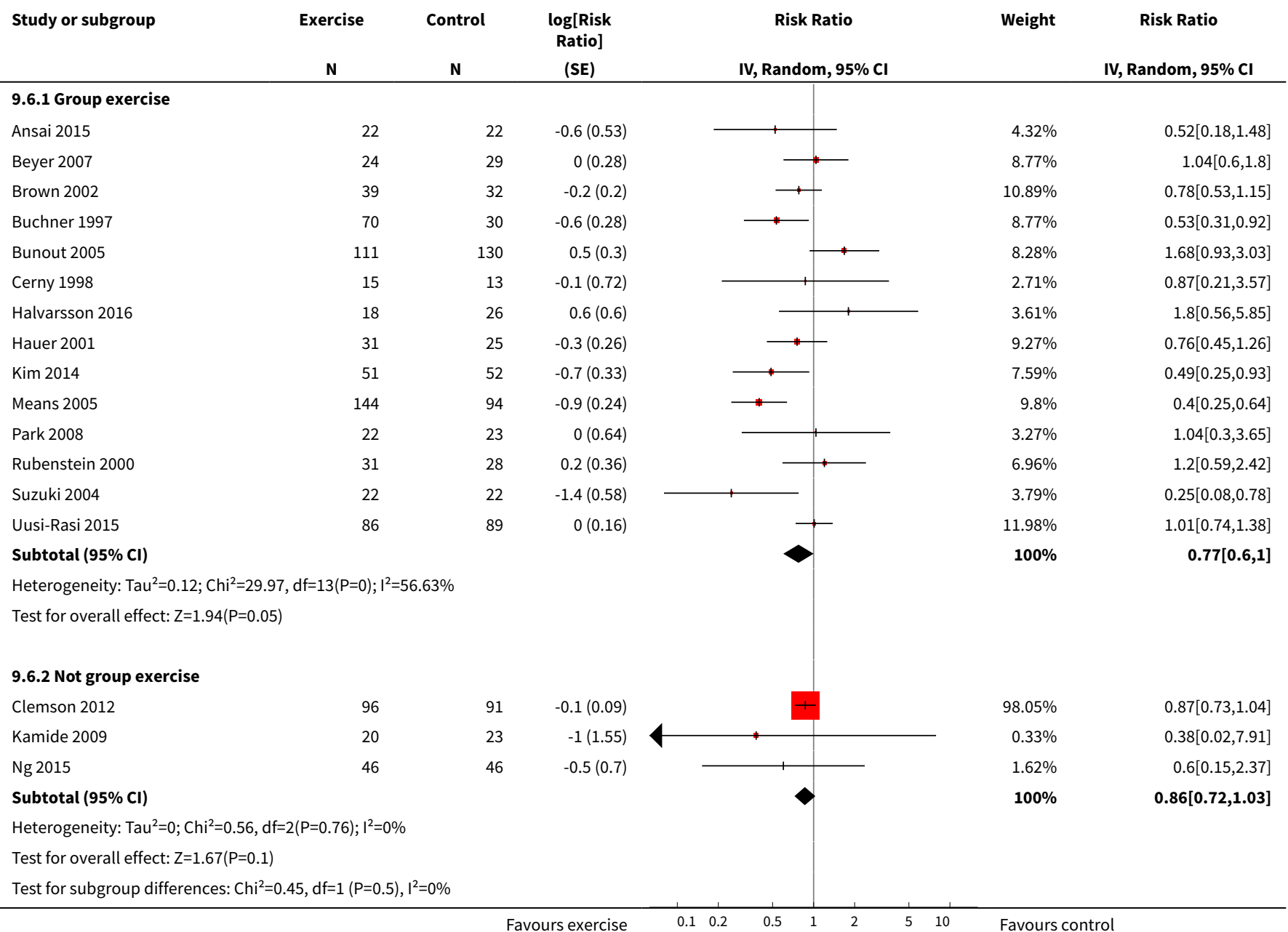


Comparison 10. Exercise versus control (by exercise type, in people after hospital stays)

\begin{tabular}{|c|c|c|c|c|}
\hline Outcome or subgroup title & No. of studies & $\begin{array}{l}\text { No. of partici- } \\
\text { pants }\end{array}$ & Statistical method & Effect size \\
\hline 1 Rate of falls & 3 & & Rate Ratio (Random, 95\% Cl) & $\begin{array}{l}\text { Totals not select- } \\
\text { ed }\end{array}$ \\
\hline 1.1 Resistance exercise vs control & 1 & & Rate Ratio (Random, 95\% Cl) & $0.0[0.0,0.0]$ \\
\hline $\begin{array}{l}\text { 1.2 Multiple categories of exercise vs } \\
\text { control }\end{array}$ & 2 & & Rate Ratio (Random, 95\% Cl) & $0.0[0.0,0.0]$ \\
\hline 2 Number of fallers & 4 & & Risk Ratio (Random, 95\% Cl) & $\begin{array}{l}\text { Totals not select- } \\
\text { ed }\end{array}$ \\
\hline 2.1 Resistance exercise vs Control & 2 & & Risk Ratio (Random, 95\% Cl) & $0.0[0.0,0.0]$ \\
\hline $\begin{array}{l}2.2 \text { Multiple categories of exercise vs } \\
\text { Control }\end{array}$ & 3 & & Risk Ratio (Random, 95\% Cl) & $0.0[0.0,0.0]$ \\
\hline 3 Health-related quality of life & 3 & & $\begin{array}{l}\text { Std. Mean Difference (IV, Ran- } \\
\text { dom, } 95 \% \mathrm{Cl} \text { ) }\end{array}$ & $\begin{array}{l}\text { Totals not select- } \\
\text { ed }\end{array}$ \\
\hline 3.1 Resistance exercise vs control & 1 & & $\begin{array}{l}\text { Std. Mean Difference (IV, Ran- } \\
\text { dom, } 95 \% \mathrm{Cl} \text { ) }\end{array}$ & $0.0[0.0,0.0]$ \\
\hline $\begin{array}{l}3.2 \text { Multiple categories of exercise } \\
\text { versus control }\end{array}$ & 2 & & $\begin{array}{l}\text { Std. Mean Difference (IV, Ran- } \\
\text { dom, } 95 \% \mathrm{Cl} \text { ) }\end{array}$ & $0.0[0.0,0.0]$ \\
\hline 4 Number of people who died & 4 & & $\begin{array}{l}\text { Risk Ratio (M-H, Random, 95\% } \\
\text { Cl) }\end{array}$ & $\begin{array}{l}\text { Totals not select- } \\
\text { ed }\end{array}$ \\
\hline 4.1 Resistance exercise vs control & 2 & & $\begin{array}{l}\text { Risk Ratio (M-H, Random, 95\% } \\
\text { Cl) }\end{array}$ & $0.0[0.0,0.0]$ \\
\hline $\begin{array}{l}\text { 4.2 Multiple categories of exercise vs } \\
\text { control }\end{array}$ & 3 & & $\begin{array}{l}\text { Risk Ratio (M-H, Random, 95\% } \\
\mathrm{Cl} \text { ) }\end{array}$ & $0.0[0.0,0.0]$ \\
\hline
\end{tabular}

\section{Analysis 10.1. Comparison 10 Exercise versus control (by exercise type, in people after hospital stays), Outcome 1 Rate of falls.}

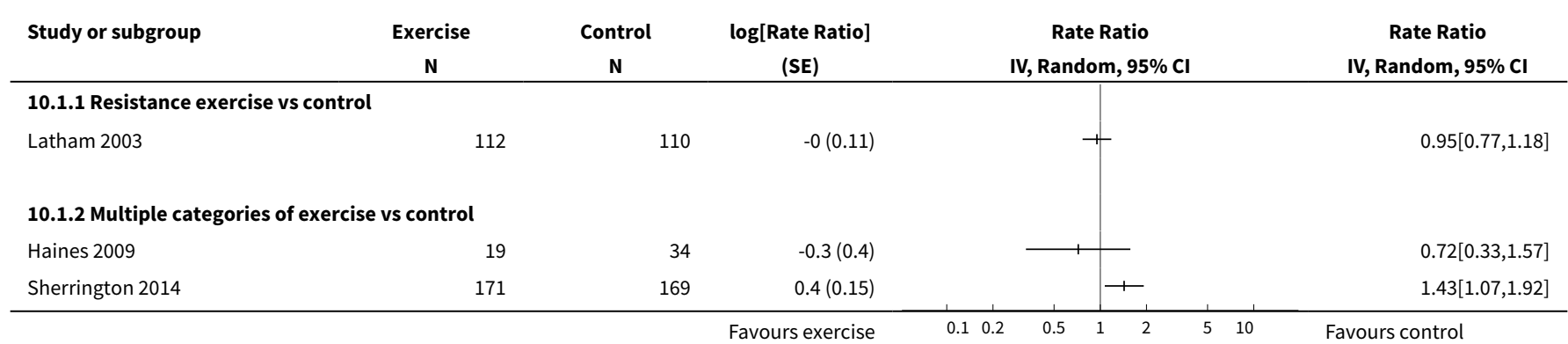


Analysis 10.2. Comparison 10 Exercise versus control (by exercise type, in people after hospital stays), Outcome 2 Number of fallers.

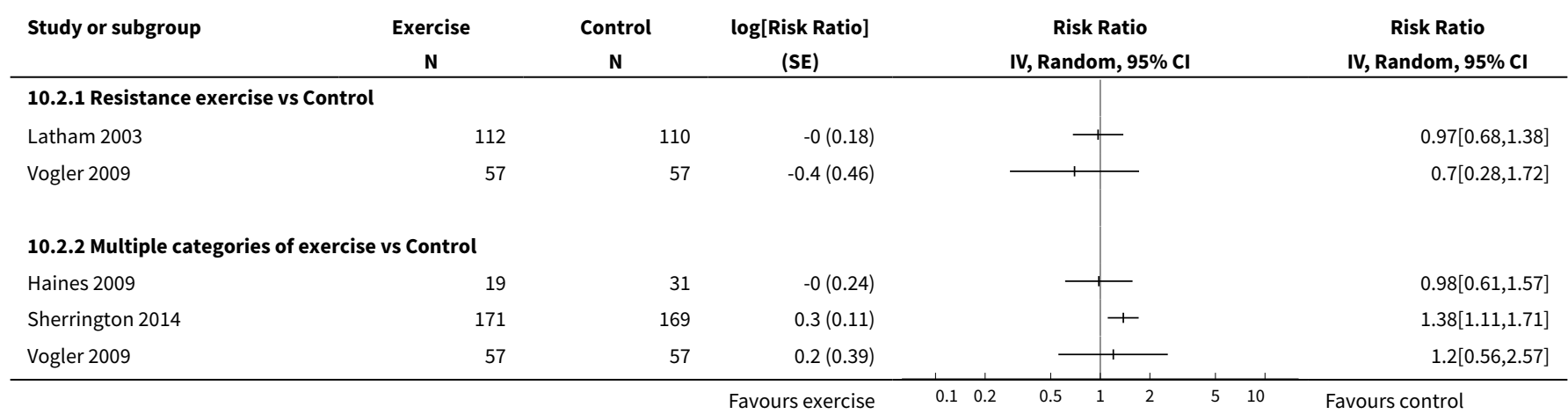

Analysis 10.3. Comparison 10 Exercise versus control (by exercise type, in people after hospital stays), Outcome 3 Health-related quality of life.

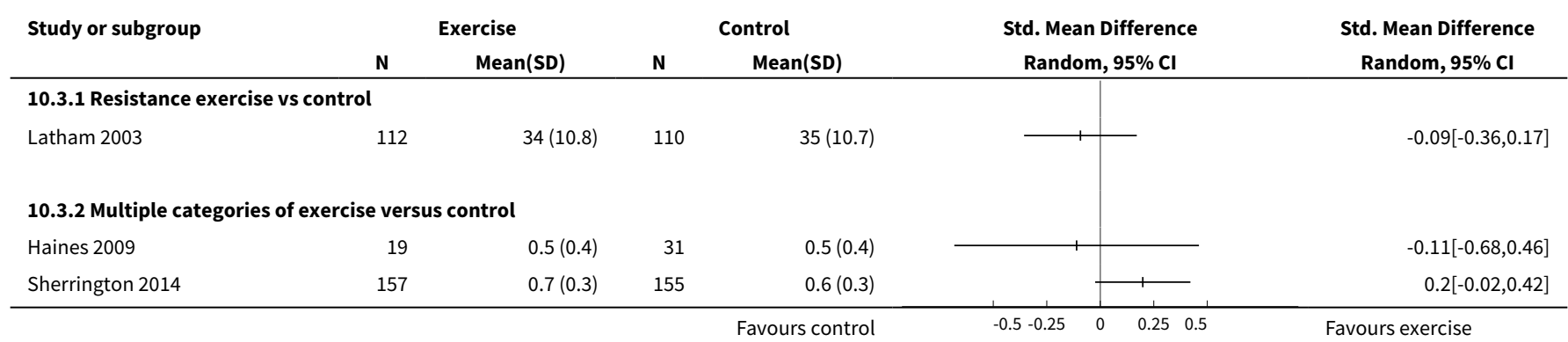

Analysis 10.4. Comparison 10 Exercise versus control (by exercise type, in people after hospital stays), Outcome 4 Number of people who died.

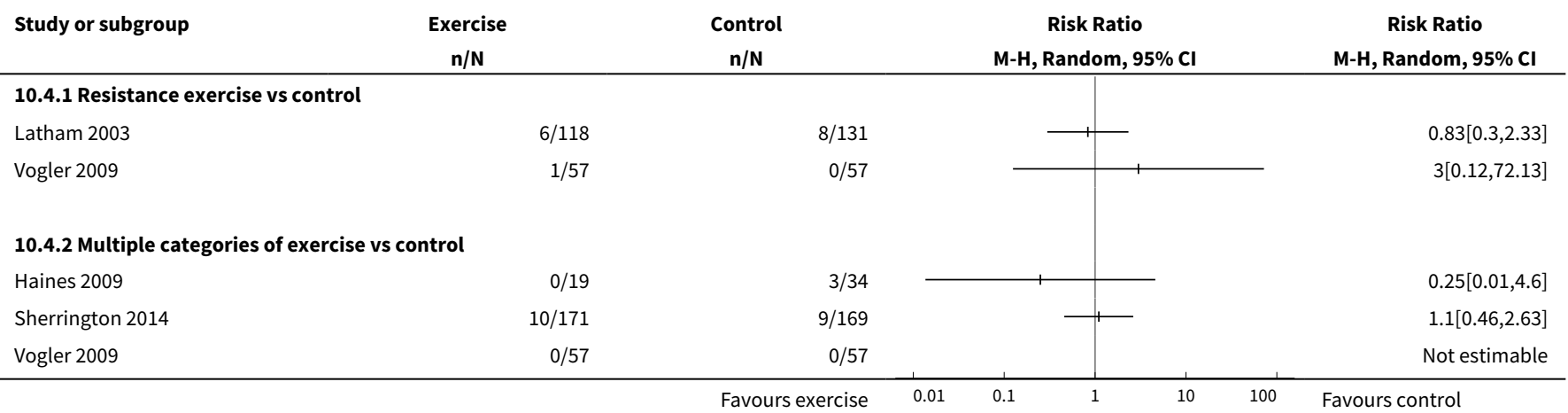




\section{Comparison 11. Exercise versus exercise}

\begin{tabular}{|c|c|c|c|c|}
\hline Outcome or subgroup title & No. of studies & $\begin{array}{l}\text { No. of partici- } \\
\text { pants }\end{array}$ & Statistical method & Effect size \\
\hline $\begin{array}{l}1 \text { Rate of falls, different types of exercise } \\
\text { compared }\end{array}$ & 20 & & $\begin{array}{l}\text { Rate Ratio (Random, } \\
95 \% \mathrm{Cl} \text { ) }\end{array}$ & $\begin{array}{l}\text { Totals not select- } \\
\text { ed }\end{array}$ \\
\hline $\begin{array}{l}1.1 \text { Balance and functional exercises vs bal- } \\
\text { ance and functional exercises }\end{array}$ & 6 & & $\begin{array}{l}\text { Rate Ratio (Random, } \\
95 \% \mathrm{Cl} \text { ) }\end{array}$ & $0.0[0.0,0.0]$ \\
\hline $\begin{array}{l}\text { 1.2 Balance and functional exercises vs re- } \\
\text { sistance exercises }\end{array}$ & 3 & & $\begin{array}{l}\text { Rate Ratio (Random, } \\
95 \% \mathrm{Cl} \text { ) }\end{array}$ & $0.0[0.0,0.0]$ \\
\hline $\begin{array}{l}1.3 \text { Balance and functional exercises vs } \\
\text { walking }\end{array}$ & 2 & & $\begin{array}{l}\text { Rate Ratio (Random, } \\
95 \% \mathrm{Cl} \text { ) }\end{array}$ & $0.0[0.0,0.0]$ \\
\hline $\begin{array}{l}\text { 1.4 Balance and functional exercises vs mul- } \\
\text { tiple categories of exercise }\end{array}$ & 1 & & $\begin{array}{l}\text { Rate Ratio (Random, } \\
95 \% \mathrm{Cl} \text { ) }\end{array}$ & $0.0[0.0,0.0]$ \\
\hline $\begin{array}{l}1.53 \mathrm{D} \text { (Tai Chi) vs balance and functional ex- } \\
\text { ercises }\end{array}$ & 2 & & $\begin{array}{l}\text { Rate Ratio (Random, } \\
95 \% \mathrm{Cl} \text { ) }\end{array}$ & $0.0[0.0,0.0]$ \\
\hline 1.6 3D (Tai Chi) vs 3D (Tai Chi) & 1 & & $\begin{array}{l}\text { Rate Ratio (Random, } \\
95 \% \mathrm{Cl} \text { ) }\end{array}$ & $0.0[0.0,0.0]$ \\
\hline $\begin{array}{l}1.7 \text { Multiple categories of exercise vs bal- } \\
\text { ance and functional exercises }\end{array}$ & 1 & & $\begin{array}{l}\text { Rate Ratio (Random, } \\
95 \% \mathrm{Cl} \text { ) }\end{array}$ & $0.0[0.0,0.0]$ \\
\hline $\begin{array}{l}1.8 \text { Multiple categories of exercise vs resis- } \\
\text { tance exercises }\end{array}$ & 2 & & $\begin{array}{l}\text { Rate Ratio (Random, } \\
95 \% \mathrm{Cl} \text { ) }\end{array}$ & $0.0[0.0,0.0]$ \\
\hline $\begin{array}{l}1.9 \text { Multiple categories of exercise vs multi- } \\
\text { ple categories of exercise }\end{array}$ & 4 & & $\begin{array}{l}\text { Rate Ratio (Random, } \\
95 \% \mathrm{Cl} \text { ) }\end{array}$ & $0.0[0.0,0.0]$ \\
\hline $\begin{array}{l}2 \text { Rate of falls }>18 \text { months, different types of } \\
\text { exercise compared }\end{array}$ & 1 & & $\begin{array}{l}\text { Rate Ratio (Random, } \\
95 \% \mathrm{Cl} \text { ) }\end{array}$ & $\begin{array}{l}\text { Totals not select- } \\
\text { ed }\end{array}$ \\
\hline $\begin{array}{l}\text { 2.1 Multiple categories of exercise vs multi- } \\
\text { ple categories of exercise }\end{array}$ & 1 & & $\begin{array}{l}\text { Rate Ratio (Random, } \\
95 \% \mathrm{Cl} \text { ) }\end{array}$ & $0.0[0.0,0.0]$ \\
\hline $\begin{array}{l}3 \text { Number of fallers, different types of exer- } \\
\text { cise compared }\end{array}$ & 17 & & $\begin{array}{l}\text { Risk Ratio (Random, } \\
95 \% \mathrm{Cl} \text { ) }\end{array}$ & $\begin{array}{l}\text { Totals not select- } \\
\text { ed }\end{array}$ \\
\hline $\begin{array}{l}3.1 \text { Balance and functional exercises vs bal- } \\
\text { ance and functional exercises }\end{array}$ & 5 & & $\begin{array}{l}\text { Risk Ratio (Random, } \\
95 \% \mathrm{Cl} \text { ) }\end{array}$ & $0.0[0.0,0.0]$ \\
\hline $\begin{array}{l}3.2 \text { Balance and functional exercises vs } \\
\text { walking }\end{array}$ & 2 & & $\begin{array}{l}\text { Risk Ratio (Random, } \\
95 \% \mathrm{Cl} \text { ) }\end{array}$ & $0.0[0.0,0.0]$ \\
\hline $\begin{array}{l}\text { 3.3 Balance and functional exercises vs mul- } \\
\text { tiple categories of exercise }\end{array}$ & 1 & & $\begin{array}{l}\text { Risk Ratio (Random, } \\
95 \% \mathrm{Cl} \text { ) }\end{array}$ & $0.0[0.0,0.0]$ \\
\hline $\begin{array}{l}\text { 3.4 3D (Tai Chi) vs balance and functional ex- } \\
\text { ercises }\end{array}$ & 1 & & $\begin{array}{l}\text { Risk Ratio (Random, } \\
95 \% \mathrm{Cl} \text { ) }\end{array}$ & $0.0[0.0,0.0]$ \\
\hline 3.5 3D (Tai Chi) vs resistance exercises & 1 & & $\begin{array}{l}\text { Risk Ratio (Random, } \\
95 \% \mathrm{Cl} \text { ) }\end{array}$ & $0.0[0.0,0.0]$ \\
\hline
\end{tabular}




\begin{tabular}{|c|c|c|c|c|}
\hline Outcome or subgroup title & No. of studies & $\begin{array}{l}\text { No. of partici- } \\
\text { pants }\end{array}$ & Statistical method & Effect size \\
\hline $\begin{array}{l}\text { 3.6 Multiple categories of exercise vs bal- } \\
\text { ance and functional exercises }\end{array}$ & 1 & & $\begin{array}{l}\text { Risk Ratio (Random, } \\
95 \% \mathrm{CI} \text { ) }\end{array}$ & $0.0[0.0,0.0]$ \\
\hline $\begin{array}{l}\text { 3.7 Multiple categories of exercise vs resis- } \\
\text { tance exercises }\end{array}$ & 1 & & $\begin{array}{l}\text { Risk Ratio (Random, } \\
95 \% \mathrm{Cl} \text { ) }\end{array}$ & $0.0[0.0,0.0]$ \\
\hline $\begin{array}{l}\text { 3.8 Multiple categories of exercise vs resis- } \\
\text { tance exercises (after hospital stays) }\end{array}$ & 1 & & $\begin{array}{l}\text { Risk Ratio (Random, } \\
95 \% \mathrm{Cl} \text { ) }\end{array}$ & $0.0[0.0,0.0]$ \\
\hline $\begin{array}{l}3.9 \text { Multiple categories of exercise vs multi- } \\
\text { ple categories of exercise }\end{array}$ & 4 & & $\begin{array}{l}\text { Risk Ratio (Random, } \\
95 \% \mathrm{Cl} \text { ) }\end{array}$ & $0.0[0.0,0.0]$ \\
\hline $\begin{array}{l}4 \text { Number of people who experienced one or } \\
\text { more fall-related fractures, different types of } \\
\text { exercise compared }\end{array}$ & 3 & & $\begin{array}{l}\text { Risk Ratio (Random, } \\
95 \% \mathrm{Cl} \text { ) }\end{array}$ & $\begin{array}{l}\text { Totals not select- } \\
\text { ed }\end{array}$ \\
\hline $\begin{array}{l}\text { 4.1 Balance and functional exercise vs bal- } \\
\text { ance and functional exercise }\end{array}$ & 2 & & $\begin{array}{l}\text { Risk Ratio (Random, } \\
95 \% \mathrm{Cl} \text { ) }\end{array}$ & $0.0[0.0,0.0]$ \\
\hline $\begin{array}{l}\text { 4.2 Balance and functional exercises vs re- } \\
\text { sistance exercises }\end{array}$ & 1 & & $\begin{array}{l}\text { Risk Ratio (Random, } \\
95 \% \mathrm{Cl} \text { ) }\end{array}$ & $0.0[0.0,0.0]$ \\
\hline $\begin{array}{l}4.3 \text { Multiple categories of exercise vs resis- } \\
\text { tance exercises }\end{array}$ & 1 & & $\begin{array}{l}\text { Risk Ratio (Random, } \\
95 \% \mathrm{Cl} \text { ) }\end{array}$ & $0.0[0.0,0.0]$ \\
\hline $\begin{array}{l}5 \text { Number of people who experienced one or } \\
\text { more falls that required medical attention, } \\
\text { different types of exercise compared }\end{array}$ & 1 & & $\begin{array}{l}\text { Risk Ratio (Random, } \\
95 \% \mathrm{Cl} \text { ) }\end{array}$ & $\begin{array}{l}\text { Totals not select- } \\
\text { ed }\end{array}$ \\
\hline $\begin{array}{l}5.1 \text { Balance and functional exercises vs re- } \\
\text { sistance exercises }\end{array}$ & 1 & & $\begin{array}{l}\text { Risk Ratio (Random, } \\
95 \% \mathrm{Cl} \text { ) }\end{array}$ & $0.0[0.0,0.0]$ \\
\hline $\begin{array}{l}5.2 \text { Multiple categories of exercise vs bal- } \\
\text { ance and functional exercises }\end{array}$ & 1 & & $\begin{array}{l}\text { Risk Ratio (Random, } \\
95 \% \mathrm{Cl} \text { ) }\end{array}$ & $0.0[0.0,0.0]$ \\
\hline $\begin{array}{l}5.3 \text { Multiple categories of exercise vs resis- } \\
\text { tance exercises }\end{array}$ & 1 & & $\begin{array}{l}\text { Risk Ratio (Random, } \\
95 \% \mathrm{Cl} \text { ) }\end{array}$ & $0.0[0.0,0.0]$ \\
\hline $\begin{array}{l}6 \text { Quality of life, different types of exercise } \\
\text { compared }\end{array}$ & 1 & & $\begin{array}{l}\text { Std. Mean Difference (IV, } \\
\text { Random, } 95 \% \mathrm{Cl} \text { ) }\end{array}$ & $\begin{array}{l}\text { Totals not select- } \\
\text { ed }\end{array}$ \\
\hline $\begin{array}{l}6.1 \text { Balance and functional exercises versus } \\
\text { balance and functional exercises }\end{array}$ & 1 & & $\begin{array}{l}\text { Std. Mean Difference (IV, } \\
\text { Random, } 95 \% \mathrm{Cl} \text { ) }\end{array}$ & $0.0[0.0,0.0]$ \\
\hline $\begin{array}{l}7 \text { Number of people who died, different } \\
\text { types of exercise compared }\end{array}$ & 2 & & $\begin{array}{l}\text { Risk Ratio (IV, Random, } \\
95 \% \mathrm{Cl})\end{array}$ & $\begin{array}{l}\text { Totals not select- } \\
\text { ed }\end{array}$ \\
\hline $\begin{array}{l}7.13 \mathrm{D} \text { (Tai Chi) vs balance and functional ex- } \\
\text { ercises }\end{array}$ & 1 & & $\begin{array}{l}\text { Risk Ratio (IV, Random, } \\
95 \% \mathrm{CI} \text { ) }\end{array}$ & $0.0[0.0,0.0]$ \\
\hline 7.2 Multiple v multiple & 1 & & $\begin{array}{l}\text { Risk Ratio (IV, Random, } \\
95 \% \mathrm{CI} \text { ) }\end{array}$ & $0.0[0.0,0.0]$ \\
\hline $\begin{array}{l}8 \text { Rate of falls, group vs individual exercise } \\
\text { delivery within the same type of exercise }\end{array}$ & 4 & & $\begin{array}{l}\text { Rate Ratio (Random, } \\
95 \% \mathrm{Cl} \text { ) }\end{array}$ & $\begin{array}{l}\text { Totals not select- } \\
\text { ed }\end{array}$ \\
\hline
\end{tabular}




\begin{tabular}{|c|c|c|c|c|}
\hline Outcome or subgroup title & No. of studies & $\begin{array}{l}\text { No. of partici- } \\
\text { pants }\end{array}$ & Statistical method & Effect size \\
\hline 8.1 Balance and functional exercises & 3 & & $\begin{array}{l}\text { Rate Ratio (Random, } \\
95 \% \mathrm{CI} \text { ) }\end{array}$ & $0.0[0.0,0.0]$ \\
\hline 8.2 3D (Tai Chi) exercise & 1 & & $\begin{array}{l}\text { Rate Ratio (Random, } \\
95 \% \mathrm{CI} \text { ) }\end{array}$ & $0.0[0.0,0.0]$ \\
\hline $\begin{array}{l}9 \text { Number of fallers, group vs individual ex- } \\
\text { ercise delivery within the same type of exer- } \\
\text { cise }\end{array}$ & 4 & & $\begin{array}{l}\text { Risk Ratio (Random, } \\
95 \% \mathrm{Cl} \text { ) }\end{array}$ & $\begin{array}{l}\text { Totals not select- } \\
\text { ed }\end{array}$ \\
\hline 9.1 Balance and functional exercises & 4 & & $\begin{array}{l}\text { Risk Ratio (Random, } \\
95 \% \mathrm{Cl} \text { ) }\end{array}$ & $0.0[0.0,0.0]$ \\
\hline $\begin{array}{l}10 \text { Number of people who experienced one } \\
\text { or more falls requiring hospital admission, } \\
\text { group vs individual exercise delivery within } \\
\text { the same type of exercise }\end{array}$ & 1 & & $\begin{array}{l}\text { Risk Ratio (IV, Random, } \\
95 \% \mathrm{CI} \text { ) }\end{array}$ & $\begin{array}{l}\text { Totals not select- } \\
\text { ed }\end{array}$ \\
\hline 10.1 Balance and functional exercises & 1 & & $\begin{array}{l}\text { Risk Ratio (IV, Random, } \\
95 \% \mathrm{CI} \text { ) }\end{array}$ & $0.0[0.0,0.0]$ \\
\hline $\begin{array}{l}11 \text { Health-related quality of life, group vs in- } \\
\text { dividual exercise delivery within the same } \\
\text { type of exercise }\end{array}$ & 1 & & $\begin{array}{l}\text { Std. Mean Difference (IV, } \\
\text { Fixed, } 95 \% \mathrm{CI} \text { ) }\end{array}$ & $\begin{array}{l}\text { Totals not select- } \\
\text { ed }\end{array}$ \\
\hline 11.1 Balance and functional exercises & 1 & & $\begin{array}{l}\text { Std. Mean Difference (IV, } \\
\text { Fixed, } 95 \% \mathrm{CI} \text { ) }\end{array}$ & $0.0[0.0,0.0]$ \\
\hline $\begin{array}{l}12 \text { Number of people who died, group vs in- } \\
\text { dividual exercise delivery within the same } \\
\text { type of exercise }\end{array}$ & 1 & & $\begin{array}{l}\text { Risk Ratio (IV, Random, } \\
95 \% \mathrm{CI} \text { ) }\end{array}$ & $\begin{array}{l}\text { Totals not select- } \\
\text { ed }\end{array}$ \\
\hline 12.1 Balance and functional exercises & 1 & & $\begin{array}{l}\text { Risk Ratio (IV, Random, } \\
95 \% \mathrm{CI} \text { ) }\end{array}$ & $0.0[0.0,0.0]$ \\
\hline $\begin{array}{l}13 \text { Rate of falls, higher vs lower dose within } \\
\text { the same type of exercise }\end{array}$ & 3 & & $\begin{array}{l}\text { Rate Ratio (Random, } \\
95 \% \mathrm{CI} \text { ) }\end{array}$ & $\begin{array}{l}\text { Totals not select- } \\
\text { ed }\end{array}$ \\
\hline 13.1 Balance and functional exercises & 1 & & $\begin{array}{l}\text { Rate Ratio (Random, } \\
95 \% \mathrm{Cl} \text { ) }\end{array}$ & $0.0[0.0,0.0]$ \\
\hline 13.2 Resistance exercises & 1 & & $\begin{array}{l}\text { Rate Ratio (Random, } \\
95 \% \mathrm{Cl} \text { ) }\end{array}$ & $0.0[0.0,0.0]$ \\
\hline 13.3 3D (Tai Chi) & 1 & & $\begin{array}{l}\text { Rate Ratio (Random, } \\
95 \% \mathrm{CI} \text { ) }\end{array}$ & $0.0[0.0,0.0]$ \\
\hline $\begin{array}{l}14 \text { Number of fallers, higher vs lower dose } \\
\text { within the same type of exercise }\end{array}$ & 1 & & $\begin{array}{l}\text { Risk Ratio (Random, } \\
95 \% \mathrm{Cl} \text { ) }\end{array}$ & $\begin{array}{l}\text { Totals not select- } \\
\text { ed }\end{array}$ \\
\hline $14.13 \mathrm{D}$ (Tai Chi) & 1 & & $\begin{array}{l}\text { Risk Ratio (Random, } \\
95 \% \mathrm{CI} \text { ) }\end{array}$ & $0.0[0.0,0.0]$ \\
\hline $\begin{array}{l}15 \text { Number of people who died, higher vs } \\
\text { lower dose within the same type of exercise }\end{array}$ & 1 & & $\begin{array}{l}\text { Risk Ratio (M-H, Ran- } \\
\text { dom, } 95 \% \mathrm{Cl})\end{array}$ & $\begin{array}{l}\text { Totals not select- } \\
\text { ed }\end{array}$ \\
\hline
\end{tabular}


Analysis 11.1. Comparison 11 Exercise versus exercise, Outcome 1 Rate of falls, different types of exercise compared.

Study or subgroup Exercise A Exercise B

$\log [$ Rate Ratio]

(SE)

N

alance and functional exercises

Hirase 2015

Iliffe 2015

Liston 2014

Steadman 2003

Yamada 2012

Yamada 2013

\section{9}

227

8

64

72

118
$-0.9(0.45)$

$-0(0.1)$

0 (0.49)

0 (0.23)

$2.2(0.73)$

$-1(0.32)$

11.1.2 Balance and functional exercises vs resistance exercises

\section{Davis 2011}

Davis 2011

Karinkanta 2007

54

52

35

Liu-Ambrose 2004

11.1.3 Balance and functional exercises vs walking

Shigematsu 2008

Yamada 2010

11.1.4 Balance and functional exercises vs multiple categories of exercise

Clemson 2012

11.1.5 3D (Tai Chi) vs balance and functional exercises

Hwang 2016

167

Wolf 1996

72

167

$-1.1(0.41)$

$-0.5(0.18)$

11.1.6 3D (Tai Chi) vs 3D (Tai Chi)

Wu 2010

Wu 2010

\section{2}

22

$-0.7(0.71)$

$0.3(0.91)$

11.1.7 Multiple categories of exercise vs balance and functional exercises

Karinkanta 2007

36

35

$0(0.33)$

11.1.8 Multiple categories of exercise vs resistance exercises

Ansai 2015

22

36

22

22
37

$-0.9(0.22)$

$0.9(0.42)$

11.1.9 Multiple categories of exercise vs multiple categories of exercise

\section{Freiberger 2007}

Kemmler 2010

Kwok 2016

LaStayo 2017

$\begin{array}{rr}65 & 6 \\ 115 & 11 \\ 40 & 4 \\ 54 & 5\end{array}$

Rate Ratio

Rate Ratio

IV, Random, 95\% CI

IV, Random, $95 \% \mathrm{CI} \quad$ IV, Random, $95 \% \mathrm{CI}$

$0.41[0.17,0.99]$

$0.97[0.8,1.18]$

$1.02[0.39,2.67]$

$1[0.64,1.57]$

$9.39[2.25,39.28]$

$0.35[0.19,0.66]$

$0.73[0.44,1.22]$

$0.88[0.67,1.16]$

$2.39[1.05,5.44]$

$0.58[0.27,1.22]$

$0.7[0.23,2.13]$

$0.45[0.14,1.49]$

$0.87[0.71,1.06]$

$0.32[0.14,0.71]$

$0.63[0.44,0.9]$

$0.5[0.12,2.02]$

$1.36[0.23,8.11]$

$1.03[0.54,1.97]$

$0.4[0.26,0.62]$

$2.44[1.07,5.55]$

$0.74[0.53,1.03]$

$0.6[0.47,0.76]$

$0.95[0.37,2.44]$

$1.63[1.24,2.15]$

Favours exercise $A$

Favours exercise $B$ 
Analysis 11.2. Comparison 11 Exercise versus exercise, Outcome 2 Rate of falls $>18$ months, different types of exercise compared.

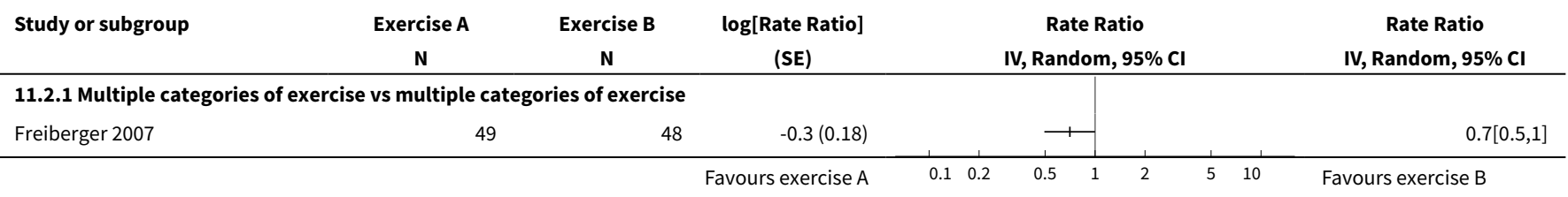

\section{Analysis 11.3. Comparison 11 Exercise versus exercise, Outcome} 3 Number of fallers, different types of exercise compared.

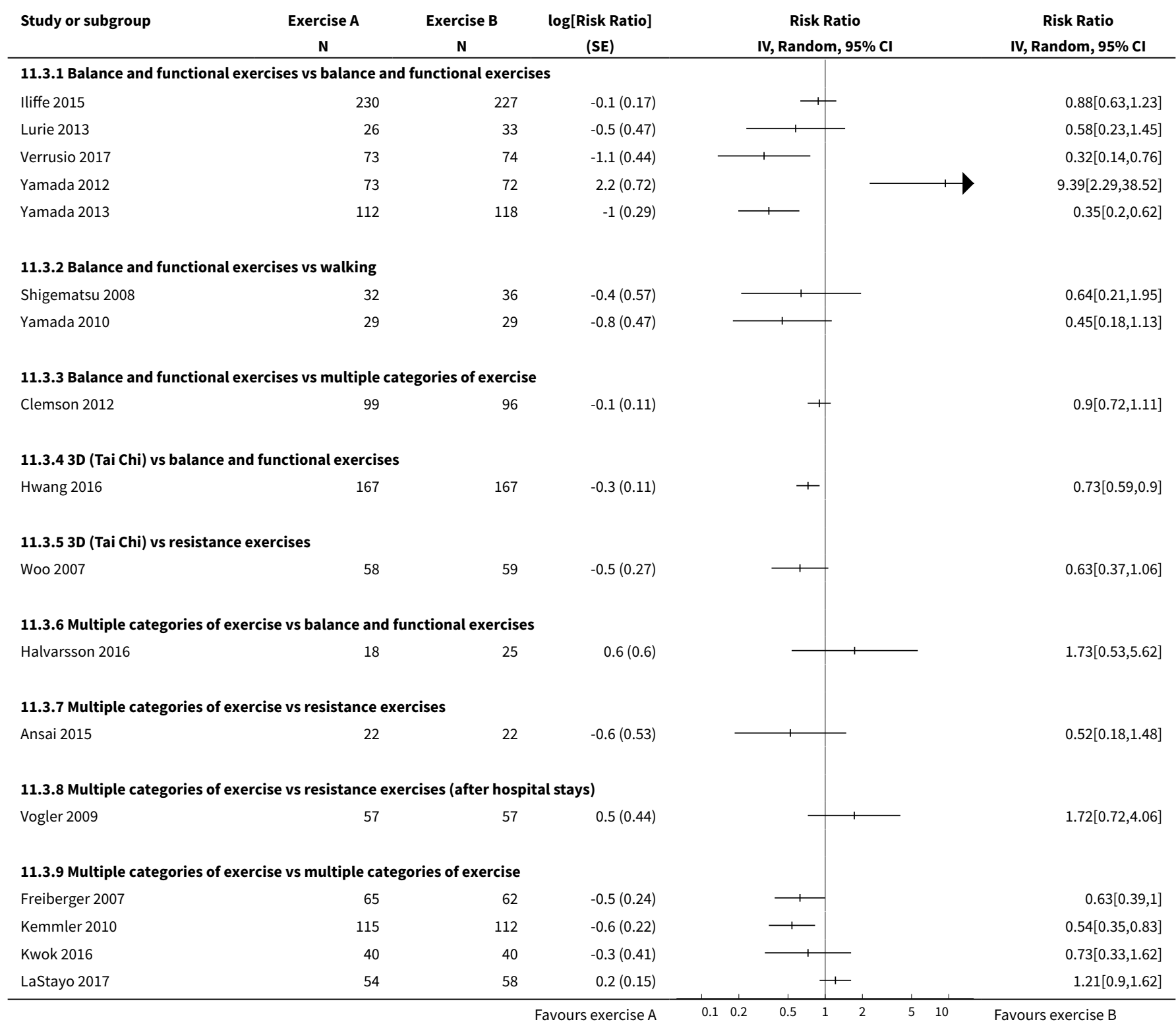


Analysis 11.4. Comparison 11 Exercise versus exercise, Outcome 4 Number of people who experienced one or more fall-related fractures, different types of exercise compared.

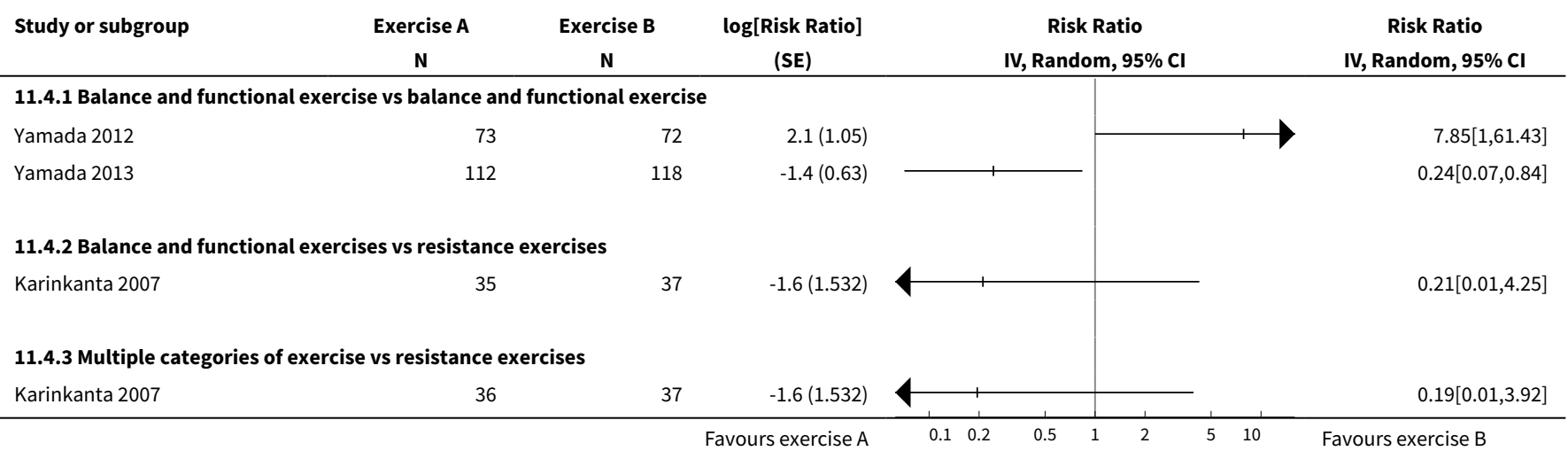

Analysis 11.5. Comparison 11 Exercise versus exercise, Outcome 5 Number of people who experienced one or more falls that required medical attention, different types of exercise compared.

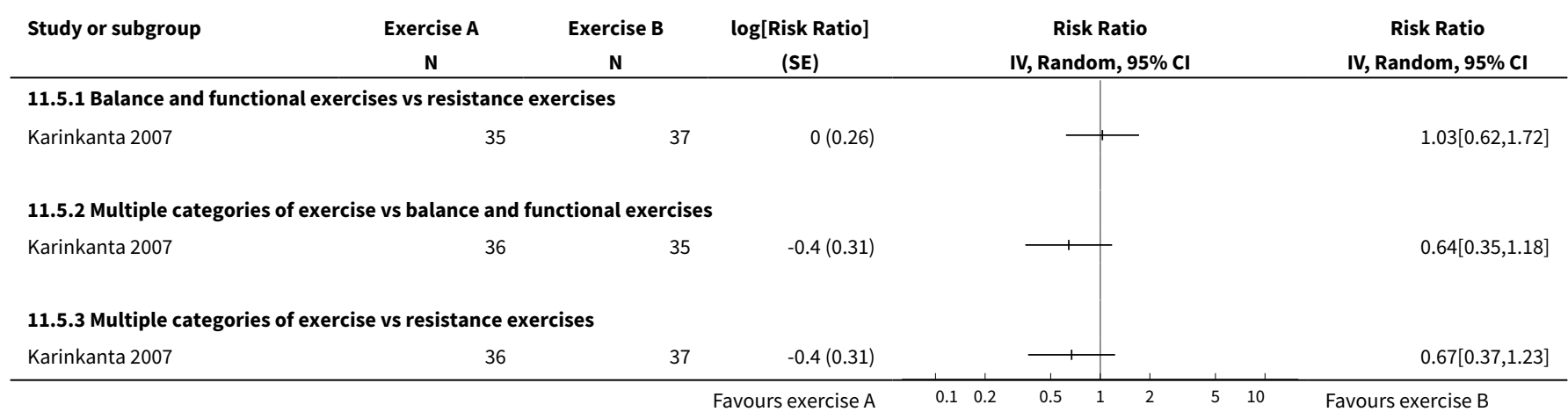

Analysis 11.6. Comparison 11 Exercise versus exercise, Outcome 6 Quality of life, different types of exercise compared.

\begin{tabular}{|c|c|c|c|c|c|c|c|}
\hline \multirow[t]{2}{*}{ Study or subgroup } & \multicolumn{2}{|c|}{ Exercise A } & \multicolumn{2}{|c|}{ Exercise B } & \multirow{2}{*}{\multicolumn{2}{|c|}{$\begin{array}{c}\text { Std. Mean Difference } \\
\text { Random, } 95 \% \mathrm{Cl}\end{array}$}} & \multirow{2}{*}{$\begin{array}{c}\text { Std. Mean Difference } \\
\text { Random, } 95 \% \mathrm{Cl}\end{array}$} \\
\hline & $\mathbf{N}$ & Mean(SD) & $\mathbf{N}$ & Mean(SD) & & & \\
\hline \multicolumn{8}{|c|}{ 11.6.1 Balance and functional exercises versus balance and functional exercises } \\
\hline Steadman 2003 & 69 & $64.4(19.9)$ & 64 & $64.5(17.4)$ & & & $-0.01[-0.35,0.33]$ \\
\hline
\end{tabular}

Analysis 11.7. Comparison 11 Exercise versus exercise, Outcome 7 Number of people who died, different types of exercise compared.

\begin{tabular}{|c|c|c|c|c|c|c|}
\hline Study or subgroup & $\begin{array}{c}\text { Exercise A } \\
\mathbf{n} / \mathbf{N}\end{array}$ & $\begin{array}{c}\text { Exercise B } \\
n / N\end{array}$ & & $\begin{array}{c}\text { Risk Ratio } \\
\text { IV, Random, } 95 \% \mathrm{CI}\end{array}$ & & \multirow[t]{2}{*}{$\begin{array}{c}\text { Risk Ratio } \\
\text { IV, Random, } 95 \% \mathrm{CI} \\
\end{array}$} \\
\hline \multicolumn{6}{|c|}{ 11.7.1 3D (Tai Chi) vs balance and functional exercises } & \\
\hline Hwang 2016 & $2 / 167$ & $3 / 167$ & & 1 & & $0.67[0.11,3.94]$ \\
\hline
\end{tabular}




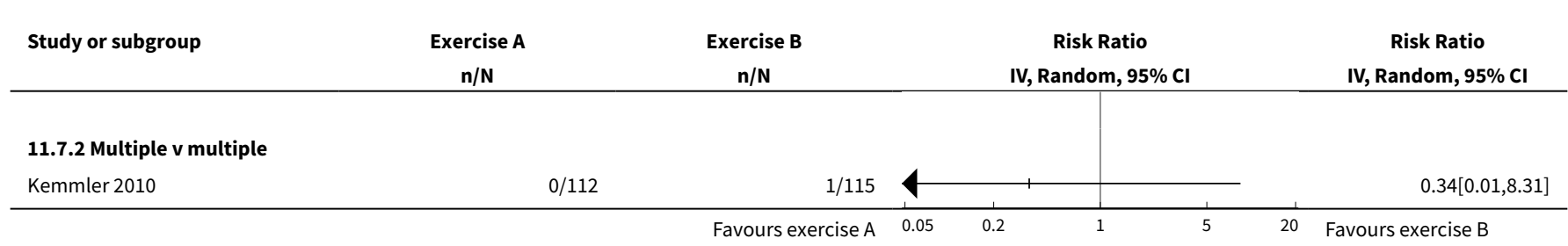

Analysis 11.8. Comparison 11 Exercise versus exercise, Outcome 8 Rate of falls, group vs individual exercise delivery within the same type of exercise.

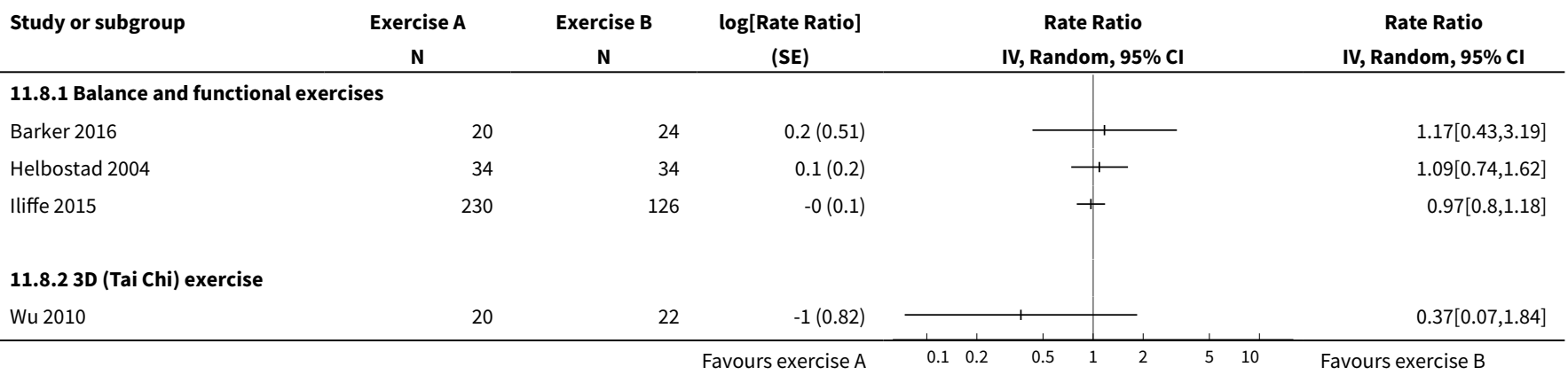

Analysis 11.9. Comparison 11 Exercise versus exercise, Outcome 9 Number of fallers, group vs individual exercise delivery within the same type of exercise.

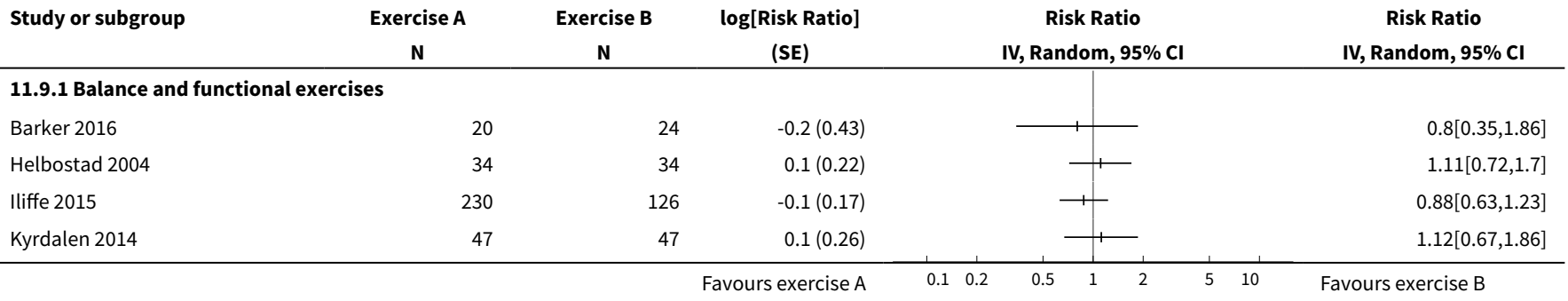

Analysis 11.10. Comparison 11 Exercise versus exercise, Outcome 10 Number of people who experienced one or more falls requiring hospital admission, group vs individual exercise delivery within the same type of exercise.

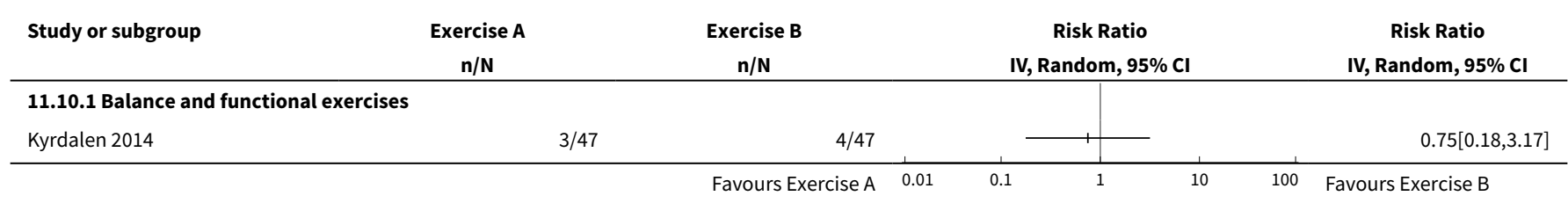


Analysis 11.11. Comparison 11 Exercise versus exercise, Outcome 11 Health-related quality of life, group vs individual exercise delivery within the same type of exercise.

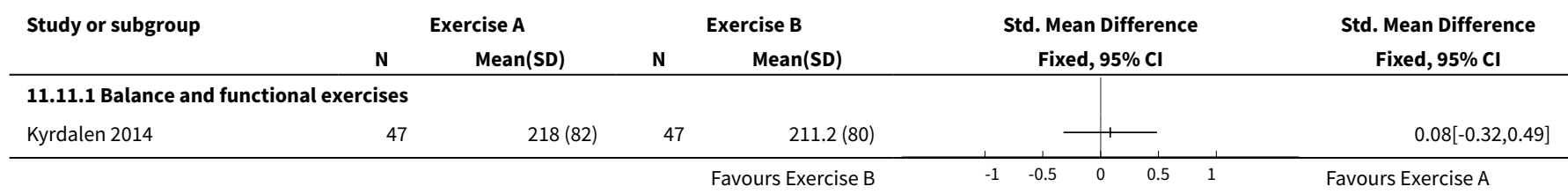

Analysis 11.12. Comparison 11 Exercise versus exercise, Outcome 12 Number of people who died, group vs individual exercise delivery within the same type of exercise.

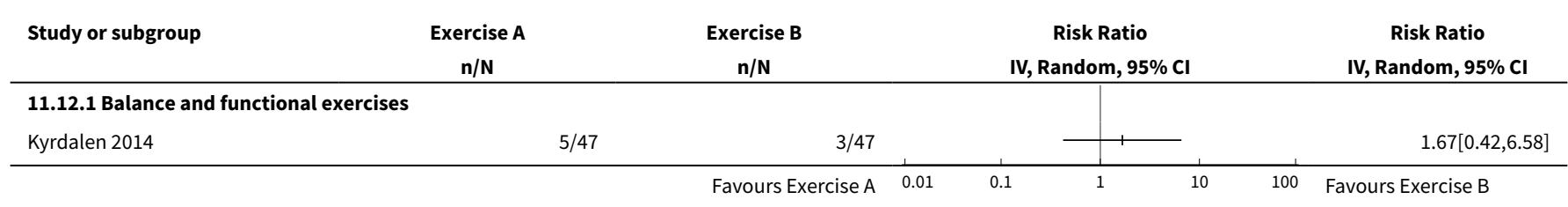

Analysis 11.13. Comparison 11 Exercise versus exercise, Outcome 13

Rate of falls, higher vs lower dose within the same type of exercise.

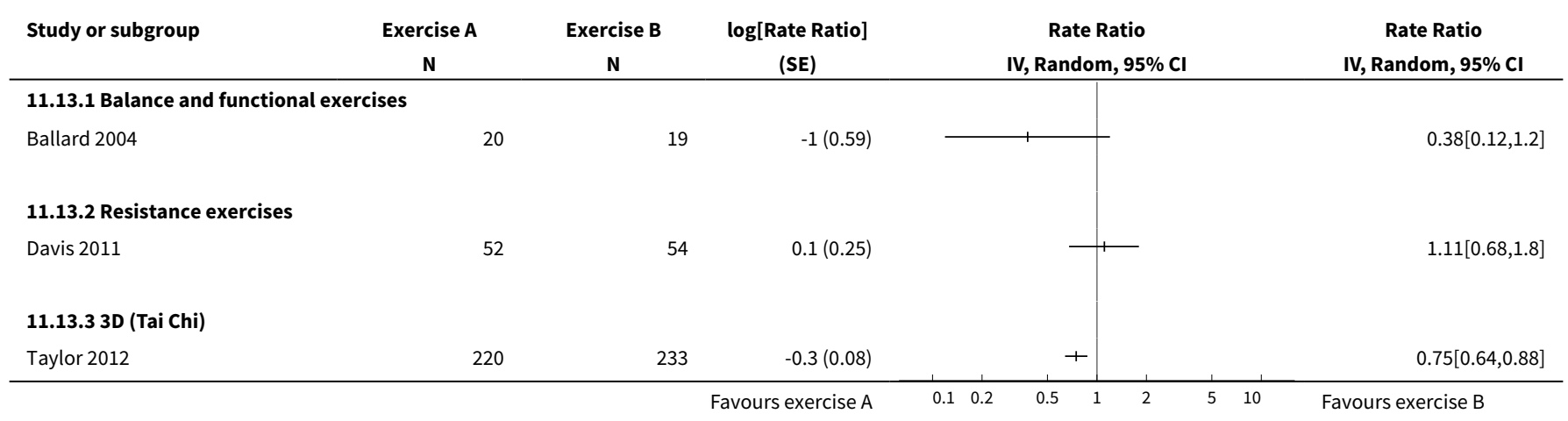

Analysis 11.14. Comparison 11 Exercise versus exercise, Outcome 14 Number of fallers, higher vs lower dose within the same type of exercise.

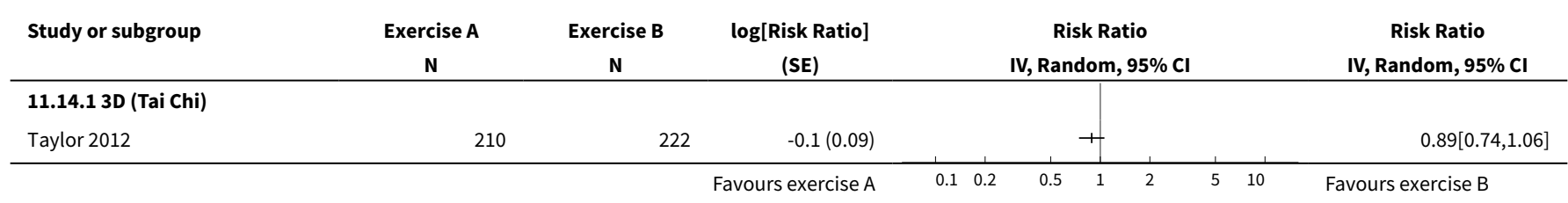


Analysis 11.15. Comparison 11 Exercise versus exercise, Outcome 15 Number of people who died, higher vs lower dose within the same type of exercise.

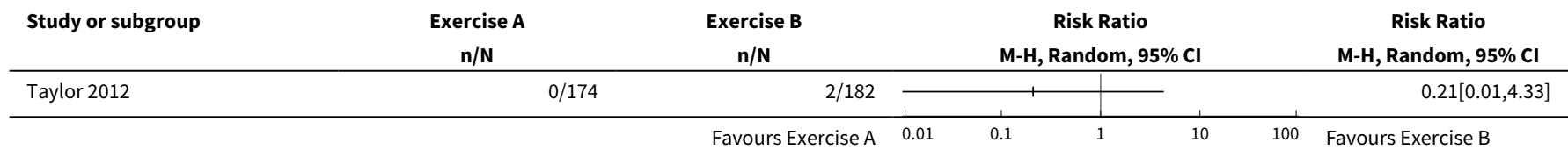

Comparison 12. Sensitivity analysis 1: exercise versus control excluding studies that included people $<65$ years

\begin{tabular}{|c|c|c|c|c|}
\hline Outcome or subgroup title & No. of studies & $\begin{array}{l}\text { No. of partici- } \\
\text { pants }\end{array}$ & Statistical method & Effect size \\
\hline 1 Rate of falls: pooled data & 53 & 11807 & $\begin{array}{l}\text { Rate Ratio (Random, 95\% } \\
\text { Cl) }\end{array}$ & $0.77[0.71,0.84]$ \\
\hline 2 Rate of falls: grouped by exercise type & 53 & & $\begin{array}{l}\text { Rate Ratio (Random, 95\% } \\
\text { Cl) }\end{array}$ & Subtotals only \\
\hline $\begin{array}{l}\text { 2.1 Balance and functional exercises vs } \\
\text { control }\end{array}$ & 34 & 7436 & $\begin{array}{l}\text { Rate Ratio (Random, 95\% } \\
\text { Cl) }\end{array}$ & $0.75[0.69,0.81]$ \\
\hline 2.2 Resistance exercise vs control & 5 & 327 & $\begin{array}{l}\text { Rate Ratio (Random, 95\% } \\
\text { Cl) }\end{array}$ & $1.14[0.67,1.97]$ \\
\hline 2.3 3D exercise (Tai Chi) vs control & 6 & 1971 & $\begin{array}{l}\text { Rate Ratio (Random, 95\% } \\
\text { Cl) }\end{array}$ & $0.83[0.67,1.03]$ \\
\hline 2.4 3D exercise (dance) vs control & 1 & 522 & $\begin{array}{l}\text { Rate Ratio (Random, 95\% } \\
\text { Cl) }\end{array}$ & $1.34[0.98,1.83]$ \\
\hline 2.5 Walking programme vs control & 2 & 441 & $\begin{array}{l}\text { Rate Ratio (Random, 95\% } \\
\text { Cl) }\end{array}$ & $1.14[0.66,1.97]$ \\
\hline $\begin{array}{l}2.6 \text { Multiple categories of exercise vs con- } \\
\text { trol }\end{array}$ & 11 & 1404 & $\begin{array}{l}\text { Rate Ratio (Random, 95\% } \\
\text { Cl) }\end{array}$ & $0.66[0.50,0.88]$ \\
\hline 3 Number of fallers: pooled data & 52 & 11576 & $\begin{array}{l}\text { Risk Ratio (Random, 95\% } \\
\text { Cl) }\end{array}$ & $0.85[0.80,0.90]$ \\
\hline $\begin{array}{l}4 \text { Number of fallers: grouped by exercise } \\
\text { type }\end{array}$ & 54 & & $\begin{array}{l}\text { Risk Ratio (Random, 95\% } \\
\text { Cl) }\end{array}$ & Subtotals only \\
\hline $\begin{array}{l}\text { 4.1 Balance and functional exercises vs } \\
\text { control }\end{array}$ & 30 & 7287 & $\begin{array}{l}\text { Risk Ratio (Random, 95\% } \\
\text { Cl) }\end{array}$ & $0.86[0.82,0.91]$ \\
\hline 4.2 Resistance exercise vs control & 2 & 163 & $\begin{array}{l}\text { Risk Ratio (Random, 95\% } \\
\text { Cl) }\end{array}$ & $0.81[0.57,1.15]$ \\
\hline 4.3 3D exercise (Tai Chi) vs control & 6 & 1915 & $\begin{array}{l}\text { Risk Ratio (Random, 95\% } \\
\text { Cl) }\end{array}$ & $0.82[0.71,0.94]$ \\
\hline 4.4 3D exercise (dance) vs control & 1 & 522 & $\begin{array}{l}\text { Risk Ratio (Random, 95\% } \\
\text { Cl) }\end{array}$ & $1.35[0.83,2.20]$ \\
\hline
\end{tabular}




\begin{tabular}{|c|c|c|c|c|}
\hline Outcome or subgroup title & No. of studies & $\begin{array}{l}\text { No. of partici- } \\
\text { pants }\end{array}$ & Statistical method & Effect size \\
\hline 4.5 Walking programme vs control & 2 & 441 & $\begin{array}{l}\text { Risk Ratio (Random, 95\% } \\
\mathrm{Cl} \text { ) }\end{array}$ & $1.05[0.71,1.54]$ \\
\hline $\begin{array}{l}4.6 \text { Multiple categories of exercise vs con- } \\
\text { trol }\end{array}$ & 17 & 1623 & $\begin{array}{l}\text { Risk Ratio (Random, 95\% } \\
\mathrm{Cl} \text { ) }\end{array}$ & $0.78[0.64,0.96]$ \\
\hline $\begin{array}{l}5 \text { Number of people who experienced one } \\
\text { or more fall-related fractures: pooled da- } \\
\text { ta }\end{array}$ & 10 & 4047 & Risk Ratio (Fixed, 95\% Cl) & $0.73[0.56,0.95]$ \\
\hline $\begin{array}{l}6 \text { Number of people who experienced one } \\
\text { or more fall-related fractures: by exercise } \\
\text { type }\end{array}$ & 10 & & Risk Ratio (Fixed, 95\% Cl) & Subtotals only \\
\hline $\begin{array}{l}\text { 6.1 Balance and functional exercises vs } \\
\text { control }\end{array}$ & 7 & 2139 & Risk Ratio (Fixed, 95\% Cl) & $0.44[0.25,0.76]$ \\
\hline 6.2 Resistance exercise vs control & 1 & 73 & Risk Ratio (Fixed, 95\% Cl) & $0.97[0.14,6.49]$ \\
\hline 6.3 Walking programme vs control & 1 & 97 & Risk Ratio (Fixed, 95\% CI) & $0.66[0.11,3.76]$ \\
\hline $\begin{array}{l}6.4 \text { Multiple categories of exercise vs con- } \\
\text { trol }\end{array}$ & 3 & 1810 & Risk Ratio (Fixed, 95\% Cl) & $0.85[0.62,1.16]$ \\
\hline $\begin{array}{l}7 \text { Number of people who experienced one } \\
\text { or more falls requiring medical attention: } \\
\text { pooled data }\end{array}$ & 5 & 1019 & $\begin{array}{l}\text { Risk Ratio (Random, 95\% } \\
\mathrm{Cl} \text { ) }\end{array}$ & $0.61[0.47,0.79]$ \\
\hline $\begin{array}{l}8 \text { Number of people who experienced one } \\
\text { or more falls requiring medical attention - } \\
\text { subgrouped by exercise type }\end{array}$ & 5 & & $\begin{array}{l}\text { Risk Ratio (Random, 95\% } \\
\mathrm{Cl} \text { ) }\end{array}$ & Subtotals only \\
\hline $\begin{array}{l}\text { 8.1 Balance and functional exercises vs } \\
\text { Control }\end{array}$ & 3 & 585 & $\begin{array}{l}\text { Risk Ratio (Random, 95\% } \\
\mathrm{Cl} \text { ) }\end{array}$ & $0.76[0.54,1.09]$ \\
\hline 8.2 Resistance exercises vs control & 1 & 73 & $\begin{array}{l}\text { Risk Ratio (Random, 95\% } \\
\mathrm{Cl} \text { ) }\end{array}$ & $0.92[0.47,1.80]$ \\
\hline 8.3 3D exercise (Tai Chi) vs Control & 1 & 188 & $\begin{array}{l}\text { Risk Ratio (Random, 95\% } \\
\mathrm{Cl} \text { ) }\end{array}$ & $0.35[0.13,0.93]$ \\
\hline $\begin{array}{l}\text { 8.4 Multiple categories of exercise vs con- } \\
\text { trol }\end{array}$ & 2 & 248 & $\begin{array}{l}\text { Risk Ratio (Random, 95\% } \\
\mathrm{Cl} \text { ) }\end{array}$ & $0.44[0.29,0.66]$ \\
\hline
\end{tabular}

Analysis 12.1. Comparison 12 Sensitivity analysis 1: exercise versus control excluding studies that included people $<65$ years, Outcome 1 Rate of falls: pooled data.

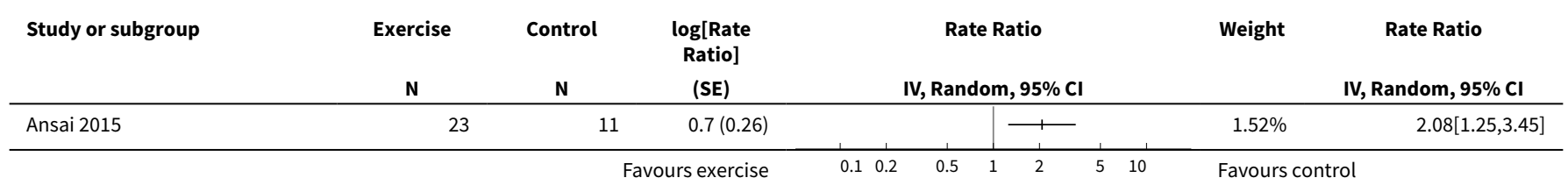




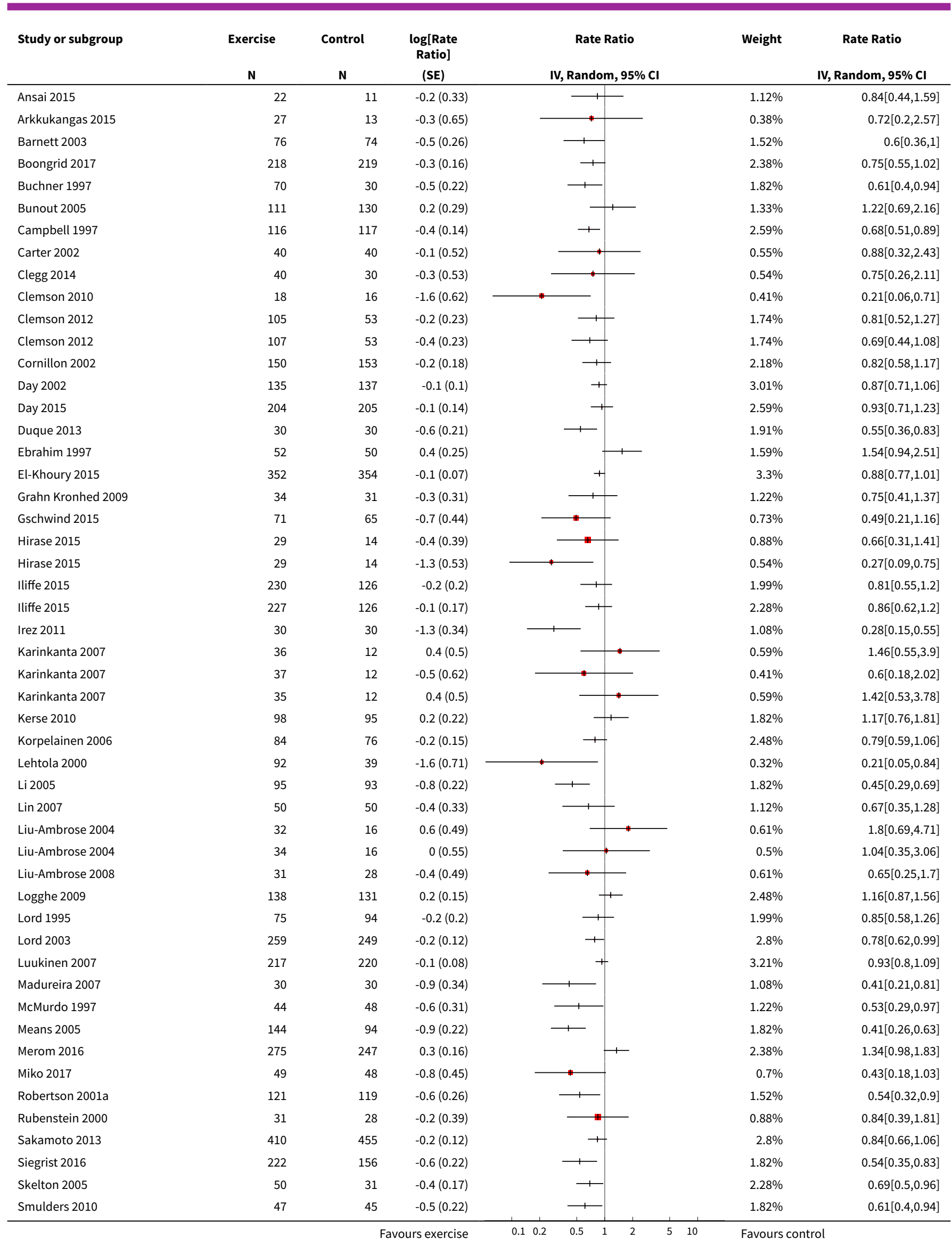




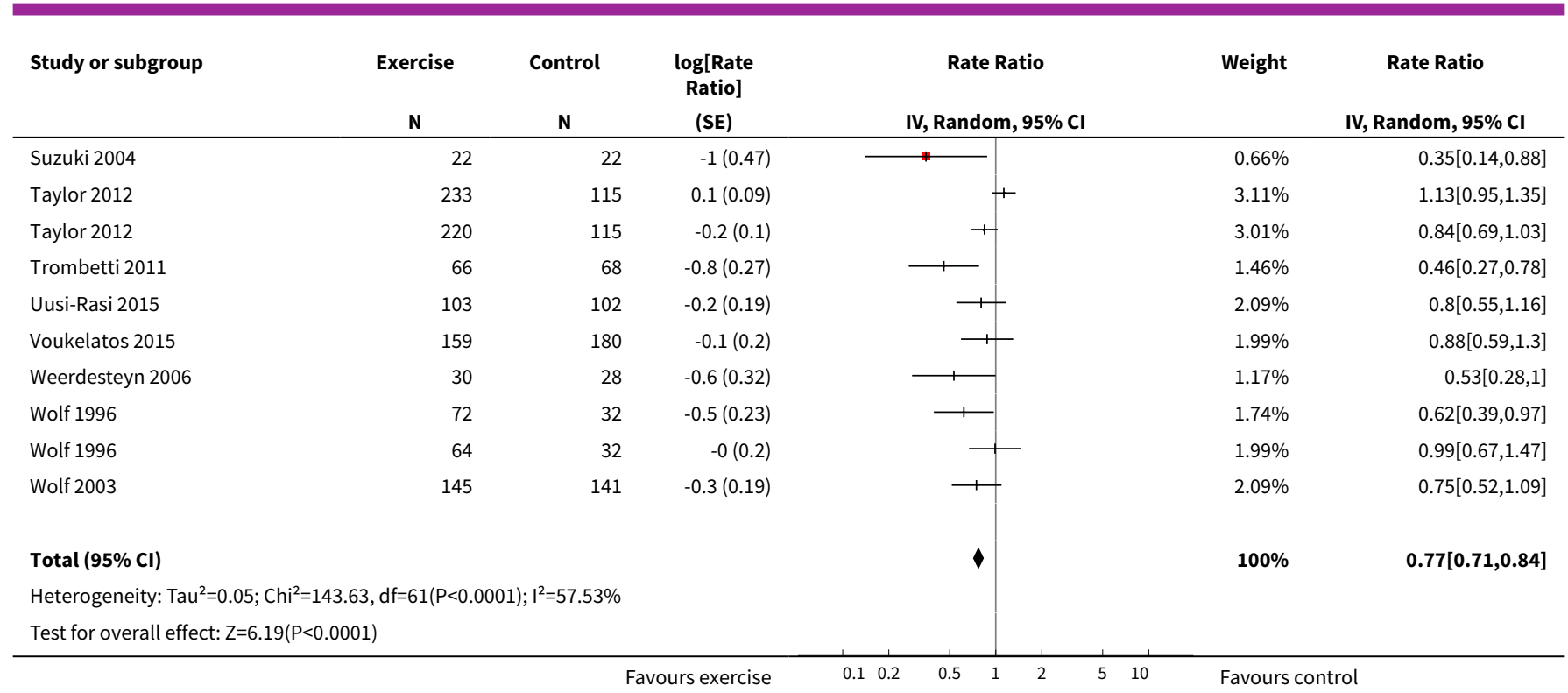

Analysis 12.2. Comparison 12 Sensitivity analysis 1 : exercise versus control excluding studies that included people $<65$ years, Outcome 2 Rate of falls: grouped by exercise type.

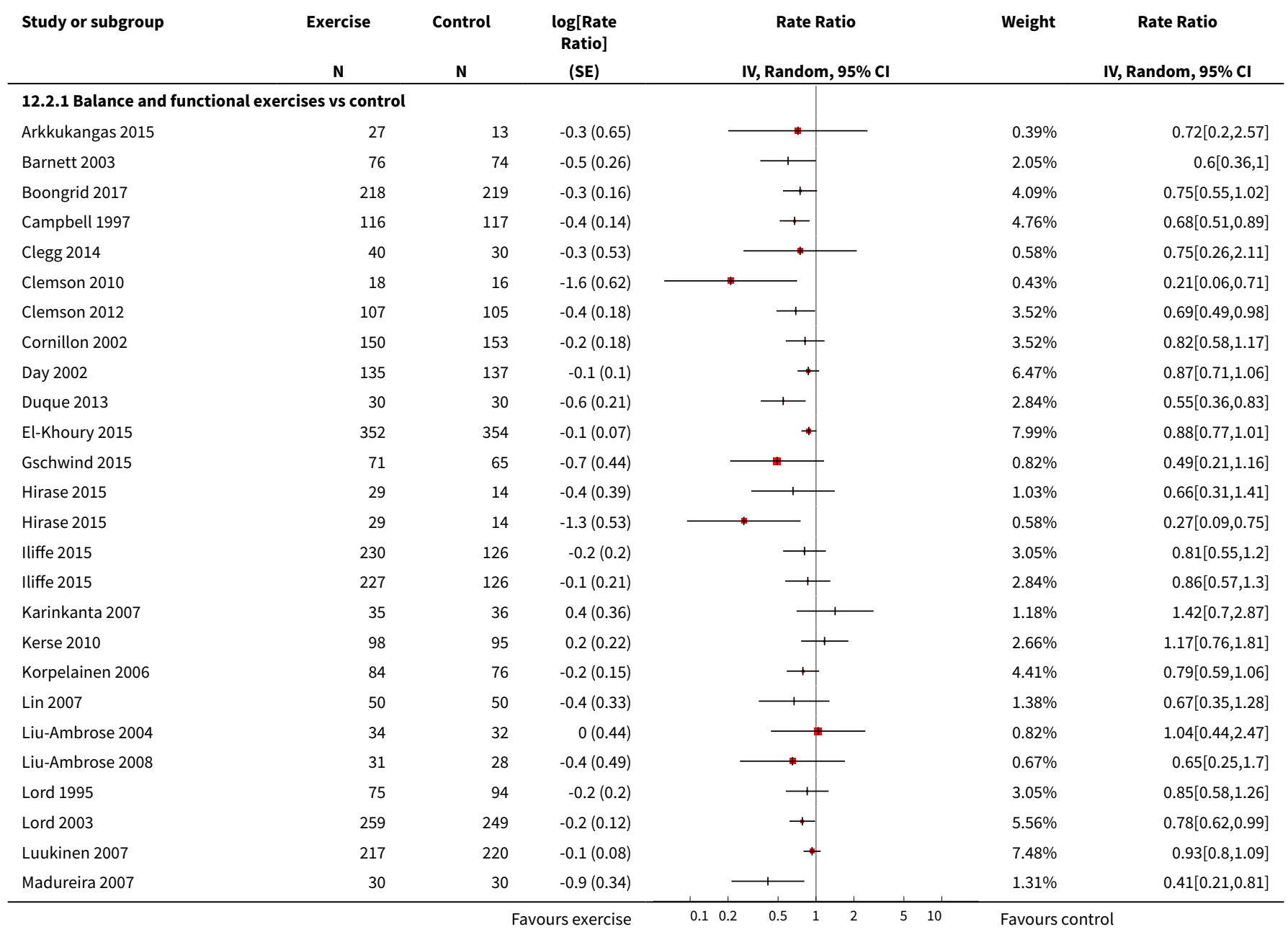




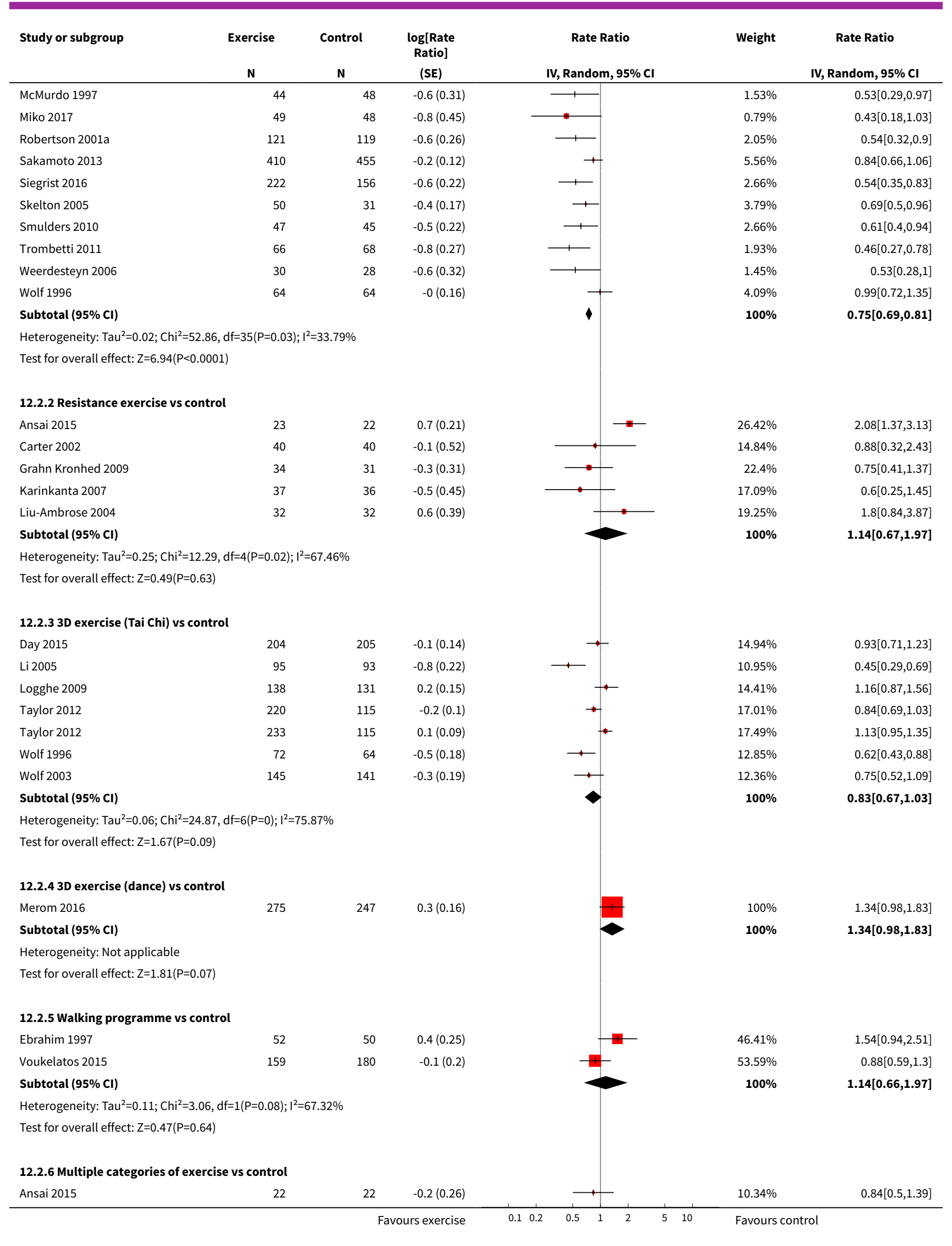




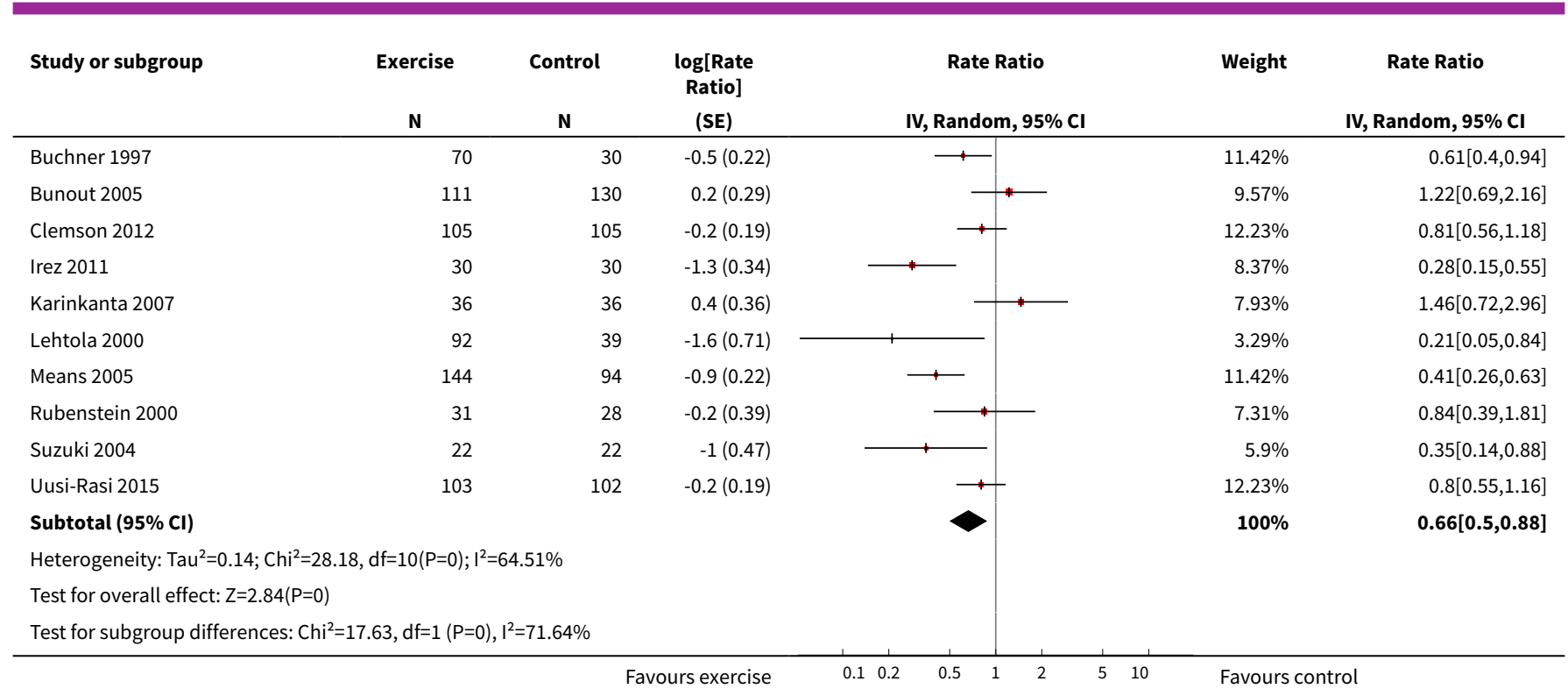

Analysis 12.3. Comparison 12 Sensitivity analysis 1: exercise versus control excluding studies that included people $<65$ years, Outcome 3 Number of fallers: pooled data.

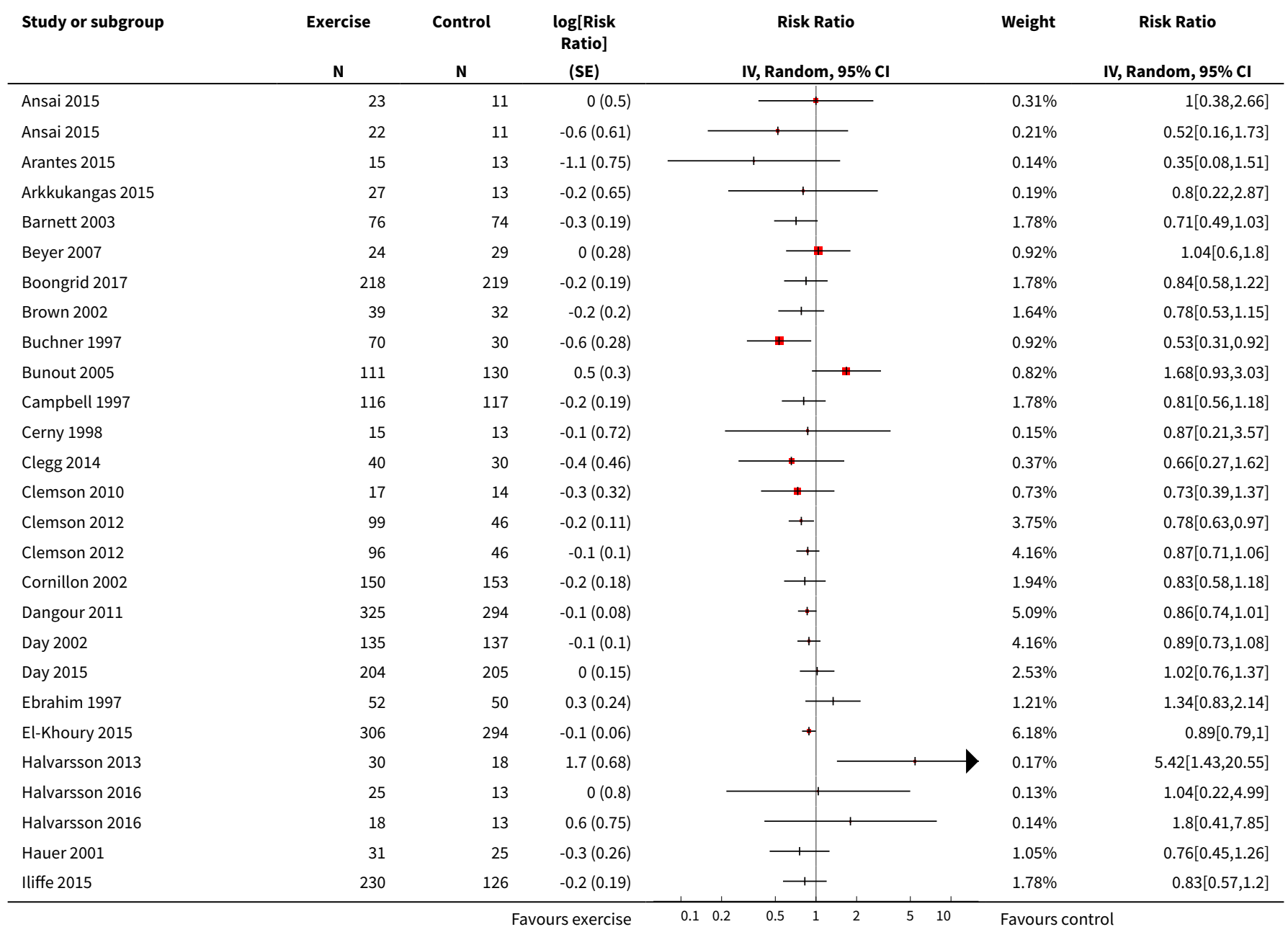




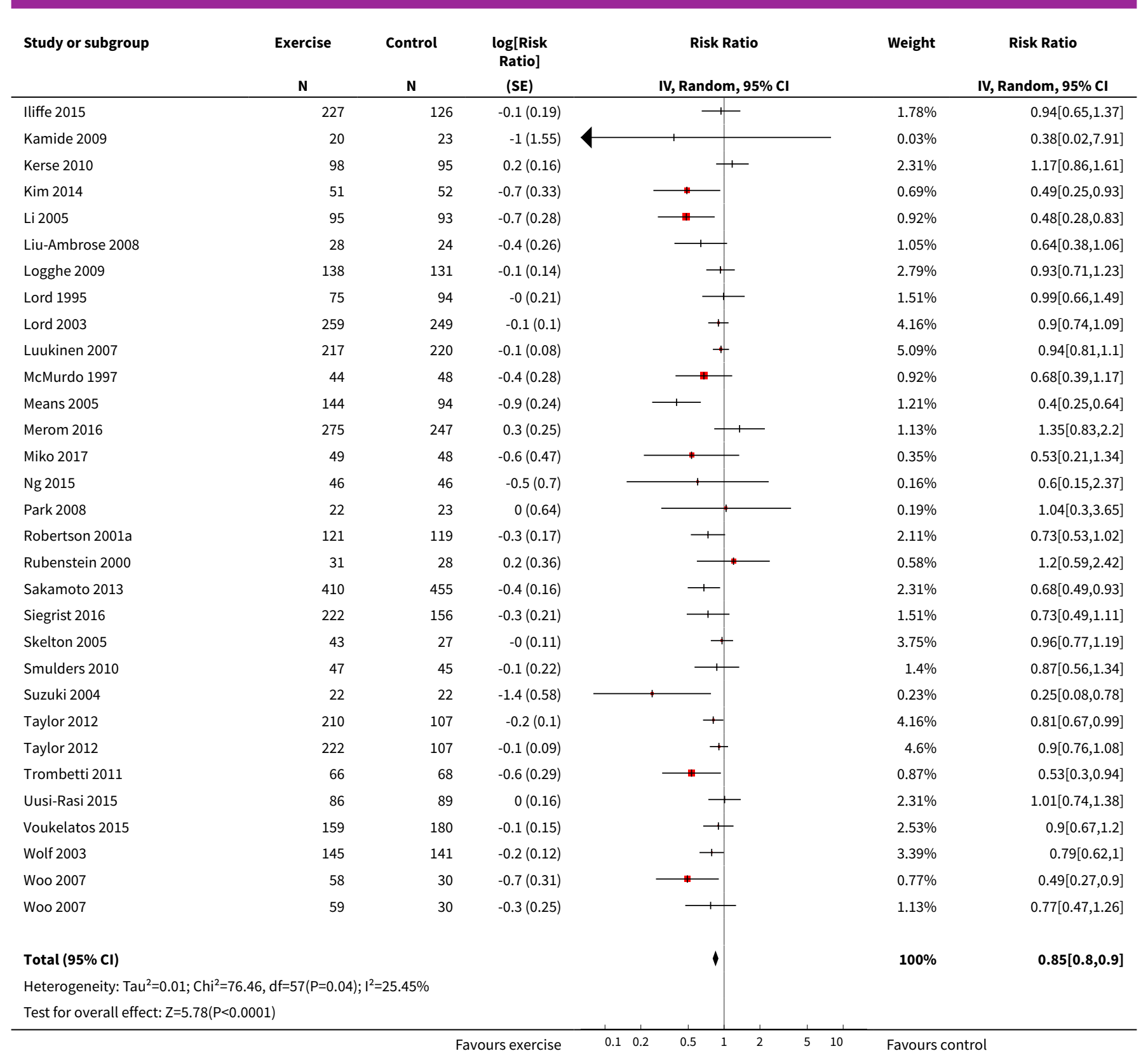
Analysis 12.4. Comparison 12 Sensitivity analysis 1: exercise versus control excluding studies
that included people < 65 years, Outcome 4 Number of fallers: grouped by exercise type.

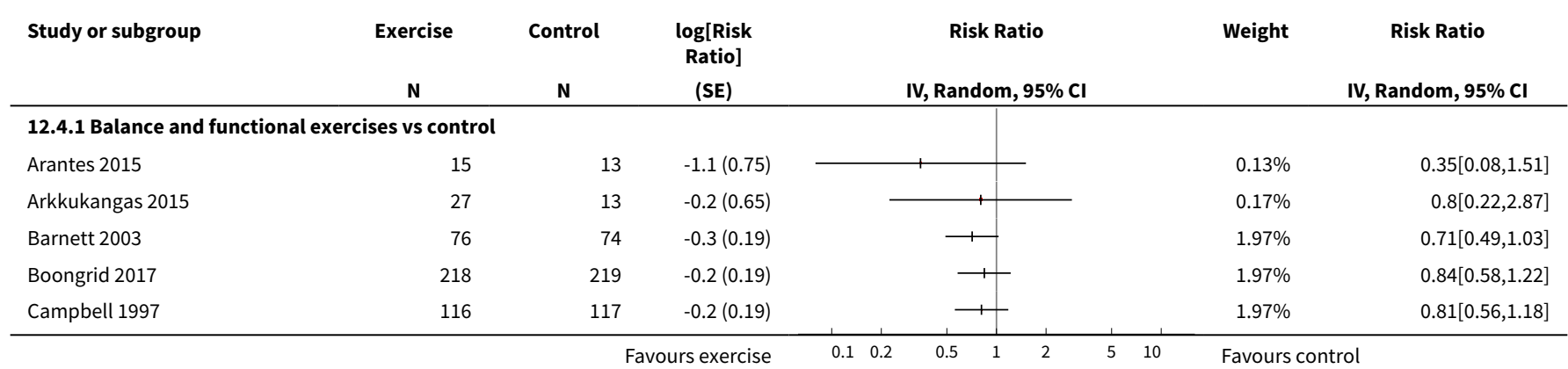




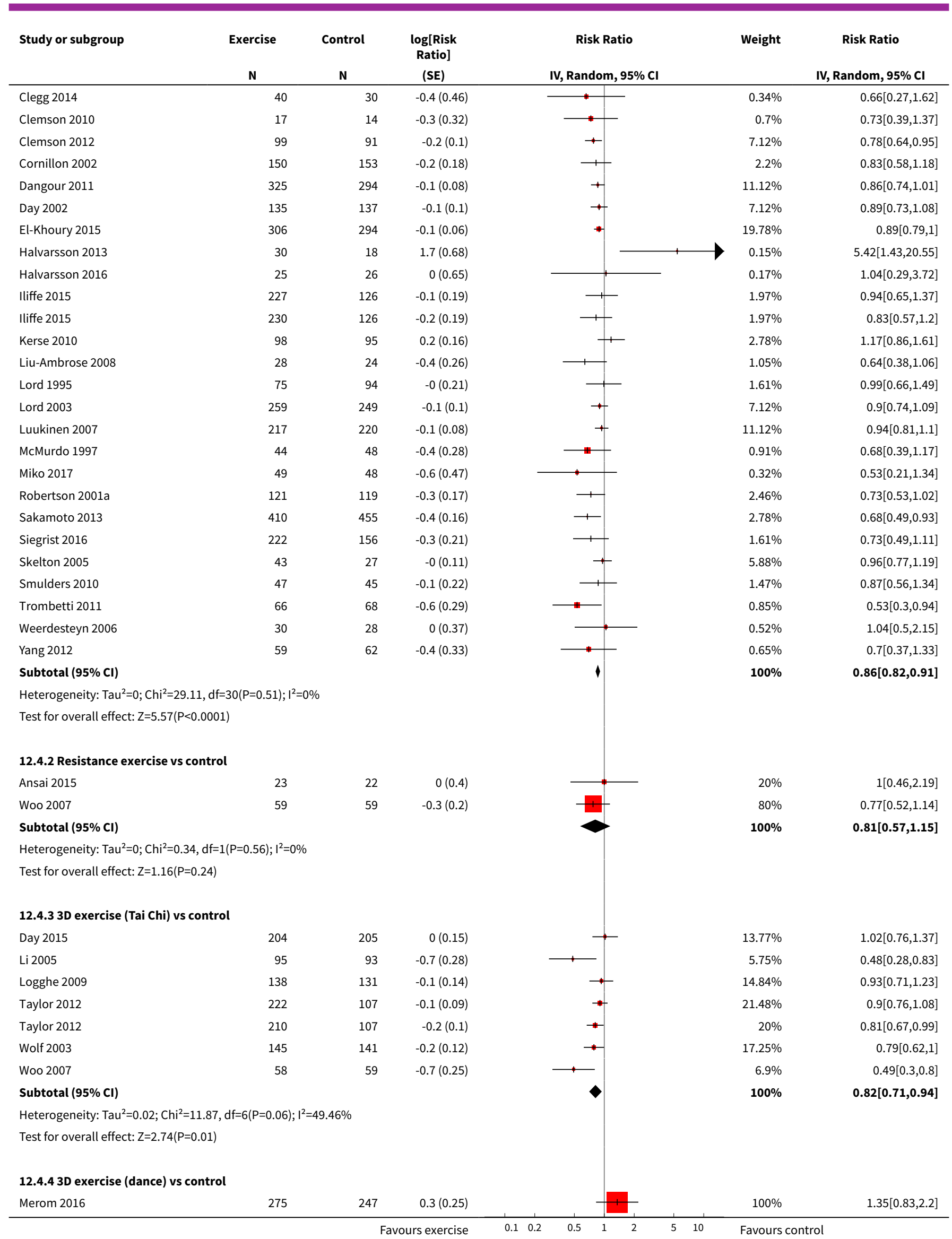




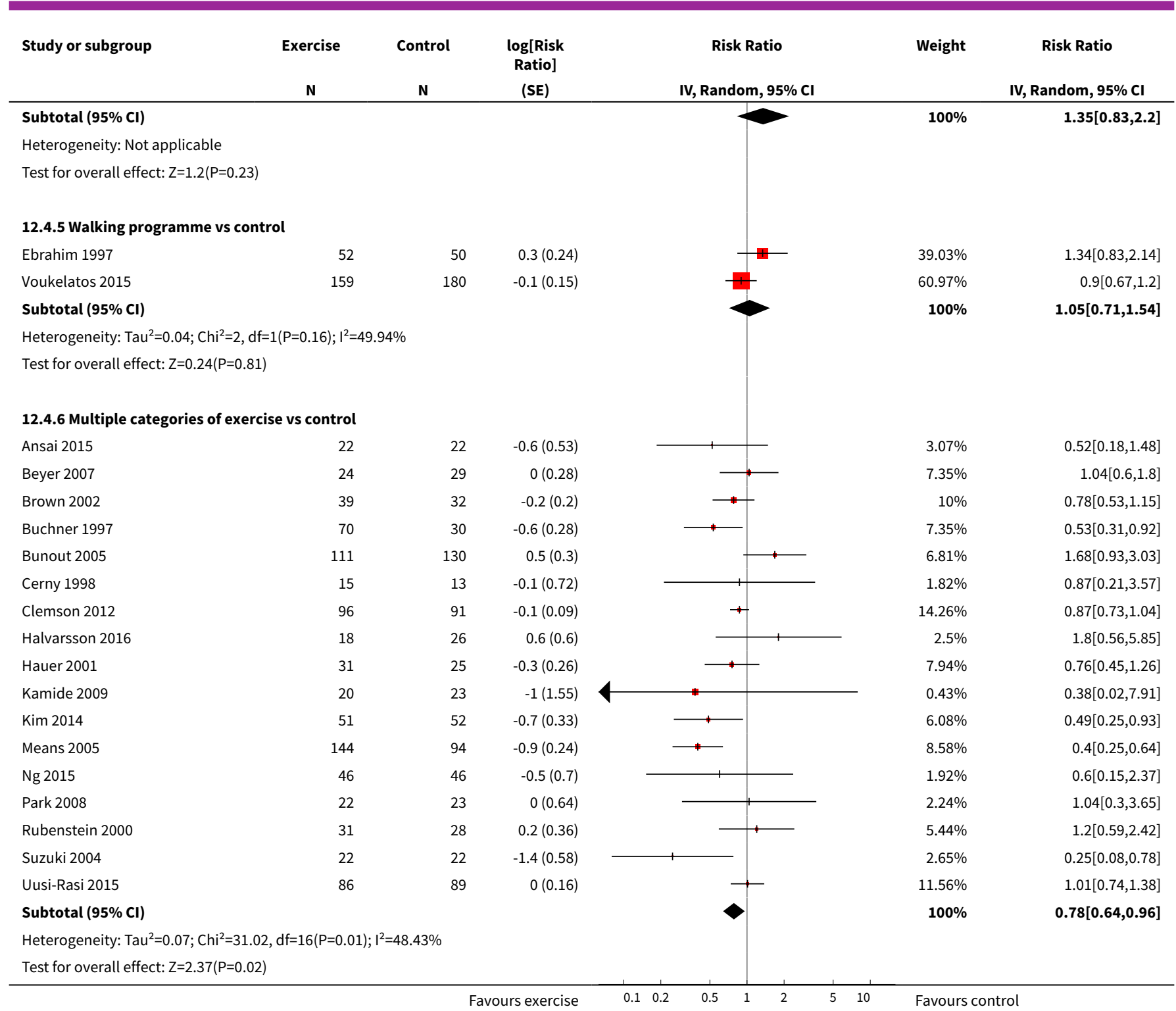

Analysis 12.5. Comparison 12 Sensitivity analysis 1: exercise versus control excluding studies that included people < 65 years, Outcome 5 Number of people who experienced one or more fall-related fractures: pooled data.

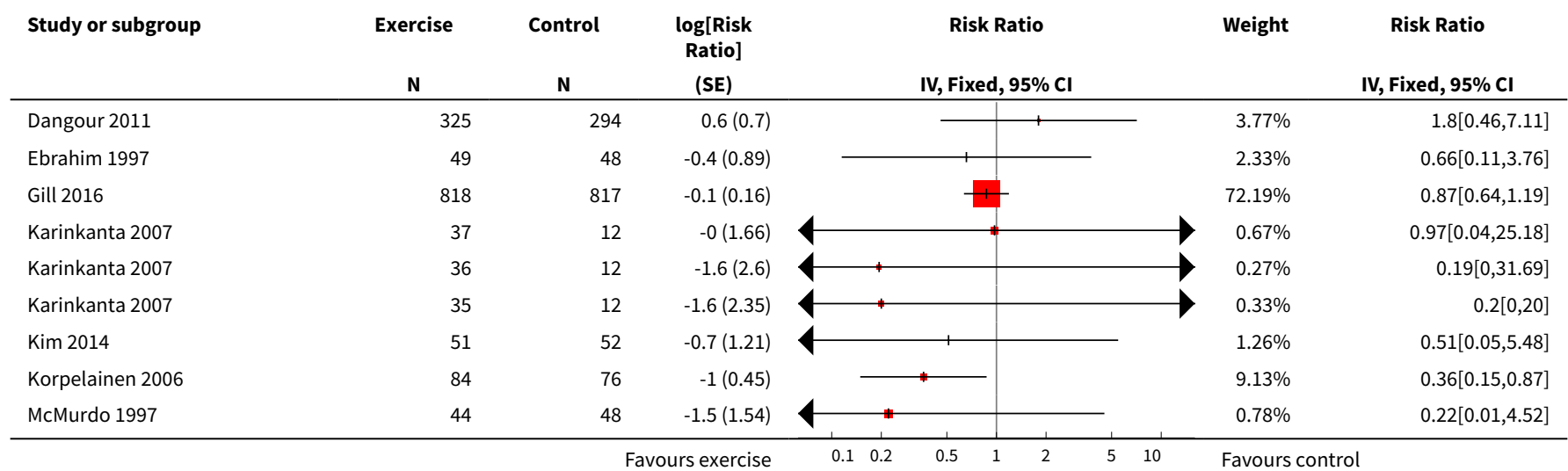




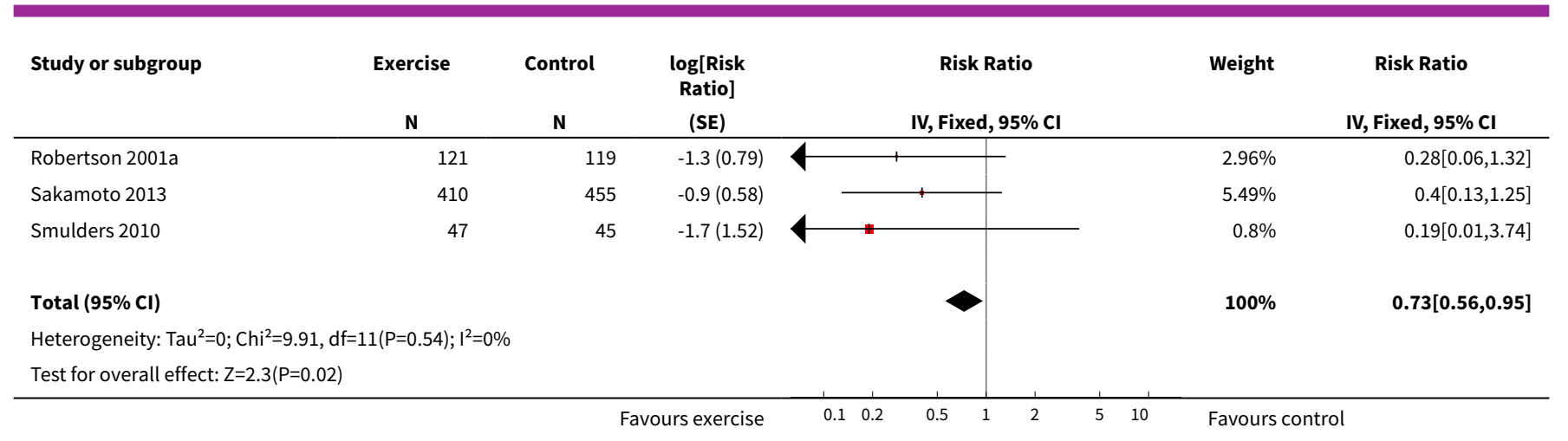

Analysis 12.6. Comparison 12 Sensitivity analysis 1: exercise versus control excluding studies that included people $<65$ years, Outcome 6 Number of people who experienced one or more fall-related fractures: by exercise type.

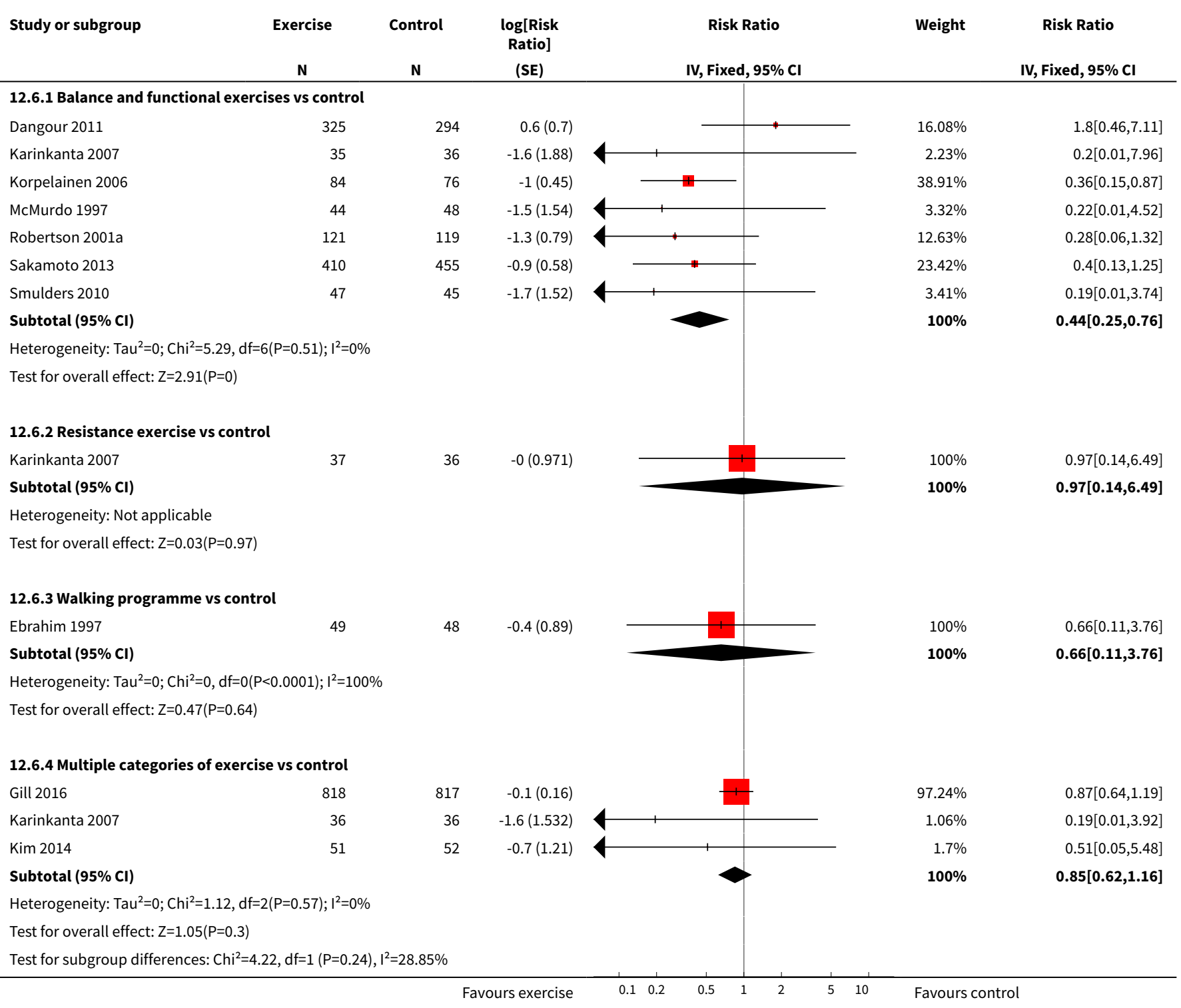


Analysis 12.7. Comparison 12 Sensitivity analysis 1 : exercise versus control excluding studies that included people $<65$ years, Outcome 7 Number of people who experienced one or more falls requiring medical attention: pooled data.

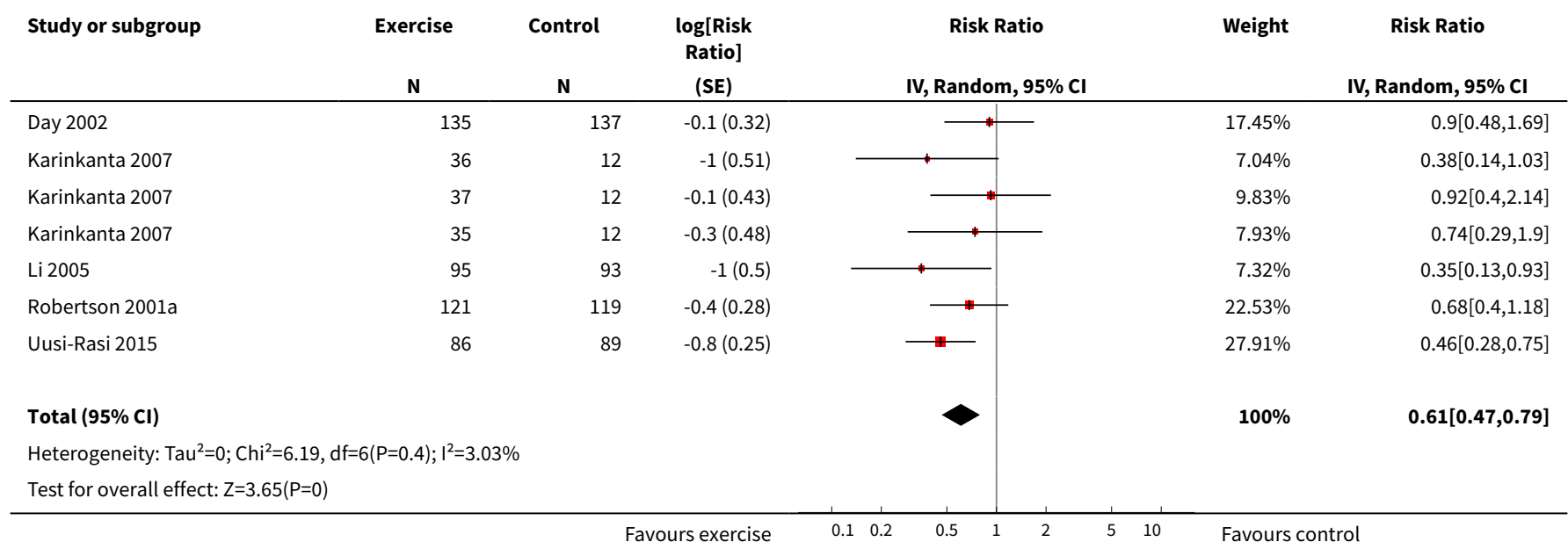

Analysis 12.8. Comparison 12 Sensitivity analysis 1: exercise versus control excluding studies that included people $<65$ years, Outcome 8 Number of people who experienced one or more falls requiring medical attention - subgrouped by exercise type.

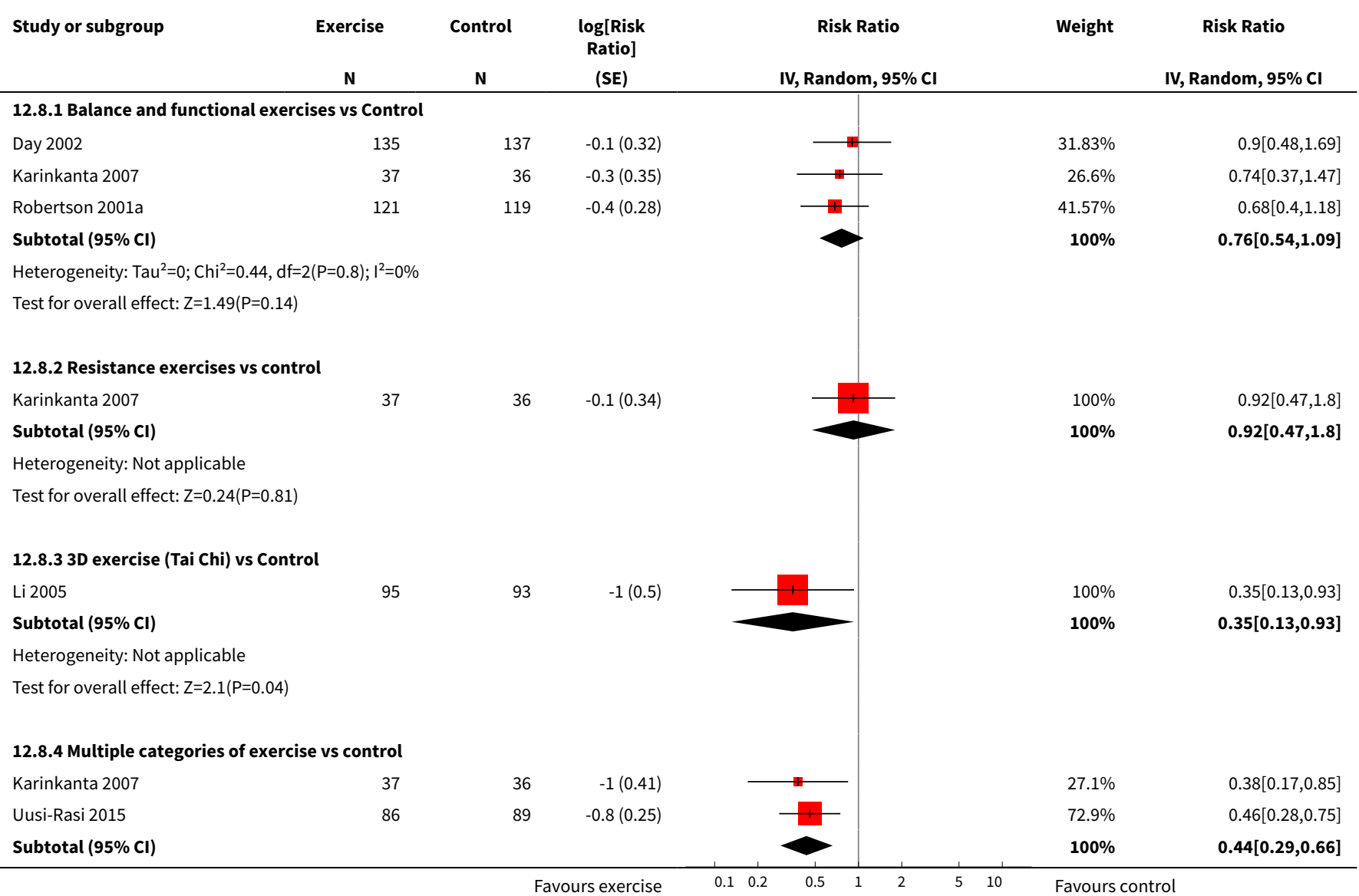




\begin{tabular}{|c|c|c|c|c|c|c|c|c|c|}
\hline \multirow[t]{2}{*}{ Study or subgroup } & \multirow{2}{*}{$\begin{array}{c}\text { Exercise } \\
\text { N }\end{array}$} & \multirow{2}{*}{$\begin{array}{c}\text { Control } \\
\text { N }\end{array}$} & \multirow{2}{*}{$\begin{array}{c}\text { log[Risk } \\
\text { Ratio] } \\
\text { (SE) }\end{array}$} & \multicolumn{4}{|c|}{ Risk Ratio } & \multirow[t]{2}{*}{ Weight } & \multirow{2}{*}{$\begin{array}{c}\text { Risk Ratio } \\
\text { IV, Random, 95\% CI }\end{array}$} \\
\hline & & & & \multicolumn{4}{|c|}{ IV, Random, $95 \% \mathrm{CI}$} & & \\
\hline \multicolumn{10}{|c|}{ Heterogeneity: Tau $^{2}=0 ; \mathrm{Chi}^{2}=0.16, \mathrm{df}=1(\mathrm{P}=0.69) ; \mathrm{I}^{2}=0 \%$} \\
\hline \multicolumn{10}{|c|}{ Test for overall effect: $Z=3.9(P<0.0001)$} \\
\hline
\end{tabular}

\section{Comparison 13. Sensitivity analysis 2: exercise versus control excluding studies at a high risk of bias}

\begin{tabular}{|c|c|c|c|c|}
\hline Outcome or subgroup title & No. of studies & $\begin{array}{l}\text { No. of partici- } \\
\text { pants }\end{array}$ & Statistical method & Effect size \\
\hline 1 Rate of falls - overall analysis & 25 & 6757 & $\begin{array}{l}\text { Rate Ratio (Random, 95\% } \\
\mathrm{Cl} \text { ) }\end{array}$ & $0.78[0.71,0.87]$ \\
\hline $\begin{array}{l}2 \text { Rate of falls - subgrouped by exercise } \\
\text { type }\end{array}$ & 25 & & $\begin{array}{l}\text { Rate Ratio (Random, 95\% } \\
\mathrm{Cl} \text { ) }\end{array}$ & Subtotals only \\
\hline $\begin{array}{l}\text { 2.1 Balance and functional exercises vs } \\
\text { control }\end{array}$ & 16 & 3184 & $\begin{array}{l}\text { Rate Ratio (Random, 95\% } \\
\mathrm{Cl} \text { ) }\end{array}$ & $0.69[0.61,0.79]$ \\
\hline 2.2 Resistance exercise vs control & 0 & 0 & $\begin{array}{l}\text { Rate Ratio (Random, 95\% } \\
\mathrm{Cl} \text { ) }\end{array}$ & $0.0[0.0,0.0]$ \\
\hline 2.3 3D exercise (Tai Chi) vs control & 5 & 2331 & $\begin{array}{l}\text { Rate Ratio (Random, 95\% } \\
\mathrm{Cl} \text { ) }\end{array}$ & $0.92[0.78,1.09]$ \\
\hline 2.4 3D exercise (dance) vs control & 1 & 522 & $\begin{array}{l}\text { Rate Ratio (Random, 95\% } \\
\mathrm{Cl} \text { ) }\end{array}$ & $1.34[0.98,1.83]$ \\
\hline 2.5 Walking programme vs control & 1 & 339 & $\begin{array}{l}\text { Rate Ratio (Random, 95\% } \\
\mathrm{Cl} \text { ) }\end{array}$ & $0.88[0.59,1.30]$ \\
\hline $\begin{array}{l}\text { 2.6 Multiple categories of exercise vs } \\
\text { control }\end{array}$ & 3 & 485 & $\begin{array}{l}\text { Rate Ratio (Random, 95\% } \\
\mathrm{Cl} \text { ) }\end{array}$ & $0.75[0.60,0.94]$ \\
\hline 3 Number of fallers - overall analysis & 26 & 6865 & $\begin{array}{l}\text { Risk Ratio (Random, 95\% } \\
\mathrm{Cl} \text { ) }\end{array}$ & $0.84[0.80,0.89]$ \\
\hline $\begin{array}{l}4 \text { Number of fallers - subgrouped by ex- } \\
\text { ercise type }\end{array}$ & 26 & & $\begin{array}{l}\text { Risk Ratio (Random, 95\% } \\
\text { Cl) }\end{array}$ & Subtotals only \\
\hline $\begin{array}{l}\text { 4.1 Balance and functional exercises vs } \\
\text { control }\end{array}$ & 16 & 3282 & $\begin{array}{l}\text { Risk Ratio (Random, 95\% } \\
\mathrm{Cl} \text { ) }\end{array}$ & $0.83[0.77,0.89]$ \\
\hline 4.2 Resistance exercise vs control & 0 & 0 & $\begin{array}{l}\text { Risk Ratio (Random, 95\% } \\
\mathrm{Cl} \text { ) }\end{array}$ & $0.0[0.0,0.0]$ \\
\hline 4.3 3D exercise (Tai Chi) vs control & 5 & 2294 & $\begin{array}{l}\text { Risk Ratio (Random, 95\% } \\
\text { Cl) }\end{array}$ & $0.85[0.77,0.94]$ \\
\hline 4.4 3D exercise (dance) vs control & 1 & 522 & $\begin{array}{l}\text { Risk Ratio (Random, 95\% } \\
\text { Cl) }\end{array}$ & $1.35[0.83,2.20]$ \\
\hline
\end{tabular}




\begin{tabular}{lllll}
\hline Outcome or subgroup title & No. of studies & $\begin{array}{l}\text { No. of partici- } \\
\text { pants }\end{array}$ & Statistical method & Effect size \\
\hline 4.5 Walking programme vs control & 1 & 339 & $\begin{array}{l}\text { Risk Ratio (Random, 95\% } \\
\text { Cl) }\end{array}$ & 0.90 [0.67, 1.20] \\
\hline $\begin{array}{l}4.6 \text { Multiple categories of exercise vs } \\
\text { control }\end{array}$ & 4 & 518 & $\begin{array}{l}\text { Risk Ratio (Random, 95\% } \\
\text { Cl) }\end{array}$ & $0.84[0.69,1.02]$ \\
\hline $\begin{array}{l}5 \text { Number of people who experienced } \\
\text { one or more fall-related fractures - over- } \\
\text { all analysis }\end{array}$ & 2 & 332 & $\begin{array}{l}\text { Risk Ratio (Random, 95\% } \\
\text { Cl) }\end{array}$ & $0.26[0.07,1.02]$ \\
\hline
\end{tabular}

Analysis 13.1. Comparison 13 Sensitivity analysis 2: exercise versus control excluding studies at a high risk of bias, Outcome 1 Rate of falls - overall analysis.

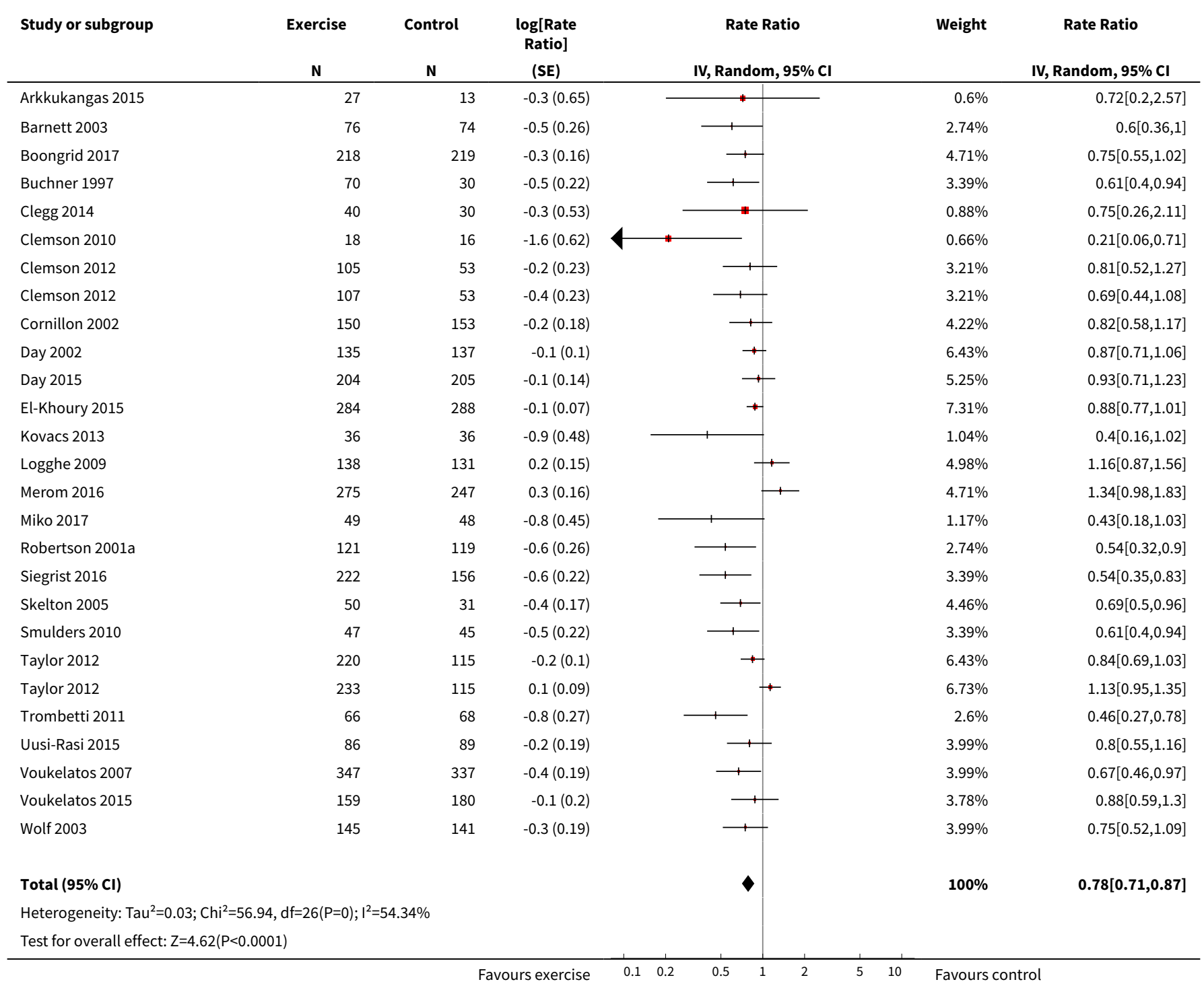




\section{Analysis 13.2. Comparison 13 Sensitivity analysis 2: exercise versus control excluding} studies at a high risk of bias, Outcome 2 Rate of falls - subgrouped by exercise type.

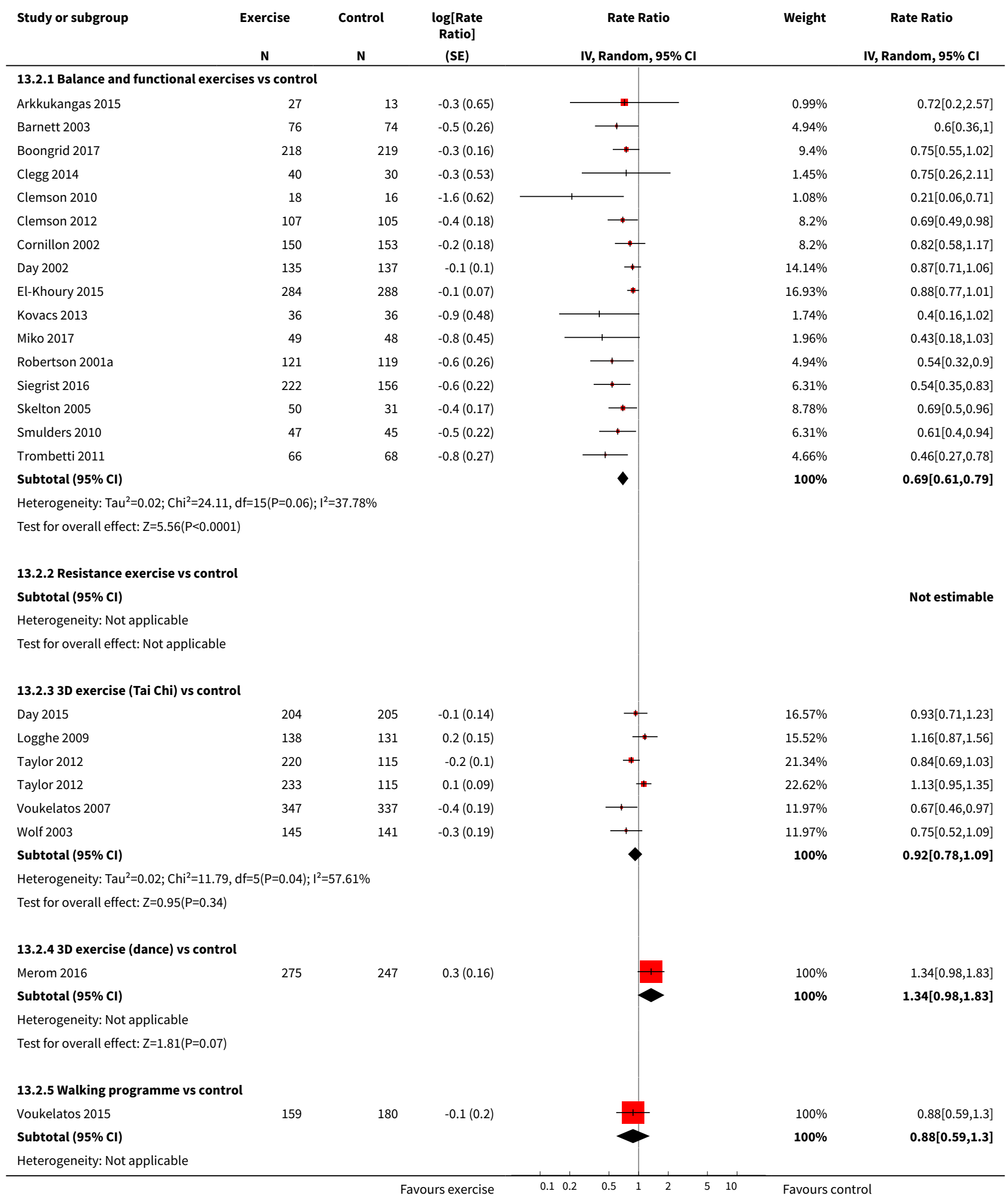




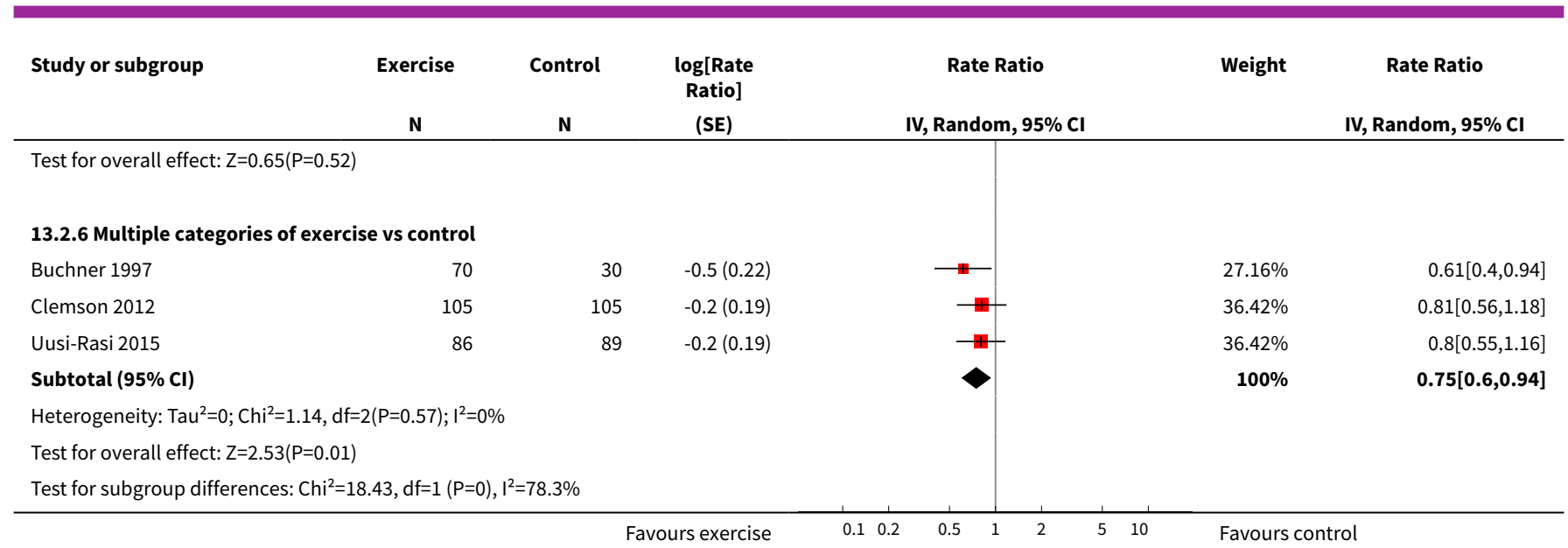

Analysis 13.3. Comparison 13 Sensitivity analysis 2: exercise versus control excluding studies at a high risk of bias, Outcome 3 Number of fallers - overall analysis.

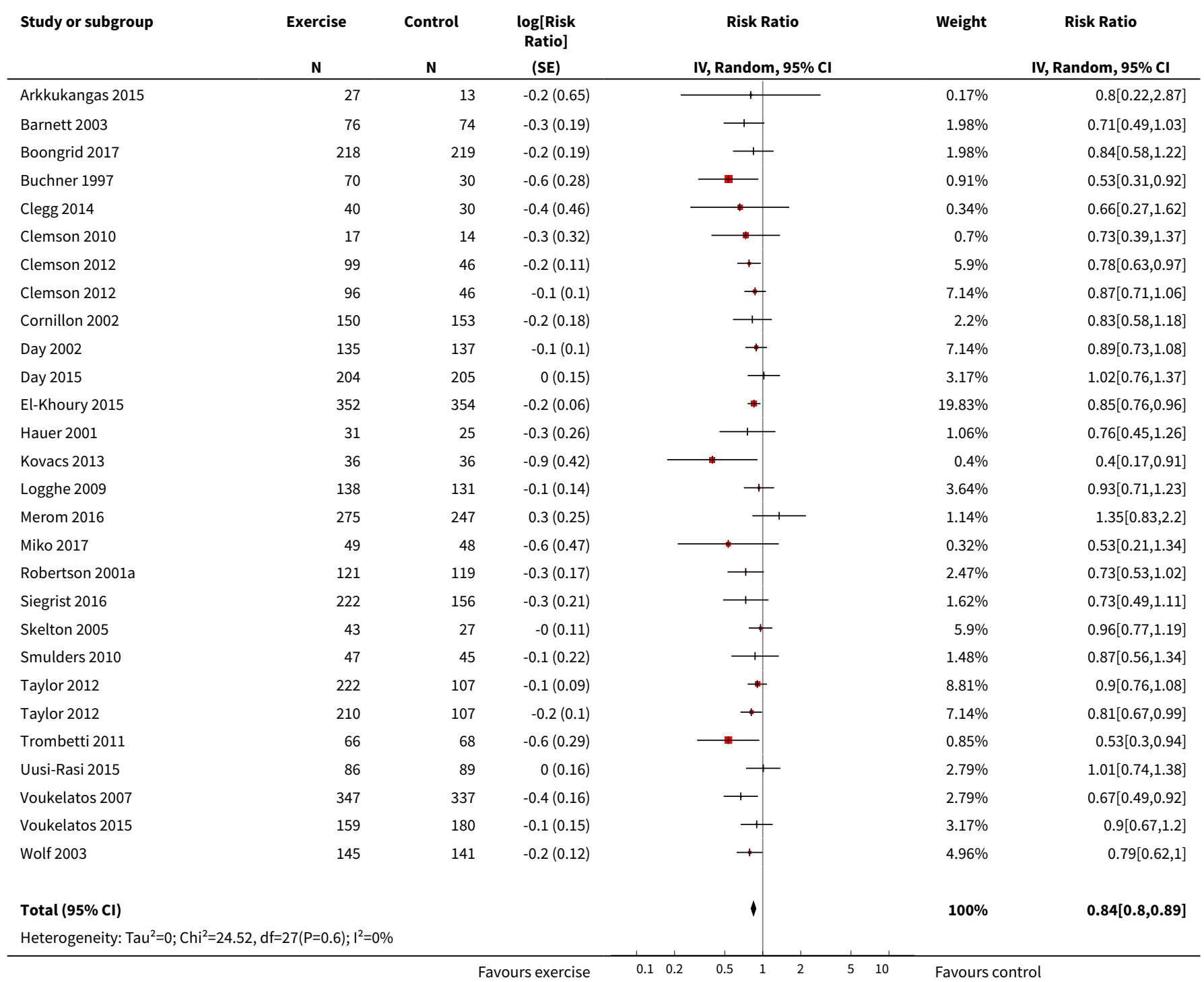




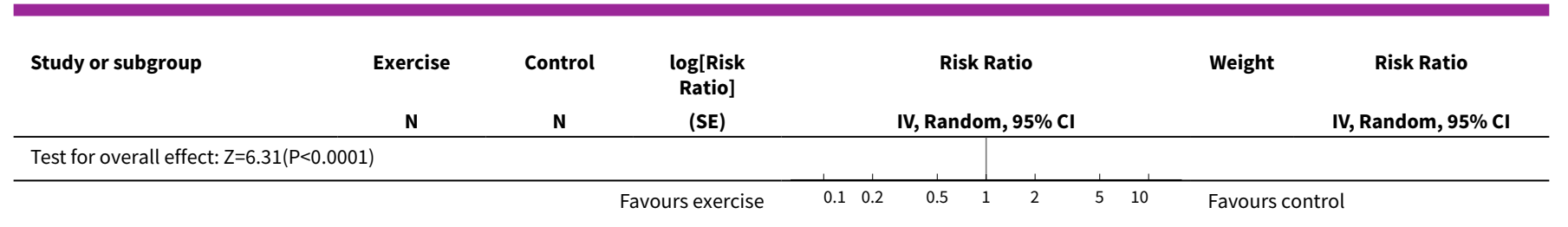

Analysis 13.4. Comparison 13 Sensitivity analysis 2 : exercise versus control excluding studies at a high risk of bias, Outcome 4 Number of fallers - subgrouped by exercise type.

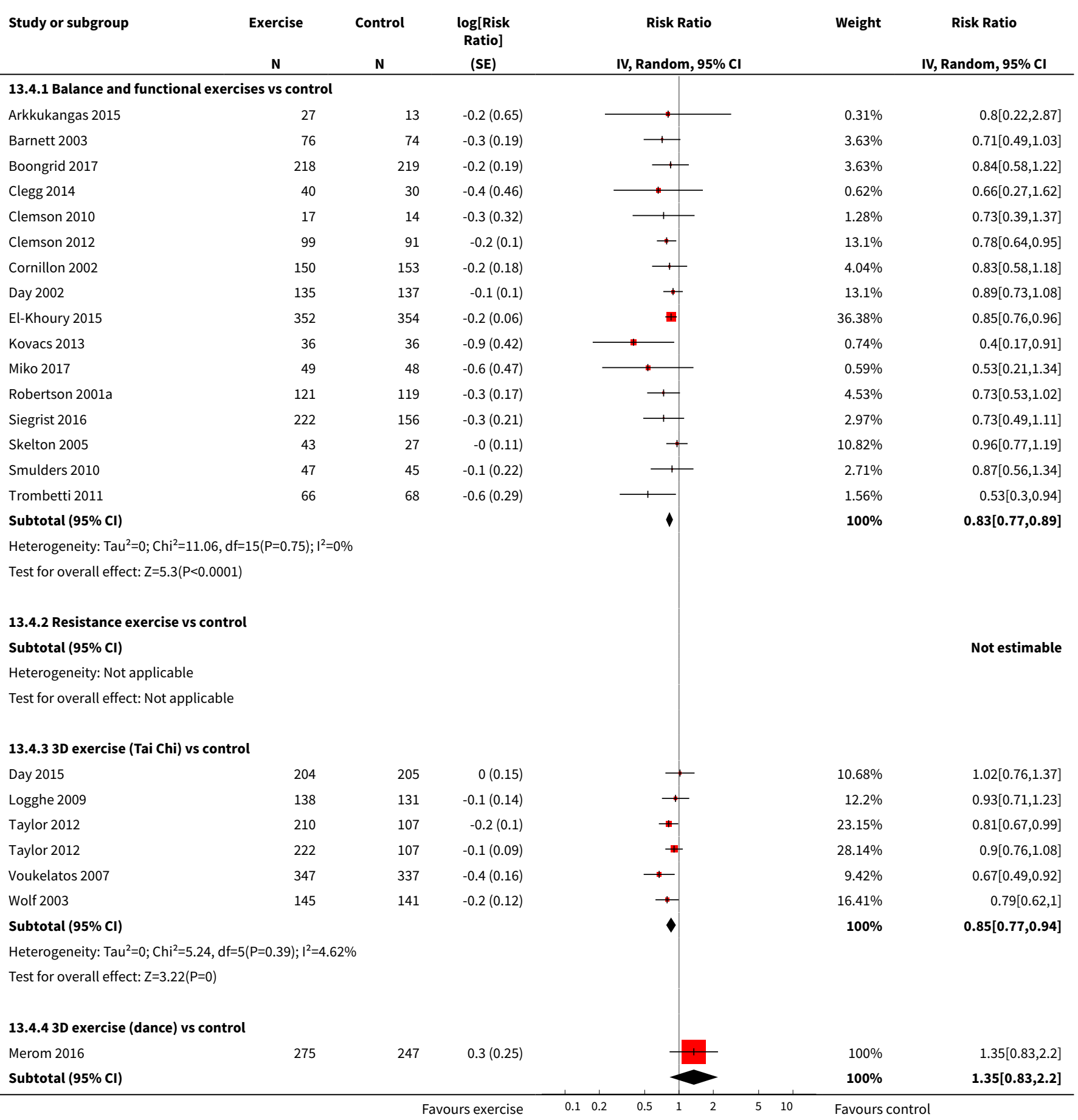




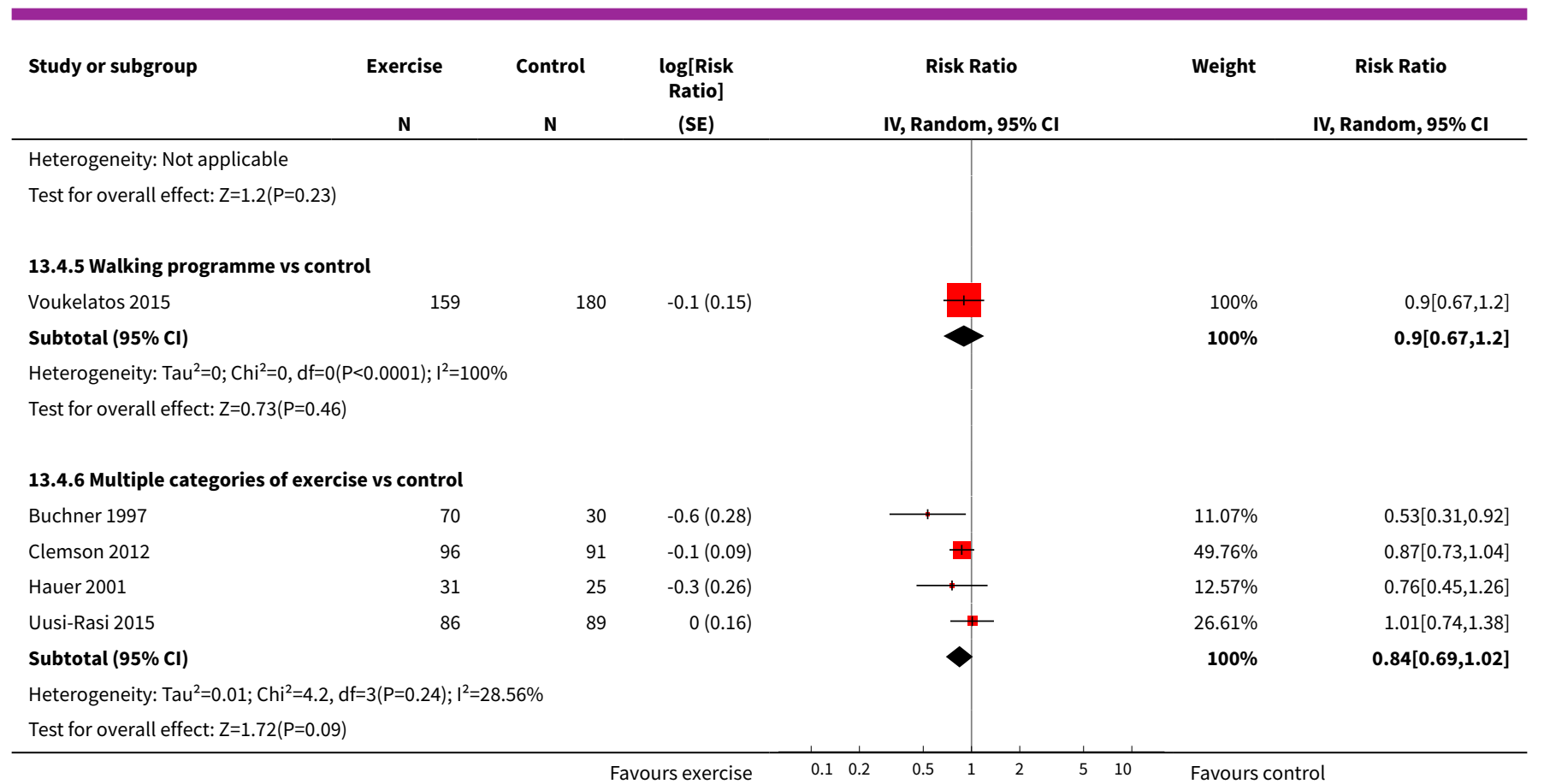

Analysis 13.5. Comparison 13 Sensitivity analysis 2: exercise versus control excluding studies at a high risk of bias, Outcome 5 Number of people who experienced one or more fall-related fractures - overall analysis.

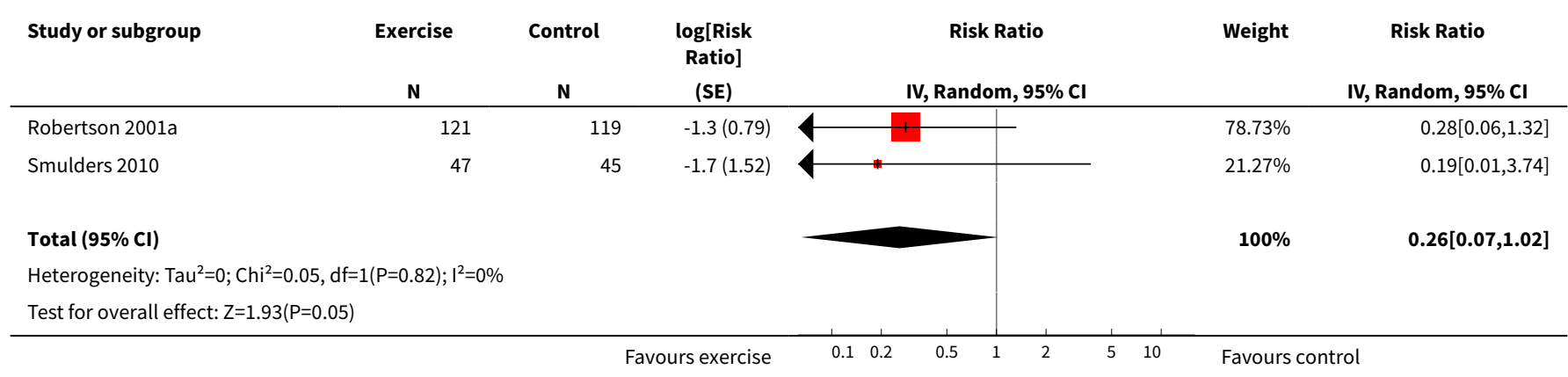

Comparison 14. Sensitivity analysis 3: exercise versus control excluding studies with unclear or high risk of bias due to allocation concealment (rate of falls)

\begin{tabular}{lllll}
\hline Outcome or subgroup title & No. of studies & $\begin{array}{l}\text { No. of partici- } \\
\text { pants }\end{array}$ & Statistical method & Effect size \\
\hline 1 Rate of falls - overall analysis & 22 & 6092 & $\begin{array}{l}\text { Rate Ratio (Random, 95\% } \\
\text { Cl) }\end{array}$ & $0.85[0.77,0.95]$ \\
\hline
\end{tabular}


Analysis 14.1. Comparison 14 Sensitivity analysis 3: exercise versus control excluding studies with unclear or high risk of bias due to allocation concealment (rate of falls), Outcome 1 Rate of falls - overall analysis.

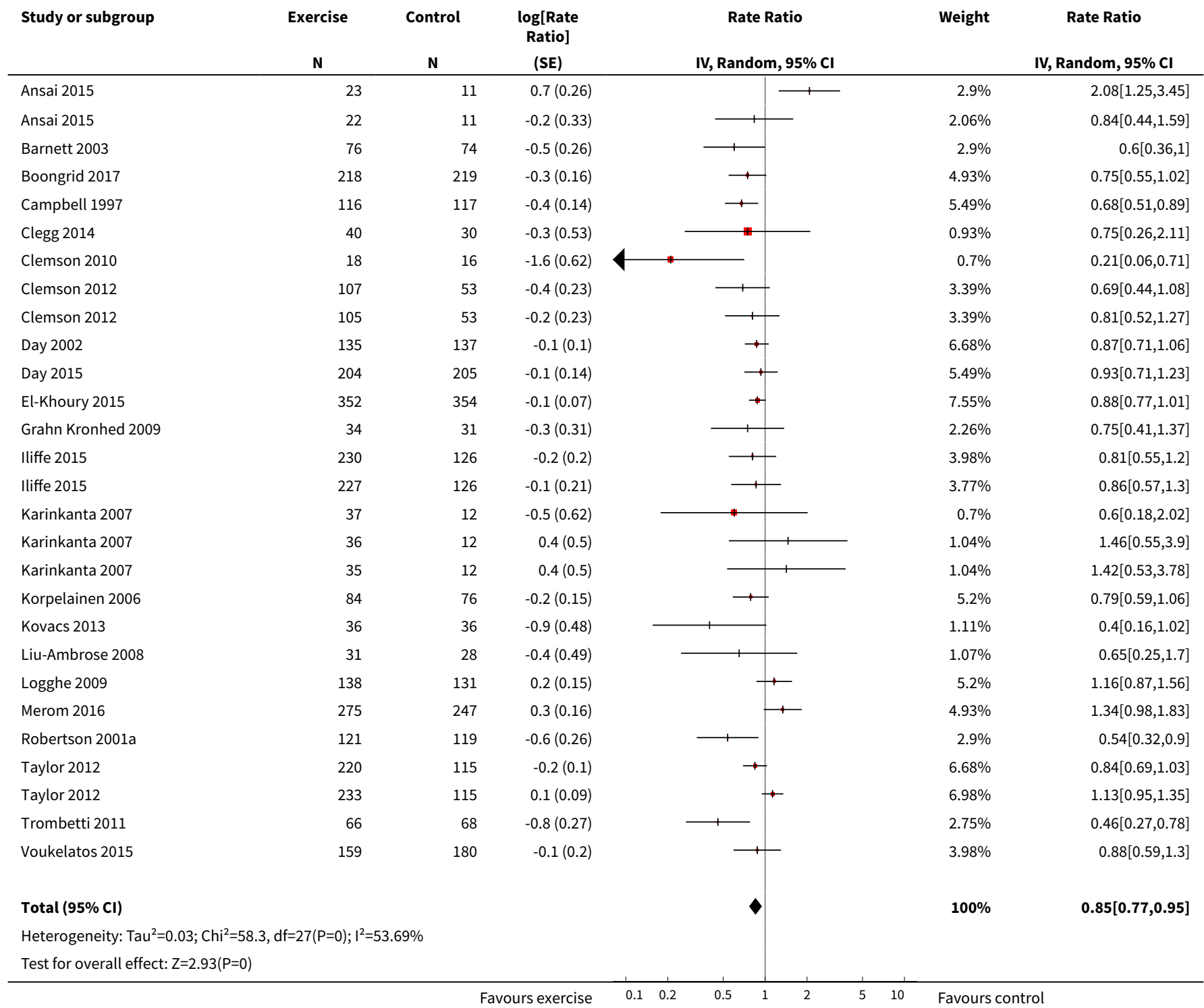

Comparison 15. Sensitivity analysis 4: exercise versus control excluding studies with unclear or high risk of bias due to assessor blinding (rate of falls)

\begin{tabular}{lllll}
\hline Outcome or subgroup title & No. of studies & $\begin{array}{l}\text { No. of partici- } \\
\text { pants }\end{array}$ & Statistical method & Effect size \\
\hline 1 Rate of falls - overall analysis & 27 & 6996 & $\begin{array}{l}\text { Rate Ratio (Random, 95\% } \\
\text { Cl) }\end{array}$ & $0.76[0.69,0.85]$ \\
\hline
\end{tabular}


Analysis 15.1. Comparison 15 Sensitivity analysis 4: exercise versus control excluding studies with unclear or high risk of bias due to assessor blinding (rate of falls), Outcome 1 Rate of falls - overall analysis.

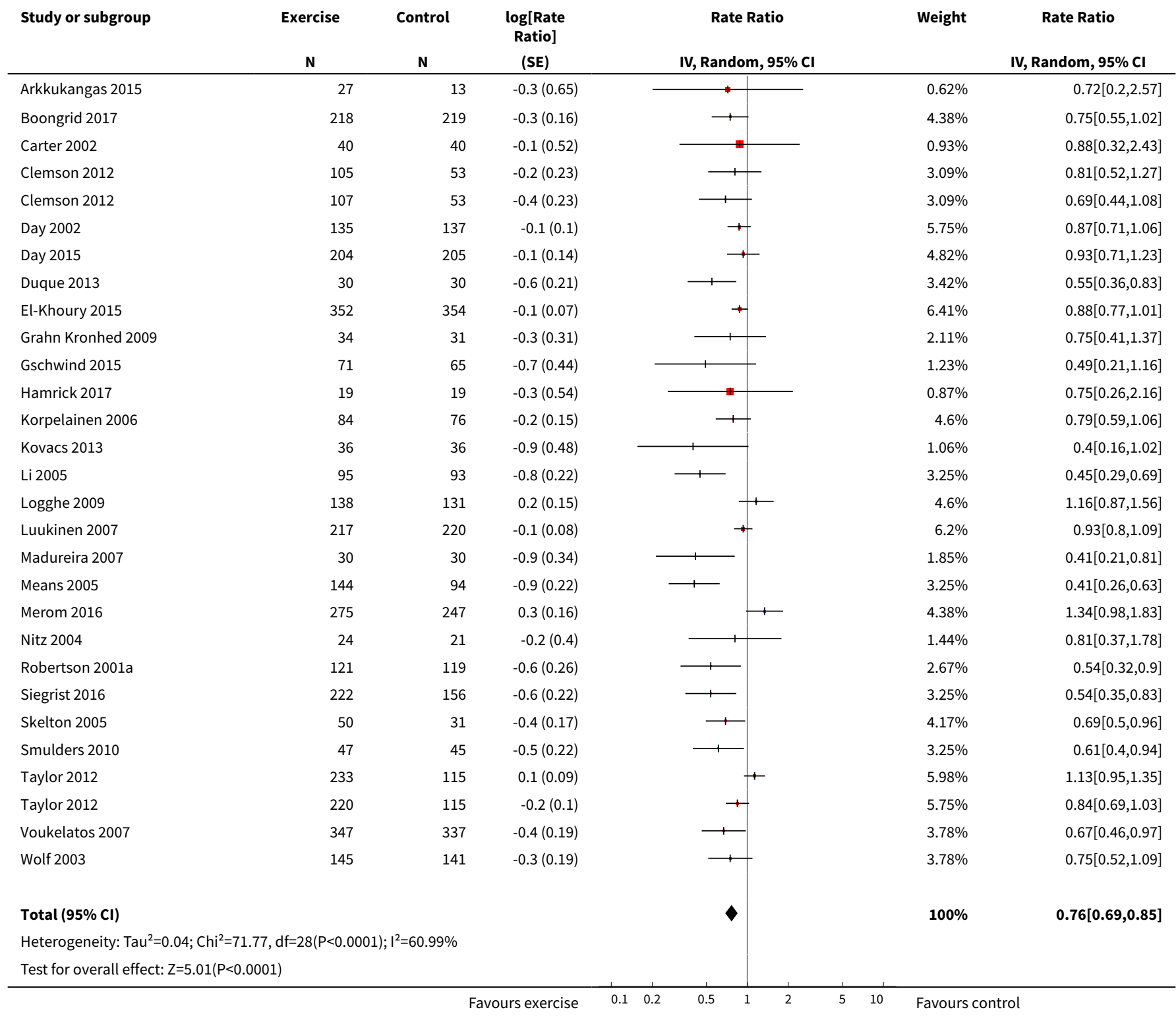

Comparison 16. Sensitivity analysis 5: exercise versus control excluding studies with unclear or high risk of bias due to incomplete outcome data (rate of falls)

\begin{tabular}{lllll}
\hline Outcome or subgroup title & No. of studies & $\begin{array}{l}\text { No. of partici- } \\
\text { pants }\end{array}$ & Statistical method & Effect size \\
\hline 1 Rate of falls - overall analysis & 36 & 7646 & $\begin{array}{l}\text { Rate Ratio (Random, 95\% } \\
\text { Cl) }\end{array}$ & $0.77[0.69,0.85]$ \\
\hline
\end{tabular}


Analysis 16.1. Comparison 16 Sensitivity analysis 5: exercise versus control excluding studies with unclear or high risk of bias due to incomplete outcome data (rate of falls), Outcome 1 Rate of falls - overall analysis.

\begin{tabular}{|c|c|c|c|c|c|c|c|}
\hline \multirow[t]{2}{*}{ Study or subgroup } & \multirow{2}{*}{$\begin{array}{c}\text { Exercise } \\
\mathrm{N} \\
\end{array}$} & \multirow{2}{*}{$\begin{array}{l}\text { Control } \\
\text { N }\end{array}$} & \multirow{2}{*}{$\begin{array}{c}\text { log[Rate } \\
\text { Ratio] } \\
\text { (SE) } \\
\end{array}$} & \multicolumn{2}{|c|}{ Rate Ratio } & Weight & \multirow{2}{*}{$\begin{array}{c}\text { Rate Ratio } \\
\text { IV, Random, } 95 \% \text { Cl }\end{array}$} \\
\hline & & & & IV, RandoI & $\mathrm{m}, \mathbf{9 5 \%} \mathrm{Cl}$ & & \\
\hline Ansai 2015 & 22 & 11 & $-0.2(0.33)$ & 1 & - & $1.73 \%$ & $0.84[0.44,1.59]$ \\
\hline Ansai 2015 & 23 & 11 & $0.7(0.26)$ & & 1 & $2.3 \%$ & $2.08[1.25,3.45]$ \\
\hline Barnett 2003 & 76 & 74 & $-0.5(0.26)$ & & & $2.3 \%$ & $0.6[0.36,1]$ \\
\hline Boongrid 2017 & 218 & 219 & $-0.3(0.16)$ & 1 & & $3.48 \%$ & $0.75[0.55,1.02]$ \\
\hline Carter 2002 & 40 & 40 & $-0.1(0.52)$ & — & & $0.88 \%$ & $0.88[0.32,2.43]$ \\
\hline Clemson 2010 & 18 & 16 & $-1.6(0.62)$ & - & & $0.65 \%$ & $0.21[0.06,0.71]$ \\
\hline Cornillon 2002 & 150 & 153 & $-0.2(0.18)$ & $\longrightarrow$ & - & $3.21 \%$ & $0.82[0.58,1.17]$ \\
\hline Day 2002 & 135 & 137 & $-0.1(0.1)$ & + & & $4.3 \%$ & $0.87[0.71,1.06]$ \\
\hline Day 2015 & 204 & 205 & $-0.1(0.14)$ & - & - & $3.75 \%$ & $0.93[0.71,1.23]$ \\
\hline Duque 2013 & 30 & 30 & $-0.6(0.21)$ & - & & $2.84 \%$ & $0.55[0.36,0.83]$ \\
\hline Grahn Kronhed 2009 & 34 & 31 & $-0.3(0.31)$ & + & - & $1.87 \%$ & $0.75[0.41,1.37]$ \\
\hline Gschwind 2015 & 71 & 65 & $-0.7(0.44)$ & & - & $1.14 \%$ & $0.49[0.21,1.16]$ \\
\hline Hamrick 2017 & 19 & 19 & $-0.3(0.54)$ & *- & & $0.82 \%$ & $0.75[0.26,2.16]$ \\
\hline Hirase 2015 & 29 & 14 & $-0.4(0.39)$ & & - & $1.37 \%$ & $0.66[0.31,1.41]$ \\
\hline Hirase 2015 & 29 & 14 & $-1.3(0.53)$ & & & $0.85 \%$ & $0.27[0.09,0.75]$ \\
\hline Irez 2011 & 30 & 30 & $-1.3(0.34)$ & & & $1.66 \%$ & $0.28[0.15,0.55]$ \\
\hline Karinkanta 2007 & 37 & 12 & $-0.5(0.62)$ & & & $0.65 \%$ & $0.6[0.18,2.02]$ \\
\hline Karinkanta 2007 & 35 & 12 & $0.4(0.5)$ & & 世 & $0.93 \%$ & $1.42[0.53,3.78]$ \\
\hline Karinkanta 2007 & 36 & 12 & $0.4(0.5)$ & & - & $0.93 \%$ & $1.46[0.55,3.9]$ \\
\hline Kerse 2010 & 98 & 95 & $0.2(0.22)$ & & 1 & $2.72 \%$ & $1.17[0.76,1.81]$ \\
\hline Korpelainen 2006 & 84 & 76 & $-0.2(0.15)$ & 1 & & $3.62 \%$ & $0.79[0.59,1.06]$ \\
\hline Kovacs 2013 & 36 & 36 & $-0.9(0.48)$ & 4 & & $1 \%$ & $0.4[0.16,1.02]$ \\
\hline Liu-Ambrose 2004 & 32 & 16 & $0.6(0.49)$ & & + & $0.96 \%$ & $1.8[0.69,4.71]$ \\
\hline Madureira 2007 & 30 & 30 & $-0.9(0.34)$ & & & $1.66 \%$ & $0.41[0.21,0.81]$ \\
\hline Merom 2016 & 275 & 247 & $0.3(0.16)$ & & - & $3.48 \%$ & $1.34[0.98,1.83]$ \\
\hline Miko 2017 & 49 & 48 & $-0.8(0.45)$ & & & $1.1 \%$ & $0.43[0.18,1.03]$ \\
\hline Robertson 2001a & 121 & 119 & $-0.6(0.26)$ & & & $2.3 \%$ & $0.54[0.32,0.9]$ \\
\hline Rubenstein 2000 & 31 & 28 & $-0.2(0.39)$ & & & $1.37 \%$ & $0.84[0.39,1.81]$ \\
\hline Siegrist 2016 & 222 & 156 & $-0.6(0.22)$ & & & $2.72 \%$ & $0.54[0.35,0.83]$ \\
\hline Skelton 2005 & 50 & 31 & $-0.4(0.17)$ & + & & $3.35 \%$ & $0.69[0.5,0.96]$ \\
\hline Smulders 2010 & 47 & 45 & $-0.5(0.22)$ & & & $2.72 \%$ & $0.61[0.4,0.94]$ \\
\hline Taylor 2012 & 233 & 115 & $0.1(0.09)$ & & + & $4.42 \%$ & $1.13[0.95,1.35]$ \\
\hline Taylor 2012 & 220 & 115 & $-0.2(0.1)$ & + & & $4.3 \%$ & $0.84[0.69,1.03]$ \\
\hline Trombetti 2011 & 66 & 68 & $-0.8(0.27)$ & & & $2.21 \%$ & $0.46[0.27,0.78]$ \\
\hline Voukelatos 2007 & 347 & 337 & $-0.4(0.19)$ & + & & $3.09 \%$ & $0.67[0.46,0.97]$ \\
\hline Weerdesteyn 2006 & 30 & 28 & $-0.6(0.32)$ & & & $1.8 \%$ & $0.53[0.28,1]$ \\
\hline Wolf 1996 & 72 & 32 & $-0.5(0.23)$ & 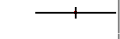 & & $2.61 \%$ & $0.62[0.39,0.97]$ \\
\hline Wolf 1996 & 64 & 32 & $-0(0.2)$ & - & - & $2.96 \%$ & $0.99[0.67,1.47]$ \\
\hline Wolf 2003 & 145 & 141 & $-0.3(0.19)$ & 1 & & $3.09 \%$ & $0.75[0.52,1.09]$ \\
\hline Total $(95 \% \mathrm{Cl})$ & & & & $\diamond$ & & $100 \%$ & $0.77[0.69,0.85]$ \\
\hline \multicolumn{8}{|c|}{ Heterogeneity: $\operatorname{Tau}^{2}=0.06 ; \mathrm{Chi}^{2}=105.94, \mathrm{df}=42(\mathrm{P}<0.0001) ; \mathrm{I}^{2}=60.35 \%$} \\
\hline Test for overall effect: & & & & & & & \\
\hline
\end{tabular}

Favours exercise

$\begin{array}{lllllll}0.2 & 0.5 & 1 & 2 & 5 & 10 & \text { Favours control }\end{array}$ 
Comparison 17. Sensitivity analysis 6: exercise versus control excluding cluster trials (rate of falls)

\begin{tabular}{lllll}
\hline Outcome or subgroup title & No. of studies & $\begin{array}{l}\text { No. of partici- } \\
\text { pants }\end{array}$ & Statistical method & Effect size \\
\hline 1 Rate of falls - overall analysis & 53 & 10261 & $\begin{array}{l}\text { Rate Ratio (Random, 95\% } \\
\text { Cl) }\end{array}$ & $0.76[0.70,0.83]$ \\
\hline
\end{tabular}

Analysis 17.1. Comparison 17 Sensitivity analysis 6: exercise versus control excluding cluster trials (rate of falls), Outcome 1 Rate of falls - overall analysis.

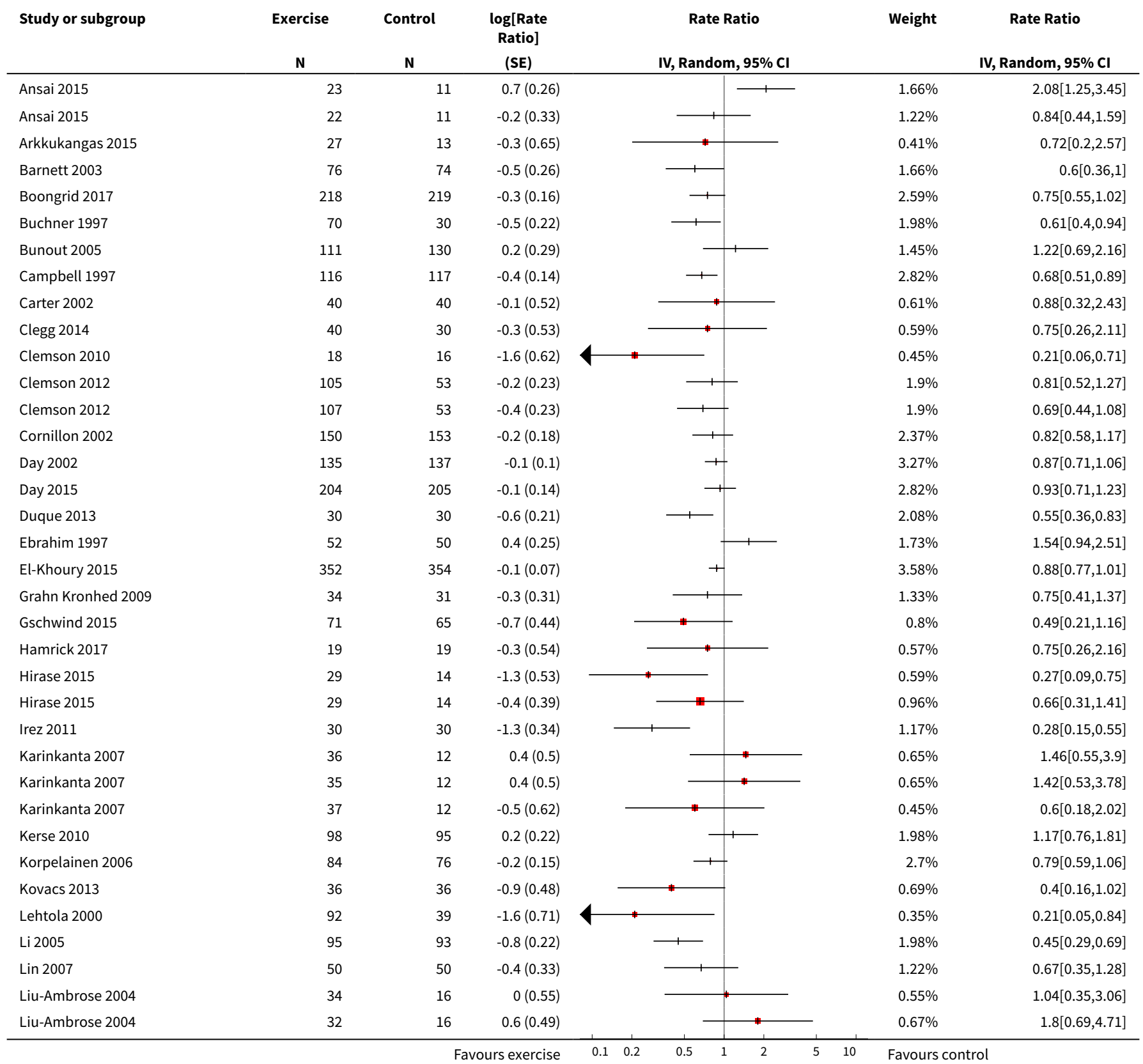




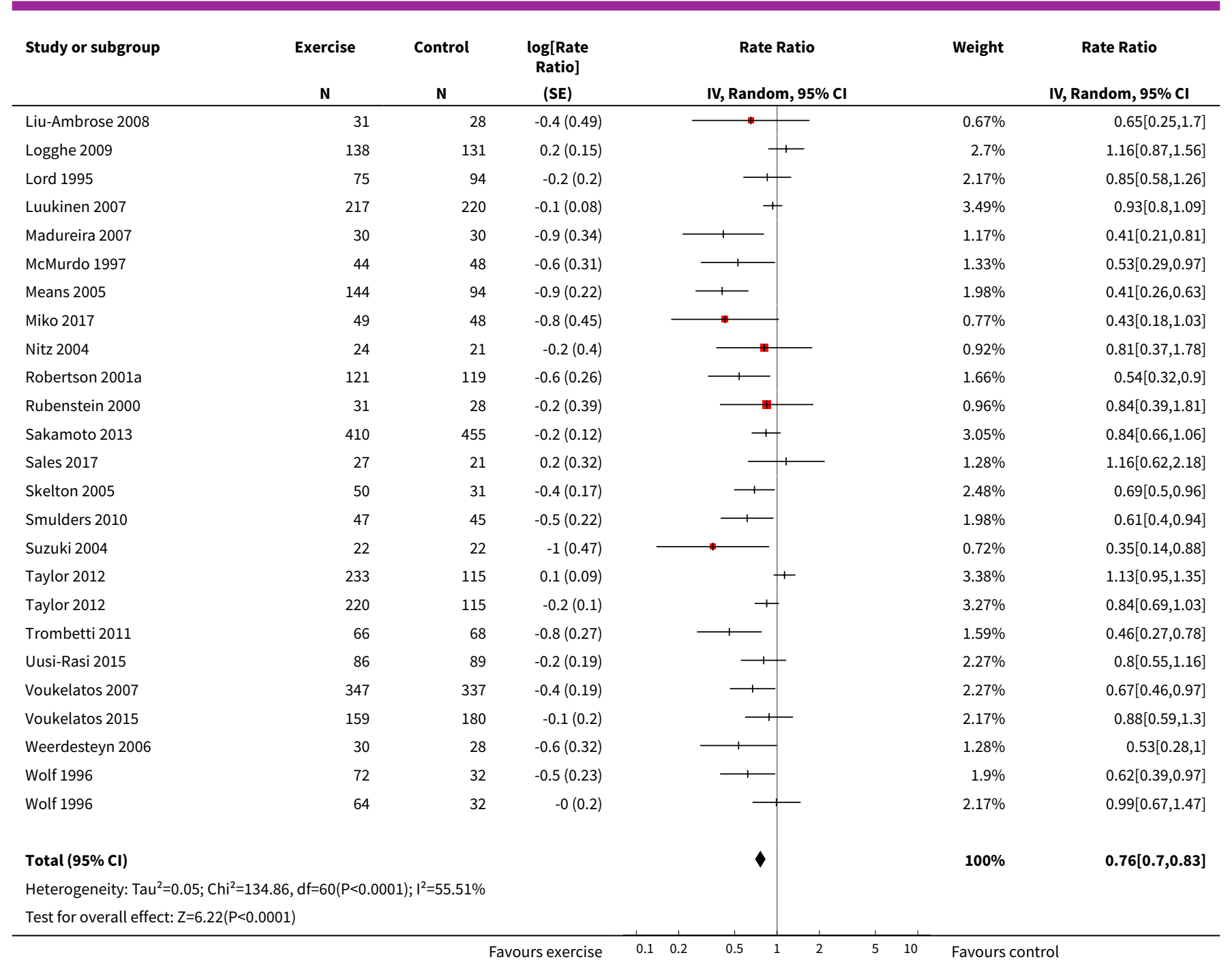

Comparison 18. Sensitivity analysis 7: exercise versus control with fixed-effect meta-analysis (rate of falls)

\begin{tabular}{lllll}
\hline Outcome or subgroup title & No. of studies & $\begin{array}{l}\text { No. of partici- } \\
\text { pants }\end{array}$ & Statistical method & Effect size \\
\hline 1 Rate of falls - overall analysis & 59 & 12981 & Rate Ratio (Fixed, 95\% Cl) & $0.82[0.79,0.86]$ \\
\hline
\end{tabular}

Analysis 18.1. Comparison 18 Sensitivity analysis 7: exercise versus control with fixed-effect meta-analysis (rate of falls), Outcome 1 Rate of falls - overall analysis.

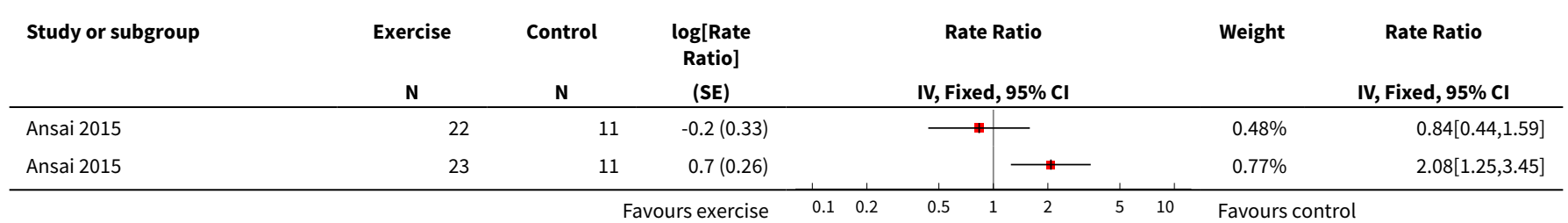




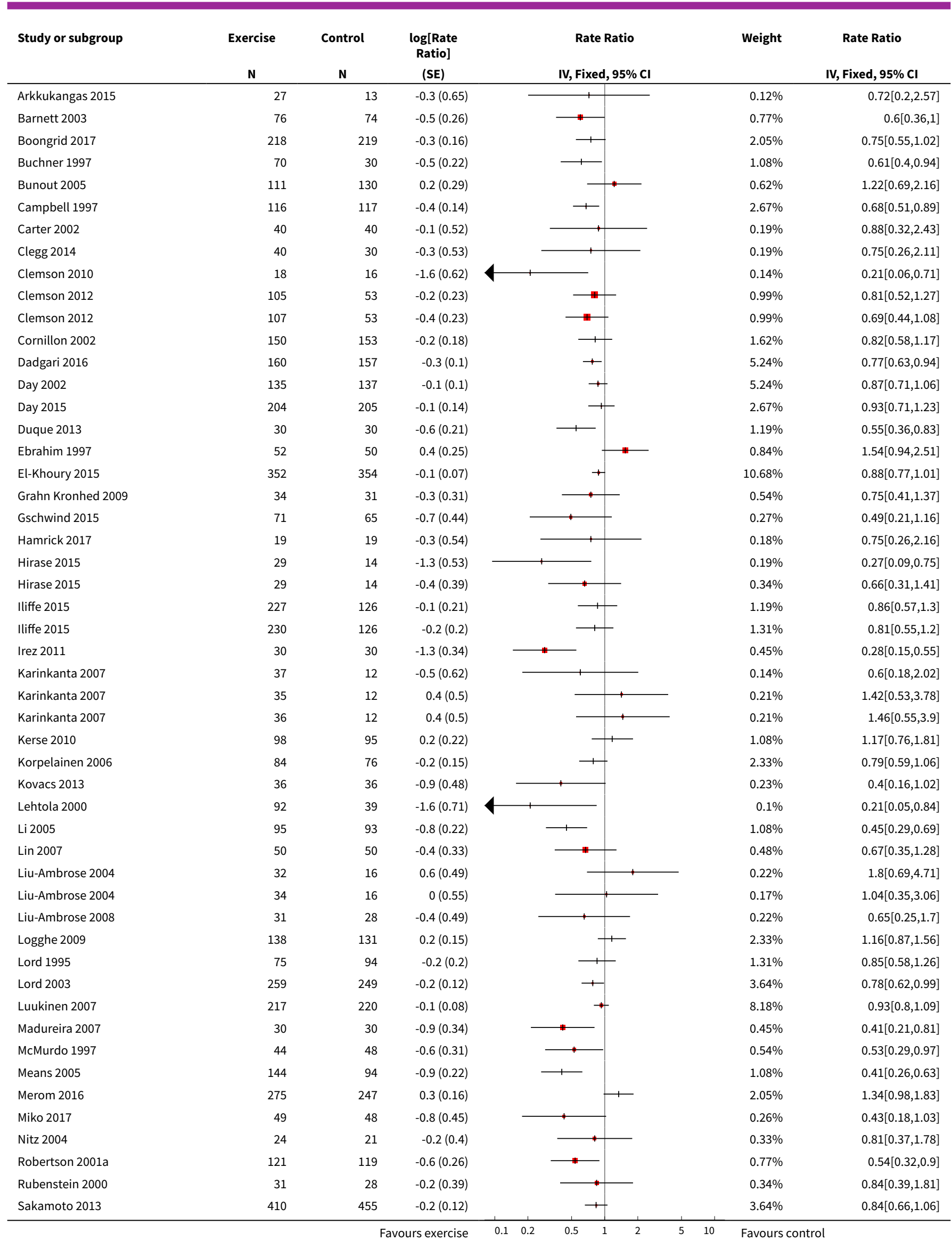




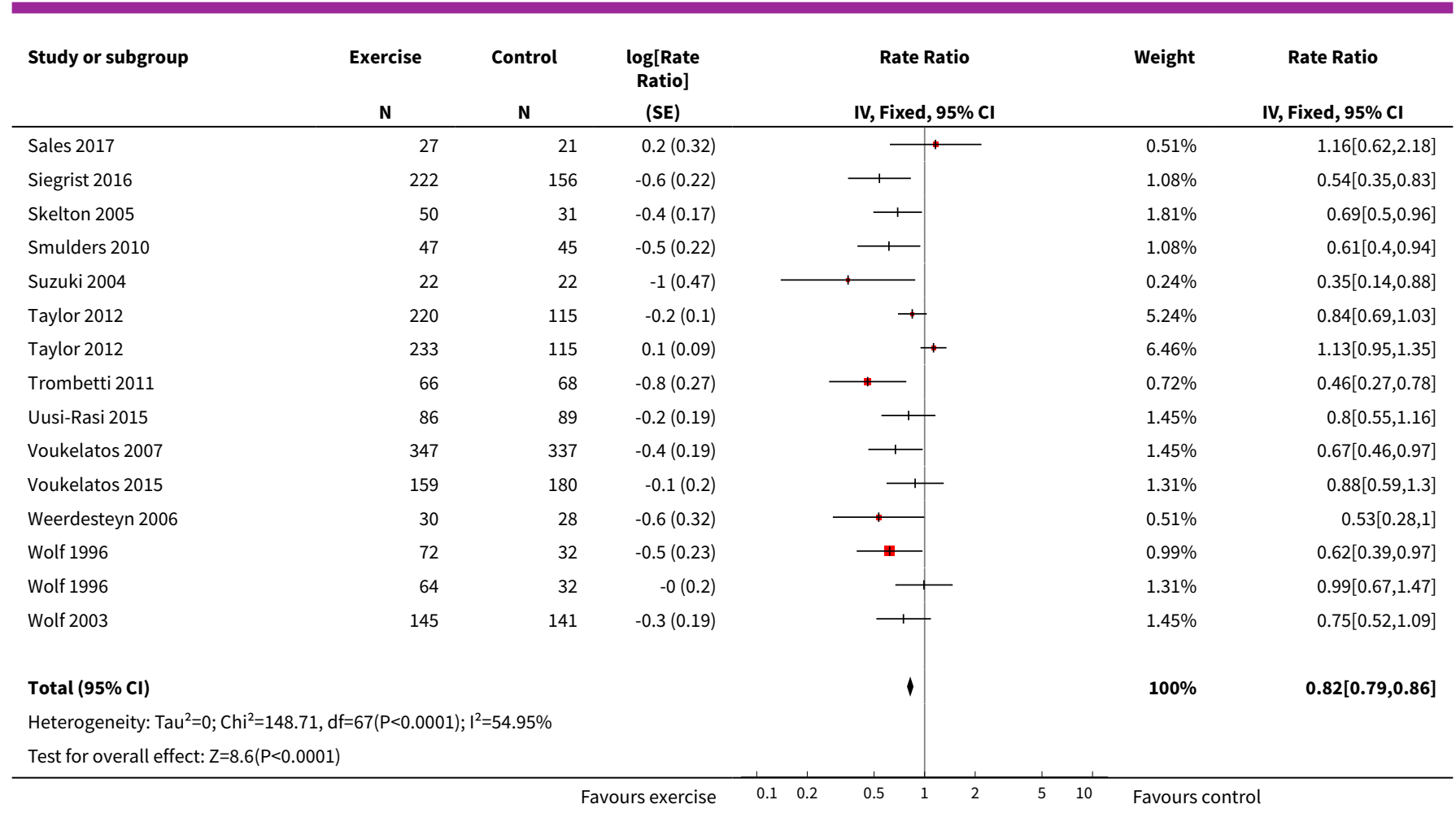

Comparison 19. Sensitivity analysis 8: multiple categories of exercise versus control excluding trials that do not include balance and strength training

\begin{tabular}{lllll}
\hline Outcome or subgroup title & No. of studies & $\begin{array}{l}\text { No. of partici- } \\
\text { pants }\end{array}$ & Statistical method & Effect size \\
\hline 1 Rate of falls & 8 & 1084 & Rate Ratio (Random, 95\% Cl) & $0.69[0.48,0.97]$ \\
\hline 2 Number of fallers & 13 & 1375 & Risk Ratio (Random, 95\% Cl) & $0.76[0.61,0.95]$ \\
\hline
\end{tabular}

Analysis 19.1. Comparison 19 Sensitivity analysis 8: multiple categories of exercise versus control excluding trials that do not include balance and strength training, Outcome 1 Rate of falls.

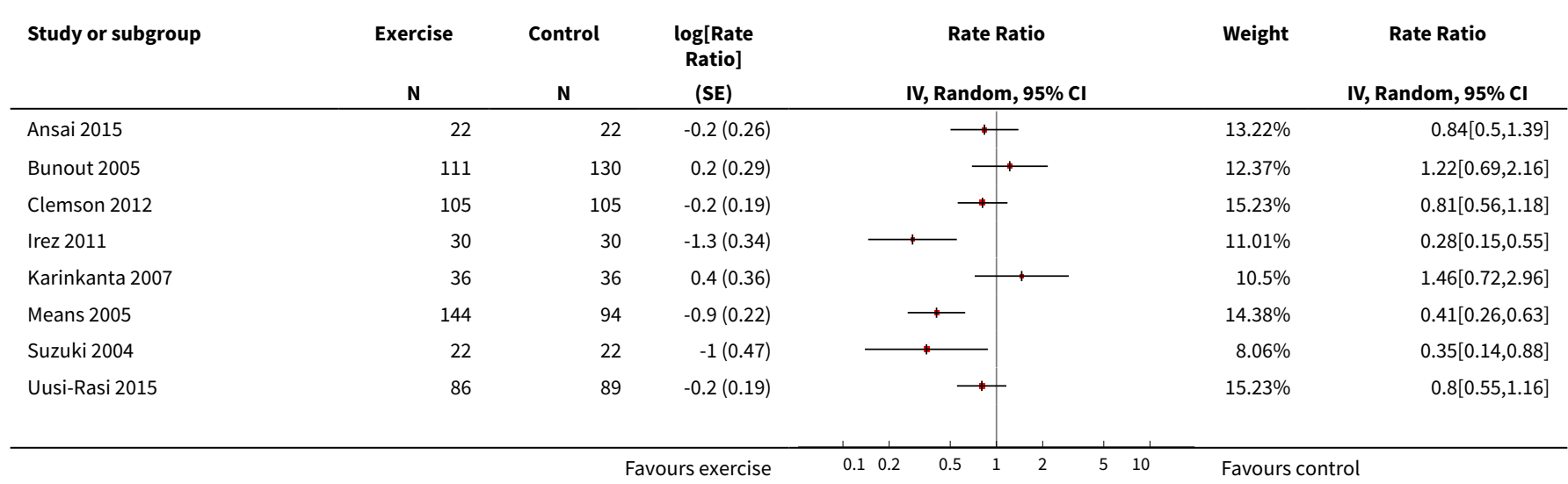




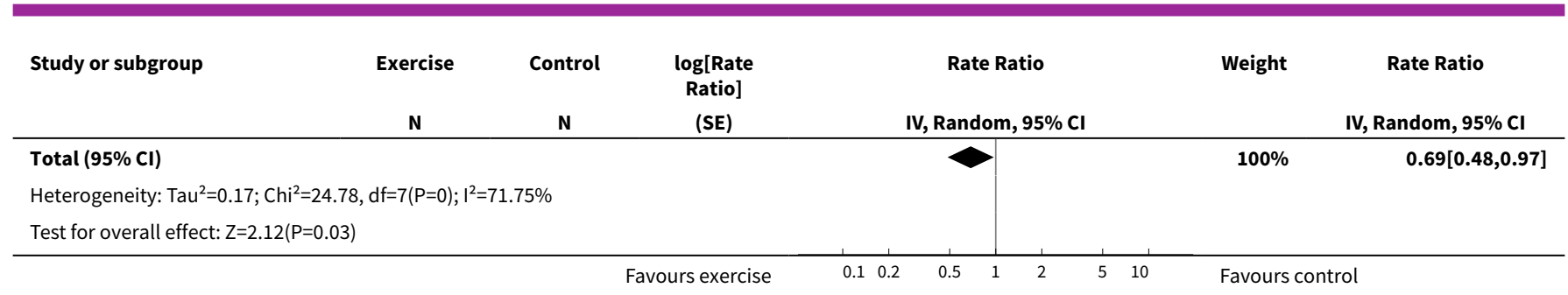

\section{Analysis 19.2. Comparison 19 Sensitivity analysis 8: multiple categories of exercise versus control excluding trials that do not include balance and strength training, Outcome 2 Number of fallers.}

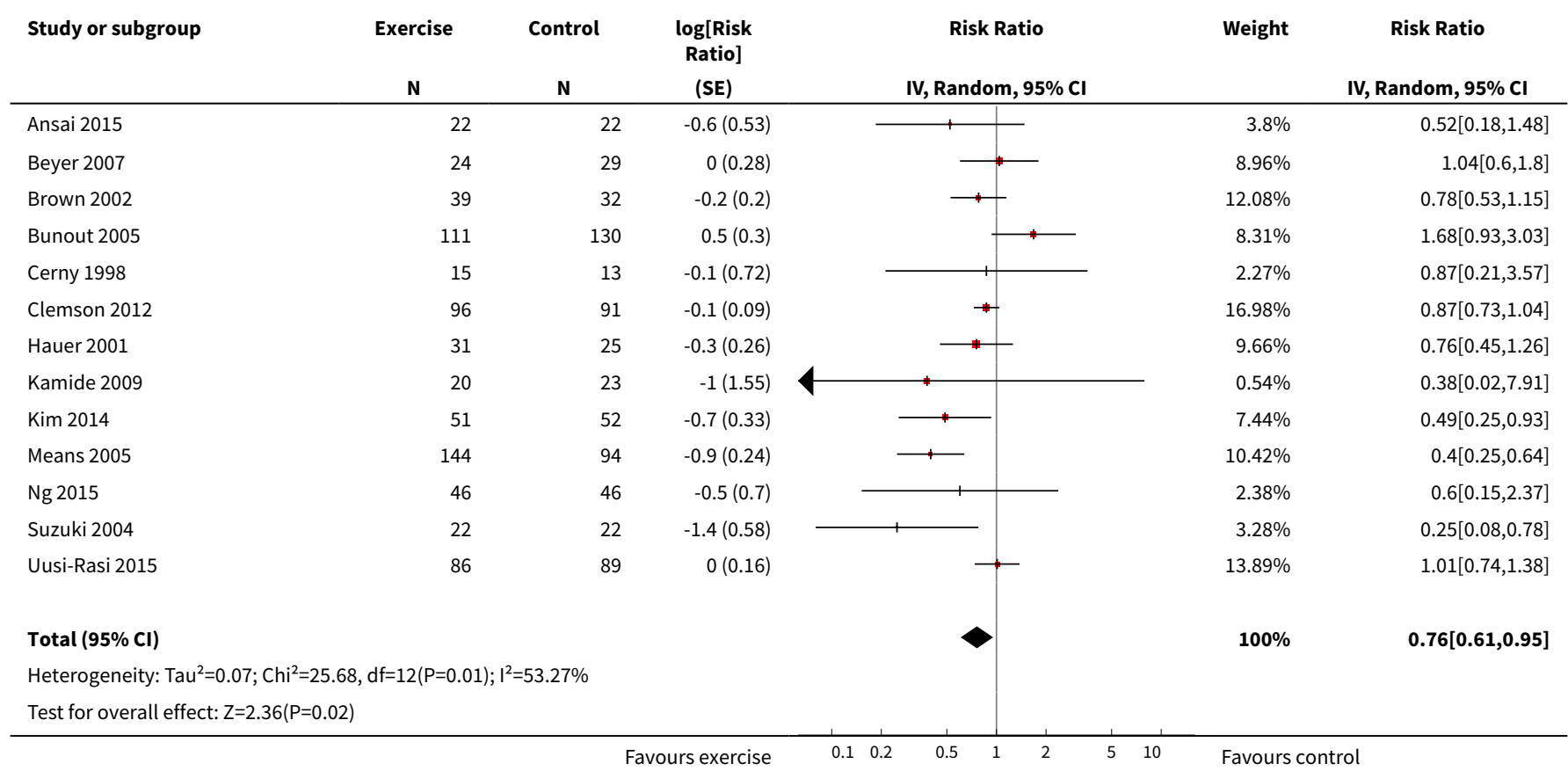

\section{Comparison 20. Sensitivity analysis 9: different exercise type coding}

\begin{tabular}{lllll}
\hline Outcome or subgroup title & No. of studies & $\begin{array}{l}\text { No. of partici- } \\
\text { pants }\end{array}$ & Statistical method & Effect size \\
\hline $\begin{array}{l}1 \text { Rate of falls - subgrouped by exercise type } \\
\text { (OEP as multiple intervention) }\end{array}$ & 48 & & $\begin{array}{l}\text { Rate Ratio (Random, } \\
95 \% \mathrm{Cl})\end{array}$ & Subtotals only \\
\hline $\begin{array}{l}1.1 \text { Balance and functional exercises vs con- } \\
\text { trol }\end{array}$ & 30 & 5556 & $\begin{array}{l}\text { Rate Ratio (Random, } \\
95 \% \mathrm{Cl})\end{array}$ & $0.75[0.68,0.82]$ \\
\hline $\begin{array}{l}1.2 \text { Multiple categories of exercise vs control } \\
20\end{array}$ & & 3738 & $\begin{array}{l}\text { Rate Ratio (Random, } \\
95 \% \mathrm{Cl})\end{array}$ & $0.72[0.62,0.83]$ \\
\hline $\begin{array}{l}2 \text { Number of fallers - subgrouped by exercise } \\
\text { type (OEP as multiple intervention) }\end{array}$ & 52 & & $\begin{array}{l}\text { Risk Ratio (Random, } \\
95 \% \mathrm{Cl})\end{array}$ \\
\hline
\end{tabular}




\begin{tabular}{|c|c|c|c|c|}
\hline Outcome or subgroup title & No. of studies & $\begin{array}{l}\text { No. of partici- } \\
\text { pants }\end{array}$ & Statistical method & Effect size \\
\hline $\begin{array}{l}2.1 \text { Balance and functional exercises vs con- } \\
\text { trol }\end{array}$ & 28 & 5946 & $\begin{array}{l}\text { Risk Ratio (Random, } \\
95 \% \mathrm{Cl} \text { ) }\end{array}$ & $0.86[0.80,0.92]$ \\
\hline 2.2 Multiple categories of exercise vs control & 26 & 3965 & $\begin{array}{l}\text { Risk Ratio (Random, } \\
95 \% \mathrm{Cl} \text { ) }\end{array}$ & $0.83[0.75,0.92]$ \\
\hline $\begin{array}{l}3 \text { Rate of falls - subgrouped by exercise type } \\
\text { (any balance+strength as multiple interven- } \\
\text { tion) }\end{array}$ & 50 & & $\begin{array}{l}\text { Rate Ratio (Random, } \\
95 \% \mathrm{Cl} \text { ) }\end{array}$ & Subtotals only \\
\hline $\begin{array}{l}\text { 3.1 Balance and functional exercises vs con- } \\
\text { trol }\end{array}$ & 16 & 2718 & $\begin{array}{l}\text { Rate Ratio (Random, } \\
95 \% \mathrm{Cl} \text { ) }\end{array}$ & $0.72[0.62,0.84]$ \\
\hline 3.2 Resistance exercise vs control & 3 & 182 & $\begin{array}{l}\text { Rate Ratio (Random, } \\
95 \% \mathrm{Cl} \text { ) }\end{array}$ & $1.42[0.71,2.82]$ \\
\hline 3.3 Multiple categories of exercise vs control & 35 & 6721 & $\begin{array}{l}\text { Rate Ratio (Random, } \\
95 \% \mathrm{Cl} \text { ) }\end{array}$ & $0.74[0.67,0.81]$ \\
\hline $\begin{array}{l}4 \text { Number of fallers - subgrouped by exercise } \\
\text { type (any balance+strength as multiple in- } \\
\text { tervention) }\end{array}$ & 53 & & $\begin{array}{l}\text { Risk Ratio (Random, } \\
95 \% \mathrm{Cl} \text { ) }\end{array}$ & Subtotals only \\
\hline $\begin{array}{l}\text { 4.1 Balance and functional exercises vs con- } \\
\text { trol }\end{array}$ & 13 & 2310 & $\begin{array}{l}\text { Risk Ratio (Random, } \\
95 \% \mathrm{CI} \text { ) }\end{array}$ & $0.79[0.65,0.96]$ \\
\hline 4.2 Resistance exercise vs control & 1 & 45 & $\begin{array}{l}\text { Risk Ratio (Random, } \\
95 \% \mathrm{Cl} \text { ) }\end{array}$ & $1.0[0.46,2.19]$ \\
\hline 4.3 Multiple categories of exercise vs control & 41 & 7719 & $\begin{array}{l}\text { Risk Ratio (Random, } \\
95 \% \mathrm{Cl} \text { ) }\end{array}$ & $0.86[0.81,0.91]$ \\
\hline
\end{tabular}

Analysis 20.1. Comparison 20 Sensitivity analysis 9: different exercise type coding, Outcome 1 Rate of falls - subgrouped by exercise type (OEP as multiple intervention).

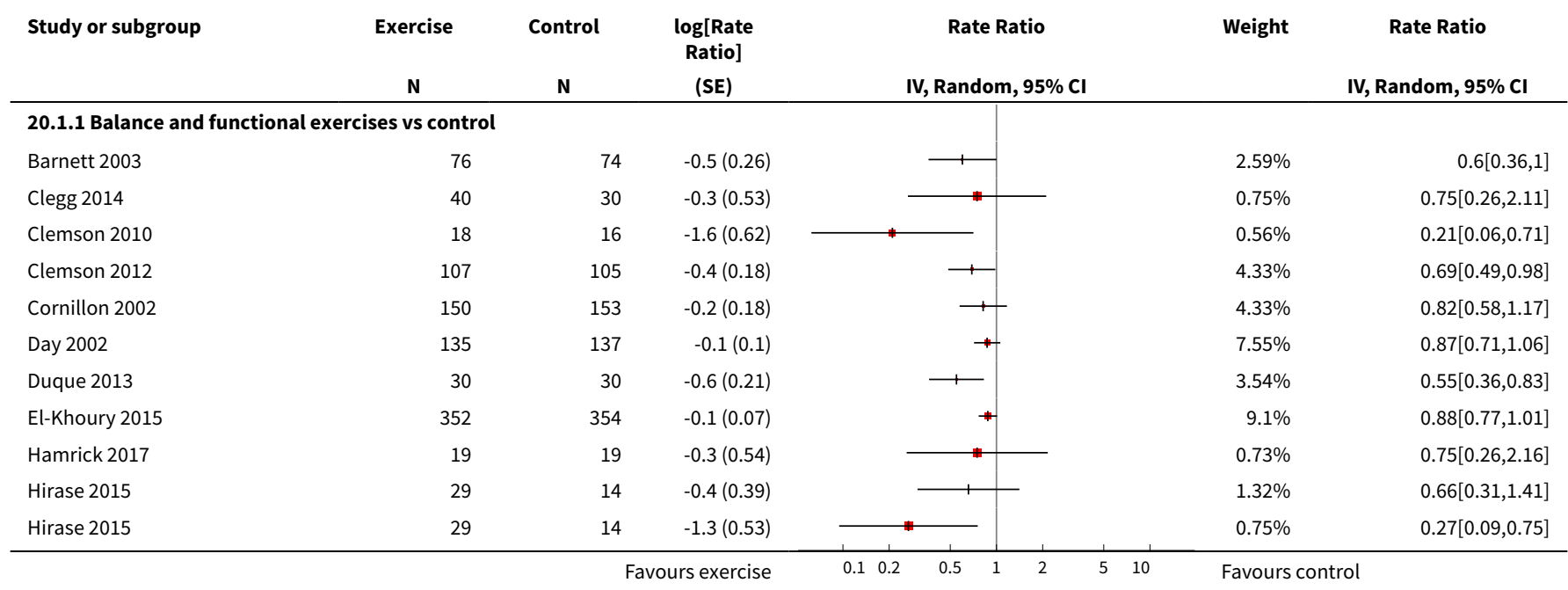




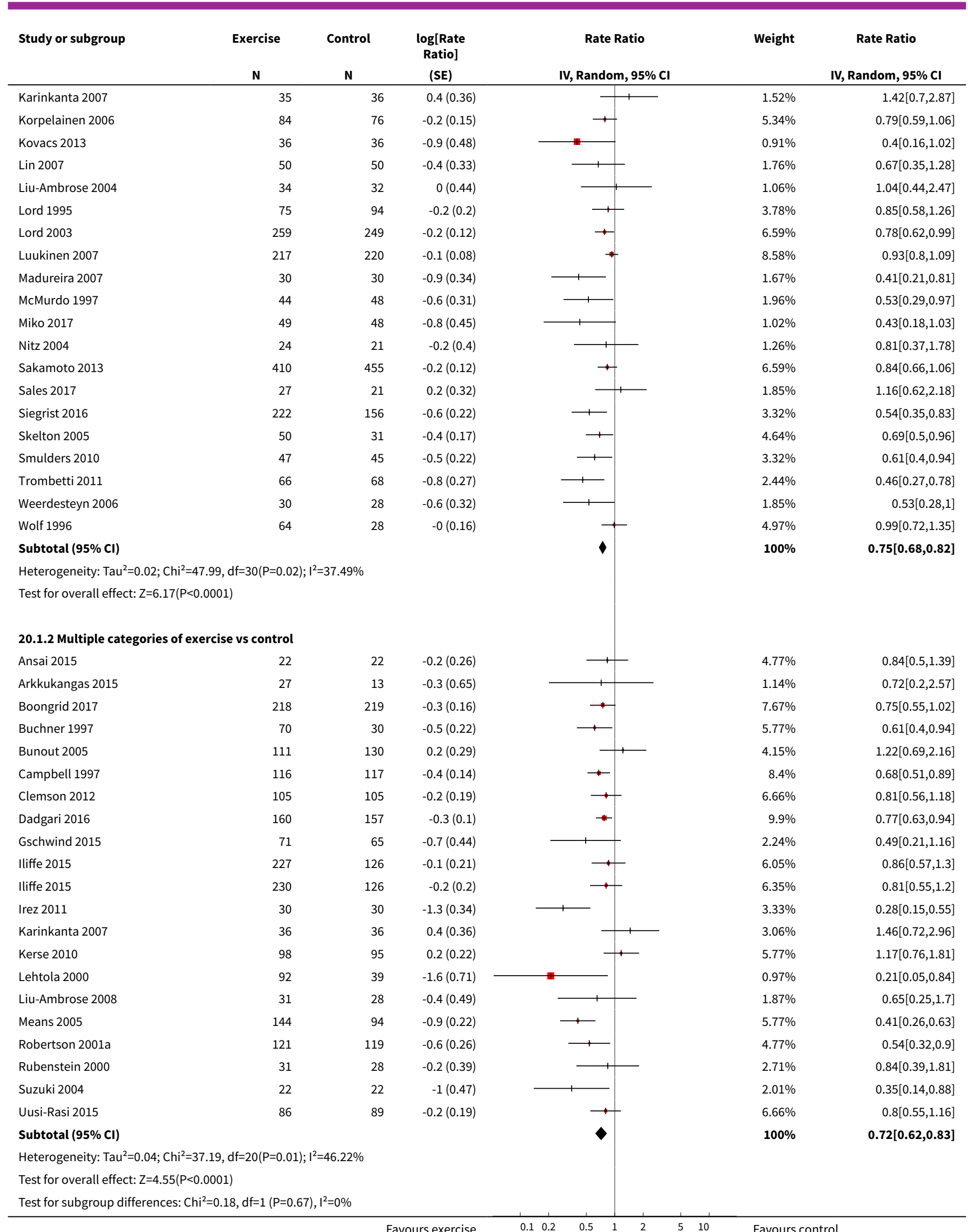


Analysis 20.2. Comparison 20 Sensitivity analysis 9: different exercise type coding, Outcome 2 Number of fallers - subgrouped by exercise type (OEP as multiple intervention).

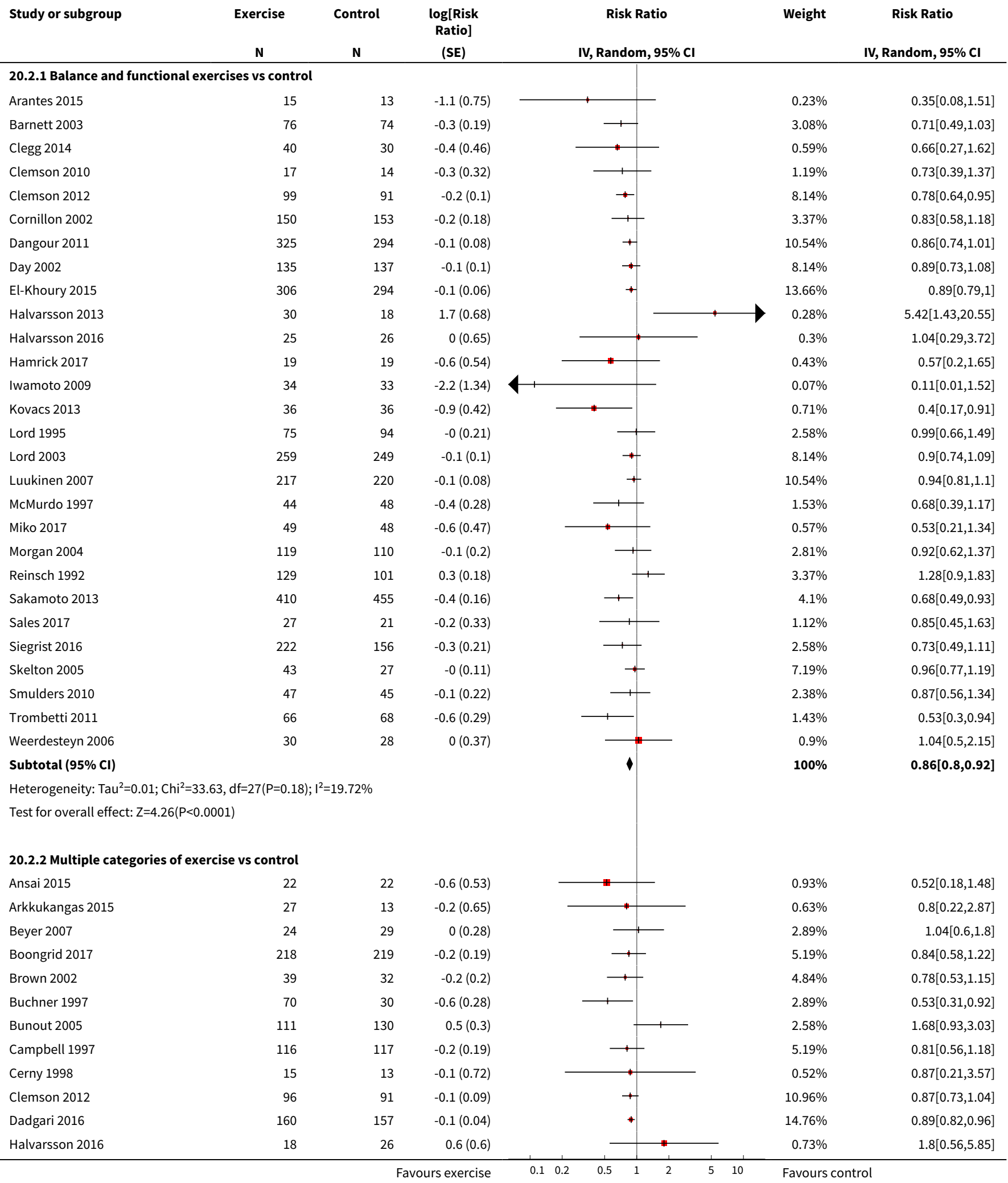




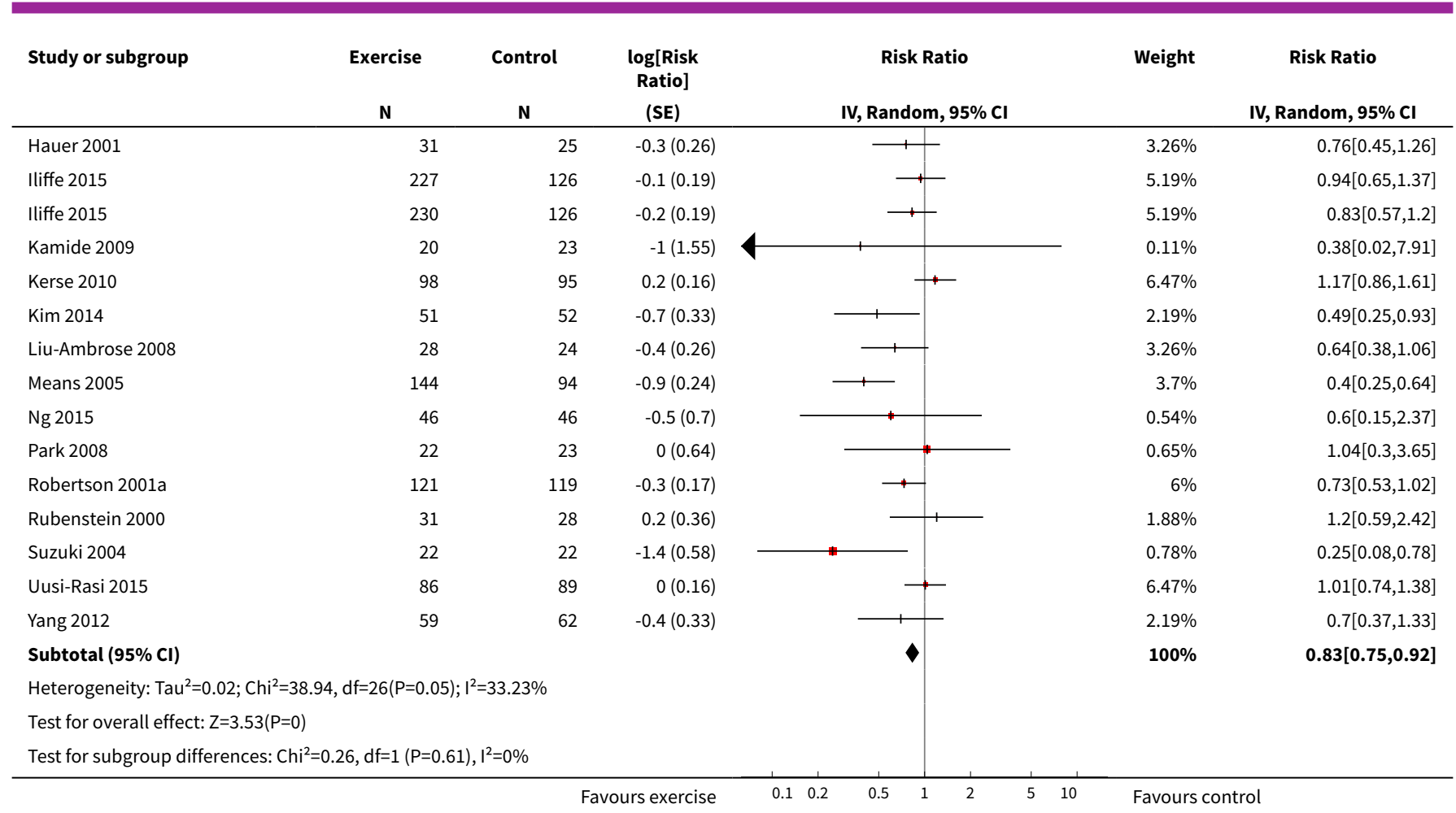

Analysis 20.3. Comparison 20 Sensitivity analysis 9: different exercise type coding, Outcome 3 Rate of falls - subgrouped by exercise type (any balance+strength as multiple intervention).

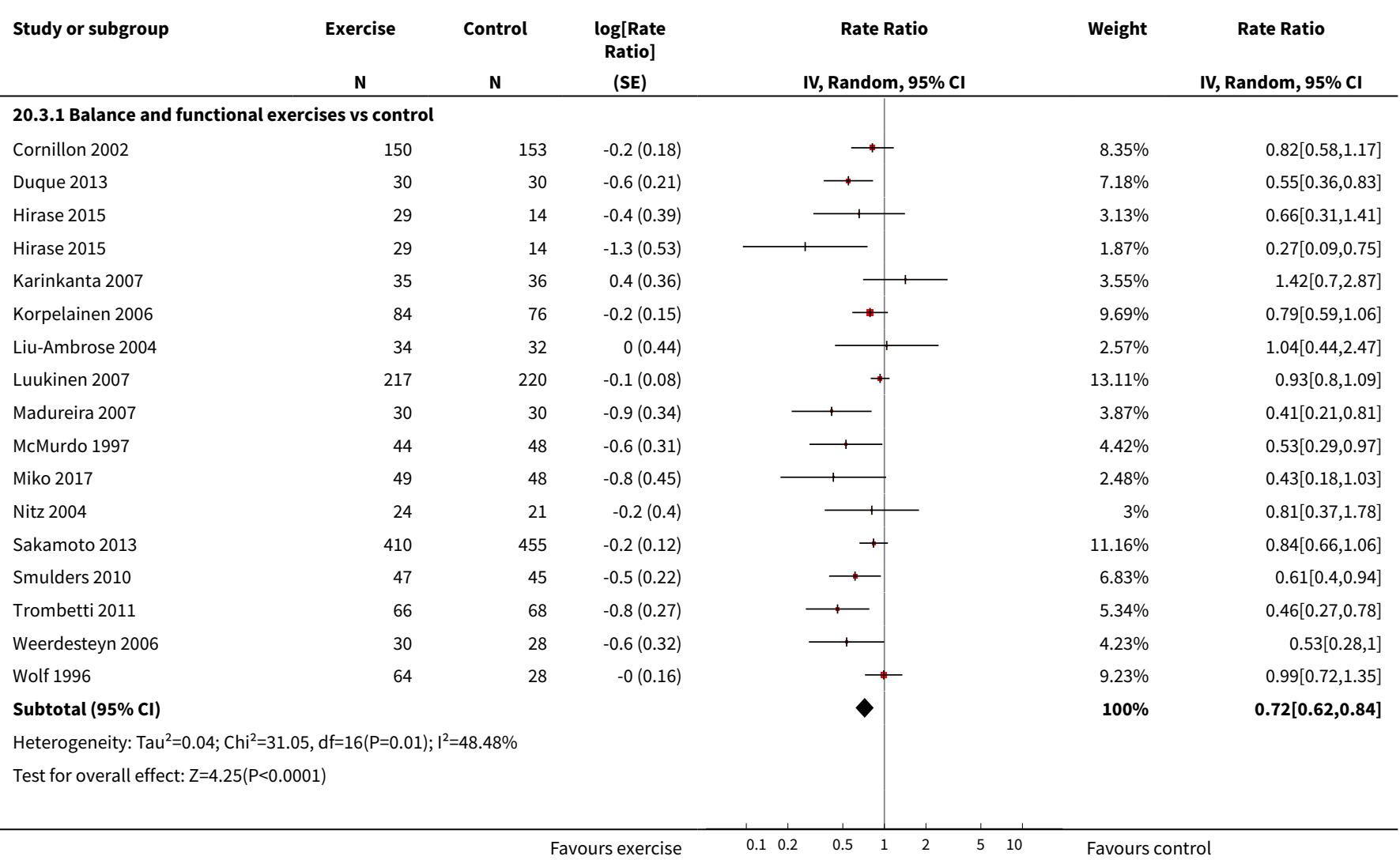




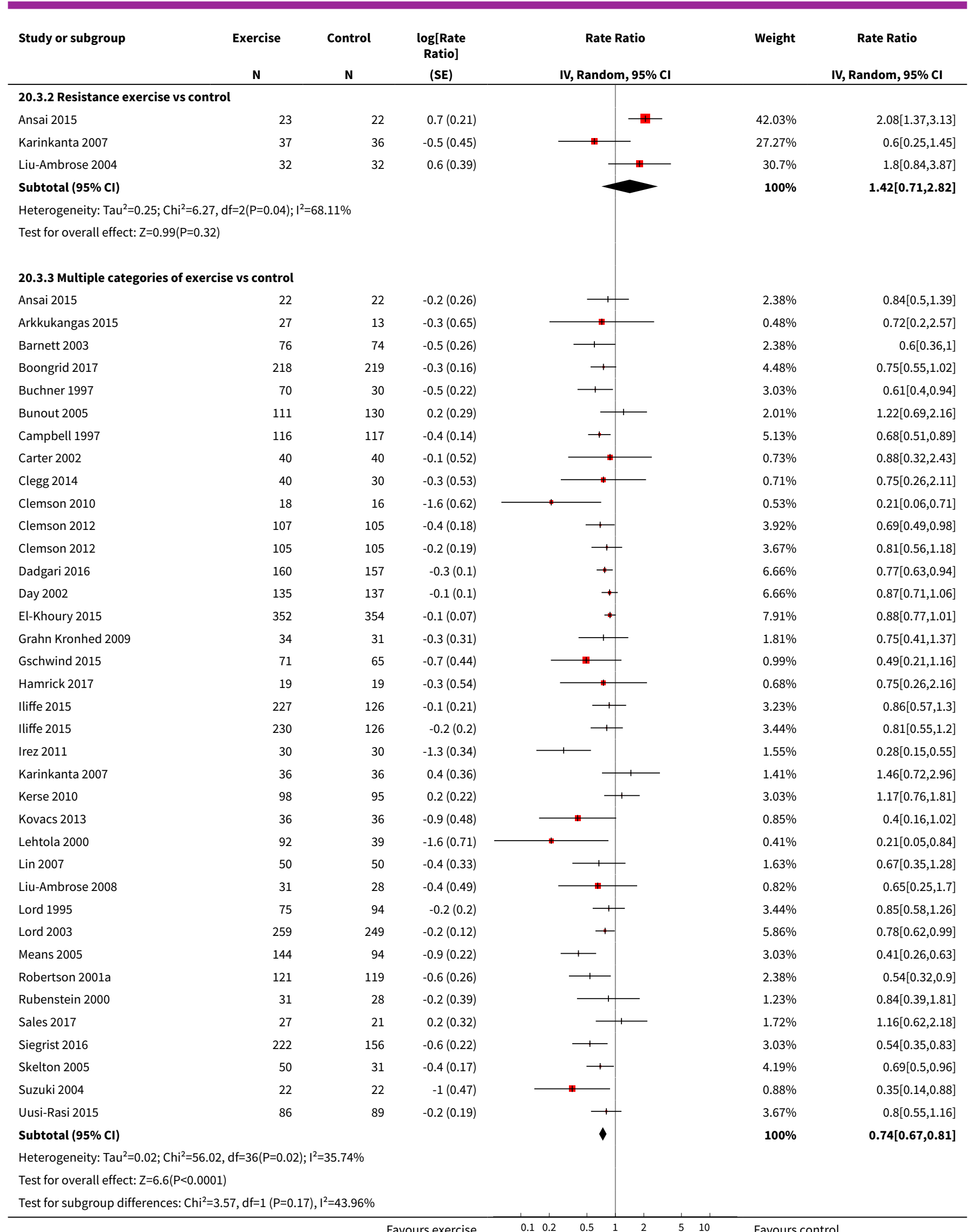


Analysis 20.4. Comparison 20 Sensitivity analysis 9: different exercise type coding, Outcome 4 Number of fallers - subgrouped by exercise type (any balance+strength as multiple intervention).

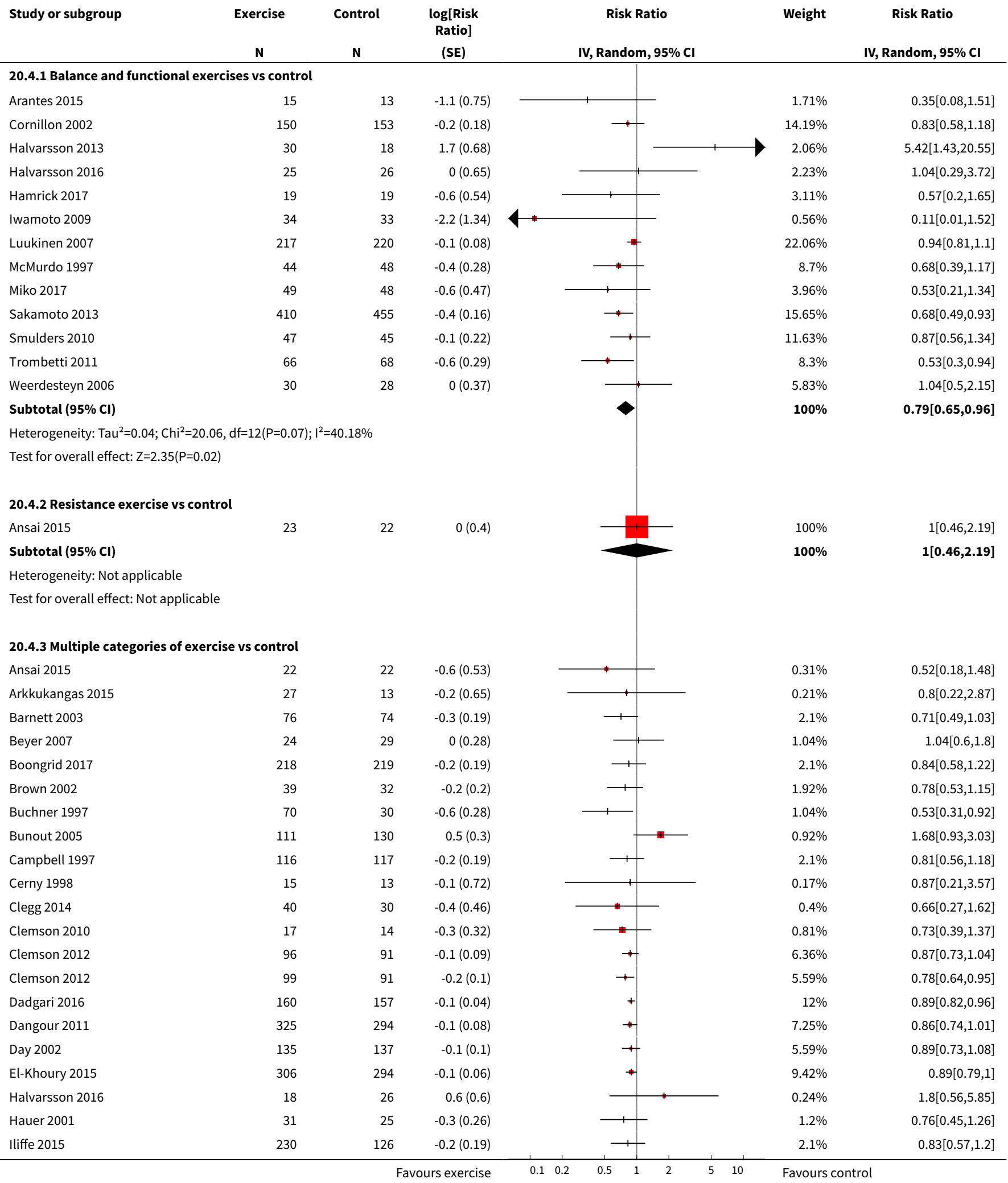




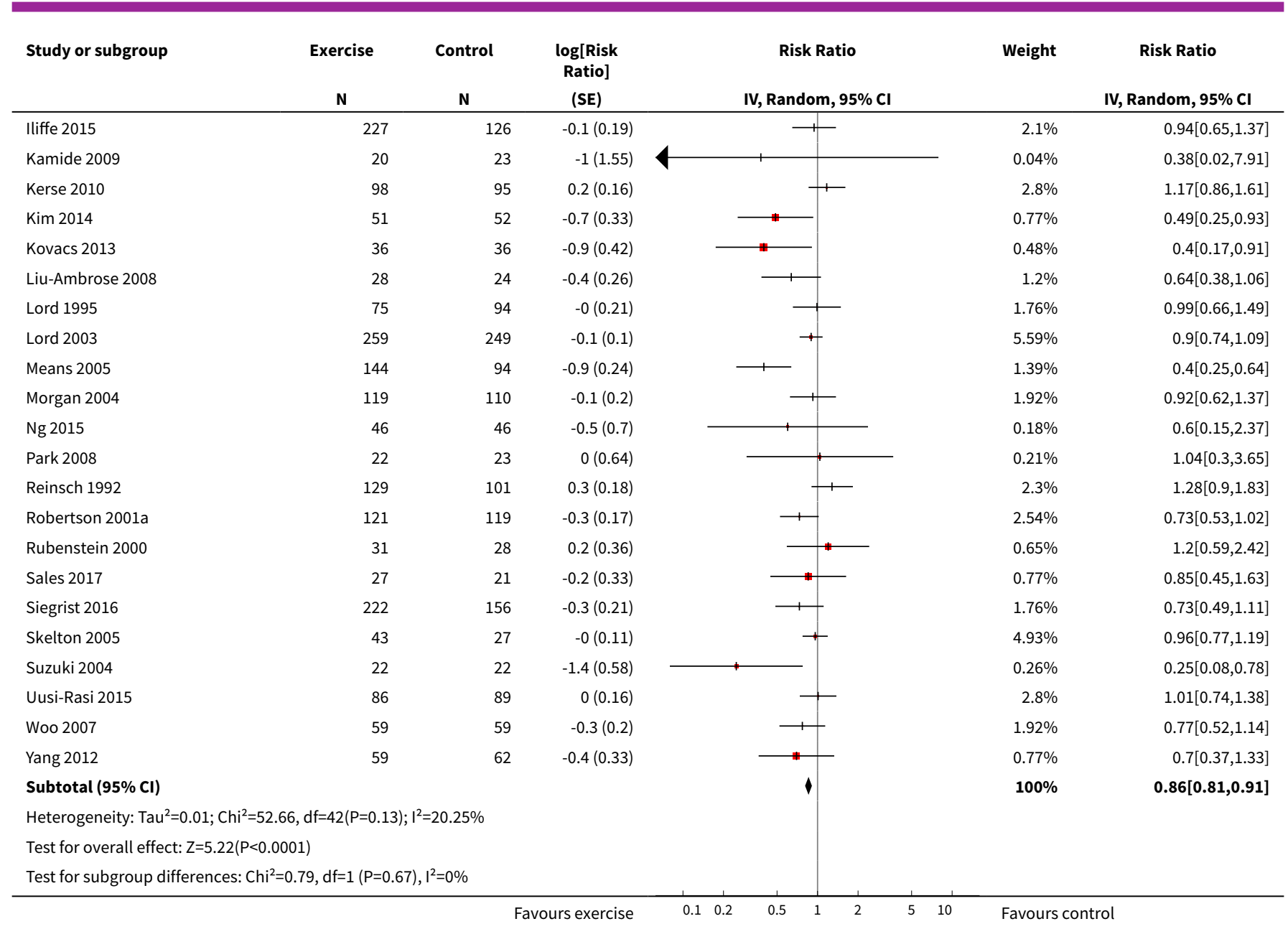




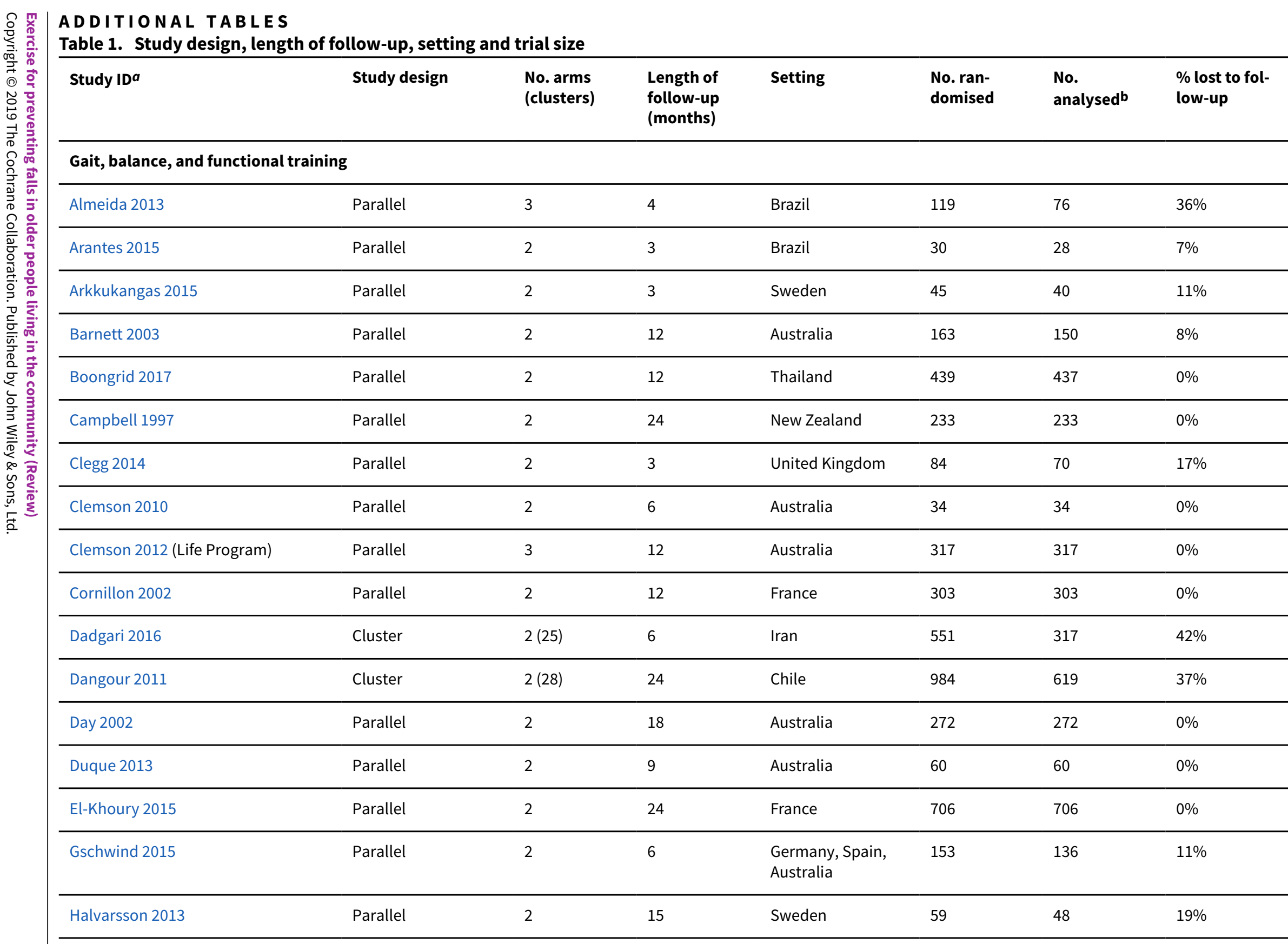

ADDITIONAL TABLES

Table 1. Study design, length of follow-up, setting and trial size

follow-up

o. ran

No.

$\%$ lost to fol-

\section{Gait, balance, and functional training}

Almeida 201

Parallel

Sweden 


\begin{tabular}{|c|c|c|c|c|c|c|c|}
\hline Halvarsson 2016 (balance group) & Parallel & 3 & 3 & Sweden & 96 & 69 & $28 \%$ \\
\hline Hamrick 2017 & Parallel & 2 & 6 & USA & 43 & 38 & $12 \%$ \\
\hline Hirase 2015 & Parallel & 3 & 4 & Japan & 93 & 86 & $8 \%$ \\
\hline Iliffe 2015 (FaME and OEP groups) & Cluster & $3(42)$ & 18 & United Kingdom & 1254 & 709 & $43 \%$ \\
\hline Iwamoto 2009 & Parallel & 2 & 5 & Japan & 68 & 67 & $1 \%$ \\
\hline Karinkanta 2007 (balance group) & Parallel & 4 & 12 & Finland & 149 & 144 & $3 \%$ \\
\hline Kerse 2010 & Parallel & 2 & 12 & New Zealand & 193 & 193 & $0 \%$ \\
\hline Korpelainen 2006 & Parallel & 2 & 30 & Finland & 160 & 160 & $0 \%$ \\
\hline Kovacs 2013 & Parallel & 2 & 12 & Hungary & 76 & 72 & $5 \%$ \\
\hline Lin 2007 & Parallel & 2 & 6 & Taiwan & 100 & 100 & $0 \%$ \\
\hline Liu-Ambrose 2008 & Parallel & 2 & 12 & Canada & 74 & 59 & $30 \%$ \\
\hline Liu-Ambrose 2004 (agility group) & Parallel & 3 & 6 & Canada & 104 & 98 & $6 \%$ \\
\hline Lord 1995 & Parallel & 2 & 12 & Australia & 197 & 169 & $14 \%$ \\
\hline Lord 2003 & Cluster & $2(20)$ & 12 & Australia & 551 & 508 & $8 \%$ \\
\hline Luukinen 2007 & Parallel & 2 & 16 & Finland & 486 & 437 & $10 \%$ \\
\hline Madureira 2007 & Parallel & 2 & 12 & Brazil & 66 & 60 & $9 \%$ \\
\hline McMurdo 1997 & Parallel & 2 & 24 & Scotland & 118 & 92 & $22 \%$ \\
\hline Miko 2017 & Parallel & 2 & 12 & Hungary & 100 & 97 & $3 \%$ \\
\hline Morgan 2004 & Parallel & 2 & 12 & USA & 294 & 229 & $22 \%$ \\
\hline Nitz 2004 & Parallel & 2 & 6 & Australia & 73 & 45 & $38 \%$ \\
\hline Reinsch 1992 & Cluster & $2(16)$ & 12 & USA & 230 & 230 & $0 \%$ \\
\hline
\end{tabular}




\begin{tabular}{|c|c|c|c|c|c|c|c|}
\hline Robertson 2001a & Parallel & 2 & 12 & New Zealand & 240 & 240 & $0 \%$ \\
\hline Sakamoto 2013 & Parallel & 2 & 6 & Japan & 1365 & 865 & $37 \%$ \\
\hline Sales 2017 & Parallel & 2 & 12 & Australia & 66 & 48 & $27 \%$ \\
\hline Siegrist 2016 & Cluster & $2(40)$ & 12 & Germany & 378 & 378 & $0 \%$ \\
\hline Skelton 2005 & Parallel & 2 & 9 & United Kingdom & 81 & 81 & $0 \%$ \\
\hline Smulders 2010 & Parallel & 2 & 12 & Netherlands & 96 & 92 & $4 \%$ \\
\hline Trombetti 2011 & Parallel & 2 & 6 & Switzerland & 134 & 134 & $0 \%$ \\
\hline Weerdesteyn 2006 & Parallel & 2 & 7 & Netherlands & 58 & 58 & $0 \%$ \\
\hline Wolf 1996 (balance group) & Parallel & 3 & 8 & USA & 200 & 200 & $0 \%$ \\
\hline Yang 2012 & Parallel & 2 & 6 & Australia & 165 & 121 & $27 \%$ \\
\hline \multicolumn{8}{|l|}{ Strength/resistance (including power) } \\
\hline Ansai 2015 (resistance group) & Parallel & 3 & 4 & Brazil & 69 & 68 & $1 \%$ \\
\hline Carter 2002 & Parallel & 2 & 5 & Canada & 93 & 80 & $14 \%$ \\
\hline Fiatarone 1997 & Parallel & 2 & 4 & USA & 34 & 0 & $\mathrm{~N} / \mathrm{A}$ \\
\hline Grahn Kronhed 2009 & Parallel & 2 & 12 & Sweden & 65 & 65 & $0 \%$ \\
\hline Karinkanta 2007 (resistance group) & Parallel & 4 & 12 & Finland & 149 & 144 & $3 \%$ \\
\hline Latham $2003 c$ & Parallel & 2 & 6 & $\begin{array}{l}\text { New Zealand and } \\
\text { Australia }\end{array}$ & 243 & 222 & $9 \%$ \\
\hline Liu-Ambrose 2004 (resistance group) & Parallel & 3 & 6 & Canada & 104 & 98 & $6 \%$ \\
\hline Vogler 2009 (seated group)c & Parallel & 3 & 12 & Australia & 180 & 171 & $5 \%$ \\
\hline Woo 2007 (resistance group) & Parallel & 3 & 12 & China & 180 & 176 & $33 \%$ \\
\hline 3D & & & & & & & \\
\hline
\end{tabular}




\begin{tabular}{|c|c|c|c|c|c|c|c|}
\hline Day 2015 & Parallel & 2 & 12 & Australia & 503 & 409 & $19 \%$ \\
\hline Huang 2010 & Cluster & $2(4)$ & 5 & Taiwan & 115 & 78 & $32 \%$ \\
\hline Li 2005 & Parallel & 2 & 6 & USA & 256 & 188 & $27 \%$ \\
\hline Logghe 2009 & Parallel & 2 & 12 & Netherlands & 269 & 269 & $0 \%$ \\
\hline Merom 2016 & Cluster & $2(23)$ & 12 & Australia & 530 & 522 & $2 \%$ \\
\hline Taylor 2012 & Parallel & 2 & 17 & New Zealand & 684 & 684 & $0 \%$ \\
\hline Voukelatos 2007 & Parallel & 2 & 6 & Australia & 702 & 684 & $3 \%$ \\
\hline Wolf 2003 & Cluster & $2(20)$ & 11 & USA & 311 & 286 & $8 \%$ \\
\hline Wolf 1996 (Tai Chi group) & Parallel & 3 & 8 & USA & 200 & 200 & $0 \%$ \\
\hline Woo 2007 (Tai Chi group) & Parallel & 3 & 12 & China & 180 & 176 & $3 \%$ \\
\hline Wu 2010 (com-ex group) & Parallel & 3 & 4 & USA & 64 & 64 & $0 \%$ \\
\hline Wu 2010 (home-ex group) & Parallel & 3 & 4 & USA & 64 & 64 & $0 \%$ \\
\hline Wu 2010 (tel-ex group) & Parallel & 3 & 4 & USA & 64 & 64 & $0 \%$ \\
\hline \multicolumn{8}{|l|}{ General physical activity } \\
\hline Ebrahim 1997 & Parallel & 2 & 24 & United Kingdom & 165 & 102 & $38 \%$ \\
\hline Resnick 2002 & Parallel & 2 & 6 & USA & 20 & 17 & $15 \%$ \\
\hline Voukelatos 2015 & Parallel & 2 & 12 & Australia & 386 & 339 & $12 \%$ \\
\hline \multicolumn{8}{|l|}{ Multiple primary exercise categories } \\
\hline Ansai 2015 (multicomponent group)d $^{d}$ & Parallel & 3 & 4 & Brazil & 69 & 68 & $1 \%$ \\
\hline Beyer 2007d & Parallel & 2 & 12 & Denmark & 65 & 53 & $18 \%$ \\
\hline Brown 2002d & Parallel & 2 & 14 & Australia & 99 & 71 & $28 \%$ \\
\hline
\end{tabular}




\begin{tabular}{|c|c|c|c|c|c|c|c|}
\hline Buchner 1997 & Parallel & 2 & 25 & USA & 105 & 100 & $5 \%$ \\
\hline Bunout 2005d & Parallel & 2 & 12 & Chile & 298 & 241 & $19 \%$ \\
\hline Cerny 1998d & Parallel & 2 & 6 & USA & 28 & 28 & $0 \%$ \\
\hline Clemson 2012 (structured group)d & Parallel & 3 & 12 & Australia & 317 & 317 & $0 \%$ \\
\hline Gill 2016d & Parallel & 2 & 42 & USA & 1635 & 1635 & $0 \%$ \\
\hline Haines 2009c,d & Parallel & 2 & 6 & Australia & 53 & 53 & $0 \%$ \\
\hline $\begin{array}{l}\text { Halvarsson } 2016 \text { (balance and physi- } \\
\text { cal activity group) }\end{array}$ & Parallel & 3 & 3 & Sweden & 96 & 69 & $28 \%$ \\
\hline Hauer $2001^{d}$ & Parallel & 2 & 6 & Germany & 57 & 56 & $2 \%$ \\
\hline Irez 2011d & Parallel & 2 & 3 & Turkey & 60 & 60 & $0 \%$ \\
\hline Kamide 2009d & Parallel & 2 & 6 & Japan & 57 & 43 & $25 \%$ \\
\hline $\begin{array}{l}\text { Karinkanta } 2007 \text { (resistance and bal- } \\
\text { ance groups)d }\end{array}$ & Parallel & 4 & 12 & Finland & 149 & 144 & $3 \%$ \\
\hline Kim 2014d & Parallel & 2 & 12 & Japan & 105 & 103 & $2 \%$ \\
\hline Lehtola 2000 & Parallel & 2 & 10 & Finland & 131 & 131 & $0 \%$ \\
\hline Means 2005d & Parallel & 2 & 6 & USA & 338 & 238 & $30 \%$ \\
\hline $\mathrm{Ng} 2015^{d}$ & Parallel & 2 & 12 & Singapore & 98 & 92 & $6 \%$ \\
\hline Park 2008 & Parallel & 2 & 11 & Korea & 50 & 45 & $10 \%$ \\
\hline Rubenstein 2000 & Parallel & 2 & 3 & USA & 59 & 59 & $0 \%$ \\
\hline Sherrington 2014c,d & Parallel & 2 & 12 & Australia & 340 & 340 & $0 \%$ \\
\hline Suzuki 2004d & Parallel & 2 & 20 & Japan & 52 & 44 & $15 \%$ \\
\hline Uusi-Rasi 2015d & Parallel & 2 & 24 & Finland & 205 & 186 & $9 \%$ \\
\hline
\end{tabular}




\begin{tabular}{|c|c|c|c|c|c|c|c|}
\hline Vogler 2009 (weightbearing group)c & Parallel & 3 & 12 & Australia & 180 & 171 & $5 \%$ \\
\hline \multicolumn{8}{|l|}{ Exercise versus exercise } \\
\hline Ballard 2004 & Parallel & 2 & 16 & USA & 40 & 39 & $3 \%$ \\
\hline Barker 2016 & Parallel & 2 & 6 & Australia & 53 & 44 & $17 \%$ \\
\hline Clemson 2012 & Parallel & 3 & 12 & Australia & 317 & 286 & $10 \%$ \\
\hline Davis 2011 & Parallel & 3 & 9 & Canada & 155 & 155 & $0 \%$ \\
\hline Freiberger 2007 & Parallel & 2 & 24 & Germany & 134 & 127 & $5 \%$ \\
\hline Helbostad 2004 & Parallel & 2 & 12 & Norway & 77 & 68 & $12 \%$ \\
\hline Hwang 2016 & Parallel & 2 & 18 & Taiwan & 456 & 334 & $27 \%$ \\
\hline Iliffe 2015 & Cluster & $3(42)$ & 18 & United Kingdom & 1254 & 709 & $43 \%$ \\
\hline Karinkanta 2007 & Parallel & 4 & 12 & Finland & 149 & 144 & $3 \%$ \\
\hline Kemmler 2010 & Parallel & 2 & 18 & Germany & 246 & 227 & $8 \%$ \\
\hline Kwok 2016 & Parallel & 2 & 12 & Singapore & 80 & 80 & $0 \%$ \\
\hline Kyrdalen 2014 & Parallel & 2 & 3 & Norway & 125 & 94 & $25 \%$ \\
\hline LaStayo 2017 & Parallel & 2 & 12 & USA & 134 & 112 & $16 \%$ \\
\hline Liston 2014 & Parallel & 2 & 6 & United Kingdom & 21 & 15 & $29 \%$ \\
\hline Liu-Ambrose 2004 & Parallel & 3 & 6 & Canada & 104 & 98 & $6 \%$ \\
\hline Lurie 2013 & Parallel & 2 & 3 & USA & 64 & 59 & $8 \%$ \\
\hline Mirelman 2016 & Parallel & 2 & N/A & $\begin{array}{l}\text { Belgium, Israel, } \\
\text { Italy, Netherlands, } \\
\text { and United King- } \\
\text { dom }\end{array}$ & 152 & 0 & $\mathrm{~N} / \mathrm{A}$ \\
\hline Morone 2016 & Parallel & 2 & 3 & Italy & 38 & 0 & $\mathrm{~N} / \mathrm{A}$ \\
\hline
\end{tabular}




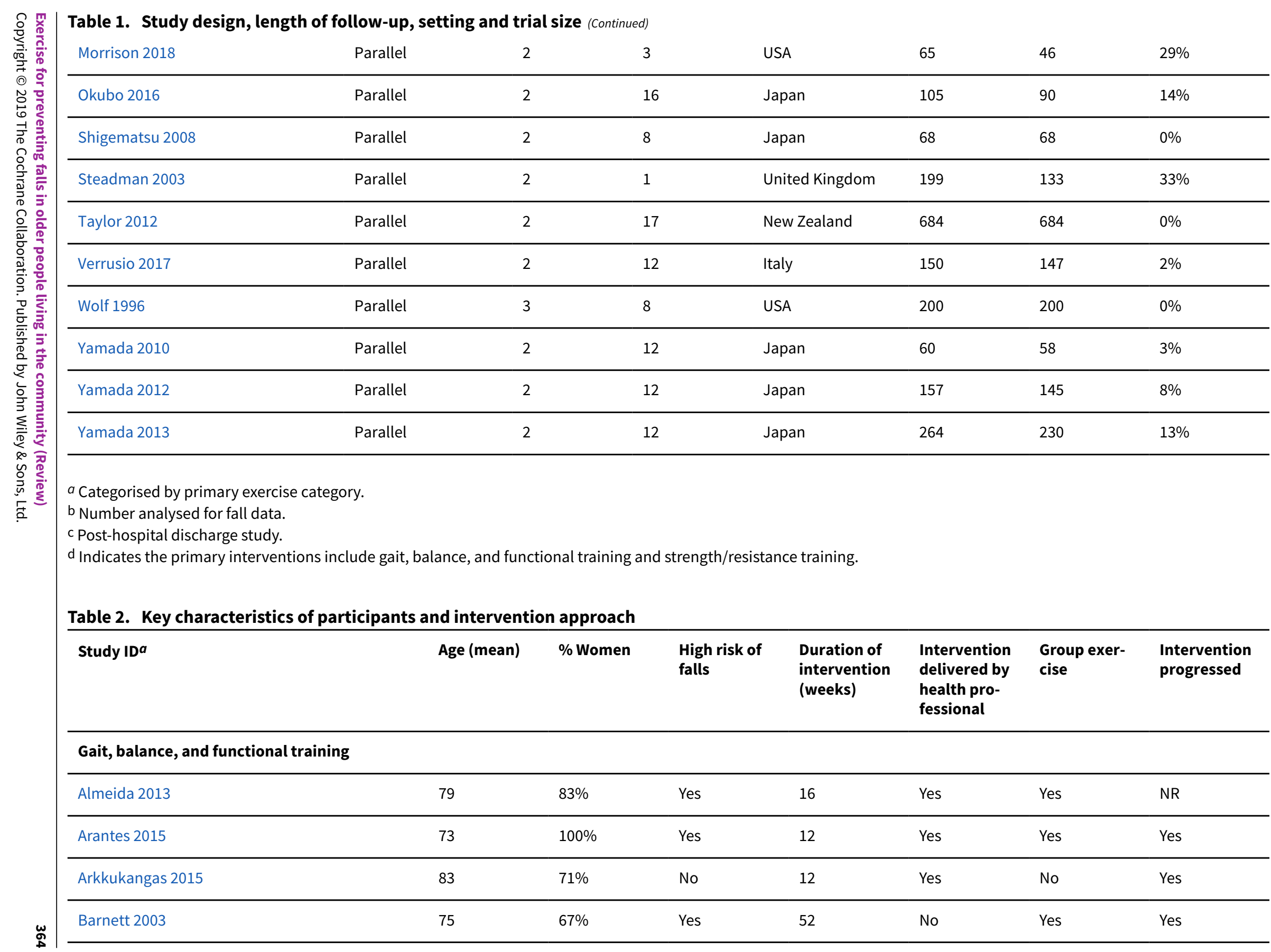




\begin{tabular}{|c|c|c|c|c|c|c|c|}
\hline Boongrid 2017 & 74 & $83 \%$ & Yes & 52 & Yes & No & Yes \\
\hline Campbell 1997 & 84 & $100 \%$ & Yes & 52 & Yes & No & Yes \\
\hline Clegg 2014 & 79 & $71 \%$ & Yes & 12 & Yes & No & Yes \\
\hline Clemson 2010 & 81 & $47 \%$ & Yes & 26 & Yes & No & Yes \\
\hline Clemson 2012 (Life Program) & 83 & $55 \%$ & Yes & 52 & Yes & No & Yes \\
\hline Cornillon 2002 & 71 & $83 \%$ & No & 52 & No & No & No \\
\hline Dadgari 2016 & 70 & $49 \%$ & Yes & 24 & No & No & Yes \\
\hline Dangour 2011 & 66 & $68 \%$ & No & 104 & No & Yes & Yes \\
\hline Day 2002 & 76 & $59 \%$ & No & 18 & No & Yes & No \\
\hline Duque 2013 & 77 & $62 \%$ & Yes & 6 & Yes & No & Yes \\
\hline El-Khoury 2015 & 79 & $100 \%$ & Yes & 104 & No & Yes & Yes \\
\hline Gschwind 2015 & 75 & $61 \%$ & No & 16 & No & No & Yes \\
\hline Halvarsson 2013 & 77 & $71 \%$ & Yes & 12 & Yes & Yes & Yes \\
\hline Halvarsson 2016 (balance group) & 76 & $98 \%$ & Yes & 12 & Yes & Yes & Yes \\
\hline Hamrick 2017 & 70 & $79 \%$ & No & 8 & No & Yes & Yes \\
\hline Hirase 2015 & 82 & $70 \%$ & Yes & 16 & Yes & No & No \\
\hline Iliffe 2015 & 73 & $62 \%$ & No & 24 & No & $\begin{array}{l}\text { OEP: no; } \\
\text { FaME: Yes }\end{array}$ & Yes \\
\hline Iwamoto 2009 & 76 & $90 \%$ & No & 20 & No & Yes & No \\
\hline Karinkanta 2007 (balance group) & 73 & $100 \%$ & No & 52 & No & Yes & No \\
\hline Kerse 2010 & 81 & $58 \%$ & No & 26 & No & No & Yes \\
\hline Korpelainen 2006 & 73 & $100 \%$ & No & 130 & Yes & Yes & Yes \\
\hline
\end{tabular}




\begin{tabular}{|c|c|c|c|c|c|c|c|}
\hline Kovacs 2013 & 69 & $100 \%$ & No & 25 & Yes & Yes & Yes \\
\hline Lin 2007 & 77 & $51 \%$ & Yes & 16 & Yes & No & Yes \\
\hline Liu-Ambrose 2004 (agility group) & 79 & $100 \%$ & No & 25 & No & Yes & No \\
\hline Liu-Ambrose 2008 & 83 & $71 \%$ & Yes & 26 & Yes & No & Yes \\
\hline Lord 1995 & 71 & $100 \%$ & No & 52 & No & Yes & No \\
\hline Lord 2003 & 80 & $86 \%$ & No & 52 & No & Yes & No \\
\hline Luukinen 2007 & 88 & $79 \%$ & Yes & 70 & Yes & No & Yes \\
\hline Madureira 2007 & 74 & $100 \%$ & No & 40 & Yes & Yes & No \\
\hline McMurdo 1997 & 65 & $100 \%$ & No & 60 & No & Yes & No \\
\hline Miko 2017 & 69 & $100 \%$ & No & 52 & Yes & Yes & Yes \\
\hline Morgan 2004 & 81 & $71 \%$ & Yes & 8 & Yes & Yes & Yes \\
\hline Nitz 2004 & 76 & $92 \%$ & Yes & 10 & Yes & Yes & No \\
\hline Reinsch 1992 & 74 & $80 \%$ & No & 52 & No & Yes & No \\
\hline Robertson 2001a & 84 & $68 \%$ & No & 52 & Yes & No & Yes \\
\hline Sakamoto 2013 & 80 & $82 \%$ & Yes & 26 & No & No & Yes \\
\hline Sales 2017 & 73 & $69 \%$ & Yes & 18 & Both & Yes & Yes \\
\hline Siegrist 2016 & 78 & $75 \%$ & Yes & 16 & Yes & Yes & Yes \\
\hline Skelton 2005 & 72 & $100 \%$ & Yes & 36 & No & Yes & Yes \\
\hline Smulders 2010 & 71 & $94 \%$ & Yes & 5.5 & Yes & Yes & Yes \\
\hline Trombetti 2011 & 76 & $96 \%$ & Yes & 26 & No & Yes & Yes \\
\hline Weerdesteyn 2006 & 74 & $77 \%$ & Yes & 5 & No & Yes & Yes \\
\hline
\end{tabular}




\begin{tabular}{|c|c|c|c|c|c|c|c|}
\hline Wolf 1996 (balance group) & 76 & $81 \%$ & No & 15 & No & No & Yes \\
\hline Yang 2012 & 81 & $44 \%$ & Yes & 26 & Yes & No & No \\
\hline \multicolumn{8}{|l|}{ Strength/resistance (including power) } \\
\hline Ansai 2015 (resistance group) & 82 & $68 \%$ & Yes & 16 & No & Yes & Yes \\
\hline Carter 2002 & 69 & $100 \%$ & No & 20 & No & Yes & No \\
\hline Fiatarone 1997 & 82 & $94 \%$ & Yes & 16 & No & No & No \\
\hline Grahn Kronhed 2009 & 71 & $100 \%$ & No & 16 & Yes & Yes & Yes \\
\hline Karinkanta 2007 (resistance group) & 73 & $100 \%$ & No & 52 & No & Yes & Yes \\
\hline Latham 2003b & 80 & $53 \%$ & Yes & 10 & Yes & No & Yes \\
\hline Liu-Ambrose 2004 (resistance group) & 79 & $100 \%$ & No & 25 & No & Yes & Yes \\
\hline Vogler 2009 (seated group) & 80 & $79 \%$ & Yes & 12 & Yes & No & Yes \\
\hline Woo 2007 (resistance group) & 69 & $50 \%$ & No & 52 & No & Yes & No \\
\hline \multicolumn{8}{|l|}{ 3D } \\
\hline Day 2015 & 77 & $70 \%$ & Yes & 48 & No & Yes & Yes \\
\hline Huang 2010 & 71 & $30 \%$ & No & 22 & No & Yes & No \\
\hline Li 2005 & 77 & $70 \%$ & No & 26 & No & Yes & No \\
\hline Logghe 2009 & 77 & $71 \%$ & Yes & 13 & No & Yes & No \\
\hline Merom 2016 & & $85 \%$ & No & 52 & No & Yes & Yes \\
\hline Taylor 2012 & 75 & $73 \%$ & Yes & 20 & No & Yes & No \\
\hline Voukelatos 2007 & 69 & $84 \%$ & No & 16 & No & Yes & No \\
\hline Wolf 1996 (Tai Chi group) & 76 & $81 \%$ & No & 15 & No & Yes & Yes \\
\hline
\end{tabular}




\begin{tabular}{|c|c|c|c|c|c|c|c|}
\hline Wolf 2003 & 81 & $94 \%$ & Yes & 48 & No & Yes & Yes \\
\hline Woo 2007 (Tai Chi group) & 69 & $50 \%$ & No & 52 & No & Yes & No \\
\hline Wu 2010 (com-ex group) & 75 & $84 \%$ & Yes & 15 & No & Yes & No \\
\hline Wu 2010 (home-ex group) & 75 & $84 \%$ & Yes & 15 & No & No & No \\
\hline Wu 2010 (tel-ex group) & 75 & $84 \%$ & Yes & 15 & No & No & No \\
\hline \multicolumn{8}{|l|}{ General physical activity } \\
\hline Ebrahim 1997 & 67 & $100 \%$ & No & 104 & Yes & No & Yes \\
\hline Resnick 2002 & 88 & $100 \%$ & No & 26 & No & Yes & Yes \\
\hline Voukelatos 2015 & 73 & $74 \%$ & No & 48 & No & No & No \\
\hline \multicolumn{8}{|l|}{ Multiple primary exercise categories } \\
\hline Ansai 2015 (multicomponent group)c & 82 & $68 \%$ & Yes & 16 & No & Yes & Yes \\
\hline Beyer 2007c & 78 & $100 \%$ & Yes & 26 & Yes & Yes & Yes \\
\hline Brown 2002c & & $79 \%$ & No & 16 & Yes & Yes & Yes \\
\hline Buchner 1997 & 75 & $51 \%$ & Yes & 25 & No & Yes & Yes \\
\hline Bunout 2005c & 75 & $70 \%$ & No & 52 & No & Yes & Yes \\
\hline Cerny $1998 \mathrm{C}$ & 71 & & No & 24 & No & Yes & NR \\
\hline Clemson 2012 (structured group)c & 83 & $55 \%$ & Yes & 52 & Yes & No & Yes \\
\hline Gill 2016c & 79 & $67 \%$ & Yes & 96 & No & Yes & Yes \\
\hline Haines 2009b,c & 81 & $60 \%$ & Yes & 8 & Yes & No & Yes \\
\hline $\begin{array}{l}\text { Halvarsson } 2016 \text { (balance and physical activity } \\
\text { group) }\end{array}$ & 76 & $98 \%$ & Yes & 12 & Yes & Yes & Yes \\
\hline
\end{tabular}




\begin{tabular}{|c|c|c|c|c|c|c|c|}
\hline Hauer $2001 \mathrm{c}$ & 82 & $100 \%$ & Yes & 12 & Yes & Yes & Yes \\
\hline Irez $2011^{c}$ & 75 & $100 \%$ & No & 12 & No & Yes & Yes \\
\hline Kamide 2009 c & 71 & $100 \%$ & No & 26 & Yes & No & No \\
\hline $\begin{array}{l}\text { Karinkanta } 2007 \text { (resistance and balance } \\
\text { groups)c }\end{array}$ & 73 & $100 \%$ & No & 52 & No & Yes & Yes \\
\hline Kim 2014c & 78 & $100 \%$ & Yes & 52 & No & Yes & Yes \\
\hline Lehtola 2000 & 74 & $80 \%$ & No & 26 & No & Yes & Yes \\
\hline Means $2005^{c}$ & 74 & $57 \%$ & No & 6 & Yes & Yes & Yes \\
\hline $\mathrm{Ng} 2015 \mathrm{C}$ & 70 & $61 \%$ & Yes & 12 & No & Yes & Yes \\
\hline Park 2008 & 68 & $100 \%$ & No & 48 & $N R$ & Yes & No \\
\hline Rubenstein 2000 & 75 & $0 \%$ & Yes & 12 & No & Yes & Yes \\
\hline Sherrington 2014b,c & 81 & $74 \%$ & Yes & 52 & Yes & No & Yes \\
\hline Suzuki 2004c & 78 & $100 \%$ & No & 26 & No & Yes & No \\
\hline Uusi-Rasi 2015c & 74 & $100 \%$ & Yes & 104 & Yes & Yes & Yes \\
\hline Vogler 2009b (weightbearing group) & 80 & $79 \%$ & Yes & 12 & Yes & No & Yes \\
\hline \multicolumn{8}{|l|}{ Exercise versus exercise } \\
\hline Ballard 2004 & 73 & $100 \%$ & Yes & $\begin{array}{l}15 \text { (Low inten- } \\
\text { sity = 2) }\end{array}$ & No & Yes & NR \\
\hline Barker 2016 & 69 & $88 \%$ & Yes & 12 & Yes & $\begin{array}{l}\text { Pilates group: } \\
\text { Yes; HEP } \\
\text { group: No }\end{array}$ & Yes \\
\hline Clemson 2012 & 83 & $55 \%$ & Yes & 52 & Yes & No & Yes \\
\hline Davis 2011 & 78 & $100 \%$ & No & 52 & No & Yes & No \\
\hline
\end{tabular}




\begin{tabular}{|c|c|c|c|c|c|c|c|}
\hline Freiberger 2007 & 76 & $44 \%$ & Yes & 16 & No & No & Yes \\
\hline Helbostad 2004 & 81 & $81 \%$ & Yes & & Yes & Yes & $\begin{array}{l}\text { Combined } \\
\text { training:No; } \\
\text { Home train- } \\
\text { ing: Yes. }\end{array}$ \\
\hline Hirase 2015 & 82 & $70 \%$ & Yes & 16 & Yes & No & No \\
\hline Hwang 2016 & 72 & $67 \%$ & Yes & 24 & $\begin{array}{l}\text { Tai Chi: No; } \\
\text { other group: } \\
\text { Yes }\end{array}$ & No & Yes \\
\hline Karinkanta 2007 & 73 & $100 \%$ & No & 52 & No & Yes & Yes \\
\hline Kemmler 2010 & 69 & $100 \%$ & No & 78 & No & Yes & $\begin{array}{l}\text { High intensi- } \\
\text { ty: Yes; low in- } \\
\text { tensity: No }\end{array}$ \\
\hline Kwok 2016 & 70 & $85 \%$ & Yes & 12 & Yes & Yes & Yes \\
\hline Kyrdalen 2014 & 83 & $73 \%$ & Yes & 12 & Yes & $\begin{array}{l}\text { Group: Yes; } \\
\text { Home: No }\end{array}$ & Yes \\
\hline LaStayo 2017 & 76 & $65 \%$ & Yes & 12 & Yes & Yes & Yes \\
\hline Liston 2014 & 77 & $85 \%$ & Yes & 8 & Yes & Yes & $\begin{array}{l}\text { OEP: Yes; } \\
\text { Stretching: } \\
\text { No. }\end{array}$ \\
\hline Liu-Ambrose 2004 & 79 & $100 \%$ & No & 25 & No & Yes & Yes \\
\hline Lurie 2013 & 80 & $59 \%$ & Yes & Variable & Yes & No & Yes \\
\hline Mirelman 2016 & 83 & $35 \%$ & Yes & 6 & No & No & Yes \\
\hline Morone 2016 & 69 & $100 \%$ & Yes & 8 & Yes & Yes & No \\
\hline Morrison 2018 & 67 & $48 \%$ & No & 12 & No & $\begin{array}{l}\text { Balance: Yes; } \\
\text { Wii: No }\end{array}$ & $\begin{array}{l}\text { Balance: No; } \\
\text { Wii: Yes }\end{array}$ \\
\hline Okubo 2016 & 71 & $63 \%$ & No & 64 & No & Yes & Yes \\
\hline
\end{tabular}




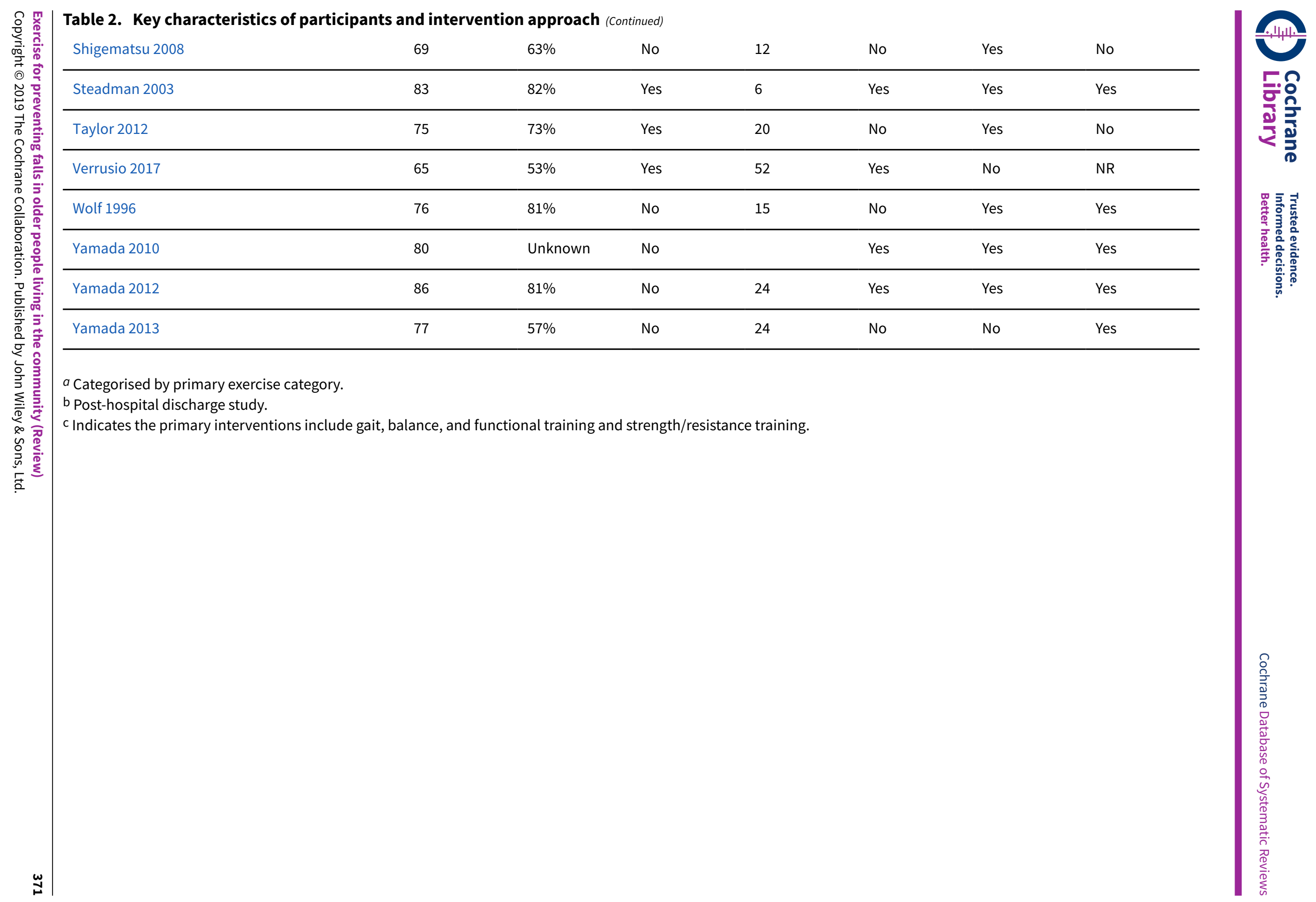


Table 3. Numbers of studies and participants included in the exercise versus control comparison for each primary exercise category

\begin{tabular}{|c|c|c|c|c|c|}
\hline Comparisona & $\begin{array}{l}\text { Number of } \\
\text { trials (clus- } \\
\text { ter) b }\end{array}$ & $\begin{array}{l}\text { Number of } \\
\text { participants } \\
\text { randomised }\end{array}$ & $\begin{array}{l}\text { Number of } \\
\text { participants } \\
\text { analysed for } \\
\text { any one out- } \\
\text { come }\end{array}$ & $\begin{array}{l}\text { Number of } \\
\text { trials (clus- } \\
\text { ter) with par- } \\
\text { ticipants } \\
\text { analysed for } \\
\text { rate of falls } \\
\text { outcome c,d }\end{array}$ & $\begin{array}{l}\text { Number of } \\
\text { participants } \\
\text { analysed for } \\
\text { rate of falls } \\
\text { outcome d }\end{array}$ \\
\hline Exercise (all types) versus control & $81(9)$ & 19684 & 13518 & $59(6)$ & 12,981 \\
\hline $\begin{array}{l}\text { Balance and functional exercises versus } \\
\text { control }\end{array}$ & $48(6)$ & 11860 & 8288 & $39(4)$ & 7920 \\
\hline Resistance exercises versus control & 7 & 694 & 327 & 5 & 327 \\
\hline Flexibility versus control & 0 & 0 & 0 & 0 & 0 \\
\hline 3D exercise (Tai Chi) versus control & $10(2)$ & 3284 & 2677 & $7(1)$ & 2655 \\
\hline 3D exercise (dance) versus control & $1(1)$ & 530 & 522 & $1(1)$ & 522 \\
\hline $\begin{array}{l}\text { General physical activity (walking pro- } \\
\text { gramme) versus control }\end{array}$ & 3 & 571 & 441 & 2 & 441 \\
\hline Endurance training versus control & 0 & 0 & 0 & 0 & 0 \\
\hline Other kinds of exercise versus control & 0 & 0 & 0 & 0 & 0 \\
\hline $\begin{array}{l}\text { Multiple categories of exercise versus } \\
\text { control }\end{array}$ & 21 & 4073 & 1623 & 11 & 1374 \\
\hline
\end{tabular}

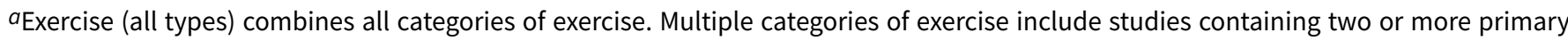
categories of exercise, as categorised using the ProFaNE taxonomy. The remaining analyses include only one primary category of exercise, as categorised using the ProFaNE taxonomy.

bStudy IDs are shown in Appendix 6.

cStudy IDs are shown in Appendix 7.

dThese data apply to the follow-up (at the time point included in main analysis) for the primary outcome (rate of falls) for the individual trials.

\section{AP PEN DICES}

Appendix 1. Categories of exercise (ProFaNE): definitions and application

\begin{tabular}{lll}
\hline Exercise category & ProFaNE description & $\begin{array}{l}\text { How the category criteria were ap- } \\
\text { plied in this reviewa }\end{array}$ \\
\hline $\begin{array}{lll}\text { Gait, balance, and func- } \\
\text { tional training }\end{array}$ & $\begin{array}{l}\text { Gait training involves specific correction of walking technique } \\
\text { (e.g. posture, stride length and cadence) and changes of pace, }, \\
\text { level and direction. Balance training involves the efficient } \\
\text { transfer of bodyweight from one part of the body to anoth- } \\
\text { er or challenges specific aspects of the balance systems (e.g. }\end{array}$ & $\begin{array}{l}\text { Selectention met the baseline assess- } \\
\text { tervent, tailoring and progression crite- } \\
\text { ria. Selected as primary category for in- } \\
\text { terventions where most exercises were }\end{array}$
\end{tabular}


(Continued)

vestibular systems). Balance retraining activities range from the re-education of basic functional movement patterns to a wide variety of dynamic activities that target more sophisticated aspects of balance. Functional training uses functional activities as the training stimulus, and is based on the theoretical concept of task specificity. All gait, balance and functional training should be based on an assessment of the participant's abilities prior to starting the programme; tailoring of the intervention to the individuals abilities; and progression of the exercise programme as ability improves
Strength/resistance (including power)
The term 'resistance training' covers all types of weight training i.e. contracting the muscles against a resistance to 'overload' and bring about a training effect in the muscular system. The resistance is an external force, which can be one's own body placed in an unusual relationship to gravity (e.g. prone back extension) or an external resistance (e.g. free weight). All strength/resistance training should be based on an assessment of the participant's abilities prior to starting the programme; tailoring the intervention to the individual's abilities; and progression of the exercise programme as ability improves
Flexibility training is the planned process by which stretching exercises are practised and progressed to restore or maintain the optimal range of movement (ROM) available to a joint or joints. The ranges of motion used by flexibility programmes may vary from restoration/maintenance of the entire physiological range of motion, or alternatively, maintenance of range that is essential to mobility or other functions conducted standing and where the intervention focus and most time spent was on exercise in this category
Selected as exercise category if the intervention met the baseline assessment, tailoring and progression criteria. Selected as primary category for interventions where additional resistance was used or where it was clear that overload was sufficient without external resistance and where the intervention focus and most time spent was on exercise in this category
Selected as exercise category if the intervention met the progression of stretching criterion. Selected as primary category for interventions where flexibility training was a stated aim of the intervention and where the intervention focus and most time spent was on exercise in this category

\section{$3 \mathrm{D}$}

3D training involves constant movement in a controlled, fluid, repetitive way through all three spatial planes or dimensions (forward and back, side to side, and up and down). Tai Chi and Qi Gong incorporate specific weight transferences and require upright posture and subtle changes of head position and gaze direction. Dance involves a wide range of dynamic movement qualities, speeds and patterns
Selected as exercise category if the intervention involved Tai Chi or dance. Selected as primary category for interventions where the intervention focus and most time spent was on exercise in this category

Selected as exercise category if the intervention included unstructured physical activity. We classed programmes that included unstructured walking as this category. Selected as primary category for interventions where the intervention focus and most time spent was on exercise in this category
Physical activity is any bodily movement produced by skeleenergy expenditure. Physical activity has both occupational, transportation and recreational components and includes pursuits like golf, tennis, and swimming. It also includes other active pastimes like gardening, cutting wood, and carpentry. Physical activity can provide progressive health benefits and is a catalyst for improving health attitudes, health habits, and lifestyle. Increasing habitual physical activity should be with specific recommendations as to duration, frequency and intensity if a physical or mental health improvement is indicated
Endurance Endurance training is aimed at cardiovascular conditioning and is aerobic in nature and simultaneously increases the heart rate and the return of blood to the heart
Selected as exercise category if the intervention focused on structured aerobic training. We classed programmes that included treadmill walking as this category. Selected as primary category for interventions where the intervention 

cise in this category

Other

Other kinds of exercises not described

Selected as exercise category if the intervention did not meet the other categories listed and where the intervention focus and most time spent was on exercise in this category

alnterventions were allocated a secondary category if some but not all criteria were met by the intervention or where the category was not the primary focus of the intervention, or both

\section{Appendix 2. Search strategies (February 2012 to 2 May 2018) \\ CENTRAL (CRS Online)}

\#1 MESH DESCRIPTOR Accidental Falls EXPLODE ALL TREES

\#2 (falls or faller $\left.{ }^{\star}\right): T I, A B, K Y$

\#3 \#1 or \#2

\#4 MESH DESCRIPTOR Aged EXPLODE ALL TREES

\#5 (senior ${ }^{\star}$ or elder $^{\star}$ or old ${ }^{*}$ or aged or ag?ing or postmenopausal or community dwelling):TI,AB,KY

\#6 \#4 or \#5

$\# 7$ \#3 and \#6

\section{MEDLINE (Ovid Interface)}

1 Accidental Falls/

2 (falls or faller ${ }^{\star} 1$ ).tw.

3 or $1-2$

4 exp Aged/

5 (senior ${ }^{\star} 1$ or elder $^{\star}$ or old $^{\star}$ or aged or ag?ing or postmenopausal or community dwelling).tw.

6 or $/ 4-5$

73 and 6

8 Randomized controlled trial.pt.

9 Controlled clinical trial.pt.

10 randomized.ab.

11 placebo.ab.

12 Clinical trials as topic/

13 randomly.ab.

14 trial.ti.

158 or 9 or 10 or 11 or 12 or 13 or 14

16 exp Animals/ not Humans/

1715 not 16

187 and 17

\section{Embase (Ovid Interface)}

1 Falling/

2 (falls or fallers).tw.

3 or $/ 1-2$

4 exp Aged/

5 (senior ${ }^{\star} 1$ or elder ${ }^{\star}$ or old ${ }^{\star}$ or aged or ag?ing or postmenopausal or community dwelling).tw.

6 or/4-5

73 and 6

8 exp Randomized Controlled Trial/ or exp Single Blind Procedure/ or exp Double Blind Procedure/ or Crossover Procedure/

9 (random* or RCT or placebo or allocat ${ }^{\star}$ or crossover ${ }^{\star}$ or $^{\prime}$ cross over' or trial or (doubl* adj1 blind $^{\star}$ ) or (singl ${ }^{\star}$ adj1 blind $\left.{ }^{\star}\right)$ ).ti,ab.

108 or 9

11 (exp Animal/ or animal.hw. or Nonhuman/) not (exp Human/ or Human cell/ or (human or humans).ti.)

1210 not 11

137 and 12 


\section{CINAHL (Ebsco)}

S1 (MH "Accidental Falls")

S2 TI ( falls or faller*) OR AB ( falls or faller ${ }^{\star}$ )

S3 S1 OR S2

S4 (MH "Aged+")

S5 TI ( senior ${ }^{\star}$ or elder ${ }^{\star}$ or old* or aged or ag?ing or postmenopausal or community dwelling ) OR AB ( senior $^{\star}$ or elder $^{\star}$ or old ${ }^{\star}$ or aged or ag?ing or postmenopausal or community dwelling )

S6 S4 OR S5

S7 S3 AND S6

S8 PT Clinical Trial

S9 (MH "Clinical Trials+")

S10 TI clinical trial* OR AB clinical trial*

S11 TI ( (single blind ${ }^{\star}$ or double blind*) ) OR AB ( (single blind* or double blind $\left.{ }^{\star}\right)$ )

S12 TI random* OR AB random*

S13 S8 OR S9 OR S10 OR S11 OR S12

S14 S7 AND S13

\section{PEDro}

Advanced search option selected

Abstract and Title: fall*

Method: clinical trial

Sub discipline: gerontology

New record added since: (date of last review entered here)

\section{ClinicalTrials.gov}

(prevent OR reduce OR reduction OR risk) AND (fall OR fallers) AND (exercise OR training)

\section{WHO ICTRP}

prevent ${ }^{\star}$ AND fall ${ }^{\star}$ AND exercise ${ }^{\star}$ OR reduc ${ }^{\star}$ AND fall ${ }^{\star}$ AND exercise ${ }^{\star}$ OR risk ${ }^{\star}$ AND fall ${ }^{\star}$ AND exercise ${ }^{\star}$ OR prevent ${ }^{\star}$ AND fall ${ }^{\star}$ AND train ${ }^{\star}$ OR reduc ${ }^{\star}$ AND fall* AND train* OR risk* AND fall* AND exercise*

\section{Appendix 3. 'Risk of bias' assessment tool}

\section{Domain Criteria for judging risk of bias}

Random sequence generation relating to selection bias (biased allocation to interventions) due to inadequate generation of a randomised sequence
- Judgement of 'low risk' if the trial authors described a random component in the sequence generation, e.g. referring to a random-number table; using a computer random-number generator; coin-tossing; shuffling cards or envelopes; throwing dice; drawing of lots; minimisation

- Judgement of 'high risk' if the trial used a systematic non-random method, e.g. date of admission; odd or even date of birth; case record number; clinician judgement; participant preference; patient risk factor score or test results; availability of intervention

- Judgement of 'unclear risk' if there is insufficient information about the sequence generation process to permit judgement of 'low risk' or 'high risk'.

\begin{abstract}
Allocation concealment relating to selection bias (biased allocation to interventions) due to inadequate concealment of allocations prior to assignment
\end{abstract}

- Judgement of 'low risk' in studies using:

- individual randomisation if the trial described allocation concealment as by central allocation (telephone, internet-based or pharmacy-controlled randomisation); sequentially-numbered identical drug containers; sequentially-numbered, opaque, sealed envelopes

- cluster-randomisation if allocation of all cluster units performed at the start of the study and individual participant recruitment was completed prior to assignment of the cluster, and the same participants were followed up over time or individual participants were recruited after cluster assignment, but recruitment carried out by a person unaware of group allocation and participant characteristics (e.g. fall history) or individual participants in intervention and control arms were invited by mail questionnaire with identical information

- Judgement of 'high risk' in studies using: 
- individual randomisation if investigators enrolling participants could possibly foresee assignments and thus introduce selection bias, e.g. using an open random allocation schedule (e.g. a list of random numbers); assignment envelopes unsealed, non-opaque, or not sequentially-numbered; alternation or rotation; date of birth; case record number; or any other explicitly unconcealed procedure

- cluster-randomisation if individual participant recruitment was undertaken after group allocation by a person who was unblinded and may have had knowledge of participant characteristics

- Judgement of 'unclear risk' if insufficient information to permit judgement of 'low risk' or 'high risk'. This is usually the case if the method of concealment is not described or not described in sufficient detail to allow a definite judgement, e.g. if the use of assignment envelopes is described, but it remains unclear whether envelopes were sequentially numbered, opaque and sealed

Blinding of participants and personnel relating to performance bias due to knowledge of the allocated interventions by participants and personnel carrying out the interventions
- Judgement of 'low risk' if blinding of participants and personnel implementing the interventions was ensured, and unlikely that the blinding could have been broken but the review authors judge that the outcomes (falls and fractures) are unlikely to be influenced by lack of blinding

- Judgement of 'high risk' if participants or intervention delivery personnel, or both, were not blinded to group allocation (e.g. exercise intervention), and the outcomes (falls and fractures) are likely to be influenced by lack of blinding

- Judgement of 'unclear risk' if there is insufficient information to make a judgement of 'low risk' or 'high risk'
Blinding of outcome assessment relating to detection bias due to knowledge of the allocated interventions by outcome assessors
- Falls

- judgement of 'low risk' if outcomes were recorded/confirmed in all allocated groups using the same method and the personnel recording/confirming outcomes were blind to group allocation

- judgement of 'high risk' if outcomes were not recorded/confirmed in all allocated groups using the same method or the personnel recording/confirming outcomes were NOT blind to group allocation

- judgement of 'unclear risk' if there is insufficient information to make a judgement of 'low risk' or 'high risk'

- Fractures:

- judgement of 'low risk' if fractures were recorded/confirmed in all allocated groups using the same method and fractures were confirmed by the results of radiological examination or from primary-care case records and the personnel recording/confirming fractures were blind to group allocation

- judgement of 'high risk' if fractures were not recorded/confirmed in all allocated groups using the same method or the only evidence for fractures was from self-reports from participants or carers

- judgement of 'unclear risk' if there is insufficient information to make a judgement of 'low risk' or 'high risk'

- Hospital admission, medical attention and adverse events:

- judgement of 'low risk' if requiring hospital admission/medical attention as a result of a fall was recorded/confirmed in all allocated groups using the same method (e.g. from primary-care records)

- judgement of 'high risk' if requiring hospital admission/medical attention as a result of a fall was not recorded/confirmed in all allocated groups using the same method (e.g. from primary-care records) or the only evidence for requiring medical attention was from self-reports from participants or carers

○ judgement of 'unclear risk' if there is insufficient information to make a judgement of 'low risk' or 'high risk'

- Health-related quality of life (self-reported outcome):

- judgement of 'low risk' if trial participants were blind to group allocation

- judgement of 'high risk' if trial participants were not blind to group allocation

○ judgement of 'unclear risk' if blinding was reported and thus trial participants may have been unaware of group 
(Continued)

Incomplete outcome data relating to attrition bias due to amount, nature or handling of incomplete outcome data
- Judgement of 'low risk' if there are no missing outcome data, or less than $20 \%$ of outcome data are missing and losses are balanced in numbers across intervention groups with similar reasons for missing data across groups or missing data have been imputed using appropriate methods

- Judgement of 'high risk' if greater than $20 \%$ of outcome data missing, or reason for missing outcome data likely to be related to true outcome, with either imbalance in numbers or reasons for missing data across intervention groups, or 'as-treated' analysis done with substantial departure of the intervention received from that assigned at randomisation or potentially inappropriate application of simple imputation

- Judgement of 'unclear risk' if there is insufficient information to make a judgement of 'low risk' or 'high risk'
Selective outcome report-

ing relating to bias due to the selective reporting or non-reporting of findings
- Judgement of 'low risk' if the trial reports rate of falls, risk of falls and adverse events (minimum set of expected outcomes) and the prospective trial registration or the study protocol are available and prespecify the same fall outcomes as those in the trial report

- Judgement of 'high risk' if there is evidence of selective outcome reporting, with important disparity between prespecified falls outcomes if the prospective trial registration or the study protocol are available; or the lack of appropriate data for both falls outcomes

- Judgement of 'unclear risk' if the trial does not report on one or more of the minimum set of expected outcomes or if there is insufficient information to make a judgement of 'low risk' or 'high risk'

\section{Method of ascertaining falls} relating to bias in the recall of falls due to unreliable methods of ascertainment
- Judgement of 'low risk' if the study used some form of concurrent collection of data about falling, e.g. participants given postcards to fill in daily and mail back monthly, calendar to mark monthly, or more frequent follow-up by the researchers

- Judgement of 'high risk' if ascertainment relied on participant recall at longer intervals than one month during the study or at its conclusion

- Judgement of 'unclear risk' if there was retrospective recall over a short period only, or if the trial authors did not describe details of ascertainment, i.e. insufficient information was provided to allow a judgement of 'low risk' or 'high risk'

\section{Cluster-randomised trials} relating to bias due to factors particular to cluster-randomised trials
- Judgement of 'low risk' if the study predominantly had the following characteristics: i) individuals were recruited to the trial prior to randomisation of the clusters; ii) baseline comparability of clusters was reported or there was statistical adjustment for baseline characteristics; iii) no loss of clusters or missing outcomes for individuals within specific clusters; iv) clustering is accounted for in the analyses; v) results are comparable with individually-randomised trials

- Judgement of 'high risk' if the study predominantly had the following characteristics: i) individuals were recruited to the trial after the randomisation of the clusters; ii) baseline comparability of clusters was not reported and there was no statistical adjustment for baseline characteristics; iii) loss of entire clusters or missing outcomes for individuals within clusters; iv) no account for clustering in analyses; v) results not comparable with individually-randomised trials

- Judgement of 'unclear risk' if there is insufficient information to make a judgement of 'low risk' or 'high risk'

We adapted this from Table 8.5.a 'The Cochrane Collaboration's tool for assessing risk of bias' and Table 8.5.d 'Criteria for judging risk of bias in the 'Risk of bias' assessment tool' (Higgins 2011).

\section{Appendix 4. Description of included studies: reference links}

\section{Study description Links to references}

Setting (country)

Australia: Barker 2016; Barnett 2003; Brown 2002; Clemson 2010; Clemson 2012; Day 2002; Day 2015; Duque 2013; Haines 2009; Lord 1995; Lord 2003; Merom 2016; Nitz 2004; Sales 2017; Sherrington 2014; Vogler 2009; Voukelatos 2015; Voukelatos 2007; Yang 2012 
Australia, New Zeland: Latham 2003

Australia, Spain and Germany: Gschwind 2015

Belgium, Israel, Italy, Netherlands, and United Kingdom: Mirelman 2016

Brazil: Almeida 2013; Ansai 2015; Arantes 2015; Madureira 2007

Canada: Carter 2002; Davis 2011; Liu-Ambrose 2004; Liu-Ambrose 2008

Chile: Bunout 2005; Dangour 2011

China: Woo 2007

Denmark: Beyer 2007

Finland: Karinkanta 2007; Korpelainen 2006; Lehtola 2000; Luukinen 2007; Uusi-Rasi 2015

France: Cornillon 2002; El-Khoury 2015

Germany: Freiberger 2007; Hauer 2001; Kemmler 2010; Siegrist 2016

Hungary: Kovacs 2013; Miko 2017

Iran: Dadgari 2016

Italy: Morone 2016; Verrusio 2017

Japan:Hirase 2015; Iwamoto 2009; Kamide 2009; Kim 2014; Okubo 2016; Sakamoto 2013; Shigematsu 2008; Suzuki 2004; Yamada 2010; Yamada 2012; Yamada 2013

Korea: Park 2008

Netherlands: Logghe 2009; Smulders 2010; Weerdesteyn 2006

New Zealand: Campbell 1997; Kerse 2010; Robertson 2001a; Taylor 2012

Norway: Helbostad 2004; Kyrdalen 2014

Singapore: Kwok 2016; Ng 2015

Sweden: Arkkukangas 2015; Grahn Kronhed 2009; Halvarsson 2013; Halvarsson 2016

Switzerland: Trombetti 2011

Taiwan: Huang 2010; Hwang 2016; Lin 2007

Thailand: Boongrid 2017

Turkey: Irez 2011

United Kingdom: Clegg 2014; Ebrahim 1997; Iliffe 2015; Liston 2014; McMurdo 1997; Skelton 2005; Steadman 2003

USA: Ballard 2004; Buchner 1997; Cerny 1998; Fiatarone 1997; Gill 2016; Hamrick 2017; LaStayo 2017; Li 2005; Lurie 2013; Means 2005; Morgan 2004; Morrison 2018; Reinsch 1992; Resnick 2002; Rubenstein 2000; Wolf 1996; Wolf 2003; Wu 2010

\section{Participants}

Trials in which all participants were women
Arantes 2015; Ballard 2004; Beyer 2007; Campbell 1997; Carter 2002; Davis 2011; Ebrahim 1997; ElKhoury 2015; Grahn Kronhed 2009; Hauer 2001; Irez 2011; Kamide 2009; Karinkanta 2007; Kemmler 2010; Kim 2014; Korpelainen 2006; Kovacs 2013; Liu-Ambrose 2004; Lord 1995; Madureira 2007; McMurdo 1997; Miko 2017; Morone 2016; Park 2008; Resnick 2002; Skelton 2005; Suzuki 2004; UusiRasi 2015

Barker 2016; Dadgari 2016; Hamrick 2017; Hwang 2016; Iwamoto 2009; Kovacs 2013; Kwok 2016; Mirelman 2016; Morgan 2004; Nitz 2004; Reinsch 1992; Sales 2017; Sherrington 2014; Steadman 2003; Verrusio 2017; Voukelatos 2007
Trials that would have been excluded if the review inclusion criteria had been set at $65+$ years of age 
(Continued)

Trials recruiting on the basis of identified falls history or one or more risk factors
Almeida 2013; Ansai 2015; Arantes 2015; Barker 2016; Ballard 2004; Barnett 2003; Beyer 2007; Boongrid 2017; Buchner 1997; Campbell 1997; Clegg 2014; Clemson 2010; Clemson 2012; Dadgari 2016; Day 2015; Duque 2013; El-Khoury 2015; Fiatarone 1997; Freiberger 2007; Gill 2016; Haines 2009; Halvarsson 2013; Halvarsson 2016; Hauer 2001; Helbostad 2004; Hirase 2015; Hwang 2016; Kim 2014; Kyrdalen 2014; Kwok 2016; LaStayo 2017; Latham 2003; Lin 2007; Liston 2014; Liu-Ambrose 2008; Logghe 2009; Lurie 2013; Luukinen 2007; Mirelman 2016; Morgan 2004; Morone 2016; Ng 2015; Nitz 2004; Rubenstein 2000; Sakamoto 2013; Sales 2017; Sherrington 2014; Siegrist 2016; Skelton 2005; Smulders 2010; Steadman 2003; Taylor 2012; Trombetti 2011; Uusi-Rasi 2015; Verrusio 2017; Vogler 2009; Weerdesteyn 2006; Wolf 2003; Wu 2010; Yang 2012

Almeida 2013; Ansai 2015; Arantes 2015; Arkkukangas 2015; Barker 2016; Barnett 2003; Beyer 2007; Boongrid 2017; Brown 2002; Bunout 2005; Campbell 1997; Clegg 2014; Clemson 2010; Clemson 2012; Cornillon 2002; Dangour 2011; Davis 2011; Day 2002; Day 2015; Duque 2013; Ebrahim 1997; Freiberger 2007; Gill 2016; Grahn Kronhed 2009; Gschwind 2015; Haines 2009; Halvarsson 2013; Halvarsson 2016; Hamrick 2017; Hauer 2001; Helbostad 2004; Hirase 2015; Hwang 2016; Iliffe 2015; Irez 2011; Kamide 2009; Karinkanta 2007; Kerse 2010; Kim 2014; Korpelainen 2006; Kwok 2016; Kyrdalen 2014; Latham 2003; LaStayo 2017; Li 2005; Liu-Ambrose 2004; Liu-Ambrose 2008; Lord 1995; Lord 2003; McMurdo 1997; Means 2005; Merom 2016; Mirelman 2016; Morgan 2004; Ng 2015; Park 2008; Resnick 2002; Robertson 2001a; Rubenstein 2000; Sakamoto 2013; Sherrington 2014; Skelton 2005; Steadman 2003; Taylor 2012; Vogler 2009; Voukelatos 2007; Voukelatos 2015; Wolf 1996; Wolf 2003; Yamada 2010; Yamada 2012; Yamada 2013

\section{Interventions}

Comparisons

\begin{abstract}
Exercise versus control (not recently discharged): Almeida 2013*; Ansai 2015*; Arantes 2015; Arkkukangas 2015; Barnett 2003; Beyer 2007; Boongrid 2017; Brown 2002; Buchner 1997; Bunout 2005; Campbell 1997; Carter 2002; Cerny 1998; Clegg 2014; Clemson 2010; Clemson 2012*; CornilIon 2002; Dadgari 2016; Dangour 2011; Day 2002; Duque 2013; Ebrahim 1997; El-Khoury 2015; Fiatarone 1997; Gill 2016; Grahn Kronhed 2009; Gschwind 2015; Halvarsson 2013; Halvarsson 2016*; Hamrick 2017; Hauer 2001; Hirase 2015; Huang 2010; Iliffe 2015*; Irez 2011; Iwamoto 2009; Kamide 2009; Karinkanta 2007; Kerse 2010; Kim 2014; Korpelainen 2006; Kovacs 2013; Day 2015; Lehtola 2000; Li 2005; Lin 2007; Liu-Ambrose 2004*; Liu-Ambrose 2008; Logghe 2009; Lord 1995; Lord 2003; Luukinen 2007; Madureira 2007; McMurdo 1997; Means 2005; Merom 2016; Miko 2017; Morgan 2004; Ng 2015; Nitz 2004; Park 2008; Reinsch 1992; Resnick 2002; Robertson 2001a; Rubenstein 2000; Sakamoto 2013; Sales 2017; Siegrist 2016; Skelton 2005; Smulders 2010; Suzuki 2004; Taylor 2012; Trombetti 2011; Uusi-Rasi 2015; Voukelatos 2007; Voukelatos 2015; Weerdesteyn 2006; Wolf 1996*; Wolf 2003; Woo 2007*; Yamada 2012
\end{abstract}

Exercise versus control (recently discharged): Haines 2009; Latham 2003; Sherrington 2014

Different types of exercise (not recently discharged): Ballard 2004; Barker 2016; Davis 2011*; Freiberger 2007; Helbostad 2004; Hwang 2016; Kemmler 2010; Kwok 2016; Kyrdalen 2014; LaStayo 2017; Liston 2014; Lurie 2013; Mirelman 2016; Morone 2016; Morrison 2018; Okubo 2016; Shigematsu 2008; Steadman 2003; Taylor 2012; Verrusio 2017; Wu 2010*; Yamada 2010; Yamada 2012; Yamada 2013

Different types of exercise (recently discharged): Vogler 2009*

Group versus individual exercise: Barker 2016; Helbostad 2004; Iliffe 2015*; Kyrdalen 2014; Wu $2010^{*}$

High- versus low-dose exercise: Ballard 2004; Davis 2011*; Taylor 2012

Exercises

Predominantly group-based: Almeida 2013 (supervised group); Ansai 2015; Arantes 2015; Ballard 2004; Beyer 2007; Brown 2002; Buchner 1997; Bunout 2005; Carter 2002; Cerny 1998; Dangour 2011; Davis 2011; Day 2002; Day 2015; Freiberger 2007; Grahn Kronhed 2009; Halvarsson 2013; Halvarsson 2016; Hauer 2001; Huang 2010; Irez 2011; Karinkanta 2007; Kemmler 2010 (low intensity group); Kim 2014; Kovacs 2013; Kwok 2016; LaStayo 2017; Li 2005; Liu-Ambrose 2004; Lord 1995; Lord 2003; Madureira 2007; McMurdo 1997; Means 2005; Merom 2016; Miko 2017; Morgan 2004; Morone 2016; Morrison 2018 (balance group); Nitz 2004; Okubo 2016; Park 2008; Reinsch 1992; Rubenstein 2000; 
Sales 2017; Shigematsu 2008; Siegrist 2016; Smulders 2010; Steadman 2003; Suzuki 2004; Taylor 2012; Trombetti 2011; Voukelatos 2007; Weerdesteyn 2006; Wolf 2003; Woo 2007; Wu 2010 (community group); Yamada 2010; Yamada 2012

Combination of group-based and individual exercise: Almeida 2013 (home based group); Barker 2016 (pilates group); Barnett 2003; El-Khoury 2015; Gill 2016; Hamrick 2017; Helbostad 2004; Iliffe 2015 (FaME group); Kemmler 2010 (high intensity group); Kyrdalen 2014 (OEP group); Lehtola 2000; Liston 2014; Logghe 2009; Resnick 2002; Skelton 2005 (FaME group); Uusi-Rasi 2015; Wolf 1996 (Tai Chi group)

Predominantly individual exercise: Arkkukangas 2015; Barker 2016 (home program group); Boongrid 2017; Campbell 1997; Clegg 2014; Clemson 2010; Clemson 2012; Cornillon 2002; Dadgari 2016; Duque 2013; Ebrahim 1997; Fiatarone 1997; Gschwind 2015; Haines 2009; Hirase 2015; Hwang 2016; Iliffe 2015 (OEP group); Iwamoto 2009; Kamide 2009; Kerse 2010; Korpelainen 2006; Kyrdalen 2014 (home based group); Latham 2003; Lin 2007; Liu-Ambrose 2008; Lurie 2013; Luukinen 2007; Mirelman 2016; Morrison 2018 (Wii group); Ng 2015; Robertson 2001a; Sakamoto 2013; Sherrington 2014; Verrusio 2017; Vogler 2009; Voukelatos 2015; Wolf 1996 (balance group); Wu 2010 (telephone and home exercise groups); Yamada 2013; Yang 2012

Personnel delivering intervention
Health professional delivering intervention: Almeida 2013; Arantes 2015; Arkkukangas 2015; Barker 2016; Beyer 2007; Boongrid 2017; Brown 2002; Campbell 1997; Clegg 2014; Clemson 2010; Clemson 2012; Duque 2013; Ebrahim 1997; Grahn Kronhed 2009; Haines 2009; Halvarsson 2013; Halvarsson 2016; Hauer 2001; Helbostad 2004; Hirase 2015; Hwang 2016 (lower limb group); Kamide 2009; Korpelainen 2006; Kovacs 2013; Kwok 2016; Kyrdalen 2014; LaStayo 2017; Latham 2003; Lin 2007; Liston 2014; Liu-Ambrose 2008; Lurie 2013; Luukinen 2007; Madureira 2007; Means 2005; Miko 2017; Morgan 2004; Morone 2016; Nitz 2004; Robertson 2001a; Sales 2017; Sherrington 2014; Siegrist 2016; Smulders 2010; Steadman 2003; Uusi-Rasi 2015; Verrusio 2017; Vogler 2009; Yamada 2010; Yamada 2012; Yang 2012

No health professional delivering intervention: Ansai 2015; Ballard 2004; Barnett 2003; Buchner 1997; Bunout 2005; Carter 2002; Cerny 1998; Cornillon 2002; Dadgari 2016; Dangour 2011; Davis 2011; Day 2002; Day 2015; El-Khoury 2015; Fiatarone 1997; Freiberger 2007; Gill 2016; Gschwind 2015; Hamrick 2017; Huang 2010; Hwang 2016 (Tai Chi group); Iliffe 2015; Irez 2011; Iwamoto 2009; Karinkanta 2007; Kemmler 2010; Kerse 2010; Kim 2014; Lehtola 2000; Li 2005; Liu-Ambrose 2004; Logghe 2009; Lord 1995; Lord 2003; McMurdo 1997; Merom 2016; Mirelman 2016; Morrison 2018; Ng 2015; Okubo 2016; Reinsch 1992; Resnick 2002; Rubenstein 2000; Sakamoto 2013; Shigematsu 2008; Skelton 2005; Suzuki 2004; Taylor 2012; Trombetti 2011; Voukelatos 2007; Voukelatos 2015; Weerdesteyn 2006; Wolf 1996; Wolf 2003; Woo 2007; Wu 2010; Yamada 2013

Not reported: Park 2008

* $=$ multigroup trial appearing in more than one category 


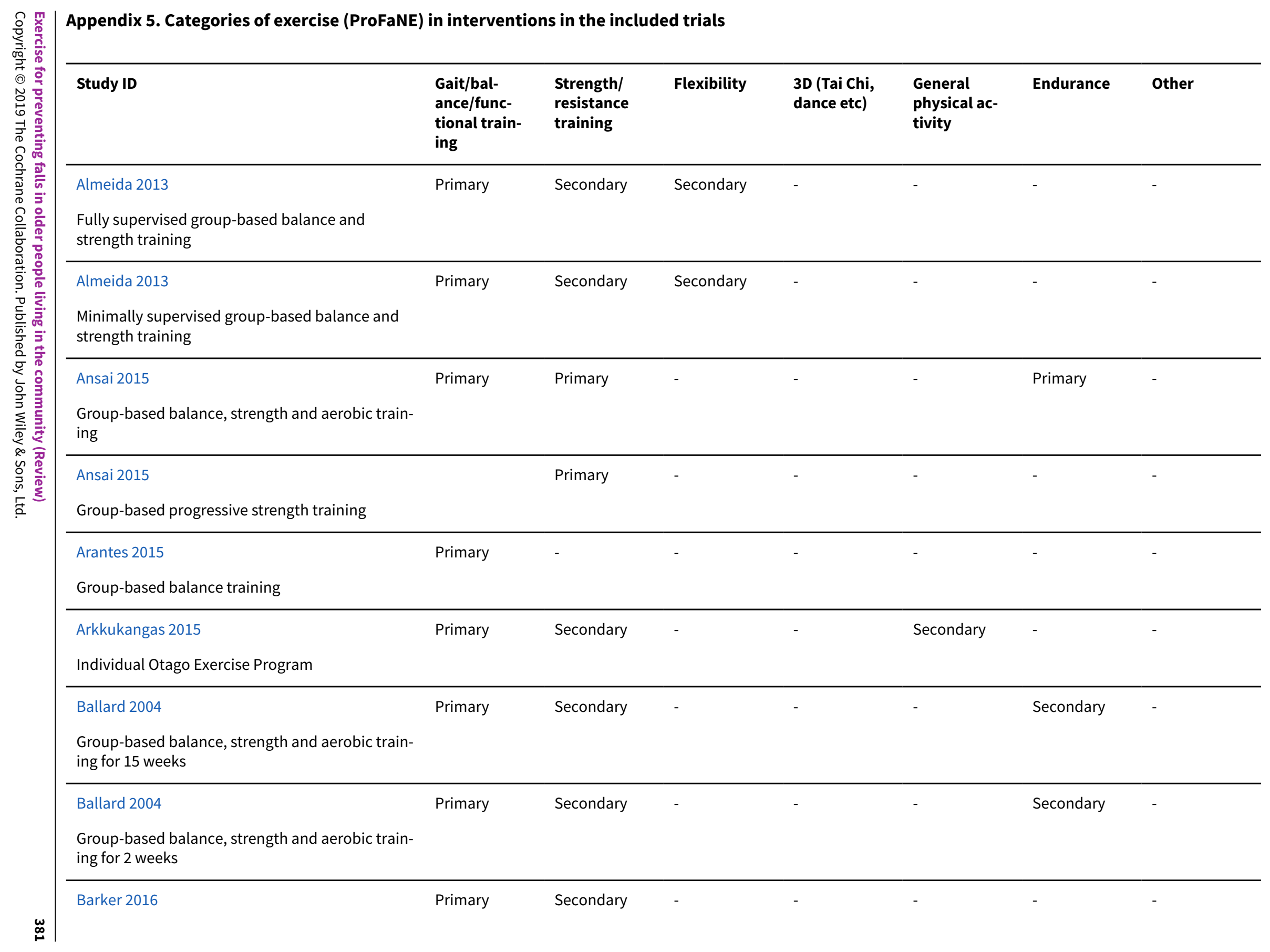




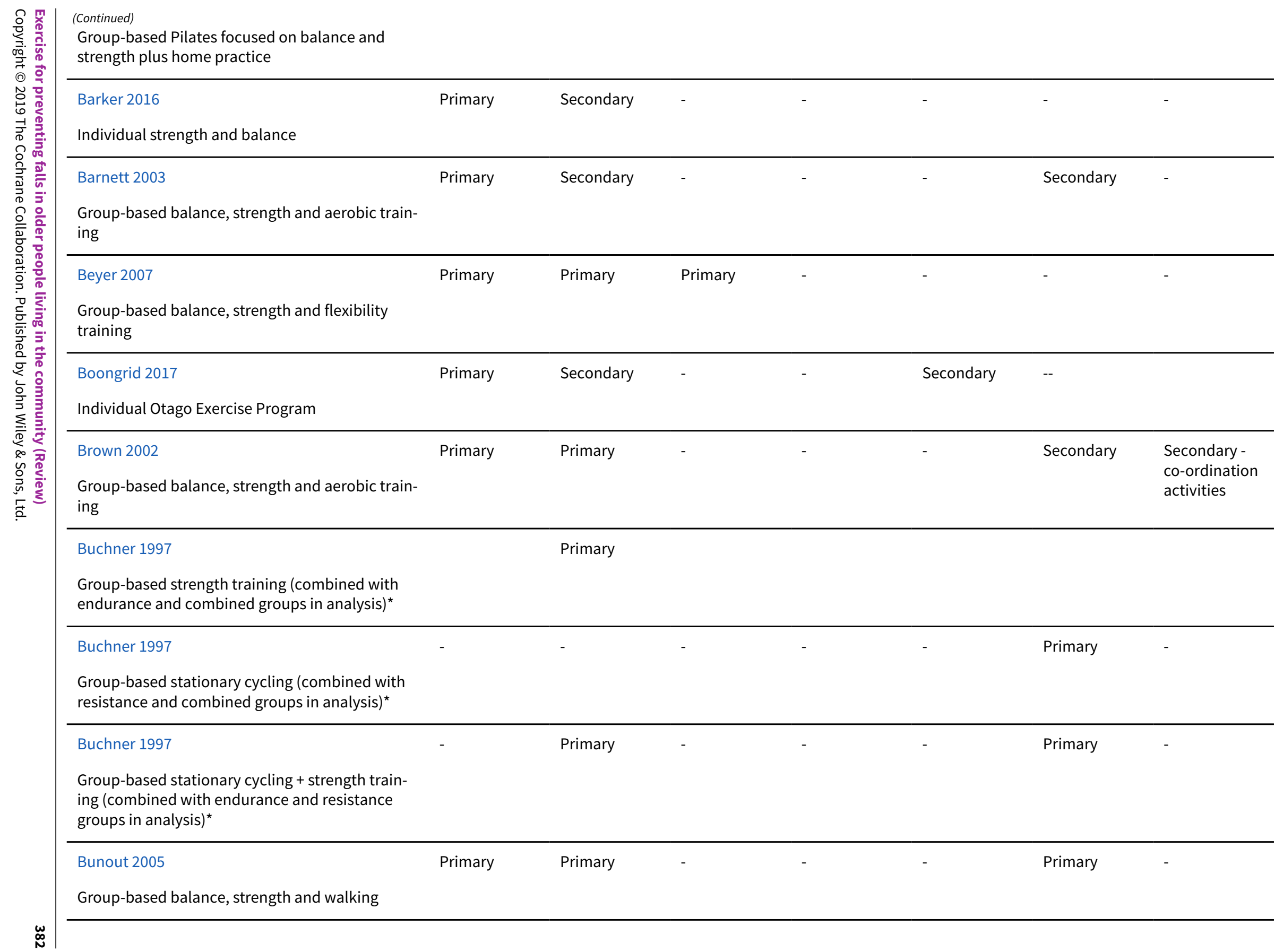




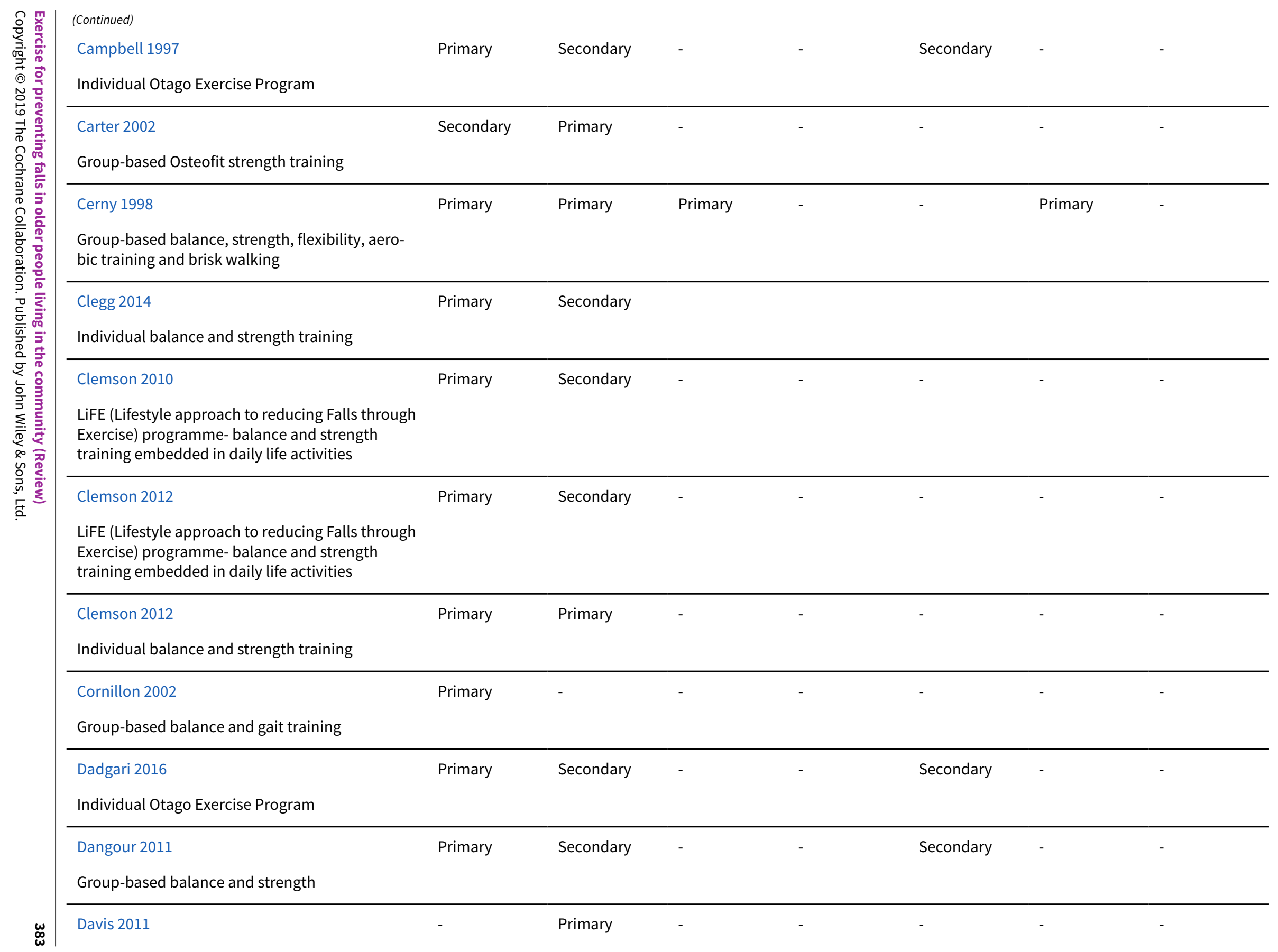




\begin{tabular}{|c|c|c|c|c|c|c|c|c|c|}
\hline 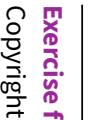 & $\begin{array}{l}\text { (Continued) } \\
\text { Group-based progressive high intensity resis- } \\
\text { tance training once weekly }\end{array}$ & & & & & & & & , \\
\hline 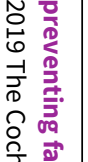 & $\begin{array}{l}\text { Davis } 2011 \\
\text { Group-based progressive high intensity resis- } \\
\text { tance training twice weekly }\end{array}$ & - & Primary & - & - & - & - & - & 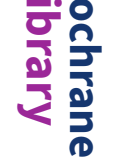 \\
\hline 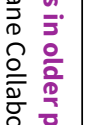 & $\begin{array}{l}\text { Davis } 2011 \\
\text { Group-based balance and tone }\end{array}$ & Primary & & Secondary & & & & & 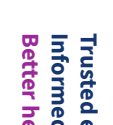 \\
\hline 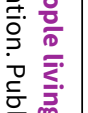 & $\begin{array}{l}\text { Day } 2002 \\
\text { Group-based balance and strength }\end{array}$ & Primary & Secondary & Secondary & - & - & - & - & 荡 \\
\hline 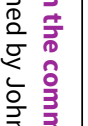 & $\begin{array}{l}\text { Day } 2015 \\
\text { Group-based Tai Chi }\end{array}$ & - & - & - & Primary & - & - & - & \\
\hline 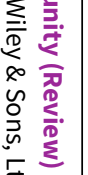 & $\begin{array}{l}\text { Duque } 2013 \\
\text { Virtual reality balance training }\end{array}$ & Primary & - & - & - & - & - & $\begin{array}{l}\text { Secondary- vi- } \\
\text { sual-vestibu- } \\
\text { lar rehabilita- } \\
\text { tion }\end{array}$ & \\
\hline & $\begin{array}{l}\text { Ebrahim } 1997 \\
\text { Brisk walking }\end{array}$ & - & - & - & - & Primary & - & - & \\
\hline & $\begin{array}{l}\text { El-Khoury } 2015 \\
\text { Group-based balance and strength plus home } \\
\text { practice }\end{array}$ & Primary & Secondary & Secondary & - & - & - & - & \\
\hline & $\begin{array}{l}\text { Fiatarone } 1997 \\
\text { Individual high-intensity progressive resistance } \\
\text { training }\end{array}$ & - & Primary & - & - & - & - & - & 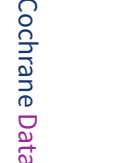 \\
\hline & $\begin{array}{l}\text { Freiberger } 2007 \\
\text { Group-based psychomotor programme }\end{array}$ & Primary & Primary & - & - & - & - & $\begin{array}{l}\text { Primary- per- } \\
\text { ceptual train- } \\
\text { ing }\end{array}$ & 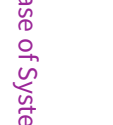 \\
\hline 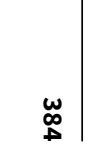 & Freiberger 2007 & Primary & Primary & Primary & & & Primary & & 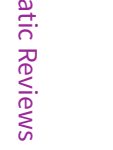 \\
\hline
\end{tabular}




\begin{tabular}{|c|c|c|c|c|c|c|c|c|}
\hline 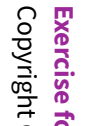 & $\begin{array}{l}\text { (Continued) } \\
\text { Group-based balance, strength, flexibility, en- } \\
\text { durance }\end{array}$ & & & & & & & \\
\hline 꿍 & Gill 2016 & Primary & Primary & Secondary & & Primary & - & - \\
\hline 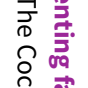 & $\begin{array}{l}\text { Group and home-based balance, strength, flexi- } \\
\text { bility and walking training }\end{array}$ & & & & & & & \\
\hline$\stackrel{气}{5}$ & Grahn Kronhed 2009 & Secondary & Primary & Secondary & - & - & Secondary & - \\
\hline 产产 & Group-based strength and balance training & & & & & & & \\
\hline$\frac{0}{0}$ & Gschwind 2015 & Primary & Secondary & - & - & - & - & - \\
\hline 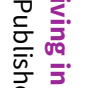 & $\begin{array}{l}\text { Individual balance and strength training using } \\
\text { exergames }\end{array}$ & & & & & & & \\
\hline 苛 & Haines 2009 & Primary & Primary & - & Primary & - & - & - \\
\hline 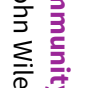 & $\begin{array}{l}\text { Home strength and balance program with DVD/ } \\
\text { workbook }\end{array}$ & & & & & & & \\
\hline 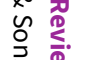 & Halvarsson 2013 & Primary & - & - & - & - & - & - \\
\hline$\leq$ & Group-based progressive balance training & & & & & & & \\
\hline & Halvarsson 2016 & Primary & - & - & - & - & - & - \\
\hline & Group-based progressive balance training & & & & & & & \\
\hline & Halvarsson 2016 & Primary & - & - & - & Primary & - & - \\
\hline & $\begin{array}{l}\text { Group-based progressive balance training plus } \\
\text { walking }\end{array}$ & & & & & & & \\
\hline & Hamrick 2017 & Primary & - & Secondary & - & - & - & - \\
\hline & Home exercise group & & & & & & & \\
\hline & Hauer 2001 & Primary & Primary & - & - & Primary & - & - \\
\hline & $\begin{array}{l}\text { Group-based progressive strength and balance } \\
\text { training }\end{array}$ & & & & & & & \\
\hline & Helbostad 2004 & Primary & Secondary & - & - & - & - & - \\
\hline
\end{tabular}




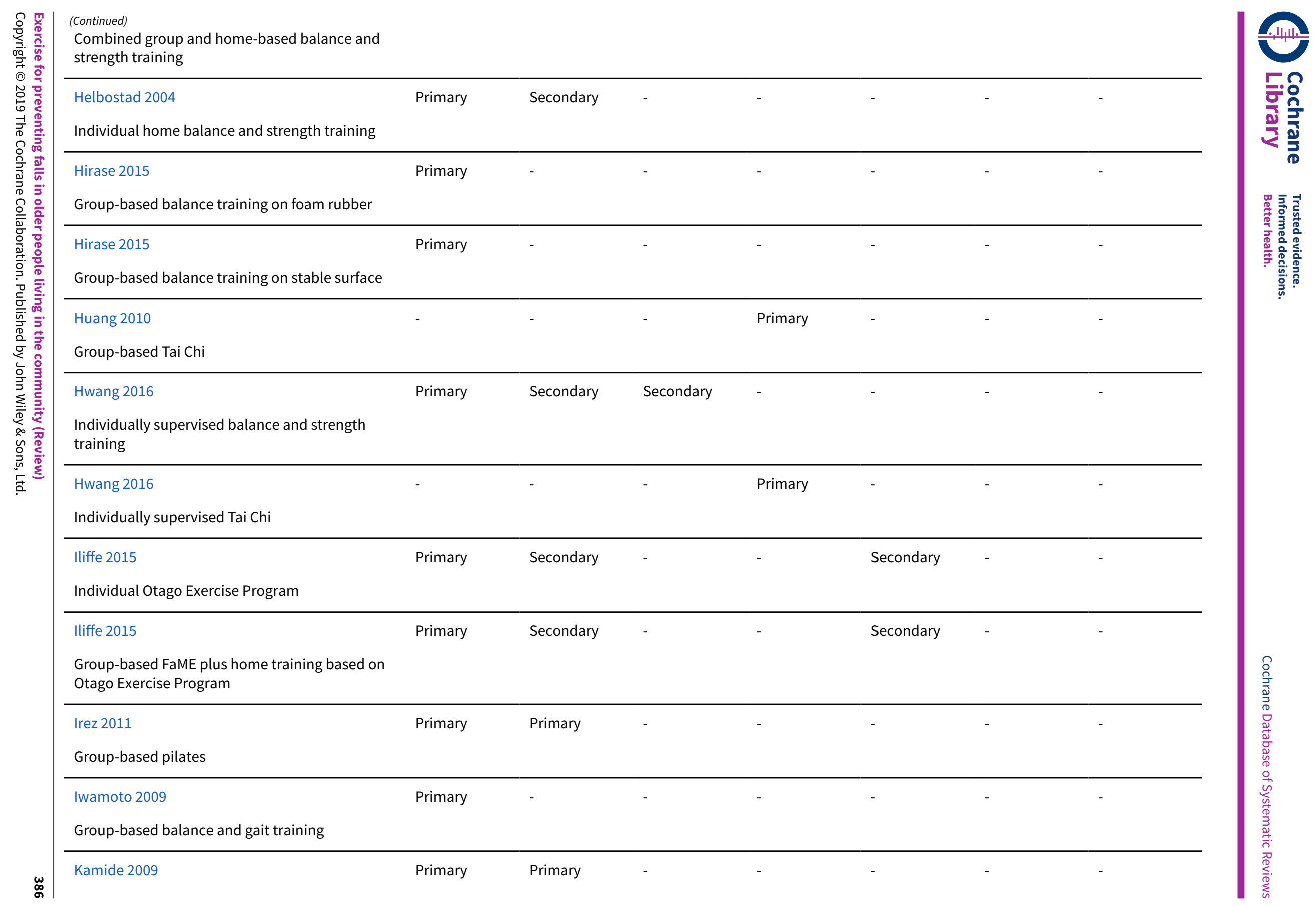




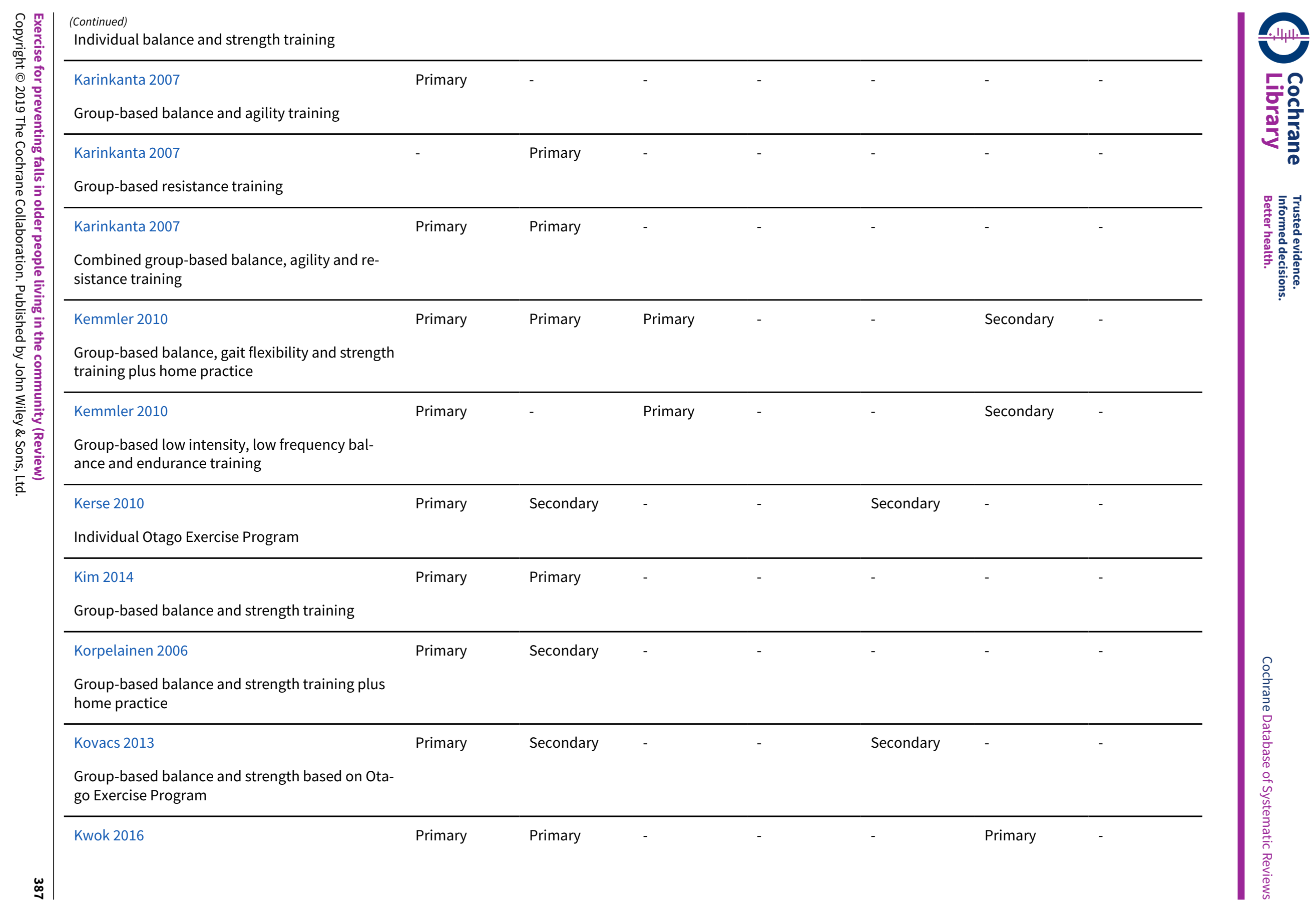




\begin{tabular}{|c|c|c|c|c|c|c|c|c|}
\hline 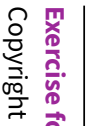 & $\begin{array}{l}\text { (Continued) } \\
\text { Group-based balance, strength and aerobic train- } \\
\text { ing plus home practice }\end{array}$ & & & & & & & \\
\hline 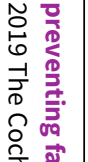 & $\begin{array}{l}\text { Kwok } 2016 \\
\text { Balance, strength and aerobic training using the } \\
\text { Nintendo WiiActive }\end{array}$ & Primary & Primary & - & - & - & Primary & - \\
\hline 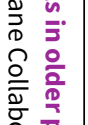 & $\begin{array}{l}\text { Kyrdalen } 2014 \\
\text { Group-based Otago Exercise Program }\end{array}$ & Primary & Secondary & - & - & Secondary & - & - \\
\hline 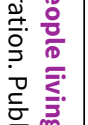 & $\begin{array}{l}\text { Kyrdalen } 2014 \\
\text { Individual Otago Exercise Program }\end{array}$ & Primary & Secondary & - & - & Secondary & - & - \\
\hline 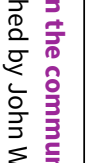 & $\begin{array}{l}\text { LaStayo } 2017 \\
\text { Resisted lower limb exercise in standing and leg } \\
\text { press }\end{array}$ & Primary & Primary & Secondary & - & Secondary & - & - \\
\hline 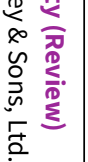 & $\begin{array}{l}\text { LaStayo } 2017 \\
\text { Resisted lower limb exercise using recumbent } \\
\text { stepper-ergometer }\end{array}$ & Primary & Primary & Secondary & - & Secondary & - & - \\
\hline & $\begin{array}{l}\text { Latham } 2003 \\
\text { Resistance exercise }\end{array}$ & - & Primary & - & - & - & - & - \\
\hline & $\begin{array}{l}\text { Lehtola } 2000 \\
\text { Group-based balance and flexibility training plus } \\
\text { walking and home practice }\end{array}$ & Primary & - & Primary & - & Primary & - & - \\
\hline & $\begin{array}{l}\text { Li } 2005 \\
\text { Group-based Tai Chi }\end{array}$ & - & - & - & Primary & - & - & - \\
\hline & $\begin{array}{l}\text { Lin } 2007 \\
\text { Individual balance, strength and flexibility train- } \\
\text { ing }\end{array}$ & Primary & Secondary & Secondary & - & - & - & - \\
\hline$\underset{\substack{\infty \\
\infty}}{\boldsymbol{\omega}}$ & Liston 2014 & Primary & Secondary & - & - & Secondary & - & - \\
\hline
\end{tabular}




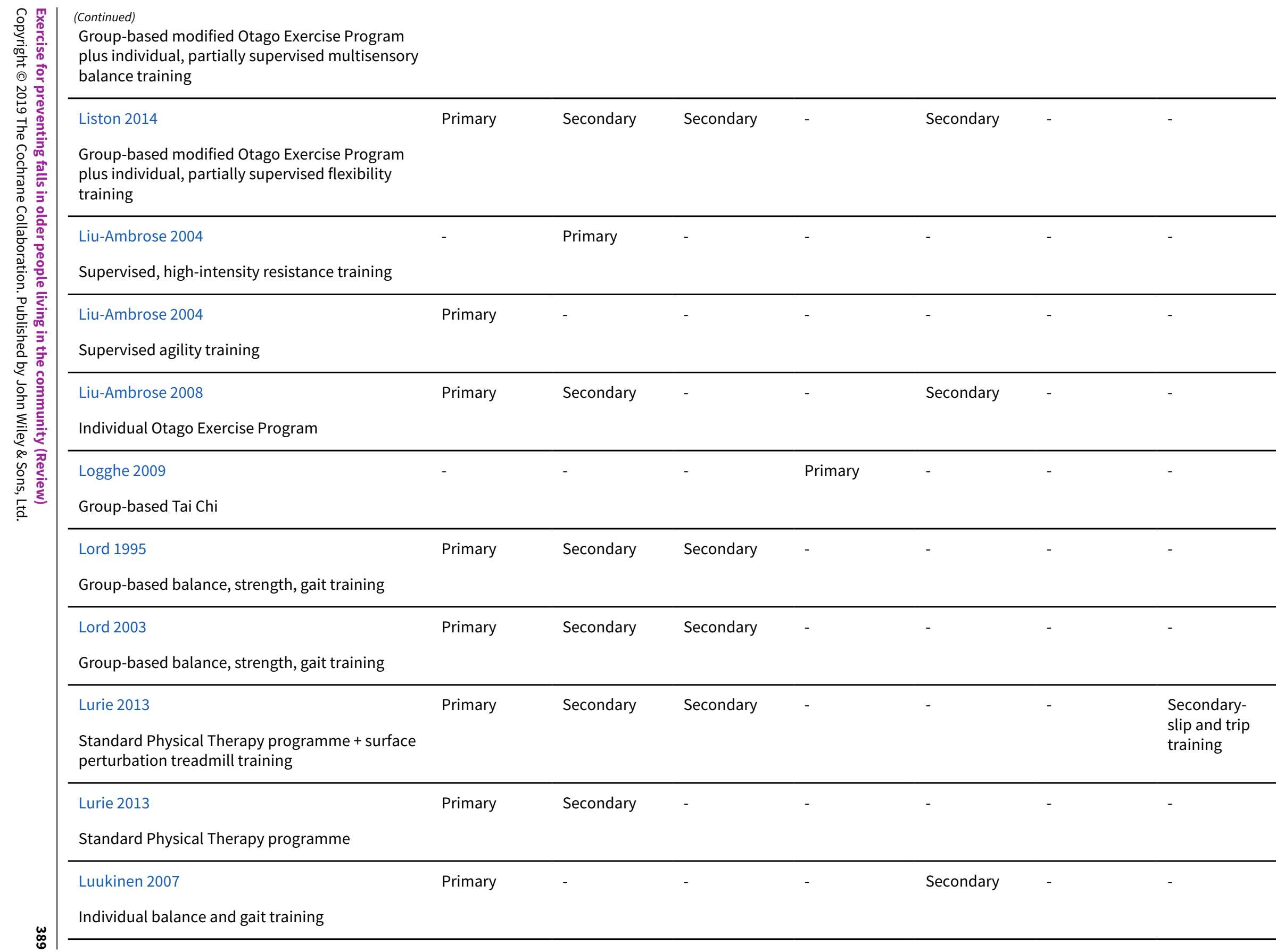




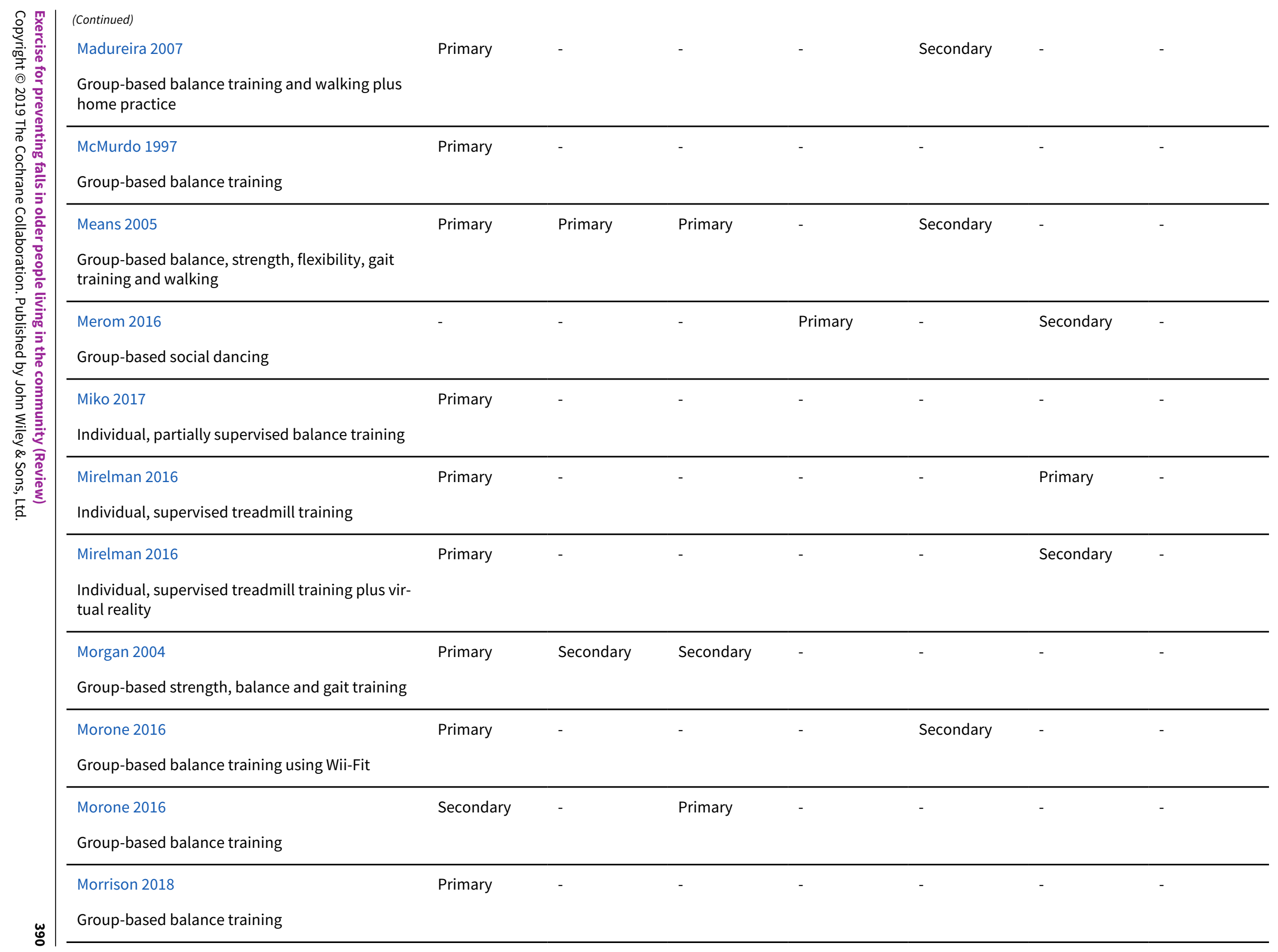




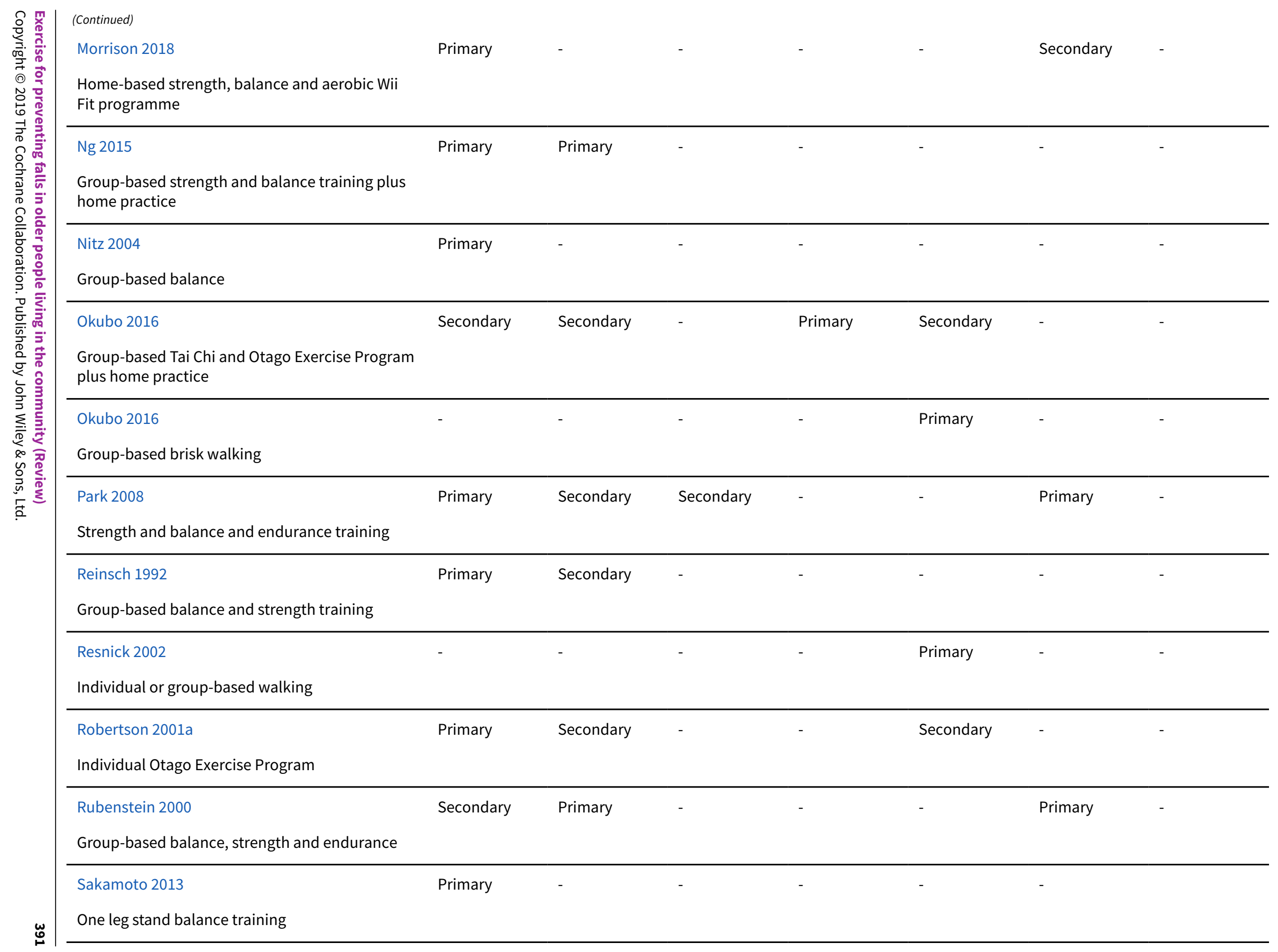




\begin{tabular}{|c|c|c|c|c|c|c|c|c|}
\hline 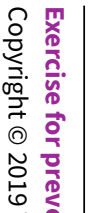 & $\begin{array}{l}\text { (Continued) } \\
\text { Sales } 2017 \\
\text { Group-based strength, balance, co-ordination, } \\
\text { mobility and flexibility }\end{array}$ & Primary & Secondary & - & - & - & - & - \\
\hline 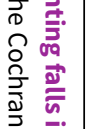 & $\begin{array}{l}\text { Sherrington } 2014 \\
\text { home-based strength and balance programme }\end{array}$ & Primary & Primary & - & - & & - & \\
\hline 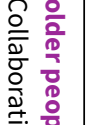 & $\begin{array}{l}\text { Shigematsu } 2008 \\
\text { Group-based stepping training on felt mat }\end{array}$ & Primary & - & - & - & - & - & - \\
\hline 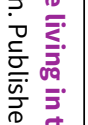 & $\begin{array}{l}\text { Shigematsu } 2008 \\
\text { Group-based walking }\end{array}$ & Primary & - & - & - & Primary & - & \\
\hline 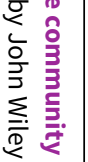 & $\begin{array}{l}\text { Siegrist } 2016 \\
\text { Group-based balance, strength, power and gait } \\
\text { training plus home practice }\end{array}$ & Primary & Secondary & - & - & - & - & - \\
\hline 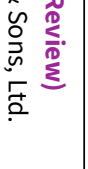 & $\begin{array}{l}\text { Skelton } 2005 \\
\text { Group-based FaME balance and strength training } \\
\text { plus home practice }\end{array}$ & Primary & Secondary & - & - & Secondary & - & - \\
\hline & $\begin{array}{l}\text { Smulders } 2010 \\
\text { Group-based balance and gait training using an } \\
\text { obstacle avoidance course }\end{array}$ & Primary & - & - & - & Secondary & - & $\begin{array}{l}\text { Secondary- } \\
\text { training in fall } \\
\text { techniques, } \\
\text { lifting tech- } \\
\text { niques }\end{array}$ \\
\hline & $\begin{array}{l}\text { Steadman } 2003 \\
\text { Standard, individualised physiotherapy focused } \\
\text { on functional training plus balance training }\end{array}$ & Primary & - & - & - & - & - & - \\
\hline & $\begin{array}{l}\text { Steadman } 2003 \\
\text { Standard, individualised physiotherapy focused } \\
\text { on functional training }\end{array}$ & Primary & - & - & - & - & - & - \\
\hline & Suzuki 2004 & Primary & Primary & Primary & Primary & - & - & - \\
\hline
\end{tabular}




\begin{tabular}{|c|c|c|c|c|c|c|c|c|}
\hline 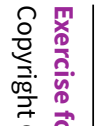 & $\begin{array}{l}\text { (Continued) } \\
\text { Group-based strength, balance and gait training } \\
\text { plus home practice }\end{array}$ & & & & & & & \\
\hline$\frac{\overline{0}}{0}$ & Taylor 2012 & - & - & - & Primary & - & - & - \\
\hline 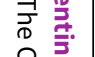 & Group-based Tai Chi, 2x/ week & & & & & & & \\
\hline 髉 & Taylor 2012 & - & - & - & Primary & - & - & - \\
\hline 官高 & Group-based Tai Chi, 1x/ week & & & & & & & \\
\hline 票 & Trombetti 2011 & Primary & - & - & - & - & - & - \\
\hline$\stackrel{\overline{0}}{=}$ & Group-based balance and gait training & & & & & & & \\
\hline$\frac{\overline{\bar{m}}}{\bar{c}}$ & Uusi-Rasi 2015 & Primary & Primary & - & - & - & - & - \\
\hline 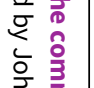 & $\begin{array}{l}\text { Group-based balance and strength training plus } \\
\text { home practice }\end{array}$ & & & & & & & \\
\hline 京竞 & Verrusio 2017 & Primary & - & - & - & - & - & - \\
\hline 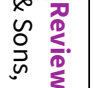 & $\begin{array}{l}\text { Individual, supervised balance and gait training } \\
\text { using exoskeleton human body posturizer }\end{array}$ & & & & & & & \\
\hline & Verrusio 2017 & Primary & - & - & - & - & - & - \\
\hline & Individual, supervised balance and gait training & & & & & & & \\
\hline & Vogler 2009 & - & Primary & - & - & - & - & - \\
\hline & $\begin{array}{l}\text { home-based seated lower limb strength exercis- } \\
\text { es }\end{array}$ & & & & & & & \\
\hline & Vogler 2009 & Primary & - & - & - & - & - & - \\
\hline & $\begin{array}{l}\text { home-based strength training with weightbear- } \\
\text { ing, functional tasks }\end{array}$ & & & & & & & \\
\hline & Voukelatos 2007 & - & - & - & Primary & - & - & - \\
\hline & Group-based Tai Chi & & & & & & & \\
\hline & Voukelatos 2015 & - & - & - & - & Primary & - & - \\
\hline & Individual walking programme & & & & & & & \\
\hline
\end{tabular}




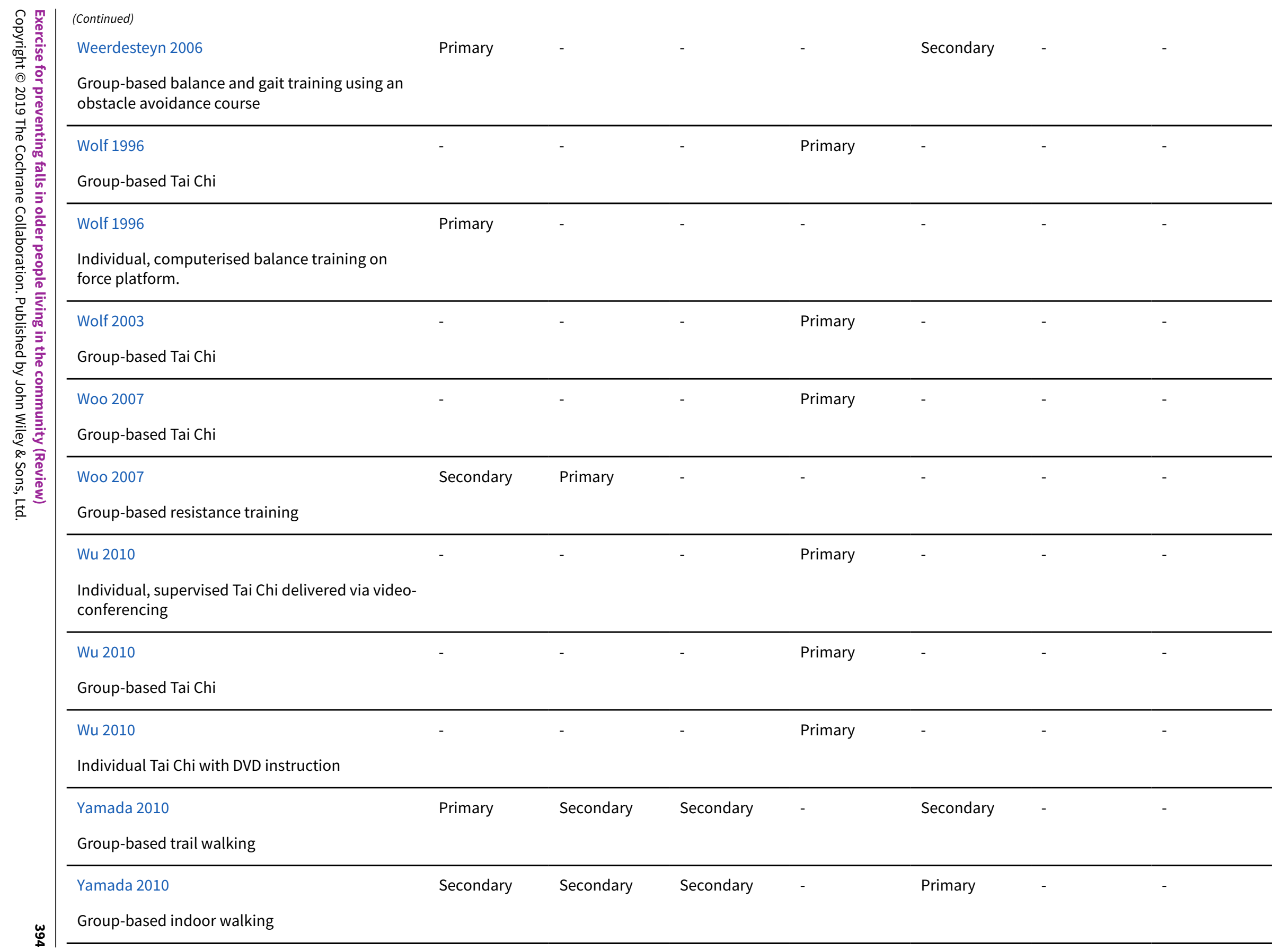




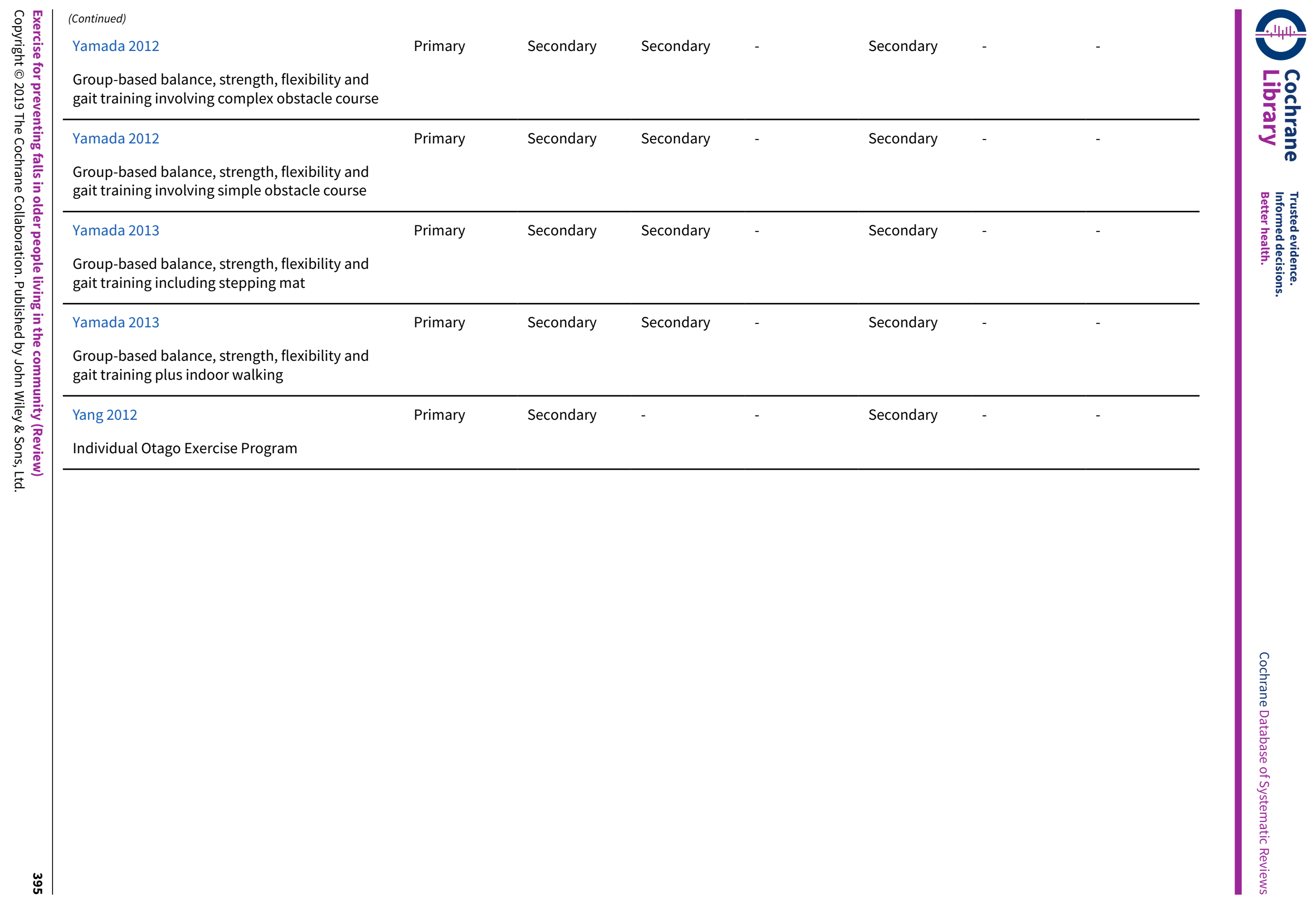


* Intervention groups combined due to fall data not being available for individual intervention groups.

\title{
Appendix 6. Study IDs for the 81 studies included in the exercise (all types) versus control comparison
}

\begin{tabular}{ll}
\hline Comparison & Study IDs \\
\hline $\begin{array}{l}\text { Exercise (all types) versus con- } \\
\text { trol }\end{array}$ & Almeida 2013*; Ansai 2015*; Arantes 2015; Arkkukangas 2015; Barnett 2003; Beyer 2007; Boongrid \\
& 2017; Brown 2002; Buchner 1997; Bunout 2005; Campbell 1997; Carter 2002; Cerny 1998; Clegg \\
& 2014; Clemson 2010; Clemson 2012*; Cornillon 2002; Dadgari 2016; Dangour 2011; Day 2002; Day \\
& 2015; Duque 2013; Ebrahim 1997; El-Khoury 2015; Fiatarone 1997; Gill 2016; Grahn Kronhed 2009; \\
& Gschwind 2015; Halvarsson 2013; Halvarsson 2016*; Hamrick 2017; Hauer 2001; Hirase 2015*; \\
& Huang 2010; Iliffe 2015*; Irez 2011; Iwamoto 2009; Kamide 2009; Karinkanta 2007*; Kerse 2010; Kim \\
& 2014; Korpelainen 2006; Kovacs 2013; Lehtola 2000; Li 2005; Lin 2007; Liu-Ambrose 2004*; Liu-Am- \\
& brose 2008; Logghe 2009; Lord 1995; Lord 2003; Luukinen 2007; Madureira 2007; McMurdo 1997; \\
& Means 2005; Merom 2016; Miko 2017; Morgan 2004; Ng 2015; Nitz 2004; Park 2008; Reinsch 1992; \\
& Resnick 2002; Robertson 2001a; Rubenstein 2000; Sakamoto 2013; Sales 2017; Siegrist 2016; Skel- \\
& ton 2005; Smulders 2010; Suzuki 2004; Taylor 2012; Trombetti 2011; Uusi-Rasi 2015; Voukelatos \\
& 2007; Voukelatos 2015; Weerdesteyn 2006; Wolf 1996*; Wolf 2003; Woo 2007*; Yang 2012
\end{tabular}

Balance and functional exercises versus control
Almeida 2013*; Arantes 2015; Arkkukangas 2015; Barnett 2003; Boongrid 2017; Campbell 1997; Clegg 2014; Clemson 2010; Clemson 2012*; Cornillon 2002; Dadgari 2016; Dangour 2011; Day 2002; Duque 2013; El-Khoury 2015; Gschwind 2015; Halvarsson 2013; Halvarsson 2016*; Hamrick 2017; Hirase 2015*; Iliffe 2015*; Iwamoto 2009; Karinkanta 2007*; Kerse 2010; Korpelainen 2006; Kovacs 2013; Lin 2007; Liu-Ambrose 2004*; Liu-Ambrose 2008; Lord 1995; Lord 2003; Luukinen 2007; Madureira 2007; McMurdo 1997; Miko 2017; Morgan 2004; Nitz 2004; Reinsch 1992; Robertson 2001a; Sakamoto 2013; Sales 2017; Siegrist 2016; Skelton 2005; Smulders 2010; Trombetti 2011; Weerdesteyn 2006; Wolf 1996*; Yang 2012

\begin{tabular}{ll}
$\begin{array}{l}\text { Resistance exercises versus } \\
\text { control }\end{array}$ & Ansai 2015*; Carter 2002; Fiatarone 1997; Grahn Kronhed 2009; Karinkanta 2007*; Liu-Ambrose \\
\hline
\end{tabular}

Ansai 2015*; Carter 2002; Fiatarone 1997; Grahn Kronhed 2009; Karinkanta 2007*; Liu-Ambrose ; Woo $2007^{\star}$

Flexibility versus control

$\begin{array}{ll}\begin{array}{l}\text { 3D exercise (Tai Chi) versus } \\ \text { control }\end{array} & \text { Day 2015; Huang 2010; Li 2005; Logghe 2009; Taylor 2012; Voukelatos 2007; Wolf 1996*; Wolf 2003; } \\ \text { Woo 2007* }\end{array}$

3D exercise (dance) versus Merom 2016
control

control

General physical activity $\quad$ Ebrahim 1997; Resnick 2002; Voukelatos 2015
(walking programme) versus

control

Endurance training versus

control

\begin{abstract}
Other kinds of exercise versus control
\end{abstract}

Multiple categories of exercise versus control
Ansai 2015*; Beyer 2007; Brown 2002; Buchner 1997; Bunout 2005; Cerny 1998; Clemson 2012*; Gill 2016; Halvarsson 2016*; Hauer 2001; Irez 2011; Kamide 2009; Karinkanta 2007*; Kim 2014; Lehtola 2000; Means 2005; Ng 2015; Park 2008; Rubenstein 2000; Suzuki 2004; Uusi-Rasi 2015

\footnotetext{
${ }^{\star}=$ multigroup trial appearing in more than one category.
} 
Appendix 7. Study IDs for the 59 exercise versus control studies included in rate of falls analysis

\begin{tabular}{ll}
\hline Comparison & Study IDs \\
\hline Exercise (all types) versus con- & Ansai 2015*; Arkkukangas 2015; Barnett 2003; Boongrid 2017; Buchner 1997; Bunout 2005; Camp- \\
trol & bell 1997; Carter 2002; Clegg 2014; Clemson 2010; Clemson 2012*; Cornillon 2002; Dadgari 2016; \\
& Day 2002; Day 2015; Duque 2013; Ebrahim 1997; El-Khoury 2015; Grahn Kronhed 2009; Gschwind \\
& 2015; Hamrick 2017; Hirase 2015*; Iliffe 2015*; Irez 2011; Karinkanta 2007*; Kerse 2010; Korpelainen \\
& 2006; Kovacs 2013; Lehtola 2000; Li 2005; Lin 2007; Liu-Ambrose 2004*; Liu-Ambrose 2008; Logghe \\
& 2009; Lord 1995; Lord 2003; Luukinen 2007; Madureira 2007; McMurdo 1997; Means 2005; Merom \\
& 2016; Miko 2017; Nitz 2004; Robertson 2001a; Rubenstein 2000; Sakamoto 2013; Sales 2017; Siegrist \\
& 2016; Skelton 2005; Smulders 2010; Suzuki 2004; Taylor 2012; Trombetti 2011; Uusi-Rasi 2015; \\
& Voukelatos 2007; Voukelatos 2015; Weerdesteyn 2006; Wolf 1996*
\end{tabular}

Balance and functional exercises versus control
Arkkukangas 2015; Barnett 2003; Boongrid 2017; Campbell 1997; Clegg 2014; Clemson 2010; Clemson 2012*; Cornillon 2002; Dadgari 2016; Day 2002; Duque 2013; El-Khoury 2015; Gschwind 2015; Hamrick 2017; Hirase 2015*; Iliffe 2015*; Karinkanta 2007*; Kerse 2010; Korpelainen 2006; Kovacs 2013; Lin 2007; Liu-Ambrose 2004*; Liu-Ambrose 2008; Lord 1995; Lord 2003; Luukinen 2007; Madureira 2007; McMurdo 1997; Miko 2017; Nitz 2004; Robertson 2001a; Sakamoto 2013; Sales 2017; Siegrist 2016; Skelton 2005; Smulders 2010; Trombetti 2011; Weerdesteyn 2006; Wolf 1996*

\section{Resistance exercises versus \\ Ansai 2015*; Carter 2002; Grahn Kronhed 2009; Karinkanta 2007*; Liu-Ambrose 2004* control}

$$
\text { Flexibility versus control }
$$

3D exercise (Tai Chi) versus Day 2015; Li 2005; Logghe 2009; Taylor 2012; Voukelatos 2007; Wolf $1996^{\star}$; Wolf 2003 control

3D exercise (dance) versus Merom 2016
control

\section{General physical activity}

(walking program) versus con-

trol

\section{Endurance training versus}

control

Other kinds of exercise versus

control

Multiple categories of exercise versus control
Ansai 2015*; Buchner 1997; Bunout 2005; Clemson 2012*; Irez 2011; Karinkanta 2007*; Lehtola 2000; Means 2005; Rubenstein 2000; Suzuki 2004; Uusi-Rasi 2015

${ }^{\star}=$ multigroup trial appearing in more than one category

\section{Appendix 8. Number of studies and participants in primary analysis (exercise versus control on rate of falls), by primary category of exercise}

\begin{tabular}{|c|c|c|c|c|}
\hline Comparisona & $\begin{array}{l}\text { Number of trials } \\
\text { (cluster) }^{b}\end{array}$ & $\begin{array}{l}\text { Number of trials } \\
\text { with no }\end{array}$ & $\begin{array}{l}\text { Number of par- } \\
\text { ticipants } \\
\text { randomised }\end{array}$ & $\begin{array}{l}\text { Number of par- } \\
\text { ticipants } \\
\text { analysedd }\end{array}$ \\
\hline
\end{tabular}


(Continued)

\section{secondary ex-}

ercise categori-

esc

\begin{tabular}{|c|c|c|c|c|}
\hline Exercise (all types) versus control & $59(6)$ & 15 & 16363 & 12981 \\
\hline Balance and functional exercises versus control & $39(4)$ & 7 & 9815 & 7920 \\
\hline Resistance exercises versus control & 5 & 3 & 480 & 327 \\
\hline Flexibility versus control & 0 & 0 & 0 & 0 \\
\hline 3D exercise (Tai Chi) versus control & $7(1)$ & 6 & 2794 & 2655 \\
\hline 3D exercise (dance) versus control & $1(1)$ & 0 & 530 & 522 \\
\hline $\begin{array}{l}\text { General physical activity (walking program) versus } \\
\text { control }\end{array}$ & 2 & 2 & 551 & 441 \\
\hline Endurance training versus control & 0 & 0 & 0 & 0 \\
\hline Other kinds of exercise versus control & 0 & 0 & 0 & 0 \\
\hline Multiple categories of exercise versus control & 11 & $\mathrm{~N} / \mathrm{A}$ & 1783 & 1374 \\
\hline
\end{tabular}

aExercise (all types) combines all categories of exercise. Multiple categories of exercise include studies containing two or more primary categories of exercise, as categorised using the ProFaNE taxonomy. The remaining analyses include only one primary category of exercise, as categorised using the ProFaNE taxonomy.

bStudy IDs are shown in Appendix 7.

CThe number of trials where the intervention programme did not include a secondary exercise from another exercise category using the ProFaNE taxonomy.

dThese data apply to the follow-up (at the time point included in main analysis) for the primary outcome (rate of falls) for the individual trials 


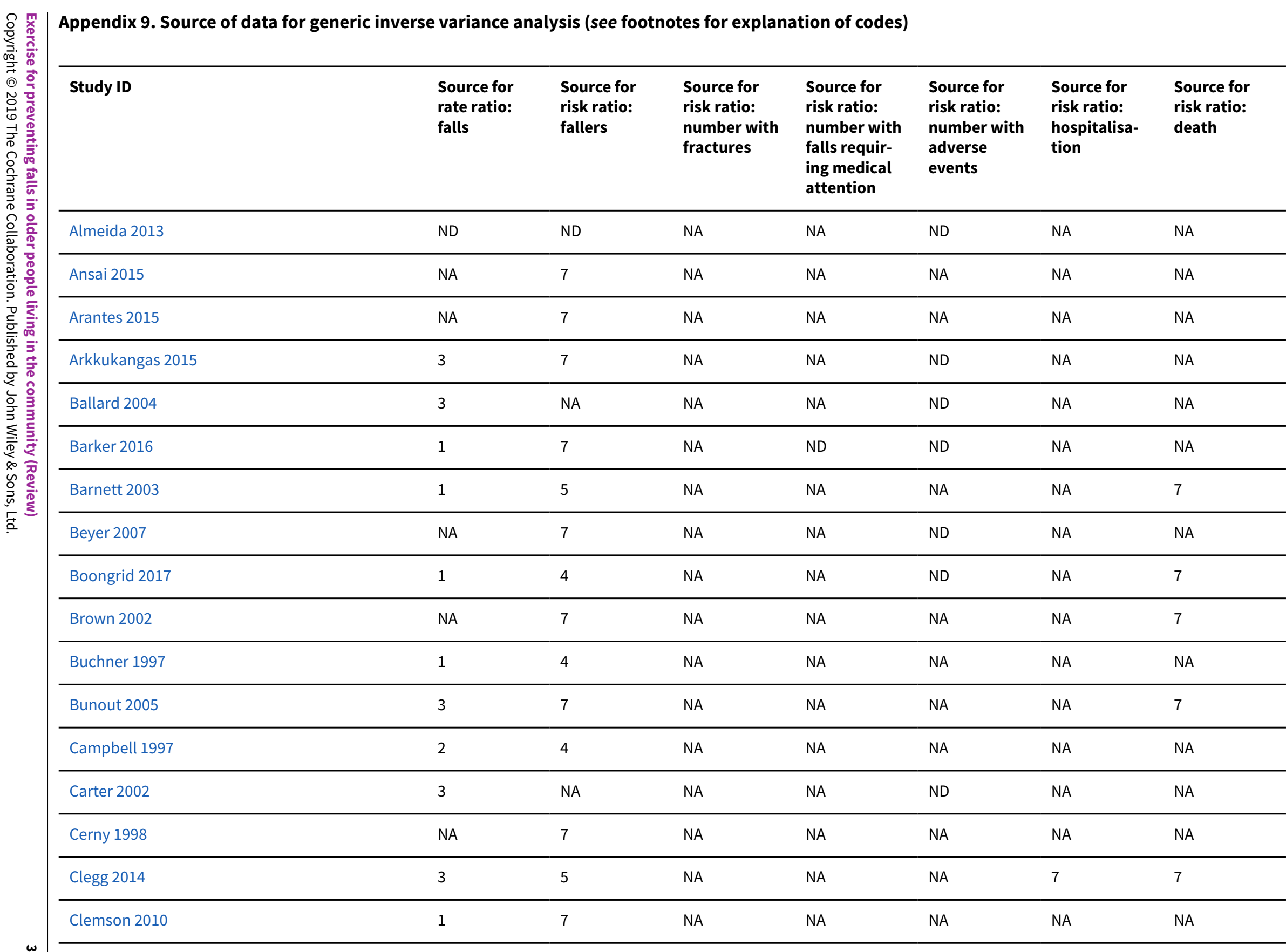




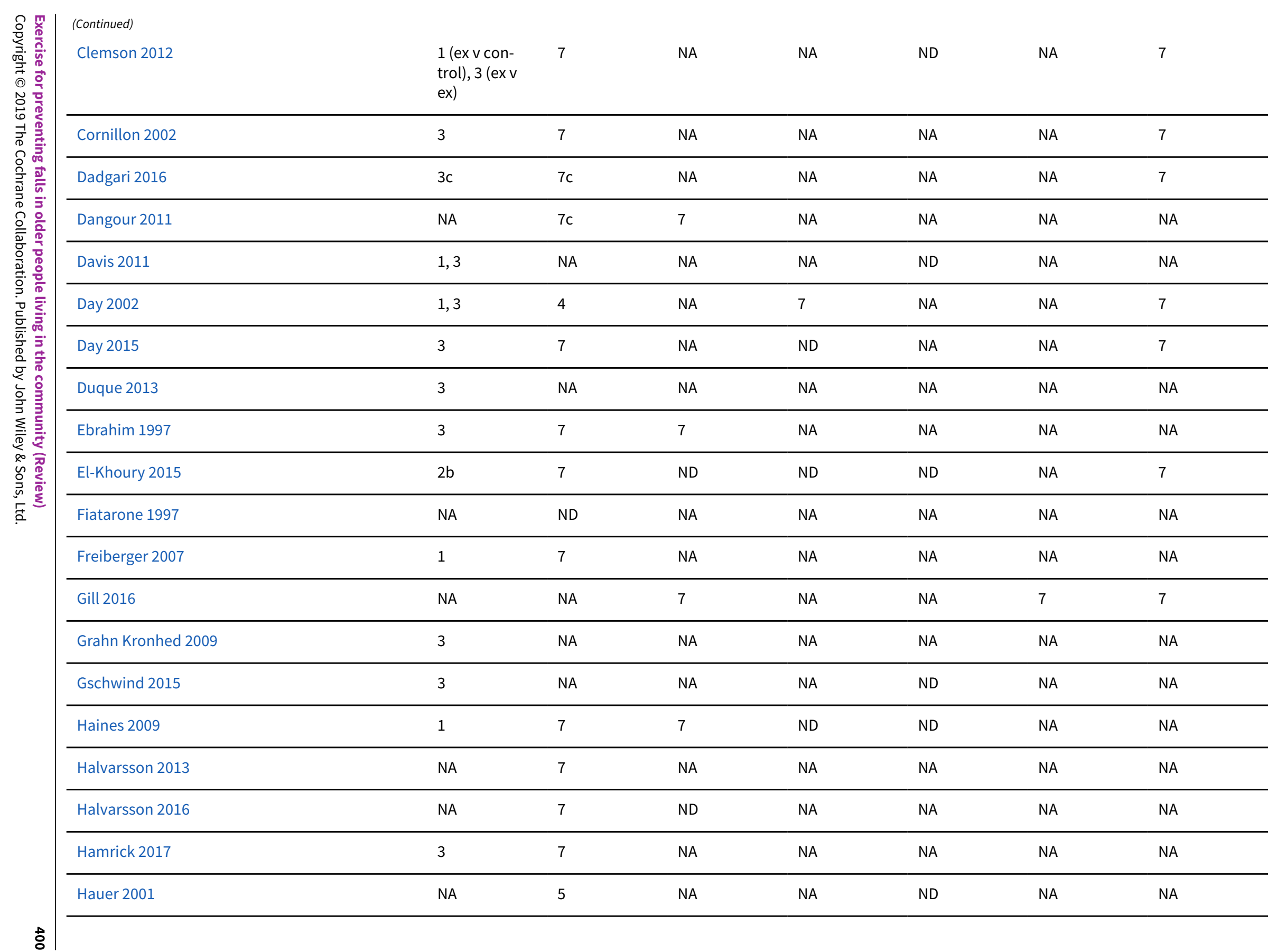




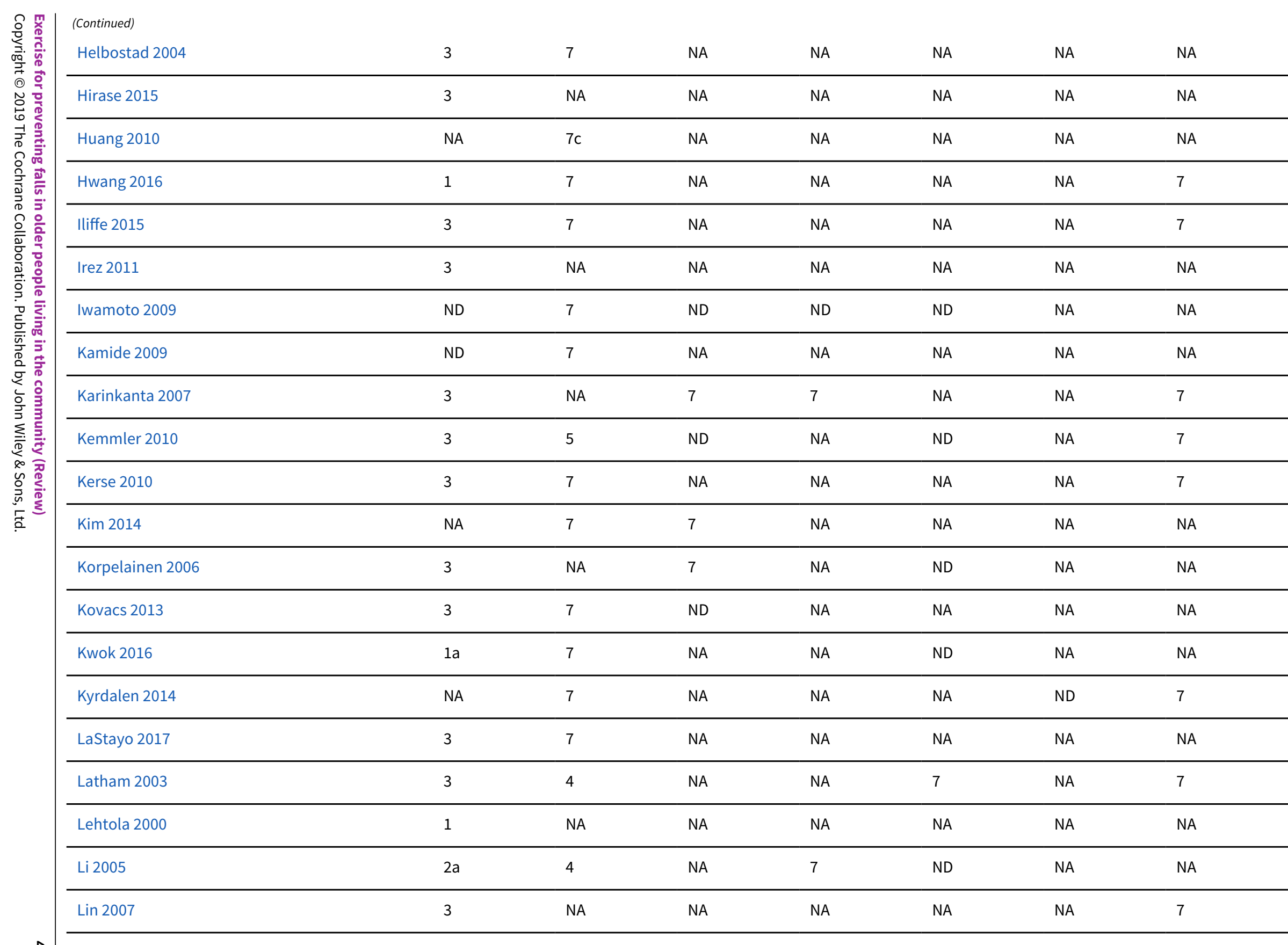




\begin{tabular}{|c|c|c|c|c|c|c|c|}
\hline \multicolumn{8}{|l|}{ 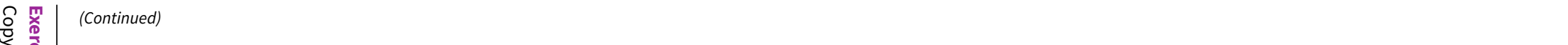 } \\
\hline 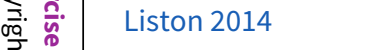 & 3 & ND & NA & NA & NA & NA & NA \\
\hline Liu-Ambrose 2004 & 3 & ND & NA & NA & ND & NA & NA \\
\hline Liu-Ambrose 2008 & 1 & 7 & NA & NA & NA & NA & 7 \\
\hline Logghe 2009 & 2 & 7 & NA & NA & NA & NA & 7 \\
\hline Lord 1995 & 3 & 5 & NA & NA & NA & NA & 7 \\
\hline Lord 2003 & $1 a$ & 7 & NA & NA & NA & NA & 7 \\
\hline Lurie 2013 & NA & 7 & NA & NA & NA & NA & NA \\
\hline Luukinen 2007 & 2 & 7 & NA & NA & NA & NA & NA \\
\hline Madureira 2007 & 3 & NA & NA & NA & NA & NA & NA \\
\hline McMurdo 1997 & 3 & 7 & 7 & NA & NA & NA & NA \\
\hline Means 2005 & 3 & 7 & NA & NA & ND & NA & 7 \\
\hline Merom 2016 & $1 b$ & $5 b$ & NA & NA & ND & NA & 7 \\
\hline Miko 2017 & 3 & 7 & NA & NA & NA & NA & NA \\
\hline Mirelman 2016 & ND & NA & NA & NA & ND & NA & NA \\
\hline Morgan 2004 & NA & 7 & NA & NA & NA & NA & NA \\
\hline Morone 2016 & ND & ND & NA & NA & NA & NA & NA \\
\hline Morrison 2018 & NA & ND & NA & NA & NA & NA & NA \\
\hline Ng 2015 & NA & 7 & NA & NA & ND & NA & 7 \\
\hline Nitz 2004 & 3 & NA & NA & NA & ND & NA & NA \\
\hline Okubo 2016 & NA & NA & NA & NA & NA & NA & NA \\
\hline Reinsch 1992 & NA & $7 c$ & NA & ND & ND & NA & NA \\
\hline
\end{tabular}




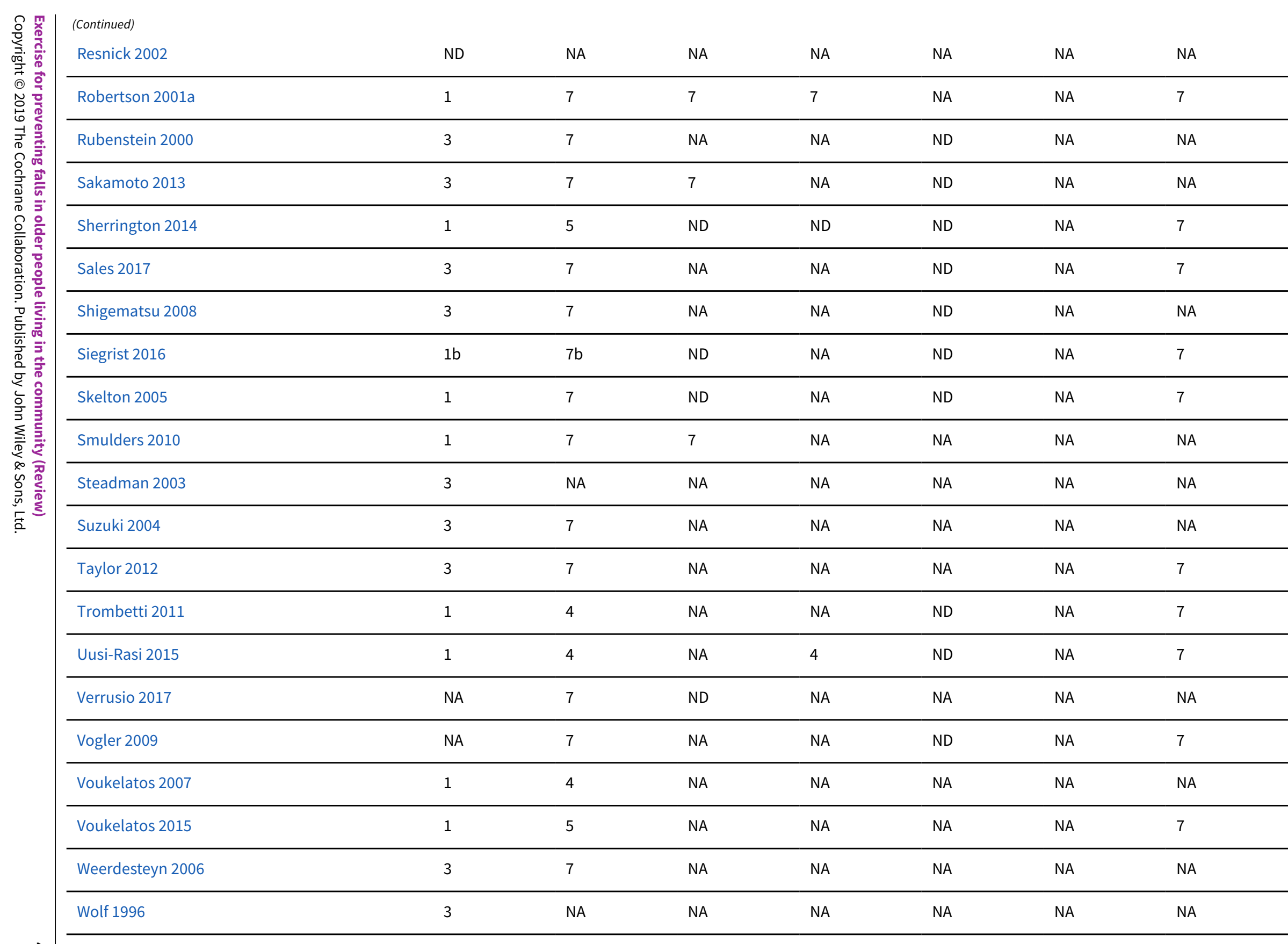




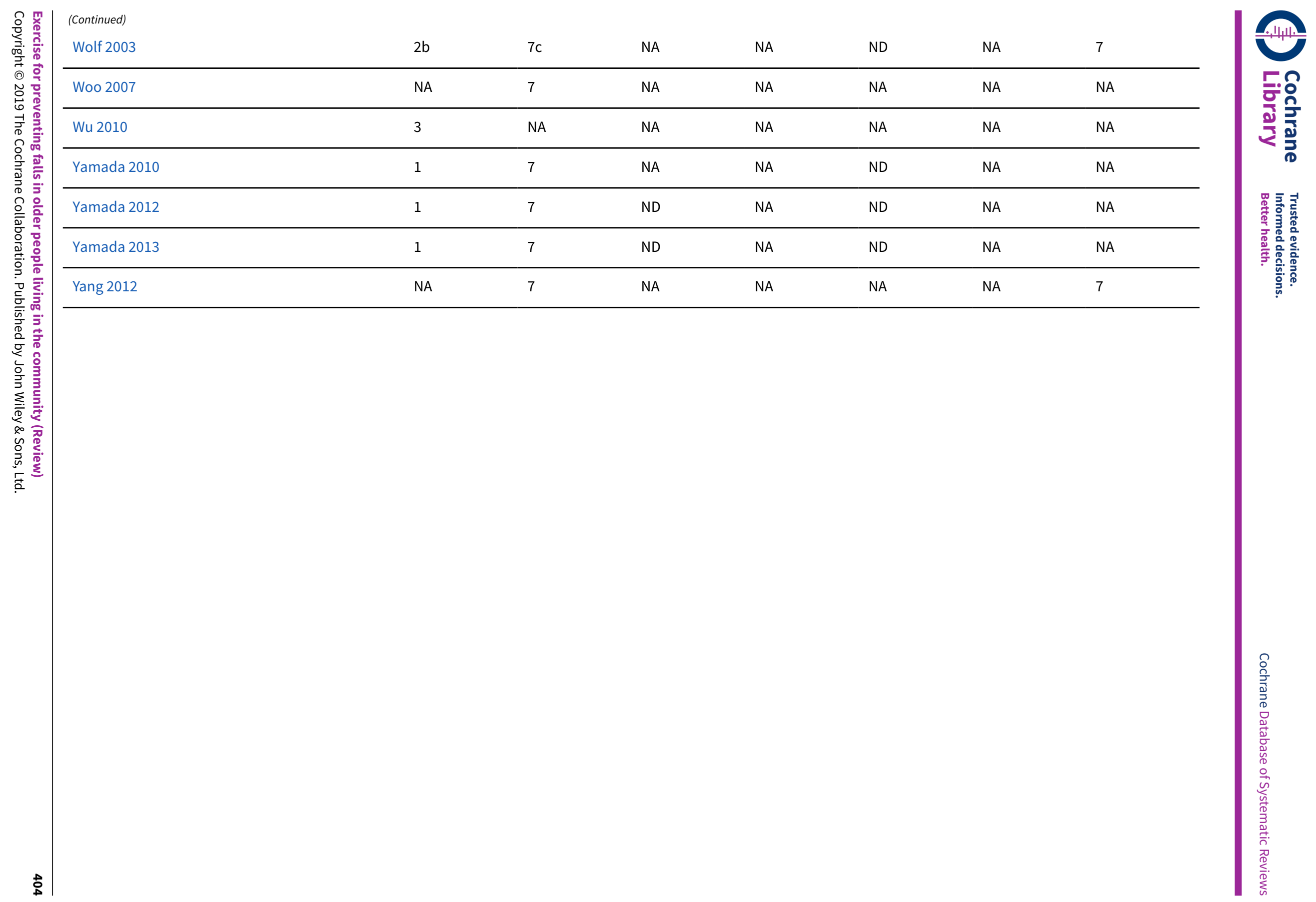




\section{Abbreviations:}

\section{Codes for source of rate ratio}

1: incidence rate ratio reported by trial authors

2: hazard ratio/relative hazard (multiple events) reported by trial authors

3: incidence rate ratio calculated by review authors

a: adjusted for confounders by trial authors

$\mathrm{b}$ : adjusted for clustering by trial authors

c: adjusted for clustering by review authors

\section{Codes for source of risk ratio:}

4: hazard ratio/relative hazard (first fall only) reported by trial authors

5: relative risk reported by trial authors

6: odds ratio reported by trial authors

7: relative risk calculated by review authors

a: adjusted for confounders by trial authors

b: adjusted for clustering by trial authors

c: adjusted for clustering by review authors

NA: not applicable. Falls (for rate ratio) or fallers (for risk ratio) or number of people sustaining a fracture (for risk ratio) or number with falls requiring medical attention (for risk ratio) or number with adverse events (for risk ratio) or number of people with falls requiring hospital admission (for risk ratio) or death (for risk ratio) not reported as an outcome in the trial

ND: outcomes relating to falls or fallers or fractures or falls requiring medical attention or adverse events or hospital admission or death were reported, but there were no useable data 







\begin{tabular}{|c|c|c|c|c|c|c|c|c|}
\hline 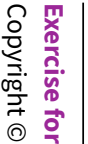 & $\begin{array}{l}\text { (Continued) } \\
\text { Clemson } 2012 \\
\text { LiFE/ structured vs control }\end{array}$ & $1.66 / 1.90$ & 2.28 & $60 / 65$ & $105 / 107$ & 71 & 106 & $12 \mathrm{mo}$ \\
\hline$\stackrel{\infty}{\infty}_{0}^{0}$ & Cornillon 2002 & 0.39 & 0.47 & 39 & 150 & 48 & 153 & $12 \mathrm{mo}$ \\
\hline 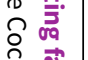 & Dadgari 2016 & 2.52 & 3.28 & 138 & 160 & 154 & 157 & $6 \mathrm{mo}$ \\
\hline$\overline{5}$ & Dangour 2011 & - & - & 189 & 325 & 198 & 294 & $24 \mathrm{mo}$ \\
\hline 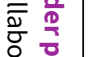 & Davis 2011 & $0.74 / 0.82$ & 1.06 & - & $52 / 54$ & - & 49 & $9 \mathrm{mo}$ \\
\hline$\frac{\circ}{0}$ & x1/x2 wkly resistance vs balance/tone & & & & & & & \\
\hline 吉े & Day 2002 & 1.05 & 1.20 & 76 & 135 & 87 & 137 & $18 \mathrm{mo}$ \\
\hline 焉富 & Day 2015 & 0.62 & 0.58 & 65 & 204 & 64 & 205 & $12 \mathrm{mo}$ \\
\hline 울 丞 & Duque 2013 & 2.2 & 4 & - & 30 & - & 40 & $9 \mathrm{mo}$ \\
\hline$\underbrace{\frac{D}{\infty}}_{\infty}$ & Ebrahim 1997 & $0.80 / 0.70$ & $0.52 / 0.55$ & $25 /-$ & $52 / 49$ & $18 /-$ & $50 / 48$ & $24 \mathrm{mo}$ \\
\hline 年. & $12 \mathrm{mo} / 24 \mathrm{mo}$ vs control & & & & & & & \\
\hline & El-Khoury 2015 & 0.79 & 0.92 & 189 & 352 & 222 & 354 & $24 \mathrm{mo}$ \\
\hline & Freiberger 2007 & $0.90 /-$ & $1.22 /-$ & $19 /-$ & $65 / 48$ & $29 /-$ & $62 / 49$ & $24 \mathrm{mo}$ \\
\hline & $12 \mathrm{mo} / 24 \mathrm{mo}$ & & & & & & & \\
\hline & Fitness vs strength \& balance & & & & & & & \\
\hline & Gill 2016 & - & - & - & 818 & - & 817 & $42 \mathrm{mo}$ \\
\hline & Grahn Kronhed 2009 & 0.6 & 0.8 & - & 31 & - & 34 & $12 \mathrm{mo}$ \\
\hline & Gschwind 2015 & 0.25 & 0.50 & - & 71 & - & 65 & $6 \mathrm{mo}$ \\
\hline & Haines 2009 & - & - & 11 & 19 & 20 & 34 & $6 \mathrm{mo}$ \\
\hline & Halvarsson 2013 & - & - & 18 & 30 & 2 & 18 & $15 \mathrm{mo}$ \\
\hline & Halvarsson 2016 & - & - & $4 / 5$ & $25 / 18$ & 4 & 26 & $3 \mathrm{mo}$ \\
\hline & balance/ balance+walking vs control & & & & & & & \\
\hline
\end{tabular}




\begin{tabular}{|c|c|c|c|c|c|c|c|c|}
\hline \multirow{22}{*}{ 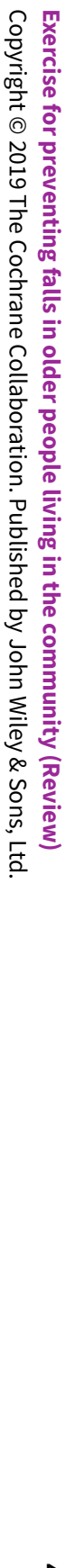 } & \multicolumn{8}{|l|}{ (Continued) } \\
\hline & Hamrick 2017 & 0.63 & 0.84 & 4 & 19 & 7 & 19 & $6 \mathrm{mo}$ \\
\hline & Hauer 2001 & - & - & 14 & 31 & 15 & 25 & $6 \mathrm{mo}$ \\
\hline & Helbostad 2004 & 1.45 & 1.33 & 20 & 34 & 18 & 34 & $12 \mathrm{mo}$ \\
\hline & $\begin{array}{l}\text { Hirase } 2015 \\
\text { foam/ stable surface vs control }\end{array}$ & $0.72 / 1.77$ & 2.7 & - & $29 / 29$ & - & $\begin{array}{l}28 \text { (14 in } \\
\text { analysis) }\end{array}$ & $4 \mathrm{mo}$ \\
\hline & Huang 2010 & - & - & 0 & 31 & 2 & 47 & $5 \mathrm{mo}$ \\
\hline & Hwang 2016 & 0.08 & 0.16 & 72 & 167 & 99 & 167 & $18 \mathrm{mo}$ \\
\hline & \multicolumn{8}{|l|}{ Tai Chi vs lower extremity } \\
\hline & Iliffe 2015 & $0.64 / 0.66$ & 0.76 & - & $230 / 227$ & - & 252 & $30 \mathrm{mo}$ \\
\hline & \multicolumn{8}{|l|}{ FAME/ OEP vs control, (18 mo/30mo) } \\
\hline & Irez 2011 & 1.48 & 5.2 & - & 30 & - & 30 & $3 \mathrm{mo}$ \\
\hline & Iwamoto 2009 & 0.00 & 0.29 & 0 & 34 & 4 & 33 & $5 \mathrm{mo}$ \\
\hline & Kamide 2009 & - & - & 0 & 20 & 1 & 23 & $6 \mathrm{mo}$ \\
\hline & Karinkanta 2007 & $0.51 / 0.21 / 0.53$ & 0.36 & - & $35 / 37 / 36$ & - & 36 & $12 \mathrm{mo}$ \\
\hline & \multicolumn{8}{|l|}{ balance/resistance/bal+resistance vs control } \\
\hline & Kemmler 2010 & 0.17 & 0.28 & - & 112 & - & 115 & $18 \mathrm{mo}$ \\
\hline & Kerse 2010 & 0.48 & 0.41 & 47 & 98 & 39 & 95 & $12 \mathrm{mo}$ \\
\hline & Kim 2014 & - & - & 10 & 51 & 21 & 52 & $12 \mathrm{mo}$ \\
\hline & Korpelainen 2006 & 0.42 & 0.53 & - & 84 & - & 76 & $30 \mathrm{mo}$ \\
\hline & Kovacs 2013 & 0.42 & 0.17 & 6 & 36 & 15 & 36 & $12 \mathrm{mo}$ \\
\hline & Kwok 2016 & - & - & 8 & 40 & 11 & 40 & $12 \mathrm{mo}$ \\
\hline & Kyrdalen 2014 & - & - & 19 & 47 & 17 & 47 & $3 \mathrm{mo}$ \\
\hline
\end{tabular}




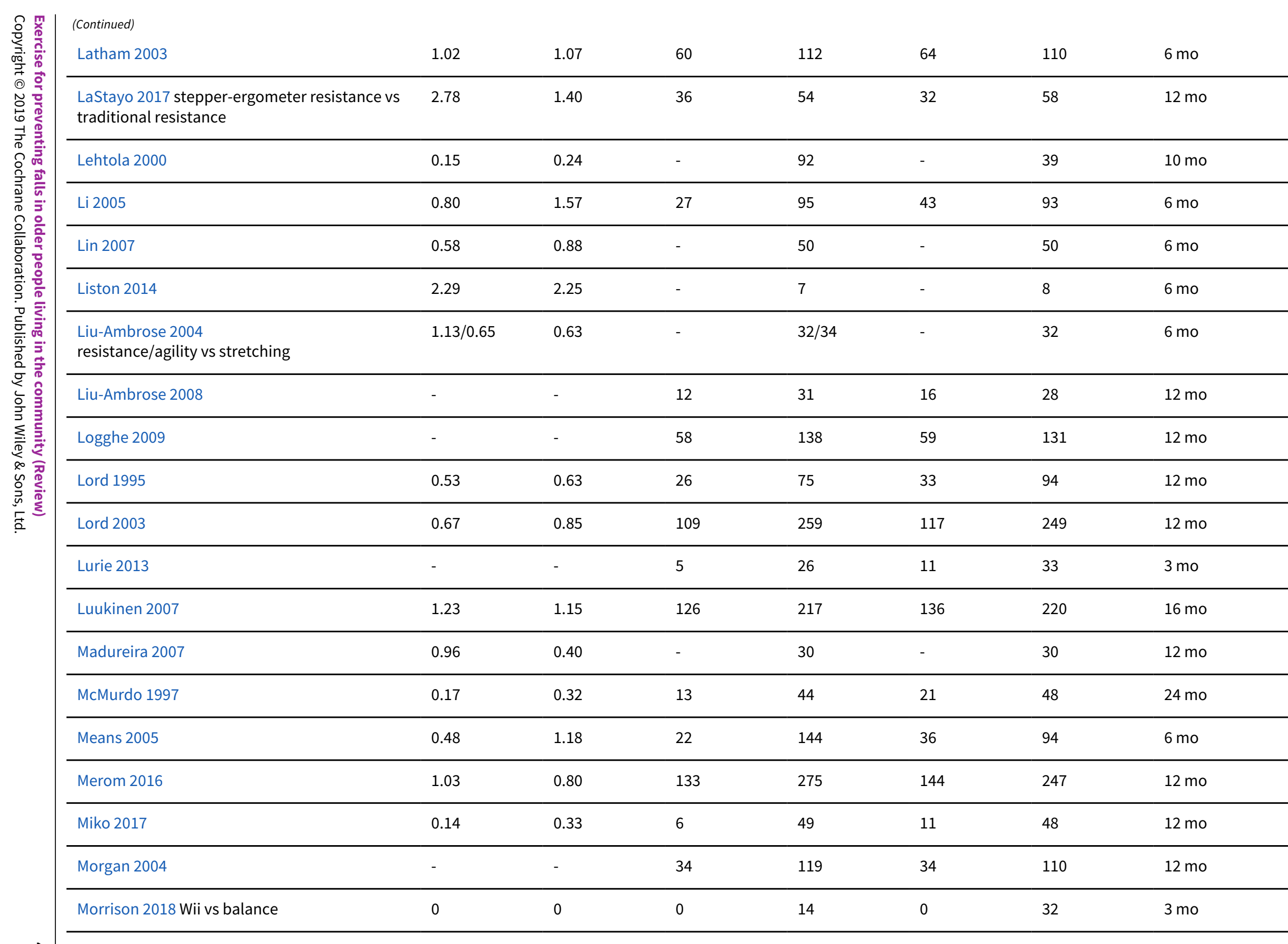




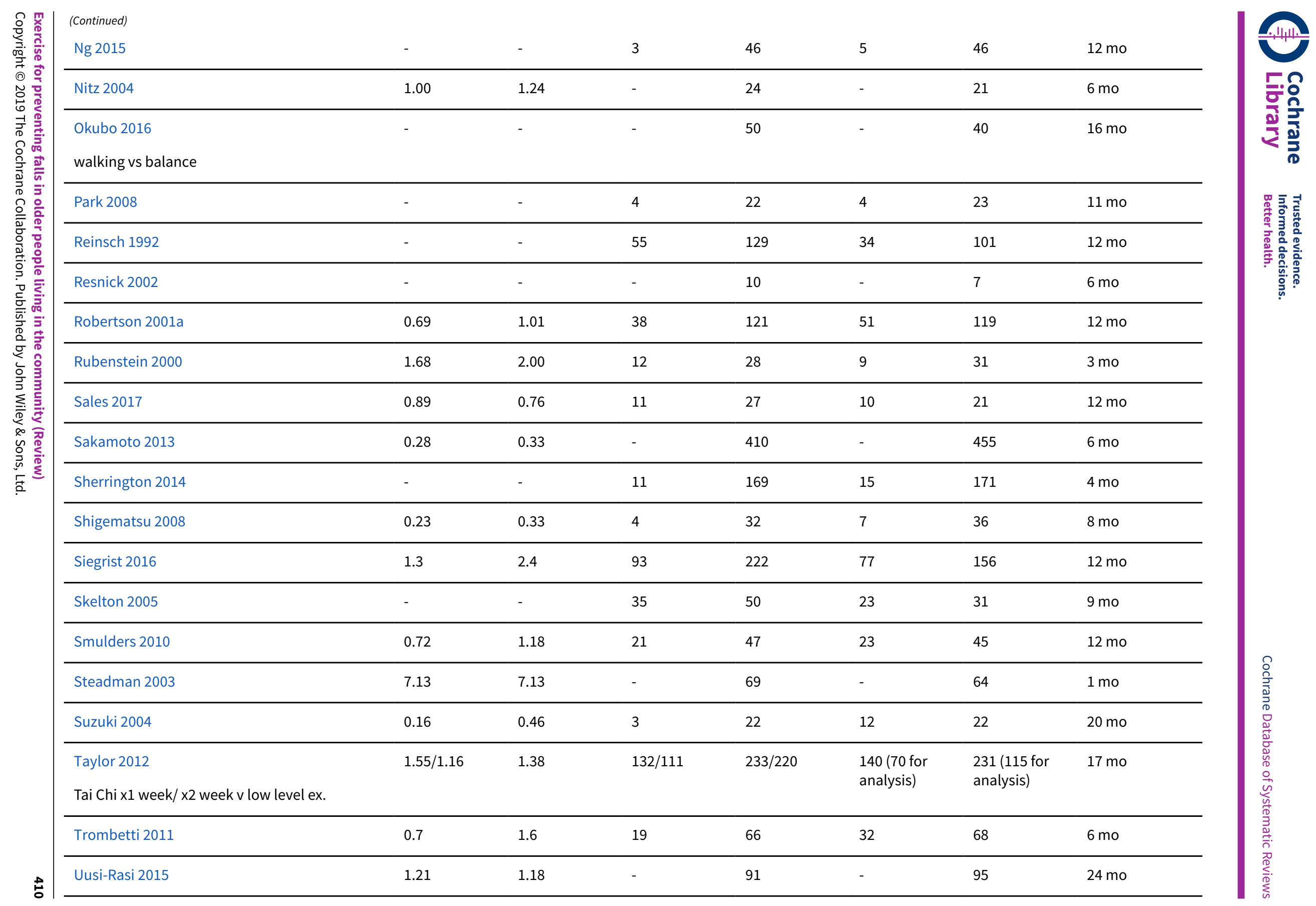




\begin{tabular}{|c|c|c|c|c|c|c|c|c|c|}
\hline 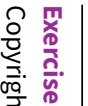 & $\begin{array}{l}\text { (Continued) } \\
\text { Verrusio } 2017\end{array}$ & - & - & 6 & 73 & 19 & 74 & $12 \mathrm{mo}$ & 0.1111 \\
\hline 雚 & Vogler 2009 & & & & & & & & \\
\hline$\stackrel{0}{\frac{0}{3}}$ & Voukelatos 2007 & 0.50 & 0.75 & 71 & 347 & 81 & 337 & $6 \mathrm{mo}$ & \\
\hline 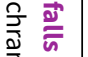 & Voukelatos 2015 & - & - & 54 & 159 & 68 & 180 & $12 \mathrm{mo}$ & \\
\hline$\stackrel{\varrho}{\equiv} \div$ & Weerdesteyn 2006 & 0.89 & 1.68 & 10 & 30 & 9 & 28 & $7 \mathrm{mo}$ & 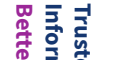 \\
\hline 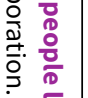 & $\begin{array}{l}\text { Wolf } 1996 \\
\text { Tai Chi/ balance training vs education }\end{array}$ & $0.86 / 1.53$ & 1.29 & - & $72 / 64$ & - & 64 & $8 \mathrm{mo}$ & 0 \\
\hline 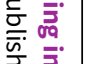 & Wolf 2003 & - & - & 69 & 145 & 85 & 141 & $11 \mathrm{mo}$ & \\
\hline 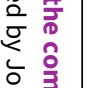 & $\begin{array}{l}\text { Woo } 2007 \\
\text { Tai Chi/ resistance vs control }\end{array}$ & - & - & $15 / 24$ & $58 / 59$ & $31 / 31$ & 59 & $12 \mathrm{mo}$ & \\
\hline 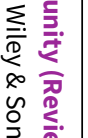 & $\begin{array}{l}\text { Wu } 2010 \\
\text { Telecommunication-based Tai Chi/ home- } \\
\text { based Tai Chi vs group Tai Chi }\end{array}$ & $0.47 / 0.94$ & 0.35 & - & $22 / 22$ & - & 20 & $4 \mathrm{mo}$ & \\
\hline & Yamada 2010 & - & - & 5 & 29 & 11 & 29 & $12 \mathrm{mo}$ & \\
\hline & Yamada 2012 & - & - & 19 & 73 & 2 & 72 & $12 \mathrm{mo}$ & \\
\hline & Yamada 2013 & - & - & 13 & 112 & 39 & 118 & $12 \mathrm{mo}$ & \\
\hline & Yang 2012 & - & - & 12 & 59 & 18 & 62 & $6 \mathrm{mo}$ & \\
\hline
\end{tabular}


mo: months 


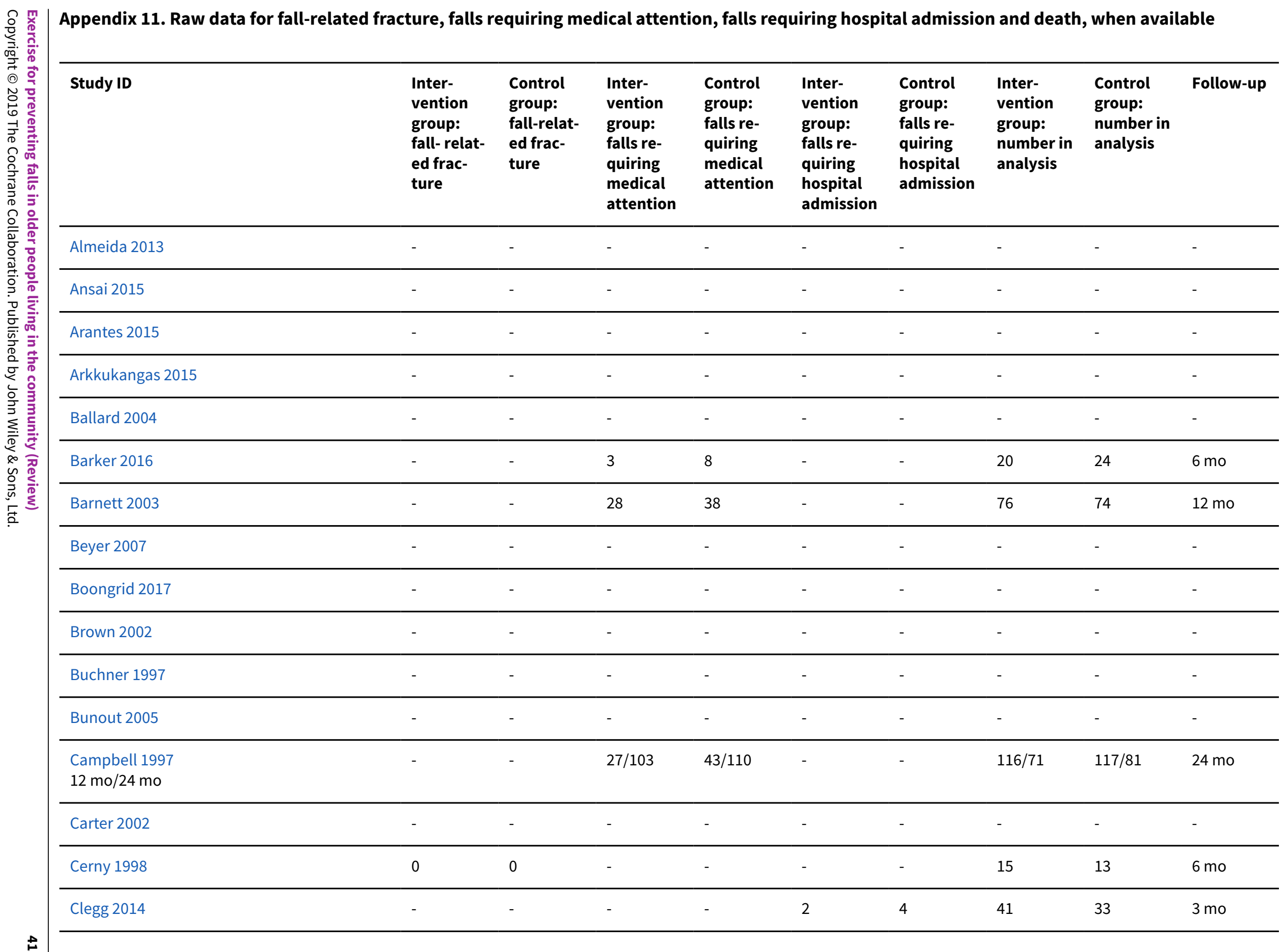




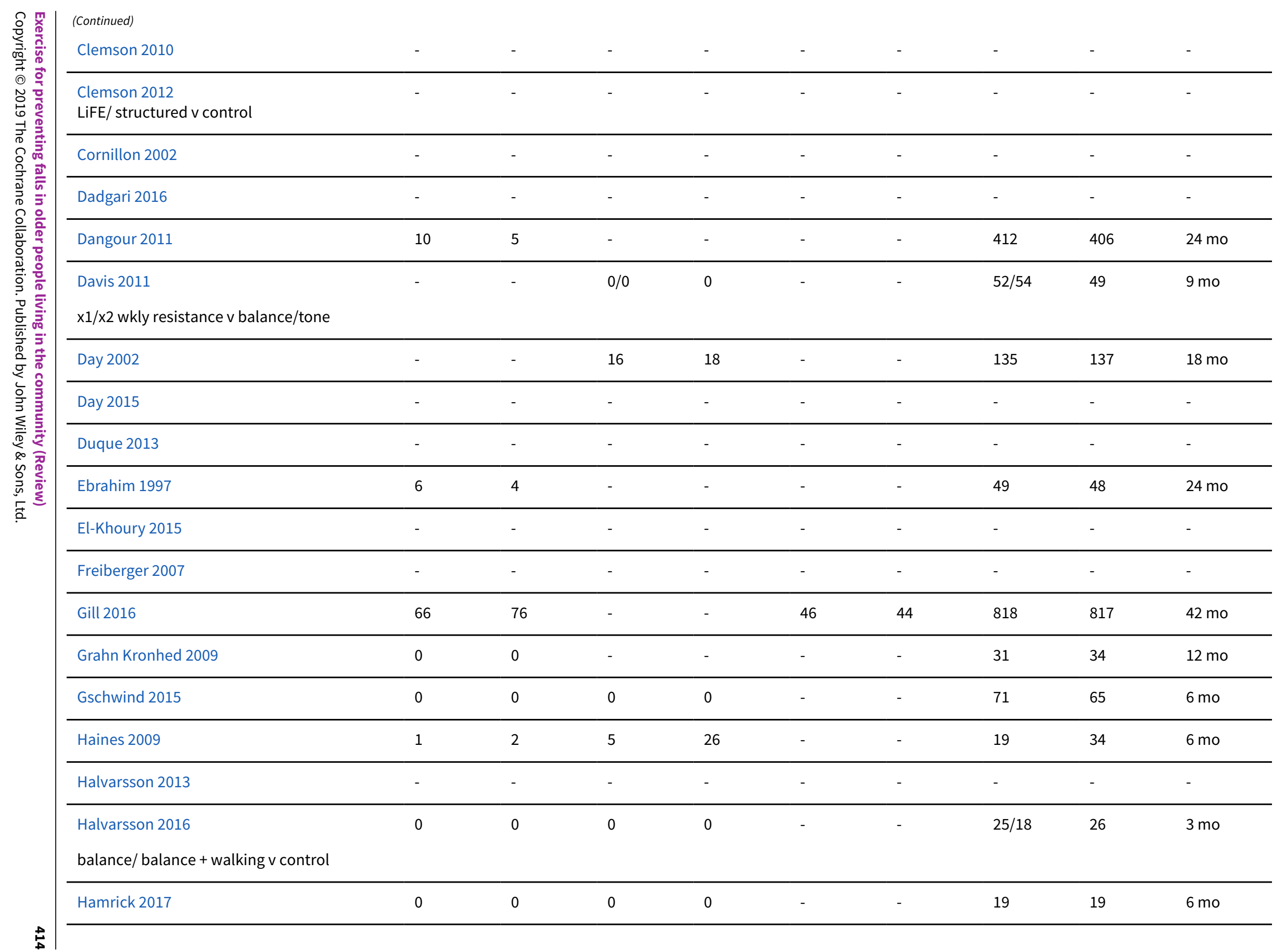




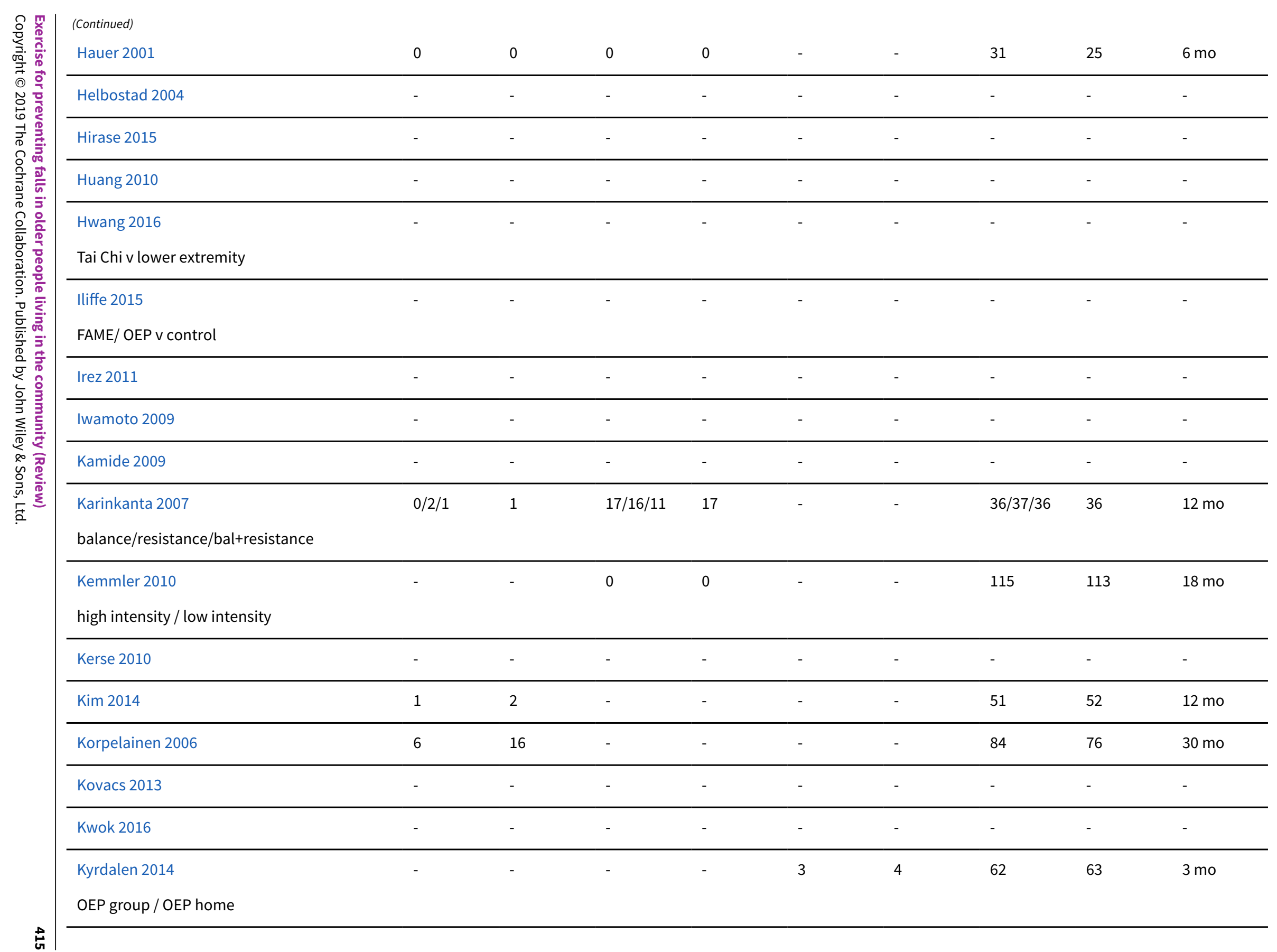




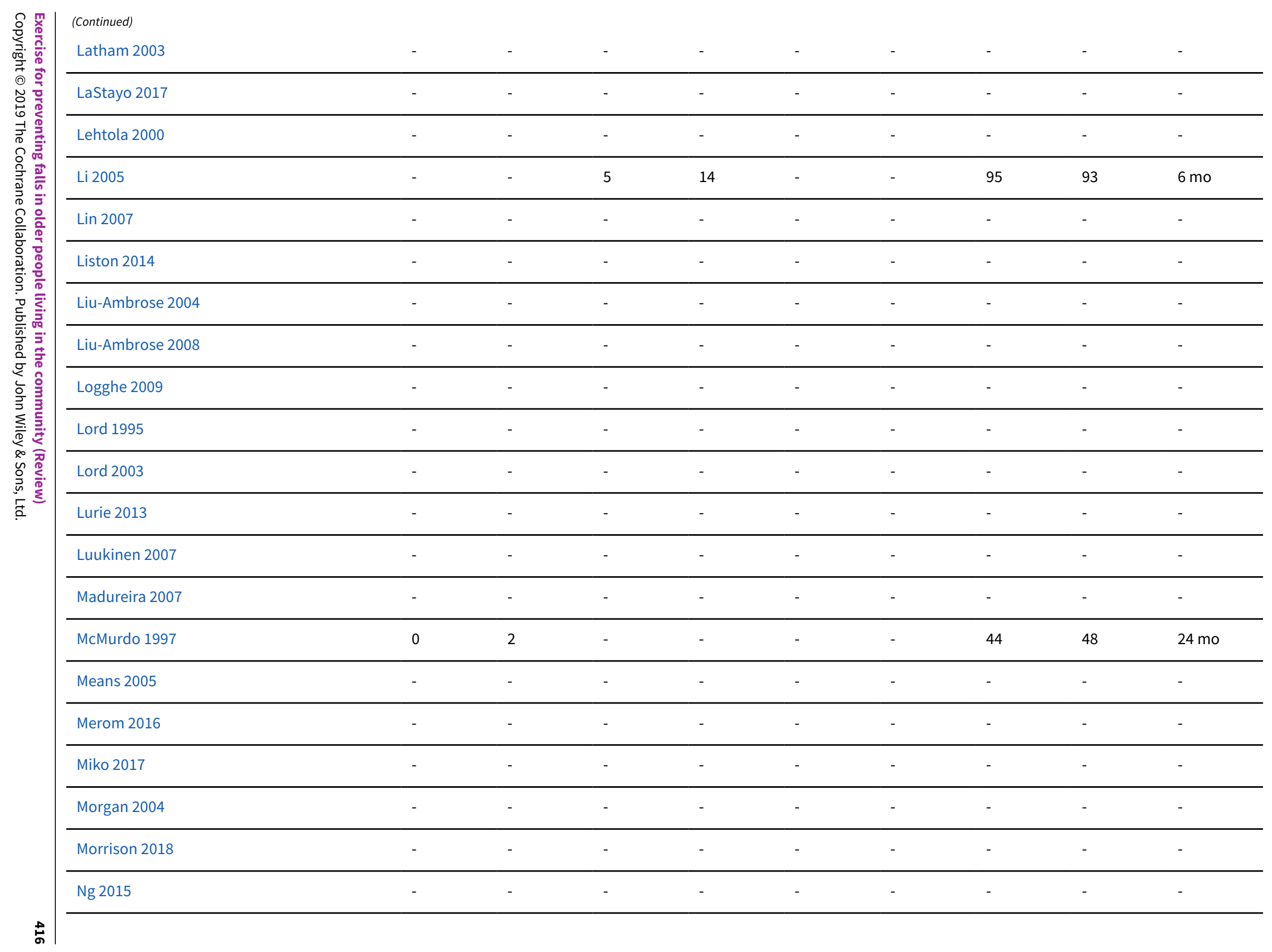




\begin{tabular}{|c|c|c|c|c|c|c|c|c|c|c|}
\hline 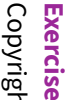 & $\begin{array}{l}\text { (Continued) } \\
\text { Nitz } 2004\end{array}$ & - & - & - & - & - & - & - & - & - \\
\hline خृ & Okubo 2016 & - & - & - & - & - & - & - & - & \\
\hline 永㗭. & Park 2008 & - & - & - & - & - & - & - & - & - \\
\hline 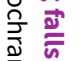 & Reinsch 1992 & - & - & 4 & 1 & - & - & 129 & 101 & $12 \mathrm{mo}$ \\
\hline 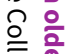 & Resnick 2002 & - & - & - & - & - & - & - & - & - \\
\hline 垔 & Robertson 2001a & 2 & 7 & 18 & 26 & - & - & 114 & 104 & $12 \mathrm{mo}$ \\
\hline$\stackrel{0}{\vdots}$ & Rubenstein 2000 & 0 & 0 & 0 & 0 & - & - & 28 & 31 & $3 \mathrm{mo}$ \\
\hline 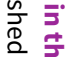 & Sales 2017 & - & - & - & - & - & - & - & - & - \\
\hline$\frac{9}{3}$ & Sakamoto 2013 & 4 & 11 & - & - & - & - & 410 & 455 & $6 \mathrm{mo}$ \\
\hline$\sum_{\substack{0 \\
0}}^{5}$ & Sherrington 2014 & 14 & 15 & 61 & 53 & - & - & 171 & 169 & $4 \mathrm{mo}$ \\
\hline$\widehat{\varrho}$ & Shigematsu 2008 & - & - & - & - & - & - & - & - & - \\
\hline & Siegrist 2016 & - & - & - & - & - & - & - & - & - \\
\hline & Skelton 2005 & $\mathrm{NDa}$ & $\mathrm{NDa}$ & - & - & - & - & - & - & $9 \mathrm{mo}$ \\
\hline & Smulders 2010 & 1 & 3 & 0 & 2 & - & - & 47 & 45 & $12 \mathrm{mo}$ \\
\hline & Steadman 2003 & - & - & - & - & - & - & - & - & - \\
\hline & Suzuki 2004 & 0 & 0 & - & - & - & - & 22 & 22 & $20 \mathrm{mo}$ \\
\hline & Taylor 2012 & - & - & - & - & - & - & - & - & - \\
\hline & Tai Chi x1 week/ x & & & & & & & & & \\
\hline & Trombetti 2011 & - & - & - & - & - & - & - & - & - \\
\hline & Uusi-Rasi 2015 & - & - & $\mathrm{HR}$ & $\mathrm{HR}$ & - & - & 91 & 97 & $24 \mathrm{mo}$ \\
\hline & Verrusio 2017 & - & - & - & - & - & - & - & - & - \\
\hline A & Vogler 2009 & - & - & - & - & - & - & - & - & - \\
\hline
\end{tabular}




\begin{tabular}{|c|c|c|c|c|c|c|c|c|c|c|c|}
\hline 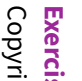 & $\begin{array}{l}\text { (Continued) } \\
\text { seated v weightbea }\end{array}$ & & & & & & & & & & , \\
\hline$\stackrel{\overrightarrow{0}}{\vec{T}}$ & Voukelatos 2007 & - & - & - & - & - & - & - & - & - & $\Gamma$ \\
\hline 离 & Voukelatos 2015 & - & - & - & - & - & - & - & - & - & \\
\hline 离 & Weerdesteyn 2006 & - & - & - & - & - & - & - & - & - & $\bar{D}$ \\
\hline $\begin{array}{ll}\overrightarrow{0} & \overline{3} \\
& 0 \\
0 & 0\end{array}$ & Wolf 1996 & - & - & - & - & - & - & - & - & - & 罥云 \\
\hline$\frac{1}{0}$ & Wolf 2003 & - & - & - & - & - & - & - & - & - & (q) \\
\hline 象 & Woo 2007 & - & - & - & - & - & - & - & - & - & \\
\hline$\frac{0}{\bar{m}} \cdot \frac{0}{5}$ & Wu 2010 & - & - & - & - & - & - & - & - & - & \\
\hline 足 & Yamada 2010 & - & - & - & - & - & - & - & - & - & \\
\hline 空. & Yamada 2012 & 1 & 8 & - & - & - & - & 73 & 72 & $12 \mathrm{mo}$ & \\
\hline 央石 & Yamada 2013 & 3 & 13 & - & - & - & - & 112 & 118 & $12 \mathrm{mo}$ & \\
\hline 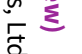 & Yang 2012 & - & - & - & - & - & - & - & - & - & \\
\hline
\end{tabular}


mo: months; HR: hazard ratio data only; NDa: no data presented by group

\section{Appendix 12. Raw data for death, when available}

\begin{tabular}{|c|c|c|c|c|c|}
\hline Study ID & $\begin{array}{l}\text { Intervention } \\
\text { group: death }\end{array}$ & $\begin{array}{l}\text { Control } \\
\text { group: death }\end{array}$ & $\begin{array}{l}\text { Intervention } \\
\text { group: num- } \\
\text { ber in analy- } \\
\text { sis }\end{array}$ & $\begin{array}{l}\text { Control } \\
\text { group: num- } \\
\text { ber in analy- } \\
\text { sis }\end{array}$ & Follow-up \\
\hline Barnett 2003 & 0 & 3 & 76 & 74 & $12 \mathrm{mo}$ \\
\hline Boongrid 2017 & 0 & 1 & 219 & 220 & $12 \mathrm{mo}$ \\
\hline Brown 2002 & 0 & 3 & 46 & 47 & $14 \mathrm{mo}$ \\
\hline Bunout 2005 & 3 & 3 & 111 & 133 & $12 \mathrm{mo}$ \\
\hline Clegg 2014 & 1 & 3 & 41 & 33 & $3 \mathrm{mo}$ \\
\hline $\begin{array}{l}\text { Clemson } 2012 \\
\text { LiFE/structured vs control }\end{array}$ & $1 / 3$ & 3 & $100 / 99$ & 94 & $12 \mathrm{mo}$ \\
\hline Cornillon 2002 & 1 & 0 & 150 & 153 & $12 \mathrm{mo}$ \\
\hline Dangour 2011 & 9 & 6 & 412 & 406 & $24 \mathrm{mo}$ \\
\hline Day 2002 & NRa & NRa & 135 & 137 & $18 \mathrm{mo}$ \\
\hline Day 2015 & 1 & 4 & 204 & 205 & $12 \mathrm{mo}$ \\
\hline El-Khoury 2015 & 5 & 6 & 352 & 354 & $24 \mathrm{mo}$ \\
\hline Gill 2016 & 42 & 37 & 818 & 817 & $42 \mathrm{mo}$ \\
\hline Haines 2009c & 0 & 3 & 19 & 34 & $6 \mathrm{mo}$ \\
\hline Hwang 2016 & 2 & 3 & 169 & 170 & $18 \mathrm{mo}$ \\
\hline \multicolumn{6}{|l|}{ Tai Chi vs lower extremity } \\
\hline Iliffe 2015 & $3 / 3$ & 4 & $243 / 256$ & 274 & $18 \mathrm{mo}$ \\
\hline \multicolumn{6}{|l|}{ FAME/OEP vs control } \\
\hline Karinkanta 2007 & $1 / 0 / 0$ & 1 & $36 / 37 / 36$ & 36 & $12 \mathrm{mo}$ \\
\hline balance/resistance/bal+resi & & & & & \\
\hline Kemmler 2010 & 0 & 1 & 115 & 113 & $18 \mathrm{mo}$ \\
\hline \multicolumn{6}{|l|}{ high intensity/low intensity } \\
\hline Kerse 2010 & 1 & 4 & 92 & 95 & $12 \mathrm{mo}$ \\
\hline Kyrdalen 2014 & 6 & 3 & 62 & 63 & $3 \mathrm{mo}$ \\
\hline OEP group/OEP home & & & & & \\
\hline
\end{tabular}


(Continued)

\begin{tabular}{|c|c|c|c|c|c|}
\hline Latham $2003 \mathrm{c}$ & 6 & 8 & 118 & 118 & $6 \mathrm{mo}$ \\
\hline Lin 2007 & 2 & 0 & 45 & 45 & $6 \mathrm{mo}$ \\
\hline Liu-Ambrose 2008 & 1 & 2 & 31 & 27 & $12 \mathrm{mo}$ \\
\hline Logghe 2009 & 1 & 0 & 127 & 117 & $12 \mathrm{mo}$ \\
\hline Lord 1995 & $N R^{b}$ & $N R^{b}$ & 75 & 94 & $12 \mathrm{mo}$ \\
\hline Lord 2003 & 5 & 1 & 264 & 250 & $12 \mathrm{mo}$ \\
\hline Means 2005 & 4 & 4 & 148 & 98 & $6 \mathrm{mo}$ \\
\hline Merom 2016 & 3 & 2 & 278 & 249 & $12 \mathrm{mo}$ \\
\hline Ng 2015 & 0 & 1 & 46 & 47 & $12 \mathrm{mo}$ \\
\hline Robertson 2001a & 1 & 6 & 114 & 104 & $12 \mathrm{mo}$ \\
\hline Sales 2017 & 0 & 1 & 31 & 22 & $12 \mathrm{mo}$ \\
\hline Sherrington 2014c & 10 & 9 & 171 & 169 & $4 \mathrm{mo}$ \\
\hline Siegrist 2016 & 8 & 10 & 222 & 156 & $12 \mathrm{mo}$ \\
\hline Skelton 2005 & 1 & 1 & 44 & 28 & $9 \mathrm{mo}$ \\
\hline Taylor 2012 & $2 / 0$ & 7 & $182 / 174$ & 181 & $17 \mathrm{mo}$ \\
\hline \multicolumn{6}{|c|}{$\begin{array}{l}\text { Tai Chi x } 1 \text { week/ x } 2 \text { week vs low-level exer- } \\
\text { cise }\end{array}$} \\
\hline Trombetti 2011 & 2 & 2 & 57 & 52 & $6 \mathrm{mo}$ \\
\hline Uusi-Rasi 2015 & 0 & 2 & 91 & 97 & $24 \mathrm{mo}$ \\
\hline Vogler $2009 c$ & 1 & 1 & 58 & 58 & $3 \mathrm{mo}$ \\
\hline \multicolumn{6}{|c|}{ seated vs weight-bearing training } \\
\hline Voukelatos 2015 & 4 & 0 & 180 & 189 & $12 \mathrm{mo}$ \\
\hline Wolf 2003 & 2 & 4 & 147 & 141 & $11 \mathrm{mo}$ \\
\hline Yang 2012 & 0 & 1 & 59 & 62 & $6 \mathrm{mo}$ \\
\hline
\end{tabular}

mo: months

NR: not reported.

adata not presented by group; total deaths $=15$.

bdata not presented by group; total deaths $=3$.

cPost-hospital discharge trials. 


\section{Appendix 13. Adverse events. Studies that reported on adverse events}

\begin{tabular}{|c|c|c|c|c|c|}
\hline \multirow[t]{2}{*}{ Study IDa } & \multirow{2}{*}{$\begin{array}{l}\text { Group in which ad- } \\
\text { verse events were } \\
\text { reported }\end{array}$} & \multicolumn{2}{|c|}{ Adverse events reported in intervention group $(s)^{\mathbf{b}}$} & \multicolumn{2}{|c|}{$\begin{array}{l}\text { Adverse events reported in con- } \\
\text { trol groupb }\end{array}$} \\
\hline & & Adverse events reported & $\begin{array}{l}\text { Number in in- } \\
\text { tervention } \\
\text { group(s) }\end{array}$ & $\begin{array}{l}\text { Adverse } \\
\text { events report- } \\
\text { ed }\end{array}$ & $\begin{array}{l}\text { Number in } \\
\text { control group }\end{array}$ \\
\hline \multicolumn{6}{|c|}{ Gait, balance, and functional training } \\
\hline Almeida 2013 & $\begin{array}{l}\text { Two intervention } \\
\text { groups and control }\end{array}$ & 0,0 & 28 & 0 & 26 \\
\hline Boongrid 2017 & $\begin{array}{l}\text { Intervention and } \\
\text { control }\end{array}$ & Knee pain $(n=2)$ & 218 & $\begin{array}{l}\text { Knee pain }(n= \\
\text { 2) }\end{array}$ & 219 \\
\hline $\begin{array}{l}\text { Clemson } 2012 \\
\text { LiFEC }\end{array}$ & Intervention only & Pelvic stress fracture $(n=1)$ & 105 & - & 106 \\
\hline $\begin{array}{l}\text { El-Khoury } \\
2015\end{array}$ & Intervention only & $\begin{array}{l}\text { Painful wrist }(n=1) \text {, twisted ankle ( } n \\
=1) \text {, bruises }(n=5) \text {, lumbago }(n=1)\end{array}$ & 352 & - & 354 \\
\hline $\begin{array}{l}\text { Gschwind } \\
2015\end{array}$ & $\begin{array}{l}\text { Intervention and } \\
\text { control }\end{array}$ & 0 & 71 & 0 & 65 \\
\hline $\begin{array}{l}\text { Iliffe } 2015 \\
\text { FaME/OEP } \\
\text { groupsd }\end{array}$ & $\begin{array}{l}\text { Two intervention } \\
\text { groups and control }\end{array}$ & $\begin{array}{l}\text { FaME: } 59 \text { (including 'pulled mus- } \\
\text { cles', exacerbation of back/knee } \\
\text { pain, muscle/joint soreness) } \\
\text { OEP: } 69 \text { (including 'pulled muscles', } \\
\text { venous problems, exacerbation of } \\
\text { back/knee/hip pain and sciatica) }\end{array}$ & $230 / 227$ & $\begin{array}{l}45 \text { (including } \\
\text { exacerbation of } \\
\text { back pain) }\end{array}$ & 252 \\
\hline Iwamoto 2009 & $\begin{array}{l}\text { Intervention and } \\
\text { control }\end{array}$ & 0 & 34 & 0 & 33 \\
\hline $\begin{array}{l}\text { Liu-Ambrose } \\
2004 \\
\text { agility groupc }\end{array}$ & $\begin{array}{l}\text { Two intervention } \\
\text { groups and control }\end{array}$ & $\begin{array}{l}\text { Agility intervention group: Muscu- } \\
\text { loskeletal complaints }(n=3) \text {, short- } \\
\text { ness of breath }(n=4)\end{array}$ & 34 & $\begin{array}{l}\text { Musculoskele- } \\
\text { tal complaint ( } \mathrm{n} \\
=1 \text { ) }\end{array}$ & 32 \\
\hline Nitz 2004 & $\begin{array}{l}\text { Intervention and } \\
\text { control }\end{array}$ & 0 & 24 & 0 & 21 \\
\hline Reinsch 1992 & $\begin{array}{l}\text { Intervention and } \\
\text { control }\end{array}$ & Pain, bruise, minor injury & 129 & $\begin{array}{l}\text { Pain, bruise, } \\
\text { minor injury }\end{array}$ & 101 \\
\hline $\begin{array}{l}\text { Sakamoto } \\
2013\end{array}$ & Intervention only & $\begin{array}{l}\text { Knee pain }(n=2) \text {, lower limb pain ( } \\
=1) \text {, palpitations }(n=1)\end{array}$ & 410 & - & 455 \\
\hline Sales 2017 & Intervention only & $\begin{array}{l}\text { Falls during exercise session, no in- } \\
\text { jury }(n=2)\end{array}$ & 27 & - & 21 \\
\hline Siegrist 2016 & $\begin{array}{l}\text { Intervention and } \\
\text { control }\end{array}$ & 0 & 222 & 0 & 156 \\
\hline
\end{tabular}


(Continued)

\begin{tabular}{lllll} 
Skelton 2005 & $\begin{array}{l}\text { Intervention and } \\
\text { control }\end{array}$ & 0 & 50 & 0 \\
\hline $\begin{array}{l}\text { Trombetti } \\
2011\end{array}$ & $\begin{array}{l}\text { Intervention and } \\
\text { control }\end{array}$ & 0 & 66 & 0
\end{tabular}

\section{Strength/resistance (including power)}

\begin{tabular}{|c|c|c|c|c|c|}
\hline Latham $2003^{f}$ & $\begin{array}{l}\text { Intervention and } \\
\text { control }\end{array}$ & $\begin{array}{l}\text { Back and knee pain directly attrib- } \\
\text { utable to the exercise programme ( } \mathrm{n} \\
=18 \text { ) }\end{array}$ & 112 & $\begin{array}{l}\mathrm{n}=5 \text { (no further } \\
\text { details) }\end{array}$ & 110 \\
\hline $\begin{array}{l}\text { Liu-Ambrose } \\
2004 \text { Resis- } \\
\text { tance groupc }\end{array}$ & $\begin{array}{l}\text { Two intervention } \\
\text { groups and control }\end{array}$ & $\begin{array}{l}\text { Resistance intervention group: Mus- } \\
\text { culoskeletal complaints }(n=10)\end{array}$ & 32 & $\begin{array}{l}\text { Musculoskeltal } \\
\text { complaint }(n= \\
1)^{4}\end{array}$ & 32 \\
\hline $\begin{array}{l}\text { Vogler } 2009 \\
\text { Seated group }\end{array}$ & $\begin{array}{l}\text { Two intervention } \\
\text { groups and control }\end{array}$ & \multicolumn{3}{|c|}{ Musculoskeletal symptoms in all groups: lower back, hip, knee pain } & $\begin{array}{l}\text { All groups } n= \\
171\end{array}$ \\
\hline \multicolumn{6}{|l|}{ 3D (Tai Chi) } \\
\hline Li 2005 & $\begin{array}{l}\text { Intervention and } \\
\text { control }\end{array}$ & 0 & 95 & 0 & 93 \\
\hline Wolf 2003 & $\begin{array}{l}\text { Intervention and } \\
\text { control }\end{array}$ & 0 & 145 & 0 & 141 \\
\hline
\end{tabular}

\section{D (Dance)}

\begin{tabular}{llllll}
\hline Merom 2016 & Intervention only & 0 & 275 & 247 \\
\hline
\end{tabular}

\section{Multiple primary exercise categories}

\begin{tabular}{|c|c|c|c|c|c|}
\hline $\begin{array}{l}\text { Arkkukangas } \\
2015\end{array}$ & Intervention only & 0 & 27 & - & 13 \\
\hline Beyer 2007e & Intervention only & $\begin{array}{l}\text { Mild and transient pain symp- } \\
\text { toms: knee }(n=3) \text {, hip }(n=1) \text {, thigh/ } \\
\text { gluteal/groin/hamstrings }(n=3) \text {, } \\
\text { back }(n=2) \text {, ankle }(n=1)\end{array}$ & 24 & - & 29 \\
\hline Carter 2002 & $\begin{array}{l}\text { Intervention and } \\
\text { control }\end{array}$ & 0 & 40 & $\begin{array}{l}\text { Grade } 1 \text { quadri- } \\
\text { ceps } \\
\text { strain }(n=1)\end{array}$ & 40 \\
\hline $\begin{array}{l}\text { Clemson } 2012 \\
\text { structuredc,e }\end{array}$ & Intervention only & $\begin{array}{l}\text { Groin strain and inguinal hernia } \\
\text { surgery }(n=1)\end{array}$ & 107 & - & 106 \\
\hline Haines 200ge,f & Intervention only & Muscle soreness $(n=1)$ & 19 & - & 34 \\
\hline Hauer $2001 \mathrm{e}$ & $\begin{array}{l}\text { Intervention and } \\
\text { control }\end{array}$ & 0 & 31 & 0 & 25 \\
\hline $\begin{array}{l}\text { Korpelainen } \\
2006\end{array}$ & Intervention only & Musculoskeletal problems $(n=3)$ & 84 & - & 76 \\
\hline
\end{tabular}


(Continued)

\begin{tabular}{|c|c|c|c|c|c|}
\hline Means 2005e & Intervention only & 0 & 144 & - & 94 \\
\hline $\operatorname{Ng} 2015^{e}$ & $\begin{array}{l}\text { Intervention and } \\
\text { control }\end{array}$ & $\begin{array}{l}\text { Joint pain, hip and knee }(n=2) \text {; re- } \\
\text { lieved after adjusting training regi- } \\
\text { men }\end{array}$ & 46 & 0 & 46 \\
\hline $\begin{array}{l}\text { Rubenstein } \\
2000\end{array}$ & $\begin{array}{l}\text { Intervention and } \\
\text { control }\end{array}$ & 0 & 28 & 0 & 31 \\
\hline $\begin{array}{l}\text { Sherrington } \\
2014 \mathrm{e}, \mathrm{f}\end{array}$ & Intervention only & $\begin{array}{l}\text { Finger pain following grip strength } \\
\text { assessment; thigh pain after assess- } \\
\text { ment, low back pain, calf pain, knee } \\
\text { pain, exacerbation of hernia symp- } \\
\text { toms, pre-existing conditions (main- } \\
\text { ly musculoskeletal) limited progres- } \\
\text { sion of exercises }(n=12 \text { ) }\end{array}$ & 169 & - & 171 \\
\hline Uusi-Rasi 2015 & $\begin{array}{l}\text { Intervention and } \\
\text { control }\end{array}$ & $\begin{array}{l}\text { Mild musculoskeletal overuse symp- } \\
\text { toms, pre-existing osteoarthritic } \\
\text { symptoms }(n=25)\end{array}$ & 91 & $\begin{array}{l}\text { Mild mus- } \\
\text { culoskeletal } \\
\text { overuse symp- } \\
\text { toms }(\mathrm{n}=1)\end{array}$ & 95 \\
\hline
\end{tabular}

\begin{tabular}{llll}
\hline $\begin{array}{l}\text { Exercise ver- } \\
\text { sus exercise } \\
\text { only }\end{array}$ & $\begin{array}{l}\text { Group in which ad- } \\
\text { verse events were } \\
\text { reported }\end{array}$ & Adverse events reported in intervention group & $\begin{array}{l}\text { Adverse events reported in in- } \\
\text { tervention group }\end{array}$
\end{tabular}

\begin{tabular}{|c|c|c|c|c|c|}
\hline & & Adverse events reported & $\begin{array}{l}\text { Number in in- } \\
\text { tervention } \\
\text { group }\end{array}$ & $\begin{array}{l}\text { Adverse } \\
\text { events report- } \\
\text { ed }\end{array}$ & $\begin{array}{l}\text { Number in in- } \\
\text { tervention } \\
\text { group }\end{array}$ \\
\hline Ballard 2004 & $\begin{array}{l}\text { One intervention } \\
\text { group }\end{array}$ & 15 weeks ex group: Hip pain $(n=1)$ & 20 & & 19 \\
\hline Barker 2016 & $\begin{array}{l}\text { One intervention } \\
\text { group }\end{array}$ & Pilates group: Hip pain $(n=1)$ & 20 & & 24 \\
\hline Davis 2011 & $\begin{array}{l}\text { Two intervention } \\
\text { groups and control }\end{array}$ & $\begin{array}{l}\text { 1x/week group: Musculoskeletal } \\
\text { complaints }(n=14)\end{array}$ & 52 & $\begin{array}{l}2 \text { a week group: } \\
\text { Musculoskele- } \\
\text { tal complaints } \\
(n=5) \\
\text { Balance and } \\
\text { tone group: } \\
\text { Musculoskele- } \\
\text { tal complaints } \\
(n=4)\end{array}$ & $\begin{array}{l}2 \text { a week } \\
\text { group = 54; } \\
\text { Balance and } \\
\text { tone group = } \\
49\end{array}$ \\
\hline Kemmler 2010 & $\begin{array}{l}\text { Two intervention } \\
\text { groups }\end{array}$ & 0 & 112 & 0 & 115 \\
\hline Kwok 2016 & $\begin{array}{l}\text { Two intervention } \\
\text { groups }\end{array}$ & 0 & 40 & 0 & 40 \\
\hline $\begin{array}{l}\text { Mirelman } \\
2016\end{array}$ & $\begin{array}{l}\text { Two intervention } \\
\text { groups }\end{array}$ & $\begin{array}{l}\text { Treadmill group: Death from nat- } \\
\text { ural causes }(n=1) \text {, myocardial in- } \\
\text { farctions }(n=2) \text {, exacerbated or- } \\
\text { thopaedic-related pain or arthritis } \\
(n=3) \text {, rhabdomyolysis }(n=4) \text {. (All }\end{array}$ & 136 & $\begin{array}{l}\text { Virtual reality } \\
\text { group: Stroke } \\
(n=1) \text {, exac- } \\
\text { erbated or- } \\
\text { thopaedic-re- } \\
\text { lated pain or }\end{array}$ & 146 \\
\hline
\end{tabular}


(Continued)

deemed not to be caused by the in-

terventions) arthritis ( $\mathrm{n}$

$=4)$, herpes

zoster $(n=1)$

(All deemed not

to be caused

by the interven-

tions)

\begin{tabular}{llllll}
\hline $\begin{array}{l}\text { Shigematsu } \\
2008\end{array}$ & $\begin{array}{l}\text { Two intervention } \\
\text { groups }\end{array}$ & 0 & 32 & 0 & 36 \\
\hline Yamada 2010 & $\begin{array}{l}\text { Two intervention } \\
\text { groups }\end{array}$ & Muscle ache and fatigue & 29 & $\begin{array}{l}\text { Muscle ache } \\
\text { and fatigue }\end{array}$ & 29 \\
\hline Yamada 2012 & $\begin{array}{l}\text { Two intervention } \\
\text { groups }\end{array}$ & Muscle ache and fatigue & 73 & $\begin{array}{l}\text { Muscle ache } \\
\text { and fatigue }\end{array}$ & 72 \\
\hline Yamada 2013 & $\begin{array}{l}\text { Two intervention } \\
\text { groups }\end{array}$ & Muscle ache and fatigue & 112 & $\begin{array}{l}\text { Muscle ache } \\
\text { and fatigue }\end{array}$ & 118 \\
\hline
\end{tabular}

aCategorised by primary exercise category.

bAt time point used in falls analysis (if available).

cStudy with two intervention groups plus a control group; intervention groups reported across multiple rows.

dincluded events classified as adverse reactions and possible adverse reactions.

eIndicates the primary interventions include gait, balance, and functional training plus strength/resistance training.

fParticipants recently discharged from hospital.

\section{Appendix 14. Adherence}

\begin{tabular}{|c|c|c|c|c|}
\hline Study IDa & $\begin{array}{l}\text { Adherence was } \\
\text { measured }\end{array}$ & $\begin{array}{l}\text { Adherence data } \\
\text { were reported }\end{array}$ & $\begin{array}{l}\text { Measurement of } \\
\text { adherence }\end{array}$ & Reported adherence results ${ }^{b}$ \\
\hline \multicolumn{5}{|c|}{ Gait, balance, and functional training } \\
\hline Almeida 2013 & No & No & - & - \\
\hline Arantes 2015 & Yes & Yes & $\begin{array}{l}\text { Adherence to } \\
\text { exercise pro- } \\
\text { gramme }\end{array}$ & $\begin{array}{l}\text { Mean (range) number of sessions attended: exer- } \\
\text { cise group }=22.1 \text { ( } 20 \text { to } 24) \text {, control group }=10.8(10 \\
\text { to } 12 \text { ) }\end{array}$ \\
\hline Barnett 2003 & Yes & Yes & Attendance & $33.7 \%$ of participants attended $>30 / 37$ classes \\
\hline Boongrid 2017 & Yes & Yes & $\begin{array}{l}\text { Repetitions, sets, } \\
\text { duration }\end{array}$ & $\begin{array}{l}56.8 \% \text { exercised } \geq 120 \text { minutes a week at } 12 \\
\text { months }\end{array}$ \\
\hline Campbell 1997 & Yes & No & - & - \\
\hline Clegg 2014 & Yes & Yes & Attendance & Mean attendance $=46 \%$ \\
\hline Clemson 2010 & No & No & - & - \\
\hline $\begin{array}{l}\text { Clemson } 2012 \\
\text { LiFE }\end{array}$ & Yes & Yes & $\begin{array}{l}\text { Adherence to } \\
\text { exercise pro- } \\
\text { gramme }\end{array}$ & $76 \%$ still exercising at 6 months \\
\hline
\end{tabular}

Exercise for preventing falls in older people living in the community (Review) 
(Continued)

\begin{tabular}{|c|c|c|c|c|}
\hline Cornillon 2002 & No & No & - & - \\
\hline Dadgari 2016 & No & No & - & - \\
\hline Dangour 2011 & Yes & Yes & Attendance & Adherence: 38\% \\
\hline Day 2002 & Yes & Yes & Attendance & $\begin{array}{l}\text { Mean (SD) number of sessions attended = } 10 \text { (3.8) } \\
\text { of } 15 \text { sessions }\end{array}$ \\
\hline Duque 2013 & Yes & Yes & $\begin{array}{l}\text { Adherence to } \\
\text { exercise pro- } \\
\text { gramme }\end{array}$ & Adherence: 97\% \\
\hline El-Khoury 2015 & Yes & Yes & $\begin{array}{l}\text { Started exercise } \\
\text { programme }\end{array}$ & $\begin{array}{l}\text { Started the programme }=84 \% . \text { Attended }>1 \text { month } \\
=73 \%\end{array}$ \\
\hline Gschwind 2015 & Yes & Yes & $\begin{array}{l}\text { Adherence to } \\
\text { exercise pro- } \\
\text { gramme }\end{array}$ & $\begin{array}{l}\text { Median (IQR): number of times iStoppFalls system } \\
\text { used }=42(57) \text {; duration }=11.7 \text { hours }(22) \text {; number } \\
\text { of times balance exergames were performed }=24 \\
\text { (30); duration }=4.0 \text { hours }(6.9) \text {; number of strength } \\
\text { exercises performed = } 20(31) \text {; duration }=7.9 \text { hours } \\
(13.4)\end{array}$ \\
\hline
\end{tabular}

\begin{tabular}{|c|c|c|c|c|}
\hline Halvarsson 2013 & Yes & Yes & Attendance & $\begin{array}{l}\text { Mean (range) adherence to the training sessions, } \\
\text { intervention group: } 87 \% \text { ( } 71 \% \text { to } 100 \%)\end{array}$ \\
\hline $\begin{array}{l}\text { Halvarsson } 2016 \\
\text { balance }\end{array}$ & Yes & Yes & Attendance & $\begin{array}{l}\text { Mean (range) attendance, intervention group: } 89 \% \\
(66 \% \text { to } 100 \%)\end{array}$ \\
\hline Hamrick 2017 & Yes & Yes & Attendance & Mean attendance at yoga classes: $92 \%$ \\
\hline Hirase 2015 & Yes & Yes & Attendance & $\begin{array}{l}\text { Proportion of classes attended, foam rubber: } \\
95.5 \% \text {; stable surface: } 93.3 \% \text {; control: } 91.2 \%\end{array}$ \\
\hline Iliffe 2015 & Yes & Yes & Attendance & $\begin{array}{l}\text { Proportion attended } \geq 75 \% \text { classes, group ex }+ \text { OEP } \\
\text { group: } 40 \% \text {. Attained } \geq 75 \% \text { home exercise pre- } \\
\text { scription of } 90 \text { minutes/week, OEP: } 37 \%\end{array}$ \\
\hline Iwamoto 2009 & Yes & Yes & Attendance & Attendance at 3-week programme: 100\% \\
\hline $\begin{array}{l}\text { Karinkanta } 2007 \\
\text { balance }\end{array}$ & Yes & Yes & Attendance & Mean attendance: 59\% \\
\hline Kerse 2010 & Yes & Yes & $\begin{array}{l}\text { Adherence to } \\
\text { exercise pro- } \\
\text { gramme }\end{array}$ & $\begin{array}{l}\text { Intervention group: exercised } \geq 2 \text { a week }=55 \% \text { of } \\
\text { participants; walked } \geq 2 \text { a week }=59 \% \text {; exercised } \\
\geq 3 \text { a week }=25 \% \text {; walked } \geq 3 \text { a week }=37 \% \text {; pro- } \\
\text { gramme almost daily = } 20 \% \text {. Control group: com- } \\
\text { pleted all visits }=86 \% \text { of participants }\end{array}$ \\
\hline Kovacs 2013 & Yes & Yes & $\begin{array}{l}\text { Adherence to } \\
\text { exercise pro- } \\
\text { gramme }\end{array}$ & $\begin{array}{l}\text { Mean (range) attendance (/50 sessions): } 80.6 \% \\
(56 \% \text { to } 100 \%)\end{array}$ \\
\hline
\end{tabular}

\begin{tabular}{llll}
\hline $\operatorname{Lin} 2007$ & No & No & - \\
\hline
\end{tabular}




\section{(Continued)}

$\begin{array}{llll}\begin{array}{l}\text { Liu-Ambrose } \\ 2008\end{array} & \text { Yes } & \begin{array}{l}\text { Adherence to } \\ \text { exercise pro- }\end{array} & \begin{array}{l}\text { Intervention group. Completed programme } \geq 1 \text { a } \\ \text { week }=68 \% \text { of participants } ; \geq 2 \text { a week }=57 \% \text { of } \\ \text { gramme }\end{array} \\ & \text { participants; } \geq 3 \text { a week }=25 \% \text { of participants }\end{array}$

\begin{tabular}{lcccc}
\hline $\begin{array}{l}\text { Liu-Ambrose } \\
\begin{array}{l}2004 \text { agility } \\
\text { group }\end{array}\end{array}$ & Yes & Attendance & $\begin{array}{l}\text { Attendance, agility training group: 87.3\%; stretch- } \\
\text { ing group: } 78.8 \%\end{array}$ \\
\hline Lord 1995 & Yes & Yes & Attendance & $\begin{array}{l}\text { Attendance at } \geq 60 \% \text { classes: } 75 \% \text {. For these atten- } \\
\text { dees, mean (range) number of classes attended: } 60 \\
(26-82)\end{array}$
\end{tabular}

\begin{tabular}{lllll}
\hline Lord 2003 & Yes & Yes & Attendance & Mean proportion of sessions attended: $42.3 \%$ \\
\hline Luukinen 2007 & No & No & - & - \\
\hline Madureira 2007 & Yes & Yes & $\begin{array}{l}\text { Adherence to } \\
\text { exercise pro- } \\
\text { gramme }\end{array}$ & $\begin{array}{l}\text { Proportion of participants who attended } 100 \% \text { of } \\
\text { sessions: } 60 \% \text {. Proportion of participants who did } \\
\text { home exercise } \geq 1 \text { a week: } 76.7 \% ; \geq 1 \text { to } 4 \text { a week: } \\
36.7 \% \text {; every day: } 40 \%\end{array}$ \\
\hline McMurdo 1997 & Yes & Yes & Attendance & $\begin{array}{l}\text { Mean (range) proportion of sessions attended: } 76 \% \\
\text { (46\% to 100\%) }\end{array}$ \\
\hline
\end{tabular}

\begin{tabular}{|c|c|c|c|c|}
\hline Miko 2017 & No & No & - & - \\
\hline Morgan 2004 & Yes & Yes & Attendance & $\begin{array}{l}\text { Mean proportion of the } 24 \text { scheduled sessions at- } \\
\text { tended: } 70 \% \text {. Participants who dropped out of the } \\
\text { study completed an average of } 31.7 \% \text { of the exer- } \\
\text { cise sessions compared with } 82.9 \% \text { completed ses- } \\
\text { sion by those who did not drop out }\end{array}$ \\
\hline
\end{tabular}

\begin{tabular}{|c|c|c|c|c|}
\hline Nitz 2004 & No & No & - & - \\
\hline Reinsch 1992 & No & No & - & - \\
\hline Robertson 2001a & Yes & Yes & $\begin{array}{l}\text { Adherence to } \\
\text { exercise pro- } \\
\text { gramme }\end{array}$ & $\begin{array}{l}\text { Performed exercises } \geq 2 x / \text { week }=72 \% \text { of partici- } \\
\text { pants } \geq 3 x / \text { week }=43 \% \text { of participants. Walked } \geq \\
2 x / \text { week }=71 \% \text { of participants }\end{array}$ \\
\hline Sakamoto 2013 & Yes & No & $\begin{array}{l}\text { Adherence to } \\
\text { exercise pro- } \\
\text { gramme }\end{array}$ & No data \\
\hline
\end{tabular}

\begin{tabular}{|c|c|c|c|c|}
\hline Sales 2017 & Yes & Yes & Attendance & Mean adherence: $79.6 \%$ \\
\hline Siegrist 2016 & Yes & Yes & Attendance & $\begin{array}{l}\text { Proportion of participants who attended }>10 \text { ses- } \\
\text { sions: } 82 \% \text {. Proportion of participants who per- } \\
\text { formed home exercise programme } \geq 10 x: 46 \%\end{array}$ \\
\hline
\end{tabular}

\begin{tabular}{llcll}
\hline Smulders 2010 & Yes & Yes & Attendance & $\begin{array}{l}\text { Proportion of sessions completed: } 92.8 \% . \text { Propor- } \\
\text { tion of participants who completed } 100 \% \text { of ses- } \\
\text { sions: } 53.2 \%\end{array}$ \\
\hline Trombetti 2011 & Yes & Yes & Attendance & Mean attendance at exercise programme: $78 \%$ \\
\hline
\end{tabular}




\section{(Continued)}

\begin{tabular}{|c|c|c|c|c|}
\hline $\begin{array}{l}\text { Weerdesteyn } \\
2006\end{array}$ & Yes & Yes & Attendance & Mean attendance at exercise sessions: $87 \%$ \\
\hline $\begin{array}{l}\text { Wolf } 1996 \text { bal- } \\
\text { ance }\end{array}$ & No & No & - & - \\
\hline Yang 2012 & Yes & Yes & $\begin{array}{l}\text { Adherence to } \\
\text { exercise pro- } \\
\text { gramme }\end{array}$ & $\begin{array}{l}\text { Proportion of intervention participants with full ad- } \\
\text { herence: } 44.1 \% \text {; exercised < 2x/week: } 13.6 \%\end{array}$ \\
\hline
\end{tabular}

\section{Strength/resistance (including power)}

\begin{tabular}{|c|c|c|c|c|}
\hline $\begin{array}{l}\text { Ansai } 2015 \text { resis- } \\
\text { tance }\end{array}$ & Yes & Yes & $\begin{array}{l}\text { Adherence to } \\
\text { exercise pro- } \\
\text { gramme }\end{array}$ & $\begin{array}{l}56.5 \% \text { performed } \geq 24 \text { sessions for } 16 \text { weeks }(50 \% \\
\text { intervention) }\end{array}$ \\
\hline
\end{tabular}

\begin{tabular}{|c|c|c|c|c|}
\hline Fiatarone 1997 & No & No & - & - \\
\hline $\begin{array}{l}\text { Grahn Kronhed } \\
2009\end{array}$ & Yes & Yes & Attendance & $\begin{array}{l}\text { Mean attendance at scheduled sessions, exercise } \\
\text { group: } 24 / 30 \text { sessions (median (range) } 25 \text { (13 - 30) }\end{array}$ \\
\hline $\begin{array}{l}\text { Karinkanta } 2007 \\
\text { resistance }\end{array}$ & Yes & Yes & Attendance & Mean attendance: $74 \%$ \\
\hline Latham 2003 & Yes & Yes & $\begin{array}{l}\text { Attendance, ex- } \\
\text { ercise intensity }\end{array}$ & $\begin{array}{l}\text { Mean adherence: } 82 \% \text { of prescribed sessions. Mean } \\
\text { (SD) exercise intensity at the end of training: } 51 \% \\
(13 \%) \text { of } 1 \text { RM; } 25 \% \text { of participants reached the high } \\
\text { intensity desired by the intervention }\end{array}$ \\
\hline $\begin{array}{l}\text { Liu-Ambrose } \\
2004 \text { resistance }\end{array}$ & Yes & Yes & Attendance & $\begin{array}{l}\text { Attendance, agility training group: } 87.3 \% \text {; stretch- } \\
\text { ing group: } 78.8 \%\end{array}$ \\
\hline $\begin{array}{l}\text { Vogler } 2009 \text { seat- } \\
\text { ed group }\end{array}$ & Yes & Yes & Attendance & Proportion of sessions completed: $70 \%$ \\
\hline $\begin{array}{l}\text { Woo } 2007 \text { resis- } \\
\text { tance }\end{array}$ & No & No & - & - \\
\hline
\end{tabular}

\section{D}

\begin{tabular}{llll}
\hline Day 2015 Yes & Yes & $\begin{array}{l}\text { Attendance, } \\
\text { hours }\end{array}$ & $\begin{array}{l}\text { Mean (SD) class attendance (/96 classes offered): } \\
\text { intervention 34.4 (SD 26.9); control 41.3 (26.1). } \\
\text { Mean intervention dose }=25.8 \text { hours }\end{array}$ \\
\hline
\end{tabular}

\begin{tabular}{lllll}
\hline Huang 2010 & No & No & - & - \\
\hline Li 2005 & Yes & Yes & Attendance & - \\
\hline Logghe 2009 & Yes & Yes & Attendance & Attendance at $\geq 80 \%$ of lessons: 47\% \\
\hline Merom 2016 & Yes & Yes & Attendance & $\begin{array}{l}\text { Median (IQR) attendance to sessions was 56\% (IQR } \\
\text { 26\% to 77\%) (approximately 45 sessions) }\end{array}$ \\
\hline Voukelatos 2007 & No & No & - & - \\
\hline Wolf 2003 & Yes & Yes & Attendance & $\begin{array}{l}\text { Mean (SD) attendance in the Tai Chi group: 76\% } \\
\text { (19); control group 81\% (17) }\end{array}$ \\
\hline
\end{tabular}


(Continued)

\begin{tabular}{lccc} 
Wolf 1996Tai Chi & No & No & - \\
\hline $\begin{array}{l}\text { Woo 2007 Tai Chi } \\
\text { No }\end{array}$ & No & - \\
\hline $\begin{array}{l}\text { Wu } 2010 \text { Com-ex } \\
\text { Wu } 2010 \text { Home- }\end{array}$ & No & No & - \\
ex & & No &
\end{tabular}

\begin{tabular}{|c|c|c|c|c|}
\hline Wu 2010 Tel-ex & Yes & Yes & Attendance & $\begin{array}{l}\text { Mean (SD) attendance in Tel-ex group: } 69 \%(27) \text {; } \\
\text { Comm-ex: } 71 \%(27) \text {; Home-ex: } 38 \%(46) \text {. Mean (SD) } \\
\text { total exercise time (hours): Tel-ex: } 30(12) \text {; Comm- } \\
\text { ex: } 31 \text { (12); Home-ex } 17(21)\end{array}$ \\
\hline
\end{tabular}

\section{General physical activity}

\begin{tabular}{|c|c|c|c|c|}
\hline Ebrahim 1997 & No & No & - & - \\
\hline Resnick 2002 & Yes & Yes & $\begin{array}{l}\text { Adherence to } \\
\text { exercise pro- } \\
\text { gramme }\end{array}$ & $\begin{array}{l}\text { 7/10 intervention participants adhered to the rec- } \\
\text { ommended walking programme ( } 20 \text { minutes } 3 \\
\text { a week). } 2 / 10 \text { engaged in a regular walking pro- } \\
\text { gramme but did not meet the recommended dose. } \\
1 \text { did not engage in any exercise. None of the } 7 \text { con- } \\
\text { trol group participants started an exercise pro- } \\
\text { gramme during the course of the study }\end{array}$ \\
\hline
\end{tabular}

\begin{tabular}{lllll}
\hline Voukelatos $2015 \quad$ No & No
\end{tabular}

\section{Exercise versus exercise}

\begin{tabular}{|c|c|c|c|c|}
\hline Ballard 2004 & No & No & - & - \\
\hline Barker 2016 & Yes & Yes & $\begin{array}{l}\text { Adherence to } \\
\text { exercise pro- } \\
\text { gramme }\end{array}$ & Proportion attended over $75 \%$ of the classes: $95 \%$ \\
\hline Davis 2011 & No & No & - & - \\
\hline Helbostad 2004 & No & No & - & - \\
\hline Hwang 2016 & Yes & Yes & Attendance & $\begin{array}{l}\text { Proportion attended }>20 \text { sessions: Tai Chi group } \\
78 \% \text {; lower limb group } 72 \%\end{array}$ \\
\hline Kemmler 2010 & No & No & - & - \\
\hline Kwok 2016 & No & - & - & - \\
\hline Kyrdalen 2014 & Yes & Yes & Attendance & $\begin{array}{l}\text { Mean(SD) attendance, OEP group: } 21.9 \text { (SD } 2.7 \text { ) out } \\
\text { of the possible } 24 \text { exercise sessions; OEP home: } \\
32.8 \text { (2.8) out of the recommended } 36 \text { exercise ses- } \\
\text { sions }\end{array}$ \\
\hline LaStayo 2017 & Yes & Yes & Attendance & $\begin{array}{l}\text { In both groups, all participants completed } \geq \text { requi- } \\
\text { site minimum } 18 / 36 \text { exercise classes and }>90 \% \text { of } \\
\text { participants who }>28 / 36 \text { exercise classes }\end{array}$ \\
\hline Liston 2014 & No & No & - & - \\
\hline
\end{tabular}


(Continued)

\begin{tabular}{|c|c|c|c|c|}
\hline Lurie 2013 & No & No & - & - \\
\hline Mirelman 2016 & No & Yes & - & - \\
\hline Morone 2016 & No & No & - & - \\
\hline Morrison 2018 & Yes & Yes & $\begin{array}{l}\text { Adherence to } \\
\text { exercise pro- } \\
\text { gramme }\end{array}$ & $\begin{array}{l}\text { Proportion who completed the training or all ses- } \\
\text { sions in Wii group: }<50 \%\end{array}$ \\
\hline
\end{tabular}

\begin{tabular}{|c|c|c|c|c|}
\hline Okubo 2016 & Yes & Yes & $\begin{array}{l}\text { Repetitions, sets, } \\
\text { duration }\end{array}$ & $\begin{array}{l}\text { Mean (SD) exercise, balance group: } 1.4 \text { (0.5) sets/ } \\
\text { day, for } 4.6 \text { ( } 2.0) \text { days/week; walking group: } 45.2 \\
\text { (24.5) min/day of walking for } 4.3 \text { (1.7) days week }\end{array}$ \\
\hline Shigematsu 2008 & No & No & - & - \\
\hline Skelton 2005 & Yes & Yes & $\begin{array}{l}\text { Started exercise } \\
\text { programme }\end{array}$ & $\begin{array}{l}\text { Proportion of intervention participants who com- } \\
\text { pleted }>1 \text { intervention session: } 73 \%\end{array}$ \\
\hline
\end{tabular}

\begin{tabular}{|c|c|c|c|c|}
\hline Steadman 2003 & No & No & - & - \\
\hline Taylor 2012 & Yes & Yes & Attendance & $\begin{array}{l}\text { Median (IQR) attendance at exercise programme: } \\
79 \%(49 \% \text { to } 90 \%)\end{array}$ \\
\hline Verrusio 2017 & No & No & - & - \\
\hline Yamada 2010 & Yes & Yes & Attendance & $\begin{array}{l}\text { Median (IQR) adherence: } 100 \% \text { ( } 74 \% \text { to } 100 \%) \text { for } \\
\text { each group }\end{array}$ \\
\hline Yamada 2012 & Yes & Yes & Attendance & $\begin{array}{l}\text { Median (IQR) adherence in complex course group: } \\
96 \%(88 \% \text { to } 100 \%) \text {; simple course group: } 96 \% \\
(88 \% \text { to } 100 \%)\end{array}$ \\
\hline Yamada 2013 & Yes & Yes & Attendance & $\begin{array}{l}\text { Median (IQR) adherence, multitarget stepping pro- } \\
\text { gramme: } 93 \%(83 \% \text { to } 96 \%) \text {; walking programme: } \\
92 \%(83 \% \text { to } 96 \%)\end{array}$ \\
\hline
\end{tabular}

\section{Multiple primary exercise categories}

\begin{tabular}{|c|c|c|c|c|}
\hline $\begin{array}{l}\text { Ansai } 2015 \text { multi- } \\
\text { component }^{\star}\end{array}$ & Yes & Yes & $\begin{array}{l}\text { Adherence to } \\
\text { exercise pro- } \\
\text { gramme }\end{array}$ & $\begin{array}{l}34.7 \% \text { performed } \geq 24 \text { sessions for } 16 \text { weeks ( } 50 \% \\
\text { intervention) }\end{array}$ \\
\hline $\begin{array}{l}\text { Arkkukangas } \\
2015\end{array}$ & Yes & Yes & $\begin{array}{l}\text { Adherence to } \\
\text { exercise pro- } \\
\text { gramme }\end{array}$ & $\begin{array}{l}\text { Adherent }=73 \text {, not adherent }=27 \text {. Definition of ad- } \\
\text { herence unclear }\end{array}$ \\
\hline Beyer $2007 c$ & Yes & Yes & Attendance & $\begin{array}{l}\text { Training compliance was on average } 79 \% \text { (42 - } \\
100 \%)\end{array}$ \\
\hline Brown $2002 c$ & Yes & Yes & Attendance & $\begin{array}{l}\text { Mean attendance } 84.6 \%(22 / 26 \text { sessions }) \text {, range } \\
62 \% \text { to } 100 \%\end{array}$ \\
\hline Buchner 1997 & No & No & - & - \\
\hline Bunout 2005c & Yes & Yes & Attendance & $58 \%$ attended $>50 \%$ of sessions \\
\hline
\end{tabular}


(Continued)

\begin{tabular}{lllll} 
Carter 2002 & Yes & Yes & Attendance & Attendance: $89 \%$ \\
\hline Cerny $1998 \mathrm{c}$ & No & No & - & - \\
\hline $\begin{array}{l}\text { Clemson } 2012 \\
\text { structuredc }\end{array}$ & Yes & Yes & $\begin{array}{l}\text { Adherence to } \\
\text { exercise pro- } \\
\text { gramme }\end{array}$ & $71 \%$ still exercising at 6 months \\
\hline
\end{tabular}

\begin{tabular}{lllll}
\hline Freiberger 2007c & Yes & Yes & Attendance & $\begin{array}{l}\text { Proportion of intervention participants participat- } \\
\text { ing in }>75 \% \text { of sessions: } 77 \%\end{array}$ \\
\hline Gill 2016c & Yes & Yes & Attendance & $\begin{array}{l}\text { Mean attendance at scheduled sessions, physi- } \\
\text { cal activity group: } 68 \% \text {; median (IQR) } 71 \% \text { (50\% to } \\
83 \%)\end{array}$ \\
\end{tabular}

\begin{tabular}{|c|c|c|c|c|}
\hline Haines $2009 \mathrm{c}$ & Yes & Yes & $\begin{array}{l}\text { Adherence to } \\
\text { exercise pro- } \\
\text { gramme }\end{array}$ & $\begin{array}{l}\text { Number of intervention participants who adhered } \\
\text { to exercise in week } 8: \geq 1 \text { a week }=8 / 19 ; \geq 2 \text { a week } \\
=4 / 19\end{array}$ \\
\hline Hauer $2001 \mathrm{c}$ & Yes & Yes & $\begin{array}{l}\text { Adherence to } \\
\text { exercise pro- } \\
\text { gramme }\end{array}$ & $\begin{array}{l}\text { Mean adherence, intervention group: } 85.4 \% \text {; con- } \\
\text { trol group: } 84.2 \%\end{array}$ \\
\hline Irez 2011c & Yes & Yes & Attendance & Proportion of sessions completed: $92 \%$ \\
\hline Kamide 2009* & Yes & Yes & $\begin{array}{l}\text { Adherence to } \\
\text { exercise pro- } \\
\text { gramme }\end{array}$ & $\begin{array}{l}\text { Intervention participants. Completed intervention } \\
\text { > } 3 \text { a week, 19/23 (82.6\%) participants; completed } \\
\text { intervention > } 2 \text { a wk, } 21 / 23(91.3 \%) \text { participants }\end{array}$ \\
\hline $\begin{array}{l}\text { Karinkanta } 2007 \\
\text { resistance and } \\
\text { balance groupsc }\end{array}$ & Yes & Yes & Attendance & Mean attendance: $67 \%$ \\
\hline Kim 2014c & Yes & Yes & $\begin{array}{l}\text { Attendance; ex- } \\
\text { ercise sessions at } \\
\text { home }\end{array}$ & $\begin{array}{l}\text { Intervention group. Mean (range) attendance at } \\
\text { sessions: } 75.3 \%(64 \%-86 \%) \text {; mean frequency of } \\
\text { home exercise programme: } 3.4 \text { a week; mean exer- } \\
\text { cise time: } 24.9 \text { minutes }\end{array}$ \\
\hline $\begin{array}{l}\text { Korpelainen } \\
2006\end{array}$ & Yes & Yes & Attendance & $\begin{array}{l}\text { Intervention group. Mean attendance at ses- } \\
\text { sions: } 75 \% \text {; mean frequency of home exercise pro- } \\
\text { gramme: } 3 \text { a week }\end{array}$ \\
\hline Lehtola 2000 & Yes & Yes & $\begin{array}{l}\text { Adherence to } \\
\text { exercise pro- } \\
\text { gramme }\end{array}$ & $\begin{array}{l}\text { "Active participants": } 52 \text { participants; "Passive par- } \\
\text { ticipants": } 20\end{array}$ \\
\hline
\end{tabular}

\begin{tabular}{lllll}
\hline Means 2005c & No & No & - & - \\
\hline Ng 2015c & Yes & Yes & Attendance & $\begin{array}{l}\text { Mean attendance: physical training 85\%, control } \\
94 \%\end{array}$ \\
\hline Park 2008 & No & No & - & - \\
\hline Rubenstein 2000 & Yes & Yes & Attendance & $\begin{array}{l}\text { Exercise participants attended 84\% of the exercise } \\
\text { sessions }\end{array}$ \\
\hline
\end{tabular}




\begin{tabular}{|c|c|c|c|c|}
\hline $\begin{array}{l}\text { Sherrington } \\
2014 \mathrm{C}\end{array}$ & Yes & Yes & $\begin{array}{l}\text { Reps, sets, dura- } \\
\text { tion }\end{array}$ & $\begin{array}{l}\text { Proportion of prescribed repetitions completed in } \\
\text { 12th month: } 47 \%\end{array}$ \\
\hline Suzuki 2004c & Yes & Yes & Attendance & Mean attendance at exercise classes: $75.3 \%$ \\
\hline Uusi-Rasi 2015c & Yes & Yes & Attendance & $\begin{array}{l}\text { Mean (range) attendance at group training: } 72.8 \% \\
\text { (0\% to } 97.4 \%) \text {; home training sessions: } 66.1 \%(0 \% \\
\text { to } 100 \%)\end{array}$ \\
\hline $\begin{array}{l}\text { Vogler } 2009 \\
\text { Weight-bearing } \\
\text { group }\end{array}$ & Yes & Yes & Attendance & Proportion of sessions completed: $62 \%$ \\
\hline
\end{tabular}

aCategorised by primary exercise category.

bAt time point used in falls analysis (if available).

CIndicates the primary interventions include gait, balance, plus functional training and strength/resistance training.

\section{Appendix 15. Description of excluded studies: reference links}

Reason for exclusion Links to references

\section{Types of participants}

\begin{tabular}{ll}
\hline Not meeting age criteria & $\mathrm{N}=1$ : Pereira 1998 \\
\hline In a single diagnostic group with increased risk of falls & $\mathrm{N}=1$ : Hsu 2017 \\
\hline Not predominantly community-dwelling & $\mathrm{N}=1$ : DeSure 2013 \\
\hline
\end{tabular}

\section{Types of intervention}

Not exercise as a single intervention

N = 15: Alkan 2011; Beling 2009; Clemson 2004b; Fahlström 2017; Gianoudis 2014; Iwamoto 2012; Lee 2013; Leung 2014; Li 2018a; Olsen 2014; Pai 2014; Rossi-Izquierdo 2017; Steinberg 2000; Swanenburg 2007; Ueda 2017

\section{Type of control}

Control did not meet inclusion criteria

\section{Type of outcome}

\begin{tabular}{ll}
\hline Falls not measured & $N=1$ : Hinrichs 2016 \\
\hline Participants with injurious falls excluded & $N=1$ : Morris 2008 \\
\hline
\end{tabular}




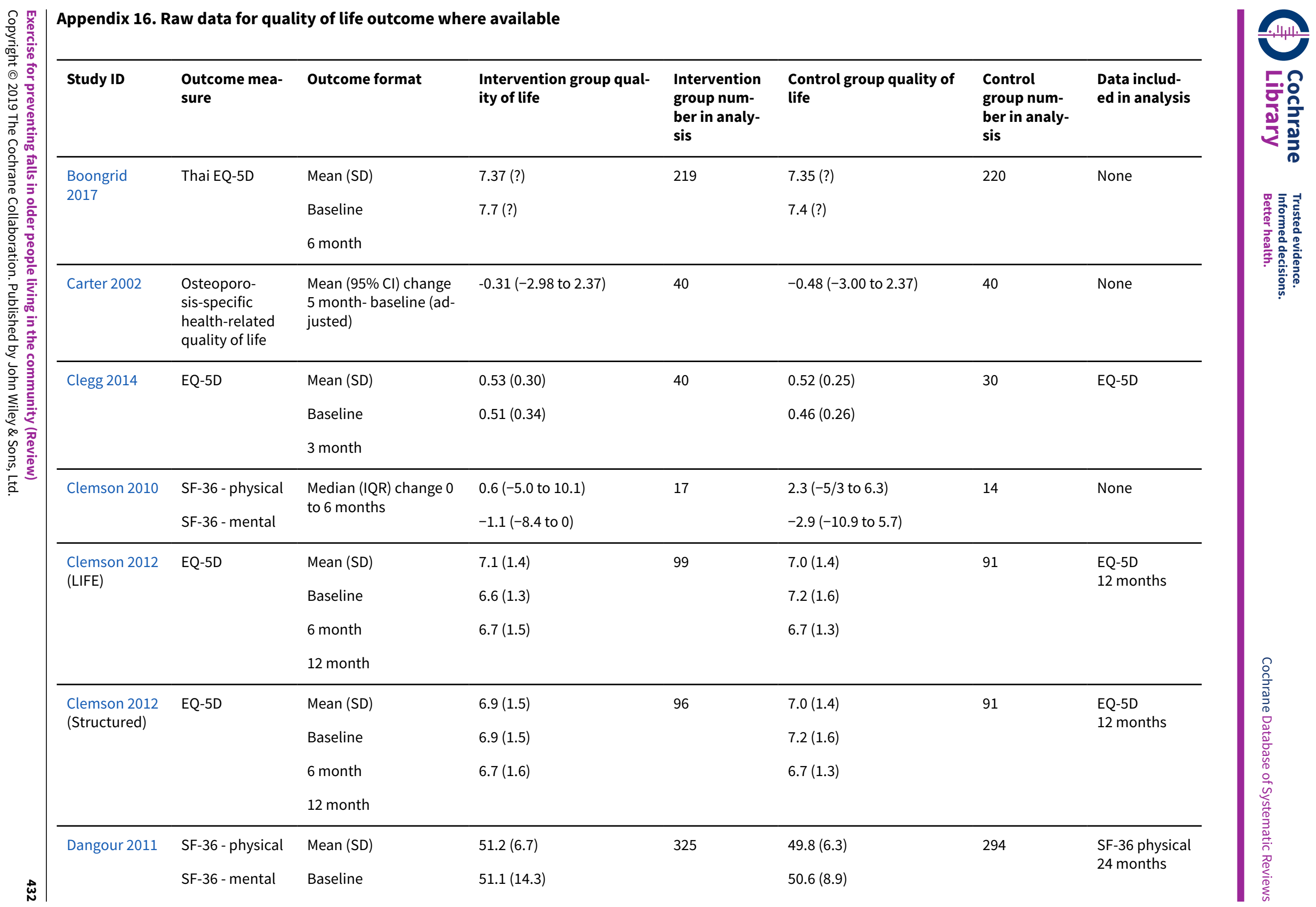




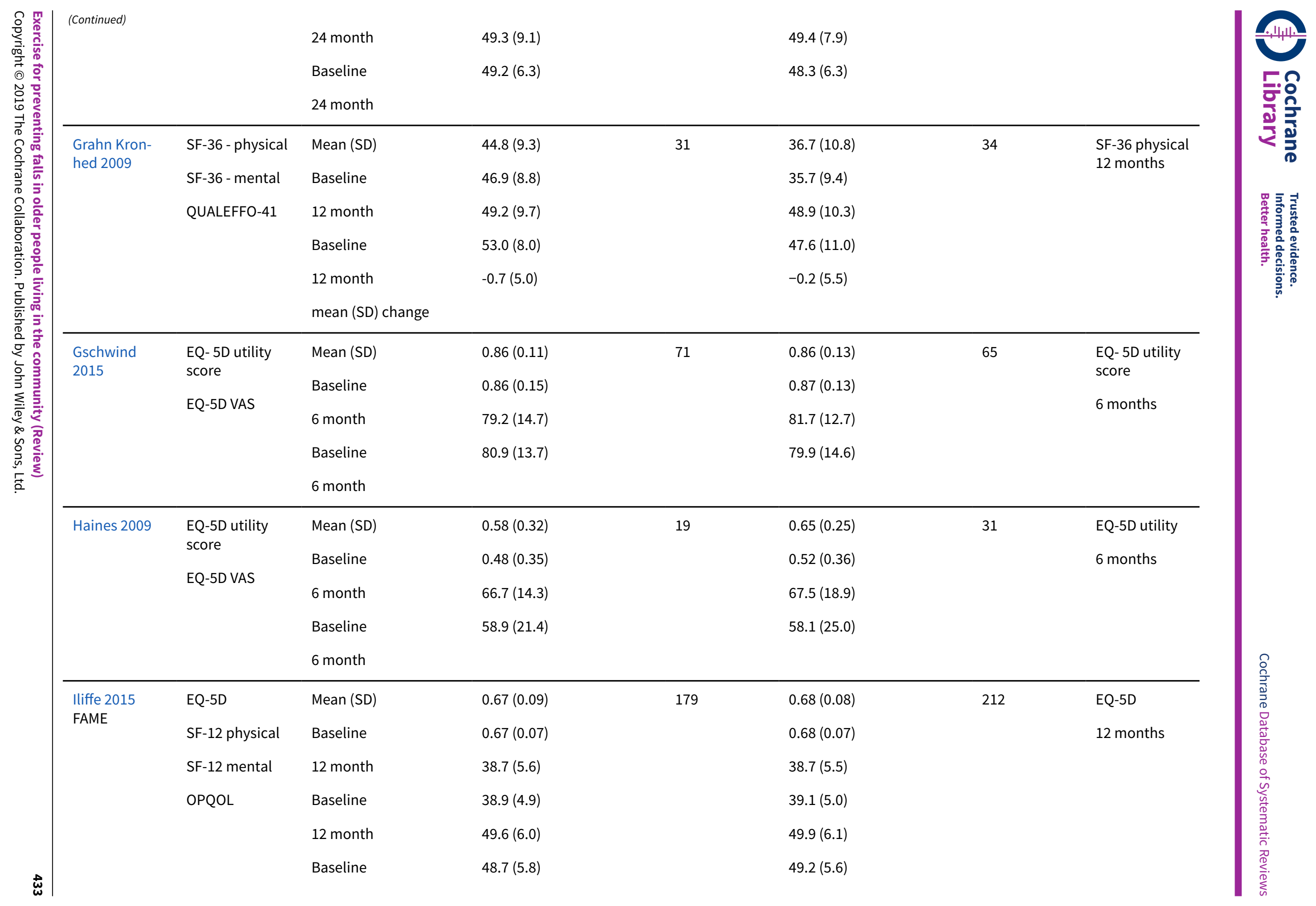




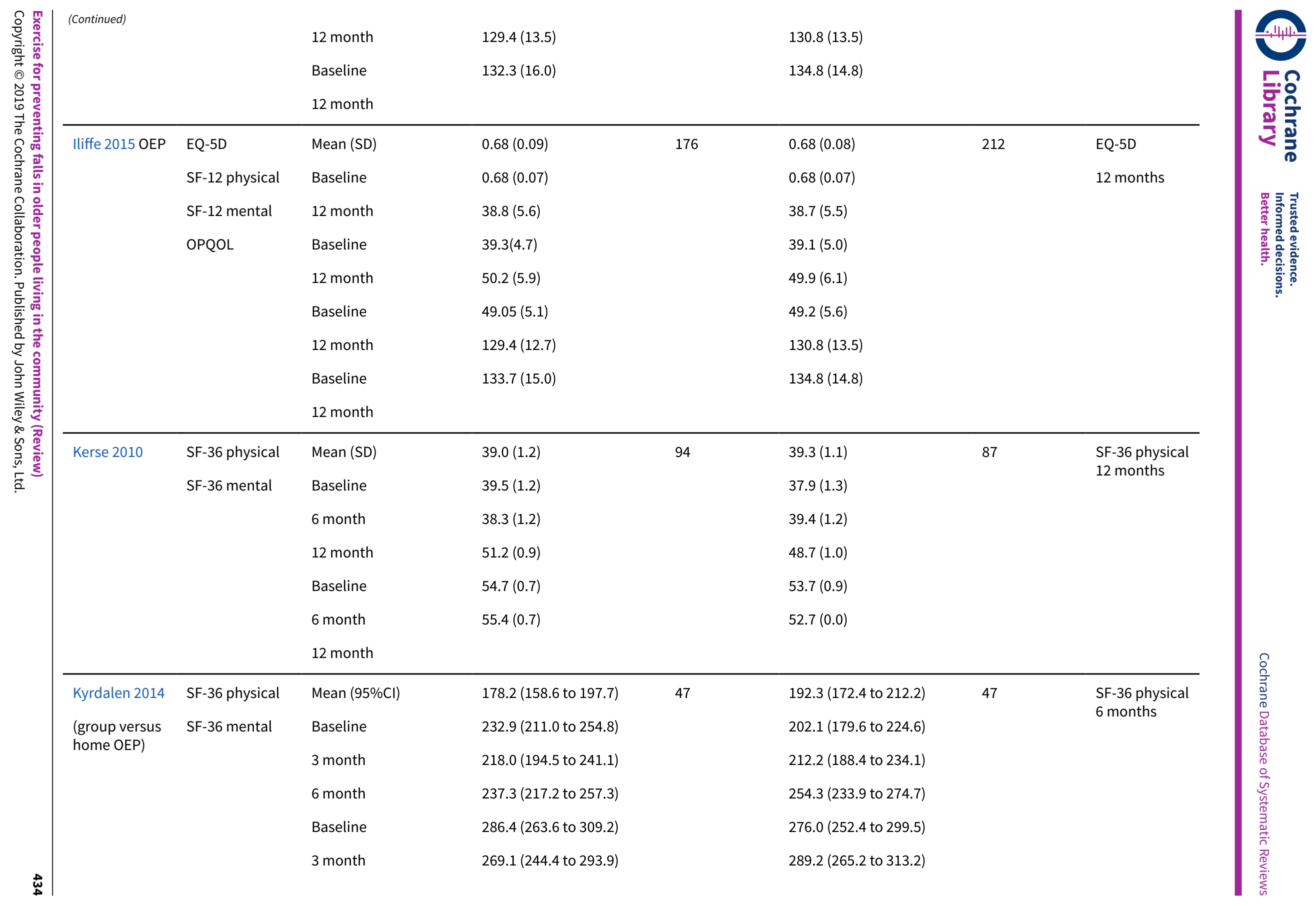




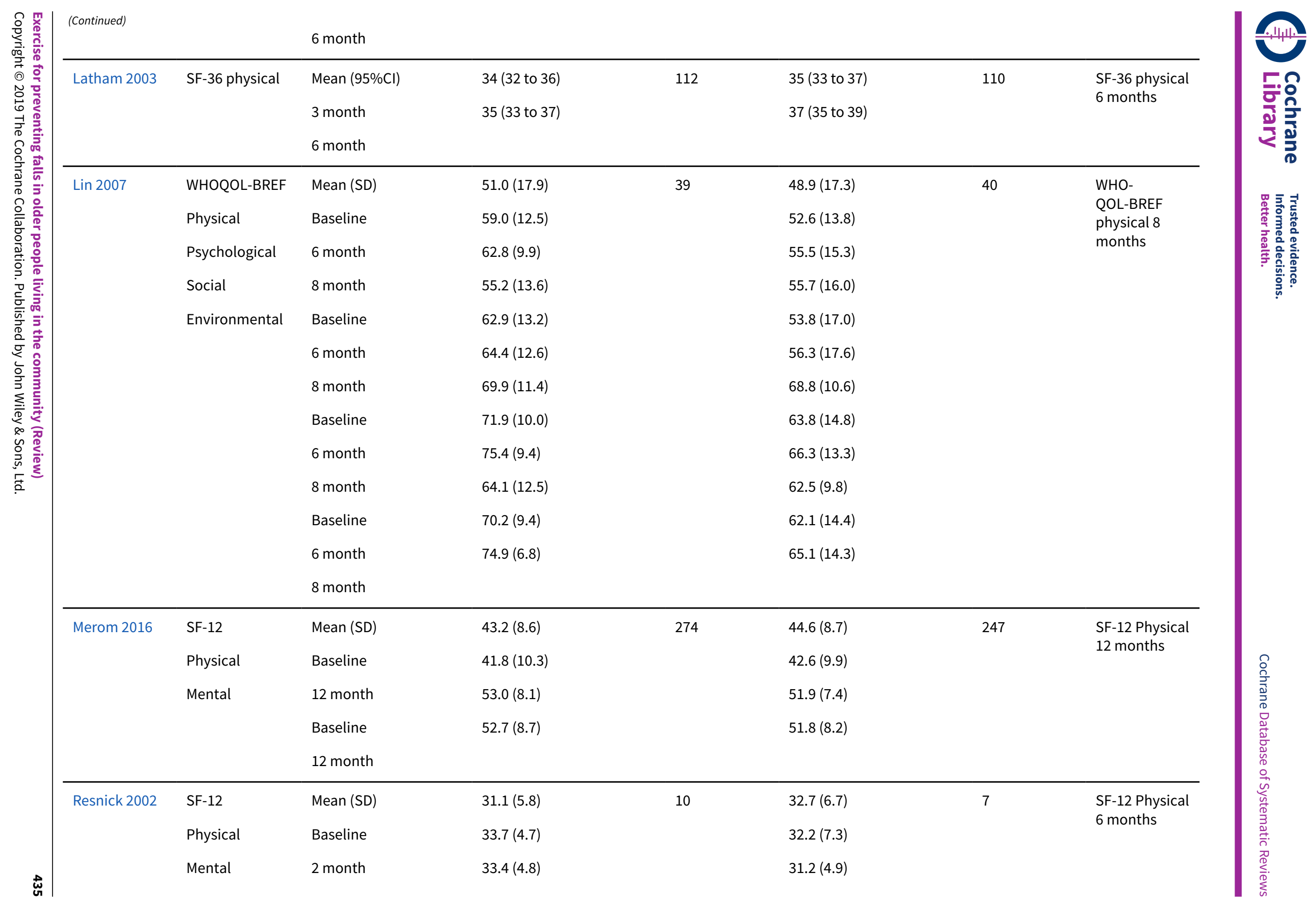









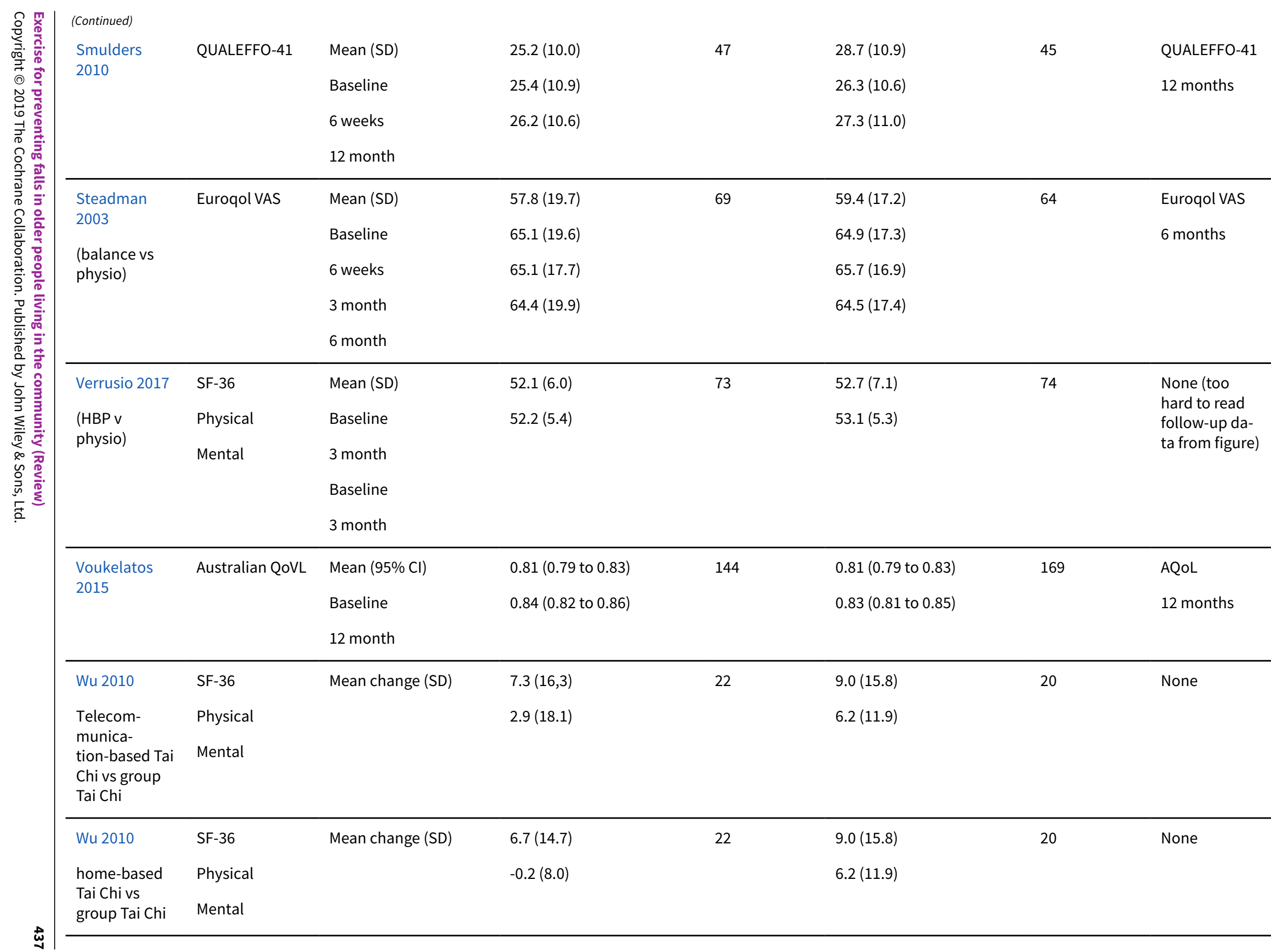




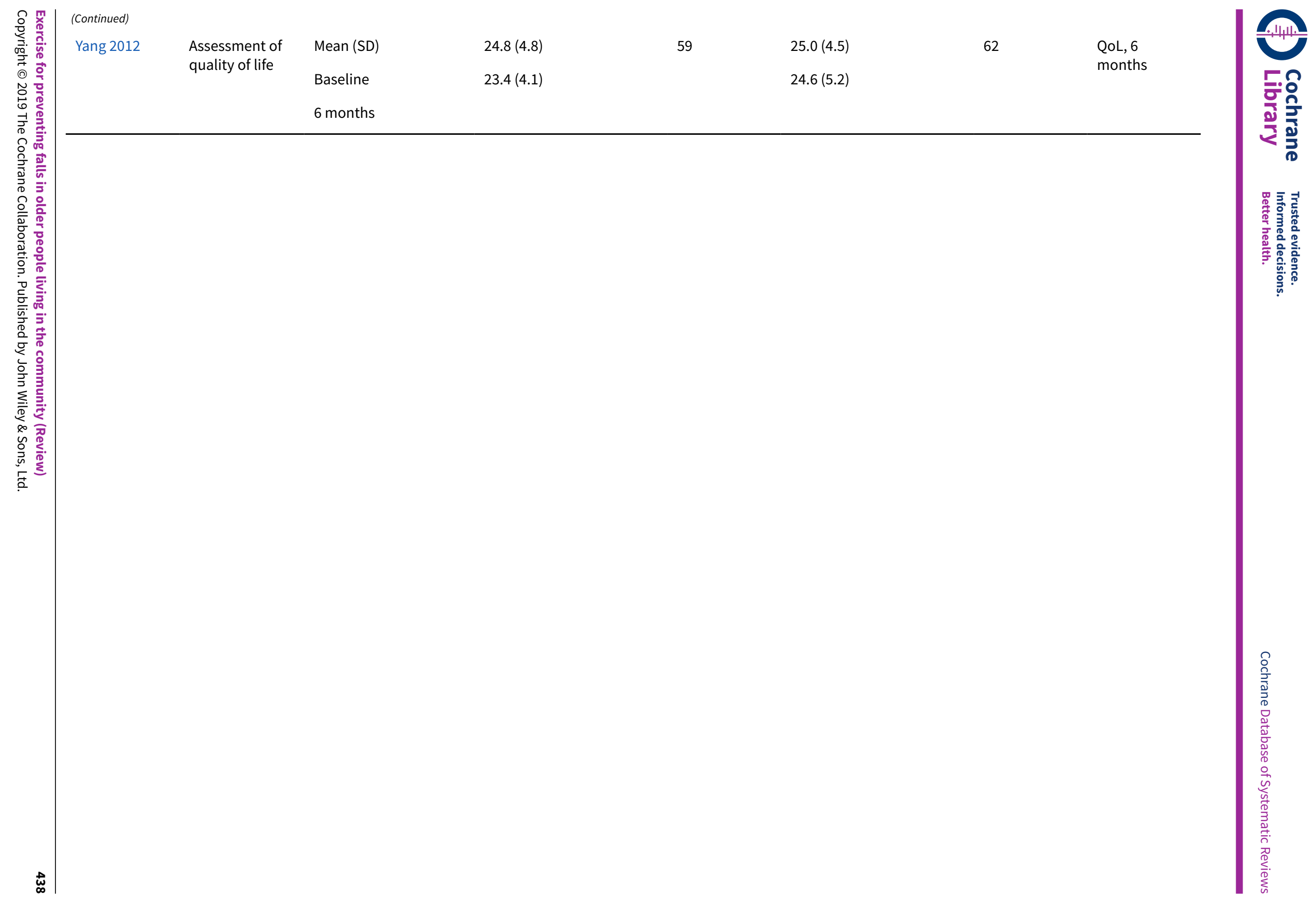


Appendix 17. Studies reporting cost-effectiveness, cost-utility, or costs (intervention and/or healthcare resource use) related to fall outcomes

\begin{tabular}{|c|c|c|c|c|c|c|}
\hline $\begin{array}{l}\text { Study ID } \\
\text { (source if not } \\
\text { primary ref- } \\
\text { erence), sam- } \\
\text { ple, efficacy } \\
\text { analyses, type } \\
\text { of evaluation }\end{array}$ & $\begin{array}{l}\text { Intervention(s) and com- } \\
\text { parator ( } \mathrm{N} \text { in analysis) }\end{array}$ & $\begin{array}{l}\text { Perspec- } \\
\text { tive(s), } \\
\text { type of } \\
\text { currency, } \\
\text { price year, } \\
\text { time hori- } \\
\text { zon }\end{array}$ & $\begin{array}{l}\text { Cost items mea- } \\
\text { sured }\end{array}$ & $\begin{array}{l}\text { Mean (SD) } \\
\text { interven- } \\
\text { tion cost } \\
\text { per person }\end{array}$ & $\begin{array}{l}\text { Health- } \\
\text { care ser- } \\
\text { vice costs }\end{array}$ & $\begin{array}{l}\text { Incremen- } \\
\text { tal cost per } \\
\text { fall prevent- } \\
\text { ed/per QALY } \\
\text { gained }\end{array}$ \\
\hline $\begin{array}{l}\text {-Buchner } 1997 \\
\text {-Patients from } \\
\text { a HMO, mild } \\
\text { deficits in } \\
\text { strength and } \\
\text { balance, mean } \\
\text { age } 75 \text { years } \\
\text {-Analysis } \\
\text {-Cost analysis }\end{array}$ & $\begin{array}{l}\text {-Centre-based endurance } \\
\text { training or strength train- } \\
\text { ing, or both, supervised for } \\
24 \text { to } 26 \text { weeks then self-su- } \\
\text { pervised }(N=75) \text { vs no in- } \\
\text { tervention }(N=30)\end{array}$ & $\begin{array}{l}\text { •HMO } \\
\text { •US dollar } \\
\text { •Not spec- } \\
\text { ified (pre- } \\
\text { sumed } \\
1992 \text { ) } \\
\text { •Period } 7 \text { to } \\
18 \text { months } \\
\text { after ran- } \\
\text { domisation }\end{array}$ & $\begin{array}{l}\text {-Hospital costs, } \\
\text { ancillary outpa- } \\
\text { tient costs (from } \\
\text { HMO computerised } \\
\text { records) }\end{array}$ & - & $\begin{array}{l}\cdot \text { Hospi- } \\
\text { talised con- } \\
\text { trol partici- } \\
\text { pants more } \\
\text { likely to } \\
\text { have hospi- } \\
\text { tal costs> } \\
\text { USD 5000 } \\
\text { (P<0.05) } \\
\text {-Ancillary } \\
\text { outpatient } \\
\text { costs } 7 \text { - } \\
18 \text { months } \\
\text { after ran- } \\
\text { domisa- } \\
\text { tion: } \\
\text { Exercise: } \\
\text { USD } 270 \\
\text { Control: } \\
\text { USD } 285 \\
\text { (no signifi- } \\
\text { cant differ- } \\
\text { ence) }\end{array}$ & - \\
\hline $\begin{array}{l}\cdot \text { Campbell } 1997 \\
\text { and Campbell } \\
1999 \text { (Robert- } \\
\text { son 2001b) } \\
\text {-Women aged } \\
\geq 80 \text { years from } \\
17 \text { general } \\
\text { practices, mean } \\
\text { age (SD) } 84.1 \\
\text { (3.3) years } \\
\text { •Analysis } \\
\text {-Cost-effective- } \\
\text { ness analysis }\end{array}$ & $\begin{array}{l}\text {-Specific set of muscle } \\
\text { strengthening and balance } \\
\text { retraining exercises individ- } \\
\text { ually prescribed at home } \\
\text { (OEP) by physiotherapist, } \\
4 \text { home visits and monthly } \\
\text { phone calls in year } 1 \text {, phone } \\
\text { contact only in year } 2(\mathrm{~N}= \\
116) \text { vs social visits and usu- } \\
\text { al care }(\mathrm{N}=117)\end{array}$ & $\begin{array}{l}\cdot \text { Societal } \\
\cdot \text { New } \\
\begin{array}{l}\text { Zealand } \\
\text { dollar }\end{array} \\
\text {-1995 } \\
\text {-During } \\
\text { participa- } \\
\text { tion in tri- } \\
\text { al (up to } 2 \\
\text { years) }\end{array}$ & $\begin{array}{l}\text {-Intervention costs } \\
\text { (recruitment, pro- } \\
\text { gramme delivery, } \\
\text { overheads) } \\
\text { •Healthcare costs } \\
\text { resulting from falls } \\
\text { (actual costs of hos- } \\
\text { pital admissions } \\
\text { and outpatient ser- } \\
\text { vices, estimates of } \\
\text { GP visits and other } \\
\text { costs) } \\
\text {-Total healthcare } \\
\text { resource use (ac- } \\
\text { tual costs of hospi- } \\
\text { tal admissions and } \\
\text { outpatient services) }\end{array}$ & $\begin{array}{l}\text { In research } \\
\text { setting: } \\
\text { •NZD } 173 \\
(0) \text { in year } 1 \\
\text {-NZD } 22(0) \\
\text { in year } 2\end{array}$ & $\begin{array}{l}\text {-No differ- } \\
\text { ence be- } \\
\text { tween the } \\
2 \text { groups } \\
\text { for health- } \\
\text { care costs } \\
\text { resulting } \\
\text { from falls } \\
\text { or for total } \\
\text { healthcare } \\
\text { costs } \\
\text {-27\% of } \\
\text { hospital } \\
\text { admission } \\
\text { costs dur- } \\
\text { ing trial re- } \\
\text { sulted from } \\
\text { falls }\end{array}$ & $\begin{array}{l}\text { At } 1 \text { year: } \\
\text {-NZD } 314 \text { per } \\
\text { fall prevented } \\
\text { (programme } \\
\text { implementa- } \\
\text { tion costs on- } \\
\text { ly) } \\
\text { At } 2 \text { years: } \\
\text {-NZD } 265 \text { per } \\
\text { fall prevented } \\
\text { (programme } \\
\text { implementa- } \\
\text { tion costs on- } \\
\text { ly) }\end{array}$ \\
\hline
\end{tabular}


(Continued)

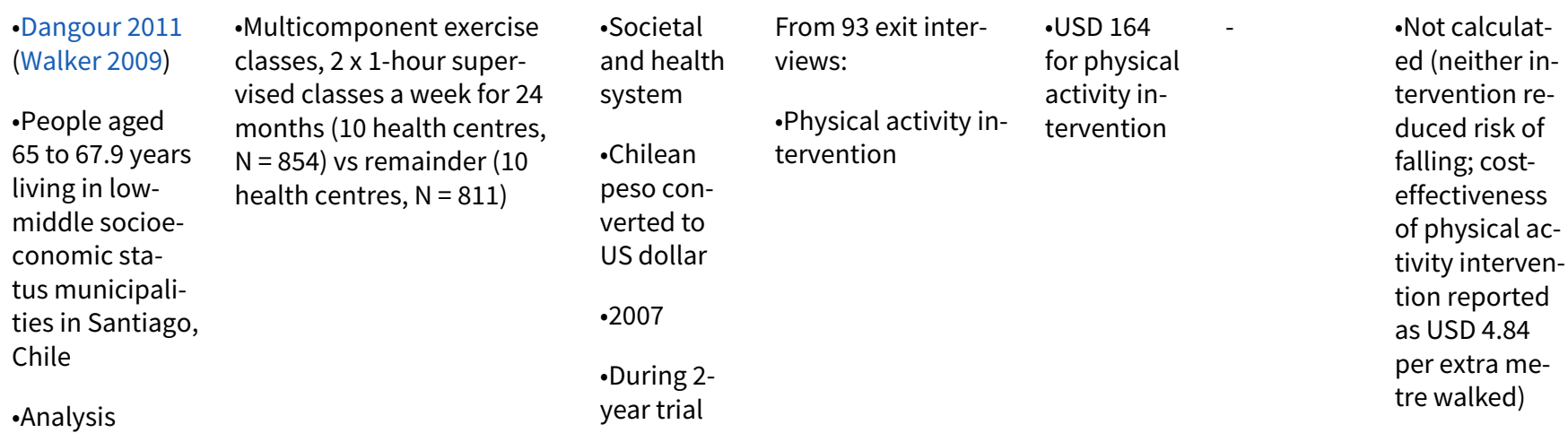

- Cost analysis

\begin{tabular}{|c|c|c|c|c|c|c|}
\hline $\begin{array}{l}\text {-Davis } 2011 \\
\text { (Liu-Ambrose } \\
\text { 2010) } \\
\text { •Commu- } \\
\text { nity-living } \\
\text { women aged } 65 \\
\text { to } 75 \text { years } \\
\text { •Analysis } \\
\text { •Cost-effec- } \\
\text { tiveness analy- } \\
\text { sis, cost-utility } \\
\text { analysis }\end{array}$ & $\begin{array}{l}\text {-Once weekly resistance } \\
\text { training }(\mathrm{N}=54) \text { vs twice- } \\
\text { weekly balance and tone } \\
\text { classes ( } N=49) \\
\text {-Twice-weekly resistance } \\
\text { training }(\mathrm{N}=51) \text { vs twice- } \\
\text { weekly balance and tone } \\
\text { classes ( } N=49)\end{array}$ & $\begin{array}{l}\cdot \text { Health ser- } \\
\text { vice } \\
\text {-Canadian } \\
\text { dollar } \\
\cdot 2008 \\
\cdot 9 \text { months }\end{array}$ & $\begin{array}{l}\text {-Costs of delivering } \\
\text { the interventions } \\
\text { (staff time, room } \\
\text { use, equipment, } \\
\text { building overhead } \\
\text { costs); visits to } \\
\text { health profession- } \\
\text { als; all visits, admis- } \\
\text { sions, and proce- } \\
\text { dures in hospital; } \\
\text { laboratory and di- } \\
\text { agnostic tests }\end{array}$ & $\begin{array}{l}\text { •CAD } 353 \\
\text { once-week- } \\
\text { ly resis- } \\
\text { tance train- } \\
\text { ing } \\
\text { •CAD } 706 \\
\text { twice- } \\
\text { weekly re- } \\
\text { sistance } \\
\text { training } \\
\text { •CAD } 706 \\
\text { twice- } \\
\text { weekly bal- } \\
\text { ance and } \\
\text { tone class- } \\
\text { es }\end{array}$ & $\begin{array}{l}\text { •Mean } \\
\text { health- } \\
\text { care costs } \\
\text { resulting } \\
\text { from falls, } \\
\text { mean to- } \\
\text { tal health- } \\
\text { care costs } \\
\text { respective- } \\
\text { ly: } \\
\text { CAD 547, } \\
\text { CAD 1379 } \\
\text { once-week- } \\
\text { ly resis- } \\
\text { tance train- } \\
\text { ing } \\
\text { •CAD 184, } \\
\text { CAD 1684 } \\
\text { twice- } \\
\text { weekly re- } \\
\text { sistance } \\
\text { training } \\
\text { •CAD } 162 \text {, } \\
\text { CAD 1772 } \\
\text { twice- } \\
\text { weekly bal- } \\
\text { ance and } \\
\text { tone class- } \\
\text { es }\end{array}$ & $\begin{array}{l}\text {-Both once- } \\
\text { and twice- } \\
\text { weekly resis- } \\
\text { tance train- } \\
\text { ing dominated } \\
\text { balance and } \\
\text { tone classes } \\
\text { in terms of } \\
\text { both falls and } \\
\text { QALYs (i.e. less } \\
\text { costly, more } \\
\text { effective) }\end{array}$ \\
\hline $\begin{array}{l}\text {-Day } 2002 \\
\text { (McLean 2015) } \\
\text {-Communi- } \\
\text { ty-dwelling } \\
\text { people identi- } \\
\text { fied from the } \\
\text { electoral roll, } \\
\text { mean age } 76.1 \\
\text { years } \\
\text {-Analysis }\end{array}$ & $\begin{array}{l}\text { Exercise group, } 1 \text {-hour class } \\
\text { a week, } 15 \text { weeks, plus daily } \\
\text { home exercises designed by } \\
\text { physiotherapist }(N=135) \text { vs } \\
\text { no intervention }(N=137)\end{array}$ & $\begin{array}{l}\text { •Healthcare } \\
\text { •Australian } \\
\text { dollar } \\
\text { (costs con- } \\
\text { verted from } \\
\text { Australian } \\
\text { Ddllar to } \\
\text { GBP us- } \\
\text { ing } 2010 \\
\text { purchas- }\end{array}$ & $\begin{array}{l}\text {-Intervention cost } \\
\text { (labour, equipment, } \\
\text { venue hire, music } \\
\text { and consumables) } \\
\text { •Healthcare costs: } \\
\text { (General Practition- } \\
\text { er, ambulance ser- } \\
\text { vices, emergency } \\
\text { department vis- } \\
\text { its, hospital admis- } \\
\text { sions) }\end{array}$ & •AUD 52 & $\begin{array}{l}\text { •AUD } 33 . \\
\text { for exercise } \\
\text { group; } \\
\text { AUD } 39 . \\
\text { for control } \\
\text { group }\end{array}$ & $\begin{array}{l}\text { ICER per: } \\
\text { •Fall prevent- } \\
\text { ed } 652 \\
\text {-Injurious fall } \\
\text { prevented } \\
1176 \\
\text { •Fracture pre- } \\
\text { vented } 26,236\end{array}$ \\
\hline
\end{tabular}


(Continued)

- Cost-effective-

ing-power

ness analysis

parity)

-QALY 51,483

Cost-utility

$\cdot 2010$

analysis

-18 months

- Iliffe 2014 and

Iliffe 2015

-Communi-

ty-dwelling

people with

mean age 73

years

-Analysis

- Cost-effective-

ness analysis

Cost-utility

analysis
1. home-based Otago exercise programme (OEP) $(\mathrm{N}=$ 410) 30 minutes, 3 a week, 24 weeks vs Control group: no intervention $(\mathrm{N}=457)$

2. Community centre-based Falls Management Exercise (FaME) group $(\mathrm{N}=387) 1$ hour, weekly + home exercises based on OEP 30 minutes, 2 a week for 24 weeks vs Control group: no intervention $(\mathrm{N}=457)$

\section{OEP vs FaME}

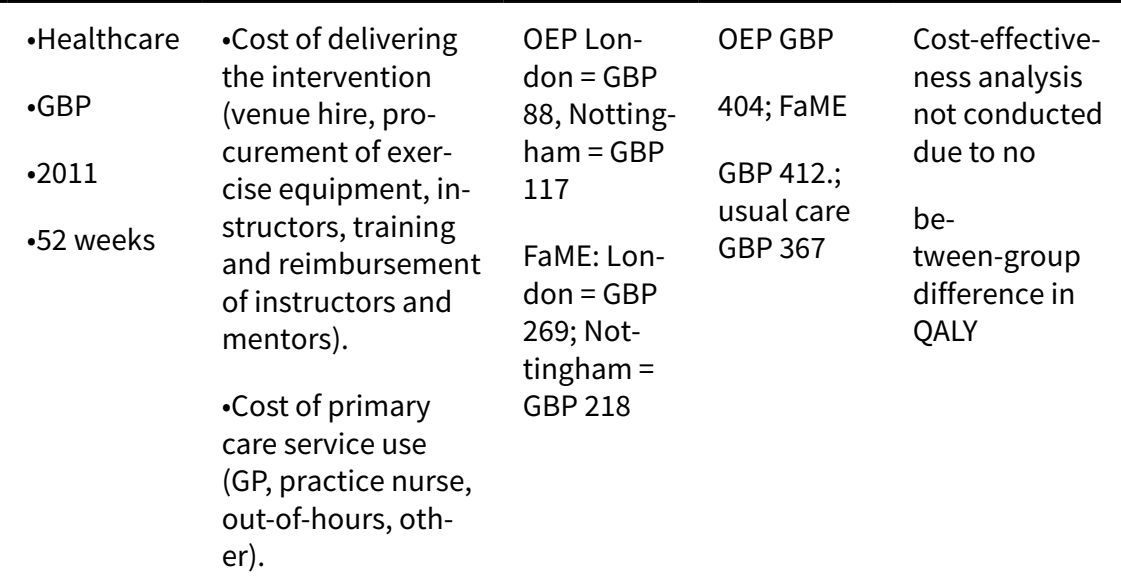

•EUR 2255

(2596) exer-

cise group

and EUR

2780 (3318)

control

group for

mean total

healthcare

costs $(\mathrm{P}=$

0.20 )
-All participants received calcium $(1500 \mathrm{~m} / \mathrm{d})$ and cholecalciferol (500 IU/d) supplements

\begin{tabular}{|c|c|c|c|c|c|c|}
\hline $\begin{array}{l}\cdot \text { Liu-Ambrose } \\
2008 \text { (Davis } \\
2009 \text { ) } \\
\text {-Women and } \\
\text { men aged } \geq 70 \\
\text { years recruit- } \\
\text { ed from } 2 \text { refer- } \\
\text { ral-based falls } \\
\text { clinics } \\
\text {-Analysis }\end{array}$ & $\begin{array}{l}\text {-Specific set of muscle } \\
\text { strengthening and balance } \\
\text { retraining exercises individ- } \\
\text { ually prescribed at home } \\
\text { (OEP) by trained physio- } \\
\text { therapist for } 1 \text { year }(\mathrm{N}=36) \\
\text { vs guideline care }(\mathrm{N}=38) \\
\text {-All participants received } \\
\text { falls risk assessment, com- } \\
\text { prehensive geriatric assess- } \\
\text { ment and treatment }\end{array}$ & $\begin{array}{l}\cdot \text { Health sys- } \\
\text { tem } \\
\text {-Canadian } \\
\text { dollar } \\
\text { •Not speci- } \\
\text { fied } \\
\text { •12 months }\end{array}$ & $\begin{array}{l}\text { - Cost of delivering } \\
\text { the intervention } \\
\text {-Cost of the falls } \\
\text { clinic }\end{array}$ & -CAD 14,285 & - & $\begin{array}{l}\text {-CAD } 247 \text { per } \\
\text { fall prevent- } \\
\text { ed (compara- } \\
\text { ble to incre- } \\
\text { mental cost- } \\
\text { effectiveness } \\
\text { ratios in New } \\
\text { Zealand stud- } \\
\text { ies of the Ota- } \\
\text { go Exercise } \\
\text { Program) }\end{array}$ \\
\hline $\begin{array}{l}\text {-Cost-effective- } \\
\text { ness analysis }\end{array}$ & & & & & & \\
\hline $\begin{array}{l}\cdot \text { Robertson } \\
2001 a \\
\cdot \text { Men and } \\
\text { women aged } \geq \\
75 \text { years from }\end{array}$ & $\begin{array}{l}\text {-Specific set of mus- } \\
\text { cle-strengthening and bal- } \\
\text { ance-retraining exercises } \\
\text { individually prescribed at } \\
\text { home (OEP) by trained dis- } \\
\text { trict nurse, supervised by }\end{array}$ & $\begin{array}{l}\text { •Health sys- } \\
\text { tem } \\
\text { •New } \\
\text { Zealand } \\
\text { dollar }\end{array}$ & $\begin{array}{l}\text {-Intervention costs } \\
\text { (training, recruit- } \\
\text { ment, programme } \\
\text { delivery, super- } \\
\text { vision of exercise }\end{array}$ & $\begin{array}{l}\text { In commu- } \\
\text { nity health } \\
\text { service set- } \\
\text { ting: }\end{array}$ & $\begin{array}{l}\cdot 5 \text { hospital } \\
\text { admissions } \\
\text { due to fall } \\
\text { injuries } \\
\text { in con- } \\
\text { trol group, }\end{array}$ & $\begin{array}{l}\text {-NZD } 1803 \text { per } \\
\text { fall prevented } \\
\text { (programme } \\
\text { implementa- } \\
\text { tion costs on- } \\
\text { ly) }\end{array}$ \\
\hline
\end{tabular}


(Continued)

17 general practices, mean

(SD) age 80.9

(4.2) years

physiotherapist, 5 home vis-

its and monthly phone calls

for 1 year $(N=121)$ vs usual care $(\mathrm{N}=119)$

-Analysis

- Cost-effective-

ness analysis

\section{.1998}

-During

participa-

tion in 1-

year trial instructor, over-
heads)

- Hospital admission costs resulting from fall injuries during trial (actual costs of hospital admissions)

•NZD 432
(0) for 1
year

$\begin{array}{ll}\text { none in } & \text { - NZD 7471 } \\ \text { exercise } & \text { per injurious } \\ \text { group (cost } & \text { fall prevented } \\ \text { savings } & \text { (programme } \\ \text { of NZD } & \text { implementa- } \\ \text { 47,818) } & \text { tion costs on- }\end{array}$ ly)

-NZD 155 per fall prevented (programme implementation costs and hospital admission cost savings)

- NZD 640 per injurious fall prevented (programme implementation costs and hospital admission cost savings)

\begin{tabular}{|c|c|c|c|c|c|c|}
\hline $\begin{array}{l}\text {-Sherrington } \\
2014 \text { (Farag } \\
2015 a) \\
\text { •Communi- } \\
\text { ty-dwelling } \\
\text { people aged } 60 \\
\text { years and over, } \\
\text { discharged } \\
\text { from hospital } \\
\text {-Analysis } \\
\text {-Cost-effective- } \\
\text { ness analysis } \\
\text { Cost-utility } \\
\text { analysis }\end{array}$ & $\begin{array}{l}\text {-Weight-bearing Exercise for } \\
\text { Better Balance (WEBB) pro- } \\
\text { gramme, } 15-20 \text { minutes } \\
\text { up to } 6 \text { times weekly for } 12 \\
\text { months }(N=171) \text { vs usual } \\
\text { care }(N=169)\end{array}$ & $\begin{array}{l}\cdot \text { Health and } \\
\text { community } \\
\text { care funder } \\
\text { perspective } \\
\text { (Australia) } \\
\text {-Australian } \\
\text { Dollar } \\
\text {-2012 } \\
\text {-1 year }\end{array}$ & $\begin{array}{l}\text {-Costs of delivering } \\
\text { the interventions } \\
\text { (travel, staff, equip- } \\
\text { ment, phone calls) } \\
\text {-Cost of health ser- } \\
\text { vice use (respite } \\
\text { care, residential } \\
\text { aged care, hospital } \\
\text { admission, emer- } \\
\text { gency department } \\
\text { presentation, gen- } \\
\text { eral practitioner, } \\
\text { specialist and nurs- } \\
\text { ing services, allied } \\
\text { health, social sup- } \\
\text { port services) }\end{array}$ & $\begin{array}{l}\text { AUD } 751 \text { for } \\
\text { WEBB } \\
\text { AUD } 0 \text { for } \\
\text { usual care }\end{array}$ & $\begin{array}{l}\text { AUD 12,029 } \\
\text { for WEBB } \\
\text { AUD 10,327 } \\
\text { for usual } \\
\text { care }\end{array}$ & $\begin{array}{l}\text { AUD } 77,403 \\
\text { per QALY } \\
\text { gained }\end{array}$ \\
\hline $\begin{array}{l}\text {-Uusi-Rasi } 2015 \\
\text { (Patil 2016) } \\
\text {-Communi- } \\
\text { ty-dwelling } \\
\text { women with } \\
\text { mean age } \cdot 74 \\
\text { years } \\
\text { - Analysis } \\
\text {-Cost-effective- } \\
\text { ness analysis }\end{array}$ & $\begin{array}{l}\text {-No exercise + placebo } \\
\text {-No exercise + vitamin D } 800 \\
\text { IU/day } \\
\text {-Exercise + placebo: super- } \\
\text { vised group training classes } \\
2 \text { a week for first year, and } 1 \\
\text { a week for second year ( } \mathrm{N}= \\
91 \text { ) vs No exercise + placebo } \\
\text { (control) ( } \mathrm{N}=95 \text { ) } \\
\text {-Exercise + vitamin D } 800 \mathrm{IU} / \\
\text { day }\end{array}$ & $\begin{array}{l}\cdot \text { Societal } \\
\cdot \text { Euros (Fin- } \\
\text { land) } \\
\cdot 2011 \\
\cdot 2 \text { years }\end{array}$ & $\begin{array}{l}\text {-Intervention costs } \\
\text { (salaries, adminis- } \\
\text { tration costs) } \\
\text { •Healthcare costs } \\
\text { (fall-related health } \\
\text { care costs for all in- } \\
\text { jurious falls report- } \\
\text { ed during the inter- } \\
\text { vention period) }\end{array}$ & 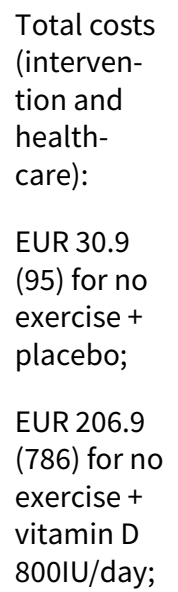 & - & $\begin{array}{l}\text { ICER all inter- } \\
\text { vention (ex- } \\
\text { cluding out- } \\
\text { liers): } \\
\text { EUR } 220.7 \\
(220.7) \text { for } \\
\text { no exercise + } \\
\text { placebo } \\
\text { EUR } 17,600 \\
\text { (exc) for no ex- } \\
\text { ercise + vita- } \\
\text { min D } 800 \text { IU/ } \\
\text { day }\end{array}$ \\
\hline
\end{tabular}




\begin{tabular}{|c|c|c|c|c|c|c|}
\hline & & & & $\begin{array}{l}\text { EUR 73.4 } \\
\text { (104) for } \\
\text { exercise + } \\
\text { placebo; } \\
\text { EUR 188.0 } \\
\text { (454) for } \\
\text { exercise + } \\
\text { vitamin D } \\
800 I U / \text { day }\end{array}$ & & $\begin{array}{l}\text { EUR } 2670 \\
(708.3) \text { for ex- } \\
\text { ercise + place- } \\
\text { bo } \\
\text { EUR } 3820 \\
\text { ( } 3820 \text { ) for ex- } \\
\text { ercise + vita- } \\
\text { min D } 800 \text { IU/ } \\
\text { day }\end{array}$ \\
\hline $\begin{array}{l}\text {-Voukelatos } \\
2007 \text { (Haas } \\
2006) \\
\text { •Healthy com- } \\
\text { munity-living } \\
\text { people aged } \geq \\
60 \text { years, mean } \\
\text { (SD) age } 69 \\
\text { (6.5) years } \\
\text {-Analysis } \\
\text {-Cost-effective- } \\
\text { ness analysis }\end{array}$ & $\begin{array}{l}\text {-Tai Chi classes } 1 \text { hour } \\
\text { weekly for } 16 \text { weeks }(\mathrm{N}= \\
\text { 347) vs no intervention ( } \mathrm{N}= \\
\text { 337) }\end{array}$ & $\begin{array}{l}\text {-Public } \\
\text { health sys- } \\
\text { tem (NSW } \\
\text { Health) } \\
\text {-Australian } \\
\text { dollar } \\
\text { - Not spec- } \\
\text { ified (pre- } \\
\text { sumed } \\
2001 \text { ) } \\
\text {-During 24- } \\
\text { week trial } \\
\text { period }\end{array}$ & $\begin{array}{l}\text {-Intervention costs } \\
\text { (cost of venues, ad- } \\
\text { vertising, instruc- } \\
\text { tors) } \\
\text {-Health service use } \\
\text { related to falls from } \\
\text { healthcare use di- } \\
\text { ary and hospital } \\
\text { records, valued at } \\
\text { standard costs (GP, } \\
\text { specialist, tests, } \\
\text { hospitalisations, } \\
\text { medications) }\end{array}$ & $\begin{array}{l}\text {-AUD } 245 \\
\text { (0) inter- } \\
\text { vention } \\
\text { group plus } \\
\text { charge AUD } \\
44 \text { per par- } \\
\text { ticipant }\end{array}$ & $\begin{array}{l}\cdot \text { Mean total } \\
\text { healthcare } \\
\text { costs high- } \\
\text { er for Tai } \\
\text { Chi group } \\
\text { (AUD 55) } \\
\text { than con- } \\
\text { trol group } \\
\text { (AUD 17) (P } \\
<0.001 \text { ) }\end{array}$ & $\begin{array}{l}\text {-AUD } 1683 \text { per } \\
\text { fall prevent- } \\
\text { ed (includes } \\
\text { cost offset by } \\
\text { charging AUD } \\
44 \text { per instruc- } \\
\text { tion course) }\end{array}$ \\
\hline
\end{tabular}

See also Davis 2010

GP: general practitioner; HMO: health maintenance organisation; OEP: Otago Exercise Program; QALY: quality-adjusted life-year

Appendix 18. Sensitivity analyses: exploring impact on results (rate of falls outcome)

\begin{tabular}{|c|c|}
\hline Sensitivity analysis & $\begin{array}{l}\text { Pooled impact of exercise on fall rate, Rate ra- } \\
\text { tio, } 95 \% \mathrm{Cl}\end{array}$ \\
\hline Primary analysis, all trials, random-effects meta-analysis & $\begin{array}{l}0.77,0.71 \text { to } 0.83 ; \text { participants }=12,981 ; \text { studies } \\
=59\end{array}$ \\
\hline Sensitivity analysis 1 , removing trials that included participants aged $<65$ years & $\begin{array}{l}0.77,0.71 \text { to } 0.84 ; \text { participants }=11,807 ; \text { studies } \\
=53\end{array}$ \\
\hline Sensitivity analysis 2 , removing trials with high risk of bias on any itema & $\begin{array}{l}0.78,0.71 \text { to } 0.87 ; \text { participants }=6757 ; \text { studies }= \\
25\end{array}$ \\
\hline $\begin{array}{l}\text { Sensitivity analysis } 3 \text {, removing trials with unclear or high risk of bias on alloca- } \\
\text { tion concealment }\end{array}$ & $\begin{array}{l}0.85,0.77 \text { to } 0.95 ; \text { participants }=6092 ; \text { studies }= \\
22\end{array}$ \\
\hline $\begin{array}{l}\text { Sensitivity analysis 4, removing trials with unclear or high risk of bias on assessor } \\
\text { blinding (falls outcome) }\end{array}$ & $\begin{array}{l}0.76,0.69 \text { to } 0.85 ; \text { participants }=6996 ; \text { studies }= \\
27\end{array}$ \\
\hline $\begin{array}{l}\text { Sensitivity analysis } 5 \text {, removing trials with unclear or high risk of bias on incom- } \\
\text { plete outcome data }\end{array}$ & $\begin{array}{l}0.77,0.69 \text { to } 0.85 ; \text { participants }=7646 ; \text { studies }= \\
36\end{array}$ \\
\hline
\end{tabular}


(Continued)

Sensitivity analysis 6 , removing cluster-randomised trials

Sensitivity analysis 7, all trials, fixed-effect meta-analysis

$0.76, \mathrm{Cl} 0.70$ to 0.83 ; participants $=10,261$; studies $=53$

$0.82,0.79$ to 0.86 ; participants $=12,981 ;$ studies

$=59$

Sensitivity analysis 8, multiple categories of exercise versus control, excluding trials that do not include balance and strength training

\begin{tabular}{|c|c|}
\hline Primary analysis, subgrouped by exercise type & $\begin{array}{l}0.76, \mathrm{Cl} 0.70 \text { to } 0.81 ; \text { participants }=7920 \text {; studies } \\
=39\end{array}$ \\
\hline \multicolumn{2}{|l|}{ Balance and functional exercises versus control } \\
\hline Multiple categories of exercise versus control & $\begin{array}{l}0.66, \mathrm{Cl} 0.50 \text { to } 0.88 ; \text { participants }=1374 \text {; studies } \\
=11\end{array}$ \\
\hline $\begin{array}{l}\text { Sensitivity analysis } 9 \text { a, classification of interventions based on the Otago Exercise } \\
\text { Program as multiple categories of exercise }\end{array}$ & $\begin{array}{l}0.75,0.68 \text { to } 0.82 ; \text { participants }=5556 ; \text { studies }= \\
30\end{array}$ \\
\hline Balance and functional exercises versus control & $0.72,0.62$ to $0.83 ;$ participants $=3738 ;$ studies $=$ \\
\hline \multicolumn{2}{|l|}{ Multiple categories of exercise versus control } \\
\hline $\begin{array}{l}\text { Sensitivity analysis } 9 b \text {, classification of interventions that included balance and } \\
\text { functional exercises plus strength exercises as multiple categories of exercise }\end{array}$ & $\begin{array}{l}0.72,0.62 \text { to } 0.84 ; \text { participants }=2718 ; \text { studies }= \\
16\end{array}$ \\
\hline Balance and functional exercises versus control & $\begin{array}{l}0.74,0.67 \text { to } 0.81 ; \text { participants }=6721 ; \text { studies }= \\
35\end{array}$ \\
\hline Multiple categories of exercise versus control & \\
\hline
\end{tabular}

aAfter removing trials assessed as high risk of bias in one or more key domains: random sequence generation (selection bias), allocation concealment (selection bias), blinding of outcome assessors (detection bias), and incomplete outcome data (attrition bias).

\section{CONTRIBUTIONS OF AUTHORS}

All authors have contributed to the production of this review.

CS was involved in screening, data extraction, data analysis, co-led the writing of the review and acted as guarantor of the review. $\mathrm{NF}$ was involved in screening, data extraction, data analysis, and co-led the writing of the review.

AT was involved in screening, data extraction, data analysis, and contributed to writing the review.

GW and ZM were involved in screening, data extraction, data analysis, and contributed to writing the review.

$\mathrm{KH}$ was involved in data extraction, data analysis, contributed to writing the review and commented on drafts of the review.

LC, SH and SL contributed to writing the review and commented on drafts of the review.

\section{DECLARATIONSOF INTEREST}

Several authors (CS, AT, SH, KH and SL) are currently running trials of fall prevention interventions; including the following ongoing trials in this review (ACTRN 12615000138583; ACTRN 12615000865516; ISRCTN71002650). These trials are all funded by national grant agencies.

No review author was involved in study selection or processing of any trials in which they were or are involved.

CS is an author of several trials considered in this review, including four included trials (Merom 2016; Sherrington 2014; Vogler 2009; Voukelatos 2015).

NF has no known conflicts of interest.

GW has no known conflicts of interest.

AT has no known conflicts of interest.

ZM has no known conflicts of interest.

$\mathrm{KH}$ is an author of several trials considered in this review, including one included trial (Sherrington 2014).

LC is an author of several trials considered in this review, including two included trials (Clemson 2010; Clemson 2012).

$\mathrm{SH}$ has no known conflicts of interest.

$\mathrm{SL}$ is lead author of the ProFaNE consensus for falls guidance and is an author of one of the trials considered in this review. 


\section{SOURCES OF SUPPORT}

\section{Internal sources}

- School of Public Health, Faculty of Medicine and Health, The University of Sydney, Sydney, Australia.

- Nuffield Department of Orthopaedics, Rheumatology and Musculoskeletal Sciences (NDORMS), University of Oxford, Oxford, UK.

- Faculty of Health Sciences, The University of Sydney, Lidcombe, Australia.

\section{External sources}

- National Institute for Health Research (NIHR) via Cochrane Infrastructure funding to the Cochrane Bone, Joint and Muscle Trauma Group, UK.

- Australian National Health and Medical Research Council fellowships contribute to the salaries of CS and AT, Australia.

- NIHR Cochrane Reviews of NICE Priority scheme, project reference NIHR127512, UK.

\section{DIFFERENCES BETWEEN PROTOCOLANDREVIEW}

\section{Changes and clarifications to protocol \\ Types of participants}

We clarified that we considered studies that focused on people who had been recently discharged from hospital - typically, trial participants would be recruited in hospital prior to discharge - were a distinct category.

\section{Types of interventions}

We clarified that our umbrella comparison was exercise (all types) versus control. We clarified that comparisons of different types, modes of delivery or doses of exercise were secondary comparisons. We redefined comparisons of different intensities of exercise as different doses of exercise to reflect the way dose was reported in the included trials.

We recoded intervention programmes in the included studies rather than using codes from Gillespie 2012, as we considered it more relevant for practice to divide studies on the basis of the primary intervention component rather than the presence of certain components. We examined the descriptions of interventions used in individual trials and categorised the intervention based on the ProFaNE taxonomy (Lamb 2011). We classified exercise programmes on the basis of the primary exercise category and noted the presence of additional, secondary, exercise categories. The exercise categories follow: i) gait, balance, co-ordination and functional task training (referred to as 'balance and functional exercises' for simplicity); ii) strength/resistance training (including power training, using resistance so referred to as 'resistance exercises'); iii) flexibility; iv) three-dimensional (3D) exercise (with Tai Chi or dance subcategories); iv) general physical activity (walking programmes); v) endurance; vi) other kind of exercises. We formed an additional category for exercise programmes that included more than one of the above categories as the primary exercise category. As indicated in our protocol, some forms of yoga were categorised as flexibility exercise and others as 3D exercise, depending on the content of the intervention in the individual trial.

\section{Types of outcomes}

We added two outcomes for consistency with a related review on multifactorial and multiple component interventions (Hopewell 2018): number of people who experienced one or more falls that resulted in hospital admission, and health-related quality of life.

While we collected all reports of adverse events, we stipulated that these needed to be monitored closely in all groups using the same methods over the entire study period to be included in the data analysis.

We clarified that outcomes collected within 18 months of randomisation would be included in the primary analyses. Outcomes collected more than 18 months after randomisation were considered long-term outcomes that would be pooled and reported separately. The 18 months threshold was a pragmatic choice that allowed for some slippage in the 12-month follow-up; these data could actually be collected later on, such as between 13 and 15 months.

\section{Data extraction and management}

In particular, we evaluated whether trials excluded participants with cognitive impairment. This was to aid assessment of the generalisability of the review's results.

We clarified that we recorded and reported data on fracture, hospitalisation, medical attention and health-related quality of life only where it was reported by group. Additionally, we returned to trial authors where data were missing for falls outcomes only.

\section{Risk of bias assessment}

We applied 'risk of bias' assessments for the primary outcome (rate of falls). In addition, we reported blinding of outcome assessment (detection bias) separately for four groups of outcomes (falls; fractures; medical attention, hospital admission and adverse events; and health-related quality of life). 
We have added an assessment of risk of bias specifically for trials using cluster-randomised trials. We assessed the risk of additional bias relating to recruitment, baseline imbalance, loss of clusters, incorrect analysis and comparability with individually-randomised trials, as described in Chapter 16 of the Cochrane Handbook for Systematic Reviews of Interventions (Higgins 2011).

In light of more recent recommendations in the interpretation of funnel plots (Sterne 2011), we did not refer to the examination of funnel plots as purely assessing publication and reporting bias.

\section{Data synthesis - decisions for pooling data}

We decided not to pool the results of studies that recruited people in hospitals and delivered interventions after discharge with the other trials of people living in the community. This was because, on reflection, we considered post-hospital patients to be distinct from general community-dwelling older adults. Thus, while the post-hospital studies are included, we analysed them separately rather than pooling together with the general community-dwelling older adults.

We followed the recommendations in the Cochrane Handbook for Systematic Reviews of Interventions (Higgins 2011), and primarily used random-effects meta-analyses (where meta-analysis was considered appropriate) as we considered it more likely that there was a range of true effects rather than a single effect of exercise on falls. We then undertook sensitivity analyses to assess the impact on the conclusions of the fixed-effect analyses.

\section{Subgroup analysis}

Given the need for caution in conducting subgroup analyses, we set out a criterion that we would only perform a subgroup analysis where there were at least 10 trials in a comparison.

\section{Sensitivity analysis}

In order to aid interpretation of the sensitivity analyses, we decided not to group three risk of bias domains together. Instead, we conducted separate sensitivity analyses for each risk of bias domain to examine the effects of including trials at high or unclear risk of selection, detection and attrition bias.

In order to assist in the interpretation of the results of the type of exercise subgroup 'multiple categories of exercise' comparisons, we undertook additional sensitivity analyses for both falls outcomes which only included trials that were coded as having the two primary components balance/functional exercises and resistance exercises.

\section{GRADE assessment}

We used the updated GRADE assessment criteria, which expressed our judgement of the quality of the evidence in terms of 'certainty' rather than 'quality'.

\section{'Summary of findings' tables}

We clarified our intention to produce a 'Summary of findings' table for our umbrella comparison (exercise (all types) versus control); the outcomes shown included the two new secondary outcomes (hospital admission and health-related quality of life); see next section. We also limited the number of outcomes in the 'Summary of findings' tables for the different primary exercise category versus control comparisons to four outcomes: rate of falls, risk of falling, fall-related fractures and adverse events. This reflected the sparse data for other outcomes and that these are subgroup comparisons.

\section{Changes to protocol in response to a commissioning brief relating to NICE guideline CG161}

To enhance the direct usefulness of the review to decisions relating to the NICE clinical guideline (CG161; NICE 2013), we made the following changes to the protocol in response to a commissioning brief (April 2018).

1. We set the umbrella comparison as 'exercise (all types) versus control'.

2. We added two new secondary outcomes to Types of outcome measures: number of people who experienced one of more falls that resulted in hospital admission, and health-related quality of life. In addition, we recorded and reported mortality data. We reported collecting these data in Data extraction and management.

3. We added in the details of the measures of treatment effect we would use for continuous outcomes in Measures of treatment effect.

4. In view of the different cut-offs used to define the populations of older people of 60 years in this review (Types of participants), and 65 years in CG161, we examined how many trials would have been excluded if the age limit was raised to 65 years and set out a sensitivity analysis to explore the effects of excluding these from the exercise (all types) versus control comparison.

5. We set out a subgroup analysis to compare the effects on falls outcomes in trials with predominantly older populations (based on the proposed threshold of 75 years) and those with predominantly younger populations (Subgroup analysis and investigation of heterogeneity).

6. We set out a subgroup analysis for the fall and fracture outcomes for the pooled (all-exercise types) versus control analyses to compare the effect of exercise on falls and fractures in trials that did and did not use an increased risk of falls as an inclusion criterion (Subgroup analysis and investigation of heterogeneity). 


\section{Peer referee feedback}

In response to peer referee feedback, we extended two subgroup analyses (qualifications of personnel delivering the exercise programmes; group versus individual exercise programmes) to the all types of exercise analyses versus control comparisons for the falls outcomes.

To explore the possible impact of how we classified exercise interventions, we conducted additional sensitivity analyses to examine the effects on both falls outcomes of the:

1. classification of interventions based on the Otago Exercise Program as multiple categories of exercise; and

2. classification of any intervention that included balance and functional exercises plus strength exercises as multiple categories of exercise.

\section{NOTES}

This review provides updated evidence for one of the intervention categories (exercise) covered in the Cochrane Review 'Interventions for preventing falls in older people living in the community' (Gillespie 2012). Some of the wording in several sections of the protocol, such as Background/Description of the condition, was taken from Gillespie 2012. This reflects shared authorship of the two publications, but also attempts to maintain a continuity with the Gillespie 2012 review, as well as links between our review and other proposed reviews that will cover other intervention categories, such as multifactorial and multiple component interventions (Hopewell 2018).

Editorial management and appraisal for this review were conducted by the Cochrane Fast-Track Service (Managing Editor: Helen Wakeford; Associate Editor: Liz Bickerdike; Information Specialist Advisor: Ruth Foxlee) with additional oversight and appraisal by the Cochrane Bone, Joint and Muscle Trauma Group (Managing Editor: Joanne Elliott; Co-ordinating Editor: Helen Handoll). Approval for publication given by Helen Handoll. This review was copy-edited by Kate Cahill and Clare Dooley.

Support to the authors for implementing the requirement by NICE for additional analyses to inform the update of their guideline on Falls in older people was provided by Helen Handoll and Liz Bickerdike, with facilitation by Joanne Elliott and Helen Wakeford. This aspect was under the aegis of Michael Brown, Senior Editor of the Cochrane Acute and Emergency Care Network.

\section{IN DEX TERMS}

\section{Medical Subject Headings (MeSH)}

*Exercise; *Independent Living; Accidental Falls [ ${ }^{*}$ prevention \& control] [statistics \& numerical data]; Dance Therapy [statistics \& numerical data]; Exercise Therapy [ ${ }^{\star}$ statistics \& numerical data]; Fractures, Bone [epidemiology] [prevention \& control]; Gait; Postural Balance; Quality of Life; Randomized Controlled Trials as Topic; Resistance Training [statistics \& numerical data]; Tai Ji [statistics \& numerical data]

\section{MeSH check words}

Aged; Female; Humans; Male; Middle Aged 